\title{
Steady-State Flammable Gas Release Rate Calculation and Lower Flammability Level Evaluation for Hanford Tank Waste
}

T. A. Hu

CH2M HILL Hanford Group Inc.

Richland, WA 99352

U.S. Department of Energy Contract DE-AC27-99RL14047

EDT/ECN: 721735-R9 UC:

Cost Center: 7T600 Charge Code: 502298

B\&R Code: $\quad$ Total Pages: 328

Key Words: hydrogen generation, flammable gas, lower flammability limit, rate equation model, ammonia, liquid/vapor equilibrium

Abstract: Assess the steady-state flammability level at normal and off-normal ventilation conditions. The hydrogen generation rate was calculated for 177 tanks using the rate equation model. Flammability calculations based on hydrogen, ammonia, and methane were performed for 177 tanks for various scenarios.

TRADEMARK DISCLAIMER. Reference herein to any specific commercial product, process, or service by trade name, trademark, manufacturer, or otherwise, does not necessarily constitute or imply its endorsement, recommendation, or favoring by the United States Government or any agency thereof or its contractors or subcontractors.

Printed in the United States of America. To obtain copies of this document, contact: Document Control Services, P.O. Box 950, Mailstop H6-08, Richland WA 99352, Phone (509) 372-2420; Fax (509) 376-4989.
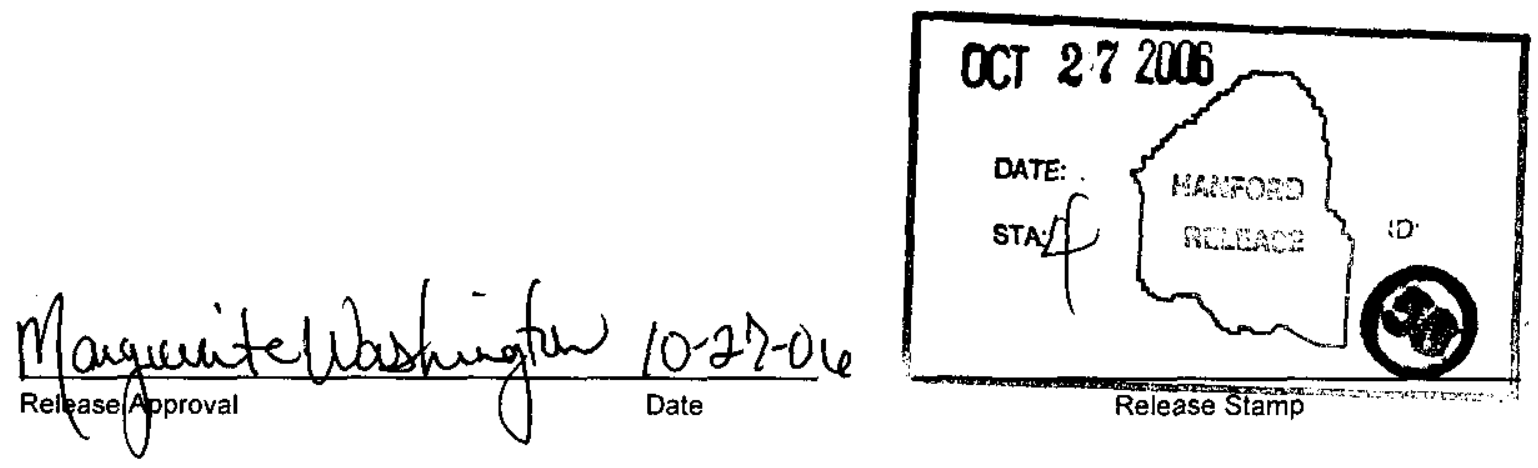
(2) Title

Steady-State Flammable Gas Release Rate Calculation and Lower Flammability Level Evaluation for Hanford Tank Waste

\begin{tabular}{|c|c|c|c|}
\hline \multicolumn{4}{|c|}{ Change Control Record } \\
\hline \multirow{2}{*}{$\begin{array}{c}\text { (3) } \\
\text { Revision }\end{array}$} & \multirow{2}{*}{ (4) Description of Change - Replace, Add, and Delete Pages } & \multicolumn{2}{|c|}{ Authorized for Release } \\
\hline & & (5) Resp. Engr. (print/sign/date) & (6) Resp. Mgr. (print/sign/date) \\
\hline 0 & Original Release via EDT 628264 & T.A. $\mathrm{Hu}$ & $\begin{array}{l}\text { K.M. Hodgson } \\
4 / 27 / 00\end{array}$ \\
\hline 1 & $\begin{array}{l}\text { Total refision to address comments } \\
\text { resulting from DOE-ORP review. (ECN } \\
664031 \text { ) }\end{array}$ & T.A. $\mathrm{Hu}$ & $\begin{array}{l}\text { J.S. Garfield } \\
2 / 23 / 01\end{array}$ \\
\hline 2 & $\begin{array}{l}\text { Total revision to update the hydrogen } \\
\text { rates and flammability to Nov. } 2001 \text { BBI } \\
\text { data. (ECN 665039) }\end{array}$ & T.A. $\mathrm{Hu}$ & $\begin{array}{l}\text { N.W. Kirch } \\
6 / 30 / 02\end{array}$ \\
\hline $2 \mathrm{~A}$ & $\begin{array}{l}\text { Add Appendix I, flammability evaluation } \\
\text { of } 5 \text { DSTs with highest LFL. (ECN 720321) }\end{array}$ & T.A. $\mathrm{Hu}$ & $\begin{array}{l}\text { N.W. Kirch } \\
3 / 20 / 03\end{array}$ \\
\hline 3 & $\begin{array}{l}\text { Total revision to update the hydrogen } \\
\text { rates and flammability to July } 2003 \mathrm{BBI} \\
\text { data. }\end{array}$ & T.A. $\mathrm{Hu}$ & $\begin{array}{l}\text { N.W. Kirch } \\
9 / 30 / 03\end{array}$ \\
\hline $3 \mathrm{~A}$ & $\begin{array}{l}\text { Add Appendix J, flammability evaluation } \\
\text { of vacuum retrieval project of } 241-\mathrm{C} \text { Tank } \\
\text { Farm } 200 \text {-Series. }\end{array}$ & T.A. Hu & $\begin{array}{l}\text { T.M. Horner } \\
11 / 19 / 03\end{array}$ \\
\hline $3 B$ & $\begin{array}{l}\text { Add Appendix } K \text {, Elammability evaluation } \\
\text { to raise DST operation waste level to } 460 \\
\text { inch. }\end{array}$ & T.A. Hu & $\begin{array}{l}\text { T.M. Horner } \\
12 / 23 / 03\end{array}$ \\
\hline $3 \mathrm{C}$ & $\begin{array}{l}\text { Add Appendix L, flammability evaluation } \\
\text { of retrieval project on } 241-C-103 \text { and } \\
241-C-105\end{array}$ & T.A. $\mathrm{Hu}$ & $\begin{array}{l}\text { T.M. Horner } \\
1 / 22 / 04\end{array}$ \\
\hline $3 D$ & $\begin{array}{l}\text { Add Appendix M, Elammability evaluation } \\
\text { of } 241-A Y-102 \text { to support receiving } \\
\text { condensate }\end{array}$ & T.A. Hu & $\begin{array}{l}\text { T.M. Hornex } \\
4 / 20 / 04\end{array}$ \\
\hline $3 \mathrm{E}$ & $\begin{array}{l}\text { Update Appendix K, (Title, See Rev 3B) } \\
\text { ECN-721735-R1 }\end{array}$ & T.A. Hu & $\begin{array}{l}\text { T.M. Horner } \\
6 / 2 / 04\end{array}$ \\
\hline 4 & $\begin{array}{l}\text { Complete revision of document as annual } \\
\text { update per ECN-721735-R2 }\end{array}$ & T.A. Hu & $\begin{array}{l}\text { T.M. Horner } \\
10 / 27 / 04\end{array}$ \\
\hline $4 \mathrm{~A}$ & $\begin{array}{l}\text { Address ORP comments on Rev } 4 \text { and correct } \\
\text { editorial errors per ECN-721735-R3 }\end{array}$ & T. A. Hu & $\begin{array}{l}\text { T.M. Horner } \\
2 / 17 / 05\end{array}$ \\
\hline $4 \mathrm{~B}$ & $\begin{array}{l}\text { Add Appendix I, flammability evaluation } \\
\text { on raising the waste level to } 460 \text { inch } \\
\text { for DSTs per ECN-721735-R4 }\end{array}$ & T. A. $\mathrm{Hu}$ & $\begin{array}{l}\text { T.M. Horner } \\
4 / 27 / 05\end{array}$ \\
\hline $4 \mathrm{C}$ & $\begin{array}{l}\text { Delete the content of Appendix I per } \\
\text { ECN-721735-R5 }\end{array}$ & T. A. Hu & $\begin{array}{l}\text { T.M. Horner } \\
6 / 15 / 05\end{array}$ \\
\hline $4 \mathrm{D}$ & $\begin{array}{l}\text { Add Appendix I content with the } \\
\text { flammability evaluation on the uncovered } \\
\text { solids for DSTs per ECN-721735-R6 }\end{array}$ & T. A. Hu & $\begin{array}{l}\text { T.M. Horner } \\
7 / 28 / 05\end{array}$ \\
\hline $4 E$ & $\begin{array}{l}\text { Add Appendix J content with the } \\
\text { flammability evaluation on tank 241-C-103 } \\
\text { retrieval per ECN-721735-R7 }\end{array}$ & T. A. Hu & $\begin{array}{l}\text { T.M. Horner } \\
9 / 12 / 05\end{array}$ \\
\hline
\end{tabular}




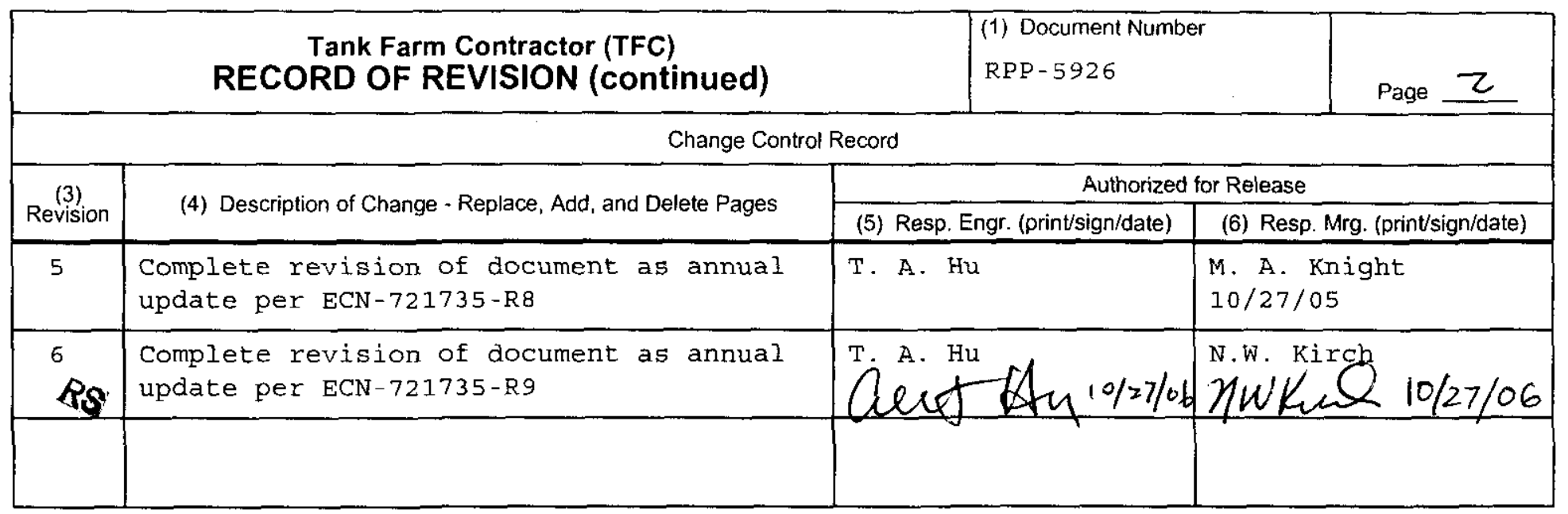


RPP-5926

Revision 6

Steady-State Flammable Gas

Release Rate Calculation and Lower Flammability Level Evaluation for Hanford Tank Waste

T. A. Hu

CH2M HILL Hanford Group, Inc.

Date Published

October 2006

Prepared for the U.S. Department of Energy

Assistant Secretary for Environmental Management

Contractor for the U.S. Department of Energy

Office of River Protection under Contract DE-AC27-99RL14047

\section{GMEAMIULL \\ Hantord Group, the}

P.O. Box 1500

Richland, Washington 


\section{RPP-5926 REV 6}

\section{EXECUTIVE SUMMARY}

Flammable gases such as hydrogen, ammonia, and methane are observed in the tank dome space of the Hanford Site high-level waste tanks. This report assesses the steady-state flammability level under normal and off-normal ventilation conditions in the tank dome space for 177 double-shell tanks and single-shell tanks at the Hanford Site. The steady-state flammability level was estimated from the gas concentration of the mixture in the dome space using estimated gas release rates, Le Chatelier's rule and lower flammability limits of fuels in an air mixture. A time-dependent equation of gas concentration, which is a function of the gas release and ventilation rates in the dome space, has been developed for both soluble and insoluble gases. With this dynamic model, the time required to reach the specified flammability level at a given ventilation condition can be calculated.

In the evaluation, hydrogen generation rates can be calculated for a given tank waste composition and its physical condition (e.g., waste density, waste volume, temperature) using the empirical rate equation model provided in HNF-3851, Empirical Rate Equation Model and Rate Calculations of Hydrogen Generation for Hanford Tank Waste. The release rate of other insoluble gases and the mass transport properties of the soluble gas can be derived from the observed steady-state gas concentration under normal ventilation conditions. The off-normal ventilation rate is assumed to be natural barometric breathing only. A large body of data is required to do both the hydrogen generation rate calculation and the flammability level evaluation. For tank waste that does not have sample data, a statistical-based value from probability distribution regression was used based on data from tanks having similar waste types.

This report (Revision 6) updates the input data of hydrogen generation rate calculations in Revision 5 for 177 tanks using the waste composition information in the Best-Basis Inventory Detail Report 'in the Tank Waste Information Network System (data effective as of

April 1, 2006), and the waste temperature data in the Surveillance Analysis Computer System $(\mathrm{SACS})^{2}$ for the time period from April 1, 2005 through April 1, 2006. However, the release rate of methane, ammonia, and nitrous oxide is based on the input data (dated October 1999) as stated in Revision 0 of this report. In the flammability evaluations with zero ventilation, an increase of $5{ }^{\circ} \mathrm{C}$ of the waste temperatures for all double-shell tanks, and water addition of $10 \mathrm{kgal}$ and $1 \mathrm{kgal}$ for 100- and 200-series tanks, respectively, are performed to address the issues of routine water additions. Annual maximum waste temperatures are used based on highest temperatures for any one day. Also the gas diffusion effect through the concrete dome space is included in the mass balance equations for single-shell tanks under the zero ventilation condition.

Overall, the flammability assessment indicates that no tank will exceed $25 \%$ of the lower flammability limit under normal ventilation conditions. Off-normal ventilation conditions

\footnotetext{
${ }^{1}$ TWINS, 2006, Tank Waste Information Network System, Internet address: http: twins.pnl.gov/twins.htm, queried September 27, 2006.

2 SACS, 2006, Surveillance Analysis Computer System, queried September 27, 2006, SACSPROD database; also available at hitp:/lwins.pnl.gov/twins. him.
} 
evaluated in this report included shutdown of the active ventilation system coupled with closure of the air inlet for actively ventilated systems and isolation of the high-efficiency particulate air breather filter for passively ventilated tanks. Considering the 10 and $1 \mathrm{kgal}$ water additions and $5{ }^{\circ} \mathrm{C}$ temperature increases for double-shell tanks with the barometric breathing rate, the shortest time to reach $25 \%$ of the lower flammability limit is 10 days for double-shell tanks (i.e., tank 241-AZ-102) and 39 days for single-shell tanks (i.e., tank 241-B-203). Considering 10 and $1 \mathrm{kgal}$ water additions and $5{ }^{\circ} \mathrm{C}$ temperature increases for double-shell tanks under zero ventilation scenario with only a diffusion mechanism for gas releases for single-shell tanks, the shortest time to reach $25 \%$ of the lower flammability limit is 10 days for double-shell tanks (i.e., tank 241-AZ-102) and 35 days for single-shell tanks (i.e., tank 241-B-203). Note that off-normal ventilation conditions rarely occur. 


\section{CONTENTS}

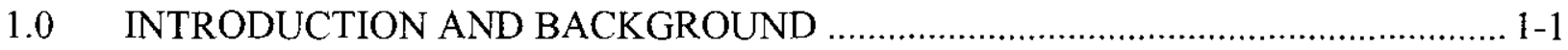

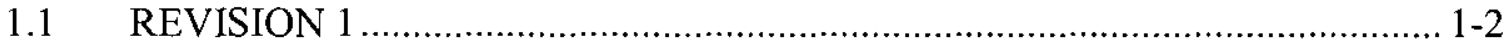

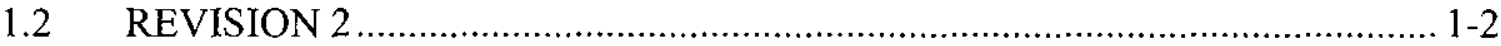

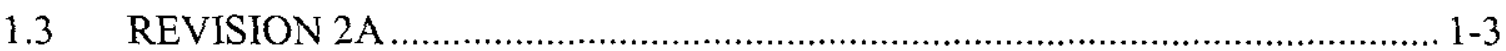

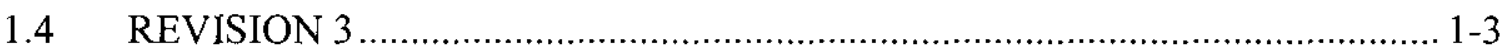

1.5 REVISIONS 3A THROUGH 3E ........................................................ 1-3

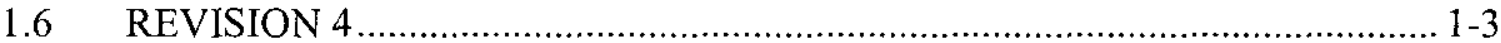

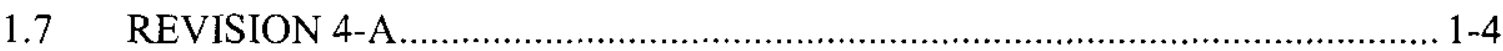

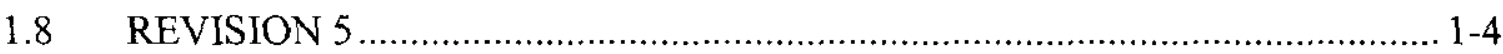

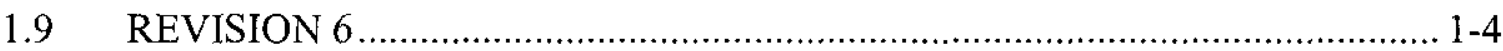

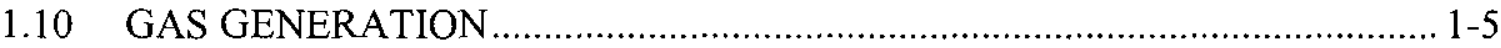

1.11 DOCUMENT ORGANIZATION …….....................................................

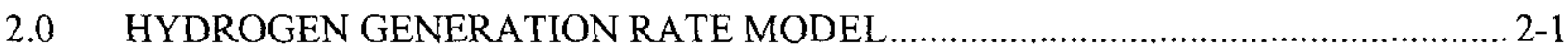

2.1 SEMI-EMPIRICAL RATE EQUATIONS ……......................................... 2-1

3.0 METHODOLOGY TO ESTIMATE STEADY-STATE

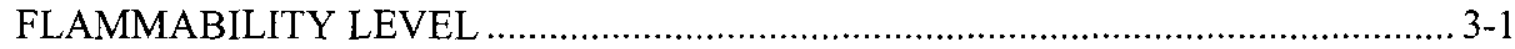

3.1 LOWER FLAMMABILITY LIMITS …….............................................. 3-1

3.2 VENTILATION CONDITIONS IN TANK DOME SPACE............................ 3-3

3.3 TIME-DEPENDENT GAS CONCENTRATION MODEL ……....................... 3-4

3.3.1 Insoluble Gas ................................................................................ $3-5$

3.3.2 Soluble Gas .............................................................................. 3-7

3.4 STEADY-STATE GAS CONCENTRATION AND GAS RELEASE

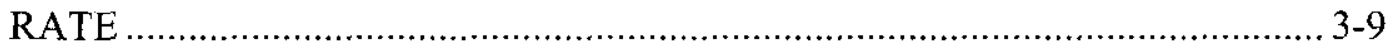

3.5 TIME TO REACH LOWER FLAMMABILITY LIMIT ……........................ 3-12

3.6 THE MINIMUM VENTILATION RATE TO MAINTAIN DOME SPACE BELOW 25 OR 100 PERCENT OF THE LOWER FLAMMABILITY LIMIT ....................................................................... 3-13

3.7 TIME-DEPENDENT GAS CONCENTRATION UNDER ZERO VENTILATION CONDITION ………................................................ 3-14

3.8 TANK WASTE TEMPERATURE INCREASE RATE .................................. 3-15

4.0 EVALUATIONS OF STEADY-STATE FLAMMABILITY LEVEL ......................... 4-1

4.1 HYDROGEN GENERATION RATE MODEL CALCULATIONS .................. 4-1

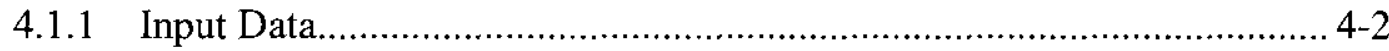

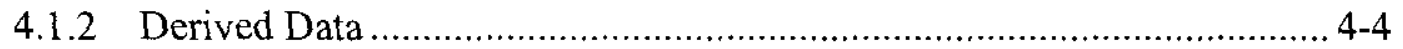

4.1.3 Calculated Hydrogen Generation Rates................................................ 4-5

4.2 TANK VENTILATION RATES AND STEADY-STATE GAS CONCENTRATIONS ............................................................................... 4

4.3 STEADY-STATE GAS RELEASE RATES FOR $\mathrm{H}_{2}, \mathrm{NH}_{3}, \mathrm{CH}_{4}$, AND $\mathrm{N}_{2} \mathrm{O}$

4.4 STEADY-STATE MASS TRANSPORT PROPERTIES "h" AND "A"

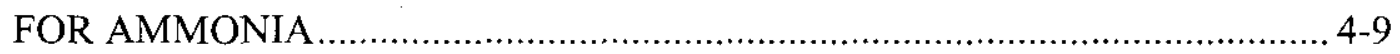




\section{APPENDICES}

A INPUT DATA FOR HYDROGEN GENERATION RATE CALCULATIONS FROM BEST BASIS UBVEBTORY DETAIL CALCULATION REPORT AND DERIVATION DOCUMENT (QUERIED ON SEPTEMBER 27, 2006)

B HYDROGEN GENERATION RATE CALCULATIONS FROM EMPIRICAL RATE EQUATION MODEL FOR 177 HANFORD TANKS

C CALCULATIONS OF THE HENRY'S LAW CONSTANTS AND THE MASS TRANPORT PRODUCTS OF h AND A.

D CALCULATION RESULTS OF STEADY-STATE FLAMMABILITY EVALUATIONS IN TANK DOME SPACES FOR 177 HANFORD TANKS

E EQUATIONS TO CALCULATE THE MASS TRANSPORT COEFFICIENT $\mathrm{h}$ FOR SOLUBLE GAS

F CALCULATIONS OF THE GAS DIFFUSION COEFFICIENTS FOR THE CONCRETE DOME OF SINGLE-SHELL TANKS

G FLAMMABILITY EVALUATION OF TIME TO 25\% OF THE LOWER FLAMMABILITY LIMIT TO SUPPORT THE VACUUM RETRIEVAL PROJECT.

H FLAMMABILITY EVALUATION OF TIME TO REACH $25 \%$ AND 100\% OF THE LOWER FLAMMABILITY LIMIT FOR DOUBLE-SHELL TANK 241-AY-102 AT VARIOUS WASTE LEVELS WHILE RECEIVING EXHAUSTER CONDENSATE COLLECTED FROM CATCH

TANK 241-AZ-151 H-i

I PEER REVIEW CHECKLIST 


\section{LIST OF FIGURES}

Figure 3-1. A Simplified Tank System for Time-Dependent Gas Concentration Model. 3-5

Figure 4-1. Ammonia Concentration and Release Rate for Double-Shell Tank 241-SY-101 Before and After Loss of Active Ventilation.

\section{LIST OF TABLES}

Table 2-1. Comparison of Model-Calculated and Field-Observed Hydrogen Generation Rates

Table 4-1. Calculated Top Ten Hydrogen Generation Rates In Dome Space for 177 Tanks With Current Waste.

Table 4-2. The Calculation of Ammonia Transport Properties

Table 4-3. Time to Reach $25 \%$ and $100 \%$ of the Lower Flammability Limit With Water Addition and $5{ }^{\circ} \mathrm{C}$ Temperature Increase Under Barometric Breathing Condition.

Table 4-4. The Hydrogen Generation Rates, Time to $25 \%$ and $100 \%$ of the Lower Flammability Limit and Minimum Vent Rate With $10 \mathrm{kgal}$ Water Addition and $5{ }^{\circ} \mathrm{C}$ Temperature Increase Under Zero Ventilation for Double-Shell Tanks

Table 4-5. The Hydrogen Generation Rates, Time to $25 \%$ and $100 \%$ of the Lower Flammability Limit and Minimum Vent Rate with Water Addition Under Zero Ventilation (Except Gas Diffusion Mechanism Through The Dome) for Single-Shell Tanks 


\section{RPP-5926 REV 6}

\section{LIST OF TERMS}

BBI

DST

GRE

HEPA

HGR

LFL

SACS

SST

TCD

TOC

TWINS best-basis inventory

double-shell tank

gas release event

high-efficiency particulate air

hydrogen generation rate

lower flammability limit

Surveillance Analysis Computer System

single-shell tank

Tank Characterization Database

total organic carbon

Tank Waste Information Network System 
RPP-5926 REV 6

\subsection{INTRODUCTION AND BACKGROUND}

This report provides the methodology to predict the steady-state gas release rate and to evaluate the steady-state flammability level of the tank dome space under both normal and off-normal ventilation operating conditions for 177 underground nuclear waste storage tanks at the Hanford Site. Revision 0 of this report replaced two previous calculation notes: HNF-SD-WM-CN-116, Calculation Note Hydrogen Generation Rates At Steady State Flammable Gas Concentrations for Single-Shell Tanks, and HNF-SD-WM-CN-117, Calculations of Hydrogen Release Rate at Steady State for Double-Shell Tanks. Revision 0 also provided the technical basis for establishing flammable gas controls. The focus of this report is the loss of ventilation transient such that technical safety requirement surveillance frequencies and action times can be established. This report also provides the following:

- Estimates of the flammable gas release rates in the tank dome space

- Estimates of the time to reach $25 \%$ of the lower flammability limit (LFL) and $100 \%$ of the LFL following the loss of normal ventilation in actively and passively ventilated double-shell tanks (DST) and single-shell tanks (SST)

- Estimates of the minimum ventilation rate required to maintain less than $25 \%$ of the LFL

- Estimates of the maximum dome space concentrations following an extended loss of normal ventilation.

The methodology presented in this report can be used in other applications.

To clarify the application of the subject document, "steady-state" has been defined as the condition of no active operational changes to the tank, and the non-GRE (gas release event) condition with GRE defined as a 500-ppm rise in hydrogen concentration followed by exponential decay of the released flammable gas. For this application the following assumptions were used: (1) long-term variations in ventilation rates brought about by seasonal effects or ventilation rate changes within the exhaust system operating range are minimal and neglected; (2) the variability of the hydrogen concentration about the baseline is less than that produced by a GRE. When the results from only one sample were available, the sample was assumed to have been taken at steady-state condition unless clear documentation shows the existence of a GRE condition.

This report is organized as follows: Chapter 2.0 describes the hydrogen generation rate (HGR) model calculation and summarizes the empirical equation of HGRs. Chapter 3.0 describes the methodology to evaluate the flammability level of the gas mixture in the dome space. Details of the validity of the LFLs in air, ammonia liquid-vapor equilibrium model, time-dependent gas concentration model, and the flammability level calculations are discussed. Chapter 4.0 discusses the input data and results of the gas generation calculations and the flammability evaluations under various ventilation conditions. Chapter 5.0 gives the overall summary and conclusions. Detailed formula, input data, and calculation results for 177 DST and SSTs are 
given in Appendices A through F. In addition, flammable gas evaluations for special cases or some scenarios are given in Appendices $\mathrm{G}$ and $\mathrm{H}$.

\subsection{REVISION 1}

Revision 1 of this report differs from Revision 0 in the following ways:

- The mass balance equation (Equation 3-5) and its derived equations in the methodology of flammability assessment section (Chapter 3.0) were modified by redefining the ventilation rate as the outlet measurement or calculated outlet flow for natural breathing plus gas generation to reflect total gas leaving the tank.

- The input data and calculations for DST 241-SY-101 were updated to reflect the tank waste status after $616 \mathrm{kgal}$ of active flammable-gas-generation waste material were transferred between December 1999 and February 2000, as provided in RPP-6517, Evaluation of Hanford High-Level Waste Tank 241-SY-101. As of April 2001, the HGR in DST 241-SY-101 was $3.1 \mathrm{E}-3 \mathrm{ft}^{3} / \mathrm{min}$, which is one-tenth the generation rate before the remediation task. Other than the data for DST 241-SY-101, all the input data used were from Revision 0, which presents data as of October 1999, unless noted in Revision 1.

- The liquid/vapor equilibrium model was added to address the behavior of the highly soluble gas ammonia.

- The off-normal ventilation calculations used in the flammability assessment were more clearly defined and text was added.

\subsection{REVISION 2}

Revision 2 was issued to cover the following items:

- The U.S. Department of Energy's comments on Revision 1 were resolved. Primarily, the off-normal ventilation conditions were redefined and it was clarified that the off-normal conditions rarely occur. Second, the discussions on the discrepancies of the ammonia mass transport properties between the field data-derived values and the direct calculation of mass transport coefficient, h, and using 100\% surface area were redone. Third, several editorial errors were identified and corrected.

- The HGRs for 177 tanks were updated using the waste composition information in the latest best-basis inventory (BBI) detailed calculation report generated by the Tank Waste Information Network System (TWINS) and temperature data in Surveillance Analysis Computer System (SACS) (dated November 2001) as the input data. In this revision, DST 241-AY-102 has the highest HGR instead of DST 241-AN-102 (Revision 1). This is because the updated input data for DST 241-AY-102 include the waste received from SST 241-C-106, and the tank also shows higher tank waste temperature than previously. 
- The flammability calculations for the 177 tanks were updated to reflect the updated HGRs. For off-normal ventilation condition, the updated flammability calculations were performed at barometric breathing rate for DSTs, and at both barometric breathing and zero ventilation rates for SST. The SSTs was sealed off as much as possible under the interim stabilization project.

- Using BBI data dated November 15, 2001, SST 241-A-101 has the greatest HGR among the SSTs, but the tank was undergoing saltwell pumping, which reduced both the HGR and flammability. The HGR and flammability are recalculated based on the latest waste volume information (February 28, 2002) to reflect the saltwell pumping activity.

- To address routine water addition and other possible waste transfer operations, flammability assessments were performed on several waste addition cases. The resulting information were used to determine the flammable gas control for the ventilation surveillance frequency and the waste compatibility program.

\subsection{REVISION 2A}

Revision 2A made no changes to the text or Appendices A through $\mathrm{H}$. It added a new Appendix I (subsequently changed to Appendix H in Revision 3. The new Appendix I contained flammability evaluations on the sensitivity of waste temperature and water addition under zero ventilation condition for top three flammability-level and aging-waste DSTs.

Appendix I contained a complete set of equations derived for flammability evaluations under zero ventilation condition for both soluble and insoluble gases. A simple and conservative heat transfer model (RPP-6213, Hanford Waste Tank Bump Accident and Consequence Analysis), used in evaluation of the steam bump hazard, was used for tank waste temperature increase analysis in the gas generation rate calculations.

\section{$1.4 \quad$ REVISION 3}

Revision 3 updated the HGR calculations and thus changed the flammability evaluations based on the latest BBI data from July 15, 2003, which resulted in changes to Appendices A, B, D, and $\mathrm{H}$. The text was modified to reflect the changes.

\subsection{REVISIONS 3A THROUGH 3E}

Revision 3A to 3E made no changes to the main text or Appendices A through I of Revision 3. It added a new Appendix $\mathrm{J}$ to $\mathrm{M}$ (Appendices $\mathrm{J}$ and $\mathrm{M}$ were subsequently changed to Appendices $F$ and $\mathrm{G}$ in Revision 4).

\subsection{REVISION 4}

In Revision 4, the HGR equation model has been upgraded and documented (HNF-3851, Empirical Rate Equation Model and Rate Calculations of Hydrogen Generation for Hanford 
Tank Waste) following the Waste Treatment Project studies (24590-WTP-RPT-RT-04-0002) on the applicability of the HGR model on the waste treatment processes. In this revision the methodology to calculate the HGR is also, updated using the latest HGR model. The improvements to the HGR model include the following:

- Development of water radiolysis equation which is more accurate and covers a wider range of tank waste concentrations

- Addition of the radiolysis term from total alpha contribution for both organic and water radiolysis

- Introduction of the hydrogen generation efficiency on the corrosion mechanism to remove conservatism from the model

- Refit of both thermolysis and radiolysis equations because of the changes to the water radiolysis equation and the new gas generation data.

Revision 4 also introduces the diffusion effect through the concrete dome of the SSTs by adding the diffusion term in the mass balance equation. A time-dependent gas concentration equation to include the diffusion mechanism has been developed and applied to the flammable gas evaluation for SSTs. The gas diffusion coefficients for the calculations are taken from RPP-18491, Flammable Gas Diffusion Through Single-Shell Tank Domes.

The HGR calculation was also updated in Revision 4 and the flammability evaluation was changed based on the latest BBI data from September 27, 2004, and the latest temperature data from SACS from April 1, 2003 to April 1, 2004.

\subsection{REVISION 4-A}

Revision 4-A is intended to address comments from the U.S. Department of Energy, Office of River Protection and correct editorial errors.

\section{$1.8 \quad$ REVISION 5}

Revision 5 updated the HGR calculations and thus changed the flammability evaluations based on the latest BBI data queried on September 21,2005, which resulted in changes to Appendices A, B, D, and I. The text was modified to reflect the changes.

\section{$1.9 \quad$ REVISION 6}

Revision 6 updated the HGR calculations and thus changed the flammability evaluations based on the latest BBI data queried on September 27, 2006 (effective as of April 1,2006), and the 
waste temperature data in $\operatorname{SACS}^{3}$ (April 1, 2005 through April 1, 2006). This data update resulted in changes to Appendices $\mathrm{A}, \mathrm{B}$, and $\mathrm{D}$. The text was modified to reflect the changes.

\subsection{GAS GENERATION}

The gases generated in the tank waste are a mixture of hydrogen, nitrogen, ammonia, nitrous oxide, methane, and other hydrocarbons. Hydrogen is the most abundant gas and contributes more than $90 \%$ of the flammable components in the dome space for most of the storage tanks containing gas-generating waste. All of these gases except ammonia have low solubility and are almost fully released to the dome space or partially trapped in the solid layers of waste. Because of its high solubility, $99 \%$ of the ammonia generated is dissolved and stored in the liquid waste (PNNL-1 1450, Composition and Quantities of Retained Gas Measured in Hanford Waste Tanks 241-AW-101, A-101, $A N-105, A N-104$, and $A N-103)$ rather than being released to the dome space. For most of the tanks, little methane is generated, but methane is the major species among the observed hydrocarbon vapors. Nitrous oxide is not flammable by itself, but it is an oxidizer. Studies provided in Flammability and Flame Propagation in $\mathrm{H}_{2}-\mathrm{N}_{2} \mathrm{O}-\mathrm{CH}_{4}-\mathrm{NH}_{3}-\mathrm{O}_{2}-\mathrm{N}_{2}$ Mixtures, Explosion Dynamics Laboratory Report FM97-4 (Pfahl and Shepherd 1997), show that concentrated nitrous oxide can lower the flammability limit of fuels in an air mixture.

Understanding gas generation phenomena and predicting the gas generation and gas release rates are important in controlling the flammable gas hazard and planning daily operations in the tank farms. For example, the overall rate of generation is needed to verify that any given tank has sufficient ventilation to ensure that the level of flammable gases is maintained below the operational flammability limit in its dome space. In the past decade, many studies (HNF-SP-1193, Flammable Gas Project Topical Report) have been conducted on the mechanisms of gas generation, gas release, and gas retention of tank waste using simulated or actual waste. Systematic tank waste characterization programs and various tank safety programs have generated a tremendous amount of tank waste data. This information provided the basis for HNF-3851. The model is tank-waste-constituent- and tank-condition-based and provides a tool to estimate the generation rate for not only the current tank waste contents, but also tanks with contents that have changed. The other observed gases-ammonia, methane, and nitrous oxideare not modeled to have a general rate equation for calculating generation rates as a function of waste constituents.

The report provides the methodology for calculating gas release rates. The HGR equation model (HNF-3851) is used to estimate the hydrogen release rate by subtracting the field-observed gas accumulation rate, if any, from the model-calculated generation rate. The gas release rates for ammonia, methane, and nitrous oxide-the minor gases of interest observed in tank dome spacesare derived from vapor concentration data, ventilation rates in the respective tank dome spaces, and other information about tank waste.

While updating the DST $241-S Y-101$ calculations for the Revision 1 of the report, the dome space steady-state ammonia concentration in DST 241-SY-101 rose from a preremediation

\footnotetext{
${ }^{3}$ SACS, 2006, Surveillance Analysis Computer System, queried September 27, 2006, SACSPROD database; also available at http:/twins.pnl.gov/twins.htm.
} 
concentration of $4 \mathrm{ppm}$ (before the waste was transferred) to $400 \mathrm{ppm}$ (January 14, 2001). Based on the value of $400 \mathrm{ppm}$, the derived ammonia release rate, if used as a constant, will predict a value of $108 \%$ of the LFL as the steady-state ammonia concentration under the off-normal condition of a loss of ventilation. This is a factor of 50 times larger than the equilibrium value predicted by the model. Apparently, treating the ammonia release rate as constant is over-conservative when the ammonia concentration in the dome space is high. Therefore, the liquid-vapor equilibrium model provided in RPP-4941, Methodology for Predicting Flammable Gas Mixtures in Double-Contained Receiver Tanks, is introduced into the methodology to address the dome space calculations for ammonia. A time-dependent equation for gas concentration in a tank dome space was developed by solving the mass balance equations for the gas volume. Once the steady-state gas release rates or the mass transport properties of soluble gas are obtained, a time-dependent gas concentration can be derived from the equation for a given ventilation condition. Therefore, the flammability level of the gas mixture in a dome space can be calculated under both normal and off-normal ventilation conditions. Also, the time required to reach a specified flammability level in a dome space and the minimum ventilation rate required to prevent reaching that level can be estimated.

\subsection{DOCUMENT ORGANIZATION}

Attachment A to TFC-ENG-DESIGN-C-10, Engineering Calculations, provides a list of certain sections to be included in engineering documents. The following notes where the sections in this document correspond to the TFC-ENG-DESIGN-C-10 attachment.

1. Title and Identifier (required) - See title page.

2. Objective/Purpose (required) - See Chapter 1.0.

3. Summary of Results and Conclusions (optional depending on calculation length or complexity) - See Executive Summary.

4. Introduction/Background (optional) - See Chapter 1.0.

5. Input Data (required) - See Chapter 4.0 and Appendix A, B, C, D, F.

6. Assumptions (required if assumptions are made) - See Chapters 2.0 and 3.0.

7. Method of Analysis (required) - Calculation methods are in Chapter 3.0 and spreadsheets in appendices.

8. Use of Computer Software (required if software is used) - The only software used are Excel $^{4}$ spreadsheets.

The cases study uses a validated Excel spreadsheet, documented in Chapters 3.0 and 4.0 , to calculate the HGRs and the time to $25 \%$ and $100 \%$ of the LFL, minimum ventilation

\footnotetext{
${ }^{4}$ Excel is a registered trademark of Microsoft Corporation, Redmond, Washington.
} 
rate. The spreadsheet was verified in compliance with TFC-ENG-CHEM-D-33, Spreadsheet Verification, and is documented in spreadsheet verification form SVF-032, Rev 4.

Spreadsheet owner: T. A. Hu Spreadsheet name: RPP-5926-8050-R4-LFL-CAL-T2-102004.xls Spreadsheet location: U:|FlamGas \RPP-5926 Rev 4\Calculations

This verified spreadsheet was used with the update input data to calculate the gas generation rates, then calculates the flammability and time to LFL for a given ventilation rate.

The input data for HGR calculation is prepared by converting the latest BBI data with the spreadsheet "InputTimeToLFL101504.xls." The spreadsheet was verified in compliance with TFC-ENG-CHEM-D-33 and is documented in spreadsheet verification form SVF-275, Rev 0.

Spreadsheet owner: T. A. Hu

Spreadsheet name: InputTimeToLFL101504.xls

Spreadsheet location: U:IFlamGas\RPP-5926 Rev 4\Calculations

9. Results (required) - See Chapter 4.0 as well as various summary tables.

10. Conclusions (required) - See Section 5.0.

11. Recommendations (optional) - None included.

${ }^{5}$ TFC-ENG-CHEM-D-33 has been replaced by TFC-ENG-DESIGN-C-32, Spreadsheet Development and Verification. 


\section{RPP-5926 REV 6}

This page intentionally left blank. 


\subsection{HYDROGEN GENERATION RATE MODEL}

Several hydrogen rate models have been proposed (WHC-EP-0702, Criteria for Flammable Gas Watch List Tanks; WHC-SD-WM-TI-724, Methodology for Flammable Gas Evaluations; WHC-SD-WM-SARR-015, Topical Report on Flammable Gases in Non-Burping Waste Tanks; PNNL-11297, Status and Integration of Studies of Gas Generation in Hanford Wastes; and HNF-SD-WM-CN-117) to estimate the HGR for Hanford tank waste, but these models are based on either the waste simulant data or very limited tank waste data. A more comprehensive HGR model (described in Empirical Rate Equation Model of Hydrogen Generation for Hanford Tank Waste, paper presented at American Nuclear Society, 1999 Winter Meeting, and HNF-3851) was developed and validated based on a large body of tank waste data. The model constructed a set of semi-empirical rate equations to simulate the gas generation mechanisms of thermal chemical reactions, radiolysis of water and organic components, and corrosion processes. The rate equations are formulated as a function of physical and chemical properties of tank waste and are able to estimate the HGR of waste under current conditions, including the rates for newly mixed waste with known properties. The model has been applied to calculate the generation rate for more than 28 SSTs and DSTs and shows good agreement with field-observed rates.

\subsection{SEMI-EMPIRICAL RATE EQUATIONS}

The empirical rate equation for hydrogen generation in Hanford Site waste developed above contains the simulation of thermal reaction, $\mathrm{HGR}_{\mathrm{thm}}$, radiolysis of water and organic, $\mathrm{HGR}_{\mathrm{rad}}$, and the corrosion process, $\mathrm{HGR}_{\text {corr }}$. This rate equation is a function of waste composition (TOC, $\mathrm{Al}^{+3}, \mathrm{NO}_{3}{ }^{-}, \mathrm{NO}_{2}{ }^{-}$and $\mathrm{Na}^{+}$), radiation dose, temperature, liquid fraction, and tank wetted area.

Both the thermal and organic radiolysis rates follow Arrhenius behavior with a derived activation energy. The equation for HGR in the units of moles per kilogram per day can be summarized as follows (Equation 2-1):

$$
H G R=H G R_{t h m}+H G R_{r a d}+H G R_{c o r r}
$$

where:

$$
\begin{aligned}
& H G R_{t h m}=a_{t h m} \times\left(r_{f} \times[T O C]\right) \times\left[\mathrm{Al}^{+3}\right]^{0.4} \times L_{f} \times \exp ^{\left(-E_{t h m} / R T\right)}
\end{aligned}
$$

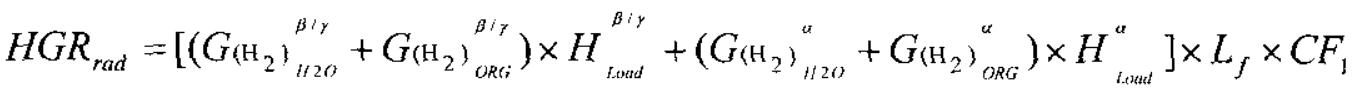

$$
\begin{aligned}
& H G R_{\text {corr }}=R_{\text {corr }} \times E_{\mathrm{H}_{2}} \times A_{\text {wetted }} / M_{\tan k} \times C F_{2}
\end{aligned}
$$

with:

$$
G_{\left(\mathrm{H}_{2}\right)^{p / y}}^{p / s}=a_{\text {rad }} \times \exp ^{\left(-E_{\text {rad }} / R T\right)} \times\left(r_{f} \times[T O C]\right)
$$




$$
\begin{aligned}
& G_{\left(\mathrm{H}_{2}\right)^{\beta \prime \prime}{ }^{\beta \prime \prime}}=\frac{0.32}{1+2.4\left[\mathrm{NO}_{3}^{-}\right]+0.62\left[\mathrm{NO}_{2}^{-}\right]+0.31\left[\mathrm{Na}^{+}\right]_{e x}^{2}}+\frac{0.13}{1+139\left[\mathrm{NO}_{3}^{-}\right]+54\left[\mathrm{NO}_{2}^{-}\right]}
\end{aligned}
$$

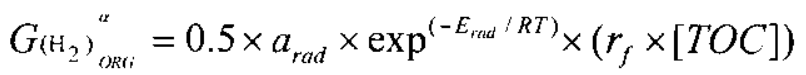

$$
\begin{aligned}
& G_{\left(\mathrm{H}_{2}\right)^{\prime \prime}{ }_{120}}=\frac{1.05}{1+2.4\left[\mathrm{NO}_{3}^{-}\right]+0.63\left[\mathrm{NO}_{2}^{-}\right]}+\frac{0.35}{1+3900\left[\mathrm{NO}_{3}^{-}\right]+1400\left[\mathrm{NO}_{2}^{-}\right]}
\end{aligned}
$$

and:

$$
\begin{aligned}
& \mathrm{E}_{\mathrm{thm}}=89.6 \mathrm{~kJ} / \text { mole, the activation energy for the thermal reaction } \\
& \mathrm{a}_{\mathrm{thm}}=3.94 \mathrm{E}+09 \mathrm{~mole} / \mathrm{kg} \text {-day, pre-exponential factor of the thermal rate } \\
& \mathrm{E}_{\mathrm{rad}}=48.8 \mathrm{~kJ} / \text { mole, activation energy in organic radiolysis, } \mathrm{G} \\
& \mathrm{a}_{\mathrm{rad}}=1.11 \mathrm{E}+07 \mathrm{H}_{2} / 100 \mathrm{eV} \text {, the pre-exponential term in organic radiolysis, } \mathrm{G} \\
& \mathrm{r}_{\mathrm{f}} \quad=0.6 \text { for DSTs and } 0.3 \text { for SSTs (unitless), the total organic carbon } \\
& \text { reactivity coefficient } \\
& \text { Note: The } r_{f}=0.3 \text { for SSTs is an average value from the tanks tested. If } \\
& \text { tank has a high fraction of energetic organic compounds, the } \mathrm{r}_{\mathrm{f}} \text { can be } \\
& \text { adjusted to as high as } 0.6 \text {. } \\
& \mathrm{R}=8.314 \mathrm{~J} / \mathrm{mole} / \mathrm{K} \text {, gas constant } \\
& \mathrm{R}_{\text {сот }}=6.0 \mathrm{E}-08 \text { for DSTs and } 2.4 \mathrm{E}-07 \text { for SSTs }\left(\mathrm{ft}^{3} / \mathrm{min} / \mathrm{ft}^{2}\right) \text {, corrosion } \\
& \text { coefficient } \\
& \mathrm{E}_{\mathrm{H} 2}=\text { the hydrogen generation efficiency coefficient from corrosion is } \\
& 20 \text { percent if }\left[\mathrm{NO}_{3}{ }^{-}\right],\left[\mathrm{NO}_{2}{ }^{-}\right] \text {, and }\left[\mathrm{OH}^{-}\right]>0.1 \text {, otherwise it is } 50 \text { percent. } \\
& {[\mathrm{TOC}]=\text { total organic carbon concentration in the liquid waste }(\mathrm{wt} \%)} \\
& \text { Note: Insoluble energetic organic compounds (excluding oxalate) in the } \\
& \text { solid layer should be considered case by case when data is available } \\
& {\left[\mathrm{Al}^{+3}\right]=\text { aluminum ion concentration in liquid waste }(\mathrm{wt} \%)} \\
& {\left[\mathrm{NO}_{3}{ }^{-}\right]=\text {nitrate ion concentration in the liquid waste }(\mathrm{moles} / \mathrm{L})} \\
& {\left[\mathrm{NO}_{2}{ }^{-}\right]=\text {nitrite ion concentration in the liquid waste }(\mathrm{moles} / \mathrm{L})} \\
& {\left[\mathrm{Na}^{+}\right]_{\mathrm{ex}}=\text { concentration of sodium minus nitrate and nitrite concentration in liquid }} \\
& \text { waste }(\mathrm{moles} / \mathrm{L}) \\
& \mathrm{H}_{\text {load }}^{\beta / \gamma}=\text { total heat load of the tank from beta/gamma (Watt } / \mathrm{kg} \text { ) } \\
& \mathrm{H}_{\text {load }}{ }^{\alpha}=\text { total heat load of the tank from total alpha (Watt } / \mathrm{kg} \text { ) } \\
& \mathrm{L}_{\mathrm{f}} \quad=\text { liquid weight fraction in the waste (unitless) } \\
& \mathrm{T} \quad=\text { temperature of waste }(\mathrm{K}) \\
& \mathrm{A}_{\text {wetted }}=\text { area of steel exposed to moisture-containing waste }\left(\mathrm{ft}^{2}\right) \\
& \mathrm{M}_{\mathrm{tank}}=\text { total mass in the waste }(\mathrm{kg}) \\
& \mathrm{CF}_{1}=\text { conversion factor from }\left(\mathrm{H}_{2} / 100 \mathrm{eV}\right)(\text { Watts } / \mathrm{kg}) \text { to (Mole } / \mathrm{kg} \text {-day) } \\
& \mathrm{CF}_{2}=\text { conversion factor from }\left(\mathrm{m}^{3} / \mathrm{kg}-\mathrm{min}\right) \text { to (Mole/kg-day). }
\end{aligned}
$$

In general, the HGR caused by chemical reactions follows Arrhenius behavior and the rate from radiolysis is proportional to the radiation dose. In addition, the HGRs from radiation effects in tank waste samples have been observed to be temperature dependent as provided in PNNL-12181, Thermal and Radiolytic Gas Generation on Material from Tanks 241-U-103, 
241-AW-101, 241-S-106, and 241-S-102: Status Report. The analysis of all available gas generation data provided in HNF-3851 shows that this temperature-dependent reaction follows Arrhenius behavior, which may result from a multi-step degradation of organic compounds initiated by radiolysis, followed by thermal reactions. The water radiolysis rate is assumed to be temperature independent, with the $\mathrm{G}_{\mathrm{H} 2 \mathrm{O}}$ value reduced by scavenging effects.

The numerical parameters in the equations are established by analyzing gas generation kinetic data from waste samples with the aid of tank field surveillance data and tank waste characterization data. The reactivity coefficient, $r_{f}$, was used to adjust for differences in reactivity of total organic carbon (TOC) among the tanks. HNF-3851 describes this model in detail. For comparison, Table 2-1 lists the field-observed and model-calculated total HGRs, and the ratio between these values for 28 tanks.

Table 2-1. Comparison of Model-Calculated and Field-Observed Hydrogen Generation Rates. (2 sheets)

\begin{tabular}{|c|c|c|c|c|c|}
\hline Tanks & $\begin{array}{c}\mathbf{G}_{\text {mod }} \\
\left(f^{3} / \text { min) }\right. \\
\text { Total HGR } \\
\text { from Model }\end{array}$ & $\begin{array}{c}\mathrm{G}_{\text {field }} \\
\left(\mathrm{ft}^{3} / \mathrm{min}\right) \\
\text { Total HGR } \\
\text { from Field }\end{array}$ & $\begin{array}{c}\mathbf{G}_{\text {mod }} \\
\text { (L/day) } \\
\text { Total HGR } \\
\text { from Model }\end{array}$ & $\begin{array}{c}\mathrm{G}_{\text {field }} \\
\text { ( } \mathrm{L} / \mathrm{day}) \\
\text { Total HGR } \\
\text { from Field }\end{array}$ & $\begin{array}{c}\text { Ratio Between } \\
\text { Model } \\
\text { and Field }\end{array}$ \\
\hline 241-AN-101 & $1.73 \mathrm{E}-04$ & $2.50 \mathrm{E}-04$ & 7 & 10 & 0.69 \\
\hline 241-AN-103 & $4.54 \mathrm{E}-03$ & $4.76 \mathrm{E}-03$ & 186 & 195 & 0.95 \\
\hline 241-AN-104 & $5.53 \mathrm{E}-03$ & $2.55 \mathrm{E}-03$ & 227 & 104 & 2.17 \\
\hline 241-AN-105 & $5.14 \mathrm{E}-03$ & $3.06 \mathrm{E}-03$ & 211 & 125 & 1.68 \\
\hline 241-AN-107 & $1.09 \mathrm{E}-02$ & $5.25 \mathrm{E}-03$ & 447 & 214 & 2.09 \\
\hline 241-AW-101 & $3.55 \mathrm{E}-03$ & $3.17 \mathrm{E}-03$ & 146 & 129 & 1.12 \\
\hline $241-\mathrm{AY}-102$ & $2.10 \mathrm{E}-02$ & $1.70 \mathrm{E}-02$ & 859 & 691 & 1.24 \\
\hline $241-A Z-101$ & $2.79 \mathrm{E}-02$ & $9.44 \mathrm{E}-03$ & 1,144 & 385 & 2.97 \\
\hline 241-AZ-102 & $2.90 \mathrm{E}-02$ & $1.90 \mathrm{E}-02$ & 1,190 & 775 & 1.54 \\
\hline $241-S Y-101$ & $5.96 \mathrm{E}-02$ & 2.44E-02 & 2,441 & 993 & 2.45 \\
\hline 241-SY-102 & $9.66 \mathrm{E}-04$ & $7.26 \mathrm{E}-04$ & 40 & 30 & 1.34 \\
\hline 241-SY-103 & $3.63 \mathrm{E}-03$ & $3.54 \mathrm{E}-03$ & 149 & 145 & 1.02 \\
\hline 241-A-101 & $5.64 \mathrm{E}-03$ & $2.14 \mathrm{E}-03$ & 231 & 87 & 2.65 \\
\hline $241-\mathrm{C}-104$ & $2.56 \mathrm{E}-03$ & $2.21 \mathrm{E}-03$ & 105 & 90 & 1.16 \\
\hline $241-\mathrm{C}-106$ & $1.62 \mathrm{E}-02$ & $9.03 \mathrm{E}-03$ & 664 & 368 & 1.80 \\
\hline $241-S-102$ & $1.25 \mathrm{E}-03$ & $1.64 \mathrm{E}-03$ & 51 & 67 & 0.77 \\
\hline 241-SX-101 & $6.64 \mathrm{E}-04$ & $4.20 \mathrm{E}-04$ & 27 & 17 & 1.59 \\
\hline 241-SX-103 & $3.03 \mathrm{E}-03$ & $1.27 \mathrm{E}-03$ & 124 & 52 & 2.40 \\
\hline 241-SX-104 & $1.31 \mathrm{E}-03$ & $2.51 \mathrm{E}-04$ & 53 & 10 & 5.23 \\
\hline 241-SX-105 & $5.77 \mathrm{E}-03$ & $4.82 \mathrm{E}-03$ & 236 & 197 & 1.20 \\
\hline 241-SX-106 & $1.53 \mathrm{E} \sim 03$ & $1.24 \mathrm{E}-03$ & 63 & 50 & 1.25 \\
\hline $241-\mathrm{U}-102$ & $1.05 \mathrm{E}-03$ & $1.10 \mathrm{E}-03$ & 43 & 45 & 0.96 \\
\hline $241-\mathrm{U}-103$ & $1.46 \mathrm{E}-03$ & $1.48 \mathrm{E}-03$ & 60 & 60 & 1.00 \\
\hline 241-U-105 & $1.37 \mathrm{E}-03$ & $1.61 \mathrm{E}-03$ & 56 & 65 & 0.86 \\
\hline
\end{tabular}


Table 2-1. Comparison of Model-Calculated and Field-Observed Hydrogen Generation Rates. (2 sheets)

\begin{tabular}{|c|c|c|c|c|c|}
\hline Tanks & $\begin{array}{c}\mathbf{G}_{\text {mod }} \\
\left(\mathbf{f t}^{3} / \mathbf{m i n}\right) \\
\text { Total HGR } \\
\text { from Model }\end{array}$ & $\begin{array}{c}\mathbf{G}_{\text {field }} \\
\left(\mathbf{f t}^{3} / \mathbf{m i n}\right) \\
\text { Total HGR } \\
\text { from Field }\end{array}$ & $\begin{array}{c}\mathbf{G}_{\text {mod }} \\
(\mathbf{L} / \mathbf{d a y}) \\
\text { Total HGR } \\
\text { from Model }\end{array}$ & $\begin{array}{c}\mathbf{G}_{\text {field }} \\
\text { (L/day) } \\
\text { Total HGR } \\
\text { from Field }\end{array}$ & $\begin{array}{c}\text { Ratio Between } \\
\text { Model } \\
\text { and Field }\end{array}$ \\
\hline $241-\mathrm{U}-106$ & $1.12 \mathrm{E}-03$ & $6.62 \mathrm{E}-04$ & 46 & 27 & 1.70 \\
\hline $241-\mathrm{U}-107$ & $4.71 \mathrm{E}-04$ & $8.27 \mathrm{E}-04$ & 19 & 34 & 0.57 \\
\hline $241-\mathrm{U}-108$ & $9.42 \mathrm{E}-04$ & $1.41 \mathrm{E}-03$ & 39 & 57 & 0.67 \\
\hline $241-\mathrm{U}-109$ & $5.44 \mathrm{E}-04$ & $7.11 \mathrm{E}-04$ & 22 & 29 & 0.77 \\
\hline
\end{tabular}

Note:

HGR $=$ hydrogen generation rate.

Among these 28 tanks, six SSTs and two DSTs are underestimated by the model comparing the field-estimated rate (i.e., the ratio is less than one). Again, these eight tanks have relatively low generation rates (below $70 \mathrm{~L} /$ day) except for DST 241-AN-103, which has a rate of $195 \mathrm{~L} /$ day with the ratio of 0.95 . Overall the model-predicted rates agree very well, within a factor of three, with field-estimated rates. 
RPP-5926 REV 6

\subsection{METHODOLOGY TO ESTIMATE STEADY-STATE FLAMMABILITY LEVEL}

This chapter describes the methodology used to evaluate the steady-state flammability level in the dome space for all DSTs and SSTs. The methodology also provides a technical basis for flammable gas controls in tank farm operations. The evaluation will address the flammability level in the dome space under both normal and off-normal ventilation conditions. In the evaluation, the steady-state gas release rates and ventilation rates are needed to derive the steady-state gas concentration under given ventilation conditions. Based on the flammable gas concentrations, the level of flammability of the mixture can be calculated. Under current normal ventilation conditions, the steady-state flammability level in the tank dome space is below $25 \%$ of the LFL for all tanks (HNF-3294, Adequacy of Ventilation Rates to Remediate Flammable Gas Levels in Headspace of Hanford Tanks - Status Report). The primary evaluation is focused on the flammable gas level under off-normal ventilation conditions.

The major gases of concern were identified as hydrogen, ammonia, nitrous oxide, and methane in the tank dome space. Among these identified gases, hydrogen is the primary flammable gas in the tank dome space. The gas release rate of hydrogen in the dome space can be determined by subtracting the gas accumulation rate from the generation rate calculated with the rate equation model described in Chapter 2.0. The other two flammable gases, ammonia and methane, have much lower concentrations in the dome space and have no general model for calculating their gas generation rates; therefore, the steady-state gas release rates for ammonia and methane are estimated by their steady-state gas concentrations under normal ventilation conditions. Nitrous oxide is an oxidizer; the concern is whether its concentration is high enough to lower the LFL of hydrogen, ammonia, and methane as established in air. The gas release rate of nitrous oxide also is derived by the steady-state gas concentration under normal ventilation conditions.

To evaluate the flammable gas level, a time-dependent gas concentration model was established. This model can calculate the steady-state flammability level for a given ventilation condition. The model also can estimate the time required to reach certain flammability levels in the dome space under different ventilation conditions and calculate the minimum ventilation rate to keep the dome space below the specified flammability limit.

\subsection{LOWER FLAMMABILITY LIMITS}

The National Fire Protection Agency codes require that operations be terminated when the concentration of flammable gases exceeds $25 \%$ of the LFL. Based on the empirical rule developed by Le Chatelier in the late 19th century, the LFL of mixtures of multiple flammable gases in air can be determined. Le Chatelier's rule (Limits of Flammability of Gases and Vapors, [Coward and Jones 1952]) can be written in terms of the LFL concentration of the fuel mixture, $\mathrm{LFL}_{\mathrm{m}}$, as follows: 


$$
L F L_{m}=\frac{100}{\sum_{i}^{n}\left(\frac{C_{i}}{L F L_{i}}\right)}
$$

where:

$\mathrm{C}_{i} \quad=$ volume percent of fuel gas $i$ in the fuel gas mixture $\mathrm{LFL}_{\mathrm{i}}=$ volume percent of fuel gas $i$ at its LFL in air alone.

In the tank dome space, hydrogen gas, ammonia, and methane are the flammable gases of concern. For tank waste conditions, the volume percents of the flammable gases vary over time depending on individual flammable gas release rate in the tank head space, and thus, Equation 3-1 cannot apply directly. WHC-SD-WM-ES-219, Laboratory Flammability Studies of Mixtures of Hydrogen, Nitrous Oxide, and Air, has shown experimentally that the total flammability level $\mathrm{LFL}_{\mathrm{f}}$ (the fraction of the LFL) of a mixture of hydrogen, ammonia, and methane in an air-nitrous oxide atmosphere can be computed using the principle of Le Chatelier's rule in terms of the individual fuel gas flammability as in Equation 3-2.

$$
L F L_{f}=\frac{\left[\mathrm{H}_{2}\right]}{L F L_{\mathrm{H} 2}}+\frac{\left[\mathrm{NH}_{3}\right]}{L F L_{\mathrm{NH} 3}}+\frac{\left[\mathrm{CH}_{4}\right]}{L F L_{\mathrm{CH} 4}}
$$

where:

$\left[\mathrm{H}_{2}\right] \quad=$ volume percent of hydrogen in the tank headspace

$\left[\mathrm{NH}_{3}\right]=$ volume percent of ammonia in the tank headspace

$\left[\mathrm{CH}_{4}\right]=$ volume percent of methane in the tank headspace

$\mathrm{LFL}_{\mathrm{H} 2}=$ volume percent of hydrogen at its LFL in air alone

$\mathrm{LFL}_{\mathrm{NH} 3}=$ volume percent of ammonia at its LFL in air alone

$\mathrm{LFL}_{\mathrm{CH} 4}=$ volume percent of methane at its LFL in air alone.

For simplicity, in Equation 3-2, flammable gases with concentrations lower than that of methane are omitted. The flammability of the individual gases in air is fairly well characterized. The $\mathrm{LFL}_{\mathrm{H} 2}, \mathrm{LFL}_{\mathrm{NH} 3}$ and $\mathrm{LFL}_{\mathrm{CH} 4}$ are 4,15 , and $5 \%$, respectively, at the upward propagation $\mathrm{LFL}$, which is more conservative than the downward propagation LFL. In addition to the flammable gases included in Equation 3-2, nitrous oxide was observed in the tank dome space. Nitrous oxide plays the role of oxidizer in the mixture and has the potential to decrease the amount of fuel required to reach the LFL. The effect of the multiple oxidizers (e.g., $\mathrm{N}_{2} \mathrm{O}$ and $\mathrm{O}_{2}$ ) was not addressed in Equation 3-2.

Pfahl and Shepherd (1997) from the California Institute of Technology have conducted a series of flammability studies on gas mixtures generated from Hanford Site tank waste. The studies show that the established LFLs of individual fuels in the air will decrease if concentrated $\mathrm{N}_{2} \mathrm{O}$ is present in the mixtures. For example, in the mixture $\mathrm{NH}_{3}-\mathrm{N}_{2} \mathrm{O}$-air with a ratio of 13.2-19.8-67.0, the system will be flammable, although the ammonia volume percent is 13.2 and is lower than the $\mathrm{LFL}_{\mathrm{NH} 3}$ of $15 \%$ in air. This fuel-oxidizer mixture will be flammable with the ammonia volume percent as low as $8 \%$ if the $\mathrm{N}_{2} \mathrm{O}$ concentration is $65 \%$. For $\mathrm{NH}_{3}-\mathrm{N}_{2} \mathrm{O}$-air mixtures, 
Pfahl and Shepherd (Figure 32 in 1997 report) suggest that the ammonia flammability limit will be $15 \%$ if the $\mathrm{N}_{2} \mathrm{O}$ does not exceed $8 \%$. These test results are for turbulent conditions, with an ignition energy of $8 \mathrm{~J}$. For a $\mathrm{CH}_{4}-\mathrm{N}_{2} \mathrm{O}$-air mixture, the data show that the ratio of 4.5-10.1-85.4 is not flammable. For an $\mathrm{H}_{2}-\mathrm{N}_{2} \mathrm{O}$-air mixture, the data show that a mixture with the ratio of 5.7-10-84.3 has incomplete combustion. It should be noted here that the ignition energy of $8 \mathrm{~J}$ used in these experiments is very large compared to the energy in most electrostatic discharge sparks (typically a few millijoules) that might occur inside a Hanford Site tank.

This information suggests that the flammability limit established in the fuel-air mixture for each gas will not be reduced unless the $\mathrm{N}_{2} \mathrm{O}$ volume percent exceeds $8 \%$. In this evaluation, the volume percents of 4,15 , and $5 \%$ will be used for $\mathrm{LFL}_{\mathrm{H} 2}, \mathrm{LFL}_{\mathrm{NH} 3}$ and $\mathrm{LFL}_{\mathrm{CH} 4}$, respectively, in Equation 3-2. In each case, the concentration of nitrous oxide was evaluated and did not exceed $8 \%$, therefore, the oxidizer effect of lowering these flammability limits can be ignored.

\subsection{VENTILATION CONDITIONS IN TANK DOME SPACE}

Under normal operating conditions, the ventilation conditions can be active ventilation or passive ventilation. The tank dome spaces of all DSTs and some SSTs are equipped with an active ventilation system. For active ventilation systems, the main ventilation flow is designed to go through an installed inlet filter. The 241-AP Tank Farm is an exception to this system; inlet air flows through cover blocks or gaps in riser covers, and other tank openings. The size of the inlet openings was designed to be either fully adjustable or to have just an on-off valve. These filtered inlets are used to control the ventilation rate and to adjust the negative pressure to prevent leakage of tank hazardous materials or to provide control for any other operational needs. For passively ventilated tanks, the ventilation flow goes mainly through high-efficiency particulate air (HEPA) breather filters. Under normal operating conditions, the active ventilation rates range from 100 to $700 \mathrm{ft}^{3} / \mathrm{min}$, while the passive ventilation rates typically range from 0.5 to $10 \mathrm{ft}^{3} / \mathrm{min}$. On actively ventilated tanks, the off-normal operating ventilation condition considered in the flammability assessment is that the ventilation system is shut down and the air inlet is closed; on passively ventilated tanks, the HEPA breather filter isolation valve is closed or a blank is installed. For these conditions, the only venting flow is through the gaps between the cover blocks or riser covers. The off-normal condition allows three possible tank ventilation conditions: no ventilation flow, ventilation flow caused only by barometric breathing, or ventilation flow caused by natural breathing, which is a combination of ventilation by barometric breathing plus thermal convection. The barometric breathing rate, $\mathrm{V}_{\mathrm{bb}}$, is estimated as $0.45 \%$ of the dome space volume per day (WHC-EP-0651, Barometric Pressure Variations) and is given in Equation 3-3:

$$
V_{b b}=0.45 \% \cdot \operatorname{Vol}_{\text {headspace }}\left(\frac{f t^{3}}{d a y}\right)
$$

where:

$$
\text { Vol }{ }_{\text {headspace }}=\text { volume of the tank dome space }\left(\mathrm{ft}^{3}\right) \text {. }
$$


During off-normal operating conditions, the most likely ventilation rate is either natural breathing or barometric breathing. The natural breathing rate is estimated to be 5 to 10 times the barometric breathing rate, but the estimation of the thermal convection flow is not available. In general, the zero-flow condition is considered to be impossible because of the many openings into the tanks. However, since 1980, a great effort has been made to seal off the openings as much as possible for SSTs under the interim stabilization project to prevent water intrusion. In this evaluation, the barometric breathing rate, which is more conservative but reasonable, will be used for the ventilation rate for DSTs when normal ventilation is not present. For SSTs, both barometric breathing rate and zero ventilation rate will be evaluated under off-normal ventilation condition. As the shown in the calculations later, there is not much difference between the zero and barometric breathing rate in terms of time to reach $25 \%$ and $100 \%$ of the LFL because the barometric breathing rate is very small and close to zero. Using a tracer gas, the measured passive ventilation rates (PNNL-11925, Waste Tank Ventilation Rates Measured with A Tracer Gas Method, Fiscal Year 1998 Summary) under normal ventilation conditions in SSTs ranged from 1.7 to $10 \mathrm{ft}^{3} / \mathrm{min}$ for most tanks. No similar measurements for the passive rates (i.e., the active ventilation system is shut down with the air-inlet open) are available for DSTs. Unfortunately, no ventilation rate measurements have been made for the condition where the air inlet is shut off. For the off-normal condition, based on Equation 3-3, the calculated barometric breathing rate for most tanks is around $0.5 \mathrm{ft}^{3} / \mathrm{min}$ or less (as shown in Table D-2 of Appendix D), which is one-third to one-twentieth of normal conditions.

\subsection{TIME-DEPENDENT GAS CONCENTRATION MODEL}

An equation was developed to calculate the time-dependent flammable gas concentration in the dome space. The concentration is a function of the gas release rate and the ventilation rate. Figure 3-1 shows a simplified tank dome space system.

In general, most of the tanks contain supernatant liquid and sludge/saltcake layers. A few tanks, including DST 241-AN-103, have a thin crust layer above the supernatant liquid. The gas release rate $\left(R_{g}\right)$ is the net result of the generation rate $\left(G_{r}\right)$ and the accumulation rate $\left(A_{r}\right)$. The gas accumulation rate refers to the rate at which gas is trapped in the sludge/saltcake layer. The ventilation flows $\left(\mathrm{V}_{\mathrm{r}}\right)$ are available as field measurements taken at the output streams. These flows are the sum of air inflows from the inlets and tank cracks and openings and the gases released from tank waste. The gas volume change rate can be formulated from a mass balance as provided in Equation 3-4:

$$
\begin{gathered}
\text { Gas Change Rate in } \\
\text { Tank Dome Space }
\end{gathered} \quad=\begin{gathered}
\text { Gas Release Rate from } \\
\text { the Waste Surface }
\end{gathered} \quad \text { Gas Exit Rate }
$$

Equation 3-4 can be changed to calculate the gas volume change rate for SSTs where the tank dome is made of concrete. Assuming that there is no ventilation flow available but the gas can diffuse out through the concrete dome, then the "Gas Exit Rate" term in Equation 3-4 is changed to the gas diffusion rate. 
Figure 3-1. A Simplified Tank System for Time-Dependent Gas Concentration Model.

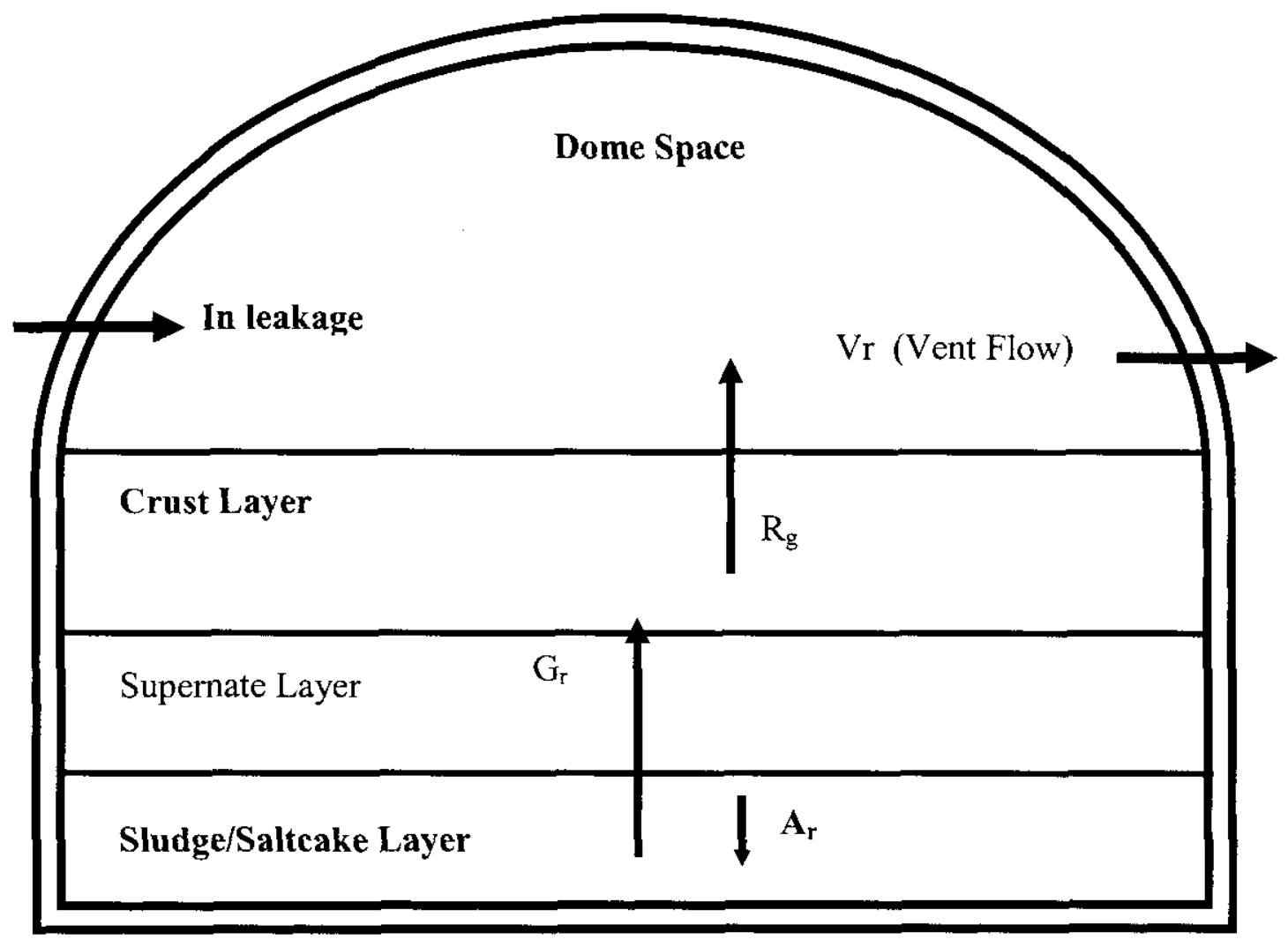

\subsubsection{Insoluble Gas}

For the less soluble gases such as hydrogen or methane, the gas release rate can be considered to be constant; therefore, the mass balance equation can be written as a differential equation as given in Equation 3-5:

$$
\operatorname{Vol} \frac{\partial\left\lfloor C_{g}\right\rfloor}{\partial t}=R_{g}-V_{r} \cdot\left[C_{g}\right]
$$

where:

$\left[\mathrm{C}_{\mathrm{g}}\right]=$ gas concentration (volume percent)

Vol = volume of the tank dome space (volume)

$\mathrm{R}_{\mathrm{g}}=$ gas release rate from the waste surface (volume/time)

$\mathrm{V}_{\mathrm{r}} \quad=$ ventilation rate in the dome space (volume/time). 
As given in Equation 3-5, the gas exhaust rate is simply the gas concentration times the output ventilation rate. By solving the differential Equation 3-5, a time-dependent gas concentration at time $t,\left[C_{g}\right](t)$, in units of volume percent is given in Equation 3-6:

$$
\left[C_{g}\right]_{\%}(t)=\left[C_{g}\right]_{\%}\left(t_{0}\right) \exp (-\lambda t)+\left[\frac{R_{g}}{V_{r}}\right]_{\%}[1-\exp (-\lambda t)]
$$

with

where:

$$
\lambda \quad=\mathrm{V}_{\mathrm{r}} / \mathrm{Vol}
$$

$$
\begin{array}{ll}
\mathrm{R}_{\mathrm{g}} & =\text { hydrogen release rate from the waste surface (volume/time) } \\
\mathrm{V}_{\mathrm{r}} & =\text { ventilation rate in the dome space (volume/time) } \\
{\left[\mathrm{C}_{\mathrm{g}}\right]\left(\mathrm{t}_{\mathrm{o}}\right)} & =\text { initial gas concentration at time } \mathrm{t} \text { (volume percent) } \\
\mathrm{Vol} & =\text { volume of the tank dome space (volume) } \\
\lambda & =\text { decay parameter from the venting (inverse time) } \\
\mathrm{t} & =\text { time. }
\end{array}
$$

In Equation 3-6, the first term represents how the initial gas concentration decays away exponentially with the factor of $\mathrm{e}^{\left(-\lambda^{t}\right)}$ and second term represents how the gas level builds up in the form of $\left[R_{g} / V_{r}\right]_{\%}$ with the factor of $\left[1-e^{(-\lambda t)}\right]$ as time goes on.

For the case of gas exiting by the diffusion effect through the dome, the mass balance equation can be written as a differential equation as given in Equation 3-6a:

$$
\operatorname{Vol} \frac{\partial\left\lfloor C_{g}\right\rfloor}{\partial t}=R_{g}-A \times Q=R_{g}-A \times \frac{1}{\sum_{i} \frac{L_{i}}{D_{i}}} \times\left(\left[C_{g}\right]-\left[C_{o}\right]\right)
$$

where:

$\left[\mathrm{C}_{\mathrm{g}}\right]=$ gas concentration in the tank headspace (volume percent)

$\left[\mathrm{C}_{\mathrm{o}}\right]=$ gas concentration outside the tank (volume percent)

$\mathrm{Vol}=$ volume of the tank dome space (volume)

$\mathrm{R}_{\mathrm{g}}=$ gas release rate from the waste surface (volume/time)

$\mathrm{Q}=$ the diffusion flux (concentration/area-time)

$\mathrm{A}=$ the area of the dome to diffuse (area)

$\mathrm{D}_{\mathrm{i}} \quad=$ the ith layer effective diffusivity (area/time)

$\mathrm{L}_{\mathrm{i}} \quad=$ the thickness of the ith layer to diffuse (length).

As given in Equation 3-6a, the gas exhaust rate is the gas diffusion rate, which is the diffusion flux, $\mathrm{Q}_{\mathrm{i}}$, times the surface area of the dome. The diffusion flux is defined as the simply the gas concentration gradient between the dome space and outside over the path-length for diffusion times the diffusivity. The summation of the diffusion rates in Equation 3-6a accounts for several diffusion resistances in series (e.g. paint, concrete, and soil for a transfer structure wall). Assuming the gas concentration outside is diluted infinitely becoming zero. By solving the 
differential Equation 3-6a, a time-dependent gas concentration at time $t,\left[C_{\mathrm{g}}\right](\mathrm{t})$, in units of volume percent is given in Equation 3-6b:

$$
\left[C_{g}\right]_{\%}(t)=\left[C_{g}\right]_{\%}\left(t_{0}\right) \exp (-\lambda t)+\left[\frac{R_{g}}{A \times \frac{1}{\sum_{i} \frac{L_{i}}{D_{i}}}}\right]_{\%}[1-\exp (-\lambda t)]
$$

with

$$
\lambda=\mathrm{A} \times\left[1 / \Sigma\left(\mathrm{L}_{\mathrm{i}} / \mathrm{D}_{\mathrm{i}}\right)\right] / \mathrm{Vol}
$$

where:

$$
\begin{array}{ll}
\mathrm{R}_{\mathrm{g}} & =\text { hydrogen release rate from the waste surface (volume/time) } \\
\mathrm{V}_{\mathrm{r}} & =\text { ventilation rate in the dome space (volume/time) } \\
{\left[\mathrm{C}_{\mathrm{g}}\right]\left(\mathrm{t}_{\mathrm{o}}\right)} & =\text { initial gas concentration at time } \mathrm{t} \text { (volume percent) } \\
\mathrm{Vol} & =\text { volume of the tank dome space (volume) } \\
\lambda & =\text { decay parameter from the diffusion (inverse time) } \\
\mathrm{t} & =\text { time. }
\end{array}
$$

\subsubsection{Soluble Gas}

For a highly soluble gas, such as ammonia, the gas release rate from liquid waste is not a constant, but depends on the material transport properties and the ammonia concentration gradient between the liquid and vapor phases and appropriate Henry's Law constants. Thus the differential equation of mass balance in Equation 3-5 and the time-dependent gas concentration Equation 3-6 have different formats. For a closed system, Henry's Law describes the relationship of a soluble gas in the liquid and vapor phases as provided in Equation 3-7:

$$
C_{l}=K_{H} \cdot P_{g}
$$

where:

$$
\begin{aligned}
& \mathrm{C}_{\mathrm{H}}=\text { ammonia concentration in the liquid phase }\left(\mathrm{kg}-\mathrm{mole} / \mathrm{m}^{3}\right) \\
& \mathrm{K}_{\mathrm{H}}=\text { Henry's Law constant }\left(\mathrm{kg}-\mathrm{mole} / \mathrm{m}^{3} \text {-atm }\right) \\
& \mathrm{P}_{\mathrm{g}}=\text { partial pressure of the ammonia }(\mathrm{atm}) .
\end{aligned}
$$

The Henry's Law constant is a function of temperature in pure water. In mixed salt solutions, Henry's Law constants are functions of both temperature and the concentrations of the ions in the solution. "The Estimation of Gas Solubility in Salt Solutions at Temperature from $273 \mathrm{~K}$ to $363 \mathrm{~K}$," AICHE Journal (Weisenberger and Schumpe 1996), provides a formula to calculate Henry's Law constant as given in Equation 3-8: 


$$
K_{H}=K_{H}^{o} \cdot 10^{-\sum_{i}\left(h_{i}+h_{g}\right) c_{i}}
$$

with

$$
\mathrm{K}_{\mathrm{H}}{ }^{\circ}=\mathrm{e}^{(-8.0964+3917.5 / \mathrm{T}-0.00314 \mathrm{~T})}
$$

where:

$$
\begin{array}{ll}
\mathrm{K}_{\mathrm{H}}^{\circ} & =\text { Henry's Law constant in the pure water }\left(\mathrm{kg}-\mathrm{mole} / \mathrm{m}^{3}-\mathrm{atm}\right) \\
\mathrm{h}_{\mathrm{i}} & =\text { ionic dependent coefficient } \\
\mathrm{h}_{\mathrm{g}} & =\text { gas specific constant for ammonia } \\
\mathrm{C}_{\mathrm{i}} & =\text { ion concentration in the solution } \\
\mathrm{T} & =\text { temperature of waste }(\mathrm{K}) .
\end{array}
$$

However, the Weisenberger and Schumpe (1996) formula is good only for the solution up to 5 M. RPP-4941 reported Henry's Law constants for DST 241-SY-101 simulant waste at different dilution conditions and temperatures. This study covers solution concentrations ranging from 5 to $21 \mathrm{M}$ at temperatures of $20^{\circ} \mathrm{C}$ to $70^{\circ} \mathrm{C}$. These results provide a correction factor to the calculated Henry's Law constant from the Weisenberger and Schumpe (1996) formula. For example, the measured Henry's Law constant is five times the calculation from the Weisenberger and Schumpe (1996) formula for the undiluted sample of $21 \mathrm{M}$ at $70^{\circ} \mathrm{C}$.

For soluble gas, the gas release rate depends on the equilibrium mechanism between the liquid and vapor phases described earlier. A comprehensive soluble gas transfer methodology applied to Hanford Site waste has been described in RPP-4941.

Consider the two-film theory of gas transfer. The ammonia release will encounter the resistance from two films, the liquid film and the gas film, between the bulk liquid and bulk vapor phases. The release rate is proportional to the difference between the ammonia liquid concentration and the ammonia vapor concentration before reaching equilibrium. It also is proportional to the mass transport coefficient, $h$, and the effective area, $A$. Therefore, the release rate can be derived as provided in Equation 3-9:

$$
R_{g}=h \cdot A \cdot\left(C_{l}-C_{l}^{E Q}\right)=h \cdot A \cdot\left(C_{l}-K_{H} \cdot P_{g}\right)=h \cdot A \cdot\left(C_{l}-K_{H} \cdot P \cdot v \cdot C_{g}\right)
$$

where:

$$
\begin{array}{ll}
\mathrm{h}= & \text { overall mass transport coefficient from liquid to vapor }(\mathrm{m} / \mathrm{sec}) \\
\mathrm{A} & =\text { effective area for the transport }\left(\mathrm{m}^{2}\right) \\
\mathrm{C}_{\mathrm{l}}^{\mathrm{EQ}}= & \text { corresponding liquid ammonia concentration in equilibrium at the } \\
& \text { current ammonia vapor pressure }\left(\mathrm{kg}-\mathrm{mole} / \mathrm{m}^{3}\right) \\
\mathrm{C}_{\mathrm{l}}= & \text { current ammonia concentration in liquid }\left(\mathrm{kg}-\mathrm{mole} / \mathrm{m}^{3}\right) \\
\mathrm{P}_{\mathrm{g}}= & \text { ammonia gas partial pressure }(\mathrm{atm}) \\
\mathrm{P} & =\text { dome space total pressure }(\mathrm{atm}) \\
\nu & =
\end{array}
$$


As Equation 3-9 shows, the ammonia gas release rate clearly is a function of the ammonia concentrations in the liquid and vapor phases and the Henry's Law constant for ammonia in the solution. In the two-film-theory of gas transfer, the overall mass transport coefficient h contains the transport coefficient $h_{1}$ for gas through the liquid film and $h_{g}$ for the gas through the gas film. Equations of the calculated $h$ are described in detail in RPP-4941 and summarized in Appendix E.

A sensitivity study indicates that the overall mass transport coefficient, $h$, varies by several factors, while the effective tank waste surface area, A, for mass transport is difficult to determine, particularly for the SSTs, where the waste surfaces are mostly crusts. Dome space ammonia concentrations would be overestimated by several orders of magnitude if the full area for the SST is used as the effective transport surface. As discussed in Section 3.4, however, the product of $h$ and $A$ can be determined by field data at steady-state conditions. Once $h$ times $A$ is determined, the gas release rate can be calculated by Equation 3-9. Applying the ammonia release rate from Equation 3-9 to the mass balance in Equation 3-5 results in Equation 3-10:

$$
\operatorname{Vol} \frac{\partial\left\lfloor C_{g}\right\rfloor}{\partial t}=h \cdot A \cdot\left(C_{l}-K_{H} \cdot P \cdot v \cdot C_{g}\right)-\left[V_{r}\right] \cdot\left[C_{g}\right]
$$

Dividing by $\mathrm{Vol}$ and rearranging the terms, Equation 3-10 becomes Equation 3-11:

$$
\frac{\partial\left\lfloor C_{g}\right\rfloor}{\partial t}=k_{2}-k_{1} \cdot\left[C_{g}\right]
$$

with

$$
k_{1}=\left[V_{r}+h \cdot A \cdot K_{H} \cdot P \cdot v\right] /[\mathrm{Vol}]
$$

and

$$
k_{2}=\left[h \cdot A \cdot C_{l}\right] /[\mathrm{Vol}] \text {. }
$$

The solution of Equation 3-11 is given as Equation 3-12:

$$
\left[C_{g}\right](t)=\left[C_{g}\right]\left(t_{0}\right) \exp \left(-k_{1} \cdot t\right)+\left[\frac{k_{2}}{k_{1}}\right]\left[1-\exp \left(-k_{1} \cdot t\right)\right]
$$

\subsection{STEADY-STATE GAS CONCENTRATION AND GAS RELEASE RATE}

For insoluble gas, when the systems reach steady state (set time, t, to infinity in Equation 3-6), the exponential term drops out from Equation 3-6 and a steady-state gas concentration is given in Equation 3-13:

$$
\left[C_{g}\right]_{\%}^{s s}=\left[\frac{R_{g}}{V_{r}}\right] \times 100
$$




\section{RPP-5926 REV 6}

where:

$$
\begin{array}{ll}
{\left[\mathrm{C}_{\mathrm{g}}\right]_{\%}{ }^{\text {ss }}} & =\text { steady-state gas concentration (volume percent) } \\
\mathrm{R}_{\mathrm{g}} & =\text { gas release rate (volume/time) } \\
\mathrm{V}_{\mathrm{r}} & =\text { ventilation rate in the dome space (volume/time) }
\end{array}
$$

Using Equation 3-13, the steady-state gas concentrations in the tank dome space can be calculated by knowing the gas release rate and the ventilation rate.

The gas release rate, $R_{\mathrm{g}}$, can be estimated by subtracting the gas accumulation rate from the gas generation rate as shown in Equation 3-14:

$$
\mathrm{R}_{\mathrm{g}}=\mathrm{G}_{\mathrm{r}}-\mathrm{A}_{\mathrm{r}}
$$

where:

$$
\begin{aligned}
& \mathrm{G}_{\mathrm{r}}=\text { gas generation rate from waste (volume/ time) } \\
& \mathrm{A}_{\mathrm{r}}=\text { gas accumulation rate due to gas trapping (volume/ time). }
\end{aligned}
$$

For hydrogen, the generation rate can be calculated using the empirical rate equation described in Section 2.1. The accumulation rate (HNF-3851) can be determined in the field, based on the waste level change rate and the gas composition data from the retained gas sampler. If the waste level does not change noticeably, the hydrogen release rate will be the same as the HGR.

For other insoluble gases, no gas generation rate model is available. Therefore, the steady-state gas release rate can be estimated from the known steady-state concentration in the dome space under normal ventilation conditions by rearranging Equation 3-13 as shown in Equation 3-15:

$$
R_{g}=\left(\frac{\left[C_{g}\right]_{\%}^{s s}}{100}\right) \cdot V_{r}
$$

where:

$$
\begin{array}{ll}
{\left[\mathrm{C}_{\mathrm{g}}\right]_{\%}{ }^{\text {ss }}} & =\text { steady-state gas concentration (volume percent) } \\
\mathrm{R}_{\mathrm{g}} & =\text { gas release rate (volume/time) } \\
\mathrm{V}_{\mathrm{r}} & =\text { ventilation rate in the dome space (volume/time) } .
\end{array}
$$

In this report, the gas release rates for methane and nitrous oxide were obtained from their steady-state concentrations under normal ventilation conditions using Equation 3-15. Then the calculated gas release rate was used to calculate the steady-state concentrations for off-normal conditions. This methodology is a reasonable way to determine methane concentrations under off-normal conditions because methane is insoluble and whatever gases are generated are released directly to the dome space. 
For highly soluble gases, from Equation 3-12, the steady-state concentration can be expressed as Equation 3-16:

$$
\left[C_{g}\right]^{s s}=\left[\frac{k_{2}}{k_{1}}\right]=\left[\frac{h \cdot A \cdot C_{l}}{V_{r}+h \cdot A \cdot K_{H} \cdot P \cdot v}\right]
$$

As discussed in Section 3.3, the mass transport properties, the product of $\mathrm{h}$ and $\mathrm{A}$, are difficult to estimate directly from tank waste properties, particularly for tank waste covered with an irregular crust layer. However, for the known steady-state vapor concentration $\mathrm{C}^{\mathrm{ss}}$, the liquid concentration $C_{l}$; along with Henry's Law constant and the ventilation rate $V_{r}$, the product of $h$ and $\mathrm{A}$ can be determined. By rearranging Equation 3-16, the product of $\mathrm{h}$ and $\mathrm{A}$ can be expressed as Equation 3-17:

$$
h \cdot A=\left[\frac{C_{g}^{s s} \cdot V_{r}}{\left(C_{l}-K_{H} \cdot P \cdot v \cdot C_{g}^{s s}\right)}\right]
$$

Once the product of $\mathrm{h}$ and $\mathrm{A}$ is determined from the steady state under normal ventilation conditions, the steady-state concentration under off-normal condition can be calculated using Equation 3-16. In addition, under the off-normal ventilation conditions, the ammonia concentration can be estimated using the time-dependent gas concentration of Equation 3-12, as well as the gas release rate of Equation 3-9.

Note that the product of $h$ and $A$ depends on the waste configuration and will be different if the waste is perturbed or changed physically. For the off-normal ventilation calculations, the overall steady-state ammonia concentration is governed by the liquid ammonia concentration. The product of $\mathrm{h}$ and $\mathrm{A}$ will change only the ammonia release rate, which indicates how fast the system will reach the steady state.

The steady-state flammability evaluation of this work is based on current tank waste data at storage conditions. For those tanks actively receiving waste, including DSTs 241-AP-108 and 241-AN-101 as dilute receiver tanks, the flammability level will be reevaluated during the waste transfer compatibility assessment based on the predicted post-transfer condition of the tank waste. For hydrogen, the release rate still can be calculated based on the predicted tank waste condition using this rate equation model. For methane, because steady-state methane vapor data are not available before the waste transfer, the release rate will be estimated as $10 \%$ of the model-calculated HGR. This is a reasonable estimate because, for all tanks, methane is less than 10 volume percent of the hydrogen in the generated gas as given in the gas generation study by PNNL-12181 and retained gas sampler results in PNNL-13000, Retained Gas Sampling Results for the Flammable Gas Program. For ammonia, instead of estimating the release rate, the ammonia vapor concentration at thermodynamic equilibrium for a given waste condition will be used as a bounding value in the flammability level evaluation. This ammonia vapor concentration can be calculated based on the measured liquid ammonia concentration and other tank waste conditions using Henry's Law and the Weisenberger and Schumpe (1996) model as 
described earlier. For the off-normal ventilation condition, which is about $0.5 \mathrm{ft}^{3} / \mathrm{min}$ or less, an equilibrium prediction of the ammonia concentration is reasonable and conservative.

\subsection{TIME TO REACH LOWER FLAMMABILITY LIMIT}

For active ventilation system interruptions, the time for the dome space to build up to flammable gas concentrations exceeding the LFL is one of the main concerns of this evaluation.

A time-dependent flammability level, $\operatorname{LFL}_{\mathrm{f}}(\mathrm{t})$ of the mixture can be calculated using the time-dependent gas concentration (Equation 3-6 for insoluble gas or Equation 3-12 for soluble gas) and Le Chatelier's rule (Equation 3-2) as provided in Equation 3-18:

$$
L F L_{f}(t)=\frac{\left[\mathrm{H}_{2}\right](t)}{L F L_{H 2}}+\frac{\left[\mathrm{NH}_{3}\right](t)}{L F L_{\mathrm{NH}}}+\frac{\left[\mathrm{CH}_{4}\right](t)}{L F L_{\mathrm{CH} 4}}
$$

where:
$\left[\mathrm{H}_{2}\right](\mathrm{t})=$ hydrogen concentration (volume percent) at time $\mathrm{t}$
$\left[\mathrm{NH}_{3}\right](\mathrm{t})=$ ammonia concentration (volume percent) at time $\mathrm{t}$
$\left[\mathrm{CH}_{4}\right](\mathrm{t})=$ methane concentration (volume percent) at time $\mathrm{t}$
$\mathrm{LFL}_{\mathrm{H} 2}=$ volume percent of hydrogen at its LFL in air alone
$\mathrm{LFL}_{\mathrm{NH} 3}=$ volume percent of ammonia at its LFL in air alone
$\mathrm{LFL}_{\mathrm{CH} 4}=$ volume percent of methane at its LFL in air alone.

The volume percent of the LFLs; $\mathrm{LFL}_{\mathrm{H} 2}, \mathrm{LFL}_{\mathrm{NH} 3}$, and $\mathrm{LFL}_{\mathrm{CH} 4}$ are 4,15 , and $5 \%$, respectively, which have been established for a fuel-air mixture.

The time to reach a specified gas concentration under given ventilation conditions for insoluble gas can be obtained by rewriting Equation 3-6 as Equation 3-19:

$$
t=\frac{\ln \left\{\frac{R_{g}-V_{r} \cdot C_{g}\left(t_{0}\right)}{R_{g}-V_{r} \cdot C_{g}(t)}\right\}}{\lambda}
$$

with

$$
\lambda \quad=\mathrm{V}_{\mathrm{r}} / \mathrm{Vol}
$$

where:

$\mathrm{R}_{\mathrm{g}} \quad=$ gas release rate (volume/time)

$\mathrm{V}_{\mathrm{r}} \quad=$ barometric breathing rate (volume/time)

$\left[\mathrm{C}_{\mathrm{g}}\right]\left(\mathrm{t}_{\mathrm{o}}\right)=$ initial gas concentration at time $\mathrm{t}_{\mathrm{o}}$ (volume percent)

$\left[\mathrm{C}_{\mathrm{g}}\right](\mathrm{t})=$ gas concentration at time $\mathrm{t}$ (volume percent). 
Similarly, for soluble gases, the time to reach a specified gas concentration under a given ventilation condition can be obtained by rewriting Equation 3-12 as Equation 3-20:

$$
t=\frac{\ln \left\{\frac{k_{2}-k_{1} \cdot C_{g}\left(t_{0}\right)}{k_{2}-k_{1} \cdot C_{g}(t)}\right\}}{k_{1}}
$$

with

$$
k_{1}=\left[V_{r}+h \cdot A \cdot K_{H} \cdot P \cdot v\right] /[V o l]
$$

and

$$
k_{2}=\left[h \cdot A \cdot C_{l}\right] /[\mathrm{Vol}]
$$

However, the time to reach a specified flammability limit of the mixture was not calculated explicitly. A customized Excel macro function, which was developed using Visual Basic ${ }^{6}$ and is based on Equation 3-19 for an insoluble gas and Equation 3-20 for a soluble gas, is used to calculate the time to reach a specific flammability limit.

\subsection{THE MINIMUM VENTILATION RATE TO MAINTAIN DOME SPACE BELOW 25 OR 100 PERCENT OF THE LOWER FLAMMABILITY LIMIT}

Whether the dome space of a tank will reach the specified flammability limit or not depends on the competition between the gas release rates and the ventilation rates of the system. For steady state, the flammability level as the fraction of the LFL of the mixture in the dome space can be calculated using Equation 3-2. For insoluble gas, the required steady-state gas concentrations in Equation 3-2 can be calculated using Equation 3-13 for insoluble gas and Equation 3-16 for soluble gas, where the ventilation rate is embedded in both equations. If the ventilation rate is zero (i.e., no gas exits the tank), the system will reach the LFL sooner or later, and then flammability will continue to increase beyond $100 \%$ of the LFL. The ventilation will reduce the steady-state concentration. A ventilation rate, defined as the minimum vent rate, which the steady-state concentrations will just reach the specified flammability limit using Equation 3-2 for parameters in Equations 3-13 and 3-16. If the dome space ventilation rate is larger than this minimum, the system will never reach the specified flammability limit. Again, the ventilation rate is embedded in the equation, and a customized Excel macro function developed using Visual Basic code is used to calculate the minimum ventilation needed to stay below the specified flammability limit.

\footnotetext{
${ }^{6} \mathrm{Visual}$ Basic is a registered trademark of Microsoft Corporation.
} 


\subsection{TIME-DEPENDENT GAS CONCENTRATION UNDER ZERO VENTILATION CONDITION}

For insoluble gas, under zero ventilation, the mass balance equation (Equation 3-5) can be rewritten as Equation 3-21:

$$
\operatorname{Vol} \frac{\partial\left\lfloor C_{g}\right\rfloor}{\partial t}=R_{g}
$$

where:

$$
\begin{aligned}
& {\left[C_{g}\right]=\text { gas concentration (volume percent) }} \\
& V o l=\text { volume of the tank dome space (volume) } \\
& R_{g}=\text { gas release rate from the waste surface (volume/time) }
\end{aligned}
$$

By solving the differential Equation 3-21, a time-dependent gas concentration at time $\mathrm{t},\left[C_{g}\right]_{\%}(t)$, in the unit of volume percent is given in Equation 3-22:

$$
\left[C_{g}\right]_{\%}(t)=\left[C_{g}\right]_{\%}\left(t_{0}\right)+\left[\frac{R_{g}}{V o l} \cdot t\right]_{\%}
$$

where:

$$
\begin{array}{ll}
R_{g} & \text { gas release rate from the waste surface (volume/time) } \\
{\left[C_{g}\right]_{\%}\left(t_{o}\right)} & =\text { initial gas concentration at time } \text { (volume percent) } \\
V o l & =\text { volume of the tank dome space (volume). }
\end{array}
$$

In Equation 3-22, $\left[C_{g}\right]_{\%}\left(t_{o}\right)$ represents initial gas concentration and $\left[R_{g} t / V_{O} l\right]_{\%}$ represents how the gas level builds up as time goes on. In the actual calculation in Chapter 4.0, the gas concentration was calculated analytically as shown in Equation 3-23 instead of as shown in Equation 3-22, because the hydrogen release rate is variable as time progresses rather than a fixed value. This is because the tank waste heats up under the zero ventilation condition and the hydrogen release rate increases.

$$
\left[C_{g}\right]_{\%}(t)=\left[C_{g}\right]_{\%}\left(t_{0}\right)+\sum_{t=0}^{t}\left[\frac{R_{g}(t)}{V o l} \cdot t\right]_{\%}
$$

The summation of gas concentration in the second term on the right side of Equation 3-23 is calculated in the time step of 1 day. As shown in Chapter 4.0, the time step of 1 day is a reasonable choice considering the change of $\mathrm{HGR}$ for the temperature increase rates used.

For a highly soluble gas such as ammonia, the mass balance in Equation 3-10 can be rewritten for the zero ventilation condition as shown in Equation 3-24:

$$
\operatorname{Vol} \frac{\partial\left\lfloor C_{g}\right\rfloor}{\partial t}=h \cdot A \cdot\left(C_{I}-K_{H} \cdot P \cdot v \cdot C_{g}\right)
$$


where:

$$
\begin{aligned}
& h=\text { overall mass transport coefficient from liquid to vapor }(\mathrm{m} / \mathrm{sec}) \\
& A=\text { effective area for the transport }\left(\mathrm{m}^{2}\right) \\
& C_{l}=\text { current ammonia concentration in liquid }\left(\mathrm{kg}-\mathrm{mole} / \mathrm{m}^{3}\right) \\
& P \quad=\quad \text { dome space total pressure (atm) } \\
& \nu=\text { specific molar volume of gas in the dome space }\left(\mathrm{m}^{3} / \mathrm{kg} \text {-mole }\right) \\
& C_{g}=\text { current ammonia concentration in the dome space (volume percent) } \\
& K_{H}=\text { Henry's Law constant }\left(\mathrm{kg}-\mathrm{mole} / \mathrm{m}^{3} \text {-atm }\right) \text {. }
\end{aligned}
$$

Dividing by $V o l$ and rearranging the terms, Equation 3-24 becomes Equation 3-25:

with

$$
\frac{\partial\left\lfloor C_{g}\right\rfloor}{\partial t}=g_{2}-g_{1} \cdot\left[C_{g}\right]
$$

and

$$
g_{1}=\frac{\left[h \cdot A \cdot K_{H} \cdot P \cdot v\right]}{V o l}
$$

$$
g_{2}=\frac{\left[h \cdot A \cdot C_{l}\right]}{V o l}
$$

The solution of Equation 3-25 is given as Equation 3-26:

$$
\left[C_{g}\right](t)=\left[C_{g}\right]\left(t_{0}\right) \exp \left(-g_{1} \cdot t\right)+\left[\frac{g_{2}}{g_{1}}\right]\left[1-\exp \left(-g_{1} \cdot t\right)\right]
$$

In some cases, particularly when $h A$ is large, the $g_{l} t$ is much greater than one in a few days. So the decay rate $\exp \left(-g_{1} . t\right)$ goes to zero in a few days, thus the vapor concentration is simply the vapor equilibrium concentration $C_{e q}$ with liquid phase as shown in Equation 3-27:

$$
C_{e q}=\frac{C_{l}}{K_{H} \cdot P \cdot v}
$$

\subsection{TANK WASTE TEMPERATURE INCREASE RATE}

Under normal operation, tank waste temperature ranged from an ambient temperature as low as $15^{\circ} \mathrm{C}$ up to $78^{\circ} \mathrm{C}$. This tank waste temperature range reflected seasonal temperature changes, which oscillate up and down throughout the year. During the off-normal operation, however, the tank waste temperature could increase due to the loss of active or passive ventilation. For DSTs, the tank waste temperature could increase even more if the ventilation of the annulus is shut down. For example, the tank waste temperature of DST $241-\mathrm{AY}-102$ increased $13{ }^{\circ} \mathrm{F}$ in one month while the annulus ventilation was shut down. It is known that the flammable gas generation rate is a function of temperature. In the HGR model (HNF-3851), the thermolysis 


\section{RPP-5926 REV 6}

rate and the organic $G$ value of the radiolysis rate are an exponential function of temperature. In this section, a simple and conservative heat transfer model (RPP-6213), used in evaluation of the steam bump hazard, is used for tank waste temperature increase analysis in the gas generation rate calculations and is described below.

With the tank under the zero ventilation condition, the temperature change with time can be estimated using a lumped capacitance solution for the transient waste temperature as shown in Equation 3-28:

$$
\left(\rho_{n c l} \cdot V_{n c l} \cdot c_{n c l}+\rho_{c l} \cdot V_{c l} \cdot c_{c l}\right) \frac{\partial T}{\partial t}=Q-\frac{k_{s} \cdot A \cdot\left(T-T_{\infty, s}\right)}{R}-\frac{k_{s} \cdot A \cdot\left(T-T_{\infty, a}\right)}{\delta}
$$

where:

$$
\begin{aligned}
& V_{n c l}=\text { volume of the nonconvective layer }\left(\mathrm{m}^{3}\right) \\
& \rho_{n c l}=\text { density of the nonconvective layer }\left(\mathrm{kg} / \mathrm{m}^{3}\right) \\
& c_{n c l}=\text { specific heat of the nonconvective layer }(\mathrm{J} / \mathrm{kg}-\mathrm{K}) \\
& V_{c l}=\text { volume of the convective layer }\left(\mathrm{m}^{3}\right) \\
& \rho_{c l}=\text { density of the convective layer }\left(\mathrm{kg} / \mathrm{m}^{3}\right) \\
& c_{c l}=\text { specific heat of the convective layer }(\mathrm{J} / \mathrm{kg}-\mathrm{K}) \\
& Q=\text { tank waste heat load }(\text { Watt }) \\
& k_{s}=\text { soil thermal conductivity }(\text { Watt } / \mathrm{m}-\mathrm{K}) \\
& A=\text { tank heat transfer area }\left(\mathrm{m}^{2}\right) \\
& T=\text { waste average temperature }(\mathrm{K}) \\
& T_{\alpha, s}=\text { average ambient soil temperature }(\mathrm{K}) \\
& T_{x, a}=\text { average ambient air temperature }(\mathrm{K}) \\
& \delta=\text { soil thickness }(\mathrm{m}) \\
& R=\text { tank radius as the length scale for heat conduction from tank } \\
& \quad \text { bottom to soil }(\mathrm{m}) .
\end{aligned}
$$

The right side of Equation 3-28 represents the net heat generation rate, which is the total heat source generation rate $Q$ from tank waste minus the downward (second term) and upward (third term) heat transfer rates. For a given tank waste temperature $T$ along with other waste conditions, the temperature increase rate $\partial T / \partial t$ can simply be calculated by dividing the net heat generation rate by the product of mass of waste and waste specific heat using Equation 3-28. The following parameter values were taken from RPP-6213 and apply to all tanks:

- Heat capacity: $3,300 \mathrm{~J} / \mathrm{kg}-\mathrm{K}$

- Air temperature: $285 \mathrm{~K}$

- Soil temperature: $287 \mathrm{~K}$

- Heat exchange area: $411 \mathrm{~m}^{2}$

- Soil thermal conductivity: $1 \mathrm{~W} / \mathrm{m}-\mathrm{K}$

- Soil overburden thickness: $4 \mathrm{~m}$

- Tank radius: $11 \mathrm{~m}$. 


\section{RPP-5926 REV 6}

Note that Equation 3-28 can be used for tanks having similar waste temperatures for convective and nonconvective layers. For tanks having distinct temperatures between layers (e.g., aging tank waste [241-AY/AZ Tank Farms]), the energy balance equation should be written for each layer as shown in Equations 3-29 and 3-30:

$$
\begin{aligned}
& \rho_{c l} \cdot V_{c l} \cdot c_{c l} \frac{\partial T_{c l}}{\partial t}=Q_{c l}-\frac{k_{s} \cdot A}{\delta}\left(T_{c l}-T_{\infty, a}\right)+h_{e x} \cdot A \cdot\left(T_{n c l}-T_{c l}\right) \\
& \rho_{n c l} \cdot V_{n c l} \cdot c_{n c l} \frac{\partial T_{n c l}}{\partial t}=Q_{n c l}-\frac{k_{s} \cdot A}{R}\left(T_{n c l}-T_{\infty, s}\right)-h_{e x} \cdot A \cdot\left(T_{n c l}-T_{c l}\right)
\end{aligned}
$$

where:

$T_{c l}=$ waste temperature of the convective layer $(\mathrm{K})$

$Q_{c l}=$ tank waste heat load of the convective layer (Watt)

$T_{n c l}=$ waste temperature of the nonconvective layer $(\mathrm{K})$

$Q_{n c l}=$ tank waste heat load of the nonconvective layer (Watt)

$h_{e x}=$ heat transfer coefficient for heat exchange between two layers $\left(\mathrm{W} / \mathrm{m}^{2} / \mathrm{K}\right)$.

Symbols that are not defined in Equations 3-29 and 3-30 are defined in Equation 3-28. As described in RPP-6213, the heat exchange coefficient was adjusted to meet the quasi-steady approximation in Equation 3-31:

$$
\frac{\partial T_{c l}}{\partial t}=\frac{\partial T_{n c l}}{\partial t}
$$

For the given waste temperatures and other waste conditions, once $h_{e x}$ is determined, the temperature increase rate $\partial T_{n c} / \partial t$ or $\partial T_{c l} / \partial t$ can be calculated.

As shown in Equations 3-28 to 3-30, this heat transfer model is relatively conservative in terms of overestimating the heat-up rate because it considers only the upward and downward heat transfer but neglects the heat transfer from the sides. Additionally, the temperature increase rates for the convective and nonconvective layers may be different throughout the heatup process while Equation 3-31 assumes they are the same. For example, while DST 241-AY-102 lost annulus cooling in August 2001, it was observed that the temperature increase rate for the convective layer was slower than the temperature increase rate for the nonconvective layer in the first few weeks; in time, the two temperature increase rates got closer. The calculated temperature increase rate $\left(0.27^{\circ} \mathrm{C} /\right.$ day from Table $\mathrm{H}-2$ of Revision 3$)$ using Equation 3-31 is comparable, but larger than the rate observed $\left(0.24^{\circ} \mathrm{C} /\right.$ day estimated from the temperature data on personal computer interface for SACS) in August 2001. Overall, the temperature data of DST 241-AY-102 validates that the assumption of Equation 3-31 is reasonable and conservative. 
RPP-5926 REV 6

This page intentionally left blank. 
RPP-5926 REV 6

4.0 EVALUATIONS OF STEADY-STATE FLAMMABILITY LEVEL

In this chapter, steady-state gas releases from the 28 DSTs and 149 SSTs are evaluated quantitatively against tank ventilation conditions using the methodology described in Chapters 2.0 and 3.0 to determine if the resulting flammability level becomes a safety concern.

Section 4.1 contains the calculated HGRs and discusses collection of input data and setup of the Excel spreadsheet for rate calculations. Section 4.2 covers the data collection, estimation of ventilation rates for both normal and off-normal conditions, and the steady-state gas concentrations for the normal ventilation rate. Section 4.3 discusses the steady-state gas release rates for the flammability level evaluation. For hydrogen, the gas release rate is the net result of the model-calculated gas generation rate minus the field-estimated gas accumulation rate. For ammonia, methane, and nitrous oxide, the gas release rates were extrapolated from steady-state concentrations at normal ventilation rates. For those tanks showing higher predicted steady-state ammonia concentrations (more than $0.5 \%$ ) under off-normal ventilation conditions and flammability levels exceeding $25 \%$ of the LFL in Revision 0 , the ammonia concentration has been reanalyzed with the liquid/vapor equilibrium model as described in Section 3.4. Section 4.4 describes the evaluation of flammability levels in the tank dome space for all 177 tanks. The evaluations include the following:

- Calculation of total flammability level, as well as the distribution from each flammable gas under both normal and off-normal ventilation conditions

- The time required for the flammable gases concentration in the tank dome space to reach various flammability levels when the normal ventilation system is shut down

- The minimum ventilation rate required to keep the dome space concentration below the specified flammability limit.

Details of the results of the inventory data from BBI and surveillance data from SACS, HGR calculations, ammonia mass transport properties, and flammability evaluations in the tank dome space for the 177 tanks are given in Appendices A through F. Appendices $\mathrm{G}$ and $\mathrm{H}$ provide flammability evaluation for C-200 series SSTs and DST 241-AY-102, respectively, under special conditions.

\subsection{HYDROGEN GENERATION RATE MODEL CALCULATIONS}

In the evaluation, the HGR was calculated for each tank based on the semi-empirical rate equation model. The total generation rate was calculated for each waste layer in each tank. The generation rate is a function of tank waste conditions; thus, a large tank waste database is needed. The analytical and surveillance data necessary to perform the rate calculations are available for some tanks. For those tanks without current measured data, process history data and waste type information were used.

An Excel spreadsheet was set up to calculate HGRs for all 177 Hanford Site DSTs and SSTs. The spreadsheet has four sections covering input data (Section 4.1.1), derived data 
(Section 4.1.2), and calculated unit rates and total rates (Section 4.1.3). The spreadsheet was set up to allow for easy upgrades and to facilitate checking the rate calculations.

\subsubsection{Input Data}

The data required to estimate hydrogen generation include the concentrations of TOC, hydroxide, nitrate ion, nitrite ion, sodium ion, and aluminate ion in the liquid phase; the total heat load for plutonium $\left({ }^{238} \mathrm{Pu},{ }^{239} \mathrm{Pu}\right.$, and $\left.{ }^{240} \mathrm{Pu}\right)$, americium $\left({ }^{241} \mathrm{Am}\right)$ cesium $\left({ }^{137} \mathrm{Cs}\right)$ and strontium $\left({ }^{90} \mathrm{Sr}\right)$; liquid and bulk densities; total solids and supernatant liquid tank waste volumes; the weight percent of water for liquid and sludge layers; tank waste temperature, and the tank dome space temperature. The organic species present provide the source term for thermolysis and organic radiolysis and the TOC data are used as an indicator of organic species. Aluminate is a catalyst in the thermal reaction and the aluminum ion concentration was used in the thermolysis rate calculation. Nitrate, nitrite, and sodium concentrations are used to estimate the scavenger effect for radiolysis of pure water. Also nitrate, nitrite, and hydroxide are used to evaluate the efficiency of hydrogen generation from the corrosion process of tank liner. Tank waste temperature is needed to calculate the radiolysis G-value and to account for the Arrhenius behavior of the thermolysis rate. The cesium and strontium concentrations are used to estimate the heat load from beta/gamma radiation of the tank waste, which is the power source for both water and organic radiolysis. Similarly, plutonium isotopes and americium are used to estimate the heat load from total alpha of the tank, and also function as the power source for both water and organic radiolysis. Weight-percent water data were used to estimate the liquid fraction of the waste because the model considers that gas generation reactions occur most effectively in the liquid phase. The density and waste volume are used to calculate the total mass of the waste and to estimate the wetted tank surface area to calculate the corrosion rates. The temperature in the tank dome space is used to convert the total gas generation rate from mole to volume per unit time.

The BBI, Tank Characterization Database (TCD), and SACS were queried to obtain the required waste properties and compositions. Data missing from the database were estimated, based on the methodology presented in SNL-000198, Flammable Gas Safety Analysis Data Review.

In Revision 0 of this report, the input data used were prepared based on data available as of October 1999 and documented in RPP-6069, Input Data for Hydrogen Generation Rate Model Calculation. In Revision 6 of this report, the input data of waste compositions including TOC, nitrate ion, nitrite ion, aluminum, cesium, strontium, density, and volume were queried from the BBI detail calculation database, and the preferred density and weight percent water in TWINS (queried on September 27, 2006). Note that the date of data quoted in the parenthesis is referred to as the date of data published, but is not the date of data gathering. The actual cut-off date of the data gathering of April 1, 2006, can be found in the inventory derivation documents. In addition, if the concentrations of TOC, nitrate ion, nitrite ion, and aluminum in liquid phase were not available from the BBI detailed calculations, the values prepared in Revision 0 were used or defaulted to the values in Revision 5, instead. 


\section{RPP-5926 REV 6}

To extract the information from the BBI detail report to the format of the required input data, several things were done:

- The required data were downloaded from BBI detail calculation database and listed in Appendix A.

- In BBI detail calculations, more than one density was used in a waste layer for a number of tanks. A single representative density labeled as preferred density was chosen for each waste layer for 177 tanks, and the preferred density along with weight percent water data were listed in Appendix A.

- In BBI detail calculations, the multiplier was introduced to compensate for differences in weight-percent water and density when the concentration data came from different data sources for a given waste layer. The concentrations used in the input data of Revision 6 of this report were recalculated by dividing the inventory of each analyte with the preferred density and the waste volume as

In BBI: $\quad$ Inventory $=$ density $\times$ Volume $\times$ Concentration $\times$ Multiplier

In this report: NewConcentration $=$ Inventory $\div($ PreferedDensity $\times$ Volume $)$

- The input data required the information from the supernatant liquid layer, solid layer (the waste below the supernatant liquid layer), and the total waste. The values of waste composition from various waste types of the solid layer were weight averaged to one value.

- The concentrations of TOC, nitrate ion, nitrite ion, and aluminum in the liquid phase and the liquid density for solid layer were assigned the same value as the supernatant liquid layer if no interstitial liquid data were available. The final waste inventory data are listed in Table B-1 of Appendix B.

In BBI development, the total waste volume is divided into three parts: solid, liquid, and retained gas (if there is any). In the detail calculation report, the volume of retained gas is not reported because it has no contribution to the analytes inventory. Although the retained gas has no contribution to the HGR calculations, it is part of the total waste volume and will impact the headspace volume calculation, and thus the flammability calculations. The reported retained gas volume is included in determining the waste depth and total waste volume for flammability calculations. Table A-3 in Appendix A lists the tanks with reported retained gas inventory from the BBI derivation document in TWINS.

The required temperature data of the tank waste and tank headspace were obtained from SACS for the time period from April 1, 2005 to April 1, 2006. The temperature data of tank waste and tank headspace were obtained from PCSACS. The maximum temperature from April 1, 2005 to April 1, 2006, was used to obtain an averaging temperature within each waste layer (supernatant and solid layers) and the headspace. The maximum value over a one year period throughout the tank was used to cover the seasonal effect. The temperature sensitivity study on the aging waste tanks was performed in Appendix $\mathrm{H}$ of Revision 3 of this report to prevent the possible 
underestimation of the temperature gradient vertically caused by cooling for using the average value.

With the given tank waste inventory data, physical properties like volume and density, and the waste and headspace temperature data as discussed above, an input data spreadsheet for HGR calculation was prepared and is listed in Table B-1 of Appendix B. For data not available from Appendix A, the information and data from previous revisions of this report are used.

In Revision 0 of this report, missing data were estimated, based on the methodology presented in SNL-000198. Waste tanks were first classified into one of six groups, based on the volumes of the liquid, saltcake, and sludge layers. The criteria for the various tank classifications also are presented in SNL-000198. Data from tanks in a given classification were used to create a distribution that was assumed to describe the total population of data for a given tank classification. This distribution then was used to provide a range of data including the missing data. In cases where insufficient data were available to create a statistically valid distribution, data from similar groups of tanks were combined to create a distribution of data (SNL-000198). For single-point calculations, the mean of the distribution is used to describe the missing values. In Monte Carlo statistical analyses, the full range of data for the tank waste classification was used.

This methodology of providing values and distributions for missing data was not optimized to provide the "best" waste properties or compositions with respect to waste chemical makeup. This methodology was prepared to determine missing data for flammable gas safety analyses. Because sufficient analytical data for all waste types are not available to estimate the missing data at the waste-type level, the methodology in SNL-000198 was used to determine overall composition ranges when a finer resolution of data was not statistically justified. As a result, the mean values used in this analysis were not optimized based on waste characteristics, but were developed to fit the available data related to flammable gas safety analyses.

The SST 241-A-105 HGR has not been calculated. The steel liner leaked and the tank has been interim stabilized. The waste moisture content is unknown, but is expected to be very low. The HGR is very sensitive to moisture content (the main hydrogen source). Because this key parameter is unknown and cannot be estimated, no attempt is made to estimate the corresponding HGR. The input data of DST 241-AY-102 are from the latest core sampling results and the HGRs of the solid layer are calculated by individual segments of the core sampling results. The latest sampling has reflected either depleted (Revision 4 using core 312 data) or very low (Revision 5 using core 319 data) nitrate and nitrite values on the bottom segment in the tank and results in a HGR increase compared to the HGR calculated by the averaged value of the BBI data.

\subsubsection{Derived Data}

Once the input data were provided, the derived data were calculated before performing the final rate calculations. In the "derived data section" the input data are converted to the system of units used in the equations and other necessary parameters are derived. The derivation includes waste level, the tank surface area wetted by the waste, total mass, liquid fractions of the layers, layer 
heat load, layer density, total heat load, conversion of nitrate ion and nitrite ion to units of "moles per liter," conversion of TOC and aluminum to units of "weight percent," and water radiolysis and total radiolysis G-values. The purpose of the derived data section is twofold. First, necessary unit conversions were built into the spreadsheet so the spreadsheet can take the input data directly from the TCD. Second, it prepares the necessary parameters, which reduces the cumbersome task of completing the rate calculations and makes error checking easier and more effective. Details of the derived data are provided in Appendix B, Table B-2.

\subsubsection{Calculated Hydrogen Generation Rates}

The HGRs were calculated for the total tank waste, as well as for different waste layers. The waste layers are the convective layer (supernatant liquid) and the nonconvective layer (sludge/saltcake); a few tanks have a crust layer on top of the convective layer. The rates for each layer are presented as radiolysis rates, thermolysis rates, corrosion rates, and total generation rates in units of mole per kilogram per day and moles per cubic meter per second.

Details of the calculated rates per unit mass or per unit volume are provided in the Appendix B, Table B-3. Note that the unit rate of the corrosion rate is a function of wetted surface area, so the unit volume rate depends on the waste volume. The unit rate from corrosion is an average rate for a given tank waste volume. The total gas generation rate for a tank (in units of cubic feet per minute and liters per day) was calculated by multiplying the unit rates by the total waste volume; the results are provided in Appendix B, Table B-4. Table 4-1 lists the 10 tanks with the highest model-calculated hydrogen generation based on the current waste content.

For the water addition study, the input data are modified by adding $10 \mathrm{kgal}$ of water to 100 -series tanks and $1 \mathrm{kgal}$ to 200 -series tanks and listed in Appendix B, Table B-5. The required input data listed in Appendix B, Table B-1, are used as the starting point before water addition. It is assumed that the water is well mixed with supernatant, and that the concentration, density, and the weight percent of water of the supernatant are adjusted accordingly by a simple dilution factor. The dilution factor is the ratio between current waste volume and current waste volume plus the volume of $10 \mathrm{kgal}$ and $1 \mathrm{kgal}$ of water for 100 - and 200 -series tanks. 
Table 4-1. Calculated Top Ten Hydrogen Generation Rates In Dome Space for

177 Tanks With Current Waste.

\begin{tabular}{|c|c|c|c|c|c|c|c|c|c|c|}
\hline Tank & $\begin{array}{c}\text { HGR from } \\
\text { radiolysis } \\
\text { Grad b/r } \\
\left(\text { ft }^{3} / \mathrm{min}\right)\end{array}$ & \begin{tabular}{|} 
HGR from \\
radiolysis \\
Grad \\
alpha \\
$\left(\mathrm{ft}^{3} / \mathrm{min}\right)$
\end{tabular} & $\begin{array}{l}\text { HGR from } \\
\text { thermo- } \\
\text { lysis } \\
\text { Gtherm } \\
\left(\mathrm{ft}^{3} / \mathrm{min}\right)\end{array}$ & $\begin{array}{c}\text { HGR from } \\
\text { corrosion } \\
\text { Gcorr } \\
\left(\mathrm{ft}^{3} / \mathrm{min}\right)\end{array}$ & $\begin{array}{c}\text { Percent of } \\
\text { HGR from } \\
\left.\text { radiolysis }^{\left(\text {Grad }^{6 / r}\right) \%}\right)\end{array}$ & $\begin{array}{l}\text { Percent of } \\
\text { HGR from } \\
\text { radiolysis } \\
\text { (Grad } \\
\text { alpha) } \%\end{array}$ & $\begin{array}{l}\text { Percent of } \\
\text { HGR from } \\
\text { thermolysis } \\
\text { (Gtherm)\% }\end{array}$ & $\begin{array}{l}\text { Percent of } \\
\text { HGR from } \\
\text { corrosion } \\
\text { (Gcorr) } \%\end{array}$ & $\begin{array}{c}\text { Total HGR } \\
\text { from model } \\
\text { Gmod } \\
\left(\mathrm{ft}^{3} / \mathrm{min}\right)\end{array}$ & $\begin{array}{c}\text { Total HGR } \\
\text { from model } \\
\text { HGR }_{\text {mod }} \\
\text { (L/day) }\end{array}$ \\
\hline $241-A Z-102$ & $2.82 \mathrm{E}-02$ & $2.11 \mathrm{E}-03$ & $2.36 \mathrm{E}-03$ & $1.53 \mathrm{E}-04$ & $85.9 \%$ & $6.44 \mathrm{E}-02$ & $7.2 \%$ & $0.5 \%$ & $3.28 \mathrm{E}-02$ & 1338 \\
\hline Supernatant & $1.21 \mathrm{E}-02$ & $3.52 \mathrm{E}-06$ & 1.83E-03 & 8.32E-05 & $86.3 \%$ & $2.52 \mathrm{E}-04$ & $13.1 \%$ & $0.6 \%$ & $1.40 \mathrm{E}-02$ & 570 \\
\hline Solid Layer & $1.61 \mathrm{E}-02$ & $2.11 \mathrm{E}-03$ & $5.30 \mathrm{E}-04$ & $6.98 \mathrm{E}-05$ & $85.6 \%$ & 1.12E-01 & $2.8 \%$ & $0.4 \%$ & $1.88 \mathrm{E}-02$ & 768 \\
\hline $241-\mathrm{AY}-102$ & $2.54 \mathrm{E}-02$ & $6.47 \mathrm{E}-04$ & $1.24 \mathrm{E}-03$ & $3.57 \mathrm{E}-04$ & $91.9 \%$ & $2.34 \mathrm{E} \cdot 02$ & $4.5 \%$ & $1.3 \%$ & $2.77 \mathrm{E}-02$ & 1128 \\
\hline Supernatant & $2.47 \mathrm{E}-04$ & $8.96 \mathrm{E}-07$ & $8.70 \mathrm{E}-04$ & $1.84 \mathrm{E}-04$ & $19.0 \%$ & $6.89 \mathrm{E}-04$ & $66.9 \%$ & $14.1 \%$ & $1.30 \mathrm{E}-03$ & 53 \\
\hline Solid Layer & $2.52 \mathrm{E}-02$ & $6.46 \mathrm{E}-04$ & $3.73 \mathrm{E}-04$ & $1.73 \mathrm{E}-04$ & $95.5 \%$ & 2.45E-02 & $1.4 \%$ & $0.7 \%$ & $2.64 \mathrm{E}-02$ & 1075 \\
\hline $241-\mathrm{AZ}-10 \mathrm{I}$ & $1.67 \mathrm{E}-02$ & $7.11 \mathrm{E}-04$ & $4.22 \mathrm{E}-03$ & $1.45 \mathrm{E}-04$ & $76.7 \%$ & $3.26 \mathrm{~F}-02$ & $19.3 \%$ & $0.7 \%$ & $2.18 \mathrm{E}-02$ & 889 \\
\hline Supernatant & $8.79 \mathrm{E}-03$ & $3.40 \mathrm{E}-07$ & $3.63 \mathrm{E}-03$ & $7.94 \mathrm{E}-05$ & $70.3 \%$ & 2.72E-05 & $29.0 \%$ & $0.6 \%$ & 1.25E-02 & 510 \\
\hline Solid Layer & $7.94 \mathrm{E}-03$ & $7.11 \mathrm{E}-04$ & $5.86 \mathrm{E}-04$ & $6.57 \mathrm{E}-05$ & $85.4 \%$ & $7.65 \mathrm{E}-02$ & $6.3 \%$ & $0.7 \%$ & $9.30 \mathrm{E}-03$ & 379 \\
\hline 241-AN-102 & $3.88 \mathrm{E}-03$ & $2.55 \mathrm{E}-05$ & $5.86 \mathrm{E}-03$ & $1.47 \mathrm{E}-04$ & $39.1 \%$ & $2.57 \mathrm{E}-03$ & $59.1 \%$ & $1.5 \%$ & $9.92 \mathrm{E}-03$ & 404 \\
\hline Supernatant & $3.19 \mathrm{E}-03$ & $1.32 \mathrm{E}-05$ & $5.03 E-03$ & $7.99 \mathrm{E}-05$ & $38.4 \%$ & $1.59 \mathrm{E}-03$ & $60.5 \%$ & $1.0 \%$ & $8.32 \mathrm{E}-03$ & 339 \\
\hline Solid Layer & $6.89 \mathrm{E}-04$ & $1.22 \mathrm{E}-05$ & $8.30 \mathrm{E}-04$ & $6.75 \mathrm{E}-05$ & $43.1 \%$ & $7.66 \mathrm{E}-03$ & $51.9 \%$ & $4.2 \%$ & $1.60 \mathrm{E}-03$ & 65 \\
\hline 24l-AN-107 & $4.45 \mathrm{E}-03$ & $9.35 \mathrm{E}-05$ & $3.56 \mathrm{E}-03$ & $1.50 \mathrm{E}-04$ & $53.9 \%$ & $1.13 \mathrm{E}-02$ & $43.1 \%$ & $1.8 \%$ & $8.25 \mathrm{E}-03$ & 336 \\
\hline Supernatant & $3.16 \mathrm{E}-03$ & $6.51 \mathrm{E}-05$ & $2.80 \mathrm{E}-03$ & $7.59 \mathrm{E}-05$ & $51.8 \%$ & $1.07 \mathrm{E}-02$ & $45.9 \%$ & $1.2 \%$ & $6.10 \mathrm{E}-03$ & 249 \\
\hline Solid Layer & $1.29 \mathrm{E}-03$ & $2.83 \mathrm{E}-05$ & $7.55 \mathrm{E}-04$ & $7.41 \mathrm{E}-05$ & $60.1 \%$ & $1.32 \mathrm{E}-02$ & $35.1 \%$ & $3.4 \%$ & $2.15 \mathrm{E}-03$ & 88 \\
\hline 24l-AN-106 & $6.61 \mathrm{E}-03$ & $2.04 \mathrm{E}-04$ & $7.87 \mathrm{E}-05$ & $1.20 \mathrm{E}-04$ & $94.3 \%$ & $2.91 \mathrm{E}-02$ & $1.1 \%$ & $1.7 \%$ & $7.01 \mathrm{E}-03$ & 286 \\
\hline Supernatant & $4.01 \mathrm{E}-04$ & $8.13 \mathrm{E}-07$ & $5.96 \mathrm{E}-05$ & $5.53 \mathrm{E}-05$ & $77.6 \%$ & $1.57 \mathrm{E}-03$ & $11.5 \%$ & $10.7 \%$ & $5.17 \mathrm{E}-04$ & 21 \\
\hline Solid Layer & $6.20 \mathrm{E}-03$ & $2.03 \mathrm{E}-04$ & $1.91 \mathrm{E}-05$ & $6.46 \mathrm{E}-05$ & $95.6 \%$ & $3.13 \mathrm{E}-02$ & $0.3 \%$ & $1.0 \%$ & $6.49 \mathrm{E}-03$ & 265 \\
\hline 241-SX-103 & $8.26 \mathrm{E}-04$ & $1.65 \mathrm{E}-05$ & $5.63 \mathrm{E}-03$ & $3.98 \mathrm{E}-04$ & $12.0 \%$ & $2.40 \mathrm{E}-03$ & $81.9 \%$ & $5.8 \%$ & $6.87 E-03$ & 280 \\
\hline Supernatant & NA & NA & $\mathrm{NA}$ & NA & NA & NA & NA & NA & NA & NA \\
\hline Solid Layer & $8.26 \mathrm{E}-04$ & $1.65 \mathrm{E}-05$ & $5.63 \mathrm{E}-03$ & $3.98 \mathrm{E}-04$ & $12.0 \%$ & $2.40 \mathrm{E}-03$ & $81.9 \%$ & $5.8 \%$ & 6.87E-03 & 280 \\
\hline $241-A Y-101$ & $5.13 \mathrm{E}-03$ & $7.44 \mathrm{E}-04$ & $2.51 \mathrm{E}-04$ & $1.89 \mathrm{E}-04$ & $81.2 \%$ & $1.18 \mathrm{E}-01$ & $4.0 \%$ & $3.0 \%$ & $6.31 \div-03$ & 257 \\
\hline Supernatant & $3.38 \mathrm{E}-05$ & $9.16 \mathrm{E}-07$ & $1.23 \mathrm{E}-04$ & $2.54 \mathrm{E}-05$ & $18.5 \%$ & $5.00 \mathrm{E}-03$ & $67.1 \%$ & $13.9 \%$ & $1.83 \mathrm{E}-04$ & 7 \\
\hline Solid Layer & $5.09 \mathrm{E}-03$ & $7.43 \mathrm{E}-04$ & $1.28 \mathrm{E}-04$ & 1. $64 \mathrm{E}-04$ & $83.1 \%$ & $1.21 \mathrm{E}-01$ & $2.1 \%$ & $2.7 \%$ & $6.13 \mathrm{E}-03$ & 250 \\
\hline $241-\mathrm{SX}-105$ & $8.20 \mathrm{E}-04$ & $2.43 \mathrm{E}-05$ & $3.95 \mathrm{E}-03$ & $3.60 \mathrm{E}-04$ & $15.9 \%$ & $4.72 \mathrm{E}-03$ & $76.6 \%$ & $7.0 \%$ & $5.15 \mathrm{E}-03$ & 210 \\
\hline Supernatant & NA & NA & NA & NA & NA & $\mathrm{NA}$ & NA & NA & NA & NA \\
\hline Solid Layer & $8.20 \mathrm{E}-04$ & $2.43 \mathrm{E} \cdot 05$ & $3.95 \mathrm{E}-03$ & $3.60 \mathrm{E}-04$ & $15.9 \%$ & $4.72 \mathrm{E}-03$ & $76.6 \%$ & $7.0 \%$ & $5.15 \mathrm{E}-03$ & 210 \\
\hline 241-SY-103 & $1.06 \mathrm{E}-03$ & $2.43 \mathrm{E}-05$ & $2.12 \mathrm{E}-03$ & $1.15 \mathrm{E}-04$ & $31.9 \%$ & $7.33 \mathrm{E}-03$ & $63.9 \%$ & $3.5 \%$ & $3.31 \mathrm{E}-03$ & 135 \\
\hline Supernatant & $5.63 \mathrm{E}-04$ & $3.06 \mathrm{E}-07$ & $5.46 \mathrm{E}-04$ & $3.35 \mathrm{E}-05$ & $49.3 \%$ & $2.68 \mathrm{E}-04$ & $47.8 \%$ & $2.9 \%$ & $1.14 \mathrm{E}-03$ & 47 \\
\hline Solid Layer & $4.93 \mathrm{E}-04$ & $2.40 \mathrm{E}-05$ & $1.57 \mathrm{E}-03$ & $8.12 \mathrm{E}-05$ & $22.7 \%$ & $1.11 \mathrm{E}-02$ & $72.5 \%$ & $3.7 \%$ & $2.17 \mathrm{E}-03$ & 89 \\
\hline
\end{tabular}

Notes:

To convert cubic feet to liters, multiply the value by $28.454 \mathrm{~L} / \mathrm{ft}^{3}$.

$\mathrm{HGR}=$ hydrogen generation rate.

NA $=$ not applicable.

DST 241-AZ-102 has the largest HGR, $3.28 \mathrm{E}-2 \mathrm{ft}^{3} / \mathrm{min}$; this rate is approximately a $30 \%$ increase over the value of $2.22 \mathrm{E}-2 \mathrm{ft}^{3} / \mathrm{min}$ calculated in Revision $4 \mathrm{~A}$ of this report. The rate difference may result from the latest $\mathrm{BBI}$ correction on the strontium concentration of tank 241-AZ-102. In addition, the DST 241-AY-102 HGR has dropped from 3.60 E-2 to $2.77 \mathrm{E}-2 \mathrm{ft}^{3} / \mathrm{min}$ due to the relatively larger nitrate and nitrite concentration results in the bottom 
segment rather than the depleted values used in the Revision 4 calculation. The generation rate of the solid layer in Revision 5 and 6 was calculated by summing the generation rate of individual layers based on each segment's data from the recent core sampling. Generation rates from radiolysis, thermolysis, and corrosion are listed in Table 4-1 for solid and liquid layers for each tank. The contribution from thermolysis and radiolysis varied from tank to tank. Corrosion contributed $7 \%$ or less to the overall HGR for the listed tanks in Table 4-1. Tanks without sample-based data use the default data from the waste grouping analysis, which have a larger uncertainty.

\subsection{TANK VENTILATION RATES AND STEADY-STATE GAS CONCENTRATIONS}

Tank ventilation and steady-state gas concentration data collection and data reduction are documented in RPP-5660, Collection and Analysis of Selected Tank Headspace Parameter Data. Ventilation rates at the tank outlet were determined by a number of methods, including tracer studies, Standard Hydrogen Monitoring System hydrogen composition decay curves, direct measurements of hydrogen, and calculations from earlier flammable gas hazard analyses. The ventilation rates were for normal ventilation conditions, either passive or active, in conjunction with steady-state gas concentration data to estimate the gas release rates.

Dome space gas composition data were based on analytical data from RPP-5660, when available. The TWINS database was queried to obtain all gas sample data. The data were reduced using mean values for single-point calculations and derived distributions for Monte Carlo analyses. Values for missing data were determined using the methodology described in SNL-000198. The ventilation rates and the steady-state gas concentrations under normal operating conditions, along with waste volumes and dome space volumes for all 177 tanks, are listed in Appendix D, Table D-1. For sludge tanks, dome space gas concentrations are very small, less than $50 \mathrm{ppm}$ hydrogen, methane, and ammonia. For these tanks, the ratios between the various gases are questionable and are not an accurate analysis of gas generation rates.

\subsection{STEADY-STATE GAS RELEASE RATES FOR $\mathrm{H}_{2}, \mathrm{NH}_{3}, \mathrm{CH}_{4}, \mathrm{AND} \mathrm{N}_{2} \mathrm{O}$}

To evaluate the steady-state flammability levels under different ventilation conditions, the steady-state gas release rates were used to calculate the flammable gas concentrations for a given ventilation condition. For hydrogen, the steady-state release rates were calculated by subtracting the gas accumulation rate from the model calculated generation rate. In this report, the accumulation rates are assumed to be zero because no noticeable waste level increase due to gas accumulation has been reported recently. The HGRs are calculated in Section 4.1 and listed in Appendix B. For all cases, the gas release rate is equal to the model calculated gas generation rate. In the past, a few tanks had a noticeable waste level increase. The waste level increase rate can be converted to a total waste volume increase rate, which then is multiplied by the hydrogen fraction obtained from the retained gas sample analysis. The steady-state gas release rates of $\mathrm{CH}_{4}, \mathrm{NH}_{3}$, and $\mathrm{N}_{2} \mathrm{O}$ were derived from the steady-state concentrations under normal ventilation rates using Equation 3-15. The historical data derived release may be too large compared to the calculated hydrogen generation rate because of the effect of waste transfers. Since the calculated hydrogen generation rate reflects the latest tank inventory data, the calculations of methane 
release were constrained to no more than $20 \%$ of the latest calculated hydrogen generation rate of that tank. The calculated gas release rates of $\mathrm{H}_{2}, \mathrm{NH}_{3}, \mathrm{CH}_{4}$, and $\mathrm{N}_{2} \mathrm{O}$ are listed in Appendix D, Table D-1.

For ammonia, however, the method described in the previous paragraph would largely overestimate the steady-state concentrations under off-normal ventilation conditions for those tanks having relatively large release rates calculated from the steady-state concentration. For example, for DST 241-SY-101, the steady-state release rate obtained from the dome space concentration of $400 \mathrm{ppm}$ under normal ventilation conditions predicts a steady-state concentration of $108 \%$ of the LFL for the off-normal ventilation condition. This value is two orders of magnitude larger than the current estimated value of $0.4 \%$ of the LFL using the liquid-vapor equilibrium mechanism. In Revision 0 , similar overestimations of ammonia concentration also were reported for DSTs 241-AN-102 and 241-AN-107.

As described in Chapter 3.0, ammonia is very soluble, and the gas release rate is not a constant but is governed by the liquid-vapor equilibrium defined by Henry's Law, the mass transport coefficient, h, and the effective transport surface area, A. The ammonia release rate is driven by the gradient between the vapor and liquid phases before reaching equilibrium. Therefore, a greater difference results in a larger release rate.

For a closed system (i.e., a zero ventilation rate), the final release rate is zero when the system reaches equilibrium. An open system will never reach the equilibrium concentration, but will reach a steady-state release condition. At steady state, the concentration is lower than the equilibrium value, and the larger the ventilation rate, the lower the steady-state concentration of ammonia. For a given system, the release rate at steady state also is proportional to the ventilation rate. Therefore, if the steady-state release rate obtained at a high ventilation rate (i.e., normal active ventilation) is used as a constant to predict the steady-state concentrations for the off-normal low ventilation condition, the ammonia dome space concentration for the off-normal ventilation condition could be greatly overestimated.

Figure 4-1 demonstrates the relationship of the equilibrium value, the steady-state concentration, and the release rate of ammonia under both normal and off-normal conditions for DST 241-SY-101 after remediation. As shown in Figure 4-1 for the normal ventilation rate of $486 \mathrm{ft}^{3} / \mathrm{min}$, the steady-state concentration of ammonia is $400 \mathrm{ppm}$, which is far below the equilibrium value of $3,895 \mathrm{ppm}$. For an off-normal ventilation rate of $0.18 \mathrm{ft}^{3} / \mathrm{min}$, the ammonia concentration is computed to increase exponentially and took less than 8 days to reach steady state with the concentration of $3,882 \mathrm{ppm}$, which is lower than but very close to the equilibrium value. Also, note that the steady-state release rate from $0.19 \mathrm{ft}^{3} / \mathrm{min}$ under normal ventilation conditions of $486 \mathrm{ft}^{3} / \mathrm{min}$ is computed to drop exponentially to $7.6 \mathrm{E}-4 \mathrm{ft}^{3} / \mathrm{min}$ for the off-normal ventilation condition of $0.18 \mathrm{ft}^{3} / \mathrm{min}$. 
Figure 4-1. Ammonia Concentration and Release Rate for Double-Shell Tank 241-SY-101 Before and After Loss of Active Ventilation.

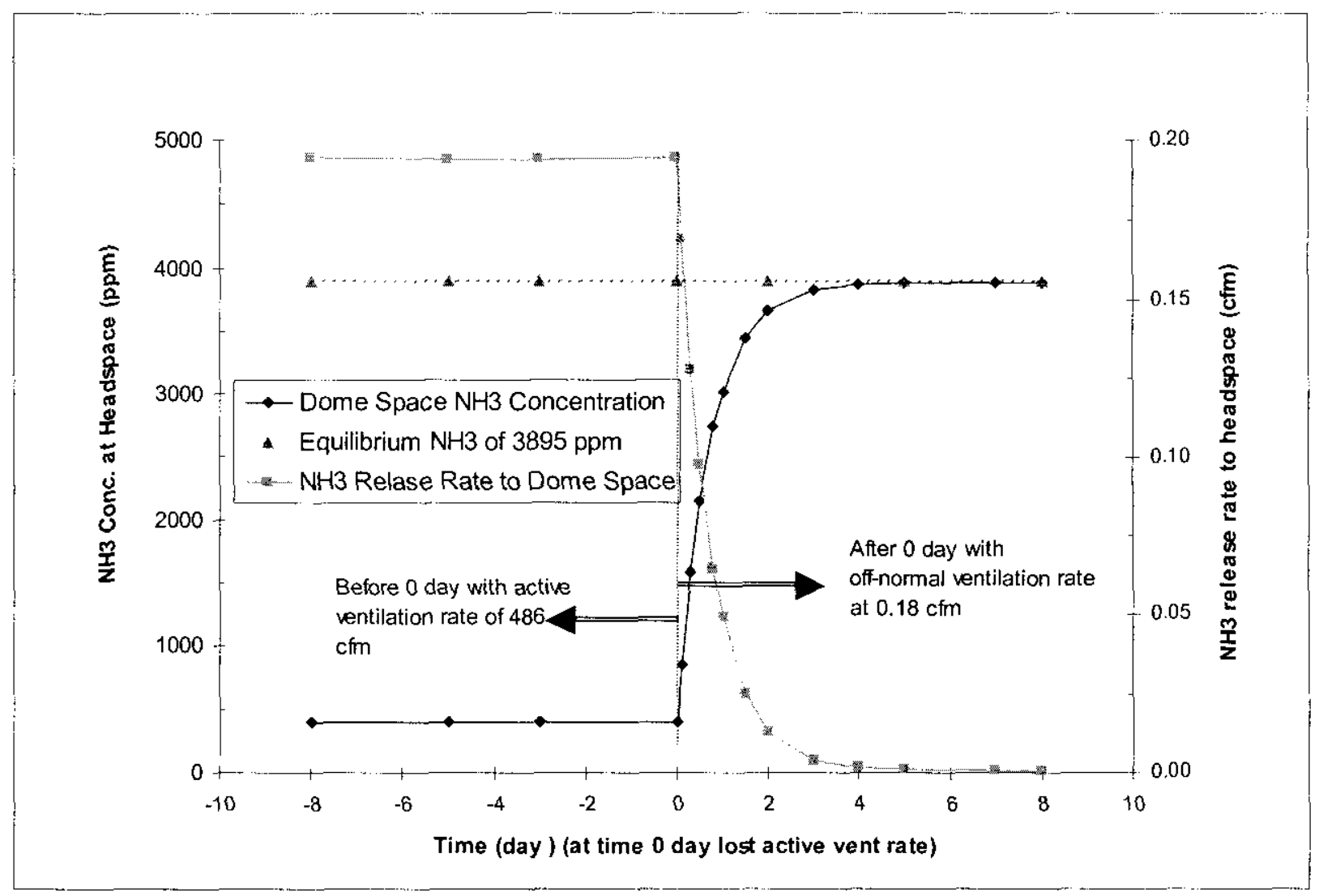

For many SSTs and some of the DSTs with lower concentrations of ammonia, the ammonia release rate was too small to contribute significantly to reaching $25 \%$ of the LFL even under off-normal ventilation conditions. Therefore, this method of considering ammonia release rates as constants would be a simple method for screening out tanks with small release rates. Using this method would prevent use of the liquid-vapor equilibrium method, which requires liquid and dome space ammonia concentration data and more complex calculations.

\subsection{STEADY-STATE MASS TRANSPORT PROPERTIES "h" AND "A" FOR AMMONIA}

As discussed in Chapter 3.0, it is difficult to estimate the effective area, A, of the mass transport in the liquid-vapor equilibrium model. The effective area is the actual waste surface area available for ammonia transport, and will be the full or partial tank cross-sectional area depending on how much true liquid surface is available. The ammonia concentration could be overestimated by two orders of magnitude in the liquid-vapor equilibrium model if $100 \%$ of the 
effective area is used. In this report, the ammonia concentration analysis follows the logic in the following steps to perform the dome space ammonia calculations:

1. The ammonia concentration in all DSTs was analyzed using the liquid-vapor equilibrium model as described in Chapter 3.0. The mass transport properties, the product of $\mathrm{h}$ and $\mathrm{A}$, were obtained from the steady-state concentration under normal conditions by applying Equation 3-17. Then, using this product of $\mathrm{h}$ and $\mathrm{A}$, the time-dependent ammonia concentration and flammability under off-normal conditions were calculated.

2. The ammonia concentrations for the SSTs were first evaluated by assuming a constant release rate. If the ammonia concentrations computed using this conservative approach were more than $0.5 \%$ and the total flammability was above $20 \%$ of the LFL, the ammonia concentration was reanalyzed more accurately using the liquid-vapor equilibrium method as was done for the DSTs.

The input data collected included the ammonia concentrations in the liquid and in the dome space under normal ventilation conditions, the waste volume and ionic composition, and the temperatures of the waste and the dome space under both normal and off-normal conditions. Appendix C, Table C-1, gives the liquid concentrations in the DSTs and selected SSTs for the ions such as nitrate, nitrite, and aluminum used in the calculation of the HGR. The additional ion constituents required are carbonate, chloride, chromium, fluorine, hydroxide, iron, nickel, phosphate, potassium, sodium, and sulfate. Other required data include the tank waste density, temperature, and weight-percent water. These data were obtained from the BBI reports. Where tank ion concentrations or waste properties were not available in the BBI detail report, an average value from the sample analysis data contained in the TCD was used. The tank waste temperature values were the only figures not taken from the BBI or the TCD. They were obtained from RPP-5660, Table 3-2. However, the input data for ammonia calculations were not updated in Revision 3 because the required liquid ammonia data is not available. The ammonia calculations remain the same as in Revision 1 of the report; however, ammonia has a minor contribution to the flammability at steady-state.

Based on Equation 3-8, the Henry's Law constants were calculated and are listed in Appendix C, Table C-2. Table C-2 also includes the summations of ion concentrations, the Henry's Law constants in pure water, and the correction factors for the calculated Henry's Law constants, $\mathrm{K}_{\mathrm{H}}$, for salt solutions. The correction factors were assigned using the tank waste concentrations and temperatures according to the study results for ammonia solubility in high-concentration salt solutions (RPP-4941). The mass transport properties of the product $\mathrm{h}$ and A were calculated using Equation 3-17 and are listed in Appendix C, Table C-3. Based on the products of $\mathrm{h}$ and A, the time-dependent ammonia concentration and percent of the LFL under off-normal ventilation conditions were calculated, as discussed in Section 4.5. As discussed in Chapter 3.0, the product of $\mathrm{h}$ and $\mathrm{A}$ depends on the waste configuration and physical and chemical properties, particularly the surface area, A, which is the effective surface for gas transport from the liquid to the vapor phase. For example, if the waste was disturbed or the configuration of the waste surface changed, both the effective surface area and $h$ could change, so the product of $h$ and $A$ would change. Therefore, what has been calculated as the product of $h$ and $A$ in this report reflects only the current ammonia transport status of storage tank waste at steady state. 
As a comparison, Table 4-2 lists the products of $h$ and $A$ from the values derived from the field data under normal conditions using Equation 3-17 and from the calculated h using the equations in Appendix E with a 100\% tank surface area, A. For most of the tanks, the values for $\mathrm{h}$ and $\mathrm{A}$ derived from field data are a few orders of magnitude smaller than the values from the calculated $\mathrm{h}$ and $100 \%$ area A. Two possible factors can contribute to this discrepancy. First, many tanks may not have $100 \%$ transport area A, particularly for those tanks having a large crust layer or solid waste on the surface. Second, from Equation 3-17, the field-data-derived values of $h$ and A rely mainly on the ammonia vapor data and ventilation rate in the headspace, and the corresponding value of liquid concentration in the waste (usually the value of $\mathrm{K}_{\mathrm{H}} \mathrm{P}_{\mathrm{V} \mathrm{C}}{ }_{\mathrm{g}}^{\text {ss }}$ is much smaller than $\mathrm{C}_{1}$ ). The uncertainties of these input data and the fact that these data are not measured simultaneously can contribute to errors in the field-derived $h$ and A values.

Next, the ammonia underneath the liquid surface could be depleted and present a large gradient throughout the tank. In this case, the measured liquid-phase ammonia data from the middle or bottom sections of the tank will be too large for the observed vapor data. Also, the equations used to calculate $\mathrm{h}$ in Appendix $\mathrm{E}$ may involve large uncertainties. In all cases, note that the values of the product of $\mathrm{h}$ and A determine how fast the ammonia will be released to the dome space. The bounding value for the flammability is the steady-state ammonia concentration in the dome space. The last column of Table 4-2 lists the steady-state ammonia concentrations under off-normal conditions. For all calculated values, the ammonia concentration is less than $8,300 \mathrm{ppm}$, which contributes less than $6 \%$ of the LFL to the total flammability.

Table 4-2. The Calculation of Ammonia Transport Properties. (2 sheets)

\begin{tabular}{|c|c|c|c|c|c|c|}
\hline Tank & $\begin{array}{c}\left(\mathrm{NH}_{3}\right)^{\mathrm{ss}} \text { in } \\
\text { Dome Under } \\
\text { Normal Vent } \\
(\mathrm{ppm})\end{array}$ & $\begin{array}{c}\text { Liquid }\left[\mathrm{NH}_{3}\right] \\
\text { in Waste } \\
(\mu \mathrm{g} / \mathrm{mL})\end{array}$ & $\begin{array}{l}\text { Normal Vent } \\
\text { Rate in the } \\
\text { Dome Space } \\
\left(\mathrm{ft}^{3} / \mathrm{min}\right)\end{array}$ & $\begin{array}{c}\text { Product } h \text { A } \\
\left(\mathrm{ft}^{3} / \mathrm{min}\right) \text { From } \\
\text { Field Data at } \\
\text { Normal Vent }\end{array}$ & $\begin{array}{c}\text { Product h A } \\
\left(\mathrm{ft}^{3} / \text { min) From }\right. \\
\text { Calculated h } \\
\text { and } 100 \% \mathrm{~A}\end{array}$ & $\begin{array}{l}\left(\mathrm{NH}_{3}\right)^{\mathrm{ss}} \text { at } \\
\text { Off-Normal } \\
\text { Vent (ppm) }\end{array}$ \\
\hline 241-AN-101 & 11 & 40 & 113 & $2.85 \mathrm{E}-02$ & $1.02 \mathrm{E}+00$ & 55 \\
\hline 241-AN-102 & 300 & 210 & 119 & $1.80 \mathrm{E}-01$ & $2.03 E+00$ & 857 \\
\hline 241-AN-103 & 7 & 345 & 100 & $1.33 \mathrm{E}-03$ & $3.50 \mathrm{E}+00$ & 2,034 \\
\hline 241-AN-104 & 21 & 246 & 100 & $5.92 \mathrm{E}-03$ & $3.08 \mathrm{E}+00$ & 2,011 \\
\hline 241-AN-105 & 15 & 210 & 100 & $5.02 \mathrm{E}-03$ & $2.91 E+00$ & 1,523 \\
\hline 241-AN-106 & 1 & 210 & 117 & 3.99 E-04 & $1.12 \mathrm{E}+00$ & 123 \\
\hline 241-AN-107 & 400 & 210 & 129 & $3.60 \mathrm{E}-01$ & $1.94 \mathrm{E}+00$ & 759 \\
\hline 241-AP-101 & 125 & 439 & 108 & 2.57 E-02 & $1.26 \mathrm{E}+00$ & 827 \\
\hline 241-AP-102 & 75 & 439 & 135 & $1.83 \mathrm{E}-02$ & $1.15 \mathrm{E}+00$ & 728 \\
\hline 241-AP-103 & 1 & 250 & 200 & $5.76 \mathrm{E}-04$ & $1.48 \mathrm{E}+00$ & 248 \\
\hline 241-AP-104 & 3 & 133 & 71 & $1.23 \mathrm{E}-03$ & $1.18 \mathrm{E}+00$ & 136 \\
\hline 241-AP-105 & 7 & 324 & 176 & $2.79 \mathrm{E}-03$ & $1.75 \mathrm{E}+00$ & 800 \\
\hline 241-AP-106 & 5 & 68 & 111 & $6.54 \mathrm{E}-03$ & $1.11 \mathrm{E}+00$ & 104 \\
\hline 241-AP-107 & 5 & 1,060 & 342 & $1.16 \mathrm{E}-03$ & $7.14 \mathrm{E}-01$ & 909 \\
\hline 241-AP-108 & 27 & 800 & 129 & $3.14 \mathrm{E}-03$ & 8.82 E-01 & 852 \\
\hline
\end{tabular}


Table 4-2. The Calculation of Ammonia Transport Properties. (2 sheets)

\begin{tabular}{|c|c|c|c|c|c|c|}
\hline Tank & $\begin{array}{c}\left(\mathrm{NH}_{3}\right)^{\mathrm{ss}} \text { in } \\
\text { Dome Under } \\
\text { Normal Vent } \\
(\mathrm{ppm})\end{array}$ & $\begin{array}{c}\text { Liquid }\left[\mathrm{NH}_{3}\right] \\
\text { in Waste } \\
(\mu \mathrm{g} / \mathrm{mL})\end{array}$ & $\begin{array}{l}\text { Normal Vent } \\
\text { Rate in the } \\
\text { Dome Space } \\
\left(\mathrm{ft}^{3} / \mathrm{min}\right)\end{array}$ & $\begin{array}{c}\text { Product h A } \\
\left(\mathrm{ft}^{3} / \mathrm{min}\right) \text { From } \\
\text { Field Data at } \\
\text { Normal Vent }\end{array}$ & $\begin{array}{c}\text { Product h A } \\
\left(\mathrm{ft}^{3} / \mathrm{min}\right) \text { From } \\
\text { Calculated } \mathrm{h} \\
\text { and } 100 \% \mathrm{~A}\end{array}$ & $\begin{array}{l}\left(\mathrm{NH}_{3}\right)^{\mathrm{ss}} \text { at } \\
\text { Off-Normal } \\
\text { Vent (ppm) }\end{array}$ \\
\hline 241-AW-101 & 5 & 10 & 100 & 3.92 E-02 & $3.05 \mathrm{E}+00$ & 89 \\
\hline 241-AW-102 & 3 & 74 & 292 & $7.16 \mathrm{E}-03$ & $7.74 \mathrm{E}-01$ & 80 \\
\hline 241-AW-103 & 138 & 512 & 134 & $3.63 \mathrm{E}-02$ & $7.06 \mathrm{E}-01$ & 437 \\
\hline 241-AW-104 & 6 & 169 & 141 & $3.64 \mathrm{E}-03$ & $6.99 \mathrm{E}-01$ & 142 \\
\hline 241-AW-105 & 43 & 1,010 & 194 & 5.97 E-03 & $5.49 \mathrm{E}-01$ & 625 \\
\hline 241-AW-106 & 28 & 355 & 158 & $8.59 \mathrm{E}-03$ & $2.06 \mathrm{E}+00$ & 1,164 \\
\hline 241-AY-101 & 5 & 113 & 250 & $7.69 \mathrm{E} \cdot 03$ & $1.55 \mathrm{E}+00$ & 286 \\
\hline 241-AY-102 & 4 & 74 & 250 & $1.05 \mathrm{E}-02$ & $1.60 \mathrm{E}+00$ & 207 \\
\hline 241-AZ-101 & 1 & 125 & 150 & $8.31 \mathrm{E}-04$ & $2.79 \mathrm{E}+00$ & 331 \\
\hline 241-AZ-102 & 6 & 438 & 112 & $1.01 \mathrm{E}-03$ & $1.96 \mathrm{E}+00$ & 1,181 \\
\hline 241-SY-101 & 400 & 1,044 & 486 & 1.42 E-01 & $2.09 \mathrm{E}+00$ & 3,870 \\
\hline 241-SY-102 & 12 & 1,150 & 363 & $2.67 \overline{E-03}$ & $1.24 \mathrm{E}+00$ & 1,771 \\
\hline 241-SY-103 & 59 & 1,290 & 116 & $3.88 \mathrm{E}-03$ & $3.14 \mathrm{E}+00$ & 5,581 \\
\hline 241-A-101 & 394 & 531 & 10 & $5.17 \mathrm{E}-03$ & $3.74 \mathrm{E}+00$ & 8,253 \\
\hline $241-\mathrm{A}-103$ & 261 & 531 & 8 & $2.61 \mathrm{E}-03$ & $3.21 \mathrm{E}+00$ & 2,922 \\
\hline $241-\mathrm{C}-103$ & 324 & 1,210 & 10 & $1.94 \mathrm{E}-03$ & $1.88 \mathrm{E}+00$ & 3,159 \\
\hline 241-S-101 & 765 & 1,210 & 6 & $2.94 \mathrm{E}-03$ & $2.59 \mathrm{E}+00$ & 5,817 \\
\hline $241-S-102$ & 588 & 1,700 & 2 & $5.47 \mathrm{E}-04$ & $3.01 \mathrm{E}+00$ & 4,312 \\
\hline 241-SX-103 & 31 & 1,800 & 53 & $6.11 \mathrm{E}-04$ & $3.55 \mathrm{E}+00$ & 4,977 \\
\hline 241-U-102 & 651 & 745 & 2 & $1.66 \mathrm{E}-03$ & $2.08 \mathrm{E}+00$ & 2,157 \\
\hline $241-U-103$ & 722 & 1,400 & 2 & $9.56 \mathrm{E}-04$ & $2.47 \mathrm{E}+00$ & 4,582 \\
\hline $241-U-105$ & 1,936 & 1,600 & 5 & $5.64 \mathrm{E}-03$ & $2.22 \mathrm{E}+00$ & 6,909 \\
\hline 241-U-107 & 448 & 403 & 12 & $1.34 \mathrm{E}-02$ & $1.98 \mathrm{E}+00$ & 1,380 \\
\hline $241-U-108$ & 684 & 1,050 & 4 & $1.87 \mathrm{E}-03$ & $2.57 \mathrm{E}+00$ & 4,345 \\
\hline
\end{tabular}

Note:

To convert cubic feet to liters, multiply the value by $28.454 \mathrm{~L} / \mathrm{ft}^{3}$.

\subsection{FLAMMABILITY EVALUATION AND RESULTS}

With the collected ventilation rates and steady-state gas concentrations, the flammable gas concentrations, expressed as a percent of the LFL, in the tank dome space under normal ventilation conditions were calculated using Equation 3-18 and are listed in Appendix D, Table D-1. For all tanks, the flammable gas concentration under normal ventilation is less than $2 \%$ except SSTs $241-\mathrm{C}-107$ with $6.76 \%$ and $241-\mathrm{U}-106$ with $2.35 \%$. This suggests that the tank dome space of all tanks under current ventilation rates, whether actively or passively ventilated, will not exceed $25 \%$ of the LFL. 
For off-normal ventilation conditions, there is no guarantee that every tank will have a pathway for barometric breathing due to the sealing-off action for interim stabilization; thus the flammability calculation will be performed under both barometric breathing rate and zero ventilation rate for water addition and temperature increase condition. A study focusing on the sensitivity of waste temperature and water addition in the flammability evaluation under zero ventilation for DSTs has been done and documented in Appendix $\mathrm{H}$ of Revision 3 of this document. The study concluded that for the flammability evaluation under zero ventilation condition, 10 and $1 \mathrm{kgal}$ of water will be added to the tank waste for 100- and 200-series DST and SSTs. Also $5{ }^{\circ} \mathrm{C}$ temperature increase will apply to all DSTs. In addition, the diffusion mechanism through concrete tank dome has been included in the flammability calculation under zero ventilation condition for 149 SSTs.

Detailed calculation results on the case of water addition and $5{ }^{\circ} \mathrm{C}$ temperature increase for DSTs are given in Appendix D. The summarized results are given in Tables 4-3 under barometric breathing condition, and Tables 4-4 and 4-5 for DSTs and SSTs, respectively under zero ventilation condition with water addition and temperature study.

\subsubsection{Off-Normal Condition With Barometric Breathing}

For off-normal ventilation rates, assuming only barometric breathing rate, $\mathrm{V}_{\mathrm{bb}}$, is available (discussed in Section 4.2), the steady-state concentration can be calculated using Equation 3-13 for insoluble gas and using Equation 3-16 for the soluble gas; the results are listed in Appendix D, Table D-2. In the calculation for the off-normal condition, the initial concentrations are assumed to be the steady-state concentrations under normal ventilation conditions; these concentrations are listed in Appendix D, Table D-1. As listed in Table D-2, under the off-normal condition with barometric breathing rate, 22 DSTs and 7 SSTs are computed to exceed $25 \%$ of the LFL when the system reaches steady state, and 5 DSTs and 2 SSTs are predicted to reach $100 \%$ of the LFL.

Equation 3-18 gives the formula to calculate the flammable gas concentrations at a given time with the aid of a time-dependent gas concentration equation (Equation 3-6 for insoluble gas and Equation 3-12 for soluble gas). Because the time variable cannot be expressed explicitly in an equation, a customized Excel macro was developed using Visual Basic to iterate the calculations by increasing the time until the specified LFLs in Equation 3-18 are met. The calculated times to reach $25 \%$ and $100 \%$ of the LFL (time-to $25 \%$ LFL and $100 \% \mathrm{LFL}$ ) under the off-normal ventilation condition with barometric breathing rate are listed in Appendix D, Tables D-3 and D-4. In the calculations, the flammability level of each flammable gas also is calculated for each case to understand the distribution of each gas's contribution to the total LFL.

Another needed variable is the minimum ventilation rate required to prevent the tank dome space from exceeding $25 \%$ of the LFL. Equation 3-18 gives the time-dependent flammable gas concentration in terms of percent of the LFL; however, the ventilation rate is embedded in the time-dependent concentration as given in Equations 3-6 and 3-12. No explicit solution was developed to find the minimum ventilation rate in Equation 3-18. For those tanks exceeding the specified flammability level, the minimum ventilation rate can be found by gradually increasing the ventilation rate from zero until a total flammability level of less than the specified $25 \%$ or 
$100 \%$ of the LFL, is obtained. A customized Excel macro was developed using Visual Basic code to iterate this equation automatically to find the minimum ventilation rate. Details of the calculated minimum ventilation rates are listed in Appendix D, Tables D-3 and D-4, for $25 \%$ and $100 \%$ of the LFL, respectively.

Table 4-3 lists all the tanks (22 DSTs and 8 SSTs) that will reach $25 \%$ of the LFL with current waste content plus water addition of $10 \mathrm{kgal}$ and $1 \mathrm{kgal}$ for 100 - and 200 -series tanks, respectively and $5{ }^{\circ} \mathrm{C}$ temperature increase under barometric breathing rate. In the off-normal condition with barometric breathing rate, DST 241-AZ-102 and SST 241-B-203 give the shortest time to $25 \%$ of the LFL as 10 and 39 days for DSTs and SSTs, respectively. Overall, under barometric breathing rate, five DSTs and two SSTs are computed to reach $100 \%$ of the LFL if the off-normal condition persists long enough. It takes 47 and 254 days to reach $100 \%$ of the LFL for DST 241-AZ-102 and SST 241-B-203, respectively. Note that the results of this calculation listed in Table 4-3 are based on the BBI information queried on September 27, 2006.

Table 4-3. Time ${ }^{\mathrm{a}, \mathrm{b}}$ to Reach $25 \%$ and $100 \%$ of the Lower Flammability Limit With Water Addition ${ }^{\mathrm{c}}$ and $5{ }^{\circ} \mathrm{C}$ Temperature Increase ${ }^{\mathrm{d}}$ Under Barometric Breathing Condition.

\begin{tabular}{|c|c|c|c|c|c|}
\hline DSTs & $\begin{array}{c}\text { Time to reach } \\
25 \% \text { LFL } \\
\text { t } 25 \% \\
\text { (day) }\end{array}$ & $\begin{array}{c}\text { Time to reach } \\
100 \% \text { LFL } \\
t_{100 \%} \\
\text { (day) }\end{array}$ & SSTs & $\begin{array}{l}\text { Time to reach } \\
25 \% \text { LFL } \\
\text { t }_{25 \%} \\
\text { (day) }\end{array}$ & $\begin{array}{c}\text { Time to reach } \\
100 \% \text { LFL } \\
t_{100 \%} \\
\text { (day) }\end{array}$ \\
\hline $241-\mathrm{AZ}-102$ & 10 & 47 & 241-B-203* & 39 & 254 \\
\hline 241-AY-102 & 13 & 59 & 241-B-204* & 42 & 292 \\
\hline 241-AZ-101 & 19 & 91 & 241-SX-103* & 123 & not occur \\
\hline 241-AN-102 & 19 & 92 & $241-S X-105^{*}$ & 251 & not occur \\
\hline 241-AN-107 & 21 & 106 & $241-T-201$ & 271 & not occur \\
\hline 241-AN-104 & 69 & not occur & 241-C-107 & 341 & not occur \\
\hline $241-\mathrm{AN}-106$ & 84 & not occur & 241-C-104 & 736 & not occur \\
\hline 241-AN-105 & 88 & not occur & \multirow{9}{*}{\multicolumn{3}{|c|}{ 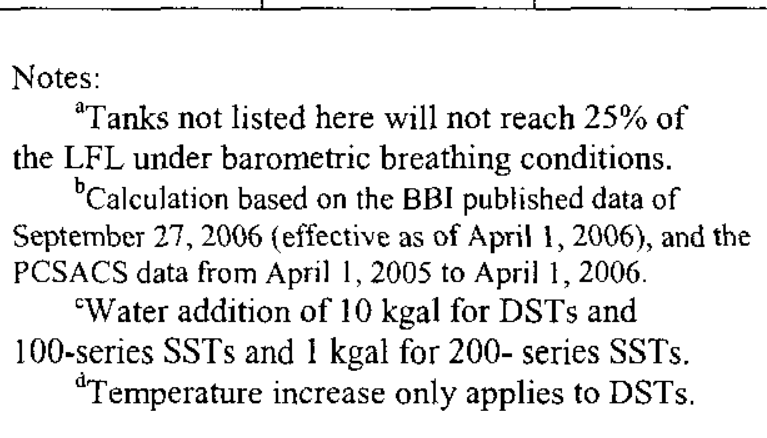 }} \\
\hline $241-\mathrm{AW}-101$ & 92 & not occur & & & \\
\hline 241-AP-102 & 116 & not occur & & & \\
\hline 241-AN-103 & 129 & not occur & & & \\
\hline $241-S Y-103$ & 129 & not occur & & & \\
\hline $241-A W-104$ & 154 & not occur & & & \\
\hline $241-\mathrm{AP}-108$ & 160 & not occur & & & \\
\hline $241-\mathrm{AP}-106$ & 174 & not occur & & & \\
\hline 241-AP-105 & 188 & not occur & & & \\
\hline 241-AW-106 & 219 & not occur & \multirow{6}{*}{$\begin{array}{l}\text { BBI } \\
\text { DST } \\
\text { LFL } \\
\text { PCSACS } \\
\text { SST }\end{array}$} & \multirow{6}{*}{$\begin{aligned}= & \text { best basis invento } \\
= & \text { double-shell tank. } \\
= & \text { lower flammabilit } \\
= & \text { personal compute } \\
& \text { Analysis Compute } \\
= & \text { single-shell tank. }\end{aligned}$} & \\
\hline $241-S Y-102$ & 239 & not occur & & & \\
\hline $241-A Y-101$ & 266 & not occur & & & \\
\hline 241-AN-101 & 284 & not occur & & & $\begin{array}{l}\text { rvelllance } \\
\text { ystem. }\end{array}$ \\
\hline 241-AP-104 & 296 & not occur & & & \\
\hline 241-AP-101 & 1038 & not occur & & & \\
\hline
\end{tabular}




\subsubsection{Off-Normal Condition with Zero Ventilation}

A zero ventilation condition means a closed system. Without considering or including other possible mechanisms for gas leaving the system, such as diffusion through the dome space concrete wall, it is expected that every tank will reach $100 \%$ of the LFL under zero ventilation given adequate time. A complete set of equations for flammability evaluation under zero ventilation is given in Section 3-7 for both soluble and insoluble gases. In this model calculation, it is reasonable to assume that the tank will not be pressurized, thus, the newly generated gas will slowly push the old gas composition out and form a new concentration of flammable gas without increasing the pressure.

For SSTs, if gas diffusion is considered as the only mechanism for ventilation, then the gas concentration such as hydrogen methane can be calculated using Equation 3-6b. The required gas diffusivity, thickness of the coating on the dome, the dome area, and the calculated effective diffusion rate are given in Table F-1 and F-2 of Appendix F. For ammonia, the diffusion mechanism is too small to impact its equilibrium concentration. So the ammonia concentrations remain unchanged during the calculation.

For DSTs, the temperature effect under zero ventilation condition is considered. Other than $5^{\circ} \mathrm{C}$ temperature increase on top of current tank temperature, it also considers the temperature's continued rising in the closed system. The temperature increase rates are calculated using Equation 3-28 for the case when temperatures of the convective and non-convective layers are the same and using Equations 3-29 to 3-31 when the temperatures are different. The required input data for the calculation for each layer of each tank includes density, volume, heat load, and waste temperature. Other input parameters commonly used in the calculations for all tanks are the following (values are taken from RPP-6213):

- Heat capacity: $3,300 \mathrm{~J} / \mathrm{kg}$

- Air temperature: $285 \mathrm{~K}$

- Soil temperature: $287 \mathrm{~K}$

- Heat exchange area: $411 \mathrm{~m}^{2}$

- Soil thermal conductivity: $1 \mathrm{~W} / \mathrm{m}-\mathrm{K}$

- Soil overburden thickness: $4 \mathrm{~m}$

- Tank radius: $11 \mathrm{~m}$.

For tanks treated as a whole, the temperature increase rate is the net heat rate, which is the tank heat load minus upward and downward heat lost, divided by the product of mass and heat capacity. For tanks with distinct temperatures between layers, the heat transfer coefficients are adjusted and determined until the calculated temperature increase rates are equal for convective and nonconvective layers. Once the heat transfer coefficients are determined, the heat-up rates are finalized.

At the given initial temperature along with the temperature increase rate, the gas concentrations for hydrogen and methane are calculated as the temperature changes in the time step of 1 day. The accumulated gas concentrations are calculated daily using Equation 3-23, since the first day of losing the normal ventilation condition. The initial concentration condition in Equation 3-23 is a steady-state concentration at the normal ventilation condition. The hydrogen release rate, 


\section{RPP-5926 REV 6}

which is assumed to be the same as the HGR, is calculated daily using the rate equation model documented in RPP-3851, or summarized in Chapter 2.0, based on the calculated temperature of each day.

Because methane does not have a rate equation model available, its daily gas release rate is calculated with the initial release rate at the given initial tank waste temperature, plus the daily increase rate due to tank waste heat-up under the zero ventilation condition. The daily increase rate of methane uses the same daily increase HGR, which is the ratio between the HGRs of the current and previous days. The initial methane rates at baseline temperature are taken from Appendix D, Table D-1. The initial methane release rate at baseline temperature plus $5^{\circ} \mathrm{C}$ is calculated in the same ratio as the hydrogen release rate increases as shown in Equation 4-3:

$$
M G R_{\text {baseline }+T}^{i}=M G R_{\text {baseline }}^{i} \cdot \frac{H G R_{\text {baseline }+T}^{i}}{H G R_{\text {baseline }}^{i}}
$$

where:

$$
\begin{array}{ll}
M G R_{\text {baseline }+T}^{i}= & \begin{array}{l}
\text { methane initial release rate at baseline temperature plus } \mathrm{T} \\
\left(\mathrm{ft}^{3} / \mathrm{min}\right)
\end{array} \\
M G R_{\text {baseline }}^{i}= & \text { Methane initial release rate at baseline temperature }\left(\mathrm{ft}^{3} / \mathrm{min}\right) \\
H G R_{\text {baseline }+T}= & \begin{array}{l}
\text { hydrogen initial release rate at baseline temperature plus } \mathrm{T} \\
\left(\mathrm{ft}^{3} / \mathrm{min}\right)
\end{array} \\
H G R_{\text {baseline }}^{i}= & \text { hydrogen initial release rate at baseline temperature }\left(\mathrm{ft}^{3} / \mathrm{min}\right) .
\end{array}
$$

Ammonia concentrations are calculated using Equation 3-25. Although ammonia concentration is also a function of temperature, to simplify the flammability evaluation the intention was to obtain one set of ammonia transport properties at the highest temperature for each tank and use it throughout the whole calculation. Calculations (Appendix H of Revision 3 of this document) show that the equilibrium ammonia vapor concentration at its highest temperatures due to temperature increase contributes to flammability is not too far from the contributions calculated at baseline temperature. In this calculation, the ammonia transport coefficients $g_{1}$ and $g_{2}$ derived at the initial temperature are used for the flammability calculations.

In the flammability calculations, there are several tanks that the $\mathrm{N}_{2} \mathrm{O}$ concentration has increased to the model limit of $8 \%$ before the total flammability reached $100 \%$ of the LFL due to zero ventilation assumption. As mentioned in Section 3.2, the flammability level for individual flammable gas is $4 \%, 15 \%$, and $5 \%$ for hydrogen, ammonia, and methane, respectively, if the $\mathrm{N}_{2} \mathrm{O}$ is less than $8 \%$. When the mixture has more than $8 \% \mathrm{~N}_{2} \mathrm{O}$, the required individual LFL gas concentrations are lower than listed above. For those tanks having a high $\mathrm{N}_{2} \mathrm{O}$ concentration, the calculations of "the time to reach $100 \%$ of the LFL" simply represents the time for the $\mathrm{N}_{2} \mathrm{O}$ to reach $8 \%$ while the total flammability is still less than $100 \%$ of the LFL, and this approach is conservative. For flammable gas control, the time to $25 \%$ of the LFL is used to determine the surveillance interval, and usually the $\mathrm{N}_{2} \mathrm{O}$ will not build up to $8 \%$ at the time to reach $25 \%$ of the LFL. It is possible to establish the LFL for each individual flammable gas as function of higher

$\mathrm{N}_{2} \mathrm{O}$ concentration by further investigating the flammability studies on gas mixtures by Pfahl and Shepherd (1997). In any case, this will complicate the calculation of time-dependent gas concentrations, and make automation of the calculations more difficult. 
Table 4-4 summarizes the flammability calculation for DSTs under off-normal ventilation condition at zero ventilation scenarios with $10 \mathrm{kgal}$ water addition and $5{ }^{\circ} \mathrm{C}$ temperature increase. Under the zero ventilation condition the ratio of time to $100 \%$ of the LFL to $25 \%$ of the LFL should be roughly four (some that are higher than four means the ammonia contribution is more significant and the ammonia concentration is not linearly increasing by time but is governed by the liquid-vapor equilibrium mechanism). Those tanks with a ratio less than four means the "time to reach $100 \%$ of the LFL" is the time to reach the $8 \% \mathrm{~N}_{2} \mathrm{O}$ model limit, but the flammability has not reached $100 \%$ of the LFL. As shown in Table 4-4, the five tanks with the shortest time to $25 \%$ of the LFL are $10,12,15,16$, and 20 days for tanks 241 -AZ-102, 241-AY-102, 241-AN-102, 241-AZ-101, and 241-AN-107, respectively. The minimum vent rates to keep the dome space below $100 \%$ of the LFL ranges between 0.92 to $0.01 \mathrm{ft}^{3} / \mathrm{min}$.

Table 4-4. The Hydrogen Generation Rates, Time to $25 \%$ and $100 \%$ of the Lower Flammability Limit and Minimum Vent Rate With $10 \mathrm{kgal}$ Water Addition and $5{ }^{\circ} \mathrm{C}$ Temperature Increase Under Zero Ventilation for Double-Shell Tanks. (2 sheets)

\begin{tabular}{|c|c|c|c|c|c|}
\hline Tank & $\begin{array}{c}\text { Hydrogen } \\
\text { generation rate } \\
\operatorname{Rr}\left(\mathbf{H}_{2}\right) \\
\left(\mathrm{ft}^{3} / \mathrm{min}\right)\end{array}$ & $\begin{array}{c}\text { Time* to } \\
\text { reach } 25 \% \\
\text { of the LFL } \\
\text { (days) }\end{array}$ & $\begin{array}{l}\text { Time* }^{*} \text { to } \\
\text { reach } 100 \% \\
\text { of the LFL } \\
\text { (days) }\end{array}$ & $\begin{array}{l}\text { Minimum } \\
\text { vent rate to } \\
\text { keep below } \\
25 \% \mathrm{LFL} \\
\left(\mathrm{ft}^{3} / \mathrm{min}\right)\end{array}$ & $\begin{array}{c}\text { Minimum } \\
\text { vent rate to } \\
\text { keep below } \\
100 \% \mathrm{LFL} \\
\left(\mathrm{ft}^{3} / \mathrm{min}\right)\end{array}$ \\
\hline 241-AN-101 & $2.38 \mathrm{E}-03$ & 151 & 522 & 0.25 & 0.06 \\
\hline 241-AN-102 & $1.56 \mathrm{E}-02$ & 15 & 47 & 1.63 & 0.40 \\
\hline 241-AN-103 & $3.84 \mathrm{E}-03$ & 91 & 313 & 0.41 & 0.10 \\
\hline 241-AN-104 & $4.91 \mathrm{E}-03$ & 56 & 201 & 0.53 & 0.13 \\
\hline 241-AN-105 & $3.14 \mathrm{E}-03$ & 69 & 259 & 0.34 & 0.08 \\
\hline 241-AN-106 & $7.25 \mathrm{E}-03$ & 68 & 248 & 0.78 & 0.20 \\
\hline 241-AN-107 & $1.24 \mathrm{E}-02$ & 20 & 78 & 1.31 & 0.32 \\
\hline 241-AP-101 & $1.00 \mathrm{E}-03$ & 208 & 316 & 0.12 & 0.03 \\
\hline 241-AP-102 & $2.80 \mathrm{E}-03$ & 88 & 340 & 0.31 & 0.08 \\
\hline 241-AP-103 & $1.75 \mathrm{E}-03$ & 234 & 798 & 0.18 & 0.05 \\
\hline 241-AP-104 & $1.68 \mathrm{E}-03$ & 151 & 490 & 0.17 & 0.04 \\
\hline 241-AP-105 & $1.68 \mathrm{E}-03$ & 127 & 425 & 0.19 & 0.05 \\
\hline 241-AP-106 & $1.98 \mathrm{E}-03$ & 114 & 390 & 0.20 & 0.05 \\
\hline 241-AP-107 & $3.33 \mathrm{E}-04$ & 1,304 & 1,345 & 0.04 & 0.01 \\
\hline 241-AP-108 & $2.96 \mathrm{E}-03$ & 114 & 467 & 0.30 & 0.07 \\
\hline 241-AW-101 & $3.16 \mathrm{E}-03$ & 72 & 255 & 0.33 & 0.08 \\
\hline 241-AW-102 & $6.50 \mathrm{E}-04$ & 1,093 & 1,500 & 0.07 & 0.02 \\
\hline 241-AW-103 & $9.70 \mathrm{E}-04$ & 245 & 993 & 0.11 & 0.03 \\
\hline 241-AW-104 & $2.32 \mathrm{E}-03$ & 107 & 393 & 0.27 & 0.07 \\
\hline 241-AW-105 & $3.19 \mathrm{E}-04$ & 1,500 & 1,500 & 0.04 & 0.01 \\
\hline 241-AW-106 & $1.58 \mathrm{E}-03$ & 136 & 534 & 0.17 & 0.04 \\
\hline $241-A Y-101$ & $6.88 \mathrm{E}-03$ & 115 & 240 & 0.70 & 0.18 \\
\hline
\end{tabular}


Table 4-4. The Hydrogen Generation Rates, Time to $25 \%$ and $100 \%$ of the Lower Flammability Limit and Minimum Vent Rate With $10 \mathrm{kgal}$ Water Addition and $5{ }^{\circ} \mathrm{C}$ Temperature Increase Under Zero Ventilation for Double-Shell Tanks. (2 sheets)

\begin{tabular}{|c|c|c|c|c|c|}
\hline Tank & $\begin{array}{c}\text { Hydrogen } \\
\text { generation rate } \\
\operatorname{Rr}\left(\mathbf{H}_{2}\right) \\
\left(\mathbf{f t}^{3} / \mathrm{min}\right)\end{array}$ & $\begin{array}{c}\text { Time* to } \\
\text { reach } 25 \% \\
\text { of the LFL } \\
\text { (days) }\end{array}$ & $\begin{array}{c}\text { Time* to } \\
\text { reach } 100 \% \\
\text { of the LFL } \\
\text { (days) }\end{array}$ & $\begin{array}{l}\text { Minimum } \\
\text { vent rate to } \\
\text { keep below } \\
25 \% \text { LFL } \\
\left(\mathrm{ft}^{3} / \mathrm{min}\right)\end{array}$ & $\begin{array}{c}\text { Minimum } \\
\text { vent rate to } \\
\text { keep below } \\
100 \% \text { LFL } \\
\left(\mathrm{ft}^{3} / \mathrm{min}\right)\end{array}$ \\
\hline 241-AY-102 & $3.00 \mathrm{E}-02$ & 12 & 46 & 3.04 & 0.76 \\
\hline 241-AZ-101 & $2.56 \mathrm{E}-02$ & 16 & 50 & 2.63 & 0.66 \\
\hline $241-A Z-102$ & $3.61 \mathrm{E}-02$ & 10 & 38 & 3.69 & 0.92 \\
\hline 241-SY-101 & $6.57 \mathrm{E}-04$ & 290 & 804 & 0.09 & 0.02 \\
\hline $241-S Y-102$ & $1.37 \mathrm{E}-03$ & 146 & 615 & 0.17 & 0.04 \\
\hline 241-SY-103 & $5.10 \mathrm{E}-03$ & 93 & 361 & 0.61 & 0.14 \\
\hline
\end{tabular}

Note:

$* 1,500$ in the Time to $25 \%$ and $100 \%$ of the LFL column means equal or longer than 1,500 days.

LFL = lower flammability limit.

Table 4-5 summarizes the flammability calculation for SSTs under off-normal ventilation condition at zero ventilation scenarios with $10 \mathrm{kgal}$ and $1 \mathrm{kgal}$ water addition for 100 - and 200 -series tanks. The gas diffusion mechanism through the concrete dome are included in the calculations. Due to the gas diffusion effect, there are 20 tanks will not reach $100 \%$ of the LFL including tank 241-A-104, 4 241-AX Tank Farm tanks and 15 241-SX Tank Farm tanks. The diffusion rates of these tanks are two orders of magnitude larger than the rest of tanks because their concrete tank domes are not coated with a coating such as Asphaltic or Gunite. These coating materials increase the gas diffusion resistance. Asphaltic coating is particularly hard for gas to pass through because its diffusivity is four orders of magnitude smaller than concrete's diffusivity. For the remaining tanks, the shortest time to $25 \%$ of the LFL are 35,37 , and 136 days from tanks 241-B-203, 241-B-204, and 241-C-107, respectively. There are 12 tanks which have the time to $25 \%$ of the LFL shorter than 365 days, and among these 12 tanks, 241-SX-103 will not reach $100 \%$ of the LFL due to the effect of gas diffusion through the dome space concrete. 
Table 4-5. The Hydrogen Generation Rates, Time to $25 \%$ and $100 \%$ of the Lower Flammability Limit and Minimum Vent Rate with Water Addition ${ }^{\mathrm{a}}$ Under Zero Ventilation (Except Gas Diffusion Mechanism Through The Dome) for Single-Shell Tanks. (5 sheets)

\begin{tabular}{|c|c|c|c|c|c|}
\hline Tank & 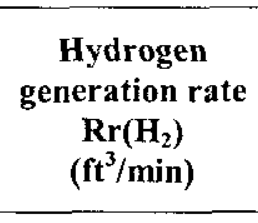 & $\begin{array}{c}\text { Time }^{b, c} \text { to } \\
\text { reach } 25 \% \\
\text { of the LFL } \\
\text { (days) }\end{array}$ & $\begin{array}{c}\text { Time }^{\mathrm{b}, \mathrm{c}} \text { to } \\
\text { reach } 100 \% \\
\text { of the LFL } \\
\text { (days) }\end{array}$ & $\begin{array}{c}\text { Minimum } \\
\text { vent rate to } \\
\text { keep below } \\
25 \% \text { LFL } \\
\left(\mathrm{ft}^{3} / \mathrm{min}\right)\end{array}$ & $\begin{array}{c}\text { Minimum } \\
\text { vent rate to } \\
\text { keep below } \\
100 \% \text { LFL } \\
\left(\mathrm{ft}^{3} / \mathrm{min}\right)\end{array}$ \\
\hline 241-A-101 & $1.53 \mathrm{E}-03$ & 451 & 1,500 & 0.18 & 0.04 \\
\hline $241-\mathrm{A}-102$ & $3.60 \mathrm{E}-04$ & 1,500 & 1,500 & 0.09 & 0.02 \\
\hline 241-A-103 & $1.52 \mathrm{E}-03$ & 515 & 1,500 & 0.18 & 0.04 \\
\hline 241-A-104 & $2.32 \mathrm{E}-04$ & 1,500 & not occur & 0.03 & 0.01 \\
\hline 241-A-105 & NA & $\mathrm{NA}$ & $\mathrm{NA}$ & $\mathrm{NA}$ & $\mathrm{NA}$ \\
\hline 241-A-106 & $1.88 \mathrm{E}-03$ & 417 & 1,500 & 0.27 & 0.07 \\
\hline 241-AX-101 & $1.02 \mathrm{E}-03$ & not occur & not occur & 0.11 & 0.03 \\
\hline $241-\mathrm{AX}-102$ & $6.17 \mathrm{E}-04$ & not occur & not occur & 0.08 & 0.02 \\
\hline $241-\mathrm{AX}-103$ & $8.92 \mathrm{E}-04$ & not occur & not occur & 0.12 & 0.03 \\
\hline 241-AX-104 & $4.50 \mathrm{E}-03$ & not occur & not occur & 0.45 & 0.11 \\
\hline 241-B-101 & $4.13 \mathrm{E}-04$ & 1,500 & 1,500 & 0.05 & 0.01 \\
\hline 241-B-102 & $2.39 \mathrm{E}-04$ & 1,500 & 1,500 & 0.03 & 0.01 \\
\hline $241-\mathrm{B}-103$ & $2.42 \mathrm{E}-04$ & 1,500 & 1,500 & 0.03 & 0.01 \\
\hline 241-B-104 & $3.57 \mathrm{E}-04$ & 1,074 & 1,500 & 0.04 & 0.01 \\
\hline $241-\mathrm{B}-105$ & $3.19 \mathrm{E}-04$ & 1,481 & 1,500 & 0.04 & 0.01 \\
\hline $241-\mathrm{B}-106$ & $3.06 \mathrm{E}-04$ & 1,500 & 1,500 & 0.04 & 0.01 \\
\hline $241-\overline{-B}-107$ & $2.80 \mathrm{E}-04$ & 1,500 & 1,500 & 0.04 & 0.01 \\
\hline $241-\mathrm{B}-108$ & $2.54 \mathrm{E}-04$ & 1,500 & 1,500 & 0.03 & $\overline{0.01}$ \\
\hline 241-B-109 & $2.64 \mathrm{E}-04$ & 1,500 & 1,500 & 0.04 & 0.01 \\
\hline $241-\mathrm{B}-110$ & $5.37 \mathrm{E}-04$ & 790 & 1,500 & 0.07 & 0.02 \\
\hline $241-\mathrm{B}-111$ & $9.21 \mathrm{E}-04$ & 505 & 1,500 & 0.11 & 0.03 \\
\hline $241-\mathrm{B}-112$ & $2.69 \mathrm{E}-04$ & 1,500 & 1,500 & 0.04 & 0.01 \\
\hline $241-\mathrm{B}-201$ & $5.91 \mathrm{E}-05$ & 350 & 1,500 & 0.01 & 0.00 \\
\hline $241-\mathrm{B}-202$ & $5.57 \mathrm{E}-05$ & 402 & 1,500 & 0.01 & 0.00 \\
\hline 241-B-203 & $1.98 \mathrm{E}-04$ & 35 & 154 & 0.02 & 0.00 \\
\hline 241-B-204 & $2.03 \mathrm{E}-04$ & 37 & 165 & 0.02 & 0.01 \\
\hline 241-BX-101 & $2.88 \mathrm{E}-04$ & 1,500 & 1,500 & 0.04 & 0.01 \\
\hline $241-\mathrm{BX}-102$ & $2.65 \mathrm{E}-04$ & 1,500 & 1,500 & 0.03 & 0.01 \\
\hline $241-B X-103$ & $3.10 \mathrm{E}-04$ & 1,500 & 1,500 & 0.05 & 0.01 \\
\hline 241-BX-104 & $3.71 \mathrm{E}-04$ & 1,211 & 1,500 & 0.09 & 0.02 \\
\hline 241-BX-105 & $2.87 \mathrm{E}-04$ & 1,500 & 1,500 & 0.07 & 0.02 \\
\hline 241-BX-106 & $2.77 \mathrm{E}-04$ & 1,500 & 1,500 & 0.04 & 0.01 \\
\hline 241-BX-107 & $4.05 \mathrm{E}-04$ & 817 & 1,500 & 0.06 & 0.01 \\
\hline
\end{tabular}


Table 4-5. The Hydrogen Generation Rates, Time to $25 \%$ and $100 \%$ of the Lower Flammability Limit and Minimum Vent Rate with Water Addition ${ }^{\mathrm{a}}$ Under Zero Ventilation (Except Gas Diffusion Mechanism Through The Dome) for Single-Shell Tanks. (5 sheets)

\begin{tabular}{|c|c|c|c|c|c|}
\hline Tank & $\begin{array}{c}\text { Hydrogen } \\
\text { generation rate } \\
\mathbf{R r}\left(\mathbf{H}_{2}\right) \\
\left(\mathrm{ft}^{3} / \mathrm{min}\right) \\
\end{array}$ & $\begin{array}{c}\text { Time } e^{b, c} \text { to } \\
\text { reach } 25 \% \\
\text { of the LFL } \\
\text { (days) }\end{array}$ & $\begin{array}{c}\text { Time } e^{b, c} \text { to } \\
\text { reach } 100 \% \\
\text { of the LFL } \\
\text { (days) } \\
\end{array}$ & $\begin{array}{c}\text { Minimum } \\
\text { vent rate to } \\
\text { keep below } \\
25 \% \text { LFL } \\
\left(\mathrm{ft}^{3} / \mathrm{min}\right)\end{array}$ & $\begin{array}{c}\text { Minimum } \\
\text { vent rate to } \\
\text { keep below } \\
100 \% \text { LFL } \\
\left(\mathrm{ft}^{3} / \mathrm{min}\right)\end{array}$ \\
\hline 241-BX-108 & $2.56 \mathrm{E}-04$ & 1,500 & 1,500 & 0.03 & 0.01 \\
\hline 241-BX-109 & $5.09 \mathrm{E}-04$ & 986 & 1,500 & 0.06 & 0.02 \\
\hline $241-\mathrm{BX}-110$ & $3.83 \mathrm{E}-04$ & 1,106 & 1,500 & 0.05 & 0.01 \\
\hline 241-BX-111 & $2.90 \mathrm{E}-04$ & 1,500 & 1,500 & 0.04 & 0.01 \\
\hline 241-BX-112 & $3.37 \mathrm{E}-04$ & 1,220 & 1,500 & 0.06 & 0.02 \\
\hline 241-BY-101 & $6.83 \mathrm{E}-04$ & 745 & 1,500 & 0.08 & 0.02 \\
\hline $241-\mathrm{BY}-102$ & $3.49 \mathrm{E}-04$ & 1,458 & 1,500 & 0.07 & 0.02 \\
\hline $241-B Y-103$ & $4.26 \mathrm{E}-04$ & 1,086 & 1,500 & 0.06 & 0.01 \\
\hline 241-BY-104 & $6.79 \mathrm{E}-04$ & 605 & 1,500 & 0.12 & 0.03 \\
\hline 241-BY-105 & $5.85 \mathrm{E}-04$ & 607 & 1,500 & 0.10 & 0.02 \\
\hline 241-BY-106 & $7.51 \mathrm{E}-04$ & 709 & 1,500 & 0.08 & 0.02 \\
\hline 241-BY-107 & $4.62 \mathrm{E}-04$ & 1,062 & 1,500 & 0.18 & 0.05 \\
\hline 241-BY-108 & $4.03 \mathrm{E}-04$ & 1,295 & 1,500 & 0.20 & 0.05 \\
\hline 241-BY-109 & $3.44 \mathrm{E}-04$ & 1,455 & 1,500 & 0.05 & 0.01 \\
\hline 241-BY-110 & $1.07 \mathrm{E}-03$ & 417 & 1,500 & 0.25 & 0.06 \\
\hline 241-BY-111 & $3.70 \mathrm{E}-04$ & 1,296 & 1,500 & 0.05 & 0.01 \\
\hline $241-\mathrm{BY}-112$ & $3.38 \mathrm{E}-04$ & 1,500 & 1,500 & 0.06 & 0.01 \\
\hline $241-\mathrm{C}-101$ & $3.33 \mathrm{E}-04$ & 1,500 & 1,500 & 0.05 & 0.01 \\
\hline $241-C-102$ & $5.07 \mathrm{E}-04$ & 625 & 1,500 & 0.12 & 0.03 \\
\hline $241-C-103$ & $9.57 \mathrm{E}-04$ & 644 & 835 & 0.12 & 0.03 \\
\hline $241-\mathrm{C}-104$ & $1.63 \mathrm{E}-03$ & 215 & 1,068 & 0.26 & 0.07 \\
\hline $241-C-105$ & $8.94 \mathrm{E}-04$ & 500 & 1,500 & 0.13 & 0.03 \\
\hline $241-C-106$ & $9.91 \mathrm{E}-04$ & 668 & 1,500 & 0.12 & 0.03 \\
\hline 241-C-107 & $2.95 \mathrm{E}-03$ & 136 & 716 & 0.30 & 0.07 \\
\hline $241-C-108$ & $3.41 \mathrm{E}-04$ & 1,500 & 1,500 & 0.03 & 0.01 \\
\hline 241-C-109 & $8.60 \mathrm{E}-04$ & 814 & 1,259 & 0.09 & 0.02 \\
\hline $241-\mathrm{C}-110$ & $7.32 \mathrm{E}-04$ & 559 & 1,500 & 0.11 & 0.03 \\
\hline $241-\mathrm{C}-111$ & $1.65 \mathrm{E}-03$ & 434 & 1,500 & 0.17 & 0.04 \\
\hline $241-C-112$ & $1.65 \mathrm{E}-03$ & 395 & 934 & 0.17 & 0.04 \\
\hline $241-C-201$ & $4.48 \mathrm{E}-05$ & 1,161 & 1,500 & 0.00 & 0.00 \\
\hline 241-C-202 & $4.54 \mathrm{E}-05$ & 1,174 & 1,500 & 0.00 & 0.00 \\
\hline 241-C-203 & $4.26 \mathrm{E}-05$ & 1,000 & 1,500 & 0.01 & 0.00 \\
\hline $241-\mathrm{C}-204$ & $1.87 \mathrm{E}-05$ & 1,500 & 1,500 & 0.00 & 0.00 \\
\hline $241-\mathrm{S}-101$ & $1.00 \mathrm{E}-03$ & 520 & 1,500 & 0.13 & 0.03 \\
\hline
\end{tabular}


Table 4-5. The Hydrogen Generation Rates, Time to $25 \%$ and $100 \%$ of the Lower Flammability Limit and Minimum Vent Rate with Water Addition ${ }^{\mathrm{a}}$ Under Zero Ventilation (Except Gas Diffusion Mechanism Through The Dome) for Single-Shell Tanks. (5 sheets)

\begin{tabular}{|c|c|c|c|c|c|}
\hline Tank & $\begin{array}{c}\text { Hydrogen } \\
\text { generation rate } \\
\operatorname{Rr}\left(\mathbf{H}_{2}\right) \\
\left(\mathrm{ft}^{3} / \mathbf{m i n}\right)\end{array}$ & $\begin{array}{c}\text { Time }^{\text {b,c }} \text { to } \\
\text { reach } 25 \% \\
\text { of the LFL } \\
\text { (days) }\end{array}$ & $\begin{array}{l}\text { Time }{ }^{\mathrm{b}, \mathrm{c}} \text { to } \\
\text { reach } 100 \% \\
\text { of the LFL } \\
\text { (days) }\end{array}$ & $\begin{array}{c}\text { Minimum } \\
\text { vent rate to } \\
\text { keep below } \\
25 \% \mathrm{LFL} \\
\left(\mathrm{ft}^{3} / \mathrm{min}\right)\end{array}$ & $\begin{array}{l}\text { Minimum } \\
\text { vent rate to } \\
\text { keep below } \\
100 \% \mathrm{LFL} \\
\left(\mathrm{ft}^{3} / \mathrm{min}\right)\end{array}$ \\
\hline $241-\mathrm{S}-102$ & $5.79 \mathrm{E}-04$ & 1,167 & 1,500 & 0.08 & 0.02 \\
\hline $241-\mathrm{S}-103$ & $5.07 \mathrm{E}-04$ & 1,254 & 1,500 & 0.06 & 0.01 \\
\hline 241-S-104 & $6.18 \mathrm{E}-04$ & 942 & 1,500 & 0.08 & 0.02 \\
\hline $241-S-105$ & $3.71 \mathrm{E}-04$ & 1,123 & 1,500 & 0.06 & 0.01 \\
\hline 241-S-106 & $5.23 \mathrm{E}-04$ & 917 & 1,500 & 0.06 & 0.02 \\
\hline $241-S-107$ & $7.07 \mathrm{E}-04$ & 666 & 1,500 & $0 . \overline{10}$ & 0.02 \\
\hline 241-S-108 & 4.28E-04 & 989 & 1,500 & 0.05 & 0.01 \\
\hline $241-S-109$ & $4.28 \mathrm{E}-04$ & 889 & 1,500 & 0.05 & 0.01 \\
\hline $241-S-110$ & $5.41 \mathrm{E}-04$ & 779 & 1,500 & 0.10 & 0.02 \\
\hline $241-S-111$ & $6.36 \mathrm{E}-04$ & 886 & 1,500 & 0.06 & 0.02 \\
\hline $241-S-112$ & $2.56 \mathrm{E}-04$ & 1,500 & 1,500 & 0.04 & 0.01 \\
\hline 241-SX-101 & $7.00 \mathrm{E}-04$ & not occur & not occur & 0.08 & 0.02 \\
\hline 241-SX-102 & $1.55 \mathrm{E}-03$ & not occur & not occur & 0.16 & 0.04 \\
\hline 241-SX-103 & $6.89 \mathrm{E}-03$ & 241 & not occur & 0.73 & 0.18 \\
\hline 241-SX-104 & $1.83 \mathrm{E}-03$ & not occur & not occur & 0.19 & 0.05 \\
\hline 241-SX-105 & $5.17 \mathrm{E}-03$ & not occur & not occur & 0.52 & 0.13 \\
\hline 241-SX-106 & $9.24 \mathrm{E}-04$ & not occur & not occur & 0.10 & 0.03 \\
\hline 241-SX-107 & $1.41 \mathrm{E}-03$ & not occur & not occur & 0.17 & 0.04 \\
\hline 241-SX-108 & 7.61E-04 & not occur & not occur & 0.08 & 0.02 \\
\hline 241-SX-109 & $2.25 \mathrm{E}-03$ & not occur & not occur & 0.24 & 0.06 \\
\hline 241-SX-110 & $2.43 \mathrm{E}-03$ & not occur & not occur & 0.26 & 0.06 \\
\hline 241-SX-111 & $1.61 \mathrm{E}-03$ & not occur & not occur & 0.19 & 0.05 \\
\hline 241-SX-112 & $1.05 \mathrm{E}-03$ & not occur & not occur & 0.13 & 0.03 \\
\hline 241-SX-113 & $2.45 \mathrm{E}-04$ & not occur & not occur & 0.03 & 0.01 \\
\hline 241-SX-114 & $2.35 \mathrm{E}-03$ & not occur & not occur & 0.28 & 0.07 \\
\hline 241-SX-115 & $1.61 \mathrm{E}-03$ & not occur & not occur & 0.16 & 0.04 \\
\hline $241-\mathrm{T}-101$ & $2.95 \mathrm{E}-04$ & 1,500 & 1,500 & 0.04 & 0.01 \\
\hline $241-\mathrm{T}-102$ & $5.91 \mathrm{E}-04$ & 1,241 & 1,500 & 0.06 & 0.02 \\
\hline $241-\mathrm{T}-103$ & $2.32 \mathrm{E}-04$ & 1,500 & 1,500 & 0.03 & 0.01 \\
\hline $241-\mathrm{T}-104$ & $3.59 \mathrm{E}-04$ & 974 & 1,500 & 0.06 & 0.01 \\
\hline $241-\mathrm{T}-105$ & $3.16 \mathrm{E}-04$ & 1,441 & 1,500 & 0.05 & 0.01 \\
\hline $241-\mathrm{T}-106$ & $2.30 \mathrm{E}-04$ & 1,500 & 1,500 & 0.04 & 0.01 \\
\hline $241-T-107$ & $4.32 \mathrm{E}-04$ & 930 & 1,500 & 0.07 & 0.02 \\
\hline $241-T-108$ & $2.27 \mathrm{E}-04$ & 1,500 & 1,500 & 0.03 & 0.01 \\
\hline
\end{tabular}


Table 4-5. The Hydrogen Generation Rates, Time to $25 \%$ and $100 \%$ of the Lower Flammability Limit and Minimum Vent Rate with Water Addition ${ }^{\mathrm{a}}$ Under Zero Ventilation (Except Gas Diffusion Mechanism Through The Dome) for Single-Shell Tanks. (5 sheets)

\begin{tabular}{|c|c|c|c|c|c|}
\hline Tank & $\begin{array}{c}\text { Hydrogen } \\
\text { generation rate } \\
\operatorname{Rr}\left(\mathbf{H}_{2}\right) \\
\left(\mathbf{f t}^{3} / \mathbf{m i n}\right) \\
\end{array}$ & $\begin{array}{c}\text { Time }^{\mathrm{b}, \mathrm{c}} \text { to } \\
\text { reach } 25 \% \\
\text { of the LFL } \\
\text { (days) }\end{array}$ & $\begin{array}{c}\text { Time }^{\mathrm{b}, \mathrm{c}} \text { to } \\
\text { reach } 100 \% \\
\text { of the LFL } \\
\text { (days) }\end{array}$ & $\begin{array}{c}\text { Minimum } \\
\text { vent rate to } \\
\text { keep below } \\
25 \% \text { LFL } \\
\left(\mathrm{ft}^{3} / \mathrm{min}\right)\end{array}$ & $\begin{array}{c}\text { Minimum } \\
\text { vent rate to } \\
\text { keep below } \\
100 \% \text { LFL } \\
\left(\mathrm{ft}^{3} / \mathrm{min}\right)\end{array}$ \\
\hline $241-\mathrm{T}-109$ & $2.41 \mathrm{E}-04$ & 1,500 & 1,500 & 0.03 & 0.01 \\
\hline $241-\mathrm{T}-110$ & $8.72 \mathrm{E}-04$ & 484 & 1,500 & 0.09 & 0.02 \\
\hline $241-\mathrm{T}-111$ & $4.22 \mathrm{E}-04$ & 560 & 1,500 & 0.08 & 0.02 \\
\hline $241-\mathrm{T}-112$ & $2.47 \mathrm{E}-04$ & 1,500 & 1,500 & 0.03 & 0.01 \\
\hline 241-T-201 & $1.43 \mathrm{E}-04$ & 157 & 508 & 0.02 & 0.00 \\
\hline 241-T-202 & $4.40 \mathrm{E}-05$ & 647 & 958 & 0.01 & 0.00 \\
\hline $241-\mathrm{T}-203$ & $6.53 \mathrm{E}-05$ & 271 & 331 & 0.01 & 0.00 \\
\hline 241-T-204 & $6.52 \mathrm{E}-05$ & 270 & 309 & 0.01 & 0.00 \\
\hline 241-TX-101 & $4.46 \mathrm{E}-04$ & 1,500 & 1,500 & 0.05 & 0.01 \\
\hline $241-\mathrm{TX}-102$ & $3.35 \mathrm{E}-04$ & 1,500 & 1,500 & 0.09 & 0.02 \\
\hline 241-TX-103 & $2.92 \mathrm{E}-04$ & 1,500 & 1,500 & 0.05 & 0.01 \\
\hline 241-TX-104 & $3.11 \mathrm{E}-04$ & 1,500 & 1,500 & 0.04 & 0.01 \\
\hline 241-TX-105 & 4.72E-04 & 795 & 1,500 & 0.06 & 0.01 \\
\hline 241-TX-106 & $4.17 \mathrm{E}-04$ & 1,068 & 1,500 & 0.09 & 0.02 \\
\hline 241-TX-107 & $2.49 \mathrm{E}-04$ & 1,500 & 1,500 & 0.06 & 0.02 \\
\hline 241-TX-108 & $2.76 \mathrm{E}-04$ & 1,500 & 1,500 & 0.03 & 0.01 \\
\hline 241-TX-109 & $5.14 \mathrm{E}-04$ & 784 & 1,111 & 0.13 & 0.03 \\
\hline 241-TX-110 & $4.13 \mathrm{E}-04$ & 937 & 1,500 & 0.22 & 0.06 \\
\hline 241-TX-111 & $3.72 \mathrm{E}-04$ & 1,237 & 1,500 & 0.20 & 0.05 \\
\hline 241-TX-112 & $4.70 \mathrm{E}-04$ & 560 & 1,500 & 0.07 & 0.02 \\
\hline $241-\mathrm{TX}-113$ & $4.45 \mathrm{E}-04$ & 739 & 1,500 & 0.05 & 0.01 \\
\hline 241-TX-114 & $4.10 \mathrm{E}-04$ & 837 & 1,500 & 0.09 & 0.02 \\
\hline 241-TX-115 & $4.34 \mathrm{E}-04$ & 967 & 1,500 & 0.05 & 0.01 \\
\hline 241-TX-116 & $4.28 \mathrm{E}-04$ & 908 & 1,500 & 0.05 & 0.01 \\
\hline $241-\mathrm{TX}-117$ & $3.87 \mathrm{E}-04$ & 1,285 & 1,500 & 0.04 & 0.01 \\
\hline 241-TX-118 & $4.74 \mathrm{E}-04$ & 1,177 & 1,500 & 0.06 & 0.02 \\
\hline 241-TY-101 & $2.58 \mathrm{E}-04$ & 1,500 & 1,500 & 0.03 & 0.01 \\
\hline $241-\mathrm{TY}-102$ & $2.50 \mathrm{E}-04$ & 1,500 & 1,500 & 0.03 & 0.01 \\
\hline $241-T Y+103$ & $3.78 \mathrm{E}-04$ & 1,500 & 1,500 & 0.05 & 0.01 \\
\hline 241-TY-104 & $2.83 \mathrm{E}-04$ & 1,500 & 1,500 & 0.05 & 0.01 \\
\hline 241-TY-105 & $5.08 \mathrm{E}-04$ & 1,306 & 1,500 & 0.06 & 0.01 \\
\hline 241-TY-106 & $2.55 \mathrm{E}-04$ & 1,500 & 1,500 & 0.03 & 0.01 \\
\hline $241-U-101$ & $3.21 \mathrm{E}-04$ & 1,500 & 1,500 & 0.05 & 0.01 \\
\hline $241-\mathrm{U}-102$ & $9.11 \mathrm{E}-04$ & 471 & 1,500 & 0.10 & 0.02 \\
\hline
\end{tabular}


Table 4-5. The Hydrogen Generation Rates, Time to $25 \%$ and $100 \%$ of the Lower Flammability Limit and Minimum Vent Rate with Water Addition ${ }^{\text {a }}$ Under Zero Ventilation (Except Gas Diffusion Mechanism Through The Dome) for Single-Shell Tanks. (5 sheets)

\begin{tabular}{|c|c|c|c|c|c|}
\hline Tank & $\begin{array}{c}\text { Hydrogen } \\
\text { generation rate } \\
\operatorname{Rr}\left(\mathbf{H}_{2}\right) \\
\left(\mathrm{ft}^{3} / \mathrm{min}\right)\end{array}$ & $\begin{array}{c}\text { Time }^{b, c} \text { to } \\
\text { reach } 25 \% \\
\text { of the LFL } \\
\text { (days) }\end{array}$ & $\begin{array}{l}\text { Time }^{\mathrm{b}, \mathrm{c}} \text { to } \\
\text { reach } 100 \% \\
\text { of the LFL } \\
\text { (days) }\end{array}$ & $\begin{array}{l}\text { Minimum } \\
\text { vent rate to } \\
\text { keep below } \\
25 \% \text { LFL } \\
\left(\mathrm{ft}^{3} / \mathrm{min}\right)\end{array}$ & $\begin{array}{c}\text { Minimum } \\
\text { vent rate to } \\
\text { keep below } \\
100 \% \text { LFL } \\
\left(\mathrm{ft}^{3} / \mathrm{min}\right)\end{array}$ \\
\hline $241-U-103$ & $1.04 \mathrm{E}-03$ & 312 & 1,500 & 0.12 & 0.03 \\
\hline 241-U-104 & 2.75E-04 & 1,500 & $1, \overline{500}$ & 0.03 & 0.01 \\
\hline $241-U-105$ & $9.31 \mathrm{E}-04$ & 338 & 554 & 0.13 & 0.03 \\
\hline $241-U-106$ & $8.42 \mathrm{E}-04$ & 625 & 1,500 & 0.09 & 0.02 \\
\hline $241-U-107$ & 4.99E-04 & 801 & 801 & 0.06 & 0.01 \\
\hline $241-\mathrm{U}-108$ & $9.40 \mathrm{E}-04$ & 340 & 1,500 & 0.11 & 0.03 \\
\hline 241-U-109 & $6.39 \mathrm{E}-04$ & 615 & 1,500 & 0.07 & 0.02 \\
\hline $241-U-110$ & $5.22 \mathrm{E}-04$ & 853 & 1,500 & 0.07 & 0.02 \\
\hline 241-U-111 & $4.68 \mathrm{E}-04$ & 868 & 1,500 & 0.08 & 0.02 \\
\hline 241-U-112 & $3.14 \mathrm{E}-04$ & 1,500 & 1,500 & 0.05 & 0.01 \\
\hline 241-U-201 & $2.61 \mathrm{E}-05$ & 1,344 & 1,500 & 0.00 & 0.00 \\
\hline 241-U-202 & $2.17 \mathrm{E}-05$ & 1,500 & 1,500 & 0.00 & 0.00 \\
\hline 241-U-203 & $2.52 \mathrm{E}-05$ & 1,500 & 1,500 & 0.003 & 0.001 \\
\hline 241-U-204 & $5.12 \mathrm{E}-05$ & 971 & 1,500 & 0.005 & 0.001 \\
\hline
\end{tabular}

Notes:

${ }^{\mathrm{a}} \mathrm{W}$ ater addition of $10 \mathrm{kgal}$ and $1 \mathrm{kgal}$ for 100 - and 200 -series, respectively.

${ }^{b} 1,500$ in the Time to $25 \%$ and $100 \%$ of the LFL column means equal or longer than 1,500 days.

"For those tanks labeled as "not occur" means the gas generation rate is so low such that the tank will not reach $25 \%$ or $100 \%$ of the LFL through the gas diffusion mechanism.

$\mathrm{LFL}=$ lower flammability limit.

\subsection{DISCUSSION}

In Revision 4-A of this report, the flammability calculations show that the time to $25 \%$ of the LFL has become shorter for several tanks compared to the calculations in Revision 3. One of the dramatic changes is from tank $241-\mathrm{C}-103$, where the time to $25 \%$ of the LFL drops from 239 days (in Revision 3) to 63 days. The decrease in time to $25 \%$ of the LFL is because the $\mathrm{HGR}$ is increased by a factor of four due to large value of $\mathrm{G}_{\mathrm{H} 2 \mathrm{O}}$. Using the newly developed water radiolysis equation (Equation 2-1) the low concentration of $\mathrm{NO}_{3}$ and $\mathrm{NO}_{2}$ in tank 241-C-103 results in the value of 0.224 for $\mathrm{G}_{\mathrm{H} 2 \mathrm{O}}$ in Revision 4-A rather then the default value of 0.005 used in Revision 3, which is based on the old HGR model. Similarly, in tank 241-C-107 the $\mathrm{G}_{\mathrm{H} 2 \mathrm{O}}$ value used is 0.069 versus the default value of 0.005 . Apparently the water radiolysis term has been underestimated for given intermediate salt condition in Revision 3 of this document.

This newly developed water radiolysis equation is more sensitive to the salt concentrations in wide range. The development of water radiolysis equation is based on a large set of laboratory data with a wide range of salt condition ( $0-12$ moles of salts). Thus, it calculates the value of 


\section{RPP-5926 REV 6}

$\mathrm{G}_{\mathrm{H} 2 \mathrm{O}}$ more accurately for intermediate and dilute $\mathrm{NO}_{3}$ and $\mathrm{NO}_{2}$ conditions. Most of the values of $\mathrm{G}_{\mathrm{H} 2 \mathrm{O}}$ have gone up from the default value of 0.005 for given intermediate or low $\mathrm{NO}_{3}$ and $\mathrm{NO}_{2}$ conditions. The $\mathrm{G}_{\mathrm{H} 2 \mathrm{O}}$ value used for tanks 241-B-203, 241-B-204 and 241-T-201 are 0.099, 0.131 , and 0.114 , respectively, rather than the default value of 0.005 in Revision 3. However, the water radiolysis change effect dramatically impacts the final HGR only when the radiation dose or heat load is also large. The total radiation dose for beta/gamma is about $10 \mathrm{Kw}$ and $14 \mathrm{Kw}$ for 241-C-103 and 241-C-107, respectively, which are enormously large for SSTs, while the total radiation dose for tank 241-B-203, 241-B-204, and 241-T-201 are only one tenth watt or less. In other words, the change of $\mathrm{G}_{\mathrm{H} 2 \mathrm{O}}$ in $241-\mathrm{C}-103$ and $241-\mathrm{C}-107$ are amplified by their huge radiation dose. This is not the case for 241-B-203, 241-B-204 and 241-T-201.

In Revision 1, the dome space ammonia concentration in DST 241-SY-101 increased from $4 \mathrm{ppm}$ (before-transfer data) to $400 \mathrm{ppm}$ (January 2001 data), even though the liquid concentration decreased by a factor of 4 comparing data from before and after the waste was transferred out of the tank. The concentration increased because the thick crust had greatly reduced the ammonia transport from the waste to the dome space. After the waste had been transferred from DST 241-SY-101, most of the crust layer was removed and the effective area, $\mathrm{A}$, for mass transport increased; therefore, the concentration in the dome space increased even though the concentration of the ammonia in the liquid decreased. Some thin crust has formed in DST 241-SY-101. Breaks in this crust appear periodically.

Following the transfer of waste from DST 241-SY-101, the dome space ammonia concentration increased from 50 to 400 ppm from July 2000 to January 2001, while the hydrogen concentration remained constant. This may be explained by changes in the effective surface area of mass transport. For example, calculations show that an effective surface area increase from $1.5 \%$ to $7 \%$ of the total tank waste surface area would account for the dome space concentration increase from 100 to $400 \mathrm{ppm}$. This effective area of $1.5 \%$ and $7 \%$ is roughly 70 and $300 \mathrm{ft}^{2}$, respectively, and is consistent with the estimated liquid pool of $10 \mathrm{ft}$ (RPP-6517) and $20 \mathrm{ft}$ or more in diameter, respectively, observed on the surface in the corresponding time period. Calculations also indicated that most of the SSTs for which calculations were done had effective surface areas as small as several tenths of $1 \%$ of the total tank waste surface. Caution should be taken in using the results of the calculated product of $\mathrm{h}$ and $\mathrm{A}$ from Appendix $\mathrm{C}$, Table C-3 because the accuracy is limited by the accuracy of the input data. Whether these data, such as dome space ammonia concentration, ventilation rate, and liquid concentration, were collected at the same time also introduces uncertainty. DST 241-SY-101 is an example that shows that the model calculations agree reasonably with field observations when the input data are accurate and are taken in the same time frame.

Another observation is that the area of liquid surface in DST 241-SY-101 seems to be changing continuously and, therefore, the ammonia transport rate will change accordingly. Calculations show that the steady-state dome space ammonia concentration under normal ventilation conditions would reach $2,573 \mathrm{ppm}$ if the effective area were $100 \%$ for a given liquid ammonia concentration of $1,040 \mu \mathrm{g} / \mathrm{mL}$. Using this concentration, the equilibrium vapor concentration of $3,895 \mathrm{ppm}$ is calculated. For the scenario of the waste surface being fully open with a $100 \%$ effective mass transport area, the total flammable gas concentration is calculated to be $1.73 \%$ under normal ventilation conditions, and the total mass transport product of $\mathrm{h}$ and $\mathrm{A}$ is $2.09 \mathrm{ft}^{3} / \mathrm{min}$. Therefore the calculated time to reach $25 \%$ of the LFL under the off-normal 
ventilation condition would become 145 days rather than the 146 days predicted by the previous methods. Monitoring data from July 2001 show that the ammonia concentration in the dome space went up to $1,700 \mathrm{ppm}$. This is a factor of four increase compared to the January 2001 data. The increase of ammonia concentration could result from the increase of open surface area as discussed earlier. It also is possible that the results are from the reduced ventilation flow rate because a similar increase also was observed for both hydrogen and nitrous oxide. In any case, the ammonia concentration of $1,700 \mathrm{ppm}$ is still within the range of the ammonia calculation, which shows that the time to $25 \%$ would be around 146 days whether the ammonia concentration is $400 \mathrm{ppm}$ or $2,573 \mathrm{ppm}$ under the off-normal ventilation condition.

These HGR models assume that hydrogen generation occurs in the liquid phase. For the supernatant layer, this model uses the supernatant composition data when calculating generation rates. For the solid layer, the interstitial liquid composition data and the liquid fraction are used to estimate the gas generation rate. The liquid fraction of the solid layer is estimated based on weight-percent-water data of interstitial liquid and bulk of the solid layer. If new data is not available, historical data from previous versions are used for each waste layer. The historical data from previous versions come from either BBI data or are statistically determined from the other tanks in the same waste group. 
RPP-5926 REV 6

This page intentionally left blank. 


\subsection{SUMMARY AND CONCLUSIONS}

The results of this report may be used to establish technical safety requirements for ventilation in tanks, establish maximum permissible down times for active ventilation systems, develop procedures for operations that require closing vent valves to HEPA filters on passively ventilated tanks, and support the technical basis for flammable gas control strategies. Revision 6 is mainly an update of the input data for HGR calculations using latest BBI data for physical and chemical properties of the waste and PCSACs data for waste temperatures.

The methodology for the HGR calculation was updated in Revision 4 with the newly upgraded HGR model (HNF-3851) as summarized in Chapter 2.0. The main thrust of applying the new model to current HGR calculations is to correctly calculate the water radiolysis term for the tanks having intermediate/dilute salt conditions. HGRs were calculated for the 177 SSTs and DSTs using this updated rate equation model and latest tank waste data. For tanks that do not have complete information, missing values were defaulted to the values used in Revision 4.

Flammable gas concentrations, in terms of percent of the LFL in the tank dome space, were evaluated under both normal and off-normal conditions for all 177 tanks. For normal ventilation conditions, all tanks are computed to remain well below $25 \%$ of the LFL with the highest flammable gas concentration level at $6.74 \%$ of the LFL in SST 241-C-107. With a barometric breathing rate, the shortest time to reach $25 \%$ of the LFL for current tank waste is 39 days for SST 241-B-203 and 10 days for DST 241-AZ-102; with zero ventilation scenario, the shortest time to reach $25 \%$ of the LFL for water addition is 35 days for SST 241-B-203 and 10 days for DST 241-AZ-102.

Calculated HGRs of current raw waste for DSTs ranged from $3.6 \mathrm{E}-2$ to $3.2 \mathrm{E}-4 \mathrm{ft}^{3} / \mathrm{min}$ with the highest rate found in DST 241-AZ-102. For SSTs, the rate ranged from $6.9 \mathrm{E}-3$ to $1.9 \mathrm{E}-5 \mathrm{ft}^{3} / \mathrm{min}$, with SST $241-\mathrm{SX}-103$ having the highest rate. For most DSTs, the tank data are sample based. For quite a few SSTs, the tanks were stabilized and no liquid samples were available.

The steady-state flammability level in the tank dome space was evaluated based on the steady-state gas concentrations of hydrogen, ammonia, and methane using Le Chatelier's rule. Overall, the calculations under off-normal conditions show that 22 DSTs and 7 SSTs will reach $25 \%$ of the LFL and 5 DSTs and 2 SSTs will reach $100 \%$ of the LFL if allowed to reach steady state with the off-normal ventilation rates of barometric breathing. 
RPP-5926 REV 6

This page intentionally left blank. 


\subsection{REFERENCES}

24590-WTP-RPT-RT-04-0002, 2004, Modifying the Hu Correlation to Predict Hydrogen Formation in the Hanford Waste Treatment and Immobilization Plant, Bechtel National, Richland, Washington.

Coward, H. F., and G. W. Jones, 1952, Limits of Flammability of Gases and Vapors, Bumines B. 503, 155 pp.

HNF-3294, 1998, Adequacy of Ventilation Rates to Remediate Flammable Gas Levels in Headspace of Hanford Tanks - Status Report, Rev. 0, Lockheed Martin Hanford Corporation, Richland, Washington.

HNF-3851, 2004, Empirical Rate Equation Model and Rate Calculations of Hydrogen Generation for Hanford Tank Waste, Rev.1, CH2M HILL Hanford Group, Inc., Richland, Washington.

HNF-SD-WM-CN-116, 1997, Calculation Note Hydrogen Generation Rates At Steady State Flammable Gas Concentrations For Single-Shell Tanks, Rev. 0A, Lockheed Martin Hanford Corporation, Richland, Washington.

HNF-SD-WM-CN-117, 1997, Calculations of Hydrogen Release Rate at Steady State for Double-Shell Tanks, Rev. 0A, Lockheed Martin Hanford Corporation, Richland, Washington.

HNF-SP-1193, 1997, Flammable Gas Project Topical Report, Rev. 2, Lockheed Martin Hanford Corporation, Richland, Washington.

Pfahl, U., and J. Shepherd, 1997, Flammability and Flame Propagation in $\mathrm{H}_{2}-\mathrm{N}_{2} \mathrm{O}-\mathrm{CH}_{4}-\mathrm{NH}_{3}-\mathrm{O}_{2}-\mathrm{N}_{2}$ Mixtures, Explosion Dynamics Laboratory Report FM97-4, Graduate Aeronautical Laboratories, California Institute of Technology, Pasadena, California.

PNNL-1 1297, 1996, Status and Integration of Studies of Gas Generation in Hanford Wastes, Rev. 0, Pacific Northwest National Laboratory, Richland, Washington.

PNNL-11450, 1997, Composition and Quantities of Retained Gas Measured in Hanford Waste Tanks 241-AW-101, $A-101, A N-105, A N-104$, and $A N-103$, Rev. 1, Pacific Northwest National Laboratory, Richland, Washington.

PNNL-1 1925, 1998, Waste Tank Ventilation Rates Measured with A Tracer Gas Method, Fiscal Year 1998 Summary, Pacific Northwest National Laboratory, Richland, Washington.

PNNL-12181, 1999, Thermal and Radiolytic Gas Generation on Material from Tanks 241-U-103, 241-AW-101, 241-S-106, and 241-S-102: Status Report, Pacific Northwest National Laboratory, Richland, Washington. 
PNNL-13000, 1999, Retained Gas Sampling Results for the Flammable Gas Program, Rev. 0, Pacific Northwest National Laboratory, Richland, Washington.

RPP-4941, 2000, Methodology for Predicting Flammable Gas Mixtures in Double-Contained Receiver Tanks, Rev. 0B, CH2M HILL Hanford Group, Inc., Richland, Washington.

RPP-5660, 2000, Collection And Analysis Of Selected Tank Headspace Parameter Data, Rev. 0, CH2M HILL Hanford Group, Inc., Richland, Washington.

RPP-6069, 2000, Input Data for Hydrogen Generation Rate Model Calculation, Rev. 0, CH2M HILL Hanford Group, Inc., Richland, Washington.

RPP-6213, 2005, Hanford Waste Tank Bump Accident and Consequence Analysis, Rev. 4, CH2M HILL Hanford Group, Inc., Richland, Washington.

RPP-6517, 2000, Evaluation of Hanford High-Level Waste Tank 241-SY-101, Rev. 0, CH2M HILL Hanford Group, Inc., Richland, Washington.

RPP-18491, 2003, Flammable Gas Diffusion Through Single-Shell Tank Domes, Rev. 0, CH2M HILL Hanford Group, Inc., Richland, Washington.

SACS, 2001, Surveillance Analysis Computer System, Queried September 2006, [Tank temperature data April 2005 through April 2006], HISI ID No. 242, CH2M HILL Hanford Group, Inc., Richland, Washington.

SNL-000198, 1999, Flammable Gas Safety Analysis Data Review, Sandia National Laboratory, Albuquerque, New Mexico.

TFC-ENG-CHEM-D-33, 2003, Spreadsheet Verification, Rev. B, CH2M HILL Hanford Group, Inc., Richland, Washington.

TFC-ENG-DESIGN-C-10, 2006, Engineering Calculations, Rev. A-16, CH2M HILL Hanford Group, Inc., Richland, Washington.

TFC-ENG-DESIGN-C-32, 2006, Spreadsheet Development and Verification, Rev. B-4, $\mathrm{CH} 2 \mathrm{M}$ HILL Hanford Group, Inc., Richland, Washington.

TWINS, 2006, Tank Waste Information Network System, queried September 2006 [Best Basis Inventory data], internet address: http://twins.pnl.gov/twins.htm.

Weisenberger, S., and A. Schumpe, 1996, "The Estimation of Gas Solubility in Salt Solutions at Temperature from $273 \mathrm{~K}$ to $363 \mathrm{~K}$," AICHE Journal, Vol. 42, 299.

WHC-EP-0651, 1993, Barometric Pressure Variations, Westinghouse Hanford Company, Richland, Washington.

WHC-EP-0702, 1994, Criteria for Flammable Gas Watch List Tanks, Rev. 0, Westinghouse Hanford Company, Richland, Washington. 


\section{RPP-5926 REV 6}

WHC-SD-WM-ES-219, 1992, Laboratory Flammability Studies of Mixtures of Hydrogen, Nitrous Oxide, and Air, Rev. 0, Westinghouse Hanford Company, Richland, Washington.

WHC-SD-WM-SARR-015, 1994, Topical Report on Flammable Gases in Non-Burping Waste Tanks, Westinghouse Hanford Company, Richland, Washington.

WHC-SD-WM-TI-724, 1996, Methodology for Flammable Gas Evaluations, Rev. 1, Westinghouse Hanford Company, Richland, Washington. 
RPP-5926 REV 6

This page intentionally left blank. 
RPP-5926 REV 6

APPENDIX A

INPUT DATA FOR HYDROGEN GENERATION RATE CALCULATIONS FROM BEST BASIS INVENTORY DETAIL CALCULATION REPORT AND DERIVATION DOCUMENT (QUERIED ON SEPTEMBER 27, 2006) 
RPP-5926 REV 6

This page intentionally left blank.

A-ii 


\section{RPP-5926 REV 6}

\section{CONTENTS}

Table A-1. Best Basis Inventory - Density and Weight Percent Water by Tank, Waste

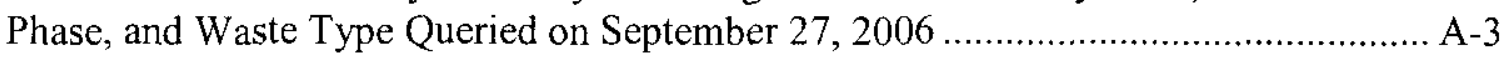

Table A-2. Tank Waste Inventory from Best Basis Inventory Detail Report (Queried on September 27, 2006) for Hydrogen Generation Rate Calculations ............................. A-15

Table A-3. Retained Gas Inventory from Best Basis Inventory Detail Report (Queried on September 27, 2006) for Hydrogen Generation Rate Calculations ............................. A-42 
RPP-5926 REV 6

This page intentionally left blank.

A-2 
Table A-1. Best Basis Inventory - Density and Weight Percent Water By Tank, Waste Phase, and Waste Type Queried on September 27, 2006. (12 sheets)

\begin{tabular}{|c|c|c|c|c|c|}
\hline Tank Name & Waste Phase & Waste Type & $\begin{array}{c}\text { Volume } \\
(\mathrm{kL})\end{array}$ & $\begin{array}{l}\text { Density } \\
(\mathrm{g} / \mathrm{mL})\end{array}$ & Wt\% Water \\
\hline $241-\mathrm{A}-101$ & Sludge (Liquid \& Solid) & P2 (Solid) & 11 & 1.36 & 9.1 \\
\hline 241-A-101 & Saltcake Solid & A1-SltCk (Solid) & 878 & 1.74 & 29.2 \\
\hline $241-\mathrm{A}-101$ & Saltcake Interstitial Liquid & A1-SltCk (Liquid) & 148 & 1.49 & 46.9 \\
\hline $241-\mathrm{A}-101$ & Retained Gas (Saltcake) & NA & 174 & NA & NA \\
\hline 241-A-102 & Supernatant & Al-SltCk (Liquid) & 12 & 1.57 & 46.2 \\
\hline 241-A-102 & Saltcake Solid & A1-SltCk (Solid) & 106 & 1.7 & 34.3 \\
\hline 241-A-102 & Saltcake Interstitial Liquid & A1-SltCk (Liquid) & 33 & 1.57 & 46.2 \\
\hline 241-A-103 & Supernatant & A1-SltCk (Liquid) & 17 & 1.51 & 50.3 \\
\hline 241-A-103 & Sludge (Liquid \& Solid) & AR (Solid) & 8 & 1.34 & 68.6 \\
\hline 241-A-103 & Saltcake Solid & A1-SltCk (Solid) & 1071 & 1.32 & 37.8 \\
\hline 241-A-103 & Saltcake Interstitial Liquid & A1-SltCk (Liquid) & 338 & 1.51 & 50.3 \\
\hline 241-A-104 & Sludge Solid & AR (Solid) & 102 & 0.946 & 0 \\
\hline 241-A-104 & Sludge Solid & P1 (Solid) & 4 & 0.946 & 0 \\
\hline 241-A-105 & Sludge Solid & P2 (Solid) & 139 & 1.54 & 0 \\
\hline $241-\mathrm{A}-106$ & Sludge (Liquid \& Solid) & SRR (Solid) & 110 & 1.7 & 34.3 \\
\hline 241-A-106 & Sludge (Liquid \& Solid) & AR (Solid) & 79 & 1.7 & 34.3 \\
\hline 241-A-106 & Saltcake Solid & A1-SltCk (Solid) & 110 & 1.7 & 34.3 \\
\hline 241-AN-101 & Supernatant & $\mathrm{NA}$ & 3506 & 1.41 & 51.5 \\
\hline 241-AN-101 & Saltcake Solid & A1-SltCk (Solid) & 90 & 1.58 & 37.8 \\
\hline 241-AN-101 & Saltcake Interstitial Liquid & A1-SltCk (Liquid) & 28 & 1.45 & 49.1 \\
\hline 241-AN-102 & Supernatant & NA & 3468 & 1.41 & 42.7 \\
\hline 241-AN-102 & Saltcake (Liquid \& Solid) & A2-SltSlr (Solid) & 584 & 1.53 & 39.9 \\
\hline 241-AN-103 & Supernatant & A2-SltSh (Liquid) & 1769 & 1.48 & 46.7 \\
\hline 241.AN-103 & Saltcake (Liquid \& Solid) & A2-SltSlr (Solid) & 1638 & 1.72 & 34.9 \\
\hline 241-AN-103 & Retained Gas (Saltcake) & NA & 220 & NA & NA \\
\hline 241-AN-104 & Supernatant & A2-SltSlr (Liquid) & 2298 & 1.4 & 51.1 \\
\hline 241-AN-104 & Saltcake (Liquid \& Solid) & A2-SltSlr (Solid) & 1566 & 1.59 & 45.7 \\
\hline 241-AN-104 & Retained Gas (Saltcake) & $\mathrm{NA}$ & 119 & NA & NA \\
\hline 241-AN-105 & Supernatant & A2-SltSlr (Liquid) & 2227 & 1.42 & 49.9 \\
\hline 241-AN-105 & Saltcake (Liquid \& Solid) & A2-SltSlr (Solid) & 1925 & 1.57 & 44.4 \\
\hline 241-AN-105 & Retained Gas (Saltcake) & $\mathrm{NA}$ & 109 & NA & $\mathrm{NA}$ \\
\hline 241-AN-106 & Supernatant & NA & 2485 & 1.1 & 83.1 \\
\hline 241-AN-106 & Sludge Interstitial Liquid & $\mathrm{NA}$ & 192 & 1.1 & 83.1 \\
\hline 241-AN-106 & Sludge (Liquid \& Solid) & NA & 299 & 1.54 & 47.3 \\
\hline 241-AN-106 & Saltcake (Liquid \& Solid) & $\mathrm{NA}$ & 65 & 1.58 & 37.8 \\
\hline 241-AN-107 & Supernatant & A2-SltSlr (Liquid) & 3297 & 1.43 & 51.5 \\
\hline 241-AN-107 & Saltcake (Liquid \& Solid) & A2-SltSlr (Solid) & 872 & 1.48 & 44.3 \\
\hline 241-AP-101 & Supernatant & A2-SltSlr (Liquid) & 4219 & 1.3 & 63.6 \\
\hline 241-AP-102 & Supernatant & Waste Transfer & 4049 & 1.39 & 58.7 \\
\hline 241-AP-102 & Sludge (Liquid \& Solid) & NA & 88 & 1.75 & 31.1 \\
\hline 241-AP-103 & Supernatant & $\mathrm{NA}$ & 3298 & 1.35 & 56.7 \\
\hline 241-AP-103 & Sludge (Liquid \& Solid) & $\mathrm{NA}$ & 80 & 1.68 & 40.9 \\
\hline 241-AP-104 & Supernatant & NA (Liquid) & 4155 & 1.28 & 66.4 \\
\hline 241-AP-105 & Supernatant & Waste Transfer & 3965 & 1.27 & 71.4 \\
\hline 241-AP-105 & Saltcake (Liquid \& Solid) & A2-SltSlr (Solid) & 337 & 1.61 & 47.7 \\
\hline 241-AP-106 & Supernatant & NA & 4301 & 1.21 & 72.4 \\
\hline 241-AP-107 & Supernatant & $\mathrm{NA}$ & 1306 & 1.31 & 63.1 \\
\hline
\end{tabular}


Table A-1. Best Basis Inventory - Density and Weight Percent Water By Tank, Waste Phase, and Waste Type Queried on September 27, 2006. (12 sheets)

\begin{tabular}{|c|c|c|c|c|c|}
\hline Tank Name & Waste Phase & Waste Type & $\begin{array}{c}\text { Volume } \\
(\mathrm{kL})\end{array}$ & $\begin{array}{l}\text { Density } \\
(\mathrm{g} / \mathrm{mL})\end{array}$ & Wt $\%$ Water \\
\hline 241-AP-108 & Supernatant & $\mathrm{NA}$ & 3385 & 1.42 & 49.7 \\
\hline 241-AP-108 & Saltcake (Liquid \& Solid) & $\mathrm{NA}$ & 487 & 1.52 & 46 \\
\hline 241-AW-101 & Supernatant & A2-SltSlr (Liquid) & 2770 & 1.47 & 43.8 \\
\hline 241-AW-101 & Saltcake (Liquid \& Solid) & A2-SltSlr (Solid) & 1403 & 1.59 & 43.9 \\
\hline 241-AW-101 & Retained Gas (Saltcake) & $\mathrm{NA}$ & $\overline{95}$ & NA & $\mathrm{NA}$ \\
\hline $241-\mathrm{AW}-102$ & Supernatant & Waste Transfer & 2268 & 1.22 & 71.1 \\
\hline 241-AW-102 & Sludge (Liquid \& Solid) & NA & 25 & 1.32 & 33.4 \\
\hline 241-AW-103 & Supernatant & NA & 2893 & 1.24 & 69.2 \\
\hline 241-AW-103 & Sludge (Liquid \& Solid) & CWZr2 (Solid) & 1105 & 1.47 & 55.8 \\
\hline $241-\mathrm{AW}-103$ & Saltcake Solid & Al-SltCk (Solid) & 115 & 1.69 & 45.2 \\
\hline $241-A W-103$ & Saltcake Interstitial Liquid & A1-SltCk (Liquid) & 36 & 1.42 & 50.5 \\
\hline 241-AW-104 & Supernatant & NA & 3221 & 1.35 & 66.6 \\
\hline 241-AW-104 & Sludge Solid & PL2 (Solid) & 188 & 1.28 & 65.8 \\
\hline 241-AW-104 & Sludge Interstitial Liquid & PL2 (Liquid) & 62 & 1.33 & 63.8 \\
\hline $241-A W+104$ & Saltcake Solid & A2-SltSlr (Solid) & 457 & 1.6 & 42 \\
\hline 241-AW-104 & Saltcake Interstitial Liquid & A2-SltSlr (Liquid) & 136 & 1.45 & 52 \\
\hline 241-AW-105 & Supernatant & NA & 587 & 1.06 & 89.9 \\
\hline 241-AW-105 & Sludge Solid & CWZr2 (Solid) & 660 & 1.47 & 49.7 \\
\hline 241-AW-105 & Sludge Solid & PL2 (Solid) & 61 & 1.41 & 37.8 \\
\hline 241-AW-105 & Sludge Interstitial Liquid & CWZr2 (Liquid) & 266 & 1.1 & 84.3 \\
\hline 241-AW-105 & Sludge Interstitial Liquid & PL2 (Liquid) & 12 & 1.17 & 76.5 \\
\hline 241-AW-106 & Supernatant & Waste Transfer & 3224 & 1.25 & 74.6 \\
\hline 241-AW-106 & Saltcake (Liquid \& Solid) & A2-SitSlr (Solid) & 1072 & 1.61 & 43.1 \\
\hline 241-AX-101 & Sludge (Liquid \& Solid) & SRR (Solid) & 11 & 1.51 & 62.2 \\
\hline $241-\mathrm{AX}-101$ & Saltcake Solid & A1-SltCk (Solid) & 1164 & 1.73 & 32.5 \\
\hline $241-\mathrm{AX}-101$ & Saltcake Interstitial Liquid & A1-SltCk (Liquid) & 178 & 1.53 & 43.1 \\
\hline $241-\mathrm{AX}-102$ & Sludge (Liquid \& Solid) & B (Solid) & 23 & 1.57 & 42.5 \\
\hline $241-\mathrm{AX}-102$ & Saltcake (Liquid \& Solid) & Al-SltCk (Solid) & 90 & 1.58 & 33.9 \\
\hline $241-\mathrm{AX}-103$ & Sludge (Liquid \& Solid) & P2 (Solid) & 30 & 1.61 & 44.2 \\
\hline $241-\mathrm{AX}-103$ & Saltcake Solid & A1-SltCk (Solid) & 298 & $1 . \overline{61}$ & 43.2 \\
\hline $241-\mathrm{AX}-103$ & Saltcake Interstitial Liquid & A1-SltCk (Liquid) & 75 & 1.45 & 49.1 \\
\hline $241-\mathrm{AX}-104$ & Sludge Solid & P2 (Solid) & 28 & 1.8 & 8.23 \\
\hline 241-AY-101 & Supernatant & NA (Liquid) & 426 & 1.13 & 85.1 \\
\hline 241-AY-101 & Sludge Solid & NA (Solid) & 339 & 1.78 & 35.1 \\
\hline 241-AY-101 & Sludge Interstitial Liquid & NA (Liquid) & 60 & 1.14 & 81.4 \\
\hline 241-AY-102 & Supernatant & NA & 3092 & 1.15 & 82.7 \\
\hline 241-AY-102 & Sludge Solid & BL (Solid) & 75 & 1.69 & 31.6 \\
\hline $241-\mathrm{AY}-102$ & Sludge Solid & NA & 376 & 1.69 & 31.5 \\
\hline 241-AY-102 & Sludge Interstitial Liquid & BL (Liquid) & 120 & 1.15 & 82 \\
\hline 241-AZ-101 & Supernatant & P3AZ1 (Liquid) & 3074 & 1.22 & 69.6 \\
\hline $241-\mathrm{AZ}-101$ & Sludge (Liquid \& Solid) & P3AZ1 (Solid) & 167 & 1.61 & 34.3 \\
\hline $241-\mathrm{AZ}-101$ & Sludge (Liquid \& Solid) & NA (Solid) & 30 & 1.61 & 34.3 \\
\hline 241-AZ-102 & Supernatant & P3AZ2 (Liquid) & 3267 & 1.13 & 84.1 \\
\hline $241-\mathrm{AZ}-102$ & Sludge (Liquid \& Solid) & P3AZ2 (Solid) & 275 & 1.41 & 54.8 \\
\hline $241-\mathrm{AZ}-102$ & Sludge (Liquid \& Solid) & PL2 (Solid) & 15 & 1.41 & 54.8 \\
\hline 241-AZ-102 & Sludge (Liquid \& Solid) & SRR (Solid) & 98 & 1.41 & 54.8 \\
\hline $241-\mathrm{AZ}-102$ & Sludge (Liquid \& Solid) & NA sludge & 8 & 1.41 & 54.8 \\
\hline
\end{tabular}


Table A-1. Best Basis Inventory - Density and Weight Percent Water By Tank, Waste Phase, and Waste Type Queried on September 27, 2006. (12 sheets)

\begin{tabular}{|c|c|c|c|c|c|}
\hline Tank Name & Waste Phase & Waste Type & $\begin{array}{c}\text { Volume } \\
(\mathrm{kL})\end{array}$ & $\begin{array}{l}\text { Density } \\
(\mathrm{g} / \mathrm{mL})\end{array}$ & Wt\% Water \\
\hline $241-\mathrm{B}-101$ & Sludge (Liquid \& Solid) & MW1 (Solid) & 11 & 1.8 & 43.8 \\
\hline $241-\mathrm{B}-101$ & Sludge (Liquid \& Solid) & B (Solid) & 19 & 1.74 & 52.7 \\
\hline 241-B-101 & Sludge (Liquid \& Solid) & BL (Solid) & 76 & 1.5 & 52.4 \\
\hline 241-B-101 & Saltcake Solid & B-SltCk (Solid) & 232 & 1.48 & 32 \\
\hline $241-\mathrm{B}-101$ & Saltcake Interstitial Liquid & B-SitCk (Liquid) & 73 & 1.53 & 50 \\
\hline 241-B-102 & Supernatant & B-SltCk (Liquid) & 15 & 1.26 & 69.6 \\
\hline $241-B-102$ & Saltcake Solid & B-SltCk (Solid) & 81 & 1.72 & 39.7 \\
\hline $241-\mathrm{B}-102$ & Saltcake Interstitial Liquid & B-SltCk (Liquid) & 25 & 1.26 & 69.6 \\
\hline 241-B-103 & Sludge (Liquid \& Solid) & MW1 (Solid) & 4 & 1.8 & 43.8 \\
\hline 241-B-103 & Saltcake Solid & B-SltCk (Solid) & 157 & 1.72 & 44.9 \\
\hline 241-B-103 & Saltcake Interstitial Liquid & B-SltCk (Liquid) & 50 & 1.26 & 69.6 \\
\hline 241-B-104 & Sludge (Liquid \& Solid) & $1 \mathrm{C}$ (Solid) & 473 & 1.39 & 46.7 \\
\hline 241-B-104 & Sludge (Liquid \& Solid) & 2C (Solid) & 697 & 1.39 & 46.7 \\
\hline 241-B-104 & Saltcake Solid & B-SltCk (Solid) & 192 & 1.39 & 46.7 \\
\hline $241-B-104$ & Saltcake Interstitial Liquid & B-SltCk (Liquid) & 55 & 1.26 & 69.6 \\
\hline 241-B-105 & Sludge (Liquid \& Solid) & 2C (Solid) & 61 & 1.28 & 66.6 \\
\hline $241-B-105$ & Sludge (Liquid \& Solid) & IC (Solid) & 45 & 1.43 & 54.1 \\
\hline $241-\mathrm{B}-105$ & Saltcake Solid & B-SltCk (Solid) & 919 & 1.72 & 40.2 \\
\hline 241-B-105 & Saltcake Interstitial Liquid & B-SltCk (Liquid) & 73 & 1.26 & 69.6 \\
\hline 241-B-106 & Supernatant & B-SitCk (Liquid) & 4 & 1.26 & 67.2 \\
\hline $241-\mathrm{B}-106$ & Sludge (Liquid \& Solid) & TBP (Solid) & 297 & 1.36 & 61.6 \\
\hline 241-B-106 & Sludge (Liquid \& Solid) & $1 \mathrm{C}$ (Solid) & 163 & 1.42 & 56.8 \\
\hline 241-B-107 & Sludge (Liquid \& Solid) & $1 \mathrm{C}($ Solid $)$ & 285 & 1.63 & 42.1 \\
\hline 241-B-107 & Sludge (Liquid \& Solid) & CWP2 (Solid) & 42 & 1.68 & 40.9 \\
\hline 241-B-107 & Saltcake Solid & B-SitCk (Solid) & 216 & 1.7 & 33.1 \\
\hline $241-\mathrm{B}-107$ & Saltcake Interstitial Liquid & $\overrightarrow{\text { B-SltCk (Liquid) }}$ & 68 & 1.34 & 57.5 \\
\hline 241-B-108 & Sludge (Liquid \& Solid) & CWP2 (Solid) & 104 & 1.8 & 25.9 \\
\hline 241-B-108 & Saltcake Solid & B-SltCk (Solid) & 204 & 1.72 & 33.3 \\
\hline 241-B-108 & Saltcake Interstitial Liquid & B-SltCk (Liquid) & 42 & 1.38 & 69.6 \\
\hline 241-B-109 & Sludge (Liquid \& Solid) & CWP2 (Solid) & 189 & 1.85 & 36.2 \\
\hline 241-B-109 & Saltcake Solid & B-SltCk (Solid) & 245 & 1.89 & 38.4 \\
\hline 241-B-109 & Saltcake Interstitial Liquid & B-SltCk (Liquid) & 41 & 1.26 & 69.6 \\
\hline $241-\mathrm{B}-110$ & Supernatant & CSR (Liquid) & 4 & 1.19 & 76.2 \\
\hline $241-\mathrm{B}-110$ & Sludge (Liquid \& Solid) & B (Solid) & 11 & 1.36 & 58.3 \\
\hline $241-\mathrm{B}-110$ & Sludge (Liquid \& Solid) & 2C(Solid) & 914 & 1.36 & 58.3 \\
\hline 241-B-111 & Supernatant & CSR (Liquid) & 4 & 1.19 & 76.2 \\
\hline $241-\mathrm{B}-111$ & Sludge (Liquid \& Solid) & 2C (Solid) & 809 & 1.27 & 63.1 \\
\hline $241-\mathrm{B}-111$ & Sludge (Liquid \& Solid) & B (Solid) & 101 & 1.27 & 63.1 \\
\hline $241-\mathrm{B}-112$ & Supernatant & BY-SltCk (Liquid) & 11 & 1.51 & 46.2 \\
\hline $241-\mathrm{B}-112$ & Sludge (Liquid \& Solid) & 2C (Solid) & 56 & 1.49 & 40.2 \\
\hline 241-B-112 & Saltcake Solid & BY-SltCk (Solid) & 49 & 1.49 & 40.2 \\
\hline $241-\mathrm{B}-112$ & Saltcake Interstitial Liquid & BY-SltCk (Liquid) & 16 & 1.51 & 46.2 \\
\hline 241-B-201 & Sludge (Liquid \& Solid) & 224-1 (Solid) & 111 & 1.26 & 64.2 \\
\hline 241-B-202 & Sludge (Liquid \& Solid) & 224-2 (Solid) & 108 & 1.22 & 75.9 \\
\hline 241-B-203 & Supernatant & NA & 2 & 1.05 & 89.4 \\
\hline 241-B-203 & Sludge (Liquid \& Solid) & 224-2 (Solid) & 188 & 1.19 & $\overline{75.7}$ \\
\hline $241-\mathrm{B}-204$ & Supernatant & NA & 3 & 1.05 & 91.4 \\
\hline
\end{tabular}


Table A-1. Best Basis Inventory - Density and Weight Percent Water By Tank, Waste Phase, and Waste Type Queried on September 27, 2006. (12 sheets)

\begin{tabular}{|c|c|c|c|c|c|}
\hline Tank Name & Waste Phase & Waste Type & $\begin{array}{c}\text { Volume } \\
(\mathrm{kL})\end{array}$ & $\begin{array}{l}\text { Density } \\
(\mathrm{g} / \mathrm{mL})\end{array}$ & Wt\% Water \\
\hline 241-B-204 & Sludge (Liquid \& Solid) & 224-2 (Solid) & 184 & 1.19 & 77.3 \\
\hline 241-BX-101 & Sludge (Liquid \& Solid) & TBP (Solid) & 59 & 1.68 & 15.5 \\
\hline 241-BX-101 & Sludge (Liquid \& Solid) & CWP2 (Solid) & 47 & 1.68 & 15.5 \\
\hline 241-BX-101 & Sludge (Liquid \& Solid) & BL (Solid) & 74 & 1.68 & 15.5 \\
\hline 241-BX-102 & Sludge Solid & CWP2 (Solid) & 81 & 1.68 & 40.9 \\
\hline 241-BX-102 & Sludge Solid & DE (Solid) & 147 & 0.384 & 0 \\
\hline 241-BX-102 & Sludge Solid & TBP (Solid) & 70 & 1.47 & 50.5 \\
\hline 241-BX-103 & Supernatant & CWP2 (Liquid) & 48 & 1.07 & 77.8 \\
\hline $241-\mathrm{BX}-103$ & Sludge (Liquid \& Solid) & TBP (Solid) & 21 & 1.47 & 49.6 \\
\hline 241-BX-103 & Sludge (Liquid \& Solid) & CWP2 (Solid) & 214 & 1.68 & 49.6 \\
\hline 241-BX-104 & Supernatant & CWR1 (Liquid) & 11 & 1.28 & 59.1 \\
\hline 241-BX-104 & Sludge (Liquid \& Solid) & CWP2 (Solid) & 51 & 1.68 & 26.9 \\
\hline 241-BX-104 & Sludge (Liquid \& Solid) & MW1 (Solid) & 155 & 1.68 & 26.9 \\
\hline $241-\mathrm{BX}-104$ & Sludge (Liquid \& Solid) & CWR1 (Solid) & 110 & 1.68 & 26.9 \\
\hline 241-BX-104 & Sludge (Liquid \& Solid) & TBP (Solid) & 53 & 1.68 & 26.9 \\
\hline 241-BX-105 & Supernatant & CWP2 (Liquid) & 18 & 1.29 & 68.7 \\
\hline 241-BX-105 & Sludge (Liquid \& Solid) & TBP (Solid) & 55 & 1.69 & 12.6 \\
\hline 241-BX-105 & Sludge (Liquid \& Solid) & MW1 (Solid) & 9 & 1.8 & 43.8 \\
\hline 241-BX-105 & Sludge (Liquid \& Solid) & CWP2 (Solid) & 96 & 1.69 & 12.6 \\
\hline 241-BX-105 & Saltcake (Liquid \& Solid) & BY-SltCk (Solid) & 94 & 1.69 & 12.6 \\
\hline 241-BX-106 & Sludge (Liquid \& Solid) & TBP (Solid) & 20 & 1.64 & 38.7 \\
\hline 241-BX-106 & Sludge (Liquid \& Solid) & CWP2 (Solid) & 18 & 1.64 & 38.7 \\
\hline 241-BX-106 & Saltcake Solid & BY-SltCk (Solid) & 80 & 1.64 & 38.7 \\
\hline 241-BX-106 & Saltcake Interstitial Liquid & BY-SltCk (Liquid) & 25 & 1.51 & 46.2 \\
\hline 241-BX-107 & Sludge (Liquid \& Solid) & 1C (Solid) & 1313 & 1.44 & 50.5 \\
\hline 241-BX-108 & Sludge (Liquid \& Solid) & 1C (Solid) & 38 & 1.43 & 17.2 \\
\hline 241-BX-108 & Sludge (Liquid \& Solid) & TBP (Solid) & 81 & 1.47 & 17.2 \\
\hline 241-BX-109 & Sludge (Liquid \& Solid) & TBP (Solid) & 730 & 1.52 & 50.6 \\
\hline $241-\mathrm{BX}-110$ & Supernatant & BY-SltCk (Liquid) & 5 & 1.44 & 53.1 \\
\hline 241-BX-110 & Sludge (Liquid \& Solid) & $\mathrm{NA}$ & 94 & 1.79 & 36.6 \\
\hline 241-BX-110 & Sludge (Liquid \& Solid) & 1C (Solid) & 151 & 1.43 & 54.1 \\
\hline $241-\overline{B X}-110$ & Saltcake Solid & BY-SltCk (Solid) & 433 & 1.79 & 36.6 \\
\hline 241-BX-110 & Saltcake Interstitial Liquid & BY-SltCk (Liquid) & 129 & 1.44 & 53.1 \\
\hline $241-\mathrm{BX}-111$ & Sludge (Liquid \& Solid) & $1 \mathrm{C}$ (Solid) & 121 & 1.43 & 54.1 \\
\hline 241-BX-111 & Saltcake Solid & BY-SltCk (Solid) & 538 & 1.45 & 15.6 \\
\hline 241-BX-111 & Saltcake Interstitial Liquid & BY-SitCk (Liquid) & 54 & 1.45 & 51.2 \\
\hline 241-BX-112 & Supernatant & 1C2 (Liquid) & 5 & 1.18 & 67.6 \\
\hline 241-BX-112 & Sludge (Liquid \& Solid) & $1 \mathrm{C}$ (Solid) & 617 & 1.31 & 63.3 \\
\hline 241-BY-101 & Sludge (Liquid \& Solid) & TFeCN (Solid) & 140 & 1.6 & 46.8 \\
\hline 241-BY-101 & Saltcake Solid & BY-SltCk (Solid) & 1208 & 1.87 & 20.4 \\
\hline 241-BY-101 & Saltcake Interstitial Liquid & BY-SltCk (Liquid) & 52 & 1.51 & 46.2 \\
\hline 241-BY-102 & Saltcake Solid & BY-SltCk (Solid) & 897 & 1.59 & 27.9 \\
\hline 241-BY-102 & Saltcake Interstitial Liquid & BY-SltCk (Liquid) & 157 & 1.46 & 50.8 \\
\hline 241-BY-103 & Sludge (Liquid \& Solid) & CWP2 (Solid) & 34 & 1.68 & 40.9 \\
\hline 241-BY-103 & Saltcake Solid & BY-SltCk (Solid) & 1316 & 1.72 & 23.7 \\
\hline 241-BY-103 & Saltcake Interstitial Liquid & BY-SltCk (Liquid) & 214 & 1.29 & 59 \\
\hline 241-BY-104 & Sludge (Liquid \& Solid) & PFeCN (Solid) & 172 & 1.64 & 29 \\
\hline
\end{tabular}


Table A-1. Best Basis Inventory - Density and Weight Percent Water By Tank, Waste Phase, and Waste Type Queried on September 27, 2006. (12 sheets)

\begin{tabular}{|c|c|c|c|c|c|}
\hline Tank Name & Waste Phase & Waste Type & $\begin{array}{l}\text { Volume } \\
\text { (kL) }\end{array}$ & $\begin{array}{l}\text { Density } \\
(\mathrm{g} / \mathrm{mL})\end{array}$ & Wt $\%$ Water \\
\hline 241-BY-104 & Saltcake Solid & BY-SltCk (Solid) & 1208 & 1.75 & 23 \\
\hline 241-BY-104 & Saltcake Interstitial Liquid & BY-SltCk (Liquid) & 153 & 1.51 & 48.9 \\
\hline 241-BY-105 & Sludge (Liquid \& Solid) & PFeCN (Solid) & 151 & 1.68 & 25.5 \\
\hline 241-BY-105 & Sludge (Liquid \& Solid) & Portland Cement (Solid) & 30 & 1.9 & 8 \\
\hline 241-BY-105 & Saltcake Solid & BY-SltCk (Solid) & 1481 & 1.85 & 14.6 \\
\hline 241-BY-105 & Saltcake Interstitial Liquid & BY-SltCk (Liquid) & 159 & 1.44 & 51.3 \\
\hline 241-BY-106 & Sludge (Liquid \& Solid) & PFeCN (Solid) & 120 & 1.68 & 37.3 \\
\hline 241-BY-106 & Saltcake Solid & BY-SltCk (Solid) & 1365 & 1.68 & 23.3 \\
\hline 241-BY-106 & Saltcake Interstitial Liquid & BY-SltCk (Liquid) & 138 & 1.31 & 59.4 \\
\hline 241-BY-107 & Sludge (Liquid \& Solid) & PFeCN (Solid) & 58 & 1.78 & 37.6 \\
\hline 241-BY-107 & Saltcake Solid & BY-SltCk (Solid) & 835 & 1.72 & 36.3 \\
\hline 241-BY-107 & Saltcake Interstitial Liquid & BY-SltCk (Liquid) & 135 & 1.46 & 48.6 \\
\hline 241-BY-108 & Sludge (Liquid \& Solid) & PFeCN (Solid) & 151 & 1.53 & 31 \\
\hline 241-BY-108 & Saltcake Solid & BY-SltCk (Solid) & 587 & 1.5 & 25.5 \\
\hline 241-BY-108 & Saltcake Interstitial Liquid & BY-SltCk (Liquid) & 103 & 1.33 & 32.7 \\
\hline 241-BY-109 & Sludge (Liquid \& Solid) & CWP2 (Solid) & 89 & 2 & 28.4 \\
\hline 241-BY-109 & Saltcake Solid & BY-SltCk (Solid) & 851 & 1.71 & 42 \\
\hline 241-BY-109 & Saltcake Interstitial Liquid & BY-SltCk (Liquid) & 146 & 1.5 & 53 \\
\hline 241-BY-110 & Sludge (Liquid \& Solid) & PFeCN (Solid) & 162 & 1.82 & 28.7 \\
\hline $241-B Y-110$ & Saltcake Solid & BY-SltCk (Solid) & 1123 & 1.54 & 27.3 \\
\hline 241-BY-110 & Saltcake Interstitial Liquid & BY-SltCk (Liquid) & 99 & 1.44 & 39.9 \\
\hline 241-BY-111 & Saltcake Solid & BY-SltCk (Solid) & 1378 & 1.7 & 33.6 \\
\hline 241-BY-111 & Saltcake Interstitial Liquid & BY-SltCk (Liquid) & 145 & $\overline{1.42}$ & 53.8 \\
\hline 241-BY-112 & Sludge (Liquid \& Solid) & MW2 (Solid) & 8 & 1.85 & 41.4 \\
\hline 241-BY-112 & Saltcake Solid & BY-SltCk (Solid) & 996 & 1.76 & 28.3 \\
\hline 241-BY-112 & Saltcake Interstitial Liquid & BY-SltCk (Liquid) & 79 & 1.47 & 48.5 \\
\hline $241-\mathrm{C}-101$ & Sludge (Liquid \& Solid) & TBP (Solid) & 125 & 1.78 & 23.4 \\
\hline $241-\mathrm{C}-101$ & Sludge (Liquid \& Solid) & CWP1 (Solid) & 208 & 1.78 & 23.4 \\
\hline $241-\mathrm{C}-102$ & Sludge (Liquid \& Solid) & MW1 (Solid) & 19 & 1.8 & 43.8 \\
\hline $241-\mathrm{C}-102$ & Sludge (Liquid \& Solid) & TBP (Solid) & 61 & 1.47 & 50.5 \\
\hline $241-\mathrm{C}-102$ & Sludge (Liquid \& Solid) & CWP1 (Solid) & 125 & 1.63 & 33.4 \\
\hline $241-\mathrm{C}-102$ & Sludge (Liquid \& Solid) & TH1 (Solid) & 98 & 1.32 & 63.5 \\
\hline $241-\mathrm{C}-102$ & Sludge (Liquid \& Solid) & CWZr1 (Solid) & 38 & 1.74 & 65.3 \\
\hline $241-\mathrm{C}-102$ & Sludge (Liquid \& Solid) & CWP2 (Solid) & 855 & 1.74 & 40.9 \\
\hline $241-\mathrm{C}-103$ & Sludge (Liquid \& Solid) & CWP1 (Solid) & 101 & 1.63 & 23.2 \\
\hline $241-\mathrm{C}-104$ & Sludge (Liquid \& Solid) & CWZr1 (Solid) & 90 & 1.68 & 47.9 \\
\hline $241-\mathrm{C}-104$ & Sludge (Liquid \& Solid) & OWW3 (Solid) & 103 & 1.68 & 47.9 \\
\hline $241-\mathrm{C}-104$ & Sludge (Liquid \& Solid) & CWP2 (Solid) & 229 & 1.68 & 47.9 \\
\hline $241-\mathrm{C}-104$ & Sludge (Liquid \& Solid) & TH2 (Solid) & 80 & 1.68 & 47.9 \\
\hline 241-C-104 & Sludge (Liquid \& Solid) & CWP1 (Solid) & 326 & 1.68 & 47.9 \\
\hline $241-\mathrm{C}-104$ & Sludge (Liquid \& Solid) & NA & 152 & 1.68 & 47.9 \\
\hline 241-C-105 & Sludge (Liquid \& Solid) & CWP1 (Solid) & 450 & 1.55 & 25.8 \\
\hline $241-\mathrm{C}-105$ & Sludge (Liquid \& Solid) & TBP (Solid) & 50 & 1.55 & 50.5 \\
\hline $241-\mathrm{C}-106$ & Supernatant & $\overrightarrow{\mathrm{NA}}$ & 0.322 & 1.02 & 97.8 \\
\hline $241-\mathrm{C}-106$ & Sludge (Liquid \& Solid) & NA & 10.166 & 1.56 & 41.9 \\
\hline $241-C-107$ & Sludge (Liquid \& Solid) & 1C (Solid) & 507 & 1.55 & 47.5 \\
\hline $241-\mathrm{C}-107$ & Sludge (Liquid \& Solid) & SRR (Solid) & 339 & 1.55 & 47.5 \\
\hline
\end{tabular}


Table A-1. Best Basis Inventory - Density and Weight Percent Water By Tank, Waste Phase, and Waste Type Queried on September 27, 2006. (12 sheets)

\begin{tabular}{|c|c|c|c|c|c|}
\hline Tank Name & Waste Phase & Waste Type & $\begin{array}{c}\text { Volume } \\
(\mathrm{kL})\end{array}$ & $\begin{array}{l}\text { Density } \\
(\mathrm{g} / \mathrm{mL})\end{array}$ & $\mathrm{Wt} \%$ Water \\
\hline $241-C-107$ & Sludge (Liquid \& Solid) & CWP2 (Solid) & 89 & 1.55 & 47.5 \\
\hline $241-\mathrm{C}-108$ & Sludge (Liquid \& Solid) & TBP (Solid) & 95 & 1.48 & 38.2 \\
\hline $241-\mathrm{C}-108$ & Sludge (Liquid \& Solid) & TFeCN (Solid) & 45 & 1.48 & 38.2 \\
\hline $241-\mathrm{C}-108$ & Sludge (Liquid \& Solid) & $1 \mathrm{C}($ Solid $)$ & 110 & 1.48 & 38.2 \\
\hline 241-C-109 & Sludge (Liquid \& Solid) & CWP1 (Solid) & 55 & 1.57 & 36.1 \\
\hline $241-\mathrm{C}-109$ & Sludge (Liquid \& Solid) & HS (Solid) & 26 & 1.57 & 36.1 \\
\hline $241-C-109$ & Sludge (Liguid \& Solid) & TFeCN (Solid) & 121 & 1.57 & 36.1 \\
\hline 241-C-109 & Sludge (Liquid \& Solid) & 1C (Solid) & 38 & 1.43 & 54.1 \\
\hline $241-\mathrm{C}-110$ & Supernatant & $1 \mathrm{Cl}$ (Liquid) & 4 & 1.1 & 82.9 \\
\hline $241-C-110$ & Sludge (Liquid \& Solid) & $1 \mathrm{C}($ Solid $)$ & 670 & 1.34 & 60.2 \\
\hline $241-\mathrm{C}-111$ & Sludge (Liquid \& Solid) & CWP1 (Solid) & 60 & 1.58 & 31.4 \\
\hline $241-\mathrm{C}-111$ & Sludge (Liquid \& Solid) & HS (Solid) & 17 & 1.58 & 31.4 \\
\hline $241-\mathrm{C}-111$ & Sludge (Liquid \& Solid) & TFeCN (Solid) & 91 & 1.58 & 31.4 \\
\hline $241-C-111$ & Sludge (Liquid \& Solid) & $1 \mathrm{C}$ (Solid) & 49 & 1.43 & 54.1 \\
\hline $241-C-112$ & Sludge (Liquid \& Solid) & CWP1 (Solid) & 60 & 1.6 & 51.5 \\
\hline $241-\mathrm{C}-112$ & Sludge (Liquid \& Solid) & TFeCN (Solid) & 272 & 1.6 & 51.5 \\
\hline $241-\mathrm{C}-112$ & Sludge (Liquid \& Solid) & $1 \mathrm{C}$ (Solid) & 57 & 1.6 & 51.5 \\
\hline $241-\mathrm{C}-112$ & Sludge (Liquid \& Solid) & HS (Solid) & 4 & 1.6 & 51.5 \\
\hline $241-\mathrm{C}-201$ & Supernatant & NA & 0.007 & 1 & 100 \\
\hline $241-\mathrm{C}-201$ & Sludge Solid & HS (Solid) & 0.537 & 1.75 & 24.4 \\
\hline $241-\mathrm{C}-202$ & Supernatant & NA & 0.009 & 1 & 100 \\
\hline $241-\mathrm{C}-202$ & Sludge (Liquid \& Solid) & HS (Solid) & 0.548 & 1.75 & 24.4 \\
\hline 241-C-203 & Supernatant & NA & 0.048 & 1 & 100 \\
\hline $241-C-203$ & Sludge (Liquid \& Solid) & HS (Solid) & 0.476 & 1.93 & 35.5 \\
\hline $241-\mathrm{C}-204$ & Sludge (Liquid \& Solid) & HS (Solid) & $5 . \overline{623}$ & 1.62 & 41.2 \\
\hline $241-S-101$ & Sludge (Liquid \& Solid) & $\mathrm{NA}$ & 890 & 1.7 & 37.5 \\
\hline $241-S-101$ & Saltcake Solid & S1-SltCk (Solid) & 246 & 1.56 & 42.4 \\
\hline $241-S-101$ & Saltcake Solid & S2-S1tSlr (Solid) & 169 & 1.56 & 42.4 \\
\hline $241-S-101$ & Saltcake Interstitial Liquid & S1-SltCk (Liquid) & 16 & 1.47 & 50.7 \\
\hline $241-\mathrm{S}-101$ & Saltcake Interstitial Liquid & S2-S1tSlr (Liquid) & 11 & 1.47 & 50.7 \\
\hline $241-S-102$ & Sludge (Liquid \& Solid) & R1 (Solid) & 71 & 1.88 & 22.2 \\
\hline $241-S-102$ & Saltcake Solid & NA SltCk & 593 & 1.69 & 36.2 \\
\hline $241-S-102$ & Retained Gas (Sludge) & NA & 13 & NA & $\mathrm{NA}$ \\
\hline $241-\mathrm{S}-102$ & Retained Gas (Saltcake) & NA & 90 & NA & $\mathrm{NA}$ \\
\hline $241-\mathrm{S}-103$ & Supernatant & S2-SitSlr (Liquid) & 4 & 1.45 & 49.8 \\
\hline 241-S-103 & Sludge (Liquid \& Solid) & R1 (Solid) & 34 & 1.77 & 22.7 \\
\hline 241-S-103 & Saltcake Solid & S1-SltCk (Solid) & 347 & 1.68 & 30.2 \\
\hline $241-S-103$ & Saltcake Solid & S2-SltSlr (Solid) & 300 & 1.63 & 34.9 \\
\hline $241-S-103$ & Saltcake Interstitial Liquid & S2-SltSlr (Liquid) & 95 & 1.45 & 49.8 \\
\hline $241-S-103$ & Saltcake Interstitial Liquid & S1-SltCk (Liquid) & 116 & 1.45 & 49.8 \\
\hline 241-S-104 & Sludge (Liquid \& Solid) & R1 (Solid) & 409 & 1.77 & 33.5 \\
\hline 241-S-104 & Sludge (Liquid \& Solid) & CWR1 (Solid) & 91 & 1.8 & 33.5 \\
\hline 241-S-104 & Saltcake Solid & R-SltCk (Solid) & 451 & 1.64 & 41 \\
\hline $241-S-104$ & Saltcake Interstitial Liquid & R-SltCk (Liquid) & 139 & 1.37 & 55.1 \\
\hline $241-\mathrm{S}-105$ & Sludge (Liquid \& Solid) & R1 (Solid) & 8 & 1.77 & 22.7 \\
\hline $241-\mathrm{S}-105$ & Saltcake Solid & S1-SltCk (Solid) & 1371 & 1.68 & 3.57 \\
\hline 241-S-105 & Saltcake Interstitial Liquid & S1-SltCk (Liquid) & 157 & 1.45 & 49.1 \\
\hline
\end{tabular}


Table A-1. Best Basis Inventory - Density and Weight Percent Water By Tank, Waste Phase, and Waste Type Queried on September 27, 2006. (12 sheets)

\begin{tabular}{|c|c|c|c|c|c|}
\hline Tank Name & Waste Phase & Waste Type & $\begin{array}{c}\text { Volume } \\
(\mathrm{kL})\end{array}$ & $\begin{array}{l}\text { Density } \\
(\mathrm{g} / \mathrm{mL})\end{array}$ & Wt $\%$ Water \\
\hline $241-\mathrm{S}-106$ & Saltcake Solid & S1-SltCk (Solid) & 1624 & 1.74 & 33.5 \\
\hline 241-S-106 & Saltcake Interstitial Liquid & S1-SltCk (Liquid) & 99 & 1.43 & 54.2 \\
\hline 241-S-107 & Sludge (Liquid \& Solid) & CWR1 (Solid) & 447 & 1.8 & 33 \\
\hline 241-S-107 & Sludge (Liquid \& Solid) & CWZr1 (Solid) & 91 & 1.8 & 33 \\
\hline 241-S-107 & Sludge (Liquid \& Solid) & CWR2 (Solid) & 211 & 1.8 & 33 \\
\hline $241-\mathrm{S}-107$ & Sludge (Liquid \& Solid) & R1 (Solid) & 462 & 1.8 & 33 \\
\hline 241-S-107 & Saltcake Solid & T2-SltCk (Solid) & 52 & 1.57 & 44.895 \\
\hline $241-\mathrm{S}-107$ & Saltcake Solid & S1-SltCk (Solid) & 63 & 1.57 & 44.895 \\
\hline 241-S-107 & Saltcake Solid & S2-SltSlr (Solid) & 30 & 1.57 & 44.895 \\
\hline $241-S-108$ & Sludge (Liquid \& Solid) & R1 (Solid) & 19 & 1.77 & 22.7 \\
\hline 241-S-108 & Saltcake Solid & S1-SltCk (Solid) & 2032 & 1.68 & 30.2 \\
\hline 241-S-108 & Saltcake Interstitial Liquid & S1-SltCk (Liquid) & 31 & 1.45 & 49.1 \\
\hline 241-S-109 & Sludge (Liquid \& Solid) & R1 (Solid) & 49 & 1.77 & 22.7 \\
\hline 241-S-109 & Saltcake Solid & S1-SltCk (Solid) & 1905 & 1.66 & 9.1 \\
\hline 241-S-109 & Saltcake Interstitial Liquid & S1-SltCk (Liquid) & 63 & 1.49 & 53.1 \\
\hline $241-\mathrm{S}-110$ & Sludge (Liquid \& Solid) & R1 (Solid) & 288 & 1.77 & 31.5 \\
\hline $241-\mathrm{S}-110$ & Sludge (Liquid \& Solid) & CWR1 (Solid) & 76 & 1.77 & 31.5 \\
\hline $241-\mathrm{S}-110$ & Saltcake Solid & S1-Sltck (Solid) & 994 & 1.65 & 12.5 \\
\hline $241-\mathrm{S}-110$ & Saltcake Interstitial Liquid & S1-Sltck (Liquid) & 115 & 1.43 & 49.3 \\
\hline $241-S-111$ & Sludge (Liquid \& Solid) & R1 (Solid) & 207 & 1.67 & 19.4 \\
\hline $241-S-111$ & Sludge (Liquid \& Solid) & CWR1 (Solid) & 38 & 1.67 & 19.4 \\
\hline $241-\mathrm{S}-111$ & Saltcake Solid & S1-SltCk (Solid) & 1028 & 1.53 & 29 \\
\hline $241-S-111$ & Saltcake Interstitial Liquid & S1-SltCk (Liquid) & 124 & 1.45 & 51 \\
\hline $241-S-111$ & Retained Gas (Sludge) & NA & 43 & NA & NA \\
\hline 241-S-111 & Retained Gas (Saltcake) & NA & 77 & $\mathrm{NA}$ & NA \\
\hline $241-\mathrm{S}-112$ & Sludge (Liquid \& Solid) & R1 (Solid) & 5 & 1.77 & 22.7 \\
\hline $241-S-112$ & Saltcake Solid & S1-SltCk (Solid) & 14 & 1.69 & 41.3 \\
\hline $241-S X-101$ & Sludge (Liquid \& Solid) & R1 (Solid) & 545 & 1.69 & 25.5521 \\
\hline 241-SX-101 & Saltcake Solid & R-SltCk (Solid) & 497 & 1.69 & 25.5521 \\
\hline 241-SX-101 & Saltcake Solid & S1-SltCk (Solid) & 296 & 1.69 & 25.5521 \\
\hline $241-S X-101$ & Saltcake Solid & S2-SltSlr (Solid) & 166 & 1.69 & 25.5521 \\
\hline 241-SX-101 & Saltcake Interstitial Liquid & R-SltCk (Liquid) & 41 & 1.48 & 49.1015 \\
\hline 241-SX-101 & Saltcake Interstitial Liquid & S1-SitCk (Liquid) & 25 & 1.48 & 49.1015 \\
\hline 241-SX-101 & Saltcake Interstitial Liquid & S2-SltSlr (Liquid) & 14 & 1.48 & 49.1015 \\
\hline 241-SX-102 & Sludge (Liquid \& Solid) & R1 (Solid) & 209 & 1.72 & 38.52 \\
\hline $241-\mathrm{SX}-102$ & Saltcake Solid & S1-SltCk (Solid) & 875 & 1.72 & 38.52 \\
\hline 241-SX-102 & Saltcake Solid & S2-SltSlr (Solid) & 92 & 1.72 & 38.52 \\
\hline 241-SX-102 & Saltcake Interstitial Liquid & S1-SltCk (Liquid) & 105 & 1.46 & 48.1 \\
\hline $241-\mathrm{SX}-102$ & Saltcake Interstitial Liquid & S2-SltSlr (Liquid) & 11 & 1.46 & 48.1 \\
\hline 241-SX-103 & Sludge (Liquid \& Solid) & R1 (Solid) & 294 & 1.88 & 21.9 \\
\hline 241-SX-103 & Saltcake Solid & S1-Sltck (Solid) & 1439 & 1.72 & 30.9 \\
\hline 241-SX-103 & Saltcake Solid & R-SltCk (Solid) & 77 & 1.72 & 37.3 \\
\hline 241-SX-103 & Saltcake Interstitial Liquid & S1-SltCk (Liquid) & 94 & 1.47 & 46.6 \\
\hline 241-SX-103 & Saltcake Interstitial Liquid & R-SltCk (Liquid) & 22 & 1.47 & 46.6 \\
\hline 241-SX-104 & Sludge (Liquid \& Solid) & R1 (Solid) & 515 & 1.77 & 22.7 \\
\hline 241-SX-104 & Saltcake Solid & S1-SltCk (Solid) & 903 & 1.68 & 30.2 \\
\hline 241-SX-104 & Saltcake Solid & R-SltCk (Solid) & 142 & 1.72 & 23.4 \\
\hline
\end{tabular}


Table A-1. Best Basis Inventory - Density and Weight Percent Water By Tank, Waste Phase, and Waste Type Queried on September 27, 2006. (12 sheets)

\begin{tabular}{|c|c|c|c|c|c|}
\hline Tank Name & Waste Phase & Waste Type & $\begin{array}{c}\text { Volume } \\
\text { (kL) }\end{array}$ & $\begin{array}{l}\text { Density } \\
(\mathrm{g} / \mathrm{mL})\end{array}$ & $\mathrm{Wt} \%$ Water \\
\hline $241-S X-104$ & Saltcake Interstitial Liquid & S1-SltCk (Liquid) & 17 & 1.47 & 45.3291 \\
\hline 241-SX-104 & Saltcake Interstitial Liquid & R-SltCk (Liquid) & 111 & 1.47 & 45.3291 \\
\hline $241-S X-105$ & Sludge (Liquid \& Solid) & R1 (Solid) & 189 & 1.67 & 22.7 \\
\hline $241-S X-105$ & Sludge (Liquid \& Solid) & R2 (Solid) & 49 & 1.67 & 22.7 \\
\hline $241-S X-105$ & Saltcake Solid & S1-SltCk (Solid) & 1061 & 1.64 & 37.1 \\
\hline $241-S X-105$ & Saltcake Interstitial Liquid & S1-SltCk (Liquid) & 123 & 1.47 & 42.9 \\
\hline 241-SX-106 & Saltcake Solid & S2-SltSlr (Solid) & 1107 & 1.61 & 39 \\
\hline $241-S X-106$ & Saltcake Solid & S1-SltCk (Solid) & 245 & 1.61 & 39 \\
\hline $241-S X-106$ & Saltcake Interstitial Liquid & S2-SltSlr (Liquid) & 122 & 1.29 & 49.4 \\
\hline $241-\mathrm{SX}-106$ & Saltcake Interstitial Liquid & S1-SltCk (Liquid) & 27 & 1.29 & 49.4 \\
\hline $241-\mathrm{SX}-107$ & Sludge (Liquid \& Solid) & R1 (Solid) & 239 & 1.77 & 22.7 \\
\hline $241-S X-107$ & Sludge (Liquid \& Solid) & R2 (Solid) & 117 & 1.77 & 22.7 \\
\hline $241-\mathrm{SX}-108$ & Sludge Solid & R1 (Solid) & 186 & 1.77 & 2.03 \\
\hline $241-\mathrm{SX}-108$ & Sludge Solid & R2 (Solid) & 94 & 1.77 & 2.03 \\
\hline $241-S X-109$ & Sludge Solid & R1 (Solid) & 170 & 1.77 & 22.7 \\
\hline $241-S X-109$ & Sludge Solid & R2 (Solid) & 81 & 1.77 & 22.7 \\
\hline 241-SX-109 & Saltcake Solid & R-SltCk (Solid) & 662 & 1.72 & 23.4 \\
\hline $241-\mathrm{SX}-110$ & Sludge Solid & R2 (Solid) & 184 & 1.77 & 22.7 \\
\hline $241-\mathrm{SX}-110$ & Saltcake Solid & R-SltCk (Solid) & 28 & 1.72 & 23.4 \\
\hline $241-\mathrm{SX}-111$ & Sludge (Liquid \& Solid) & R1 (Solid) & 164 & 1.77 & 22.7 \\
\hline $241-\mathrm{SX}-111$ & Sludge (Liquid \& Solid) & R2 (Solid) & 205 & 1.77 & 22.7 \\
\hline $241-\mathrm{SX}-111$ & Saltcake Solid & R-SltCk (Solid) & 67 & 1.72 & 23.4 \\
\hline 241-SX-112 & Sludge (Liquid \& Solid) & R1 (Solid) & 144 & 1.77 & 22.7 \\
\hline $241-S X-112$ & Sludge (Liquid \& Solid) & R2 (Solid) & 139 & 1.77 & 22.7 \\
\hline 241-SX-113 & Sludge Solid & R1 (Solid) & 8 & 1.43 & 46 \\
\hline $241-\mathrm{SX}-113$ & Sludge Solid & DE (Solid) & 64 & 1.43 & $\overline{46}$ \\
\hline $241-\mathrm{SX}-114$ & Sludge (Liquid \& Solid) & R1 (Solid) & 298 & 1.77 & 22.7 \\
\hline 241-SX-114 & Sludge (Liquid \& Solid) & R2 (Solid) & 180 & 1.77 & 22.7 \\
\hline $241-\mathrm{SX}-114$ & Saltcake Solid & R-SltCk (Solid) & 84 & 1.72 & 23.4 \\
\hline $241-S X-114$ & Saltcake Interstitial Liquid & R-SltCk (Liquid) & 26 & 1.5 & 51.9 \\
\hline $241-\mathrm{SX}-115$ & Sludge Solid & R2 (Solid) & 16 & 1.77 & 10.1 \\
\hline $241-S Y-101$ & Supernatant & NA & 3300 & 1.28 & 69.1 \\
\hline 241-SY-101 & Saltcake (Liquid \& Solid) & S2-SltSlr (Solid) & 893 & 1.52 & 50.4 \\
\hline 241-SY-101 & Retained Gas (Saltcake) & NA & 87 & NA & NA \\
\hline $241-S Y-102$ & Supernatant & NA & 3688 & 1.2 & 66.8 \\
\hline $241-\mathrm{SY}-102$ & Sludge Solid & Z(Solid) & 186 & 1.65 & 41.3 \\
\hline 241-SY-102 & Sludge Solid & $\mathrm{NA}$ & 232 & 1.65 & 41.3 \\
\hline $241-S Y-102$ & Sludge Interstitial Liquid & Z(Liquid) & 83 & 1.36 & 63.2 \\
\hline $241-S Y-102$ & Sludge Interstitial Liquid & NA & 104 & 1.36 & 63.2 \\
\hline $241-S Y-103$ & Supernatant & S2-S1tSlr (Liquid) & 1503 & 1.47 & 43.1 \\
\hline 241-SY-103 & Saltcake (Liquid \& Solid) & S2-S1tSlr (Solid) & 1203 & 1.61 & 36.5 \\
\hline 241-SY-103 & Retained Gas (Saltcake) & NA & 91 & NA & NA \\
\hline $241-\mathrm{T}-101$ & Sludge (Liquid \& Solid) & CWR2 (Solid) & 140 & 1.46 & 51.7 \\
\hline $241-T-101$ & Saltcake Solid & T2-ShtCk (Solid) & 179 & 1.64 & 28.3 \\
\hline $241-\mathrm{T}-101$ & Saltcake Interstitial Liquid & T2-SltCk (Liquid) & 57 & 1.45 & 49.1 \\
\hline $241-\mathrm{T}-102$ & Supernatant & CSR (Liquid) & 48 & 1.14 & 79.77 \\
\hline $241-\mathrm{T}-102$ & Sludge (Liquid \& Solid) & CWP2 (Solid) & 64 & 1.79 & 28.075 \\
\hline
\end{tabular}


Table A-1. Best Basis Inventory - Density and Weight Percent Water By Tank, Waste Phase, and Waste Type Queried on September 27, 2006. (12 sheets)

\begin{tabular}{|c|c|c|c|c|c|}
\hline Tank Name & Waste Phase & Waste Type & $\begin{array}{c}\text { Volume } \\
(\mathrm{kL})\end{array}$ & $\begin{array}{l}\text { Density } \\
(\mathrm{g} / \mathrm{mL})\end{array}$ & Wt $\%$ Water \\
\hline $241-\mathrm{T}-102$ & Sludge (Liquid \& Solid) & MW2 (Solid) & 8 & 1.85 & 41.4 \\
\hline $241-\mathrm{T}-103$ & Supernatant & CSR (Liquid) & 15 & 1.19 & 71.6 \\
\hline $241-\mathrm{T}-103$ & Sludge (Liquid \& Solid) & CWR1 (Solid) & 19 & 1.8 & 24.5 \\
\hline $241-\mathrm{T}-103$ & Sludge (Liquid \& Solid) & CWP2 (Solid) & 64 & 1.68 & 40.9 \\
\hline $241-\mathrm{T}-103$ & Sludge (Liquid \& Solid) & MW2 (Solid) & 4 & 1.85 & 41.4 \\
\hline $241-\mathrm{T}-104$ & Sludge (Liquid \& Solid) & $1 \mathrm{C}$ (Solid) & 1199 & 1.29 & 70.525 \\
\hline $241-\mathrm{T}-105$ & Sludge (Liquid \& Solid) & $2 \mathrm{C}$ (Solid) & 273 & 1.51 & 53.5 \\
\hline $241-T-105$ & Sludge (Liquid \& Solid) & $1 \mathrm{C}$ (Solid) & 6 & 1.32 & 51.4 \\
\hline $241-\mathrm{T}-105$ & Sludge (Liquid \& Solid) & CWR1 (Solid) & 92 & 1.32 & 51.4 \\
\hline $241-\mathrm{T}-106$ & Sludge (Liquid \& Solid) & $1 \mathrm{C}$ (Solid) & 38 & 1.43 & 16.7 \\
\hline $241-T-106$ & Sludge (Liquid \& Solid) & CWR1 (Solid) & 34 & 1.8 & 16.7 \\
\hline $241-\mathrm{T}-106$ & Sludge (Liquid \& Solid) & CWR2 (Solid) & 10 & 1.46 & 16.7 \\
\hline $241-T-107$ & Sludge (Liquid \& Solid) & CWP2 (Solid) & 32 & 1.56 & 45.7 \\
\hline $241-\mathrm{T}-107$ & Sludge (Liquid \& Solid) & $1 \mathrm{C}($ Solid $)$ & 559 & 1.56 & 45.7 \\
\hline $241-\mathrm{T}-107$ & Sludge (Liquid \& Solid) & TBP (Solid) & 64 & 1.56 & 45.7 \\
\hline $241-\mathrm{T}-108$ & Sludge (Liquid \& Solid) & $1 \mathrm{C}$ (Solid) & 20 & 1.43 & 54.1 \\
\hline $241-T-108$ & Saltcake Solid & T1-SltCk (Solid) & 30 & 1.72 & 15.1 \\
\hline $241-T-108$ & Saltcake Interstitial Liquid & T1-SltCk (Liquid) & 10 & 1.26 & 69.6 \\
\hline $241-\mathrm{T}-109$ & Saltcake Solid & T1-SitCk (Solid) & 197 & 1.72 & 47.9083 \\
\hline $241-T-109$ & Saltcake Interstitial Liquid & T1-SltCk (Liquid) & 38 & 1.26 & 69.6 \\
\hline $241-T-110$ & Supernatant & $2 \mathrm{Cl}$ (Liquid) & 3 & 1.05 & 86.8 \\
\hline $241-\mathrm{T}-110$ & Sludge (Liquid \& Solid) & 224-2 (Solid) & 37 & 1.25 & 75.5 \\
\hline $241-T-110$ & Sludge (Liquid \& Solid) & $2 \mathrm{C}$ (Solid) & 1360 & 1.25 & 75.5 \\
\hline $241-\mathrm{T}-111$ & Sludge (Liquid \& Solid) & $2 \mathrm{C}$ (Solid) & 787 & 1.24 & 75.3 \\
\hline $241-T-111$ & Sludge (Liquid \& Solid) & $224-2$ (Solid) & 904 & 1.24 & 75.3 \\
\hline $241-T-112$ & Supernatant & DW (Liquid) & 13 & 1.1 & 85.05 \\
\hline $241-T-112$ & Supernatant & CSR (Liquid) & 13 & 1.1 & 85.05 \\
\hline $241-\mathrm{T}-112$ & Sludge (Liquid \& Solid) & $2 \mathrm{C}$ (Solid) & 135 & 1.28 & 73.9 \\
\hline $241-\mathrm{T}-112$ & Sludge (Liquid \& Solid) & $224-2$ (Solid) & 91 & 1.28 & 73.9 \\
\hline $241-\mathrm{T}-201$ & Supernatant & 224-1 (Liquid) & 8 & 1.06 & 91.2 \\
\hline $241-T-201$ & Sludge (Liquid \& Solid) & 224-1 (Solid) & 107 & 1.31 & 67.5 \\
\hline $241-\mathrm{T}-202$ & Sludge (Liquid \& Solid) & 224-2 (Solid) & 77 & 1.18 & 75.8 \\
\hline $241-\mathrm{T}-203$ & Sludge (Liquid \& Solid) & 224-2 (Solid) & 136 & 1.22 & 76.2 \\
\hline $241-T-204$ & Sludge (Liquid \& Solid) & 224-2 (Solid) & 136 & 1.18 & 75 \\
\hline $241-\mathrm{TX}-101$ & Sludge (Liquid \& Solid) & R1 (Solid) & 265 & 1.77 & 22.7 \\
\hline $241-\mathrm{TX}-101$ & Sludge (Liquid \& Solid) & MW2 (Solid) & 11 & 1.85 & 41.4 \\
\hline 241-TX-101 & Sludge (Liquid \& Solid) & Z(Solid) & 4 & 1.76 & 29.1 \\
\hline $241-\mathrm{TX}-101$ & Saltcake Solid & T2-SltCk (Solid) & 49 & 1.64 & 28.3 \\
\hline $241-\mathrm{TX}-101$ & Saltcake Interstitial Liquid & T2-SltCk (Liquid) & 15 & 1.45 & 49.1 \\
\hline $241-\mathrm{TX}-102$ & Sludge (Liquid \& Solid) & MW2 (Solid) & 8 & 1.85 & 41.4 \\
\hline $241-\mathrm{TX}-102$ & Saltcake Solid & T2-SltCk (Solid) & 692 & 1.64 & 28.3 \\
\hline $241-\mathrm{TX}-102$ & Saltcake Interstitial Liquid & T2-SltCk (Liquid) & 121 & 1.45 & 49.1 \\
\hline $241-\mathrm{TX}-103$ & Saltcake Solid & T1-SltCk (Solid) & 9 & 1.72 & 23.5 \\
\hline $241-\mathrm{TX}-103$ & Saltcake Solid & T2-SltCk (Solid) & 454 & 1.64 & 28.3 \\
\hline $241-\mathrm{TX}-103$ & Saltcake Interstitial Liquid & T2-S1tCk (Liquid) & 82 & 1.45 & 49.1 \\
\hline $241-\mathrm{TX}-103$ & Saltcake Interstitial Liquid & T1-SltCk (Liquid) & 3 & 1.26 & 69.6 \\
\hline $241-\mathrm{TX}-104$ & Supernatant & T2-SliCk (Liquid) & 9 & 1.44 & 51.9 \\
\hline
\end{tabular}


Table A-1. Best Basis Inventory - Density and Weight Percent Water By Tank, Waste Phase, and Waste Type Queried on September 27, 2006. (12 sheets)

\begin{tabular}{|c|c|c|c|c|c|}
\hline Tank Name & Waste Phase & Waste Type & $\begin{array}{c}\text { Volume } \\
(\mathrm{kL})\end{array}$ & $\begin{array}{l}\text { Density } \\
(\mathrm{g} / \mathrm{mL})\end{array}$ & Wt\% Water \\
\hline 241-TX-104 & Sludge (Liquid \& Solid) & R1 (Solid) & 130 & 1.89 & 44.6 \\
\hline 241-TX-104 & Saltcake Solid & T2-SltCk (Solid) & 93 & 1.62 & 45.2 \\
\hline 241-TX-104 & Saltcake Interstitial Liquid & T2-SltCk (Liquid) & 30 & 1.44 & 51.9 \\
\hline 241-TX-105 & Sludge (Liquid \& Solid) & MW2 (Solid) & 31 & 1.85 & 41.4 \\
\hline 241-TX-105 & Saltcake Solid & T2-SltCk (Solid) & 2044 & 1.64 & 28.3 \\
\hline 241-TX-105 & Saltcake Interstitial Liquid & T2-SltCk (Liquid) & 106 & 1.45 & 49.1 \\
\hline 241-TX-106 & Sludge (Liquid \& Solid) & MW2 (Solid) & 4 & 1.85 & 41.4 \\
\hline 241-TX-106 & Sludge (Liquid \& Solid) & R1 (Solid) & 15 & 1.77 & 22.7 \\
\hline 241-TX-106 & Saltcake Solid & T2-SltCk (Solid) & 1147 & 1.64 & 28.3 \\
\hline 241-TX-106 & Saltcake Interstitial Liquid & T2-SltCk (Liquid) & 152 & 1.45 & 49.1 \\
\hline 241-TX-107 & Saltcake Solid & T2-SltCk (Solid) & 62 & 1.94 & 22.2 \\
\hline 241-TX-107 & Saltcake Solid & R-SltCk (Solid) & 24 & 1.72 & 23.4 \\
\hline 241-TX-107 & Saltcake Interstitial Liquid & T2-SltCk (Liquid) & 19 & 1.45 & 49.1 \\
\hline 241-TX-107 & Saltcake Interstitial Liquid & R-SltCk (Liquid) & 7 & 1.5 & 51.9 \\
\hline 241-TX-108 & Sludge (Liquid \& Solid) & MW2 (Solid) & 8 & 1.85 & 41.4 \\
\hline $241-\mathrm{TX}-108$ & Sludge (Liquid \& Solid) & TBP (Solid) & 15 & 1.47 & 50.5 \\
\hline 241-TX-108 & Saltcake Solid & T2-SltCk (Solid) & 415 & 1.64 & 28.3 \\
\hline 241-TX-108 & Saltcake Interstitial Liquid & T2-SltCk (Liquid) & 40 & 1.45 & 49.1 \\
\hline 241-TX-109 & Sludge (Liquid \& Solid) & 1C(Solid) & 1375 & 1.43 & 54.1 \\
\hline 241-TX-110 & Sludge (Liquid \& Solid) & 1C (Solid) & 140 & 1.43 & 54.1 \\
\hline 241-TX-110 & Saltcake Solid & T2-S1tCk (Solid) & 1580 & 1.64 & 28.3 \\
\hline $241-\mathrm{TX}-110$ & Saltcake Interstitial Liquid & T2-SltCk (Liquid) & 49 & 1.45 & 49.1 \\
\hline 241-TX-111 & Sludge (Liquid \& Solid) & 1C (Solid) & 163 & 1.43 & 54.1 \\
\hline 241-TX-111 & Saltcake Solid & T2-SltCk (Solid) & 1194 & 1.64 & 28.3 \\
\hline 241-TX-111 & Saltcake Interstitial Liquid & T2-SltCk (Liquid) & 24 & 1.45 & 49.1 \\
\hline 241-TX-112 & Saltcake Solid & T2-SltCk (Solid) & 2215 & 1.64 & 28.3 \\
\hline $241-\mathrm{TX}-112$ & Saltcake Solid & T1-SltCk (Solid) & 75 & 1.72 & 23.5 \\
\hline 241-TX-112 & Saltcake Interstitial Liquid & T1-SltCk (Liquid) & 15 & 1.26 & 69.6 \\
\hline 241-TX-112 & Saltcake Interstitial Liquid & T2-SltCk (Liquid) & 93 & 1.45 & 49.1 \\
\hline 241-TX-113 & Sludge (Liquid \& Solid) & $1 \mathrm{C}$ (Solid) & 351 & 1.43 & 54.1 \\
\hline 241-TX-113 & Saltcake Solid & T2-SltCk (Solid) & 2045 & 1.64 & 9.43 \\
\hline 241-TX-113 & Saltcake Interstitial Liquid & T2-SltCk (Liquid) & 20 & 1.45 & 49.1 \\
\hline 241-TX-114 & Sludge (Liquid \& Solid) & 1C (Solid) & 15 & 1.43 & 54.1 \\
\hline 241-TX-114 & Saltcake Solid & T2-SltCk(Solid) & 1750 & 1.64 & 28.3 \\
\hline 241-TX-114 & Saltcake Solid & T1-SltCk (Solid) & 173 & 1.72 & 23.5 \\
\hline 241-TX-114 & Saltcake Interstitial Liquid & T1-SltCk (Liquid) & 46 & 1.26 & 69.6 \\
\hline 241-TX-114 & Saltcake Interstitial Liquid & T2-SltCk (Liquid) & 28 & 1.45 & 49.1 \\
\hline 241-TX-115 & Sludge (Liquid \& Solid) & TBP (Solid) & 30 & 1.47 & 50.5 \\
\hline 241-TX-115 & Saltcake Solid & T2-SltCk (Solid) & 1960 & 1.64 & 28.3 \\
\hline 241-TX-115 & Saltcake Interstitial Liquid & T2-SltCk (Liquid) & 103 & 1.45 & 49.1 \\
\hline 241-TX-116 & Sludge (Liquid \& Solid) & DE (Solid) & 248 & 1.6 & 38.8 \\
\hline 241-TX-116 & Saltcake Solid & T2-SltCk (Solid) & 926 & 1.64 & 28.5 \\
\hline $241-\mathrm{TX}-116$ & Saltcake Solid & T1-SltCk (Solid) & 977 & 1.72 & 7.5 \\
\hline 241-TX-116 & Saltcake Interstitial Liquid & T1-SltCk (Liquid) & 113 & 1.39 & 53 \\
\hline 241-TX-117 & Sludge Solid & DE (Solid) & 110 & 0.384 & 0 \\
\hline 241-TX-117 & Saltcake Solid & T1-SltCk (Solid) & 626 & 1.72 & 23.5 \\
\hline 241-TX-117 & Saltcake Solid & T2-SltCk (Solid) & 1033 & 1.64 & 28.3 \\
\hline
\end{tabular}


Table A-1. Best Basis Inventory - Density and Weight Percent Water By Tank, Waste Phase, and Waste Type Queried on September 27, 2006. (12 sheets)

\begin{tabular}{|c|c|c|c|c|c|}
\hline Tank Name & Waste Phase & Waste Type & $\begin{array}{c}\text { Volume } \\
(\mathrm{kL})\end{array}$ & $\begin{array}{l}\text { Density } \\
(\mathrm{g} / \mathrm{mL})\end{array}$ & Wt $\%$ Water \\
\hline 241-TX-117 & Saltcake Interstitial Liquid & T1-SltCk (Liquid) & 48 & 1.26 & 69.6 \\
\hline 241-TX-118 & Saltcake Solid & T2-SltCk (Solid) & 671 & 1.77 & 43.6 \\
\hline $241-\mathrm{TX}-118$ & Saltcake Solid & $\overrightarrow{\mathrm{NA}}$ & 125 & 1.54 & 46.8 \\
\hline $241-\mathrm{TX}-118$ & Saltcake Interstitial Liquid & T2-SltCk (Liquid) & 139 & 1.45 & 49.1 \\
\hline 241-TY-101 & Sludge (Liquid \& Solid) & $1 \mathrm{CFeCN}$ (Solid) & 273 & 1.64 & 43.5 \\
\hline $241-\mathrm{TY}-101$ & Saltcake Solid & T1-SltCk (Solid) & 159 & 1.64 & 43.5 \\
\hline 241-TY-101 & Saltcake Interstitial Liquid & T1-SltCk (Liquid) & 15 & 1.26 & 69.6 \\
\hline 241-TY-102 & Saltcake Solid & T1-SltCk (Solid) & 90 & 1.88 & 23.5 \\
\hline $241-\mathrm{TY}-102$ & Saltcake Solid & T2-SItCk (Solid) & 109 & 1.88 & 28.3 \\
\hline 241-TY-102 & Saltcake Interstitial Liquid & T1-SltCk (Liquid) & 29 & 1.26 & 69.6 \\
\hline $241-\mathrm{TY}-102$ & Saltcake Interstitial Liquid & T2-SltCk (Liquid) & 34 & 1.45 & 49.1 \\
\hline 241-TY-103 & Sludge (Liquid \& Solid) & TBP (Solid) & 220 & 1.7 & 51.9 \\
\hline $241-\mathrm{TY}-103$ & Sludge (Liquid \& Solid) & $1 \mathrm{CFeCN}$ (Solid) & 170 & 1.7 & 51.9 \\
\hline 241-TY-103 & Saltcake Solid & T2-SltCk (Solid) & 150 & 1.7 & 51.9 \\
\hline 241-TY-103 & Saltcake Interstitial Liquid & T2-SItCk (Liquid) & 45 & 1.45 & 49.1 \\
\hline 241-TY-104 & Supernatant & DW (Liquid) & 5 & 1.18 & 77.5 \\
\hline 241-TY-104 & Sludge (Liquid \& Solid) & $1 \mathrm{CFeCN}$ (Solid) & 114 & 1.65 & 51.9 \\
\hline 241-TY-104 & Sludge (Liquid \& Solid) & TBP (Solid) & 49 & 1.65 & 51.9 \\
\hline 241-TY-105 & Sludge (Liquid \& Solid) & TBP (Solid) & 874 & 1.53 & 39.4 \\
\hline 241-TY-106 & Sludge (Liquid \& Solid) & DE (Solid) & $\overline{47}$ & 1.4 & 38.1 \\
\hline 241-TY-106 & Sludge (Liquid \& Solid) & TBP (Solid) & 15 & 1.4 & 38.1 \\
\hline $241-\mathrm{U}-101$ & Sludge (Liquid \& Solid) & R1 (Solid) & 87 & 1.77 & 29.8 \\
\hline $241-\mathrm{U}-102$ & Supernatant & T2-SltCk (Liquid) & 4 & 1.48 & 47.5 \\
\hline $241-\mathrm{U}-102$ & Sludge (Liquid \& Solid) & R1 (Solid) & 163 & 1.77 & 22.7 \\
\hline $241-U-102$ & Saltcake Solid & T2-SltCk (Solid) & 647 & 1.68 & 34.7 \\
\hline $241-\mathrm{U}-102$ & Saltcake Solid & S2-SltSlr (Solid) & 307 & 1.68 & 34.7 \\
\hline $241-U-102$ & Saltcake Interstitial Liquid & T2-SltCk (Liquid) & 117 & 1.48 & 47.5 \\
\hline $241-\mathrm{U}-103$ & Supernatant & S1-SltCk (Liquid) & 2 & 1.44 & 49.9 \\
\hline $241-\mathrm{U}-103$ & Sludge (Liquid \& Solid) & R1 (Solid) & $\overline{42}$ & 1.9 & 47.4 \\
\hline $241-U-103$ & Saltcake Solid & S2-SltSlr (Solid) & 356 & 1.72 & 38.4 \\
\hline $241-\mathrm{U}-103$ & Saltcake Solid & S1-SltCk (Solid) & 891 & 1.72 & 38.4 \\
\hline $241-\mathrm{U}-103$ & Saltcake Interstitial Liquid & S1-SltCk (Liquid) & 119 & 1.44 & 49.9 \\
\hline $241-\mathrm{U}-103$ & Retained Gas (Sludge) & NA & 5 & $\mathrm{NA}$ & NA \\
\hline $241-\mathrm{U}-103$ & Retained Gas (Saltcake) & NA & 163 & NA & NA \\
\hline $241-\mathrm{U}-104$ & Sludge (Liquid \& Solid) & MW2 (Solid) & 4 & 1.85 & 41.4 \\
\hline $241-U-104$ & Sludge (Liquid \& Solid) & DE (Solid) & 201 & 1.11 & 72.4 \\
\hline $241-\mathrm{U}-105$ & Sludge (Liquid \& Solid) & CWR1 (Solid) & 121 & 1.7 & 22.4 \\
\hline $241-U-105$ & Saltcake Solid & S2-S1tS1r (Solid) & 827 & 1.7 & 28.4 \\
\hline $241-\mathrm{U}-105$ & Saltcake Solid & T2-SltCk (Solid) & 223 & 1.7 & 28.4 \\
\hline $241-\mathrm{U}-105$ & Saltcake Interstitial Liquid & T2-SltCk (Liquid) & 35 & 1.46 & 51.6 \\
\hline $241-\mathrm{U}-105$ & Saltcake Interstitial Liquid & S2-SltSlr (Liquid) & 130 & 1.46 & 51.6 \\
\hline $241-\mathrm{U}-106$ & Supernatant & S1-SltCk (Liquid) & 6 & 1.34 & 48.6 \\
\hline $241-U-106$ & Saltcake Solid & S1-SltCk (Solid) & 484 & 1.62 & 39.8 \\
\hline 241-U-106 & Saltcake Interstitial Liquid & S1-SltCk (Liquid) & 154 & 1.34 & 48.6 \\
\hline 241-U-107 & Sludge (Liquid \& Solid) & CWR1 (Solid) & 57 & 1.8 & 24.5 \\
\hline $241-\mathrm{U}-107$ & Saltcake Solid & S2-SltSlr (Solid) & 902 & 1.77 & 31.5 \\
\hline $241-\mathrm{U}-107$ & Saltcake Solid & T2-SltCk (Solid) & 44 & 1.77 & 31.5 \\
\hline
\end{tabular}


Table A-1. Best Basis Inventory - Density and Weight Percent Water By Tank, Waste Phase, and Waste Type Queried on September 27, 2006. (12 sheets)

\begin{tabular}{|c|c|c|c|c|c|}
\hline Tank Name & Waste Phase & Waste Type & $\begin{array}{c}\text { Volume } \\
\text { (kL) }\end{array}$ & $\begin{array}{l}\text { Density } \\
(\mathrm{g} / \mathrm{mL})\end{array}$ & Wt\% Water \\
\hline 241-U-107 & Saltcake Interstitial Liquid & S2-S1tSlr (Liquid) & 105 & 1.43 & 49.6 \\
\hline $241-U-107$ & Saltcake Interstitial Liquid & T2-SltCk (Liquid) & 5 & 1.43 & 49.6 \\
\hline 241-U-108 & Sludge (Liquid \& Solid) & CWR2 (Solid) & 110 & 1.46 & 51.7 \\
\hline 241-U-108 & Saltcake Solid & S1-SltCk (Solid) & 766 & $1 . \overline{73}$ & 34.7 \\
\hline 241-U-108 & Saltcake Solid & S2-SltSlr (Solid) & 610 & 1.73 & 34.7 \\
\hline 241-U-108 & Saltcake Interstitial Liquid & S2-S1tSlr (Liquid) & 69 & 1.4 & 49.9 \\
\hline 241-U-108 & Saltcake Interstitial Liquid & S1-SltCk (Liquid) & 86 & 1.4 & 49.9 \\
\hline 241-U-109 & Sludge (Liquid \& Solid) & CWR1 (Solid) & 103 & 1.71 & 22.7 \\
\hline 241-U-109 & Saltcake Solid & S1-SltCk (Solid) & 600 & 1.67 & 27 \\
\hline 241-U-109 & Saltcake Solid & S2-SltS1r (Solid) & 512 & 1.67 & 27 \\
\hline 241-U-109 & Saltcake Interstitial Liquid & S1-SltCk (Liquid) & 137 & 1.47 & 50.7 \\
\hline 241-U-109 & Retained Gas (Sludge) & $\overline{\mathrm{NA}}$ & 29 & NA & NA \\
\hline 241-U-109 & Retained Gas (Saltcake) & $\mathrm{NA}$ & 137 & NA & NA \\
\hline $241-\mathrm{U}-110$ & Sludge (Liquid \& Solid) & CWR1 (Solid) & 149 & 1.8 & 3.08 \\
\hline $241-U-110$ & Sludge (Liquid \& Solid) & R1 (Solid) & 396 & 1.77 & 38.8 \\
\hline $241-U-110$ & Sludge (Liquid \& Solid) & 1C(Solid) & 120 & 1.43 & 39.7 \\
\hline 241-U-111 & Sludge (Liquid \& Solid) & R1 (Solid) & 49 & 1.77 & 22.7 \\
\hline 241-U-111 & Sludge (Liquid \& Solid) & 1C (Solid) & 49 & 1.43 & 54.1 \\
\hline $241-U-111$ & Saltcake Solid & S1-SltCk (Solid) & 228 & 1.68 & 30.2 \\
\hline 241-U-111 & Saltcake Solid & S2-SltSIr (Solid) & 401 & 1.63 & 34.9 \\
\hline $241-\mathrm{U}-111$ & Saltcake Interstitial Liquid & S1-SltCk (Liquid) & 41 & 1.4 & 52.6 \\
\hline 241-U-111 & Saltcake Interstitial Liquid & S2-S1tSlr (Liquid) & 72 & 1.4 & 52.6 \\
\hline $241-U-112$ & Sludge (Liquid \& Solid) & CWR1 (Solid) & 58 & 1.86 & 26 \\
\hline $241-U-112$ & Sludge (Liquid \& Solid) & $1 \mathrm{C}$ (Solid) & 47 & 1.43 & 54.1 \\
\hline 241-U-112 & Sludge (Liquid \& Solid) & R1 (Solid) & 67 & 1.86 & 26 \\
\hline $241-\mathrm{U}-201$ & Supernatant & CWR1 (Liquid) & 4 & 1.26 & 69.3 \\
\hline $241-U-201$ & Sludge (Liquid \& Solid) & CWR1 (Solid) & 11 & 1.63 & 27.1 \\
\hline 241-U-202 & Supernatant & CWR1 (Liquid) & 4 & 1.28 & 69.1 \\
\hline 241-U-202 & Sludge (Liquid \& Solid) & CWR1 (Solid) & 10 & 1.51 & 27.8 \\
\hline $241-\mathrm{U}-203$ & Supernatant & CWR1 (Liquid) & 4 & 1.28 & 68.7 \\
\hline 241-U-203 & Sludge (Liquid \& Solid) & CWR1 (Solid) & 9 & 1.59 & 40.2 \\
\hline $241-\mathrm{U}-204$ & Supernatant & CWR1 (Liquid) & 4 & 1.11 & 87.0 \\
\hline $241-U-204$ & Sludge (Liquid \& Solid) & CWR1 (Solid) & 7 & 1.47 & 26.2 \\
\hline
\end{tabular}

Note: TWINS, 2006, Tank Waste Information Network System, internet address: http://twins.pnl.gov:8001, Data from prefer density and weight percent water queried September 27, 2006 
Table A-2. Tank Waste Inventory from Best Basis Inventory Detail Report (Queried on September 27, 2006) for Hydrogen Generation Rate Calculations. (27 sheets)

\begin{tabular}{|c|c|c|c|c|c|c|c|c|c|c|c|c|c|c|}
\hline $\begin{array}{l}\text { Tank } \\
\text { Name }\end{array}$ & Was & ype & $\begin{array}{c}\text { Vol } \\
(\mathrm{kL})\end{array}$ & $\begin{array}{l}\text { TOC } \\
(\mathrm{Kg})\end{array}$ & $\begin{array}{l}\mathrm{NO}_{3} \\
(\mathrm{Kg})\end{array}$ & $\begin{array}{l}\mathrm{NO}_{2} \\
(\mathrm{Kg})\end{array}$ & $\begin{array}{c}\mathrm{Na} \\
(\mathrm{Kg})\end{array}$ & $\begin{array}{c}A \mathbf{l} \\
(\mathbf{K g})\end{array}$ & $\begin{array}{l}{ }^{90} \mathrm{Sr} \\
(\mathrm{Ci})\end{array}$ & $\begin{array}{c}{ }^{241} \mathrm{Am} \\
\text { (Ci) }\end{array}$ & $\begin{array}{c}{ }^{240} \mathrm{Pu} \\
(\mathrm{Ci})\end{array}$ & $\begin{array}{c}{ }^{239} \mathbf{P u} \\
\text { (Ci) }\end{array}$ & $\begin{array}{c}{ }^{238} \mathbf{P u} \\
(\mathrm{Ci})\end{array}$ & $\begin{array}{c}{ }^{137} \mathrm{Cs} \\
(\mathrm{Ci})\end{array}$ \\
\hline $241-A-101$ & Total & & & $1.26 \mathrm{E}+04$ & $45 \mathrm{E}+05$ & $1.33 E+05$ & 95 & 04 & $\mathrm{E}+05$ & $4.76 \mathrm{E}+02$ & $2.35 \mathrm{E}+01$ & $9.75 \mathrm{E}+01$ & +00 & $3.03 \mathrm{E}+05$ \\
\hline 241-A-101 & Sludge (Liquid \& Solid) & P2 (Solid) & 11 & $0.00 \mathrm{E}+00$ & $1.08 \mathrm{E}+03$ & $4.24 E+01$ & $1.26 \mathrm{E}+03$ & $2.95 \mathrm{E}+03$ & $8.12 E+04$ & $3.57 \mathrm{E}+02$ & $1.58 \mathrm{E}+01$ & $6.43 \mathrm{E}+01$ & $3.02 \mathrm{E}+00$ & $1.57 \mathrm{E}+03$ \\
\hline 241-A-101 & iid & 1-SltCk (Solid) & 878 & $E+04$ & $9 \mathrm{E}+05$ & $1.08 \mathrm{E}+05$ & $3.44 \mathrm{E}+05$ & $3.52 \mathrm{E}+04$ & $6.18 \mathrm{E}+04$ & $7 E+02$ & $7.67 \mathrm{E}+00$ & $.31 E+01$ & $1.36 \mathrm{E}+00$ & $2.50 \mathrm{E}+05$ \\
\hline 241-A-10! & Saltcake Interstitial Liquid & Al-SltCk (Liquid) & 148 & $5.54 \mathrm{E}+02$ & $3.45 \mathrm{E}+04$ & $2.44 \mathrm{E}+04$ & $4.11 E+04$ & $7.66 \mathrm{E}+03$ & $1.03 \mathrm{E}+01$ & $2.26 \mathrm{E}+00$ & $2.26 \mathrm{E}-02$ & $9.71 \mathrm{E}-02$ & $3.98 \mathrm{E}-03$ & $5.16 \mathrm{E}+04$ \\
\hline $241-$ & Total & & & $\mathrm{E}+03$ & +04 & $E+04$ & $22 \mathrm{E}+04$ & $E+03$ & $7.76 \mathrm{E}+04$ & $E+02$ & $5 \mathrm{E}+01$ & $\mathrm{E}+02$ & $E+01$ & $4.06 \mathrm{E}+04$ \\
\hline $241-\mathrm{A}-102$ & Supernatant & A1-SltCk (Liquid) & 12 & $5.30 \mathrm{E}+01$ & $2.27 \mathrm{E}+03$ & $2.07 \mathrm{E}+03$ & $2.40 \mathrm{E}+03$ & $4.92 \mathrm{E}+02$ & $1.33 \mathrm{E}+01$ & $1.29 \mathrm{E}-02$ & $1.02 \mathrm{E}-02$ & $4.41 \mathrm{E}-02$ & $1.81 \mathrm{E}-03$ & $5.95 \mathrm{E}+03$ \\
\hline $241-\mathrm{A}-102$ & Saltcake Solid & A1-SltCk (Solid) & 106 & $2.56 \mathrm{E}+03$ & $1.63 \mathrm{E}+04$ & $1.50 \mathrm{E}+04$ & $2.32 \mathrm{E}+04$ & $5.71 \mathrm{E}+03$ & $7.75 \mathrm{E}+04$ & $2.48 \mathrm{E}+02$ & $7.24 \mathrm{E}+01$ & $3.12 \mathrm{E}+02$ & $1.28 \mathrm{E}+01$ & $1.83 \mathrm{E}+04$ \\
\hline $241-\mathrm{A}-102$ & Interstit & uid) & 33 & $1.46 \mathrm{E}+02$ & $24 \mathrm{E}+03$ & $5.69 \mathrm{E}+03$ & $6.60 \mathrm{E}+03$ & $1.35 E+03$ & $3.66 \mathrm{E}+01$ & $3.54 \mathrm{E}-02$ & $2.82 \mathrm{E}-02$ & $1.21 \mathrm{E}-01$ & 4.97E-03 & $1.64 \mathrm{E}+04$ \\
\hline $241-\mathrm{A}-103$ & Total & & & $1.48 \mathrm{E}+04$ & $2.26 \mathrm{E}+0.5$ & $1.62 \mathrm{E}+05$ & $3.92 E+05$ & $3.39 E+04$ & $1.24 \mathrm{E}+05$ & $2.35 \mathrm{E}+02$ & $5.06 \mathrm{E}+01$ & $2.20 \mathrm{E}+02$ & $8.75 \mathrm{E}+00$ & $2.81 \mathrm{E}+05$ \\
\hline $24 \mathrm{I} \cdot \mathrm{A}-103$ & $\underline{s}$ & 1-SltCk (Liquid) & 17 & $1.41 E+02$ & $4.11 \mathrm{E}+03$ & $2.11 E+03$ & $4.00 \mathrm{E}+03$ & $6.04 \mathrm{E}+02$ & $3.29 \mathrm{E}+01$ & $5.31 \mathrm{E}-02$ & $8.59 \mathrm{E}-02$ & $3.70 \mathrm{E}-01$ & $1.52 \mathrm{E}-02$ & $4.59 E+03$ \\
\hline 241-A-103 & ludge (Liquid \& Solid) & AR (Solid) & 8 & $0.00 \mathrm{E}+00$ & $5.11 \mathrm{E}-07$ & $2.35 \mathrm{E}+02$ & $1.11 \mathrm{E}+03$ & $1.53 \mathrm{E}+01$ & $6.78 \mathrm{E}+04$ & $2.47 \mathrm{E}+01$ & $7.91 \mathrm{E}+00$ & $3.64 \mathrm{E}+01$ & $1.19 E+00$ & $5.32 \mathrm{E}+03$ \\
\hline $241-\mathrm{A}-103$ & Saltcake Solid & 1-SItCk (Solid) & 1071 & $1.18 \mathrm{E}+04$ & $1.40 \mathrm{E}+05$ & $1.17 \mathrm{E}+05$ & $3.07 \mathrm{E}+05$ & $2.12 \mathrm{E}+04$ & $5.51 \mathrm{E}+04$ & $2.09 \mathrm{E}+02$ & $4.09 \mathrm{E}+01$ & $1.76 \mathrm{E}+02$ & $7.25 \mathrm{E}+00$ & $1.80 \mathrm{E}+05$ \\
\hline $241-\mathrm{A}-103$ & 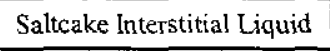 & A1-SltCk (Liquid) & 338 & $2.81 \mathrm{E}+03$ & $8.18 \mathrm{E}+04$ & $4.19 \mathrm{E}+04$ & $7.95 E+04$ & $1.20 \mathrm{E}+04$ & $6.54 \mathrm{E}+02$ & $1.06 \mathrm{E}+00$ & $1.71 \mathrm{E}+00$ & $7.36 \mathrm{E}+00$ & $3.02 \mathrm{E}-01$ & $9.12 \mathrm{E}+04$ \\
\hline 241-A-104 & Total & & & $0.00 \mathrm{E}+00$ & $3.02 \mathrm{E}+02$ & $6.74 \mathrm{E}+03$ & $3.23 \mathrm{E}+04$ & $8.06 \mathrm{E}+03$ & $2.29 \mathrm{E}+06$ & $8.07 \mathrm{E}+02$ & $1.49 \mathrm{E}+02$ & $6.81 \mathrm{E}+02$ & $2.26 \mathrm{E}+01$ & $6.19 E+04$ \\
\hline 241-A-104 & $d$ & 2. & 4 & $0.00 \mathrm{E}+00$ & 02 & $1.18 \mathrm{E}+01$ & $3.50 \mathrm{E}+02$ & $3.04 \mathrm{E}+02$ & $8.66 \mathrm{E}+04$ & $9.95 \mathrm{E}+01$ & $6.17 \mathrm{E}+00$ & $2.51 \mathrm{E}+01$ & $1.18 \mathrm{E}+00$ & $2.34 \mathrm{E}+03$ \\
\hline $241-\mathrm{A}-104$ & Sludge Solid & AR (Solid) & 102 & $0.00 \mathrm{E}+00$ & $1.46 \mathrm{E}-05$ & $6.73 \mathrm{E} \div 03$ & $3.19 \mathrm{E}+04$ & $7.76 \mathrm{E}+03$ & $2.21 \mathrm{E}+06$ & $7.07 \mathrm{E}+02$ & $1.43 \mathrm{E}+02$ & $6.56 \mathrm{E}+02$ & $2.14 \mathrm{E}+01$ & $5.96 \mathrm{E}+04$ \\
\hline $241-\mathrm{A}-105$ & Total & & & $0.00 \mathrm{E}+00$ & $1.71 \mathrm{E}+04$ & $6.67 \mathrm{E}+02$ & $5.50 \mathrm{E}+04$ & $8.28 \mathrm{E}+03$ & $2.88 \mathrm{E}+06$ & $5.62 \mathrm{E}+03$ & $1.36 \mathrm{E}+02$ & $5.56 \mathrm{E}+02$ & $2.61 \mathrm{E}+01$ & $1.07 \mathrm{E}+05$ \\
\hline $241-\mathrm{A}-105$ & 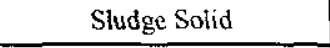 & 12 (Oovini) & 139 & $0.00 \mathrm{E}+00$ & $.71 \mathrm{E}+04$ & $6.67 \mathrm{E}+02$ & $5.50 \mathrm{E}+04$ & $8.28 E+03$ & $\mathrm{E}+06$ & $E+03$ & $1.36 \mathrm{E}+02$ & $5.56 \mathrm{E}+02$ & $2.61 \mathrm{E}+01$ & $1.07 \mathrm{E}+05$ \\
\hline 241-A-106 & Total & & & $4.31 \mathrm{E}+03$ & $5.03 \mathrm{E}+04$ & $4.57 \mathrm{E}+04$ & $7.86 \mathrm{E}+04$ & $1.47 \mathrm{E}+04$ & $4.90 \mathrm{E}+05$ & $5.81 \mathrm{E}+02$ & $2.36 \mathrm{E}+02$ & $1.03 \mathrm{E}+03$ & $4.03 \mathrm{E}+01$ & $6.43 E+04$ \\
\hline 241-A-106 & Sludge (Liquid \& Solid) & RR (Solid) & 110 & $1.59 \mathrm{E}+03$ & $1.85 \mathrm{E}+04$ & $1.68 \mathrm{E}+04$ & $2.89 \mathrm{E}+04$ & $5.40 \mathrm{E}+03$ & $1.80 \mathrm{E}+05$ & $2.15 \mathrm{E}+02$ & $8.79 \mathrm{E}+01$ & $3.79 \mathrm{E}+02$ & $1.57 \mathrm{E}+01$ & $2.36 \mathrm{E}+04$ \\
\hline $241-\mathrm{A}-106$ & Sludge (Liquid \& Solid) & AR (Solid) & 79 & $1.14 \mathrm{E}+03$ & $1.33 \mathrm{E}+04$ & $1.21 \mathrm{E}+04$ & $2.08 \mathrm{E}+04$ & $3.87 \mathrm{E}+03$ & $1.29 \mathrm{E}+05$ & $1.49 \mathrm{E}+02$ & $5.99 \mathrm{E}+01$ & $2.75 \mathrm{E}+02$ & $8.99 \mathrm{E}+00$ & $1.70 \mathrm{E}+04$ \\
\hline 241-A-106 & Saltcake Solid & Al-SltCk (Solid) & 110 & $1.59 \mathrm{E}+03$ & $1.85 \mathrm{E}+04$ & $1.68 \mathrm{E}+04$ & $2.89 \mathrm{E}+04$ & $5.40 \mathrm{E}+03$ & $1.80 \mathrm{E}+05$ & $2.17 \mathrm{E}+02$ & $8.79 \mathrm{E}+01$ & $3.79 \mathrm{E}+02$ & $1.56 \mathrm{E}+01$ & $2.36 \mathrm{E}+04$ \\
\hline 241-AN-101 & Total & & & $1.89 \mathrm{E}+04$ & $5.91 \mathrm{E}+05$ & $4.22 \mathrm{E}+05$ & $7.87 E+05$ & $7.07 \mathrm{E}+04$ & $1.08 \mathrm{E}+04$ & $9.74 \mathrm{E}+00$ & 4.74E-01 & $2.13 \mathrm{E}+00$ & $8.26 \mathrm{E}-02$ & $1.01 \mathrm{E}+06$ \\
\hline 241-AN-101 & Supernatant & $\mathrm{NA}$ & 3506 & $1.79 \mathrm{E}+04$ & $5.68 \mathrm{E}+05$ & $4.07 \mathrm{E}+05$ & $7.51 \mathrm{E}+05$ & $6.64 \mathrm{E}+04$ & $6.29 \mathrm{E}+03$ & $3.25 \mathrm{E}+00$ & $5.10 \mathrm{E}-02$ & $3.07 \mathrm{E}-01$ & $7.60 \mathrm{E}-03$ & $9.80 \mathrm{E}+05$ \\
\hline 241-AN-101 & Saltcake Solid & A1-SltCk (Solid) & 90 & $8.52 \mathrm{E}+02$ & $1.82 \mathrm{E}+04$ & $1.18 \mathrm{E}+04$ & $2.96 \mathrm{E}+04$ & $3.20 \mathrm{E}+03$ & $4.46 \mathrm{E}+03$ & $6.41 \mathrm{E}+00$ & $4.22 \mathrm{E}-01$ & $1.82 \mathrm{E}+00$ & $7.48 \mathrm{E}-02$ & $2.36 \mathrm{E}+04$ \\
\hline 241-AN-10I & Saltcake Interstitial Liquid & Al-SitCk (Liquid) & 28 & $1.13 \mathrm{E}+02$ & $5.32 \mathrm{E}+03$ & $3.42 \mathrm{E}+03$ & $6.61 \mathrm{E}+03$ & $1.06 E+03$ & $2.79 E+01$ & $7.89 \mathrm{E}-02$ & $7.87 \mathrm{E}-04$ & $3.39 \mathrm{E}-03$ & $1.39 \mathrm{E}-04$ & $9.96 \mathrm{E}+03$ \\
\hline 241-AN-102 & Total & & & $1.04 \mathrm{E}+05$ & $9.23 \mathrm{E}+05$ & $3.86 \mathrm{E}+05$ & $8.64 E+05$ & $5.64 \mathrm{E}+04$ & $3.96 \mathrm{E}+05$ & $1.10 \mathrm{E}+03$ & $1.37 \mathrm{E}+01$ & $5.26 \mathrm{E}+01$ & $3.82 \mathrm{E}+00$ & $1.59 \mathrm{E}+06$ \\
\hline
\end{tabular}




\section{RPP-5926 REV 6}

Table A-2. Tank Waste Inventory from Best Basis Inventory Detail Report (Queried on September 27, 2006) for Hydrogen Generation Rate Calculations. (27 sheets)

\begin{tabular}{|c|c|c|c|c|c|c|c|c|c|c|c|c|c|c|}
\hline $\begin{array}{l}\text { Tank } \\
\text { Name }\end{array}$ & Wast & Wasto & $\begin{array}{c}\text { Vol } \\
(\mathbf{k L})\end{array}$ & $\begin{array}{l}\text { TOC } \\
(\mathrm{Kg})\end{array}$ & $\begin{array}{l}\mathrm{NO}_{3} \\
\text { (Kg) }\end{array}$ & $\begin{array}{l}\mathrm{NO}_{2} \\
(\mathrm{Kg})\end{array}$ & $\begin{array}{c}\mathrm{Na} \\
(\mathrm{Kg})\end{array}$ & $\begin{array}{c}\mathbf{A l} \\
(\mathbf{K g})\end{array}$ & $\begin{array}{l}{ }^{90} \mathrm{Sr} \\
\text { (Ci) }\end{array}$ & $\begin{array}{c}{ }^{241} \mathrm{Am} \\
\text { (Ci) }\end{array}$ & $\begin{array}{c}{ }^{240} \mathrm{Pu} \\
(\mathrm{Ci})\end{array}$ & $\begin{array}{c}{ }^{239} \mathrm{Pu} \\
(\mathrm{Ci})\end{array}$ & $\begin{array}{l}{ }^{238} \mathrm{Pu} \\
(\mathrm{Ci})\end{array}$ & $\begin{array}{c}{ }^{137} \mathrm{Cs} \\
(\mathrm{Ci})\end{array}$ \\
\hline 241-AN-102 & ernatant & NA & 468 & $7 \mathrm{E}+04$ & $7.24 \mathrm{E}+05$ & $02 \mathrm{E}+05$ & $17 \mathrm{E}+05$ & $56 \mathrm{E}+04$ & $2.74 \mathrm{E}+05$ & $5.63 \mathrm{E}+02$ & $4.29 \mathrm{E}+00$ & $1.65 \mathrm{E}+01$ & $1.20 \mathrm{E}+00$ & $1.36 \mathrm{E}+06$ \\
\hline 241-AN-102 & Saltcake (Liquid \& Solid) & A2-SltSlr (Solid) & 584 & $2.07 \mathrm{E}+04$ & $1.98 \mathrm{E}+05$ & $8.35 \mathrm{E}+04$ & $1.47 \mathrm{E}+05$ & $1.08 \mathrm{E}+04$ & $1.22 \mathrm{E}+05$ & $5.35 \mathrm{E}+02$ & $9.40 \mathrm{E}+00$ & $3.61 \mathrm{E}+01$ & $2.62 \mathrm{E}+00$ & $2.36 \mathrm{E}+05$ \\
\hline 241-AN-103 & Total & & & $5 \mathrm{E}+04$ & $6.63 \mathrm{E}+05$ & $4.27 \mathrm{E}+05$ & $1.07 \mathrm{E}+06$ & $2.26 \mathrm{E}+05$ & $6.29 \mathrm{E}+03$ & $2.07 \mathrm{E}+01$ & $1.13 E+00$ & $4.34 \mathrm{E}+00$ & $3.15 \mathrm{E}-01$ & $1.70 \mathrm{E}+06$ \\
\hline 241-AN-103 & Supernatant & A2-SltSlr (Liquid) & 1769 & $5.39 \mathrm{E}+03$ & $2.27 \mathrm{E}+05$ & $2.30 \mathrm{E}+05$ & $4.78 \mathrm{E}+05$ & $5.30 \mathrm{E}+04$ & $3.13 \mathrm{E}+01$ & $1.08 \mathrm{E}+01$ & $1.17 \mathrm{E}-01$ & $4.51 \mathrm{E}-01$ & $3.28 \mathrm{E}-02$ & $1.12 \mathrm{E}+06$ \\
\hline 241-AN-103 & Saltcake ( & A2-Sltslr & 1638 & $E+03$ & $6 \mathrm{E}+05$ & $1.97 \mathrm{E}+05$ & $38 \mathrm{E}+05$ & $73 \mathrm{E}+05$ & $6.26 \mathrm{E}+03$ & $89 E+00$ & $1.01 \mathrm{E}+00$ & $3.89 \mathrm{E}+00$ & $2.82 \mathrm{E}-01$ & $5.79 \mathrm{E}+05$ \\
\hline 241-AN-104 & Total & & & $1.93 \mathrm{E}+04$ & $7.32 \mathrm{E}+05$ & $4.32 \mathrm{E}+05$ & $1.07 \mathrm{E}+06$ & $1.41 \mathrm{E}+05$ & $7.18 \mathrm{E}+04$ & $4.79 \mathrm{E}+01$ & $1.90 \mathrm{E}+00$ & $7.28 \mathrm{E}+00$ & $5.29 \mathrm{E}-01$ & $1.88 \mathrm{E}+06$ \\
\hline 241-AN-104 & pernatant & iid) & 98 & $7.22 \mathrm{E}+03$ & $4.35 \mathrm{E}+05$ & $2.74 \mathrm{E}+05$ & +05 & $8.96 \mathrm{E}+04$ & $E+02$ & $\mathrm{E}-01$ & $\mathrm{E}-02$ & -01 & $1.20 \mathrm{E}-02$ & $1.13 \mathrm{E}+06$ \\
\hline 24l-AN-104 & Saltcake (Liquid \& Solid) & A2-SitSlr & 1566 & $1.20 \mathrm{E}+04$ & $2.96 \mathrm{E}+05$ & $1.58 \mathrm{E}+05$ & $4.81 \mathrm{E}+05$ & $5.13 \mathrm{E}+04$ & $7.16 \mathrm{E}+04$ & $4.71 \mathrm{E}+01$ & $1.85 \mathrm{E}+00$ & $7.12 \mathrm{E}+00$ & $5.16 \mathrm{E}-01$ & $7.48 \mathrm{E}+05$ \\
\hline 241-AN-105 & Total & & & $E+04$ & $6.91 \mathrm{E}+05$ & $4.59 \mathrm{E}+05$ & $1.10 \mathrm{E}+06$ & $1.60 \mathrm{E}+05$ & $2.88 \mathrm{E}+04$ & $5.29 \mathrm{E}+01$ & $1.76 \mathrm{E}+00$ & $6.75 \mathrm{E}+00$ & $4.89 \mathrm{E}-01$ & $1.41 \mathrm{E}+06$ \\
\hline 241-AN-105 & pernatant & uid) & 27 & $E+03$ & $E+05$ & $2.64 \mathrm{E}+05$ & $E+05$ & $9.28 \mathrm{E}+04$ & $E+01$ & $E+01$ & $\mathrm{E}-01$ & $+\infty$ & $9.72 \mathrm{E}-02$ & $6.52 \mathrm{E}+05$ \\
\hline 241-AN-105 & Saltcake (Liquid \& Solid) & A2-SitSlr (Solid) & 1925 & $1.62 \mathrm{E}+04$ & $3.43 \mathrm{E}+05$ & $1.94 \mathrm{E}+05$ & $5.52 \mathrm{E}+05$ & $6.76 \mathrm{E}+04$ & $2.87 \mathrm{E}+04$ & $2.09 \mathrm{E}+01$ & $1.41 \mathrm{E}+00$ & $5.41 \mathrm{E}+00$ & $3.92 \mathrm{E}-01$ & $7.56 \mathrm{E}+05$ \\
\hline 106 & Total & & & 04 & $7.96 \mathrm{E}+04$ & 6 & $1.75 \mathrm{E}+05$ & $2.38 \mathrm{E}+04$ & $1.90 \mathrm{E}+06$ & $2.37 \mathrm{E}+03$ & $2.28 \mathrm{E}+02$ & $\mathrm{E}+03$ & +01 & $1.95 \mathrm{E}+05$ \\
\hline 241-AN-106 & Supernatant & NA & 2485 & $4.86 \mathrm{E}+03$ & $5.84 \mathrm{E}+04$ & $4.65 \mathrm{E}+04$ & $1.04 \mathrm{E}+05$ & $9.57 \mathrm{E}+03$ & $1.47 \mathrm{E}+03$ & $1.08 \mathrm{E}+00$ & $1.22 \mathrm{E}+00$ & $7.86 \mathrm{E}+00$ & $1.25 \mathrm{E}-01$ & $1.19 \mathrm{E}+05$ \\
\hline 241-AN-106 & Sludge Interstitial Liquid & NA & 192 & $3.76 \mathrm{E}+02$ & $4.52 \mathrm{E}+03$ & $3.59 \mathrm{E}+03$ & $8.04 \mathrm{E}+03$ & $7.39 E+02$ & $1.14 \mathrm{E}+02$ & $8.32 \mathrm{E}-02$ & $9.42 \mathrm{E}-02$ & $6.07 \mathrm{E}-01$ & $9.67 \mathrm{E}-03$ & $9.22 \mathrm{E}+03$ \\
\hline-106 & Sluds & 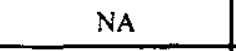 & 299 & $\mathrm{E}+04$ & $8 \mathrm{E}+03$ & +03 & $12 \mathrm{E}+04$ & $1.12 \mathrm{E}+04$ & $1.90 \mathrm{E}+06$ & $2.36 \mathrm{E}+03$ & $2.27 \mathrm{E}+02$ & $1.05 \mathrm{E}+03$ & $3.71 \mathrm{E}+01$ & $4.95 \mathrm{E}+04$ \\
\hline 241-AN-106 & Saltcake (Liquid \& Solid) & NA & 65 & $6.15 \mathrm{E}+02$ & $1.31 \mathrm{E}+04$ & $8.53 \mathrm{E}+03$ & $2.14 E+04$ & $2.31 \mathrm{E}+03$ & $3.22 \mathrm{E}+03$ & $4.63 \mathrm{E}+00$ & $3.05 \mathrm{E}-01$ & $1.31 \mathrm{E}+00$ & $5.41 \mathrm{E}-02$ & $1.71 E+04$ \\
\hline 241-AN-107 & Total & & & $1.47 \mathrm{E}+05$ & $8.37 \mathrm{E}+05$ & $2.74 \mathrm{E}+05$ & $8.72 \mathrm{E}+05$ & $2.60 \mathrm{E}+04$ & 4.67E+05 & $3.62 \mathrm{E}+03$ & $5.71 \mathrm{E}+01$ & $2.20 \mathrm{E}+02$ & $1.59 \mathrm{E}+01$ & $1.29 \mathrm{E}+06$ \\
\hline 241-AN-107 & 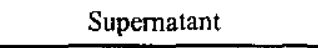 & a) & 3297 & $07 \mathrm{E}+05$ & 05 & +05 & 05 & $3.70 \mathrm{E}+03$ & $53 E+05$ & $E+03$ & $3.87 \mathrm{E}+01$ & $1.49 E+02$ & $1.08 E+01$ & $9.85 E+05$ \\
\hline 241-AN-107 & Saltcake (Liquid \& Solid) & A2-SltSlr (Solid) & 872 & $4.03 E+04$ & $1.47 \mathrm{E}+05$ & $5.38 \mathrm{E}+04$ & $1.88 \mathrm{E}+05$ & $2.23 \mathrm{E}+04$ & $2.14 \mathrm{E}+05$ & $1.20 \mathrm{E}+03$ & $1.84 \mathrm{E}+01$ & $7.08 \mathrm{E}+01$ & $5.14 \mathrm{E}+00$ & $3.01 \mathrm{E}+05$ \\
\hline 241-AP-101 & Total & & & $8.00 \mathrm{E}+03$ & $4.96 \mathrm{E}+05$ & $1.72 \mathrm{E}+05$ & $6 \mathrm{E}+05$ & $3.08 \mathrm{E}+04$ & $4.12 \mathrm{E}+02$ & $8.13 \mathrm{E}-01$ & $1.15 \mathrm{E}-01$ & $4.42 \mathrm{E}-01$ & $3.21 \mathrm{E}-02$ & $5.87 \mathrm{E}+05$ \\
\hline 241-AP-101 & Supernatant & 2-SltSlr (Liquid) & 4219 & $8.00 \mathrm{E}+03$ & $4.96 \mathrm{E}+05$ & $1.72 \mathrm{E}+05$ & $5.46 \mathrm{E}+05$ & $3.08 \mathrm{E}+04$ & $4.12 \mathrm{E}+02$ & $8.13 \mathrm{E}-01$ & $1.15 \mathrm{E}-01$ & $4.42 \mathrm{E}-01$ & $3.21 \mathrm{E}-02$ & $5.87 \mathrm{E}+05$ \\
\hline 241-AP-102 & Total & & & $1.24 \mathrm{E}+04$ & $6.86 \mathrm{E}+05$ & $4.03 \mathrm{E}+05$ & $7.98 \mathrm{E}+05$ & $1.41 \mathrm{E}+05$ & $1.62 \mathrm{E}+03$ & $7.1 \mathrm{tE}+00$ & $2.23 \mathrm{E}-01$ & $1.32 \mathrm{E}+00$ & $3.43 \mathrm{E}-02$ & $9.80 E+05$ \\
\hline 241-AP-102 & Supernatant & Waste Transfer & 4049 & $1.22 \mathrm{E}+04$ & $6.78 \mathrm{E}+05$ & $3.98 \mathrm{E}+05$ & $7.89 \mathrm{E}+05$ & $1.25 \mathrm{E}+05$ & $1.56 \mathrm{E}+03$ & $7.00 \mathrm{E}+00$ & $2.11 \mathrm{E}-01$ & $1.25 \mathrm{E}+00$ & $3.24 \mathrm{E}-02$ & $9.67 E+05$ \\
\hline $241-\mathrm{AP}-102$ & Sludge (Liquid \& Solid) & NA & 88 & $2.16 \mathrm{E}+02$ & $7.58 \mathrm{E}+03$ & $4.67 \mathrm{E}+03$ & $9.18 \mathrm{E}+03$ & $1.57 \mathrm{E}+04$ & $5.66 \mathrm{E}+01$ & $1.07 \mathrm{E}-01$ & $1.22 \mathrm{E}-02$ & $7.21 \mathrm{E}-02$ & $1.85 \mathrm{E}-03$ & $1.25 \mathrm{E}+04$ \\
\hline 241-AP-103 & Total & & & $2.46 \mathrm{E}+04$ & $5.05 \mathrm{E}+05$ & $2.71 \mathrm{E}+0 \mathrm{~s}$ & $6.01 \mathrm{E}+05$ & $8.36 \mathrm{E}+04$ & $8.13 \mathrm{E}+03$ & $3.74 \mathrm{E}+01$ & $5.23 \mathrm{E}-01$ & $3.14 \mathrm{E}+00$ & $9.69 \mathrm{E}-02$ & $7.72 \mathrm{E}+05$ \\
\hline 241-AP-103 & Supernatant & NA & 3298 & $2.43 \mathrm{E}+04$ & $4.98 \mathrm{E}+05$ & $2.65 \mathrm{E}+05$ & $5.90 \mathrm{E}+05$ & $6.13 E+04$ & $8.10 \mathrm{E}+03$ & $3.73 \mathrm{E}+01$ & $5.02 \mathrm{E}-01$ & $3.01 \mathrm{E}+00$ & $9.26 \mathrm{E}-02$ & $7.57 \mathrm{E}+05$ \\
\hline 241-AP-103 & Sludge (Liquid \& Solid) & NA & 80 & $2.55 \mathrm{E}+02$ & $6.76 \mathrm{E}+03$ & $5.73 \mathrm{E}+03$ & $1.10 \mathrm{E}+04$ & $2.23 \mathrm{E}+04$ & $3.28 \mathrm{E}+01$ & $3.35 \mathrm{E}-02$ & $2.18 \mathrm{E}-02$ & $1.29 \mathrm{E}-01$ & 4.26E-03 & $1.50 \mathrm{E}+04$ \\
\hline
\end{tabular}




\section{RPP-5926 REV 6}

Table A-2. Tank Waste Inventory from Best Basis Inventory Detail Report (Queried on September 27, 2006) for Hydrogen Generation Rate Calculations. (27 sheets)

\begin{tabular}{|c|c|c|c|c|c|c|c|c|c|c|c|c|c|c|}
\hline $\begin{array}{c}\text { Tank } \\
\text { Name }\end{array}$ & Waste Phase & laste Type & $\begin{array}{l}\text { Vol } \\
\text { (kL) }\end{array}$ & $\begin{array}{l}\text { TOC } \\
(\mathrm{Kg})\end{array}$ & $\begin{array}{l}\mathrm{NO}_{3} \\
(\mathrm{Kg})\end{array}$ & $\begin{array}{l}\mathrm{NO}_{2} \\
\text { (Kg) }\end{array}$ & $\begin{array}{c}\mathrm{Na} \\
(\mathrm{Kg})\end{array}$ & $\begin{array}{c}\mathrm{Al} \\
(\mathrm{Kg})\end{array}$ & $\begin{array}{l}{ }^{90} \mathrm{Sr} \\
\text { (Ci) }\end{array}$ & ${ }_{(\mathrm{Ci})}^{241} \mathrm{Am}$ & $\begin{array}{c}{ }^{240} \mathrm{Pu} \\
(\mathrm{Ci})\end{array}$ & $\begin{array}{c}{ }^{239} \mathrm{Pu} \\
(\mathrm{Ci})\end{array}$ & $\begin{array}{r}{ }^{238} \mathrm{Pu} \\
(\mathrm{Ci})\end{array}$ & $\begin{array}{c}{ }^{137} \mathrm{Cs} \\
(\mathrm{Ci})\end{array}$ \\
\hline 241-AP-104 & Total & & & $E+04$ & $E+05$ & $6 \mathrm{E}+05$ & +05 & $E+04$ & $6.94 \mathrm{E}+03$ & $.17 \mathrm{E}+01$ & $\mathrm{E}-01$ & 1 & 1.6 & $7.71 \mathrm{E}+05$ \\
\hline 241-AP-104 & Supematant & NA (Liquid) & 4155 & $1.73 E+04$ & $4.19 \mathrm{E}+05$ & $2.66 \mathrm{E}+05$ & $5.34 \mathrm{E}+05$ & $6.98 \mathrm{E}+04$ & $6.94 \mathrm{E}+03$ & $1.17 \mathrm{E}+01$ & $1.25 \mathrm{E}-01$ & $7.33 \mathrm{E}-01$ & $1.65 \mathrm{E}-0 \mathrm{I}$ & $7.71 \mathrm{E}+05$ \\
\hline 241-AP-105 & Total & & & $8.88 \mathrm{E}+03$ & $4.84 \mathrm{E}+05$ & $2.04 \mathrm{E}+05$ & $6.35 \mathrm{E}+05$ & $8.11 E+04$ & $1.08 \mathrm{E}+04$ & $5.57 \mathrm{E}+00$ & $6.67 \mathrm{E}-01$ & $2.98 \mathrm{E}+00$ & $1.98 \mathrm{E}-01$ & $6.61 \mathrm{E}+05$ \\
\hline 241-AP-105 & Super & aste & 965 & $5.94 \mathrm{E}+03$ & $4.21 \mathrm{E}+05$ & $1.88 \mathrm{E}+05$ & $31 \mathrm{E}+05$ & $7.63 \mathrm{E}+04$ & $1.08 \mathrm{E}+03$ & $2.75 E+00$ & $1.84 \mathrm{E}-01$ & $1.12 \mathrm{E}+00$ & $6.38 \mathrm{E}-02$ & $5.39 E+05$ \\
\hline 241-AP-105 & Saltcake (Liquid \& Solid) & A2-SltSIr (Solid) & 337 & $2.94 E+03$ & $6.31 E+04$ & $1.62 \mathrm{E}+04$ & $1.04 E+05$ & $4.73 E+03$ & $9.69 \mathrm{E}+03$ & $2.83 \mathrm{E}+00$ & $4.83 \mathrm{E}-01$ & $1.86 \mathrm{E}+00$ & $1.35 \mathrm{E}-01$ & $1.22 \mathrm{E}+05$ \\
\hline 241-AP-106 & Total & & & $1.49 \mathrm{E}+04$ & $3.04 \mathrm{E}+05$ & $1.78 \mathrm{E}+05$ & $4.45 E+05$ & $5.81 \mathrm{E}+04$ & $5.12 \mathrm{E}+03$ & $2.61 \mathrm{E}+00$ & $5.47 \mathrm{E}-02$ & $3.18 \mathrm{E}-01$ & $8.93 \mathrm{E}-03$ & $7.54 \mathrm{E}+05$ \\
\hline 241-AP-106 & Supernatant & NA & 301 & $1.49 \mathrm{E}+04$ & $3.04 \mathrm{E}+05$ & $1.78 \mathrm{E}+05$ & $45 \mathrm{E}+05$ & $5.81 \mathrm{E}+04$ & $5.12 \mathrm{E}+03$ & $2.61 \mathrm{E}+00$ & $5.47 \mathrm{E}-02$ & $3.18 \mathrm{E}-01$ & $8.93 \mathrm{E}-03$ & $7.54 \mathrm{E}+05$ \\
\hline 241-AP-107 & Total & & & $3.63 \mathrm{E}+03$ & $1.64 \mathrm{E}+05$ & $7.53 \mathrm{E}+04$ & $1.82 \mathrm{E}+05$ & $2.14 \mathrm{E}+04$ & $1.10 \mathrm{E}+03$ & $5.53 \mathrm{E}-01$ & $3.77 \mathrm{E}-02$ & $1.99 \mathrm{E}-01$ & $1.16 \mathrm{E}-02$ & $1.91 \mathrm{E}+05$ \\
\hline 241-AP-107 & Supe & NA & 306 & $3.63 E+03$ & $1.64 \mathrm{E}+05$ & $7.53 \mathrm{E}+04$ & $1.82 \mathrm{E}+05$ & $2.14 \mathrm{E}+04$ & $1.10 \mathrm{E}+03$ & $5.53 \mathrm{E}-01$ & $3.77 \mathrm{E}-02$ & $1.99 \mathrm{E}-01$ & $1.16 \mathrm{E}-02$ & $1.91 E+05$ \\
\hline 241-AP-108 & Total & & & $1.10 \mathrm{E}+04$ & $6.83 \mathrm{E}+05$ & $3.04 \mathrm{E}+05$ & $8.56 \mathrm{E}+05$ & $9.25 E+04$ & $2.80 \mathrm{E}+03$ & $1.31 \mathrm{E}+00$ & $2.60 \mathrm{E}-01$ & $1.28 \mathrm{E}+00$ & $9.61 \mathrm{E}-02$ & $7.83 \mathrm{E}+05$ \\
\hline 241-AP-108 & Supernatant & NA & 3385 & $9.47 \mathrm{E}+03$ & $6.02 \mathrm{E}+05$ & $2.68 \mathrm{E}+05$ & $7.20 \mathrm{E}+05$ & $11 \mathrm{E}+04$ & $2.45 \mathrm{E}+03$ & $1.15 \mathrm{E}+00$ & $2.27 \mathrm{E}-01$ & $1.12 \mathrm{E}+00$ & $8.41 \mathrm{E}-02$ & $6.85 \mathrm{E}+05$ \\
\hline 241-AP-108 & Saltcake (Liquid \& Solid) & NA & 487 & $1.56 \mathrm{E}+03$ & $8.07 E+04$ & $3.56 \mathrm{E}+04$ & $1.35 \mathrm{E}+05$ & $1.13 \mathrm{E}+04$ & $3.53 \mathrm{E}+02$ & $1.65 \mathrm{E}-01$ & $3.27 \mathrm{E}-02$ & $1.61 \mathrm{E}-01$ & $1.21 \mathrm{E}-02$ & $9.85 \mathrm{E}+04$ \\
\hline 241-AW-101 & Total & & & $2.04 \mathrm{E}+04$ & $8.19 E+05$ & $4.22 \mathrm{E}+05$ & $1.02 \mathrm{E}+06$ & $1.15 E+05$ & $8.30 \mathrm{E}+04$ & $1.05 \mathrm{E}+02$ & $2.61 \mathrm{E}+01$ & $1.00 \mathrm{E}+02$ & $7.29 E+00$ & $1.52 \mathrm{E}+06$ \\
\hline 241-AW-101 & Supernatant & 2-SitSlr (Liquid) & 2770 & $7.24 \mathrm{E}+03$ & $4.74 \mathrm{E}+05$ & $2.92 \mathrm{E}+05$ & $6.45 \mathrm{E}+05$ & $8.17 \mathrm{E}+04$ & $1.04 \mathrm{E}+03$ & $7.21 \mathrm{E}-01$ & $2.96 \mathrm{E}-01$ & $1.14 \mathrm{E}+00$ & $8.25 \mathrm{E}-02$ & $1.05 \mathrm{E}+06$ \\
\hline 241-AW-101 & Saltcake (Liquid \& Solid) & A2-SltSlr (Solid) & 1403 & $1.32 \mathrm{E}+04$ & $3.46 \mathrm{E}+05$ & $1.30 \mathrm{E}+05$ & $3.77 \mathrm{E}+05$ & $3.34 \mathrm{E}+04$ & $8.20 \mathrm{E}+04$ & $1.04 \mathrm{E}+02$ & $2.58 \mathrm{E}+01$ & $9.93 E+01$ & $7.20 \mathrm{E}+00$ & $4.75 \mathrm{E}+05$ \\
\hline $241-\mathrm{AW}-102$ & Total & & & $4.86 \mathrm{E}+03$ & $2.20 \mathrm{E}+05$ & $1.01 \mathrm{E}+05$ & $E+05$ & $3.50 \mathrm{E}+04$ & $1.97 \mathrm{E}+04$ & 6.1 & +00 & $E+01$ & $2.75 \mathrm{E}-01$ & $2.61 E+05$ \\
\hline 241-AW-102 & Supematant & Waste Transfer & 2268 & $4.67 E+03$ & $2.20 \mathrm{E}+05$ & $1.01 \mathrm{E}+05$ & $2.40 \mathrm{E}+05$ & $2.75 \mathrm{E}+04$ & $1.82 \mathrm{E}+03$ & $9.35 \mathrm{E}-01$ & $3.52 \mathrm{E}-02$ & $2.01 \mathrm{E}-01$ & $2.21 \mathrm{E}-02$ & $2.59 \mathrm{E}+05$ \\
\hline $241-\mathrm{AW}-102$ & Sludge (Liquid \& Solid) & NA & 25 & $1.89 \mathrm{E}+02$ & $2.20 \mathrm{E}+02$ & $3.01 \mathrm{E}+02$ & $1.67 \mathrm{E}+03$ & $7.54 \mathrm{E}+03$ & $1.79 \mathrm{E}+04$ & $6.05 \mathrm{E}+01$ & $3.05 \mathrm{E}+00$ & $1.47 \mathrm{E}+01$ & $2.53 \mathrm{E}-01$ & $2.35 \mathrm{E}+03$ \\
\hline 241-AW-103 & Total & & & $9.41 \mathrm{E}+03$ & $3.62 \mathrm{E}+05$ & $1.36 \mathrm{E}+05$ & $5.17 E+05$ & $3.46 \mathrm{E}+04$ & $1.34 \mathrm{E}+04$ & $1.67 \mathrm{E}+02$ & $1.15 \mathrm{E}+02$ & $4.09 \mathrm{E}+02$ & $4.04 \mathrm{E}+01$ & $3.76 \mathrm{E}+05$ \\
\hline 241-AW-103 & Supernatant & NA & 2893 & $5.42 \mathrm{E}+03$ & $2.79 E+05$ & $9.69 \mathrm{E}+04$ & $2.92 \mathrm{E}+05$ & $2.34 \mathrm{E}+04$ & $6.38 \mathrm{E}+02$ & $1.48 \mathrm{E}-01$ & $7.34 \mathrm{E}-02$ & $3.45 \mathrm{E}-01$ & $3.12 \mathrm{E}-02$ & $3.25 \mathrm{E}+05$ \\
\hline 241-AW-103 & Sludge (Liquid \& Solid) & $\mathrm{CWZ} 2$ (Solid) & 1105 & $2.02 \mathrm{E}+03$ & $4.51 \mathrm{E}+04$ & $2.14 \mathrm{E}+04$ & $1.88 \mathrm{E}+05$ & $3.84 \mathrm{E}+03$ & $1.15 \mathrm{E}+04$ & $1.57 \mathrm{E}+02$ & $1.14 \mathrm{E}+02$ & $4.06 \mathrm{E}+02$ & $4.03 E+01$ & $3.55 \mathrm{E}+04$ \\
\hline 241-AW-103 & Saltcake Solid & Al-SltCk (Solid) & 115 & $1.76 \mathrm{E}+03$ & $2.95 E+04$ & $1.41 \mathrm{E}+04$ & $3.04 E+04$ & $7.05 \mathrm{E}+03$ & $1.20 \mathrm{E}+03$ & $1.01 \mathrm{E}+01$ & $6.62 \mathrm{E}-01$ & $2.85 \mathrm{E}+00$ & 1.17E-01 & $3.69 E+03$ \\
\hline 241-AW-103 & Saltcake Interstitial Liquid & Al-SltCk (Liquid) & 36 & $2.20 \mathrm{E}+02$ & $8.88 \mathrm{E}+03$ & $3.35 \mathrm{E}+03$ & $7.23 \mathrm{E}+03$ & $2.91 \mathrm{E}+02$ & $3.41 \mathrm{E}+01$ & $9.65 \mathrm{E}-02$ & $9.62 \mathrm{E}-04$ & 4.14E-03 & $1.70 \mathrm{E}-04$ & $1.22 \mathrm{E}+04$ \\
\hline 241-AW-104 & Total & & & $2.19 \mathrm{E}+04$ & $4.56 \mathrm{E}+05$ & $3.03 \mathrm{E}+05$ & $7.82 \mathrm{E}+05$ & $1.06 \mathrm{E}+05$ & $5.98 \mathrm{E}+03$ & $6.78 E+01$ & $1.46 \mathrm{E}+02$ & $5.18 \mathrm{E}+02$ & $5.16 \mathrm{E}+01$ & $9.81 E+05$ \\
\hline 241-AW-104 & Supernatant & NA & 3221 & $1.39 \mathrm{E}+04$ & $3.62 \mathrm{E}+05$ & $2.40 \mathrm{E}+05$ & $5.47 \mathrm{E}+05$ & $8.19 \mathrm{E}+04$ & $5.24 \mathrm{E}+03$ & $5.05 \mathrm{E}+00$ & $8.17 \mathrm{E}-02$ & $4.58 \mathrm{E}-01$ & $1.46 \mathrm{E}-02$ & $7.69 E+05$ \\
\hline 241-AW-104 & Sludge Solid & PL2 (Solid) & 188 & $2.84 \mathrm{E}+03$ & $1.02 \mathrm{E}+04$ & $5.82 \mathrm{E}+03$ & $2.31 \mathrm{E}+04$ & $2.57 \mathrm{E}+03$ & $1.20 \mathrm{E}+01$ & $1.83 \mathrm{E}+01$ & $1.38 \mathrm{E}+02$ & $4.90 \mathrm{E}+02$ & $4.95 \mathrm{E}+01$ & $1.37 \mathrm{E}+01$ \\
\hline 241-AW-104 & Sludge Interstitial Liquid & PL2 (Liquid) & 62 & $1.63 \mathrm{E}+02$ & $4.38 \mathrm{E}+03$ & $2.72 \mathrm{E}+03$ & $1.17 \mathrm{E}+04$ & $8.37 E+02$ & $4.02 \mathrm{E}+01$ & $2.57 \mathrm{E}-01$ & $2.80 \mathrm{E}-03$ & $1.08 \mathrm{E}-02$ & $7.81 \mathrm{E}-04$ & $1.44 \mathrm{E}+04$ \\
\hline
\end{tabular}




\section{RPP-5926 REV 6}

Table A-2. Tank Waste Inventory from Best Basis Inventory Detail Report (Queried on September 27, 2006) for

Hydrogen Generation Rate Calculations. (27 sheets)

\begin{tabular}{|c|c|c|c|c|c|c|c|c|c|c|c|c|c|c|}
\hline $\begin{array}{c}\text { Tank } \\
\text { Name }\end{array}$ & Waste Phase & Waste Type & $\begin{array}{l}\text { Vol } \\
(\mathrm{kL})\end{array}$ & $\begin{array}{l}\text { TOC } \\
(\mathrm{Kg})\end{array}$ & $\begin{array}{l}\mathrm{NO}_{3} \\
(\mathrm{Kg})\end{array}$ & $\begin{array}{l}\mathrm{NO}_{2} \\
(\mathrm{Kg})\end{array}$ & $\begin{array}{c}\mathbf{N a} \\
(\mathrm{Kg})\end{array}$ & $\begin{array}{c}\mathrm{Al} \\
(\mathrm{Kg})\end{array}$ & $\begin{array}{l}{ }^{90} \mathrm{Sr} \\
\text { (Ci) }\end{array}$ & $\begin{array}{c}{ }^{241} \mathrm{Am} \\
(\mathrm{Ci})\end{array}$ & $\begin{array}{c}{ }^{240} \mathrm{Pu} \\
(\mathrm{Ci})\end{array}$ & $\begin{array}{l}{ }^{239} \mathbf{P u} \\
\text { (Ci) }\end{array}$ & $\begin{array}{c}{ }^{238} \mathrm{Pu} \\
\text { (Ci) }\end{array}$ & $\begin{array}{c}{ }^{137} \mathrm{Cs} \\
\text { (Ci) }\end{array}$ \\
\hline 241-AW-104 & Saltcake Solid & A2-SltSlr (Solid) & 457 & $4.51 \mathrm{E}+03$ & $7 \mathrm{E}+04$ & $4.17 \mathrm{E}+04$ & $1.65 E+05$ & $1.46 \mathrm{E}+04$ & $56 \mathrm{E}+02$ & $4.29 \mathrm{E}+01$ & $32 \mathrm{E}+00$ & $2.81 \mathrm{E}+01$ & $2.04 \mathrm{E}+00$ & $1.52 \mathrm{E}+05$ \\
\hline 241-AW-104 & Saltcake Interstitial Liquid & 2-SltSlr & 136 & $5.14 \mathrm{E}+02$ & $89 \mathrm{E}+04$ & 1.32E+04 & $3.50 \mathrm{E}+04$ & $5.67 \mathrm{E}+03$ & $1.28 \mathrm{E}+02$ & $1.38 \mathrm{E}+00$ & $1.51 \mathrm{E}-02$ & $5.80 \mathrm{E}-02$ & $4.21 \mathrm{E}-03$ & $4.56 \mathrm{E}+04$ \\
\hline 241-AW-105 & Total & & & $4.90 \mathrm{E}+03$ & $5.35 \mathrm{E}+04$ & $1.37 \mathrm{E}+04$ & $1.46 \mathrm{E}+05$ & 4.12E+03 & $3.13 \mathrm{E}+04$ & $3.86 \mathrm{E}+02$ & $1.84 \mathrm{E}+02$ & $6.52 \mathrm{E}+02$ & $6.47 \mathrm{E}+01$ & $5.03 \mathrm{E}+04$ \\
\hline 241-AW-105 & Supernatant & NA & 587 & $2.42 \mathrm{E}+02$ & $1.46 \mathrm{E}+04$ & $1.51 \mathrm{E}+03$ & $1.34 \mathrm{E}+04$ & $8.50 \mathrm{E}+0 \mathrm{l}$ & $1.31 E+01$ & $3.78 \mathrm{E}-02$ & $3.83 \mathrm{E}-02$ & $1.36 \mathrm{E}-01$ & $1.35 \mathrm{E}-02$ & $5.92 \mathrm{E}+03$ \\
\hline 241-AW-105 & olid & PL2 (Solid) & 61 & $1.89 \mathrm{E}+03$ & $2.89 \mathrm{E}+03$ & $1.10 \mathrm{E}+03$ & $9.29 \mathrm{E}+03$ & $1.49 \mathrm{E}+03$ & $2.27 \mathrm{E}+04$ & $1.45 \mathrm{E}+02$ & $1.27 \mathrm{E}+01$ & $4.49 \mathrm{E}+01$ & $4.55 \mathrm{E}+00$ & $5.10 \mathrm{E}+03$ \\
\hline 241-AW-105 & Sludge Solid & CWZr2 (Solid) & 660 & $2.66 \mathrm{E}+03$ & $2.05 \mathrm{E}+04$ & $6.28 \mathrm{E}+03$ & $1.09 \mathrm{E}+05$ & $2.10 \mathrm{E}+03$ & $7.52 \mathrm{E}+03$ & $2.37 \mathrm{E}+02$ & $1.70 \mathrm{E}+02$ & $6.03 E+02$ & $5.98 \mathrm{E}+01$ & $2.23 \mathrm{E}+04$ \\
\hline 241-AW-105 & Sludge Interstitial Liquid & PL2 (Liquid) & 12 & $3.40 \mathrm{E}+01$ & $6.15 \mathrm{E}+02$ & $2.68 \mathrm{E}+02$ & $9.78 \mathrm{E}+02$ & $4.93 \mathrm{E}+01$ & $4.72 \mathrm{E}+00$ & $3.59 \mathrm{E}-02$ & $9.68 \mathrm{E}-02$ & $3.44 \mathrm{E}-01$ & $3.47 \mathrm{E}-02$ & $5.39 \mathrm{E}+00$ \\
\hline 241-AW-105 & Sludge Interstitial Liquid & $\mathrm{CWZr2}$ & 266 & $7.98 \mathrm{E}+01$ & $1.49 \mathrm{E}+04$ & $4.55 \mathrm{E}+03$ & $1.33 \mathrm{E}+04$ & $3.95 \mathrm{E}+02$ & $1.11 \mathrm{E}+03$ & $4.84 \mathrm{E}+00$ & $9.84 \mathrm{E}-01$ & $3.50 \mathrm{E}+00$ & $3.46 \mathrm{E}-01$ & $1.69 \mathrm{E}+04$ \\
\hline 241-AW-106 & Total & & & $1.44 \mathrm{E}+04$ & $5.01 \mathrm{E}+05$ & $2.44 \mathrm{E}+05$ & $7.12 \mathrm{E}+05$ & $8.24 \mathrm{E}+04$ & $3.52 \mathrm{E}+04$ & $3.53 \mathrm{E}+01$ & $6.19 \mathrm{E}+00$ & $2.52 \mathrm{E}+01$ & $1.63 \mathrm{E}+00$ & $8.27 \mathrm{E}+05$ \\
\hline 241-AW-106 & Supernatant & insfer & 3224 & $5.90 \mathrm{E}+03$ & $2.87 \mathrm{E}+05$ & $1.45 \mathrm{E}+05$ & $3.75 E+05$ & $5.25 \mathrm{E}+04$ & $1.62 \mathrm{E}+03$ & $2.15 \mathrm{E}+00$ & $5.32 \mathrm{E}-01$ & $3.42 \mathrm{E}+00$ & $5.73 \mathrm{E}-02$ & $4.05 \mathrm{E}+05$ \\
\hline 241-AW-106 & Saltcake (Liquid \& Solid) & A2-SitSlr (Solid) & 1072 & $8.54 \mathrm{E}+03$ & $2.14 \mathrm{E}+05$ & $9.89 E+04$ & $3.37 \mathrm{E}+05$ & $2.99 \mathrm{E}+04$ & $3.36 \mathrm{E}+04$ & $3.31 \mathrm{E}+01$ & $5.66 \mathrm{E}+00$ & $2.17 \mathrm{E}+01$ & $1.58 \mathrm{E}+00$ & $4.22 \mathrm{E}+05$ \\
\hline 241-AX-101 & Total & & & $1.13 \mathrm{E}+04$ & $3.35 \mathrm{E}+05$ & $1.65 \mathrm{E}+05$ & $4.90 \mathrm{E}+05$ & $5.12 \mathrm{E}+04$ & $2.21 \mathrm{E}+05$ & $3.93 \mathrm{E}+02$ & $2.68 \mathrm{E}+01$ & $1.16 \mathrm{E}+02$ & $4.78 \mathrm{E}+00$ & $4.07 \mathrm{E}+05$ \\
\hline 241-AX-101 & Sludge (Liquid \& Solid) & SRR (Solid) & 11 & $3.79 \mathrm{E}-02$ & $1.23 \mathrm{E}-08$ & $2.57 E+02$ & $2.03 \mathrm{E}+03$ & $0.00 \mathrm{E}+00$ & $1.45 \mathrm{E}+05$ & $2.78 \mathrm{E}+02$ & $1.93 \mathrm{E}+01$ & $8.30 \mathrm{E}+01$ & $3.44 \mathrm{E}+00$ & $1.51 \mathrm{E}+03$ \\
\hline 241-AX-I01 & Saltcake Solid & Al-SltCk (Solid) & 1164 & $1.06 \mathrm{E}+04$ & $2.87 \mathrm{E}+05$ & $1.34 \mathrm{E}+05$ & $4.38 \mathrm{E}+05$ & 4.23E+04 & $7.59 \mathrm{E}+04$ & $1.15 \mathrm{E}+02$ & $7.54 \mathrm{E}+00$ & $3.25 E+01$ & $1.34 \mathrm{E}+00$ & $3.44 \mathrm{E}+05$ \\
\hline 241-AX-101 & Saltcake Interstitial Liquid & Al-SltCk (Liquid) & 178 & $7.08 \mathrm{E}+02$ & $4.84 \mathrm{E}+04$ & $3.08 \mathrm{E}+04$ & $5.02 \mathrm{E}+04$ & $8.95 \mathrm{E}+03$ & $4.06 \mathrm{E}+01$ & $7.02 \mathrm{E}-02$ & $E-03$ & $2.54 \mathrm{E}-02$ & $1.04 \mathrm{E}-03$ & $6.09 \mathrm{E}+04$ \\
\hline $241-\mathrm{AX}-102$ & Total & & & $6.05 \mathrm{E}+03$ & $2.58 \mathrm{E}+04$ & $5.07 \mathrm{E}+03$ & $2.43 E+04$ & $2.37 \mathrm{E}+03$ & $2.30 \mathrm{E}+05$ & $2.14 \mathrm{E}+03$ & $4.87 \mathrm{E}+01$ & $1.52 \mathrm{E}+02$ & $1.41 \mathrm{E}+01$ & $2.45 \mathrm{E}+04$ \\
\hline 241-AX-102 & Sludge (Liquid \& Solid) & B (Solid) & 23 & $8.71 \mathrm{E}+00$ & $5.08 \mathrm{E}-08$ & $1.57 \mathrm{E}+02$ & $6.96 \mathrm{E}+03$ & $8.10 \mathrm{E}+02$ & $2.02 \mathrm{E}+05$ & $1.87 \mathrm{E}+03$ & $3.98 \mathrm{E}+01$ & $1.14 \mathrm{E}+02$ & $1.25 \mathrm{E}+01$ & $8.12 \mathrm{E}+03$ \\
\hline $24 \mathrm{i}-\mathrm{AX}-102$ & Saltcake (Liquid \& Solid) & A1-SltCk (Solid) & 90 & $6.04 E+03$ & $\mathrm{E}+04$ & +03 & $1.73 \mathrm{E}$ & +03 & $\mathrm{E}+04$ & $E+02$ & +00 & $3.84 \mathrm{E}+01$ & $1.58 \mathrm{E}+00$ & $1.64 \mathrm{E}+04$ \\
\hline 241-AX-103 & Total & & & $2.54 \mathrm{E}+03$ & $6.83 \mathrm{E}+04$ & $5.39 \mathrm{E}+04$ & $1.07 \mathrm{E}+05$ & $1.51 E+04$ & $4.45 E+05$ & $7.28 \mathrm{E}+02$ & $2.34 \mathrm{E}+01$ & $9.26 \mathrm{E}+01$ & $4.33 \mathrm{E}+00$ & $1.16 \mathrm{E}+05$ \\
\hline 241-AX-103 & Sludge (Liquid \& Solid) & P2 (Solid) & 30 & $0.00 \mathrm{E}+00$ & $2.25 \mathrm{E}+03$ & $8.40 \mathrm{E}+01$ & $1.74 \mathrm{E}+03$ & $1.53 \mathrm{E}+03$ & $4.31 \mathrm{E}+05$ & $7.07 \mathrm{E}+02$ & $2.18 \mathrm{E}+01$ & $8.90 \mathrm{E}+01$ & $4.18 E+00$ & $1.60 \mathrm{E}+04$ \\
\hline 241-AX-103 & Saltcake Solid & A1-SitCk (Solid) & 298 & $2.23 E+03$ & $5.18 \mathrm{E}+04$ & $4.47 \mathrm{E}+04$ & $8.72 \mathrm{E}+04$ & $1.07 \mathrm{E}+04$ & $1.37 \mathrm{E}+04$ & $2.00 \mathrm{E}+01$ & $1.52 \mathrm{E}+00$ & $3.60 \mathrm{E}+00$ & $1.48 \mathrm{E}-01$ & $7.28 \mathrm{E}+04$ \\
\hline 241-AX-103 & Saltcake Interstitial Liquid & Al-SltCk (Liquid) & 75 & $3.02 E+02$ & $1.43 E+04$ & $9.15 \mathrm{E}+03$ & $1.77 \mathrm{E}+04$ & $2.84 \mathrm{E}+03$ & $7.47 \mathrm{E}+01$ & $2.11 \mathrm{E}-01$ & $2.11 \mathrm{E}-03$ & $9.07 \mathrm{E}-03$ & $3.72 \mathrm{E}-04$ & $2.67 \mathrm{E}+04$ \\
\hline 241-AX-104 & Total & & & $0.00 \mathrm{E}+00$ & $2.30 \mathrm{E}+03$ & $1.13 E+02$ & $2.17 \mathrm{E}+03$ & $2.67 \mathrm{E}+03$ & $2.05 E+06$ & $9.48 \mathrm{E}+02$ & $6.61 \mathrm{E}+01$ & $2.69 \mathrm{E}+02$ & $1.27 \mathrm{E}+01$ & $4.97 \mathrm{E}+04$ \\
\hline 241-AX-104 & Sludge Solid & P2 (Solid) & 28 & $0.00 \mathrm{E}+00$ & $2.30 \mathrm{E}+03$ & $1.13 E+02$ & $2.17 \mathrm{E}+03$ & $2.67 \mathrm{E}+03$ & $2.05 \mathrm{E}+06$ & $9.48 \mathrm{E}+02$ & $6.61 \mathrm{E}+01$ & $2.69 \mathrm{E}+02$ & $1.27 E+01$ & $4.97 \mathrm{E}+04$ \\
\hline 241-AY-101 & Total & & & $8.54 \mathrm{E}+03$ & $3.62 \mathrm{E}+03$ & $2.72 \mathrm{E}+04$ & $1.06 \mathrm{E}+05$ & $3.97 \mathrm{E}+04$ & $2.13 \mathrm{E}+06$ & $1.44 \mathrm{E}+04$ & $2.35 \mathrm{E}+02$ & $7.64 \mathrm{E}+02$ & $6.51 \mathrm{E}+01$ & $8.47 \mathrm{E}+04$ \\
\hline 241-AY-101 & Supernatant & NA (Liquid) & 426 & $3.95 \mathrm{E}+02$ & $1.34 \mathrm{E}+03$ & $9.72 \mathrm{E}+03$ & $3.36 \mathrm{E}+04$ & $1.38 \mathrm{E}+03$ & $2.69 \mathrm{E}+02$ & $7.72 \mathrm{E}-01$ & $1.16 \mathrm{E}+00$ & $4.97 \mathrm{E}+00$ & $2.13 \mathrm{E}-01$ & $1.35 \mathrm{E}+04$ \\
\hline 241-AY-101 & Sludge Solid & NA (Solid) & 339 & $7.97 \mathrm{E}+03$ & $1.87 \mathrm{E}+03$ & $1.36 \mathrm{E}+04$ & $6.79 E+04$ & $3.83 \mathrm{E}+04$ & $2.13 E+06$ & $1.44 \mathrm{E}+04$ & $2.28 \mathrm{E}+02$ & $7.37 \mathrm{E}+02$ & $6.40 \mathrm{E}+01$ & $6.60 \mathrm{E}+04$ \\
\hline
\end{tabular}




\section{RPP-5926 REV 6}

Table A-2. Tank Waste Inventory from Best Basis Inventory Detail Report (Queried on September 27, 2006) for Hydrogen Generation Rate Calculations. (27 sheets)

\begin{tabular}{|c|c|c|c|c|c|c|c|c|c|c|c|c|c|c|}
\hline $\begin{array}{c}\text { Tank } \\
\text { Name }\end{array}$ & Was & e & $\begin{array}{c}\text { Vol } \\
(\mathbf{k L})\end{array}$ & $\begin{array}{l}\text { TOC } \\
\text { (Kg) }\end{array}$ & $\begin{array}{l}\mathrm{NO}_{3} \\
(\mathrm{Kg})\end{array}$ & $\begin{array}{l}\mathrm{NO}_{2} \\
(\mathrm{Kg})\end{array}$ & $\begin{array}{c}\mathrm{Na} \\
(\mathrm{Kg})\end{array}$ & $\begin{array}{c}\mathrm{Al} \\
(\mathrm{Kg})\end{array}$ & $\begin{array}{l}{ }^{{ }^{90}} \mathrm{Sr} \\
(\mathrm{Ci})\end{array}$ & ${ }_{(\mathrm{Ci})}^{241}$ & $\begin{array}{c}{ }^{240} \mathrm{Pu} \\
(\mathrm{Ci})\end{array}$ & $\begin{array}{c}{ }^{239} \mathrm{Pu} \\
(\mathrm{Ci})\end{array}$ & $\begin{array}{c}{ }^{238} \mathbf{P u} \\
\text { (Ci) }\end{array}$ & $\begin{array}{c}{ }^{137} \mathrm{Cs} \\
\text { (Ci) }\end{array}$ \\
\hline-101 & Sludge Interstiti & & 60 & $1.77 \mathrm{E}+02$ & $05 \mathrm{E}+02$ & $3.92 \mathrm{E}+03$ & $68 \mathrm{E}+03$ & $18 E+01$ & $4.62 \mathrm{E}+01$ & $3.08 \mathrm{E}+00$ & $5.22 \mathrm{E}+00$ & $.23 \mathrm{E}+01$ & $9.54 \mathrm{E}-01$ & $5.26 \mathrm{E}+03$ \\
\hline $241-\mathrm{AY}-102$ & Total & & & $6.58 \mathrm{E}+03$ & $1.19 \mathrm{E}+03$ & $8.93 E+04$ & $2.26 \mathrm{E}+05$ & $5.49 \mathrm{E}+04$ & $4.64 E+06$ & $4.97 \mathrm{E}+03$ & $3.65 E+02$ & $1.58 \mathrm{E}+03$ & $6.12 \mathrm{E}+01$ & $3.06 \mathrm{E}+05$ \\
\hline $241-\mathrm{AY}-102$ & Supernatant & NA & 992 & $2.33 \mathrm{E}+03$ & $80 \mathrm{E}+02$ & $8.59 \mathrm{E}+04$ & $1.66 \mathrm{E}+05$ & $3.28 \mathrm{E}+03$ & $3.21 \mathrm{E}+03$ & $5.98 \mathrm{E}-01$ & $9.71 \mathrm{E}-01$ & $48 \mathrm{E}+00$ & $7.19 \mathrm{E}-01$ & $5.69 \mathrm{E}+04$ \\
\hline $241-\mathrm{AY}-102$ & Sludge Solid & NA & 376 & $3.40 \mathrm{E}+03$ & $40 \mathrm{E}+02$ & $2.10 \mathrm{E}+03$ & $4.33 \mathrm{E}+04$ & $4.30 E+04$ & $3.86 \mathrm{E}+06$ & $4.14 E+03$ & $2.98 \mathrm{E}+02$ & $1.32 \mathrm{E}+03$ & $4.64 \mathrm{E}+01$ & $2.05 \mathrm{E}+05$ \\
\hline-102 & $s$ & ) & 75 & $E+02$ & $\mathrm{E}+01$ & $9 \mathrm{E}+02$ & $64 \mathrm{E}+03$ & $E+03$ & $7.71 E+05$ & $8 \mathrm{E}+02$ & $6.56 \mathrm{E}+01$ & $7 \mathrm{E}+02$ & $1.41 \mathrm{E}+01$ & $4.09 \mathrm{E}+04$ \\
\hline $241-\mathrm{AY}-102$ & Sludge Interstitial Liq & BL (Liq & 120 & $1.83 \mathrm{E}+02$ & $4.65 \mathrm{E}+01$ & $8.70 \mathrm{E}+02$ & $8.44 E+03$ & $4.09 \mathrm{E}+01$ & $2.05 \mathrm{E}+02$ & $3.81 \mathrm{E}-02$ & $7.20 \mathrm{E}-02$ & $2.82 \mathrm{E}-01$ & $1.54 \mathrm{E}-02$ & $3.70 \mathrm{E}+03$ \\
\hline 241-AZ-101 & Total & & & $1.92 \mathrm{E}+03$ & $.78 \mathrm{E}+05$ & $2.08 \mathrm{E}+05$ & $3.83 \mathrm{E}+05$ & $5.90 \mathrm{E}+04$ & $5.52 \mathrm{E}+06$ & $2.56 \mathrm{E}+04$ & $3.32 \mathrm{E}+02$ & $1.18 \mathrm{E}+03$ & $1.15 \mathrm{E}+02$ & $5.31 E+06$ \\
\hline $241-A Z-101$ & Supernatant & A21 (Liquid) & 3074 & $1.66 \mathrm{E}+03$ & $71 \mathrm{E}+05$ & $1.99 \mathrm{E}+05$ & $3.60 \mathrm{E}+05$ & $1.96 \mathrm{E}+04$ & $2.81 \mathrm{E}+03$ & $4.67 \mathrm{E}-01$ & $1.30 \mathrm{E}+00$ & $4.55 \mathrm{E}+00$ & $4.70 \mathrm{E}-01$ & $5.06 \mathrm{E}+06$ \\
\hline 241-AZ-101 & Siudge (Liquid \& Sc & P3AZ1 (Solid) & 167 & $2.20 \mathrm{E}+02$ & $6.65 \mathrm{E}+03$ & $7.92 \mathrm{E}+03$ & $1.94 \mathrm{E}+04$ & $3.34 \mathrm{E}+04$ & $4.68 \mathrm{E}+06$ & $2.17 \mathrm{E}+04$ & $2.84 \mathrm{E}+02$ & $9.89 \mathrm{E}+02$ & $1.02 \mathrm{E}+02$ & $2.06 \mathrm{E}+05$ \\
\hline $241-\mathrm{AZ}-101$ & Sludge (Liquid \& Solid) & olid) & 30 & $3.95 \mathrm{E}+01$ & +03 & $\mathrm{E}+03$ & $48 \mathrm{E}+03$ & $E+03$ & $\mathrm{E}+05$ & $\mathrm{E}+03$ & $4.72 \mathrm{E}+01$ & $1 \mathrm{E}+02$ & $E+01$ & $3.70 \mathrm{E}+04$ \\
\hline 241-AZ-102 & Total & & & $3.72 \mathrm{E}+03$ & $83 \mathrm{E}+04$ & $1.28 \mathrm{E}+05$ & $2.15 \mathrm{E}+05$ & $3.14 \mathrm{E}+04$ & $3.92 \mathrm{E}+06$ & $2.84 \mathrm{E}+04$ & $3.51 \mathrm{E}+02$ & $1.33 E+03$ & $1.97 \mathrm{E}+02$ & $3.50 \mathrm{E}+06$ \\
\hline $241-\mathrm{AZ}-102$ & Supernatant & 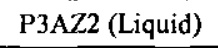 & 3267 & $3.43 E+03$ & $4.40 E+04$ & $1.11 \mathrm{E}+05$ & $1.89 \mathrm{E}+05$ & $1.83 \mathrm{E}+03$ & $6.51 \mathrm{E}+03$ & $2.07 \mathrm{E}+00$ & $6.61 E+00$ & $2.42 \mathrm{E}+01$ & $2.34 \mathrm{E}+00$ & $3.13 \mathrm{E}+06$ \\
\hline 241-AZ-102 & Sludge (Liquid \& Solid) & SRR (Solid) & 98 & $7.02 \mathrm{E}+01$ & $05 \mathrm{E}+03$ & $4.09 \mathrm{E}+03$ & $6.60 \mathrm{E}+03$ & $7.31 \mathrm{E}+03$ & $9.67 \mathrm{E}+05$ & $7.01 \mathrm{E}+03$ & $7.70 \mathrm{E}+01$ & $3.32 \mathrm{E}+02$ & $4.81 \mathrm{E}+01$ & $9.15 \mathrm{E}+04$ \\
\hline $241-A Z-102$ & Sludge (Liquid \& Solid) & PL2 (Solid) & 15 & $1.07 \mathrm{E}+01$ & $1.61 \mathrm{E}+02$ & $6.26 \mathrm{E}+02$ & $1.01 \mathrm{E}+03$ & $1.12 \mathrm{E}+03$ & $1.48 \mathrm{E}+05$ & $1.07 \mathrm{E}+03$ & $1.38 \mathrm{E}+01$ & $4.88 \mathrm{E}+01$ & $7.36 \mathrm{E}+00$ & $1.40 \mathrm{E}+04$ \\
\hline 241-AZ-102 & Sludg & rJAE & 275 & Cruz & $6 \mathrm{E}+03$ & $\mathrm{E}+04$ & $1.85 \mathrm{E}+04$ & $2.05 \mathrm{E}+04$ & $2.71 \mathrm{E}+06$ & $97 \mathrm{E}+04$ & $2.47 \mathrm{E}+02$ & $.01 \mathrm{E}+02$ & $1.35 \mathrm{E}+02$ & $2.57 \mathrm{E}+05$ \\
\hline $241-\mathrm{AZ}-102$ & Sludge (Liquid \& Solid) & NA sludge & 8 & $5.73 \mathrm{E}+00$ & $8.61 \mathrm{E}+01$ & $3.34 \mathrm{E}+02$ & $5.39 \mathrm{E}+02$ & $5.97 \mathrm{E}+02$ & $7.90 \mathrm{E}+04$ & $5.72 \mathrm{E}+02$ & $6.29 \mathrm{E}+00$ & $2.71 \mathrm{E}+01$ & $3.93 \mathrm{E}+00$ & $7.47 \mathrm{E}+03$ \\
\hline 241-B-101 & Total & & & $3.96 \mathrm{E}+03$ & $.12 \mathrm{E}+04$ & $2.62 \mathrm{E}+04$ & $1.23 \mathrm{E}+05$ & $1.52 \mathrm{E}+04$ & $2.03 E+05$ & $1.42 \mathrm{E}+03$ & $9.65 \mathrm{E}+01$ & $3.13 \mathrm{E}+02$ & $2.73 \mathrm{E}+01$ & $2.16 \mathrm{E}+03$ \\
\hline $241-\mathrm{B}-101$ & Sludge (Liquid \& Solid) & MWI (Solid) & 11 & $0.00 \mathrm{E}+00$ & $2.28 \mathrm{E}+01$ & $1.08 \mathrm{E}+01$ & $1.33 \mathrm{E}+03$ & $0.00 \mathrm{E}+00$ & $7.63 \mathrm{E}+02$ & $2.49 \mathrm{E}-03$ & $8.08 \mathrm{E}-04$ & $1.20 \mathrm{E}-02$ & $4.04 \mathrm{E}-05$ & $3.88 \mathrm{E}+01$ \\
\hline $241-\mathrm{B}-101$ & Sludge (Liquid \& Solid) & BL (Solid) & 76 & $3.31 \mathrm{E}+03$ & $2.31 \mathrm{E}+03$ & $4.46 \mathrm{E}+02$ & $2.09 \mathrm{E}+04$ & $4.38 E+03$ & $3.63 \mathrm{E}+03$ & $5.37 \mathrm{E}+00$ & $2.61 \mathrm{E}+01$ & $1.02 \mathrm{E}+02$ & $5.61 \mathrm{E}+00$ & $0.00 \mathrm{E}+00$ \\
\hline $241-\mathrm{B}-101$ & Sludge (Liquid \& Solid) & B (Solid) & 19 & $6.58 \mathrm{E}+00$ & $83 \mathrm{E}-08$ & $1.18 \mathrm{E}+02$ & $5.26 \mathrm{E}+03$ & $6.12 \mathrm{E}+02$ & $1.98 \mathrm{E}+05$ & +03 & $6.87 \mathrm{E}+01$ & $1.96 \mathrm{E}+02$ & $2,16 \mathrm{E}+01$ & $1.76 \mathrm{E}+02$ \\
\hline 241-B-101 & Saltcake Solid & B-SitCk (Solid) & 232 & $6.50 \mathrm{E}+02$ & $6.28 \mathrm{E}+04$ & $1.60 \mathrm{E}+04$ & $7.92 \mathrm{E}+04$ & $1.01 \mathrm{E}+04$ & $1.34 \mathrm{E}+02$ & $9.12 \mathrm{E}-02$ & $1.54 \mathrm{E}+00$ & $1.37 \mathrm{E}+01$ & $1.01 \mathrm{E}-01$ & $6.37 \mathrm{E}+02$ \\
\hline 241-B-101 & Saltcake Interstitial Liquid & B-SitCk (Liquid) & 73 & $4.94 \mathrm{E}-01$ & $2.60 \mathrm{E}+04$ & $9.60 \mathrm{E}+03$ & $1.65 \mathrm{E}+04$ & $5.28 \mathrm{E}+01$ & $3.10 \mathrm{E}+02$ & $2.26 \mathrm{E}-01$ & $8.64 \mathrm{E}-02$ & 7.64E-01 & $5.67 \mathrm{E}-03$ & $1.31 \mathrm{E}+03$ \\
\hline 241-B-102 & Total & & & $8.12 \mathrm{E}+02$ & $2.22 \mathrm{E}+04$ & $1.35 \mathrm{E}+03$ & $3.28 \mathrm{E}+04$ & $3.64 \mathrm{E}+03$ & $1.33 \mathrm{E}+02$ & $9.45 \mathrm{E}-02$ & $5.75 \mathrm{E}-01$ & $5.08 \mathrm{E}+00$ & $3.77 \mathrm{E}-02$ & $5.84 \mathrm{E}+02$ \\
\hline $241-\mathrm{B}-102$ & Supernatant & B-SitCk (Liquid) & 15 & $5.07 \mathrm{E}-02$ & $3.25 \mathrm{E}+03$ & $1.27 \mathrm{E}+02$ & $1.69 \mathrm{E}+03$ & $5.42 \mathrm{E}+00$ & $3.18 \mathrm{E}+01$ & $2.32 \mathrm{E}-02$ & $8.86 \mathrm{E}-03$ & $7.83 \mathrm{E}-02$ & $5.82 \mathrm{E}-04$ & $1.34 \mathrm{E}+02$ \\
\hline $241-\mathrm{B}-102$ & Saltcake Solid & B-SltCk (Solid) & 81 & $8.12 \mathrm{E}+02$ & $1.36 \mathrm{E}+04$ & $1.01 E+03$ & $2.83 \mathrm{E}+04$ & $3.62 \mathrm{E}+03$ & $4.80 \mathrm{E}+01$ & $3.25 \mathrm{E}-02$ & $5.52 \mathrm{E}-01$ & $4.88 \mathrm{E}+00$ & $3.62 \mathrm{E}-02$ & $2.28 \mathrm{E}+02$ \\
\hline $241-\mathrm{B}-102$ & Saltcake Interstitial Liquid & B-SltCk (Liquid) & 25 & $8.45 \mathrm{E}-02$ & $5.43 \mathrm{E}+03$ & $2.13 \mathrm{E}+02$ & $2.82 \mathrm{E}+03$ & $9.03 \mathrm{E}+00$ & $5.30 \mathrm{E}+01$ & $3.87 \mathrm{E}-02$ & $1.48 \mathrm{E}-02$ & 1.30E-01 & $9.69 \mathrm{E}-04$ & $2.23 E+02$ \\
\hline 241-B-103 & Total & & & $1.83 \mathrm{E}+02$ & $3.71 E+04$ & $2.38 \mathrm{E}+03$ & $6.10 \mathrm{E}+04$ & $7.04 \mathrm{E}+03$ & $4.76 \mathrm{E}+02$ & $4.18 \mathrm{E}-01$ & $5.79 E+00$ & $5.12 \mathrm{E}+0 \mathrm{I}$ & $3.80 \mathrm{E}-01$ & $9.01 \mathrm{E}+02$ \\
\hline
\end{tabular}


Table A-2. Tank Waste Inventory from Best Basis Inventory Detail Report (Queried on September 27, 2006) for Hydrogen Generation Rate Calculations. (27 sheets)

\begin{tabular}{|c|c|c|c|c|c|c|c|c|c|c|c|c|c|c|}
\hline $\begin{array}{c}\text { Tank } \\
\text { Name }\end{array}$ & Was & pe & $\begin{array}{c}\text { Vol } \\
(\mathrm{kL})\end{array}$ & $\begin{array}{l}\text { TOC } \\
(\mathrm{Kg})\end{array}$ & $\begin{array}{l}\mathrm{NO}_{3} \\
(\mathrm{Kg})\end{array}$ & $\begin{array}{l}\mathrm{NO}_{2} \\
(\mathrm{Kg})\end{array}$ & $\begin{array}{c}\mathrm{Na} \\
(\mathrm{Kg})\end{array}$ & $\begin{array}{c}\mathbf{A l} \\
(\mathbf{K g})\end{array}$ & $\begin{array}{l}{ }^{90} \mathrm{Sr} \\
\text { (Ci) }\end{array}$ & $\begin{array}{c}{ }^{241} \mathrm{Am} \\
\text { (Ci) }\end{array}$ & $\begin{array}{c}{ }^{240} \mathrm{Pu} \\
(\mathrm{Ci})\end{array}$ & $\begin{array}{c}{ }^{239} \mathrm{Pu} \\
\text { (Ci) }\end{array}$ & $\begin{array}{c}{ }^{238} \mathbf{P u} \\
(\mathrm{Ci})\end{array}$ & $\begin{array}{l}{ }^{137} \mathrm{Cs} \\
\text { (Ci) }\end{array}$ \\
\hline 241-B-103 & Sludge (Liquid & Solid) & 4 & $0.00 \mathrm{E}+00$ & $28 \mathrm{E}+00$ & $3.92 \mathrm{E}+00$ & $85 \mathrm{E}+02$ & $0.00 \mathrm{E}+00$ & $2.77 \mathrm{E}+02$ & $9.04 E-04$ & $2.94 \mathrm{E}-04$ & $4.36 \mathrm{E}-03$ & $1.47 \mathrm{E}-05$ & $1.41 \mathrm{E}+01$ \\
\hline $241-B-103$ & Saltcake Solid & SltCk (Solid) & 157 & $1.83 \mathrm{E}+02$ & $.63 E+04$ & $1.96 \mathrm{E}+03$ & $48 E+04$ & $7.02 \mathrm{E}+03$ & $9.30 \mathrm{E}+01$ & $3.40 \mathrm{E}-01$ & $5.76 \mathrm{E}+00$ & $5.09 \mathrm{E}+01$ & $3.78 \mathrm{E}-01$ & $4.41 \mathrm{E}+02$ \\
\hline $241-\mathrm{B}-103$ & Saltcake Interstitial Liquid & B-SltCk (Liquid) & 50 & $1.69 \mathrm{E}-01$ & $1.09 E+04$ & $4.25 \mathrm{E}+02$ & $5.65 \mathrm{E}+03$ & $1.81 E+01$ & $1.06 \mathrm{E}+02$ & $7.74 \mathrm{E}-02$ & $2.95 \mathrm{E}-02$ & $2.61 \mathrm{E}-01$ & $1.94 \mathrm{E}-03$ & $4.46 \mathrm{E}+02$ \\
\hline $241-\mathrm{B}-104$ & Total & & & $2.55 \mathrm{E}+03$ & $59 \mathrm{E}+05$ & $5.16 \mathrm{E}+03$ & $28 \mathrm{E}+05$ & $3.50 \mathrm{E}+03$ & $3.88 \mathrm{E}+03$ & $4.79 \mathrm{E}+00$ & $9.26 \mathrm{E}+00$ & $8.65 \mathrm{E}+01$ & $5.95 \mathrm{E}-01$ & $1.30 \mathrm{E}+04$ \\
\hline $241-B-104$ & Liquid \& Solid) & lid) & 697 & $91 \mathrm{E}+03$ & $80 \mathrm{E}+05$ & $2.40 \mathrm{E}+03$ & $13 \mathrm{E}+05$ & $.78 \mathrm{E}+03$ & $2.61 \mathrm{E}+00$ & $43 \mathrm{E}-02$ & $4.82 \mathrm{E}+00$ & $4.62 \mathrm{E}+01$ & $3.07 \mathrm{E}-01$ & $3.04 \mathrm{E}+00$ \\
\hline $241-B-104$ & Sludge (Liquid \& Solid) & 1C (Solid) & 473 & $4.02 \mathrm{E}+02$ & $1.90 \mathrm{E}+05$ & $1.63 \mathrm{E}+03$ & $7.69 \mathrm{E}+04$ & $1.21 \mathrm{E}+03$ & $3.68 \mathrm{E}+03$ & $4.53 \mathrm{E}+00$ & $2.97 \mathrm{E}+00$ & $2.73 \mathrm{E}+01$ & $1.92 \mathrm{E}-01$ & $1.21 \mathrm{E}+04$ \\
\hline $241-B-104$ & lid & (olid) & 192 & $2.40 \mathrm{E}+02$ & $.72 E+04$ & $.62 \mathrm{E}+02$ & $2 \mathrm{E}+04$ & +02 & $E+01$ & E-02 & $E+\infty$ & $E+01$ & $\mathrm{E}-02$ & $\mathrm{E}+02$ \\
\hline $241-B-104$ & Saltcake Interstitial Liquid & vid) & 55 & $1.86 \mathrm{E}-01$ & $19 E+04$ & $4.67 \mathrm{E}+02$ & $6.21 \mathrm{E}+03$ & $1.99 \mathrm{E}+01$ & $1.17 \mathrm{E}+02$ & $8.51 \mathrm{E}-02$ & $3.25 \mathrm{E}-02$ & $2.87 \mathrm{E}-01$ & $2.13 \mathrm{E}-03$ & $4.91 \mathrm{E}+02$ \\
\hline 241-B-105 & Total & & & $1.73 \mathrm{E}+03$ & $1.84 \mathrm{E}+05$ & $1.43 \mathrm{E}+04$ & $3.41 \mathrm{E}+05$ & $4.22 \mathrm{E}+04$ & $1.01 \mathrm{E}+03$ & $1.37 \mathrm{E}+00$ & $7.02 \mathrm{E}+00$ & $6.23 \mathrm{E}+01$ & $4.60 \mathrm{E}-01$ & $4.25 \mathrm{E}+03$ \\
\hline 241-B-105 & ludge (Liquid & olid) & 61 & $9.68 \mathrm{E}+01$ & $E+03$ & $E+03$ & $E+03$ & $1.55 \mathrm{E}+02$ & $2 \mathrm{E}-01$ & $2.58 \mathrm{E}-03$ & $2 \mathrm{E}-01$ & $1.26 \mathrm{E}+00$ & $9 \mathrm{E}-03$ & $1.54 \mathrm{E}-01$ \\
\hline 241-B-105 & Sludge (Liquid \& Solid) & 1C (Solid) & 45 & $3.38 \mathrm{E}+01$ & $11 E+03$ & $6.25 \mathrm{E}+02$ & $6.39 \mathrm{E}+03$ & $9.59 \mathrm{E}+02$ & $3.10 \mathrm{E}+02$ & $8.89 \mathrm{E}-01$ & $5.83 \mathrm{E}-0 \mathrm{I}$ & $5.36 \mathrm{E}+00$ & $3.77 \mathrm{E}-02$ & $1.02 \mathrm{E}+03$ \\
\hline $241-B-105$ & Saltcake Solid & 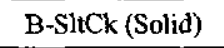 & 919 & $1.60 \mathrm{E}+03$ & $1.54 \mathrm{E}+05$ & $1.14 \mathrm{E}+04$ & $3.21 \mathrm{E}+05$ & $4.11 \mathrm{E}+04$ & $5.44 \mathrm{E}+02$ & $3.69 \mathrm{E}-01$ & $6.26 \mathrm{E}+00$ & $5.53 \mathrm{E}+01$ & $4.11 \mathrm{E}-01$ & $2.58 \mathrm{E}+03$ \\
\hline 241-B-105 & Saltcake Interstitial Liquid & iquid) & 73 & $2.47 \mathrm{E}-01$ & $1.58 \mathrm{E}+04$ & $6.21 \mathrm{E}+02$ & $8.25 E+03$ & $2.64 \mathrm{E}+01$ & $1.55 \mathrm{E}+02$ & $1.13 E-01$ & $4.31 \mathrm{E}-02$ & $3.81 \mathrm{E}-01$ & $2.83 \mathrm{E}-03$ & $6.51 \mathrm{E}+02$ \\
\hline $241-B-106$ & Total & & & $2.67 \mathrm{E}+02$ & $1.15 \mathrm{E}+05$ & $4.63 \mathrm{E}+03$ & $7.07 \mathrm{E}+04$ & $3.79 \mathrm{E}+03$ & $3.12 \mathrm{E}+04$ & $6.33 \mathrm{E}+00$ & $2.00 \mathrm{E}+00$ & $1.85 \mathrm{E}+01$ & $1.30 \mathrm{E}-01$ & $9.90 \mathrm{E}+03$ \\
\hline 241-B-106 & II & 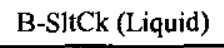 & 4 & $1.35 \mathrm{E}-02$ & $.68 \mathrm{E}+02$ & $E+01$ & $E+02$ & $.44 \mathrm{E}+00$ & $8.48 \mathrm{E}+00$ & $.19 \mathrm{E}-03$ & $2.36 \mathrm{E}-03$ & $2.09 \mathrm{E}-02$ & $1.55 \mathrm{E}-04$ & $3.57 \mathrm{E}+01$ \\
\hline $241-B-106$ & Sludge (Liquid \& Solid) & TBP (Solid) & 297 & $2.18 \mathrm{E}+02$ & $7.48 \mathrm{E}+04$ & $3.06 \mathrm{E}+03$ & $4.22 \mathrm{E}+04$ & $6.11 \mathrm{E}+02$ & $3.01 E+04$ & $4.65 \mathrm{E}+00$ & $9.06 \mathrm{E}-01$ & $8.46 \mathrm{E}+00$ & $5.89 \mathrm{E}-02$ & $7.52 \mathrm{E}+03$ \\
\hline 241-B-106 & ludge (Liquid \& Solid) & IC (Solid) & 163 & $4.81 \mathrm{E}+01$ & $.94 E+04$ & $1.54 \mathrm{E}+03$ & $81 \mathrm{E}+04$ & $3.18 \mathrm{E}+03$ & $1.14 \mathrm{E}+03$ & $1.67 \mathrm{E}+00$ & $1.09 \mathrm{E}+00$ & $1.01 \mathrm{E}+01$ & $7.07 \mathrm{E}-02$ & $2.35 \mathrm{E}+03$ \\
\hline 24I-B-107 & Total & & & $7.57 \mathrm{E}+02$ & $1.56 \mathrm{E}+05$ & $3.46 \mathrm{E}+03$ & $1.50 \mathrm{E}+05$ & $2.59 \mathrm{E}+04$ & $3.20 \mathrm{E}+03$ & $4.56 \mathrm{E}+00$ & $4.70 \mathrm{E}+00$ & $4.00 \mathrm{E}+01$ & $3.24 \mathrm{E}-01$ & $1.09 \mathrm{E}+04$ \\
\hline $241-B-107$ & Sludge (Liquid \& Solid) & CWP2 (Solid) & 42 & $3.37 \mathrm{E}+01$ & $1.73 E+03$ & $5.45 \mathrm{E}+01$ & $1.19 \mathrm{E}+03$ & $1.09 \mathrm{E}+04$ & $2.32 \mathrm{E}+01$ & $2.74 \mathrm{E}-01$ & $5.43 \mathrm{E}-01$ & $2.30 \mathrm{E}+00$ & $5.43 \mathrm{E}-02$ & $2.73 \mathrm{E}+01$ \\
\hline 241-B-107 & ludge (Liquid \& Solid) & $-($ (soliu) & 285 & $3.08 \mathrm{E}+02$ & $9.65 \mathrm{E}+04$ & $2.10 \mathrm{E}+03$ & $6.63 \mathrm{E}+04$ & $9.77 \mathrm{E}+03$ & +03 & $4.20 \mathrm{E}+00$ & $2.76 \mathrm{E}+00$ & $2.53 \mathrm{E}+01$ & $1.78 \mathrm{E}-01$ & $9.30 \mathrm{E}+03$ \\
\hline 241-B-107 & Saltcake Solid & B-SltCk (Solid) & 216 & $4.15 \mathrm{E}+02$ & $3.56 \mathrm{E}+04$ & $8.62 \mathrm{E}+02$ & $7.22 \mathrm{E}+04$ & $5.26 \mathrm{E}+03$ & $1.41 \mathrm{E}+02$ & $8.27 \mathrm{E}-02$ & $1.40 \mathrm{E}+00$ & $1.24 \mathrm{E}+01$ & $9.20 \mathrm{E}-02$ & $6.71 \mathrm{E}+02$ \\
\hline $241-\mathrm{B}-107$ & Saltcake Interstitial Liquid & B-SItCk (Liquid) & 68 & $3.43 \mathrm{E}-01$ & $2.25 \mathrm{E}+04$ & $4.43 \mathrm{E}+02$ & $9.96 \mathrm{E}+03$ & $1.37 \mathrm{E}+00$ & $2.15 E+02$ & $2.44 \mathrm{E}-03$ & $9.32 \mathrm{E}-04$ & $8.23 \mathrm{E}-03$ & $6.11 \mathrm{E}-05$ & $9.05 E+02$ \\
\hline 241-B-108 & Total & & & $5.50 \mathrm{E}+02$ & $4.12 \mathrm{E}+04$ & $7.31 \mathrm{E}+03$ & $1.17 \mathrm{E}+05$ & $3.38 \mathrm{E}+04$ & $9.29 \mathrm{E}+02$ & $1.08 \mathrm{E}-01$ & $2.88 \mathrm{E}-01$ & $1.96 \mathrm{E}+00$ & $2.33 \mathrm{E}-02$ & $7.54 \mathrm{E}+03$ \\
\hline 241-B-108 & Sludge (Liquid \& Solid) & CWP2 (Solid) & 104 & $1.54 \mathrm{E}+02$ & $9.09 \mathrm{E}+03$ & $2.42 E+03$ & $2.35 \mathrm{E}+04$ & $2.55 \mathrm{E}+04$ & $6.96 \mathrm{E}+02$ & $6.45 \mathrm{E}-02$ & $1.28 \mathrm{E}-01$ & $5.42 \mathrm{E}-01$ & $1.28 \mathrm{E}-02$ & $3.17 \mathrm{E}+03$ \\
\hline $241-B-108$ & Saltcake Solid & 3-SltCk (Solid) & 204 & $3.95 \mathrm{E}+02$ & $1.63 E+04$ & $2.64 \mathrm{E}+03$ & $8.60 \mathrm{E}+04$ & $8.33 E+03$ & $1.35 \mathrm{E}+02$ & $8.66 \mathrm{E}-03$ & $1.47 \mathrm{E}-01$ & $1.30 \mathrm{E}+00$ & $9.64 \mathrm{E}-03$ & $3.95 \mathrm{E}+03$ \\
\hline $241-B-108$ & Saltcake Interstitial Liquid & B-SitCk (Liquid) & 42 & $1.56 \mathrm{E}-01$ & $1.58 E+04$ & $2.26 \mathrm{E}+03$ & $7.31 E+03$ & $3.77 \mathrm{E}+01$ & $9.79 \mathrm{E}+01$ & $3.44 \mathrm{E}-02$ & $1.31 \mathrm{E}-02$ & $1.16 \mathrm{E}-01$ & $8.62 \mathrm{E}-04$ & $4.12 \mathrm{E}+02$ \\
\hline $241-\mathrm{B}-109$ & Total & & & $1.09 \mathrm{E}+03$ & $9.38 \mathrm{E}+04$ & $5.04 \mathrm{E}+03$ & $1.68 \mathrm{E}+05$ & $4.29 \mathrm{E}+04$ & $3.75 E+02$ & $6.27 \mathrm{E}-01$ & $2.89 E+00$ & $2.14 \mathrm{E}+01$ & $2.20 \mathrm{E}-01$ & $1.29 \mathrm{E}+03$ \\
\hline
\end{tabular}


Table A-2. Tank Waste Inventory from Best Basis Inventory Detail Report (Queried on September 27, 2006) for Hydrogen Generation Rate Calculations. (27 sheets)

\begin{tabular}{|c|c|c|c|c|c|c|c|c|c|c|c|c|c|c|}
\hline $\begin{array}{c}\text { Tank } \\
\text { Name }\end{array}$ & Waste Phase & Was & $\begin{array}{r}\text { Vol } \\
(k \mathbf{L})\end{array}$ & $\begin{array}{l}\text { TOC } \\
(\mathrm{Kg})\end{array}$ & $\begin{array}{l}\mathrm{NO}_{3} \\
(\mathrm{Kg})\end{array}$ & $\begin{array}{l}\mathrm{NO}_{2} \\
(\mathrm{Kg})\end{array}$ & $\begin{array}{c}\mathrm{Na} \\
(\mathrm{Kg})\end{array}$ & $\begin{array}{c}\mathrm{Al} \\
(\mathbf{K g})\end{array}$ & $\begin{array}{l}{ }^{90} \mathrm{Sr} \\
(\mathrm{Ci})\end{array}$ & $\begin{array}{l}{ }_{(\mathrm{Ci})}^{241} \mathrm{Am} \\
\text { (1) }\end{array}$ & $\begin{array}{l}{ }_{(\mathrm{Ci})} \mathrm{Pu} \\
{ }^{240}\end{array}$ & $\begin{array}{l}{ }^{239} \mathbf{P u} \\
\text { (Ci) }\end{array}$ & $\begin{array}{l}{ }^{238} \mathbf{P u} \\
(\mathrm{Ci})\end{array}$ & $\begin{array}{l}{ }^{137} \mathrm{Cs} \\
(\mathrm{Ci})\end{array}$ \\
\hline 241-B-109 & Sludge & olid) & 189 & $8 \mathrm{E}+02$ & +04 & $3 \mathrm{E}+03$ & $5.50 \mathrm{E}+04$ & $3.63 \mathrm{E}+04$ & $4 \mathrm{E}+02$ & 1 & 1 & 0 & 2 & $6 \mathrm{E}+02$ \\
\hline $241-B-109$ & Saltcake Solid & B-SltCk (Solid) & 245 & $1.74 \mathrm{E}+02$ & $7.28 \mathrm{E}+04$ & $2.56 \mathrm{E}+03$ & $1.09 \mathrm{E}+05$ & $6.59 \mathrm{E}+03$ & $1.64 \mathrm{E}+02$ & $1.16 \mathrm{E}-01$ & $1.97 \mathrm{E}+00$ & $1.74 \mathrm{E}+01$ & $1.29 \mathrm{E}-01$ & $7.79 \mathrm{E}+02$ \\
\hline $241-B-109$ & Saltcake Interstitial Liquid & B-SItCk (Liquid) & 41 & $1.39 \mathrm{E}-01$ & $8.90 \mathrm{E}+03$ & $3.49 \mathrm{E}+02$ & $4.63 \mathrm{E}+03$ & $1.48 \mathrm{E}+01$ & $8.70 E+01$ & $6.35 \mathrm{E}-02$ & $2.42 \mathrm{E}-02$ & $2.14 \mathrm{E}-01$ & $1.59 \mathrm{E}-03$ & $3.66 \mathrm{E}+02$ \\
\hline $241-B-110$ & Total & & & $5.01 \mathrm{E}+02$ & $2.36 \mathrm{E}+05$ & $1.31 E+04$ & $1.23 E+05$ & $1.46 \mathrm{E}+03$ & $9.77 \mathrm{E}+04$ & $9.11 E+01$ & $1.35 \mathrm{E}+01$ & $1.27 \mathrm{E}+02$ & $3.94 \mathrm{E}+00$ & $1.37 \mathrm{E}+04$ \\
\hline 241 & t & ) & 4 & $2 E+01$ & $\mathrm{E}+02$ & $8 \mathrm{E}+02$ & $E+02$ & 11 & 00 & $\mathrm{E}-02$ & $E-03$ & $\mathrm{E}-02$ & $8.44 \mathrm{E}-04$ & $5.60 \mathrm{E}+01$ \\
\hline $241-\mathrm{B}-110$ & Sludge (Liquid \& Solid) & B (Solid) & 11 & $5.70 \mathrm{E}+00$ & $2.80 \mathrm{E}+03$ & $1.54 \mathrm{E}+02$ & $1.46 \mathrm{E}+03$ & $1.69 \mathrm{E}+01$ & $1.16 \mathrm{E}+03$ & $1.28 \mathrm{E}+00$ & $4.35 \mathrm{E}-01$ & $1.24 \mathrm{E}+00$ & $4.31 \mathrm{E}-02$ & $1.63 \mathrm{E}+02$ \\
\hline $241-\mathrm{B}-110$ & Sludge (Liquid \& Solid) & $2 \mathrm{C}$ (Solid) & 914 & $4.74 \mathrm{E}+02$ & $2.32 \mathrm{E}+05$ & $1.28 \mathrm{E}+04$ & $1.21 E+05$ & $1.40 \mathrm{E}+03$ & $9.66 \mathrm{E}+04$ & $8.98 \mathrm{E}+01$ & $1.31 E+01$ & $1.26 \mathrm{E}+02$ & $3.90 \mathrm{E}+00$ & $1.35 \mathrm{E}+04$ \\
\hline $241-B-111$ & Total & & & $1.26 \mathrm{E}+03$ & $9.51 \mathrm{E}+04$ & $5.22 \mathrm{E}+04$ & $1.02 \mathrm{E}+05$ & $1.08 \mathrm{E}+03$ & $2.22 \mathrm{E}+05$ & $9.85 \mathrm{E}+01$ & $1.26 \mathrm{E}+01$ & $9.98 \mathrm{E}+01$ & $3.42 \mathrm{E}+00$ & $1.43 \mathrm{E}+05$ \\
\hline 241-B-111 & Supernatant & d) & 4 & $2.22 \mathrm{E}+01$ & $3.11 E+02$ & $1.58 \mathrm{E}+02$ & $3.82 \mathrm{E}+02$ & $4.16 \mathrm{E}+01$ & $9.23 \mathrm{E}+00$ & $2.70 \mathrm{E}-02$ & $4.68 \mathrm{E}-03$ & $2.02 \mathrm{E}-02$ & $8.44 \mathrm{E}-04$ & $5.60 \mathrm{E}+01$ \\
\hline $241-B-111$ & Sludge (Liquid \& Solid) & B (Solid) & 101 & $1.38 \mathrm{E}+02$ & $1.05 E+04$ & $5.77 \mathrm{E}+03$ & $1.13 E+04$ & $1.15 \mathrm{E}+02$ & $2.46 E+04$ & $1.18 \mathrm{E}+01$ & $3.24 \mathrm{E}+00$ & $9.23 E+00$ & $3.60 \mathrm{E}-01$ & $1.59 \mathrm{E}+04$ \\
\hline $241-\mathrm{B}-111$ & Sludge (Liquid \& & id) & 809 & $1.10 \mathrm{E}+03$ & $8.42 \mathrm{E}+04$ & $4.62 \mathrm{E}+04$ & $9.03 E+04$ & $9.23 \mathrm{E}+02$ & $1.97 \mathrm{E}+05$ & $8.66 \mathrm{E}+01$ & $9.40 \mathrm{E}+00$ & $9.06 \mathrm{E}+01$ & $3.06 \mathrm{E}+00$ & $1.27 \mathrm{E}+05$ \\
\hline $241-\mathrm{B}-112$ & Total & & & $9.30 \mathrm{E}+02$ & $3.34 E+04$ & $7.90 \mathrm{E}+03$ & $3.17 E+04$ & $3.10 \mathrm{E}+03$ & $7.88 \mathrm{E}+02$ & $2.84 \mathrm{E}-01$ & $7.76 \mathrm{E}-02$ & $5.11 \mathrm{E}-01$ & $9.11 \mathrm{E}-03$ & $7.90 \mathrm{E}+03$ \\
\hline $241-B-112$ & Supernatant & BY-SICCK $(1$ & 11 & $2.00 \mathrm{E}+02$ & $\mathrm{E}+03$ & $1.29 \mathrm{E}+03$ & $2.80 \mathrm{E}+03$ & $\mathrm{E}+02$ & $2.54 \mathrm{E}+01$ & $7.42 \mathrm{E}-02$ & $1.33 \mathrm{E}-02$ & $5.54 \mathrm{E}-02$ & $2.14 \mathrm{E}-03$ & $1.68 \mathrm{E}+03$ \\
\hline $241-\mathrm{B}-112$ & Sludge (Liquid \& Solid) & 2C (Solid) & 56 & $1.85 \mathrm{E}+02$ & $1.22 \mathrm{E}+04$ & $3.13 E+03$ & $1.09 \mathrm{E}+04$ & $2.96 \mathrm{E}+02$ & $2.52 \mathrm{E}-01$ & $6.78 \mathrm{E}-04$ & $3.46 \mathrm{E}-02$ & $3.32 \mathrm{E}-01$ & $2.20 \mathrm{E}-03$ & $2.93 \mathrm{E}-01$ \\
\hline $241-B-112$ & Itcake Solid & BY-SILK (SOIIO) & 49 & $2.54 \mathrm{E}+02$ & $1.54 \mathrm{E}+04$ & $61 \mathrm{E}+03$ & $1.40 \mathrm{E}+04$ & $1.53 \mathrm{E}+03$ & $26 \mathrm{E}+02$ & $1.01 \mathrm{E}-01$ & 02 & $E-02$ & $E-03$ & $3.77 \mathrm{E}+03$ \\
\hline $241-\mathrm{B}-112$ & altcake Interstitial Liquid & Y-SltCk (Liquid) & 16 & $2.91 E+02$ & $3.47 \mathrm{E}+03$ & $1.87 \mathrm{E}+03$ & $4.08 \mathrm{E}+03$ & $7.57 \mathrm{E}+02$ & $3.69 \mathrm{E}+01$ & $1.08 \mathrm{E}-01$ & $1.94 \mathrm{E}-02$ & $8.06 \mathrm{E}-02$ & $3.11 \mathrm{E}-03$ & $2.45 \mathrm{E}+03$ \\
\hline $241-B-201$ & Total & & & $3.31 \mathrm{E}+02$ & $7.06 \mathrm{E}+03$ & $1.23 \mathrm{E}+02$ & $4.79 \mathrm{E}+03$ & $1.52 \mathrm{E}+02$ & $2.39 \mathrm{E}+02$ & $3.97 \mathrm{E}+00$ & $6.05 E+00$ & $1.05 \mathrm{E}+02$ & $8.79 \mathrm{E}-01$ & $1.90 \mathrm{E}+01$ \\
\hline $241-B$ & Studge (L & 224-1 (Solla) & 111 & 02 & $06 \mathrm{E}+03$ & +02 & 03 & -02 & 02 & +00 & 100 & +02 & E-01 & $1.90 \mathrm{E}+01$ \\
\hline $241-\mathrm{B}-202$ & Total & & & $3.89 E+02$ & $8.10 \mathrm{E}+03$ & $1.44 \mathrm{E}+02$ & $5.17 E+03$ & $2.25 E+02$ & $3.95 \mathrm{E}+02$ & $8.79 \mathrm{E}+00$ & $2.86 \mathrm{E}+00$ & $1.69 \mathrm{E}+01$ & $2.67 \mathrm{E}-01$ & $1.01 \mathrm{E}+01$ \\
\hline -B-202 & ludge (Liquid & 4-2 (Solid) & 108 & $3.89 \mathrm{E}+02$ & $8.10 \mathrm{E}+03$ & $1.44 E+02$ & $5.17 \mathrm{E}+03$ & $2.25 E+02$ & $3.95 \mathrm{E}+02$ & $8.79 \mathrm{E}+00$ & $2.86 \mathrm{E}+00$ & $1.69 \mathrm{E}+01$ & $2.67 \mathrm{E}-01$ & $1.01 E+01$ \\
\hline 241-B-203 & Total & & & $1.21 \mathrm{E}+02$ & $1.40 \mathrm{E}+04$ & $1.61 \mathrm{E}+02$ & $6.53 E+03$ & $1.16 \mathrm{E}+01$ & $1.55 \mathrm{E}+01$ & $7.73 \mathrm{E}+00$ & $6.59 \mathrm{E}+00$ & $5.23 \mathrm{E}+01$ & $4.52 \mathrm{E}-01$ & $1.48 \mathrm{E}+00$ \\
\hline $241-B-203$ & Supernatant & NA & 2 & $1.92 \mathrm{E}-01$ & $1.13 \mathrm{E}+02$ & $3.14 \mathrm{E}+00$ & $6.23 \mathrm{E}+01$ & $0.00 \mathrm{E}+00$ & $1.63 \mathrm{E}-04$ & $8.54 \mathrm{E}-05$ & $1.02 \mathrm{E}-03$ & $8.04 \mathrm{E}-03$ & $6.96 \mathrm{E}-05$ & $1.89 \mathrm{E}-04$ \\
\hline 241-B-203 & ludge (Liquid \& Solid) & 24-2 (Solid) & 188 & $1.21 E+02$ & $1.39 \mathrm{E}+04$ & $1.58 \mathrm{E}+02$ & $6.47 \mathrm{E}+03$ & $1.16 E+01$ & $1.55 \mathrm{E}+01$ & $7.73 E+00$ & $6.59 \mathrm{E}+00$ & $5.22 \mathrm{E}+01$ & $4.52 \mathrm{E}-01$ & $1.48 \mathrm{E}+00$ \\
\hline $241-B-204$ & Total & & & $1.01 \mathrm{E}+02$ & $1.09 E+04$ & $1.30 \mathrm{E}+02$ & $5.84 \mathrm{E}+03$ & $1.28 \mathrm{E}+01$ & $5.73 \mathrm{E}-01$ & $8.41 \mathrm{E}+00$ & $5.50 \mathrm{E}+00$ & $4.36 \mathrm{E}+01$ & $3.77 \mathrm{E}-01$ & $5.73 \mathrm{E}+00$ \\
\hline 241-B-204 & Supernatant & NA & 3 & $2.26 \mathrm{E}-01$ & $1.04 \mathrm{E}+02$ & $4.09 \mathrm{E}+00$ & $8.35 \mathrm{E}+01$ & $0.00 \mathrm{E}+00$ & $1.09 \mathrm{E}-04$ & 4.92E-07 & $1.48 \mathrm{E}-05$ & $2.20 \mathrm{E}-04$ & $7.43 \mathrm{E}-07$ & $1.25 \mathrm{E}-04$ \\
\hline $241-\mathrm{B}-204$ & ludge (Liquid \& Solid) & $224-2$ (Solid) & 184 & $1.00 \mathrm{E}+02$ & $1.08 \mathrm{E}+04$ & $1.26 \mathrm{E}+02$ & $5.75 \mathrm{E}+03$ & $1.28 \mathrm{E}+01$ & $5.73 \mathrm{E}-01$ & $8.41 \mathrm{E}+00$ & $5.50 \mathrm{E}+00$ & $4.36 \mathrm{E}+01$ & $3.77 \mathrm{E}-01$ & $5.73 \mathrm{E}+00$ \\
\hline $241-B X-101$ & Total & & & $6.55 \mathrm{E}+03$ & $1.14 \mathrm{E}+04$ & $6.97 \mathrm{E}+03$ & $2.29 \mathrm{E}+04$ & $4.13 E+04$ & $7.72 E+04$ & $4.15 \mathrm{E}+01$ & $4.01 E+01$ & $1.93 E+02$ & $6.37 E+00$ & $2.75 \mathrm{E}+04$ \\
\hline
\end{tabular}




\section{RPP-5926 REV 6}

Table A-2. Tank Waste Inventory from Best Basis Inventory Detail Report (Queried on September 27, 2006) for Hydrogen Generation Rate Calculations. (27 sheets)

\begin{tabular}{|c|c|c|c|c|c|c|c|c|c|c|c|c|c|c|}
\hline $\begin{array}{l}\text { Tank } \\
\text { Name }\end{array}$ & $\mathbf{W a}$ & e & $\begin{array}{c}\text { Vol } \\
(\mathrm{kL})\end{array}$ & $\begin{array}{l}\text { TOC } \\
\text { (Kg) }\end{array}$ & $\begin{array}{l}\mathrm{NO}_{3} \\
(\mathrm{Kg})\end{array}$ & $\begin{array}{l}\mathrm{NO}_{2} \\
(\mathrm{Kg})\end{array}$ & $\begin{array}{c}\mathrm{Na} \\
(\mathrm{Kg})\end{array}$ & $\begin{array}{c}\mathbf{A l} \\
(\mathbf{K g})\end{array}$ & $\begin{array}{l}{ }^{90} \mathrm{Sr} \\
(\mathrm{Ci})\end{array}$ & $\begin{array}{c}{ }_{(\mathrm{Ci})}^{241} \mathrm{Am} \\
{ }^{2}\end{array}$ & $\begin{array}{c}{ }^{240} \mathrm{Pu} \\
(\mathrm{Ci})\end{array}$ & $\begin{array}{c}{ }^{239} \mathbf{P u} \\
(\mathrm{Ci})\end{array}$ & $\begin{array}{c}{ }^{238} \mathbf{P u} \\
(\mathrm{Ci})\end{array}$ & $\begin{array}{c}{ }^{137} \mathrm{Cs} \\
(\mathrm{Ci})\end{array}$ \\
\hline 101 & Sludge ( & blid) & 59 & $1.15 \mathrm{E}+02$ & $3.73 E+03$ & $28 \mathrm{E}+03$ & $7.52 \mathrm{E}+03$ & $1.35 \mathrm{E}+04$ & $1.86 \mathrm{E}+04$ & $10 \mathrm{E}+0 \mathrm{I}$ & $6.03 \mathrm{E}+00$ & $63 \mathrm{E}+01$ & $3.92 \mathrm{I}$ & $9.00 \mathrm{E}+03$ \\
\hline 241-BX-101 & Sludge (Liquid \& Solid) & CWP2 (Solid) & 47 & OF+01 & $2.97 \mathrm{E}+03$ & $2 \mathrm{E}+03$ & $5.99 \mathrm{E}+03$ & $\mathrm{E}+04$ & $6 \mathrm{E}+04$ & $\mathrm{E}+00$ & $1.16 \mathrm{E}+01$ & $4.92 \mathrm{E}+01$ & $+\infty$ & 3 \\
\hline 2 & Sludge ( & ) & 74 & 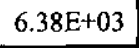 & 33 & $2+03$ & $\mathrm{E}+03$ & +04 & $7.00 \mathrm{E}+03$ & $4.62 \mathrm{E}+00$ & $4 \mathrm{E}+01$ & $8.78 \mathrm{E}+01$ & $4.82 \mathrm{E}+00$ & $.13 \mathrm{E}+04$ \\
\hline 241-BX-102 & Total & & & $1.35 \mathrm{E}+02$ & $2.23 \mathrm{E}+04$ & $31 \mathrm{E}+03$ & $1.50 \mathrm{E}+04$ & $2.33 E+04$ & $1.13 E+04$ & $1.16 \mathrm{E}+00$ & $1.17 \mathrm{E}+00$ & $5.59 \mathrm{E}+00$ & $1.13 E-01$ & $1.27 \mathrm{E}+03$ \\
\hline $241-B X-102$ & 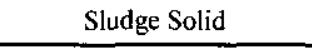 & TBP (Solid) & 70 & $7.00 \mathrm{E}+01$ & 4 & 3 & 4 & +02 & 44 & $\mathrm{E}-01$ & $\mathrm{E}-01$ & $1.15 E+00$ & $\mathrm{E}-03$ & $1.22 \mathrm{E}+03$ \\
\hline $241-\mathrm{BX}-102$ & Sludge & id) & 147 & $0.00 \mathrm{E}+00$ & $0.00 \mathrm{E}+00$ & $0.00 \mathrm{E}+00$ & $0.00 \mathrm{E}+00$ & $5.54 \mathrm{E}+02$ & $0.00 \mathrm{E}+00$ & $0.00 \mathrm{E}+00$ & $0.00 \mathrm{E}+00$ & $0.00 \mathrm{E}+00$ & $0.00 \mathrm{E}+00$ & $0.00 \mathrm{E}+00$ \\
\hline 102 & $\mathrm{~s}$ & 0 & 81 & +01 & +03 & +02 & +03 & +04 & +01 & $7-01$ & +00 & +00 & 1 & +01 \\
\hline 103 & Total & & & $3.60 \mathrm{E}+03$ & $1.51 \mathrm{E}+04$ & $3.32 \mathrm{E}+03$ & $1.40 \mathrm{E}+04$ & $5.18 \mathrm{E}+04$ & $3.58 \mathrm{E}+03$ & $1.29 \mathrm{E}+02$ & $1.93 E+02$ & $8.50 \mathrm{E}+02$ & $1.90 \mathrm{E}+01$ & $5.22 \mathrm{E}+02$ \\
\hline 241-BX-103 & inernat & CWP2 (Liquid) & 48 & $1.82 \mathrm{E}+02$ & $2.22 \mathrm{E}+03$ & $\mathrm{E}+02$ & $1.94 \mathrm{E}+03$ & $8.85 \mathrm{E}+02$ & $2.61 \mathrm{E}+01$ & $2.90 \mathrm{E}-01$ & $6.20 \mathrm{E}-02$ & 2.63E-01 & $\mathrm{E}-03$ & $3.07 \mathrm{E}+01$ \\
\hline 241-BX-103 & Sludge (Liquid \& Solid) & ) & 21 & 02 & +03 & +02 & +03 & +01 & +03 & $:+01$ & +00 & +01 & $\mathrm{E}-01$ & $3.72 E+02$ \\
\hline $241-\mathrm{BX}-103$ & ETquide & id) & 214 & $3.11 E+03$ & $7.05 \mathrm{E}+03$ & $2.24 \mathrm{E}+03$ & $8.62 E+03$ & $5.09 \mathrm{E}+04$ & $1.01 \mathrm{E}+02$ & $9.37 \mathrm{E}+01$ & $1.86 \mathrm{E}+02$ & $7.87 \mathrm{E}+02$ & $1.86 \mathrm{E}+01$ & $1.18 \mathrm{E}+02$ \\
\hline 2 & otal & & & $2.72 \mathrm{E}+03$ & +04 & +04 & +04 & 04 & 5 & $E+02$ & $E+01$ & $\mathrm{E}+02$ & $2+\infty$ & +04 \\
\hline -DA-104 & periatatil & (L) (2) & 11 & $51 E+01$ & $1.47 \mathrm{E}+03$ & $7.01 \mathrm{E}+02$ & $1.54 \mathrm{E}+03$ & $3.12 \mathrm{E}+01$ & $1.86 \mathrm{E}+01$ & $2.96 \mathrm{E}-03$ & $4.49 \mathrm{E}-04$ & $2.36 \mathrm{E}-03$ & $3.67 \mathrm{E}-05$ & $1.36 \mathrm{E}+03$ \\
\hline $241-B X-104$ & Sludge (Liquid \& Solid) & TBP (Solid) & 53 & $3.86 \mathrm{E}+02$ & $4.09 \mathrm{E}+03$ & $2.02 \mathrm{E}+03$ & $5.91 \mathrm{E}+03$ & $1.20 \mathrm{E}+04$ & $1.73 \mathrm{E}+04$ & $4.97 \mathrm{E}+01$ & $3.40 \mathrm{E}+00$ & $3.17 \mathrm{E}+01$ & $2.21 \mathrm{E}-01$ & $5.41 E+03$ \\
\hline - & Sludge (Liquid \& S & MW1 (Solid) & 1 & 03 & 4 & +03 & 44 & -04 & +04 & $1.46 \mathrm{E}+02$ & $8 \mathrm{E}+00$ & $9.61 \mathrm{E}+01$ & $3.24 \mathrm{E}-01$ & $1.58 \mathrm{E}+04$ \\
\hline 241-BX-104 & Sludge (Liquid \& Solid) & CWR1 (Solid) & 110 & $8.01 E+02$ & $8.49 \mathrm{E}+03$ & $4.19 \mathrm{E}+03$ & $1.23 \mathrm{E}+04$ & $2.48 \mathrm{E}+04$ & $3.58 \mathrm{E}+04$ & $1.03 E+02$ & $1.16 \mathrm{E}+01$ & $6.12 \mathrm{E}+01$ & $9.53 \mathrm{E}-01$ & $1.12 E+04$ \\
\hline 241-BX-104 & udge (Liquid $\delta$ & lid) & 51 & $3.72 \mathrm{E}+02$ & $3.94 \mathrm{E}+03$ & $1.94 \mathrm{E}+03$ & $5.68 \mathrm{E}+03$ & $1.15 \mathrm{E}+04$ & $1.66 \mathrm{E}+04$ & $3.39 \mathrm{E}+01$ & $6.45 \mathrm{E}+00$ & $2.73 \mathrm{E}+01$ & $6.45 \mathrm{E}-01$ & $5.21 E+03$ \\
\hline 241-BX-105 & Totol & & & $58 \mathrm{E}+02$ & $3.01 \mathrm{E}+04$ & $9.71 \mathrm{E}+03$ & $9.58 \mathrm{E}+04$ & $2.86 \mathrm{E}+04$ & $7.89 \mathrm{E}+04$ & $2.58 \mathrm{E}+01$ & $8.34 \mathrm{E}+00$ & $4.18 \mathrm{E}+01$ & $8.89 \mathrm{E}-01$ & $3.37 E+04$ \\
\hline $241-\mathrm{BX}-105$ & Supematant & CWP2 (Liquid) & 18 & $1.70 \mathrm{E}+02$ & $2.11 \mathrm{E}+03$ & $7.20 \mathrm{E}+02$ & $2.23 \mathrm{E}+03$ & $1.07 \mathrm{E}+02$ & $1.35 \mathrm{E}+02$ & $4.44 \mathrm{E}-01$ & $5.88 \mathrm{E}-02$ & $2.50 \mathrm{E}-01$ & $5.89 \mathrm{E}-03$ & $2.14 \mathrm{E}+03$ \\
\hline 2 & Sludg & id) & 55 & $\mathrm{E}+02$ & $6.28 \mathrm{E}+03$ & $E+03$ & +04 & 6.40 & $1.75 \mathrm{E}+04$ & $6.57 \mathrm{E}+00$ & $8 \mathrm{E}+00$ & +01 & E-02 & $7.07 \mathrm{E}+03$ \\
\hline 241-BX-105 & Sludge (Liquid \& Solid) & MW1 (Solid) & 9 & $0.00 \mathrm{E}+00$ & $1.86 \mathrm{E}+01$ & $8.81 E+00$ & $1.09 \mathrm{E}+03$ & $0.00 \mathrm{E}+00$ & $6.24 \mathrm{E}+02$ & $2.03 \mathrm{E}-03$ & $6.61 \mathrm{E}-04$ & $9.80 \mathrm{E}-03$ & $3.31 \mathrm{E}-05$ & $3.17 \mathrm{E}+01$ \\
\hline 241-BX-105 & Sludge (Liquid \& Solid) & CWP2 (Solid) & 96 & $2.70 \mathrm{E}+02$ & $1.10 \mathrm{E}+04$ & $1.75 \mathrm{E}+03$ & $3.62 \mathrm{E}+04$ & $1.12 \mathrm{E}+04$ & $3.06 \mathrm{E}+04$ & $2.70 \mathrm{E}+00$ & $5.36 \mathrm{E}+00$ & $2.27 \mathrm{E}+01$ & $5.36 \mathrm{E}-01$ & $1.23 \mathrm{E}+04$ \\
\hline-105 & Saltcake (Liquid \& Solid) & Solid) & 94 & $2.64 \mathrm{E}+02$ & $1.07 \mathrm{E}+04$ & $5.13 \mathrm{E}+03$ & $3.55 \mathrm{E}+04$ & $1.09 \mathrm{E}+04$ & $3.00 \mathrm{E}+04$ & $1.61 \mathrm{E}+01$ & $1.64 \mathrm{E}+00$ & $6.87 \mathrm{E}+00$ & $2.64 \mathrm{E}-01$ & $1.21 \mathrm{E}+04$ \\
\hline 241-BX-106 & Total & & & $9.65 \mathrm{E}+02$ & $4.19 \mathrm{E}+04$ & $6.64 \mathrm{E}+03$ & $3.73 E+04$ & $9.16 \mathrm{E}+03$ & $5.86 \mathrm{E}+03$ & $5.77 \mathrm{E}+01$ & $9.27 \mathrm{E}+00$ & $4.52 \mathrm{E}+01$ & $1.20 \mathrm{E}+00$ & $1.13 E+04$ \\
\hline 241-BX-106 & Sludge (Liquid \& Solid) & TBP (Solid) & 20 & $2.76 \mathrm{E}+01$ & $7.56 \mathrm{E}+03$ & $5.20 \mathrm{E}+02$ & $4.43 \mathrm{E}+03$ & $7.31 \mathrm{E}+01$ & $4.46 \mathrm{E}+03$ & $6.26 \mathrm{E}+00$ & $1.22 \mathrm{E}+00$ & $1.14 \mathrm{E}+01$ & 7.92E-02 & $4.82 \mathrm{E}+02$ \\
\hline 241-BX-106 & udge (Liquid \& Solid) & CWP2 (Solid) & 18 & $1.46 \mathrm{E}+01$ & $7.05 \mathrm{E}+02$ & $2.24 \mathrm{E}+02$ & $8.61 \mathrm{E}+02$ & $5.09 \mathrm{E}+03$ & $1.01 \mathrm{E}+01$ & $1.49 \mathrm{E}+00$ & $2.96 \mathrm{E}+00$ & $1.25 E+01$ & $2.96 \mathrm{E}-01$ & $1.18 \mathrm{E}+01$ \\
\hline 241-BX-106 & Saltcake Solid & BY-SltCk (Solid) & 80 & $4.68 \mathrm{E}+02$ & $2.83 \mathrm{E}+04$ & $2.97 \mathrm{E}+03$ & $2.57 \mathrm{E}+04$ & $2.81 \mathrm{E}+03$ & $1.34 \mathrm{E}+03$ & $4.98 \mathrm{E}+01$ & $5.07 \mathrm{E}+00$ & $2.12 \mathrm{E}+01$ & $8.17 \mathrm{E}-01$ & $6.94 \mathrm{E}+03$ \\
\hline
\end{tabular}




\section{RPP-5926 REV 6}

Table A-2. Tank Waste Inventory from Best Basis Inventory Detail Report (Queried on September 27, 2006) for

Hydrogen Generation Rate Calculations. (27 sheets)

\begin{tabular}{|c|c|c|c|c|c|c|c|c|c|c|c|c|c|c|}
\hline $\begin{array}{l}\text { Tank } \\
\text { Name }\end{array}$ & e & e & $\begin{array}{c}\text { Vol } \\
(\mathbf{k L})\end{array}$ & $\begin{array}{l}\text { TOC } \\
(\mathrm{Kg})\end{array}$ & $\begin{array}{l}\mathrm{NO}_{3} \\
(\mathrm{Kg})\end{array}$ & $\begin{array}{l}\mathrm{NO}_{2} \\
(\mathrm{Kg})\end{array}$ & $\begin{array}{c}\mathrm{Na} \\
(\mathrm{Kg})\end{array}$ & $\begin{array}{c}\text { Al } \\
\text { (Kg) }\end{array}$ & $\begin{array}{l}{ }^{90} \mathrm{Sr} \\
\text { (Ci) }\end{array}$ & $\begin{array}{l}{ }_{(\mathbf{C i})}^{24 \mathrm{Am}} \\
\end{array}$ & $\begin{array}{c}{ }^{240} \mathrm{Pu} \\
(\mathrm{Ci})\end{array}$ & $\begin{array}{c}{ }^{239} \mathbf{P u} \\
\text { (Ci) }\end{array}$ & $\begin{array}{l}{ }^{238} \mathrm{Pu} \\
\text { (Ci) }\end{array}$ & $\begin{array}{l}{ }^{137} \mathrm{Cs} \\
(\mathrm{Ci})\end{array}$ \\
\hline 106 & quid & uid) & 25 & $5 \mathrm{E}+02$ & $\varepsilon+03$ & $3 \mathrm{E}+03$ & $7 \mathrm{E}+03$ & $18 \mathrm{E}+03$ & $7 \mathrm{E}+01$ & $1.69 \mathrm{E}-0 !$ & $3.02 \mathrm{E}-02$ & $26 \mathrm{E}-01$ & $4.86 \mathrm{E}-03$ & $3.83 \mathrm{E}+03$ \\
\hline $241-\mathrm{BX}-107$ & Total & & & $1.13 \mathrm{E}+03$ & $2.59 \mathrm{E}+05$ & $1.54 \mathrm{E}+04$ & $1.92 \mathrm{E}+05$ & $2.70 \mathrm{E}+04$ & $1.42 \mathrm{E}+04$ & $2.45 \mathrm{E}+01$ & $1.06 \mathrm{E}+01$ & $9.75 \mathrm{E}+01$ & $6.86 \mathrm{E}-01$ & $2.54 \mathrm{E}+04$ \\
\hline 241-BX-107 & Sludge (Liquid \& & (Solid) & 1313 & $1.13 \mathrm{E}+03$ & $2.59 \mathrm{E}+0.5$ & $1.54 \mathrm{E}+04$ & $1.92 \mathrm{E}+05$ & $2.70 \mathrm{E}+04$ & $1.42 \mathrm{E}+04$ & $2.45 \mathrm{E}+01$ & $1.06 \mathrm{E}+01$ & $9.75 \mathrm{E}+01$ & $6.86 \mathrm{E}-01$ & $2.54 \mathrm{E}+04$ \\
\hline 241-BX-108 & Total & & & $1.87 E+02$ & $4.94 \mathrm{E}+04$ & $3.50 \mathrm{E}+03$ & $3.14 \mathrm{E}+04$ & $1.82 \mathrm{E}+03$ & $2.23 \mathrm{E}+04$ & $3.25 \mathrm{E}+00$ & $8.95 \mathrm{E}-01$ & $8.30 \mathrm{E}+00$ & $5.80 \mathrm{E}-02$ & $3.92 \mathrm{E}+03$ \\
\hline 241-BX-108 & Sludge (Liquid \& Solid) & TBP (Solid) & 81 & $1.35 \mathrm{E}+02$ & $3.71 \mathrm{E}+04$ & $2.55 \mathrm{E}+03$ & $2.17 E+04$ & $3.59 \mathrm{E}+02$ & $2.19 \mathrm{E}+04$ & $2.68 \mathrm{E}+00$ & $5.21 \mathrm{E}-01$ & $4.87 \mathrm{E}+00$ & $3.39 \mathrm{E}-02$ & $2.36 \mathrm{E}+03$ \\
\hline 241-BX-108 & Sludge (Liquid \& Solid) & (Solid) & 38 & $5.16 \mathrm{E}+01$ & $1.24 \mathrm{E}+04$ & $9.52 \mathrm{E}+02$ & $9.73 \mathrm{E}+03$ & $1.46 \mathrm{E}+03$ & $4.72 \mathrm{E}+02$ & $5.69 \mathrm{E}-01$ & $3.74 \mathrm{E}-01$ & $3.43 \mathrm{E}+00$ & $2.41 \mathrm{E}-02$ & $1.56 \mathrm{E}+03$ \\
\hline 241-BX-109 & Total & & & $7.83 \mathrm{E}+02$ & $2.17 \mathrm{E}+05$ & $2.00 \mathrm{E}+04$ & $1.17 \mathrm{E}+05$ & $2.21 \mathrm{E}+03$ & $1.19 \mathrm{E}+05$ & $5.95 \mathrm{E}+00$ & $1.16 \mathrm{E}+00$ & $1.08 \mathrm{E}+01$ & $7.53 \mathrm{E}-02$ & $1.20 \mathrm{E}+04$ \\
\hline 241-BX-109 & Sludge (Liquid \& Solid) & lid) & 730 & $7.83 \mathrm{E}+02$ & $2.17 \mathrm{E}+05$ & $2.00 \mathrm{E}+04$ & $1.17 \mathrm{E}+05$ & $2.21 E+03$ & $1.19 \mathrm{E}+05$ & $5.95 \mathrm{E}+00$ & $1.16 \mathrm{E}+00$ & $1.08 \mathrm{E}+01$ & $7.53 \mathrm{E}-02$ & $1.20 \mathrm{E}+04$ \\
\hline $241-\mathrm{BX}-110$ & Total & & & $3.87 E+03$ & $4.44 \mathrm{E}+05$ & $1.88 \mathrm{E}+04$ & $2.71 \mathrm{E}+05$ & $2.60 \mathrm{E}+04$ & $1.06 \mathrm{E}+04$ & $6.40 \mathrm{E}+00$ & $2.44 E+00$ & $2.04 \mathrm{E}+01$ & $1.95 \mathrm{E}-01$ & $6.66 \mathrm{E}+04$ \\
\hline 241-BX-110 & Supernatant & BY-SltCk (Liquid) & 5 & $7.58 \mathrm{E}+01$ & $2.06 \mathrm{E}+03$ & $2.61 \mathrm{E}+02$ & $1.12 \mathrm{E}+03$ & $2.20 \mathrm{E}+01$ & $9.61 E+00$ & $2.81 \mathrm{E}-02$ & $5.04 \mathrm{E}-03$ & $2.10 \mathrm{E}-02$ & $8.09 \mathrm{E}-04$ & $6.37 \mathrm{E}+02$ \\
\hline $241-\mathrm{BX}-110$ & Sludge (Liquid \& Solid) & NA & 94 & $3.08 \mathrm{E}+02$ & $6.45 \mathrm{E}+04$ & $1.74 \mathrm{E}+03$ & $3.93 \mathrm{E}+04$ & $3.96 \mathrm{E}+03$ & $1.12 \mathrm{E}+03$ & 1.31E-01 & $8.63 \mathrm{E}-02$ & $7.93 \mathrm{E}-01$ & $5.58 \mathrm{E}-03$ & $3.69 \mathrm{E}+03$ \\
\hline 241-BX-110 & Sludge (Liquid \& Solid) & IC (Solid) & 151 & $1.14 \mathrm{E}+02$ & $2.72 \mathrm{E}+04$ & $2.10 \mathrm{E}+03$ & $2.14 \mathrm{E}+04$ & $3.22 \mathrm{E}+03$ & $1.04 \mathrm{E}+03$ & $2.98 \mathrm{E}+00$ & $1.96 \mathrm{E}+00$ & $1.80 \mathrm{E}+01$ & $1.27 \mathrm{E}-01$ & $3.43 \mathrm{E}+03$ \\
\hline $241-B X-110$ & Saltcake Solid & Y-SltCk (Solid) & 433 & $1.42 \mathrm{E}+03$ & $2.97 \mathrm{E}+05$ & $8.00 \mathrm{E}+03$ & $1.81 \mathrm{E}+05$ & $1.82 \mathrm{E}+04$ & $8.16 \mathrm{E}+03$ & $2.53 \mathrm{E}+00$ & $2.57 \mathrm{E}-01$ & $1.08 \mathrm{E}+00$ & $4.15 \mathrm{E}-02$ & $4.24 \mathrm{E}+04$ \\
\hline $241-\mathrm{BX}-110$ & Saltcake Interstitial Liquid & BY-SItCk (Liquid) & 129 & $1.96 \mathrm{E}+03$ & $5.32 \mathrm{E}+04$ & $6.72 \mathrm{E}+03$ & $2.88 \mathrm{E}+04$ & $5.67 \mathrm{E}+02$ & $2.48 \mathrm{E}+02$ & 7.25E-01 & $1.30 \mathrm{E}-01$ & $5.42 \mathrm{E}-01$ & $2.09 \mathrm{E}-02$ & $1.64 E+04$ \\
\hline $241-\mathrm{BX}-111$ & Total & & & $1.13 \mathrm{E}+03$ & $4.06 \mathrm{E}+05$ & $1.75 \mathrm{E}+04$ & $2.49 \mathrm{E}+05$ & $1.62 \mathrm{E}+04$ & $1.19 \mathrm{E}+04$ & $3.54 \mathrm{E}+00$ & $1.69 \mathrm{E}+00$ & $1.49 \mathrm{E}+01$ & $1.22 \mathrm{E}-01$ & $6.67 \mathrm{E}+04$ \\
\hline 24I-BX-111 & Sludge (Liquid \& Solid) & $1 \mathrm{C}$ (Solid) & 121 & $9.10 \mathrm{E}+01$ & $2.18 \mathrm{E}+04$ & $1.68 \mathrm{E}+03$ & $1.72 \mathrm{E}+04$ & $2.58 \mathrm{E}+03$ & $8.34 E+02$ & $2.39 \mathrm{E}+00$ & $1.57 \mathrm{E}+00$ & $1.44 \mathrm{E}+01$ & $1.01 \mathrm{E}-01$ & $2.74 \mathrm{E}+03$ \\
\hline 241-BX-111 & Saltcake Solid & Y-SltCk (Solid) & 538 & $9.52 \mathrm{E}+02$ & $3.75 \mathrm{E}+05$ & $1.01 \mathrm{E}+04$ & $2.20 \mathrm{E}+05$ & $1.10 \mathrm{E}+04$ & $1.09 \mathrm{E}+04$ & $1.05 E+00$ & $1.07 \mathrm{E}-01$ & $4.49 \mathrm{E}-01$ & $1.73 \mathrm{E}-02$ & $5.68 \mathrm{E}+04$ \\
\hline 241-BX-111 & Saltcake Interstitial Liquid & BY-SltCk (Liquid) & 54 & $9.18 \mathrm{E}+01$ & $1.00 \mathrm{E}+04$ & $5.73 \mathrm{E}+03$ & $1.22 \mathrm{E}+04$ & $2.63 \mathrm{E}+03$ & $1.09 \mathrm{E}+02$ & $9.94 \mathrm{E}-02$ & $1.78 \mathrm{E}-02$ & $7.42 \mathrm{E}-02$ & $2.86 \mathrm{E}-03$ & $7.21 \mathrm{E}+03$ \\
\hline 241-BX-112 & Total & & & $4.70 \mathrm{E}+02$ & $6.19 \mathrm{E}+04$ & $2.24 \mathrm{E}+04$ & $6.77 \mathrm{E}+04$ & $1.00 \mathrm{E}+04$ & $4.70 \mathrm{E}+03$ & $1.95 \mathrm{E}+01$ & $1.28 \mathrm{E}+01$ & $1.17 \mathrm{E}+02$ & $8.26 \mathrm{E}-01$ & $3.28 \mathrm{E}+04$ \\
\hline $241-\mathrm{BX}-112$ & Supernatant & 1C2 (Liquid) & 5 & $0.00 \mathrm{E}+00$ & $4.93 \mathrm{E}+02$ & $1.76 \mathrm{E}+02$ & $3.89 \mathrm{E}+02$ & $2.39 \mathrm{E}-01$ & $3.67 \mathrm{E}+00$ & $8.06 \mathrm{E}-04$ & $1.13 \mathrm{E}-03$ & $7.96 \mathrm{E}-03$ & $7.90 \mathrm{E}-05$ & $4.31 \mathrm{E}+00$ \\
\hline 241-BX-112 & Sludge (Liquid \& Solid) & $1 C$ (Solid) & 617 & $4.70 \mathrm{E}+02$ & $6.14 \mathrm{E}+04$ & $2.22 \mathrm{E}+04$ & $6.73 \mathrm{E}+04$ & $1.00 \mathrm{E}+04$ & $4.70 \mathrm{E}+03$ & $1.95 \mathrm{E}+01$ & $1.28 \mathrm{E}+01$ & $1.17 \mathrm{E}+02$ & $8.26 \mathrm{E}-01$ & $3.28 \mathrm{E}+04$ \\
\hline 241-BY-101 & Total & & & $7.00 \mathrm{E}+03$ & $8.77 \mathrm{E}+05$ & $1.90 \mathrm{E}+04$ & $6.09 \mathrm{E}+05$ & $5.47 \mathrm{E}+04$ & $1.89 \mathrm{E}+05$ & $1.24 \mathrm{E}+02$ & $1.83 \mathrm{E}+00$ & $1.46 \mathrm{E}+01$ & $1.56 \mathrm{E}-01$ & $3.05 E+05$ \\
\hline 241-BY-101 & Sludge (Liquid \& Solid) & $\mathrm{TFeCN}$ (Solid) & 140 & $7.86 \mathrm{E}+02$ & $1.22 \mathrm{E}+04$ & $1.05 \mathrm{E}+04$ & $2.06 \mathrm{E}+04$ & $6.34 \mathrm{E}+03$ & $1.59 \mathrm{E}+05$ & $1.20 \mathrm{E}+02$ & $1.45 E+00$ & $1.30 \mathrm{E}+01$ & \begin{tabular}{|l|l|}
$9.49 \mathrm{E}-02$ \\
\end{tabular} & $1.42 \mathrm{E}+05$ \\
\hline 241-BY-101 & Saltcake Solid & BY-SttCk (Solid) & 1208 & $5.27 \mathrm{E}+03$ & $8.54 \mathrm{E}+05$ & $2.48 \mathrm{E}+03$ & $5.75 \mathrm{E}+05$ & $4.59 \mathrm{E}+04$ & $2.99 \mathrm{E}+04$ & $3.10 \mathrm{E}+00$ & $3.15 \mathrm{E}-01$ & $1.32 \mathrm{E}+00$ & \begin{tabular}{|l|l|}
$5.08 \mathrm{E}-02$ \\
\end{tabular} & $1.55 \mathrm{E}+05$ \\
\hline 241-BY-101 & Saltcake Interstitial Liquid & BY-SItCk (Liquid) & 52 & $9.46 \mathrm{E}+02$ & $1.13 \mathrm{E}+04$ & $6.08 \mathrm{E}+03$ & $1.33 \mathrm{E}+04$ & $2.46 \mathrm{E}+03$ & $1.20 \mathrm{E}+02$ & $3.51 \mathrm{E}-01$ & $6.29 \mathrm{E}-02$ & $2.62 \mathrm{E}-01$ & $1.01 \mathrm{E}-02$ & $7.96 \mathrm{E}+03$ \\
\hline 241-BY-102 & Total & & & $9.23 \mathrm{E}+03$ & $1.82 \mathrm{E}+05$ & $3.62 \mathrm{E}+04$ & $4.30 \mathrm{E}+05$ & $5.03 \mathrm{E}+04$ & $1.74 \mathrm{E}+04$ & $8.36 \mathrm{E}+01$ & $8.53 E+00$ & $3.57 \mathrm{E}+01$ & $1.37 \mathrm{E}+00$ & $1.10 \mathrm{E}+05$ \\
\hline 241-BY-102 & Saltcake Solid & BY-SltCk (Solid) & 897 & $8.99 \mathrm{E}+03$ & $1.59 \mathrm{E}+05$ & $2.31 E+04$ & $3.94 \mathrm{E}+05$ & $4.14 \mathrm{E}+04$ & $1.71 \mathrm{E}+04$ & $8.34 \mathrm{E}+01$ & $8.48 E+00$ & $3.55 \mathrm{E}+01$ & $1.37 \mathrm{E}+00$ & $8.88 \mathrm{E}+04$ \\
\hline
\end{tabular}




\section{RPP-5926 REV 6}

Table A-2. Tank Waste Inventory from Best Basis Inventory Detail Report (Queried on September 27, 2006) for

Hydrogen Generation Rate Calculations. (27 sheets)

\begin{tabular}{|c|c|c|c|c|c|c|c|c|c|c|c|c|c|c|}
\hline $\begin{array}{c}\text { Tank } \\
\text { Name }\end{array}$ & Waste & ype & $\begin{array}{c}\text { Vol } \\
(\mathrm{kL})\end{array}$ & $\begin{array}{l}\text { TOC } \\
\text { (Kg) }\end{array}$ & $\begin{array}{l}\mathrm{NO}_{3} \\
(\mathrm{Kg})\end{array}$ & $\begin{array}{l}\mathrm{NO}_{2} \\
(\mathrm{Kg})\end{array}$ & $\begin{array}{c}\mathrm{Na} \\
(\mathrm{Kg})\end{array}$ & $\begin{array}{c}\text { Al } \\
(\mathbf{K g})\end{array}$ & $\begin{array}{l}{ }^{90} \mathrm{Sr} \\
(\mathrm{Ci})\end{array}$ & $\begin{array}{l}{ }_{(\mathrm{Ci})}^{241} \mathrm{Am} \\
\end{array}$ & $\begin{array}{l}{ }^{240} \mathrm{Pu} \\
(\mathrm{Ci})\end{array}$ & $\begin{array}{c}{ }^{239} \mathrm{Pu} \\
\text { (Ci) }\end{array}$ & $\begin{array}{c}{ }^{238} \mathbf{P u} \\
(\mathrm{Ci})\end{array}$ & $\begin{array}{c}{ }^{137} \mathrm{Cs} \\
\text { (Ci) }\end{array}$ \\
\hline-102 & Itcake Intersti & id) & 157 & $2.43 \mathrm{E}+02$ & $32 \mathrm{E}+04$ & $32 \mathrm{E}+04$ & $68 \mathrm{E}+04$ & $8.88 \mathrm{E}+03$ & $3.21 \mathrm{E}+02$ & $2.74 \mathrm{E}-01$ & $4.92 \mathrm{E}-02$ & $2.05 \mathrm{E}-01$ & $7.90 \mathrm{E}-03$ & $2.13 \mathrm{E}+04$ \\
\hline $241-\mathrm{BY}-103$ & Total & & & $3.32 \mathrm{E}+04$ & $2 \mathrm{E}+05$ & $2.62 \mathrm{E}+04$ & $06 \mathrm{E}+05$ & $E+04$ & $2.95 \mathrm{E}+04$ & 01 & $.47 \mathrm{E}+00$ & $E+01$ & 5.3 & 1.74 \\
\hline 241-BY-103 & Sludge (Liquid \& Solid) & (olid) & 34 & $2.72 \mathrm{E}+01$ & $31 \mathrm{E}+03$ & $4.17 E+02$ & $1.61 E+03$ & $9.48 \mathrm{E}+03$ & $1.88 \mathrm{E}+0 \mathrm{I}$ & $2.22 \mathrm{E}-01$ & $4.40 \mathrm{E}-01$ & $1.86 \mathrm{E}+00$ & $4.40 \mathrm{E}-02$ & $2.21 E+01$ \\
\hline $241-\mathrm{BY}-103$ & Saltcake Solid & BY-SltCk (Solid) & 1316 & $3.28 \mathrm{E}+04$ & $5.41 \mathrm{E}+05$ & $1.61 \mathrm{E}+04$ & $5.66 \mathrm{E}+05$ & $6.20 \mathrm{E}+04$ & $2.94 \mathrm{E}+04$ & $2.98 \mathrm{E}+01$ & $3.03 E+00$ & $1.27 \mathrm{E}+01$ & $4.89 \mathrm{E}-01$ & $1.53 \mathrm{E}+05$ \\
\hline $241-1$ & Saltca & BY & 214 & +02 & +04 & +03 & 04 & $E+03$ & $1.62 \mathrm{E}+01$ & 1 & 03 & 02 & $5.84 \mathrm{E}-04$ & $2.06 \mathrm{E}+04$ \\
\hline 24!-BY-104 & Total & & & $1.78 E+04$ & $5.85 \mathrm{E}+05$ & $1.06 \mathrm{E}+05$ & $5.38 \mathrm{E}+05$ & $7.14 \mathrm{E}+04$ & $3.63 E+05$ & $1.45 \mathrm{E}+02$ & $1.53 \mathrm{E}+01$ & $7.72 \mathrm{E}+01$ & $2.23 \mathrm{E}+00$ & $2.28 \mathrm{E}+05$ \\
\hline $241-$ & Sludge (Liquid & d) & 172 & $3.23 \mathrm{E}+03$ & $3.58 \mathrm{E}+04$ & $1.20 \mathrm{E}+04$ & $4.35 \mathrm{E}+04$ & $1.70 \mathrm{E}+04$ & $1.59 \mathrm{E}+05$ & $2.01 E+01$ & $2.55 \mathrm{E}+00$ & $2.36 \mathrm{E}+01$ & 01 & 1 \\
\hline 241-BY-104 & Saltcak & BY-SltCk (Solid) & 1208 & $1.42 \mathrm{E}+04$ & $5.18 \mathrm{E}+05$ & $7.66 \mathrm{E}+04$ & $4.56 \mathrm{E}+05$ & $4.34 \mathrm{E}+04$ & $2.04 \mathrm{E}+05$ & $1.24 \mathrm{E}+02$ & $1.26 \mathrm{E}+01$ & $5.28 \mathrm{E}+01$ & $2.03 \mathrm{E}+00$ & $1.89 \mathrm{E}+05$ \\
\hline 241-BY-104 & Saltcake Interstitial L & BY-SltCk (Liquid) & 153 & $\mathrm{E}+02$ & $E+04$ & $1.70 \mathrm{E}+04$ & $3.79 \mathrm{E}+04$ & $E+04$ & $3.36 \mathrm{E}+02$ & $9.82 \mathrm{E}-01$ & $1.76 \mathrm{E}-01$ & $7.33 \mathrm{E}-01$ & $2.83 \mathrm{E}-02$ & $2.23 \mathrm{E}+04$ \\
\hline 241-BY-105 & Total & & & $9.24 \mathrm{E}+03$ & $1.40 \mathrm{E}+06$ & $4.16 \mathrm{E}+04$ & $6.95 \mathrm{E}+05$ & $8+04$ & $1.40 \mathrm{E}+05$ & $\mathrm{E}+02$ & $E+01$ & $7.80 \mathrm{E}+01$ & $1.60 \mathrm{E}+00$ & $1.53 \mathrm{E}+05$ \\
\hline 241-BY-105 & Sludge (Liquid \& Solid) & prtland Cement (Soli & 30 & $0.00 \mathrm{E}+00$ & $0.00 \mathrm{E}+00$ & $0.00 \mathrm{E}+00$ & $0.00 \mathrm{E}+00$ & $1.57 \mathrm{E}+03$ & $0.00 \mathrm{E}+00$ & $0.00 \mathrm{E}+00$ & $0.00 \mathrm{E}+00$ & $0.00 \mathrm{E}+00$ & $0.00 \mathrm{E}+00$ & $0.00 \mathrm{E}+00$ \\
\hline $241-$ & Sludg & d) & 151 & $90 \mathrm{E}+03$ & $E+04$ & $6.75 \mathrm{E}+03$ & +04 & $E+03$ & $1.25 \mathrm{E}+05$ & $3.77 \mathrm{E}+01$ & +00 & $\mathrm{E}+01$ & $3.09 \mathrm{E}-01$ & +04 \\
\hline 241-BY-105 & Saltcak & BY-SltCk (Solid) & 1481 & $4.96 \mathrm{E}+03$ & $1.33 E+06$ & $2.39 \mathrm{E}+04$ & $6.22 \mathrm{E}+05$ & $5.80 \mathrm{E}+04$ & $1.48 \mathrm{E}+04$ & $7.89 \mathrm{E}+01$ & $8.02 \mathrm{E}+00$ & $3.36 \mathrm{E}+01$ & $1.29 \mathrm{E}+00$ & $8.21 \mathrm{E}+04$ \\
\hline $241-B Y-105$ & Saltcake Interstitial Liquid & iquid) & 159 & $3.86 \mathrm{E}+02$ & $3.17 E+04$ & $1.09 \mathrm{E}+04$ & $3.30 \mathrm{E}+04$ & $6.23 \mathrm{E}+03$ & $1.53 \mathrm{E}+01$ & $1.63 \mathrm{E}+00$ & 7.32E-03 & $3.05 \mathrm{E}-02$ & $1.18 \mathrm{E}-03$ & $2.99 \mathrm{E}+04$ \\
\hline 0 & Ioral & & & $E+04$ & $\mathrm{E}+05$ & $7.90 \mathrm{E}+04$ & $5.17 \mathrm{E}+05$ & $5.43 \mathrm{E}+04$ & $1.36 \mathrm{E}+05$ & $3.85 \mathrm{E}+01$ & $3.21 \mathrm{E}+00$ & $2.34 \mathrm{E}+01$ & $3.28 \mathrm{E}-01$ & $3.01 \mathrm{E}+05$ \\
\hline 241-BY-106 & Sludge (Liquid \& Solid) & $\mathrm{PFeCN}$ (Solid) & 120 & $4.11 \mathrm{E}+03$ & $2.54 \mathrm{E}+04$ & $6.30 \mathrm{E}+03$ & $2.61 \mathrm{E}+04$ & $6.21 \mathrm{E}+03$ & $1.28 \mathrm{E}+05$ & $2.62 \mathrm{E}+01$ & $1.96 \mathrm{E}+00$ & $1.81 \mathrm{E}+01$ & $1.26 \mathrm{E}-01$ & $8.56 \mathrm{E}+04$ \\
\hline 241-BY-106 & Saltcake & olid) & 1365 & +03 & $7.69 \mathrm{E}+05$ & +04 & +05 & +04 & E+03 & $1.23 \mathrm{E}$ & 00 & $E+00$ & $2.02 \mathrm{E}-01$ & $\varepsilon+05$ \\
\hline 241-BY-106 & Saltcake Interstitial Liquid & BY-SItCk (Liquid) & 138 & $3.69 \mathrm{E}+02$ & $1.52 \mathrm{E}+04$ & $1.08 \mathrm{E}+04$ & $2.27 \mathrm{E}+04$ & $5.49 \mathrm{E}+03$ & $4.32 \mathrm{E}+00$ & $6.40 \mathrm{E}-03$ & $2.15 \mathrm{E}-04$ & $8.95 \mathrm{E}-04$ & $3.45 \mathrm{E}-05$ & $2.40 \mathrm{E}+04$ \\
\hline 241-BY-107 & Total & & & $6.78 \mathrm{E}+03$ & $3.25 \mathrm{E}+05$ & $7.49 \mathrm{E}+04$ & 4.25E+05 & $6.08 \mathrm{E}+04$ & $7.45 \mathrm{E}+04$ & $2.06 \mathrm{E}+01$ & $2.31 E+00$ & $1.41 E+01$ & $2.89 \mathrm{E}-01$ & $1.99 \mathrm{E}+05$ \\
\hline 241-BY-107 & Sludge (Liquid & id) & 58 & +02 & $9.85 \mathrm{E}+03$ & 4.21 & +04 & +03 & +04 & $\mathrm{E}+00$ & $\mathrm{E}-01$ & $8.02 \mathrm{E}+00$ & $5.59 \mathrm{E}-02$ & $2.83 \mathrm{E}+04$ \\
\hline 241-BY-107 & Saltcake Solid & BY-SltCk (Solid) & 835 & $5.60 \mathrm{E}+03$ & $2.97 \mathrm{E}+05$ & $5.48 \mathrm{E}+04$ & $3.70 \mathrm{E}+05$ & $4.91 E+04$ & $2.67 \mathrm{E}+04$ & $1.31 \mathrm{E}+01$ & $1.33 \mathrm{E}+00$ & $5.58 \mathrm{E}+00$ & $2.15 \mathrm{E}-01$ & $1.52 \mathrm{E}+05$ \\
\hline 241-BY-107 & Saltcake Interstitial Liquid & BY-SltCk (Liquid) & 135 & $3.24 \mathrm{E}+02$ & $1.77 \mathrm{E}+04$ & $1.58 \mathrm{E}+04$ & $3.28 \mathrm{E}+04$ & $6.91 E+03$ & $2.88 \mathrm{E}+02$ & $6.16 \mathrm{E}-01$ & $1.10 \mathrm{E}-01$ & $4.60 \mathrm{E}-01$ & $1.77 \mathrm{E}-02$ & $1.91 \mathrm{E}+04$ \\
\hline 241-BY-108 & Total & & & $5.58 \mathrm{E}+03$ & $2.29 \mathrm{E}+05$ & $3.81 \mathrm{E}+04$ & $2.32 \mathrm{E}+05$ & $8.49 \mathrm{E}+04$ & $1.29 \mathrm{E}+05$ & $3.81 \mathrm{E}+01$ & $4.29 \mathrm{E}+00$ & $2.82 \mathrm{E}+01$ & $4.94 \mathrm{E}-01$ & $5.26 \mathrm{E}+04$ \\
\hline 241-BY-108 & Sludge (Liquid \& Solid) & PFeCN (Solid) & 151 & $2.32 \mathrm{E}+03$ & $1.94 \mathrm{E}+04$ & $9.87 \mathrm{E}+03$ & $3.39 \mathrm{E}+04$ & $5.41 \mathrm{E}+03$ & $1.18 \mathrm{E}+05$ & $1.60 \mathrm{E}+01$ & $2.03 E+00$ & $1.88 \mathrm{E}+01$ & $1.31 \mathrm{E}-01$ & $2.01 \mathrm{E}+04$ \\
\hline 241-BY-108 & Saltcake Solid & BY-SltCk (Solid) & 587 & $3.01 \mathrm{E}+03$ & $1.79 \mathrm{E}+05$ & $2.01 \mathrm{E}+04$ & $1.70 \mathrm{E}+05$ & $7.57 \mathrm{E}+04$ & $1.09 \mathrm{E}+04$ & $2.19 \mathrm{E}+01$ & $2.23 \mathrm{E}+00$ & $9.34 \mathrm{E}+00$ & $3.60 \mathrm{E}-01$ & $1.51 \mathrm{E}+04$ \\
\hline 241-BY-108 & Saltcake Interstitial Liquid & BY-SltCk (Liquid) & 103 & $2.44 E+02$ & $3.03 E+04$ & $8.09 \mathrm{E}+03$ & $2.90 \mathrm{E}+04$ & $3.74 \mathrm{E}+03$ & $2.62 \mathrm{E}+02$ & $1.19 \mathrm{E}-01$ & $2.14 \mathrm{E}-02$ & $8.90 \mathrm{E}-02$ & $3.43 \mathrm{E}-03$ & $1.74 \mathrm{E}+04$ \\
\hline 241-BY-109 & Total & & & $1.01 \mathrm{E}+04$ & $1.33 E+05$ & $5.20 \mathrm{E}+04$ & $3.38 \mathrm{E}+05$ & $7.37 \mathrm{E}+04$ & $1.44 \mathrm{E}+04$ & $6.84 \mathrm{E}+01$ & $8.07 \mathrm{E}+00$ & $3.38 \mathrm{E}+01$ & $1.23 E+00$ & $9.23 E+04$ \\
\hline
\end{tabular}


Table A-2. Tank Waste Inventory from Best Basis Inventory Detail Report (Queried on September 27, 2006) for Hydrogen Generation Rate Calculations. (27 sheets)

\begin{tabular}{|c|c|c|c|c|c|c|c|c|c|c|c|c|c|c|}
\hline $\begin{array}{c}\text { Tank } \\
\text { Name }\end{array}$ & Y & $\mathbf{e}$ & $\begin{array}{c}\text { Vol } \\
(k L)\end{array}$ & $\begin{array}{l}\text { TOC } \\
\text { (Kg) }\end{array}$ & $\begin{array}{l}\mathrm{NO}_{\mathbf{3}} \\
(\mathrm{Kg})\end{array}$ & $\begin{array}{l}\mathrm{NO}_{2} \\
(\mathrm{Kg})\end{array}$ & $\begin{array}{c}\mathrm{Na} \\
(\mathrm{Kg})\end{array}$ & $\begin{array}{c}\mathbf{A l} \\
(\mathbf{K g})\end{array}$ & $\begin{array}{l}{ }_{(\mathrm{S}}^{90} \mathrm{Cr} \\
\text { (C) }\end{array}$ & $\begin{array}{c}{ }_{(\mathrm{Ci})}^{241} \mathrm{Am} \\
\end{array}$ & $\begin{array}{l}{ }_{(\mathrm{Ci})}^{240} \mathrm{Pu} \\
\end{array}$ & $\begin{array}{c}{ }^{239} \mathrm{Pu} \\
(\mathrm{Ci})\end{array}$ & $\begin{array}{c}{ }^{238} \mathrm{Pu} \\
(\mathrm{Ci})\end{array}$ & $\begin{array}{c}{ }^{137} \mathrm{Cs} \\
\text { (Ci) }\end{array}$ \\
\hline & a & & 89 & & $8 \mathrm{E}+03$ & $03 \mathrm{E}+03$ & $6 \mathrm{E}+04$ & $E+04$ & $7.08 \mathrm{E}+01$ & & 0 & 0 & & C701 \\
\hline 109 & lid & solid) & 51 & $2 \mathrm{E}+03$ & $.64 \mathrm{E}+04$ & $69 \mathrm{E}+04$ & $96 \mathrm{E}+05$ & $3.29 \mathrm{E}+04$ & $1.40 \mathrm{E}+04$ & $6.70 \mathrm{E}+01$ & $6.81 \mathrm{E}+00$ & $2.85 \mathrm{E}+01$ & $1.10 \mathrm{E}+00$ & $7.28 \mathrm{E}+04$ \\
\hline & 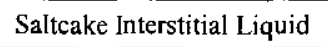 & (Liquid) & 146 & IJETVE & $2.78 \mathrm{E}+04$ & $1.21 \mathrm{E}+04$ & $3.06 \mathrm{E}+04$ & $5.66 \mathrm{E}+03$ & $2.93 E+02$ & -01 & -01 & $\mathrm{E}-01$ & -02 & $1.94 \mathrm{E}+04$ \\
\hline 241-BY-110 & Total & & & $1.41 \mathrm{E}+04$ & +05 & $7 E+04$ & -05 & +04 & $1.58 \mathrm{E}+05$ & $5.98 \mathrm{E}+0 \mathrm{I}$ & $8.27 \mathrm{E}+00$ & $4.81 \mathrm{E}+01$ & $1.08 \mathrm{E}+00$ & $1.90 \mathrm{E}+05$ \\
\hline $241-B Y \cdot 110$ & Sludge (Liquid \& Solid) & PFeCN (Solid) & 5 & 03 & 04 & 4 & $4.42 E+04$ & 3 & 5 & 1 & 0 & 01 & 01 & +04 \\
\hline 241 & & olid) & 1123 & $54 \mathrm{E}+03$ & $98 \mathrm{E}+05$ & $\varepsilon+04$ & 05 & +04 & $4.86 \mathrm{E}+04$ & $E+01$ & $E+00$ & $E+01$ & $E-01$ & $1.39 E+05$ \\
\hline 241-BY-110 & Itcake Interstitia & $\omega_{1}$ & 99 & $1.92 \mathrm{E}+03$ & $1.23 \mathrm{E}+04$ & $1.23 \mathrm{E}+04$ & $2.51 E+04$ & $6.51 \mathrm{E}+03$ & $2.44 E+02$ & $6.60 \mathrm{E}-02$ & $1.18 \mathrm{E}-02$ & $4.93 \mathrm{E}-02$ & $1.90 \mathrm{E}-03$ & $1.62 \mathrm{E}+04$ \\
\hline 11 & $a_{1}$ & & & $8+04$ & +05 & $\mathrm{E}+04$ & . & +04 & $2.61 \mathrm{E}+04$ & $1.57 \mathrm{E}+02$ & $E+01$ & $\mathrm{E}+01$ & $E+\infty$ & $\mathrm{E}+05$ \\
\hline 241-BY-111 & 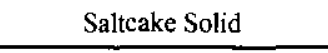 & & 1378 & $1.42 \mathrm{E}+04$ & $3.39 \mathrm{E}+05$ & $3.66 \mathrm{E}+04$ & $.75 \mathrm{E}+05$ & $5.85 \mathrm{E}+04$ & $2.58 \mathrm{E}+04$ & $56 \mathrm{E}+02$ & $1.59 \mathrm{E}+01$ & $6.66 \mathrm{E}+01$ & $2.56 \mathrm{E}+00$ & $1.34 \mathrm{E}+05$ \\
\hline & 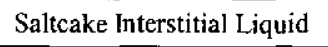 & 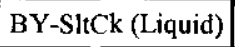 & 145 & $1.85 \mathrm{E}+02$ & & $92 \mathrm{E}+03$ & $3.14 \mathrm{E}+04$ & & $2.71 \mathrm{E}+02$ & $5.29 \mathrm{E}-01$ & 2 & 1 & $1.52 \mathrm{E}-02$ & $1.80 \mathrm{E}+04$ \\
\hline $241-B Y-112$ & 1otal & & & $1.50 \mathrm{E}+04$ & $1.44 \mathrm{E}+05$ & $23 \mathrm{E}+04$ & $5.92 \mathrm{E}+05$ & $3.92 \mathrm{E}+04$ & $2.22 \mathrm{E}+04$ & $8.11 \mathrm{E}+01$ & $8.29 \mathrm{E}+00$ & $48 \mathrm{E}+01$ & $1.34 \mathrm{E}+00$ & $1.20 \mathrm{E}+05$ \\
\hline 1112 & -1 & & 8 & $0 \mathrm{E}+00$ & $6.99 \mathrm{E}+00$ & +00 & +03 & -00 & $2 \mathrm{~F}+03$ & $5.47 \mathrm{E}-03$ & $1.06 \mathrm{E}-02$ & $8.38 \mathrm{E}-02$ & 04 & +01 \\
\hline D 10116 & Thake sullu & 1-DIICK (JOHI) & 996 & $49 \mathrm{E}+04$ & $1.29 \mathrm{E}+05$ & $13 E+04$ & $71 \mathrm{~T}+\mathrm{US}$ & +04 & 04 & 1 & 0 & 01 & 00 & $1.08 \mathrm{E}+05$ \\
\hline $241-\mathrm{B}$ & altcake Interstitial I & Y-Slt & 79 & $36 \mathrm{E}+02$ & $1.50 \mathrm{E}+04$ & $11 \mathrm{E}+04$ & $.93 \mathrm{E}+04$ & +03 & $1.70 \mathrm{E}+02$ & 4.97E-01 & $8.92 \mathrm{E}-02$ & $3.71 \mathrm{E}-01$ & $1.43 \mathrm{E}-02$ & $1.13 \mathrm{E}+04$ \\
\hline 2 & al & & & 3 & 94 & 3 & 4 & (4) & 04 & 1 & 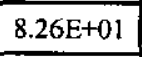 & $E+02$ & $\mathrm{E}+00$ & $E+04$ \\
\hline 241-C-101 & Sludge (Liquid \& Solid) & $\mathrm{BP}$ (Solid) & 125 & $2.34 \mathrm{E}+02$ & $6.40 \mathrm{E}+04$ & $4.41 \mathrm{E}+03$ & $3.75 \mathrm{E}+04$ & $6.20 \mathrm{E}+02$ & $3.78 \mathrm{E}+04$ & $7.89 \mathrm{E}+01$ & $1.54 \mathrm{E}+01$ & $1.43 \mathrm{E}+02$ & $9.98 \mathrm{E}-01$ & $4.08 \mathrm{E}+03$ \\
\hline $241-C-101$ & udge (Liquid \& Solid) & d) & 208 & $1.43 \mathrm{E}+03$ & $2.30 \mathrm{E}+03$ & $E+03$ & $1.75 \mathrm{E}+04$ & $7.88 \mathrm{E}+04$ & $2.40 \mathrm{E}+02$ & $\varepsilon+00$ & $73 E+01$ & $3.23 \mathrm{E}+02$ & $5.58 \mathrm{E}+00$ & $2.59 \mathrm{E}+04$ \\
\hline 241-C-102 & Total & & & $1.86 \mathrm{E}+03$ & $6.52 \mathrm{E}+04$ & $1.90 \mathrm{E}+04$ & $1.06 \mathrm{E}+05$ & $2.78 \mathrm{E}+05$ & $2.80 \mathrm{E}+04$ & $1.02 \mathrm{E}+03$ & $8.15 \mathrm{E}+02$ & $3.40 \mathrm{E}+03$ & $8.80 \mathrm{E}+01$ & $2.59 \mathrm{E}+04$ \\
\hline 102 & olid) & olid) & 98 & $4.73 \mathrm{E}+01$ & $9.18 \mathrm{E}+03$ & $83 \mathrm{E}+03$ & $9.16 \mathrm{E}+03$ & $1.80 \mathrm{E}+03$ & $1.53 \mathrm{E}+04$ & $6.81 \mathrm{E}-12$ & $1.19 \mathrm{E}-11$ & $1.73 \mathrm{E}-10$ & $3.34 \mathrm{E}-06$ & $8.05 E+02$ \\
\hline $2+11-2-102$ & HOE (L & De & 61 & 0.102101 & $1.67 \mathrm{E}+04$ & $.15 \mathrm{E}+03$ & 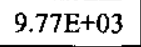 & $1.02 \mathrm{~L} 102$ & $9.84 \mathrm{E}+03$ & Ca & $\mathrm{E}-01$ & $1.00 \mathrm{E}+00$ & $6.99 \mathrm{E}-03$ & $1.06 \mathrm{E}+03$ \\
\hline $241-C-102$ & Sludge (Liquid \& Solid) & MW1 (Solid) & 19 & $0.00 \mathrm{E}+00$ & $3.93 \mathrm{E}+01$ & $1.86 \mathrm{E}+01$ & $2.30 \mathrm{E}+03$ & $0.00 \mathrm{E}+00$ & $1.32 \mathrm{E}+03$ & $4.29 \mathrm{E}-03$ & $1.39 \mathrm{E}-03$ & $2.07 \mathrm{E}-02$ & $6.98 \mathrm{E}-05$ & $6.70 \mathrm{E}+01$ \\
\hline $241-C$ & udge (Liquid \& Solid) & olid) & 38 & $4.54 \mathrm{E}+01$ & $1.63 \mathrm{E}+03$ & $31 \mathrm{E}+02$ & $3.27 \mathrm{E}+03$ & $0.00 \mathrm{E}+00$ & $9.74 \mathrm{E}+02$ & $6.73 \mathrm{E}+01$ & $4.68 \mathrm{E}+01$ & $1.21 \mathrm{E}+02$ & $1.20 \mathrm{E}+01$ & $4.92 \mathrm{E}+02$ \\
\hline $241-\mathrm{C}-102$ & Sludge (Liquid \& Solid) & WP2 (Solid) & 855 & $1.02 \mathrm{E}+03$ & $3.66 \mathrm{E}+04$ & $1.20 \mathrm{E}+04$ & $7.35 \mathrm{E}+04$ & $2.38 \mathrm{E}+05$ & $4.72 \mathrm{E}+02$ & $9.50 \mathrm{E}+02$ & $7.21 \mathrm{E}+02$ & $3.05 E+03$ & $7.2 \mathrm{iE}+01$ & $1.11 \mathrm{E}+04$ \\
\hline 241-C-102 & udge (Liquid \& Solid) & olid) & 125 & $6.83 \mathrm{E}+02$ & $1.10 \mathrm{E}+03$ & $1.51 \mathrm{E}+03$ & $8.35 \mathrm{E}+03$ & $3.77 \mathrm{E}+04$ & $1.15 \mathrm{E}+02$ & $4.28 \mathrm{E}+00$ & $4.7 ! \mathrm{E}+01$ & $2.26 \mathrm{E}+02$ & $3.90 \mathrm{E}+00$ & $1.24 \mathrm{E}+04$ \\
\hline $241-\mathrm{C}-103$ & Total & & & $7.42 \mathrm{E}+02$ & $5.84 \mathrm{E}+01$ & $3.91 \mathrm{E}+02$ & $4.34 \mathrm{E}+03$ & $3.18 \mathrm{E}+04$ & $7.93 E+04$ & $4.35 \mathrm{E}+00$ & $4.79 \mathrm{E}+01$ & $2.30 \mathrm{E}+02$ & $3.97 \mathrm{E}+00$ & $7.68 \mathrm{E}+03$ \\
\hline 241-C-103 & ludge (Liquid \& Solid) & CWP1 (Solid) & 101 & $7.42 \mathrm{E}+02$ & $5.84 \mathrm{E}+01$ & $3.91 \mathrm{E}+02$ & $4.34 \mathrm{E}+03$ & $3.18 E+04$ & $7.93 \mathrm{E}+04$ & $4.35 E+00$ & $4.79 \mathrm{E}+01$ & $2.30 \mathrm{E}+02$ & $3.97 \mathrm{E}+00$ & $7.68 \mathrm{E}+03$ \\
\hline $241-C-104$ & Total & & & $41 \mathrm{E}+04$ & $1.95 \mathrm{E}+04$ & $63 \mathrm{E}+04$ & $1.77 \mathrm{E}+05$ & $8.96 \mathrm{E}+04$ & $4.47 \mathrm{E}+05$ & $6.34 \mathrm{E}+03$ & $1.33 \mathrm{E}+03$ & $5.18 \mathrm{E}+03$ & $2.29 \mathrm{E}+02$ & $8.86 \mathrm{E}+04$ \\
\hline
\end{tabular}




\section{RPP-5926 REV 6}

Table A-2. Tank Waste Inventory from Best Basis Inventory Detail Report (Queried on September 27, 2006) for Hydrogen Generation Rate Calculations. (27 sheets)

\begin{tabular}{|c|c|c|c|c|c|c|c|c|c|c|c|c|c|c|}
\hline $\begin{array}{l}\text { Tank } \\
\text { Name }\end{array}$ & sse & ype & $\begin{array}{c}\text { Vol } \\
(\mathrm{kL})\end{array}$ & $\begin{array}{l}\text { TOC } \\
\text { (Kg) }\end{array}$ & $\begin{array}{l}\mathrm{NO}_{3} \\
(\mathrm{Kg})\end{array}$ & $\begin{array}{l}\mathrm{NO}_{2} \\
(\mathrm{Kg})\end{array}$ & $\begin{array}{c}\mathrm{Na} \\
(\mathrm{Kg})\end{array}$ & $\begin{array}{c}\mathrm{Al} \\
(\mathrm{Kg})\end{array}$ & $\begin{array}{l}{ }^{90} \mathrm{Sr} \\
\text { (Ci) }\end{array}$ & ${ }_{(\mathrm{Ci})}^{241}$ & $\begin{array}{l}{ }^{240} \mathrm{Pu} \\
\text { (Ci) }\end{array}$ & $\begin{array}{c}{ }^{239} \mathbf{P u} \\
(\mathrm{Ci})\end{array}$ & $\begin{array}{c}{ }^{238} \mathbf{P u} \\
\text { (Ci) }\end{array}$ & $\begin{array}{c}{ }^{137} \mathrm{Cs} \\
\text { (Ci) }\end{array}$ \\
\hline-104 & Slud & d) & 80 & $E+03$ & $9 \mathrm{E}+03$ & $E+03$ & 4 & 3 & $\mathrm{E}+04$ & 2 & 91 & 12 & 0 & 7 \\
\hline $241-\mathrm{C}-104$ & Sludge (Liquid \& Solid) & owW3 (Solid) & 103 & $1.48 \mathrm{E}+03$ & $2.04 \mathrm{E}+03$ & $3.81 E+03$ & $1.86 \mathrm{E}+04$ & $9.42 \mathrm{E}+03$ & $4.70 \mathrm{E}+04$ & $6.88 \mathrm{E}+02$ & $1.77 \mathrm{E}+02$ & $5.07 \mathrm{E}+02$ & $5.59 \mathrm{E}+01$ & $9.31 \mathrm{E}+03$ \\
\hline $241-C-104$ & Sludge (Liquid \& Solid) & NA & 152 & $2.18 \mathrm{E}+03$ & $3.02 \mathrm{E}+03$ & $5.63 \mathrm{E}+03$ & $2.75 \mathrm{E}+04$ & $1.39 E+04$ & $6.93 E+04$ & $1.00 \mathrm{E}+03$ & $2.34 \mathrm{E}+02$ & $7.76 \mathrm{E}+02$ & $6.28 \mathrm{E}+01$ & $1.37 \mathrm{E}+04$ \\
\hline $241-\mathrm{C}-104$ & Sludge (Liquid \& Solid) & CWZr1 (Solid) & 90 & $1.29 \mathrm{E}+03$ & $1.79 \mathrm{E}+03$ & $3.33 \mathrm{E}+03$ & $1.63 \mathrm{E}+04$ & $8.23 E+03$ & $4.11 \mathrm{E}+04$ & $6.07 \mathrm{E}+02$ & $1.67 \mathrm{E}+02$ & $4.31 E+02$ & $4.28 \mathrm{E}+01$ & $8.14 \mathrm{E}+03$ \\
\hline $241-C-104$ & Sludge (Liquid \& Solid) & CWP2 (Solid) & 229 & $3.29 \mathrm{E}+03$ & $4.55 \mathrm{E}+03$ & $8.48 E+03$ & $4.14 \mathrm{E}+04$ & $2.09 \mathrm{E}+04$ & $1.04 E+05$ & $1.47 \mathrm{E}+03$ & $2.91 \mathrm{E}+02$ & $1.23 E+03$ & $2.90 \mathrm{E}+01$ & $2.07 E+04$ \\
\hline $241-C-104$ & Sludge (Liquid \& Solid) & CWP1 (Solid) & 326 & $4.68 \mathrm{E}+03$ & $6.47 \mathrm{E}+03$ & $1.21 E+04$ & $5.89 E+04$ & $2.98 \mathrm{E}+04$ & $1.49 E+05$ & $2.07 E+03$ & $3.73 \mathrm{E}+02$ & $1.79 \mathrm{E}+03$ & $3.09 \mathrm{E}+01$ & $2.95 \mathrm{E}+04$ \\
\hline $241-C-105$ & Total & & & $4.31 \mathrm{E}+03$ & $8.14 \mathrm{E}+03$ & $6.73 \mathrm{E}+03$ & $3.81 E+04$ & $1.55 \mathrm{E}+05$ & $4.4 \mathrm{lE}+05$ & $1.19 \mathrm{E}+03$ & $3.90 \mathrm{E}+02$ & $1.98 \mathrm{E}+03$ & $3.19 \mathrm{E}+01$ & $7.64 \mathrm{E}+04$ \\
\hline 241-C-105 & Sludge (Liquid \& Solid) & TBP (Solid) & 50 & $5.27 \mathrm{E}+01$ & $8.14 \mathrm{E}+02$ & $9.92 \mathrm{E}+02$ & $8.44 E+03$ & $1.39 \mathrm{E}+02$ & $4.41 E+04$ & $1.15 \mathrm{E}+02$ & $2.29 \mathrm{E}+01$ & $2.14 \mathrm{E}+02$ & $1.49 E+00$ & $7.64 \mathrm{E}+03$ \\
\hline $24 !-C-105$ & Sludge (Liquid \& Solid) & CWP1 (Solid) & 450 & $4.26 \mathrm{E}+03$ & $7.32 \mathrm{E}+03$ & $5.74 \mathrm{E}+03$ & $2.97 \mathrm{E}+04$ & $1.55 \mathrm{E}+05$ & $3.97 E+05$ & $1.08 \mathrm{E}+03$ & $3.67 E+02$ & $1.77 \mathrm{E}+03$ & $3.04 \mathrm{E}+01$ & $6.87 \mathrm{E}+04$ \\
\hline $241-\mathrm{C}-106$ & Tota] & & & $9.07 \mathrm{E}+01$ & $3.48 \mathrm{E}+01$ & $4.14 \mathrm{E}+01$ & $1.88 \mathrm{E}+02$ & $3.82 \mathrm{E}+02$ & $6.61 \mathrm{E}+04$ & $6.52 \mathrm{E}+01$ & $3.57 E+00$ & $1.67 E+01$ & $2.70 \mathrm{E}+00$ & $1.45 \mathrm{E}+03$ \\
\hline $24 !-C-106$ & - & NA & 322 & $\mathrm{E}-01$ & $94 \mathrm{E}-02$ & $75 \mathrm{E}-02$ & $15 E+00$ & $E-02$ & $1.42 E-02$ & $1.35 \mathrm{E}-06$ & $2.79 \mathrm{E}-07$ & $1.31 \mathrm{E}-06$ & $2.10 \mathrm{E}-07$ & $1.40 \mathrm{E}-01$ \\
\hline $241-C-106$ & Sludge (Liquid \& Solid) & NA & 0.166 & $9.06 \mathrm{E}+01$ & $3.48 \mathrm{E}+01$ & $4.14 \mathrm{E}+01$ & $1.85 \mathrm{E}+02$ & $3.82 \mathrm{E}+02$ & $6.61 \mathrm{E}+04$ & $6.52 \mathrm{E}+01$ & $3.57 \mathrm{E}+00$ & $1.67 \mathrm{E}+01$ & $2.70 \mathrm{E}+00$ & $1.45 E+03$ \\
\hline $241-\mathrm{C}-107$ & Total & & & $7.32 \mathrm{E}+02$ & $4.75 \mathrm{E}+04$ & $3.51 \mathrm{E}+04$ & $9.47 \mathrm{E}+04$ & $5.83 E+04$ & $2.08 \mathrm{E}+06$ & $6.69 \mathrm{E}+03$ & $3.55 \mathrm{E}+02$ & $2.18 \mathrm{E}+03$ & $4.43 \mathrm{E}+01$ & $5.89 \mathrm{E}+04$ \\
\hline $241-C-107$ & Sludge (Liquid \& Solid) & $\mathrm{RR}$ (Solid) & 339 & $2.66 \mathrm{E}+02$ & $1.00 \mathrm{E}+04$ & $1.21 E+04$ & $2.83 E+04$ & $2.29 E+04$ & $1.68 \mathrm{E}+06$ & $2.43 \mathrm{E}+03$ & $1.73 \mathrm{E}+02$ & $7.47 \mathrm{E}+02$ & $3.09 \mathrm{E}+01$ & $2.14 \mathrm{E}+04$ \\
\hline $241-C-107$ & Sludge (Liquid \& Solid) & CWP2 (Solid) & 89 & $6.97 \mathrm{E}+01$ & $3.21 \mathrm{E}+03$ & $2.58 \mathrm{E}+03$ & $7.88 \mathrm{E}+03$ & $1.64 \mathrm{E}+04$ & $5.97 \mathrm{E}+04$ & $6.37 \mathrm{E}+02$ & $4.62 \mathrm{E}+01$ & $1.95 \mathrm{E}+02$ & $4.61 E+00$ & $5.61 \mathrm{E}+03$ \\
\hline $241-C-107$ & Sludge (Liquid \& Solid) & IC (Solid) & 507 & $3.97 \mathrm{E}+02$ & $3.43 \mathrm{E}+04$ & $2.05 E+04$ & $5.85 E+04$ & $1.89 \mathrm{E}+04$ & $3.40 \mathrm{E}+05$ & $3.63 E+03$ & $1.35 \mathrm{E}+02$ & $1.24 \mathrm{E}+03$ & $8.73 E+00$ & $3.20 E+04$ \\
\hline $241-C-108$ & Total & & & $3.50 \mathrm{E}+02$ & $1.61 \mathrm{E}+04$ & $8.92 \mathrm{E}+03$ & $3.48 \mathrm{E}+04$ & $1.93 \mathrm{E}+04$ & $8.00 \mathrm{E}+03$ & $1.07 E+01$ & $3.39 \mathrm{E}-01$ & $3.12 \mathrm{E}+00$ & $2.20 \mathrm{E}-02$ & $7.77 \mathrm{E}+04$ \\
\hline $241-C-108$ & Sludge (Liquid \& Solid) & TFeCN (Solid) & 45 & $6.30 \mathrm{E}+01$ & $2.89 \mathrm{E}+03$ & $1.61 E+03$ & $6.27 \mathrm{E}+03$ & $3.47 \mathrm{E}+03$ & $1.44 \mathrm{E}+03$ & $1.93 \mathrm{E}+00$ & $6.25 \mathrm{E}-02$ & $5.60 \mathrm{E}-01$ & $4.10 \mathrm{E}-03$ & $1.40 \mathrm{E}+04$ \\
\hline $241-\mathrm{C}-108$ & Sludge (Liquid \& Solid) & TBP (Solid) & 95 & $1.33 \mathrm{E}+02$ & $6.10 \mathrm{E}+03$ & $3.39 \mathrm{E}+03$ & $1.32 \mathrm{E}+04$ & $7.32 \mathrm{E}+03$ & $3.04 \mathrm{E}+03$ & $4.08 \mathrm{E}+00$ & $1.27 \mathrm{E}-01$ & $1.19 \mathrm{E}+00$ & 8.27E-03 & $2.95 \mathrm{E}+04$ \\
\hline $241-C-108$ & Sludge (Liquid \& Solid) & IC (Solid) & 110 & $1.54 \mathrm{E}+02$ & $7.07 \mathrm{E}+03$ & $3.92 \mathrm{E}+03$ & $1.53 \mathrm{E}+04$ & $8.48 \mathrm{E}+03$ & $3.52 \mathrm{E}+03$ & $4.73 \mathrm{E}+00$ & $1.50 \mathrm{E}-01$ & $1.37 \mathrm{E}+00$ & $9.66 \mathrm{E}-03$ & $3.42 \mathrm{E}+04$ \\
\hline $241-C-109$ & Total & & & $8.15 E+02$ & $1.96 \mathrm{E}+04$ & $1.32 \mathrm{E}+04$ & $3.76 \mathrm{E}+04$ & $2.52 \mathrm{E}+04$ & $2.53 \mathrm{E}+05$ & $5.01 \mathrm{E}+01$ & $1.12 \mathrm{E}+01$ & $6.54 \mathrm{E}+01$ & $1.65 \mathrm{E}+00$ & $1.73 \mathrm{E}+05$ \\
\hline $241-C-109$ & Studge (Liquid \& Solid) & TFeCN (Solid) & 121 & $4.71 E+02$ & $7.65 \mathrm{E}+03$ & $7.60 \mathrm{E}+03$ & $1.93 \mathrm{E}+04$ & $1.46 \mathrm{E}+04$ & $1.51 E+05$ & $2.96 \mathrm{E}+01$ & $6.43 \mathrm{E}+00$ & $3.65 \mathrm{E}+01$ & $9.68 \mathrm{E}-01$ & $1.03 \mathrm{E}+05$ \\
\hline $241-C-109$ & Sludge (Liquid \& Solid) & HS (Solid) & 26 & $1.01 \mathrm{E}+02$ & $1.64 \mathrm{E}+03$ & $1.63 \mathrm{E}+03$ & $4.15 \mathrm{E}+03$ & $3.14 E+03$ & $3.25 \mathrm{E}+04$ & $6.36 \mathrm{E}+00$ & $1.38 \mathrm{E}+00$ & $7.83 \mathrm{E}+00$ & $2.08 \mathrm{E}-01$ & $2.21 \mathrm{E}+04$ \\
\hline $241-C-109$ & Studge (Liquid \& Solid) & CWP1 (Solid) & 55 & $2.14 \mathrm{E}+02$ & $3.48 \mathrm{E}+03$ & $3.45 \mathrm{E}+03$ & $8.78 \mathrm{E}+03$ & $6.65 \mathrm{E}+03$ & $6.87 \mathrm{E}+04$ & 1.34E+01 & $2.92 E+00$ & $1.66 \mathrm{E}+01$ & $4.40 \mathrm{E}-01$ & $4.67 \mathrm{E}+04$ \\
\hline $241-C \cdot 109$ & Sludge (Liquid \& Solid) & 1C (Solid) & 38 & $2.86 \mathrm{E}+01$ & $6.85 \mathrm{E}+03$ & $5.28 \mathrm{E}+02$ & $5.40 \mathrm{E}+03$ & $8.10 \mathrm{E}+02$ & $2.62 \mathrm{E}+02$ & $7.51 \mathrm{E}-01$ & $4.93 \mathrm{E}-01$ & $4.53 \mathrm{E}+00$ & $3.18 \mathrm{E}-02$ & $8.62 \mathrm{E}+02$ \\
\hline $241-C-110$ & Total & & & $4.12 \mathrm{E}+02$ & $9.80 \mathrm{E}+04$ & $6.53 E+03$ & $7.53 \mathrm{E}+04$ & $1.31 \mathrm{E}+04$ & $3.45 \mathrm{E}+03$ & $3.86 \mathrm{E}+01$ & $7.05 E+00$ & $6.48 \mathrm{E}+01$ & $4.56 \mathrm{E}-01$ & $1.35 \mathrm{E}+04$ \\
\hline $241-C-110$ & Supematant & IC1 (Liquid) & 4 & $2.32 \mathrm{E}+00$ & $2.41 \mathrm{E}+02$ & $1.84 \mathrm{E}+01$ & $1.22 E+02$ & $8.34 \mathrm{E}-01$ & $8.46 \mathrm{E}-02$ & $7.58 \mathrm{E}-05$ & $4.44 \mathrm{E}-04$ & $6.59 \mathrm{E}-03$ & $2.23 E-05$ & $1.50 \mathrm{E}+01$ \\
\hline
\end{tabular}


Table A-2. Tank Waste Inventory from Best Basis Inventory Detail Report (Queried on September 27, 2006) for

Hydrogen Generation Rate Calculations. (27 sheets)

\begin{tabular}{|c|c|c|c|c|c|c|c|c|c|c|c|c|c|c|}
\hline $\begin{array}{c}\text { Tank } \\
\text { Name }\end{array}$ & Waste & Was & $\begin{array}{c}\text { Vol } \\
(\mathbf{k L})\end{array}$ & $\begin{array}{c}\text { TOC } \\
(\mathrm{Kg})\end{array}$ & $\begin{array}{l}\mathrm{NO}_{3} \\
(\mathrm{Kg})\end{array}$ & $\begin{array}{l}\mathrm{NO}_{2} \\
\text { (Kg) }\end{array}$ & $\begin{array}{c}\mathrm{Na} \\
(\mathrm{Kg})\end{array}$ & $\begin{array}{c}\mathbf{A l} \\
(\mathbf{K g})\end{array}$ & $\begin{array}{l}{ }^{96} \mathrm{Sr} \\
(\mathrm{Ci})\end{array}$ & $\begin{array}{c}{ }_{(\mathrm{Ci})}^{241} \mathrm{Am} \\
\text {. }\end{array}$ & $\begin{array}{l}{ }^{240} \mathrm{Pu} \\
(\mathrm{Ci})\end{array}$ & $\begin{array}{c}{ }^{239} \mathbf{P u} \\
(\mathrm{Ci})\end{array}$ & $\begin{array}{c}{ }_{(\mathrm{Ci}}^{238} \mathbf{P u} \\
\text { ( })\end{array}$ & $\begin{array}{c}{ }^{137} \mathrm{Cs} \\
(\mathrm{Ci})\end{array}$ \\
\hline-110 & Sludg & ) & 670 & +02 & +04 & $\mathrm{E}+03$ & 34 & $1.31 \mathrm{E}+04$ & $E+03$ & $3.86 \mathrm{E}+01$ & 7.05E+00 & $6.48 \mathrm{E}+01$ & $4.56 \mathrm{E}-01$ & $1.35 \mathrm{E}+04$ \\
\hline 241-C-111 & Total & & & $2.58 \mathrm{E}+02$ & $2.05 \mathrm{E}+04$ & $06 \mathrm{E}+04$ & $1.38 \mathrm{E}+04$ & $3.13 \mathrm{E}+04$ & $9.07 \mathrm{E}+05$ & $1.90 \mathrm{E}+02$ & $3.30 \mathrm{E}+01$ & $1.70 \mathrm{E}+02$ & $3.12 \mathrm{E}+00$ & $1.98 \mathrm{E}+04$ \\
\hline $241-C-111$ & Sludge (Liquid \& Solid) & 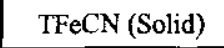 & 91 & $1.20 \mathrm{E}+02$ & $9.87 \mathrm{E}+03$ & $.46 \mathrm{E}+03$ & $3.68 \mathrm{E}+03$ & $1.64 \mathrm{E}+04$ & $4.91 \mathrm{E}+05$ & $1.86 \mathrm{E}+02$ & $2.24 E+00$ & $2.01 \mathrm{E}+01$ & $1.47 \mathrm{E}-01$ & $1.01 \mathrm{E}+04$ \\
\hline 241-C-111 & Sludge (Liquid \& Solid) & HS (Solid) & 17 & $2.24 \mathrm{E}+01$ & $1.32 \mathrm{E}+03$ & $7.95 \mathrm{E}+02$ & $6.88 \mathrm{E}+02$ & $6 \mathrm{E}+03$ & $9.17 \mathrm{E}+04$ & $1.24 \mathrm{E}+00$ & $6.68 \mathrm{E}+00$ & $3.09 \mathrm{E}+01$ & $9.83 \mathrm{E}-01$ & $1.89 \mathrm{E}+03$ \\
\hline 2 & Slud & $\mathrm{C}$ & 60 & 01 & +02 & +02 & 3 & +04 & $E+05$ & $\mathrm{E}+00$ & +01 & +02 & $1.95 \mathrm{E}+00$ & $6.66 \mathrm{E}+03$ \\
\hline $241-C-111$ & Solid) & 1) & 49 & $3.69 \mathrm{E}+01$ & $8.83 E+03$ & $6.80 \mathrm{E}+02$ & $6.96 \mathrm{E}+03$ & $1.04 \mathrm{E}+03$ & $3.38 \mathrm{E}+02$ & $9.68 \mathrm{E}-01$ & 6.35E-01 & $5.84 \mathrm{E}+00$ & $4.11 \mathrm{E}-02$ & $1.11 \mathrm{E}+03$ \\
\hline $241-C-112$ & Total & & & $2.42 \mathrm{E}+03$ & $3.72 E+04$ & $2.78 \mathrm{E}+04$ & $5.01 \mathrm{E}+04$ & $1.24 \mathrm{E}+04$ & $6.02 \mathrm{E}+05$ & $4.71 \mathrm{E}+02$ & $1.06 \mathrm{E}+01$ & $8.43 \mathrm{E}+01$ & $7.84 \mathrm{E}+00$ & $2.66 \mathrm{E}+05$ \\
\hline $241-\mathrm{C}-112$ & Sludge (Liq & $\mathrm{TFeC}$ & 272 & $1.67 \mathrm{E}+03$ & $2.57 \mathrm{E}+04$ & $1.92 \mathrm{E}+04$ & $3.47 \mathrm{E}+04$ & $.55 \mathrm{E}+03$ & 4.16E+05 & $3.26 \mathrm{E}+02$ & $6.59 \mathrm{E}+00$ & $5.91 \mathrm{E}+01$ & $5.42 \mathrm{E}+00$ & $1.84 \mathrm{E}+05$ \\
\hline $241-C-112$ & Sludge (Liquid \& Solid) & $S$ (Solid) & 4 & $2.46 \mathrm{E}+01$ & $3.78 \mathrm{E}+02$ & $2.83 \mathrm{E}+02$ & $5.10 \mathrm{E}+02$ & $1.26 \mathrm{E}+02$ & $6.12 \mathrm{E}+03$ & $4.81 \mathrm{E}+00$ & 1.72E-01 & $7.94 \mathrm{E}-01$ & $7.98 \mathrm{E}-02$ & $2.71 E+03$ \\
\hline $241-\mathrm{C}-112$ & Slud & CWP1 (Solid) & 60 & $3.69 \mathrm{E}+02$ & $5.67 \mathrm{E}+03$ & $4.25 \mathrm{E}+03$ & $7.65 \mathrm{E}+03$ & $1.89 \mathrm{E}+03$ & $9.18 \mathrm{E}+04$ & $7.21 E+01$ & $2.50 \mathrm{E}+00$ & $1.20 \mathrm{E}+01$ & $1.20 \mathrm{E}+00$ & $4.06 \mathrm{E}+04$ \\
\hline $241-\mathrm{C}-112$ & Sludge (Liquic & id) & 57 & $3.51 E+02$ & $5.39 \mathrm{E}+03$ & $4.03 \mathrm{E}+03$ & $7.27 \mathrm{E}+03$ & $1.79 \mathrm{E}+03$ & $8.73 E+04$ & $6.83 \mathrm{E}+01$ & $1.35 \mathrm{E}+00$ & $1.24 \mathrm{E}+01$ & $1.14 \mathrm{E}+00$ & $3.86 \mathrm{E}+04$ \\
\hline $241-C-201$ & Total & & & $3.46 \mathrm{E}+01$ & $1.35 \mathrm{E}+00$ & $27 \mathrm{E}-01$ & $4.91 \mathrm{E}+01$ & $4.11 \mathrm{E}+00$ & $2.52 \mathrm{E}+02$ & $2.42 \mathrm{E}+00$ & $3.40 \mathrm{E}+00$ & $1.58 \mathrm{E}+01$ & $5.01 \mathrm{E}-01$ & $1.01 \mathrm{E}+01$ \\
\hline 24 & 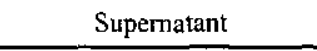 & NA & 007 & $0.00 \mathrm{E}+00$ & $0.00 \mathrm{E}+00$ & $0.00 \mathrm{E}+00$ & $0.00 \mathrm{E}+00$ & $0.00 \mathrm{E}+00$ & $0.00 \mathrm{E}+00$ & $0.00 \mathrm{E}+00$ & $0.00 \mathrm{E}+00$ & $0.00 \mathrm{E}+00$ & $0.00 \mathrm{E}+00$ & $0.00 \mathrm{E}+00$ \\
\hline $241-C-201$ & Sludge Solid & (Solid) & 0.537 & $3.46 \mathrm{E}+01$ & $1.35 \mathrm{E}+00$ & $5.27 \mathrm{E}-01$ & $4.91 \mathrm{E}+01$ & $4.11 \mathrm{E}+00$ & $2.52 \mathrm{E}+02$ & $2.42 \mathrm{E}+00$ & $3.40 \mathrm{E}+00$ & $1.58 \mathrm{E}+01$ & $5.01 \mathrm{E}-01$ & $1.01 \mathrm{E}+01$ \\
\hline 241 & Total & & & $3.53 \mathrm{E}+01$ & +00 & $4.52 \mathrm{E}-01$ & $58 \mathrm{E}+01$ & $8.48 E+00$ & $4.87 E+02$ & $1.15 \mathrm{E}+00$ & $3.08 \mathrm{E}+00$ & $1.43 \mathrm{E}+01$ & 4.54E-01 & $8.93 \mathrm{E}+00$ \\
\hline $241-\mathrm{C}-202$ & supermatamt & NA & 0.009 & $0.00 \mathrm{E}+00$ & $0.00 \mathrm{E}+00$ & $0.00 \mathrm{E}+00$ & $0.00 \mathrm{E}+00$ & $0.00 \mathrm{E}+00$ & $0.00 \mathrm{E}+00$ & $0.00 \mathrm{E}+00$ & $0.00 \mathrm{E}+00$ & $0.00 \mathrm{E}+00$ & $0.00 \mathrm{E}+00$ & $0.00 \mathrm{E}+00$ \\
\hline $241-C \cdot 202$ & Sludge (Liquid \& Solid) & HS (Solid) & 0.548 & $3.53 \mathrm{E}+01$ & $1.25 \mathrm{E}+00$ & $4.52 \mathrm{E}-01$ & $4.58 \mathrm{E}+01$ & $8.48 \mathrm{E}+00$ & $4.87 \mathrm{E}+02$ & $1.15 \mathrm{E}+00$ & $3.08 \mathrm{E}+00$ & $1.43 \mathrm{E}+01$ & $4.54 \mathrm{E}-01$ & $8.93 E+00$ \\
\hline $241-\mathrm{C}-203$ & Total & & & $.97 \mathrm{E}+00$ & $76 \mathrm{E}+00$ & 01 & $59 \mathrm{E}+01$ & $+\infty$ & $36 \mathrm{E}+00$ & $E-02$ & -01 & E-01 & $1.55 \mathrm{E}-02$ & $1.31 E+01$ \\
\hline $241-\mathrm{C}-203$ & Supernatant & NA & 0.048 & $0.00 \mathrm{E}+00$ & $0.00 \mathrm{E}+00$ & $0.00 \mathrm{E}+00$ & $0.00 \mathrm{E}+00$ & $0.00 \mathrm{E}+00$ & $0.00 \mathrm{E}+00$ & $0.00 \mathrm{E}+00$ & $0.00 \mathrm{E}+00$ & $0.00 \mathrm{E}+00$ & $0.00 \mathrm{E}+00$ & $0.00 \mathrm{E}+00$ \\
\hline $241-\mathrm{C}-203$ & Sludge (Liquid \& & Solid) & 0.476 & $4.97 \mathrm{E}+00$ & $3.76 \mathrm{E}+00$ & $9.94 \mathrm{E}-01$ & $5.59 \mathrm{E}+01$ & $0.00 \mathrm{E}+00$ & $2.36 \mathrm{E}+00$ & $2.91 \mathrm{E}-02$ & $1.05 \mathrm{E}-01$ & $4.86 \mathrm{E}-01$ & $1.55 \mathrm{E}-02$ & $1.31 \mathrm{E}+01$ \\
\hline $241-\mathrm{C}-204$ & Total & & & $9.44 \mathrm{E}+02$ & $2.03 E+02$ & $1.41 \mathrm{E}+02$ & $4.55 \mathrm{E}+02$ & $1.10 \mathrm{E}+02$ & $9.89 \mathrm{E}+01$ & $1.27 \mathrm{E}-02$ & $1.12 \mathrm{E}-02$ & $5.19 \mathrm{E}-02$ & $1.65 \mathrm{E}-03$ & 4.30E+01 \\
\hline $241-\mathrm{C}-204$ & Sludge (Liquid \& Solid) & HS (Solid) & 5.623 & $9.44 \mathrm{E}+02$ & $2.03 E+02$ & $1.41 \mathrm{E}+02$ & $4.55 \mathrm{E}+02$ & $1.10 \mathrm{E}+02$ & $9.89 \mathrm{E}+01$ & $1.27 \mathrm{E}-02$ & $1.12 \mathrm{E}-02$ & $5.19 \mathrm{E}-02$ & $1.65 \mathrm{E}-03$ & $4.30 \mathrm{E}+01$ \\
\hline $241-S-101$ & Total & & & $5.54 \mathrm{E}+03$ & $3.11 E+05$ & $1.08 \mathrm{E}+05$ & $3.27 \mathrm{E}+05$ & $1.86 \mathrm{E}+05$ & $4.53 \mathrm{E}+05$ & $2.21 \mathrm{E}+02$ & $6.32 \mathrm{E}+01$ & $3.21 \mathrm{E}+02$ & $6.71 \mathrm{E}+00$ & $2.45 \mathrm{E}+05$ \\
\hline 241-S-101 & Sludge (Liquid \& Solid) & NA & 890 & $2.93 \mathrm{E}+03$ & $2.35 \mathrm{E}+05$ & $6.61 \mathrm{E}+04$ & $1.99 \mathrm{E}+05$ & $1.70 \mathrm{E}+05$ & $3.09 \mathrm{E}+05$ & $1.68 \mathrm{E}+02$ & $5.82 \mathrm{E}+01$ & $2.98 \mathrm{E}+02$ & $5.91 \mathrm{E}+00$ & $1.42 \mathrm{E}+05$ \\
\hline $241-S-101$ & Saltcake Solid & S2-SltSlr (Solid) & 169 & $1.04 \mathrm{E}+03$ & $2.88 \mathrm{E}+04$ & $1.57 \mathrm{E}+04$ & $5.00 \mathrm{E}+04$ & $6.08 \mathrm{E}+03$ & $5.86 \mathrm{E}+04$ & $1.82 \mathrm{E}+01$ & $2.61 \mathrm{E}+00$ & $1.22 \mathrm{E}+01$ & $4.24 \mathrm{E}-01$ & $3.93 E+04$ \\
\hline 241-S-101 & Saltcake Solid & S1-SltCk (Solid) & 246 & $1.52 \mathrm{E}+03$ & $4.19 \mathrm{E}+04$ & $2.28 \mathrm{E}+04$ & $7.29 \mathrm{E}+04$ & $8.85 \mathrm{E}+03$ & $8.53 \mathrm{E}+04$ & $3.49 \mathrm{E}+01$ & $2.36 \mathrm{E}+00$ & $1.08 \mathrm{E}+01$ & $3.81 \mathrm{E}-01$ & $5.71 \mathrm{E}+04$ \\
\hline 241-S-101 & Saltcake Interstitial Liquid & S2-SItSIr (Liquid) & 11 & $1.97 \mathrm{E}+01$ & $2.35 \mathrm{E}+03$ & $1.24 \mathrm{E}+03$ & $2.44 \mathrm{E}+03$ & $3.29 \mathrm{E}+02$ & $4.17 \mathrm{E}-01$ & $9.16 \mathrm{E}-04$ & $5.51 \mathrm{E}-05$ & $2.57 \mathrm{E}-04$ & $8.98 \mathrm{E}-06$ & $3.00 \mathrm{E}+03$ \\
\hline
\end{tabular}




\section{RPP-5926 REV 6}

Table A-2. Tank Waste Inventory from Best Basis Inventory Detail Report (Queried on September 27, 2006) for Hydrogen Generation Rate Calculations. (27 sheets)

\begin{tabular}{|c|c|c|c|c|c|c|c|c|c|c|c|c|c|c|}
\hline $\begin{array}{c}\text { Tank } \\
\text { Name }\end{array}$ & Waste Phase & Waste Type & $\begin{array}{c}\text { Vol } \\
(k L)\end{array}$ & $\begin{array}{l}\text { TOC } \\
(\mathrm{Kg})\end{array}$ & $\begin{array}{l}\mathrm{NO}_{3} \\
(\mathrm{Kg})\end{array}$ & $\begin{array}{l}\mathrm{NO}_{2} \\
(\mathrm{Kg})\end{array}$ & $\begin{array}{c}\mathrm{Na} \\
(\mathbf{K g})\end{array}$ & $\begin{array}{c}\mathbf{A l} \\
(\mathbf{K g})\end{array}$ & $\begin{array}{l}{ }^{90} \mathrm{Sr} \\
\text { (Ci) }\end{array}$ & $\begin{array}{c}{ }^{241} \mathrm{Am} \\
\text { (Ci) }\end{array}$ & $\begin{array}{c}{ }^{240} \mathrm{Pu} \\
(\mathrm{Ci})\end{array}$ & $\begin{array}{c}{ }^{239} \mathrm{Pu} \\
(\mathrm{Ci})\end{array}$ & $\begin{array}{c}{ }^{238} \mathrm{Pu} \\
(\mathrm{Ci})\end{array}$ & $\begin{array}{c}{ }^{137} \mathrm{Cs} \\
\text { (Ci) }\end{array}$ \\
\hline 241-S-101 & Saltcal & id) & 16 & $86 \mathrm{E}+01$ & $3.42 \mathrm{E}+03$ & $1.80 \mathrm{E}+03$ & $.55 E+03$ & $4.79 \mathrm{E}+02$ & $6.07 \mathrm{E}-01$ & $1.33 \mathrm{E}-03$ & $8.12 \mathrm{E}-05$ & $3.73 \mathrm{E}-04$ & $1.31 \mathrm{E}-05$ & $4.36 \mathrm{E}+03$ \\
\hline $241-S-102$ & Total & & & $97 \mathrm{E}+03$ & $24 \mathrm{E}+05$ & $3.89 \mathrm{E}+04$ & $2.08 \mathrm{E}+05$ & $3.73 E+04$ & $7.46 \mathrm{E}+04$ & $1.12 \mathrm{E}+02$ & $1.35 \mathrm{E}+01$ & $6.35 \mathrm{E}+01$ & $2.08 \mathrm{E}+00$ & $1.09 \mathrm{E}+05$ \\
\hline $241-S-102$ & Sludge (Liquid \& Solid) & Rl (Solid) & 71 & $1.06 \mathrm{E}+03$ & $6.47 \mathrm{E}+03$ & $4.76 \mathrm{E}+03$ & $1.41 \mathrm{E}+04$ & $2.51 \mathrm{E}+04$ & $5.50 \mathrm{E}+04$ & $4.17 \mathrm{E}+00$ & $1.60 \mathrm{E}+00$ & $8.25 \mathrm{E}+00$ & $1.53 \mathrm{E}-01$ & $8.47 E+03$ \\
\hline $241-S-102$ & Saltcake Solid & $\mathrm{Ck}$ & 593 & $3.91 \mathrm{E}+03$ & $3.17 \mathrm{E}+05$ & $3.41 \mathrm{E}+04$ & $1.94 \mathrm{E}+05$ & $1.22 \mathrm{E}+04$ & $1.96 \mathrm{E}+04$ & $1.08 \mathrm{E}+02$ & $1.19 \mathrm{E}+01$ & $5.52 \mathrm{E}+01$ & $1.93 E+00$ & $1.00 \mathrm{E}+05$ \\
\hline $241-S-103$ & Total & & & $5.74 \mathrm{E}+03$ & $64 \mathrm{E}+05$ & $8.30 \mathrm{E}+04$ & $75 \mathrm{E}+05$ & $3.66 \mathrm{E}+04$ & $15 \mathrm{E}+04$ & $1.35 E+02$ & $96 \mathrm{E}+01$ & $9.40 \mathrm{E}+01$ & $2.80 \mathrm{E}+00$ & $1.95 \mathrm{E}+05$ \\
\hline $241-S-103$ & Supernatant & $-\mathrm{S} 1 \mathrm{tS}$ & 4 & $1.87 \mathrm{E}+01$ & $8.64 \mathrm{E}+02$ & $5.88 \mathrm{E}+02$ & $9.12 \mathrm{E}+02$ & $1.28 \mathrm{E}+02$ & $1.48 \mathrm{E}+00$ & $4.72 \mathrm{E}-03$ & $4.90 \mathrm{E}-05$ & $2.29 \mathrm{E}-04$ & $7.98 \mathrm{E}-06$ & $1.29 \mathrm{E}+03$ \\
\hline $241-S-103$ & Sludge (Liquid \& Solid) & Rl (Solid) & 34 & $6.32 \mathrm{E}+01$ & $6.74 \mathrm{E}+03$ & $1.90 \mathrm{E}+03$ & $6.08 \mathrm{E}+03$ & $1.13 E+04$ & $2.46 \mathrm{E}+04$ & $1.51 \mathrm{E}+01$ & $5.78 \mathrm{E}+00$ & $2.98 \mathrm{E}+01$ & $5.52 \mathrm{E}-01$ & $3.80 \mathrm{E}+03$ \\
\hline $241-S-103$ & Saltcake Solid & S2-SitSlr (Solid) & 300 & $2.58 \mathrm{E}+03$ & $1.32 \mathrm{E}+05$ & $2.47 \mathrm{E}+04$ & $9.49 \mathrm{E}+04$ & $9.29 \mathrm{E}+03$ & $6.64 \mathrm{E}+03$ & $7.53 \mathrm{E}+01$ & $1.08 \mathrm{E}+01$ & $5.04 \mathrm{E}+01$ & $1.76 \mathrm{E}+00$ & $6.71 E+04$ \\
\hline $241-S-103$ & Saltcake Solid & S1-SltCk (Solid) & 347 & $2.09 \mathrm{E}+03$ & $1.79 \mathrm{E}+05$ & $2.48 \mathrm{E}+04$ & $25 \mathrm{E}+05$ & $9.21 E+03$ & $E+04$ & $4.45 \mathrm{E}+01$ & $3.01 \mathrm{E}+00$ & $\mathrm{E}+01$ & -01 & $5.49 \mathrm{E}+04$ \\
\hline $241-S-103$ & Saltcake Interstitial Liquid & S2-S1tSIr & 95 & $4.44 \mathrm{E}+02$ & $2.05 \mathrm{E}+04$ & $1.40 \mathrm{E}+04$ & $2.17 \mathrm{E}+04$ & $3.04 \mathrm{E}+03$ & $3.51 \mathrm{E}+01$ & $1.12 \mathrm{E}-01$ & $1.16 \mathrm{E}-03$ & $5.43 \mathrm{E}-03$ & $1.90 \mathrm{E}-04$ & $3.07 \mathrm{E}+04$ \\
\hline $241-S-103$ & Saltcake Interstitial Liquid & S1-SitCk (Liquid) & 116 & $5.43 \mathrm{E}+02$ & $2.51 \mathrm{E}+04$ & $1.70 \mathrm{E}+04$ & $2.64 \mathrm{E}+04$ & $3.72 \mathrm{E}+03$ & $4.29 \mathrm{E}+01$ & $1.37 \mathrm{E}-01$ & $1.44 \mathrm{E}-03$ & $6.61 \mathrm{E}-03$ & $2.33 \mathrm{E}-04$ & $3.75 E+04$ \\
\hline $241-S-104$ & Total & & & 103 & 5 & +04 & 05 & 5 & $2 E+05$ & $2.01 \mathrm{E}+02$ & $9 \mathrm{E}+01$ & $3 E+02$ & $9 \mathrm{E}+00$ & +04 \\
\hline $241-S-104$ & Sludge (Liquid \& Solid) & R1 (Solid) & 409 & $1.10 \mathrm{E}+03$ & $1.67 \mathrm{E}+05$ & $1.66 \mathrm{E}+04$ & $9.00 \mathrm{E}+04$ & $8.47 \mathrm{E}+04$ & $1.73 \mathrm{E}+05$ & $8.56 \mathrm{E}+01$ & $2.58 \mathrm{E}+01$ & $1.33 \mathrm{E}+02$ & $2.46 \mathrm{E}+00$ & $3.10 \mathrm{E}+04$ \\
\hline $241-S-104$ & Sludge (Liquid \& Solid) & CWR1 (Solid) & 91 & $2.45 \mathrm{E}+02$ & $3.72 \mathrm{E}+04$ & $3.70 \mathrm{E}+03$ & $2.00 \mathrm{E}+04$ & $1.88 \mathrm{E}+04$ & $3.85 \mathrm{E}+04$ & $1.91 \mathrm{E}+01$ & $5.63 E+00$ & $2.96 \mathrm{E}+01$ & $4.61 \mathrm{E}-01$ & $6.90 E+03$ \\
\hline $241-S-104$ & id & SitCk (Solid) & 451 & $1.21 \mathrm{E}+03$ & $1.52 \mathrm{E}+05$ & $1.66 \mathrm{E}+04$ & $.80 \mathrm{E}+04$ & $9.34 \mathrm{E}+04$ & $1.91 \mathrm{E}+05$ & $9.68 \mathrm{E}+01$ & $3.95 \mathrm{E}+01$ & $1.86 \mathrm{E}+02$ & $7.07 E+00$ & $3.08 \mathrm{E}+04$ \\
\hline $241-S-104$ & Saltcake Interstitial Liquid & R-SitCk (Liquid) & 139 & $5.51 E+01$ & $3.73 E+04$ & $5.31 E+03$ & $2.75 E+04$ & $1.66 \mathrm{E}+03$ & $5.57 \mathrm{E}-01$ & $1.04 \mathrm{E}-02$ & $2.19 \mathrm{E}-03$ & $1.03 \mathrm{E}-02$ & $3.92 \mathrm{E}-04$ & $8.77 E+03$ \\
\hline $241-S-105$ & Total & & & $2.08 \mathrm{E}+03$ & $1.38 \mathrm{E}+06$ & $2.92 E+04$ & $6.32 \mathrm{E}+05$ & $1.71 \mathrm{E}+04$ & $6.51 E+03$ & $7.77 \mathrm{E}+00$ & $1.85 \mathrm{E}+00$ & $9.24 \mathrm{E}+00$ & $2.08 \mathrm{E}-01$ & $8.70 \mathrm{E}+04$ \\
\hline 105 & sludige (Liquic & j & 8 & $1.49 \mathrm{E}+01$ & $59 \mathrm{E}+03$ & $4.47 \mathrm{E}+02$ & $43 \mathrm{E}+03$ & $2.65 \mathrm{E}+03$ & $80 \mathrm{E}+03$ & $3.54 \mathrm{E}+00$ & $36 \mathrm{E}+00$ & $7.01 \mathrm{E}+00$ & $1.30 \mathrm{E}-01$ & $8.93 \mathrm{E}+02$ \\
\hline $241-S-105$ & Saltcake Solid & S1-SltCk (Solid) & 1371 & $1.43 \mathrm{E}+03$ & $1.35 \mathrm{E}+06$ & $9.61 \mathrm{E}+03$ & $5.94 \mathrm{E}+05$ & $8.55 \mathrm{E}+03$ & $5.59 \mathrm{E}+02$ & $3.78 \mathrm{E}+00$ & $4.82 \mathrm{E}-01$ & $2.21 \mathrm{E}+00$ & $7.78 \mathrm{E}-02$ & $3.03 \mathrm{E}+04$ \\
\hline 241-S-105 & Saltcake Interstitial Liquid & S1-SltCk (Liquid) & 157 & $6.33 \mathrm{E}+02$ & $2.98 \mathrm{E}+04$ & $1.92 \mathrm{E}+04$ & $3.71 \mathrm{E}+04$ & $5.93 \mathrm{E}+03$ & $1.56 \mathrm{E}+02$ & $4.42 \mathrm{E}-01$ & $4.17 \mathrm{E}-03$ & $1.92 \mathrm{E}-02$ & $6.75 \mathrm{E}-04$ & $5.58 \mathrm{E}+04$ \\
\hline $241-S-106$ & Total & & & $6.33 \mathrm{E}+03$ & $9.84 \mathrm{E}+05$ & $7.75 \mathrm{E}+04$ & $6.01 \mathrm{E}+05$ & $5.15 \mathrm{E}+04$ & $3.64 \mathrm{E}+04$ & $5.82 \mathrm{E}+01$ & $3.91 \mathrm{E}+00$ & $1.79 \mathrm{E}+01$ & $6.30 \mathrm{E}-01$ & $2.71 \mathrm{E}+05$ \\
\hline $241-S-106$ & Saltcake Solid & S1-SttCk (Solid) & 1624 & $6.13 \mathrm{E}+03$ & $9.61 \mathrm{E}+05$ & $6.73 E+04$ & $5.76 \mathrm{E}+05$ & $4.78 \mathrm{E}+04$ & $3.64 E+04$ & $5.77 \mathrm{E}+01$ & $3.90 \mathrm{E}+00$ & $1.79 \mathrm{E}+01$ & $6.30 \mathrm{E}-01$ & $2.48 \mathrm{E}+05$ \\
\hline $241-S-106$ & Saltcake Interstitial Liquid & S1-SltCk (Liquid) & 99 & $1.96 \mathrm{E}+02$ & $2.34 \mathrm{E}+04$ & $1.03 E+04$ & $2.42 \mathrm{E}+04$ & $3.76 \mathrm{E}+03$ & $4.85 \mathrm{E}+00$ & $5.42 \mathrm{E}-01$ & $5.11 \mathrm{E}-03$ & $2.34 \mathrm{E}-02$ & $8.26 \mathrm{E}-04$ & $2.30 \mathrm{E}+04$ \\
\hline $241-S-107$ & Total & & & $4.43 E+03$ & $1.41 \mathrm{E}+05$ & $7.40 \mathrm{E}+04$ & $2.22 E+05$ & $3.61 \mathrm{E}+05$ & $3.12 \mathrm{E}+05$ & $7.46 \mathrm{E}+02$ & $2.54 \mathrm{E}+02$ & $1.21 \mathrm{E}+03$ & $2.94 E+01$ & $1.63 E+05$ \\
\hline $241-S-107$ & Sludge (Liquid \& Solid) & R1 (Solid) & 462 & $1.17 \mathrm{E}+03$ & $5.00 \mathrm{E}+04$ & $2.65 E+04$ & $7.29 \mathrm{E}+04$ & $1.35 \mathrm{E}+05$ & $1.13 \mathrm{E}+05$ & $2.16 \mathrm{E}+02$ & $8.29 \mathrm{E}+01$ & $4.27 \mathrm{E}+02$ & $7.92 \mathrm{E}+00$ & $5.84 \mathrm{E}+04$ \\
\hline $241-S-107$ & Sludge (Liquid \& Solid) & CWZrl (Solid) & 91 & $2.30 \mathrm{E}+02$ & $9.84 \mathrm{E}+03$ & $5.23 \mathrm{E}+03$ & $1.43 \mathrm{E}+04$ & $0.00 \mathrm{E}+00$ & $2.22 \mathrm{E}+04$ & $5.87 \mathrm{E}+01$ & $2.37 \mathrm{E}+01$ & $6.12 \mathrm{E}+01$ & $6.08 E+00$ & $1.15 \mathrm{E}+04$ \\
\hline $241-S-107$ & Sludge (Liquid \& Solid) & CWR2 (Solid) & 211 & $5.33 \mathrm{E}+02$ & $2.28 \mathrm{E}+04$ & $1.21 \mathrm{E}+04$ & $3.33 E+04$ & $5.70 \mathrm{E}+04$ & $5.14 \mathrm{E}+04$ & $2.29 \mathrm{E}+01$ & $5.57 \mathrm{E}+01$ & $2.54 \mathrm{E}+02$ & $6.33 E+00$ & $2.67 \mathrm{E}+04$ \\
\hline
\end{tabular}




\section{RPP-5926 REV 6}

Table A-2. Tank Waste Inventory from Best Basis Inventory Detail Report (Queried on September 27, 2006) for

Hydrogen Generation Rate Calculations. (27 sheets)

\begin{tabular}{|c|c|c|c|c|c|c|c|c|c|c|c|c|c|c|}
\hline $\begin{array}{c}\text { Tank } \\
\text { Name }\end{array}$ & Was & ype & $\begin{array}{l}\text { Vol } \\
(k L)\end{array}$ & $\begin{array}{l}\text { TOC } \\
\text { (Kg) }\end{array}$ & $\begin{array}{l}\mathrm{NO}_{3} \\
(\mathrm{Kg})\end{array}$ & $\begin{array}{l}\mathrm{NO}_{2} \\
(\mathrm{Kg})\end{array}$ & $\begin{array}{c}\mathrm{Na} \\
(\mathrm{Kg})\end{array}$ & $\begin{array}{c}\mathrm{Al} \\
(\mathrm{Kg})\end{array}$ & $\begin{array}{l}{ }^{90} \mathrm{Sr} \\
\text { (Ci) }\end{array}$ & $\begin{array}{l}{ }_{(\mathrm{Ci})}^{241} \mathrm{Am} \\
\end{array}$ & $\begin{array}{l}{ }^{240} \mathbf{P u} \\
\text { Ci) }\end{array}$ & $\begin{array}{c}{ }^{239} \mathbf{P u} \\
(\mathrm{Ci})\end{array}$ & ${ }_{(\mathrm{Ci})}^{{ }^{238} \mathrm{Pu}}$ & $\begin{array}{c}{ }^{137} \mathrm{Cs} \\
\text { (Ci) }\end{array}$ \\
\hline $241-S-107$ & Sludge (Liquid \& Solid) & CWR1 (Solid) & 447 & $3 \mathrm{E}+03$ & $.83 \mathrm{E}+04$ & $57 \mathrm{E}+04$ & $.05 E+04$ & $65 E+05$ & $.09 E+05$ & $2.47 \mathrm{E}+02$ & $29 \mathrm{E}+01$ & $3.84 \mathrm{E}+02$ & $5.97 \mathrm{E}+00$ & $5.65 \mathrm{E}+04$ \\
\hline $241-S-107$ & Saltcake Solid & T2-SltCk (Solid) & 52 & $4.93 E+02$ & $3.44 \mathrm{E}+03$ & $1.60 \mathrm{E}+03$ & $1.12 E+04$ & $1.69 \mathrm{E}+03$ & $6.04 \mathrm{E}+03$ & $6.91 \mathrm{E}+01$ & $7.19 \mathrm{E}+00$ & $3.43 E+01$ & $1.21 E+00$ & $3.53 \mathrm{E}+03$ \\
\hline $241-S-107$ & Saltcake Solid & S2-SltSlr (Solid) & 30 & $4 \mathrm{E}+02$ & $1.99 \mathrm{E}+03$ & $9.22 \mathrm{E}+02$ & $47 \mathrm{E}+03$ & $3 \mathrm{E}+02$ & $3.48 \mathrm{E}+03$ & $3.51 E+01$ & $5.04 \mathrm{E}+00$ & $2.35 \mathrm{E}+01$ & $8.20 \mathrm{E}-01$ & $2.03 \mathrm{E}+03$ \\
\hline $241-S-107$ & olid & id) & 63 & $7 E+02$ & $17 \mathrm{E}+03$ & $94 \mathrm{E}+03$ & $36 \mathrm{E}+04$ & $04 \mathrm{E}+03$ & $7.31 \mathrm{E}+03$ & $9.72 \mathrm{E}+01$ & $6.57 \mathrm{E}+00$ & $3.01 E+01$ & $1.06 \mathrm{E}+00$ & $4.27 \mathrm{E}+03$ \\
\hline $241-S-108$ & Total & & & $1.24 \mathrm{E}+04$ & $1.06 \mathrm{E}+06$ & $1.50 \mathrm{E}+05$ & $7.45 E+05$ & $6.14 \mathrm{E}+04$ & $7.32 \mathrm{E}+04$ & $2.69 \mathrm{E}+02$ & $2.09 \mathrm{E}+01$ & $9.75 \mathrm{E}+01$ & $3.16 \mathrm{E}+00$ & $3.35 \mathrm{E}+05$ \\
\hline $241-S-108$ & Sludge (Liquid \& & d) & 19 & $3.53 \mathrm{E}+01$ & $3.77 \mathrm{E}+03$ & $1.06 \mathrm{E}+03$ & $E+03$ & $6.29 \mathrm{E}+03$ & $1.38 \mathrm{E}+04$ & $8.42 \mathrm{E}+00$ & $E+00$ & $\mathrm{E}+01$ & 01 & $2.12 \mathrm{E}+03$ \\
\hline 241-S-108 & Saltcake Solid & S1-SltCk (Solid) & 2032 & $23 E+04$ & $1.05 \mathrm{E}+06$ & $1.45 \mathrm{E}+05$ & $7.34 \mathrm{E}+05$ & $39 \mathrm{E}+04$ & $5.94 \mathrm{E}+04$ & $2.61 \mathrm{E}+02$ & $1.76 \mathrm{E}+01$ & $8.09 \mathrm{E}+01$ & $2.85 \mathrm{E}+00$ & $3.22 \mathrm{E}+05$ \\
\hline $241-S-108$ & Saltcake Interstitial L & -SltCk (Liquid) & 31 & $5 \mathrm{E}+02$ & $\mathrm{E}+03$ & $\mathrm{E}+03$ & $7.32 \mathrm{E}+03$ & $1.17 \mathrm{E}+03$ & $3.09 \mathrm{E}+01$ & $8.73 \mathrm{E}-02$ & $8.24 \mathrm{E}-04$ & $3.78 \mathrm{E}-03$ & $1.33 \mathrm{E}-04$ & $1.10 \mathrm{E}+04$ \\
\hline $241-S-109$ & Total & & & +03 & $1.72 \mathrm{E}+06$ & $2.27 \mathrm{E}+04$ & $7.70 \mathrm{E}+05$ & +04 & $E+04$ & +01 & \pm+00 & +01 & 01 & $4.36 \mathrm{E}+04$ \\
\hline 241-S-109 & Sludge (Liquid \& Solid) & R1 (Solid) & 49 & $9.11 \mathrm{E}+01$ & $9.71 \mathrm{E}+03$ & $2.74 \mathrm{E}+03$ & $8.76 \mathrm{E}+03$ & $1.62 \mathrm{E}+04$ & $3.55 \mathrm{E}+04$ & $2.17 \mathrm{E}+01$ & $8.33 \mathrm{E}+00$ & $4.29 \mathrm{E}+01$ & $7.96 \mathrm{E}-01$ & $5.47 \mathrm{E}+03$ \\
\hline $24 !-S-109$ & iत & 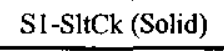 & 005 & $E+03$ & $E+06$ & $\mathrm{~F}+\mathrm{A}$ & $7.48 \mathrm{E}+05$ & $5.44 \mathrm{E}+03$ & $1.40 \mathrm{E}+04$ & $1.66 \mathrm{E}+01$ & $1.12 \mathrm{E}+00$ & $5.14 \mathrm{E}+00$ & $1.81 \mathrm{E}-01$ & $2.02 \mathrm{E}+04$ \\
\hline 241-S-109 & altcake Interstitial Liquid & S1-SltCk & 63 & $7.02 \mathrm{E}+01$ & $8.29 E+03$ & $5.35 \mathrm{E}+03$ & $1.38 \mathrm{E}+04$ & $2.89 \mathrm{E}+03$ & $1.53 \mathrm{E}+01$ & $1.69 \mathrm{E}-02$ & $6.04 \mathrm{E}-04$ & $2.77 \mathrm{E}-03$ & $9.77 \mathrm{E}-05$ & $1.79 E+04$ \\
\hline $241-S-110$ & Total & & & $5.49 \mathrm{E}+03$ & $9.05 \mathrm{E}+05$ & $5.41 \mathrm{E}+04$ & $4.82 \mathrm{E}+05$ & $1.25 \mathrm{E}+05$ & $2.30 \mathrm{E}+05$ & $3.02 \mathrm{E}+02$ & $6.11 \mathrm{E}+01$ & $3.10 \mathrm{E}+02$ & $6.43 \mathrm{E}+00$ & $1.78 \mathrm{E}+05$ \\
\hline 241-S-110 & Slue & 1) & 288 & +02 & 5.10 & 34 & +04 & $8.57 E+04$ & $1.48 \mathrm{E}+05$ & $1.05 E+02$ & $4.04 \mathrm{E}+01$ & $E \div 02$ & $3.86 \mathrm{E}+00$ & $3.86 \mathrm{E}+04$ \\
\hline $241-S-110$ & Sludge (Liquid \& Solid) & CWR1 (Solid) & 76 & $4.92 \mathrm{E}+01$ & $1.35 \mathrm{E}+04$ & $3.71 \mathrm{E}+03$ & $1.25 \mathrm{E}+04$ & $2.26 \mathrm{E}+04$ & $3.90 \mathrm{E}+04$ & $3.28 \mathrm{E}+01$ & $9.69 \mathrm{E}+00$ & $5.10 \mathrm{E}+01$ & $7.93 \mathrm{E}-01$ & $1.02 \mathrm{E}+04$ \\
\hline $241-S-110$ & Saltcake Solid & S1-Sltck (Solid) & 994 & $4.99 \mathrm{E}+03$ & $8.15 \mathrm{E}+05$ & $2.65 \mathrm{E}+04$ & $3.98 \mathrm{E}+05$ & $1.28 \mathrm{E}+04$ & $4.32 \mathrm{E}+04$ & $1.63 \mathrm{E}+02$ & $1.11 \mathrm{E}+01$ & $5.07 \mathrm{E}+01$ & $1.78 \mathrm{E}+00$ & $8.91 \mathrm{E}+04$ \\
\hline $241-S-110$ & Saltcake Interstitial $\mathrm{Li}$ & 1-Sltck (Liquid) & 115 & $2.62 \mathrm{E}+02$ & $2.50 \mathrm{E}+04$ & $9.87 \mathrm{E}+03$ & $2.43 \mathrm{E}+04$ & $3.56 \mathrm{E}+03$ & $1.12 \mathrm{E}+02$ & $8.28 \mathrm{E}-01$ & $7.81 \mathrm{E}-03$ & $3.58 \mathrm{E}-02$ & $1.26 \mathrm{E}-03$ & $4.02 \mathrm{E}+04$ \\
\hline $241-S-111$ & Total & & & $4.07 \mathrm{E}+03$ & $5.03 \mathrm{E}+05$ & $6.64 \mathrm{E}+04$ & $3.84 \mathrm{E}+05$ & $1.23 E+05$ & $3.95 \mathrm{E}+05$ & $3.20 \mathrm{E}+01$ & $3.10 \mathrm{E}+00$ & $1.49 \mathrm{E}+0 !$ & $4.23 \mathrm{E}-01$ & $2.22 \mathrm{E}+05$ \\
\hline $241-S-111$ & Sludge (Liquid \& Solid) & R1 (Solid) & 207 & $3.17 \mathrm{E}+02$ & $1.38 \mathrm{E}+04$ & $8.48 \mathrm{E}+03$ & $2.05 \mathrm{E}+04$ & $7.45 \mathrm{E}+04$ & $1.48 \mathrm{E}+05$ & $2.56 \mathrm{E}+00$ & $9.82 \mathrm{E}-01$ & $5.06 \mathrm{E}+00$ & $9.38 \mathrm{E}-02$ & $2.57 \mathrm{E}+04$ \\
\hline $241-S-111$ & Sludge (Liquid \& Solid) & CWR1 (Solid) & 38 & $5.81 \mathrm{E}+01$ & $2.53 \mathrm{E}+03$ & $1.56 \mathrm{E}+03$ & $3.76 E+03$ & $1.37 \mathrm{E}+04$ & $1.26 \mathrm{E}+02$ & $5.55 \mathrm{E}-01$ & $1.64 \mathrm{E}-01$ & $8.62 \mathrm{E}-01$ & $1.34 \mathrm{E}-02$ & $4.72 \mathrm{E}+03$ \\
\hline $241-S-111$ & Saltcake Solid & S1-SltCk (Solid) & 1028 & $3.51 \mathrm{E}+03$ & $4.62 \mathrm{E}+05$ & $4.60 \mathrm{E}+04$ & $3.32 \mathrm{E}+05$ & $3.04 \mathrm{E}+04$ & $2.48 \mathrm{E}+05$ & $2.89 \mathrm{E}+01$ & $1.96 \mathrm{E}+00$ & $8.97 \mathrm{E}+00$ & $3.16 \mathrm{E}-01$ & $1.54 \mathrm{E}+05$ \\
\hline $241-S-111$ & altcake Interstitial Liquid & 1-SltCk (Liquid) & 124 & $1.85 \mathrm{E}+02$ & $2.39 \mathrm{E}+04$ & $1.04 E+04$ & $2.76 \mathrm{E}+04$ & $4.51 \mathrm{E}+03$ & $1.40 \mathrm{E}+01$ & $1.91 \mathrm{E}-02$ & $3.18 \mathrm{E}-03$ & $1.46 \mathrm{E}-02$ & $5.13 \mathrm{E}-04$ & $3.72 \mathrm{E}+04$ \\
\hline $241-S-112$ & Total & & & $5.74 \mathrm{E}+02$ & $9.91 \mathrm{E}+02$ & $2.80 \mathrm{E}+02$ & $2.71 \mathrm{E}+03$ & $1.65 \mathrm{E}+03$ & $2.04 \mathrm{E}+04$ & $1.70 \mathrm{E}+01$ & $1.85 \mathrm{E}+00$ & $1.04 \mathrm{E}+01$ & $2.35 \mathrm{E}-01$ & $5.58 \mathrm{E}+02$ \\
\hline 241-S-112 & Sludge (Liquid \& Solid) & R1 (Solid) & 5 & $9.29 \mathrm{E}+00$ & $9.91 \mathrm{E}+02$ & $2.80 \mathrm{E}+02$ & $8.94 \mathrm{E}+02$ & $1.65 E+03$ & $3.62 \mathrm{E}+03$ & $2.22 \mathrm{E}+00$ & $8.50 \mathrm{E}-01$ & $4.38 \mathrm{E}+00$ & $8.12 \mathrm{E}-02$ & $5.58 \mathrm{E}+02$ \\
\hline $241-S-112$ & Saltcake Solid & 1-SltCk (Solid) & 14 & $5.65 \mathrm{E}+02$ & $0.00 \mathrm{E}+00$ & $0.00 \mathrm{E}+00$ & $1.81 \mathrm{E}+03$ & $0.00 \mathrm{E}+00$ & $1.68 \mathrm{E}+04$ & $1.48 E+01$ & $9.97 \mathrm{E}-01$ & $6.03 E+00$ & $1.54 \mathrm{E}-01$ & $0.00 \mathrm{E}+00$ \\
\hline 241-SX-101 & Total & & & $5.13 \mathrm{E}+03$ & $7.76 \mathrm{E}+05$ & $6.50 \mathrm{E}+04$ & $5.28 \mathrm{E}+05$ & $1.62 \mathrm{E}+05$ & $3.02 \mathrm{E}+05$ & $5.08 \mathrm{E}+02$ & $9.02 \mathrm{E}+01$ & $4.42 \mathrm{E}+02$ & $1.22 \mathrm{E}+01$ & $2.67 \mathrm{E}+05$ \\
\hline $241-S X-101$ & Sludge (Liquid \& Solid) & R1 (Solid) & 545 & $1.85 \mathrm{E}+03$ & $2.78 \mathrm{E}+05$ & $2.14 \mathrm{E}+04$ & $1.84 \mathrm{E}+05$ & 5.63E+04 & $1.09 \mathrm{E}+05$ & $1.13 \mathrm{E}+02$ & $4.32 \mathrm{E}+01$ & $2.23 E+02$ & $4.13 E+00$ & $8.66 \mathrm{E}+04$ \\
\hline
\end{tabular}




\section{RPP-5926 REV 6}

Table A-2. Tank Waste Inventory from Best Basis Inventory Detail Report (Queried on September 27, 2006) for Hydrogen Generation Rate Calculations. (27 sheets)

\begin{tabular}{|c|c|c|c|c|c|c|c|c|c|c|c|c|c|c|}
\hline $\begin{array}{l}\text { Tank } \\
\text { Name }\end{array}$ & Waste Phase & Type & $\begin{array}{l}\text { Vol } \\
(k L)\end{array}$ & $\begin{array}{l}\text { TOC } \\
\text { (Kg) }\end{array}$ & $\begin{array}{l}\mathrm{NO}_{3} \\
(\mathrm{Kg})\end{array}$ & $\begin{array}{l}\mathrm{NO}_{2} \\
(\mathrm{Kg})\end{array}$ & $\begin{array}{c}\mathbf{N a} \\
(\mathbf{K g})\end{array}$ & $\begin{array}{c}\mathrm{Al} \\
(\mathrm{Kg})\end{array}$ & $\begin{array}{l}{ }^{90} \mathrm{Sr} \\
(\mathrm{Ci})\end{array}$ & $\begin{array}{c}{ }^{241} \mathrm{Am} \\
\text { (Ci) }\end{array}$ & ${ }^{{ }^{240}} \mathrm{Pu}$ & $\begin{array}{c}{ }^{{ }^{339}} \mathrm{Pu} \\
\mathrm{Ci})\end{array}$ & 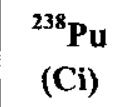 & $\begin{array}{c}{ }^{137} \mathrm{Cs} \\
\text { (Ci) }\end{array}$ \\
\hline 01 & id & lid) & 166 & 02 & 4 & $51 E+03$ & $1 \mathrm{E}+04$ & +04 & $3.33 \mathrm{E}+04$ & $31 \mathrm{E}+01$ & $05 \mathrm{E}+00$ & $4.22 \mathrm{E}+01$ & $7 \mathrm{E}+00$ & 04 \\
\hline $24 I-S X-101$ & Saltcake Solid & S1-SltCk (Solid) & 296 & $1.00 \mathrm{E}+03$ & $\overline{E+05}$ & $16 \mathrm{E}+04$ & $1.00 \mathrm{E}+05$ & $3.06 \mathrm{E}+04$ & $5.94 \mathrm{E}+04$ & $1.49 E+02$ & $1.00 \mathrm{E}+01$ & $4.61 E+01$ & $1.62 \mathrm{E}+00$ & $4.70 \mathrm{E}+04$ \\
\hline 101 & Saltc & olid) & 497 & $9 \mathrm{E}+03$ & +05 & $E+04$ & $3 E+05$ & +04 & $8 \mathrm{E}+04$ & $E+02$ & $8 \mathrm{E}+01$ & $1 \mathrm{E}+02$ & $.99 \mathrm{E}+00$ & $7.89 E+04$ \\
\hline 241-SX-101 & Saltcake Interstitial Liquid & S2-SitSir (Liquid) & 14 & $4.69 \mathrm{E}+00$ & $1.51 E+03$ & $1.05 E+03$ & $3.39 \mathrm{E}+03$ & $1.12 \mathrm{E}+03$ & $9.81 \mathrm{E}-01$ & $7.67 \mathrm{E}-03$ & $2.88 \mathrm{E}-04$ & $1.34 \mathrm{E}-0.3$ & 4.69E-05 & $4.96 \mathrm{E}+03$ \\
\hline 241-SX-101 & Saltcake Interstitial Liquid & S1-SltCk (Liquid) & 25 & $8.37 \mathrm{E}+00$ & $70 \mathrm{E}+03$ & $1.87 \mathrm{E}+03$ & $6.06 \mathrm{E}+03$ & $2.00 \mathrm{E}+03$ & $1.75 E+00$ & $1.37 \mathrm{E}-02$ & $5.21 \mathrm{E}-04$ & $2.39 \mathrm{E}-03$ & $8.43 \mathrm{E}-05$ & $8.85 E+03$ \\
\hline-101 & Saltcake Interstitial Liquid & $\mathrm{R}-\mathrm{Slt}$ & 41 & $1.37 \mathrm{E}+01$ & $2 \mathrm{E}+03$ & $3.07 \mathrm{E}+03$ & $93 \mathrm{E}+03$ & $3.28 \mathrm{E}+03$ & $2.87 \mathrm{E}+00$ & $2.24 E-02$ & $8.38 \mathrm{E}-04$ & $.94 \mathrm{E}-03$ & $.50 \mathrm{E}-04$ & $1.45 \mathrm{E}+04$ \\
\hline $241-\mathrm{SX}-102$ & Total & & & $\mathrm{E}+03$ & $4.88 \mathrm{E}+05$ & $1.60 \mathrm{E}+05$ & $.05 \mathrm{E}+05$ & $5.70 \mathrm{E}+04$ & $1.80 \mathrm{E}+05$ & $2.68 \mathrm{E}+02$ & $2.65 \mathrm{E}+01$ & $1.27 \mathrm{E}+02$ & $3.71 E+00$ & $3.07 \mathrm{E}+05$ \\
\hline 102 & (Liquil & id) & 209 & $1.48 \mathrm{E}+03$ & $35 \mathrm{E}+04$ & $2.53 \mathrm{E}+04$ & $71 \mathrm{E}+04$ & $9.19 \mathrm{E}+03$ & $3.19 \mathrm{E}+04$ & $2.23 \mathrm{E}+01$ & $8.55 \mathrm{E}+00$ & $4.41 \mathrm{E}+01$ & $8.17 \mathrm{E}-01$ & $4.65 \mathrm{E}+04$ \\
\hline 241-SX-102 & Saltcake Solid & S2-SltSlr (Solid) & 92 & $6.50 \mathrm{E}+02$ & $3.68 \mathrm{E}+04$ & $1.11 \mathrm{E}+04$ & $2.95 \mathrm{E}+04$ & $4.05 \mathrm{E}+03$ & $1.41 \mathrm{E}+04$ & $1.81 \mathrm{E}+01$ & $2.59 \mathrm{E}+00$ & $1.21 \mathrm{E}+01$ & $4.21 \mathrm{E}-01$ & $2.05 \mathrm{E}+04$ \\
\hline $24 \mathrm{I}-\mathrm{SX}-102$ & Saltcake Solid & S1-SltCk (Solid) & 875 & $6.18 \mathrm{E}+03$ & $.50 \mathrm{E}+05$ & $1.06 \mathrm{E}+05$ & $2.81 \mathrm{E}+05$ & $3.85 \mathrm{E}+04$ & $1.34 \mathrm{E}+05$ & $2.27 \mathrm{E}+02$ & $1.53 \mathrm{E}+01$ & $7.03 \mathrm{E}+01$ & $2.47 \mathrm{E}+00$ & $1.95 E+05$ \\
\hline 241-SX-102 & Saltcake interstital Liquid & S2-S1tSlr (Liquid) & 11 & $1.66 \mathrm{E}+01$ & $1.73 \mathrm{E}+03$ & $1.66 \mathrm{E}+03$ & $2.59 \mathrm{E}+03$ & $5.02 \mathrm{E}+02$ & $2.28 \mathrm{E}-01$ & $9.18 \mathrm{E}-02$ & $8.60 \mathrm{E}-04$ & $4.01 \mathrm{E}-03$ & $1.40 \mathrm{E}-04$ & $4.29 \mathrm{E}+03$ \\
\hline 241-SX-102 & Saltcake Interstitial Liquid & S1-SItCk & 105 & $1.59 \mathrm{E}+02$ & $1.65 \mathrm{E}+04$ & $1.59 \mathrm{E}+04$ & $2.47 \mathrm{E}+04$ & $4.79 \mathrm{E}+03$ & $2.18 \mathrm{E}+00$ & $8.77 \mathrm{E}-01$ & $8.28 \mathrm{E}-03$ & $3.80 \mathrm{E}-02$ & $1.34 \mathrm{E}-03$ & $4.10 \mathrm{E}+04$ \\
\hline $241-\mathrm{SX}-103$ & Total & & & $1.29 \mathrm{E}+04$ & $89 \mathrm{E}+05$ & $1.80 \mathrm{E}+05$ & $42 \mathrm{E}+05$ & $1.52 \mathrm{E}+05$ & $E+05$ & $7.20 \mathrm{E}+02$ & $\mathrm{E}+01$ & $\mathrm{E}+02$ & $2 \mathrm{E}+00$ & $4.12 \mathrm{E}+05$ \\
\hline $241-S X-103$ & Sludge (Liquid \& Solid) & Rl (Solid) & 294 & $1.83 \mathrm{E}+03$ & $8.90 \mathrm{E}+04$ & $2.26 \mathrm{E}+04$ & $5.67 \mathrm{E}+04$ & $1.02 \mathrm{E}+05$ & $3.50 \mathrm{E}+05$ & $6.75 \mathrm{E}+01$ & $2.59 E+01$ & $1.33 \mathrm{E}+02$ & $2.48 \mathrm{E}+00$ & $4.45 \mathrm{E}+04$ \\
\hline 241-SX-103 & Saltonk Colid & 1-Sltck (Solid) & 1439 & $9.91 \mathrm{E}+03$ & $8.60 \mathrm{E}+05$ & $1.30 \mathrm{E}+05$ & $5.34 \mathrm{E}+05$ & $3.96 \mathrm{E}+04$ & $1.00 \mathrm{E}+05$ & $5.98 \mathrm{E}+02$ & $1.78 \mathrm{E}+01$ & $E+01$ & $.88 \mathrm{E}+00$ & $2.99 \mathrm{E}+05$ \\
\hline $241-S X-103$ & Saltcake Solid & R-SltCk (Solid) & 77 & $7.26 \mathrm{E}+02$ & $2.22 E+04$ & $9.34 \mathrm{E}+03$ & $2.40 \mathrm{E}+04$ & $5.57 \mathrm{E}+03$ & $6.29 \mathrm{E}+04$ & $5.42 \mathrm{E}+01$ & $8.20 E+00$ & $3.86 \mathrm{E}+01$ & $1.47 \mathrm{E}+00$ & $2.07 E+04$ \\
\hline 241-SX-103 & Saltcake Interstitial Liquid & -SltCk (Liquid) & 94 & $3.18 \mathrm{E}+02$ & $1.47 \mathrm{E}+04$ & $1.48 \mathrm{E}+04$ & $2.26 \mathrm{E}+04$ & $4.17 \mathrm{E}+03$ & $7.75 \mathrm{E}+00$ & $4.37 \mathrm{E}-02$ & $1.65 \mathrm{E}-03$ & $7.58 \mathrm{E}-03$ & $2.67 \mathrm{E}-04$ & $3.81 E+04$ \\
\hline $241-S X-103$ & Saltca & R-Sl & 22 & +01 & 03 & $E+03$ & 03 & +02 & $\mathrm{E}+00$ & $1.02 \mathrm{E}-02$ & $3.79 \mathrm{E}-04$ & $1.78 \mathrm{E}-03$ & $6.78 \mathrm{E}-05$ & $8.92 \mathrm{E}+03$ \\
\hline 241-SX-104 & Total & & & $7.04 \mathrm{E}+03$ & $7.13 \mathrm{E}+05$ & $1.13 \mathrm{E}+05$ & $5.05 \mathrm{E}+05$ & $2.10 \mathrm{E}+05$ & $4.05 \mathrm{E}+05$ & $3.60 \mathrm{E}+02$ & $9.78 \mathrm{E}+01$ & $4.98 \mathrm{E}+02$ & $1.01 \mathrm{E}+01$ & $2.49 E+05$ \\
\hline 241-SX-104 & Sludge (Liqui & (Solid) & 515 & $9.57 \mathrm{E}+02$ & $1.02 \mathrm{E}+05$ & $E+04$ & $9.21 \mathrm{E}+04$ & $1.70 \mathrm{E}+05$ & $3.73 E+05$ & $E+02$ & $6 \mathrm{E}+01$ & $4.51 \mathrm{E}+02$ & $8.37 \mathrm{E}+00$ & $5.75 \mathrm{E}+04$ \\
\hline 241-SX-104 & Saltcake Solid & Si-SitCk (Solid) & 903 & $5.45 \mathrm{E}+03$ & $4.66 \mathrm{E}+05$ & $6.45 \mathrm{E}+04$ & $3.26 \mathrm{E}+05$ & $2.40 \mathrm{E}+04$ & $2.64 \mathrm{E}+04$ & $1.16 \mathrm{E}+02$ & $7.84 \mathrm{E}+00$ & $3.60 \mathrm{E}+01$ & $1.27 E+00$ & $1.43 E+05$ \\
\hline $241-S X-104$ & Saltcake Solid & R-SltCk (Solid) & 142 & $3.66 \mathrm{E}+02$ & $1.08 \mathrm{E}+05$ & $4.49 \mathrm{E}+03$ & $5.47 \mathrm{E}+04$ & $1.10 \mathrm{E}+04$ & $5.68 \mathrm{E}+03$ & $1.59 \mathrm{E}+01$ & $2.41 \mathrm{E}+00$ & $1.13 E+01$ & $4.32 \mathrm{E}-01$ & $1.91 E+04$ \\
\hline 241-SX-104 & altcake Interstitial Liquid & 1-SltCk (Liquid) & 17 & $3.61 \mathrm{E}+01$ & $4.93 E+03$ & $1.99 \mathrm{E}+03$ & $4.26 E+03$ & $6.04 \mathrm{E}+02$ & $1.35 \mathrm{E}+00$ & $4.83 \mathrm{E}-03$ & $4.78 \mathrm{E}-04$ & $2.19 \mathrm{E}-03$ & $7.72 \mathrm{E}-05$ & $3.90 \mathrm{E}+03$ \\
\hline 241-SX-104 & Saltcake Interstitial Liquid & R-SltCk (Liquid) & 111 & $2.36 \mathrm{E}+02$ & $3.22 \mathrm{E}+04$ & $1.30 \mathrm{E}+04$ & $2.78 \mathrm{E}+04$ & $3.95 \mathrm{E}+03$ & $8.79 \mathrm{E}+00$ & $3.15 \mathrm{E}-02$ & $3.05 \mathrm{E}-03$ & $1.44 \mathrm{E}-02$ & $5.47 \mathrm{E}-04$ & $2.55 \mathrm{E}+04$ \\
\hline $241-S X-105$ & Total & & & $6.64 \mathrm{E}+03$ & $4.84 \mathrm{E}+05$ & $1.60 \mathrm{E}+05$ & $4.28 \mathrm{E}+05$ & $1.01 \mathrm{E}+05$ & $5.86 \mathrm{E}+05$ & $5.96 \mathrm{E}+02$ & $1.33 \mathrm{E}+02$ & $6.69 \mathrm{E}+02$ & $1.54 \mathrm{E}+01$ & $2.19 E+05$ \\
\hline $241-\mathrm{SX}-105$ & Sludge (Liquid \& Solid) & R2 (Solid) & 49 & $1.37 \mathrm{E}+02$ & $1.16 \mathrm{E}+04$ & 4.48E+03 & $1.09 \mathrm{E}+04$ & $1.16 \mathrm{E}+04$ & $4.29 \mathrm{E}+05$ & $1.93 \mathrm{E}+02$ & $1.18 \mathrm{E}+01$ & $5.04 \mathrm{E}+01$ & $3.37 \mathrm{E}+00$ & $9.87 \mathrm{E}+03$ \\
\hline $24 !-S X-105$ & Sludge (Liquid \& Solid) & R1.(Solid) & 189 & $5.30 \mathrm{E}+02$ & $4.48 E+04$ & $1.73 \mathrm{E}+04$ & $4.20 \mathrm{E}+04$ & $4.48 \mathrm{E}+04$ & $1.29 \mathrm{E}+05$ & $2.96 \mathrm{E}+02$ & $1.14 \mathrm{E}+02$ & $5.86 \mathrm{E}+02$ & $1.09 \mathrm{E}+01$ & $1.99 \mathrm{E}+04$ \\
\hline
\end{tabular}




\section{RPP-5926 REV 6}

Table A-2. Tank Waste Inventory from Best Basis Inventory Detail Report (Queried on September 27, 2006) for

Hydrogen Generation Rate Calculations. (27 sheets)

\begin{tabular}{|c|c|c|c|c|c|c|c|c|c|c|c|c|c|c|}
\hline $\begin{array}{c}\text { Tank } \\
\text { Name }\end{array}$ & Was & Type & $\begin{array}{c}\text { Vol } \\
\text { (kL) }\end{array}$ & $\begin{array}{l}\text { TOC } \\
\text { (Kg) }\end{array}$ & $\begin{array}{l}\mathrm{NO}_{3} \\
(\mathrm{Kg})\end{array}$ & $\begin{array}{l}\mathrm{NO}_{2} \\
(\mathrm{Kg})\end{array}$ & $\begin{array}{c}\mathrm{Na} \\
(\mathrm{Kg})\end{array}$ & $\begin{array}{c}\mathrm{Al} \\
(\mathrm{Kg})\end{array}$ & $\begin{array}{l}{ }^{90} \mathrm{Sr} \\
(\mathrm{Ci})\end{array}$ & $\begin{array}{c}{ }^{241} \mathrm{Am} \\
\text { (Ci) }\end{array}$ & $\begin{array}{c}{ }^{240} \mathrm{Pu} \\
\text { Ci) }\end{array}$ & $\begin{array}{c}{ }^{239} \mathrm{Pu} \\
(\mathrm{Ci})\end{array}$ & $\begin{array}{c}{ }^{238} \mathrm{Pu} \\
(\mathrm{Ci})\end{array}$ & $\begin{array}{c}{ }^{137} \mathrm{Cs} \\
\text { (Ci) }\end{array}$ \\
\hline $241-\$ X-105$ & Saltcake Solid & S1-SltCk (Solid) & 1061 & $5.57 \mathrm{E}+03$ & $4.07 \mathrm{E}+05$ & $1.21 E+05$ & $3.46 \mathrm{E}+05$ & $3.88 \mathrm{E}+04$ & $2.73 E+04$ & $1.06 \mathrm{E}+02$ & $7.19 E+00$ & $3.30 \mathrm{E}+01$ & $1.16 \mathrm{E}+00$ & $1.48 \mathrm{E}+05$ \\
\hline $241-5$ & Saltcake Interstit & $\mathrm{s}$ & 123 & $4.01 \mathrm{E}+02$ & $2 E+04$ & $6 \mathrm{E}+04$ & $90 \mathrm{E}+04$ & $2 \mathrm{E}+03$ & $1.73 \mathrm{E}+01$ & $6.12 \mathrm{E}-01$ & $50 \mathrm{E}-03$ & $1.61 \mathrm{E}-02$ & $5.66 \mathrm{E}-04$ & $4.17 \mathrm{E}+04$ \\
\hline 241-SX-106 & Total & & & $8.24 \mathrm{E}+03$ & $5.52 \mathrm{E}+05$ & $1.69 \mathrm{E}+05$ & $4.44 \mathrm{E}+05$ & $4.93 \mathrm{E}+04$ & $2.87 \mathrm{E}+04$ & $3.49 \mathrm{E}+02$ & $4.41 \mathrm{E}+01$ & $2.05 \mathrm{E}+02$ & $7.17 \mathrm{E}+00$ & $3.11 \mathrm{E}+05$ \\
\hline $241-\mathrm{SX}-106$ & Saltcake Solid & $\mathrm{S} 2-\mathrm{Sl}$ & 1107 & $6.36 \mathrm{E}+03$ & $22 \mathrm{E}+05$ & $1.20 \mathrm{E}+05$ & $3.37 \mathrm{E}+05$ & $3.76 \mathrm{E}+04$ & $2.27 \mathrm{E}+04$ & $2.70 \mathrm{E}+02$ & $3.88 \mathrm{E}+01$ & $1.81 \mathrm{E}+02$ & $6.31 \mathrm{E}+00$ & $2.29 \mathrm{E}+05$ \\
\hline 241-SX-106 & Saltcake Solid & S1-SitCk (Solid) & 245 & $1.41 \mathrm{E}+03$ & $9.35 \mathrm{E}+04$ & $2.67 \mathrm{E}+04$ & $7.46 \mathrm{E}+04$ & $8.32 \mathrm{E}+03$ & $6.00 \mathrm{E}+03$ & $7.89 \mathrm{E}+01$ & $5.34 \mathrm{E}+00$ & $2.45 \mathrm{E}+01$ & $8.62 \mathrm{E}-01$ & $3.25 \mathrm{E}+04$ \\
\hline $241-5 X-106$ & Saltcake Interstitial Liquid & S2-S1tS1r (Liquid) & 122 & $3.83 \mathrm{E}+02$ & $2.99 \mathrm{E}+04$ & $1.82 \mathrm{E}+04$ & $2.65 E+04$ & $2.76 \mathrm{E}+03$ & $3.64 \mathrm{E}+01$ & $3.83 \mathrm{E}-02$ & $1.09 \mathrm{E}-03$ & $5.07 \mathrm{E}-03$ & $1.77 \mathrm{E}-04$ & $4.06 \mathrm{E}+04$ \\
\hline $241-\mathrm{SX}-106$ & Saltcake Interstitial Liquid & S1-SltCk (Liquid) & 27 & $8.48 \mathrm{E}+01$ & $6.61 \mathrm{E}+03$ & $4.02 \mathrm{E}+03$ & $5.87 \mathrm{E}+03$ & $6.12 \mathrm{E}+02$ & $8.06 \mathrm{E}+00$ & $8.47 \mathrm{E}-03$ & $2.44 \mathrm{E}-04$ & $1.12 \mathrm{E}-03$ & $3.94 \mathrm{E}-05$ & $8.99 E+03$ \\
\hline $241-\mathrm{SX}-107$ & Total & & & $6.62 \mathrm{E}+02$ & $7.06 \mathrm{E}+04$ & $1.99 \mathrm{E}+04$ & $6.36 \mathrm{E}+04$ & $1.18 E+05$ & $1.26 \mathrm{E}+06$ & $5.59 \mathrm{E}+02$ & $6.83 \mathrm{E}+01$ & $3.27 \mathrm{E}+02$ & $1.18 \mathrm{E}+01$ & $5.17 \mathrm{E}+04$ \\
\hline 241-SX-107 & Sludge (Liquic & R2 (Solid) & 117 & $2.17 \mathrm{E}+02$ & $2.32 \mathrm{E}+04$ & $6.54 \mathrm{E}+03$ & $2.09 E+04$ & $3.87 \mathrm{E}+04$ & $1.09 \mathrm{E}+06$ & $4.53 \mathrm{E}+02$ & $2.77 \mathrm{E}+01$ & $1.18 \mathrm{E}+02$ & $7.89 \mathrm{E}+00$ & $2.50 \mathrm{E}+04$ \\
\hline $241-S X-107$ & Sludge (Liquid & Rl & 239 & $4.44 \mathrm{E}+02$ & $4.74 \mathrm{E}+04$ & $1.34 \mathrm{E}+04$ & $4.27 \mathrm{E}+04$ & $7.91 \mathrm{E}+04$ & $1.73 \mathrm{E}+05$ & $1.06 \mathrm{E}+02$ & $4.06 \mathrm{E}+01$ & $2.09 \mathrm{E}+02$ & $3.88 \mathrm{E}+00$ & $2.67 E+04$ \\
\hline 241-SX-108 & Total & & & $3.16 \mathrm{E}+02$ & $1.84 \mathrm{E}+05$ & $7.76 \mathrm{E}+03$ & $9.14 \mathrm{E}+04$ & $7.04 \mathrm{E}+04$ & $1.27 \mathrm{E}+06$ & $7.23 \mathrm{E}+02$ & $1.47 \mathrm{E}+02$ & $7.36 \mathrm{E}+02$ & $1.87 \mathrm{E}+01$ & $8.17 \mathrm{E}+04$ \\
\hline $241-S$ & Sludg & d) & 94 & $1.06 \mathrm{E}+02$ & $6.19 \mathrm{E}+04$ & $2.60 \mathrm{E}+03$ & $3.07 \mathrm{E}+04$ & $2.36 \mathrm{E}+04$ & $4.28 \mathrm{E}+05$ & $4.05 \mathrm{E}+02$ & $2.47 \mathrm{E}+01$ & $1.06 \mathrm{E}+02$ & $7.05 \mathrm{E}+00$ & $2.74 E+04$ \\
\hline $24 !-\$ X-108$ & Sludge Solid & R1 (Solid) & 186 & $2.10 \mathrm{E}+02$ & $1.22 \mathrm{E}+05$ & $5.15 \mathrm{E}+03$ & $6.07 \mathrm{E}+04$ & $4.67 E+04$ & $8.47 E+05$ & $3.19 \mathrm{E}+02$ & $1.22 \mathrm{E}+02$ & $6.30 \mathrm{E}+02$ & $1.17 \mathrm{E}+01$ & $5.43 \mathrm{E}+04$ \\
\hline 241-SX-109 & Total & & & $2.17 \mathrm{E}+03$ & $5.52 \mathrm{E}+05$ & $3.50 \mathrm{E}+04$ & $3.00 \mathrm{E}+05$ & 1.34E+05 & $9.01 \mathrm{E}+05$ & $4.63 \mathrm{E}+02$ & $5.93 E+01$ & $2.84 \mathrm{E}+02$ & $1.02 \mathrm{E}+01$ & $1.25 \mathrm{E}+05$ \\
\hline 241-SX-109 & Sludge Solid & R2 (Solid) & 81 & $1.51 \mathrm{E}+02$ & $1.61 \mathrm{E}+04$ & $4.53 \mathrm{E}+03$ & $1.45 \mathrm{E}+04$ & $2.68 \mathrm{E}+04$ & $7.52 \mathrm{E}+05$ & $3.13 \mathrm{E}+02$ & $1.92 \mathrm{E}+01$ & $8.18 \mathrm{E}+01$ & $5.46 \mathrm{E}+00$ & $1.73 \mathrm{E}+04$ \\
\hline 241-5X-109 & Sludge Solid & R1 (Solid) & 170 & $3.16 \mathrm{E}+02$ & $3.37 \mathrm{E}+04$ & $9.51 \mathrm{E}+03$ & $3.04 \mathrm{E}+04$ & $5.63 \mathrm{E}+04$ & $1.23 \mathrm{E}+05$ & $7.53 \mathrm{E}+01$ & $2.89 \mathrm{E}+01$ & $1.49 \mathrm{E}+02$ & $2.76 \mathrm{E}+00$ & $1.90 \mathrm{E}+04$ \\
\hline $241-5 X-109$ & Saltcake Solid & R-SltCk (Solid) & 662 & $1.71 \mathrm{E}+03$ & $5.02 \mathrm{E}+05$ & $2.10 \mathrm{E}+04$ & $2.55 \mathrm{E}+05$ & $5.14 \mathrm{E}+04$ & $2.65 E+04$ & $7.43 \mathrm{E}+01$ & $1.12 \mathrm{E}+01$ & $5.28 \mathrm{E}+01$ & $2.01 \mathrm{E}+00$ & $8.89 E+04$ \\
\hline $241-S X-110$ & Total & & & $4.14 \mathrm{E}+02$ & $5.77 \mathrm{E}+04$ & $1.12 \mathrm{E}+04$ & $4.37 \mathrm{E}+04$ & $6.31 \mathrm{E}+04$ & $1.71 E+06$ & $7.15 E+02$ & $4.40 \mathrm{E}+01$ & $1.88 \mathrm{E}+02$ & $1.25 \mathrm{E}+01$ & $4.30 \mathrm{E}+04$ \\
\hline $241-5 X-110$ & Sludge Solid & R2 (Solid) & 184 & $3.42 \mathrm{E}+02$ & $3.65 \mathrm{E}+04$ & $1.03 E+04$ & $3.29 \mathrm{E}+04$ & $6.09 \mathrm{E}+04$ & $1.71 \mathrm{E}+06$ & $7.12 \mathrm{E}+02$ & $4.35 \mathrm{E}+01$ & $1.86 \mathrm{E}+02$ & $1.24 \mathrm{E}+01$ & $3.93 \mathrm{E}+04$ \\
\hline $241-5 X-110$ & Saltcake Solid & R-SltCk (Solid) & 28 & $7.22 \mathrm{E}+01$ & $2.12 \mathrm{E}+04$ & $8.86 \mathrm{E}+02$ & $1.08 \mathrm{E}+04$ & $2.17 \mathrm{E}+03$ & $1.12 \mathrm{E}+03$ & $3.14 \mathrm{E}+00$ & $4.75 \mathrm{E}-0 \mathrm{l}$ & $2.23 \mathrm{E}+00$ & $8.51 \mathrm{E}-02$ & $3.76 \mathrm{E}+03$ \\
\hline 241-SX-111 & Total & & & $8.59 \mathrm{E}+02$ & $1.24 \mathrm{E}+05$ & $2.28 \mathrm{E}+04$ & $9.18 \mathrm{E}+04$ & $1.27 \mathrm{E}+05$ & $2.02 \mathrm{E}+06$ & $8.74 \mathrm{E}+02$ & $7.75 \mathrm{E}+01$ & $3.56 \mathrm{E}+02$ & $1.67 \mathrm{E}+01$ & $7.11 E+04$ \\
\hline 241-SX-111 & Sludge (Liquid \& Solid) & R2 (Solid) & 205 & $3.81 \mathrm{E}+02$ & $4.06 \mathrm{E}+04$ & $1.15 \mathrm{E}+04$ & $3.66 \mathrm{E}+04$ & $6.79 \mathrm{E}+04$ & $1.90 \mathrm{E}+06$ & $7.93 E+02$ & $4.85 \mathrm{E}+01$ & $2.07 \mathrm{E}+02$ & $1.38 \mathrm{E}+01$ & $4.38 \mathrm{E}+04$ \\
\hline 241-SX-111 & Studge (Liquid \& Solid) & R1 (Solid) & 164 & $3.05 \mathrm{E}+02$ & $3.25 \mathrm{E}+04$ & $9.17 \mathrm{E}+03$ & $2.93 \mathrm{E}+04$ & $5.43 \mathrm{E}+04$ & $1.19 \mathrm{E}+05$ & $7.27 \mathrm{E}+01$ & $2.79 \mathrm{E}+01$ & $1.44 \mathrm{E}+02$ & $2.66 \mathrm{E}+00$ & $1.83 \mathrm{E}+04$ \\
\hline 241-SX-111 & Saltcake Solid & R-SltCk (Solid) & 67 & $1.73 E+02$ & $5.08 \mathrm{E}+04$ & $2.12 \mathrm{E}+03$ & $2.58 \mathrm{E}+04$ & $5.20 \mathrm{E}+03$ & $2.68 \mathrm{E}+03$ & $7.52 \mathrm{E}+00$ & $1.14 \mathrm{E}+00$ & $5.35 \mathrm{E}+00$ & $2.04 \mathrm{E}-01$ & $9.00 \mathrm{E}+03$ \\
\hline 241-SX-112 & Total & & & $5.26 \mathrm{E}+02$ & $5.61 \mathrm{E}+04$ & $1.58 \mathrm{E}+04$ & $5.06 \mathrm{E}+04$ & $9.37 \mathrm{E}+04$ & $1.39 \mathrm{E}+06$ & $6.02 \mathrm{E}+02$ & $5.74 \mathrm{E}+01$ & $2.66 \mathrm{E}+02$ & 1.17E+01 & $4.58 \mathrm{E}+04$ \\
\hline $241-S X-112$ & Sludge (Liquid \& Solid) & R2 (Solid) & 139 & $2.58 \mathrm{E}+02$ & $2.76 \mathrm{E}+04$ & $7.77 \mathrm{E}+03$ & $2.48 \mathrm{E}+04$ & $4.60 \mathrm{E}+04$ & $1.29 \mathrm{E}+06$ & $5.38 \mathrm{E}+02$ & $3.29 \mathrm{E}+01$ & $1.40 \mathrm{E}+02$ & $9.37 \mathrm{E}+00$ & $2.97 \mathrm{E}+04$ \\
\hline 241-SX-112 & Sludge (Liquid \& Solid) & R1 (Solid) & 144 & $2.68 \mathrm{E}+02$ & $2.85 \mathrm{E}+04$ & $8.05 \mathrm{E}+03$ & $2.57 \mathrm{E}+04$ & $4.77 \mathrm{E}+04$ & $1.04 \mathrm{E}+05$ & $6.38 \mathrm{E}+01$ & $2.45 E+01$ & $1.26 \mathrm{E}+02$ & $2.34 \mathrm{E}+00$ & $1.61 \mathrm{E}+04$ \\
\hline
\end{tabular}


Table A-2. Tank Waste Inventory from Best Basis Inventory Detail Report (Queried on September 27, 2006) for Hydrogen Generation Rate Calculations. (27 sheets)

\begin{tabular}{|c|c|c|c|c|c|c|c|c|c|c|c|c|c|c|}
\hline $\begin{array}{c}\text { Tank } \\
\text { Name }\end{array}$ & Waste Phase & Waste Type & $\begin{array}{c}\text { Vol } \\
(\mathrm{kL})\end{array}$ & $\begin{array}{l}\text { TOC } \\
(\mathrm{Kg})\end{array}$ & $\begin{array}{l}\mathrm{NO}_{3} \\
(\mathrm{Kg})\end{array}$ & $\begin{array}{l}\mathrm{NO}_{2} \\
(\mathbf{K g})\end{array}$ & $\begin{array}{c}\mathbf{N a} \\
(\mathbf{K g})\end{array}$ & $\begin{array}{c}\text { Al } \\
(\mathbf{K g})\end{array}$ & $\begin{array}{l}{ }^{90} \mathrm{Sr} \\
(\mathrm{Ci})\end{array}$ & ${ }_{(\mathrm{Ci})}^{{ }^{241} \mathrm{Am}}$ & $\begin{array}{c}{ }^{240} \mathrm{Pu} \\
(\mathrm{Ci})\end{array}$ & $\begin{array}{c}{ }^{239} \mathrm{Pu} \\
(\mathrm{Ci})\end{array}$ & $\begin{array}{c}{ }^{238} \mathbf{P u} \\
(\mathrm{Ci})\end{array}$ & $\begin{array}{c}{ }^{137} \mathrm{Cs} \\
\text { (Ci) }\end{array}$ \\
\hline $241-\mathrm{SX}-113$ & Total & & & $1.49 \mathrm{E}+01$ & $8.75 \mathrm{E}+03$ & $2.26 \mathrm{E}+03$ & $9.51 \mathrm{E}+01$ & $1.18 \mathrm{E}+03$ & $2.84 \mathrm{E}+03$ & $1.12 \mathrm{E}+01$ & $9.76 \mathrm{E}-01$ & $5.03 \mathrm{E}+00$ & $9.32 \mathrm{E}-02$ & $2.08 \mathrm{E}+03$ \\
\hline 241-SX-113 & Sludge Solid & 1 (Solid) & 8 & $49 \mathrm{E}+01$ & $1.59 \mathrm{E}+03$ & $.47 E+02$ & $1.06 \mathrm{E}+01$ & $1.31 E+02$ & $3.15 \mathrm{E}+02$ & $.25 \mathrm{E}+00$ & $1.08 \mathrm{E}-01$ & $5.59 \mathrm{E}-01$ & $1.04 \mathrm{E}-02$ & $2.31 \mathrm{E}+02$ \\
\hline $241-S X-113$ & Sludge Solid & DE (Solid) & 64 & $00 \mathrm{E}+00$ & $7.17 \mathrm{E}+03$ & $1.81 \mathrm{E}+03$ & $8.45 \mathrm{E}+01$ & $1.05 \mathrm{E}+03$ & $2.52 \mathrm{E}+03$ & $9.98 \mathrm{E}+00$ & $8.67 \mathrm{E}-01$ & $4.47 \mathrm{E}+00$ & $8.29 \mathrm{E}-02$ & $1.85 \mathrm{E}+03$ \\
\hline $241-S X-114$ & Total & & & $1.13 \mathrm{E}+03$ & $1.64 \mathrm{E}+05$ & $3.20 \mathrm{E}+04$ & $1.24 \mathrm{E}+05$ & $1.66 \mathrm{E}+05$ & $1.89 \mathrm{E}+06$ & $8.38 \mathrm{E}+02$ & $9.47 \mathrm{E}+01$ & $4.50 \mathrm{E}+02$ & $1.72 \mathrm{E}+01$ & $9.02 E+04$ \\
\hline 114 & udge (Liquid & lid) & 180 & $35 \mathrm{E}+02$ & $3.57 \mathrm{E}+04$ & $1.01 E+04$ & $3.22 \mathrm{E}+04$ & $5.96 \mathrm{E}+04$ & $.67 \mathrm{E}+06$ & $6.97 \mathrm{E}+02$ & $4.26 \mathrm{E}+01$ & $1.82 \mathrm{E}+02$ & $1.21 \mathrm{E}+01$ & $3.84 \mathrm{E}+04$ \\
\hline $241-\mathrm{SX}-114$ & Sludge (Liquid \& Solid) & RI (Solid) & 298 & $5.54 \mathrm{E}+02$ & $5.91 \mathrm{E}+04$ & $1.67 \mathrm{E}+04$ & $5.33 \mathrm{E}+04$ & $9.86 \mathrm{E}+04$ & $2.16 \mathrm{E}+05$ & $1.32 \mathrm{E}+02$ & $5.07 \mathrm{E}+01$ & $2.6 \mathrm{IE}+02$ & $4.84 E+00$ & $3.33 \mathrm{E}+04$ \\
\hline 241-SX-114 & Saltcake Solid & R-SltCk (Solid) & 84 & $2.17 \mathrm{E}+02$ & $6.37 E+04$ & $2.66 \mathrm{E}+03$ & $3.24 E+04$ & $6.52 \mathrm{E}+03$ & $3.36 \mathrm{E}+03$ & $9.43 \mathrm{E}+00$ & $1.43 \mathrm{E}+00$ & $6.70 \mathrm{E}+00$ & $2.55 \mathrm{E}-01$ & $1.13 \mathrm{E}+04$ \\
\hline 241-SX-114 & Itcake Interstit & R-SltCk & 26 & $2.63 \mathrm{E}+01$ & $5.64 \mathrm{E}+03$ & $2.57 \mathrm{E}+03$ & $6.53 \mathrm{E}+03$ & $1.23 \mathrm{E}+03$ & $6.00 \mathrm{E}+01$ & $1.75 \mathrm{E}-01$ & $2.81 \mathrm{E}-02$ & $1.32 \mathrm{E}-01$ & $5.03 \mathrm{E}-03$ & $7.25 \mathrm{E}+03$ \\
\hline $241-\mathrm{SX}-115$ & Total & & & $3.17 \mathrm{E}+01$ & $3.69 \mathrm{E}+03$ & $5.89 E+01$ & $7.06 \mathrm{E}+02$ & $6.16 E+03$ & $3.83 \mathrm{E}+05$ & $4.02 E+02$ & $1.07 E+02$ & $4.55 \mathrm{E}+02$ & $3.04 \mathrm{E}+01$ & $5.81 \mathrm{E}+02$ \\
\hline $241-\mathrm{SX}-115$ & Sludge Solid & R2 (Solid) & 16 & $3.17 E+01$ & $3.69 E+03$ & $5.89 \mathrm{E}+01$ & $7.06 \mathrm{E}+02$ & $6.16 \mathrm{E}+03$ & $3.83 \mathrm{E}+05$ & $4.02 E+02$ & $1.07 \mathrm{E}+02$ & $4.55 \mathrm{E}+02$ & $3.04 \mathrm{E}+01$ & $5.81 E+02$ \\
\hline 241-SY-101 & Total & & & $2.87 \mathrm{E}+04$ & $6.54 \mathrm{E}+05$ & $1.38 \mathrm{E}+05$ & $6.01 E+05$ & $9.26 \mathrm{E}+04$ & $5.37 \mathrm{E}+04$ & 4.14E+02 & $7.80 \mathrm{E}+00$ & $3.64 \mathrm{E}+01$ & $1.27 \mathrm{E}+00$ & $3.91 \mathrm{E}+05$ \\
\hline 241-SY-101 & Supernatant & $\mathrm{NA}$ & 3300 & $3.68 \mathrm{E}+03$ & $5.56 E+05$ & $7.09 \mathrm{E}+04$ & $4.04 \mathrm{E}+05$ & $2.00 \mathrm{E}+04$ & $1.54 \mathrm{E}+03$ & $8.05 \mathrm{E}-01$ & 02 & $2.76 \mathrm{E}-01$ & $9.58 \mathrm{E}-03$ & $2.11 E+05$ \\
\hline 241-SY-101 & Saltcake (Liquid \& Solid) & S2-SltSlr (Solid) & 893 & $2.50 \mathrm{E}+04$ & $9.76 \mathrm{E}+04$ & $6.73 E+04$ & $1.97 \mathrm{E}+05$ & $7.26 \mathrm{E}+04$ & $5.22 \mathrm{E}+04$ & $4.13 E+02$ & $7.74 \mathrm{E}+00$ & $3.61 E+01$ & $1.26 \mathrm{E}+00$ & $1.80 \mathrm{E}+05$ \\
\hline 241-SY-102 & Total & & & $1.87 \mathrm{E}+04$ & $6.82 \mathrm{E}+05$ & $9.22 \mathrm{E}+04$ & $5.14 \mathrm{E}+05$ & $5.12 \mathrm{E}+04$ & $1.64 \mathrm{E}+05$ & $2.23 E+04$ & $1.19 \mathrm{E}+03$ & $5.19 \mathrm{E}+03$ & $2.43 \mathrm{E}+02$ & $2.55 E+05$ \\
\hline $241-S$ & II & N & 688 & $5 \mathrm{E}+03$ & $E+05$ & $\mathrm{E}+04$ & $4.03 \mathrm{E}+05$ & $2 \mathrm{E}+04$ & $2.60 \mathrm{E}+03$ & $2.29 \mathrm{E}+00$ & $2.38 \mathrm{E}-01$ & $1.34 \mathrm{E}+00$ & $3.07 \mathrm{E}-02$ & $1.48 \mathrm{E}+05$ \\
\hline 241-SY-102 & Sludge Solid & (Solid) & 186 & $6.80 \mathrm{E}+03$ & $2.36 \mathrm{E}+04$ & $1.06 \mathrm{E}+04$ & $3.40 \mathrm{E}+04$ & $8.87 \mathrm{E}+03$ & $3.05 \mathrm{E}+04$ & $9.94 \mathrm{E}+03$ & $5.67 \mathrm{E}+02$ & $2.27 \mathrm{E}+03$ & $1.41 \mathrm{E}+02$ & $3.20 \mathrm{E}+04$ \\
\hline $241-S Y-102$ & Sludge Solid & $\mathrm{NA}$ & 232 & $8.49 \mathrm{E}+03$ & $2.28 \mathrm{E}+04$ & $1.49 \mathrm{E}+04$ & $4.85 \mathrm{E}+04$ & $2.56 \mathrm{E}+04$ & $1.31 \mathrm{E}+05$ & $1.24 \mathrm{E}+04$ & $6.25 \mathrm{E}+02$ & $2.91 \mathrm{E}+03$ & $1.02 \mathrm{E}+02$ & $3.99 \mathrm{E}+04$ \\
\hline 241-SY-102 & Sludge Interstitial Li & $Z$ (Liquid) & 83 & $3.86 \mathrm{E}+02$ & $1.11 \mathrm{E}+04$ & $6.07 \mathrm{E}+03$ & $1.26 \mathrm{E}+04$ & $6.29 \mathrm{E}+02$ & $1.24 \mathrm{E}+02$ & $1.09 \mathrm{E}+00$ & $1.58 \mathrm{E}-02$ & $7.34 \mathrm{E}-02$ & $2.57 \mathrm{E}-03$ & $1.55 \mathrm{E}+04$ \\
\hline 241-SY-102 & Sludge Interstitial Liquid & NA & 104 & $4.83 E+02$ & $1.39 \mathrm{E}+04$ & $7.60 \mathrm{E}+03$ & $1.58 \mathrm{E}+04$ & $7.89 \mathrm{E}+02$ & $1.55 \mathrm{E}+02$ & $1.37 \mathrm{E}+00$ & 1.97E-02 & $9.20 \mathrm{E}-02$ & $3.21 \mathrm{E}-03$ & $1.94 \mathrm{E}+04$ \\
\hline $241-S Y-103$ & Total & & & $2.64 \mathrm{E}+04$ & $5.00 \mathrm{E}+05$ & $3.92 \mathrm{E}+05$ & $6.98 \mathrm{E}+05$ & $1.27 \mathrm{E}+05$ & $6.81 \mathrm{E}+04$ & $1.25 \mathrm{E}+03$ & $2.44 \mathrm{E}+01$ & $1.14 \mathrm{E}+02$ & $3.97 \mathrm{E}+00$ & $1.07 \mathrm{E}+06$ \\
\hline 241-SY-103 & Supernatant & S2-S1tSlr (Liquid) & 1503 & $9.35 \mathrm{E}+03$ & $2.52 \mathrm{E}+05$ & $2.29 \mathrm{E}+05$ & $3.23 \mathrm{E}+05$ & $5.65 \mathrm{E}+04$ & $4.53 \mathrm{E}+03$ & $1.53 \mathrm{E}+01$ & $1.76 \mathrm{E}-02$ & $8.20 \mathrm{E}-02$ & $2.87 \mathrm{E}-03$ & $6.45 \mathrm{E}+05$ \\
\hline 241-SY-103 & Saltcake (Liquid \& Solid) & S2-SltSlr (Solid) & 1203 & $1.71 \mathrm{E}+04$ & $2.48 \mathrm{E}+05$ & $1.63 E+05$ & $3.76 \mathrm{E}+05$ & $7.09 \mathrm{E}+04$ & $6.36 \mathrm{E}+04$ & $1.23 E+03$ & $2.44 \mathrm{E}+01$ & $1.14 \mathrm{E}+02$ & $3.97 \mathrm{E}+00$ & $4.26 \mathrm{E}+05$ \\
\hline 241-T-101 & Total & & & $1.31 \mathrm{E}+03$ & $1.20 \mathrm{E}+05$ & $1.56 \mathrm{E}+04$ & $7.54 \mathrm{E}+04$ & $2.98 \mathrm{E}+04$ & $3.85 \mathrm{E}+02$ & $6.65 \mathrm{E}+01$ & $4.54 \mathrm{E}+01$ & $2.08 \mathrm{E}+02$ & $5.44 \mathrm{E}+00$ & $3.92 \mathrm{E}+04$ \\
\hline $241-\mathrm{T}-101$ & Sludge (Liquid \& Solid) & CWR2 (Solid) & 140 & $0.00 \mathrm{E}+00$ & $5.74 \mathrm{E}+03$ & $1.4 ! \mathrm{E}+03$ & $4.42 \mathrm{E}+03$ & $2.21 \mathrm{E}+04$ & $1.10 \mathrm{E}+02$ & $1.65 \mathrm{E}+01$ & $4.03 E+01$ & $1.83 \mathrm{E}+02$ & $4.57 \mathrm{E}+00$ & $1.28 \mathrm{E}+02$ \\
\hline $241-\mathrm{T}-101$ & Saltcake Solid & $\mathrm{T} 2-\mathrm{Slt} C \mathrm{Ck}$ (Solid) & 179 & $1.08 \mathrm{E}+03$ & $1.04 \mathrm{E}+05$ & $7.22 \mathrm{E}+03$ & $5.75 \mathrm{E}+04$ & $5.55 \mathrm{E}+03$ & $2.18 \mathrm{E}+02$ & $4.99 \mathrm{E}+01$ & $5.19 \mathrm{E}+00$ & $2.47 \mathrm{E}+01$ & $8.72 \mathrm{E}-01$ & $1.88 \mathrm{E}+04$ \\
\hline $241-T-101$ & Saltcake Interstitial Liquid & T2-SltCk (Liquid) & 57 & $2.30 \mathrm{E}+02$ & $1.08 \mathrm{E}+04$ & $6.95 \mathrm{E}+03$ & $1.35 \mathrm{E}+04$ & $2.15 \mathrm{E}+03$ & $5.67 \mathrm{E}+01$ & $1.61 \mathrm{E}-01$ & $1.47 \mathrm{E}-03$ & $7.01 \mathrm{E}-03$ & $2.48 \mathrm{E}-04$ & $2.03 \mathrm{E}+04$ \\
\hline $241-T-102$ & Total & & & $7.62 \mathrm{E}+01$ & $8.16 \mathrm{E}+03$ & $1.78 \mathrm{E}+03$ & $6.83 \mathrm{E}+03$ & $2.44 \mathrm{E}+04$ & $1.66 \mathrm{E}+04$ & $2.14 \mathrm{E}+01$ & $9.48 \mathrm{E}-01$ & $4.06 \mathrm{E}+00$ & $9.90 \mathrm{E}-02$ & $4.59 \mathrm{E}+03$ \\
\hline
\end{tabular}


Table A-2. Tank Waste Inventory from Best Basis Inventory Detail Report (Queried on September 27, 2006) for Hydrogen Generation Rate Calculations. (27 sheets)

\begin{tabular}{|c|c|c|c|c|c|c|c|c|c|c|c|c|c|c|}
\hline $\begin{array}{l}\text { Tank } \\
\text { Name }\end{array}$ & Waste Phase & Waste Type & $\begin{array}{r}\text { Vol } \\
(\mathbf{k L})\end{array}$ & $\begin{array}{l}\text { TOC } \\
(\mathrm{Kg})\end{array}$ & $\begin{array}{l}\mathrm{NO}_{3} \\
(\mathrm{Kg})\end{array}$ & $\begin{array}{l}\mathrm{NO}_{2} \\
(\mathrm{Kg})\end{array}$ & $\begin{array}{c}\mathrm{Na} \\
(\mathrm{Kg})\end{array}$ & $\begin{array}{c}\mathrm{Al} \\
(\mathrm{Kg})\end{array}$ & $\begin{array}{l}{ }^{90} \mathrm{Sr} \\
\text { (Ci) }\end{array}$ & $\begin{array}{c}{ }^{241} \mathrm{Am} \\
\text { (Ci) }\end{array}$ & $\begin{array}{c}{ }^{240} \mathrm{Pu} \\
(\mathrm{Ci})\end{array}$ & $\begin{array}{c}{ }^{239} \mathbf{P u} \\
(\mathrm{Ci})\end{array}$ & $\begin{array}{c}{ }^{238} \mathbf{P u} \\
(\mathrm{Ci})\end{array}$ & $\begin{array}{l}{ }^{137} \mathrm{Cs} \\
\text { (Ci) }\end{array}$ \\
\hline 241-T-102 & Supernatant & CSR (Liquid) & 48 & $2.16 \mathrm{E}+01$ & $5.23 \mathrm{E}+03$ & $10 \mathrm{E}+03$ & $3.10 \mathrm{E}+03$ & $4.06 \mathrm{E}+02$ & $4.68 \mathrm{E}+01$ & $2.05 \mathrm{E}-01$ & $5.68 \mathrm{E}-02$ & $2.45 \mathrm{E}-01$ & $1.03 \mathrm{E}-02$ & $2.47 \mathrm{E}+03$ \\
\hline $241-T-102$ & Sludge (Liquid \& Solid) & Solid) & 8 & $00 \mathrm{E}+00$ & $6.99 \mathrm{E}+00$ & $5.82 \mathrm{E}+00$ & $1.21 \mathrm{E}+03$ & $.00 \mathrm{E}+00$ & $1.12 \mathrm{E}+03$ & $5.47 \mathrm{E}-03$ & $1.06 \mathrm{E}-02$ & $8.38 \mathrm{E}-02$ & $7.26 \mathrm{E}-04$ & $2.86 \mathrm{E}+01$ \\
\hline 241-T-102 & Sludge (Liquid \& Solid) & CWP2 (Solid) & 64 & $5.46 \mathrm{E}+01$ & $2.92 \mathrm{E}+03$ & $6.67 \mathrm{E}+02$ & $2.52 \mathrm{E}+03$ & $2.39 \mathrm{E}+04$ & $1.55 \mathrm{E}+04$ & $2.12 \mathrm{E}+01$ & $8.81 \mathrm{E}-01$ & $3.73 \mathrm{E}+00$ & $8.80 \mathrm{E}-02$ & $2.09 \mathrm{E}+03$ \\
\hline 241-T-103 & Total & & & $56 \mathrm{E}+02$ & $4.48 \mathrm{E}+03$ & $1.75 \mathrm{E}+03$ & $6.38 \mathrm{E}+03$ & $2.59 \mathrm{E}+04$ & $6.95 \mathrm{E}+02$ & $6.39 \mathrm{E}-01$ & $8.79 \mathrm{E}-01$ & $3.76 \mathrm{E}+00$ & $8.95 \mathrm{E}-02$ & $4.00 \mathrm{E}+02$ \\
\hline 241-T-103 & Supernatant & CSR (Liquid) & 15 & $8.33 E+01$ & $1.17 \mathrm{E}+03$ & $5.94 \mathrm{E}+02$ & $1.43 \mathrm{E}+03$ & $1.56 \mathrm{E}+02$ & $3.46 \mathrm{E}+01$ & $1.53 \mathrm{E}-01$ & $2.65 \mathrm{E}-02$ & $1.14 \mathrm{E}-01$ & $4.78 \mathrm{E}-03$ & $2.10 \mathrm{E}+02$ \\
\hline $241-\mathrm{T}-103$ & Sludge (Liquid \& Solid) & MW2 (Solid) & 4 & $0.00 \mathrm{E}+00$ & $3.49 \mathrm{E}+00$ & $2.91 \mathrm{E}+00$ & $6.06 \mathrm{E}+02$ & $0.00 \mathrm{E}+00$ & $5.62 \mathrm{E}+02$ & $2.73 \mathrm{E}-03$ & $5.31 \mathrm{E}-03$ & $4.19 \mathrm{E}-02$ & $3.63 \mathrm{E}-04$ & $1.43 \mathrm{E}+01$ \\
\hline $241-T-103$ & Sludge (Liquid \& Solid) & CWRI (Solid) & 19 & $2.18 \mathrm{E}+0 \mathrm{I}$ & $8.38 \mathrm{E}+02$ & $3.66 \mathrm{E}+02$ & $1.32 \mathrm{E}+03$ & $7.90 \mathrm{E}+03$ & $6.36 \mathrm{E}+01$ & $6.67 \mathrm{E}-02$ & $1.97 \mathrm{E}-02$ & $1.04 \mathrm{E}-01$ & $1.61 \mathrm{E}-03$ & $1.34 \mathrm{E}+02$ \\
\hline $241-T-103$ & Sludge (Liquid \& Solid) & CWP2 (Solid) & 64 & $5.13 \mathrm{E}+01$ & $2.47 \mathrm{E}+03$ & $7.85 \mathrm{E}+02$ & $3.02 \mathrm{E}+03$ & $1.78 \mathrm{E}+04$ & $3.53 \mathrm{E}+01$ & $4.17 \mathrm{E}-01$ & $8.28 \mathrm{E}-01$ & $3.50 \mathrm{E}+00$ & $8.27 \mathrm{E}-02$ & $4.15 \mathrm{E}+01$ \\
\hline 241-T-104 & Total & & & $5.23 \mathrm{E}+02$ & $8.97 \mathrm{E}+04$ & $6.55 \mathrm{E}+03$ & $8 \mathrm{E}+04$ & $E+04$ & $3.14 \mathrm{E}+03$ & $2.80 \mathrm{E}+01$ & $2.33 \mathrm{E}+01$ & $1.93 E+02$ & $2.51 \mathrm{E}+00$ & $2.40 \mathrm{E}+02$ \\
\hline $241-\mathrm{T}-104$ & Sludge (Liquid \& Solid) & 1C (Solid) & 1199 & $5.23 \mathrm{E}+02$ & $8.97 \mathrm{E}+04$ & $6.55 \mathrm{E}+03$ & $9.98 \mathrm{E}+04$ & $2.51 \mathrm{E}+04$ & $3.14 \mathrm{E}+03$ & $2.80 \mathrm{E}+01$ & $2.33 \mathrm{E}+01$ & $1.93 \mathrm{E}+02$ & $2.51 \mathrm{E}+00$ & $2.40 \mathrm{E}+02$ \\
\hline $241-T-105$ & Total & & & $1.30 \mathrm{E}+03$ & $1.25 \mathrm{E}+04$ & $1.76 \mathrm{E}+04$ & $3.95 \mathrm{E}+04$ & $2.43 \mathrm{E}+04$ & $2.81 \mathrm{E}+04$ & $3.15 \mathrm{E}+01$ & $1.59 \mathrm{E}+01$ & $1.50 \mathrm{E}+02$ & $1.13 \mathrm{E}+00$ & $5.00 \mathrm{E}+03$ \\
\hline $241-\mathrm{T}-105$ & Sludge (Liquid & CWR & 92 & $5.58 \mathrm{E}+02$ & $2.76 E+03$ & 03 & 3 & $E+04$ & $4 E+04$ & $E+01$ & $4 \mathrm{E}+00$ & $E+01$ & E-01 & $4.69 E+03$ \\
\hline $241-T-105$ & Sludge (Liquid \& Solid) & $2 \mathrm{C}$ (Solid) & 273 & $7.09 \mathrm{E}+02$ & $9.52 \mathrm{E}+03$ & $1.34 \mathrm{E}+04$ & $3.27 E+04$ & $9.17 \mathrm{E}+03$ & $9.69 \mathrm{E}-01$ & $2.73 \mathrm{E}-01$ & $1.39 \mathrm{E}+01$ & $1.34 \mathrm{E}+02$ & $8.87 \mathrm{E}-01$ & $1.13 \mathrm{E}+00$ \\
\hline $241-T-105$ & Sludge (Liquid \& Solid) & $1 \mathrm{C}$ (Solid) & 6 & $3.64 \mathrm{E}+01$ & $1.80 \mathrm{E}+02$ & $2.56 \mathrm{E}+02$ & $4.16 \mathrm{E}+02$ & $9.29 \mathrm{E}+02$ & $1.72 \mathrm{E}+03$ & $1.91 \mathrm{E}+00$ & $1.20 \mathrm{E}-01$ & $9.74 \mathrm{E}-01$ & $6.85 \mathrm{E}-03$ & $3.06 \mathrm{E}+02$ \\
\hline $241-\mathrm{T}-106$ & Total & & & $9.57 E+01$ & $1.61 \mathrm{E}+04$ & $1.89 \mathrm{E}+03$ & $1.38 \mathrm{E}+04$ & $1.84 \mathrm{E}+04$ & $6.54 \mathrm{E}+02$ & $5.55 \mathrm{E}+00$ & $2.60 \mathrm{E}+00$ & $1.74 \mathrm{E}+01$ & $2.10 \mathrm{E}-01$ & $1.99 \mathrm{E}+03$ \\
\hline $241-T-106$ & Sludge (Liquid \& Solid) & CWR2 (Solid) & 10 & $0.00 \mathrm{E}+00$ & $7.72 E+02$ & $1.90 \mathrm{E}+02$ & $5.94 \mathrm{E}+02$ & $2.97 \mathrm{E}+03$ & $1.47 \mathrm{E}+01$ & $1.86 \mathrm{E}-01$ & $4.54 \mathrm{E}-01$ & $2.07 E+00$ & $5.16 \mathrm{E}-02$ & $1.72 \mathrm{E}+01$ \\
\hline $241-T-106$ & Sludge (Liquid \& Solid) & CWR1 (Solid) & 34 & $3.81 E+01$ & $1.46 \mathrm{E}+03$ & $6.38 \mathrm{E}+02$ & $2.30 \mathrm{E}+03$ & $1.38 \mathrm{E}+04$ & $1.11 \mathrm{E}+02$ & $3.80 \mathrm{E}+00$ & $1.12 \mathrm{E}+00$ & $5.90 \mathrm{E}+00$ & $9.19 \mathrm{E}-02$ & $2.34 \mathrm{E}+02$ \\
\hline $241 \cdot \mathrm{T}-106$ & Sludge (Liquid \& Solid) & $1 \mathrm{C}$ (Solid) & 38 & $5.77 \mathrm{E}+01$ & $1.38 \mathrm{E}+04$ & $1.06 \mathrm{E}+03$ & $1.09 \mathrm{E}+04$ & $1.63 \mathrm{E}+03$ & $5.28 \mathrm{E}+02$ & $1.57 \mathrm{E}+00$ & $1.03 \mathrm{E}+00$ & $9.45 \mathrm{E}+00$ & $6.65 \mathrm{E}-02$ & $1.74 \mathrm{E}+03$ \\
\hline $241-T-107$ & Total & & & $8.82 \mathrm{E}+02$ & $7.21 \mathrm{E}+04$ & $1.13 \mathrm{E}+04$ & $9.45 \mathrm{E}+04$ & $3.83 \mathrm{E}+04$ & $1.03 \mathrm{E}+05$ & $6.01 E+01$ & $3.56 \mathrm{E}+01$ & $3.11 \mathrm{E}+02$ & $2.42 \mathrm{E}+00$ & $1.51 \mathrm{E}+04$ \\
\hline $241-\mathrm{T}-107$ & Sludge (Liquid \& Solid) & TBP & 64 & $8.62 \mathrm{E}+01$ & $7.04 \mathrm{E}+03$ & $1.10 \mathrm{E}+03$ & $9.23 \mathrm{E}+03$ & $3.74 \mathrm{E}+03$ & $1.00 \mathrm{E}+04$ & $1.32 \mathrm{E}+01$ & $2.57 \mathrm{E}+00$ & $2.40 \mathrm{E}+01$ & $1.67 \mathrm{E}-01$ & $1.47 E+03$ \\
\hline $241-T-107$ & Sludge (Liquid \& Solid) & CWP2 (Solid) & 32 & $4.31 E+01$ & $3.52 \mathrm{E}+03$ & $5.52 \mathrm{E}+02$ & $4.61 E+03$ & $1.87 \mathrm{E}+03$ & $5.02 \mathrm{E}+03$ & $1.68 \mathrm{E}+00$ & $3.33 \mathrm{E}+00$ & $1.41 \mathrm{E}+01$ & $3.33 \mathrm{E}-01$ & $7.35 \mathrm{E}+02$ \\
\hline $241-\mathrm{T}-107$ & Sludge (Liquid \& Solid) & $1 \mathrm{C}$ (Solid) & 559 & $7.53 \mathrm{E}+02$ & $6.15 \mathrm{E}+04$ & $9.65 \mathrm{E}+03$ & $8.06 \mathrm{E}+04$ & $3.27 \mathrm{E}+04$ & $8.77 \mathrm{E}+04$ & $4.53 \mathrm{E}+01$ & $2.97 \mathrm{E}+01$ & $2.73 \mathrm{E}+02$ & $1.92 \mathrm{E}+00$ & $1.28 \mathrm{E}+04$ \\
\hline $241-T-108$ & Total & & & $1.51 \mathrm{E}+01$ & $2.60 \mathrm{E}+04$ & $6.82 \mathrm{E}+02$ & $1.55 \mathrm{E}+04$ & $5.48 \mathrm{E}+02$ & $1.64 \mathrm{E}+02$ & $4.32 \mathrm{E}-01$ & $6.26 \mathrm{E}-01$ & $5.62 \mathrm{E}+00$ & $4.08 \mathrm{E}-02$ & $6.28 \mathrm{E}+02$ \\
\hline $241-\mathrm{T}-108$ & Sludge (Liquid \& Solid) & $1 \mathrm{C}$ (Solid) & 20 & $1.50 \mathrm{E}+01$ & $3.60 \mathrm{E}+03$ & $2.78 \mathrm{E}+02$ & $2.84 \mathrm{E}+03$ & $4.26 \mathrm{E}+02$ & $1.38 \mathrm{E}+02$ & $3.95 \mathrm{E}-01$ & $2.59 \mathrm{E}-01$ & $2.38 \mathrm{E}+00$ & $1.68 \mathrm{E}-02$ & $4.54 \mathrm{E}+02$ \\
\hline $241-T-108$ & Saltcake Solid & T1-SltCk (Solid) & 30 & $4.54 \mathrm{E}-02$ & $2.02 \mathrm{E}+04$ & $3.19 \mathrm{E}+02$ & $1.15 \mathrm{E}+04$ & $1.18 \mathrm{E}+02$ & $5.15 \mathrm{E}+00$ & $2.13 \mathrm{E}-02$ & $3.61 \mathrm{E}-01$ & $3.18 \mathrm{E}+00$ & $2.37 \mathrm{E}-02$ & $8.50 \mathrm{E}+01$ \\
\hline $241-\mathrm{T}-108$ & Saltcake Interstitial Liquid & T1-SltCk (Liquid) & 10 & $3.38 \mathrm{E}-02$ & $2.17 \mathrm{E}+03$ & $8.50 \mathrm{E}+01$ & $1.13 E+03$ & $3.61 \mathrm{E}+00$ & $2.12 \mathrm{E}+01$ & $1.55 \mathrm{E}-02$ & $5.91 \mathrm{E}-03$ & $5.22 \mathrm{E}-02$ & $3.88 \mathrm{E}-04$ & $8.92 E+01$ \\
\hline $241-\mathrm{T}-109$ & Total & & & $3.11 \mathrm{E}-01$ & $1.33 \mathrm{E}+04$ & $4.90 \mathrm{E}+02$ & $6.74 \mathrm{E}+04$ & $4.20 \mathrm{E}+02$ & $1.01 \mathrm{E}+02$ & $8.11 \mathrm{E}-02$ & $4.00 \mathrm{E}-01$ & $3.53 \mathrm{E}+00$ & $2.63 \mathrm{E}-02$ & $8.24 \mathrm{E}+02$ \\
\hline
\end{tabular}


Table A-2. Tank Waste Inventory from Best Basis Inventory Detail Report (Queried on September 27, 2006) for Hydrogen Generation Rate Calculations. (27 sheets)

\begin{tabular}{|c|c|c|c|c|c|c|c|c|c|c|c|c|c|c|}
\hline $\begin{array}{c}\text { Tank } \\
\text { Name }\end{array}$ & Waste Phase & Waste Type & $\begin{array}{l}\text { Vol } \\
(k L)\end{array}$ & $\begin{array}{l}\text { TOC } \\
\text { (Kg) }\end{array}$ & $\begin{array}{l}\mathrm{NO}_{3} \\
\text { (Kg) }\end{array}$ & $\begin{array}{l}\mathrm{NO}_{2} \\
(\mathrm{Kg})\end{array}$ & $\begin{array}{c}\mathrm{Na} \\
(\mathrm{Kg})\end{array}$ & $\begin{array}{c}\mathbf{A l} \\
(\mathbf{K g})\end{array}$ & $\begin{array}{l}{ }^{90} \mathrm{Sr} \\
(\mathrm{Ci})\end{array}$ & $\begin{array}{c}{ }^{241} \mathrm{Am} \\
\text { (Ci) }\end{array}$ & $\begin{array}{c}{ }^{240} \mathrm{Pu} \\
(\mathrm{Ci})\end{array}$ & $\begin{array}{c}{ }^{239} \mathrm{Pu} \\
\text { (Ci) }\end{array}$ & $\begin{array}{c}{ }^{238} \mathrm{Pu} \\
(\mathrm{Ci})\end{array}$ & $\begin{array}{c}{ }^{137} \mathrm{Cs} \\
\text { (Ci) }\end{array}$ \\
\hline $241-\mathrm{T}-109$ & Saltcake Solid & T1-SltCk (Solid) & 197 & $1.83 \mathrm{E}-01$ & $5.08 \mathrm{E}+03$ & $1.67 \mathrm{E}+02$ & $6.31 \mathrm{E}+04$ & $4.06 \mathrm{E}+02$ & $.07 \mathrm{E}+01$ & $2.23 \mathrm{E}-02$ & $3.78 \mathrm{E}-01$ & $3.33 \mathrm{E}+00$ & $2.48 \mathrm{E}-02$ & $4.84 \mathrm{E}+02$ \\
\hline $241-\mathrm{T}-109$ & altcake Interstitial Liquid & $1-\mathrm{SltC}$ & 38 & $1.28 \mathrm{E}-01$ & $25 \mathrm{E}+03$ & $23 \mathrm{E}+02$ & $29 \mathrm{E}+03$ & $37 \mathrm{E}+01$ & $8.06 \mathrm{E}+01$ & E-02 & $2.25 \mathrm{E}-02$ & $1.98 \mathrm{E}-01$ & $1.47 \mathrm{E}-03$ & $3.39 \mathrm{E}+02$ \\
\hline $241-T-110$ & Total & & & $1.56 \mathrm{E}+03$ & $1.05 \mathrm{E}+05$ & $2.61 E+04$ & $9.26 \mathrm{E}+04$ & $2.48 \mathrm{E}+03$ & $4.76 \mathrm{E}+01$ & $8.63 \mathrm{E}+00$ & $1.03 \mathrm{E}+01$ & $9.79 E+01$ & $6.55 \mathrm{E}-01$ & $2.60 \mathrm{E}+01$ \\
\hline $241-\mathrm{T}-110$ & Supernatant & 2Cl (Liquid) & 3 & $1.35 \mathrm{E}-01$ & $5.49 \mathrm{E}+01$ & $1.77 \mathrm{E}-01$ & $1.11 \mathrm{E}+02$ & $0.00 \mathrm{E}+00$ & $1.35 \mathrm{E}-03$ & $9.00 \mathrm{E}-06$ & $3.14 \mathrm{E}-05$ & $4.66 \mathrm{E}-04$ & $5.97 \mathrm{E}-06$ & $8.79 \mathrm{E}-03$ \\
\hline $241-T-110$ & Sludge (Liquid \& Solid) & $2 \mathrm{C}$ (Solid) & 1360 & $1.54 \mathrm{E}+03$ & $1.02 E+05$ & $2.61 \mathrm{E}+04$ & $9.10 \mathrm{E}+04$ & $2.47 \mathrm{E}+03$ & $4.64 \mathrm{E}+01$ & $8.40 \mathrm{E}+00$ & $9.95 \mathrm{E}+00$ & $9.54 \mathrm{E}+01$ & $6.33 \mathrm{E}-01$ & $2.53 \mathrm{E}+01$ \\
\hline $241-\mathrm{T}-110$ & Sludge (Liquid \& & 224-2 (Solid) & 37 & $1.37 \mathrm{E}+01$ & $2.72 \mathrm{E}+03$ & $2.41 \mathrm{E}+01$ & $1.53 \mathrm{E}+03$ & $2.89 \mathrm{E}+00$ & $1.26 \mathrm{E}+00$ & $2.29 \mathrm{E}-01$ & $3.21 \mathrm{E}-01$ & $2.55 \mathrm{E}+00$ & $2.20 \mathrm{E}-02$ & $6.88 \mathrm{E}-01$ \\
\hline $241-T-111$ & Total & & & $7.26 \mathrm{E}+03$ & $1.22 \mathrm{E}+05$ & $1.57 \mathrm{E}+03$ & $6.76 \mathrm{E}+04$ & $7.36 \mathrm{E}+02$ & $E+03$ & 9.85 & +01 & $2.61 \mathrm{E}$ & $1.30 \mathrm{E}+00$ & $2.13 E+02$ \\
\hline $241-\mathrm{T}-111$ & Sludge (Liquid \& Solid) & $2 \mathrm{C}$ (Solid) & 787 & $3.38 \mathrm{E}+03$ & $5.66 \mathrm{E}+04$ & $7.31 E+02$ & $3.15 \mathrm{E}+04$ & $3.42 \mathrm{E}+02$ & $3.98 \mathrm{E}+03$ & $4.57 \mathrm{E}+01$ & $1.28 \mathrm{E}+01$ & $1.23 E+02$ & $1.08 \mathrm{E}-01$ & $9.93 \mathrm{E}+01$ \\
\hline $241-\mathrm{T}-111$ & Sludge (Liquid \& Solid) & $224-2$ & 904 & $3.88 \mathrm{E}+03$ & $6.50 \mathrm{E}+04$ & $8.40 \mathrm{E}+02$ & $3.61 \mathrm{E}+04$ & $3.93 \mathrm{E}+02$ & $4.57 \mathrm{E}+03$ & $5.28 \mathrm{E}+01$ & $1.74 \mathrm{E}+01$ & $1.38 \mathrm{E}+02$ & $1.19 \mathrm{E}+00$ & $1.14 \mathrm{E}+02$ \\
\hline $241-\mathrm{T}-112$ & Total & & & $2.45 \mathrm{E}+02$ & $5.62 \mathrm{E}+03$ & $9.13 E+03$ & $2.09 \mathrm{E}+04$ & $1.48 \mathrm{E}+03$ & $1.80 \mathrm{E}+01$ & $4.70 \mathrm{E}+00$ & $7.01 \mathrm{E}+00$ & $6.23 E+01$ & $4.66 \mathrm{E}-01$ & $1.07 \mathrm{E}+02$ \\
\hline $24 !-T-112$ & Supernatant & DW (Liquid) & 13 & $0.00 \mathrm{E}+00$ & $3.03 \mathrm{E}+02$ & $5.05 \mathrm{E}+02$ & $7.44 \mathrm{E}+02$ & $0.00 \mathrm{E}+00$ & $0.00 \mathrm{E}+00$ & $1.47 \mathrm{E}-01$ & $2.55 \mathrm{E}-02$ & $1.10 \mathrm{E}-01$ & $4.60 \mathrm{E}-03$ & $0.00 \mathrm{E}+00$ \\
\hline $241-\mathrm{T}-112$ & ant & CSR & 13 & $19 \mathrm{E}+01$ & $3.03 \mathrm{E}+02$ & $5.05 E+02$ & $7.44 \mathrm{E}+02$ & $1.31 E-01$ & $1.74 \mathrm{E}+01$ & $1.47 \mathrm{E}-01$ & $2.55 \mathrm{E}-02$ & $1.10 \mathrm{E}-01$ & $4.60 \mathrm{E}-03$ & $1.06 \mathrm{E}+02$ \\
\hline $24 !-T-112$ & Sludge (Liquid \& Solid) & 2C (Solid) & 135 & $1.67 \mathrm{E}+02$ & $3.00 \mathrm{E}+03$ & $4.85 \mathrm{E}+03$ & $1.16 \mathrm{E}+04$ & $8.84 E+02$ & $2.28 \mathrm{E}-01$ & $8.08 \mathrm{E}-02$ & $4.13 \mathrm{E}+00$ & $3.96 \mathrm{E}+01$ & $2.63 \mathrm{E}-01$ & $2.65 \mathrm{E}-01$ \\
\hline $241-\mathrm{T}-112$ & Sludge (Liquid \& Solid) & 224-2 (Solid) & 91 & $3.66 \mathrm{E}+01$ & $2.02 \mathrm{E}+03$ & $3.27 \mathrm{E}+03$ & $7.83 \mathrm{E}+03$ & $5.96 \mathrm{E}+02$ & $3.50 \mathrm{E}-01$ & $4.32 \mathrm{E}+00$ & $2.83 \mathrm{E}+00$ & $2.24 E+01$ & $1.94 \mathrm{E}-0 \mathrm{I}$ & $8.50 \mathrm{E}-01$ \\
\hline 241-T-201 & Total & & & $4.01 \mathrm{E}+01$ & $6.50 \mathrm{E}+03$ & $5.90 \mathrm{E}+01$ & $4.08 \mathrm{E}+03$ & $E+01$ & $9.44 \mathrm{E}+00$ & $5.56 \mathrm{E}+00$ & $6.34 \mathrm{E}+00$ & $9.40 \mathrm{E}+01$ & $3.18 \mathrm{E}-01$ & $3.10 E+00$ \\
\hline $241-\mathrm{T}-201$ & Supernatant & 224-1 (Liquid) & 8 & $6.22 \mathrm{E}-01$ & $3.69 \mathrm{E}+02$ & $2.68 E+00$ & $2.01 E+02$ & $0.00 \mathrm{E}+00$ & $3.00 \mathrm{E}-04$ & $4.72 \mathrm{E}-07$ & $1.42 \mathrm{E}-05$ & $2.11 \mathrm{E}-04$ & $7.13 \mathrm{E}-07$ & $3.45 \mathrm{E}-04$ \\
\hline $241-\mathrm{T}-201$ & Sludge (Liquid \& Solid) & 224-1 (Solid) & 107 & $3.95 \mathrm{E}+01$ & $6.13 E+03$ & $5.63 \mathrm{E}+01$ & $3.88 \mathrm{E}+03$ & $1.15 \mathrm{E}+01$ & $9.44 \mathrm{E}+00$ & $5.56 \mathrm{E}+00$ & $6.34 \mathrm{E}+00$ & $9.40 \mathrm{E}+01$ & $3.18 \mathrm{E}-0 \mathrm{l}$ & $3.10 \mathrm{E}+00$ \\
\hline $241-\mathrm{T}-202$ & Total & & & $\mathrm{E}+01$ & $E+03$ & $E+01$ & $\mathrm{E}+03$ & 00 & $3 \mathrm{E}-01$ & $2.93 E+00$ & $1.92 \mathrm{E}+00$ & $1.52 \mathrm{E}+01$ & 1.32E-01 & $6.15 \mathrm{E}-01$ \\
\hline $241-\mathrm{T}-202$ & Sludge (Liquid \& Solid) & 224-2 (Solid) & 77 & $3.31 \mathrm{E}+01$ & $5.60 \mathrm{E}+03$ & $5.81 \mathrm{E}+01$ & $3.14 \mathrm{E}+03$ & $6.67 \mathrm{E}+00$ & $2.03 \mathrm{E}-01$ & $2.93 \mathrm{E}+00$ & $1.92 \mathrm{E}+00$ & $1.52 \mathrm{E}+01$ & $1.32 \mathrm{E}-01$ & $6.15 \mathrm{E}-01$ \\
\hline $241-\mathrm{T}-203$ & Total & & & $7.03 \mathrm{E}+01$ & $9.73 E+03$ & $5.54 \mathrm{E}+01$ & $5.73 \mathrm{E}+03$ & $9.41 \mathrm{E}+00$ & $3.67 \mathrm{E}-01$ & $6.22 \mathrm{E}+00$ & $4.67 \mathrm{E}+00$ & $3.71 \mathrm{E}+01$ & $3.20 \mathrm{E}-01$ & $1.10 \mathrm{E}+00$ \\
\hline 241-T-203 & Sludge (Liquid \& Solid) & 224-2 (Solid) & 136 & $7.03 \mathrm{E}+01$ & $9.73 \mathrm{E}+03$ & $5.54 \mathrm{E}+01$ & $5.73 \mathrm{E}+03$ & $9.41 \mathrm{E}+00$ & $3.67 \mathrm{E}-01$ & $6.22 \mathrm{E}+00$ & $4.67 \mathrm{E}+00$ & $3.71 \mathrm{E}+01$ & $3.20 \mathrm{E}-01$ & $1.10 \mathrm{E}+00$ \\
\hline $241-\mathrm{T}-204$ & Total & & & $5.97 \mathrm{E}+01$ & $9.08 \mathrm{E}+03$ & $4.67 \mathrm{E}+01$ & $5.23 \mathrm{E}+03$ & $8.82 \mathrm{E}+00$ & $6.47 \mathrm{E}-01$ & $3.64 \mathrm{E}+00$ & $3.84 \mathrm{E}+00$ & $3.05 \mathrm{E}+01$ & $2.63 \mathrm{E}-01$ & $1.10 \mathrm{E}+00$ \\
\hline 241-T-204 & Sludge (Liquid \& Solid) & 224-2 (Solid) & 136 & $5.97 \mathrm{E}+01$ & $9.08 \mathrm{E}+03$ & $4.67 \mathrm{E}+01$ & $5.23 \mathrm{E}+03$ & $8.82 \mathrm{E}+00$ & $6.47 \mathrm{E}-01$ & $3.64 \mathrm{E}+00$ & $3.84 \mathrm{E}+00$ & $3.05 E+01$ & $2.63 \mathrm{E}-01$ & $1.10 \mathrm{E}+00$ \\
\hline 241-TX-101 & Total & & & $8.49 \mathrm{E}+02$ & $8.41 \mathrm{E}+04$ & $1.86 \mathrm{E}+04$ & $6.85 \mathrm{E}+04$ & $9.10 \mathrm{E}+04$ & $1.94 \mathrm{E}+05$ & $2.52 \mathrm{E}+02$ & $7.51 \mathrm{E}+01$ & $3.53 \mathrm{E}+02$ & $1.17 \mathrm{E}+01$ & $4.01 \mathrm{E}+04$ \\
\hline $241-\mathrm{TX}-101$ & Sludge (Liquid \& Solid) & $\mathrm{Z}$ (Solid) & 4 & $0.00 \mathrm{E}+00$ & $3.38 \mathrm{E}+02$ & $1.37 \mathrm{E}+00$ & $1.72 \mathrm{E}+02$ & $1.19 \mathrm{E}+03$ & $0.00 \mathrm{E}+00$ & $1.21 \mathrm{E}+02$ & $2.86 \mathrm{E}+01$ & $1.14 \mathrm{E}+02$ & $7.15 \mathrm{E}+00$ & $0.00 \mathrm{E}+00$ \\
\hline 241-TX-101 & Sludge (Liquid \& Solid) & R1 (Solid) & 265 & $4.93 \mathrm{E}+02$ & $5.25 E+04$ & $1.48 \mathrm{E}+04$ & $4.74 \mathrm{E}+04$ & $8.77 \mathrm{E}+04$ & $1.92 \mathrm{E}+05$ & $1.17 \mathrm{E}+02$ & $4.51 \mathrm{E}+01$ & $2.32 \mathrm{E}+02$ & $4.31 \mathrm{E}+00$ & $2.96 \mathrm{E}+04$ \\
\hline 241-TX-101 & Sludge (Liquid \& Solid) & MW2 (Solid) & 11 & $0.00 \mathrm{E}+00$ & $9.61 \mathrm{E}+00$ & $8.00 \mathrm{E}+00$ & $1.67 \mathrm{E}+03$ & $0.00 \mathrm{E}+00$ & $1.54 \mathrm{E}+03$ & $7.52 \mathrm{E}-03$ & $1.46 \mathrm{E}-02$ & $1.15 \mathrm{E}-01$ & $9.98 \mathrm{E}-04$ & $3.93 \mathrm{E}+01$ \\
\hline
\end{tabular}




\section{RPP-5926 REV 6}

Table A-2. Tank Waste Inventory from Best Basis Inventory Detail Report (Queried on September 27, 2006) for

Hydrogen Generation Rate Calculations. (27 sheets)

\begin{tabular}{|c|c|c|c|c|c|c|c|c|c|c|c|c|c|c|}
\hline $\begin{array}{c}\text { Tank } \\
\text { Name }\end{array}$ & & & $\begin{array}{r}\text { Vol } \\
\text { (kL) }\end{array}$ & $\begin{array}{l}\text { TOC } \\
(\mathrm{Kg})\end{array}$ & $\begin{array}{l}\mathrm{NO}_{3} \\
(\mathrm{Kg})\end{array}$ & $\begin{array}{l}\mathrm{NO}_{2} \\
(\mathrm{Kg})\end{array}$ & $\begin{array}{c}\mathrm{Na} \\
(\mathrm{Kg})\end{array}$ & $\begin{array}{c}\mathbf{A l} \\
(\mathbf{K g})\end{array}$ & $\begin{array}{l}{ }^{90} \mathrm{Sr} \\
(\mathrm{Ci})\end{array}$ & $\begin{array}{l}{ }_{(\mathrm{Ci})}^{241} \mathrm{Am} \\
.\end{array}$ & $\begin{array}{c}{ }^{240} \mathrm{Pu} \\
(\mathrm{Ci})\end{array}$ & $\begin{array}{c}{ }^{{ }^{239}} \mathbf{P u} \\
\mathrm{Ci})\end{array}$ & ${ }_{(\mathrm{Ci})}^{{ }^{238} \mathrm{Pu}}$ & $\begin{array}{c}{ }^{137} \mathrm{Cs} \\
\text { (Ci) }\end{array}$ \\
\hline 1 & & T2-SltCk (Solid) & 49 & 2 & 4 & 3 & & 3 & 1 & & 0 & & & $5.16 \mathrm{E}+03$ \\
\hline 241-TX-101 & quid & id) & 15 & $5 E+01$ & $2.85 \mathrm{E}+03$ & $83 \mathrm{E}+03$ & $3.54 \mathrm{E}+03$ & $5.67 \mathrm{E}+02$ & $1.49 \mathrm{E}+01$ & $4.23 \mathrm{E}-02$ & $3.87 \mathrm{E}-04$ & $1.84 \mathrm{E}-03$ & $6.52 \mathrm{E}-05$ & +03 \\
\hline 241-TX-102 & Total & & & $68 \mathrm{E}+03$ & $.24 \mathrm{E}+05$ & $27 \mathrm{E}+04$ & $2.52 \mathrm{E}+05$ & $2.60 \mathrm{E}+04$ & $2.09 \mathrm{E}+03$ & $1.92 \mathrm{E}+02$ & $2.01 \mathrm{E}+01$ & $9.58 \mathrm{E}+01$ & $3.37 \mathrm{E}+00$ & $1.16 \mathrm{E}+05$ \\
\hline $241-$ & ludge (Liquid \& Solid) & ) & 8 & $\mathrm{E}+00$ & 00 & $\therefore+00$ & +03 & 00 & $E+03$ & 36E-03 & $1.06 \mathrm{E}-02$ & $38 \mathrm{E}-02$ & $26 \mathrm{E}-04$ & $86 \mathrm{E}+01$ \\
\hline 241-TX-102 & lid & T2-SltCk (Solid) & 692 & $9 \mathrm{E}+03$ & $4.01 \mathrm{E}+05$ & $79 \mathrm{E}+04$ & $2.22 \mathrm{E}+05$ & $2.14 \mathrm{E}+04$ & $8.45 \mathrm{E}+02$ & $1.92 \mathrm{E}+02$ & $2.01 \mathrm{E}+01$ & $9.57 \mathrm{E}+01$ & $3.37 \mathrm{E}+00$ & $7.29 \mathrm{E}+04$ \\
\hline 102 & 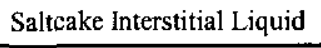 & T2-SitCk (Liquid) & 121 & 2 & 4 & 04 & 4 & 3 & $\varepsilon+02$ & 1 & 3 & 2 & 4 & 04 \\
\hline 241-TX-103 & Total & & & $8 \mathrm{E}+03$ & $2.84 \mathrm{E}+05$ & +04 & $1.69 \mathrm{E}+05$ & +04 & $E+02$ & $1.27 \mathrm{E}+02$ & $1.32 \mathrm{E}+01$ & $6.33 E+01$ & $2.22 \mathrm{E}+00$ & $7.70 \mathrm{E}+04$ \\
\hline 103 & d & T2-SltCk (Solid) & 454 & +03 & 05 & 04 & 05 & 04 & +02 & -02 & +01 & +01 & $E+\infty$ & $E+04$ \\
\hline 241-TX-103 & 110 & olid) & 9 & $3 \mathrm{E}-02$ & $2 \mathrm{E}+03$ & $08 \mathrm{E}+01$ & $9 \mathrm{E}+03$ & $2.83 E+01$ & $39 \mathrm{E}+00$ & $.70 \mathrm{E}-03$ & $6.27 \mathrm{E}-02$ & $5.53 \mathrm{E}-01$ & $4.11 \mathrm{E}-03$ & $25 \mathrm{E}+01$ \\
\hline 241 & S & T2-SltCk (Liquid) & 82 & $\mathrm{E}+02$ & +04 & +04 & +04 & +03 & $\mathrm{E}+01$ & E-01 & -03 & 02 & $\mathrm{E}-04$ & $2.92 E+04$ \\
\hline 103 & $\mathrm{~s}$ & id) & 3 & $\mathrm{E}-02$ & +02 & $E+01$ & +02 & +00 & $\mathrm{E}+00$ & -03 & $\mathrm{E}-03$ & E-02 & -04 & +01 \\
\hline 241-TX-104 & Total & & & $68 \mathrm{E}+02$ & $1.07 \mathrm{E}+05$ & $15 E+04$ & $5.22 \mathrm{E}+04$ & $4.15 \mathrm{E}+04$ & $7.22 \mathrm{E}+04$ & $7.04 \mathrm{E}+01$ & $9.55 \mathrm{E}+00$ & $4.67 \mathrm{E}+01$ & $1.38 \mathrm{E}+00$ & $3.15 E+04$ \\
\hline 2 & upernatant & - & 9 & 1 & $3.19 E+03$ & 2 & $1.85 \mathrm{E}+03$ & 2 & 0 & 1 & 3 & 3 & 4 & $00 \mathrm{E}+03$ \\
\hline 241-TX-104 & uoge (Liquil & (10) & 130 & $77 \mathrm{E}+02$ & $E+04$ & $.05 E+03$ & $2.07 \mathrm{E}+04$ & $3 \mathrm{E}+04$ & $7.21 \mathrm{E}+04$ & $\mathrm{E}+00$ & $3.11 \mathrm{E}+00$ & $0 \mathrm{E}+01$ & $2.97 \mathrm{E}-01$ & $1 \mathrm{E}+04$ \\
\hline 241-TX-104 & Itcake Solid & $\mathrm{T} 2-\mathrm{SltC}$ & 93 & $38 \mathrm{E}+02$ & $4.42 \mathrm{E}+04$ & $80 \mathrm{E}+03$ & $2.36 \mathrm{E}+04$ & $2.92 \mathrm{E}+03$ & $8.57 \mathrm{E}+01$ & $6.18 \mathrm{E}+01$ & $6.43 \mathrm{E}+00$ & $3.07 \mathrm{E}+01$ & $1.08 \mathrm{E}+00$ & $7.39 \mathrm{E}+03$ \\
\hline $10-104$ & 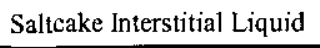 & 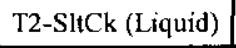 & $x^{2}+2>$ & 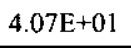 & 1 & 年 & N & $9.57 \mathrm{E}+01$ & in & 1 & $3.50 \mathrm{E}-03$ & 2 & 34 & $E+04$ \\
\hline 241-TX-105 & Total & & & $1.28 \mathrm{E}+04$ & $1.20 \mathrm{E}+06$ & $9.54 \mathrm{E}+04$ & $6.87 \mathrm{E}+05$ & $6.74 \mathrm{E}+04$ & $6.95 \mathrm{E}+03$ & $5.70 \mathrm{E}+02$ & $5.94 \mathrm{E}+01$ & $2.83 \mathrm{E}+02$ & $9.96 \mathrm{E}+00$ & $2.53 \mathrm{E}+05$ \\
\hline TX-105 & Liani & id) & 31 & $0.00 \mathrm{E}+00$ & $2.71 \mathrm{E}+01$ & $2.25 E+01$ & $4.70 \mathrm{E}+03$ & $0.00 \mathrm{E}+00$ & $4.35 \mathrm{E}+03$ & $2.12 \mathrm{E}-02$ & $4.12 \mathrm{E}-02$ & $3.25 \mathrm{E}-01$ & $2.81 \mathrm{E}-03$ & $1.11 E+02$ \\
\hline 241-TX-105 & Saitcake Solid & 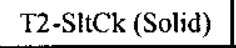 & 4 & +04 & +06 & +04 & 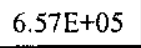 & 6. & $E+03$ & 02 & 701 & $E+02$ & $9.95 \mathrm{E}+00$ & $5 E+05$ \\
\hline 241-TX-105 & Saltcake Interstitial Liquid & T2-SltCk (Liquid) & 106 & 4.27E+02 & $2.01 \mathrm{E}+04$ & $1.29 \mathrm{E}+04$ & $2.50 \mathrm{E}+04$ & $4.01 \mathrm{E}+03$ & $1.06 \mathrm{E}+02$ & $2.99 \mathrm{E}-01$ & $2.73 \mathrm{E}-03$ & $1.30 \mathrm{E}-02$ & $4.61 \mathrm{E}-04$ & $3.77 \mathrm{E}+04$ \\
\hline 106 & tal & & & $88 \mathrm{E}+03$ & $6.96 \mathrm{E}+05$ & +04 & $4.08 \mathrm{E}+05$ & $4.63 \mathrm{E}+04$ & $1.30 \mathrm{E}+04$ & $3.27 \mathrm{E}+02$ & $3.58 \mathrm{E}+01$ & $1.72 \mathrm{E}+02$ & $5.83 \mathrm{E}+00$ & $1.77 \mathrm{E}+05$ \\
\hline 241-TX-106 & Sluage (Liquia \& solla) & 1 (3olia) & 15 & $2.79 \mathrm{E}+01$ & $2.97 \mathrm{E}+03$ & $8.39 \mathrm{E}+02$ & $2.68 \mathrm{E}+03$ & $4.96 \mathrm{E}+03$ & $1.09 \mathrm{E}+04$ & $6.65 \mathrm{E}+00$ & $2.55 \mathrm{E}+00$ & $1.31 \mathrm{E}+01$ & $2.44 \mathrm{E}-01$ & $1.67 \mathrm{E}+03$ \\
\hline 241-TX-106 & udge (Liquid \& Solid) & MW2 (Solid) & 4 & $0.00 \mathrm{E}+00$ & $3.49 \mathrm{E}+00$ & $2.91 \mathrm{E}+00$ & $6.06 \mathrm{E}+02$ & $0.00 \mathrm{E}+00$ & $5.62 \mathrm{E}+02$ & $2.73 \mathrm{E}-03$ & $5.31 \mathrm{E}-03$ & $4.19 \mathrm{E}-02$ & $3.63 \mathrm{E}-04$ & $1.43 \mathrm{E}+01$ \\
\hline 241-TX-106 & Saltcake Solid & T2-SitCk (Solid) & 1147 & $6.94 \mathrm{E}+03$ & $6.64 \mathrm{E}+05$ & $4.63 \mathrm{E}+04$ & $3.69 \mathrm{E}+05$ & $3.56 \mathrm{E}+04$ & $1.40 \mathrm{E}+03$ & $3.19 \mathrm{E}+02$ & $3.33 \mathrm{E}+01$ & $1.59 \mathrm{E}+02$ & $5.58 \mathrm{E}+00$ & $1.21 \mathrm{E}+05$ \\
\hline 241-TX-106 & Saltcake Interstitial Liquid & T2-S1tCk (Liquid) & 152 & $6.13 \mathrm{E}+02$ & $2.89 \mathrm{E}+04$ & $1.85 \mathrm{E}+04$ & $3.59 \mathrm{E}+04$ & $5.75 \mathrm{E}+03$ & $1.51 \mathrm{E}+02$ & $4.28 \mathrm{E}-01$ & $3.92 \mathrm{E}-03$ & $1.87 \mathrm{E}-02$ & $6.61 \mathrm{E}-04$ & $5.40 \mathrm{E}+04$ \\
\hline 241-TX-107 & Total & & & $6.27 \mathrm{E}+02$ & $6.94 \mathrm{E}+04$ & $6.98 \mathrm{E}+03$ & $4.11 \mathrm{E}+04$ & $5.38 \mathrm{E}+03$ & $1.09 \mathrm{E}+03$ & $3.38 \mathrm{E}+02$ & $3.53 \mathrm{E}+01$ & $1.68 \mathrm{E}+02$ & $5.93 \mathrm{E}+00$ & $2.03 \mathrm{E}+04$ \\
\hline 241-TX-107 & Saltcake Solid & -SltCk (Solid) & 62 & $4.82 \mathrm{E}+02$ & $4.61 \mathrm{E}+04$ & $3.21 \mathrm{E}+03$ & $2.56 \mathrm{E}+04$ & $2.47 \mathrm{E}+03$ & $9.72 \mathrm{E}+01$ & $3.35 \mathrm{E}+02$ & $3.49 \mathrm{E}+01$ & $1.66 \mathrm{E}+02$ & $5.85 \mathrm{E}+00$ & $8.38 \mathrm{E}+03$ \\
\hline
\end{tabular}


Table A-2. Tank Waste Inventory from Best Basis Inventory Detail Report (Queried on September 27, 2006) for Hydrogen Generation Rate Calculations. (27 sheets)

\begin{tabular}{|c|c|c|c|c|c|c|c|c|c|c|c|c|c|c|}
\hline $\begin{array}{c}\text { Tank } \\
\text { Name }\end{array}$ & v & e & $\begin{array}{c}\text { Vol } \\
(k L)\end{array}$ & $\begin{array}{l}\text { TOC } \\
(\mathrm{Kg})\end{array}$ & $\begin{array}{l}\mathrm{NO}_{3} \\
(\mathrm{Kg})\end{array}$ & $\begin{array}{l}\mathrm{NO}_{2} \\
(\mathrm{Kg})\end{array}$ & $\begin{array}{c}\mathrm{Na} \\
(\mathrm{Kg})\end{array}$ & $\begin{array}{c}\text { Al } \\
(\mathbf{K g})\end{array}$ & $\begin{array}{l}{ }^{90} \mathrm{Sr} \\
(\mathrm{Ci})\end{array}$ & $\begin{array}{c}{ }^{241} \mathrm{Am} \\
\text { (Ci) }\end{array}$ & $\begin{array}{c}{ }^{240} \mathrm{Pu} \\
(\mathrm{Ci})\end{array}$ & $\begin{array}{c}{ }^{239} \mathrm{Pu} \\
(\mathrm{Ci})\end{array}$ & ${ }_{(\mathrm{Ci})}^{{ }^{238} \mathrm{Pu}}$ & $\begin{array}{c}{ }^{137} \mathrm{Cs} \\
\text { (Ci) }\end{array}$ \\
\hline 07 & & & 24 & $9 \mathrm{E}+01$ & $32 E+04$ & $60 \mathrm{E}+02$ & $9.25 \mathrm{E}+03$ & $1.86 \mathrm{E}+03$ & $60 \mathrm{E}+02$ & $2.69 \mathrm{E}+00$ & $1.07 \mathrm{E}-01$ & $92 \mathrm{E}+00$ & $7.30 \mathrm{E}-02$ & $22 \mathrm{E}+03$ \\
\hline $241-7$ & 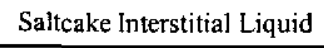 & d) & 19 & 1 & 33 & 13 & 33 & 22 & 1 & 2 & 4 & 03 & 5 & +03 \\
\hline $241-$ & Saltcake In & uid) & 7 & $7.07 \mathrm{E}+00$ & $1.52 \mathrm{E}+03$ & $92 E+02$ & $1.76 \mathrm{E}+03$ & $3.30 \mathrm{E}+02$ & $1.61 E+01$ & $4.71 \mathrm{E}-02$ & $7.56 \mathrm{E}-03$ & $3.56 \mathrm{E}-02$ & $1.35 \mathrm{E}-03$ & $1.95 E+03$ \\
\hline 241-7 & Total & & & $69 \mathrm{E}+03$ & $.52 \mathrm{E}+05$ & $19 E+04$ & $1.46 \mathrm{E}+05$ & $1.44 \mathrm{E}+04$ & $4.09 \mathrm{E}+03$ & $1.16 \mathrm{E}+02$ & $1.21 \mathrm{E}+01$ & $5.77 \mathrm{E}+01$ & +00 & $5.82 \mathrm{E}+04$ \\
\hline $2410^{-}$ & a) & d) & 15 & $50 \mathrm{E}+01$ & +03 & $32 \mathrm{E}+02$ & $2.40 \mathrm{E}+03$ & $3.97 \mathrm{E}+01$ & $2.42 \mathrm{E}+03$ & $.36 \mathrm{E}-01$ & $2.65 \mathrm{E}-02$ & $2.47 \mathrm{E}-01$ & $1.72 \mathrm{E}-03$ & $2.61 \mathrm{E}+02$ \\
\hline 241-1 & Slud & id) & 8 & $0.00 \mathrm{E}+00$ & $6.99 \mathrm{E}+00$ & $5.82 \mathrm{E}+00$ & $1.21 \mathrm{E}+03$ & $0.00 \mathrm{E}+00$ & $1.12 \mathrm{E}+03$ & $5.47 \mathrm{E}-03$ & $1.06 \mathrm{E}-02$ & $8.38 \mathrm{E}-02$ & $7.26 \mathrm{E}-04$ & $2.86 \mathrm{E}+01$ \\
\hline 241 & & a) & 15 & $51 E+03$ & $.40 \mathrm{E}+05$ & $1.67 \mathrm{E}+04$ & $1.33 \mathrm{E}+05$ & $1.29 \mathrm{E}+04$ & $5.07 \mathrm{E}+02$ & $1.16 \mathrm{E}+02$ & $1.20 \mathrm{E}+01$ & $5.74 \mathrm{E}+01$ & 00 & 04 \\
\hline $241-7$ & Interstit1 & T2-Slt & 40 & $1.61 \mathrm{E}+02$ & $7.60 \mathrm{E}+03$ & $88 \mathrm{E}+03$ & $9.44 \mathrm{E}+03$ & $1.51 \mathrm{E}+03$ & $3.98 \mathrm{E}+01$ & $1.13 \mathrm{E}-01$ & $1.03 \mathrm{E}-03$ & $4.92 \mathrm{E}-03$ & $1.74 \mathrm{E}-04$ & $1.42 \mathrm{E}+04$ \\
\hline-109 & Total & & & $03 E+03$ & $2.48 \mathrm{E}+05$ & $1.91 \mathrm{E}+04$ & $1.95 \mathrm{E}+05$ & $2.93 \mathrm{E}+04$ & $9.48 \mathrm{E}+03$ & $2.72 \mathrm{E}+01$ & $1.78 \mathrm{E}+01$ & $1.64 \mathrm{E}+02$ & $8+00$ & $E+04$ \\
\hline 241 & wor & & 375 & +03 & $E+05$ & $91 \mathrm{E}+04$ & $1.95 E+05$ & $3 E+04$ & $9.48 \mathrm{E}+03$ & $2.72 \mathrm{E}+01$ & $1.78 \mathrm{E}+01$ & $4 E+02$ & $E+00$ & $E+04$ \\
\hline 241-TX-110 & Total & & & $6 \mathrm{E}+03$ & $49 \mathrm{E}+05$ & $17 \mathrm{E}+04$ & $9 \mathrm{E}+05$ & $8 \mathrm{E}+04$ & $94 E+03$ & $4.43 \mathrm{E}+02$ & $4.77 \mathrm{E}+01$ & $35 \mathrm{E}+02$ & $7.81 \mathrm{E}+00$ & $1.87 \mathrm{E}+05$ \\
\hline $241-\mathrm{T}$ & (IIU) & id) & 40 & $E+02$ & $2.52 \mathrm{E}+04$ & $94 \mathrm{E}+03$ & $1.99 \mathrm{E}+04$ & $2.98 \mathrm{E}+03$ & $9.65 \mathrm{E}+02$ & $2.77 \mathrm{E}+00$ & $1.82 \mathrm{E}+00$ & $E+01$ & $E-01$ & $E+03$ \\
\hline 241-TX-110 & lid & T2-SitCk (Solid) & 1580 & $9.56 \mathrm{E}+03$ & $9.15 \mathrm{E}+05$ & $6.37 \mathrm{E}+04$ & $5.08 \mathrm{E}+05$ & $4.90 \mathrm{E}+04$ & $1.93 \mathrm{E}+03$ & $4.40 \mathrm{E}+02$ & $4.58 \mathrm{E}+01$ & $2.18 \mathrm{E}+02$ & $7.69 \mathrm{E}+00$ & $1.66 \mathrm{E}+05$ \\
\hline-110 & Inters & T2-SltCk & 49 & $7 \mathrm{E}+02$ & +03 & $5.98 \mathrm{E}+03$ & 1.100104 & $5 \mathrm{E}+03$ & $\mathrm{E}+01$ & $20 L=0$ & $\mathrm{E}-0$ & $\mathrm{E}-03$ & 04 & $1.74 \mathrm{E}+04$ \\
\hline 24 & lotal & & & $7.44 \mathrm{E}+03$ & $7.25 \mathrm{E}+05$ & $34 E+04$ & $4.13 \mathrm{E}+05$ & $4.14 \mathrm{E}+04$ & $2.60 \mathrm{E}+03$ & $3.36 \mathrm{E}+02$ & $3.68 \mathrm{E}+01$ & $1.84 \mathrm{E}+02$ & $5.95 \mathrm{E}+00$ & $1.38 \mathrm{E}+05$ \\
\hline 241-TX-111 & dge (Liquid \& Solid) & lid) & 163 & $1.23 \mathrm{E}+02$ & $.94 \mathrm{E}+04$ & $.26 \mathrm{E}+03$ & $2.31 E+04$ & $3.47 \mathrm{E}+03$ & $1.12 \mathrm{E}+03$ & $3.22 \mathrm{E}+00$ & $2.11 \mathrm{E}+00$ & $1.94 \mathrm{E}+01$ & $1.37 \mathrm{E}-01$ & $3.70 \mathrm{E}+03$ \\
\hline 2 & & T2-SltCk (Solid) & 1194 & 1.4 & 3 & $4.82 \mathrm{E}+04$ & $3.04 \mathrm{~b}$ & 4 & 1.4 & 2 & 1 & 02 & 00 & 05 \\
\hline 241-TX-111 & Saltcake Interstitial Liquid & T2-SitCk (Liquid) & 24 & $9.67 \mathrm{E}+01$ & $4.56 \mathrm{E}+03$ & $2.93 \mathrm{E}+03$ & $5.66 \mathrm{E}+03$ & $9.07 \mathrm{E}+02$ & $2.39 \mathrm{E}+01$ & $6.76 \mathrm{E}-02$ & $6.19 \mathrm{E}-04$ & $2.95 \mathrm{E}-03$ & $1.04 \mathrm{E}-04$ & $8.53 \mathrm{E}+03$ \\
\hline 241-TX-112 & Total & & & $1.38 \mathrm{E}+04$ & $35 \mathrm{E}+06$ & $E+05$ & $7.64 \mathrm{E}+05$ & $7.24 \mathrm{E}+04$ & $2.84 \mathrm{E}+03$ & $6.17 \mathrm{E}+02$ & $6.48 \mathrm{E}+01$ & $3.11 \mathrm{E}+02$ & $1.08 \mathrm{E}+01$ & $2.67 \mathrm{E}+05$ \\
\hline 12 & Sollid & 12-SltCk (Solid) & 2215 & $1.34 \mathrm{t}+04$ & 06 & $8.94 \mathrm{E}+04$ & $7.12 \mathrm{E}+05$ & $6.87 \mathrm{E}+04$ & 2. $.0 \mathrm{E}+\mathrm{US}$ & $6.17 \mathrm{E}+02$ & $6.43 \mathrm{E}+01$ & $3.06 \mathrm{E}+02$ & $1.08 \mathrm{E}+01$ & $2.33 \mathrm{E}+05$ \\
\hline 241-TX-112 & Saltcake Solid & T1-SltCk (Solid) & 75 & $1.02 \mathrm{E}-01$ & $4.18 \mathrm{E}+04$ & $2.57 \mathrm{E}+02$ & $2.83 \mathrm{E}+04$ & $2.36 \mathrm{E}+02$ & $1.16 \mathrm{E}+01$ & $3.09 \mathrm{E}-02$ & $5.22 \mathrm{E}-01$ & $4.60 \mathrm{E}+00$ & $3.43 \mathrm{E}-02$ & $2.71 \mathrm{E}+02$ \\
\hline $241-\mathrm{TX}-112$ & Saltcake Interstitial Liquid & T2-SitCk (Liquid) & 93 & $3.75 E+02$ & $1.77 \mathrm{E}+04$ & $1.13 E+04$ & $2.19 \mathrm{E}+04$ & $3.52 \mathrm{E}+03$ & $9.26 \mathrm{E}+01$ & $2.62 \mathrm{E}-01$ & $2.40 E-03$ & $1.14 \mathrm{E}-02$ & $4.04 \mathrm{E}-04$ & $3.31 \mathrm{E}+04$ \\
\hline $241-\mathrm{TX}-112$ & Saltcake Interstitial Liquid & uid) & 15 & E-02 & $5+03$ & $1.27 \mathrm{E}+02$ & $1.69 \mathrm{E}+03$ & $5.42 \mathrm{E}+00$ & $3.18 \mathrm{E}+01$ & $2.32 \mathrm{E}-02$ & $8.86 \mathrm{E}-03$ & $7.83 \mathrm{E}-02$ & $5.82 \mathrm{E}-04$ & $1.34 E+02$ \\
\hline $241-\mathrm{TX}-113$ & Total & & & $2.07 \mathrm{E}+03$ & $1.66 \mathrm{E}+06$ & $1.55 \mathrm{E}+04$ & $9.06 \mathrm{E}+05$ & $1.66 \mathrm{E}+04$ & $5.42 \mathrm{E}+03$ & $2.61 \mathrm{E}+01$ & $6.54 \mathrm{E}+00$ & $5.13 E+01$ & $6.28 \mathrm{E}-01$ & $3.18 \mathrm{E}+04$ \\
\hline 241 & udge (Liquid \& Solid) & (Solid) & 51 & $2.64 \mathrm{E}+02$ & +04 & $4.87 \mathrm{E}+03$ & $4.98 \mathrm{E}+04$ & $7.48 \mathrm{E}+03$ & $2.42 \mathrm{E}+03$ & $6.93 E+00$ & $4.55 E+00$ & $4.18 \mathrm{E}+01$ & $2.94 \mathrm{E}-01$ & $7.96 \mathrm{E}+03$ \\
\hline 241-TX-113 & Saltcake Solid & T2-SitCk (Solid) & 2045 & $1.73 E+03$ & $1.59 \mathrm{E}+06$ & $8.21 \mathrm{E}+03$ & $8.51 \mathrm{E}+05$ & $8.41 E+03$ & $2.98 \mathrm{E}+03$ & $1.91 \mathrm{E}+01$ & $1.99 \mathrm{E}+00$ & $9.49 \mathrm{E}+00$ & $3.34 \mathrm{E}-01$ & $1.67 \mathrm{E}+04$ \\
\hline 241-TX-113 & Itcake Interstitial Liquid & uid) & 20 & $8.06 \mathrm{E}+01$ & $.80 \mathrm{E}+03$ & $2.44 \mathrm{E}+03$ & $72 \mathrm{E}+03$ & $7.56 \mathrm{E}+02$ & $1.99 \mathrm{E}+01$ & $5.63 \mathrm{E}-02$ & $8-04$ & $2.46 \mathrm{E}-03$ & $8.69 \mathrm{E}-05$ & $7.11 \mathrm{E}+03$ \\
\hline
\end{tabular}




\section{RPP-5926 REV 6}

Table A-2. Tank Waste Inventory from Best Basis Inventory Detail Report (Queried on September 27, 2006) for

Hydrogen Generation Rate Calculations. (27 sheets)

\begin{tabular}{|c|c|c|c|c|c|c|c|c|c|c|c|c|c|c|}
\hline $\begin{array}{c}\text { Tank } \\
\text { Name }\end{array}$ & se & jpe & $\begin{array}{r}\text { Vol } \\
(k \mathbf{k})\end{array}$ & $\begin{array}{l}\text { TOC } \\
(\mathrm{Kg})\end{array}$ & $\begin{array}{l}\mathrm{NO}_{3} \\
(\mathrm{Kg})\end{array}$ & $\begin{array}{l}\mathrm{NO}_{2} \\
(\mathrm{Kg})\end{array}$ & $\begin{array}{c}\mathrm{Na} \\
(\mathbf{K g})\end{array}$ & $\begin{array}{c}\mathbf{A l} \\
(\mathbf{K g})\end{array}$ & $\begin{array}{l}{ }^{90} \mathrm{Sr} \\
\text { (Ci) }\end{array}$ & $\begin{array}{c}{ }^{241} \mathrm{Am} \\
\text { (Ci) }\end{array}$ & $\begin{array}{c}{ }^{240} \mathbf{P u} \\
(\mathrm{Ci})\end{array}$ & $\begin{array}{c}{ }^{239} \mathrm{Pu} \\
\text { (Ci) }\end{array}$ & $\begin{array}{c}{ }^{238} \mathrm{Pu} \\
(\mathrm{Ci})\end{array}$ & $\begin{array}{c}{ }^{137} \mathrm{Cs} \\
\text { (Ci) }\end{array}$ \\
\hline 14 & tal & & & +04 & $1.13 \mathrm{E}+06$ & $2 \mathrm{E}+04$ & $2 \mathrm{E}+05$ & $5.62 \mathrm{E}+04$ & $39 \mathrm{E}+03$ & 02 & $22 \mathrm{E}+01$ & -02 & $61 \mathrm{E}+00$ & 05 \\
\hline 241-TX-114 & Sludge (Liquid \& & d) & 15 & $1.13 \mathrm{E}+01$ & $2.70 \mathrm{E}+03$ & $.08 E+02$ & $2.13 E+03$ & $3.20 \mathrm{E}+02$ & $1.03 \mathrm{E}+02$ & $2.96 \mathrm{E}-01$ & $1.94 \mathrm{E}-01$ & $1.79 \mathrm{E}+00$ & $1.26 \mathrm{E}-02$ & $3.40 \mathrm{E}+02$ \\
\hline $241-1$ & ke Solid & $\varepsilon$ & 50 & $E+04$ & $1 \mathrm{E}+06$ & $6 \mathrm{E}+04$ & 05 & $E+04$ & $E+03$ & $E+02$ & $E+01$ & +02 & $8+00$ & $8+05$ \\
\hline 241-TX-114 & lid & lid) & 173 & $2.36 \mathrm{E}-01$ & $9.64 \mathrm{E}+04$ & $5.92 \mathrm{E}+02$ & $6.52 \mathrm{E}+04$ & $5.45 \mathrm{E}+02$ & $2.67 \mathrm{E}+01$ & $7.12 \mathrm{E}-02$ & $1.20 \mathrm{E}+00$ & $1.06 \mathrm{E}+01$ & $7.90 \mathrm{E}-02$ & $6.25 \mathrm{E}+02$ \\
\hline 241-TX-114 & Saltcake Interst & T2-SItCk (Liquid) & 28 & $13 E+02$ & $5.32 \mathrm{~F}+03$ & $3.42 \mathrm{E}+03$ & $6.61 E+03$ & $1.06 \mathrm{E}+03$ & $2.79 \mathrm{E}+01$ & $7.89 \mathrm{E}-02$ & $7.22 \mathrm{E}-04$ & $3.44 \mathrm{E}-03$ & $1.22 \mathrm{E}-04$ & $9.96 \mathrm{E}+03$ \\
\hline 241 . & Saltcake I & Tl-SItCl & 46 & $55 \mathrm{E}-01$ & $9.98 \mathrm{E}+03$ & $3.91 \mathrm{E}+02$ & $5.20 \mathrm{E}+03$ & $1.66 \mathrm{E}+01$ & $9.76 \mathrm{E}+01$ & $7.12 \mathrm{E}-02$ & $2.72 \mathrm{E}-02$ & $2.40 \mathrm{E}-01$ & $1.78 \mathrm{E}-03$ & $4.10 \mathrm{E}+02$ \\
\hline 241-TX-115 & Total & & & $\mathrm{E}+04$ & 1.1 & $9.22 \mathrm{E}+04$ & +05 & +04 & $7.34 \mathrm{E}+03$ & $5.46 \mathrm{E}+02$ & $5.69 \mathrm{E}+01$ & $E+02$ & $9.55 \mathrm{E}+00$ & $2.44 \mathrm{E}+05$ \\
\hline 241-T & Slud & ) & 30 & $E+01$ & $8.20 E+03$ & $5.64 \mathrm{E}+02$ & $4.81 E+03$ & $7.94 \mathrm{E}+01$ & $4.84 \mathrm{E}+03$ & $2.72 \mathrm{E}-01$ & $5.29 \mathrm{E}-02$ & 4.94E-01 & 03 & $E+02$ \\
\hline 241-TX-115 & Sallcake & T2-SltCk (Solid) & 960 & $19 E+04$ & $1.13 \mathrm{E}+06$ & $7.91 \mathrm{E}+04$ & $6.30 \mathrm{E}+05$ & $6.08 \mathrm{E}+04$ & $2.39 \mathrm{E}+03$ & $5.46 \mathrm{E}+02$ & $5.69 \mathrm{E}+01$ & $2.71 \mathrm{E}+02$ & $9.54 \mathrm{E}+00$ & $2.06 \mathrm{E}+05$ \\
\hline 241-TX-115 & Saltcake Interstit & T2-S1tC & 103 & $4.15 \mathrm{E}+02$ & $1.96 \mathrm{E}+04$ & $1.26 \mathrm{E}+04$ & $E+04$ & $3.89 \mathrm{E}+03$ & $\mathrm{E}+02$ & $2.90 \mathrm{E}-01$ & 3 & -02 & $\mathrm{E}-04$ & $3.66 \mathrm{E}+04$ \\
\hline $241-\mathrm{TX}-116$ & Total & & & $6.45 \mathrm{E}+03$ & $1.56 \mathrm{E}+06$ & $4.31 E+04$ & $7.64 \mathrm{E}+05$ & $6.25 \mathrm{E}+04$ & $1.28 \mathrm{E}+03$ & $2.58 \mathrm{E}+02$ & $3.50 \mathrm{E}+01$ & $2.00 \mathrm{E}+02$ & $5.04 \mathrm{E}+00$ & $1.32 \mathrm{E}+05$ \\
\hline 241-TX-116 & udge (Liquid \& Solid) & $\mathrm{DE}$ (Solid) & 248 & $8.25 E+02$ & $3.89 E+04$ & $2.50 \mathrm{E}+04$ & $4.83 \mathrm{E}+04$ & $5.54 \mathrm{E}+02$ & $2.04 \mathrm{E}+02$ & $5.76 \mathrm{E}-01$ & $5.28 \mathrm{E}-03$ & $2.52 \mathrm{E}-02$ & $8.89 \mathrm{E}-04$ & $7.27 \mathrm{E}+04$ \\
\hline 241-TX-116 & Saltcake Solid & T2-S1tCk (Solid) & 926 & 3 & 5 & 4 & $2.84 \mathrm{E}+05$ & 4 & 2 & 22 & +01 & 02 & -00 & +04 \\
\hline 241-TX-116 & Saltcake Solid & Tl-SltCk (Solid) & 977 & $1.61 \mathrm{E}+00$ & $9.48 \mathrm{E}+05$ & $4.04 \mathrm{E}+03$ & 4.15E+05 & $3.34 \mathrm{E}+03$ & $1.51 \mathrm{E}+02$ & $4.86 \mathrm{E}-01$ & $8.22 \mathrm{E}+00$ & $7.25 \mathrm{E}+01$ & $5.39 \mathrm{E}-01$ & $4.27 \mathrm{E}+03$ \\
\hline 116 & s & T1-SltCk (Liquid) & 113 & $3.60 \mathrm{E}+01$ & 04 & $1.19 \mathrm{E}+03$ & $1.71 \mathrm{E}+04$ & $93 \mathrm{E}-01$ & $E-01$ & $50 \mathrm{E}-02$ & $\mathrm{E}-04$ & $1.43 \mathrm{E}-03$ & $.06 \mathrm{E}-05$ & $2.33 E+03$ \\
\hline 241-TX-117 & $10 \mathrm{cal}$ & & & $6.25 \mathrm{E}+03$ & $9.57 \mathrm{E}+05$ & $4.42 \mathrm{E}+04$ & $5.73 \mathrm{E}+05$ & $3.43 \mathrm{E}+04$ & $1.46 \mathrm{E}+03$ & $2.88 \mathrm{E}+02$ & $3.44 \mathrm{E}+01$ & $1.81 \mathrm{E}+02$ & $5.32 \mathrm{E}+00$ & $1.11 E+05$ \\
\hline 241-TX-117 & udge Solid & DE (Solid) & 110 & $0.00 \mathrm{E}+00$ & $0.00 \mathrm{E}+00$ & $0.00 \mathrm{E}+00$ & $0.00 \mathrm{E}+00$ & $2.46 \mathrm{E}+02$ & $0.00 \mathrm{E}+00$ & $0.00 \mathrm{E}+00$ & $0.00 \mathrm{E}+00$ & $0.00 \mathrm{E}+00$ & $0.00 \mathrm{E}+00$ & $0.00 \mathrm{E}+00$ \\
\hline 241-TX-117 & $\mathrm{C}$ & 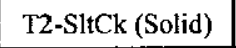 & 033 & $6.25 \mathrm{E}+03$ & $5.98 \mathrm{E}+05$ & $.17 \mathrm{E}+04$ & $32 \mathrm{E}+05$ & 04 & +03 & $2.88 \mathrm{E}+02$ & +01 & 02 & +00 & $9 \mathrm{E}+05$ \\
\hline 241-TX-117 & Saltcake Solid & T1-SltCk (Solid) & 626 & $8.54 \mathrm{E}-01$ & $3.49 \mathrm{E}+05$ & $2.14 \mathrm{E}+03$ & $2.36 \mathrm{E}+05$ & $1.97 \mathrm{E}+03$ & $9.68 \mathrm{E}+01$ & $2.58 \mathrm{E}-01$ & $4.36 \mathrm{E}+00$ & $3.84 \mathrm{E}+01$ & $2.86 \mathrm{E}-01$ & $2.26 \mathrm{E}+03$ \\
\hline 241-TX-117 & Saltcake Interstitial Liquid & T1-SltCk (Liquid) & 48 & $1.62 \mathrm{E}-01$ & $1.04 \mathrm{E}+04$ & $4.08 \mathrm{E}+02$ & $5.42 \mathrm{E}+03$ & $1.73 \mathrm{E}+01$ & $1.02 \mathrm{E}+02$ & $7.43 \mathrm{E}-02$ & $2.84 \mathrm{E}-02$ & $2.51 \mathrm{E}-01$ & $1.86 \mathrm{E}-03$ & $4.28 \mathrm{E}+02$ \\
\hline 118 & Total & & & +03 & $1.21 \mathrm{E}$ & $3.57 \mathrm{E}+04$ & $8 \mathrm{E}+05$ & $28 \mathrm{E}+04$ & $1.92 \mathrm{E}+05$ & $5.71 \mathrm{E}+03$ & $7.79 \mathrm{E}+02$ & $3.71 E+03$ & $1.31 \mathrm{E}+02$ & $2 \mathrm{E}+04$ \\
\hline 241-TX-118 & Saltcake Solid & T2-SltCk (Solid) & 671 & $3.79 \mathrm{E}+03$ & $9.33 \mathrm{E}+04$ & $1.52 \mathrm{E}+04$ & $2.42 \mathrm{E}+05$ & $5.70 \mathrm{E}+03$ & $1.39 \mathrm{E}+05$ & $1.01 E+03$ & $1.05 \mathrm{E}+02$ & $4.99 \mathrm{E}+02$ & $1.76 \mathrm{E}+01$ & $3.45 \mathrm{E}+04$ \\
\hline 241-TX-118 & Saltcake Solid & NA & 125 & $4.04 E+03$ & $1.59 \mathrm{E}+03$ & $3.51 E+03$ & $3.27 \mathrm{E}+04$ & $1.84 \mathrm{E}+03$ & $5.24 \mathrm{E}+04$ & $4.71 \mathrm{E}+03$ & $6.74 \mathrm{E}+02$ & $3.21 \mathrm{E}+03$ & $1.13 \mathrm{E}+02$ & $1.31 E+03$ \\
\hline $241-\mathrm{TX}-118$ & Saltcake Interstitial Liquid & T2-SltCk (Liquid) & 139 & $5.60 \mathrm{E}+02$ & $2.64 \mathrm{E}+04$ & $1.70 \mathrm{E}+04$ & $3.28 \mathrm{E}+04$ & $5.25 \mathrm{E}+03$ & $1.38 \mathrm{E}+02$ & $3.92 \mathrm{E}-01$ & $3.59 \mathrm{E}-03$ & $1.71 \mathrm{E}-02$ & $6.04 \mathrm{E}-04$ & $4.94 \mathrm{E}+04$ \\
\hline 241-TY-101 & Total & & & $4.70 \mathrm{E}+02$ & $1.06 \mathrm{E}+05$ & $8.35 E+03$ & $8.76 \mathrm{E}+04$ & $2.06 \mathrm{E}+04$ & $6.94 \mathrm{E}+03$ & $9.18 \mathrm{E}+00$ & $1.05 E+01$ & j. $25 \mathrm{E}+02$ & $6.16 \mathrm{E}-01$ & $2.94 \mathrm{E}+02$ \\
\hline 241-TY-101 & Sludge (Liquid \& Solid) & ICFeCN (Solid) & 273 & $2.97 \mathrm{E}+02$ & $6.49 \mathrm{E}+04$ & $7.84 \mathrm{E}+03$ & $5.43 \mathrm{E}+04$ & $1.30 \mathrm{E}+04$ & $4.36 \mathrm{E}+03$ & $5.72 \mathrm{E}+00$ & $5.43 \mathrm{E}+00$ & $8.01 E+01$ & $2.83 \mathrm{E}-01$ & $1.01 \mathrm{E}+02$ \\
\hline 241-TY-101 & Saltcake Solid & T1-SltCk (Solid) & 159 & $1.73 \mathrm{E}+02$ & $3.78 \mathrm{E}+04$ & $3.83 E+02$ & $3.16 \mathrm{E}+04$ & $7.59 \mathrm{E}+03$ & $2.54 \mathrm{E}+03$ & $3.44 \mathrm{E}+00$ & $5.07 \mathrm{E}+00$ & $4.47 \mathrm{E}+01$ & $3.33 \mathrm{E}-01$ & $5.88 \mathrm{E}+01$ \\
\hline
\end{tabular}


Table A-2. Tank Waste Inventory from Best Basis Inventory Detail Report (Queried on September 27, 2006) for Hydrogen Generation Rate Calculations. (27 sheets)

\begin{tabular}{|c|c|c|c|c|c|c|c|c|c|c|c|c|c|c|}
\hline $\begin{array}{c}\text { Tank } \\
\text { Name }\end{array}$ & $\mathbf{W}$ & pe & $\begin{array}{c}\text { Vol } \\
(\mathrm{kL})\end{array}$ & $\begin{array}{l}\text { TOC } \\
(\mathrm{Kg})\end{array}$ & $\begin{array}{l}\mathrm{NO}_{3} \\
(\mathrm{Kg})\end{array}$ & $\begin{array}{l}\mathrm{NO}_{2} \\
(\mathrm{Kg})\end{array}$ & $\begin{array}{c}\mathbf{N a} \\
(\mathbf{K g})\end{array}$ & $\begin{array}{c}\text { Al } \\
\text { (Kg) }\end{array}$ & $\begin{array}{l}{ }^{90} \mathrm{Sr} \\
\text { (Ci) }\end{array}$ & $\begin{array}{c}{ }^{241} \mathrm{Am} \\
\text { (Ci) }\end{array}$ & $\begin{array}{c}{ }^{240} \mathrm{Pu} \\
\text { (Ci) }\end{array}$ & $\begin{array}{c}{ }^{239} \mathrm{Pu} \\
(\mathrm{Ci})\end{array}$ & $\begin{array}{c}{ }^{238} \mathrm{Pu} \\
\text { (Ci) }\end{array}$ & $\begin{array}{c}{ }^{137} \mathrm{Cs} \\
\text { (Ci) }\end{array}$ \\
\hline 41-TY-101 & Saltca & ) & 15 & 7E-02 & 03 & $1.27 \mathrm{E}+02$ & $1.69 \mathrm{E}+03$ & $5.42 \mathrm{E}+00$ & $3.18 \mathrm{E}+01$ & $2.32 \mathrm{E}-02$ & $8.86 \mathrm{E}-03$ & 02 & 44 & 2 \\
\hline 241-TY-102 & Total & & & $2.59 \mathrm{E}+02$ & $1.40 \mathrm{E}+05$ & $77 \mathrm{E}+03$ & $8.84 \mathrm{E}+04$ & $45 E+03$ & $21 \mathrm{E}+03$ & $1.15 \mathrm{E}+00$ & $9.29 \mathrm{E}-02$ & $12 \mathrm{E}-01$ & $1.13 \mathrm{E}-02$ & $1.32 \mathrm{E}+04$ \\
\hline 102 & 10 & T2-SltCk (Solid) & 99 & $0 \mathrm{E}+01$ & 4 & $4 \mathrm{E}+03$ & $4.22 \mathrm{E}+04$ & $E+01$ & $6.08 \mathrm{E}+02$ & 74E-01 & $5.04 \mathrm{E}-02$ & $40 \mathrm{E}-01$ & $8.46 \mathrm{E}-03$ & $1 \mathrm{E}+02$ \\
\hline 241-TY-102 & Saltcake Solid & $\mathrm{Tl}$-SltCk (Solid) & 90 & $5.53 \mathrm{E}+01$ & $5.48 \mathrm{E}+04$ & $37 \mathrm{E}+02$ & $3.49 \mathrm{E}+04$ & $6.94 \mathrm{E}+01$ & $5.02 \mathrm{E}+02$ & $3.46 \mathrm{E}-02$ & $2.45 \mathrm{E}-02$ & $2.16 \mathrm{E}-01$ & $1.60 \mathrm{E}-03$ & $3.73 E+02$ \\
\hline 24 & lats & T2-SltCk (Liquid) & 34 & 2 & 3 & 3 & 33 & $\mathrm{E}+03$ & +01 & 2 & $\mathrm{E}-04$ & 33 & E-04 & +04 \\
\hline 102 & Saltca & T1-S & 29 & $80 \mathrm{E}-02$ & $6.29 \mathrm{E}+03$ & $2.46 \mathrm{E}+02$ & $3.28 \mathrm{E}+03$ & $1.05 \mathrm{E}+01$ & $6.15 \mathrm{E}+01$ & $4.49 \mathrm{E}-02$ & $1.71 \mathrm{E}-02$ & $1.51 \mathrm{E}-01$ & $1.12 \mathrm{E}-03$ & $2.59 \mathrm{E}+02$ \\
\hline-103 & Total & & & $1.24 \mathrm{E}+03$ & $1.49 \mathrm{E}+05$ & $1.30 \mathrm{E}+04$ & $1.17 \mathrm{E}+05$ & $4.45 \mathrm{E}+03$ & $6.89 \mathrm{E}+04$ & $2.38 \mathrm{E}+01$ & $1.90 \mathrm{E}+01$ & $1.58 \mathrm{E}+02$ & $2.07 \mathrm{E}+00$ & $2.73 E+04$ \\
\hline 241-TY-103 & Slud & (j) & 20 & $33 \mathrm{E}+02$ & $5.71 \mathrm{E}+04$ & $7 \mathrm{E}+03$ & $4.34 \mathrm{E}+04$ & $2 \mathrm{E}+03$ & $2.80 \mathrm{E}+04$ & $E+00$ & $6.97 \mathrm{E}+00$ & $E+01$ & $4.53 \mathrm{E}-01$ & $4.61 \mathrm{E}+03$ \\
\hline-103 & Sludge (Liquid \& Solid) & $1 \mathrm{CFeCN}$ (Solid) & 170 & $3.35 \mathrm{E}+02$ & $4.42 \mathrm{E}+04$ & $.37 \mathrm{E}+03$ & $3.35 \mathrm{E}+04$ & $8.65 \mathrm{E}+02$ & $2.17 \mathrm{E}+04$ & $9.95 \mathrm{E}+00$ & $3.53 \mathrm{E}+00$ & $5.21 \mathrm{E}+01$ & $1.84 \mathrm{E}-01$ & $3.56 \mathrm{E}+03$ \\
\hline $241-7$ & id & d) & 150 & +02 & $3.90 \mathrm{E}+04$ & $\mathrm{E}+03$ & $\mathrm{E}+04$ & $\mathrm{E}+02$ & $1.91 \mathrm{E}+04$ & $\mathrm{E}+0 \mathrm{l}$ & $8.52 \mathrm{E}+00$ & 01 & $E+00$ & $3.14 \mathrm{E}+03$ \\
\hline 103 & 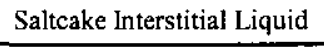 & iid) & 45 & $1.81 \mathrm{E}+02$ & $8.55 E+03$ & $49 \mathrm{E}+03$ & $1.06 \mathrm{E}+04$ & $1.70 \mathrm{E}+03$ & $4.48 \mathrm{E}+01$ & $27 \mathrm{E}-01$ & $1.16 \mathrm{E}-03$ & $5.53 \mathrm{E}-03$ & $1.96 \mathrm{E}-04$ & $1.60 \mathrm{E}+04$ \\
\hline 241-TY-104 & Total & & & $2.43 \mathrm{E}+02$ & $1.40 \mathrm{E}+04$ & $58 \mathrm{E}+03$ & $2.95 \mathrm{E}+04$ & $2.39 \mathrm{E}+03$ & $2.33 \mathrm{E}+04$ & $2.14 \mathrm{E}+01$ & $3.68 \mathrm{E}+00$ & $4.63 \mathrm{E}+01$ & $2.11 \mathrm{E}-01$ & $9.50 \mathrm{E}+03$ \\
\hline $241-7$ & Supernatant & DW (Liquid) & 5 & $E+01$ & +02 & $E+01$ & $4.17 \mathrm{E}+02$ & $\mathrm{E}+00$ & $3.02 \mathrm{E}-01$ & $2.16 \mathrm{E}-03$ & $1.56 \mathrm{E}-03$ & $6.53 \mathrm{E}-03$ & $3.70 \mathrm{E}-04$ & $3 \mathrm{E}+01$ \\
\hline 241-TY-104 & Sludge (Liquid \& Solid) & TBP (Solid) & 49 & $7.00 \mathrm{E}+01$ & $4.07 E+03$ & $1.06 \mathrm{E}+03$ & $8.73 \mathrm{E}+03$ & $7.18 \mathrm{E}+02$ & $7.02 \mathrm{E}+03$ & $6.45 \mathrm{E}+00$ & $1.45 \mathrm{E}+00$ & $1.36 \mathrm{E}+01$ & $9.45 \mathrm{E}-02$ & $2.84 \mathrm{E}+03$ \\
\hline 24 & 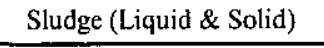 & 1CFeCN (Solid) & 114 & $3 \mathrm{E}+02$ & 9.46 & $2.46 \mathrm{E}+03$ & $2.03 E+04$ & $1.67 \mathrm{E}+03$ & $1.63 \mathrm{E}+04$ & $50 \mathrm{E}+01$ & $2.22 \mathrm{E}+00$ & $E+01$ & $\mathrm{E}-01$ & $1 \mathrm{E}+03$ \\
\hline 241-TY-105 & Total & & & $1.08 \mathrm{E}+03$ & $2.38 \mathrm{E}+05$ & $2.10 \mathrm{E}+04$ & $1.54 \mathrm{E}+05$ & $2.55 \mathrm{E}+03$ & $1.98 \mathrm{E}+05$ & $1.01 \mathrm{E}+01$ & $3.04 \mathrm{E}+00$ & $2.84 \mathrm{E}+01$ & $1.97 \mathrm{E}-01$ & $8.35 \mathrm{E}+03$ \\
\hline 241-TY-105 & (Liquid 8 & id) & 874 & $1.08 \mathrm{E}+03$ & $2.38 E+05$ & $2.10 \mathrm{E}+04$ & $1.54 \mathrm{E}+05$ & $2.55 \mathrm{E}+03$ & $1.98 \mathrm{E}+05$ & $1.01 \mathrm{E}+01$ & $3.04 \mathrm{E}+00$ & $2.84 \mathrm{E}+01$ & $1.97 \mathrm{E}-01$ & $8.35 \mathrm{E}+03$ \\
\hline 241-TY-106 & 1 & & & $48 E+02$ & +04 & +02 & 6 & $1.91 \mathrm{E}+02$ & $1.05 \mathrm{E}+04$ & $1.44 E+00$ & $1.77 \mathrm{E}-01$ & $1.65 \mathrm{E}+00$ & $1.15 \mathrm{E}-02$ & $4.90 \mathrm{E}+03$ \\
\hline 241-TY-106 & Sludge (Liquid \& Solid) & TBP (Solid) & 15 & $5.99 \mathrm{E}+01$ & $3.46 E+03$ & $1.75 \mathrm{E}+02$ & $1.67 \mathrm{E}+03$ & $4.62 \mathrm{E}+01$ & $2.54 \mathrm{E}+03$ & $3.49 \mathrm{E}-01$ & $4.27 \mathrm{E}-02$ & $3.99 \mathrm{E}-01$ & $2.78 \mathrm{E}-03$ & $1.19 E+03$ \\
\hline 241-TY-106 & (Liquid & d) & 47 & $1.88 \mathrm{E}+02$ & $1.09 \mathrm{E}+04$ & $5.48 \mathrm{E}+02$ & +03 & $1.45 \mathrm{E}+02$ & $7.97 \mathrm{E}+03$ & $1.09 \mathrm{E}+00$ & $E-01$ & $1.25 \mathrm{E}+00$ & $0 \mathrm{E}-03$ & $3.72 E+03$ \\
\hline $241-U-101$ & Total & & & $1.47 \mathrm{E}+02$ & $1.57 \mathrm{E}+04$ & $4.42 \mathrm{E}+03$ & $1.41 \mathrm{E}+04$ & $2.61 \mathrm{E}+04$ & $5.72 \mathrm{E}+04$ & $3.16 \mathrm{E}+00$ & $1.21 \mathrm{E}+00$ & $6.25 \mathrm{E}+00$ & $1.16 \mathrm{E}-01$ & $8.82 \mathrm{E}+03$ \\
\hline $241-U-101$ & ludge (Liquid \& Solid) & lid) & 87 & $1.47 \mathrm{E}+02$ & $1.57 E+04$ & $4.42 \mathrm{E}+03$ & $1.41 \mathrm{E}+04$ & $2.61 \mathrm{E}+04$ & $5.72 \mathrm{E}+04$ & $3.16 \mathrm{E}+00$ & $1.21 \mathrm{E}+00$ & $6.25 \mathrm{E}+00$ & $1.16 \mathrm{E}-01$ & $8.82 \mathrm{E}+03$ \\
\hline $241-\mathrm{U}-102$ & Total & & & $1.40 \mathrm{E}+04$ & $5.29 E+05$ & $9.94 E+04$ & $3.54 \mathrm{E}+05$ & $8.23 \mathrm{E}+04$ & $1.75 \mathrm{E}+05$ & $2.44 \mathrm{E}+02$ & $4.75 \mathrm{E}+01$ & $2.36 \mathrm{E}+02$ & $5.92 \mathrm{E}+00$ & $2.91 \mathrm{E}+05$ \\
\hline 241-U-102 & Supematant & -SltCk (Liquid) & 4 & $4.48 \mathrm{E}+01$ & $7.92 \mathrm{E}+02$ & $5.84 E+02$ & $8.92 \mathrm{E}+02$ & $1.33 \mathrm{E}+02$ & $2.69 \mathrm{E}+01$ & $4.65 \mathrm{E}-02$ & $3.90 \mathrm{E}-04$ & $1.86 \mathrm{E}-03$ & $6.58 \mathrm{E}-05$ & $1.80 \mathrm{E}+03$ \\
\hline $241-U-102$ & Sludge (Liquid \& Solid) & R1 (Solid) & 163 & $3.03 E+02$ & $3.23 \mathrm{E}+04$ & $9.12 \mathrm{E}+03$ & $2.91 E+04$ & $5.40 \mathrm{E}+04$ & $1.18 \mathrm{E}+05$ & $7.22 \mathrm{E}+01$ & $2.77 \mathrm{E}+01$ & $1.43 E+02$ & $2.65 \mathrm{E}+00$ & $1.82 \mathrm{E}+04$ \\
\hline $241-\mathrm{U}-102$ & Saltcake Solid & T2-SltCk (Solid) & 647 & $8.37 \mathrm{E}+03$ & $3.21 \mathrm{E}+05$ & $4.92 \mathrm{E}+04$ & $2.02 \mathrm{E}+05$ & $1.65 \mathrm{E}+04$ & $3.78 \mathrm{E}+04$ & $1.20 \mathrm{E}+02$ & $1.25 \mathrm{E}+01$ & $5.96 \mathrm{E}+01$ & $2.10 \mathrm{E}+00$ & $1.48 \mathrm{E}+05$ \\
\hline 241-U-102 & Saltcake Solid & S2-S1tS1r (Solid) & 307 & $3.97 \mathrm{E}+03$ & $1.52 \mathrm{E}+05$ & $2.34 \mathrm{E}+04$ & $9.59 \mathrm{E}+04$ & $7.84 \mathrm{E}+03$ & $1.79 \mathrm{E}+04$ & $5.03 \mathrm{E}+01$ & $7.21 \mathbf{E}+00$ & $3.36 \mathrm{E}+01$ & $1.17 \mathrm{E}+00$ & $7.02 \mathrm{E}+04$ \\
\hline
\end{tabular}


Table A-2. Tank Waste Inventory from Best Basis Inventory Detail Report (Queried on September 27, 2006) for Hydrogen Generation Rate Calculations. (27 sheets)

\begin{tabular}{|c|c|c|c|c|c|c|c|c|c|c|c|c|c|c|}
\hline $\begin{array}{c}\text { Tank } \\
\text { Name }\end{array}$ & $\mathbf{W}$ & $\mathbf{V}$ & $\begin{array}{c}\text { Vol } \\
(k L)\end{array}$ & $\begin{array}{l}\text { TOC } \\
(\mathrm{Kg})\end{array}$ & $\begin{array}{l}\mathrm{NO}_{3} \\
(\mathrm{Kg})\end{array}$ & $\begin{array}{l}\mathrm{NO}_{2} \\
(\mathrm{Kg})\end{array}$ & $\begin{array}{c}\mathbf{N a} \\
(\mathbf{K g})\end{array}$ & $\begin{array}{c}\mathrm{Al} \\
(\mathrm{Kg})\end{array}$ & $\begin{array}{l}{ }^{90} \mathrm{Sr} \\
(\mathrm{Ci})\end{array}$ & $\begin{array}{c}{ }_{(\mathrm{Ci})}^{241} \mathrm{Am} \\
\end{array}$ & $\begin{array}{c}{ }^{240} \mathrm{Pu} \\
(\mathrm{Ci})\end{array}$ & $\begin{array}{c}{ }^{239} \mathbf{P u} \\
(\mathrm{Ci})\end{array}$ & $\begin{array}{c}{ }^{238} \mathbf{P u} \\
(\mathrm{Ci})\end{array}$ & $\begin{array}{c}{ }^{137} \mathrm{Cs} \\
\text { (Ci) }\end{array}$ \\
\hline $241-U-102$ & Saltcake Interstitial Liquid & id) & 117 & $1.31 E+03$ & $32 E+04$ & $1.71 E+04$ & $2.61 E+04$ & $3.88 \mathrm{E}+03$ & $7.88 \mathrm{E}+02$ & $1.36 \mathrm{E}+00$ & $1.14 \mathrm{~L}-62$ & $5.44 \mathrm{E}-02$ & $1.92 \mathrm{E}-03$ & $5.27 \mathrm{E}+04$ \\
\hline $241-U-103$ & Total & & & $1.91 E+04$ & $5.04 \mathrm{E}+05$ & $1.24 \mathrm{E}+05$ & $4.72 \mathrm{E}+05$ & $4.86 \mathrm{E}+04$ & $5.48 \mathrm{E}+04$ & $2.04 \mathrm{E}+02$ & $2.17 \mathrm{E}+01$ & $1.03 E+02$ & $3.13 \mathrm{E}+00$ & $2.64 \mathrm{E}+05$ \\
\hline $241-U-103$ & Supcittatant & 40 & 2 & $48 \mathrm{E}+01$ & $04 \mathrm{E}+02$ & $74 \mathrm{E}+02$ & $.61 \mathrm{E}+02$ & $8.18 \mathrm{E}+01$ & $1.87 \mathrm{E}+01$ & $3.70 \mathrm{E}-02$ & $1.12 \mathrm{~L}-\mathrm{T}$ & $7.88 \mathrm{E}-04$ & E-05 & $E+02$ \\
\hline 241 - & $\underline{s}$ & ) & 42 & +02 & 103 & $\mathrm{a}+03$ & $9.10 \mathrm{E}+03$ & +03 & $22 \mathrm{E}+04$ & $1.50 \mathrm{E}+01$ & $5.76 \mathrm{E}+00$ & $2.97 \mathrm{E}+01$ & $5.50 \mathrm{E}-01$ & $3.42 \mathrm{E}+03$ \\
\hline $241-\mathrm{U}-103$ & lid & 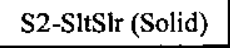 & 356 & $84 \mathrm{E}+03$ & $1.37 \mathrm{E}+05$ & $.98 \mathrm{E}+04$ & $1.24 \mathrm{E}+05$ & $1.01 \mathrm{E}+04$ & $7.87 \mathrm{E}+03$ & $4.35 \mathrm{E}+01$ & $6.24 \mathrm{E}+00$ & 1 & +00 & $7.94 \mathrm{E}+04$ \\
\hline $241-U-103$ & lid & lid) & 1 & $21 E+04$ & $42 \mathrm{E}+05$ & $7.45 \mathrm{E}+04$ & +05 & $2 \mathrm{E}+04$ & $5 \mathrm{E}+04$ & $\mathrm{E}+02$ & $71 \mathbf{E}+00$ & $E+01$ & $1.57 \mathrm{E}+00$ & $1.28 \mathrm{E}+05$ \\
\hline $241-U-103$ & $\mathrm{~S}$ & SI-SltCk (Liquid) & 19 & $1.48 \mathrm{E}+03$ & $1.81 \mathrm{E}+04$ & $1.63 \mathrm{E}+04$ & +04 & $7 \mathrm{E}+03$ & $E+03$ & +00 & -02 & $\mathrm{E}-02$ & $1.65 \mathrm{E}-03$ & $5.30 \mathrm{E}+04$ \\
\hline 4 & Total & & & $00 \mathrm{E}+00$ & $12 \mathrm{E}+02$ & $E+01$ & $37 \mathrm{E}+03$ & $3.16 \mathrm{E}+02$ & +02 & 1 & $\mathrm{E}-01$ & 00 & 03 & $E+01$ \\
\hline $241-U-104$ & Sludge (Liquid \& & W2 (Solid) & 4 & $0.00 \mathrm{E}+00$ & $3.49 \mathrm{E}+00$ & $2.91 \mathrm{E}+00$ & $6.06 \mathrm{E}+02$ & $0.00 \mathrm{E}+00$ & $5.62 \mathrm{E}+02$ & $2.73 \mathrm{E}-03$ & $5.31 \mathrm{E}-03$ & $4.19 \mathrm{E}-02$ & $3.63 \mathrm{E}-04$ & $1.43 \mathrm{E}+01$ \\
\hline $241-1+2>3$ & Sludge (Liquid \& Solid) & E (Solid) & 201 & 00 & 02 & +01 & -03 & $02-20$ & $\mathrm{E}+01$ & 01 & -01 & +00 & -03 & $6 \mathrm{E}+01$ \\
\hline 105 & Total & & & $4.03 \mathrm{E}+04$ & $\mathrm{E}+05$ & $1 \mathrm{E}+05$ & $2 E+05$ & $6.25 \mathrm{E}+04$ & $2.54 \mathrm{E}+04$ & $0 \mathrm{E}+02$ & $\mathrm{E}+02$ & $79 E+02$ & $1.78 E+01$ & $3.05 \mathrm{E}+05$ \\
\hline $241-\mathrm{U}-105$ & Sludge (Liquid \& Solid) & CWR1 (Solid) & 121 & $2.02 \mathrm{E}+03$ & $3.33 \mathrm{E}+04$ & $4.09 \mathrm{E}+03$ & $2.05 \mathrm{E}+04$ & $3.17 \mathrm{E}+04$ & $3.93 \mathrm{E}+02$ & $8.20 \mathrm{E}+0 \mathrm{l}$ & $2.42 E+01$ & $1.27 \mathrm{E}+02$ & $1.98 \mathrm{E}+00$ & $1.45 \mathrm{E}+04$ \\
\hline 2 & Saltcake Solid & T2-SltCk (Solid) & 223 & 3 & $1.23 \mathrm{E}+05$ & 4 & 4 & S & 2 & 22 & 1 & 11 & 00 & 04 \\
\hline $241-U-105$ & Saltcake Solid & S2-SltSlr (Solid) & 827 & $2.80 \mathrm{E}+04$ & $4.56 \mathrm{E}+05$ & $6.24 \mathrm{E}+04$ & $2.79 \mathrm{E}+05$ & $2.04 \mathrm{E}+04$ & $2.10 \mathrm{E}+04$ & $5.50 \mathrm{E}+02$ & $7.89 \mathrm{E}+01$ & $3.68 \mathrm{E}+02$ & $1.28 \mathrm{E}+01$ & $1.84 \mathrm{E}+05$ \\
\hline 241-U-105 & Saltcake Interstitial Liquid & 2-SltCk (Liquid) & 3. & $5.74 \mathrm{E}+02$ & $6.34 \mathrm{E}+03$ & $3.78 \mathrm{E}+03$ & $7.95 \mathrm{E}+03$ & $1.04 \mathrm{E}+03$ & $7.89 \mathrm{E}+02$ & $2.06 \mathrm{E}+00$ & $1.09 \mathrm{E}-02$ & $5.21 \mathrm{E}-02$ & $1.84 \mathrm{E}-03$ & $1.22 \mathrm{E}+04$ \\
\hline $241-\mathrm{L}$ & 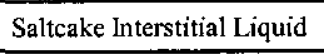 & S2-S1tSlr (Liquid) & 130 & $E+03$ & $2.35 \mathrm{E}+04$ & $\mathrm{E}+04$ & $2.95 \mathrm{E}+04$ & $\mathrm{E}+03$ & 3 & $7 \mathrm{E}+00$ & 2 & $93 \mathrm{E}-01$ & 03 & $E+04$ \\
\hline $241-U-106$ & Total & & & $2.20 \mathrm{E}+04$ & $2.04 \mathrm{E}+05$ & $5.26 \mathrm{E}+04$ & $1.77 \mathrm{E}+05$ & $1.11 \mathrm{E}+04$ & $5.92 \mathrm{E}+04$ & $6.81 \mathrm{E}+02$ & $4.31 \mathrm{E}+01$ & $1.98 \mathrm{E}+02$ & $6.96 \mathrm{E}+00$ & $1.45 \mathrm{E}+05$ \\
\hline 241-U-106 & rnatant & - & 6 & $2.03 \mathrm{E}+02$ & $1.49 \mathrm{E}+03$ & $94 \mathrm{E}+02$ & $.34 \mathrm{E}+03$ & $7.22 \mathrm{E}+01$ & $3.30 \mathrm{E}+02$ & $1.74 \mathrm{E}+00$ & $7.39 \mathrm{E}-03$ & $3.39 \mathrm{E}-02$ & $1.19 \mathrm{E}-03$ & $1.69 \mathrm{E}+03$ \\
\hline 241-U-106 & Saltcake Solid & S1-SltCk (Solid) & 484 & $1.65 \mathrm{E}+04$ & $1.64 \mathrm{E}+05$ & $3.68 \mathrm{E}+04$ & $1.41 \mathrm{E}+05$ & $9.22 \mathrm{E}+03$ & $5.04 \mathrm{E}+04$ & $6.34 \mathrm{E}+02$ & $4.29 \mathrm{E}+01$ & $1.97 \mathrm{E}+02$ & $6.93 \mathrm{E}+00$ & $9.98 \mathrm{E}+04$ \\
\hline 241-U-106 & itcake Interstitial Liquid & -SltCk (Liquid) & 154 & $5.21 \mathrm{E}+03$ & $3.83 \mathrm{E}+04$ & $1.52 \mathrm{E}+04$ & $3.43 \mathrm{E}+04$ & $1.85 \mathrm{E}+03$ & $8.47 \mathrm{E}+03$ & $4.45 \mathrm{E}+01$ & $1.90 \mathrm{E}-01$ & $8.71 \mathrm{E}-01$ & $3.07 \mathrm{E}-02$ & $4.35 \mathrm{E}+04$ \\
\hline 241-U-107 & IOtal & & & $3.17 \mathrm{E}+03$ & $6.37 \mathrm{E}+05$ & $7.15 \mathrm{E}+04$ & +05 & $5.13 \mathrm{E}+04$ & $4.05 \mathrm{E}+03$ & +02 & +01 & $\mathrm{E}+02$ & $+\infty$ & $6 \mathrm{E}+05$ \\
\hline 241-U-107 & Sludge (Liquid \& Solid) & CWRI (Solid) & 57 & $6.55 \mathrm{E}+01$ & $2.51 \mathrm{E}+03$ & $1.10 \mathrm{E}+03$ & $3.96 \mathrm{E}+03$ & $2.37 \mathrm{E}+04$ & $1.91 \mathrm{E}+02$ & $2.00 \mathrm{E}-01$ & $5.91 \mathrm{E}-02$ & $3.11 \mathrm{E}-01$ & $4.84 \mathrm{E}-03$ & $4.03 \mathrm{E}+02$ \\
\hline $241-U-107$ & Saltcake Solid & 2-SltCk (Solid) & 44 & $1.12 \mathrm{E}+02$ & $2.88 \mathrm{E}+04$ & $.62 \mathrm{E}+03$ & $1.58 \mathrm{E}+04$ & $1.07 \mathrm{E}+03$ & $1.77 \mathrm{E}+02$ & $2.18 \mathrm{E}+01$ & $2.27 \mathrm{E}+00$ & $1.08 \mathrm{E}+01$ & $3.81 \mathrm{E}-01$ & $5.04 \mathrm{E}+03$ \\
\hline 241-U-107 & Saltcake Solid & S2-SltSlr (Solid) & 902 & $2.30 \mathrm{E}+03$ & $5.90 \mathrm{E}+05$ & $5.38 \mathrm{E}+04$ & $3.25 \mathrm{E}+05$ & $2.20 \mathrm{E}+04$ & $3.63 \mathrm{E}+03$ & $3.94 \mathrm{E}+02$ & $5.65 \mathrm{E}+01$ & $2.64 \mathrm{E}+02$ & $9.20 \mathrm{E}+00$ & $1.03 \mathrm{E}+05$ \\
\hline $241-U-107$ & Saltcake Interstitial Liquid & T2-SltCk (Liquid) & 5 & $3.14 \mathrm{E}+01$ & $7.11 \mathrm{E}+02$ & $6.37 \mathrm{E}+02$ & $1.02 \mathrm{E}+03$ & $2.05 \mathrm{E}+02$ & $2.22 \mathrm{E}+00$ & $1.38 \mathrm{E}-02$ & $4.22 \mathrm{E}-05$ & $2.01 \mathrm{E}-04$ & $7.11 \mathrm{E}-06$ & $1.71 \mathrm{E}+03$ \\
\hline $241-\mathrm{U} \cdot 107$ & altcake Interstitial Liquid & S2-SltSlr (Liquid) & 105 & $6.59 \mathrm{E}+02$ & $1.49 \mathrm{E}+04$ & $1.34 \mathrm{E}+04$ & $2.15 \mathrm{E}+04$ & $4.30 \mathrm{E}+03$ & 4.67E+01 & $2.89 \mathrm{E}-01$ & $9.03 \mathrm{E}-04$ & $4.21 \mathrm{E}-03$ & $1.47 \mathrm{E}-04$ & $3.59 \mathrm{E}+04$ \\
\hline $241-U-108$ & Total & & & $1.32 \mathrm{E}+04$ & $7.26 \mathrm{E}+05$ & $1.43 \mathrm{E}+05$ & $6.17 \mathrm{E}+05$ & $5.99 \mathrm{E}+04$ & $2.08 \mathrm{E}+04$ & $9.45 \mathrm{E}+01$ & $3.94 \mathrm{E}+01$ & $1.80 \mathrm{E}+02$ & $4.86 \mathrm{E}+00$ & $3.39 \mathrm{E}+05$ \\
\hline
\end{tabular}


Table A-2. Tank Waste Inventory from Best Basis Inventory Detail Report (Queried on September 27, 2006) for Hydrogen Generation Rate Calculations. (27 sheets)

\begin{tabular}{|c|c|c|c|c|c|c|c|c|c|c|c|c|c|c|}
\hline $\begin{array}{l}\text { Tank } \\
\text { Name }\end{array}$ & Was & aste Type & $\begin{array}{l}\text { Vol } \\
\text { (kL) }\end{array}$ & $\begin{array}{l}\text { TOC } \\
(\mathrm{Kg})\end{array}$ & $\begin{array}{l}\mathrm{NO}_{3} \\
(\mathrm{Kg})\end{array}$ & $\begin{array}{l}\mathrm{NO}_{2} \\
(\mathrm{Kg})\end{array}$ & $\begin{array}{c}\mathrm{Na} \\
(\mathrm{Kg})\end{array}$ & $\begin{array}{c}\mathrm{Al} \\
(\mathrm{Kg})\end{array}$ & $\begin{array}{l}{ }^{90} \mathrm{Sr} \\
\text { (Ci) }\end{array}$ & $\begin{array}{c}{ }^{241} \mathrm{Am} \\
(\mathrm{Ci})\end{array}$ & $\begin{array}{c}{ }_{(\mathrm{Ci})} \mathrm{Pu} \\
{ }^{240}\end{array}$ & $\begin{array}{c}{ }_{(\mathrm{Ci})} \mathrm{Pu} \\
{ }^{239}\end{array}$ & $\begin{array}{c}{ }^{238} \mathbf{P u} \\
(\mathrm{Ci})\end{array}$ & $\begin{array}{c}{ }^{137} \mathrm{Cs} \\
(\mathrm{Ci})\end{array}$ \\
\hline & Sludge (Liquid \& Solid) & CWR2 (Solid) & 110 & $0.00 \mathrm{E}+00$ & $51 \mathrm{E}+03$ & $1 E+03$ & $47 \mathrm{E}+03$ & $3 E+04$ & $8.62 \mathrm{E}+01$ & $30 \mathrm{E}+01$ & $3.16 \mathrm{E}+01$ & $44 \mathrm{E}+02$ & $59 \mathrm{E}+00$ & $1.01 \mathrm{E}+02$ \\
\hline 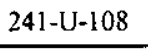 & d & S2-SltSlr (Solid) & 610 & 3 & 5 & 4 & 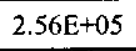 & 4 & 3 & 1 & 0 & 1 & 1 & $8+05$ \\
\hline $241-\mathrm{U}-108$ & Saltcake Solid & S1-SltCk (Solid) & 766 & $6.72 \mathrm{E}+03$ & $3.86 \mathrm{E}+05$ & $6.75 \mathrm{E}+04$ & $3.22 \mathrm{E}+05$ & $2.11 \mathrm{E}+04$ & $1.15 \mathrm{E}+04$ & $5.06 \mathrm{E}+01$ & $3.42 \mathrm{E}+00$ & $1.57 \mathrm{E}+01$ & $5.52 \mathrm{E}-01$ & $1.59 \mathrm{E}+05$ \\
\hline $241-U-108$ & altcake Interstitial Liquid & 2-SltSlr (Liquid) & 69 & $88 \mathrm{E}+02$ & $1.27 \mathrm{E}+04$ & $.25 \mathrm{E}+03$ & $1.57 \mathrm{E}+04$ & $2.10 \mathrm{E}+03$ & $1.93 \mathrm{E}+01$ & $1.63 \mathrm{E}-01$ & $9.85 \mathrm{E}-04$ & $4.59 \mathrm{E}-03$ & 1.60E-04 & $E+04$ \\
\hline 108 & altce & ) & 86 & $08 \mathrm{E}+02$ & $58 \mathrm{E}+04$ & $1.15 \mathrm{E}+04$ & $.96 \mathrm{E}+04$ & $2.62 \mathrm{E}+03$ & $2.41 \mathrm{E}+01$ & $2.03 \mathrm{E}-01$ & $1.24 \mathrm{E}-03$ & $5.71 \mathrm{E}-03$ & $1 \mathrm{E}-04$ & $.94 \mathrm{E}+04$ \\
\hline $241-U-109$ & Total & & & $8.81 E+03$ & $5.98 \mathrm{E}+05$ & $1.07 \mathrm{E}+05$ & $4.31 \mathrm{E}+05$ & $5.77 \mathrm{E}+04$ & $1.55 \mathrm{E}+04$ & $5.27 \mathrm{E}+01$ & $5.30 \mathrm{E}+00$ & $2.49 \mathrm{E}+01$ & $\mathrm{E}-01$ & $2.52 \mathrm{E}+05$ \\
\hline $241-U-109$ & $40 \mathrm{gC}(2 \mathrm{~T})$ & Solid) & 103 & $4.84 \mathrm{E}+02$ & $7.78 \mathrm{E}+03$ & $5.20 \mathrm{E}+03$ & $1.55 \mathrm{E}+04$ & $2.99 \mathrm{E}+04$ & $1.70 \mathrm{E}+03$ & $1.68 \mathrm{E}+00$ & $4.95 \mathrm{E}-01$ & $2.61 E+00$ & $4.06 \mathrm{E}-02$ & $1.14 \mathrm{E}+04$ \\
\hline 241-U-109 & Saltcake Solid & S2-SltSlr (Solid) & 512 & $3.42 E+03$ & $2.60 \mathrm{E}+05$ & $3.90 \mathrm{E}+04$ & $1.77 \mathrm{E}+05$ & $1.09 \mathrm{E}+04$ & $6.31 E+03$ & $1.93 \mathrm{E}+01$ & $2.77 \mathbf{E}+00$ & $1.29 \mathrm{E}+01$ & $4.51 \mathrm{E}-01$ & $9.04 E+04$ \\
\hline 241-U-109 & Saltcake Solid & -SltCk (Solid) & 600 & $4.01 E+03$ & $3.05 \mathrm{E}+05$ & $4.57 \mathrm{E}+04$ & $2.07 \mathrm{E}+05$ & $1.28 \mathrm{E}+04$ & $7.40 \mathrm{E}+03$ & $2.99 \mathrm{E}+01$ & $2.02 \mathrm{E}+00$ & $9.27 \mathrm{E}+00$ & -01 & -05 \\
\hline $241-$ & iid & d) & 137 & $95 \mathrm{E}+02$ & $2.53 \mathrm{E}+04$ & $75 \mathrm{E}+04$ & $3.12 E+04$ & $4.00 \mathrm{E}+03$ & $13 \mathrm{E}+02$ & $1.79 \mathrm{E}+00$ & $1.69 \mathrm{E}-02$ & $7.77 \mathrm{E}-02$ & $2.74 \mathrm{E}-03$ & $4.45 \mathrm{E}+04$ \\
\hline $241-U \cdot 110$ & Total & & & $8 \mathrm{E}+03$ & $4.10 \mathrm{E}+04$ & $18 \mathrm{E}+04$ & $7.15 \mathrm{E}+04$ & $6 \mathrm{E}+05$ & $5 E+05$ & $39 \mathrm{E}+01$ & $0 \mathrm{E}+01$ & $2.10 \mathrm{E}+02$ & $3.23 \mathrm{E}+00$ & $2.07 \mathrm{E}+04$ \\
\hline 110 & 10) & id) & 396 & $E+02$ & $38 \mathrm{E}+04$ & $.05 E+04$ & $4.90 \mathrm{E}+04$ & $1.09 \mathrm{E}+05$ & $4 \mathrm{E}+05$ & $4 E+01$ & $4 E+01$ & 02 & 00 & 04 \\
\hline $241-U-110$ & Sludge (Liquid \& Solid) & CWR1 (Solid) & 149 & $1.45 \mathrm{E}+02$ & $2.76 \mathrm{E}+01$ & $1.24 \mathrm{E}+01$ & $2.88 \mathrm{E}+02$ & $8.29 \mathrm{E}+04$ & $1.63 \mathrm{E}+02$ & $5.24 \mathrm{E}+00$ & $1.55 \mathrm{E}+00$ & $8.14 \mathrm{E}+00$ & 1.27E-01 & $7.68 \mathrm{E}+01$ \\
\hline 110 & Sludge (Liquid & id) & 120 & $1.43 \mathrm{E}+02$ & $7.13 \mathrm{~F}+03$ & $27 \mathrm{E}+03$ & $2.22 \mathrm{E}+04$ & $1.35 \mathrm{E}+04$ & $1.09 \mathrm{E}+03$ & $9.21 \mathrm{E}+00$ & $6.05 E+00$ & $.55 \mathrm{E}+01$ & $3.91 \mathrm{E}-01$ & $5.68 \mathrm{E}+03$ \\
\hline $241-U-111$ & 10ial & & & $E+03$ & ( & +04 & 0 & $E+04$ & $6 \mathrm{E}+04$ & $E+02$ & $4 \mathrm{E}+01$ & $25 \mathrm{E}+02$ & $\mathrm{E}+00$ & $1.71 \mathrm{E}+05$ \\
\hline $241-U-111$ & Sludge (Liquid \& Solid) & $\mathrm{Rl}$ (Solid) & 49 & $9.11 \mathrm{E}+01$ & $9.71 \mathrm{E}+03$ & $2.74 \mathrm{E}+03$ & $8.76 \mathrm{E}+03$ & $1.62 \mathrm{E}+04$ & $3.55 \mathrm{E}+04$ & $2.17 \mathrm{E}+01$ & $8.33 \mathrm{E}+00$ & $4.29 \mathrm{E}+01$ & $7.96 \mathrm{E}-01$ & $5.47 \mathrm{E}+03$ \\
\hline $241-U-111$ & Sludge (Liquid \& Solid) & 1C (Solid) & 49 & $3.69 \mathrm{E}+01$ & $8.83 E+03$ & $80 \mathrm{E}+02$ & $6.96 \mathrm{E}+03$ & $1.04 \mathrm{E}+03$ & $3.38 \mathrm{E}+02$ & $9.68 \mathrm{E}-01$ & $6.35 \mathrm{E}-01$ & $.84 \mathrm{E}+00$ & $4.11 \mathrm{E}-02$ & $1.11 \mathrm{E}+03$ \\
\hline 241-U-111 & Saltcake Solid & S2-S1tSlr (Solid) & 401 & $3.45 \mathrm{E}+03$ & $1.76 \mathrm{E}+05$ & $3.31 \mathrm{E}+04$ & $1.27 \mathrm{E}+05$ & $1.24 \mathrm{E}+04$ & $8.88 \mathrm{E}+03$ & $1.01 \mathrm{E}+02$ & $1.44 \mathrm{E}+01$ & $6.73 E+01$ & $2.35 \mathrm{E}+00$ & $8.97 E+04$ \\
\hline 241-U-111 & Saltcake Solid & S1-SItCk (Solid) & 228 & $1.38 \mathrm{E}+03$ & $1.18 \mathrm{E}+05$ & $1.63 \mathrm{E}+04$ & $8.24 \mathrm{E}+04$ & $6.05 \mathrm{E}+03$ & $6.66 \mathrm{E}+03$ & $2.93 \mathrm{E}+01$ & $1.98 \mathrm{E}+00$ & $9.08 \mathrm{E}+00$ & $3.19 \mathrm{E}-01$ & $3.61 \mathrm{E}+04$ \\
\hline 241-U-111 & Saltcake Interstitial Liquid & 2-SitSlr (Liquid) & 7 & $3.89 \mathrm{E}+02$ & $20 \mathrm{E}+04$ & $.64 \mathrm{E}+03$ & $1.72 \mathrm{E}+04$ & $2.64 \mathrm{E}+03$ & $1.21 \mathrm{E}+02$ & $98 \mathrm{E}-01$ & 03 & 2 & 94 & $E+04$ \\
\hline 241-U-111 & Saltcake Interstitial Liquid & S1-SltCk (Liquid) & 41 & $2.22 \mathrm{E}+02$ & $6.82 \mathrm{E}+03$ & $4.92 \mathrm{E}+03$ & $9.78 \mathrm{E}+03$ & $1.50 \mathrm{E}+03$ & $6.90 \mathrm{E}+01$ & $1.70 \mathrm{E}-01$ & $2.15 \mathrm{E}-03$ & $9.86 \mathrm{E}-03$ & 3.47E-04 & $1.41 \mathrm{E}+04$ \\
\hline $241-\mathrm{U}-112$ & Total & & & $2.28 \mathrm{E}+02$ & $1.89 \mathrm{E}+04$ & $9.92 \mathrm{E}+02$ & $2.29 \mathrm{E}+04$ & 4.12E+04 & $4.94 \mathrm{E}+04$ & $1.01 \mathrm{E}+00$ & $6.33 \mathrm{E}-01$ & $5.72 \mathrm{E}+00$ & $4.13 \mathrm{E}-02$ & $9.01 \mathrm{E}+03$ \\
\hline $241-U-112$ & Sludge (Liquid \& Solid) & R1 (Solid) & 67 & $1.25 \mathrm{E}+02$ & $5.61 \mathrm{E}+03$ & $1.82 \mathrm{E}+02$ & $8.70 \mathrm{E}+03$ & $2.15 E+04$ & $4.88 \mathrm{E}+04$ & $2.96 \mathrm{E}-04$ & $1.14 \mathrm{E}-04$ & $5.85 \mathrm{E}-04$ & $1.08 \mathrm{E}-05$ & $7.53 \mathrm{E}+03$ \\
\hline 241-U-112 & Sludge (Liquid \& Solid) & CWR1 (Solid) & 58 & $6.75 \mathrm{E}+01$ & $4.86 \mathrm{E}+03$ & $1.58 \mathrm{E}+02$ & $7.53 \mathrm{E}+03$ & $1.86 \mathrm{E}+04$ & $1.97 \mathrm{E}+02$ & $7.94 \mathrm{E}-02$ & $2.35 \mathrm{E}-02$ & $1.23 \mathrm{E}-01$ & $1.92 \mathrm{E}-03$ & $4.15 \mathrm{E}+02$ \\
\hline $241-U-112$ & Sludge (Liquid \& Solid) & 1C (Solid) & 47 & $3.54 \mathrm{E}+01$ & $8.47 \mathrm{E}+03$ & $6.53 \mathrm{E}+02$ & $6.67 \mathrm{E}+03$ & $1.00 \mathrm{E}+03$ & $3.24 \mathrm{E}+02$ & $9.28 \mathrm{E}-01$ & $6.09 \mathrm{E}-01$ & $5.60 \mathrm{E}+00$ & $3.94 \mathrm{E}-02$ & $1.07 \mathrm{E}+03$ \\
\hline 241-U-201 & Total & & & $2.26 \mathrm{E}+00$ & $8.79 \mathrm{E}+02$ & $1.29 \mathrm{E}+02$ & $1.61 \mathrm{E}+03$ & $3.52 \mathrm{E}+03$ & $2.64 \mathrm{E}+00$ & $2.97 \mathrm{E}-02$ & $6.50 \mathrm{E}-03$ & $3.42 \mathrm{E}-02$ & $5.32 \mathrm{E}-04$ & $1.93 E+02$ \\
\hline 241-U-201 & upernatant & R1 (Liquid) & 4 & $.00 \mathrm{E}+00$ & $5.71 \mathrm{E}+02$ & $28 \mathrm{E}+01$ & $4.63 \mathrm{E}+02$ & $5.65 \mathrm{E}-01$ & $5.10 \mathrm{E}-02$ & $2.18 \mathrm{E}-03$ & $1.74 \mathrm{E}-04$ & $9.16 \mathrm{E}-04$ & $1.42 \mathrm{E}-05$ & $1.09 \mathrm{E}+02$ \\
\hline
\end{tabular}




\section{RPP-5926 REV 6}

Table A-2. Tank Waste Inventory from Best Basis Inventory Detail Report (Queried on September 27, 2006) for Hydrogen Generation Rate Calculations. (27 sheets)

\begin{tabular}{|c|c|c|c|c|c|c|c|c|c|c|c|c|c|c|}
\hline $\begin{array}{c}\text { Tank } \\
\text { Name }\end{array}$ & Waste Phase & Waste Type & $\begin{array}{c}\text { Vol } \\
(k L)\end{array}$ & $\begin{array}{l}\text { TOC } \\
(\mathbf{K g})\end{array}$ & $\begin{array}{l}\mathrm{NO}_{3} \\
(\mathrm{Kg})\end{array}$ & $\begin{array}{l}\mathrm{NO}_{2} \\
(\mathrm{Kg})\end{array}$ & $\begin{array}{c}\mathrm{Na} \\
(\mathrm{Kg})\end{array}$ & $\begin{array}{c}\mathrm{Al} \\
(\mathrm{Kg})\end{array}$ & $\begin{array}{l}{ }^{90} \mathrm{Sr} \\
(\mathrm{Ci})\end{array}$ & $\begin{array}{c}{ }^{241} \mathrm{Am} \\
\text { (Ci) }\end{array}$ & $\begin{array}{c}{ }^{240} \mathrm{Pu} \\
(\mathrm{Ci})\end{array}$ & $\begin{array}{l}{ }^{239} \mathbf{P u} \\
(\mathrm{Ci})\end{array}$ & $\begin{array}{c}{ }^{238} \mathbf{P u} \\
(\mathrm{Ci})\end{array}$ & $\begin{array}{c}{ }^{137} \mathrm{Cs} \\
\text { (Ci) }\end{array}$ \\
\hline $241-U-201$ & Sludge (Liquid \& Solid) & CWRI (Solid) & 11 & $2.26 \mathrm{E}+00$ & $3.08 \mathrm{E}+02$ & $4.64 \mathrm{E}+01$ & $1.14 \mathrm{E}+03$ & $3.52 \mathrm{E}+03$ & $2.59 \mathrm{E}+00$ & $2.75 \mathrm{E}-02$ & $6.32 \mathrm{E}-03$ & $3.33 \mathrm{E}-02$ & $5.18 \mathrm{E}-04$ & $8.47 \mathrm{E}+01$ \\
\hline $241-\mathrm{U}-202$ & Total & & & $2.09 \mathrm{E}+00$ & $8.43 \mathrm{E}+02$ & $1.37 \mathrm{E}+02$ & $1.42 \mathrm{E}+03$ & $3.04 \mathrm{E}+03$ & $9.34 \mathrm{E}-01$ & $2.87 \mathrm{E}-02$ & $7.45 \mathrm{E}-03$ & $3.92 \mathrm{E}-02$ & $6.10 \mathrm{E}-04$ & $1.41 \mathrm{E}+02$ \\
\hline 241-U-202 & Supernatant & CWRI (Liquid) & $\overline{4}$ & $5.64 \mathrm{E}-01$ & $6.07 \mathrm{E}+02$ & $9.81 \mathrm{E}+01$ & $4.78 \mathrm{E}+02$ & $9.54 \mathrm{E}-01$ & $2.47 \mathrm{E}-03$ & $3.70 \mathrm{E}-05$ & 4.47E-06 & $2.35 \mathrm{E}-05$ & $3.66 \mathrm{E}-07$ & $7.83 \mathrm{E}+01$ \\
\hline $241-U-202$ & Sludge (Liquid \& Solid) & CWR1 (Solid) & 10 & $1.52 \mathrm{E}+00$ & $2.36 \mathrm{E}+02$ & $3.89 \mathrm{E}+01$ & $9.38 \mathrm{E}+02$ & $3.03 \mathrm{E}+03$ & $9.32 \mathrm{E}-01$ & $2.87 \mathrm{E}-02$ & $7.44 \mathrm{E}-03$ & $3.92 \mathrm{E}-02$ & $6.10 \mathrm{E}-04$ & $6.24 \mathrm{E}+01$ \\
\hline $241-U-203$ & Total & & & $1.14 \mathrm{E}+00$ & $8.08 \mathrm{E}+02$ & $1.12 \mathrm{E}+02$ & $2.08 E+03$ & $1.37 \mathrm{E}+03$ & $1.80 \mathrm{E}+00$ & $2.21 \mathrm{E}-02$ & $4.14 \mathrm{E}-03$ & $2.18 \mathrm{E}-02$ & $3.39 \mathrm{E}-04$ & $1.29 \mathrm{E}+02$ \\
\hline 241-U-203 & Supernatant & CWR1 (Liquid) & 4 & $0.00 \mathrm{E}+00$ & $5.76 \mathrm{E}+02$ & $7.74 \mathrm{E}+01$ & $4.01 \mathrm{E}+02$ & $7.50 \mathrm{E}-01$ & $2.17 \mathrm{E}-03$ & $3.55 \mathrm{E}-05$ & $1.90 \mathrm{E}-05$ & $9.98 \mathrm{E}-05$ & $1.55 \mathrm{E}-06$ & $7.34 \mathrm{E}+01$ \\
\hline $241-U-203$ & Sludge (Liquid \& Solid) & CWRI (Solid) & 9 & $1.14 \mathrm{E}+00$ & $2.32 \mathrm{E}+02$ & $3.49 \mathrm{E}+01$ & $1.68 \mathrm{E}+03$ & $1.37 \mathrm{E}+03$ & $1.80 \mathrm{E}+00$ & $2.21 \mathrm{E}-02$ & $4.12 \mathrm{E}-03$ & $2.17 \mathrm{E}-02$ & $3.37 \mathrm{E}-04$ & $5.57 \mathrm{E}+01$ \\
\hline $241-U-204$ & Total & & & $6.35 \mathrm{E}+00$ & $3.54 \mathrm{E}+02$ & $4.74 \mathrm{E}+01$ & $2.91 \mathrm{E}+02$ & $2.27 \mathrm{E}+03$ & $3.92 \mathrm{E}+01$ & $2.27 \mathrm{E}-01$ & $1.24 \mathrm{E}-01$ & $6.53 \mathrm{E}-01$ & $1.02 \mathrm{E}-02$ & $7.85 \mathrm{E}+01$ \\
\hline $241-U-204$ & Supernatant & CWRI (Liquid) & 4 & $3.24 \mathrm{E}-01$ & $2.21 \mathrm{E}+02$ & $2.81 E+01$ & $1.57 \mathrm{E}+02$ & $5.85 \mathrm{E}-02$ & $7.86 \mathrm{E}-04$ & $3.77 \mathrm{E}-05$ & $2.98 \mathrm{E}-06$ & $1.57 \mathrm{E}-05$ & $2.43 \mathrm{E}-07$ & $2.72 \mathrm{E}+01$ \\
\hline 241-U-204 & Sludge (Liquid \& Solid) & CWRI (Solid) & 7 & $6.02 \mathrm{E}+00$ & $1.34 \mathrm{E}+02$ & $1.93 \mathrm{E}+01$ & $1.34 \mathrm{E}+02$ & $2.27 \mathrm{E}+03$ & $3.92 \mathrm{E}+01$ & $2.27 \mathrm{E}-01$ & $1.24 \mathrm{E}-01$ & $6.53 \mathrm{E}-01$ & $1.02 \mathrm{E}-02$ & $5.12 \mathrm{E}+01$ \\
\hline
\end{tabular}




\section{RPP-5926 REV 6}

Table A-3. Retained Gas Inventory from Best Basis Inventory Detail Report (Queried on September 27, 2006) for Hydrogen

Generation Rate Calculations.

\begin{tabular}{|c|c|c|c|}
\hline Tank Name & Waste Phase & $\begin{array}{c}\text { Waste } \\
\text { Type }\end{array}$ & $\begin{array}{c}\text { Volume } \\
\text { (kL) }\end{array}$ \\
\hline $241-\mathrm{A}-101$ & Retained Gas - Salt Cake & NA & 174 \\
\hline $241-\mathrm{AN}-103$ & Retained Gas - Salt Cake & NA & 220 \\
\hline 241-AN-104 & Retained Gas - Salt Cake & NA & 119 \\
\hline $241-\mathrm{AN}-105$ & Retained Gas - Salt Cake & NA & 109 \\
\hline $241-A W-101$ & Retained Gas - Salt Cake & NA & 95 \\
\hline $241-S-102$ & Retained Gas - Salt Cake & NA & 90 \\
\hline $241-\mathrm{S}-102$ & Retained Gas - Sludge & NA & 13 \\
\hline $241-S-111$ & Retained Gas - Salt Cake & NA & 77 \\
\hline $241-S-111$ & Retained Gas - Sludge & $\mathrm{NA}$ & 43 \\
\hline $241-S Y-101$ & Retained Gas - Salt Cake & NA & 87 \\
\hline $241-S Y-103$ & Retained Gas - Salt Cake & NA & 91 \\
\hline $241-U-103$ & Retained Gas - Salt Cake & NA & 163 \\
\hline 241-U-103 & Retained Gas - Sludge & NA & 5 \\
\hline 241-U-109 & Retained Gas - Salt Cake & NA & 137 \\
\hline 241-U-109 & Retained Gas - Sludge & NA & 29 \\
\hline
\end{tabular}

Note:

$\mathrm{NA}=$ not applicable. 


\section{RPP-5926 REV 6}

APPENDIX B

HYDROGEN GENERATION RATE CALCULATIONS FROM EMPIRICAL RATE EQUATION MODEL FOR 177 HANFORD TANKS 


\section{RPP-5926 REV 6}

This page intentionally left blank.

\section{B-ii}




\section{RPP-5926 REV 6}

\section{CONTENTS}

Table B-1 Input Data for Hydrogen Generation Rate Model Calculations

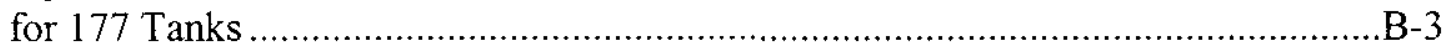

Table B-2 Derived Data for Hydrogen Generation Rate Model Calculations

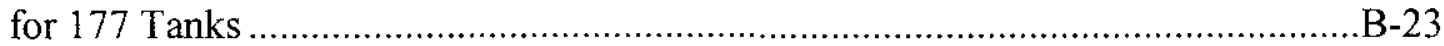

Table B-3 Calculated Hydrogen Generation Rate as Mole Per Unit Waste Weight and Volume

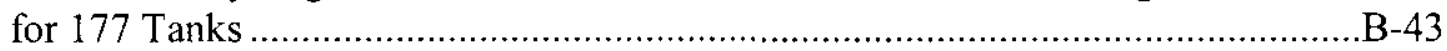

Table B-4 Calculated Total Hydrogen Generation Rate in Dome Space for 177 Tanks ..........B-63

Table B-5 Input Data with Water Addition for Hydrogen Generation Rate Model Calculations for 177 Tanks. B-83 
RPP-5926 REV 6

This page intentionally left blank.

B-2 
RPP-5926 REV 6

Table B-1. Input Data for Hydrogen Generation Rate Model Calculations for 177 Tanks. (20 sheets)

\begin{tabular}{|c|c|c|c|c|c|c|c|c|c|c|c|c|c|c|c|c|c|c|c|}
\hline Tank & $\begin{array}{c}\mathrm{OH} \\
\text { in tiquid } \\
{[\mathrm{OH}]} \\
(\mu \mathrm{g} / \mathrm{mL})\end{array}$ & $\begin{array}{c}\text { TOC } \\
\text { in liquid } \\
\text { [TOC] } \\
(\mu \mathrm{g} / \mathrm{mL})\end{array}$ & $\begin{array}{c}\mathrm{NO}_{2} \\
\text { in liquid } \\
{[\mathrm{NO} /} \\
(\mu \mathrm{g} / \mathrm{mL})\end{array}$ & $\begin{array}{c}\mathrm{NO}_{3} \\
\text { in liquid } \\
{\left[\mathrm{NO}_{3}\right\}} \\
(\mu \mathrm{g} / \mathrm{mL})\end{array}$ & $\begin{array}{c}\mathrm{Na} \\
\text { in tiquid } \\
\text { |Na| } \\
(\mathrm{ug} / \mathrm{mL})\end{array}$ & $\begin{array}{c}\mathrm{Al} \\
\text { in liquid } \\
{[\mathrm{A} \mid]} \\
(\mu \mathrm{g} / \mathrm{mL})\end{array}$ & $\begin{array}{c}{ }^{90} \mathrm{Sr} \\
\text { in waste } \\
{[\mathrm{Sr}]} \\
(\mu \mathrm{Ci} / \mathrm{g})\end{array}$ & $\begin{array}{c}{ }^{241} \mathrm{Am} \\
\text { in waste } \\
{[\mathrm{Am} 241]} \\
(\mu \mathrm{Ci} / \mathrm{g})\end{array}$ & $\begin{array}{c}{ }^{240} \mathrm{Pu} \\
\text { in waste } \\
{[\mathrm{Pu240}]} \\
(\mu \mathrm{Ci} / \mathrm{g})\end{array}$ & $\begin{array}{c}{ }^{239} \mathrm{Pu} \\
\text { in waste } \\
{[\mathrm{Pu240|}} \\
(\mu \mathrm{Ci} / \mathrm{g})\end{array}$ & $\begin{array}{c}{ }^{238} \mathrm{Pu} \\
\text { in waste } \\
{[\mathrm{Pu238}]} \\
(\mu \mathrm{Ci} / \mathrm{g})\end{array}$ & $\begin{array}{c}{ }^{137} \mathrm{Cs} \\
\text { in waste } \\
|\mathrm{Cs}| \\
(\mu \mathrm{Ci} / \mathrm{g})\end{array}$ & $\begin{array}{c}\text { Bulk } \\
\text { density } \\
\text { D } \\
(\mathrm{g} / \mathrm{mL})\end{array}$ & $\begin{array}{c}\text { Liquid } \\
\text { Density } \\
D_{\mathrm{L}} \\
(\mathrm{g} / \mathrm{ml})\end{array}$ & $\begin{array}{c}\text { Non-RGS } \\
\text { waste } \\
\text { volume } \\
(\mathrm{kL})\end{array}$ & $\begin{array}{c}\text { Bulk } \\
\text { water } \\
{\left[\mathrm{H}_{2} \mathrm{O}\right]} \\
(\mathrm{wt} \%)\end{array}$ & $\begin{array}{l}\text { Liquid } \\
\text { water } \\
{\left[\mathrm{H}_{2} \mathrm{O}\right]} \\
(\mathrm{wt} \%)\end{array}$ & $\begin{array}{c}\text { Waste } \\
\text { temp. } \\
\text { Tw } \\
\left({ }^{\circ} \mathrm{C}\right)\end{array}$ & $\begin{array}{c}\text { Dome } \\
\text { temp. } \\
\text { Td } \\
\left.{ }^{\circ} \mathrm{C}\right)\end{array}$ \\
\hline 241-AN-101 & $3.51 \mathrm{E}+04$ & $5.07 E+03$ & $1.16 \mathrm{E}+05$ & $1.63 \mathrm{E}+05$ & $2.15 \mathrm{E}+05$ & $1.96 \mathrm{E}+04$ & $2.03 \mathrm{E}+00$ & $1.79 \mathrm{E}-03$ & $8.53 \mathrm{E}-05$ & 3.85E-04 & $1.48 \mathrm{E}-05$ & $1.98 \mathrm{E}+02$ & 1.41 & 1.41 & 3624 & $51 \%$ & $51 \%$ & 27 & 26 \\
\hline Supeniatant & $3.51 \mathrm{E}+04$ & $5.11 \mathrm{E}+03$ & $1.16 \mathrm{E}+05$ & $1.62 \mathrm{E}+05$ & $2.14 \mathrm{E}+05$ & $1.89 \mathrm{E}+04$ & $1.27 \mathrm{E}+00$ & $6.57 \mathrm{E}-04$ & $1.03 \mathrm{E}-05$ & $6.21 \mathrm{E}-05$ & $1.54 \mathrm{E}-06$ & $1.98 \mathrm{E}+02$ & 1.41 & 1.41 & 3506 & $52 \%$ & $52 \%$ & 27 & 26 \\
\hline Solid Layer & $3.51 \mathrm{E}+04$ & $4.04 \mathrm{E}+03$ & $1.22 \mathrm{E}+05$ & $1.90 \mathrm{E}+05$ & $2.36 \mathrm{E}+05$ & $3.79 \mathrm{E}+04$ & $2.46 \mathrm{E}+01$ & $3.55 \mathrm{E}-02$ & $2.31 \mathrm{E}-03$ & $9.97 \mathrm{E}-03$ & $4.10 \mathrm{E}-04$ & $1.84 \mathrm{E}+02$ & 1.55 & 1.45 & $\$ 18$ & $40 \%$ & $49 \%$ & 26 & 26 \\
\hline Supernatant & $8.85 \mathrm{E}+03$ & $2.41 E+04$ & $8.71 \mathrm{E}+04$ & $2.09 \mathrm{E}+05$ & $2.07 \mathrm{E}+0.5$ & $1.31 \mathrm{E}+04$ & $5.60 \mathrm{E}+01$ & $1.15 \mathrm{E}-01$ & $8.77 \mathrm{E}-04$ & $3.37 \mathrm{E}-03$ & $2.45 \mathrm{E}-04$ & $2.78 \mathrm{E}+02$ & 1.41 & 1.41 & 3468 & $43 \%$ & $43 \%$ & 32 & 30 \\
\hline Solid Layer & $8.85 \mathrm{E}+03$ & $2.41 \mathrm{E}+04$ & $8.71 E+04$ & $2.09 \mathrm{E}+05$ & $2.07 \mathrm{E}+05$ & $1.31 \mathrm{E}+04$ & $1.37 \mathrm{E}+02$ & $5.99 \mathrm{E}-01$ & $1.05 \mathrm{E}-02$ & $4.04 \mathrm{E}-02$ & $2.93 \mathrm{E}-03$ & $2.64 E+02$ & 1.53 & 1.41 & 584 & $40 \%$ & $43 \%$ & 31 & 30 \\
\hline $24 \mathrm{~J}-\mathrm{AN}-103$ & $6.72 E+04$ & $3.05 \mathrm{E}+03$ & $1.30 \mathrm{E}+05$ & $1.28 E+05$ & $2.70 \mathrm{E}+05$ & $3.00 \mathrm{E}+04$ & $1.07 \mathrm{E}+00$ & 3.83E-03 & $1.96 \mathrm{E}-04$ & $7.53 \mathrm{E}-04$ & $5.46 \mathrm{E}-05$ & $3.21 E+02$ & 1.60 & 1.48 & 3407 & $41 \%$ & $47 \%$ & 37 & 27 \\
\hline Supernatant & $6.72 \mathrm{E}+04$ & $3.05 E+03$ & $1.30 \mathrm{E}+05$ & $1.28 \mathrm{E}+05$ & $2.70 \mathrm{E} \div 05$ & $3.00 E+04$ & $1.20 \mathrm{E}-02$ & $4.13 \mathrm{E}-03$ & 4.47E-05 & $1.72 \mathrm{E}-04$ & $1.25 \mathrm{E}-05$ & $4.28 \mathrm{E}+02$ & 1.48 & 1.48 & 1769 & $47 \%$ & $47 \%$ & 37 & 27 \\
\hline Solid Layer & $6.72 \mathrm{E}+04$ & $3.05 \mathrm{E}+03$ & $1.30 \mathrm{E}+05$ & $1.28 \mathrm{E}+05$ & $2.70 \mathrm{E}+05$ & $3.00 \mathrm{E}+04$ & $2.22 \mathrm{E}+00$ & $3.51 \mathrm{E}-03$ & $3.58 \mathrm{E}-04$ & $1.38 \mathrm{E}-03$ & $1.00 \mathrm{E}-04$ & $2.06 \mathrm{E}+02$ & 1.72 & 1.48 & 1638 & $35 \%$ & $47 \%$ & 37 & 27 \\
\hline $241-\mathrm{AN}-104$ & $6.53 \mathrm{E}+04$ & $3.14 \mathrm{E}+03$ & $1.19 \mathrm{E}+05$ & $1.89 E+05$ & $2.58 \mathrm{E}+0.5$ & $3.90 \mathrm{E}+04$ & $1.17 \mathrm{E}+01$ & 7.82E-03 & $3.09 \mathrm{E}-34$ & $4.19 E-03$ & $8.62 \mathrm{E}-05$ & $3.31 \mathrm{E}+02$ & 1.48 & 1.40 & 3864 & $49 \%$ & $51 \%$ & 36 & 28 \\
\hline Solid Layer & $6.53 \mathrm{E}+04$ & $3.14 \mathrm{E}+03$ & $1.19 \mathrm{E}+05$ & $1.89 \mathrm{E}+05$ & $2.58 E+05$ & $3.90 \mathrm{E}+04$ & $2.88 \mathrm{E}+01$ & $1.89 \mathrm{E}-02$ & $7.43 \mathrm{E}-04$ & $2.86 \mathrm{E}-03$ & $2.07 \mathrm{E}-04$ & $3.00 \mathrm{E}+02$ & 1.59 & 1.40 & 1566 & $46 \%$ & $51 \%$ & 38 & 28 \\
\hline 241-AN-105 & $5.97 \mathrm{E}+04$ & $2.76 \mathrm{E}+03$ & $1.19 \mathrm{E}+05$ & $1.56 \mathrm{E}+05$ & $2.47 \mathrm{E}+05$ & $4.17 \mathrm{E}+04$ & $4.42 \mathrm{E}+00$ & 8.63E-03 & $2.75 \mathrm{E}-04$ & $1.06 \mathrm{E}-03$ & $7.66 \mathrm{E}-05$ & $2.27 \mathrm{E}+02$ & 1.49 & 1.42 & 4152 & $47 \%$ & $50 \%$ & 32 & 26 \\
\hline Supenatant & $5.97 \mathrm{E}+04$ & $2.76 \mathrm{E}+03$ & $1.19 \mathrm{E}+05$ & $1.56 \mathrm{E}+05$ & $2.47 \mathrm{E}+05$ & $4.17 \mathrm{E}+04$ & $2.44 \mathrm{E}-02$ & $1.01 \mathrm{E}-02$ & $1.30 \mathrm{E}-04$ & $4.24 \mathrm{E}-04$ & $3.07 \mathrm{E}-05$ & $2.06 \mathrm{E}+02$ & 1.42 & 1.42 & 2227 & $50 \%$ & $50 \%$ & 31 & 26 \\
\hline Solid Layer & $5.97 \mathrm{E}+04$ & $2.76 \mathrm{E}+03$ & $1.19 \mathrm{E}+05$ & $1.56 \mathrm{E} \div 05$ & $2.47 \mathrm{E} \div 05$ & $4.17 \mathrm{E}+04$ & $9.50 \mathrm{E}+00$ & $6.92 \mathrm{E}-03$ & 4.67E-04 & $1.79 \mathrm{E}-03$ & $1.30 \mathrm{E}-04$ & $2.50 \mathrm{E}+02$ & 1.57 & 1.42 & 1925 & $44 \%$ & $50 \%$ & 33 & 26 \\
\hline $24 !-A N-106$ & $5.09 \mathrm{E}+03$ & $1.96 \mathrm{E}+03$ & $1.87 \mathrm{E}+04$ & $2.35 \mathrm{E}+04$ & $4.19 \mathrm{E}+04$ & $3.85 E+03$ & $4.50 \mathrm{E} \div 02$ & $5.59 \mathrm{E}-01$ & $5.41 \mathrm{E}-02$ & $2.51 \mathrm{E}-01$ & $8.81 \mathrm{E}-03$ & $5.35 \mathrm{E}+01$ & 1.15 & 1.10 & 3041 & $77 \%$ & $83 \%$ & 23 & 20 \\
\hline Supernatant & $5.09 \mathrm{E}+03$ & $1.96 \mathrm{E}+03$ & $1.87 \mathrm{E}+04$ & $2.35 \mathrm{E}+04$ & 4.19E+04 & $3.85 \mathrm{E}+03$ & $5.38 \mathrm{E}-01$ & $3.95 \mathrm{E}-04$ & $4.46 \mathrm{E}-04$ & $2.88 \mathrm{E}-03$ & 4.57E-05 & $4.35 \mathrm{E}+0 \mathrm{l}$ & 1.10 & 1.10 & 2485 & $83 \%$ & $83 \%$ & 22 & 20 \\
\hline Solid Layer & $5.09 \mathrm{E}+03$ & $1.96 \mathrm{E}+03$ & $E \div 04$ & $2.35 \mathrm{E}$ & $4.19 \mathrm{E}+04$ & $3.85 \mathrm{E}+03$ & $2.46 \mathrm{E}$ & 3. & $2.94 \mathrm{E}-01$ & $1.36 \mathrm{E}$ & $4.80 \mathrm{E}-02$ & $9.79 \mathrm{E}+01$ & 1.39 & 1.10 & 556 & $56 \%$ & $83 \%$ & 26 & 20 \\
\hline 241-AN-107 & $1.80 \mathrm{E}+04$ & $3.25 \mathrm{E}+04$ & $6.67 \mathrm{E}+04$ & $2.09 \mathrm{E}+05$ & $2.08 E+05$ & $1.12 \mathrm{E}+03$ & $7.71 \mathrm{E}+01$ & $5.99 \mathrm{E}-01$ & $9.47 \mathrm{E}-03$ & $3.65 \mathrm{E}-02$ & $2.64 \mathrm{E}-03$ & $2.14 \mathrm{E}+02$ & 1.44 & 1.43 & 4169 & $50 \%$ & $52 \%$ & 33 & 30 \\
\hline Supernatant & $1.80 \mathrm{E}+04$ & $3.25 \mathrm{E}+04$ & $6.67 \mathrm{E}+04$ & $2.09 \mathrm{E}+05$ & $2.08 \mathrm{E}+05$ & $1.12 \mathrm{E}+03$ & $5.37 \mathrm{E}+01$ & $5.11 \mathrm{E}-01$ & $8.21 \mathrm{E}-03$ & $3.16 \mathrm{E}-02$ & $2.29 \mathrm{E}-03$ & $2.09 \mathrm{E}+02$ & 1.43 & 1.43 & 3297 & $52 \%$ & $52 \%$ & 33 & 30 \\
\hline Solid Layer & $1.80 \mathrm{E}+04$ & $3.25 \mathrm{E}+04$ & $6.67 \mathrm{E}+04$ & $2.09 \mathrm{E}+05$ & $2.08 \mathrm{E}+05$ & $1.12 \mathrm{E}+03$ & $1.66 \mathrm{E}+02$ & $9.30 \mathrm{E}-01$ & $1.43 \mathrm{E}-02$ & $5.49 \mathrm{E}-02$ & $3.98 \mathrm{E}-03$ & $2.33 \mathrm{E}+02$ & 1.48 & 1.43 & 872 & $44 \%$ & $52 \%$ & 34 & 30 \\
\hline 241-AP-101 & $3.98 \mathrm{E}+04$ & $1.90 \mathrm{E}+03$ & $4.08 \mathrm{E}+04$ & $1.18 \mathrm{E}+05$ & $1.29 \mathrm{E}+05$ & $7.30 \mathrm{E}+03$ & $7.51 \mathrm{I}-02$ & $1.48 \mathrm{E}-04$ & $2.10 \mathrm{E}-05$ & $8.06 \mathrm{E}-05$ & $5.85 \mathrm{E}-06$ & $1.07 \mathrm{E}+02$ & 1.30 & 1.30 & 4219 & $64 \%$ & $64 \%$ & 22 & 23 \\
\hline Supetnatant & $3.98 \mathrm{E}+04$ & $1.90 \mathrm{E}+03$ & $4.08 \mathrm{E}+04$ & $1.18 \mathrm{E}+05$ & $1.29 \mathrm{E}+05$ & $7.30 \mathrm{E}+03$ & $7.51 \mathrm{E}-02$ & $1.48 \mathrm{E}-04$ & $2.10 \mathrm{E}-05$ & $8.06 \mathrm{E}-05$ & $5.85 \mathrm{E}-06$ & $1.07 \mathrm{E}+02$ & 1.30 & 1.30 & 4219 & $64 \%$ & $64 \%$ & 22 & 23 \\
\hline Solid Layer & $0.00 \mathrm{E}+00$ & 0.0 & 0.0 & 0.0 & 0.0 & 0.0 & 0.0 & $0.00 \mathrm{E}+00$ & $0.00 \mathrm{E}+00$ & $0.00 \mathrm{E}+00$ & $0.00 \mathrm{E}+00$ & 0.0 & 0.00 & 0.00 & 0 & $0 \%$ & $0 \%$ & 0 & 0 \\
\hline Solid Layer & $3.21 \mathrm{E}+04$ & 3013.1 & 98295.9 & 167448.8 & 194862.9 & 30871.8 & 0.4 & $6.95 \mathrm{E}-04$ & $7,92 \mathrm{E}-05$ & $4.68 \mathrm{E}-04$ & $1.20 \mathrm{E}-05$ & 81.2 & 1.75 & 1.39 & 88 & $31 \%$ & $59 \%$ & 24 & 31 \\
\hline
\end{tabular}


RPP-5926 REV 6

Table B-1. Input Data for Hydrogen Generation Rate Model Calculations for 177 Tanks. (20 sheets)

\begin{tabular}{|c|c|c|c|c|c|c|c|c|c|c|c|c|c|c|c|c|c|c|c|}
\hline Tank & $\begin{array}{c}\mathrm{OH} \\
\text { in liquid } \\
{[\mathrm{OH}]} \\
(\mu \mathrm{g} / \mathrm{mL})\end{array}$ & $\begin{array}{c}\text { TOC } \\
\text { in liquid } \\
\text { [TOC] } \\
(\mu \mathrm{g} / \mathrm{mL})\end{array}$ & $\begin{array}{c}\mathrm{NO}_{2} \\
\text { in liquid } \\
\left|\mathrm{NO}_{2}\right| \\
(\mu \mathrm{g} / \mathrm{mL})\end{array}$ & $\begin{array}{c}\mathrm{NO}_{3} \\
\text { in liquid } \\
{\left[\mathrm{NO}_{3}\right]} \\
(\mu \mathrm{g} / \mathrm{mL})\end{array}$ & $\begin{array}{c}\mathrm{Na} \\
\text { in liquid } \\
\text { |Na| } \\
\text { (ug/mL) }\end{array}$ & $\begin{array}{c}\mathrm{Al} \\
\text { in liquid } \\
{[\mathrm{Al}]} \\
(\mu \mathrm{g} / \mathrm{mL})\end{array}$ & $\begin{array}{c}{ }^{96} \mathrm{Sr} \\
\text { in waste } \\
{[\mathrm{Sr}]} \\
(\mu \mathrm{Ci} / \mathrm{g})\end{array}$ & $\begin{array}{c}{ }^{241} \mathrm{Am} \\
\text { in waste } \\
{[\mathrm{Am} 241]} \\
(\mu \mathrm{Ci} / \mathrm{g})\end{array}$ & $\begin{array}{c}{ }^{240} \mathrm{Pu} \\
\text { in waste } \\
{[\mathrm{Pu} 240]} \\
(\mu \mathrm{Ci} / \mathrm{g})\end{array}$ & $\begin{array}{c}{ }^{239} \mathrm{Pu} \\
\text { in waste } \\
{[\mathrm{Pu} 240]} \\
(\mu \mathrm{Ci} / \mathrm{g})\end{array}$ & $\begin{array}{c}{ }^{238} \mathrm{Pu} \\
\text { in waste } \\
{[\mathrm{Pu} 238]} \\
(\mu \mathrm{Ci} / \mathrm{g})\end{array}$ & $\begin{array}{c}{ }^{137} \mathrm{Cs} \\
\text { in waste } \\
|\mathrm{Cs}| \\
(\mu \mathrm{Ci} / \mathrm{g})\end{array}$ & $\begin{array}{c}\text { Bulk } \\
\text { density } \\
\text { D } \\
(\mathrm{g} / \mathrm{mL})\end{array}$ & $\begin{array}{c}\text { Liquid } \\
\text { Density } \\
D_{\mathrm{L}} \\
(\mathrm{g} / \mathrm{ml})\end{array}$ & $\begin{array}{c}\text { Non-RGS } \\
\text { waste } \\
\text { volume } \\
\text { (kL) }\end{array}$ & $\begin{array}{c}\text { Bulk } \\
\text { water } \\
{\left[\mathrm{H}_{2} \mathrm{O} \mid\right.} \\
(\mathrm{wt} \%)\end{array}$ & $\begin{array}{c}\text { Liquid } \\
\text { water } \\
\left|\mathrm{H}_{2} \mathrm{O}\right| \\
(w t \%)\end{array}$ & $\begin{array}{l}\text { Waste } \\
\text { temp. } \\
\mathrm{Tw} \\
\left({ }^{\circ} \mathrm{C}\right)\end{array}$ & $\begin{array}{c}\text { Dome } \\
\text { temp. } \\
\text { Td } \\
\left({ }^{\circ} \mathrm{C}\right)\end{array}$ \\
\hline 241-AP-103 & $1.67 \mathrm{E}+04$ & $7.37 \mathrm{E}+03$ & $8.04 E+04$ & $1.51 \mathrm{E}+05$ & $1.79 \mathrm{E}+05$ & $1.86 \mathrm{E}+04$ & $1.78 \mathrm{E}+00$ & $8.19 \mathrm{E}-03$ & 1.14E-04 & $6.83 \mathrm{E}-04$ & $2.11 \mathrm{E}-05$ & $1.69 \mathrm{E}+02$ & 1.36 & 1.35 & 3378 & $56 \%$ & $57 \%$ & 21 & 22 \\
\hline Supematant & $1.67 \mathrm{E}+04$ & $7.37 \mathrm{E}+03$ & $8.04 \mathrm{E}+04$ & $1.51 \mathrm{E}+05$ & $1.79 \mathrm{E}+05$ & $1.86 \mathrm{E}+04$ & $1.82 \mathrm{E}+00$ & $8.38 \mathrm{E}-03$ & 1.13E-04 & $6.76 \mathrm{E}-04$ & $2.08 \mathrm{E}-05$ & $1.70 \mathrm{E}+02$ & 1.35 & 1.35 & 3298 & $57 \%$ & $57 \%$ & 21 & 22 \\
\hline Solid Layer & $1.67 \mathrm{E}+04$ & 7368.1 & 80351.7 & 151000.6 & 178896.3 & 18587.0 & 0.2 & $2.49 \mathrm{E}-04$ & $1.62 \mathrm{E}-04$ & $9.60 \mathrm{E}-04$ & $3.17 \mathrm{E}-05$ & 111.6 & 1.68 & 1.35 & 80 & $41 \%$ & $57 \%$ & 24 & 22 \\
\hline Supernatant & $1.96 \mathrm{E}+04$ & $4.16 \mathrm{E}+03$ & $6.40 \mathrm{E}+04$ & $1.01 E+05$ & $1.29 \mathrm{E}+05$ & $1.68 \mathrm{E}+04$ & $1.30 \mathrm{E}+00$ & $2.20 \mathrm{E}-03$ & $2.35 \mathrm{E}-05$ & $1.38 \mathrm{E}-04$ & $3.10 \mathrm{E}-05$ & $1.45 \mathrm{E}+02$ & 1.28 & 1.28 & 4155 & $66 \%$ & $66 \%$ & 21 & 22 \\
\hline Solid Layer & $0.00 \mathrm{E}+00$ & 0.0 & 0.0 & 0.0 & 0.0 & 0.0 & 0.0 & $0.00 \mathrm{E}+00$ & $0.00 \mathrm{E}+00$ & $0.00 \mathrm{E}+00$ & $0.00 \mathrm{E}+00$ & 0.0 & 0.00 & 0.00 & 0 & $0 \%$ & $0 \%$ & 0 & 0 \\
\hline 241-AP-105 & $2.59 \mathrm{E}+04$ & $1.50 \mathrm{E}+03$ & $4.74 \mathrm{E}+04$ & $1.06 \mathrm{E}+05$ & $1.34 \mathrm{E} \div 05$ & $1.92 \mathrm{E}+04$ & $1.60 \mathrm{E}+00$ & $9.12 \mathrm{E}-04$ & $1.03 \mathrm{E}-04$ & $4.74 \mathrm{E}-04$ & $3.12 \mathrm{E}-05$ & $1.16 \mathrm{E}+02$ & 1.30 & 1.27 & 4302 & $69 \%$ & $71 \%$ & 31 & 32 \\
\hline Supenlatant & $2.59 \mathrm{E}+04$ & $1.50 \mathrm{E}+03$ & $4.74 \mathrm{E}+04$ & $1.06 \mathrm{E}+05$ & $1.34 \mathrm{E}+05$ & $1.92 \mathrm{E}+04$ & $2.14 \mathrm{E}-01$ & $5.46 \mathrm{E}-04$ & $3.65 \mathrm{E}-05$ & $2.22 \mathrm{E}-04$ & $1.27 \mathrm{E}-05$ & $1.07 \mathrm{E}+02$ & 1.27 & 1.27 & 3965 & $71 \%$ & $71 \%$ & 32 & 32 \\
\hline Solid Layer & $2.59 \mathrm{E}+04$ & $1.50 E+03$ & $4.74 \mathrm{E}+04$ & $1.06 \mathrm{E}+05$ & $1.34 \mathrm{E}+05$ & $1.92 \mathrm{E}+04$ & $1.79 \mathrm{E}+01$ & $5.22 \mathrm{E}-03$ & $8.90 \mathrm{E}-04$ & $3.43 \mathrm{E}-03$ & $2.49 \mathrm{E}-04$ & $2.25 \mathrm{E}+02$ & 1.61 & 1.27 & 337 & $48 \%$ & $71 \%$ & 20 & 32 \\
\hline 241-AP-106 & $7.37 \mathrm{E}+03$ & $3.46 E+03$ & $4.14 \mathrm{E}+04$ & $7.07 \mathrm{E}+04$ & $1.03 \mathrm{E}+05$ & $1.35 \mathrm{E}+04$ & $9.84 \mathrm{E}-01$ & $5.02 \mathrm{E}-04$ & $1.05 \mathrm{E}-05$ & $6.11 \mathrm{E}-05$ & $1.72 \mathrm{E}-06$ & $1.45 \mathrm{E}+02$ & 1.21 & $1.2 \mathrm{~J}$ & 4301 & $72 \%$ & $72 \%$ & 24 & 19 \\
\hline Solid Layer & $0.00 \mathrm{E}+00$ & 0.0 & 0.0 & 0.0 & 0.0 & 0.0 & 0.0 & $0.00 \mathrm{E}+00$ & $0.00 \mathrm{E}+00$ & $0.00 \mathrm{E}+00$ & $0.00 \mathrm{E}+00$ & 0.0 & 0.00 & 0.00 & 0 & $0 \%$ & $0 \%$ & 0 & 0 \\
\hline $241-\mathrm{AP}-107$ & $1.81 \mathrm{E}+04$ & $2.78 \mathrm{E}+03$ & $5.77 \mathrm{E}+04$ & $1.26 \mathrm{E}+05$ & $1.39 \mathrm{E}+05$ & $1.64 \mathrm{E}+04$ & $6.43 \mathrm{E}-01$ & $3.23 \mathrm{E}-04$ & $2.20 \mathrm{E}-05$ & $1.16 \mathrm{E}-04$ & $6.78 \mathrm{E}-06$ & $1.12 \mathrm{E}+02$ & 1.31 & 1.31 & 1306 & $63 \%$ & $63 \%$ & 15 & 14 \\
\hline Supernatant & $1.81 \mathrm{E}+04$ & $2.78 \mathrm{E}+03$ & $5.77 \mathrm{E}+04$ & $1.26 \mathrm{E}+05$ & $1.39 \mathrm{E}+05$ & $1.64 \mathrm{E}+04$ & $6.43 \mathrm{E}-0 \mathrm{I}$ & $3.23 \mathrm{E}-04$ & $2.20 \mathrm{E}-05$ & $1.16 \mathrm{E}-04$ & $6.78 \mathrm{E}-06$ & $1.12 E+02$ & 1.31 & 1.31 & 1306 & $63 \%$ & $63 \%$ & 15 & 14 \\
\hline Solid Layer & $0.00 \mathrm{E}+00$ & 0.0 & 0.0 & 0.0 & 0.0 & 0.0 & 0.0 & $0.00 \mathrm{E}+00$ & $0.00 \mathrm{E}+00$ & $0.00 \mathrm{E}+00$ & $0.00 \mathrm{E}+00$ & 0.0 & 0.00 & 0.00 & 0 & $0 \%$ & $0 \%$ & 0 & 0 \\
\hline 241-AP-108 & $4.91 \mathrm{E}+04$ & $2.80 \mathrm{E}+03$ & $7.92 \mathrm{E}+04$ & $1.78 \mathrm{E}+05$ & $2.13 \mathrm{E}+05$ & $2.40 \mathrm{E}+04$ & $5.06 \mathrm{E}-01$ & $2.37 E-04$ & $4.68 \mathrm{E}-05$ & $2.31 \mathrm{E}-04$ & $1.74 \mathrm{E}-05$ & $1.41 \mathrm{E}+02$ & 1.43 & 1.42 & 3872 & $49 \%$ & $50 \%$ & 34 & 36 \\
\hline Supernatant & $3.95 \mathrm{E}+04$ & $2.80 \mathrm{E}+03$ & $7.92 \mathrm{E}+04$ & $1.78 \mathrm{E}+05$ & $2.13 \mathrm{E}+05$ & $2.40 \mathrm{E}+04$ & $5.10 \mathrm{E}-01$ & $2.39 \mathrm{E}-04$ & $4.72 \mathrm{E}-05$ & $2.33 \mathrm{E}-04$ & $1.75 \mathrm{E}-05$ & $1.43 \mathrm{E}+02$ & 1.42 & 1.42 & 3385 & $50 \%$ & $50 \%$ & 36 & 36 \\
\hline Solid Layer & $3.95 \mathrm{E}+04$ & 2797.6 & 79172.8 & 177843.4 & 212703.1 & 23958.6 & 0.5 & $2.23 \mathrm{E}-04$ & $4.42 \mathrm{E}-05$ & $2.17 \mathrm{E}-04$ & $1.63 \mathrm{E}-05$ & 133.1 & 1.52 & 1.42 & 487 & $46 \%$ & $50 \%$ & 23 & 36 \\
\hline 241-AW-101 & $9.96 \mathrm{E}+04$ & $2.61 \mathrm{E}+03$ & $1.05 E+05$ & $1.71 \mathrm{E}+05$ & $2.33 \mathrm{E}+05$ & $2.95 E+04$ & $1.25 \mathrm{E}+01$ & 1.58E-02 & $3.94 \mathrm{E}-03$ & $1.52 \mathrm{E}-02$ & $1.10 \mathrm{E}-03$ & $2.43 \mathrm{E}+02$ & 1.51 & 1.47 & 4173 & $44 \%$ & $44 \%$ & 33 & 22 \\
\hline Supernatant & $9.96 \mathrm{E}+04$ & $2.61 E+03$ & $1.05 \mathrm{E}+05$ & $1.71 \mathrm{E}+05$ & $2.33 \mathrm{E}+05$ & $2.95 \mathrm{E}+04$ & $2.55 \mathrm{E}-01$ & $1.77 \mathrm{E}-04$ & $7.27 \mathrm{E}-05$ & $2.80 \mathrm{E}-04$ & $2.03 \mathrm{E}-05$ & $2.58 \mathrm{E}+02$ & 1.47 & 1.47 & 2770 & $44 \%$ & $44 \%$ & 33 & 22 \\
\hline Solid Layer & $9.96 \mathrm{E}+04$ & $2.61 \mathrm{E}+03$ & $1.05 \mathrm{E}+05$ & $1.71 \mathrm{E}+05$ & $2.33 \mathrm{E}+05$ & $2.95 \mathrm{E}+04$ & $3.68 \mathrm{E}+01$ & $4.66 \mathrm{E}-02$ & $1.16 \mathrm{E}-02$ & $4.45 \mathrm{E}-02$ & $3.23 \mathrm{E}-03$ & $2.13 \mathrm{E}+02$ & 1.59 & 1.47 & 1403 & $44 \%$ & $44 \%$ & 34 & 22 \\
\hline 241-AW-102 & $1.85 \mathrm{E}+04$ & $2.06 \mathrm{E}+03$ & $4.45 \mathrm{E}+04$ & $9.70 \mathrm{E}+04$ & $1.06 \mathrm{E}+05$ & $1.21 \mathrm{E}+04$ & $6.56 \mathrm{E}+00$ & $2.03 \mathrm{E}-02$ & $1.02 \mathrm{E}-03$ & $4.93 \mathrm{E}-03$ & $9.15 \mathrm{E}-05$ & $9.34 \mathrm{E}+01$ & 1.22 & 1.22 & 2293 & $71 \%$ & $71 \%$ & 22 & 21 \\
\hline Supematant & $1.85 \mathrm{E}+04$ & $2.06 \mathrm{E}+03$ & $4.45 \mathrm{E}+04$ & $9.70 \mathrm{E}+04$ & $1.06 \mathrm{E}+05$ & $1.21 E+04$ & $6.58 \mathrm{E}-01$ & $3.38 \mathrm{E}-04$ & $3.27 \mathrm{E}-05$ & $7.26 \mathrm{E}-05$ & $7.99 \mathrm{E}-06$ & $9.36 \mathrm{E}+01$ & 1.22 & 1.22 & 2268 & $71 \%$ & $71 \%$ & 22 & 21 \\
\hline Solid Layer & $1.85 \mathrm{E}+04$ & $2.06 \mathrm{E}+03$ & $4.45 \mathrm{E}+04$ & $9.70 \mathrm{E}+04$ & $1.06 \mathrm{E}+05$ & $1.21 \mathrm{E}+04$ & $5.42 \mathrm{E}+02$ & $1.83 \mathrm{E}+00$ & $9.24 \mathrm{E}-02$ & $4.45 \mathrm{E}-01$ & $7.67 \mathrm{E}-03$ & $7.12 \mathrm{E}+01$ & 1.32 & 1.22 & 25 & $33 \%$ & $71 \%$ & 28 & 21 \\
\hline Solid Layer & $4.81 \mathrm{E}+04$ & $6.11 \mathrm{E}+03$ & $9.31 E+04$ & $2.47 \mathrm{E}+05$ & $2.01 \mathrm{E}+05$ & $8.08 \mathrm{E}+03$ & $6.81 E+00$ & $8.94 \mathrm{E}-02$ & $6.13 \mathrm{E}-02$ & $2.19 \mathrm{E}-01$ & $2.16 \mathrm{E}-02$ & $2.75 \mathrm{E}+01$ & 1.49 & 1.42 & 1256 & $55 \%$ & $51 \%$ & 20 & 21 \\
\hline
\end{tabular}


RPP-5926 REV 6

Table B-1. Input Data for Hydrogen Generation Rate Model Calculations for 177 Tanks. (20 sheets)

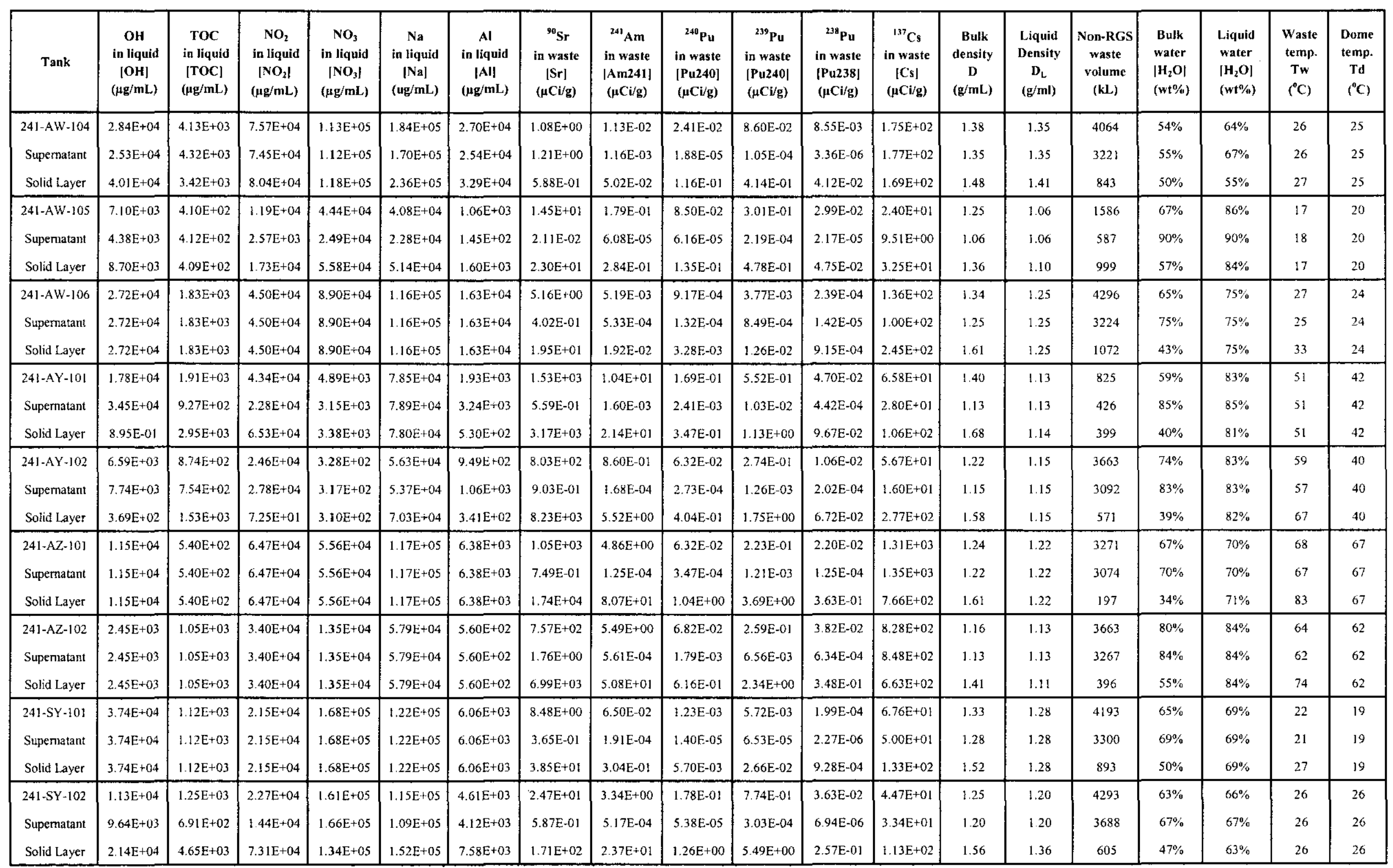


RPP-5926 REV 6

Table B-1. Input Data for Hydrogen Generation Rate Model Calculations for 177 Tanks. (20 sheets)

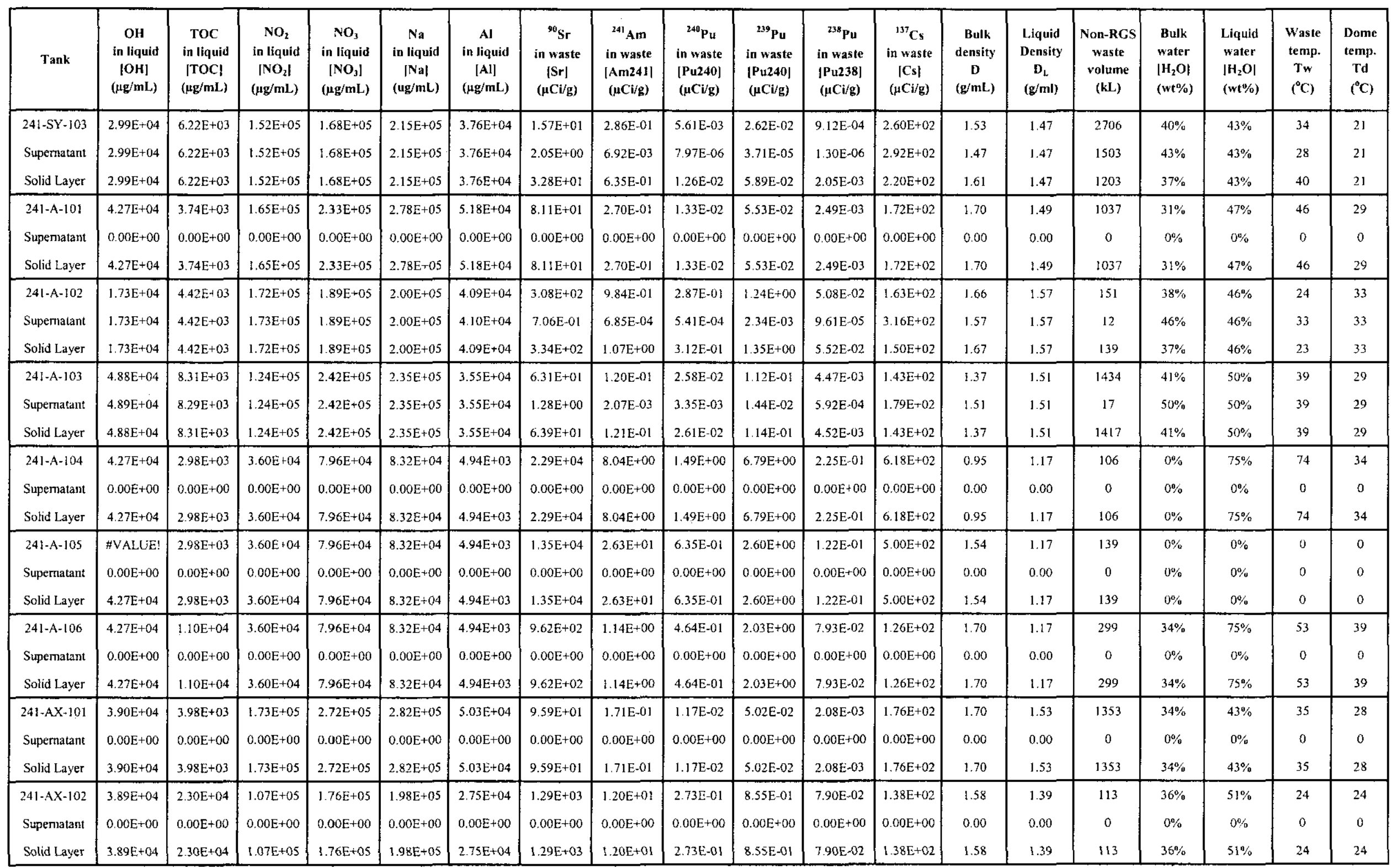




\section{RPP-5926 REV 6}

Table B-1. Input Data for Hydrogen Generation Rate Model Calculations for 177 Tanks. (20 sheets)

\begin{tabular}{|c|c|c|c|c|c|c|c|c|c|c|c|c|c|c|c|c|c|c|c|}
\hline Tank & $\begin{array}{c}\mathrm{OH} \\
\text { in liquid } \\
{[\mathrm{OH}]} \\
(\mu \mathrm{g} / \mathrm{mL})\end{array}$ & $\begin{array}{c}\text { TOC } \\
\text { in liquid } \\
{[\mathrm{TOC}]} \\
(\mu \mathrm{g} / \mathrm{mL})\end{array}$ & $\begin{array}{c}\mathrm{NO}_{2} \\
\text { in liquid } \\
{\left[\mathrm{NO}_{2}\right]} \\
(\mu \mathrm{g} / \mathrm{mL})\end{array}$ & $\begin{array}{c}\mathrm{NO}_{3} \\
\text { in liquid } \\
{\left[\mathrm{NO}_{3}\right]} \\
(\mu \mathrm{g} / \mathrm{mL})\end{array}$ & $\begin{array}{c}\mathrm{Na} \\
\text { in liquid } \\
|\mathrm{Na}| \\
\text { (ug/mL) }\end{array}$ & $\begin{array}{c}\mathrm{Al} \\
\text { in líquid } \\
{[\mathrm{Al}} \\
(\mu \mathrm{g} / \mathrm{mL})\end{array}$ & $\begin{array}{c}{ }^{90} \mathrm{Sr} \\
\text { in waste } \\
{[\mathrm{Sr}\rceil} \\
(\mu \mathrm{Ci} / \mathrm{g})\end{array}$ & $\begin{array}{c}{ }^{24 t} \mathrm{Am} \\
\text { in waste } \\
{\left[\begin{array}{c}\mathrm{Am} 241] \\
(\mu \mathrm{Ci} / \mathrm{g})\end{array}\right.}\end{array}$ & $\begin{array}{c}{ }^{240} \mathrm{Pu} \\
\text { in waste } \\
{\left[\begin{array}{l}\mathrm{Pu240}] \\
(\mu \mathrm{Ci} / \mathrm{g})\end{array}\right.}\end{array}$ & $\begin{array}{c}{ }^{239} \mathrm{Pu} \\
\text { in waste } \\
|\mathrm{Pu} 240| \\
(\mu \mathrm{Ci} / \mathrm{g})\end{array}$ & $\begin{array}{c}{ }^{2.39} \mathrm{Pu} \\
\text { in waste } \\
{[\mathrm{Pu238}]} \\
(\mu \mathrm{Ci} / \mathrm{g})\end{array}$ & $\begin{array}{c}{ }^{37} \mathrm{Cs} \\
\text { in waste } \\
{[\mathrm{Cs} \mid} \\
(\mu \mathrm{Ci} / \mathrm{g})\end{array}$ & $\begin{array}{c}\text { Bulk } \\
\text { density } \\
D \\
(\mathrm{~g} / \mathrm{mL})\end{array}$ & $\begin{array}{c}\text { Liquid } \\
\text { Density } \\
\mathrm{D}_{\mathrm{L}} \\
(\mathrm{g} / \mathrm{ml})\end{array}$ & $\begin{array}{c}\text { Non-RGS } \\
\text { waste } \\
\text { volume } \\
(\mathrm{kL})\end{array}$ & $\begin{array}{l}\text { Bulk } \\
\text { water } \\
{\left[\mathrm{H}_{2} \mathrm{O}\right]} \\
(\mathbf{w t} \%)\end{array}$ & $\begin{array}{c}\text { Liquid } \\
\text { water } \\
{\left[\mathrm{H}_{2} \mathrm{O}\right]} \\
(\mathrm{wt} \%)\end{array}$ & $\begin{array}{c}\text { Waste } \\
\text { temp. } \\
T w \\
\left({ }^{\circ} \mathrm{C}\right)\end{array}$ & $\begin{array}{c}\text { Dome } \\
\text { temp } \\
\mathrm{Td} \\
\left({ }^{\mathrm{C}} \mathrm{C}\right)\end{array}$ \\
\hline 241-AX-103 & $9 \mathrm{E}+04$ & $3 E+03$ & $1.22 \mathrm{E}+05$ & $1.91 \mathrm{E}+05$ & $2.36 \mathrm{E}+05$ & $3.79 \mathrm{E}+04$ & $98 \mathrm{E}+02$ & 00 & $3.66 \mathrm{E}-02$ & $1.45 \mathrm{E}-01$ & $80 \mathrm{E}-03$ & $1.81 \mathrm{E}+02$ & 1.58 & 1.45 & 403 & $44 \%$ & $49 \%$ & 38 & 31 \\
\hline Supernatant & $0.00 \mathrm{E}+00$ & $0.00 \mathrm{E}+00$ & $0.00 \mathrm{E}+00$ & $0.00 \mathrm{E}+00$ & $0.00 \mathrm{E}+00$ & $0.00 \mathrm{E}+00$ & $0.00 \mathrm{E}+00$ & $0.00 \mathrm{E}+00$ & $0.00 \mathrm{E}+00$ & $0.00 \mathrm{E}+00$ & $0.00 \mathrm{E}+00$ & $0.00 \mathrm{E}+00$ & 0.00 & 0.00 & 0 & $0 \%$ & $0 \%$ & 0 & 0 \\
\hline Solid Layer & $3.89 \mathrm{E}+04$ & $4.03 E+03$ & $1.22 \mathrm{E}+05$ & $1.91 \mathrm{E}+05$ & $2.36 \mathrm{E}+05$ & $3.79 \mathrm{E}+04$ & $6.98 \mathrm{E}+02$ & 1. $14 \mathrm{E}+00$ & $3.66 \mathrm{E}-02$ & $1.45 \mathrm{E}-01$ & $6.80 \mathrm{E}-03$ & $1.81 \mathrm{E}+02$ & 1.58 & 1.45 & 403 & $44 \%$ & $49 \%$ & 38 & 31 \\
\hline $241-\mathrm{AX}-104$ & $3.89 \mathrm{E}+04$ & $2.98 \mathrm{E}+03$ & $3.60 \mathrm{E}+04$ & $7.96 \mathrm{E}+04$ & $8.32 \mathrm{E}+04$ & $4.94 \mathrm{E}+03$ & $4.07 \mathrm{E}+04$ & $1.88 \mathrm{E}+01$ & $1.31 E+00$ & $5.34 \mathrm{E}+00$ & $2.52 \mathrm{E}-01$ & $9.86 \mathrm{E}+02$ & 1.80 & 1.17 & 28 & $8 \%$ & $75 \%$ & 32 & 31 \\
\hline Supematant & $0.00 \mathrm{E}+00$ & $0.00 \mathrm{E}+00$ & $0.00 \mathrm{E}+00$ & $0.00 \mathrm{E}+00$ & $0.00 \mathrm{E}+00$ & $0.00 \mathrm{E}+00$ & $0.00 \mathrm{E}+00$ & $0.00 \mathrm{E}+00$ & $0.00 \mathrm{E}+00$ & $0.00 \mathrm{E}+00$ & $0.00 \mathrm{E}+00$ & $0.00 \mathrm{E}+00$ & 0.00 & 0.00 & 0 & $0 \%$ & $0 \%$ & 0 & 0 \\
\hline Solid Layer & $3.89 E+04$ & $2.98 \mathrm{E}+03$ & $3.60 \mathrm{E}+04$ & $7.96 \mathrm{E}+04$ & $8.32 \mathrm{E}+04$ & $4.94 \mathrm{E}+03$ & $4.07 \mathrm{E}+04$ & $1.88 \mathrm{E}+01$ & $1.31 \mathrm{E}+00$ & $5.34 \mathrm{E}+00$ & $2.52 \mathrm{E}-01$ & $9.86 \mathrm{E}+02$ & 1.80 & 1.17 & 28 & $8 \%$ & $75 \%$ & 32 & 31 \\
\hline 241-B-101 & $1.70 \mathrm{E}+04$ & $6.77 \mathrm{E}+00$ & $1.32 \mathrm{E}+05$ & $3.56 \mathrm{E}+05$ & $2.26 \mathrm{E}+05$ & $7.23 \mathrm{E}+02$ & $3.26 \mathrm{E}+02$ & $2.28 \mathrm{E}+00$ & 1.55E-01 & $5,02 \mathrm{E}-01$ & $4.39 \mathrm{E}-02$ & $3.48 \mathrm{E}+00$ & 1.51 & 1.53 & 411 & $40 \%$ & $50 \%$ & 38 & 33 \\
\hline Supenatant & $0.00 \mathrm{E}+00$ & $0.00 \mathrm{E}+00$ & $0.00 \mathrm{E}+00$ & $0.00 \mathrm{E} \div 00$ & $0.00 \mathrm{E}+00$ & $0.00 \mathrm{E}+00$ & $0.00 \mathrm{E}+00$ & $0.00 \mathrm{E}+00$ & $0.00 \mathrm{E}+00$ & $0.00 \mathrm{E}+00$ & $0.00 \mathrm{E}+00$ & $0.00 \mathrm{E}+00$ & 0.00 & 0.00 & 0 & $0 \%$ & $0 \%$ & 0 & 0 \\
\hline Solid Layer & $1.70 \mathrm{E}+04$ & $6.77 \mathrm{E}+00$ & $1.32 \mathrm{E}+05$ & $3.56 \mathrm{E}+05$ & $2.26 \mathrm{E}+05$ & $7.23 \mathrm{E}+02$ & $3.26 \mathrm{E}+02$ & $2.28 \mathrm{E}+00$ & $1.55 \mathrm{E}-01$ & $5.02 \mathrm{E}-01$ & 4.39E-02 & $3.48 \mathrm{E}+00$ & 1.51 & 1.53 & 411 & $40 \%$ & $50 \%$ & 38 & 33 \\
\hline 24 !-B-102 & $1.70 \mathrm{E}+04$ & $3.38 \mathrm{E}+00$ & $8.51 E+03$ & $2.17 \mathrm{E}+05$ & $1.13 \mathrm{E}+05$ & $3.61 \mathrm{E}+02$ & $7.27 \mathrm{E}-01$ & $5.17 \mathrm{E}-04$ & $2.96 \mathrm{E}-03$ & $2.62 \mathrm{E}-02$ & $1.94 \mathrm{E}-04$ & $3.19 \mathrm{E} \div 00$ & 1.57 & 1.26 & 121 & $48 \%$ & $70 \%$ & 18 & 22 \\
\hline Supcriatant & $1.70 \mathrm{E}+04$ & $3.38 E+00$ & $8.47 \mathrm{E} \div 03$ & $2.17 \mathrm{E}+05$ & $1.13 \mathrm{E}+05$ & $3.61 \mathrm{E}+02$ & $1.68 \mathrm{E}+00$ & $1.23 \mathrm{E}-03$ & 4.69E-04 & 4.14E-03 & $3.08 \mathrm{E}-05$ & $7.09 \mathrm{E}+00$ & 1.26 & 1.26 & 15 & $70 \%$ & $70 \%$ & 18 & 22 \\
\hline Solid Layer & $1.70 E+04$ & 350 & $8.92 \mathrm{tos}$ & 2.1705 & H.15005 & $3.012+02$ & $5.91 \mathrm{E}-01$ & $4.175-04$ & 3.32E-0. & $2.95 \mathrm{E}=02$ & $2.18 E-04$ & $2.64 \mathrm{E}+00$ & 1.61 & 1.26 & 106 & $45 \%$ & $70 \%$ & 18 & 22 \\
\hline $241-\mathrm{B}-103$ & 4 & $3.38 \mathrm{E} \div 00$ & $8.50 \mathrm{E}+03$ & $E+05$ & $\overline{E+05}$ & 3 & $1.40 \mathrm{E}+00$ & 3 & -02 & 01 & 3 & $2.65 \mathrm{E}+00$ & $i$ & 1.26 & 2 & $49 \%$ & $70 \%$ & 17 & 23 \\
\hline Supernatant & $0.00 \mathrm{E}+00$ & $0.00 \mathrm{E}+00$ & $0.00 \mathrm{E}+00$ & $0.00 \mathrm{E}+00$ & $0.00 \mathrm{E}+00$ & $0.00 \mathrm{E}+00$ & $0.00 \mathrm{E}+00$ & $0.00 \mathrm{E}+00$ & $0.00 \mathrm{E}+00$ & $0.00 \mathrm{E}+00$ & $0.00 \mathrm{E}+00$ & $0.00 \mathrm{E}+00$ & 0.00 & 0.00 & 0 & $0 \%$ & $0 \%$ & 0 & 0 \\
\hline Solid Layer & $1.70 \mathrm{E}+04$ & $3.38 \mathrm{E}+00$ & $8.50 \mathrm{E}+03$ & $2.18 \mathrm{E}+05$ & 1.13E +05 & $3.62 \mathrm{E}+02$ & $1.40 \mathrm{E}+00$ & 1.23E-03 & 1.70E-02 & $1.50 \mathrm{E}-0$ & $1.12 \mathrm{E}-03$ & $2.65 \mathrm{E}+00$ & 1.61 & 1.26 & 211 & $49 \%$ & $70 \%$ & 17 & 23 \\
\hline 241-B-104 & $1.70 \mathrm{E}+04$ & $3.38 \mathrm{E}+00$ & $8.49 \mathrm{E}+03$ & $2.16 \mathrm{E}+05$ & $1.13 \mathrm{E}+05$ & $3.62 \mathrm{E}+02$ & $1.98 \mathrm{E}+00$ & $.44 \mathrm{E}-03$ & $71 \mathrm{E}-03$ & $4.40 \mathrm{E}-02$ & $3.03 \mathrm{E}-04$ & $6.62 \mathrm{E}+00$ & 120 & 2 & 1417 & $48 \%$ & $70 \%$ & 17 & 20 \\
\hline Supematant & $0.00 \mathrm{E}+00$ & $0.00 E+00$ & $0.00 \mathrm{E}+00$ & $0.00 \mathrm{E}+00$ & $0.00 \mathrm{E}+00$ & $0.00 \mathrm{E}+00$ & $0.00 \mathrm{E}+00$ & $0.00 \mathrm{E}+00$ & $0.00 \mathrm{E}+00$ & $0.00 \mathrm{E}+00$ & $0.00 \mathrm{E}+00$ & $0.00 \mathrm{E}+00$ & 0.00 & 0.00 & 0 & $0 \%$ & $0 \%$ & 0 & 0 \\
\hline Solid Layer & $1.70 \mathrm{E}+04$ & $3.38 \mathrm{E}+00$ & $8.49 \mathrm{E}+03$ & $2.16 \mathrm{E}+05$ & $1.13 \mathrm{E}+05$ & $3.62 \mathrm{E}+02$ & $1.98 \mathrm{E}+00$ & $2.44 \mathrm{E}-03$ & $4.71 \mathrm{E}-03$ & 4.40E-02 & $3.03 \mathrm{E}-04$ & $6.62 \mathrm{E}+00$ & 1.38 & 1.26 & 1417 & $48 \%$ & $70 \%$ & 17 & 20 \\
\hline 241-B-105 & $1.70 \mathrm{E}+04$ & $3.38 \mathrm{E}+00$ & $8.51 \mathrm{~J}+03$ & $2.16 \mathrm{E}+05$ & $1.13 \mathrm{E}+05$ & $3.62 \mathrm{E}+02$ & $5.56 \mathrm{E}-0 \mathrm{l}$ & $7.57 \mathrm{E}-04$ & $3.87 \mathrm{E}-03$ & $3.43 \mathrm{E}-02$ & $2.53 \mathrm{E}-04$ & $2.34 \mathrm{E}+00$ & 1.65 & 1.26 & 1098 & $43 \%$ & $70 \%$ & 19 & 19 \\
\hline Supernatant & $0.00 \mathrm{E} \div 00$ & $0.00 \mathrm{E}+00$ & $0.00 \mathrm{E}+00$ & $0.00 \mathrm{E}+00$ & $0.00 \mathrm{E}+00$ & $0.00 \mathrm{E}+00$ & $0.00 \mathrm{E}+00$ & $0.00 \mathrm{E}+00$ & $0.00 \mathrm{E}+00$ & $0.00 \mathrm{E}+00$ & $0.00 \mathrm{E}+00$ & $0.00 \mathrm{E}+00$ & 0.00 & 0.00 & 0 & $0 \%$ & $0 \%$ & 0 & 0 \\
\hline Solid Layer & $1.70 \mathrm{E}+04$ & $3.38 \mathrm{E}+00$ & $8.5 i \mathrm{E}+03$ & $2.16 \mathrm{E}+05$ & $1.13 \mathrm{E}+05$ & $3.62 \mathrm{E}+02$ & $5.56 \mathrm{E}-01$ & $7.57 \mathrm{E}-04$ & $3.87 \mathrm{E}-03$ & $3.43 \mathrm{E}-02$ & $2.53 \mathrm{E}-04$ & $2.34 \mathrm{E}+00$ & 1.65 & 1.26 & 1098 & $43 \%$ & $70 \%$ & 19 & 19 \\
\hline 241-B-106 & $1.70 \mathrm{E}+04$ & $3.38 \mathrm{E}+00$ & $8.50 \mathrm{E}+03$ & $2.17 E+05$ & $1.13 \mathrm{E}+05$ & $3.60 \mathrm{E}+02$ & $4.88 \mathrm{E}+01$ & $9.87 \mathrm{E}-03$ & 3.12E-03 & $2.90 \mathrm{E}-02$ & $2.02 \mathrm{E}-04$ & $1.55 \mathrm{E}+01$ & 1.38 & 1.26 & 464 & $59 \%$ & $67 \%$ & 23 & 21 \\
\hline Supematant & $1.70 \mathrm{E}+04$ & $3.38 \mathrm{E}+00$ & $8.50 \mathrm{E}+03$ & $2.17 \mathrm{E}+05$ & $1.13 \mathrm{E}+05$ & $3.60 \mathrm{E}+02$ & $1.68 \mathrm{E}+00$ & $1.23 \mathrm{E}-03$ & $4.68 \mathrm{E}-04$ & $4.15 \mathrm{E}-03$ & $3.08 \mathrm{E}-05$ & $7.08 \mathrm{E}+00$ & 1.26 & 1.26 & 4 & $67 \%$ & $67 \%$ & 18 & 21 \\
\hline Solid Layer & $1.70 \mathrm{E}+04$ & $3.38 \mathrm{E}+00$ & $8.50 \mathrm{E}+03$ & $2.17 \mathrm{E}+05$ & $1.13 \mathrm{E}+05$ & $3.60 \mathrm{E}+02$ & $4.92 \mathrm{E}+01$ & $9.95 \mathrm{E}-03$ & $3.14 \mathrm{E}-03$ & $2.92 \mathrm{E}-02$ & $2.04 \mathrm{E}-04$ & $1.55 \mathrm{E}+01$ & 1.38 & 1.19 & 460 & $59 \%$ & $67 \%$ & 23 & 21 \\
\hline 241-B-107 & $1.70 \mathrm{E}+04$ & $5.04 \mathrm{E}+00$ & $6.51 \mathrm{E}+03$ & $3.31 \mathrm{E}+05$ & $1.46 \mathrm{E}+05$ & $2.01 \mathrm{E}+01$ & $3.22 \mathrm{E}+00$ & $4.59 \mathrm{E}-03$ & $4.74 \mathrm{E}-03$ & $4.03 \mathrm{E}-02$ & $3.27 \mathrm{E}-04$ & $1.10 \mathrm{E}+01$ & 1.63 & 1.34 & 611 & $40 \%$ & $58 \%$ & 16 & 20 \\
\hline Supernatant & $0.00 \mathrm{E}+00$ & $0.00 \mathrm{E}+00$ & $0.00 \mathrm{E}+00$ & $0.00 E+00$ & $0.00 \mathrm{E}+00$ & $0.00 \mathrm{E}+00$ & $0.00 \mathrm{E}+00$ & $0.00 \mathrm{E}+00$ & $0.00 \mathrm{E}+00$ & $0.00 \mathrm{E}+00$ & $0.00 \mathrm{E}+00$ & $0.00 \mathrm{E}+00$ & 0.00 & 0.00 & 0 & $0 \%$ & $0 \%$ & 0 & 0 \\
\hline Solid Layer & $1.70 E \div 04$ & $5.04 \mathrm{E}+00$ & $6.51 E+03$ & $3.31 \mathrm{E}+05$ & $1.46 \mathrm{E}+05$ & $2.01 \mathrm{E}+01$ & $3.22 \mathrm{E}+00$ & $4.59 \mathrm{E}-03$ & $4.74 \mathrm{E}-03$ & $4.03 \mathrm{E}-02$ & $3.27 \mathrm{E}-04$ & $1.10 \mathrm{E}+01$ & 1.63 & 1.34 & 611 & $40 \%$ & $58 \%$ & 16 & 20 \\
\hline
\end{tabular}




\section{RPP-5926 REV 6}

Table B-1. Input Data for Hydrogen Generation Rate Model Calculations for 177 Tanks. (20 sheets)

\begin{tabular}{|c|c|c|c|c|c|c|c|c|c|c|c|c|c|c|c|c|c|c|c|}
\hline Tank & $\begin{array}{c}\text { OH } \\
\text { in liquid } \\
{[\mathrm{OH}]} \\
(\mu \mathrm{g} / \mathrm{mL})\end{array}$ & $\begin{array}{c}\text { TOC } \\
\text { in liquid } \\
{[\mathrm{TOC}]} \\
(\mu \mathrm{g} / \mathrm{mL})\end{array}$ & $\begin{array}{c}\mathrm{NO}_{2} \\
\text { in liquid } \\
{\left[\mathrm{NO} \mathrm{O}_{2} \mid\right.} \\
(\mu \mathrm{g} / \mathrm{mL})\end{array}$ & $\begin{array}{c}\mathrm{NO}_{3} \\
\text { in liquid } \\
{\left[\mathrm{NO}_{3}\right]} \\
(\mu \mathrm{g} / \mathrm{mL})\end{array}$ & $\begin{array}{c}\mathrm{Na} \\
\text { in liquid } \\
{[\mathrm{Na}]} \\
(\mathrm{ug} / \mathrm{mL})\end{array}$ & $\begin{array}{c}\mathrm{Al} \\
\text { in liquid } \\
\mid \mathrm{Al}] \\
(\mu \mathrm{g} / \mathrm{mL})\end{array}$ & $\begin{array}{c}{ }^{90} \mathrm{Sr} \\
\text { in waste } \\
{[\mathrm{Sr}]} \\
(\mu \mathrm{Ci} / \mathrm{g})\end{array}$ & $\begin{array}{c}{ }^{241} \mathrm{Am} \\
\text { in waste } \\
{[\mathrm{Am} 241]} \\
(\mu \mathrm{Ci} / \mathrm{g})\end{array}$ & $\begin{array}{c}{ }^{240} \mathrm{Pu} \\
\text { in waste } \\
{[\mathrm{Pu} 240]} \\
(\mu \mathrm{Ci} / \mathrm{g})\end{array}$ & $\begin{array}{c}{ }^{239} \mathrm{Pu} \\
\text { in waste } \\
{\left[\begin{array}{l}\text { Pu240] } \\
(\mu \mathrm{Ci} / \mathrm{g})\end{array}\right.}\end{array}$ & $\begin{array}{c}{ }^{238} \mathrm{Pu} \\
\text { in waste } \\
{[\mathrm{Pu} 238]} \\
(\mu \mathrm{Ci} / \mathrm{g})\end{array}$ & $\begin{array}{c}{ }^{137} \mathrm{Cs} \\
\text { in waste } \\
{[\mathrm{Cs}]} \\
(\mu \mathrm{Ci} / \mathrm{g})\end{array}$ & $\begin{array}{c}\text { Bulk } \\
\text { density } \\
\text { D } \\
(\mathrm{g} / \mathrm{mL})\end{array}$ & $\begin{array}{c}\text { Liquid } \\
\text { Density } \\
\mathrm{D}_{\mathrm{L}} \\
(\mathrm{g} / \mathrm{ml})\end{array}$ & $\begin{array}{c}\text { Non-RGS } \\
\text { waste } \\
\text { volume } \\
\text { (kL) }\end{array}$ & $\begin{array}{l}\text { Bulk } \\
\text { water } \\
{\left[\mathrm{H}_{2} \mathrm{O} \mid\right.} \\
(\mathrm{wt} \%)\end{array}$ & $\begin{array}{c}\text { Liquid } \\
\text { water } \\
\left|\mathrm{H}_{2} \mathrm{O}\right| \\
(\mathrm{wt} \%)\end{array}$ & $\begin{array}{c}\text { Waste } \\
\text { temp. } \\
\mathrm{Tw} \\
\left({ }^{\circ} \mathrm{C}\right)\end{array}$ & $\begin{array}{c}\text { Dome } \\
\text { temp. } \\
\text { Td } \\
\left.\text { ( }{ }^{\circ} \mathrm{C}\right)\end{array}$ \\
\hline $241-B-108$ & $0 \mathrm{E}+04$ & $3.71 \mathrm{E}+00$ & $5.38 \mathrm{E}+04$ & $3.76 \mathrm{E}+05$ & $1.74 \mathrm{E}+05$ & $98 \mathrm{E}+02$ & $56 \mathrm{E}+00$ & $80 \mathrm{E}-04$ & 83E-04 & $3.29 \mathrm{E}-03$ & 91E-05 & $1.26 \mathrm{E}+01$ & 1.70 & 1.38 & 350 & $35 \%$ & $70 \%$ & 21 & 21 \\
\hline Supematant & $0.00 \mathrm{E}+00$ & $0.00 \mathrm{E}+00$ & $0.00 \mathrm{E}+00$ & $0.00 \mathrm{E}+00$ & $0.00 \mathrm{E}+00$ & $0.00 \mathrm{E}+00$ & $0.00 \mathrm{E}+00$ & $0.00 \mathrm{E}+00$ & $0.00 \mathrm{E}+00$ & $0.00 \mathrm{E}+00$ & $0.00 E+00$ & $0.00 \mathrm{E}+00$ & 0.00 & 0.00 & 0 & $0 \%$ & $0 \%$ & 0 & 0 \\
\hline Solid Layer & $1.70 \mathrm{E}+04$ & $3.71 E+00$ & $5.38 \mathrm{E}+04$ & $3.76 \mathrm{E}+05$ & $1.74 \mathrm{E}+05$ & $8.98 \mathrm{E}+02$ & $1.56 \mathrm{E}+00$ & $1.80 \mathrm{E}-04$ & 4.83E-04 & $3.29 \mathrm{E}-03$ & $3.91 \mathrm{E}-05$ & $1.26 \mathrm{E}+01$ & 1.70 & 1.38 & 350 & $35 \%$ & $70 \%$ & 21 & 21 \\
\hline 241 -B- 109 & $1.70 \mathrm{E}+04$ & $3.39 \mathrm{E}+00$ & $8.51 \mathrm{E}+03$ & $2.17 \mathrm{E}+05$ & $1.13 \mathrm{E}+05$ & $3.61 \mathrm{E}+02$ & $4.34 \mathrm{E}-01$ & $7.25 \mathrm{E}-04$ & 3.33E-03 & $2.47 \mathrm{E}-02$ & $2.54 \mathrm{E}-04$ & $1.49 \mathrm{E}+00$ & 1.82 & 1.26 & 475 & $39 \%$ & $70 \%$ & 20 & 21 \\
\hline Supernatant & $0.00 \mathrm{E}+00$ & $0.00 \mathrm{E}+00$ & $0.00 \mathrm{E}+00$ & $0.00 \mathrm{E}+00$ & $0.00 \mathrm{E}+00$ & $0.00 \mathrm{E}+00$ & $0.00 \mathrm{E}+00$ & $0.00 \mathrm{E}+00$ & $0.00 \mathrm{E}+00$ & $0.00 \mathrm{E}+00$ & $0.00 \mathrm{E}+00$ & $0.00 \mathrm{E}+00$ & 0.00 & 0.00 & 0 & $0 \%$ & $0 \%$ & 0 & 0 \\
\hline Solid Layer & $1.70 \mathrm{E}+04$ & $3.39 \mathrm{E}+00$ & $8.51 \mathrm{E}+03$ & $2.17 \mathrm{E}+05$ & $1.13 \mathrm{E}+05$ & $3.61 \mathrm{E}+02$ & 4.34E-01 & 7.25E-04 & $3.33 \mathrm{E}-03$ & $2.47 \mathrm{E}-02$ & $2.54 \mathrm{E}-04$ & $1.49 \mathrm{E}+00$ & 1.82 & 1.26 & 475 & $39 \%$ & $70 \%$ & 20 & 21 \\
\hline $241-B-110$ & $1.70 \mathrm{E}+04$ & $5.55 \mathrm{E}+03$ & $3.95 \mathrm{E}+04$ & $7.78 \mathrm{E}+04$ & $9.55 \mathrm{E}+04$ & $1.04 \mathrm{E}+04$ & $7.74 \mathrm{E}+01$ & $7.21 \mathrm{E}-02$ & $1.07 \mathrm{E}-02$ & $1.01 \mathrm{E}-01$ & $3.12 \mathrm{E}-03$ & $1.09 \mathrm{E}+01$ & 1.36 & 1.19 & 929 & $58 \%$ & $76 \%$ & 21 & 21 \\
\hline Supenatant & $1.70 \mathrm{E}+04$ & $5.55 \mathrm{E}+03$ & $3.95 E+04$ & $7.78 E+04$ & $9.55 \mathrm{E}+04$ & $1.04 \mathrm{E}+04$ & $1.94 \mathrm{E}+00$ & $5.67 \mathrm{E}-03$ & $9.83 \mathrm{E}-04$ & $4.24 \mathrm{E}-03$ & $1.77 \mathrm{E}-04$ & $1.18 \mathrm{E}+0 \mathrm{l}$ & 1.19 & 1.19 & 4 & $76 \%$ & $76 \%$ & 21 & 21 \\
\hline Solid Layer & $1.70 \mathrm{E}+04$ & $5.55 \mathrm{E}+03$ & $3.95 \mathrm{E}+04$ & $7.78 \mathrm{E}+04$ & $9.55 \mathrm{E}+04$ & $1.04 E+04$ & $7.77 \mathrm{E}+01$ & $7.24 \mathrm{E}-02$ & $1.08 \mathrm{E}-02$ & $1.01 \mathrm{E}-01$ & $3.13 \mathrm{E}-03$ & $1.09 \mathrm{E}+01$ & 1.36 & 1.01 & 925 & $58 \%$ & $76 \%$ & 21 & 21 \\
\hline $241 \cdot B-111$ & $1.70 \mathrm{E}+04$ & $5.55 \mathrm{E}+03$ & $3.95 \mathrm{E}+04$ & $7.78 \mathrm{E}+04$ & $9.55 \mathrm{E}+04$ & $1.04 E+04$ & $1.91 \mathrm{E}+02$ & $8.48 \mathrm{E}-02$ & $1.09 \mathrm{E}-02$ & $8.60 \mathrm{E}-02$ & $2.95 \mathrm{E}-03$ & $1.23 \mathrm{E}+02$ & 1.27 & 1.19 & 914 & $63 \%$ & $76 \%$ & 25 & 25 \\
\hline Stpematant & $1.70 \mathrm{E}+04$ & $5.55 \mathrm{E}+03$ & $3.95 E+04$ & $7.78 \mathrm{E}+04$ & $9.55 \mathrm{E}+04$ & $1.04 \mathrm{E}+04$ & $1.94 \mathrm{E}+00$ & $5.67 \mathrm{E}-03$ & $9.83 \mathrm{E}-04$ & $4.24 \mathrm{E}-03$ & $1.77 \mathrm{E}-04$ & $1.18 \mathrm{E}+01$ & 1.19 & 1.19 & 4 & $76 \%$ & $76 \%$ & 24 & 25 \\
\hline Solid Layer & $1.70 \mathrm{E}+04$ & $5.55 \mathrm{E}+03$ & $3.95 E+04$ & $7.78 \mathrm{E}+04$ & $9.55 \mathrm{E}+04$ & $1.04 \mathrm{E}+04$ & $1.92 \mathrm{E}+02$ & $8.51 \mathrm{E}-02$ & $1.09 \mathrm{E}-02$ & $8.64 \mathrm{E}-02$ & $2.96 \mathrm{E}-03$ & $1.24 \mathrm{E}+02$ & 1.27 & 1.01 & 910 & $63 \%$ & $76 \%$ & 25 & 25 \\
\hline $241-B-112$ & $10 \mathrm{E}+04$ & $1.82 \mathrm{E}+04$ & $1.17 \mathrm{E}+05$ & $8+05$ & $2.55 \mathrm{E} \div 05$ & $73 \mathrm{E}+04$ & $E+00$ & 3 & 44 & $9 \mathrm{E}-03$ & 5 & 4 & ! & 1.51 & 132 & $41 \%$ & $46 \%$ & 21 & 22 \\
\hline Supernatant & $1.70 \mathrm{E}+04$ & $1.82 \mathrm{E}+04$ & $1.17 \mathrm{E}+05$ & $2.17 \mathrm{E}+05$ & $2.55 \mathrm{E}+05$ & $4.73 \mathrm{E}+04$ & $1.53 \mathrm{E}+00$ & $4.47 \mathrm{E}-03$ & $8.01 E-04$ & $3.34 \mathrm{E}-03$ & $1.29 \mathrm{E}-04$ & $1.01 E+02$ & 1.51 & 1.51 & 11 & $46 \%$ & $46 \%$ & 21 & 22 \\
\hline Solid Layer & $1.70 \mathrm{E}+04$ & $1.82 E+04$ & $1.17 \mathrm{E}+05$ & $2.17 \mathrm{E}+05$ & $2.55 \mathrm{E}+05$ & $4.73 \mathrm{E}+04$ & $4.23 \mathrm{E}+00$ & $1.16 \mathrm{E}-03$ & $3.56 \mathrm{E}-04$ & $2.52 \mathrm{E}-03$ & $3.86 \mathrm{E}-05$ & $3.44 \mathrm{E}+01$ & 1.49 & 1.51 & 121 & $41 \%$ & $46 \%$ & 21 & 22 \\
\hline $241-B-201$ & $1.70 \mathrm{E}+04$ & $2.98 \mathrm{E}+03$ & $3.60 \mathrm{E}+04$ & $7.96 \mathrm{E}-04$ & $8.32 \mathrm{E}+04$ & $4.94 \mathrm{E}+03$ & $1.71 \mathrm{E}+00$ & $2.84 \mathrm{E}-02$ & $4.33 \mathrm{E}-02$ & $7.51 \mathrm{E}-01$ & $6.28 \mathrm{E}-03$ & $1.36 \mathrm{E}-01$ & 1.26 & 1.17 & 1 & $64 \%$ & $75 \%$ & 17 & 19 \\
\hline Supensatant & $0.00 E+00$ & $0.00 \mathrm{E}+00$ & $0.00 \mathrm{E}+00$ & $0.00 \mathrm{E}+00$ & $0.00 \mathrm{E}+00$ & $0.00 \mathrm{E}+00$ & $0.00 \mathrm{E}+00$ & $0.00 \mathrm{E}+00$ & $0.00 \mathrm{E}+00$ & $0.00 \mathrm{E}+00$ & $0.00 \mathrm{E}+00$ & $0.00 \mathrm{E}+00$ & 0.00 & 0.00 & 0 & $0 \%$ & $0 \%$ & 0 & 0 \\
\hline Solid Layer & $1.70 \mathrm{E}+04$ & $2.98 \mathrm{E}+03$ & $3.60 \mathrm{E}+04$ & $7.96 \mathrm{E}+04$ & $8.32 E+04$ & $4.94 \mathrm{E}+03$ & $1.71 \mathrm{E}+00$ & $2.84 \mathrm{E}-02$ & $4,33 \mathrm{E}-02$ & $7.51 \mathrm{E}-01$ & $6.28 \mathrm{E}-03$ & $1.36 \mathrm{E}-01$ & 1.26 & 1.17 & 111 & $64 \%$ & $75 \%$ & 17 & 19 \\
\hline $241-B-202$ & $1.70 \mathrm{E}+04$ & $2.98 \mathrm{E}+03$ & $3.60 \mathrm{E}+04$ & $7.96 \mathrm{E}+04$ & $8.32 \mathrm{E}+04$ & $4.94 \mathrm{E}+03$ & $3.00 \mathrm{E}+00$ & $6.67 \mathrm{E}-02$ & $2.17 \mathrm{E}-02$ & $1.28 \mathrm{E}-01$ & $2.03 \mathrm{E}-03$ & $7.67 \mathrm{E}-02$ & 1.2 & 1.17 & 108 & $76 \%$ & $83 \%$ & 17 & 19 \\
\hline Supernatant & $0.00 \mathrm{E}+00$ & $0.00 \mathrm{E}+00$ & $0.00 \mathrm{E}+00$ & $0.00 \mathrm{E}+00$ & $0.00 \mathrm{E} \div 00$ & $0.00 \mathrm{E}+00$ & $0.00 \mathrm{E}+00$ & $0.00 \mathrm{E}+00$ & $0.00 \mathrm{E}+00$ & $0.00 \mathrm{E}+00$ & $0.00 \mathrm{E}+00$ & $0.00 \mathrm{E}+00$ & 0.00 & 0.00 & 0 & $0 \%$ & $0 \%$ & 0 & 0 \\
\hline Solid Layer & $1.70 \mathrm{E}+04$ & $2.98 \varepsilon+03$ & $3.60 \mathrm{E}+04$ & $7.96 \mathrm{E}+04$ & $8.32 \mathrm{E}+04$ & $4.94 E+03$ & $3.00 \mathrm{E}+00$ & $6.67 \mathrm{E}-02$ & $2.17 \mathrm{E}-02$ & $1.28 \mathrm{E}-01$ & $2.03 \mathrm{E}-03$ & $7.67 \mathrm{E}-02$ & 1.22 & 1.17 & 108 & $76 \%$ & $83 \%$ & 17 & 19 \\
\hline $241-B-203$ & $1.70 \mathrm{E}+04$ & $9.60 \mathrm{E}+01$ & $1.57 \mathrm{E}+03$ & $5.65 \mathrm{E}+04$ & $3.12 \mathrm{E}+04$ & $0.00 \mathrm{E}+00$ & $6.86 \mathrm{E}-02$ & $3.42 \mathrm{E}-02$ & $2.92 \mathrm{E}-02$ & $2.31 \mathrm{E}-01$ & $2.00 \mathrm{E}-03$ & $6.55 \mathrm{E}-03$ & 1.19 & 1.05 & 190 & $76 \%$ & $89 \%$ & 18 & 12 \\
\hline Supematant & $1.70 \mathrm{E}+04$ & $9.60 \mathrm{E}+01$ & $1.57 \mathrm{E}+03$ & $5.65 \mathrm{E}+04$ & $3.12 \mathrm{E}+04$ & $0.00 \mathrm{E}+00$ & $7.76 \mathrm{E}-05$ & $4.07 \mathrm{E}-05$ & $4.86 \mathrm{E}-04$ & $3.83 \mathrm{E}-03$ & $3.31 \mathrm{E}-05$ & $9.00 \mathrm{E}-05$ & 1.05 & 1.05 & 2 & $89 \%$ & $89 \%$ & 22 & 12 \\
\hline Solid Layer & 1. $70 \mathrm{E}+04$ & $9.60 \mathrm{E}+01$ & $1.57 \mathrm{E}+03$ & $5.65 \mathrm{E}+04$ & $3.12 \mathrm{E}+04$ & $0.00 \mathrm{E}+00$ & $6.93 \mathrm{E}-02$ & $3.46 \mathrm{E}-02$ & $2.95 \mathrm{E}-02$ & $2.33 \mathrm{E}-01$ & $2.02 \mathrm{E}-03$ & $6.62 \mathrm{E}-03$ & 1.19 & 1.05 & 188 & $76 \%$ & $89 \%$ & 18 & 12 \\
\hline $241-B-204$ & $1.70 \mathrm{E}+04$ & $7.53 \mathrm{E}+01$ & $1.36 \mathrm{E}+03$ & $3.47 \mathrm{E}+04$ & $2.78 \mathrm{E}+04$ & $0.00 \mathrm{E}+00$ & $2.58 \mathrm{E}-03$ & $3.78 \mathrm{E}-02$ & $2.47 \mathrm{E}-02$ & $1.96 \mathrm{E}-01$ & $1.69 \mathrm{E}-03$ & $2.57 \mathrm{E}-02$ & 1.19 & 1.05 & 187 & $77 \%$ & $91 \%$ & 18 & 22 \\
\hline Supernatant & $1.70 \mathrm{E}+04$ & $7.53 \mathrm{E}+01$ & $1.36 \mathrm{E}+03$ & $3.47 \mathrm{E}+04$ & $2.78 \mathrm{E}+04$ & $0.00 \mathrm{E}+00$ & $3.46 \mathrm{E}-05$ & $3.56 \mathrm{E}-07$ & $4.70 \mathrm{E}-06$ & $6.98 \mathrm{E}-05$ & $2.36 \mathrm{E}-07$ & $3.97 \mathrm{E}-05$ & 1.05 & 1.05 & 3 & $91 \%$ & $91 \%$ & 19 & 22 \\
\hline Solid Layer & $1.70 \mathrm{E}+04$ & $7.53 \mathrm{E}+0 \mathrm{f}$ & $1.36 \mathrm{E} \div 03$ & $3.47 \mathrm{E}+04$ & $2.78 \mathrm{E}+04$ & $0.00 \mathrm{E}+00$ & $2.62 \mathrm{E}-03$ & 3.84E-02 & $2.51 \mathrm{E}-02$ & โ. $99 \mathrm{E}-01$ & $1.72 \mathrm{E}-03$ & $2.62 \mathrm{E}-02$ & 1.19 & 1.05 & 184 & $77 \%$ & $91 \%$ & 18 & 22 \\
\hline
\end{tabular}




\section{RPP-5926 REV 6}

Table B-1. Input Data for Hydrogen Generation Rate Model Calculations for 177 Tanks. (20 sheets)

\begin{tabular}{|c|c|c|c|c|c|c|c|c|c|c|c|c|c|c|c|c|c|c|c|}
\hline Tank & $\begin{array}{c}\text { OH } \\
\text { in liquid } \\
\text { jOH] } \\
(\mu \mathrm{g} / \mathrm{mL})\end{array}$ & $\begin{array}{c}\text { TOC } \\
\text { in liquid } \\
\text { |TOC] } \\
(\mu \mathrm{g} / \mathrm{mL})\end{array}$ & $\begin{array}{c}\mathrm{NO}_{2} \\
\text { in liquid } \\
{\left[\mathrm{NO}_{2}\right]} \\
(\mu \mathrm{g} / \mathrm{mL})\end{array}$ & $\begin{array}{c}\mathrm{NO}_{3} \\
\text { in liquid } \\
{\left[\mathrm{NO}_{3}\right]} \\
(\mu \mathrm{g} / \mathrm{mL})\end{array}$ & $\begin{array}{c}\mathrm{Na} \\
\text { in liquid } \\
{[\mathrm{Na} \mid} \\
(\mathrm{ug} / \mathrm{mL})\end{array}$ & $\begin{array}{c}\text { Al } \\
\text { in liquid } \\
{[A \mid]} \\
(\mu \mathrm{g} / \mathrm{mL})\end{array}$ & $\begin{array}{c}{ }^{90} \mathrm{Sr} \\
\text { in waste } \\
{[\mathrm{Sr}]} \\
(\mu \mathrm{Ci} / \mathrm{g})\end{array}$ & $\begin{array}{c}{ }^{241} \mathrm{Am} \\
\text { in waste } \\
\mid \mathrm{Am} 241\} \\
(\mu \mathrm{Ci} / \mathrm{g})\end{array}$ & $\begin{array}{c}{ }^{240} \mathrm{Pu} \\
\text { in waste } \\
{[\mathrm{Pu} 240]} \\
(\mu \mathrm{Ci} / \mathrm{g})\end{array}$ & $\begin{array}{c}{ }^{239} \mathrm{Pu} \\
\text { in waste } \\
{[\mathrm{Pu} 240]} \\
\left({ }^{2} \mathrm{Ci} / \mathrm{g}\right)\end{array}$ & $\begin{array}{c}{ }^{238} \mathrm{Pu} \\
\text { in waste } \\
|\mathrm{Pu} 238| \\
(\mu \mathrm{Ci} / \mathrm{g})\end{array}$ & $\begin{array}{c}{ }^{137} \mathrm{Cs} \\
\text { in waste } \\
{[\mathrm{Cs}]} \\
(\mu \mathrm{Ci} / \mathrm{g})\end{array}$ & $\begin{array}{c}\text { Bulk } \\
\text { density } \\
D \\
(\mathrm{~g} / \mathrm{mL})\end{array}$ & $\begin{array}{c}\text { Liquid } \\
\text { Density } \\
\mathrm{D}_{\mathrm{L}} \\
(\mathrm{g} / \mathrm{ml})\end{array}$ & $\begin{array}{c}\text { Non-RGS } \\
\text { waste } \\
\text { volume } \\
(\mathbf{k L})\end{array}$ & $\begin{array}{l}\text { Bulk } \\
\text { water } \\
{\left[\mathrm{H}_{2} \mathrm{O}\right]} \\
(\mathbf{w 1} \%)\end{array}$ & $\begin{array}{c}\text { Liquid } \\
\text { water } \\
{\left[\mathrm{H}_{2} \mathrm{O}\right]} \\
(\mathrm{wt} \%)\end{array}$ & $\begin{array}{l}\text { Waste } \\
\text { temp. } \\
\text { Tw } \\
\left({ }^{\circ} \mathrm{C}\right)\end{array}$ & $\begin{array}{c}\text { Dome } \\
\text { temp. } \\
\text { Td } \\
\left.{ }^{\circ} \mathrm{C}\right)\end{array}$ \\
\hline 241-BX-101 & $0 \mathrm{E}+04$ & $4.98 \mathrm{E}+03$ & $3 \mathrm{E}+04$ & $21 \mathrm{E}+05$ & $42 E+05$ & $17 E+03$ & $55 \mathrm{E}+02$ & $1.37 \mathrm{E}-01$ & $1.32 \mathrm{E}-01$ & $6.39 \mathrm{E}-01$ & $2.11 \mathrm{E}-02$ & $9.08 \mathrm{E}+01$ & 1.68 & 1.28 & 180 & $16 \%$ & $75 \%$ & 23 & 22 \\
\hline Supenatant & $0.00 \mathrm{E}+00$ & $0.00 \mathrm{E}+00$ & $0.00 \mathrm{E}+00$ & $0.00 \mathrm{E}+00$ & $0.00 \mathrm{E}+00$ & $0.00 \mathrm{E}+00$ & $0.00 E+00$ & $0.00 \mathrm{E}+00$ & $0.00 \mathrm{E}+00$ & $0.00 \mathrm{E}+00$ & $0.00 \mathrm{E} \div 00$ & $0.00 \mathrm{E}+00$ & 0.00 & 0.00 & 0 & $0 \%$ & $0 \%$ & 0 & 0 \\
\hline Solid Layer & $1.70 \mathrm{E}+04$ & $4.98 \mathrm{E}+03$ & $6.43 E+04$ & $1.21 \mathrm{E}+05$ & $1.42 \mathrm{E}+05$ & $5.17 \mathrm{E}+03$ & $2.55 \mathrm{E}+02$ & $1.37 \mathrm{E}-01$ & $1.32 \mathrm{E}-01$ & $6.39 \mathrm{E}-0 \mathrm{]}$ & $2.11 \mathrm{E}-02$ & $9.08 \mathrm{E}+01$ & 1.68 & 1.28 & 180 & $16 \%$ & $75 \%$ & 23 & 22 \\
\hline 241-BX-102 & $1.70 \mathrm{E}+04$ & $2.98 \mathrm{E}+03$ & $3.60 \mathrm{E} \div 04$ & $7.96 \mathrm{E}+04$ & $8.32 \mathrm{E}+04$ & $4.94 \mathrm{E}+03$ & $3.84 \mathrm{E}+01$ & $3.93 \mathrm{E}-03$ & $3.97 \mathrm{E}-03$ & $1.89 \mathrm{E}-02$ & $3.83 \mathrm{E}-04$ & $4.31 \mathrm{E}+00$ & 0.99 & 1.17 & 298 & $36 \%$ & $75 \%$ & 20 & 21 \\
\hline Supernatant & $0.00 \mathrm{E}+00$ & $0.00 \mathrm{E}+00$ & $0.00 \mathrm{E}+00$ & $0.00 \mathrm{E}+00$ & $0.00 \mathrm{E}+00$ & $0.00 \mathrm{E}+00$ & $0.00 \mathrm{E}+00$ & $0.00 \mathrm{E}+00$ & $0.00 \mathrm{E}+00$ & $0.00 \mathrm{E}+00$ & $0.00 \mathrm{E}+00$ & $0.00 \mathrm{E}+00$ & 0.00 & 0,00 & 0 & $0 \%$ & $0 \%$ & 0 & 0 \\
\hline Solid Layer & $1.70 \mathrm{E}+04$ & $2.98 \mathrm{E}+03$ & $3.60 \mathrm{E}+04$ & $7.96 \mathrm{E}+04$ & $8.32 \mathrm{E}+04$ & $4.94 \mathrm{E}+03$ & $3.84 \mathrm{E}+01$ & $3.93 \mathrm{E}-03$ & $3.97 \mathrm{E}-03$ & $1.89 \mathrm{E}-02$ & $3.83 \mathrm{E}-04$ & $4.3 ! \mathrm{E}+00$ & 0.99 & 1.17 & 298 & $36 \%$ & $75 \%$ & 20 & 21 \\
\hline $241-\mathrm{BX}-103$ & $1.70 \mathrm{E}+04$ & $3.79 \mathrm{E}+03$ & $42 \mathrm{E}+04$ & $63 E+04$ & $04 E+04$ & $84 E+04$ & $7.64 \mathrm{E}+00$ & $2.74 \mathrm{E}-01$ & $4.10 \mathrm{E}-0 \mathrm{l}$ & $1.81 \mathrm{E}+00$ & $4.05 \mathrm{E}-02$ & $1.14 \mathrm{E}+00$ & 1.56 & 1.07 & 283 & $53 \%$ & $78 \%$ & 21 & 21 \\
\hline Supernatant & $1.70 \mathrm{E}+04$ & $3.79 E+03$ & $1.42 \mathrm{E}+04$ & $63 \mathrm{E}+04$ & $4.04 E+04$ & $84 \mathrm{E}+04$ & $5.08 \mathrm{E}-01$ & $5.65 \mathrm{E}-03$ & $1.21 \mathrm{E}-03$ & $5.12 \mathrm{E}-03$ & $1.21 \mathrm{E}-04$ & $5.98 \mathrm{E}-01$ & 1.07 & 1.07 & 48 & $78 \%$ & $78 \%$ & 21 & 21 \\
\hline Solid Layer & $1.70 \mathrm{E}+04$ & $3.79 \mathrm{E}+03$ & $1.42 \mathrm{E}+04$ & $4.63 \mathrm{E}+04$ & $4.04 \mathrm{E}+04$ & $1.84 \mathrm{E}+04$ & $9.10 \mathrm{E}+00$ & $3.29 \mathrm{E}-01$ & $4.94 \mathrm{E}-01$ & $2.18 E+00$ & $4.88 \mathrm{E}-02$ & $1.26 \mathrm{E}+00$ & 1.66 & 1.11 & 235 & $50 \%$ & $78 \%$ & 21 & 21 \\
\hline 241-BX-104 & $0 \mathrm{E}+04$ & $19 \mathrm{E}+03$ & $7 \mathrm{E}+04$ & $34 \mathrm{E}+05$ & $40 \mathrm{E}+05$ & $84 E+03$ & $88 E+02$ & $5.21 \mathrm{E}-01$ & $4.38 \mathrm{E}-02$ & $3.39 \mathrm{E}-01$ & $3.36 \mathrm{E}-03$ & $6.17 \mathrm{E}+01$ & 1.67 & 1.28 & 380 & $28 \%$ & $59 \%$ & 30 & 25 \\
\hline Supernatant & $1.70 \mathrm{E}+04$ & $3.19 \mathrm{E}+03$ & $6.37 \mathrm{E}+04$ & $1.34 \mathrm{E}+05$ & $1.40 \mathrm{E}+05$ & $2.84 \mathrm{E}+03$ & $1.32 E+00$ & $2.10 \mathrm{E}-04$ & $3.19 \mathrm{E}-05$ & $1.68 \mathrm{E}-04$ & $2.61 \mathrm{E}-06$ & $9.66 \mathrm{E}+01$ & 1.28 & 1.28 & 11 & $59 \%$ & $59 \%$ & 30 & 25 \\
\hline Solid Layer & $1.70 \mathrm{E}+04$ & $3.19 \mathrm{E}+03$ & $6.37 \mathrm{E}+04$ & $1.34 \mathrm{E}+05$ & $1.40 \mathrm{E}+05$ & $2.84 \mathrm{E}+03$ & $1.94 \mathrm{E}+02$ & $5.37 \mathrm{E}-0 \mathrm{I}$ & $4.5 ! \mathrm{E}-02$ & $3.49 E-01$ & $3.46 \mathrm{E}-03$ & $6.07 \mathrm{E}+01$ & 1.68 & 1.28 & 369 & $27 \%$ & $59 \%$ & 30 & 25 \\
\hline $241-B X-105$ & $1.70 E+04$ & $9.44 E+03$ & $4.00 E+04$ & $E+05$ & $24 E+05$ & $34 E+03$ & $71 \mathrm{E}+02$ & $5.63 E-02$ & $1.81 \mathrm{E}-02$ & $9.07 \mathrm{E}-02$ & $1.93 \mathrm{E}-03$ & $7.45 \mathrm{E}+01$ & 1.67 & 1.29 & 272 & $17 \%$ & $69 \%$ & 20 & 21 \\
\hline Supernatant & $1.70 \mathrm{E}+04$ & $9.44 \mathrm{E}+03$ & $4.00 \mathrm{E}+04$ & $1.17 \mathrm{E}+05$ & $1.24 \mathrm{E}+05$ & $5.94 E+03$ & $5.81 \mathrm{E}+00$ & $1.91 \mathrm{E}-02$ & $2.53 \mathrm{E}-03$ & $1.08 \mathrm{E}-02$ & $2.54 \mathrm{E}-04$ & $9.22 \mathrm{E}+01$ & 1.29 & 1.29 & 18 & $69 \%$ & $69 \%$ & 20 & 21 \\
\hline Solid Layer & $1.70 \mathrm{E}+04$ & $9.44 \mathrm{E}+03$ & $4.00 \mathrm{E}+04$ & i. $17 \mathrm{E}+05$ & $1.24 \mathrm{E}+05$ & $5.94 \mathrm{E}+03$ & $1.83 \mathrm{E}+02$ & $5.90 \mathrm{E}-02$ & $1.92 \mathrm{E}-02$ & $9.64 \mathrm{E}-02$ & $2.05 \mathrm{E}-03$ & $7.32 \mathrm{E}+01$ & 1.69 & 1.29 & 254 & $14 \%$ & $69 \%$ & 20 & 21 \\
\hline 241-B & 04 & $1.82 \mathrm{E}+04$ & +05 & +05 & +05 & $72 \mathrm{E}+04$ & $54 \mathrm{E}+01$ & $2.50 \mathrm{E}-01$ & 4.01E-02 & $1,96 \mathrm{E}-01$ & $5.18 \mathrm{E}-03$ & $87 E+01$ & 1.62 & 1.17 & 143 & $40 \%$ & $46 \%$ & 20 & 20 \\
\hline Supernatant & $0.00 \mathrm{E}+00$ & $0.00 \mathrm{E}+00$ & $0.00 \mathrm{E}+00$ & $0.00 \mathrm{E}+00$ & $0.00 \mathrm{E}+00$ & $0.00 \mathrm{E}+00$ & $0.00 E+00$ & $0.00 \mathrm{E}+00$ & $0.00 \mathrm{E}+00$ & $0.00 \mathrm{E}+00$ & $0.00 \mathrm{E}+00$ & $0.00 \mathrm{E}+00$ & 0.00 & 0.00 & 0 & $0 \%$ & $0 \%$ & 0 & 0 \\
\hline Solid Layer & $1.70 E+04$ & $1.82 \mathrm{E}+04$ & $1.17 \mathrm{E}+05$ & $2.17 \mathrm{E}+05$ & $2.55 \mathrm{E}+05$ & $4.72 \mathrm{E}+04$ & $2.54 \mathrm{E}+01$ & $2.50 \mathrm{E}-01$ & $4.01 \mathrm{E}-02$ & $1.96 \mathrm{E}-0 \mathrm{~J}$ & $5.18 \mathrm{E}-03$ & $4.87 \mathrm{E}+01$ & 1.62 & 1.51 & 143 & $40 \%$ & $46 \%$ & 20 & 20 \\
\hline $241-B X-107$ & $1.70 \mathrm{E}+04$ & $2.98 \mathrm{E}+03$ & $3.60 E+04$ & $7.96 \mathrm{E}+04$ & $8.32 E+04$ & $4.94 \mathrm{E}+03$ & $7.51 \mathrm{E}+00$ & $1.30 \mathrm{E}-02$ & $5.61 \mathrm{E}-03$ & $5.16 \mathrm{E}-02$ & $3.63 \mathrm{E}-04$ & $1.34 \mathrm{E}+01$ & 1.44 & 1.17 & 1313 & $51 \%$ & $75 \%$ & 19 & 21 \\
\hline Supernatant & $0.00 \mathrm{E}+00$ & $0.00 \mathrm{E}+00$ & $0.00 \mathrm{E}+00$ & $0.00 \mathrm{E}+00$ & $0.00 E+00$ & $0.00 \bar{E}+00$ & $0.00 E+00$ & $0.00 \mathrm{E}+00$ & $0.00 \mathrm{E}+00$ & $0.00 \mathrm{E}+00$ & $0.00 \mathrm{E}+00$ & $0.00 \mathrm{E}+00$ & 0.00 & 0.00 & 0 & $0 \%$ & $0 \%$ & 0 & 0 \\
\hline Solid Layer & $1.70 \mathrm{E}+04$ & $2.98 \mathrm{E}+03$ & $50 \mathrm{E}+04$ & $7.96 \mathrm{E}+04$ & $8.32 \mathrm{E} \div 04$ & $4.94 \mathrm{E}+03$ & $7.51 \mathrm{E}+00$ & $1.30 \mathrm{E}-02$ & $5.61 \mathrm{E}-03$ & $5.16 \mathrm{E}-02$ & $3.63 \mathrm{E}-04$ & $1.34 \mathrm{E}+01$ & 1.44 & 1.17 & 1313 & $51 \%$ & $75 \%$ & 19 & 21 \\
\hline $241 \cdot \mathrm{BX}-108$ & $1.70 \mathrm{E}+04$ & $2.98 \mathrm{E}+03$ & $3.60 \mathrm{E}+04$ & $7.96 \mathrm{E}+04$ & $8.32 \mathrm{E}+04$ & $4.94 \mathrm{E}+03$ & $1.29 \mathrm{E}+02$ & $1.87 \mathrm{E}-02$ & $5.16 \mathrm{E}-03$ & $4.79 \mathrm{E}-02$ & $3.34 \mathrm{E}-04$ & $2.26 \mathrm{E}+01$ & 1.46 & 1.17 & 119 & $17 \%$ & $75 \%$ & 20 & 20 \\
\hline Supernatant & $0.00 \mathrm{E}+00$ & $0.00 \mathrm{E}+00$ & $0.00 \mathrm{E}+00$ & $0.00 \mathrm{E}+00$ & $0.00 \mathrm{E}+00$ & $0.00 \mathrm{E}+00$ & $0.00 \mathrm{E}+00$ & $0.00 \mathrm{E}+00$ & $0.00 \mathrm{E}+00$ & $0.00 \mathrm{E}+00$ & $0.00 \mathrm{E}+00$ & $0.00 \mathrm{E}+00$ & 0.00 & 0.00 & 0 & $0 \%$ & $0 \%$ & 0 & 0 \\
\hline Solid Layer & $1.70 \mathrm{E}+04$ & $2.98 \mathrm{E}+03$ & $3.60 \mathrm{E}+04$ & $7.96 \mathrm{E}+04$ & $8.32 \mathrm{E}+04$ & $4.94 \mathrm{E}+03$ & $1.29 \mathrm{E}+02$ & $1.87 \mathrm{E}-02$ & $5.16 \mathrm{E}-03$ & $4.79 \mathrm{E}-02$ & $3.34 \mathrm{E}-04$ & $2.26 \mathrm{E}+01$ & 1.46 & 1.17 & 119 & $17 \%$ & $75 \%$ & 20 & 20 \\
\hline 241-BX-109 & $1.70 \mathrm{E}+04$ & $2.98 \mathrm{E}+03$ & $3.60 \mathrm{E}+04$ & $7.96 \mathrm{E}+04$ & $8.32 E+04$ & $4.94 \mathrm{E}+03$ & $1.07 \mathrm{E}+02$ & $5.36 \mathrm{E}-03$ & $1.05 \mathrm{E}-03$ & $9.73 \mathrm{E}-03$ & $6.79 \mathrm{E}-05$ & $1.08 \mathrm{E}+01$ & 1.52 & 1.17 & 730 & $51 \%$ & $72 \%$ & 21 & 22 \\
\hline Supernatant & $0.00 \mathrm{E} \div 00$ & $0.00 \mathrm{E}+00$ & $0.00 \mathrm{E}+00$ & $0.00 \mathrm{E}+00$ & $0.00 \mathrm{E}+00$ & $0.00 \mathrm{E}+00$ & $0.00 \mathrm{E}+00$ & $0.00 \mathrm{E}+00$ & $0.00 \mathrm{E}+00$ & $0.00 \mathrm{E}+00$ & $0.00 \mathrm{E}+00$ & 00 & 0.00 & 0.00 & 0 & $\%$ & $\%$ & 0 & 0 \\
\hline Solid Layet & $1.70 \mathrm{E}+04$ & $2.98 \mathrm{E}+03$ & $3.60 \mathrm{E}+04$ & $7.96 \mathrm{E}+04$ & $8.32 \mathrm{E}+04$ & $4.94 \mathrm{E}+03$ & $1.07 \mathrm{E}+02$ & $5.36 \mathrm{E}-03$ & $1.05 \mathrm{E}-03$ & $9.73 \mathrm{E}-03$ & $6.79 \mathrm{E}-05$ & $1.08 \mathrm{E}+01$ & 1.52 & 1.17 & 730 & $51 \%$ & $72 \%$ & 21 & 22 \\
\hline
\end{tabular}




\section{RPP-5926 REV 6}

Table B-1. Input Data for Hydrogen Generation Rate Model Calculations for 177 Tanks. (20 sheets)

\begin{tabular}{|c|c|c|c|c|c|c|c|c|c|c|c|c|c|c|c|c|c|c|c|}
\hline Tank & $\begin{array}{c}\mathrm{OH} \\
\text { in liquid } \\
{[\mathrm{OH} \mid} \\
(\mu \mathrm{g} / \mathrm{mL})\end{array}$ & $\begin{array}{l}\text { TOC } \\
\text { in liquid } \\
\text { [TOC] } \\
(\mu \mathrm{g} / \mathrm{mL})\end{array}$ & $\begin{array}{c}\mathrm{NO}_{2} \\
\text { in liquid } \\
\left|\mathrm{NO}_{2}\right| \\
(\mu \mathrm{g} / \mathrm{mL})\end{array}$ & $\begin{array}{c}\mathrm{NO}_{3} \\
\text { in liquid } \\
{\left[\mathrm{NO}_{3}\right]} \\
(\mu \mathrm{g} / \mathrm{mL})\end{array}$ & $\begin{array}{c}\mathrm{Na} \\
\text { in liquid } \\
{[\mathrm{Na}]} \\
\text { (ug/mL) }\end{array}$ & $\begin{array}{c}\mathrm{A}] \\
\text { in liquid } \\
{[\mathrm{AI}]} \\
(\mu \mathrm{g} / \mathrm{mL})\end{array}$ & $\begin{array}{c}{ }^{90} \mathrm{Sr} \\
\text { in waste } \\
{[\mathrm{Sr}]} \\
(\mu \mathrm{Ci} / \mathrm{g})\end{array}$ & $\begin{array}{c}{ }^{241} \mathrm{Am} \\
\text { in waste } \\
{[\mathrm{Am} 241]} \\
(\mu \mathrm{Ci} / \mathrm{g})\end{array}$ & $\begin{array}{c}{ }^{240} \mathrm{Pu} \\
\text { in waste } \\
{\left[\begin{array}{l}\text { Pu240] } \\
(\mu \mathrm{Ci} / \mathrm{g})\end{array}\right.}\end{array}$ & $\begin{array}{c}{ }^{239} \mathrm{Pu} \\
\text { in waste } \\
|\mathrm{Pu} 240| \\
(\mu \mathrm{Ci} / \mathrm{g})\end{array}$ & $\begin{array}{c}{ }^{238} \mathrm{Pu} \\
\text { in waste } \\
{[\mathrm{Pu} 238]} \\
(\mu \mathrm{Ci} / \mathrm{g})\end{array}$ & $\begin{array}{c}{ }^{337} \mathrm{Cs} \\
\text { in waste } \\
{[\mathrm{Cs}]} \\
(\mu \mathrm{Cl} / \mathrm{g})\end{array}$ & $\begin{array}{c}\text { Bulk } \\
\text { density } \\
\text { D } \\
(\mathrm{g} / \mathrm{mL})\end{array}$ & $\begin{array}{c}\text { Liquid } \\
\text { Density } \\
D_{\mathrm{L}} \\
(\mathrm{g} / \mathrm{ml})\end{array}$ & $\begin{array}{c}\text { Non-RGS } \\
\text { waste } \\
\text { volume } \\
\text { (kL) }\end{array}$ & $\begin{array}{l}\text { Bulk } \\
\text { water } \\
{\left[\mathrm{H}_{2} \mathrm{O}\right]} \\
(\mathrm{wt} \%)\end{array}$ & $\begin{array}{c}\text { Liquid } \\
\text { water } \\
{\left[\mathrm{H}_{2} \mathrm{O}\right]} \\
(w t \%)\end{array}$ & $\begin{array}{c}\text { Waste } \\
\text { temp. } \\
\mathrm{Tw}_{w} \\
\left({ }^{\circ} \mathrm{C}\right)\end{array}$ & $\begin{array}{l}\text { Dome } \\
\text { temp. } \\
\mathrm{Td} \\
\left(^{\circ} \mathrm{C}\right)\end{array}$ \\
\hline $241-B X-110$ & $70 \mathrm{E}+04$ & $1.52 \mathrm{E}+04$ & $21 E+04$ & $12 E+05$ & $23 \mathrm{E}+05$ & $4.40 \mathrm{E}+03$ & $82 \mathrm{E}+00$ & $4.73 \mathrm{E}-03$ & $1.80 \mathrm{E}-03$ & $1.51 \mathrm{E}-02$ & $1.45 \mathrm{E}-04$ & $4.93 \mathrm{E}+01$ & 1.67 & 1.44 & 812 & $41 \%$ & $53 \%$ & 20 & 20 \\
\hline Supematant & $1.70 \mathrm{E}+04$ & $1.52 \mathrm{E}+04$ & $5.22 \mathrm{E}+04$ & 4. $12 \mathrm{E}+05$ & $2.24 \mathrm{E}+05$ & $4.40 \mathrm{E}+03$ & $1.33 \mathrm{E}+00$ & $3.90 \mathrm{E}-03$ & $7.00 \mathrm{E}-04$ & $2.92 \mathrm{E}-03$ & $1.12 \mathrm{E}-04$ & $8.85 \mathrm{E}+01$ & 1.44 & 1.44 & 5 & $53 \%$ & $53 \%$ & 20 & 20 \\
\hline Solid Layer & $1.70 \mathrm{E}+04$ & $1.52 \mathrm{E}+04$ & $5.21 \mathrm{E}+04$ & 4. $12 \mathrm{E}+05$ & $2.23 E+05$ & $4.40 \mathrm{E}+03$ & $7.86 \mathrm{E}+00$ & 4.73E-03 & $1.81 \mathrm{E}-03$ & $1.52 \mathrm{E}-02$ & $1.45 \mathrm{E}-04$ & $4.90 \mathrm{E}+0 \mathrm{t}$ & 1.67 & 1.44 & 807 & $41 \%$ & $53 \%$ & 20 & 20 \\
\hline $241-\mathrm{BX}-111$ & $1.70 \mathrm{E}+04$ & $1.70 \mathrm{E}+03$ & $1.06 \mathrm{E}+05$ & $1.85 \mathrm{E}+05$ & $2.26 \mathrm{E}+05$ & $4.87 \mathrm{E}+04$ & $1.15 \mathrm{E}+01$ & $3.43 \mathrm{E}-03$ & $1.64 \mathrm{E}-03$ & $1.45 \mathrm{E}-02$ & 1.17E-04 & $6.47 E+01$ & 1.45 & 1.45 & 713 & $25 \%$ & $51 \%$ & 20 & 19 \\
\hline Supernatant & $0.00 \mathrm{E}+00$ & $0.00 \mathrm{E}+00$ & $0.00 \mathrm{E}+00$ & $0.00 E+00$ & $0.00 E+00$ & $0.00 \mathrm{E}+00$ & $0.00 \mathrm{E}+00$ & $0.00 \mathrm{E}+00$ & $0.00 \mathrm{E}+00$ & $0.00 \mathrm{E}+00$ & $0.00 \mathrm{E}+00$ & $0.00 \mathrm{E}+00$ & 0.00 & 0.00 & 0 & $0 \%$ & $0 \%$ & 0 & 0 \\
\hline Solid Layer & $1.70 \mathrm{E}+04$ & $1.70 \mathrm{E}+03$ & $1.06 \mathrm{E}+05$ & $1.85 \mathrm{E}+05$ & $2.26 \mathrm{E}+05$ & $4.87 \mathrm{E}+04$ & $1.15 \mathrm{E}+01$ & $3.43 \mathrm{E}-03$ & $1.64 \mathrm{E}-03$ & $1.45 \mathrm{E}-02$ & $1.17 \mathrm{E}-04$ & $6.47 \mathrm{E}+01$ & 1.45 & 1.45 & 713 & $25 \%$ & $51 \%$ & 20 & 19 \\
\hline $241-\mathrm{BX}-112$ & $1.70 \mathrm{E}+04$ & $0.00 \mathrm{E}+00$ & $3.52 \mathrm{E}+04$ & $9.86 \mathrm{E}+04$ & $7.78 \mathrm{E}+04$ & $4.78 \mathrm{E}+01$ & $5.77 \mathrm{E}+00$ & $2.39 \mathrm{E}-02$ & $1.57 \mathrm{E}-02$ & $1.44 \mathrm{E}-01$ & $1.01 \mathrm{E}-03$ & $4.03 \mathrm{E}+01$ & 1.31 & 1.18 & 622 & $63 \%$ & $68 \%$ & 20 & 19 \\
\hline Supernatant & $1.70 \mathrm{E}+04$ & $0.00 \mathrm{E}+00$ & $3.52 \mathrm{E}+04$ & $9.86 \mathrm{E}+04$ & $7.78 \mathrm{E}+04$ & $4.78 \mathrm{E}+01$ & $6.22 \mathrm{E}-01$ & $1.37 \mathrm{E}-04$ & $1.92 \mathrm{E}-04$ & $1.35 \mathrm{E}-03$ & $1.34 \mathrm{E}-05$ & $7.31 \mathrm{E}-01$ & 1.18 & 1.18 & 5 & $68 \%$ & $68 \%$ & 20 & 19 \\
\hline Solid Layer & $1.70 \mathrm{E}+04$ & $0.00 \bar{E}+00$ & $3.52 \mathrm{E}+04$ & $9.86 \mathrm{E}+04$ & $7.78 \mathrm{E}+04$ & $4.78 \mathrm{E}+01$ & $5.81 \mathrm{E} \div 00$ & $2.41 \mathrm{E}-02$ & $1.58 \mathrm{E}-02$ & $1.45 \mathrm{E}-01$ & $1.02 \mathrm{E}-03$ & $4.06 \mathrm{E}+01$ & 1.31 & 1.18 & 617 & $63 \%$ & $68 \%$ & 20 & 19 \\
\hline $241-\mathrm{BY}-10 \mathrm{t}$ & $3.83 \mathrm{E}+04$ & $1.82 \mathrm{E}+04$ & $1.17 \mathrm{E}+05$ & 2175605 & $2.56 \mathrm{E}+05$ & $473 F+04$ & $738 \mathrm{~F}+01$ & $4.82 \mathrm{E}-02$ & $7.14 \mathrm{E}-04$ & $5.69 \mathrm{E}-03$ & $6.08 \mathrm{E}-05$ & $1.19 \mathrm{E}+02$ & 1.83 & 1.51 & 1400 & $23 \%$ & $46 \%$ & 25 & 24 \\
\hline Supernatant & $0.00 \mathrm{E}+00$ & $0.00 \mathrm{E}+00$ & $0.00 \mathrm{E}+00$ & $0.00 E+00$ & $0.00 \mathrm{E}+00$ & $0.00 \mathrm{E}+00$ & $0.00 \mathrm{E}+00$ & $0.00 \mathrm{E}+00$ & $0.00 \mathrm{E}+00$ & $0.00 \mathrm{E}+00$ & $0.00 \mathrm{E}+00$ & $0.00 \mathrm{E}+00$ & 0.00 & 0.00 & 0 & $0 \%$ & $0 \%$ & 0 & 0 \\
\hline Solid Layer & $3.83 \mathrm{E}+04$ & $1.82 \mathrm{E}+04$ & $1.17 \mathrm{E}+05$ & $2.17 \mathrm{E}+05$ & $2.56 \mathrm{E}+05$ & $4.73 \mathrm{E}+04$ & $7.38 \mathrm{E}+01$ & $4.82 \mathrm{E}-02$ & $7.14 \mathrm{E}-04$ & $5.69 \mathrm{E}-03$ & $6.08 \mathrm{E}-05$ & $1.19 \mathrm{E}+02$ & 1.83 & 1.51 & 1400 & $23 \%$ & $46 \%$ & 25 & 24 \\
\hline $241-B Y-102$ & $3.83 \mathrm{E}+04$ & $1.55 \mathrm{E}+03$ & $1 \mathrm{E}+04$ & $3 E+05$ & $2.34 \mathrm{E} \div 05$ & $66 \mathrm{E}+04$ & $E+01$ & $5.05 E-02$ & $15 \mathrm{E}-03$ & $16 \mathrm{E}-02$ & $8.32 \mathrm{E}-04$ & $6.65 E+01$ & 1.57 & 1.44 & 1054 & $31 \%$ & $51 \%$ & 22 & 22 \\
\hline Supernatant & $0.00 \mathrm{E}+00$ & $0.00 \mathrm{E}+00$ & $0.00 \mathrm{E}+00$ & $0.00 \mathrm{E}+00$ & $0.00 \mathrm{E}+00$ & $0.00 \mathrm{E}+00$ & $0.00 \mathrm{E}+00$ & $0.00 \mathrm{E}-00$ & $0.00 \mathrm{E}+00$ & $0.00 \mathrm{E}+00$ & $0.00 \mathrm{E}+00$ & $0.00 \mathrm{E}+00$ & 0.00 & 0.00 & 0 & $0 \%$ & $0 \%$ & 0 & 0 \\
\hline Solid Layer & $3.83 \mathrm{E}+04$ & $1.55 \mathrm{E}+03$ & $8.41 E+04$ & $1.48 \mathrm{E}+05$ & $2.34 \mathrm{E}+05$ & $5.66 \mathrm{E}+04$ & $1.05 \mathrm{E}+01$ & $5.05 \mathrm{E}-02$ & $5.15 \mathrm{E}-03$ & $2.16 \mathrm{E}-02$ & $8.32 \mathrm{E}-04$ & $6.65 \mathrm{E}+01$ & 1.57 & 1.46 & 1054 & $31 \%$ & $51 \%$ & 22 & 22 \\
\hline $241-\mathrm{BY}-103$ & $3.15 \mathrm{E}+04$ & $1.59 \mathrm{E}+03$ & $4.49 \mathrm{E}+04$ & $41 E+05$ & $1.80 \mathrm{E}+05$ & $3.46 \mathrm{E}+04$ & $1.13 \mathrm{E}+01$ & $16 \mathrm{E}-02$ & $1.34 \mathrm{E}-03$ & $5.61 \mathrm{E}-03$ & $2.05 \mathrm{E}-04$ & $6.69 \mathrm{E}+01$ & 1.66 & 1.29 & 1564 & $28 \%$ & $59 \%$ & 23 & 20 \\
\hline Supernatant & $0.00 \mathrm{E}+00$ & $0.00 \mathrm{E}+00$ & $0.00 \mathrm{E}+00$ & $0.00 \mathrm{E}+00$ & $0.00 E+00$ & $0.00 \mathrm{E}+00$ & $0.00 \mathrm{E}+00$ & $0.00 \mathrm{E}+00$ & $0.00 \mathrm{E}+00$ & $0.00 \mathrm{E}+00$ & $0.00 \mathrm{E}+00$ & $0.00 \mathrm{E}+00$ & 0.00 & 0.00 & 0 & $0 \%$ & $0 \%$ & 0 & 0 \\
\hline Solid Layer & $3.15 \mathrm{E}+04$ & $1.59 \mathrm{E}+03$ & $4.49 \mathrm{E}+04$ & $1.41 \mathrm{E}+05$ & $1.80 \mathrm{E}+05$ & $3.46 \mathrm{E}+04$ & $1.13 \mathrm{E}+01$ & $1.16 \mathrm{E}-02$ & $1.34 \mathrm{E}-03$ & $5.61 \mathrm{E}-03$ & $2.05 \mathrm{E}-04$ & $6.69 \mathrm{E}+01$ & 1.66 & 1.29 & 1564 & $28 \%$ & $59 \%$ & 23 & 20 \\
\hline $24 !-B Y-104$ & $3.83 E+04$ & $2.37 \mathrm{E}+03$ & $1.11 \mathrm{E}+05$ & $2.07 \mathrm{E}+05$ & $2.48 \mathrm{E}+05$ & $7.19 \mathrm{E}+04$ & $1.38 \mathrm{E}+02$ & $5.52 \mathrm{E}-02$ & $5.83 \mathrm{E}-03$ & $2.94 \mathrm{E}-02$ & $8.46 \mathrm{E}-04$ & $8.65 \mathrm{E}+01$ & 1.71 & 1.51 & 1533 & $26 \%$ & $49 \%$ & 41 & 27 \\
\hline Supernatant & $0.00 E+00$ & $0.00 \mathrm{E}+00$ & $0.00 \mathrm{E}+00$ & $0.00 \mathrm{E}+00$ & $0.00 \mathrm{E}+00$ & $0.00 \mathrm{E}+00$ & $0.00 \mathrm{E}+00$ & $0.00 \mathrm{E}+00$ & $0.00 \mathrm{E}+00$ & $0.00 \mathrm{E}+00$ & $0.00 \mathrm{E}+00$ & $0.00 \mathrm{E}+00$ & 0.00 & 0.00 & 0 & $0 \%$ & $0 \%$ & 0 & 0 \\
\hline Solid Layer & $3.83 \mathrm{E}+04$ & $2.37 \mathrm{E}+03$ & $1.11 \mathrm{E}+05$ & $2.07 E+05$ & $2.48 \mathrm{E}+05$ & $7.19 \mathrm{E}+04$ & $1.38 \mathrm{E}+02$ & $5.52 \mathrm{E}-02$ & $5.83 \mathrm{E}-03$ & $2.94 \mathrm{E}-02$ & $8.46 \mathrm{E}-04$ & $8.65 \mathrm{E}+01$ & 1.71 & 1.51 & 1533 & $26 \%$ & $49 \%$ & 41 & 27 \\
\hline $241 \cdot \mathrm{BY}-105$ & $3.82 \mathrm{E}+04$ & $2.43 E+03$ & $6.86 \mathrm{E}+04$ & $1.99 \mathrm{E}+05$ & $2.08 \mathrm{E}+05$ & $3.92 \mathrm{E}+04$ & $4.26 \mathrm{E}+01$ & $3.61 \mathrm{E}-02$ & $3.91 \mathrm{E}-03$ & $2.38 \mathrm{E}-02$ & $4.88 \mathrm{E}-04$ & $4.65 \mathrm{E}+01$ & 1.80 & 1.44 & 1821 & $18 \%$ & $51 \%$ & 33 & 31 \\
\hline Supernatant & $0.00 \mathrm{E}+00$ & $0.00 \mathrm{E}+00$ & $0.00 \mathrm{E}+00$ & $0.00 \mathrm{E}+00$ & $0.00 \mathrm{E}+00$ & $0.00 E+00$ & $0.00 \mathrm{E}+00$ & $0.00 \mathrm{E}+00$ & $0.00 \mathrm{E}+00$ & $0.00 \mathrm{E}+00$ & $0.00 E+00$ & $0.00 \mathrm{E}+00$ & 0.00 & 0.00 & 0 & $0 \%$ & $0 \%$ & 0 & 0 \\
\hline Solid Layer & $3.82 \mathrm{E}+04$ & $2.43 E+03$ & $6.86 \mathrm{E}+04$ & $1.99 \mathrm{E}+05$ & $2.08 \mathrm{E}+05$ & $3.92 E+04$ & 4.26E+01 & $3.61 \mathrm{E}-02$ & $3.91 \mathrm{E}-03$ & $2.38 \mathrm{E}-02$ & $4.88 \mathrm{E}-04$ & $4.65 \mathrm{E}+01$ & 1.80 & 1.44 & 1821 & $18 \%$ & $51 \%$ & 33 & 31 \\
\hline $241-B Y-106$ & $3.58 \mathrm{E}+04$ & $2.67 \mathrm{E}+03$ & $7.83 E+04$ & $1.10 \mathrm{E}+05$ & $1.64 \mathrm{E}+05$ & $3.98 \mathrm{E}+04$ & $5.09 \mathrm{E}+01$ & $1.44 \mathrm{E}-02$ & $1.20 \mathrm{E}-03$ & $8.72 \mathrm{E}-03$ & $1.23 E-04$ & $1.12 \mathrm{E}+02$ & 1.65 & 1.31 & 1623 & $27 \%$ & $59 \%$ & 34 & 34 \\
\hline Supernatant & $0.00 \mathrm{E}+00$ & $0.00 E+00$ & $0.00 \mathrm{E}+00$ & $0.00 \mathrm{E}+00$ & $0.00 \mathrm{E} \div 00$ & $0.00 \mathrm{E}+00$ & $0.00 \mathrm{E}+00$ & $0.00 \mathrm{E}+00$ & $0.00 \mathrm{E}+00$ & $0.00 \mathrm{E}+00$ & $0.00 \mathrm{E}+00$ & $0.00 \mathrm{E}+00$ & 0.00 & 0.00 & 0 & $0 \%$ & $0 \%$ & 0 & 0 \\
\hline Solid Layer & $3.58 \mathrm{E}+04$ & $2.67 \mathrm{E}+03$ & $7.83 E+04$ & $1.10 \mathrm{E}+05$ & $1.64 \mathrm{E}+05$ & $3.98 \mathrm{E}+04$ & $5.09 \mathrm{E}+01$ & $1.44 \mathrm{E}-02$ & $1.20 \mathrm{E}-03$ & 8.72E-03 & 1.23E-04 & $1.12 \mathrm{E}+02$ & 1.65 & 1.31 & 1623 & $27 \%$ & $59 \%$ & 34 & 34 \\
\hline
\end{tabular}




\section{RPP-5926 REV 6}

Table B-1. Input Data for Hydrogen Generation Rate Model Calculations for 177 Tanks. (20 sheets)

\begin{tabular}{|c|c|c|c|c|c|c|c|c|c|c|c|c|c|c|c|c|c|c|c|}
\hline Tank & $\begin{array}{c}\text { OH } \\
\text { in liquid } \\
{[\mathrm{OH}]} \\
(\mu \mathrm{g} / \mathrm{mL})\end{array}$ & $\begin{array}{c}\text { TOC } \\
\text { in liquid } \\
\text { [TOC] } \\
(\mu \mathrm{g} / \mathrm{mL})\end{array}$ & $\begin{array}{c}\mathrm{NO}_{2} \\
\text { in liquid } \\
{\left[\mathrm{NO}_{2}\right]} \\
(\mu \mathrm{g} / \mathrm{mL})\end{array}$ & $\begin{array}{c}\mathrm{NO}_{3} \\
\text { in liquid } \\
{\left[\mathrm{NO}_{3}\right]} \\
(\mu \mathrm{g} / \mathrm{mL})\end{array}$ & $\begin{array}{c}\mathrm{Na} \\
\text { in liquid } \\
{[\mathrm{Na}]} \\
\text { (ug/mL) }\end{array}$ & $\begin{array}{c}\text { Al } \\
\text { in liquid } \\
{[\mathrm{Al}]} \\
(\mu \mathrm{g} / \mathrm{mL})\end{array}$ & $\begin{array}{c}{ }^{90} \mathrm{Sr} \\
\text { in waste } \\
{[\mathrm{Sr}]} \\
(\mu \mathrm{Ci} / \mathrm{g})\end{array}$ & $\begin{array}{c}{ }^{241} \mathrm{Am} \\
\text { in waste } \\
{[\mathrm{Am} 241]} \\
(\mu \mathrm{Ci} / \mathrm{g})\end{array}$ & $\begin{array}{c}{ }^{240} \mathrm{Pu} \\
\text { in waste } \\
{\left[\mathrm{Pu}^{240]}\right]} \\
(\mu \mathrm{Ci} / \mathrm{g})\end{array}$ & $\begin{array}{c}{ }^{239} \mathrm{Pu} \\
\text { in waste } \\
{[\mathrm{Pu} 240]} \\
(\mu \mathrm{Cl} / \mathrm{g})\end{array}$ & $\begin{array}{c}{ }^{238} \mathrm{Pu} \\
\text { in waste } \\
{\left[\mathrm{Pu}^{238}\right]} \\
(\mu \mathrm{Ci} / \mathrm{g})\end{array}$ & $\begin{array}{c}{ }^{137} \mathrm{Cs} \\
\text { in waste } \\
{[\mathrm{Cs} \mid} \\
(\mu \mathrm{Ci} / \mathrm{g})\end{array}$ & $\begin{array}{c}\text { Bulk } \\
\text { density } \\
\text { D } \\
(\mathrm{g} / \mathrm{mL})\end{array}$ & $\begin{array}{c}\text { Liquid } \\
\text { Density } \\
\mathrm{D}_{\mathrm{L}} \\
(\mathrm{g} / \mathrm{ml})\end{array}$ & $\begin{array}{c}\text { Yon-RGS } \\
\text { waste } \\
\text { volume } \\
(\mathrm{kL})\end{array}$ & $\begin{array}{c}\text { Bulk } \\
\text { water } \\
\left|\mathrm{H}_{2} \mathrm{O}\right| \\
(\mathrm{wt} \%)\end{array}$ & $\begin{array}{c}\text { Liquid } \\
\text { water } \\
{\left[\mathrm{H}_{2} \mathrm{O}\right]} \\
(\mathrm{wt} \%)\end{array}$ & $\begin{array}{c}\text { Waste } \\
\text { temp. } \\
\text { Tw } \\
\left({ }^{\circ} \mathrm{C}\right)\end{array}$ & $\begin{array}{c}\text { Dome } \\
\text { temp. } \\
\text { Td } \\
\text { (C) }\end{array}$ \\
\hline $24 !-B Y-107$ & $3.83 \mathrm{E}+04$ & $2.40 \mathrm{E}+03$ & $1.17 \mathrm{E}+05$ & 1.31E+05 & $2.43 \mathrm{E}+05$ & $5.12 \mathrm{E}+04$ & $4.29 \mathrm{E}+01$ & $1.18 \mathrm{E}-02$ & $1.33 \mathrm{E}-03$ & $8.10 \mathrm{E}-03$ & $1.66 \mathrm{E}-04$ & $1.15 \mathrm{E}+02$ & 1.69 & 1.46 & 1028 & $38 \%$ & $49 \%$ & 31 & 26 \\
\hline Supernatant & $0.00 \mathrm{E}+00$ & $0.00 \mathrm{E}+00$ & $0.00 \mathrm{E} \div 00$ & $0.00 \mathrm{E}+00$ & $0.00 \mathrm{E}+00$ & $0.00 \mathrm{E}+00$ & $0.00 \mathrm{E}+00$ & $0.00 \mathrm{E}+00$ & $0.00 \mathrm{E}+00$ & $0.00 \mathrm{E}+00$ & $0.00 \mathrm{E}+00$ & $0.00 \mathrm{E}+00$ & 0.00 & 0.00 & 0 & $0 \%$ & $0 \%$ & 0 & 0 \\
\hline Solid Layer & $3.83 \mathrm{E}+04$ & $2.40 \mathrm{E}+03$ & $1.17 \mathrm{E}+05$ & $1.31 \mathrm{E}+05$ & $2.43 \mathrm{E}+05$ & $5.12 \mathrm{E}+04$ & $4.29 \mathrm{E}+0 \mathrm{I}$ & 1. $18 \mathrm{E}-02$ & $1.33 \mathrm{E}-03$ & 8.10E-03 & $1.66 \mathrm{E}-04$ & $1.15 \mathrm{E}+02$ & 1.69 & 1.46 & 1028 & $38 \%$ & $49 \%$ & 31 & 26 \\
\hline $241-B Y-108$ & $3.83 \mathrm{E}+04$ & $2.37 \mathrm{E}+03$ & $7.85 \mathrm{E}+04$ & $2.94 \mathrm{E}+05$ & $2.82 \mathrm{E}+05$ & $3.63 \mathrm{E}+04$ & $1.03 \mathrm{E}+02$ & $3.05 \mathrm{E}-02$ & 3.43E-03 & $2.26 \mathrm{E}-02$ & $3.96 \mathrm{E}-04$ & $4.21 E+01$ & 1.48 & 1.33 & 841 & $27 \%$ & $33 \%$ & 31 & 26 \\
\hline Supematant & $0.00 \mathrm{E}+00$ & $0.00 \mathrm{E}+00$ & $0.00 \mathrm{E}+00$ & $0.00 \mathrm{E}+00$ & $0.00 \mathrm{E}+00$ & $0.00 \mathrm{E}+00$ & $0.00 \mathrm{E}+00$ & $0.00 \mathrm{E}+00$ & $0.00 \mathrm{E}+00$ & $0.00 \mathrm{E}+00$ & $0.00 \mathrm{E}+00$ & $0.00 \mathrm{E}+00$ & 0.00 & 0.00 & 0 & $0 \%$ & $0 \%$ & 0 & 0 \\
\hline Solid Layer & $3.83 \mathrm{E}+04$ & $2.37 \mathrm{E}+03$ & $7.85 \mathrm{E}+04$ & $2.94 \mathrm{E}+05$ & $2.82 \mathrm{E}+05$ & $3.63 \mathrm{E}+04$ & $1.03 \mathrm{E}+02$ & $3.05 \mathrm{E}-02$ & $3.43 \mathrm{E}-03$ & $2.26 \mathrm{E}-02$ & $3.96 \mathrm{E}-04$ & $4.21 \mathrm{E}+01$ & 1.48 & 1.33 & 841 & $27 \%$ & $33 \%$ & 31 & 26 \\
\hline $241-B Y-109$ & $3.83 \mathrm{E}+04$ & $1.88 \mathrm{E}+03$ & $8.29 \mathrm{E}+04$ & $1.90 \mathrm{E}+05$ & $2.10 \mathrm{E}+05$ & $3.88 \mathrm{E}+04$ & $7.75 E+00$ & $3.69 \mathrm{E}-02$ & $4.35 \mathrm{E}-03$ & $1.82 \mathrm{E}-02$ & $6.67 \mathrm{E}-04$ & $4.98 \mathrm{E}+01$ & 1.71 & 1.50 & 1086 & $42 \%$ & $53 \%$ & 18 & 17 \\
\hline Supernatant & $0.00 \mathrm{E}+00$ & $0.00 \mathrm{E}+00$ & $0.00 \mathrm{E}+00$ & $0.00 \mathrm{E}+00$ & $0.00 \mathrm{E}+00$ & $0.00 \mathrm{E}+00$ & $0.00 \mathrm{E}+00$ & $0.00 \mathrm{E}+00$ & $0.00 \mathrm{E}+00$ & $0.00 \mathrm{E}+00$ & $0.00 \mathrm{E}+00$ & $0.00 \mathrm{E}+00$ & 0.00 & 0.00 & 0 & $0 \%$ & $0 \%$ & 0 & 0 \\
\hline Solid Layer & $3.83 \mathrm{E}+04$ & $1.88 \mathrm{E}+03$ & $8.29 \mathrm{E}+04$ & $1.90 \mathrm{E}+05$ & $2.10 \mathrm{E}+05$ & $3.88 E+04$ & $7.75 \mathrm{E}+00$ & $3.69 \mathrm{E}-02$ & $4.35 \mathrm{E}-03$ & $1.82 \mathrm{E}-02$ & $6.67 \mathrm{E}-04$ & $4.98 \mathrm{E}+01$ & 1.71 & 1.50 & 1086 & $42 \%$ & $53 \%$ & 18 & 17 \\
\hline $24 \mathrm{i}-\mathrm{BY}-110$ & $3.83 \mathrm{E}+04$ & $1.94 \mathrm{E}+04$ & $1.24 \mathrm{E}+05$ & $1.24 \mathrm{E}+05$ & $2.54 \mathrm{E}+05$ & $6.58 \mathrm{E}+04$ & $7.28 \mathrm{E}+01$ & $2.76 \mathrm{E}-02$ & $3.82 \mathrm{E}-03$ & $2.22 \mathrm{E}-02$ & $4.97 \mathrm{E}-04$ & $8.75 E+0 \mathrm{f}$ & 1.57 & 1.44 & 1384 & $28 \%$ & $40 \%$ & 34 & 21 \\
\hline Supernatant & $0.00 \mathrm{E}+00$ & $0.00 \mathrm{E}+00$ & $0.00 \mathrm{E}+00$ & $0.00 \mathrm{E}+00$ & $0.00 \mathrm{E}+00$ & $0.00 \mathrm{E}+00$ & $0.00 \mathrm{E}+00$ & $0.00 \mathrm{E}+00$ & $0.00 \mathrm{E}+00$ & $0.00 \mathrm{E}+00$ & $0.00 \mathrm{E}+00$ & $0.00 \mathrm{E}+00$ & 0.00 & 0.00 & 0 & $0 \%$ & $0 \%$ & 0 & 0 \\
\hline Solid Layer & $3.83 \mathrm{E}+04$ & $1.94 \mathrm{E}+04$ & $1.24 \mathrm{E}+05$ & $1.24 \mathrm{E}+05$ & $2.54 \mathrm{E}+05$ & $6.58 \mathrm{E}+04$ & $7.28 \mathrm{E}+0$ & $2.76 \mathrm{E}-02$ & $3.82 \mathrm{E}-03$ & $2.22 \mathrm{E}-02$ & $4.97 \mathrm{E}-04$ & $8.75 \mathrm{E}+01$ & 1.57 & 1.44 & 1384 & $28 \%$ & $40 \%$ & 34 & 21 \\
\hline 241-BY-111 & $3.83 E+04$ & $1.28 \mathrm{E}+03$ & $5.46 \mathrm{E}+04$ & $2.01 \mathrm{E}+05$ & $2.17 \mathrm{E}+05$ & $4.30 \mathrm{E}+04$ & $1.02 E+01$ & 6. $14 \mathrm{E}-02$ & $6.28 \mathrm{E}-03$ & $2.63 \mathrm{E}-02$ & $1.01 \mathrm{E}-03$ & $5.96 \mathrm{E}+01$ & 1.67 & 1.42 & 1523 & $35 \%$ & $54 \%$ & 25 & 20 \\
\hline Supernatant & $0.00 \mathrm{E}+00$ & $0.00 \mathrm{E}+00$ & $0.00 E+00$ & $0.00 E+00$ & $0.00 \mathrm{E} \div 00$ & $0.00 \mathrm{E}+00$ & $0.00 \mathrm{E} \div 00$ & $0.00 \mathrm{E}+00$ & $0.00 \mathrm{E}+00$ & $0.00 \mathrm{E}+00$ & $0.00 \mathrm{E}+00$ & $0.00 \mathrm{E}+00$ & 0.00 & 0.00 & 0 & $0 \%$ & $0 \%$ & 0 & 0 \\
\hline Solid Layer & $3.83 \mathrm{E}+04$ & $1.28 \mathrm{E}+03$ & $5.46 \mathrm{E}+04$ & $2.01 \mathrm{E}+05$ & $2.17 \mathrm{E}+05$ & $4.30 \mathrm{E}+04$ & $1.02 \mathrm{E}+01$ & $6.14 \mathrm{E}-02$ & $6.28 \mathrm{E}-03$ & $2.63 \mathrm{E}-02$ & 1.01E-03 & $5.96 \mathrm{E}+01$ & 1.67 & 1.42 & 1523 & $35 \%$ & $54 \%$ & 25 & 20 \\
\hline $241-B Y-112$ & $3.83 E+04$ & $1.72 \mathrm{E}+03$ & $1.41 E+05$ & $1.90 \mathrm{E}+05$ & $2.44 \mathrm{E}+05$ & $5.54 \mathrm{E}+04$ & $1.18 \mathrm{E}+01$ & $4.31 \mathrm{E}-02$ & $4.40 \mathrm{E}-03$ & $1.84 \mathrm{E}-02$ & $7.09 \mathrm{E}-04$ & $6.33 E+01$ & 1.74 & 1.47 & 1083 & $30 \%$ & $49 \%$ & 28 & 21 \\
\hline Supernatant & $0.00 \mathrm{E}+00$ & $0.00 \mathrm{E}+00$ & $0.00 \mathrm{E}+00$ & $0.00 \mathrm{E}+00$ & $0.00 \mathrm{E}+00$ & $0.00 \mathrm{E}+00$ & $0.00 \mathrm{E}+00$ & $0.00 E+00$ & $0.00 \mathrm{E}+00$ & $0.00 \mathrm{E}+00$ & $0.00 \mathrm{E}+00$ & $0.00 \mathrm{E}+00$ & 0.00 & 0.00 & 0 & $0 \%$ & $0 \%$ & 0 & 0 \\
\hline Solid Layer & $3.83 \mathrm{E}+04$ & $1.72 \mathrm{E}+03$ & $1.41 \mathrm{E}+05$ & 1.90 & 2.44 & 5. & 1.18 & 4 & 4.40 & -02 & 24 & 6. & 1 & 1.47 & 1083 & $30 \%$ & $49 \%$ & 28 & 21 \\
\hline $241-C-101$ & $1.70 \mathrm{E}+04$ & $2.98 \mathrm{E}+03$ & $3.60 E+04$ & $7.96 \mathrm{E}+04$ & $8.32 \mathrm{E}+04$ & $4.94 E+03$ & $6.42 \mathrm{E}+01$ & $1.43 \mathrm{E}-01$ & $1.40 \mathrm{E}-01$ & $7.86 \mathrm{E}-01$ & $1.11 \mathrm{E}-02$ & $5.06 \mathrm{E}+01$ & 1.78 & 1.17 & 333 & $23 \%$ & $74 \%$ & 32 & 32 \\
\hline Supematant & $0.00 \mathrm{E}+00$ & $0.00 \mathrm{E}+00$ & $0.00 \mathrm{E}+00$ & $0.00 \mathrm{E}+00$ & $0.00 \mathrm{E}+00$ & $0.00 \mathrm{E}+00$ & $0.00 \mathrm{E}+00$ & $0.00 \mathrm{E}+00$ & $0.00 \mathrm{E}+00$ & $0.00 \mathrm{E}+00$ & $0.00 \mathrm{E}+00$ & $0.00 \mathrm{E}+00$ & 0.00 & 0.00 & 0 & $0 \%$ & $0 \%$ & 0 & 0 \\
\hline Solid Layer & $1.70 \mathrm{E}+04$ & $2.98 \mathrm{E}+03$ & $3.60 \mathrm{E}+04$ & $7.96 \mathrm{E}+04$ & $8.32 \mathrm{E}+04$ & $4.94 \mathrm{E}+03$ & $6.42 \mathrm{E}+$ & $1.43 \mathrm{E}-01$ & $1.40 \mathrm{E}-01$ & $7.86 \mathrm{E}-01$ & $1.11 \mathrm{E}-02$ & $5.06 \mathrm{E}+01$ & 1.78 & 1.17 & 333 & $23 \%$ & $74 \%$ & 32 & 32 \\
\hline $241-C-102$ & $1.70 \mathrm{E}+04$ & $2.98 \mathrm{E}+03$ & $3.60 \mathrm{E}+04$ & $7.96 \mathrm{E}+04$ & $8.32 \mathrm{E}+04$ & $4.94 \mathrm{E}+03$ & $1.39 \mathrm{E}+01$ & $5.08 \mathrm{E}-01$ & $4.05 \mathrm{E}-01$ & $1.69 E+00$ & $4.38 \mathrm{E}-02$ & $1.29 \mathrm{E}+01$ & 1.68 & 1.17 & 1196 & $50 \%$ & $75 \%$ & 29 & 26 \\
\hline Supernatant & $0.00 \mathrm{E} \div 00$ & $0.00 \mathrm{E}+00$ & $0.00 \mathrm{E}+00$ & $0.00 \mathrm{E}+00$ & $0.00 \mathrm{E}+00$ & $0.00 E+00$ & $0.00 \mathrm{E}+00$ & $0.00 \mathrm{E}+00$ & $0.00 \mathrm{E}+00$ & $0.00 \mathrm{E}+00$ & $0.00 \mathrm{E}+00$ & $0.00 E+00$ & 0.00 & 0.00 & 0 & $0 \%$ & $0 \%$ & 0 & 0 \\
\hline Solid Layer & $1.70 \mathrm{E}+04$ & $2.98 \mathrm{E}+03$ & $3.60 E+04$ & $7.96 \mathrm{E}+04$ & $8.32 \mathrm{E}+04$ & $4.94 \mathrm{E}+03$ & $1.39 \mathrm{E}+01$ & $5.08 \mathrm{E}-01$ & $4.05 \mathrm{E}-01$ & $1.69 \mathrm{E}+00$ & $4.38 \mathrm{E}-02$ & $1.29 \mathrm{E}+01$ & 1.68 & 1.17 & 1196 & $50 \%$ & $75 \%$ & 29 & 26 \\
\hline $241-C-103$ & $1.70 E+04$ & $5.60 \mathrm{E}+03$ & $1.47 \mathrm{E}+04$ & $8.20 \mathrm{E}+02$ & $3.21 \mathrm{E}+04$ & $0.00 \mathrm{E}+00$ & $4.82 \mathrm{E}+02$ & $2.64 \mathrm{E}-02$ & $2.91 \mathrm{E}-01$ & $1.40 \mathrm{E}+00$ & $2.41 \mathrm{E}-02$ & $4.67 \mathrm{E}+01$ & 1.63 & 1.07 & 101 & $23 \%$ & $89 \%$ & 43 & 37 \\
\hline Supematant & $0.00 E+00$ & $0.00 \mathrm{E}+00$ & $0.00 \mathrm{E}+00$ & $0.00 \mathrm{E}+00$ & $0.00 \mathrm{E}+00$ & $0.00 \mathrm{E}+00$ & $0.00 \mathrm{E}+00$ & $0.00 \mathrm{E}+00$ & $0.00 \mathrm{E}+00$ & $0.00 \mathrm{E}+00$ & $0.00 \mathrm{E}+00$ & $0.00 \mathrm{E}+00$ & 0.00 & 0.00 & 0 & $0 \%$ & $0 \%$ & 0 & 0 \\
\hline Solid Layer & $1.70 \mathrm{E}+04$ & $5.60 \mathrm{E}+03$ & $1.47 \mathrm{E}+04$ & $8.20 \mathrm{E}+02$ & $3.21 \mathrm{E}+04$ & $0.00 \mathrm{E}+00$ & $4.82 \mathrm{E}+02$ & $2.64 \mathrm{E}-02$ & 2.9 !E-01 & $1.40 E+00$ & $2.41 \mathrm{E}-02$ & $4.67 \mathrm{E}+01$ & 1.63 & 1.11 & 101 & $23 \%$ & $89 \%$ & 43 & 37 \\
\hline
\end{tabular}




\section{RPP-5926 REV 6}

Table B-1. Input Data for Hydrogen Generation Rate Model Calculations for 177 Tanks. (20 sheets)

\begin{tabular}{|c|c|c|c|c|c|c|c|c|c|c|c|c|c|c|c|c|c|c|c|}
\hline Tank & $\begin{array}{c}\text { OH } \\
\text { in liquid } \\
\text { |OH| } \\
(\mu \mathrm{g} / \mathrm{mL})\end{array}$ & $\begin{array}{c}\text { TOC } \\
\text { in liquid } \\
\text { (TOC) } \\
(\mu \mathrm{g} / \mathrm{mL})\end{array}$ & $\begin{array}{c}\mathrm{NO}_{2} \\
\text { in liquid } \\
\left|\mathrm{NO}_{2}\right| \\
(\mu \mathrm{g} / \mathrm{mL})\end{array}$ & $\begin{array}{c}\mathrm{NO}_{3} \\
\text { in liquid } \\
{\left[\mathrm{NO}_{3}\right]} \\
(\mu \mathrm{g} / \mathrm{mL})\end{array}$ & $\begin{array}{c}\mathrm{Na} \\
\text { in liquid } \\
|\mathrm{Na}| \\
\text { (ug/mL) }\end{array}$ & $\begin{array}{c}\text { Al } \\
\text { In liquid } \\
\{\mathrm{Al}\} \\
(\mu \mathrm{g} / \mathrm{mL})\end{array}$ & $\begin{array}{c}{ }^{90} \mathrm{Sr} \\
\text { in waste } \\
{[\mathrm{Sr}]} \\
(\mu \mathrm{Ci} / \mathrm{g})\end{array}$ & $\begin{array}{c}{ }^{24 t} \mathrm{Am} \\
\text { in waste } \\
\text { |Am241] } \\
(\mu \mathrm{Ci} / \mathrm{g})\end{array}$ & $\begin{array}{c}{ }^{240} \mathrm{Pu} \\
\text { in waste } \\
\text { [Pu240| } \\
(\mu \mathrm{Ci} / \mathrm{g})\end{array}$ & $\begin{array}{c}{ }^{239} \mathrm{Pu} \\
\text { in waste } \\
{[\mathrm{Pu240]}} \\
(\mu \mathrm{Ci} / \mathrm{g})\end{array}$ & $\begin{array}{c}{ }^{238} \mathrm{Pu} \\
\text { in waste } \\
{\left[\begin{array}{c}\mathrm{Pu} 238 \mid \\
(\mu \mathrm{Ci} / \mathrm{g})\end{array}\right.}\end{array}$ & $\begin{array}{c}{ }^{137} \mathrm{Cs} \\
\text { in waste } \\
\mid \mathrm{Cs}] \\
(\mu \mathrm{Ci} / \mathrm{g})\end{array}$ & $\begin{array}{c}\text { Bulk } \\
\text { density } \\
D \\
(\mathrm{~g} / \mathrm{mL})\end{array}$ & $\begin{array}{c}\text { Liquid } \\
\text { Density } \\
D_{2} \\
(\mathrm{~g} / \mathrm{ml})\end{array}$ & $\begin{array}{c}\text { Non-RGS } \\
\text { waste } \\
\text { volume } \\
(\mathbf{k L})\end{array}$ & $\begin{array}{l}\text { Bulk } \\
\text { water } \\
{\left[\mathrm{H}_{2} \mathrm{O}\right]} \\
(\mathrm{wt} " \%)\end{array}$ & $\begin{array}{l}\text { Liquid } \\
\text { water } \\
\left|\mathrm{H}_{2} \mathrm{O}\right| \\
(\mathrm{wt} \%)\end{array}$ & $\begin{array}{c}\text { Waste } \\
\text { temp. } \\
\text { Tw } \\
\left({ }^{\circ} \mathrm{C}\right)\end{array}$ & $\begin{array}{l}\text { Dome } \\
\text { temp. } \\
\text { Td } \\
\left({ }^{\circ} \mathrm{C}\right)\end{array}$ \\
\hline $241-C \cdot 104$ & $E+04$ & $\mathrm{OE}+03$ & $0 E+04$ & $66+04$ & 04 & $94 E+03$ & $7 \pm E+02$ & $85 E+00$ & $10 \mathrm{E}-01$ & $+\infty$ & $39 \mathrm{E}-01$ & 1] & 1.68 & 1.17 & 980 & $48 \%$ & $81 \%$ & 36 & 34 \\
\hline Supernatant & $0.00 E+00$ & $0.00 \mathrm{E}+00$ & $0.00 \mathrm{E}+00$ & $0.00 \mathrm{E}+00$ & $0.00 \mathrm{E}+00$ & $0.00 \mathrm{E}+00$ & $0.00 \mathrm{E} \times 00$ & $0.00 \mathrm{E}+00$ & $0.00 \mathrm{E}+00$ & $0.00 E+00$ & $0.00 \mathrm{E}+00$ & $0.00 E+00$ & 0.00 & 0.00 & 0 & $0 \%$ & $0 \%$ & 0 & 0 \\
\hline Solid Layer & $1.70 \mathrm{E}+04$ & $5.60 \mathrm{E}+03$ & $3.60 \mathrm{E}+04$ & $7.96 \mathrm{E}+04$ & $8.32 \mathrm{E}+04$ & $4.94 \mathrm{E}+03$ & $2.71 \mathrm{E}+02$ & $3.85 \mathrm{E}+00$ & $8.10 \mathrm{E}-01$ & $3.14 \mathrm{E}+00$ & $1.39 \mathrm{E}-01$ & $5.38 \mathrm{E}+01$ & 1.68 & 1.17 & 980 & $48 \%$ & $81 \%$ & 36 & 34 \\
\hline $241-C-105$ & $1.70 \mathrm{E}+04$ & $2.87 \mathrm{E}+03$ & $3.70 \mathrm{E}+04$ & $3.43 E+04$ & $1.14 \mathrm{E}+05$ & $9.02 \mathrm{E}+00$ & $5.69 \mathrm{E}+02$ & $1.54 \mathrm{E}+00$ & $5.03 \mathrm{E}-01$ & $2.56 \mathrm{E}+00$ & $4.11 \mathrm{E}-02$ & $9.85 \mathrm{E}+01$ & 1.55 & 1.23 & 500 & $28 \%$ & $67 \%$ & 47 & 41 \\
\hline Supernatant & $0.00 \mathrm{E}+00$ & $0.00 \mathrm{E}+00$ & $0.00 \mathrm{E}+00$ & $0,00 \mathrm{E}+00$ & $.00 \mathrm{E}+00$ & $0.00 \mathrm{E}+00$ & $00 \mathrm{E}+00$ & $0.00 \mathrm{E}+00$ & $0.00 \mathrm{E}+00$ & $0.00 \mathrm{E}+00$ & $0.00 \mathrm{E}+00$ & $0.00 \mathrm{E}+00$ & 0.00 & 0.00 & 0 & $0 \%$ & $0 \%$ & 0 & 0 \\
\hline Solid Layer & $1.70 \mathrm{E}+04$ & $2.87 \mathrm{E}+03$ & $3.70 \mathrm{E}+04$ & $3.43 E+04$ & $1.14 \mathrm{E} \div 05$ & $9.02 \mathrm{E}+00$ & $5.69 \mathrm{E}+02$ & $1.54 \mathrm{E}+00$ & $5.03 \mathrm{E}-01$ & $2.56 \mathrm{E}+00$ & 4.11E-02 & $9.85 \mathrm{E}+01$ & 1.55 & 1.23 & 500 & $28 \%$ & $67 \%$ & 47 & 41 \\
\hline $241-C-106$ & $7.02 \mathrm{E}+03$ & $3.32 \mathrm{E}+02$ & $5.43 E+01$ & $6.02 \mathrm{E}+01$ & $9.78 \mathrm{E}+03$ & $9.38 \mathrm{E}+0 \mathrm{I}$ & $4.04 E+03$ & $3.99 \mathrm{E}+00$ & $2.18 \mathrm{E}-0 \mathrm{I}$ & $1.02 \mathrm{E}+00$ & $1.65 \mathrm{E}-01$ & $8.86 \mathrm{E}+01$ & 1.54 & 1.02 & 10 & $43 \%$ & $98 \%$ & 30 & 25 \\
\hline Supernatant & $7.02 \mathrm{E}+03$ & $3.32 \mathrm{E}+02$ & $5.43 \mathrm{E}+01$ & $6.02 E+01$ & $9.78 \mathrm{E}+03$ & $9.38 \mathrm{E}+01$ & $4.32 \mathrm{E}-02$ & $4.11 \mathrm{E}-06$ & $8.49 \mathrm{E}-07$ & $3.99 \mathrm{E}-06$ & $6.39 \mathrm{E}-07$ & $4.26 \mathrm{E}-01$ & 1.02 & 1.02 & 0 & $98 \%$ & $98 \%$ & $3 !$ & 25 \\
\hline Solid Layer & $7.02 \mathrm{E}+03$ & $3.32 \mathrm{E}+02$ & $5.43 \mathrm{E}+01$ & $6.02 \mathrm{E}+01$ & $9.78 \mathrm{E}+03$ & $9.38 \mathrm{E}+01$ & $4.17 \mathrm{E}+03$ & $4.11 \mathrm{E}+00$ & $2.25 \mathrm{E}-01$ & $1.05 E+00$ & $1.70 \mathrm{E}-01$ & $9.14 E+01$ & 1.56 & 1.14 & 10 & $42 \%$ & $98 \%$ & 30 & 25 \\
\hline $241-C-107$ & $1.70 \mathrm{E}+04$ & $2.98 \mathrm{E}+03$ & $3.60 \mathrm{E}+04$ & $7.96 \mathrm{E}+04$ & $32 \mathrm{E}+04$ & $4.94 \mathrm{E}+03$ & $.44 \mathrm{E}+03$ & $4.62 \mathrm{E} \div 00$ & $2.44 \mathrm{E}-01$ & $1.51 E+00$ & $3.05 \mathrm{E}-02$ & $4.07 \mathrm{E}+01$ & 1.55 & 1.17 & 935 & $48 \%$ & $79 \%$ & 43 & 41 \\
\hline Supeniatant & $0.008+00$ & $0.00 E+00$ & $0.00 E+00$ & $0.00 \mathrm{E}+00$ & $.00 \mathrm{E}+00$ & $0.00 \mathrm{E}+00$ & $0.00 \mathrm{E}+00$ & $0.00 \mathrm{E}+00$ & $0.00 \mathrm{E}+00$ & $0.00 E+00$ & $0.00 E+00$ & $0.00 \mathrm{E}+00$ & 0.00 & 0.00 & 0 & $0 \%$ & $0 \%$ & 0 & 0 \\
\hline Solid Layer & $1.70 \mathrm{E}+04$ & $2.98 \mathrm{E}+03$ & $3.60 \mathrm{E}+04$ & $7.96 \mathrm{E}+04$ & $8.32 \mathrm{~F}+04$ & $4.94 \mathrm{E}+03$ & $1.44 \mathrm{E}+03$ & $4.62 \mathrm{E}+00$ & $2.44 \mathrm{E}-01$ & $1.51 \mathrm{E}+00$ & $3.05 \mathrm{E}-02$ & $4.07 \mathrm{E}+01$ & 1.55 & 1.17 & 935 & $48 \%$ & $79 \%$ & 43 & 41 \\
\hline $241-C-108$ & $1.70 \mathrm{E}+04$ & $8 \mathrm{E}+03$ & $0 \mathrm{E}+04$ & $7.96 \mathrm{E} \div 04$ & $2 \mathrm{E}+04$ & $4.94 \mathrm{E}+03$ & $\mathrm{E}+01$ & $90 \mathrm{E}-02$ & I8E-04 & 8.43E-03 & $5.95 \mathrm{E}-05$ & $2.10 \mathrm{E}+02$ & 1. & 1.1 & 250 & $38 \%$ & $75 \%$ & 26 & 27 \\
\hline Supernatant & $0.00 \mathrm{E}+00$ & $0.00 \mathrm{E}+00$ & $0.00 \mathrm{E}+00$ & $0.00 \mathrm{E}+00$ & $0.00 \mathrm{E}+00$ & $0.00 \mathrm{E} \div 00$ & $0.00 E+00$ & $0.00 E+00$ & $0.00 E+00$ & $0.00 \mathrm{E}+00$ & $0.00 \mathrm{E} \div 00$ & $0.00 \mathrm{E}+00$ & 0.00 & 0.00 & 0 & $0 \%$ & $0 \%$ & 0 & 0 \\
\hline Solid Layer & $1.70 \mathrm{E}+04$ & $2.98 E+03$ & $3.60 \mathrm{E}+04$ & $7.96 \mathrm{E}+04$ & $8.32 \mathrm{E}+04$ & $4.94 \mathrm{E}+03$ & $2.16 \mathrm{E}+01$ & $2.90 \mathrm{E}-02$ & $9.18 \mathrm{E}-04$ & $8.43 \mathrm{E}-03$ & $5.95 E-05$ & $2.10 \mathrm{E}+02$ & 1.48 & 1.17 & 250 & $38 \%$ & $75 \%$ & 26 & 27 \\
\hline 241-C-109 & $1705+04$ & $98 \mathrm{E}+03$ & $3.60 \mathrm{E}+04$ & $7.96 \mathrm{E}+04$ & $32 \mathrm{E}+04$ & $4.94 \mathrm{E}+03$ & $80 \mathrm{E}+02$ & $35 E-01$ & $.02 \mathrm{E}-02$ & $1.76 \mathrm{E}-0 !$ & $4.44 \mathrm{E}-03$ & $4.65 \mathrm{E}+02$ & 1.55 & 1.17 & 24 & $45 \%$ & $75 \%$ & 26 & 26 \\
\hline Supernatant & $0.00 \mathrm{E}+00$ & $0.00 \mathrm{E}+00$ & $0.00 \mathrm{E}+00$ & $0.00 \mathrm{E}+00$ & $0.00 \mathrm{E}+00$ & $0.00 \mathrm{E}+00$ & $0.00 \Sigma+00$ & $0.00 \mathrm{E}+00$ & $0.00 \mathrm{E}+00$ & $0.00 E+00$ & $0.00 \mathrm{E}+00$ & $0.00 E+00$ & 0.00 & 0.00 & 0 & $0 \%$ & $0 \%$ & 0 & 0 \\
\hline Solid Layer & $1.70 \mathrm{E}+04$ & $2.98 \mathrm{E}+03$ & $3.60 \mathrm{E}+04$ & $7.96 \mathrm{E}+04$ & $8.32 \mathrm{E}+04$ & $4.94 E+03$ & $6.80 \mathrm{E}+02$ & $1.35 \mathrm{E}-0 \mathrm{l}$ & $3.02 \mathrm{E}-02$ & $1.76 \mathrm{E}-01$ & $4.44 \mathrm{E}-03$ & $4.65 \mathrm{E}+02$ & 1.55 & 1.17 & 240 & $45 \%$ & $75 \%$ & 26 & 26 \\
\hline $24 \mathrm{I}-\mathrm{C}-110$ & $1.70 \mathrm{E}+04$ & $5.80 \mathrm{E}+02$ & $4.60 \mathrm{E}+03$ & $6.03 \mathrm{E}+04$ & $3.05 E+04$ & $2.09 \mathrm{E}+02$ & $3.82 E+00$ & $4.27 \mathrm{E}-02$ & $7.81 \mathrm{E}-03$ & $7.18 \mathrm{E}-02$ & $5.05 \mathrm{E}-04$ & $1.50 \mathrm{E}+01$ & 1.34 & 1.10 & 674 & $60 \%$ & $83 \%$ & 21 & 22 \\
\hline Superuatant & $1.70 \mathrm{E}+04$ & $5.80 \mathrm{E}+02$ & $4.60 \mathrm{E}+03$ & $6.03 \mathrm{E}+04$ & $3.05 E+04$ & $2.09 \mathrm{E} \div 02$ & $1.92 \mathrm{E}-02$ & $1.72 \mathrm{E}-05$ & $1.01 \mathrm{E}-04$ & $1.50 \mathrm{E}-03$ & $5.07 \mathrm{E}-06$ & $3.41 \mathrm{E}+00$ & 1.10 & 1.10 & 4 & $83 \%$ & $83 \%$ & 21 & 22 \\
\hline Solid Layer & $1.70 \mathrm{E}+04$ & $5.80 \mathrm{E}+02$ & $4.60 \mathrm{E}+03$ & $6.03 E+04$ & $3.05 \mathrm{E}+04$ & $2.09 \mathrm{E}+02$ & $3.84 \mathrm{E}+00$ & $4.30 \mathrm{E}-02$ & $7.85 \mathrm{E}-03$ & $7.22 \mathrm{E}-02$ & $5.08 \mathrm{E}-04$ & $1.50 \mathrm{E}+01$ & 1.34 & 1.10 & 670 & $60 \%$ & $83 \%$ & 21 & 22 \\
\hline $241 \cdot \mathrm{C}-111$ & $1.70 E+04$ & $2.98 \mathrm{E}+03$ & $3.60 \mathrm{E}+04$ & $7.96 \mathrm{E}+04$ & $8.32 \mathrm{E} \div 04$ & $4.94 \mathrm{E}+03$ & $2.70 \mathrm{E}+03$ & $5.67 \mathrm{E}-01$ & $9.85 \mathrm{E}-02$ & $5.06 \mathrm{E}-01$ & $9.30 \mathrm{E}-03$ & $5.89 \mathrm{E}+01$ & 1.55 & 1.17 & 217 & $41 \%$ & $75 \%$ & 25 & 25 \\
\hline Supernatant & $0.00 \mathrm{E}+00$ & $0.00 \mathrm{E}+00$ & $0.00 \mathrm{E}, 1-00$ & $0.00 \mathrm{E}+00$ & $0.00 \mathrm{E}+00$ & $0.00 \mathrm{E}+00$ & $0.00 \mathrm{E}+00$ & $0.00 \mathrm{E}+00$ & $0.00 \mathrm{E}+00$ & $0.00 \mathrm{E}+00$ & $0.00 \mathrm{E}+00$ & $0.00 E+00$ & 0.00 & 0.00 & 0 & $0 \%$ & $0 \%$ & 0 & 0 \\
\hline Solid Layer & $1.70 \mathrm{E}+04$ & $2.98 \mathrm{E}+03$ & $3.60 \mathrm{E}+04$ & $7.96 \mathrm{E}+04$ & $8.32 \mathrm{E}+04$ & $4.94 \mathrm{E}+03$ & $2.70 \mathrm{E}+03$ & $5.67 \mathrm{E}-01$ & $9.85 \mathrm{E}-02$ & $5.06 \mathrm{E}-01$ & $9.30 \mathrm{E}-03$ & $5.89 \mathrm{E}+01$ & 1.55 & 1.17 & 217 & $41 \%$ & $75 \%$ & 25 & 25 \\
\hline $241-C-112$ & $1.70 \mathrm{E}+04$ & $2.98 \mathrm{E} \div 03$ & $3.60 \mathrm{E} \div 04$ & $7.96 \mathrm{E}+04$ & $8.32 E+04$ & $4.94 \mathrm{E} \div 03$ & $9.56 \mathrm{E}+02$ & $7.49 \mathrm{E}-01$ & $1.69 \mathrm{E}-02$ & $1.34 \mathrm{E}-01$ & $1.25 \mathrm{E}-02$ & $4.23 \mathrm{E}+02$ & 1.60 & 1.17 & 393 & $52 \%$ & $75 \%$ & 27 & 28 \\
\hline Supernatant & $0.00 \mathrm{E}+00$ & $0.00 \mathrm{E}+00$ & $0.00 \mathrm{E}+00$ & $0.00 \mathrm{E}+00$ & $0.00 \mathrm{E}+00$ & $0.00 \mathrm{E}+00$ & $0.00 \mathrm{E}+00$ & $0.00 \mathrm{E}+00$ & $0.00 \mathrm{E}+00$ & $0.00 \mathrm{E}+00$ & $0.00 \mathrm{E}+00$ & $0.00 \mathrm{E}+00$ & 0.00 & 0.00 & 0 & $0 \%$ & $0 \%$ & 0 & 0 \\
\hline Solid Layer & $1.70 E+04$ & $2.98 \mathrm{E}+03$ & $3.60 \mathrm{E}+04$ & $7.96 \mathrm{E}+04$ & $8.32 \mathrm{E}+04$ & $4.94 \mathrm{E}+03$ & $9.56 \mathrm{E}+02$ & $7.49 \mathrm{E}-01$ & $1.69 \mathrm{E}-02$ & $1.34 \mathrm{E}-0 \mathrm{~J}$ & $1.25 \mathrm{E}-02$ & $4.23 \mathrm{E}+02$ & 1.60 & 1.17 & 393 & $52 \%$ & $75 \%$ & 27 & 28 \\
\hline
\end{tabular}




\section{RPP-5926 REV 6}

Table B-1. Input Data for Hydrogen Generation Rate Model Calculations for 177 Tanks. (20 sheets)

\begin{tabular}{|c|c|c|c|c|c|c|c|c|c|c|c|c|c|c|c|c|c|c|c|}
\hline Tank & $\begin{array}{c}\mathrm{OH} \\
\text { in liquid } \\
{[\mathrm{OH} \mid} \\
(\mu \mathrm{g} / \mathrm{mL})\end{array}$ & $\begin{array}{c}\text { TOC } \\
\text { in liquid } \\
\text { |TOC| } \\
(\mu \mathrm{g} / \mathrm{mL})\end{array}$ & $\begin{array}{c}\mathrm{NO}_{2} \\
\text { in liquid } \\
{\left[\mathrm{NO}_{2}\right]} \\
(\mu \mathrm{g} / \mathrm{mL})\end{array}$ & $\begin{array}{c}\mathrm{NO}_{3} \\
\text { in liquid } \\
{\left[\mathrm{NO}_{3}\right]} \\
(\mu \mathrm{g} / \mathrm{mL})\end{array}$ & $\begin{array}{c}\mathrm{Na} \\
\text { in liquid } \\
{[\mathrm{Na}]} \\
(\mathrm{ug} / \mathrm{mL})\end{array}$ & $\begin{array}{c}\text { Al } \\
\text { in liquid } \\
{[\mathrm{Al}]} \\
(\mu \mathrm{g} / \mathrm{mL})\end{array}$ & $\begin{array}{c}{ }^{90} \mathrm{Sr} \\
\text { in waste } \\
{[\mathrm{Sr}]} \\
(\mu \mathrm{Ci} / \mathrm{g})\end{array}$ & $\begin{array}{c}{ }^{241} \mathrm{Am} \\
\text { in waste } \\
\text { |Am241] } \\
(\mu \mathrm{Ci} / \mathrm{g})\end{array}$ & 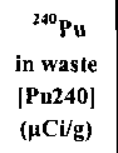 & 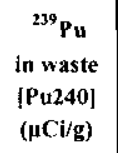 & $\begin{array}{c}{ }^{238} \mathrm{Pu} \\
\text { in waste } \\
{[\mathrm{Pu238} \mid} \\
(\mu \mathrm{Ci} / \mathrm{g})\end{array}$ & $\begin{array}{c}{ }^{137} \mathrm{Cs} \\
\text { in waste } \\
{[\mathrm{Cs}]} \\
(\mu \mathrm{Ci} / \mathrm{g})\end{array}$ & $\begin{array}{c}\text { Bulk } \\
\text { density } \\
\text { D } \\
(\mathrm{g} / \mathrm{mL})\end{array}$ & $\begin{array}{c}\text { Liquid } \\
\text { Density } \\
D_{\mathrm{L}} \\
(\mathrm{g} / \mathrm{ml})\end{array}$ & $\begin{array}{c}\text { Non-RGS } \\
\text { waste } \\
\text { volume } \\
(\mathrm{kL})\end{array}$ & $\begin{array}{l}\text { Bulk } \\
\text { water } \\
{\left[\mathrm{H}_{2} \mathrm{O}\right]} \\
(\mathrm{wt} \%)\end{array}$ & $\begin{array}{l}\text { Liquid } \\
\text { water } \\
{\left[\mathrm{H}_{2} \mathrm{O} \mid\right.} \\
\left(w_{t} \%\right)\end{array}$ & $\begin{array}{l}\text { Waste } \\
\text { temp. } \\
\text { Tw } \\
\left({ }^{\circ} \mathrm{C}\right)\end{array}$ & $\begin{array}{l}\text { Dome } \\
\text { temp. } \\
\text { Td } \\
\left({ }^{\circ} \mathrm{C}\right)\end{array}$ \\
\hline $241-C \cdot 201$ & $70 E+04$ & $0.00 \mathrm{E}+00$ & $00 \mathrm{E}+00$ & $00 \mathrm{E}+00$ & $.00 \mathrm{E}+00$ & $0.00 \mathrm{E}+00$ & $2.65 \mathrm{E}+02$ & $2.54 \mathrm{E}+00$ & $3.57 \mathrm{E}+00$ & $1.66 \mathrm{E}+01$ & $5.26 \mathrm{E}-01$ & $1.06 \mathrm{E}+01$ & 1.74 & 1.00 & 1 & $25 \%$ & $100 \%$ & 16 & 20 \\
\hline Supernatant & $1.70 \mathrm{E}+04$ & $0.00 E+00$ & $0.00 \mathrm{E}+00$ & $0.00 \mathrm{E}+00$ & $0.00 \mathrm{E}+00$ & $0.00 \mathrm{E}+00$ & $0.00 \mathrm{E}+00$ & $0.00 \mathrm{E}+00$ & $0.00 \mathrm{E}+00$ & $0.00 \mathrm{E}+00$ & $0.00 \mathrm{E}+00$ & $0.00 \mathrm{E}+00$ & 1.00 & 1.00 & 0.01 & $100 \%$ & $100 \%$ & 16 & 20 \\
\hline Solid Layer & $1.70 \mathrm{E}+04$ & $0.00 \mathrm{E}+00$ & $0.00 \mathrm{E}+00$ & $0.00 \mathrm{E}+00$ & $0.00 \mathrm{E}+00$ & $0.00 \mathrm{E}+00$ & $2.68 \mathrm{E}+02$ & $2.58 \mathrm{E}+00$ & $3.62 \mathrm{E}+00$ & $1.68 \mathrm{E}+01$ & $5.33 \mathrm{E}-01$ & $1.07 \mathrm{E}+01$ & 1.75 & 1.00 & 1 & $24 \%$ & $100 \%$ & 16 & 20 \\
\hline $241-C-202$ & $1.70 E+04$ & $0.00 \mathrm{E}+00$ & $0.00 E+00$ & $0.00 \mathrm{E}+00$ & $0.00 \mathrm{E}+00$ & $0.00 \mathrm{E}+00$ & $5.00 \mathrm{E}+02$ & $1.18 \mathrm{E}+00$ & $3.16 \mathrm{E}+00$ & $1.47 \mathrm{E}+01$ & $4.66 \mathrm{E}-01$ & $9.16 \mathrm{E}+00$ & 1.74 & 1.00 & 1 & $25 \%$ & $100 \%$ & 16 & 19 \\
\hline Supernatant & $1.70 \mathrm{E}+04$ & $0.00 \mathrm{E}+00$ & $0.00 \mathrm{E}+00$ & $0.00 \mathrm{E}+00$ & $0.00 \mathrm{E}+00$ & $0.00 \mathrm{E}+00$ & $0.00 \mathrm{E}+00$ & $0.00 \mathrm{E}+00$ & $0.00 \mathrm{E}+00$ & $0.00 E+00$ & $0.00 \mathrm{E}+00$ & $0.00 \mathrm{E}+00$ & 1.00 & 1.00 & 0.01 & $100 \%$ & $100 \%$ & 16 & 19 \\
\hline Solid Layer & $1.70 \mathrm{E}+04$ & $0.00 \mathrm{E}+00$ & $0.00 \mathrm{E}+00$ & $0.00 \mathrm{E}+00$ & $0.00 \mathrm{E}+00$ & $0.00 \mathrm{E}+00$ & $5.08 \mathrm{E}+02$ & $1.20 \mathrm{E}+00$ & $3.21 \mathrm{E}+00$ & $1.49 \mathrm{E}+01$ & $4.73 \mathrm{E}-01$ & $9.31 \mathrm{E}+00$ & 1.75 & 1.00 & 1 & $24 \%$ & $100 \%$ & 16 & 19 \\
\hline $241-C-203$ & $1.70 E+04$ & $0.00 \mathrm{E}+00$ & $0.00 \mathrm{E}+00$ & $0.00 \mathrm{E}+00$ & $0.00 E+00$ & $0.00 \mathrm{E}+00$ & $2.33 \mathrm{E}+00$ & $2.88 \mathrm{E}-02$ & $1.04 \mathrm{E}-01$ & $4.81 \mathrm{E}-01$ & $1.53 \mathrm{E}-02$ & $1.30 \mathrm{E}+01$ & 1.84 & 1.00 & 1 & $39 \%$ & $100 \%$ & 15 & 19 \\
\hline Supeniatant & $1.70 \mathrm{E}+04$ & $0.00 \mathrm{E}+00$ & $0.00 \mathrm{E}+00$ & $0.00 \mathrm{E}+00$ & $0.00 \mathrm{E}+00$ & $0.00 \mathrm{E}+00$ & $00 \mathrm{E}+00$ & $0.00 \mathrm{E}+00$ & $0.00 E+00$ & $0.00 \mathrm{E}+00$ & $0.00 \mathrm{E}+00$ & $0.00 \mathrm{E}+00$ & 1.00 & 1.00 & 0.05 & $100 \%$ & $100 \%$ & 19 & 19 \\
\hline Solid Layer & $1.70 \mathrm{E}+04$ & $0.00 \mathrm{E}+00$ & $0.00 \mathrm{E}+00$ & $0.00 \mathrm{E}+00$ & $0.00 \mathrm{E}+00$ & $0.00 \mathrm{E}+00$ & $2.57 E+00$ & $3.17 \mathrm{E}-02$ & $1.14 \mathrm{E}-01$ & $5.29 \mathrm{E}-01$ & $3.69 \mathrm{E}-02$ & $1.43 \mathrm{E}+01$ & 1.93 & 1.00 & 0 & $36 \%$ & $100 \%$ & 15 & 19 \\
\hline $241 \cdot \mathrm{C}-204$ & $1.70 \mathrm{E}+04$ & $2.98 \mathrm{E}+03$ & $3.60 \mathrm{E}-04$ & $7.96 \mathrm{E}+04$ & $8.32 \mathrm{E}+04$ & $4.94 \mathrm{E}+03$ & $3.09 \mathrm{E}+01$ & $1.39 \mathrm{E}-03$ & $1.23 \mathrm{E}-03$ & $5.70 \mathrm{E}-03$ & $1.81 \mathrm{E}-04$ & $4.72 \mathrm{E}+00$ & 1.62 & 1.17 & 6 & $41 \%$ & $75 \%$ & 17 & 16 \\
\hline Supernatant & $0.00 E+00$ & $0.00 \mathrm{E}+00$ & $0.00 \mathrm{E}+00$ & $0.00 \mathrm{E} \div 00$ & $0.00 E+00$ & $0.00 \mathrm{E}+00$ & $0.00 \mathrm{E}+00$ & $0.00 E+00$ & $0.00 \mathrm{E}+00$ & $0.00 \mathrm{E}+00$ & $0.00 \mathrm{E}+00$ & $0.00 \mathrm{E}+00$ & 0.00 & 0.00 & 0 & $0 \%$ & $0 \%$ & 0 & 0 \\
\hline Solid Layer & $1.70 \mathrm{E}+04$ & $2.98 \mathrm{E}+03$ & $3.60 \mathrm{E}+04$ & $7.96 \mathrm{E}+04$ & $8.32 \mathrm{E}+04$ & $4.94 \mathrm{E}+03$ & $1.09 \mathrm{E}+01$ & $1.39 \mathrm{E}-03$ & $1.23 \mathrm{E}-03$ & $5.70 \mathrm{E}-03$ & $1.81 \mathrm{E}-04$ & $4.72 \mathrm{E}+00$ & 1.62 & 1.17 & 6 & $41 \%$ & $75 \%$ & 17 & 16 \\
\hline $241-S-10 t$ & $\div 04$ & $1.79 \mathrm{E}+03$ & $13 \mathrm{E}+05$ & $14 E+05$ & $2.22 \mathrm{E}+05$ & $2.99 \mathrm{E}+04$ & $2.06 \mathrm{E}+02$ & J.00E-0I & $2.87 \mathrm{E}-02$ & $1.46 \mathrm{E}-01$ & $3.05 \mathrm{E}-03$ & $1.12 \mathrm{E}+02$ & 1.65 & 1.47 & 1332 & $39 \%$ & $51 \%$ & 39 & 31 \\
\hline Supernatant & $0.00 \mathrm{E}+00$ & $0.00 \mathrm{E}+00$ & $0.00 \mathrm{E}+00$ & $0.00 \mathrm{E}+00$ & $0.00 \mathrm{E}+00$ & $0.00 \mathrm{E}+00$ & $0.00 E+00$ & $0.00 \mathrm{E}+00$ & $0.00 \mathrm{E}+00$ & $0.00 E+00$ & $0.00 \mathrm{E}+00$ & $0.00 \mathrm{E}+00$ & 0.00 & 0.00 & 0 & $0 \%$ & $0 \%$ & 0 & 0 \\
\hline Solid Layer & $4.76 \mathrm{E}+04$ & $1.79 \mathrm{E}+03$ & $1.13 \mathrm{E}+05$ & $2.14 \mathrm{E}+05$ & $2.22 \mathrm{E}+05$ & $2.99 \mathrm{E}+04$ & $2.06 \mathrm{E}+02$ & $1.00 \mathrm{E}-0 \mathrm{t}$ & $2.87 \mathrm{E}-02$ & $1.46 \mathrm{E}-01$ & $3.05 \mathrm{E}-03$ & $1.12 \mathrm{E}+02$ & 1.65 & 1.47 & 1332 & $39 \%$ & $51 \%$ & 39 & 31 \\
\hline $241-$ & 4 & 3 & 05 & $55 E+05$ & +05 & $5.62 \mathrm{E}+04$ & $6.57 \mathrm{E}+01$ & $\mathrm{E}-02$ & $1.19 \mathrm{E}-02$ & $5.59 \mathrm{E}-02$ & $1.83 \mathrm{E}-03$ & $9.55 \mathrm{E}+01$ & 1.71 & 1.46 & 664 & $35 \%$ & $49 \%$ & 35 & 33 \\
\hline Supenatant & $0.00 \mathrm{E}+00$ & $0.00 \mathrm{E}+00$ & $0.00 \mathrm{E}+00$ & $0.00 \mathrm{E}+00$ & $0.00 \mathrm{E}+00$ & $0.00 \mathrm{E}+00$ & $0.00 \mathrm{E}+00$ & $0.00 \mathrm{E}+00$ & $0.00 \mathrm{E}+00$ & $0.00 \mathrm{E}+00$ & $0.00 \mathrm{E}+00$ & $0.00 \mathrm{E}+00$ & 0.00 & 0.00 & 0 & $0 \%$ & $0 \%$ & 0 & 0 \\
\hline Solid Layer & $3.76 \mathrm{E}+04$ & $3.76 \mathrm{E}+03$ & $1.27 \mathrm{E}+05$ & $1.55 \mathrm{E}+05$ & $2.34 \mathrm{E}+05$ & $5.62 \mathrm{E}+04$ & $6.57 \mathrm{E}+01$ & $9.88 \mathrm{E}-02$ & $1.19 \mathrm{E}-02$ & $5.59 \mathrm{E}-02$ & $1.83 \mathrm{E}-03$ & $9.55 \mathrm{E}+01$ & 1.71 & 1.46 & 664 & $35 \%$ & $49 \%$ & 35 & 33 \\
\hline $241-S-103$ & $3.64 \mathrm{E}+04$ & 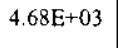 & $47 \mathrm{E}+05$ & $2.16 \mathrm{E}+05$ & $2.28 \mathrm{E}+05$ & $3.20 \mathrm{E}+04$ & $2.87 E+0 !$ & $9.36 \mathrm{E}-02$ & $1.36 \mathrm{E}-02$ & $6.51 \mathrm{E}-02$ & $1.94 \mathrm{E}-03$ & $1.35 \mathrm{E}+02$ & 1.61 & 1.45 & 896 & $36 \%$ & $50 \%$ & 27 & 25 \\
\hline Supernatant & $3.65 \mathrm{E}+04$ & $4.68 \mathrm{E}+03$ & $1.47 \mathrm{E}+0.5$ & $2.16 \mathrm{E}+05$ & $2.28 \mathrm{E}+05$ & $3.20 \mathrm{E}+04$ & $2.55 \mathrm{E}-01$ & $8.14 \mathrm{E}-04$ & $8.45 \mathrm{E}-06$ & $3.95 \mathrm{E}-05$ & $1.38 \mathrm{E}-06$ & $2.22 \mathrm{E}+02$ & 1.45 & 1.45 & 4 & $50 \%$ & $50 \%$ & 27 & 25 \\
\hline Solid Layer & $3.64 \mathrm{E}+04$ & $4.68 \mathrm{E}+03$ & $1.47 \mathrm{E}+05$ & $2.16 \mathrm{E}+05$ & $2.28 \mathrm{E}+05$ & $3.20 \mathrm{E}+04$ & $2.88 \mathrm{E}+01$ & $9.40 \mathrm{E}-02$ & $1.36 \mathrm{E}-02$ & $6.54 \mathrm{E}-02$ & $1.95 \mathrm{E}-03$ & $1.35 \mathrm{E}+02$ & 1.61 & 1.45 & 892 & $36 \%$ & $50 \%$ & 27 & 25 \\
\hline 241-S-104 & $5.10 \mathrm{E}+03$ & $3.96 \mathrm{E}+02$ & $3.82 \mathrm{E}+04$ & $2.68 \mathrm{E}+05$ & $1.98 \mathrm{E}+05$ & $1.19 \mathrm{E}+04$ & $2.21 \mathrm{E}+02$ & $1.11 \mathrm{E}-01$ & $3.90 \mathrm{E}-02$ & $1.92 \mathrm{E}-01$ & $5.50 \mathrm{E}-03$ & $4.26 \mathrm{E}+01$ & 1.67 & 1.37 & 1090 & $39 \%$ & $55 \%$ & 38 & 32 \\
\hline Supenatant & $0.00 \mathrm{E}+00$ & $0.00 \mathrm{E}+00$ & $0.00 \mathrm{E}+00$ & $0.00 \mathrm{E}+00$ & $0.00 \mathrm{E}+00$ & $0.00 \mathrm{E}+00$ & $0.00 \mathrm{E}+00$ & $0.00 \mathrm{E}+00$ & $0.00 E+00$ & $0.00 \mathrm{E}+00$ & $0.00 \mathrm{E}+00$ & $0.00 \mathrm{E}+00$ & 0.00 & 0.00 & 0 & $0 \%$ & $0 \%$ & 0 & 0 \\
\hline Solid Layer & $5.10 \mathrm{E}+03$ & $3.96 \mathrm{E}+02$ & $3.82 \mathrm{E}+04$ & $2.68 \mathrm{E}+05$ & $1.98 \mathrm{E}+05$ & $1.19 \mathrm{E}+04$ & $2.21 \mathrm{E}+02$ & $1.11 \mathrm{E}-01$ & $3.90 \mathrm{E}-02$ & $1.92 \mathrm{E}-01$ & $5.50 \mathrm{E}-03$ & $4.26 \mathrm{E}+01$ & 1.67 & 1.37 & 1090 & $39 \%$ & $55 \%$ & 38 & 32 \\
\hline 24 i-S-105 & $8.18 E+04$ & $4.03 \mathrm{E}+03$ & $1.22 \mathrm{E}+05$ & $1.90 \mathrm{E}+05$ & $2.36 \mathrm{E}+05$ & $3.78 E+04$ & $2.56 \mathrm{E}+00$ & $3.05 \mathrm{E}-03$ & $7.25 \mathrm{E}-04$ & $3.63 \mathrm{E}-03$ & $8.19 \mathrm{E}-05$ & $3.42 \mathrm{E}+01$ & 1.66 & 1.45 & 1536 & $8 \%$ & $49 \%$ & 25 & 23 \\
\hline Supernatant & $0.00 \mathrm{E}+00$ & $0.00 \mathrm{E}+00$ & $0.00 \mathrm{E}+00$ & $0.00 \mathrm{E}+00$ & $0.00 \mathrm{E}+00$ & $0.00 \mathrm{E}+00$ & $0.00 \mathrm{E}+00$ & $0.00 \mathrm{E}+00$ & $0.00 \mathrm{E}+00$ & $0.00 \mathrm{E}+00$ & $0.00 \mathrm{E}+00$ & $0.00 \mathrm{E}+00$ & 0.00 & 0.00 & 0 & $0 \%$ & $0 \%$ & 0 & 0 \\
\hline Solid Layer & $8.18 \mathrm{E}+04$ & $4.03 E+03$ & $1.22 \mathrm{E}+05$ & $1.90 \mathrm{E}+05$ & $2.36 \mathrm{E}+05$ & $3.78 \mathrm{E}+04$ & $2.56 \mathrm{E}+00$ & $3.05 \mathrm{E}-03$ & $7.25 \mathrm{E}-04$ & $3.63 \mathrm{E}-03$ & $8.19 \mathrm{E}-05$ & $3.42 \mathrm{E}+01$ & 1.66 & 1.45 & 1536 & $8 \%$ & $49 \%$ & 25 & 23 \\
\hline
\end{tabular}




\section{RPP-5926 REV 6}

Table B-1. Input Data for Hydrogen Generation Rate Model Calculations for 177 Tanks. (20 sheets)

\begin{tabular}{|c|c|c|c|c|c|c|c|c|c|c|c|c|c|c|c|c|c|c|c|}
\hline Tank & $\begin{array}{c}\mathrm{OH} \\
\text { in liquid } \\
{[\mathrm{OH}]} \\
(\mu \mathrm{g} / \mathrm{mL})\end{array}$ & $\begin{array}{c}\text { TOC } \\
\text { in liquid } \\
\text { [TOC] } \\
(\mu \mathrm{g} / \mathrm{mL})\end{array}$ & $\begin{array}{c}\mathrm{NO}_{2} \\
\text { in liquid } \\
\left.\text { [NO } \mathrm{NO}_{2}\right] \\
(\mu \mathrm{g} / \mathrm{mL})\end{array}$ & $\begin{array}{c}\mathrm{NO}_{3} \\
\text { in liquid } \\
{\left[\mathrm{NO}_{3}\right]} \\
(\mu \mathrm{g} / \mathrm{mL})\end{array}$ & $\begin{array}{c}\mathrm{Na} \\
\text { in liquìd } \\
{[\mathrm{Na}]} \\
\text { (ug/mL) }\end{array}$ & $\begin{array}{c}\text { Al } \\
\text { in liquid } \\
{[\mathrm{Al}]} \\
(\mu \mathrm{g} / \mathrm{mL})\end{array}$ & $\begin{array}{c}{ }^{90} \mathrm{Sr} \\
\text { in waste } \\
{[\mathrm{Sr}]} \\
(\mu \mathrm{Ci} / \mathrm{g})\end{array}$ & $\begin{array}{c}{ }^{241} \mathrm{Am} \\
\text { in waste } \\
{[\mathrm{Am} 241]} \\
(\mu \mathrm{Ci} / \mathrm{g})\end{array}$ & $\begin{array}{c}{ }^{240} \mathrm{Pu} \\
\text { in waste } \\
|\mathrm{Pu} 240| \\
(\mu \mathrm{Ci} / \mathrm{g})\end{array}$ & 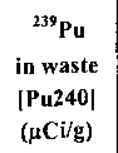 & $\begin{array}{c}{ }^{238} \mathrm{Pu} \\
\text { in waste } \\
{[\mathrm{Pu} 238]} \\
(\mu \mathrm{Ci} / \mathrm{g})\end{array}$ & $\begin{array}{c}{ }^{137} \mathrm{Cs} \\
\text { in waste } \\
{[\mathrm{Cs}]} \\
(\mu \mathrm{Cig})\end{array}$ & $\begin{array}{c}\text { Bulk } \\
\text { density } \\
\text { D } \\
(\mathrm{g} / \mathrm{mL})\end{array}$ & $\begin{array}{c}\text { Liquid } \\
\text { Density } \\
D_{\mathrm{L}} \\
(\mathrm{g} / \mathrm{ml})\end{array}$ & $\begin{array}{c}\text { Non-RGS } \\
\text { waste } \\
\text { volume } \\
\text { (kL) }\end{array}$ & $\begin{array}{l}\text { Bulk } \\
\text { water } \\
\left|\mathrm{H}_{2} \mathrm{O}\right| \\
(\mathrm{wt} \%)\end{array}$ & $\begin{array}{c}\text { Liquid } \\
\text { water } \\
{\left[\mathrm{H}_{2} \mathrm{O} \mid\right.} \\
(w t \%)\end{array}$ & $\begin{array}{l}\text { Waste } \\
\text { temp. } \\
\text { Tw } \\
\left({ }^{\circ} \mathrm{C}\right)\end{array}$ & $\begin{array}{c}\text { Dome } \\
\text { temp. } \\
\text { Td } \\
\left({ }^{\circ} \mathrm{C}\right)\end{array}$ \\
\hline $24 !-S-106$ & $5.55 \mathrm{E}+04$ & $1.98 \mathrm{E}+03$ & $1.04 \mathrm{E}+05$ & $2.36 \mathrm{E}+05$ & $2.44 \mathrm{E}+05$ & $3.80 \mathrm{E}+04$ & $1.23 \mathrm{E}+01$ & $1.96 \mathrm{E}-02$ & $1.32 \mathrm{E}-03$ & $6.04 \mathrm{E}-03$ & $2.13 E-04$ & $9.13 \mathrm{E}+01$ & 1.72 & 1.43 & 1723 & $34 \%$ & $54 \%$ & 23 & 23 \\
\hline Supeniatant & $0.00 E+00$ & $0.00 E+00$ & $0.00 \mathrm{E}+00$ & $0.00 \mathrm{E}=00$ & $0.00 \mathrm{E}+00$ & $0.00 \mathrm{E}+00$ & $0.00 \mathrm{E}+00$ & $0.00 \mathrm{E}+00$ & $0.00 \mathrm{E}+00$ & $0.00 \mathrm{E}+00$ & $0.00 \mathrm{E}+00$ & $0.00 \mathrm{E}+00$ & 0.00 & 0.00 & 0 & $0 \%$ & $0 \%$ & 0 & 0 \\
\hline Solid Layer & $5.55 \mathrm{E}+04$ & $1.98 \mathrm{E}+03$ & $1.04 \mathrm{E}+05$ & $2.36 \mathrm{E}+05$ & $2.44 \mathrm{E}+05$ & $3.80 \mathrm{E}+04$ & $1.23 \mathrm{E}+01$ & $1.96 \mathrm{E}-02$ & $1.32 \mathrm{E}-03$ & $6.04 E-03$ & $2.13 \mathrm{E}-04$ & $9.13 E+01$ & 1.72 & 1.43 & 1723 & $34 \%$ & $54 \%$ & 23 & 23 \\
\hline $241-S-107$ & $2.99 \mathrm{E}+04$ & $1.18 \mathrm{E}+03$ & $7.11 \mathrm{E}+04$ & $1.14 \mathrm{E}+05$ & $1.56 \mathrm{E}+05$ & $1.07 \mathrm{E}+04$ & $1.30 E+02$ & $3.10 \mathrm{E}-01$ & $1.06 \mathrm{E}-0$ & $5.04 \mathrm{E}-01$ & $1.22 \mathrm{E}-02$ & $6.77 \mathrm{E}+01$ & 1.78 & 1.31 & 1356 & $34 \%$ & $64 \%$ & 33 & 25 \\
\hline Supernatant & $0.00 \mathrm{E}+00$ & $0.00 \mathrm{E}+00$ & $0.00 \mathrm{E}+00$ & $0.00 E+00$ & $0.00 \mathrm{E}+00$ & $0.00 \mathrm{E}+00$ & $0.00 \mathrm{E}+00$ & $0.00 \mathrm{E}+00$ & $0.00 \mathrm{E}+00$ & $0.00 \mathrm{E}+00$ & $0.00 \mathrm{E}+00$ & $0.00 \mathrm{E}+00$ & 0.00 & 0.00 & 0 & $0 \%$ & $0 \%$ & 0 & 0 \\
\hline Sotid Layer & $2.99 \mathrm{E}+04$ & $1.18 \mathrm{E}+03$ & $7.11 \mathrm{E}+04$ & $1.14 \mathrm{E}+05$ & $1.56 \mathrm{E}+05$ & $1.07 \mathrm{E}+04$ & $1.30 \mathrm{E}+02$ & $3.10 \mathrm{E}-01$ & $1.06 \mathrm{E}-01$ & $5.04 \mathrm{E}-01$ & $1.22 \mathrm{E}-02$ & $6.77 \mathrm{E}+01$ & 1.78 & 1.31 & 1356 & $34 \%$ & $64 \%$ & 33 & 25 \\
\hline 241-S-108 & $4.81 E+04$ & $4.03 \mathrm{E}+03$ & $1.22 \mathrm{E}+05$ & $1.90 \mathrm{E}+05$ & $2.36 \mathrm{E}+05$ & $3.77 \mathrm{E}+04$ & $2.10 \mathrm{E}+01$ & $7.72 \mathrm{E}-02$ & $5.96 \mathrm{E}-03$ & $2.79 \mathrm{E}-02$ & $9.05 \mathrm{E}-04$ & $9.60 \mathrm{E}+01$ & 1.68 & 1.45 & 2082 & $30 \%$ & $49 \%$ & 27 & 24 \\
\hline Supernatant & $0.00 \mathrm{E}+00$ & $0.00 \mathrm{E}+00$ & $0.00 \mathrm{E}+00$ & $0.00 \mathrm{E}+00$ & $0.00 \mathrm{E}+00$ & $0.00 \mathrm{E}+00$ & $0.00 \mathrm{E}+00$ & $0.00 \mathrm{E}+00$ & $0.00 \mathrm{E}+00$ & $0.00 E+00$ & $0.00 \mathrm{E}+00$ & $0.00 \mathrm{E}+00$ & 0.00 & 0.00 & 0 & $0 \%$ & $0 \%$ & 0 & 0 \\
\hline Solid Layer & $4.81 \mathrm{E}+04$ & $4.03 \mathrm{E}+03$ & $1.22 \mathrm{E}+05$ & $1.90 \mathrm{E}+05$ & $2,36 \mathrm{E}+05$ & $3.77 \mathrm{E}+04$ & $2.10 E+01$ & $7.72 \mathrm{E}-02$ & $5.96 \mathrm{E}-03$ & $2.79 \mathrm{E}-02$ & $9.05 \mathrm{E}-04$ & $9.60 \mathrm{E}+01$ & 1.68 & 1.45 & 2082 & $30 \%$ & $49 \%$ & 27 & 24 \\
\hline $241-\mathrm{S}-109$ & $8.19 E+04$ & $1.11 \mathrm{E}+03$ & $8.49 \mathrm{E}+04$ & $1.32 \mathrm{E}+05$ & $2.19 \mathrm{E}+05$ & $4.59 \mathrm{E}+04$ & $1.48 \mathrm{E}+01$ & $1.15 \mathrm{E}-02$ & $2.83 \mathrm{E}-03$ & $1.44 \mathrm{E}-02$ & $2.92 \mathrm{E}-04$ & $1.30 \mathrm{E}+01$ & 1.66 & 1.49 & 2017 & $11 \%$ & $53 \%$ & 26 & 23 \\
\hline Supernatant & $0.00 E \div 00$ & $0.00 \mathrm{E} \div 00$ & $0.00 E+00$ & $0.00 \mathrm{E}+00$ & $0.00 \mathrm{E}+00$ & $0.00 \mathrm{E}+00$ & $0.00 \mathrm{E}+00$ & $0.00 \mathrm{E}+00$ & $0.00 \mathrm{E}+00$ & $0.00 E+00$ & $0.00 \mathrm{E}+00$ & $0.00 \mathrm{E}+00$ & 0.00 & 0.00 & 0 & $0 \%$ & $0 \%$ & 0 & 0 \\
\hline Solid Layer & 8.19E+04 & $1.11 \mathrm{E}+03$ & $8.49 \mathrm{E}+04$ & $1.32 \mathrm{E}+05$ & $2.19 \mathrm{E}+05$ & $4.59 \mathrm{E}+04$ & $1.48 \mathrm{E}+01$ & $1.15 \mathrm{E}-02$ & $2.83 E-03$ & 1. $.44 \mathrm{E}-02$ & $2.92 \mathrm{E}-04$ & $1.30 \mathrm{E}+01$ & 1.60 & 1.49 & 2017 & $11 \%$ & $53 \%$ & 26 & 23 \\
\hline $241-S-110$ & $8.18 \mathrm{E}+04$ & $2.28 \mathrm{E}+03$ & $8.58 \mathrm{E}+04$ & $2.17 \mathrm{E}+05$ & $2.11 E+05$ & $3.10 \mathrm{E}+04$ & $9.40 \mathrm{E}+01$ & $1.23 \mathrm{E}-01$ & $2.50 \mathrm{E}-02$ & $1.26 \mathrm{E}-01$ & $2.63 \mathrm{E}-03$ & $7.27 \mathrm{E}+01$ & 1.66 & 1.43 & 1473 & $20 \%$ & $49 \%$ & 40 & 24 \\
\hline Supernatant & $0.00 \mathrm{E}+00$ & $0.00 \mathrm{~F}+00$ & $0.00 \mathrm{E}+00$ & $0.00 \mathrm{E}+00$ & $0.00 \mathrm{E}+00$ & $0.00 \mathrm{E}+00$ & $0.00 \mathrm{E}+00$ & $0.00 \mathrm{E}+00$ & $0.00 \mathrm{E}+00$ & $0.00 E+00$ & $0.00 \mathrm{E}+00$ & $0.00 \mathrm{E}+00$ & 0.00 & 0.00 & 0 & $0 \%$ & $0 \%$ & 0 & 0 \\
\hline Solid Layer & $8.18 \mathrm{E}+04$ & $2.28 \mathrm{E}+03$ & $8.58 \mathrm{E}+04$ & $2.17 \mathrm{E}+05$ & $2.11 E+05$ & $3.10 \mathrm{E}+04$ & $9.40 \mathrm{E}+0 \mathrm{t}$ & $1.23 \mathrm{E}-01$ & $2.50 \mathrm{E}-02$ & $1.26 \mathrm{E}-01$ & $2.63 \mathrm{E}-03$ & $7.27 \mathrm{E}+01$ & 1.66 & 1.43 & 1473 & $20 \%$ & $49 \%$ & 40 & 24 \\
\hline $241-S-11]$ & $5.10 \mathrm{E}+04$ & $1.49 \mathrm{E}+03$ & $8.39 \mathrm{E}+04$ & $1.93 \mathrm{E}+05$ & $2.23 \mathrm{E}+05$ & $3.64 \mathrm{E}+04$ & $1.83 \mathrm{E}+02$ & $1.48 \mathrm{E}-02$ & $1.44 \mathrm{E}-03$ & $6.90 \mathrm{E}-03$ & $1.96 \mathrm{E}-04$ & $1.03 \mathrm{E}+02$ & 1.55 & 1.45 & 1397 & $29 \%$ & $51 \%$ & 28 & 24 \\
\hline Supenatant & $0.00 \mathrm{E}+00$ & $0.00 \mathrm{E}+00$ & $0.00 \mathrm{E}+00$ & $0.00 \mathrm{E}+00$ & $0.00 \mathrm{E}+00$ & $0.00 \mathrm{E}+00$ & $0.00 \mathrm{E}+00$ & $0.00 \mathrm{E}+00$ & $0.00 \mathrm{E}+00$ & $0.00 \mathrm{E}+00$ & $0.00 \mathrm{E}+00$ & $0.00 \mathrm{E}+00$ & 0.00 & 0.00 & 0 & $0 \%$ & $0 \%$ & 0 & 0 \\
\hline Solid Layer & $5.10 \mathrm{E}+04$ & $1.49 \mathrm{E}+03$ & $8.39 \mathrm{E}+04$ & $1.93 \mathrm{E}+05$ & $2.23 \mathrm{E}+05$ & $3.64 \mathrm{E}+04$ & $1.83 \mathrm{E}+02$ & $1.48 \mathrm{E}-02$ & $1.44 \mathrm{E}-03$ & $6.90 \mathrm{E}-03$ & 1.96E-04 & $1.03 E+02$ & 1.55 & 1.45 & 1397 & $29 \%$ & $51 \%$ & 28 & 24 \\
\hline $241-S-112$ & $4.86 \mathrm{E}+04$ & $8.31 \mathrm{E}+02$ & $6.2 \mathrm{JE}+04$ & $1.68 \mathrm{E}+05$ & $2.29 \mathrm{E}+05$ & $3.69 \mathrm{E}+04$ & $6.28 \mathrm{E}+02$ & $5.24 \mathrm{E}-01$ & $5.68 \mathrm{E}-02$ & $3.20 \mathrm{E}-01$ & $7.23 \mathrm{E}-03$ & $1.72 \mathrm{E}+01$ & 1.71 & 1.45 & 19 & $36 \%$ & $52 \%$ & 22 & 26 \\
\hline Supernatant & $0.00 \mathrm{E}+00$ & $0.00 E+00$ & $0.00 \mathrm{E}+00$ & $0.00 \mathrm{E}+00$ & $0.00 \mathrm{E}+00$ & $0.00 \mathrm{E}+00$ & $0.00 \mathrm{E}+00$ & $0.00 \mathrm{E}+00$ & $0.00 \mathrm{E}+00$ & $0.00 \mathrm{E}+00$ & $0.00 \mathrm{E}+00$ & $0.00 E+00$ & 0.00 & 0.00 & 0 & $0 \%$ & $0 \%$ & 0 & 0 \\
\hline Solid Layer & $4.86 E+04$ & $8.31 E+02$ & $6.21 E+04$ & $1.68 \mathrm{E}+05$ & $2.29 E+05$ & $3.69 \mathrm{E}+04$ & $6.28 \mathrm{E}+02$ & $5.24 \mathrm{E}-0 \mathrm{1}$ & $5.68 \mathrm{E}-02$ & $3.20 \mathrm{E}-01$ & $7.23 \mathrm{E}-03$ & $1.72 \mathrm{E}+01$ & 1.71 & 1.45 & 19 & $36 \%$ & $52 \%$ & 22 & 26 \\
\hline $24 t-S X-101$ & $4.88 \mathrm{E}+04$ & $3.35 \mathrm{E}+02$ & $7.49 \mathrm{E}+04$ & $1.08 \mathrm{E}+05$ & $2.42 E+05$ & $8.00 \mathrm{E}+04$ & $1.13 E+02$ & $1.91 \mathrm{E}-01$ & $3.39 \mathrm{E}-02$ & $1.66 \mathrm{E}-01$ & $4.59 \mathrm{E}-03$ & $1.00 \mathrm{E}+02$ & 1.68 & 1.48 & 1584 & $27 \%$ & $49 \%$ & 49 & 39 \\
\hline Supeniatant & $0.00 \mathrm{E}+00$ & $0.00 \mathrm{E}+00$ & $0.00 \mathrm{E}+00$ & $0.00 \mathrm{E}+00$ & $0.00 \mathrm{E}+00$ & $0.00 \mathrm{E}+00$ & $0.00 E+00$ & $0.00 \mathrm{E}+00$ & $0.00 \mathrm{E}+00$ & $0.00 \mathrm{E}+00$ & $0.00 \mathrm{E}+00$ & $0.00 \mathrm{E}+00$ & 0.00 & 0.00 & 0 & $0 \%$ & $0 \%$ & 0 & 0 \\
\hline Solid Layer & $4.88 \mathrm{E}+04$ & $3.35 \mathrm{E}+02$ & $7.49 \mathrm{E}+04$ & $1.08 \mathrm{E}+05$ & $2.42 \mathrm{E}+05$ & $8.00 \mathrm{E}+04$ & $1.13 \mathrm{E}+02$ & $1.91 \mathrm{E}-01$ & $3.39 \mathrm{E}-02$ & $1.66 \mathrm{E}-01$ & $4.59 \mathrm{E}-03$ & $1.00 \mathrm{E}+02$ & 1.68 & 1.48 & 1584 & $27 \%$ & $49 \%$ & 49 & 39 \\
\hline 241-SX-102 & $5.01 \mathrm{E}+04$ & $1.51 E+03$ & $1.51 \mathrm{E}+05$ & $1.57 \mathrm{E}+05$ & $2.35 \mathrm{E}+05$ & $4.56 \mathrm{E}+04$ & $8.21 \mathrm{E}+01$ & $1.22 \mathrm{E}-01$ & $1.21 \mathrm{E}-02$ & $5.77 \mathrm{E}-02$ & $1.69 \mathrm{E}-03$ & $1.40 \mathrm{E}+02$ & 1.70 & 1.46 & 1292 & $39 \%$ & $48 \%$ & 52 & 32 \\
\hline Supernatant & $0.00 \mathrm{E}+00$ & $0.00 \mathrm{E}+00$ & $0.00 \mathrm{E}+00$ & $0.00 \mathrm{E}+00$ & $0.00 E+00$ & $0.00 \mathrm{E}+00$ & $0.00 \mathrm{E}+00$ & $0.00 \mathrm{E}+00$ & $0.00 \mathrm{E}+00$ & $0.00 E+00$ & $0.00 \mathrm{E}+00$ & $0.00 \mathrm{E} \div 00$ & 0.00 & 0.00 & 0 & $0 \%$ & $\%$ & 0 & 0 \\
\hline Solid Layer & $5.01 \mathrm{E}+04$ & $1.51 \mathrm{E}+03$ & $1.51 \mathrm{E}+05$ & $1.57 \mathrm{E}+05$ & $2.35 \mathrm{E}+05$ & $4.56 \mathrm{E}+04$ & $8.21 \mathrm{E}+0 \mathrm{I}$ & 1.22E-01 & $1.21 \mathrm{E}-02$ & $5.77 \mathrm{E}-02$ & $1.69 \mathrm{E}-03$ & $1.40 \mathrm{E}+02$ & 1.70 & 1.46 & 1292 & $39 \%$ & $48 \%$ & 52 & 32 \\
\hline
\end{tabular}




\section{RPP-5926 REV 6}

Table B-1. Input Data for Hydrogen Generation Rate Model Calculations for 177 Tanks. (20 sheets)

\begin{tabular}{|c|c|c|c|c|c|c|c|c|c|c|c|c|c|c|c|c|c|c|c|}
\hline Tank & $\begin{array}{c}\mathrm{OH} \\
\text { in liquid } \\
{[\mathrm{OH}]} \\
(\mu \mathrm{g} / \mathrm{mL})\end{array}$ & $\begin{array}{c}\text { Toc } \\
\text { in liquid } \\
\text { |TOC| } \\
(\mu \mathrm{g} / \mathrm{mL})\end{array}$ & $\begin{array}{c}\mathrm{NO}_{2} \\
\text { in liquid } \\
\left|\mathrm{NO}_{2}\right| \\
(\mu \mathrm{g} / \mathrm{mL})\end{array}$ & $\begin{array}{c}\mathrm{NO}_{3} \\
\text { in liquid } \\
{\left[\mathrm{NO}_{3}\right]} \\
(\mu \mathrm{g} / \mathrm{mL})\end{array}$ & $\begin{array}{c}\mathrm{Na} \\
\text { in liquid } \\
\text { [Na] } \\
\text { (ug/mL) }\end{array}$ & $\begin{array}{c}\text { Al } \\
\text { in liquid } \\
\mid \mathrm{A}] \mid \\
(\mu \mathrm{g} / \mathrm{mL})\end{array}$ & $\begin{array}{c}{ }^{90} \mathrm{Sr} \\
\text { in waste } \\
|\mathrm{Sr}| \\
(\mu \mathrm{Ci} / \mathrm{g})\end{array}$ & $\begin{array}{c}{ }^{241} \mathrm{Am} \\
\text { in waste } \\
{[\mathrm{Am} 241]} \\
(\mu \mathrm{Ci} / \mathrm{g})\end{array}$ & $\begin{array}{c}{ }^{240} \mathrm{Pu} \\
\text { in waste } \\
|\mathrm{Pu} 240| \\
(\mu \mathrm{Ci} / \mathrm{g})\end{array}$ & $\begin{array}{c}{ }^{239} \mathrm{Pu} \\
\text { in waste } \\
{[\mathrm{Pu240}]} \\
(\mu \mathrm{Ci} / \mathrm{g})\end{array}$ & $\begin{array}{c}{ }^{238} \mathrm{Pu} \\
\text { in waste } \\
{\left[\begin{array}{l}\mathrm{Pu} 238 \mid \\
(\mu \mathrm{Cu} / \mathrm{g})\end{array}\right.}\end{array}$ & $\begin{array}{c}{ }^{137} \mathrm{Cs} \\
\text { in waste } \\
{[\mathrm{Cs} \mid} \\
(\mu \mathrm{Ci} / \mathrm{g})\end{array}$ & $\begin{array}{c}\text { Bulk } \\
\text { density } \\
0 \\
(\mathrm{~g} / \mathrm{mL})\end{array}$ & $\begin{array}{c}\text { Liquid } \\
\text { Density } \\
\mathrm{D}_{\mathrm{L}} \\
(\mathrm{g} / \mathrm{ml})\end{array}$ & $\begin{array}{c}\text { Non-RGS } \\
\text { waste } \\
\text { volume } \\
\text { (kL) }\end{array}$ & $\begin{array}{l}\text { Bulk } \\
\text { water } \\
{\left[\mathrm{H}_{2} \mathrm{O}\right]} \\
(\mathbf{w t} \%)\end{array}$ & $\begin{array}{c}\text { Liquid } \\
\text { water } \\
\left|\mathrm{H}_{2} \mathrm{O}\right| \\
(w t " \%)\end{array}$ & $\begin{array}{l}\text { Waste } \\
\text { temp. } \\
T_{w} \\
\left({ }^{\circ} \mathrm{C}\right)\end{array}$ & $\begin{array}{l}\text { Dome } \\
\text { temp. } \\
\text { Td } \\
\left(^{\circ} \mathrm{C}\right)\end{array}$ \\
\hline $241-\mathrm{SX}-103$ & 4.52E+04 & $3.38 \mathrm{E}+03$ & $.57 \mathrm{E}+05$ & $56 \mathrm{E}+05$ & $40 \mathrm{E}+05$ & $44 \mathrm{E}+04$ & $.54 \mathrm{E}+02$ & $2.16 \mathrm{E}-01$ & $1.56 \mathrm{E}-02$ & $7.61 \mathrm{E}-02$ & $2.05 \mathrm{E}-03$ & $1.23 \mathrm{E}+02$ & 1.73 & 1.47 & 1926 & $35 \%$ & $47 \%$ & 60 & 27 \\
\hline Supernatant & $0.00 \mathrm{E}+00$ & $0.00 \mathrm{E}+00$ & $0.00 \mathrm{E}+00$ & $0.00 \mathrm{E}+00$ & $0.00 \mathrm{E}+00$ & $0.00 \mathrm{E}+00$ & $0.00 \mathrm{E}+00$ & $0.00 \mathrm{E}+00$ & $0.00 \mathrm{E}+00$ & $0.00 \mathrm{E}+00$ & $0.00 \mathrm{E}+00$ & $0.00 \mathrm{E}+00$ & 0.00 & 0.00 & 0 & $0 \%$ & $0 \%$ & 0 & 0 \\
\hline Solid Layer & $4.52 \mathrm{E}+04$ & $3.38 \mathrm{E}+03$ & $1.57 \mathrm{E}+05$ & 1.56E+05 & $2.40 \mathrm{E}+05$ & $4.44 \mathrm{E}+04$ & $1.54 \mathrm{E}+02$ & $2.16 \mathrm{E}-01$ & $1.56 \mathrm{E}-02$ & $7.61 \mathrm{E}-02$ & $2.05 \mathrm{E}-0.3$ & $1.23 \mathrm{E}+02$ & 1.73 & 1.47 & 1926 & $35 \%$ & $47 \%$ & 60 & 27 \\
\hline $24 \mathrm{~J}-\mathrm{SX}-104$ & $3.05 \mathrm{E}+04$ & $2.13 \mathrm{E}+03$ & $1.17 \mathrm{E}+05$ & $2.90 \mathrm{E}+05$ & $2.50 \mathrm{E}+05$ & $3.56 \mathrm{E}+04$ & $1.42 \mathrm{E}+02$ & $1.26 \mathrm{E}-01$ & $3.42 \mathrm{E}-02$ & $1.74 \mathrm{E}-0 \mathrm{I}$ & $3.52 \mathrm{E}-03$ & $8.70 E+01$ & 1.69 & 1.47 & 1688 & $25 \%$ & $45 \%$ & 54 & 35 \\
\hline Supernatant & $0.00 E+00$ & $0.00 \mathrm{E}+00$ & $0.00 \mathrm{E}+00$ & $0.00 \mathrm{E}+00$ & $0.00 \mathrm{E}+00$ & $0.00 \mathrm{E}+00$ & $0.00 \mathrm{E}^{+}+00$ & $0.00 \mathrm{E}+00$ & $0.00 \mathrm{E}+00$ & $0.00 \mathrm{E}+00$ & $0.00 \mathrm{E}+00$ & $0.00 \mathrm{E}+00$ & 0.00 & 0.00 & 0 & $0 \%$ & $0 \%$ & 0 & 0 \\
\hline Solid Layer & $3.05 \mathrm{E}+04$ & $2.13 \mathrm{E}+03$ & $1.17 \mathrm{E}+05$ & $2.90 E+05$ & $2.50 \mathrm{E}+05$ & $3.56 \mathrm{E}+04$ & $1.42 \mathrm{E}+02$ & $1.26 \mathrm{E}-01$ & $3.42 \mathrm{E}-02$ & $1.74 \mathrm{E}-01$ & $3.52 \mathrm{E}-03$ & $8.70 \mathrm{E}+01$ & 1.69 & 1.47 & 1688 & $25 \%$ & $45 \%$ & 54 & 35 \\
\hline $241-5 X-105$ & $4.10 \mathrm{E}+04$ & $3.26 \mathrm{E}+03$ & $1.43 \mathrm{E}+05$ & $1.64 E+05$ & $2.36 \mathrm{E}+05$ & $4.41 E+04$ & $2.52 \mathrm{E}+02$ & $2.57 \mathrm{E}-01$ & $5.74 \mathrm{E}-02$ & $2.89 \mathrm{E}-0 \mathrm{~J}$ & $6.66 \mathrm{E}-03$ & $9.47 \mathrm{E}+01$ & 1.63 & 1.47 & 1422 & $35 \%$ & $43 \%$ & 59 & 34 \\
\hline Supernatant & $0.00 \mathrm{E}+00$ & $0.00 \mathrm{E}+00$ & $0.00 \mathrm{E}+00$ & $0.00 \mathrm{E}+00$ & $0.00 \mathrm{E}+00$ & $0.00 \mathrm{E}+00$ & $0.00 \mathrm{E}+00$ & $0.00 \mathrm{E}+00$ & $0.00 \mathrm{E}+00$ & $0.00 \mathrm{E}+00$ & $0.00 \mathrm{E}+00$ & $0.00 \mathrm{E}+00$ & 0.00 & 0.00 & 0 & $0 \%$ & $0 \%$ & 0 & 0 \\
\hline Solid Layer & $4.10 E+04$ & $3.26 \mathrm{E}+03$ & $1.43 E+05$ & $1.64 \mathrm{E}+05$ & $2.36 \mathrm{E}+05$ & $4.41 \mathrm{E}+04$ & $2.52 E+02$ & $2.57 \mathrm{E}-01$ & $5.74 \mathrm{E}-02$ & $2.89 \mathrm{E}-0 \mathrm{I}$ & $6.66 \mathrm{E}-03$ & $9.47 \mathrm{E}+01$ & 1.63 & 1.47 & 1422 & $35 \%$ & $43 \%$ & 59 & 34 \\
\hline $241-S X-106$ & $3.20 \mathrm{E}+04$ & $3.14 \mathrm{E}+03$ & $1.49 \mathrm{E} \div 05$ & $2.45 \mathrm{E}+05$ & $2.17 \mathrm{E} \div 05$ & $2.26 \mathrm{E}+04$ & $1.21 \mathrm{E}+01$ & $1.47 \mathrm{E}-0 !$ & $1.86 \mathrm{E}-02$ & $8.68 \mathrm{E}-02$ & 3.03E-03 & $1.31 E+02$ & 1.58 & 1.29 & 1501 & $40 \%$ & $49 \%$ & 38 & 26 \\
\hline Supernatant & $0.00 \mathrm{E}+00$ & $0.00 \mathrm{E}+00$ & $0.00 \mathrm{E}+00$ & $0.00 \mathrm{E}+00$ & $0.00 \mathrm{E}+00$ & $0.00 \mathrm{E}+00$ & $0.00 \mathrm{E}+00$ & $0.00 \mathrm{E}+00$ & $0.00 \mathrm{E}+00$ & $0.00 \mathrm{E}+00$ & $0.00 \mathrm{E}+00$ & $0.00 E+00$ & 0.00 & 0.00 & 0 & $0 \%$ & $0 \%$ & 0 & 0 \\
\hline Solid Layer & $3.20 \mathrm{E}+04$ & $3.14 \mathrm{E}+03$ & $1.49 \mathrm{E}+05$ & $2.45 \mathrm{E}+05$ & $2.17 \mathrm{E}+05$ & $2.26 \mathrm{E}+04$ & $1.21 E+01$ & $1.47 \mathrm{E}-01$ & $1.86 \mathrm{E}-02$ & $8.68 \mathrm{E}-02$ & $3.03 \mathrm{E}-03$ & $1.31 \mathrm{E}+02$ & 1.58 & 1.29 & 1501 & $40 \%$ & $49 \%$ & 38 & 26 \\
\hline 241-SX-107 & $6.07 \mathrm{E}+04$ & $1.01 E+03$ & $9.86 \mathrm{E}+04$ & $17 \mathrm{E}+05$ & $2.55 \mathrm{E}+05$ & $4.72 E+04$ & $2.00 \mathrm{E}+03$ & $8.87 \mathrm{E}-0 \mathrm{l}$ & $1.08 \mathrm{E}-01$ & $5.19 \mathrm{E}-01$ & $1.87 \mathrm{E}-02$ & $8.20 \mathrm{E}+01$ & 1.77 & 1.50 & 356 & $23 \%$ & $52 \%$ & 70 & 53 \\
\hline Supernatant & $0.00 E+00$ & $0.00 \mathrm{E} \div 00$ & $0.00 E+00$ & $0.00 \mathrm{E}+00$ & $0.00 \mathrm{E}+00$ & $0.00 \mathrm{E}+00$ & $0.00 \mathrm{E}+00$ & $0.00 \mathrm{E} \times 00$ & $0.00 \mathrm{E}+00$ & $0.00 \mathrm{E}+00$ & $0.00 \mathrm{E}+00$ & $0.00 \mathrm{E}+00$ & 0.00 & 0.00 & 0 & $0 \%$ & $0 \%$ & 0 & 0 \\
\hline Solid Layer & $6.07 E \div 04$ & $1.01 \mathrm{E}+03$ & $9.86 \mathrm{E} \div 04$ & $2.17 \mathrm{E}+05$ & $2.55 \mathrm{E}+05$ & $4.72 \mathrm{E} \div 04$ & $2.00 \mathrm{E}+03$ & $8.87 \mathrm{E}-01$ & $1.08 \mathrm{E}-01$ & $5.19 \mathrm{E}-01$ & $1.87 \mathrm{E}-02$ & $8.20 \mathrm{E}+01$ & 1.77 & 1.50 & 356 & $23 \%$ & $52 \%$ & 70 & 53 \\
\hline 241-SX-108 & $6.07 \mathrm{E}+04$ & $2.98 \mathrm{E}+03$ & $3.60 \mathrm{E}+04$ & $96 \mathrm{E}+04$ & $8.32 \mathrm{E}+04$ & $4.94 \mathrm{E}+03$ & +03 & $1.46 \mathrm{E}+00$ & $2.96 \mathrm{E}-01$ & $1.49 E+00$ & $3.78 \mathrm{E}-02$ & $1.65 \mathrm{E}+02$ & 177 & 1.17 & 280 & $2 \%$ & $75 \%$ & 77 & 59 \\
\hline Supernatant & $0.00 \mathrm{E}+00$ & $0.00 \mathrm{E}+00$ & $0.00 \mathrm{E}+00$ & $0.00 \mathrm{E}+00$ & $0.00 \mathrm{E}+00$ & $0.00 \mathrm{E}+00$ & $0.00 \mathrm{E}+00$ & $0.00 \mathrm{E}+00$ & $0.00 \mathrm{E}+00$ & $0.00 \mathrm{E}+00$ & $0.00 \mathrm{E}+00$ & $0.00 \mathrm{E}+00$ & 0.00 & 0.00 & 0 & $0 \%$ & $0 \%$ & 0 & 0 \\
\hline Solid Layer & $6.07 \mathrm{E}+04$ & $2.98 \mathrm{E}+03$ & $3.60 \mathrm{E}+04$ & $7.96 \mathrm{E}+04$ & $8.32 \mathrm{E}+04$ & $4.94 \mathrm{E}+03$ & $2.57 \mathrm{E}+03$ & $1.46 \mathrm{E}+00$ & 2.96E-01 & $1.49 \mathrm{E}+00$ & $3.78 \mathrm{E}-02$ & $1.65 E+02$ & 1.77 & 1.17 & 280 & $2 \%$ & $75 \%$ & 77 & 59 \\
\hline 241-SX-109 & $6.07 \mathrm{E}+04$ & $9.20 \mathrm{E}+03$ & $1.07 \mathrm{E}+05$ & $1.76 \mathrm{E}+05$ & $1.98 \mathrm{E}+05$ & $2.75 E+04$ & $5.70 \mathrm{E}+02$ & $2.92 \mathrm{E}-0 \mathrm{I}$ & $3.75 \mathrm{E}-02$ & $1.79 \mathrm{E}-01$ & $6.46 \mathrm{E}-03$ & $7.91 \mathrm{E}+01$ & 17 & 1. & 91 & $23 \%$ & $51 \%$ & 53 & 48 \\
\hline Supematant & $0.00 \mathrm{E}+00$ & $0.00 \mathrm{E}+00$ & $0.00 \mathrm{E}+00$ & $0.00 \mathrm{E}+00$ & $0.00 \mathrm{E}+00$ & $0.00 \mathrm{E}+00$ & $0.00 \mathrm{E}+00$ & $0.00 E+00$ & $0.00 \mathrm{E}+00$ & $0.00 \mathrm{E}+00$ & $0.00 E+00$ & $0.00 \mathrm{E}+00$ & 0.00 & 0.00 & 0 & $0 \%$ & $0 \%$ & 0 & 0 \\
\hline Solid Layer & $6.07 \mathrm{E}+04$ & $9.20 \mathrm{E}+03$ & $1.07 \mathrm{E}+05$ & $1.76 \mathrm{E}+05$ & $1.98 \mathrm{E}+05$ & $2.75 E \div-04$ & $5.70 \mathrm{E}+02$ & $2.92 \mathrm{E}-01$ & $3.75 \mathrm{E}-02$ & $1.79 \mathrm{E}-01$ & $6.46 E-03$ & $7.91 \mathrm{E}+01$ & 1.73 & 1.39 & 913 & $23 \%$ & $51 \%$ & 53 & 48 \\
\hline 241-SX-110 & $6.07 \mathrm{E}+04$ & $2.98 \mathrm{E}+03$ & $3.60 E+04$ & $7.96 \mathrm{E}+04$ & $8.32 \mathrm{E}+04$ & $4.94 \mathrm{E}+03$ & $4.58 \mathrm{E}+03$ & $1.91 \mathrm{E}+00$ & $1.18 \mathrm{E}-01$ & $5.04 \mathrm{E}-01$ & $3.34 \mathrm{E}-02$ & $1.15 \mathrm{E}+02$ & 1.76 & 1.17 & 212 & $23 \%$ & $75 \%$ & 55 & 65 \\
\hline Supenatant & $0.00 \mathrm{E}+00$ & $0.00 \mathrm{E}+00$ & $0.00 \mathrm{E}+00$ & $0.00 \mathrm{E}+00$ & $0.00 E+00$ & $0.00 \mathrm{E}+00$ & $0.00 \mathrm{E}+00$ & $0.00 \mathrm{E}+00$ & $0.00 \mathrm{E}+00$ & $0.00 \mathrm{E}+00$ & $0.00 \mathrm{E}+00$ & $0.00 \mathrm{E}+00$ & 0.00 & 0.00 & 0 & $0 \%$ & $0 \%$ & 0 & 0 \\
\hline Solid Layer & $6.07 \mathrm{E}+04$ & $2.98 \mathrm{E}+03$ & $3,60 \mathrm{E}+04$ & $7.96 \mathrm{E}+04$ & $8.32 \mathrm{E}+04$ & $4.94 E+03$ & $4.58 \mathrm{E}+03$ & $1.91 \mathrm{E}+00$ & $1.18 \mathrm{E}-0 !$ & $5.04 \mathrm{E}-0 \mathrm{I}$ & $3.34 \mathrm{E}-02$ & $1.15 \mathrm{E}+02$ & 1.76 & 1.17 & 212 & $23 \%$ & $75 \%$ & 55 & 65 \\
\hline 241-SX-111 & $6.07 E+04$ & $1.01 \mathrm{E}+03$ & $9.89 \mathrm{E}+04$ & $2.17 \mathrm{E}+05$ & $2.55 \mathrm{E}+05$ & $4.71 E+04$ & $2.63 \mathrm{E}+03$ & $1.14 \mathrm{E}+00$ & $1.01 \mathrm{E}-01$ & $4.64 \mathrm{E}-01$ & $2.17 \mathrm{E}-02$ & $9.25 \mathrm{E}+01$ & 1.76 & 1.50 & 436 & $23 \%$ & $52 \%$ & 65 & 62 \\
\hline Supematant & $0.00 \mathrm{E}+00$ & $0.00 \mathrm{E}+00$ & $0.00 \mathrm{E}+00$ & $0.00 \mathrm{E}+00$ & $0.00 \mathrm{E}+00$ & $0.00 \mathrm{E}+00$ & $0.00 \mathrm{E}+00$ & $0.00 \mathrm{E}+00$ & $0.00 \mathrm{E}+00$ & $0.00 \mathrm{E}+00$ & $0.00 \mathrm{E}+00$ & $0.00 \mathrm{E}+00$ & 0.00 & 0.00 & 0 & $0 \%$ & $0 \%$ & 0 & 0 \\
\hline Solid Layer & $6.07 \mathrm{E}+04$ & $1.01 \mathrm{E}+03$ & $9.89 \mathrm{E}+04$ & $2.17 E+05$ & $2.55 \mathrm{E}+05$ & $4.71 \mathrm{E}+04$ & $2.63 \mathrm{E}+03$ & $1.14 \mathrm{E}+00$ & $1.01 \mathrm{E}-01$ & 4.64E-01 & $2.17 \mathrm{E}-02$ & $9.25 \mathrm{E}+01$ & 1.76 & 1.50 & 436 & $23 \%$ & $52 \%$ & 65 & 62 \\
\hline
\end{tabular}


RPP-5926 REV 6

Table B-1. Input Data for Hydrogen Generation Rate Model Calculations for 177 Tanks. (20 sheets)

\begin{tabular}{|c|c|c|c|c|c|c|c|c|c|c|c|c|c|c|c|c|c|c|c|}
\hline Tank & $\begin{array}{c}\mathrm{OH} \\
\text { in liquid } \\
{[\mathrm{OH}]} \\
(\mu \mathrm{g} / \mathrm{mL})\end{array}$ & $\begin{array}{c}\text { TOC } \\
\text { in liquid } \\
{[\mathrm{TOC}]} \\
(\mu \mathrm{g} / \mathrm{mL})\end{array}$ & $\begin{array}{c}\mathrm{NO}_{2} \\
\text { in liquid } \\
{\left[\mathrm{NO}_{2}\right]} \\
(\mu \mathrm{g} / \mathrm{mL})\end{array}$ & $\begin{array}{c}\mathrm{NO}_{3} \\
\text { in liquid } \\
{\left[\mathrm{NO}_{3}\right]} \\
(\mu \mathrm{g} / \mathrm{mL})\end{array}$ & $\begin{array}{c}\mathrm{Na} \\
\text { in liquid } \\
\text { [Na| } \\
\text { (ug/mL) }\end{array}$ & $\begin{array}{c}\mathrm{Al} \\
\text { in liquid } \\
{[\mathrm{A}]} \\
(\mu \mathrm{g} / \mathrm{mL})\end{array}$ & $\begin{array}{c}{ }^{90} \mathrm{Sr} \\
\text { in waste } \\
{[\mathrm{Sr}]} \\
(\mu \mathrm{Ci} / \mathrm{g})\end{array}$ & $\begin{array}{c}{ }^{241} \mathrm{Am} \\
\text { in waste } \\
{[\mathrm{Am} 241]} \\
(\mu \mathrm{Ci} / \mathrm{g})\end{array}$ & $\begin{array}{c}{ }^{240} \mathrm{Pu} \\
\text { in waste } \\
\mid \mathrm{Pu240|} \\
(\mu \mathrm{Ci} / \mathrm{g})\end{array}$ & $\begin{array}{c}{ }^{239} \mathrm{Pu} \\
\text { in waste } \\
{[\mathrm{Pu} 240]} \\
(\mu \mathrm{Ci} / \mathrm{g})\end{array}$ & $\begin{array}{c}{ }^{238} \mathrm{Pu} \\
\text { in waste } \\
{\left[\begin{array}{c}\mathrm{Pu} 238] \\
(\mu \mathrm{Ci} / \mathrm{g})\end{array}\right.}\end{array}$ & $\begin{array}{c}{ }^{137} \mathrm{Cs} \\
\text { in waste } \\
{[\mathrm{Cs}]} \\
(\mu \mathrm{Ci} / \mathrm{g})\end{array}$ & $\begin{array}{c}\text { Bulk } \\
\text { density } \\
\text { D } \\
(\mathrm{g} / \mathrm{mL})\end{array}$ & $\begin{array}{c}\text { Liquid } \\
\text { Density } \\
\mathrm{D}_{\mathrm{L}} \\
(\mathrm{g} / \mathrm{ml})\end{array}$ & $\begin{array}{c}\text { Non-RGS } \\
\text { waste } \\
\text { volume } \\
\text { (kL) }\end{array}$ & $\begin{array}{l}\text { Bulk } \\
\text { water } \\
{\left[\mathrm{H}_{2} \mathrm{O}\right]} \\
\left(\mathrm{wt}^{\%} \%\right)\end{array}$ & $\begin{array}{l}\text { Liquid } \\
\text { water } \\
{\left[\mathrm{H}_{2} \mathrm{O}\right]} \\
\left(\mathrm{wt}_{\mathrm{t}} \%\right)\end{array}$ & $\begin{array}{c}\text { Waste } \\
\text { temp. } \\
\text { Tw } \\
\text { ('C) }\end{array}$ & $\begin{array}{c}\text { Dome } \\
\text { temp. } \\
\text { Td } \\
\left({ }^{\circ} \mathrm{C}\right)\end{array}$ \\
\hline 241-SX-112 & $6.07 \mathrm{E}+04$ & $1.01 \mathrm{E}+03$ & $9.88 \mathrm{E}+04$ & $2.17 \mathrm{E}+05$ & $2.55 \mathrm{E} \div 05$ & 4. $72 \mathrm{E}+04$ & $2.78 \mathrm{E}+03$ & $1.20 \mathrm{E}+00$ & $1.15 E-01$ & 5.3!E-01 & $2.34 \mathrm{E}-02$ & $9.14 \mathrm{E}+01$ & 1.77 & 1.50 & 283 & $23 \%$ & $52 \%$ & 60 & 52 \\
\hline Supematant & $0.00 \mathrm{E}+00$ & $0.00 \mathrm{E}+00$ & $0.00 \mathrm{E}+00$ & $0.00 \mathrm{E}+00$ & $0.00 \mathrm{E}+00$ & $0.00 \mathrm{E}+00$ & $0.00 \mathrm{E}+00$ & $0.00 \mathrm{E}+00$ & $0.00 \mathrm{E}+00$ & $0.00 \mathrm{E}+00$ & $0.00 \mathrm{E}+00$ & $0.00 \mathrm{E}+00$ & 0.00 & 0.00 & 0 & $0 \%$ & $0 \%$ & 0 & 0 \\
\hline Solid Layer & $6.07 E+04$ & $1.01 E+03$ & $9.88 \mathrm{E}+04$ & $2.17 \mathrm{E}+05$ & $2.55 \mathrm{E}+0.5$ & $4.72 \mathrm{E}+04$ & $2.78 \mathrm{E}+03$ & $1.20 \mathrm{E}+00$ & $1.15 \mathrm{E}-01$ & $5.31 \mathrm{E}-01$ & $2.34 \mathrm{E}-02$ & $9.14 \mathrm{E}+01$ & 1.77 & 1.50 & 283 & $23 \%$ & $52 \%$ & 60 & 52 \\
\hline $241-S X-113$ & $6.07 \mathrm{E}+04$ & $2.98 \mathrm{E}+03$ & $3.60 \mathrm{E}+04$ & $7.96 \mathrm{E}+04$ & $8.32 E+04$ & $4.94 \mathrm{E}+03$ & $2.75 \mathrm{E}+01$ & $1.09 \mathrm{E}-01$ & $9.47 \mathrm{E}-03$ & $4.88 \mathrm{E}-02$ & $9.06 \mathrm{E}-04$ & $2.02 \mathrm{E}+01$ & 1.43 & 1.17 & 72 & $46 \%$ & $75 \%$ & 28 & 28 \\
\hline Supernatant & $0.00 \mathrm{E}+00$ & $0.00 \mathrm{E}+00$ & $0.00 \mathrm{E}+00$ & $0.00 \mathrm{E}+00$ & $0.00 \mathrm{E}+00$ & $0.00 \mathrm{E}+00$ & $0.00 \mathrm{E}+00$ & $0.00 \mathrm{E}+00$ & $0.00 \mathrm{E}+00$ & $0.00 \mathrm{E}+00$ & $0.00 \mathrm{E}+00$ & $0.00 \mathrm{E}+00$ & 0.00 & 0.00 & 0 & $0 \%$ & $0 \%$ & 0 & 0 \\
\hline Solid Layer & $6.07 \mathrm{E}+04$ & $2.98 E+03$ & $3.60 \mathrm{E}+04$ & $7.96 \mathrm{E}+04$ & $8.32 E+04$ & $4.94 E+03$ & $2.75 \mathrm{E}+01$ & $1.09 \mathrm{E}-01$ & $9.47 \mathrm{E}-03$ & $4.88 \mathrm{E}-02$ & $9.06 \mathrm{E}-04$ & $2.02 \mathrm{E}+01$ & 1.43 & 1.17 & 72 & $46 \%$ & $75 \%$ & 28 & 28 \\
\hline $241-S X-114$ & $6.07 \mathrm{E}+04$ & $1.01 E+03$ & $9.88 \mathrm{E}+04$ & $2.17 \mathrm{E}+05$ & $2.51 \mathrm{E}+05$ & $4.73 \mathrm{E}+04$ & $1.84 \mathrm{E}+03$ & $15 \mathrm{E}-01$ & $9.20 \mathrm{E}-02$ & $4.37 \mathrm{E}-01$ & $1.67 \mathrm{E}-02$ & $8.77 \mathrm{E}+01$ & 1.75 & 1.50 & 588 & $24 \%$ & $52 \%$ & 72 & 52 \\
\hline Supernatant & $0.00 E+00$ & $0.00 \mathrm{E}+00$ & $0.00 \mathrm{E}+00$ & $0.00 \mathrm{E}+00$ & $0.00 \mathrm{E}+00$ & $0.00 \mathrm{E}+00$ & $0.00 \mathrm{E}+00$ & $0.00 \mathrm{E}+00$ & $0.00 \mathrm{E}+00$ & $0.00 \mathrm{E}+00$ & $0.00 \mathrm{E}+00$ & $0.00 \mathrm{E}+00$ & 0.00 & 0.00 & 0 & $0 \%$ & $0 \%$ & 0 & 0 \\
\hline Solid Layer & $6.07 \mathrm{E}+04$ & $1.01 \mathrm{E}+03$ & $9.88 \mathrm{E}+04$ & $2.17 \mathrm{E}+05$ & $2.51 \mathrm{E}+05$ & $4.73 \mathrm{E}+04$ & $1.84 \mathrm{E}+03$ & $8.15 \mathrm{E}-01$ & $9.20 \mathrm{E}-02$ & $4.37 \mathrm{E}-01$ & $1.67 \mathrm{E}-02$ & $8.77 \mathrm{E}+01$ & 1.75 & 1.50 & 588 & $24 \%$ & $52 \%$ & 72 & 52 \\
\hline $241-S X-115$ & $6.07 \mathrm{E}+04$ & $2.98 \mathrm{E}+03$ & $3.60 \mathrm{E}+04$ & $7.96 \mathrm{E}+04$ & $8.32 E+04$ & $4.94 \mathrm{E}+03$ & $1.35 E+04$ & $.42 \mathrm{E}+01$ & $3.78 \mathrm{E}+00$ & $1.61 \mathrm{E}+01$ & $1.07 \mathrm{E}+00$ & $2.05 \mathrm{E}+01$ & 1.77 & 1.17 & 16 & $10 \%$ & $75 \%$ & 25 & 25 \\
\hline Supernatant & $0.00 \mathrm{E}+00$ & $0.00 \mathrm{E}+00$ & $0.00 \mathrm{E}+00$ & $0.00 \mathrm{E}+00$ & $0.00 \mathrm{E}+00$ & $0.00 \mathrm{E}+00$ & $0.00 \mathrm{E}+00$ & $0.00 \mathrm{E} \div 00$ & $0.00 \mathrm{E}+00$ & $0.00 \mathrm{E}+00$ & $0.00 \mathrm{E}+00$ & $0.00 \mathrm{E}+00$ & 0.00 & 0.00 & 0 & $0 \%$ & $0 \%$ & 0 & 0 \\
\hline Sotid Layer & $6.07 \mathrm{E}+04$ & $2.98 \mathrm{E}+03$ & $3.60 \mathrm{E}+04$ & $7.96 \mathrm{E}+04$ & $8.32 E+04$ & $4.94 \mathrm{E}+03$ & $1.35 \mathrm{E} \div 04$ & $1.42 \mathrm{E}+01$ & $3.78 \mathrm{E}+00$ & $1.61 \mathrm{E}+01$ & $1.07 \mathrm{E}+00$ & $2.05 \mathrm{E}+01$ & 1.77 & 1.17 & 16 & $10 \%$ & $75 \%$ & 25 & 25 \\
\hline 241-T-101 & $4.81 E+04$ & $4.04 \mathrm{E}+03$ & $1.22 \mathrm{E}+05$ & $1.89 E+05$ & $2.37 \mathrm{E}+05$ & $3.77 \mathrm{E}+04$ & $6.63 \mathrm{E}-01$ & $1.15 \mathrm{E}-01$ & $7.84 \mathrm{E}-02$ & $3.58 \mathrm{E}-01$ & $9.37 \mathrm{E}-03$ & $6.76 \mathrm{E}+01$ & 1.54 & 1.45 & 376 & $39 \%$ & $49 \%$ & 21 & 22 \\
\hline Supernatant & $0.00 \mathrm{E}+00$ & $0.00 \mathrm{E}+00$ & $0.00 \mathrm{E}+00$ & $0.00 \mathrm{E}+00$ & $0.00 \mathrm{E}+00$ & $0.00 \mathrm{E}+00$ & $0.00 \mathrm{E}+00$ & $0.00 E+00$ & $0.00 \mathrm{E}+00$ & $0.00 \mathrm{E}+00$ & $0.00 \mathrm{E}+00$ & $0.00 \mathrm{E}+00$ & 0.00 & 0.00 & 0 & $0 \%$ & $0 \%$ & 0 & 0 \\
\hline Solid Layer & $4.81 \mathrm{E}+04$ & $4.04 \mathrm{E}+03$ & 1.22E+05 & $1.89 \mathrm{E}+05$ & $2.37 \mathrm{E}+05$ & $3.77 \mathrm{E}+04$ & $6.63 \mathrm{E}-01$ & $1.15 \mathrm{E}-01$ & $7.84 \mathrm{E}-02$ & $3.58 \mathrm{E}-01$ & $9.37 \mathrm{E}-03$ & $6.76 \mathrm{E}+0 \mathrm{t}$ & 1.54 & 1.45 & 376 & $39 \%$ & $49 \%$ & 21 & 22 \\
\hline 241-T-102 & $6.25 \mathrm{E}+0 !$ & $4.50 \mathrm{E}+02$ & $2.29 \mathrm{E}+04$ & $1.09 \mathrm{E}+05$ & $6.46 \mathrm{E}+04$ & $8.46 \mathrm{E}+03$ & $7.74 \mathrm{E}+0$ & $9.99 \mathrm{E}-02$ & $4.55 \mathrm{E}-03$ & $1.95 \mathrm{E}-02$ & $4.87 \mathrm{E}-04$ & $2.79 \mathrm{E}+01$ & 1.53 & 1.14 & 120 & $45 \%$ & $80 \%$ & 18 & 18 \\
\hline Supematant & $6.25 E+01$ & $4.50 \mathrm{E}+02$ & $2.29 \mathrm{E}+04$ & $1.09 \mathrm{E}+05$ & $6.46 \mathrm{E}+04$ & $8.46 \mathrm{E}+03$ & $8.55 \mathrm{E}-01$ & $3.75 \mathrm{E}-03$ & $1.04 \mathrm{E}-03$ & $4.48 \mathrm{E}-03$ & $1.88 \mathrm{E}-04$ & $4.51 \mathrm{E}+01$ & 1.14 & 1.14 & 48 & $80 \%$ & $80 \%$ & 18 & 18 \\
\hline Solid Layer & $6.25 E+01$ & $4.50 \mathrm{E}+02$ & $2.29 \mathrm{E}+04$ & $1.09 \mathrm{E}+05$ & 6 & 8.46 & $8 E+02$ & 1.64 & $6.89 \mathrm{E}-03$ & 2.95 & 6.86 & $1.64 \mathrm{I}$ & 1.80 & 14 & 7 & $30 \%$ & $80 \%$ & 18 & 18 \\
\hline $241-T-103$ & $1.70 \mathrm{E}+04$ & $5.55 \mathrm{E}+03$ & $3.96 \mathrm{E}+04$ & $7.80 \mathrm{E}+04$ & $9.53 E+04$ & $1.04 \mathrm{E}+04$ & $4.07 \mathrm{E}+00$ & 4.04E-03 & $5.10 \mathrm{E}-03$ & $2.18 \mathrm{E}-02$ & $5.24 \mathrm{E}-04$ & $2.82 \mathrm{E}+00$ & 1.64 & 1.19 & 102 & $34 \%$ & $72 \%$ & 19 & 20 \\
\hline Supenaatant & $1.70 \mathrm{E}+04$ & $5.55 \mathrm{E}+03$ & $3.96 \mathrm{E}+04$ & $7.80 \mathrm{E}+04$ & $9.53 E+04$ & $1.04 \mathrm{E}+04$ & $1.94 \mathrm{E}+00$ & $8.57 \mathrm{E}-03$ & $1.48 E-03$ & $6.39 \mathrm{E}-03$ & $2.68 \mathrm{E}-04$ & $1.18 \mathrm{E}+01$ & 1.19 & 1.19 & 15 & $72 \%$ & $72 \%$ & 19 & 20 \\
\hline Solid Layer & $1.70 \mathrm{~F}+04$ & $5.55 \mathrm{E}+03$ & $3.96 \mathrm{E}+04$ & $7.80 \mathrm{E}+04$ & $9.53 E+04$ & $1.04 \mathrm{E}+04$ & $4.43 E+00$ & $3.26 \mathrm{E}-03$ & 5.72E-03 & $2.44 \mathrm{E}-02$ & $5.68 \mathrm{E}-04$ & $1.27 \mathrm{E}+00$ & 1.71 & 1.18 & 87 & $29 \%$ & $72 \%$ & 19 & 20. \\
\hline 241-T-104 & $1.70 \mathrm{E}+04$ & $2.98 \mathrm{E}+03$ & $3.60 \mathrm{E}+04$ & $7.96 E+04$ & $8.32 \mathrm{E}+04$ & $4.94 E+03$ & $2.03 \mathrm{E}+00$ & $1.81 \mathrm{E}-02$ & 1.5IE-02 & $1.25 \mathrm{E}-01$ & $1.62 \mathrm{E}-03$ & $1.55 \mathrm{E}-01$ & 1.29 & 1.17 & 1199 & $71 \%$ & $84 \%$ & 19 & 22 \\
\hline Supeniatant & $0.00 \mathrm{E}+00$ & $0.00 \mathrm{E}+00$ & $0.00 \mathrm{E}+00$ & $0.00 \mathrm{E}+00$ & $0.00 \mathrm{E}+00$ & $0.00 \mathrm{E}+00$ & $0.00 \mathrm{E}+00$ & $0.00 \mathrm{E}+00$ & $0.00 \mathrm{E}+00$ & $0.00 \mathrm{E}+00$ & $0.00 \mathrm{E}+00$ & $0.00 \mathrm{E}+00$ & 0.00 & 0.00 & 0 & $0 \%$ & $0 \%$ & 0 & 0 \\
\hline Solid Layer & $1.70 \mathrm{E}+04$ & $2.98 \mathrm{E}+03$ & $3.60 \mathrm{E}+04$ & $7.96 \mathrm{E}+04$ & $8.32 \mathrm{E}+04$ & $4.94 \mathrm{E}+03$ & $2.03 \mathrm{E}+00$ & $1.81 \mathrm{E}-02$ & $1.51 \mathrm{E}-02$ & $1.25 \mathrm{E}-01$ & $1.62 \mathrm{E}-03$ & $1.55 \mathrm{E}-0 \mathrm{t}$ & 1.29 & 1.17 & 1199 & $71 \%$ & $84 \%$ & 19 & 22 \\
\hline $241-T-105$ & $1.70 \mathrm{E}+04$ & $2.98 \mathrm{E}+03$ & $5.42 E+04$ & $3.71 \mathrm{E}+04$ & $8.84 E+04$ & $3.62 \mathrm{E}+02$ & $5.19 \mathrm{E}+01$ & $5.81 \mathrm{E}-02$ & $2.93 \mathrm{E}-02$ & $2.77 \mathrm{E}-01$ & $2.08 \mathrm{E}-03$ & $9.23 E+00$ & 1.46 & 1.18 & 371 & $51 \%$ & $80 \%$ & 17 & 16 \\
\hline Supematant & $0.00 \mathrm{E}+00$ & $0.00 \mathrm{E}+00$ & $0.00 \mathrm{E}+00$ & $0.00 \mathrm{E}+00$ & $0.00 E+00$ & $0.00 \mathrm{E}+00$ & $0.00 \mathrm{E}+00$ & $0.00 \mathrm{E}+00$ & $0.00 \mathrm{E}+00$ & $0.00 \mathrm{E}+00$ & $0.00 \mathrm{E}+00$ & $0.00 \mathrm{E}+00$ & 0.00 & 0.00 & 0 & $0 \%$ & $0 \%$ & 0 & 0 \\
\hline Solid Layer & $1.70 \mathrm{E}+04$ & $2.98 \mathrm{E}+03$ & $5.42 \mathrm{E}+04$ & $3.71 E+04$ & $8.84 \mathrm{E}+04$ & $3.62 \mathrm{E}+02$ & $5.19 \mathrm{E}+01$ & $5.81 \mathrm{E}-02$ & $2.93 \mathrm{E}-02$ & $2.77 \mathrm{E}-01$ & $2.08 \mathrm{E}-03$ & $9.23 \mathrm{E}+00$ & 1.46 & 1.18 & 371 & $51 \%$ & $80 \%$ & 17 & 16 \\
\hline
\end{tabular}




\section{RPP-5926 REV 6}

Table B-1. Input Data for Hydrogen Generation Rate Model Calculations for 177 Tanks. (20 sheets)

\begin{tabular}{|c|c|c|c|c|c|c|c|c|c|c|c|c|c|c|c|c|c|c|c|}
\hline Tank & $\begin{array}{c}\text { OH } \\
\text { in liquid } \\
\text { |OH| } \\
(\mu \mathrm{g} / \mathrm{mL})\end{array}$ & $\begin{array}{c}\text { TOC } \\
\text { in liquid } \\
\text { |TOC| } \\
(\mu \mathrm{g} / \mathrm{mL})\end{array}$ & $\begin{array}{c}\mathrm{NO}_{2} \\
\text { in liquid } \\
{\left[\mathrm{NO}_{2}\right]} \\
(\mu \mathrm{g} / \mathrm{mL})\end{array}$ & $\begin{array}{c}\mathrm{NO}_{3} \\
\text { in liquid } \\
{\left[\mathrm{NO}_{3}\right]} \\
(\mu \mathrm{g} / \mathrm{mL})\end{array}$ & $\begin{array}{c}\mathrm{Na} \\
\text { in liquid } \\
{[\mathrm{Na}]} \\
(\mathrm{ng} / \mathrm{mL})\end{array}$ & $\begin{array}{c}\mathrm{Al} \\
\text { in Jiquid } \\
{[\mathrm{AI}]} \\
(\mu \mathrm{g} / \mathrm{mL})\end{array}$ & $\begin{array}{c}{ }^{90} \mathrm{Sr} \\
\text { in waste } \\
{[\mathrm{Sr}]} \\
(\mu \mathrm{Ci} / \mathrm{g})\end{array}$ & $\begin{array}{c}{ }^{24 t} \mathrm{Am} \\
\text { in waste } \\
\text { [Am241] } \\
(\mu \mathrm{Ci} / \mathrm{g})\end{array}$ & $\begin{array}{l}{ }^{240} \mathrm{Pu} \\
\text { in waste } \\
{[\mathrm{Pu} 240]} \\
(\mu \mathrm{Ci} / \mathrm{g})\end{array}$ & $\begin{array}{c}{ }^{239} \mathrm{Pu} \\
\text { in waste } \\
{\left[\begin{array}{c}\mathrm{Pu} 240] \\
(\mu \mathrm{Ci} / \mathrm{g})\end{array}\right.}\end{array}$ & $\begin{array}{c}{ }^{238} \mathrm{Pu} \\
\text { in waste } \\
{[\mathrm{Pu} 238]} \\
(\mu \mathrm{Ci} / \mathrm{g})\end{array}$ & $\begin{array}{c}{ }^{137} \mathrm{Cs} \\
\text { in waste } \\
|\mathrm{Cs}| \\
(\mu \mathrm{Ci} / \mathrm{g})\end{array}$ & $\begin{array}{c}\text { Bulk } \\
\text { density } \\
\text { D } \\
(\mathrm{g} / \mathrm{mL})\end{array}$ & $\begin{array}{c}\text { Liquid } \\
\text { Density } \\
D_{\mathrm{L}} \\
(\mathrm{g} / \mathrm{ml})\end{array}$ & $\begin{array}{c}\text { Non-KGS } \\
\text { waste } \\
\text { volume } \\
(k L)\end{array}$ & $\begin{array}{l}\text { Bulk } \\
\text { water } \\
{\left[\mathrm{H}_{2} \mathrm{O}\right]} \\
(\mathrm{wt} \%)\end{array}$ & $\begin{array}{c}\text { Liquid } \\
\text { water } \\
{\left[\mathrm{H}_{2} \mathrm{O}\right]} \\
(\mathrm{wt} \%)\end{array}$ & $\begin{array}{c}\text { Waste } \\
\text { temp. } \\
T_{w} \\
\left({ }^{\circ} \mathrm{C}\right)\end{array}$ & $\begin{array}{l}\text { Dome } \\
\text { temp. } \\
\mathrm{Td} \\
\left({ }^{\circ} \mathrm{C}\right)\end{array}$ \\
\hline 241-T-106 & $0 E+04$ & $2.98 \mathrm{E}+03$ & $60 E+04$ & $7.96 \mathrm{E}+04$ & $.32 E+04$ & $94 E+03$ & $.02 E+00$ & 4.27E-02 & $2.00 \mathrm{E}-02$ & $1.34 \mathrm{E}-01$ & $1.61 \mathrm{E}-03$ & $1.53 \mathrm{E}+0 \mathrm{l}$ & 1.59 & 1.17 & 82 & $17 \%$ & $75 \%$ & 20 & 21 \\
\hline Supentatant & $0.00 \mathrm{E}+00$ & $0.00 \mathrm{E}+00$ & $0.00 \mathrm{E}+00$ & $0.00 \mathrm{E}+00$ & $0.00 \mathrm{E}+00$ & $0.00 \mathrm{E}+00$ & $0.00 \mathrm{E} \div 00$ & $0.00 \mathrm{E}+00$ & $0.00 \mathrm{E}+00$ & $0.00 \mathrm{E}+00$ & $0.00 \mathrm{E}+00$ & $0.00 \mathrm{E}+00$ & 0.00 & 0.00 & 0 & $0 \%$ & $0 \%$ & 0 & 0 \\
\hline Solid Layer & $1.70 \mathrm{E}+04$ & $2.98 E+03$ & $3.60 \mathrm{E}+04$ & $7.96 \mathrm{E}+04$ & $8.32 \mathrm{E}+04$ & $4.94 \mathrm{E}+03$ & $5.02 \mathrm{E}+00$ & $4.27 \mathrm{E}-02$ & $2.00 \mathrm{E}-02$ & $1.34 \mathrm{E}-01$ & 1.61E-03 & $1.53 \mathrm{E}+01$ & 1.59 & 1.17 & 82 & $17 \%$ & $75 \%$ & 20 & 21 \\
\hline $241-T-107$ & $1.70 \mathrm{E}+04$ & $2.98 \mathrm{E}+03$ & $3.60 \mathrm{E}+04$ & $7.96 \mathrm{E}+04$ & $8.32 E+04$ & $4.94 \mathrm{E}+03$ & $1.01 \mathrm{E}+02$ & $5.89 \mathrm{E}-02$ & $3.48 \mathrm{E}-02$ & $3.04 \mathrm{E}-01$ & $2.37 \mathrm{E}-03$ & $1.47 \mathrm{E}+01$ & 1.56 & 1.17 & 655 & $46 \%$ & $84 \%$ & 20 & 22 \\
\hline Supeniatant & $0.00 \mathrm{E}+00$ & $0.00 \mathrm{E}+00$ & $0.00 \mathrm{E}+00$ & $0.00 \mathrm{E}+00$ & $0.00 \mathrm{E}+00$ & $0.00 \mathrm{E}+00$ & $0.00 E+00$ & $0.00 \mathrm{E}+00$ & $0.00 \mathrm{E}+00$ & $0.00 \mathrm{E}+00$ & $0.00 \mathrm{E}+00$ & $0.00 \mathrm{E}+00$ & 0.00 & 0.00 & 0 & $0 \%$ & $0 \%$ & 0 & 0 \\
\hline Solid Layer & $1.70 \mathrm{E}+04$ & $2.98 \mathrm{E}+03$ & $3.60 \mathrm{E}+04$ & $7.96 \mathrm{E}+04$ & $8.32 \mathrm{E}+04$ & $4.94 \mathrm{E}+03$ & $1.01 \mathrm{E}+02$ & $5.89 \mathrm{E}-02$ & $3.48 \mathrm{E}-02$ & $3.04 \mathrm{E}-0 !$ & $2.37 \mathrm{E}-03$ & $1.47 \mathrm{E}+0 \mathrm{l}$ & 1.56 & 1.17 & 655 & $46 \%$ & $84 \%$ & 20 & 22 \\
\hline $241-T-108$ & $10 \mathrm{E}+04$ & $3.38 \mathrm{E}+00$ & $8.50 \mathrm{E}+03$ & $2.17 \mathrm{E}+05$ & $1.13 \mathrm{E}+05$ & $3.61 \mathrm{E}+02$ & $77 \mathrm{E}+00$ & $4.65 \mathrm{E}-03$ & $6.74 \mathrm{E}-03$ & $6.05 \mathrm{E}-02$ & $.41 \mathrm{E}-04$ & $6.77 \mathrm{E}+00$ & 1.55 & 1.26 & 60 & $35 \%$ & $70 \%$ & 16 & 23 \\
\hline Supernatant & $0.00 E+00$ & $0.00 \mathrm{E}+00$ & $0.00 E+00$ & $0.00 \mathrm{E}+00$ & $0.00 E+00$ & $0.00 \mathrm{E}+00$ & $0.00 \mathrm{E}+00$ & $0.00 \mathrm{E}+00$ & $0.00 \mathrm{E} \div 00$ & $0.00 \mathrm{E}+00$ & $0.00 \mathrm{E}+00$ & $0.00 \mathrm{E}+00$ & 0.00 & 0.00 & 0 & $0 \%$ & $0 \%$ & 0 & 0 \\
\hline Solid Layer & $1.70 \mathrm{E}+04$ & $3.38 \mathrm{E}+00$ & $8.50 \mathrm{E}+03$ & $2.17 \mathrm{E}+05$ & $1.13 E+05$ & $3.61 E+02$ & $1.77 \mathrm{E}+00$ & 4.65E-03 & $6.74 \mathrm{E}-03$ & $6.05 \mathrm{E}-02$ & $4.41 E-04$ & $6.77 \mathrm{E}+00$ & 1.55 & 1.26 & 60 & $35 \%$ & $70 \%$ & 16 & 23 \\
\hline $241-T-109$ & $70 \mathrm{E}+04$ & $3.37 \mathrm{E}+00$ & $8.50 \mathrm{E}+03$ & $2.17 \mathrm{E}+05$ & $3.13 \mathrm{E}+05$ & $3.61 \mathrm{E}+02$ & $2.62 \mathrm{E}-01$ & $2.10 \mathrm{E}-04$ & $1.04 \mathrm{E}-03$ & $9.12 \mathrm{E}-03$ & $.79 \mathrm{E}-05$ & $2.13 E+00$ & 1.65 & 1.26 & 235 & $51 \%$ & $70 \%$ & 19 & 20 \\
\hline Supenatant & $0.00 \mathrm{E}+00$ & $0.00 E+00$ & $0.00 \mathrm{E}+00$ & $0.00 \mathrm{E}+00$ & $0.00 \mathrm{E}+00$ & $0.00 \mathrm{E}+00$ & $0.00 \mathrm{E}+00$ & $0.00 \mathrm{E}+00$ & $0.00 \mathrm{E}+00$ & $0.00 \mathrm{E}+00$ & $0.00 \mathrm{E}+00$ & $0.00 \mathrm{E}+00$ & 0.00 & 0.00 & 0 & $0 \%$ & $0 \%$ & 0 & 0 \\
\hline Solid Laycr & $1.70 E+04$ & $3.37 \mathrm{E}+00$ & $8.50 \mathrm{E}+03$ & $2.17 \mathrm{E}+05$ & $1.13 E+05$ & $3.61 \mathrm{E}+02$ & 2.62E-0I & $2.10 \mathrm{E}-04$ & $1.04 \mathrm{E}-03$ & $9.12 \mathrm{E}-03$ & $6.79 \mathrm{E}-05$ & $2.13 \mathrm{E}+00$ & 1.65 & 1.26 & 235 & $51 \%$ & $70 \%$ & 19 & 20 \\
\hline $24 !-T-110$ & $1.70 \mathrm{E}+04$ & $4.50 \mathrm{E}+01$ & $5.90 \mathrm{E}+0 \mathrm{i}$ & $1.83 \mathrm{E}+04$ & $3.70 \mathrm{E}+04$ & $0.00 \mathrm{E}+00$ & $2.72 \mathrm{E}-02$ & $4.93 \mathrm{E}-03$ & $5.87 \mathrm{E}-03$ & $5.60 \mathrm{E}-02$ & $3.74 \mathrm{E}-04$ & $1.49 \mathrm{E}-02$ & 1.25 & 1.05 & 1400 & $76 \%$ & $87 \%$ & 17 & 21 \\
\hline Supernatant & $1.70 \mathrm{E}+04$ & $4.50 \mathrm{E}+01$ & $5.90 \mathrm{E}+01$ & $1.83 \mathrm{E}+04$ & $3.70 E+04$ & $0.00 E+00$ & $4.29 \mathrm{E}-04$ & $2.86 \mathrm{E}-06$ & $9.97 \mathrm{E}-06$ & $1.48 E-04$ & $1.90 \mathrm{E}-06$ & $2.79 \mathrm{E}-03$ & 1.05 & 1.05 & 3 & $87 \%$ & $87 \%$ & 19 & 21 \\
\hline Solid Layer & $1.70 \mathrm{E}+04$ & $4.50 \mathrm{E}+01$ & $5.90 \mathrm{E}+01$ & $1.83 \mathrm{E}+04$ & $3.70 \mathrm{E}+04$ & $0.00 \mathrm{E}+00$ & $2.73 \mathrm{E}-02$ & $4.94 \mathrm{E}-03$ & $5.88 \mathrm{E}-03$ & $5.61 \mathrm{E}-02$ & $3.75 \mathrm{E}-04$ & $1.49 \mathrm{E}-02$ & 1.25 & 1.05 & 1397 & $76 \%$ & $87 \%$ & 17 & 21 \\
\hline 24I-T-111 & $1.70 E^{+}+04$ & $2.98 E+03$ & $3.60 \mathrm{E}+04$ & $7.96 \mathrm{E}+04$ & $8.32 \mathrm{E}+04$ & $4.94 \mathrm{E}+03$ & $4.08 \mathrm{E}+00$ & $4.70 \mathrm{E}-02$ & $1.44 \mathrm{E}-02$ & $3.24 \mathrm{E}-01$ & $6.19 \mathrm{E}-04$ & $1.02 E-01$ & 1.24 & 1.17 & 1691 & $75 \%$ & $75 \%$ & 18 & 22 \\
\hline Supernatant & $0.00 \mathrm{E}+00$ & $0.00 \mathrm{E}+00$ & $0.00 \mathrm{E}+00$ & $0.00 \mathrm{E}+00$ & $0.00 \mathrm{E}+00$ & $0.00 \mathrm{E}+00$ & $0.00 \mathrm{E}+00$ & $0.00 \mathrm{E}+00$ & $0.00 \mathrm{E}+00$ & $0.00 \mathrm{E}+00$ & $0.00 \mathrm{E}+00$ & $0.00 \mathrm{E}+00$ & 0.00 & 0.00 & 0 & $0 \%$ & $0 \%$ & 0 & 0 \\
\hline Solid Layer & $1.70 \mathrm{E}+04$ & $2.98 \mathrm{E}+03$ & $3.60 \mathrm{E}+04$ & $7.96 \mathrm{E}+04$ & $8.32 \mathrm{E}+04$ & $4.94 E+03$ & $4.08 \mathrm{E}+00$ & $4,70 \mathrm{E}-02$ & $1.44 \mathrm{E}-02$ & $1.24 \mathrm{E}-01$ & $6.19 \mathrm{E}-04$ & $1.02 \mathrm{E}-01$ & 1.24 & 1.17 & 1691 & $75 \%$ & $75 \%$ & 18 & 22 \\
\hline $24 \mid-T-112$ & $1.70 \mathrm{E}+04$ & $1.6 ! E+03$ & $3.88 \mathrm{E}+04$ & $2.33 \mathrm{E}+04$ & $5.72 \mathrm{E}+04$ & $5.04 \mathrm{E}+00$ & $6.46 \mathrm{E}-02$ & $1.47 E-02$ & $2.18 \mathrm{E}-02$ & $1.93 \mathrm{E}-01$ & $1.45 \mathrm{E}-03$ & $3.86 \mathrm{E}-01$ & 1.26 & 1.10 & 252 & $75 \%$ & $85 \%$ & 19 & 20 \\
\hline Supernatant & $1.70 E+04$ & $1.61 \mathrm{E} \div 03$ & $3.88 \mathrm{E}+04$ & $2.33 \mathrm{E}+04$ & $5.72 \mathrm{E}+04$ & $5.04 \mathrm{E}+00$ & $6.08 \mathrm{E}-01$ & $1.03 E-02$ & $1.78 \mathrm{E}-03$ & $7.69 \mathrm{E}-03$ & $3.22 \mathrm{E}-04$ & $3.71 E+00$ & 1.10 & 1.10 & 26 & $85 \%$ & $85 \%$ & 19 & 20 \\
\hline Solid Layer & $1.70 \mathrm{E} \div 04$ & $1.61 \mathrm{E}+03$ & $3.88 \mathrm{E}+04$ & $2.33 \mathrm{E} \div 04$ & $5.72 E+04$ & $5.04 \mathrm{E}+00$ & $2.00 \mathrm{E}-03$ & $1.52 \mathrm{E}-02$ & $2.41 \mathrm{E}-02$ & $2.14 \mathrm{E}-01$ & $1.58 \mathrm{E}-03$ & $3.85 \mathrm{E}-03$ & 1.28 & 1.10 & 226 & $74 \%$ & $85 \%$ & 19 & 20 \\
\hline $24 !-T-20 !$ & $1.70 \mathrm{E}+04$ & $7.78 \mathrm{E}+01$ & $3.35 \mathrm{E}+02$ & $4.61 \mathrm{E}+04$ & $2.51 \mathrm{E}+04$ & $0.00 E+00$ & $6.27 \mathrm{E}-02$ & $3.69 \mathrm{E}-02$ & $4.21 \mathrm{E}-02$ & $6.24 \mathrm{E}-01$ & $2.11 \mathrm{E}-03$ & $2.06 \mathrm{E}-02$ & 1.29 & 1.06 & 115 & $69 \%$ & $91 \%$ & 17 & 20 \\
\hline Supernatant & $1.70 \mathrm{E}+04$ & $7.78 E+01$ & $3.35 \mathrm{E}+02$ & $4.61 \mathrm{E}+04$ & $2.51 \mathrm{E}+04$ & $0.00 \mathrm{E}+00$ & $3.54 \mathrm{E}-05$ & $5.57 \mathrm{E}-08$ & $1.67 \mathrm{E}-06$ & $2.49 \mathrm{E}-05$ & $8.41 \mathrm{E}-08$ & $4.07 \mathrm{E}-05$ & 1.06 & 1.06 & 8 & $91 \%$ & $91 \%$ & 19 & 20 \\
\hline Solid Layer & $1.70 \mathrm{E}+04$ & $7.78 \mathrm{E}+01$ & $3.35 \mathrm{E}+02$ & $4.61 \mathrm{E}+04$ & $2.51 \mathrm{E}+04$ & $0.00 \mathrm{E}+00$ & $6.73 E-02$ & 3.97E-02 & $4.52 \mathrm{E}-02$ & $6.71 \mathrm{E}-01$ & $2.27 \mathrm{E}-03$ & $2.21 \mathrm{E}-02$ & 1.31 & 1.06 & 107 & $68 \%$ & $91 \%$ & 17 & 20 \\
\hline $241-T-202$ & $1.70 \mathrm{E}+04$ & $2.98 \mathrm{E}+03$ & $3.60 \mathrm{E}+04$ & $7.96 \mathrm{E}+04$ & $8.32 E+04$ & $4.94 \mathrm{E}+03$ & $2.23 \mathrm{E}-03$ & $3.22 \mathrm{E}-02$ & $2.11 \mathrm{E}-02$ & $1.67 \mathrm{E}-01$ & $1.45 \mathrm{E}-03$ & $6.77 \mathrm{E}-03$ & 1.18 & 1.17 & 77 & $76 \%$ & $75 \%$ & 17 & 19 \\
\hline Supernatant & $0.00 \mathrm{E}+00$ & $0.00 \mathrm{E}+00$ & $0.00 \mathrm{E}+00$ & $0.00 \mathrm{E}+00$ & $0.00 \mathrm{E}+00$ & $0.00 \mathrm{E}+00$ & $0.00 \mathrm{E}+00$ & $0.00 \mathrm{E}+00$ & $0.00 \mathrm{E}+00$ & $0.00 E+00$ & $0.00 \mathrm{E}+00$ & $0.00 \mathrm{E}+00$ & 0.00 & 0.00 & 0 & $0 \%$ & $0 \%$ & 0 & 0 \\
\hline Solid Layer & $1.70 \mathrm{E}+04$ & $2.98 E+03$ & $3.60 \mathrm{E}+04$ & $7.96 \mathrm{E}+04$ & $8.32 \mathrm{E}+04$ & $4.94 \mathrm{E}+03$ & $2.23 \mathrm{E}-03$ & $3.22 \mathrm{E}-02$ & $2.11 \mathrm{E}-02$ & $1.67 \mathrm{E}-01$ & $1.45 \mathrm{E}-03$ & 6.77E-03 & 1.18 & 1.17 & 77 & $76 \%$ & $75 \%$ & 17 & 19 \\
\hline
\end{tabular}




\section{RPP-5926 REV 6}

Table B-1. Input Data for Hydrogen Generation Rate Model Calculations for 177 Tanks. (20 sheets)

\begin{tabular}{|c|c|c|c|c|c|c|c|c|c|c|c|c|c|c|c|c|c|c|c|}
\hline Tank & $\begin{array}{c}\mathrm{OH} \\
\text { in liquid } \\
{[\mathrm{OH}]} \\
(\mu \mathrm{g} / \mathrm{mL})\end{array}$ & $\begin{array}{c}\text { TOC } \\
\text { in tiquid } \\
{[\mathrm{TOC}]} \\
(\mu \mathrm{g} / \mathrm{mL})\end{array}$ & $\begin{array}{c}\mathrm{NO}_{2} \\
\text { in liquid } \\
{\left[\mathrm{NO}_{2}\right]} \\
(\mu \mathrm{g} / \mathrm{mL})\end{array}$ & $\begin{array}{c}\mathrm{NO}_{3} \\
\text { in liquid } \\
{\left[\mathrm{NO}_{3}\right]} \\
(\mu \mathrm{g} / \mathrm{mL})\end{array}$ & $\begin{array}{c}\mathrm{Na} \\
\text { in liquid } \\
{[\mathrm{Na}]} \\
(\mathrm{ug} / \mathrm{mL})\end{array}$ & $\begin{array}{c}\text { Al } \\
\text { in liquid } \\
{[\mathrm{Al}]} \\
(\mu \mathrm{g} / \mathrm{mL})\end{array}$ & $\begin{array}{c}{ }^{90} \mathrm{Sr} \\
\text { in waste } \\
{[\mathrm{Sr}]} \\
(\mu \mathrm{Cl} / \mathrm{g})\end{array}$ & $\begin{array}{c}{ }^{241} \mathrm{Am} \\
\text { in waste } \\
|\mathrm{Am} 241| \\
(\mu \mathrm{Cl} / \mathrm{g})\end{array}$ & $\begin{array}{c}{ }^{240} \mathrm{Pu} \\
\text { in waste } \\
{[\mathrm{Pu240}]} \\
(\mu \mathrm{Ci} / \mathrm{g})\end{array}$ & $\begin{array}{c}{ }^{239} \mathrm{Pu} \\
\text { in waste } \\
\mid \mathrm{Pu240|} \\
(\mu \mathrm{Cv} / \mathrm{g})\end{array}$ & $\begin{array}{c}{ }^{238} \mathrm{Pu} \\
\text { in waste } \\
{[\mathrm{Pu238}]} \\
(\mu \mathrm{Ci} / \mathrm{g})\end{array}$ & $\begin{array}{c}{ }^{137} \mathrm{Cs} \\
\text { in waste } \\
{[\mathrm{Cs} \mid} \\
(\mu \mathrm{Ci} / \mathrm{g})\end{array}$ & $\begin{array}{c}\text { Bulk } \\
\text { density } \\
\text { D } \\
(\mathrm{g} / \mathrm{mL})\end{array}$ & $\begin{array}{c}\text { Liquid } \\
\text { Density } \\
\mathrm{D}_{\mathrm{L}} \\
(\mathrm{g} / \mathrm{ml})\end{array}$ & $\begin{array}{c}\text { Non-RGS } \\
\text { waste } \\
\text { volume } \\
(\mathrm{kL})\end{array}$ & $\begin{array}{l}\text { Bulk } \\
\text { water } \\
{\left[\mathrm{H}_{2} \mathrm{O}\right]} \\
(\mathrm{wt} \%)\end{array}$ & $\begin{array}{l}\text { Liquid } \\
\text { water } \\
{\left[\mathrm{H}_{2} \mathrm{O} \mid\right.} \\
(\mathrm{wt} \%)\end{array}$ & $\begin{array}{l}\text { Waste } \\
\text { temp. } \\
\text { Tw } \\
\left({ }^{\circ} \mathrm{C}\right)\end{array}$ & $\begin{array}{c}\text { Dome } \\
\text { temp. } \\
\mathrm{Td} \\
\left({ }^{\circ} \mathrm{C}\right)\end{array}$ \\
\hline 241-T-203 & $1.70 \mathrm{E}+04$ & $2.98 \mathrm{E}+03$ & $60 \mathrm{E}+04$ & $96 \mathrm{E}+04$ & $32 \mathrm{E}+04$ & $94 \mathrm{E}+03$ & $2.21 \mathrm{E}-03$ & $3.75 \mathrm{E}-02$ & $2.81 \mathrm{E}-02$ & $2.24 \mathrm{E}-01$ & $1.93 \mathrm{E}-03$ & $6.63 \mathrm{E}-03$ & 1.22 & 3.17 & 136 & $76 \%$ & $85 \%$ & 17 & 20 \\
\hline Supematant & $0,00 \mathrm{E}+00$ & $0.00 \mathrm{E}+00$ & $0.00 \mathrm{E}+00$ & $0.00 \mathrm{E}+00$ & $0.00 \mathrm{E}+00$ & $0.00 \mathrm{E}+00$ & $0.00 \mathrm{E}+00$ & $0.00 \mathrm{E}+00$ & $0.00 \mathrm{E}+00$ & $0.00 \mathrm{E}+00$ & $0.00 E+00$ & $0.00 \mathrm{E}+00$ & 0.00 & 0.00 & 0 & $0 \%$ & $0 \%$ & 0 & 0 \\
\hline Solid Layer & $1.70 \mathrm{E}+04$ & $2.98 \mathrm{E}+03$ & $3.60 \mathrm{E}+04$ & $7.96 \mathrm{E}+04$ & $8.32 \mathrm{E}+04$ & $4.94 E+03$ & $2.21 E-03$ & $3.75 \mathrm{E}-02$ & $2.81 E-02$ & $2.24 \mathrm{E}-01$ & $1.93 \mathrm{E}-03$ & $6.63 \mathrm{E}-03$ & 1.22 & 1.17 & 136 & $76 \%$ & $85 \%$ & 17 & 20 \\
\hline $241-T-204$ & $1.70 \mathrm{E}+04$ & $2.98 \mathrm{E}+03$ & $3.60 \mathrm{E}+04$ & $7.96 \mathrm{E}+04$ & $8.32 \mathrm{E} \div 04$ & $4.94 \mathrm{E}+03$ & $4.03 \mathrm{E}-03$ & $2.27 \mathrm{E}-02$ & $2.39 \mathrm{E}-02$ & $1.90 \mathrm{E}-01$ & $1.64 \mathrm{E}-03$ & $6.85 \mathrm{E}-03$ & 1.18 & 1.17 & 136 & $75 \%$ & $75 \%$ & 17 & 20 \\
\hline Supenratant & $0.00 \mathrm{E}+00$ & $0.00 \mathrm{E}+00$ & $0.00 \mathrm{E}+00$ & $0.00 \mathrm{E}-00$ & $0.00 \mathrm{E}+00$ & $0.00 \mathrm{E}+00$ & $0.00 \mathrm{E}+00$ & $0.00 \mathrm{E}+00$ & $0.00 \mathrm{E}+00$ & $0.00 \mathrm{E}+00$ & $0.00 \mathrm{E}+00$ & $0.00 \mathrm{E}+00$ & 0.00 & 0.00 & 0 & $0 \%$ & $0 \%$ & 0 & 0 \\
\hline Solid Layer & $1.70 \mathrm{E}+04$ & $2.98 \mathrm{E}+03$ & $3.60 \mathrm{E}+04$ & $7.96 \mathrm{E}+04$ & $8.32 \mathrm{E}+04$ & $4.94 \mathrm{E}+03$ & $4.03 \mathrm{E}-03$ & $2.27 \mathrm{E}-02$ & $2.39 \mathrm{E}-02$ & $1.90 \mathrm{E}-01$ & $1.64 \mathrm{E}-03$ & $6.85 E-03$ & 1.18 & 1.17 & 136 & $75 \%$ & $75 \%$ & 17 & 20 \\
\hline $241-\mathrm{TX}-101$ & $1.70 \mathrm{E}+04$ & $4.03 E+03$ & $1.22 \mathrm{E}+05$ & $1.90 \mathrm{E}+05$ & $2.36 \mathrm{\varepsilon}+05$ & $3.78 \mathrm{E}+04$ & $3.23 \mathrm{E}+02$ & $4.20 E-01$ & $1.26 \mathrm{E}-0 \mathrm{I}$ & $5.90 \mathrm{E}-01$ & $1.95 \mathrm{E}-02$ & $6.70 \mathrm{E}+01$ & 1.74 & 1.45 & 344 & $39 \%$ & $49 \%$ & 25 & 23 \\
\hline Supematant & $0.00 E+00$ & $0.00 \mathrm{E}+00$ & $0.00 \mathrm{E}+00$ & $0.00 \mathrm{E}+00$ & $0.00 \mathrm{E}+00$ & $0.00 \mathrm{E}+00$ & $0.00 \mathrm{E}+00$ & $0.00 E+00$ & $0.00 \mathrm{E}+00$ & $0.00 \mathrm{E}+00$ & $0.00 \mathrm{E}+00$ & $0.00 \mathrm{E}+00$ & 0.00 & 0.00 & 0 & $0 \%$ & $0 \%$ & 0 & 0 \\
\hline Solid Layer & $1.70 \mathrm{E}+04$ & $4.03 E+03$ & $1.22 \mathrm{E}+05$ & $1.90 \mathrm{E}+05$ & $2.36 \mathrm{E}+0.5$ & $3.78 \mathrm{E}+04$ & $3.23 \mathrm{E}+02$ & $4.20 \mathrm{E}-01$ & $1,26 \mathrm{E}-0 !$ & $5.90 \mathrm{E}-01$ & $1.95 \mathrm{E}-02$ & $6.70 \mathrm{E}+01$ & 1.74 & 1.45 & 344 & $39 \%$ & $49 \%$ & 25 & 23 \\
\hline $241-\mathrm{TX}-102$ & $1.70 \mathrm{E}+04$ & $4.03 \mathrm{E}+03$ & $1.22 \mathrm{E}+05$ & $1.90 \mathrm{E}+05$ & $2.36 \mathrm{E}+05$ & $3.78 E+04$ & $1.57 \mathrm{E}+00$ & $1.45 \mathrm{E}-01$ & $1.52 \mathrm{E}-02$ & $7.23 \mathrm{E}-02$ & $2.54 \mathrm{E}-03$ & $8.75 E+01$ & 1.61 & 1.45 & 821 & $31 \%$ & $49 \%$ & 26 & 25 \\
\hline Supematant & $0.00 \mathrm{E}+00$ & $0.00 \mathrm{E}+00$ & $0.00 \mathrm{E}+00$ & $0.00 E+00$ & $0.00 \mathrm{E}+00$ & $0.00 \mathrm{E}+00$ & $0.00 \mathrm{E}+00$ & $0.00 \mathrm{E}+00$ & $0.00 \mathrm{E}+00$ & $0.00 \mathrm{E}+00$ & $0.00 \mathrm{E}+00$ & $0.00 \mathrm{E}+00$ & 0.00 & 0.00 & 0 & $0 \%$ & $0 \%$ & 0 & 0 \\
\hline Solid Layer & $1.70 \mathrm{E}+04$ & $4.03 \mathrm{E}+03$ & $1.22 \mathrm{E}+05$ & $1.90 \mathrm{E}+05$ & $2.36 \mathrm{E}+05$ & $3.78 \mathrm{E}+04$ & $1.57 \mathrm{E}+00$ & 1.45E-01 & $1.52 \mathrm{E}-02$ & $7.23 \mathrm{E}-02$ & $2.54 \mathrm{E}-03$ & $8.75 \mathrm{E}+01$ & 1.61 & 1.45 & 821 & $31 \%$ & $49 \%$ & 26 & 25 \\
\hline $241-\mathrm{TX}-103$ & $1.70 \mathrm{E}+04$ & $3.88 E+03$ & $1.18 E+05$ & $1.91 \mathrm{E}+05$ & $2.32 \mathrm{E}+05$ & $3.65 \mathrm{E}+04$ & $7.29 \mathrm{E}-01$ & $1.43 \mathrm{E}-01$ & $1.50 \mathrm{E}-02$ & $7.18 \mathrm{E}-02$ & $2.51 \mathrm{E}-03$ & $8.73 \mathrm{E}+01$ & 1.61 & 1.44 & 548 & $34 \%$ & $69 \%$ & 22 & 21 \\
\hline Supenratant & $0.00 \mathrm{E}+00$ & $0.00 \mathrm{E}+00$ & $0.00 \mathrm{E}+00$ & $0.00 \mathrm{E}+00$ & $0.00 \mathrm{E}+00$ & $0.00 E+00$ & $0.00 \mathrm{E}+00$ & $0.00 \mathrm{E}+00$ & $0.00 \mathrm{E}+00$ & $0.00 \mathrm{E}+00$ & $0.00 \mathrm{E}+00$ & $0.00 \mathrm{E} \times 00$ & 0.00 & 0.00 & 0 & $0 \%$ & $0 \%$ & 0 & 0 \\
\hline Solid Layer & $1.70 E+04$ & $3.88 \mathrm{E}+03$ & $1.18 \mathrm{E}+05$ & $1.91 E+05$ & $2.32 \mathrm{E}+05$ & $3.65 \mathrm{E} \div 04$ & $7.29 \mathrm{E}-01$ & $1.43 \mathrm{E}-01$ & $1.50 \mathrm{E}-02$ & $7.18 \mathrm{E}-02$ & $2.51 \mathrm{IE}-03$ & $8.73 E+01$ & 1.61 & 1.44 & 548 & $34 \%$ & $69 \%$ & 22 & 21 \\
\hline $24 \mathrm{I}-\mathrm{TX}-104$ & $1.70 \mathrm{E}+04$ & $1.36 \mathrm{E}+03$ & $9.43 \mathrm{E}+04$ & $3.53 E+05$ & $2.05 \mathrm{E}+05$ & $3.19 \mathrm{E}+03$ & $1.59 \mathrm{E}+02$ & $1.55 \mathrm{E}-01$ & $2.10 \mathrm{E}-02$ & $1.03 \mathrm{E}-01$ & $3.03 E-03$ & $7.05 \mathrm{E}+0 \mathrm{~J}$ & 1.73 & 1.44 & 262 & $46 \%$ & $52 \%$ & 21 & 21 \\
\hline Supernatant & $1.70 \mathrm{E}+04$ & $1.36 \mathrm{E}+03$ & $9.42 E+04$ & $3.54 \mathrm{E}+05$ & $2.06 \mathrm{E}+05$ & $3.19 \mathrm{E}+03$ & $6.48 \mathrm{E}-0 \mathrm{t}$ & $8.87 \mathrm{E}-03$ & $8.10 \mathrm{E}-05$ & $3.86 \mathrm{E}-04$ & $1.37 \mathrm{E}-05$ & $2.31 E+02$ & 1.44 & 1.44 & 9 & $52 \%$ & $52 \%$ & 21 & 21 \\
\hline Solid Layer & $1.70 \mathrm{E}+04$ & $1.36 \mathrm{E}+03$ & $9.43 E+04$ & $3.53 \mathrm{E}+05$ & $2.05 E+05$ & $3.19 \mathrm{E}+03$ & $1.64 \mathrm{E}+02$ & $1.60 \mathrm{E}-01$ & $2.17 \mathrm{E}-02$ & $1.06 \mathrm{E}-01$ & $3.13 \mathrm{E}-03$ & $6.48 \mathrm{E}+01$ & 1.74 & 1.44 & 253 & $46 \%$ & $52 \%$ & 21 & 21 \\
\hline $241-T X-105$ & $1.70 \mathrm{E}+04$ & $4.03 \mathrm{E}+03$ & $1.22 \mathrm{E}+05$ & $1.90 E+05$ & $2.36 \mathrm{E}+05$ & $3.78 \mathrm{E}+04$ & $1.95 \mathrm{E}+00$ & $1.60 \mathrm{E}-01$ & $1.67 \mathrm{E}-02$ & $7.95 \mathrm{E}-02$ & $2.79 \mathrm{E}-03$ & $7.10 \mathrm{E}+01$ & 1.63 & 1.45 & 2181 & $29 \%$ & $49 \%$ & 34 & 23 \\
\hline Supenaatant & $0.00 \mathrm{E}+00$ & $0.00 \mathrm{E}+00$ & $0.00 \mathrm{E}+00$ & $0.00 \mathrm{E}+00$ & $0.00 E+00$ & $0.00 \mathrm{E}+00$ & $0.00 \mathrm{E}+00$ & $0.00 \mathrm{E}+00$ & $0.00 \mathrm{E} \div 00$ & $0.00 \mathrm{E}+00$ & $0.00 \mathrm{E}+00$ & $0.00 \mathrm{E}+00$ & 0.00 & 0.00 & 0 & $0 \%$ & $0 \%$ & 0 & 0 \\
\hline Solid Layer & $1.70 \mathrm{E}+04$ & $4.03 \mathrm{E}+03$ & $1.22 E+05$ & $1.90 \mathrm{E}+05$ & $2.36 \mathrm{E}+05$ & $3.78 \mathrm{E}+04$ & $1.95 \mathrm{E}+00$ & $1.60 \mathrm{E}-01$ & $1.67 \mathrm{E}-02$ & $7.95 \mathrm{E}-02$ & $2.79 \mathrm{E}-03$ & $7.10 \mathrm{E}+0 \mathrm{~J}$ & 1.63 & 1.45 & 2181 & $29 \%$ & $49 \%$ & 34 & 23 \\
\hline $241-\mathrm{TX}-106$ & $1.70 \mathrm{E}+04$ & $4.03 \mathrm{E}+03$ & $1.22 \mathrm{E}+05$ & $1.90 \mathrm{E}+05$ & $2.36 \mathrm{E}+05$ & $3.78 \mathrm{E}+04$ & $6.09 \mathrm{E}+00$ & $1.53 \mathrm{E}-01$ & $1.68 \mathrm{E}-02$ & $8.06 \mathrm{E}-02$ & $2.73 \mathrm{E}-03$ & $8.27 \mathrm{E}+01$ & 1.62 & 1.45 & 1318 & $30 \%$ & $49 \%$ & 26 & 24 \\
\hline Supernatant & $0.00 \mathrm{E}+00$ & $0.00 \mathrm{E}+00$ & $0.00 \mathrm{E}+00$ & $0.00 \mathrm{E}+00$ & $0.00 E+00$ & $0.00 \mathrm{E} \div 00$ & $0.00 \mathrm{E}+00$ & $0.00 \mathrm{E}+00$ & $0.00 \mathrm{E}+00$ & $0.00 \mathrm{E}+00$ & $0.00 \mathrm{E}+00$ & $0.00 \mathrm{E}+00$ & 0.00 & 0.00 & 0 & $0 \%$ & $0 \%$ & 0 & 0 \\
\hline Solid Layer & $1.70 \mathrm{E}+04$ & $4.03 \mathrm{E}+03$ & $1.22 \mathrm{E}+05$ & $1.90 \mathrm{E}+05$ & $2.36 \mathrm{E}+05$ & $3.78 \mathrm{E}+04$ & $6.09 \mathrm{E}+00$ & $1.53 \mathrm{E}-01$ & $1.68 \mathrm{E}-02$ & $8.06 \mathrm{E}-02$ & $2.73 E-03$ & $8.27 \mathrm{E}+01$ & 1.62 & 1.45 & 1318 & $30 \%$ & $49 \%$ & 26 & 24 \\
\hline $241-T X-107$ & $1.70 \mathrm{E}+04$ & $3.22 \mathrm{E}+03$ & $1.16 E+05$ & $1.97 \mathrm{E}+05$ & $2.40 \mathrm{E}+05$ & $4.03 \mathrm{E}+04$ & $5.47 \mathrm{E}+00$ & $1.69 \mathrm{E}+00$ & $1.77 \mathrm{E}-01$ & $8.41 \mathrm{E}-01$ & $2.97 \mathrm{E}-02$ & $1.02 \mathrm{E}+02$ & 1.78 & 1.46 & 112 & $28 \%$ & $51 \%$ & 22 & 20 \\
\hline Supenatant & $0.00 \mathrm{E}+00$ & $0.00 \mathrm{E}+00$ & $0.00 \mathrm{E} * 00$ & $0.00 \mathrm{E}+00$ & $0.00 \mathrm{E}+00$ & $0.00 E+00$ & $0.00 \mathrm{E}+00$ & $0.00 \mathrm{E}-00$ & $0.00 \mathrm{E}+00$ & $0.00 \mathrm{E}+00$ & $0.00 \mathrm{E}+00$ & $0.00 \mathrm{E}+00$ & 0.00 & 0.00 & 0 & $0 \%$ & $0 \%$ & 0 & 0 \\
\hline Solid Layer & $1.70 \mathrm{E}+04$ & $3.22 E+03$ & 1. $16 \mathrm{E}+05$ & $1.97 \mathrm{E}+05$ & $2.40 \mathrm{E}+05$ & $4.03 \mathrm{E}+04$ & $5.47 \mathrm{E}+00$ & $1.69 \mathrm{E}+00$ & $1.77 \mathrm{E}-01$ & $8.41 \mathrm{E}-01$ & $2.97 \mathrm{E}-02$ & $1.02 \mathrm{E}+02$ & 1.78 & 1.46 & 112 & $28 \%$ & $51 \%$ & 22 & 20 \\
\hline
\end{tabular}




\section{RPP-5926 REV 6}

Table B-1. Input Data for Hydrogen Generation Rate Model Calculations for 177 Tanks. (20 sheets)

\begin{tabular}{|c|c|c|c|c|c|c|c|c|c|c|c|c|c|c|c|c|c|c|c|}
\hline Tank & $\begin{array}{c}\mathrm{OH} \\
\text { in liquid } \\
|\mathrm{OH}| \\
(\mu \mathrm{g} / \mathrm{mL})\end{array}$ & $\begin{array}{c}\text { TOC } \\
\text { in liquid } \\
\text { |TOC| } \\
(\mu \mathrm{g} / \mathrm{mL})\end{array}$ & $\begin{array}{c}\mathrm{NO}_{2} \\
\text { in liquid } \\
{\left[\mathrm{NO}_{2}\right]} \\
(\mu \mathrm{g} / \mathrm{mL})\end{array}$ & $\begin{array}{c}\mathrm{NO}_{3} \\
\text { in liquid } \\
{\left[\mathrm{NO}_{3}\right]} \\
(\mu \mathrm{g} / \mathrm{mL})\end{array}$ & $\begin{array}{c}\mathrm{Na} \\
\text { in liquid } \\
|\mathrm{Na}| \\
(\mathrm{ug} / \mathrm{mL})\end{array}$ & $\begin{array}{c}\text { Al } \\
\text { in liquid } \\
{[A \mid]} \\
(\mu \mathrm{g} / \mathrm{mL})\end{array}$ & $\begin{array}{c}{ }^{90} \mathrm{Sr} \\
\text { in waste } \\
{[\mathrm{Sr}]} \\
(\mu \mathrm{Cl} / g)\end{array}$ & $\begin{array}{c}{ }^{241} \mathrm{Am} \\
\text { in waste } \\
|\mathrm{Am} 241| \\
(\mu \mathrm{Ci} / \mathrm{g})\end{array}$ & $\begin{array}{c}{ }^{240} \mathrm{Pu}_{\mathrm{u}} \\
\text { in waste } \\
\text { [Pu240] } \\
(\mu \mathrm{Ci} / \mathrm{g})\end{array}$ & $\begin{array}{c}{ }^{239} \mathrm{Pu} \\
\text { in waste } \\
{[\text { Pu240] }} \\
(\mu \mathrm{Ci} / \mathrm{g})\end{array}$ & $\begin{array}{c}{ }^{238} \mathrm{Pu} \\
\text { in waste } \\
{[\mathrm{Pu238} \mid} \\
(\mu \mathrm{Ci} / \mathrm{g})\end{array}$ & $\begin{array}{c}{ }^{137} \mathrm{Cs} \\
\text { in waste } \\
|\mathrm{Cs}| \\
(\mu \mathrm{Ci} / \mathrm{g})\end{array}$ & $\begin{array}{c}\text { Bulk } \\
\text { density } \\
D \\
(\mathrm{~g} / \mathrm{mL})\end{array}$ & $\begin{array}{c}\text { Liquid } \\
\text { Density } \\
\mathrm{D}_{\mathrm{L}} \\
(\mathrm{g} / \mathrm{ml})\end{array}$ & $\begin{array}{c}\text { Non-RGS } \\
\text { waste } \\
\text { volume } \\
\text { (kL) }\end{array}$ & $\begin{array}{l}\text { Bulk } \\
\text { water } \\
\left|\mathrm{H}_{2} \mathrm{O}\right| \\
(\mathrm{wt} \%)\end{array}$ & $\begin{array}{l}\text { Liquid } \\
\text { water } \\
\left|\mathrm{H}_{2} \mathrm{O}\right| \\
(\mathrm{wt} / \%)\end{array}$ & $\begin{array}{l}\text { Waste } \\
\text { temp. } \\
\text { Tw } \\
\left.\text { ( }{ }^{\circ} \mathrm{C}\right)\end{array}$ & $\begin{array}{c}\text { Dome } \\
\text { temp. } \\
\text { Td } \\
\left({ }^{\circ} \mathrm{C}\right)\end{array}$ \\
\hline $241-\mathrm{TX}-108$ & $1.70 \mathrm{E}+04$ & $4.03 E+03$ & $1.22 \mathrm{E}+05$ & $90 \mathrm{E}+05$ & $36 \mathrm{E}+05$ & $78 \mathrm{E}+04$ & $.27 \mathrm{E}+00$ & $1.50 \mathrm{E}-01$ & \$.55E-02 & $7.45 \mathrm{E}-02$ & $2.61 \mathrm{E}-03$ & $7.50 \mathrm{E}+01$ & 1.62 & 1.45 & 478 & $31 \%$ & $49 \%$ & 21 & 21 \\
\hline Supenatant & $0.00 \mathrm{E}+00$ & $0.00 \mathrm{E}+00$ & $0.00 \mathrm{E}+00$ & $0.00 \mathrm{E}+00$ & $0.00 \mathrm{E}+00$ & $0.00 \mathrm{E}+00$ & $0.00 \mathrm{E}+00$ & $0.00 \mathrm{E}+00$ & $0.00 \mathrm{E}+00$ & $0.00 \mathrm{E}+00$ & $0.00 \mathrm{E}+00$ & $0.00 \mathrm{E}+00$ & 0.00 & 0.00 & 0 & $0 \%$ & $0 \%$ & 0 & 0 \\
\hline Solid Layer & $1.70 \mathrm{E}+04$ & $4.03 \mathrm{E}+03$ & $1.22 \mathrm{E}+05$ & $1.90 \mathrm{E}+05$ & $2.36 E+05$ & $3.78 \mathrm{E}+04$ & $5.27 \mathrm{E}+00$ & $1.50 \mathrm{E}-01$ & $1.55 \mathrm{E}-02$ & $7.45 \mathrm{E}-02$ & $2.61 \mathrm{E}-03$ & $7.50 \mathrm{E}+0 \mathrm{I}$ & 1.62 & 1.45 & 478 & $31 \%$ & $49 \%$ & $2 !$ & 21 \\
\hline 241-TX-109 & $1.70 \mathrm{E}+04$ & $2.98 \mathrm{E}+03$ & $3.60 \mathrm{E}+04$ & $7.96 \mathrm{E}+04$ & $8.32 \mathrm{E}+04$ & $4.94 \mathrm{E}+03$ & $4.82 \mathrm{E}+00$ & $1.38 \mathrm{E}-02$ & $9.05 \mathrm{E}-03$ & $8.34 \mathrm{E}-02$ & $5.85 \mathrm{E}-04$ & $1.59 \mathrm{E}+01$ & 1.43 & 1.17 & 1375 & $54 \%$ & $75 \%$ & 34 & 39 \\
\hline Supernatant & $0.00 \mathrm{E}+00$ & $0.00 \mathrm{E} \div 00$ & $0.00 \mathrm{E}+00$ & $0.00 \mathrm{E}+00$ & $0.00 \mathrm{E}+00$ & $0.00 \mathrm{E}+00$ & $0.00 \mathrm{E}+00$ & $0.00 \mathrm{E}+00$ & $0.00 \mathrm{E}+00$ & $0.00 \mathrm{E}+00$ & $0.00 \mathrm{E}+00$ & $0.00 \mathrm{E}+00$ & 0.00 & 0.00 & 0 & $0 \%$ & $0 \%$ & 0 & 0 \\
\hline Solid Layer & $1.70 \mathrm{E}+04$ & $2.98 \mathrm{E}+03$ & $3.60 \mathrm{E}+04$ & $7.96 \mathrm{E} \div 04$ & $8.32 \mathrm{E}+04$ & $4.94 \mathrm{E}+03$ & $4.82 \mathrm{E}+00$ & $1.38 \mathrm{E}-02$ & $9.05 \mathrm{E}-03$ & $8.34 \mathrm{E}-02$ & $5.85 \mathrm{E}-04$ & $1.59 \mathrm{E}+01$ & 1.43 & 1.17 & 1375 & $54 \%$ & $75 \%$ & 34 & 39 \\
\hline $241-\mathrm{TX}+110$ & $1.70 \mathrm{E}+04$ & $4.02 \mathrm{E}+03$ & $1.22 \mathrm{E}+05$ & $90 \mathrm{E}+05$ & $2.37 \mathrm{E}+05$ & $3.78 \mathrm{E}+04$ & $1.03 E+00$ & $.55 \mathrm{E}-01$ & $1.66 \mathrm{E}-02$ & $8.20 \mathrm{E}-02$ & $2.73 \mathrm{E}-03$ & $6.52 \mathrm{E}+01$ & 1.62 & 1.45 & 1769 & $31 \%$ & $49 \%$ & 28 & 22 \\
\hline Supenatant & $0.00 \mathrm{E}+00$ & $0,00 \mathrm{E}+00$ & $0.00 \mathrm{E}+00$ & $0.00 E+00$ & $0.00 \mathrm{E}+00$ & $0.00 \mathrm{E}+00$ & $0.00 \mathrm{E}+00$ & $0.00 \mathrm{E}+00$ & $0.00 \mathrm{E}+00$ & $0.00 \mathrm{E}+00$ & $0.00 \mathrm{E}+00$ & $0.00 \mathrm{E}+00$ & 0.00 & 0.00 & 0 & $0 \%$ & $0 \%$ & 0 & 0 \\
\hline Solid Layer & $1.70 \mathrm{E} \div 04$ & $4.02 \mathrm{E}+03$ & $1.22 \mathrm{E}+05$ & $1.90 \mathrm{E}+05$ & $2.37 \mathrm{E}+05$ & $3.78 \mathrm{E}+04$ & $1.03 \mathrm{E}+00$ & $1.55 \mathrm{E}-01$ & $1.66 \mathrm{E}-02$ & 8.20E-02 & $2.73 \mathrm{E}-03$ & $6.52 \mathrm{E}+01$ & 1.62 & 1.45 & 1769 & $31 \%$ & $49 \%$ & 28 & 22 \\
\hline $241-T X-111$ & $1.70 \mathrm{E}+04$ & $4.03 E+03$ & $1.22 \mathrm{E}+05$ & $1.90 \mathrm{E}+05$ & $2.36 \mathrm{E}+05$ & $3.78 \mathrm{E}+04$ & $1.17 \mathrm{E}+00$ & $1.51 \mathrm{E}-01$ & $1.65 \mathrm{E}-02$ & $8.28 \mathrm{E}-02$ & $2.67 \mathrm{E}-03$ & $6.21 \mathrm{E}+01$ & 1.61 & 1.45 & 1381 & $31 \%$ & $49 \%$ & 26 & 22 \\
\hline Supernatant & $0.00 \mathrm{E}+00$ & $0.00 \mathrm{~F}+00$ & $0.00 \mathrm{E}+00$ & $0.00 \mathrm{E}+00$ & $0.00 \mathrm{E}+00$ & $0.00 \mathrm{E}+00$ & $0.00 \mathrm{E}+00$ & $0.00 E+00$ & $0.00 \mathrm{E}+00$ & $0.00 \mathrm{E}+00$ & $0.00 \mathrm{E}+00$ & $0.00 E+00$ & 0.00 & 0.00 & 0 & $0 \%$ & $0 \%$ & 0 & 0 \\
\hline Solid Layer & $1.70 E+04$ & $4.03 \mathrm{E} \div 03$ & $1.22 \mathrm{E}+05$ & $1.90 \mathrm{E}+05$ & $2.36 \mathrm{E}+05$ & $3.78 \mathrm{E}+04$ & 1.17E+00 & $1.51 \mathrm{E}-01$ & $1.65 \mathrm{E}-02$ & $8.28 \mathrm{E}-02$ & $2.67 \mathrm{E}-03$ & $6.21 E+01$ & 1.61 & 1.45 & 1381 & $31 \%$ & $49 \%$ & 26 & 22 \\
\hline $241-T X-112$ & $4.81 \mathrm{E}+04$ & $3.47 \mathrm{E}+03$ & $1.06 \mathrm{E}+05$ & $1.94 \mathrm{E}+05$ & $2.18 \mathrm{E}+05$ & $3.26 \mathrm{E} \cdot 04$ & $7.24 \mathrm{E}-01$ & $1.58 E-01$ & $1.66 \mathrm{E}-02$ & $7.94 \mathrm{E}-02$ & $2.77 \mathrm{E}-03$ & $6.81 \mathrm{E}+01$ & 1.63 & 1.42 & 2398 & $25 \%$ & $52 \%$ & 22 & 19 \\
\hline Supenratant & $0.00 \mathrm{E}+00$ & $0.00 \mathrm{E}+00$ & $0.00 \mathrm{E}+00$ & $0.00 \mathrm{E}+00$ & $0.00 \mathrm{E}+00$ & $0.00 \mathrm{E}+00$ & $0.00 \mathrm{E}+00$ & $0.00 \mathrm{E}+00$ & $0.00 \mathrm{E}+00$ & $0.00 \mathrm{E}+00$ & $0.00 \mathrm{E}+00$ & $0.00 \mathrm{E}+00$ & 0.00 & 0.00 & 0 & $0 \%$ & $0 \%$ & 0 & 0 \\
\hline Solid Layer & $4.81 \mathrm{E}+04$ & $3.47 \mathrm{E}+03$ & $06 \mathrm{E}+05$ & $1.94 \mathrm{E}+05$ & $2.18 \mathrm{E}+05$ & $3.26 \mathrm{E}+04$ & 7.24E-01 & $1.58 \mathrm{E}-01$ & $1.66 \mathrm{E}-02$ & $7.94 \mathrm{E}-02$ & $2.77 \mathrm{E}-03$ & $6.81 \mathrm{E}+01$ & 1.63 & 1.42 & 2398 & $25 \%$ & $52 \%$ & 22 & 19 \\
\hline $241-T X-113$ & $4.81 E+04$ & $4.03 E^{+03}$ & $1.22 \mathrm{E}+05$ & $1.90 \mathrm{E}+05$ & $2.36 \mathrm{E}+05$ & $3.78 \mathrm{E}+04$ & $1.40 \mathrm{E}+00$ & $6.72 \mathrm{E}-03$ & $1.68 \mathrm{E}-03$ & $1.32 \mathrm{E}-02$ & $1.62 \mathrm{E}-04$ & $8.18 \mathrm{E}+00$ & 1.61 & 1.45 & 2416 & $15 \%$ & $49 \%$ & 22 & 20 \\
\hline Superriatant & $0.00 \mathrm{E}+00$ & $0.00 \mathrm{E}+00$ & $0.00 \mathrm{E}+00$ & $0.00 \mathrm{E}+00$ & $0.00 \mathrm{E}+00$ & $0.00 \mathrm{E}+00$ & $0.00 \mathrm{E}+00$ & $0.00 \mathrm{E}+00$ & $0.00 \mathrm{E}+00$ & $0.00 \mathrm{E}+00$ & $0.00 E+00$ & $0.00 \mathrm{E}+00$ & 0.00 & 0.00 & 0 & $0 \%$ & $0 \%$ & 0 & 0 \\
\hline Solid Layer & $4.81 \mathrm{E}+04$ & $03 \mathrm{E}+03$ & $22 \mathrm{E}+05$ & 90E $\div 05$ & $2.36 \mathrm{E}+05$ & $3.78 \mathrm{E}+04$ & $40 \mathrm{E}+00$ & $6.72 E-03$ & $1.68 \mathrm{E}-03$ & $1.32 \mathrm{E}-02$ & $1.62 \mathrm{E}-04$ & $8.18 \mathrm{E}+00$ & 1.61 & 1.45 & 2416 & $15 \%$ & $49 \%$ & 22 & 20 \\
\hline $241-T X-114$ & $1.70 \mathrm{E}+04$ & $1.53 \mathrm{E}+03$ & $5.15 E+04$ & $2.07 \mathrm{E}+05$ & $1.60 \mathrm{~F}+05$ & $1.45 \mathrm{E}+04$ & $7.29 \mathrm{E}-01$ & $1.48 \mathrm{E}-01$ & $1.59 \mathrm{E}-02$ & $7.75 \mathrm{E}-02$ & $2.62 \mathrm{E}-03$ & $5.94 \mathrm{E}+01$ & 1.63 & 1.33 & 2012 & $25 \%$ & $61 \%$ & 21 & 18 \\
\hline Supenatant & $0.00 \mathrm{E}+00$ & $0.00 E+00$ & $0.00 \mathrm{E}+00$ & $0.00 \mathrm{E}+00$ & $0.00 \mathrm{E}+00$ & $0.00 \mathrm{E}+00$ & $0.00 E+00$ & $0.00 \mathrm{E}+00$ & $0.00 \mathrm{E}+00$ & $0.00 \mathrm{E}+00$ & $0.00 \mathrm{E}+00$ & $0.00 \mathrm{E}+00$ & 0.00 & 0.00 & 0 & $0 \%$ & $0 \%$ & 0 & 0 \\
\hline Solid Layer & $1.70 \mathrm{E}+04$ & $1.53 \mathrm{E}+03$ & $5.15 \mathrm{E}+04$ & $2.07 \mathrm{E}+05$ & $1.60 \mathrm{E}+05$ & $1.45 \mathrm{E}+04$ & $7.29 \mathrm{E}-01$ & $1.48 \mathrm{E}-01$ & $1.59 \mathrm{E}-02$ & $7.75 \mathrm{E}-02$ & $2.62 \mathrm{E}-03$ & $5.94 \mathrm{E}+01$ & 1.63 & 1.33 & 2012 & $25 \%$ & $61 \%$ & 21 & 18 \\
\hline $241-\mathrm{TX}-115$ & $4.81 \mathrm{E}+04$ & $4.03 \mathrm{E}+03$ & $1.22 E+05$ & $1.90 \mathrm{E}+05$ & $2.36 \mathrm{E}+05$ & $3.78 \mathrm{E}+04$ & $2.15 \mathrm{E}+00$ & 1.60E-01 & $1.67 \mathrm{E}-02$ & $7.97 \mathrm{E}-02$ & $2,80 \mathrm{E}-03$ & $7.13 \mathrm{E}+01$ & 1.63 & 1.45 & 2093 & $29 \%$ & $49 \%$ & 22 & 21 \\
\hline Supernatant & $0.00 \mathrm{E}+00$ & $0.00 \mathrm{E}+00$ & $0.00 \mathrm{E}+00$ & $0.00 \mathrm{E}+00$ & $0.00 \mathrm{E}+00$ & $0.00 \mathrm{E}+00$ & $0.00 \mathrm{E}+00$ & $0.00 \mathrm{E}+00$ & $0.00 \mathrm{E}+00$ & $0.00 \mathrm{E}+00$ & $0.00 \mathrm{E}+00$ & $0.00 \mathrm{E}+00$ & 0.00 & 0.00 & 0 & $0 \%$ & $0 \%$ & 0 & 0 \\
\hline Solid Layer & $4.81 \mathrm{E}+04$ & $4.03 E+03$ & $1.22 \mathrm{E}+05$ & $1.90 \mathrm{E}+05$ & $2.36 \mathrm{E}+05$ & $3.78 E+04$ & $2.15 E+00$ & $1.60 \mathrm{E}-01$ & $1.67 \mathrm{E}-02$ & $7.97 \mathrm{E}-02$ & $2.80 \mathrm{E}-03$ & $7.13 \mathrm{E}+01$ & 1.63 & 1.45 & 2093 & $29 \%$ & $49 \%$ & 22 & 21 \\
\hline $241 \cdot \mathrm{TX} \cdot 116$ & $2.54 E+03$ & $3.19 \mathrm{E}+02$ & $1.05 E+04$ & 3. $79 \mathrm{E}+05$ & 1. $51 \mathrm{E} \div 05$ & $8.79 \mathrm{E}+00$ & $3.42 \mathrm{E}-01$ & $6.88 \mathrm{E}-02$ & $9.33 \mathrm{E}-03$ & $5.34 \mathrm{E}-02$ & $1.34 \mathrm{E}-03$ & $3.51 E+01$ & 1.66 & I. 39 & 2264 & $22 \%$ & $53 \%$ & 21 & 20 \\
\hline Supernatant & $0.00 \mathrm{E}+00$ & $0.00 \mathrm{E}+00$ & $0.00 \mathrm{E}+00$ & $0.00 \mathrm{E} \div 00$ & $0.00 \mathrm{E}+00$ & $0.00 \mathrm{E}+00$ & $0.00 \mathrm{E}+00$ & $0.00 \mathrm{E}+00$ & $0.00 E+00$ & $0.00 \mathrm{E}+00$ & $0.00 \mathrm{E}+00$ & $0.00 \mathrm{E}+00$ & 0.00 & 0.00 & 0 & $0 \%$ & $0 \%$ & 0 & 0 \\
\hline Solid Eayer & $2.54 \mathrm{E}+03$ & $3.19 \mathrm{E}+02$ & $1.05 \mathrm{E}+04$ & $3.79 \mathrm{E}+05$ & $1.51 \mathrm{E}+05$ & $8.79 \mathrm{E}+00$ & $3.42 \mathrm{E}-01$ & $6.88 \mathrm{E}-02$ & $9.33 \mathrm{E}-03$ & $5.34 \mathrm{E}-02$ & $1.34 \mathrm{E}-03$ & $3.51 \mathrm{E}+0 \mathrm{l}$ & 1.66 & 1.39 & 2264 & $22 \%$ & $53 \%$ & $2 t$ & 20 \\
\hline
\end{tabular}


RPP-5926 REV 6

Table B-1. Input Data for Hydrogen Generation Rate Model Calculations for 177 Tanks. (20 sheets)

\begin{tabular}{|c|c|c|c|c|c|c|c|c|c|c|c|c|c|c|c|c|c|c|c|}
\hline Tank & $\begin{array}{c}\mathrm{OH} \\
\text { in liquid } \\
{[\mathrm{OH}]} \\
(\mu \mathrm{g} / \mathrm{mL})\end{array}$ & $\begin{array}{c}\text { TOC } \\
\text { in liquid } \\
\text { |TOC| } \\
(\mu \mathrm{g} / \mathrm{mL})\end{array}$ & $\begin{array}{c}\mathrm{NO}_{2} \\
\text { in liquid } \\
{\left[\mathrm{NO}_{2}\right]} \\
(\mu \mathrm{g} / \mathrm{mL})\end{array}$ & $\begin{array}{c}\mathrm{NO}_{3} \\
\text { in liquid } \\
{\left[\mathrm{NO}_{3}\right]} \\
(\mu \mathrm{g} / \mathrm{mL})\end{array}$ & $\begin{array}{c}\mathrm{Na} \\
\text { in liquid } \\
{[\mathrm{Na}]} \\
(\mathrm{ug} / \mathrm{mL})\end{array}$ & $\begin{array}{c}\text { Al } \\
\text { in liquid } \\
{[\mathrm{Al}]} \\
(\mu \mathrm{g} / \mathrm{mL})\end{array}$ & $\begin{array}{c}{ }^{90} \mathrm{Sr} \\
\text { in waste } \\
{[\mathrm{Sr}]} \\
(\mu \mathrm{Ci} / \mathrm{g})\end{array}$ & $\begin{array}{c}{ }^{241} \mathrm{Am} \\
\text { in waste } \\
\text { |Am241| } \\
(\mu \mathrm{Ci} / \mathrm{g})\end{array}$ & $\begin{array}{c}{ }^{240} \mathrm{Pu} \\
\text { in waste } \\
{[\mathrm{Pu} 240]} \\
(\mu \mathrm{Ci} / \mathrm{g})\end{array}$ & $\begin{array}{l}{ }^{239} \mathrm{Pu} \\
\text { in waste } \\
{\left[\begin{array}{l}\text { Pu240| } \\
(\mu \mathrm{Ci} / \mathrm{g})\end{array}\right.}\end{array}$ & $\begin{array}{c}{ }^{238} \mathrm{Pu} \\
\text { in waste } \\
{[\mathrm{Pu} 238]} \\
(\mu \mathrm{Ci} / \mathrm{g})\end{array}$ & $\begin{array}{c}{ }^{137} \mathrm{Cs} \\
\text { in waste } \\
{[\mathrm{Cs}]} \\
(\mu \mathrm{Ci} / \mathrm{g})\end{array}$ & $\begin{array}{c}\text { Bulk } \\
\text { density } \\
\text { D } \\
(\mathrm{g} / \mathrm{mL})\end{array}$ & $\begin{array}{c}\text { Liquid } \\
\text { Density } \\
\mathrm{D}_{\mathrm{L}} \\
(\mathrm{g} / \mathrm{ml})\end{array}$ & $\begin{array}{c}\text { Non-RGS } \\
\text { waste } \\
\text { volume } \\
(\mathrm{kL})\end{array}$ & $\begin{array}{l}\text { Bulk } \\
\text { water } \\
{\left[\mathrm{H}_{2} \mathrm{O}\right]} \\
(w t \%)\end{array}$ & $\begin{array}{c}\text { Liquid } \\
\text { water } \\
\left|\mathrm{H}_{2} \mathrm{O}\right| \\
(\mathrm{wt} \%)\end{array}$ & $\begin{array}{l}\text { Waste } \\
\text { temp. } \\
\text { Tw } \\
\left.{ }^{\circ} \mathrm{C}\right)\end{array}$ & $\begin{array}{c}\text { Dome } \\
\text { temp. } \\
\text { Td } \\
\left({ }^{\circ} \mathrm{C}\right)\end{array}$ \\
\hline $24 !-T X-117$ & $1.70 \mathrm{E}+04$ & $3.38 \mathrm{E}+00$ & $0 \mathrm{E}+03$ & $2.17 \mathrm{E}+05$ & $1.13 E+05$ & $3.60 \mathrm{E}+02$ & $5.08 \mathrm{E}-01$ & $1.00 \mathrm{E}-01$ & $1.20 \mathrm{E}-02$ & $6.32 \mathrm{E}-02$ & $1.85 \mathrm{E}-03$ & $3.89 E+01$ & 1.58 & 1.26 & 1817 & $27 \%$ & $70 \%$ & 21 & 20 \\
\hline Supernatant & $0.00 E+00$ & $0.00 \mathrm{E}+00$ & $0.00 E+00$ & $0.00 \mathrm{E}+00$ & $0.00 \mathrm{E}+00$ & $0.00 \mathrm{E}+00$ & $0.00 \mathrm{E}+00$ & $0.00 E+00$ & $0.00 \mathrm{E}+00$ & $0.00 E^{ \pm}+00$ & $0.00 \mathrm{E}+00$ & $0.00 \mathrm{E}+00$ & 0.00 & 0.00 & 0 & $0 \%$ & $0 \%$ & 0 & 0 \\
\hline Solid Layer & $1.70 \mathrm{E}+04$ & $3.38 \mathrm{E} \div 00$ & $8.50 \mathrm{E}+03$ & $2.17 \mathrm{E}+05$ & $1.13 \mathrm{E}+05$ & $3.60 \mathrm{E}+02$ & $5.08 \mathrm{E}-01$ & $1.00 \mathrm{E}-01$ & $1.20 \mathrm{E}-02$ & $6.32 \mathrm{E}-02$ & $1.85 \mathrm{E}-03$ & $3.89 \mathrm{E}+01$ & 1.58 & 1.26 & 1817 & $27 \%$ & $70 \%$ & 21 & 20 \\
\hline $241-\mathrm{TX}-118$ & $4.81 \mathrm{E}+04$ & $4.03 E+03$ & $1.22 \mathrm{E}+05$ & $1.90 \mathrm{E}+05$ & $2.36 \mathrm{E}+05$ & $3.78 \mathrm{E}+04$ & $1.21 \mathrm{E}+02$ & $3.62 \mathrm{E}+00$ & $4.93 \mathrm{E}-01$ & $2.34 \mathrm{E}+00$ & $8.26 \mathrm{E}-02$ & $5.39 \mathrm{E}+01$ & 1.69 & 1.45 & 935 & $45 \%$ & $49 \%$ & 25 & 24 \\
\hline Supematant & $0.00 \mathrm{E}+00$ & $0.00 \mathrm{E}+00$ & $0.00 \mathrm{E}+00$ & $0.00 \mathrm{E}+00$ & $0.00 \mathrm{E}+00$ & $0.00 \mathrm{E}+00$ & $0.00 \mathrm{E}+00$ & $0.00 \mathrm{E}+00$ & $0.00 \mathrm{E}+00$ & $0.00 \mathrm{E}+00$ & $0.00 \mathrm{E}+00$ & $0.00 \mathrm{E}+00$ & 0.00 & 0.00 & 0 & $0 \%$ & $0 \%$ & 0 & 0 \\
\hline Solid Layer & $4.81 E+04$ & $4.03 \mathrm{E}+03$ & $1.22 \mathrm{E}+05$ & $1.90 \mathrm{E}+05$ & $2.36 \mathrm{E}+05$ & $3.78 \mathrm{E}+04$ & $1.21 \mathrm{E}+02$ & $3.62 \mathrm{E}+00$ & $4.93 \mathrm{E}-01$ & $2.34 \mathrm{E}+00$ & $8.26 \mathrm{E}-02$ & $5.39 \mathrm{E}+01$ & 1.69 & 1.45 & 935 & $45 \%$ & $49 \%$ & 25 & 24 \\
\hline 241-TY-101 & $1.70 \mathrm{E}+04$ & $3.38 \mathrm{E}+00$ & $8.47 E+03$ & $2.17 \mathrm{E}+05$ & $1.13 \mathrm{E}+05$ & $3.61 \mathrm{E}+02$ & $9.53 \mathrm{E}+00$ & $1.26 \mathrm{E}-02$ & $1.44 \mathrm{E}-02$ & $1.72 E-01$ & $8.48 \mathrm{E}-04$ & $4.04 \mathrm{E}-01$ & 1.63 & 1.26 & 447 & $44 \%$ & $70 \%$ & 18 & 17 \\
\hline Supertatant & $0.00 \mathrm{E}+00$ & $0.00 \mathrm{E}+00$ & $0.00 \mathrm{E}+00$ & $0.00 \mathrm{E}+00$ & $0.00 \mathrm{E}+00$ & $0.00 \mathrm{E}+00$ & $0.00 \mathrm{E}+00$ & $0.00 \mathrm{E}+00$ & $0.00 \mathrm{E}+00$ & $0.00 \mathrm{E}+00$ & $0.00 \mathrm{E}+00$ & $0.00 \mathrm{E}+00$ & 0.00 & 0.00 & 0 & $0 \%$ & $0 \%$ & 0 & 0 \\
\hline Solid Layer & $1.70 \mathrm{E}+04$ & $3.38 \mathrm{E}+00$ & $8.47 \mathrm{E}+03$ & $2.17 \mathrm{E}+05$ & $1.13 \mathrm{E}+05$ & $3.61 \mathrm{E} \div 02$ & $9.53 \mathrm{E}+00$ & $1.26 \mathrm{E}-02$ & $1.44 \mathrm{E}-02$ & 1.72E- 01 & $8.48 \mathrm{E}-04$ & $4.04 \mathrm{E}-01$ & 1.63 & 1.26 & 447 & $44 \%$ & $70 \%$ & 18 & 17 \\
\hline $241-\mathrm{TY}-102$ & 4. $82 E+04$ & $2.18 \mathrm{E}+03$ & $6.98 \mathrm{E}+04$ & $2.02 \mathrm{E}+05$ & $1.79 \mathrm{E}+05$ & $2.06 \mathrm{E}+04$ & $2.62 \mathrm{E}+00$ & $2.50 \mathrm{E}-03$ & $2.02 \mathrm{E}-04$ & $1.33 \mathrm{E}-03$ & $2.46 \mathrm{E}-05$ & $2.87 \mathrm{E}+01$ & 1.76 & 1.36 & 262 & $32 \%$ & $58 \%$ & 16 & 19 \\
\hline Supenuatan: & $0.00 E \div 00$ & $0.00 \mathrm{E}+00$ & $0.00 \Sigma+00$ & $0.00 \mathrm{E}+00$ & $0.00 E+00$ & $0.00 \mathrm{E}+00$ & $0.00 E+00$ & $0.00 E+00$ & $0.00 E+00$ & $0.00 E+00$ & $0.00 \mathrm{E}+00$ & $0.00 E+00$ & 0.00 & 0.00 & 0 & $0 \%$ & $0 \%$ & 0 & 0 \\
\hline Solid Layer & $4.82 \mathrm{E}+04$ & $2.18 \mathrm{E}+03$ & $6.98 \mathrm{E}+04$ & $2.02 \mathrm{E}+05$ & $1.79 E+05$ & $2.06 \mathrm{E}+04$ & $2.62 E+00$ & $2.50 \mathrm{E}-03$ & $2.02 \mathrm{E}-04$ & $1.33 \mathrm{E}-03$ & $2.46 \mathrm{E}-05$ & $2.87 \mathrm{E} \div 01$ & 1.76 & 1.36 & 262 & $32 \%$ & $58 \%$ & 16 & 19 \\
\hline $241-\mathrm{TY}-103$ & $4.80 \mathrm{E}+04$ & $4.02 E+03$ & $1.22 \mathrm{E}+05$ & $1.90 \mathrm{E}+05$ & $2.36 \mathrm{E}+05$ & $3.78 \mathrm{E}+04$ & $7.00 \mathrm{E}-\mathrm{r}-0 \mathrm{I}$ & $2.42 E-02$ & $1.93 \mathrm{E}-02$ & $1.60 \mathrm{E}-01$ & $2.10 \mathrm{E}-03$ & $2.78 \mathrm{E}+01$ & 1.68 & 1.45 & 585 & $52 \%$ & $49 \%$ & 22 & 22 \\
\hline Supernatant & $0.00 \mathrm{E}+00$ & $0.00 \mathrm{E}+00$ & $0.00 \mathrm{E}+00$ & $0.00 \mathrm{E}+00$ & $0.00 \mathrm{E}+00$ & $0.00 \mathrm{E}+00$ & $0.00 \mathrm{E}+00$ & $0.00 \mathrm{E}+00$ & $0.00 \mathrm{E}+00$ & $0.00 \mathrm{E}+00$ & $0.00 \mathrm{E}+00$ & $0.00 \mathrm{E}+00$ & 0.00 & 0.00 & 0 & $0 \%$ & $0 \%$ & 0 & 0 \\
\hline Solid Layer & $4.80 \mathrm{E}+04$ & $4.02 \mathrm{E}+03$ & $1.22 E+05$ & $1.90 \mathrm{E}+05$ & $2.36 \mathrm{E}+05$ & $3.78 \mathrm{E}+04$ & $7.00 \mathrm{E}+01$ & $2.42 \mathrm{E}-02$ & $1.93 E-02$ & $1.60 \mathrm{E}-01$ & $2,10 \mathrm{E}-03$ & $2.78 \mathrm{E}+01$ & 1.68 & 1.45 & 585 & $52 \%$ & $49 \%$ & 22 & 22 \\
\hline $241-T Y-104$ & $1.70 \mathrm{E}+04$ & $2.12 \mathrm{E}+03$ & $1.13 \mathrm{E}+04$ & $9.90 \mathrm{E}+04$ & $8.34 \mathrm{E}+04$ & $5.70 \mathrm{E}+02$ & $8.4 ! E+0 !$ & 7.74E-02 & $1.32 \mathrm{E}-02$ & $1.67 \mathrm{E}-01$ & $7.61 \mathrm{E}-04$ & $3.44 E+0 !$ & 1.64 & 1.18 & 168 & $52 \%$ & $78 \%$ & 20 & 21 \\
\hline Supematant & $1.70 \mathrm{E}+04$ & $2.12 \mathrm{E}+03$ & $1.13 E+04$ & $9.90 \mathrm{E}+04$ & $8.34 E+04$ & $5.70 \mathrm{E}+02$ & $5.12 \mathrm{E}-02$ & $3.66 \mathrm{E}-04$ & $2.64 \mathrm{E}-04$ & $1.11 \mathrm{E}-03$ & $6.27 \mathrm{E}-05$ & $9.71 \mathrm{E}+00$ & 1.18 & 1.18 & 5 & $78 \%$ & $78 \%$ & 20 & 21 \\
\hline Solid Layer & $1.70 \mathrm{E}+04$ & $2.12 E+03$ & $1.13 \mathrm{E}+04$ & $9.90 \mathrm{E}+04$ & $8.34 \mathrm{E}+04$ & $5.70 \mathrm{E}+02$ & $8.67 \mathrm{E}+01$ & $7.98 \mathrm{E}-02$ & $1.36 \mathrm{E}-02$ & $1.72 \mathrm{E}-01$ & $7.83 \mathrm{E}-04$ & $3.51 \mathrm{E}+01$ & 1.65 & 1.18 & 163 & $52 \%$ & $78 \%$ & 20 & 21 \\
\hline $24 I-T Y-105$ & $1.70 \mathrm{E}+04$ & $2.98 E+03$ & $3.60 \mathrm{E}+04$ & $7.96 \mathrm{E}+04$ & $8.32 \mathrm{E}+04$ & $4.94 \mathrm{E}+03$ & $1.48 \mathrm{E}+02$ & $7.55 \mathrm{E}-03$ & $2.27 \mathrm{E}-03$ & 2.12E-02 & $1.47 \mathrm{E}-04$ & $6.24 \mathrm{E}+00$ & 1.53 & 1.17 & 874 & $39 \%$ & $75 \%$ & 25 & 23 \\
\hline Supenatant & $0.00 E+00$ & $0.00 \mathrm{E}+00$ & $0.00 E+00$ & $0.00 \mathrm{E}+00$ & $0.00 \mathrm{E}+00$ & $0.00 \mathrm{E}+00$ & $0.00 \mathrm{E}+00$ & $0.00 \mathrm{E}+00$ & $0.00 E+00$ & $0.00 \mathrm{E}+00$ & $0.00 E+00$ & $0.00 \mathrm{E}+00$ & 0.00 & 0.00 & 0 & $0 \%$ & $0 \%$ & 0 & 0 \\
\hline Solid Layer & $1.70 \mathrm{E}+04$ & $2.98 \mathrm{E}+03$ & $3.60 \mathrm{E}+04$ & $7.96 \mathrm{E}+04$ & $8.32 \mathrm{E}+04$ & $4.94 \mathrm{E}+03$ & $1.48 \mathrm{E}+02$ & $7.55 \mathrm{E}-03$ & $2.27 \mathrm{E}-03$ & $2.12 \mathrm{E}-02$ & $1.47 \mathrm{E}-04$ & $6.24 \mathrm{E}+00$ & 1.53 & 1.17 & 874 & $39 \%$ & $75 \%$ & 25 & 23 \\
\hline 241-TY-106 & $1.70 \mathrm{E}+04$ & $2.98 \mathrm{E}+03$ & $3.60 \mathrm{E}+04$ & $7.96 \mathrm{E}+04$ & $8.32 \mathrm{E}+04$ & $4.94 E+03$ & $1.21 E+02$ & $1.66 \mathrm{E}-02$ & $2.04 \mathrm{E}-03$ & $1.90 \mathrm{E}-02$ & $1.32 \mathrm{E}-04$ & $5.66 \mathrm{E}+01$ & 1.40 & 1.17 & 62 & $38 \%$ & $75 \%$ & 16 & 20 \\
\hline Supernatant & $0.00 \mathrm{E}+00$ & $0.00 \mathrm{E}+00$ & $0.00 \mathrm{E}+00$ & $0.00 \mathrm{E}+00$ & $0.00 \mathrm{E}+00$ & $0.00 \mathrm{E}+00$ & $0.00 \mathrm{E}+00$ & $0.00 \mathrm{E}+00$ & $0.00 \mathrm{E}+00$ & $0.00 \mathrm{E}+00$ & $0.00 \mathrm{E}+00$ & $0.00 \mathrm{E}+00$ & 0.00 & 0.00 & 0 & $0 \%$ & $0 \%$ & 0 & 0 \\
\hline Solid Layer & $1.70 \mathrm{E}+04$ & $2.98 \mathrm{E}+03$ & $3.60 \mathrm{E}+04$ & $7.96 \mathrm{E}+04$ & $8.32 \mathrm{E}+04$ & $4.94 \mathrm{E}+03$ & $1.21 \mathrm{E}+02$ & $1.66 \mathrm{E}-02$ & $2.04 \mathrm{E}-03$ & $1.90 \mathrm{E}-02$ & $1.32 \mathrm{E}-04$ & $5.66 \mathrm{E}+01$ & 1.40 & 1.17 & 62 & $38 \%$ & $75 \%$ & 16 & 20 \\
\hline $241-\mathrm{b}-101$ & $4.91 E \div 04$ & $2.98 \mathrm{E}+03$ & $3.60 \mathrm{E}+04$ & $7.96 \mathrm{E}+04$ & $8.32 \mathrm{E}+04$ & $4.94 \mathrm{E}+03$ & $3.71 \mathrm{E}+02$ & $2.05 \mathrm{E}-02$ & $7.86 \mathrm{E}-03$ & $4.06 \mathrm{E}-02$ & $7.53 \mathrm{E}-04$ & $5.73 \mathrm{E}+01$ & 1.77 & 1.17 & 87 & $30 \%$ & $76 \%$ & 22 & 21 \\
\hline Supematant & $0.00 \mathrm{E}+00$ & $0.00 E+00$ & $0.00 \mathrm{E}+00$ & $0.00 \mathrm{E}+00$ & $0.00 \mathrm{E}+00$ & $0.00 \mathrm{E}+00$ & $0.00 \mathrm{E}+00$ & $0.00 \mathrm{E}+00$ & $0.00 \mathrm{E}+00$ & $0.00 \mathrm{E}+00$ & $0.00 \mathrm{E}+00$ & $0.00 \mathrm{E}+00$ & 0.00 & 0.00 & 0 & $0 \%$ & $0 \%$ & 0 & 0 \\
\hline Solid Layer & $4.91 \mathrm{E}+04$ & $2.98 E+03$ & $3.60 \mathrm{E}+04$ & $7.96 \mathrm{E}+04$ & $8.32 \mathrm{E}+04$ & $4.94 \mathrm{E}+03$ & $3.71 \mathrm{E}+02$ & $2.05 \mathrm{E}-02$ & $7.86 \mathrm{E}-03$ & $4.06 \mathrm{E}-02$ & $7.53 \mathrm{E}-04$ & $5.73 E+01$ & 1.77 & 1.17 & 87 & $30 \%$ & $76 \%$ & 22 & 21 \\
\hline
\end{tabular}




\section{RPP-5926 REV 6}

Table B-1. Input Data for Hydrogen Generation Rate Model Calculations for 177 Tanks. (20 sheets)

\begin{tabular}{|c|c|c|c|c|c|c|c|c|c|c|c|c|c|c|c|c|c|c|c|}
\hline Tank & $\begin{array}{c}\text { OH } \\
\text { in liquid } \\
{[\mathrm{OH}]} \\
(\mu \mathrm{g} / \mathrm{mL})\end{array}$ & $\begin{array}{c}\text { ToC } \\
\text { in liquid } \\
{[\mathrm{TOC}]} \\
(\mu \mathrm{g} / \mathrm{mL})\end{array}$ & $\begin{array}{c}\mathrm{NO}_{2} \\
\text { in liquid } \\
{\left[\mathrm{NO}_{2}\right\rfloor} \\
(\mu \mathrm{g} / \mathrm{mL})\end{array}$ & $\begin{array}{c}\mathrm{NO}_{3} \\
\text { in liquid } \\
{\left[\mathrm{NO} \mathrm{O}_{3}\right]} \\
(\mu \mathrm{g} / \mathrm{mL})\end{array}$ & $\begin{array}{c}\mathrm{Na} \\
\text { in liquid } \\
\text { [Na| } \\
\text { (ug/mL) }\end{array}$ & $\begin{array}{c}\text { Al } \\
\text { in liquid } \\
{[A \mid} \\
(\mu \mathrm{g} / \mathbf{m L})\end{array}$ & $\begin{array}{c}{ }^{90} \mathrm{Sr} \\
\text { in waste } \\
{[\mathrm{Sr}]} \\
(\mu \mathrm{Ci} / \mathrm{g})\end{array}$ & $\begin{array}{c}{ }^{241} \mathrm{Am} \\
\text { in waste } \\
{[\mathrm{Amz41}]} \\
(\mu \mathrm{Cl} / \mathrm{g})\end{array}$ & $\begin{array}{c}{ }^{240} \mathrm{Pu} \\
\text { in waste } \\
|\mathrm{Pu} 240| \\
(\mu \mathrm{Ci} / \mathrm{g})\end{array}$ & $\begin{array}{c}{ }^{239} \mathrm{Pu} \\
\text { in waste } \\
{[\mathrm{Pu240}} \\
(\mu \mathrm{Ci} / \mathrm{g})\end{array}$ & $\begin{array}{c}{ }^{238} \mathrm{Pu} \\
\text { in waste } \\
|\mathrm{Pu} 238| \\
(\mu \mathrm{Ci} / \mathrm{g})\end{array}$ & $\begin{array}{c}{ }^{137} \mathrm{Cs} \\
\text { in waste } \\
{[\mathrm{Cs}]} \\
(\mu \mathrm{Cig})\end{array}$ & $\begin{array}{c}\text { Bulk } \\
\text { density } \\
\text { D } \\
(\mathrm{g} / \mathrm{ml})\end{array}$ & $\begin{array}{c}\text { Liquid } \\
\text { Density } \\
D_{L} \\
(g / m l)\end{array}$ & $\begin{array}{c}\text { Non-RGS } \\
\text { waste } \\
\text { volume } \\
\text { (kL) }\end{array}$ & $\begin{array}{c}\text { Bulk } \\
\text { water } \\
{\left[\mathrm{H}_{2} \mathrm{O}\right]} \\
(\mathrm{w} t \%)\end{array}$ & $\begin{array}{c}\text { Liquid } \\
\text { water } \\
\left|\mathrm{H}_{2} \mathrm{O}\right| \\
\left(\mathrm{wt}_{0} \%\right)\end{array}$ & $\begin{array}{c}\text { Wastc } \\
\text { temp. } \\
\text { Tw } \\
\left({ }^{\circ} \mathrm{C}\right)\end{array}$ & $\begin{array}{c}\text { Dome } \\
\text { temp. } \\
\text { Td } \\
\left({ }^{\circ} \mathrm{C}\right)\end{array}$ \\
\hline $241-U \cdot 102$ & $2.93 \mathrm{E}+04$ & $1.12 \mathrm{E}+04$ & $46 \mathrm{E}+05$ & $1.98 \mathrm{E}+05$ & $23 \mathrm{E}+05$ & $32 E+04$ & $43 \mathrm{E}+01$ & $1.18 \mathrm{E}-01$ & $2.29 \mathrm{E}-02$ & f.14E-0! & $2.86 \mathrm{E}-03$ & $1.41 \mathrm{E}+02$ & 1.67 & 1.48 & 1238 & $34 \%$ & $48 \%$ & 26 & 28 \\
\hline Supernatant & $2.93 E+04$ & $1.12 \mathrm{E}+04$ & $1.46 \mathrm{E}+05$ & $1.98 \mathrm{E}+05$ & $2.23 \mathrm{E}+05$ & $3.33 \mathrm{E}+04$ & $4.54 \mathrm{E}+00$ & $7.85 \mathrm{E}-03$ & $6.59 \mathrm{E}-05$ & $3.14 \mathrm{E}-04$ & $1.11 \mathrm{E}-05$ & $3.04 \mathrm{E}+02$ & 1.48 & 1.48 & 4 & $48 \%$ & $48 \%$ & 21 & 28 \\
\hline Solid Layer & $2.93 \mathrm{E}+04$ & $1.12 \mathrm{E}+04$ & $1.46 E+05$ & $1.98 \mathrm{E}+05$ & $2.23 E+05$ & $3.32 \mathrm{E}+04$ & $8.45 \mathrm{E}+01$ & $1.18 \mathrm{E}-01$ & $2.30 \mathrm{E}-02$ & $1.14 \mathrm{E}-01$ & $2.87 \mathrm{E}-03$ & $1.40 \mathrm{E}+02$ & 1.67 & 1.48 & 1234 & $34 \%$ & $48 \%$ & 26 & 28 \\
\hline $241-\mathrm{U} \cdot 103$ & $3.08 \mathrm{E}+04$ & $1.24 \mathrm{E}+04$ & $1.37 \mathrm{E}+05$ & $1.52 \mathrm{E}+05$ & $2.30 \mathrm{E}+05$ & $09 E+04$ & $2.28 \mathrm{E}+01$ & $8.53 \mathrm{E}-02$ & $9.05 \mathrm{E}-03$ & $4.31 \mathrm{E}-02$ & $1.31 E-03$ & $1.10 \mathrm{E}+02$ & 1.70 & 1.44 & 1410 & $40 \%$ & $50 \%$ & 27 & 25 \\
\hline Supeniatant & $3.08 \mathrm{E}+04$ & $1.24 \mathrm{E}+04$ & $1.37 \mathrm{E}+05$ & $1.52 \mathrm{E}+05$ & $2.31 \mathrm{E}+05$ & $4.09 \mathrm{E}+04$ & $6.49 \mathrm{E}+00$ & $1.28 \mathrm{E}-02$ & $5.97 \mathrm{E}-05$ & $2.74 \mathrm{E}-04$ & $9.65 \mathrm{E}-06$ & $3.09 \mathrm{E}+02$ & 1.44 & 1.44 & 2 & $50 \%$ & $50 \%$ & 27 & 25 \\
\hline Solid Layer & $3.08 E+04$ & $1.24 E+04$ & $1.37 \mathrm{E}+05$ & $1.52 \mathrm{E}+05$ & $2.30 \mathrm{E}+05$ & $4.09 \mathrm{E}+04$ & $2.28 \mathrm{E}+01$ & $8.54 \mathrm{E}-02$ & $9.07 \mathrm{E}-03$ & $4.31 \mathrm{E}-02$ & $1.31 \mathrm{E}-03$ & $1.10 \mathrm{E}+02$ & 1.70 & 1.44 & 1408 & $40 \%$ & $50 \%$ & 27 & 25 \\
\hline $241-\mathrm{U}-104$ & $1 E+04$ & $9.58 \mathrm{E}+03$ & $1.05 E+05$ & $07 \mathrm{E}+05$ & $2.03 \mathrm{E}+05$ & $21 \mathrm{E}+04$ & $.52 \mathrm{E}+00$ & $1.27 \mathrm{E}-03$ & $6.00 \mathrm{E}-04$ & $4.74 \mathrm{E}-03$ & 4.10E-05 & $21 E-01$ & 1.12 & 1.40 & 205 & $42 \%$ & $55 \%$ & 28 & 24 \\
\hline Supernatant & $0.00 \mathrm{E}+00$ & $0.00 \mathrm{E}+00$ & $0.00 E+00$ & $0.00 \mathrm{E}+00$ & $0.00 \mathrm{E}+00$ & $0.00 \mathrm{E}+00$ & $0.00 \mathrm{E}+00$ & $0.00 \mathrm{E}+00$ & $0.00 \mathrm{E}+00$ & $0.00 \mathrm{E}+00$ & $0.00 \mathrm{E}+00$ & $0.00 \mathrm{E}+00$ & 0.00 & 0.00 & 0 & $0 \%$ & $0 \%$ & 0 & 0 \\
\hline Solid L.ayer & $4.91 \mathrm{E}+04$ & $9.58 \mathrm{E}+03$ & $1.05 \mathrm{E}+0.05$ & $2.07 \mathrm{E}+05$ & $2.03 \mathrm{E}+05$ & $3.21 E+04$ & $2.52 \mathrm{E}+00$ & $1.27 \mathrm{E}-03$ & $6.00 \mathrm{E}-04$ & $4.74 \mathrm{E}-03$ & $4.10 \mathrm{E}-05$ & $2.21 \mathrm{E}-01$ & 1.12 & 1.40 & 205 & $42 \%$ & $55 \%$ & 28 & 24 \\
\hline $241-\mathrm{U}-105$ & $19 \mathrm{E}+04$ & $1.64 \mathrm{E}+04$ & $08 \mathrm{E}+05$ & $81 E+05$ & $27 \mathrm{E}+05$ & $98 \mathrm{E}+04$ & $14 E+01$ & $3.63 \mathrm{E}-01$ & $5.41 \mathrm{E}-02$ & $.59 E-01$ & $7.94 E-03$ & $37 \mathrm{E}+02$ & 1.67 & 1.46 & 1336 & $30 \%$ & $52 \%$ & 28 & 26 \\
\hline Supernatant & $0.00 \mathrm{E}+00$ & $0.00 \mathrm{E}+00$ & $0.00 \mathrm{E}+00$ & $0.00 \mathrm{E}+00$ & $0.00 \mathrm{E} \div 00$ & $0.00 \mathrm{E}+00$ & $0.00 E+00$ & $0.00 \mathrm{E}+00$ & $0.00 E+00$ & $0.00 \mathrm{E}+00$ & $0.00 \mathrm{E}+00$ & $0.00 \mathrm{E}+00$ & 0.00 & 0.00 & 0 & $0 \%$ & $0 \%$ & 0 & 0 \\
\hline Solid Layer & $2.09 \mathrm{E}+04$ & $1.64 E+04$ & $1.08 \mathrm{E}+05$ & $1.81 E+05$ & $2.27 \mathrm{E}+05$ & $2.98 \mathrm{E}+04$ & $1.14 \mathrm{E}+01$ & $3.63 \mathrm{E}-01$ & $5.41 \mathrm{E}-02$ & $2.59 \mathrm{E}-01$ & 7.94E-03 & $1.37 \mathrm{E}+02$ & 1.67 & 1.46 & 1336 & $30 \%$ & $52 \%$ & 28 & 26 \\
\hline 241-U-106 & $5.11 \mathrm{E}+03$ & $3.38 \mathrm{E}+04$ & $9.87 \mathrm{E}+04$ & $2.49 \mathrm{E}+05$ & $2.23 \mathrm{E}+05$ & $1.20 \mathrm{E}+04$ & $5.93 \mathrm{E}+01$ & $6.81 \mathrm{E}-01$ & 4.31E-02 & $1.98 \mathrm{E}-01$ & $6.96 \mathrm{E}-03$ & $1.45 \mathrm{E}+02$ & 1.55 & 1.34 & 644 & $42 \%$ & $49 \%$ & 26 & 25 \\
\hline Supernatant & $5.11 \mathrm{E}+03$ & $3.38 \mathrm{E}+04$ & $9.90 \mathrm{E}+04$ & $2.48 \mathrm{E}+05$ & $2.23 \mathrm{E}+05$ & $1.20 \mathrm{E}+04$ & 4. $10 \mathrm{E}+01$ & $2.16 \mathrm{E}-01$ & $9.19 \mathrm{E}-04$ & 4.22E-03 & $1.48 \mathrm{E}-04$ & $2.10 \mathrm{E}+02$ & 1.34 & 1.34 & 6 & $49 \%$ & $49 \%$ & 24 & 25 \\
\hline Solid Layer & $5.11 \mathrm{E}+03$ & $3.38 \mathrm{E}+04$ & $9.87 \mathrm{E}+04$ & $.49 \mathrm{E}+05$ & $2.23 \mathrm{E}+05$ & $1.20 E+04$ & $5.94 \mathrm{E}+0 !$ & $6.85 \mathrm{E}-01$ & $4.35 \mathrm{E}-02$ & $2.00 \mathrm{E}-01$ & 7.03E-03 & $1.45 \mathrm{E}+02$ & 1.55 & 1.34 & 638 & $42 \%$ & $49 \%$ & 26 & 25 \\
\hline $241-\mathrm{L}-107$ & $3.12 E+04$ & $6.28 E+03$ & $1.28 \mathrm{E}+05$ & $1.42 \mathrm{E}+05$ & $2.05 \mathrm{E} \sim 05$ & $4.10 \mathrm{E}+04$ & $2.09 \bar{E}+00$ & $2.15 \mathrm{E}-0 \mathrm{~J}$ & $3.04 \mathrm{E}-02$ & $1.42 \mathrm{E}-01$ & $4.96 \mathrm{E}-03$ & $7.55 \mathrm{E}+01$ & 1.74 & 1.39 & 1113 & $33 \%$ & $50 \%$ & 23 & 23 \\
\hline Supematant & $0.00 \mathrm{E}+00$ & $0.00 \mathrm{E} \div 00$ & $0.00 \mathrm{E}+00$ & $0.00 \mathrm{E}+00$ & $0.00 \mathrm{E}+00$ & $0.00 \mathrm{E}+00$ & $0.00 \mathrm{E}+00$ & $0.00 \mathrm{E}+00$ & $0.00 \mathrm{E}+00$ & $0.00 \mathrm{E}+00$ & $0.00 \mathrm{E}+00$ & $0.00 \mathrm{E}+00$ & 0.00 & 0.00 & 0 & $0 \%$ & $0 \%$ & 0 & 0 \\
\hline Solid Layer & $3.12 \mathrm{E}+04$ & $6.28 \mathrm{E}+03$ & $1.28 \mathrm{E}+05$ & $1.42 \mathrm{E}+05$ & DSE & $10 \mathrm{E}+04$ & $09 \mathrm{E}+00$ & $2.15 \mathrm{E}-01$ & $3.04 \mathrm{E}-02$ & 1.42E-01 & 4 & $7.55 \mathrm{E}+01$ & 74 & 1.43 & 1113 & $33 \%$ & $50 \%$ & 23 & 23 \\
\hline 241-U-108 & $4.9 ! \mathrm{E}+04$ & $7.07 \mathrm{E}+03$ & $1.34 \mathrm{E}+05$ & $1.84 \mathrm{E}+05$ & $2.28 \mathrm{E}+05$ & $3.05 E+04$ & $7.54 \mathrm{E}+00$ & $3.43 \mathrm{E}-02$ & $1.43 \mathrm{E}-02$ & $6.53 \mathrm{E}-02$ & $1.76 \mathrm{E}-03$ & $1.23 \mathrm{E}+02$ & 1.68 & 1.40 & 1641 & $37 \%$ & $50 \%$ & 30 & 27 \\
\hline Supernatant & $0.00 E+00$ & $0.00 \mathrm{E}+00$ & $0.00 \mathrm{E}+00$ & $0.00 E+00$ & $0.00 \mathrm{E}+00$ & $0.00 \mathrm{E}+00$ & $0.00 \mathrm{E}+00$ & $0.00 \mathrm{E}+00$ & $0.00 \mathrm{E}+00$ & $0.00 E+00$ & $0.00 \mathrm{E}+00$ & $0.00 \mathrm{E}+00$ & 0.00 & 0.00 & 0 & $0 \%$ & $0 \%$ & 0 & 0 \\
\hline Solid Layer & $4.91 \mathrm{E}+04$ & $7.07 \mathrm{E}+03$ & $1.34 \mathrm{E}+05$ & $1.84 \mathrm{E}+05$ & $2.28 \mathrm{E}+05$ & $3.05 E+04$ & $7.54 \mathrm{E}+00$ & $3.43 \mathrm{E}-02$ & $1.43 \mathrm{E}-02$ & $6.53 \mathrm{E}-02$ & $1.76 \mathrm{E}-03$ & 02 & 1.68 & 1. & 1641 & $37 \%$ & $50 \%$ & 30 & 27 \\
\hline $241-U-109$ & $4.42 \mathrm{E}+04$ & $6.53 \mathrm{E}+03$ & $1.28 \mathrm{E}+05$ & $1.85 \mathrm{E}^{+05}$ & $2.28 \mathrm{E}+05$ & $2.92 \mathrm{E}+04$ & $6.95 E+00$ & $2.36 \mathrm{E}-02$ & $2.37 \mathrm{E}-03$ & $1.11 \mathrm{E}-02$ & $3.67 E-04$ & $1.13 E+02$ & 1.65 & 1.47 & 1352 & $29 \%$ & $51 \%$ & 29 & 25 \\
\hline Supernatant & $0.00 \mathrm{E}+00$ & $0.00 \mathrm{E}+00$ & $0.00 \mathrm{E}+00$ & $0.00 \mathrm{E}+00$ & $0.00 \mathrm{E}+00$ & $0.00 \mathrm{E}+00$ & $0.00 \mathrm{E}+00$ & $0.00 \mathrm{E}+00$ & $0.00 \mathrm{E}+00$ & $0.00 \mathrm{E}+00$ & $0.00 \mathrm{E}+00$ & $0.00 \mathrm{E}+00$ & 0.00 & 0.00 & 0 & $0 \%$ & $0 \%$ & 0 & 0 \\
\hline Solid Layer & $4.42 \mathrm{E}+04$ & $6.53 \mathrm{E}+03$ & $1.28 \mathrm{E}+05$ & $1.85 \mathrm{E}+0 \mathrm{~S}$ & $2.28 \mathrm{E}+05$ & $2.92 \mathrm{E}+04$ & $6.95 \mathrm{~F}+00$ & $2.36 \mathrm{E}-02$ & $2.37 \mathrm{E}-03$ & $1.11 \mathrm{E}-02$ & 3.67E-04 & $1.13 \mathrm{E}+02$ & 1.65 & 1.47 & 1352 & $29 \%$ & $51 \%$ & 29 & 25 \\
\hline $241 \cdot \mathrm{J}-110$ & $4.91 E+04$ & $2.98 \mathrm{E}+03$ & $3.60 \mathrm{E}+04$ & $7.96 \mathrm{E}+04$ & $8.32 \mathrm{E}+04$ & $4.94 E+03$ & $1.62 \mathrm{E}+02$ & $7.35 \mathrm{E}-02$ & $3.16 \mathrm{E}-02$ & $1.84 \mathrm{E}-01$ & $2.84 \mathrm{E}-03$ & $1.81 \mathrm{E}+01$ & 1.72 & 1.17 & 665 & $34 \%$ & $75 \%$ & 25 & 24 \\
\hline Supernatant & $0.00 \mathrm{E} \div 00$ & $0.00 E+00$ & $0,00 \mathrm{E}+00$ & $0.00 \mathrm{E}+00$ & $0.00 \mathrm{E}-00$ & $0.00 \mathrm{E}+00$ & $0.00 \mathrm{E}+00$ & $0.00 E+00$ & $0.00 \mathrm{E}+00$ & $0.00 E+00$ & $0.00 \mathrm{E}+00$ & $0.00 \mathrm{E}+00$ & 0.00 & 0.00 & 0 & $0 \%$ & $0 \%$ & 0 & 0 \\
\hline Solid Layer & $4.91 \mathrm{E}+04$ & $2.98 \mathrm{~F}+03$ & $3.60 E+04$ & $7.96 \mathrm{E}+04$ & $8.32 \mathrm{E}+04$ & $4.94 \mathrm{E}+03$ & $1.62 \mathrm{E}+02$ & $7.35 \mathrm{E}-02$ & $3.16 \mathrm{E}-02$ & $1.84 \mathrm{E}_{-01}$ & 2.84E-03 & $1.81 E+01$ & 1.72 & 1.17 & 665 & $34 \%$ & $75 \%$ & 25 & 24 \\
\hline
\end{tabular}




\section{RPP-5926 REV 6}

Table B-1. Input Data for Hydrogen Generation Rate Model Calculations for 177 Tanks. (20 sheets)

\begin{tabular}{|c|c|c|c|c|c|c|c|c|c|c|c|c|c|c|c|c|c|c|c|}
\hline Tank & $\begin{array}{c}\mathrm{OH} \\
\text { in liquidd } \\
{[\mathrm{OH}]} \\
(\mu \mathrm{g} / \mathrm{mL})\end{array}$ & $\begin{array}{c}\text { roC } \\
\text { in liquid } \\
\text { [TOC] } \\
(\mu \mathrm{g} / \mathrm{mL})\end{array}$ & $\begin{array}{c}\mathrm{NO}_{2} \\
\text { in liquid } \\
{\left[\mathrm{NO}_{2}\right]} \\
(\mu \mathrm{g} / \mathrm{mL})\end{array}$ & $\begin{array}{c}\mathrm{NO}_{3} \\
\text { in liquid } \\
{\left[\mathrm{NO}_{3} !\right.} \\
(\mu \mathrm{g} / \mathrm{mL})\end{array}$ & $\begin{array}{c}\mathrm{Na} \\
\text { in liquid } \\
{[\mathrm{Na}]} \\
(\mathrm{ug} / \mathrm{mL})\end{array}$ & $\begin{array}{c}\text { Al } \\
\text { in liquid } \\
|A|\} \\
(\mu \mathrm{g} / \mathrm{mL})\end{array}$ & $\begin{array}{c}{ }^{90} \mathrm{~S} r \\
\text { in waste } \\
{[\mathrm{Sr} \mid} \\
(\mu \mathrm{Ci} / \mathrm{g})\end{array}$ & $\begin{array}{c}{ }^{241} \mathrm{Am} \\
\text { in waste } \\
{[\mathrm{Am} 241]} \\
(\mu \mathrm{Ci} / \mathrm{g})\end{array}$ & $\begin{array}{c}{ }^{240} \mathrm{Pu} \\
\text { in waste } \\
{[\mathrm{Pu} 240]} \\
(\mu \mathrm{Ci} / \mathrm{g})\end{array}$ & $\begin{array}{c}{ }^{239} \mathrm{Pu} \\
\text { in waste } \\
{[\mathrm{Pu} 240]} \\
(\mu \mathrm{Ci} / \mathrm{g})\end{array}$ & $\begin{array}{c}{ }^{238} \mathrm{Pu} \\
\text { in waste } \\
{[\mathrm{Pu} 238]} \\
(\mu \mathrm{Ci} / \mathrm{g})\end{array}$ & $\begin{array}{c}{ }^{137} \mathrm{Cs} \\
\text { in waste } \\
|\mathrm{Cs}| \\
(\mu \mathrm{Ci} / \mathrm{g})\end{array}$ & $\begin{array}{c}\text { Bulk } \\
\text { density } \\
D \\
(\mathrm{~g} / \mathrm{ml})\end{array}$ & $\begin{array}{c}\text { Liquid } \\
\text { Density } \\
\mathrm{D}_{\mathrm{L}} \\
(\mathrm{g} / \mathrm{ml})\end{array}$ & $\begin{array}{c}\text { Non-RGS } \\
\text { waste } \\
\text { volume } \\
(\mathrm{kL})\end{array}$ & $\begin{array}{c}\text { Bulk } \\
\text { water } \\
\left|\mathrm{H}_{2} \mathrm{O}\right| \\
(w t \%)\end{array}$ & $\begin{array}{l}\text { Liquid } \\
\text { water } \\
\left|\mathrm{H}_{2} \mathrm{O}\right| \\
(\mathrm{wt} \%)\end{array}$ & $\begin{array}{c}\text { Waste } \\
\text { temp. } \\
\text { Tw } \\
\left({ }^{\circ} \mathrm{C}\right)\end{array}$ & $\begin{array}{c}\text { Dome } \\
\text { temp. } \\
\text { Td } \\
\left({ }^{\circ} \mathrm{C}\right)\end{array}$ \\
\hline $241-1 ;-111$ & $3.39 E+04$ & $5.41 E+03$ & $1.20 \mathrm{E}+05$ & $1.67 \mathrm{E}+05$ & $2.39 \mathrm{E}+05$ & $3.66 \mathrm{E}+04$ & $3.82 \mathrm{E}+01$ & $1.14 \mathrm{E}-01$ & $1.88 \mathrm{E}-02$ & $9.26 \mathrm{E}-02$ & $2.59 \mathrm{E}-03$ & $1.27 \mathrm{E}+02$ & 1.61 & 1.40 & 840 & $36 \%$ & $53 \%$ & 24 & 25 \\
\hline Supernatant & $0.00 \mathrm{E} \div 00$ & $0.00 \mathrm{E}+00$ & $0.00 \mathrm{E}+00$ & $0.00 \mathrm{E}+00$ & $0.00 \mathrm{E}+00$ & $0.00 \mathrm{E}+00$ & $0.00 \mathrm{E}+00$ & $0.00 \mathrm{E}+00$ & $0.00 \mathrm{E}+00$ & $0.00 \mathrm{E}+00$ & $0.00 \mathrm{E}+00$ & $0.00 \mathrm{E}+00$ & 0.00 & 0.00 & 0 & $0 \%$ & $0 \%$ & 0 & 0 \\
\hline Solid Layer & $3.39 \mathrm{E}+04$ & $5.41 \mathrm{E}+03$ & $1.20 \mathrm{E}+05$ & $1.67 \mathrm{E}+05$ & $2.39 \mathrm{E}+05$ & $3.66 \mathrm{E}+04$ & $3.82 E+01$ & $1.14 \mathrm{E}-01$ & $1.88 \mathrm{E}-02$ & $9.26 \mathrm{E}-02$ & $2.59 \mathrm{E}-03$ & $1.27 \mathrm{E}+02$ & 1,61 & 1.40 & 840 & $36 \%$ & $53 \%$ & 24 & 25 \\
\hline $241-U-112$ & $4.91 \mathrm{E}+04$ & $2.98 E+03$ & $3.60 \mathrm{E}+04$ & $7.96 \mathrm{E}+04$ & $8.32 \mathrm{E}+04$ & $4.94 E+03$ & $1.65 E+02$ & $3.36 \mathrm{E}-03$ & $2.11 \mathrm{E}-03$ & $1.91 \mathrm{E}-02$ & $1.38 \mathrm{E}-04$ & $3.01 \mathrm{E}+01$ & 1.74 & 1.17 & 172 & $36 \%$ & $75 \%$ & 21 & 21 \\
\hline Supenatant & $0.00 \mathrm{E}+00$ & $0.00 E+00$ & $0.00 \mathrm{E}+00$ & $0.00 \mathrm{E}+00$ & $0.00 \mathrm{E}+00$ & $0.00 \mathrm{E}+00$ & $0.00 \mathrm{E}+00$ & $0.00 \mathrm{E} \div 00$ & $0.00 \mathrm{E}+00$ & $0.00 \mathrm{E}+00$ & $0.00 \mathrm{E}+00$ & $0.00 \mathrm{E}+00$ & 0.00 & 0.00 & 0 & $0 \%$ & $0 \%$ & 0 & 0 \\
\hline Solid Layer & $4.91 E+04$ & $2.98 E+03$ & $3.60 \mathrm{E}+04$ & $7.96 \mathrm{E}+04$ & $8.32 \mathrm{E} \div 04$ & $4.94 \mathrm{E}+03$ & $1.65 \mathrm{E}+02$ & $3.36 \mathrm{E}-03$ & $2.11 \mathrm{E}-03$ & $1.91 \mathrm{E}-02$ & $1.38 \mathrm{E}-04$ & $3.01 \mathrm{E}+01$ & 1.74 & 1.17 & 172 & $36 \%$ & $75 \%$ & 21 & 21 \\
\hline $241-U-201$ & $1.27 \mathrm{E}+04$ & $0.00 \mathrm{E}+00$ & $2.07 \mathrm{E}+04$ & $1.43 \mathrm{E}+05$ & $1.16 \mathrm{E}+05$ & $1.41 \mathrm{E}+02$ & $1.09 \mathrm{E}-01$ & 1. $24 \mathrm{E}-03$ & $2.68 \mathrm{E}-04$ & $1,4 ! \mathrm{E}-03$ & $2.19 \mathrm{E}-05$ & $9.23 \mathrm{E}+00$ & 1.53 & 1.26 & 15 & $36 \%$ & $69 \%$ & 20 & 21 \\
\hline Supernatant & 1.27E +04 & $0.00 E+00$ & $2.07 \mathrm{E}+04$ & $1.43 E+05$ & $1.16 \mathrm{E}+05$ & $1.41 \mathrm{E}+02$ & $1.01 \mathrm{E}-02$ & $4.33 \mathrm{E}-04$ & $3.45 \mathrm{E}-05$ & $1.82 \mathrm{E}-04$ & $2.82 E-06$ & $2.16 \mathrm{E}+01$ & 1.26 & 1.26 & 4 & $69 \%$ & $69 \%$ & 21 & 21 \\
\hline Solid Layer & $1.27 E+04$ & $0.00 \mathrm{E}+00$ & $2.07 E+04$ & $1.43 E+05$ & $1.16 \mathrm{E}+05$ & $1.41 E+02$ & $1.44 \mathrm{E}-01$ & $1.53 \mathrm{E}-03$ & $3.52 \mathrm{E}-04$ & $1.86 \mathrm{E}-03$ & $2.89 \mathrm{E}-05$ & $4.72 E+00$ & 1.63 & 1.22 & 11 & $27 \%$ & $69 \%$ & 20 & 21. \\
\hline $241-\mathrm{U}-202$ & $1.79 \mathrm{E}+04$ & $1.41 E+02$ & $2.45 \mathrm{E}+04$ & $1.52 \mathrm{E}+05$ & $1.20 E+05$ & $2.39 \mathrm{E}+02$ & $4.42 \mathrm{E}-02$ & $1.36 \mathrm{E}-0.3$ & 3.52E-04 & $1.86 \mathrm{E}-03$ & $2.89 \mathrm{E}-05$ & $7.32 \mathrm{E}+00$ & 1.44 & 1.28 & 14 & $38 \%$ & $69 \%$ & 18 & 19 \\
\hline Supernatant & $1.79 \mathrm{E}+04$ & $1.41 E+02$ & $2.45 E+04$ & $1.52 \mathrm{E}+05$ & $1.20 \mathrm{E}+05$ & $2.39 \mathrm{E}+02$ & $4.82 \mathrm{E}-04$ & 7.23E-06 & $8.73 \mathrm{E}-07$ & $4.59 \mathrm{E}-06$ & $7.15 \mathrm{E}-08$ & $1.53 \mathrm{E}+01$ & 1.28 & 1.28 & 4 & $69 \%$ & $69 \%$ & 18 & 19 \\
\hline Solid Layer & $1.79 \mathrm{E}+04$ & $1.41 E+02$ & $2.45 \mathrm{E}+04$ & $1.52 \mathrm{E}+05$ & $1.20 \mathrm{E}+05$ & $2.39 \mathrm{E}+02$ & $6.17 \mathrm{E}-02$ & $1.90 \mathrm{E}-03$ & $4.93 \mathrm{E}-04$ & $2.60 \mathrm{E}-03$ & $4.04 \mathrm{E}-05$ & $4.13 \mathrm{E}+00$ & 1.51 & 1.22 & 10 & $28 \%$ & $69 \%$ & 19 & 19 \\
\hline $241-\mathrm{C}-203$ & $4.75 E+03$ & $0.00 \mathrm{E}+00$ & $1.94 \mathrm{E}+04$ & $1.44 E+05$ & $1.00 \mathrm{E}+05$ & $1.88 \mathrm{E}+02$ & $8.72 \mathrm{E}-02$ & $1.07 \mathrm{E}-03$ & $2.00 \mathrm{E}-04$ & $1.06 \mathrm{E}-03$ & $1.64 E-05$ & $7.11 \mathrm{E}+00$ & 1.49 & 1.28 & 13 & $48 \%$ & $69 \%$ & 19 & 19 \\
\hline Supernatant & $4.75 E+03$ & $0.00 E+00$ & $1.94 E+04$ & $1.44 \mathrm{E} \div 05$ & $1.00 E+05$ & $1.88 \mathrm{E}+02$ & $4.24 \mathrm{E}-04$ & $6.93 \mathrm{E}-06$ & $3.71 \mathrm{E}-06$ & $1.95 \mathrm{E}-05$ & $3.03 \mathrm{E}-07$ & $1.43 E+01$ & 1.28 & 1.28 & 4 & $69 \%$ & $69 \%$ & 19 & 19 \\
\hline Solid Layer & $4.75 \mathrm{E}+03$ & $0.00 \mathrm{E}+00$ & $1.94 \mathrm{E}+04$ & $1.44 E+05$ & $1.00 \mathrm{E}+05$ & $1.88 \mathrm{E}+02$ & $1.26 \mathrm{E}-01$ & $1.54 \mathrm{E}-03$ & $2.88 \mathrm{E}-04$ & $1.52 \mathrm{E}-03$ & $2.35 \mathrm{E}-05$ & $3.89 \mathrm{E}+00$ & 1.59 & 1.28 & 9 & $40 \%$ & $69 \%$ & 19 & 19 \\
\hline $241-U-204$ & $1.61 \mathrm{E}+03$ & $8.10 E+01$ & $7.03 \mathrm{E}+03$ & $5.53 \mathrm{E}+04$ & $3.93 \mathrm{E}+04$ & $1.46 \mathrm{E}+01$ & $2.42 \mathrm{E}+00$ & $1.40 \mathrm{E}-02$ & $7.67 \mathrm{E}-03$ & $4.04 \mathrm{E}-02$ & $6.31 \mathrm{E}-04$ & $5.39 \mathrm{E}+00$ & 1.34 & 1.11 & II & $45 \%$ & $87 \%$ & 18 & 18 \\
\hline Supenatant & $1.61 E+03$ & $8.10 €+01$ & $7.03 E+03$ & $5.53 \mathrm{E}+04$ & $3.93 \mathrm{E}+04$ & $1.46 \mathrm{E}+01$ & $1.77 \mathrm{E}-04$ & $8.49 \mathrm{E}-06$ & $6.71 \mathrm{E}-07$ & $3.54 \mathrm{E}-06$ & $5.47 \mathrm{E}-08$ & $6.13 \mathrm{E}+00$ & 1.11 & 1.11 & 4 & $87 \%$ & $87 \%$ & 18 & 18 \\
\hline Solid Layer & $1.61 \mathrm{E}+03$ & $8.10 \mathrm{E} \div 01$ & $7.03 E+03$ & $5.53 \mathrm{E}+04$ & $3.93 E+04$ & $1.46 \mathrm{E}+01$ & $3.81 \mathrm{E}+00$ & $2.21 \mathrm{E}-02$ & $1.21 E-02$ & 6. $35 \mathrm{E}-02$ & $9.91 \mathrm{E}-04$ & $4.98 \mathrm{E}+00$ & 1.47 & 1.11 & 7 & $26 \%$ & $87 \%$ & 18 & 18 \\
\hline AY $102-S-14 U$ & $1.50 \mathrm{E}+03$ & $1.35 E+03$ & $1.79 \mathrm{E}+04$ & $3.97 \mathrm{E}+02$ & 8. $63 \mathrm{E}+04$ & $8.85 \mathrm{E}+02$ & $6.72 \mathrm{E}+0.3$ & $5.64 \mathrm{E}+00$ & $4.16 \mathrm{E}-01$ & $1.80 \mathrm{E}+00$ & $6.92 \mathrm{E}-02$ & $3.54 \mathrm{E}+02$ & 1.61 & 1.10 & 120 & $35 \%$ & $78 \%$ & 55 & 44 \\
\hline AY $102-S-14 L$ & $8.18 \mathrm{E}+02$ & $1.39 E+03$ & $1.29 \mathrm{E}+04$ & $3.33 \mathrm{E}+02$ & $8.62 \mathrm{E}+04$ & $5.84 \mathrm{E}>02$ & $9.06 \mathrm{E}+03$ & $5.64 \mathrm{E}+00$ & $4.16 \mathrm{E}-01$ & $1.80 \mathrm{E}+00$ & $6.92 \mathrm{E}-02$ & $3.50 \mathrm{E}+02$ & 1.58 & 1.20 & 89 & $40 \%$ & $793 \%$ & 67 & 44 \\
\hline AY $102-S-15 U$ & $2.50 \mathrm{E}+02$ & $1.35 E+03$ & $8.52 \mathrm{E}+03$ & $2.36 \mathrm{E}+02$ & $7.65 \mathrm{E}+04$ & $2.28 \mathrm{E}+02$ & $4.07 \mathrm{E}+03$ & $5.64 \mathrm{E}+00$ & $4.16 \mathrm{E}-01$ & $1.80 \mathrm{E}+00$ & $6.92 \mathrm{E}-02$ & $3.62 \mathrm{E}+02$ & 1.55 & 1.17 & 36 & $36 \%$ & $81 \%$ & 67 & 44 \\
\hline$A Y 102-S-15 L$ & $4.07 E+02$ & $1.80 \mathrm{E}+03$ & $3.72 E+03$ & $1.82 E+02$ & $6.88 \mathrm{E}+04$ & $2.46 \mathrm{E}+02$ & $6.15 \mathrm{E}+03$ & $5.64 \mathrm{E}+00$ & $4.16 \mathrm{E}-01$ & $1.80 \mathrm{E}+00$ & $6.92 \mathrm{E}-02$ & $4.03 E+02$ & 1.60 & 1.18 & 125 & $42 \%$ & $83 \%$ & 67 & 44 \\
\hline$A Y-102-S-16$ & $2.51 E+03$ & $1.86 \mathrm{E}+03$ & $4.46 \mathrm{E}+02$ & $1.22 \mathrm{E}+02$ & $5.42 \mathrm{E}+04$ & 4.55E+01 & $7.66 \mathrm{E}+03$ & $5.64 \mathrm{E}+00$ & 4.16E-01 & $1.80 \mathrm{E}+00$ & $6.92 \mathrm{E}-02$ & $3.24 \mathrm{E}+02$ & 1.40 & 1.15 & 109 & $43 \%$ & $86 \%$ & 72 & 44 \\
\hline AY 102-Orig-9" & $2.50 \mathrm{E}+02$ & $1.17 \mathrm{E}+03$ & $4.67 \mathrm{E}+01$ & $1.22 \mathrm{E}+02$ & $5.01 \mathrm{E}+04$ & $5.88 \mathrm{E}+01$ & $1.40 \mathrm{E}+04$ & $5.64 \mathrm{E}+00$ & $4.16 \mathrm{E}-01$ & $1.80 \mathrm{E}+00$ & $6.92 \mathrm{E}-02$ & $2.01 E+02$ & 1.44 & 1.13 & 94 & $43 \%$ & $86 \%$ & 73 & 44 \\
\hline
\end{tabular}


RPP-5926 REV 6

Table B-2. Derived Data for Hydrogen Generation Rate Model Calculations for 177 Tanks. (20 sheets)

\begin{tabular}{|c|c|c|c|c|c|c|c|c|c|c|c|c|c|c|c|}
\hline Tank & $\begin{array}{c}\text { Waste } \\
\text { level } \\
\text { Dw } \\
\text { (inch) }\end{array}$ & $\begin{array}{c}\text { Total } \\
\text { mass } \\
M \\
(\mathbf{k g})\end{array}$ & $\begin{array}{c}\text { Liquid } \\
\text { in waste } \\
(w 1 \%)\end{array}$ & $\begin{array}{c}\text { Wetted } \\
\text { area } \\
\text { Awet } \\
\left(\text { fft }^{2}\right)\end{array}$ & $\begin{array}{c}\text { Tank Heat } \\
\text { load } \\
\mathrm{H}_{\mathrm{L}}{ }^{g / t} \\
\text { (watt } / \mathrm{kg} \text { ) }\end{array}$ & $\begin{array}{c}\text { Tank } \\
\text { heat load } \\
\mathrm{H}_{\mathrm{L}}^{\text {alpha }} \\
\text { (watt } / \mathrm{kg} \text { ) }\end{array}$ & $\begin{array}{c}\mathrm{NO}_{3} \\
\text { in liquid } \\
{\left[\mathrm{NO}_{3}\right]} \\
(\mathrm{mole} / \mathrm{L})\end{array}$ & $\begin{array}{c}\mathrm{NO}_{2} \\
\text { in liquid } \\
{\left[\mathrm{NO}_{2}\right]} \\
(\mathrm{mole} / \mathrm{L})\end{array}$ & $\begin{array}{c}\text { Excess } \mathrm{Na} \\
\text { in liquid } \\
{[\mathrm{Na}]_{\mathrm{ex}}} \\
(\mathrm{mole} / \mathrm{L})\end{array}$ & $\begin{array}{c}\mathrm{OH} \\
\text { in liquid } \\
{[\mathrm{OH}]} \\
(\text { mole } / \mathrm{L})\end{array}$ & $\begin{array}{l}\text { TOC } \\
\text { in liquid } \\
\text { [TOC]\% } \\
\text { (wt } \% \text { ) }\end{array}$ & $\begin{array}{c}\text { Al } \\
\text { in liquid } \\
\mid A]] \% \\
(\mathbf{w t} \%)\end{array}$ & $\begin{array}{c}\mathrm{E}_{\mathrm{H} 2} \text { Efficiency } \\
\text { of } \mathrm{H}_{2} \text { by } \\
\text { corrosion }\end{array}$ & $\begin{array}{c}\text { Total } \\
\text { G values } \\
\mathrm{G}_{\mathrm{TOT}}^{\mathrm{B} / \gamma} \\
\left(\mathrm{H}_{2 / 1} 100 \mathrm{eV}\right)\end{array}$ & $\begin{array}{c}\text { Total } \\
\text { G values } \\
\mathrm{G}_{\text {TOT }}{ }^{\text {alpha }} \\
\left(\mathrm{H}_{2} / 100 \mathrm{eV}\right)\end{array}$ \\
\hline $241-A N-101$ & 347.6 & $5.13 E+06$ & $99 \%$ & 11244 & $9.49 \mathrm{E}-04$ & $7.83 \mathrm{E}-08$ & 2.63 & 2.53 & 4.19 & 2.06 & 0.36 & 1.39 & 0.20 & 0.030 & 0.122 \\
\hline Supernatant & 336.3 & $4.94 E+06$ & $100 \%$ & 6603 & $9.46 \mathrm{E}-04$ & $2.38 \mathrm{E}-08$ & 2.61 & 2.52 & 4.18 & 2.06 & 0.36 & 1.34 & 0.20 & 0.030 & 0.122 \\
\hline Solid Layer & 11.3 & $1.83 \mathrm{E}+05$ & $82 \%$ & 4640.1 & $1.03 \mathrm{E}-03$ & $1.55 \mathrm{E}-06$ & 3.06 & 2.66 & 4.54 & 2.06 & 0.3 & 2.6 & 0.20 & 0.0 & 0.107 \\
\hline $241-\mathrm{AN}-102$ & 388.7 & $5.78 \mathrm{E}+06$ & $99 \%$ & 12050 & 1.76E-03 & $6.60 \mathrm{E}-06$ & 3.37 & 1.89 & 3.73 & 0.52 & 1.71 & 0.93 & 0.20 & 0.071 & 0.126 \\
\hline Superiatant & 332.7 & $4.89 \mathrm{E}+06$ & $100 \%$ & 6532 & $1.69 \mathrm{E}-03$ & $3.91 \mathrm{E}-06$ & 3.37 & 1.89 & 3.73 & 0.52 & 1.71 & 0.93 & 0.20 & 0.071 & 0.126 \\
\hline Solid Layer & 56.0 & $8.94 \mathrm{E}+05$ & $93 \%$ & 5518 & $2.16 \mathrm{E}-03$ & $2.13 \mathrm{E}-05$ & 3.37 & 1.89 & 3.73 & 0.52 & 1.71 & 0.93 & 0.20 & 0.070 & 0.126 \\
\hline 241-AN-103 & 347.9 & $5.44 \mathrm{E}+06$ & $87 \%$ & 11249 & $1.49 \mathrm{E}-03$ & $1.57 \mathrm{E}-07$ & 2.07 & 2.83 & 6.85 & 3.95 & 0.21 & 2.02 & 0.20 & 0.023 & 0.140 \\
\hline Supernatant & 169.7 & $2.62 \mathrm{E}+06$ & $100 \%$ & 3332 & $2.02 \mathrm{E}-03$ & $1.42 \mathrm{E}-07$ & 2.07 & 2.83 & 6.85 & 3.95 & 0.21 & 2.02 & 0.20 & 0.023 & 0.140 \\
\hline Solid Layer & 178.2 & $2.82 \mathrm{E}+06$ & $75 \%$ & 7917.3 & $9.87 \mathrm{E}-04$ & $1.71 \mathrm{E}-07$ & 2.07 & 2,83 & 6.85 & 3.95 & 0.21 & 2.02 & 0.20 & 0.023 & 0.140 \\
\hline $241-\mathrm{AN}-104$ & 382.1 & $5.71 \mathrm{E}+06$ & $95 \%$ & 11920 & $1.64 \mathrm{E}-03$ & $3.27 \mathrm{E}-07$ & 3.05 & 2.59 & 5.59 & 3.84 & 0.22 & 2.79 & 0.20 & 0.025 & 0.110 \\
\hline Supenatant & 220.4 & $3.22 \mathrm{E}+06$ & $100 \%$ & 4328 & $1.66 \mathrm{E}-03$ & $1.03 \mathrm{E}-08$ & 3.05 & 2.59 & 5.59 & 3.84 & 0.22 & 2.79 & 0.20 & 0.024 & 0.109 \\
\hline Solid Layer & 161.6 & $2.49 \mathrm{E}+06$ & $89 \%$ & 7591 & $1.61 \mathrm{E}-03$ & $7.37 \mathrm{E}-07$ & 3.05 & 2.59 & 5.59 & 3.84 & 0.22 & 2.79 & 0.20 & 0.026 & 0.110 \\
\hline $24 \mathrm{I}-\mathrm{AN}-105$ & 408.7 & $6.18 \mathrm{E}+06$ & $95 \%$ & 12443 & $1.11 \mathrm{E}-03$ & $3.25 \mathrm{E}-07$ & 2.52 & 2.58 & 5.64 & 3.51 & 0.19 & 2.93 & 0.20 & 0.023 & 0.124 \\
\hline Supenatant & 213.6 & $3.16 \mathrm{E}+06$ & $100 \%$ & 4194 & $9.75 \mathrm{E}-04$ & $3.49 \mathrm{E}-07$ & 2.52 & 2.58 & 5.64 & 3.51 & 0.19 & 2.93 & 0.20 & 0.023 & 0.124 \\
\hline Solid Layer & 195.1 & $3.02 E+06$ & $89 \%$ & 8249 & $1.25 \mathrm{E}-03$ & $3.00 \mathrm{E}-07$ & 2.52 & 2.58 & 5.64 & 3.51 & 0.19 & 2.93 & 0.20 & 0.024 & 0.124 \\
\hline 241-AN-106 & 291.7 & $3.51 \mathrm{E}+06$ & $93 \%$ & 10145 & $3.90 \mathrm{E}-03$ & $3.37 \mathrm{E}-05$ & 0.38 & 0.41 & 1.03 & 0.30 & 0.18 & 0.35 & 0.20 & 0.133 & 0.486 \\
\hline Supematant & 238.4 & $2.73 \mathrm{E}+06$ & $100 \%$ & 4680 & $2.10 \mathrm{E}-04$ & $1.16 \mathrm{E}-07$ & 0.38 & 0.41 & 1.03 & 0.30 & 0.18 & 0.35 & 0.20 & 0.133 & 0.486 \\
\hline Solid Layer & 53.3 & $7.74 \mathrm{E}+05$ & $67 \%$ & 5465 & $1.69 \mathrm{E}-02$ & $1,52 \mathrm{E}-04$ & 0.38 & 0.41 & 1.03 & 0.30 & 0.18 & 0.35 & 0.20 & 0.134 & 0.487 \\
\hline $24 !-A N-107$ & 399.9 & $6.01 E+06$ & $97 \%$ & 12270 & 1.53E-03 & $2,12 \mathrm{E}-05$ & 3.37 & 1.45 & 4.21 & 1.06 & 2.27 & 0.08 & 0.20 & 0.092 & 0.141 \\
\hline Supentatant & 316.3 & $4.71 \mathrm{E}+06$ & $100 \%$ & 6210 & $1.35 \mathrm{E}-03$ & $1,81 \mathrm{E}-05$ & 3.37 & 1.45 & 4.21 & 1.06 & 2.27 & 0.08 & 0.20 & 0.091 & 0.140 \\
\hline Solid Layer & 83.6 & $1.29 \mathrm{E}+06$ & $86 \%$ & 6060 & $2.21 \mathrm{E}-03$ & $3.27 \mathrm{E}-05$ & 3.37 & 1.45 & 4.21 & 1.06 & 2.27 & 0.08 & 0.20 & 0.096 & 0.143 \\
\hline 241-AP-101 & 404.7 & $5.48 \mathrm{E}+06$ & $100 \%$ & 12364 & $5.07 \mathrm{E}-04$ & 8.15E-09 & 1.90 & 0.89 & 2.84 & 2.34 & 0.15 & 0.56 & 0.20 & 0.040 & 0.173 \\
\hline Supcriatant & 404.7 & $5.48 \mathrm{E}+06$ & $100 \%$ & 12364 & $5.07 \mathrm{E}-04$ & $8.15 \mathrm{E}-09$ & 1.90 & 0.89 & 2.84 & 2.34 & 0.15 & 0.56 & 0.20 & 0.040 & 0.173 \\
\hline Solid Layer & па & na & na & na & па & na & na & na & na & na & na & na & na & па & na \\
\hline $241-\mathrm{AP}+102$ & 396.8 & $5.78 \mathrm{E}+06$ & $99 \%$ & 12210 & 8.03E-04 & $4.87 \mathrm{E}-08$ & 2.70 & 2.14 & 3.63 & 1.89 & 0.22 & 2.22 & 0.20 & 0.031 & 0.122 \\
\hline Supenlatant & 388.4 & $5.63 \mathrm{E}+06$ & $100 \%$ & 7626 & $8.15 \mathrm{E}-04$ & $4.89 \mathrm{E}-08$ & 2.70 & 2.14 & 3.63 & 1.89 & 0.22 & 2.22 & 0.20 & 0.031 & 0.122 \\
\hline Solid Layer & 8.4 & $1.54 \mathrm{E}+05$ & $53 \%$ & 4584 & $3.86 \mathrm{E}-04$ & $3.99 \mathrm{E}-08$ & 2.70 & 2.14 & 3.63 & 1.89 & 0.22 & 2.22 & 0.20 & 0.029 & 0.121 \\
\hline
\end{tabular}


RPP-5926 REV 6

Table B-2. Derived Data for Hydrogen Generation Rate Model Calculations for 177 Tanks. (20 sheets)

\begin{tabular}{|c|c|c|c|c|c|c|c|c|c|c|c|c|c|c|c|}
\hline Tank & $\begin{array}{c}\text { Waste } \\
\text { level } \\
\text { Dw } \\
\text { (inch) }\end{array}$ & $\begin{array}{c}\text { Total } \\
\text { mass } \\
M \\
(\mathrm{~kg})\end{array}$ & $\begin{array}{l}\text { Liquid } \\
\text { in waste } \\
\text { (wt"\%) }\end{array}$ & $\begin{array}{l}\text { Wetted } \\
\text { area } \\
\text { Awet } \\
\left(\mathrm{ft}^{2}\right)\end{array}$ & $\begin{array}{c}\text { Tank Heat } \\
\text { load } \\
\mathbf{H}_{\mathbf{2}}{ }^{\mu / r} \\
\text { (watt } / \mathrm{kg} \text { ) }\end{array}$ & $\begin{array}{c}\text { Tank } \\
\text { heat load } \\
\mathrm{H}_{\mathrm{L}}^{\text {alpaz }} \\
\text { (watt } / \mathbf{k g} \text { ) }\end{array}$ & $\begin{array}{c}\mathrm{NO}_{3} \\
\text { in liquid } \\
{\left[\mathrm{NO}_{3}\right]} \\
(\text { mole } / \mathrm{L})\end{array}$ & $\begin{array}{c}\mathrm{NO}_{2} \\
\text { in liquid } \\
{\left[\mathrm{NO}_{2}\right]} \\
(\text { mole } / \mathrm{L})\end{array}$ & $\begin{array}{c}\text { Excess } \mathrm{Na} \\
\text { in liquid } \\
{[\mathrm{Na}]_{\text {ex }}} \\
(\mathrm{mole} / \mathrm{L})\end{array}$ & $\begin{array}{c}\mathrm{OH} \\
\text { in liquid } \\
\text { [OH] } \\
(\text { mole } / \mathrm{L})\end{array}$ & $\begin{array}{c}\text { Toc } \\
\text { in liquid } \\
\text { [TOC|\% } \% \\
(w t \%)\end{array}$ & $\begin{array}{c}\text { Al } \\
\text { in liquid } \\
{[\mathrm{Al}] \%} \\
(\mathrm{wt} \%)\end{array}$ & $\begin{array}{c}\mathrm{E}_{\mathrm{H} 2} \text { Efficiency } \\
\text { of } \mathrm{H}_{2} \text { by } \\
\text { corrosion }\end{array}$ & 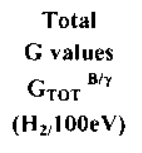 & $\begin{array}{c}\text { Total } \\
\text { G values } \\
\mathbf{G}_{\mathrm{TO}}{ }^{\text {apha }} \\
\left(\mathrm{H}_{2} / 100 \mathrm{eV}\right)\end{array}$ \\
\hline 241-AP- 103 & 324.0 & $4.59 \mathrm{E}+06$ & $99 \%$ & 10780 & $8.08 \mathrm{E}-04$ & $2.92 \mathrm{E}-07$ & 2.44 & 1.75 & 3.60 & 0.98 & 0.55 & 1.38 & 0.20 & 0.035 & 0.136 \\
\hline Supernatant & 316.4 & $4.45 \mathrm{E}+06$ & $100 \%$ & 6212 & $8.16 \mathrm{E}-04$ & $3.00 \mathrm{E}-07$ & 2.44 & 1.75 & 3.60 & 0.98 & 0.55 & 1.38 & 0.20 & 0.035 & 0.136 \\
\hline Solid Layer & 7.7 & $1.34 \mathrm{E}+05$ & $72 \%$ & 4569 & $5.30 \mathrm{E}-04$ & 4. $34 \mathrm{E}-08$ & 2.44 & 1.75 & 3.60 & 0.98 & 0.55 & 1.38 & 0.20 & 0.036 & 0.137 \\
\hline 241-AP-104 & 398.6 & $5.32 \mathrm{E}+06$ & $100 \%$ & 12244 & $6.94 \mathrm{E}-04$ & $7.81 \mathrm{E}-08$ & 1.63 & 1.39 & 2.57 & 1.16 & 0.33 & 1.31 & 0.20 & 0.046 & 0.184 \\
\hline Supernatant & 398.6 & $5.32 \mathrm{E}+06$ & $100 \%$ & 12244 & $6.94 \mathrm{E}-04$ & $7,81 \mathrm{E}-08$ & 1.63 & 1.39 & 2.57 & 1.16 & 0.33 & 1.31 & 0.20 & 0.046 & 0.184 \\
\hline Solid Layer & $\mathrm{na}$ & na & na & na & na & $\mathrm{na}$ & na & na & $\mathrm{na}$ & na & na & $\mathrm{na}$ & na & na & na \\
\hline 241-AP-105 & 412.7 & $5.58 \mathrm{E}+06$ & $97 \%$ & 12520 & $5.73 \mathrm{E}-04$ & $5.39 \mathrm{E}-08$ & 1.71 & 1.03 & 3.08 & 1.52 & 0.12 & 1.52 & 0.20 & 0.040 & 0.184 \\
\hline Supernatant & 380.3 & $5.04 \mathrm{E}+06$ & $100 \%$ & 7468 & $5.08 \mathrm{E}-04$ & $2.62 \mathrm{E}-08$ & 1.71 & 1.03 & 3.08 & 1.52 & 0.12 & 1.52 & 0.20 & 0.041 & 0.184 \\
\hline Solid Layer & 32.3 & $5.43 \mathrm{E}+05$ & $67 \%$ & 5053 & $\$ .18 \mathrm{E}-03$ & $3.11 \mathrm{E}-07$ & 1.71 & 1.03 & 3.08 & 1.52 & 0.12 & 1.52 & 0.20 & 0.039 & 0.183 \\
\hline $241-\mathrm{AP}-106$ & 412.6 & $5.20 \mathrm{E}+06$ & $100 \%$ & 12519 & $6.92 \mathrm{E}-04$ & $1.87 \mathrm{E}-08$ & 1.14 & 0.90 & 2.46 & 0.43 & 0.29 & 1.12 & 0.20 & 0.057 & 0.247 \\
\hline Supernatant & $4 ! 2.6$ & $5.20 \mathrm{E}+06$ & $100 \%$ & 12519 & $6.92 \mathrm{E}-04$ & $1.87 \mathrm{E}-08$ & 1.14 & 0.90 & 2.46 & 0.43 & 0.29 & 1.12 & 0.20 & 0.057 & 0.247 \\
\hline Solid Layer & na & na & na & na & na & na & na & na & na & na & na & na & na & na & na \\
\hline $241-A^{P}-107$ & 125.3 & $1.71 \mathrm{E}+06$ & $100 \%$ & 6878 & $5.32 \mathrm{E}-04$ & $1.50 \mathrm{E}-08$ & 2.03 & 1.25 & 2.78 & 1.07 & 0.21 & 1.25 & 0.20 & 0.038 & 0.159 \\
\hline Supernatant & 125.3 & $1.71 E+06$ & $100 \%$ & 6878 & $5.32 \mathrm{E}-04$ & 1.50E-08 & 2.03 & 1.25 & 2.78 & 1.07 & 0.21 & 1.25 & 0.20 & 0.038 & 0.159 \\
\hline Solid Layer & na & na & na & na & па & na & na & na & na & na & na & na & na & na & na \\
\hline $241 \cdot \mathrm{AP}^{\mathrm{P}} 108$ & 371.4 & $5.55 E+06$ & $99 \%$ & 11711 & $6.71 \mathrm{E}-04$ & $1.68 \mathrm{E}-08$ & 2.87 & 1.72 & 4.66 & 2.33 & 0.20 & 1.69 & 0.20 & 0.027 & 0.120 \\
\hline Supernatant & 324.7 & $4.81 \mathrm{E}+06$ & $100 \%$ & 6375 & $6.77 \mathrm{E}-04$ & $1.70 \mathrm{E}-08$ & 2.87 & 1.72 & 4.66 & 2.33 & 0.20 & 1.69 & 0.20 & 0.028 & 0.121 \\
\hline Solid Layer & 46.7 & $7.40 \mathrm{E}+05$ & $93 \%$ & 5335 & $6.33 E-04$ & 1.58E-08 & 2.87 & 1.72 & 4.66 & 2.33 & 0.20 & 1.69 & 0.20 & 0.024 & 0.119 \\
\hline 241-AW-101 & 409.4 & $6.30 E+06$ & $100 \%$ & 12456 & $1.23 \mathrm{E}-03$ & $1.20 \mathrm{E}-06$ & 2.76 & 2.29 & 5.07 & 5.86 & 0.18 & 2.01 & 0.20 & 0.025 & 0.119 \\
\hline Supernatant & 265.7 & $4.07 \mathrm{E}+06$ & $100 \%$ & $\$ 217$ & $1.22 \mathrm{E}-03$ & $1.72 \mathrm{E}-08$ & 2.76 & 2.29 & 5.07 & 5.86 & 0.18 & 2.01 & 0.20 & 0.024 & 0.119 \\
\hline Solid Layer & 143.7 & $2.23 \mathrm{E}+06$ & $100 \%$ & 7239 & $1.25 \mathrm{E}-03$ & $3.35 \mathrm{E}-06$ & 2.76 & 2.29 & 5.07 & 5.86 & 0.18 & 2.01 & 0.20 & 0.025 & 0.119 \\
\hline 241-AW-102 & 220.0 & $2.80 \mathrm{E}+06$ & $99 \%$ & 8737 & $4.89 \mathrm{E}-04$ & $9.19 \mathrm{E}-07$ & 1.56 & 0.97 & 2.07 & 1.09 & 0.17 & 0.99 & 0.20 & 0.051 & 0.197 \\
\hline Supematant & 217.6 & $2.77 \mathrm{E}+06$ & $100 \%$ & 4272 & $4.47 \mathrm{E}-04$ & $1.39 \mathrm{E}-08$ & 1.56 & 0.97 & 2.07 & 1.09 & 0.17 & 0.99 & 0.20 & 0.051 & 0.197 \\
\hline Solid Layer & 2.4 & $3.30 \mathrm{E}+04$ & $47 \%$ & 4465 & $3.97 \mathrm{E}-03$ & $7.68 \mathrm{E}-05$ & 1.56 & 0.97 & 2.07 & 1.09 & 0.17 & 0.99 & 0.20 & 0.052 & 0.198 \\
\hline $241-A W-103$ & 398.0 & $5.46 \mathrm{E}+06$ & $103 \%$ & 12232 & $3.43 \mathrm{E}-04$ & $4.18 \mathrm{E}-06$ & 2.29 & 1.12 & 2.32 & 1.50 & 0.25 & 0.62 & 0.20 & 0.040 & 0.147 \\
\hline Supernatant & 277.5 & $3.59 \mathrm{E}+06$ & $100 \%$ & 5449 & $4.30 \mathrm{E}-04$ & $5.20 \mathrm{E}-09$ & 1.56 & 0.73 & 2.10 & 0.93 & 0.15 & 0.65 & 0.20 & 0.051 & 0.203 \\
\hline Solid Layer & 120.5 & $1.87 \mathrm{E}+06$ & $108 \%$ & 6783 & $1.76 \mathrm{E}-04$ & $1.22 \mathrm{E}-05$ & 3.98 & 2.02 & 2.73 & 2.83 & 0.43 & 0.57 & 0.20 & 0.029 & 0.092 \\
\hline
\end{tabular}


RPP-5926 REV 6

Table B-2. Derived Data for Hydrogen Generation Rate Model Calculations for 177 Tanks. (20 sheets)

\begin{tabular}{|c|c|c|c|c|c|c|c|c|c|c|c|c|c|c|c|}
\hline Tank & $\begin{array}{c}\text { Waste } \\
\text { level } \\
\text { Dw } \\
\text { (inch) }\end{array}$ & $\begin{array}{c}\text { Total } \\
\text { mass } \\
M \\
(\mathrm{~kg})\end{array}$ & $\begin{array}{c}\text { Liquid } \\
\text { in waste } \\
\text { (wt\%) }\end{array}$ & $\begin{array}{c}\text { Wetted } \\
\text { area } \\
\text { Awet } \\
\left(\mathrm{ft}^{2}\right)\end{array}$ & $\begin{array}{c}\text { Tank Heat } \\
\text { load } \\
\mathbf{H}_{\mathbf{L}}{ }^{a / r} \\
\text { (watt } / \mathbf{k g} \text { ) }\end{array}$ & $\begin{array}{c}\text { Tank } \\
\text { heat load } \\
H_{\mathrm{L}}^{\text {alpha }} \\
\text { (watt/kg) }\end{array}$ & $\begin{array}{c}\mathrm{NO}_{3} \\
\text { in liquid } \\
\text { ! } \mathrm{NO}_{3} ! \\
(\mathrm{mole} / \mathrm{L})\end{array}$ & $\begin{array}{c}\mathrm{NO}_{2} \\
\text { in liquid } \\
{\left[\mathrm{NO}_{2} \mid\right.} \\
(\text { mole/L) }\end{array}$ & $\begin{array}{c}\text { Excess } \mathrm{Na} \\
\text { in liquid } \\
\text { [Nalex } \\
\text { (mole/L) }\end{array}$ & $\begin{array}{c}\mathrm{OH} \\
\text { in liquid } \\
\text { [OH] } \\
\text { (mole } / \mathrm{L})\end{array}$ & $\begin{array}{c}\text { TOC } \\
\text { in lik|uid } \\
\text { |TOC|"\% } \\
\text { (wt \%) }\end{array}$ & $\begin{array}{c}\text { Al } \\
\text { in liquid } \\
|A| \mid \% \\
(w t \%)\end{array}$ & $\mid \begin{array}{c}E_{\mathrm{H}_{2}} \text { Efficiency } \\
\text { of } \mathrm{H}_{2} \text { by } \\
\text { corrosion }\end{array}$ & $\begin{array}{c}\text { Total } \\
\text { G values } \\
\mathrm{G}_{\mathrm{TOT}}^{\mathrm{B} / \mathrm{\gamma}} \\
\left(\mathrm{H}_{2 /} 100 \mathrm{eV}\right)\end{array}$ & $\begin{array}{c}\text { Total } \\
\text { G values } \\
\mathbf{G}_{\text {TOT }}{ }^{\text {alpha }} \\
\left(\mathrm{H}_{2} / 100 \mathrm{eV}\right)\end{array}$ \\
\hline $241-\mathrm{AW}-104$ & 389.8 & $5.60 \mathrm{E}+06$ & $98 \%$ & 12072 & $8.36 \mathrm{E}-04$ & $4.32 \mathrm{E}-06$ & 1.83 & 1.65 & 4.55 & 1.67 & 0.30 & 1.98 & 0.20 & 0.031 & 0.166 \\
\hline Supematant & 309.0 & $4.35 \mathrm{E}+06$ & $100 \%$ & 6067 & $8.45 \mathrm{E}-04$ & $4.20 \mathrm{E}-08$ & 1.81 & 1.62 & 3.95 & 1.49 & 0.32 & 1.88 & 0.20 & 0.035 & 0.168 \\
\hline Solid Layer & 80.9 & $1.25 \mathrm{E}+06$ & $89 \%$ & 6006 & $8.05 \mathrm{E}-04$ & $1.92 \mathrm{E}-05$ & 1.90 & 1.75 & 6.61 & 2.36 & 0.24 & 2.33 & 0.20 & 0.021 & 0.161 \\
\hline 241-AW-105 & 152.1 & $1.99 \mathrm{E}+06$ & $78 \%$ & 7405 & $2.25 \mathrm{E}-04$ & $2.03 \mathrm{E}-05$ & 0.72 & 0.26 & 0.82 & 0.42 & 0.04 & 0.10 & 0.20 & 0.105 & 0.365 \\
\hline Supernatant & 56.3 & $6.22 E+05$ & $100 \%$ & 1106 & $4.51 \mathrm{E}-05$ & $1.12 E-08$ & 0.40 & 0.06 & 0.54 & 0.26 & 0.04 & 0.01 & 0.50 & 0.156 & 0.526 \\
\hline Solid Layer & 95.8 & $1.36 \mathrm{E}+06$ & $67 \%$ & 6299 & $3.08 \mathrm{E}-04$ & $2.96 \mathrm{E}-05$ & 0.90 & 0.38 & 0.96 & 0.51 & 0.04 & 0.14 & 0.20 & 0.088 & 0.309 \\
\hline $241-\mathrm{AW}-106$ & $4 ! 2.1$ & $5.76 \mathrm{E}+06$ & $87 \%$ & 12509 & $7.21 \mathrm{E}-04$ & $3.76 \mathrm{E}-07$ & 1.44 & 0.98 & 2.64 & 1.60 & 0.15 & 1.30 & 0.20 & 0.048 & 0.209 \\
\hline Superuatant & 309.3 & $4.03 E+06$ & $100 \%$ & 6072 & $4.78 \mathrm{E}-04$ & $4.79 \mathrm{E}-08$ & 1.44 & 0.98 & 2.64 & 1.60 & 0.15 & 1.30 & 0.20 & 0.048 & 0.209 \\
\hline Solid Layer & 102.8 & $1.73 \mathrm{E}+06$ & $58 \%$ & 6437 & $1.29 \mathrm{E}-03$ & $1.14 \mathrm{E}-06$ & 1.44 & 0.98 & 2.64 & 1.60 & 0.15 & 1.30 & 0.20 & 0.049 & 0.210 \\
\hline $24 !-\mathrm{AY}-101$ & 79.1 & $1.15 \mathrm{E}+06$ & $70 \%$ & 5972 & 1.27E-02 & 4. $38 \mathrm{E}-04$ & 0.05 & 0.94 & 2.32 & 1.05 & 0.19 & 0.15 & 0.50 & 0.113 & 0.619 \\
\hline Supenatant & 40.9 & $4.81 E \div 05$ & $100 \%$ & 802 & $1.36 \mathrm{E}-04$ & $4.56 \mathrm{E}-07$ & 0.05 & 0.50 & 2.88 & 2.03 & 0.08 & 0.29 & 0.50 & 0.091 & 0.737 \\
\hline Solid Layer & 38.3 & $6.72 \mathrm{E}+05$ & $49 \%$ & 5169 & $2.17 \mathrm{E}-02$ & $7.51 \mathrm{E}-04$ & 0.05 & 1.42 & 1.92 & 0.00 & 0.26 & 0.05 & 0.50 & 0.126 & 0.530 \\
\hline $241-\mathrm{AY}-102$ & 351.4 & $4.46 \mathrm{E}+06$ & $89 \%$ & 11317 & $1.15 \mathrm{E}-02$ & $5.04 \mathrm{E}-05$ & 0.01 & 0.51 & 1.99 & 0.39 & 0.08 & 0.08 & 0.50 & 0.140 & 0.794 \\
\hline Supernatant & 296.6 & $3.56 \mathrm{E}+06$ & $100 \%$ & 5824 & $8.17 \mathrm{E}-05$ & $5.89 \mathrm{E}-08$ & 0.01 & 0.60 & 1.73 & 0.46 & 0.07 & 0.09 & 0.50 & 0.151 & 0.759 \\
\hline Solid Layer & 54.8 & $9.00 \mathrm{E}+05$ & $48 \%$ & 5493 & $5.64 \mathrm{E}-02$ & $2.49 \mathrm{E}-04$ & 0.01 & 0.00 & 3.05 & 0.02 & 0.13 & 0.03 & 0.50 & 0.184 & 1.067 \\
\hline $241-A Z-101$ & 313.8 & $4.07 \mathrm{E}+06$ & $96 \%$ & 10579 & $1.53 \mathrm{E}-02$ & $2.19 \mathrm{E}-04$ & 0.90 & 1.41 & 2.79 & 0.68 & 0.04 & 0.52 & 0.20 & 0.060 & 0.265 \\
\hline Supernatant & 294.9 & $3.75 \mathrm{E}+06$ & $100 \%$ & 5790 & $6.39 \mathrm{E}-03$ & $5.58 \mathrm{E}-08$ & 0.90 & 1.41 & 2.79 & 0.68 & 0.04 & 0.52 & 0.20 & 0.060 & 0.265 \\
\hline Solid Layer & 18.9 & $3.17 \mathrm{E}+05$ & $48 \%$ & 4789 & $1.20 \mathrm{E}-01$ & $2.80 \mathrm{E}-03$ & 0.90 & $1.4 !$ & 2.79 & 0.68 & 0.04 & 0.52 & 0.20 & 0.070 & 0.270 \\
\hline $241-\mathrm{AZ}-102$ & 351.4 & $4.25 \mathrm{E}+06$ & $95 \%$ & 11317 & $1.01 \mathrm{E}-02$ & $2.32 \mathrm{E}-04$ & 0.22 & 0.74 & 1.56 & 0.14 & 0.09 & 0.05 & 0.20 & 0.136 & 0.537 \\
\hline Supematant & 313.4 & $3.69 \mathrm{E}+06$ & $100 \%$ & 6153 & $4.02 \mathrm{E}-03$ & $2.94 \mathrm{E}-07$ & 0.22 & 0.74 & 1.56 & 0.14 & 0.09 & 0.05 & 0.20 & 0.134 & 0.537 \\
\hline Solid Layer & 38.0 & $5.58 \mathrm{E}+05$ & $65 \%$ & 5164 & $4.99 \mathrm{E}-02$ & $1.77 \mathrm{E}-03$ & 0.22 & 0.74 & 1.56 & 0.14 & 0.09 & 0.05 & 0.20 & 0.147 & 0.543 \\
\hline 241-SY-101 & 410.6 & $5.58 \mathrm{E}+06$ & $93 \%$ & 12479 & $3.96 \mathrm{E}-04$ & $2.68 \mathrm{E}-06$ & 2.72 & 0.47 & 2.14 & 2.20 & 0.09 & 0.47 & 0.20 & 0,036 & 0.135 \\
\hline Supernatant & 316.5 & $4.22 \mathrm{E}+06$ & $100 \%$ & 6215 & $2.39 \mathrm{E}-04$ & $8.75 \mathrm{E}-09$ & 2.72 & 0.47 & 2.14 & 2.20 & 0.09 & 0.47 & 0.20 & 0.036 & 0.135 \\
\hline Solid Layer & 94.0 & $1.36 \mathrm{E}+06$ & $73 \%$ & 6264 & $8.85 \mathrm{E}-04$ & $1.10 \mathrm{E}-05$ & 2.72 & 0.47 & 2.14 & 2.20 & 0.09 & 0.47 & 0.20 & 0.037 & 0.135 \\
\hline $241-S Y-102$ & 411.8 & $5.37 \mathrm{E}+06$ & $96 \%$ & 12504 & $4.29 \mathrm{E}-04$ & $1.74 \mathrm{E}-04$ & 2.60 & 0.49 & 1.96 & 0.66 & 0.11 & 0.38 & 0.20 & 0.039 & 0.140 \\
\hline Supernatant & 353.8 & $4.43 \mathrm{E}+06$ & $100 \%$ & 6946 & $1.62 \mathrm{E}-04$ & $2.81 \mathrm{E}-08$ & 2.67 & 0.31 & 1.77 & 0.57 & 0.06 & 0.34 & 0.20 & 0.039 & 0.139 \\
\hline Solid Layer & 58.0 & $9.44 E+05$ & $75 \%$ & 5557 & $1.68 \mathrm{E}-03$ & $9.91 E-04$ & 2.16 & 1.59 & 2.85 & 1.26 & 0.34 & 0.56 & 0.20 & 0.040 & 0.150 \\
\hline
\end{tabular}


RPP-5926 REV 6

Table B-2. Derived Data for Hydrogen Generation Rate Model Calculations for 177 Tanks. (20 sheets)

\begin{tabular}{|c|c|c|c|c|c|c|c|c|c|c|c|c|c|c|c|}
\hline Tank & $\begin{array}{c}\text { Waste } \\
\text { level } \\
\text { Dw } \\
\text { (inch) }\end{array}$ & $\begin{array}{c}\text { Total } \\
\text { mass } \\
M \\
(\mathrm{~kg})\end{array}$ & $\begin{array}{c}\text { Liquid } \\
\text { in waste } \\
(\mathbf{w t} \%)\end{array}$ & $\begin{array}{c}\text { Wetted } \\
\text { area } \\
\text { Awet } \\
\left(\mathrm{ft}^{2}\right)\end{array}$ & $\begin{array}{c}\text { Tank Heat } \\
\text { load } \\
\mathbf{H}_{\mathrm{L}}{ }^{\beta / \mathrm{r}} \\
\text { (watt/kg) }\end{array}$ & $\begin{array}{c}\text { Tank } \\
\text { heat load } \\
\mathrm{H}_{\mathrm{L}}^{\text {2lpha }} \\
\text { (watt } / \mathrm{kg} \text { ) }\end{array}$ & $\begin{array}{c}\mathrm{NO}_{3} \\
\text { in liquid } \\
{\left[\mathrm{NO}_{3}\right]} \\
(\text { mole } / \mathrm{L})\end{array}$ & $\begin{array}{c}\mathrm{NO}_{2} \\
\text { in liquid } \\
\left|\mathrm{NO}_{2}\right| \\
(\mathrm{mole} / \mathrm{L})\end{array}$ & $\begin{array}{c}\text { Excess } \mathrm{Na} \\
\text { in liquid } \\
|\mathrm{Na}|_{\text {ex }} \\
(\mathrm{mole} / \mathrm{L})\end{array}$ & $\begin{array}{c}\mathrm{OH} \\
\text { in liquid } \\
{[\mathrm{OH}]} \\
(\mathrm{mole} / \mathrm{L})\end{array}$ & $\begin{array}{c}\text { TOC } \\
\text { in liquid } \\
\text { [TOC|\% } \\
\text { (wt \%) }\end{array}$ & $\begin{array}{c}\text { Al } \\
\text { in liquid } \\
|A| \mid \% \\
(w \mid \%)\end{array}$ & $\begin{array}{c}E_{\mathrm{H} 2} \text { Efficiency } \\
\text { of } \mathrm{H}_{2} \text { by } \\
\text { corrosion }\end{array}$ & $\begin{array}{c}\text { Total } \\
\text { G values } \\
\text { G }_{\text {Tot }}{ }^{\mathrm{B} / \mathrm{\gamma}} \\
\left(\mathrm{H}_{2} / 100 \mathrm{eV}\right)\end{array}$ & $\begin{array}{c}\text { Total } \\
\text { G values } \\
\text { G }_{\text {Tot }}^{2 \text { lpha }} \\
\left(\mathrm{H}_{2} / 100 \mathrm{eV}\right)\end{array}$ \\
\hline $24 \mathrm{I}-\mathrm{SY}-103$ & 268.3 & $4.15 E+06$ & $93 \%$ & 9686 & $1.33 \mathrm{E}-03$ & $1.09 \mathrm{E}-05$ & 2.70 & 3.31 & 3.33 & 1.76 & 0.42 & 2.56 & 0.20 & 0.039 & 0.117 \\
\hline Supernatant & 144.2 & $2.21 \mathrm{E}+06$ & $100 \%$ & 2831 & 1.39E- -03 & $2.29 \mathrm{E}-07$ & 2.70 & 3.31 & 3.33 & 1.76 & 0.42 & 2.56 & 0.20 & 0.035 & 0.114 \\
\hline Solid Layer & 124.1 & $1.94 \mathrm{E}+06$ & $85 \%$ & 6855 & $1.26 \mathrm{E}-03$ & $2.31 \mathrm{E}-05$ & 2.70 & 3.31 & 3.33 & 1.76 & 0.42 & 2.56 & 0.20 & 0.045 & 0.120 \\
\hline $24 \mid-A-101$ & 116.2 & $1.76 \mathrm{E}+06$ & $67 \%$ & 6699 & $1.36 \mathrm{E}-03$ & $1.10 \mathrm{E}-05$ & 3.76 & 3.58 & 4.73 & $2.5 !$ & 0.25 & 3.47 & 0.20 & 0.026 & 0.090 \\
\hline Supematant & na & na & na & na & na & na & na & na & na & na & na & na & 0.20 & na & na \\
\hline Solid Layer & 116.2 & $1.76 \mathrm{E}+06$ & $67 \%$ & 6699 & $1.36 \mathrm{E}-03$ & $1.10 \mathrm{E}-0.5$ & 3.76 & 3.58 & 4.73 & 2.51 & 0.25 & 3.47 & 0.20 & 0.026 & 0.090 \\
\hline $241-A-102$ & 14.5 & 2.51E+05 & $81 \%$ & 4702 & $2.83 \mathrm{E}-03$ & $8.09 \mathrm{E}-05$ & 3.05 & 3.75 & 1.90 & 1.02 & 0.28 & 2.61 & 0.20 & 0.030 & 0.100 \\
\hline Supernatant & 1.2 & 1. $88 \mathrm{E}+04$ & $100 \%$ & 23 & $1.50 \mathrm{E}-03$ & $1.14 \mathrm{E}-07$ & 3.05 & 3.75 & 1.89 & 1.02 & 0.28 & 2.61 & 0.20 & 0,032 & 0.101 \\
\hline Solid Layer & 13.3 & $2.32 \mathrm{E}+05$ & $80 \%$ & 4680 & $2.94 \mathrm{E}-03$ & $8.74 \mathrm{E}-05$ & 3.05 & 3.75 & 1.90 & 1.02 & 0.28 & $2.6 !$ & 0.20 & 0.030 & 0.099 \\
\hline $241-\mathrm{A}-103$ & 137.6 & $1.96 E+06$ & $82 \%$ & 7119 & $1.10 \mathrm{E}-03$ & $8.29 \mathrm{E}-06$ & 3.90 & 2.69 & 3.63 & 2.87 & 0.55 & 2.35 & 0.20 & 0.032 & 0.093 \\
\hline Supernatant & 1.6 & $2.57 \mathrm{E}+04$ & $100 \%$ & 32 & $8.54 \mathrm{E}-04$ & $6.29 \mathrm{E}-07$ & 3.90 & 2.70 & 3.63 & 2.88 & 0.55 & 2.35 & 0.20 & 0.032 & 0.093 \\
\hline Solid Layer & 135.9 & $1.93 E+06$ & $82 \%$ & 7087 & $1.10 \mathrm{E}-03$ & $8.39 \mathrm{E}-06$ & 3.90 & 2.69 & 3.63 & 2.87 & 0.55 & 2.35 & 0.20 & 0.032 & 0.093 \\
\hline $241-A-104$ & 10.2 & $1.00 \mathrm{E}+05$ & $0 \%$ & 4618 & $1.56 \mathrm{E}-01$ & $5.24 \mathrm{E}-04$ & 1.28 & 0.78 & 1.55 & 2.51 & 0.26 & 0.42 & 0.20 & 0.099 & 0.249 \\
\hline Supernatant & na & na & na & na & na & na & na & na & na & na & na & nа & 0.20 & na & na \\
\hline Solid Layer & 10.2 & $1.00 \mathrm{E}+05$ & $0 \%$ & 4618 & 1. $56 \mathrm{E}-01$ & $5.24 \mathrm{E}-04$ & 1.28 & 0.78 & 1.55 & 2.51 & 0.26 & 0.42 & 0.20 & 0.099 & 0.249 \\
\hline $241-\mathrm{A}-105$ & 13.3 & $2.14 E+05$ & $0 \%$ & 4680 & $9.24 \mathrm{E}-02$ & $9.64 \mathrm{E}-04$ & 1.28 & 0.78 & 1.55 & na & 0.26 & 0.42 & 0.20 & 0.422 & 0.422 \\
\hline Supernatant & na & na & na & na & na & na & na & na & na & na & na & nad & na & nat & 11a \\
\hline Solid Layer & 13.3 & $2.14 \mathrm{E}+05$ & $0 \%$ & 4680 & $9.24 \mathrm{E}-02$ & $9.64 \mathrm{E}-04$ & 1.28 & 0.78 & 1.55 & na & 0.26 & 0.42 & 0.20 & 0.061 & 0.230 \\
\hline $241-\mathrm{A}-106$ & 28.7 & $5.08 \mathrm{E}+05$ & $46 \%$ & 4981 & $7.03 \mathrm{E}-03$ & $1.16 \mathrm{E}-04$ & 1.28 & 0.78 & 1.55 & $2.5 \mathrm{t}$ & 0.94 & 0.42 & 0.20 & 0.108 & 0.253 \\
\hline Supernatant & na & na & na & na & na & na & na & na & na & na & па & na & 0.20 & na & na \\
\hline Solid Layer & 28.7 & $5.08 \mathrm{E}+05$ & $46 \%$ & 4981 & $7.03 \mathrm{E}-03$ & $1.16 \mathrm{E}-04$ & 1.28 & 0.78 & 1.55 & 2.51 & 0.94 & 0.42 & 0.20 & 0.108 & 0.253 \\
\hline $241-\mathrm{AX}-101$ & 129.8 & $2.30 \mathrm{E}+06$ & $79 \%$ & 6966 & 1.48E-03 & $7.55 \mathrm{E}-06$ & 4.39 & 3.76 & 4.13 & 2.29 & 0.26 & 3.29 & 0.20 & 0.022 & 0.078 \\
\hline Supernatanı & na & na & na & na & na & па & na & na & па & na & na & nа & 0.20 & na & na \\
\hline Solid Layer & 129.8 & $2.30 \mathrm{E}+06$ & $79 \%$ & 6966 & $1.48 \mathrm{E}-03$ & $7.55 \mathrm{E}-06$ & 4.39 & 3.76 & 4.11 & 2.29 & 0.26 & 3.29 & 0.20 & 0.022 & 0.078 \\
\hline $241-\mathrm{AX}-102$ & 10.8 & $1.78 \mathrm{E}+05$ & $70 \%$ & 4631 & $9.31 \mathrm{E}-03$ & 4. 32E-04 & 2.83 & 2.33 & 3.43 & 2.29 & 1.65 & 1.98 & 0.20 & 0.039 & 0.120 \\
\hline Supenaatant & na & na & па & na & na & na & na & na & na & па & na & na & 0.20 & na & na \\
\hline Solid Layer & 10.8 & $1.78 \mathrm{E}+05$ & $70 \%$ & 4631 & $9.31 \mathrm{E}-03$ & $4.32 \mathrm{E}-04$ & 2.83 & 2.33 & 3.43 & 2.29 & 1.65 & 1.98 & 0.20 & 0.039 & 0.120 \\
\hline
\end{tabular}


RPP-5926 REV 6

Table B-2. Derived Data for Hydrogen Generation Rate Model Calculations for 177 Tanks. (20 sheets)

\begin{tabular}{|c|c|c|c|c|c|c|c|c|c|c|c|c|c|c|c|}
\hline Tank & $\begin{array}{l}\text { Waste } \\
\text { level } \\
\text { Dw } \\
\text { (inch) }\end{array}$ & $\begin{array}{c}\text { Total } \\
\text { mass } \\
M \\
(\mathrm{~kg})\end{array}$ & $\begin{array}{c}\text { Liquid } \\
\text { in waste } \\
\text { (wt } \%)\end{array}$ & $\begin{array}{c}\text { Wetted } \\
\text { area } \\
\text { Awet } \\
\left(\mathbf{f t}^{2}\right)\end{array}$ & $\begin{array}{c}\text { Tank Heat } \\
\text { load } \\
\mathrm{H}_{\mathrm{L}}^{\beta / r} \\
\text { (watt/kg) }\end{array}$ & $\begin{array}{c}\text { Tank } \\
\text { heat load } \\
\mathbf{H}_{\mathrm{L}}^{\text {alpha }} \\
\text { (watt } / \mathbf{k g} \text { ) }\end{array}$ & $\begin{array}{c}\mathrm{NO}_{3} \\
\text { in liquid } \\
{\left[\mathrm{NO}_{3}\right]} \\
(\text { mole/L) }\end{array}$ & $\begin{array}{c}\mathrm{NO}_{2} \\
\text { in liquid } \\
{\left[\mathrm{NO}_{2}\right]} \\
\text { (mole } / \mathrm{L} \text { ) }\end{array}$ & $\begin{array}{c}\text { Excess } \mathrm{Na} \\
\text { in liquid } \\
\left.\quad \mathrm{Na}\right|_{\mathrm{es}} \\
(\mathrm{mole} / \mathrm{L})\end{array}$ & $\begin{array}{c}\mathrm{OH} \\
\text { in liquid } \\
|\mathrm{OH}| \\
(\mathrm{mole} / \mathrm{L})\end{array}$ & $\begin{array}{c}\text { TOC } \\
\text { in liquid } \\
\text { [TOC]\% } \\
\text { (wt } \% \text { ) }\end{array}$ & $\begin{array}{c}\text { Al } \\
\text { in liquid } \\
|A| \mid \% \\
(w t \%)\end{array}$ & $\left\{\begin{array}{c}\mathrm{E}_{\mathrm{Hz}} \text { Efficiency } \\
\text { of } \mathbf{H}_{2} \mathbf{b y} \\
\text { corrosion }\end{array}\right.$ & $\begin{array}{c}\text { Total } \\
\text { G values } \\
\text { G }_{\text {TOT }}{ }^{\text {/ } ~} \\
\left(\mathrm{H}_{2 / 10 \mathrm{eV}}\right)\end{array}$ & $\begin{array}{c}\text { Total } \\
\text { G values } \\
\mathrm{G}_{\mathrm{TOT}} \\
\left(\mathrm{H}_{2} / 100 \mathrm{eV}\right)\end{array}$ \\
\hline 241-AX-103 & 38.7 & $6.37 \mathrm{E}+05$ & $90 \%$ & 5177 & $5.53 \mathrm{E}-03$ & $4.32 \mathrm{E}-05$ & 3.08 & 2.65 & 4.53 & 2.29 & 0.28 & 2.61 & 0.20 & 0.025 & 0.107 \\
\hline Supernatant & na & na & na & na & na & na & na & $\mathrm{na}$ & na & $\mathrm{na}$ & na & na & 0.20 & na & na \\
\hline Solid Layer & 38.7 & $6.37 \mathrm{E}+05$ & $90 \%$ & 5177 & $5.53 \mathrm{E}-03$ & $4.32 \mathrm{E}-05$ & 3.08 & 2.65 & 4.53 & 2.29 & 0.28 & 2.61 & 0.20 & 0.025 & 0.107 \\
\hline 241-AX-104 & 2.7 & $5.04 \mathrm{E}+04$ & $11 \%$ & 4471 & $2.77 \mathrm{E}-01$ & $8.28 \mathrm{E}-04$ & 1.28 & 0.78 & 1.55 & 2.29 & 0.26 & 0.42 & 0.20 & 0.064 & 0.231 \\
\hline Supernatant & na & na & $\mathrm{na}$ & na & na & na & na & na & $\mathrm{na}$ & nа & па & na & 0.20 & na & $\mathrm{na}$ \\
\hline Solid Layer & 2.7 & $5.04 \mathrm{E}+04$ & $11 \%$ & 4471 & $2.77 \mathrm{E}-01$ & $8.28 \mathrm{E}-04$ & 1.28 & 0.78 & 1.55 & 2.29 & 0.26 & 0.42 & 0.20 & 0.064 & 0.231 \\
\hline $24 !-B-101$ & 46.9 & $6.22 \mathrm{E}+05$ & $81 \%$ & 5339 & $2.20 \mathrm{E}-03$ & $9.62 \mathrm{E}-05$ & 5.74 & 2.86 & 1.22 & 1.00 & 0.00 & 0.05 & 0.20 & 0.019 & 0.063 \\
\hline Supernatant & na & na & $\mathrm{na}$ & na & na & na & па & na & na & na & na & na & 0.20 & na & na \\
\hline Solid Layer & 46.9 & $6.22 \mathrm{E}+05$ & $81 \%$ & 5339 & $2.20 \mathrm{E}-03$ & $9.62 \mathrm{E}-05$ & 5.74 & 2.86 & 1.22 & 1.00 & 0.00 & 0.05 & 0.20 & 0.019 & 0.063 \\
\hline 241-B-102 & 19.1 & $1.90 \mathrm{E}+05$ & $68 \%$ & 4792 & $1.93 E-05$ & $9.34 \mathrm{E}-07$ & 3.50 & 0.19 & 1.22 & 1.00 & 0.00 & 0.03 & 0.20 & 0.032 & 0.110 \\
\hline Supematant & 1.4 & $1.89 \mathrm{E}+04$ & $100 \%$ & 28 & 4.48E-05 & $1.82 \mathrm{E}-07$ & 3.49 & 0.18 & 1.22 & 1.00 & 0.00 & 0.03 & 0.20 & 0.032 & 0.111 \\
\hline Solid Layer & 17.6 & $1.71 \mathrm{E}+05$ & $65 \%$ & 4764 & $1.64 \mathrm{E}-05$ & $1.02 \mathrm{E}-06$ & 3.50 & 0.19 & 1.22 & 1.00 & 0.00 & 0.03 & 0.20 & 0.032 & 0.110 \\
\hline $241-B-103$ & 27.7 & $3.40 \mathrm{E}+05$ & $71 \%$ & 4962 & 2.19E-0.5 & $5.18 \mathrm{E}-06$ & 3.52 & 0.18 & 1.21 & 1.00 & 0.00 & 0.03 & 0.20 & 0.032 & 0.110 \\
\hline Supenatant & na & na & ra & па & na & na & па & na & na & na & na & па & 0.20 & na & ra \\
\hline Solid Layer & 27.7 & $3.40 \mathrm{E}+05$ & $71 \%$ & 4962 & $2.19 \mathrm{E}-05$ & $5.18 \mathrm{E}-06$ & 3.52 & 0.18 & 1.21 & 1.00 & 0.00 & 0.03 & 0.20 & 0.032 & 0.110 \\
\hline $241-B-104$ & 143.6 & $1.96 \mathrm{E}+06$ & $68 \%$ & 7237 & $4.45 \mathrm{E}-0.5$ & $1.58 \mathrm{E}-06$ & 3.49 & 0.18 & 1.23 & 1.00 & 0.00 & 0.03 & 0.20 & 0.032 & 0.111 \\
\hline Supernatant & na & na & па & па & na & na & na & na & na & па & na & na & 0.20 & na & na \\
\hline Solid Layer & 143.6 & $1.96 E+06$ & $68 \%$ & 7237 & $4.45 \mathrm{E}-05$ & $1.58 \mathrm{E}-06$ & 3.49 & 0.18 & 1.23 & 1.00 & 0.00 & 0.03 & 0.20 & 0.032 & 0.111 \\
\hline $241-B-105$ & $1 ! 2.9$ & $1.82 \mathrm{E}+06$ & $62 \%$ & 6635 & $1.48 \mathrm{E}-05$ & $1.20 \mathrm{E}-06$ & 3.49 & 0.18 & 1.24 & 1.00 & 0.00 & 0.03 & 0.20 & 0.032 & 0.111 \\
\hline Supernatant & na & na & na & nа & па & na & na & na & na & na & na & na & 0.20 & na & na \\
\hline Solid Layer & 112.9 & $1.82 \mathrm{E}+06$ & $62 \%$ & 6635 & $1.48 \mathrm{E}-05$ & $1.20 \mathrm{E}-06$ & 3.49 & 0.18 & 1.24 & 1.00 & 0.00 & 0.03 & 0.20 & 0.032 & 0.111 \\
\hline $241-B-106$ & 52.0 & $6.40 \mathrm{E}+05$ & $87 \%$ & 5439 & $4.00 \mathrm{E}-04$ & $1.31 \mathrm{E}-06$ & 3.50 & 0.18 & 1.23 & 1.00 & 0.00 & 0.03 & 0.20 & 0.032 & 0.110 \\
\hline Supernatant & 0.4 & $5.04 \mathrm{E}+03$ & $100 \%$ & 8 & $4.48 \mathrm{E}-05$ & $1.82 \mathrm{E}-07$ & 3.50 & 0.18 & 1.23 & 1.00 & 0.00 & 0.03 & 0.20 & 0.032 & 0.110 \\
\hline Solid Layer & 51.6 & $6.35 \mathrm{E}+05$ & $87 \%$ & 5432 & $4.02 \mathrm{E}-04$ & $1.32 \mathrm{E}-06$ & 3.50 & 0.18 & 1.23 & 1.00 & 0.00 & 0.03 & 0.20 & 0.032 & 0.110 \\
\hline $241-B-107$ & 66.1 & $9.93 \mathrm{E}+05$ & $70 \%$ & 5717 & $7.35 \mathrm{E}-05$ & $1.53 \mathrm{E}-06$ & 5.34 & 0.14 & 0.89 & 1.00 & 0.00 & 0.00 & 0.20 & 0.023 & 0.076 \\
\hline Supernalant & na & na & na & na & na & na & na & na & na & na & na & na & 0.20 & na & na \\
\hline Solid Layer & 66.1 & $9.93 E+05$ & $70 \%$ & 5717 & $7.35 \mathrm{E}-05$ & $1.53 \mathrm{E}-06$ & 5.34 & 0.14 & 0.89 & 1.00 & 0,00 & 0.00 & 0.20 & 0.023 & 0.076 \\
\hline
\end{tabular}


RPP-5926 REV 6

Table B-2. Derived Data for Hydrogen Generation Rate Model Calculations for 177 Tanks. (20 sheets)

\begin{tabular}{|c|c|c|c|c|c|c|c|c|c|c|c|c|c|c|c|}
\hline Tank & $\begin{array}{c}\text { Waste } \\
\text { level } \\
\text { Dw } \\
\text { (inch) }\end{array}$ & $\begin{array}{c}\text { Total } \\
\text { mass } \\
M \\
(\mathrm{~kg})\end{array}$ & $\begin{array}{c}\text { Liquid } \\
\text { in waste } \\
(w+\%)\end{array}$ & $\begin{array}{l}\text { Wetted } \\
\text { area } \\
\text { Awet } \\
\left(\mathrm{it}^{2}\right)\end{array}$ & $\begin{array}{c}\text { Tank Heat } \\
\text { load } \\
\mathrm{H}_{\mathrm{L}}{ }^{\beta / r} \\
(\text { watt } / \mathrm{kg})\end{array}$ & $\begin{array}{c}\text { Tank } \\
\text { heat load } \\
H_{L}^{\text {alpha }} \\
\text { (watt } / \mathrm{kg} \text { ) }\end{array}$ & $\begin{array}{c}\mathrm{NO}_{3} \\
\text { in liquid } \\
\left\{\mathrm{NO}_{3}\right\} \\
(\text { mole } / \mathrm{L})\end{array}$ & $\begin{array}{c}\mathrm{NO}_{2} \\
\text { in liquid } \\
{\left[\mathrm{NO}_{2}\right]} \\
(\mathrm{mole} / \mathrm{L})\end{array}$ & $\begin{array}{c}\text { Excess } \mathrm{Na} \\
\text { in liquid } \\
{\left[\left.\mathrm{Na}\right|_{\mathrm{ex}}\right.} \\
\text { (mole/L) }\end{array}$ & $\begin{array}{c}\text { OH } \\
\text { in liquid } \\
{[\mathrm{OH}]} \\
(\mathrm{mole} / \mathrm{L})\end{array}$ & $\begin{array}{c}\text { TOC } \\
\text { in liquid } \\
\text { [TOC]\% } \\
\text { (wt"w) }\end{array}$ & $\begin{array}{c}\text { Al } \\
\text { in liquid } \\
|A| \mid \% \\
(w t \%)\end{array}$ & $\begin{array}{c}\mathbf{E}_{\mathrm{H} 2} \text { Efficiency } \\
\text { of } \mathrm{H}_{2} \text { by } \\
\text { corrosion }\end{array}$ & $\begin{array}{c}\text { Total } \\
\text { G values } \\
\mathrm{G}_{\text {ror }}^{\mathrm{B} / \mathrm{\gamma}} \\
\left(\mathrm{H}_{2 j} 100 \mathrm{eV}\right)\end{array}$ & $\begin{array}{c}\text { Total } \\
G \text { values } \\
\mathrm{G}_{\text {TOT }}^{\text {alpha }} \\
\left(\mathrm{H}_{2} / 100 \mathrm{eV}\right)\end{array}$ \\
\hline $241-13-108$ & 41.1 & $5.96 \mathrm{E}+05$ & $50 \%$ & 5224 & 7.02E-05 & $1.22 \mathrm{E}-07$ & 6.07 & 1.17 & 0.33 & 1.00 & 0.00 & 0.07 & 0.20 & 0.020 & 0.064 \\
\hline Supematant & na & na & na & na & na & na & na & na & na & na & na & na & 0.20 & na & па \\
\hline Solid Layer & 41.1 & $5.96 \mathrm{E}+05$ & $50 \%$ & 5224 & $7.02 \mathrm{E}-05$ & $1.22 \mathrm{E}-07$ & 6.07 & 1.17 & 0.33 & 1.00 & 0.00 & 0.07 & 0.20 & 0.020 & 0.064 \\
\hline 24 J-B-109 & 53.1 & $8.64 \mathrm{E}+05$ & $57 \%$ & 5460 & $9.97 \mathrm{E}-06$ & $8.88 \mathrm{E}-07$ & 3.50 & 0.19 & 1.22 & 1.00 & 0.00 & 0.03 & 0.20 & 0.032 & 0.110 \\
\hline Supematant & na & na & na & na & na & na & na & na & па & na & na & na & 0.20 & na & nа \\
\hline Solid Layer & 53.1 & $8.64 \mathrm{E}+05$ & $57 \%$ & 5460 & 9.97E-06 & $8.88 \mathrm{E}-07$ & 3.50 & 0.19 & 1.22 & 1.00 & 0.00 & 0.03 & 0.20 & 0.032 & 0.110 \\
\hline $241-B-110$ & 96.7 & 1.26E +06 & $76 \%$ & 6317 & $5.69 \mathrm{E}-04$ & $5.87 \mathrm{E}-06$ & 1.25 & 0.86 & 2.04 & 1.00 & 0.55 & 1.03 & 0.20 & 0.059 & 0.233 \\
\hline Supernatant & 0.4 & $4.76 \mathrm{E}+03$ & $100 \%$ & 8 & $6.86 \mathrm{E}-05$ & $3.51 \mathrm{E}-07$ & 1.25 & 0.86 & 2.04 & 1.00 & 0.47 & 0.87 & 0.20 & 0.059 & 0.233 \\
\hline Solid Layer & 96.3 & $1.26 \mathrm{E}+06$ & $76 \%$ & 6309 & $5.71 \mathrm{E}-04$ & $5.89 \mathrm{E}-06$ & 1.25 & 0.86 & 2.04 & 1.00 & 0.55 & 1.03 & 0.20 & 0.059 & 0.233 \\
\hline $241-\mathrm{B}-111$ & 95.3 & $1.16 \mathrm{E}+06$ & $76 \%$ & 6288 & $1.86 \mathrm{E}-03$ & $5.84 \mathrm{E}-06$ & 1.25 & 0.86 & 2.04 & 1.00 & 0.55 & 1.03 & 0.20 & 0.061 & 0.233 \\
\hline Supernatant & 0.4 & $4.76 \mathrm{E}+03$ & $100 \%$ & 8 & $6.86 \mathrm{E}-05$ & $3.51 \mathrm{E}-07$ & 1.25 & 0.86 & 2.04 & 1.00 & 0.47 & 0.87 & 0.20 & 0.059 & 0.233 \\
\hline Solid Layer & 94.9 & $1.16 E+06$ & $76 \%$ & 6281 & $1.87 \mathrm{E}-03$ & $5.86 \mathrm{E}-06$ & 1.25 & 0.86 & 2.04 & 1.00 & 0.55 & 1.03 & 0.20 & 0.061 & 0.233 \\
\hline $241-\mathrm{B}-112$ & 20.1 & $1.97 \mathrm{E}+05$ & $90 \%$ & 4813 & $2.16 \mathrm{E}-04$ & $1.40 E-07$ & 3.50 & 2.54 & 5.05 & 1.00 & 1.20 & 3.13 & 0.20 & 0.026 & 0.100 \\
\hline Supematant & 1.1 & $1.66 \mathrm{E}+04$ & $100 \%$ & 21 & $4.89 \mathrm{E}-04$ & $2.77 \mathrm{E}-07$ & 3.50 & 2.55 & 5.01 & 1.00 & 1.20 & 3.13 & 0.20 & 0.026 & 0.100 \\
\hline Solid Layer & 19.1 & $1.81 \mathrm{E}+05$ & $89 \%$ & 4792 & $1.91 \mathrm{E}-04$ & $1.27 \mathrm{E}-07$ & 3.50 & 2.54 & 5.05 & 1.00 & 1.20 & 3.13 & 0.20 & 0.026 & 0.100 \\
\hline $24 \mathrm{i}-\mathrm{B}-201$ & 152.6 & $1.40 \mathrm{E}+05$ & $86 \%$ & 1113 & $1.21 \mathrm{E}-05$ & $2.54 \mathrm{E}-05$ & 1.28 & 0.78 & 1.55 & 1.00 & 0.26 & 0.42 & 0.20 & 0.062 & 0.230 \\
\hline Supenatant & na & na & na & na & na & na & na & na & na & na & na & na & 0.20 & na & na \\
\hline Solid Layer & 152.6 & $1.40 E+05$ & $86 \%$ & 1113 & $1.21 \mathrm{E}-05$ & $2.54 \mathrm{E}-05$ & 1.28 & 0.78 & 1.55 & 1.00 & 0.26 & 0.42 & 0.20 & 0.062 & 0.230 \\
\hline $241-B-202$ & 148.6 & $1.32 \mathrm{E}+05$ & $91 \%$ & 1092 & $2.04 \mathrm{E}-05$ & $6.83 E-06$ & 1.28 & 0.78 & 1.55 & 1.00 & 0.26 & 0.42 & 0.20 & 0.062 & 0.230 \\
\hline Supernatant & na & na & na & na & na & na & na & na & na & na & na & na & 0.20 & na & na \\
\hline Solid Layer & 148.6 & $1.32 E+05$ & $91 \%$ & 1092 & $2.04 \mathrm{E}-05$ & $6.83 \mathrm{E}-06$ & 1.28 & 0.78 & 1.55 & 1.00 & 0.26 & 0.42 & 0.20 & 0.062 & 0.230 \\
\hline 241-B-203 & 259.1 & $2.26 \mathrm{E}+05$ & $85 \%$ & 1671 & $4.90 \mathrm{E}-07$ & $9.13 E-06$ & 0.91 & 0.03 & 0.41 & 1.00 & 0.01 & 0.00 & 0.50 & 0.099 & 0.327 \\
\hline Supenatant & 2.7 & $2.10 E+03$ & $100 \%$ & 14 & $9.45 \mathrm{E}-10$ & $1.34 \mathrm{E}-07$ & 0.91 & 0.03 & 0.41 & 1.00 & 0.01 & 0.00 & 0.50 & 0.099 & 0.327 \\
\hline Solid Layer & 256.4 & $2.24 \mathrm{E}+05$ & $85 \%$ & 1657 & $4.95 \mathrm{E}-07$ & $9.22 \mathrm{E}-06$ & 0.91 & 0.03 & 0.41 & 1.00 & 0.01 & 0.00 & 0.50 & 0.099 & 0.327 \\
\hline 241-B-204 & 255.0 & $2.22 \mathrm{E}+05$ & $85 \%$ & 1650 & $1.39 \mathrm{E}-07$ & $8.04 \mathrm{E}-06$ & 0.56 & 0.03 & 0.62 & 1.00 & 0.01 & 0.00 & 0.50 & 0.131 & 0.445 \\
\hline Supernatant & 4.0 & $3.15 E+03$ & $100 \%$ & 21 & 4. $19 \mathrm{E}-10$ & $2.29 \mathrm{E}-09$ & 0.56 & 0.03 & 0.62 & 1.00 & 0.01 & 0.00 & 0.50 & 0.131 & 0.445 \\
\hline Solid Layer & 251.0 & $2.19 \mathrm{E}+05$ & $85 \%$ & 1628 & $1.41 \mathrm{E}-07$ & $8.16 \mathrm{E}-06$ & 0.56 & 0.03 & 0.62 & 1.00 & 0.01 & 0.00 & 0.50 & 0.131 & 0.445 \\
\hline
\end{tabular}


RPP-5926 REV 6

Table B-2. Derived Data for Hydrogen Generation Rate Model Calculations for 177 Tanks. (20 sheets)

\begin{tabular}{|c|c|c|c|c|c|c|c|c|c|c|c|c|c|c|c|}
\hline Tank & $\begin{array}{c}\text { Waste } \\
\text { level } \\
\text { Dw } \\
\text { (inch) }\end{array}$ & $\begin{array}{c}\text { Total } \\
\text { mass } \\
M \\
(\mathrm{~kg})\end{array}$ & $\begin{array}{l}\text { Liquid } \\
\text { in waste } \\
\text { (w1\%) }\end{array}$ & $\begin{array}{c}\text { Wetted } \\
\text { area } \\
\text { Awet } \\
\left(\mathrm{ft}^{2}\right)\end{array}$ & $\begin{array}{c}\text { Tank Heat } \\
\text { ioad } \\
\mathrm{H}_{\mathrm{L}}{ }^{\beta / \mathrm{r}} \\
\text { (watt } / \mathrm{kg} \text { ) }\end{array}$ & $\begin{array}{c}\text { Tank } \\
\text { heat load } \\
\mathbf{H}_{\mathrm{L}}^{\text {alpha }} \\
\text { (watt } / \mathrm{kg} \text { ) }\end{array}$ & $\begin{array}{c}\mathrm{NO}_{3} \\
\text { in liquid } \\
{\left[\mathrm{NO}_{3} \mid\right.} \\
(\text { mole } / \mathrm{L})\end{array}$ & $\begin{array}{c}\mathrm{NO}_{2} \\
\text { in liquid } \\
{\left[\mathrm{NO}_{2}\right]} \\
(\text { mole/L) }\end{array}$ & $\begin{array}{c}\text { Excess } \mathrm{Na} \\
\text { in liquid } \\
{[\mathrm{Na}]_{\text {ex }}} \\
(\mathrm{mole} / \mathrm{L})\end{array}$ & $\begin{array}{c}\text { OH } \\
\text { in liquid } \\
\text { 'OH| } \\
(\text { mole } / \mathbf{L})\end{array}$ & $\begin{array}{c}\text { TOC } \\
\text { in liquid } \\
\text { |roC] }]^{\%} \% \\
(w+\%)\end{array}$ & $\begin{array}{c}\text { Al } \\
\text { in liquid } \\
{[A \mid \% \%} \\
(w t \%)\end{array}$ & {$\left[\begin{array}{c}E_{\mathrm{t} 2} \text { Efficiency } \\
\text { of } \mathrm{H}_{2} \mathrm{by} \\
\text { corrosion }\end{array}\right.$} & $\begin{array}{c}\text { Total } \\
\text { G values } \\
\mathrm{G}_{\mathrm{TOT}}^{\mathrm{B} / \gamma} \\
\left(\mathrm{H}_{z} 100 \mathrm{eV}\right)\end{array}$ & $\begin{array}{c}\text { Total } \\
\mathrm{G}^{\text {values }} \\
\mathrm{G}_{\text {Tor }}^{\text {alpha }} \\
\left(\mathrm{H}_{2} / 100 \mathrm{eV}\right)\end{array}$ \\
\hline 241-BX-101 & 24.7 & $3.02 \mathrm{E}+05$ & $21 \%$ & 4904 & $2.14 \mathrm{E}-03$ & $2.87 \mathrm{E}-05$ & 1.95 & 1.40 & 2.83 & 1.00 & 0.39 & 0.40 & 0.20 & 0.039 & 0.161 \\
\hline Supernatant & na & na & na & na & па & na & na & na & na & na & na & na & 0.20 & na & na \\
\hline Solid Layer & 24.7 & $3.02 \mathrm{E}+05$ & $21 \%$ & 4904 & $2.14 \mathrm{E}-03$ & $2.87 E-05$ & 1.95 & 1.40 & 2.83 & 1.00 & 0.39 & 0.40 & 0.20 & 0.039 & 0.161 \\
\hline $241-\mathrm{BX}-102$ & 36.1 & $2.95 \mathrm{E}+0.5$ & $49 \%$ & 5126 & $2.77 \mathrm{E}-04$ & $8.40 \mathrm{E}-07$ & 1.28 & 0.78 & 1.55 & 1.00 & 0.26 & 0.42 & 0.20 & 0.063 & 0.230 \\
\hline Supernatant & na & na & na & na & na & na & na & na & na & na & na & па & 0.20 & na & na \\
\hline Solid Layer & 36.1 & $2.95 \mathrm{E}+05$ & $49 \%$ & 5126 & $2.77 \mathrm{E}-04$ & $8.40 \mathrm{E}-07$ & 1.28 & 0.78 & 1.55 & 1.00 & 0.26 & 0.42 & 0.20 & 0.063 & 0.230 \\
\hline $241-\mathrm{BX}-103$ & 34.6 & $4.42 \mathrm{E}+0.5$ & $68 \%$ & 5098 & $5.97 \mathrm{E}-05$ & $8.30 \mathrm{E}-05$ & 0.75 & 0.31 & 0.70 & 1.00 & 0.34 & 1.67 & 0.20 & 0.106 & 0.353 \\
\hline Supernatant & 4.6 & $5.14 \mathrm{E}+04$ & $100 \%$ & 90 & $6.23 \mathrm{E}-06$ & $3.82 \mathrm{E}-07$ & 0.75 & 0.31 & 0.70 & 1.00 & 0.35 & $! .72$ & 0.20 & 0.106 & 0.353 \\
\hline Solid Layer & 30.0 & $3.90 \mathrm{E}+05$ & $64 \%$ & 5008 & $6.68 \mathrm{E}-05$ & $9.39 \mathrm{E}-05$ & 0.75 & 0.31 & 0.70 & 1.00 & 0.34 & 1.66 & 0.20 & 0.106 & 0.353 \\
\hline 24$]-8 X-104$ & 44.0 & $6.34 \mathrm{E}+0.5$ & $47 \%$ & 5281 & $1.56 \mathrm{E}-03$ & $2.91 \mathrm{E}-05$ & 2.16 & 1.39 & 2.55 & 1.00 & 0.25 & 0.22 & 0.20 & 0.039 & 0.151 \\
\hline Supenatant & 1.1 & $1.41 \mathrm{E}+04$ & $100 \%$ & 21 & $4.66 \mathrm{E}-04$ & $1.31 \mathrm{E}-08$ & 2.16 & 1.39 & 2.55 & 1.00 & 0.25 & 0.22 & 0.20 & 0.039 & 0.151 \\
\hline Solid layer & 42.9 & $6.20 \mathrm{E}+05$ & $46 \%$ & 5260 & $1.58 \mathrm{E}-03$ & $2.97 \mathrm{E}-05$ & 2.16 & 1.39 & 2.55 & 1.00 & 0.25 & 0.22 & 0.20 & 0.039 & 0.151 \\
\hline $241-B X-105$ & 33.6 & $4.53 \mathrm{E}+05$ & $24 \%$ & 5077 & $1.51 \mathrm{E}-03$ & $5.30 \mathrm{E}-06$ & 1.89 & 0.87 & 2.63 & 1.00 & 0.73 & 0.46 & 0.20 & 0.044 & 0.175 \\
\hline Supernatant & 1.7 & $2.32 \mathrm{E}+04$ & $100 \%$ & 34 & $4.75 \mathrm{E}-04$ & $1,04 \mathrm{E}-06$ & 1.89 & 0.87 & 2.63 & 1.00 & 0.73 & 0.46 & 0.20 & 0.044 & 0.175 \\
\hline Solid Layer & 31.9 & $4.30 \mathrm{E}+05$ & $20 \%$ & 5043 & $1.57 \mathrm{E}-03$ & $5.53 \mathrm{E}-06$ & 1.89 & 0.87 & 2.63 & 1.00 & 0.73 & 0.46 & 0.20 & 0.044 & 0.175 \\
\hline $241-B X-106$ & 21.2 & 2.31E+05 & $86 \%$ & 4834 & $4.00 \mathrm{E}-04$ & $1.55 \mathrm{E}-05$ & 3.50 & 2.55 & 5.03 & 1.00 & 1.21 & 3.13 & 0.20 & 0.025 & 0.100 \\
\hline Supentatant & na & па & та & na & na & na & na & na & $n a$ & ra & na & na & 0.20 & na & na \\
\hline Solid Layer & 21.2 & $2.31 \mathrm{E}+05$ & $86 \%$ & 4834 & $4.00 \mathrm{E}-04$ & $1.55 \mathrm{E}-05$ & 3.50 & 2.55 & 5.03 & 1.00 & 1.21 & 3.13 & 0.20 & 0.025 & 0.100 \\
\hline $241-\mathrm{BX}-107$ & 133.6 & $1.89 E+06$ & $67 \%$ & 7041 & $1.14 \mathrm{E}-04$ & $2.18 \mathrm{E}-06$ & 1.28 & 0.78 & 1.55 & 1.00 & 0.26 & 0.42 & 0.20 & 0.062 & 0.230 \\
\hline Supernatant & na & na & na & па & na & na & na & na & na & na & nа & na & 0.20 & na & na \\
\hline Solid Layer & 133.6 & $1.89 \mathrm{E}+06$ & $67 \%$ & 7041 & 1. $14 \mathrm{E}-04$ & $2.18 \mathrm{E}-06$ & 1.28 & 0.78 & 1.55 & 1.00 & 0.26 & 0.42 & 0.20 & 0.062 & 0.230 \\
\hline $241-B X-108$ & 18.9 & $1.73 E+05$ & $23 \%$ & 4789 & $9.70 E-04$ & $2.24 \mathrm{E}-06$ & 1.28 & 0.78 & 1.55 & 1.00 & 0.26 & 0.42 & 0.20 & 0.062 & 0.230 \\
\hline Supenatant & na & na & na & na & na & na & nа & na & na & na & na & na & 0.20 & na & na \\
\hline Solid Layer & 18.9 & $1.73 \mathrm{E}+05$ & $23 \%$ & 4789 & $9.70 \mathrm{E}-04$ & $2.24 \mathrm{E}-06$ & 1.28 & 0.78 & 1.55 & 1.00 & 0.26 & 0.42 & 0.20 & 0.062 & 0.230 \\
\hline 241-BX-109 & 77.6 & $1.11 \mathrm{E}+06$ & $70 \%$ & 5941 & $7.69 \mathrm{E}-04$ & $5.07 \mathrm{E}-07$ & 1.28 & 0.78 & 1.55 & 1.00 & 0.26 & 0.42 & 0.20 & 0.063 & 0.231 \\
\hline Supenatant & na & na & na & na & na & na & na & na & na & na & na & na & 0.20 & na & ra \\
\hline Solid Layer & 77.6 & $1.11 \mathrm{E}+06$ & $70 \%$ & $594 !$ & $7.69 \mathrm{E}-04$ & $5.07 \mathrm{E}-07$ & 1.28 & 0.78 & 1.55 & 1.00 & 0.26 & 0.42 & 0.20 & 0.063 & 0.231 \\
\hline
\end{tabular}


RPP-5926 REV 6

Table B-2. Derived Data for Hydrogen Generation Rate Model Calculations for 177 Tanks. (20 sheets)

\begin{tabular}{|c|c|c|c|c|c|c|c|c|c|c|c|c|c|c|c|}
\hline Tank & $\begin{array}{c}\text { Waste } \\
\text { level } \\
\text { Dw } \\
\text { (inch) }\end{array}$ & $\begin{array}{c}\text { Total } \\
\text { mass } \\
M \\
(\mathrm{~kg})\end{array}$ & $\begin{array}{l}\text { Liquid } \\
\text { in waste } \\
\text { (wt\%) }\end{array}$ & $\begin{array}{c}\text { Wetted } \\
\text { area } \\
\text { Awet } \\
\left(\mathrm{ft}^{2}\right)\end{array}$ & $\begin{array}{c}\text { Tank Heat } \\
\text { load } \\
\mathrm{H}_{\mathrm{L}}{ }^{\beta i r} \\
\text { (watt } / \mathrm{kg} \text { ) }\end{array}$ & $\begin{array}{c}\text { Tank } \\
\text { heat load } \\
\mathrm{H}_{1}{ }^{\text {alpha }} \\
\text { (watt } / \mathrm{kg} \text { ) }\end{array}$ & $\begin{array}{c}\mathrm{NO}_{3} \\
\text { in liquid } \\
{\left[\mathrm{NO}_{3} \mid\right.} \\
(\text { mole } / \mathrm{L})\end{array}$ & $\begin{array}{c}\mathrm{NO}_{2} \\
\text { in liquid } \\
{\left[\mathrm{NO}_{2} !\right.} \\
\text { (mole/L) }\end{array}$ & $\begin{array}{c}\text { Excess } \mathrm{Na} \\
\text { in liquid } \\
{\left[\left.\mathrm{Na}\right|_{\text {ex }}\right.} \\
(\mathrm{mole} / \mathrm{L})\end{array}$ & $\begin{array}{c}\text { OH } \\
\text { in liquid } \\
{[\mathrm{OH}]} \\
\text { (mole } / \mathrm{L})\end{array}$ & $\begin{array}{c}\text { TOC } \\
\text { in liquid } \\
\text { |TOC| } \% \\
\text { (wt } \% \text { ) }\end{array}$ & $\begin{array}{c}\text { Al } \\
\text { in liquid } \\
\mid \mathrm{Al} / \% \\
(w t \%)\end{array}$ & $\left|\begin{array}{c}E_{\mathrm{H} 2} \text { Efficiency } \\
\text { of } \mathrm{H}_{2} \text { by } \\
\text { corrosion }\end{array}\right|$ & $\begin{array}{c}\text { Total } \\
\text { G values } \\
\mathbf{G}_{\text {TOT }}{ }^{B / \gamma} \\
\left(\mathrm{H}_{2 f} 100 \mathrm{cV}\right)\end{array}$ & $\begin{array}{c}\text { Total } \\
\mathrm{G} \text { values } \\
\mathrm{G}_{\mathrm{TOT}}{ }^{\text {alpha }} \\
\left(\mathrm{H}_{2} / 100 \mathrm{eV}\right)\end{array}$ \\
\hline $241-B X-110$ & 85.5 & $1.35 \mathrm{E}+06$ & $77 \%$ & 6096 & $2.85 \mathrm{E}-04$ & $6.76 \mathrm{E}-07$ & 6.65 & 1.13 & 1.92 & 1.00 & 1.06 & 0.31 & 0.20 & 0.024 & 0.063 \\
\hline Supenatant & 0.5 & $7.20 \mathrm{E}+03$ & $100 \%$ & 9 & $4.27 \mathrm{E}-04$ & $2.42 \mathrm{E}-07$ & 6.65 & 1.13 & 1.96 & 1.00 & 1.05 & 0.31 & 0.20 & 0.024 & 0.063 \\
\hline Solid Layer & 85.0 & $1.35 E+06$ & $77 \%$ & 6086 & $2.84 \mathrm{E}-04$ & $6.78 \mathrm{E}-07$ & 6.65 & 1.13 & 1.92 & 1.00 & 1.06 & 0.31 & 0.20 & 0.024 & 0.063 \\
\hline $241-\mathrm{BX}-111$ & 75.9 & $1.03 \mathrm{E}+06$ & $10 \%$ & 5909 & $3.83 \mathrm{E}-04$ & $6.08 \mathrm{E}-07$ & 2.99 & 2.31 & 4.53 & 1.00 & 0.12 & 3.36 & 0.20 & 0.021 & 0.110 \\
\hline Supernatant & na & na & na & na & па & na & па & na & na & па & na & na & 0.20 & nat & $\mathrm{na}$ \\
\hline Solid Layer & 75.9 & $1.03 \mathrm{E}+06$ & $10 \%$ & 5909 & $3.83 \mathrm{E}-04$ & $6.08 \mathrm{E}-07$ & 2.99 & 2.31 & 4.53 & 1.00 & 0.12 & 3.36 & 0.20 & 0.021 & 0.110 \\
\hline $241-B X-112$ & 67.2 & $8.14 \mathrm{E}+05$ & $94 \%$ & 5737 & $2.29 \mathrm{E}-04$ & $5.68 \mathrm{E}-06$ & 1.59 & 0.77 & 1.03 & 1.00 & 0.00 & 0.00 & 0.20 & 0.057 & 0.198 . \\
\hline Supernatant & 0.5 & $5.90 \mathrm{E}+03$ & $100 \%$ & 9 & $7.62 \mathrm{E}-06$ & $5.19 \mathrm{E}-08$ & 1.59 & 0.77 & 1.03 & 1.00 & 0.00 & 0.00 & 0.20 & 0.057 & 0.198 \\
\hline Solid Layer & 66.7 & $8.08 E+05$ & $94 \%$ & 5728 & $2.31 \mathrm{E}-04$ & $5.72 \mathrm{E}-06$ & 1.59 & 0.77 & 1.03 & 1.00 & 0.00 & 0.00 & 0.20 & 0.057 & 0.198 \\
\hline 24 I-BY-101 & 141.9 & $2.56 \mathrm{E}+06$ & $31 \%$ & 7205 & $1.06 \mathrm{E}-03$ & $1.78 \mathrm{E}-06$ & 3.50 & 2.54 & 5.07 & 2.25 & 1.20 & 3.13 & 0.20 & 0.028 & 0.101 \\
\hline Supematant & na & na & na & na & na & na & na & na & na & na & na & na & 0.20 & na & na \\
\hline Solid Layer & 141.9 & $2.56 \mathrm{E}+06$ & $31 \%$ & 7205 & $1.06 \mathrm{E}-03$ & $1.78 \mathrm{E}-06$ & 3.50 & 2.54 & 5.07 & 2.25 & 1.20 & 3.13 & 0.20 & 0.028 & 0.101 \\
\hline $241-8 Y-102$ & 108.7 & $1.66 \mathrm{E}+06$ & $34 \%$ & 6552 & $3.85 \mathrm{E}-04$ & $2.50 \mathrm{E}-06$ & 2.38 & 1.83 & 5.98 & 2.25 & 0.11 & 3.87 & 0.20 & 0.018 & 0.134 \\
\hline Supernatant & na & na & na & na & na & ма & na & na & na & па & na & na & 0.20 & na & па \\
\hline Solid Layer & 108.7 & $1.66 \mathrm{E}+06$ & $34 \%$ & 6552 & $3.85 \mathrm{E}-04$ & $2.50 \mathrm{E}-06$ & 2.38 & 1.83 & 5.98 & 2.25 & 0.11 & 3.87 & 0.20 & 0.018 & 0.134 \\
\hline $24 !-8 Y-103$ & 157.7 & $2.60 \mathrm{E}-06$ & $34 \%$ & 7514 & 3.92E-04 & $6.00 \mathrm{E}-07$ & 2.28 & 0.98 & 4.57 & 1.85 & 0.12 & 2.68 & 0.20 & 0.025 & 0.149 \\
\hline Supematant & па & na & na & na & na & na & na & na & na & nà & na & na & 0.20 & na & na \\
\hline Solid Layer & 157.7 & $2.60 \mathrm{E}+06$ & $34 \%$ & 7514 & $3.92 E-04$ & $6.00 \mathrm{E}-07$ & 2.28 & 0.98 & 4.57 & 1.85 & 0.12 & 2.68 & 0.20 & 0.025 & 0.149 \\
\hline $241-B Y-104$ & 154.7 & $2.63 \mathrm{E}+06$ & $29 \%$ & 7456 & $1.33 \mathrm{E}-03$ & $2.91 \mathrm{E}-06$ & 3.33 & 2.42 & 5.02 & 2.25 & 0.16 & 4.76 & 0.20 & 0.022 & 0.102 \\
\hline Supernatant & na & na & na & na & na & na & na & na & na & na & na & na & 0.20 & na & na \\
\hline Solid Layer & 154.7 & $2.63 \mathrm{E}+06$ & $29 \%$ & 7456 & 1.33E-03 & $2.91 \mathrm{E}-06$ & 3.33 & 2.42 & 5.02 & 2.25 & 0.16 & 4.76 & 0.20 & 0.022 & 0.102 \\
\hline $241-B Y-105$ & 182.4 & $3.28 E+06$ & $35 \%$ & 7999 & $5.05 \mathrm{E}-04$ & $2.04 \mathrm{E}-06$ & 3.22 & 1.49 & 4.32 & 2.25 & 0.17 & 2.72 & 0.20 & 0.024 & 0.110 \\
\hline Supernatant & na & na & na & na & na & na & na & na & na & na & na & na & 0.20 & na & na \\
\hline Solid Layer & 182.4 & $3.28 \mathrm{E}+06$ & $35 \%$ & 7999 & $5.05 \mathrm{E}-04$ & $2.04 \mathrm{E}-06$ & 3.22 & 1.49 & 4.32 & 2.25 & 0.17 & 2.72 & 0.20 & 0.024 & 0.110 \\
\hline $241-B Y-106$ & 163.4 & $2.68 \mathrm{E} \pm 06$ & $45 \%$ & 7626 & $8.72 \mathrm{E}-04$ & $7.79 \mathrm{E}-07$ & 1.78 & 1.70 & 3.67 & 2.11 & 0.20 & 3.04 & 0.20 & 0.034 & 0.167 \\
\hline Supernatant & na & na & na & na & na & na & na & na & na & na & na & na & 0.20 & na & na \\
\hline Solid Layer & 163.4 & $2.68 \mathrm{E}+06$ & $45 \%$ & 7626 & $8.72 \mathrm{E}-04$ & $7.79 \mathrm{E}-07$ & 1.78 & 1.70 & 3.67 & 2.11 & 0.20 & 3.04 & 0.20 & 0.034 & 0.167 \\
\hline
\end{tabular}


RPP-5926 REV 6

Table B-2. Derived Data for Hydrogen Generation Rate Model Calculations for 177 Tanks. (20 sheets)

\begin{tabular}{|c|c|c|c|c|c|c|c|c|c|c|c|c|c|c|c|}
\hline Tank & $\begin{array}{c}\text { Waste } \\
\text { level } \\
\text { Dw } \\
\text { (inch) }\end{array}$ & $\begin{array}{c}\text { Total } \\
\text { mass } \\
M \\
(\mathrm{~kg})\end{array}$ & $\begin{array}{c}\text { Liquid } \\
\text { in waste } \\
\text { (wt\%) }\end{array}$ & $\begin{array}{c}\text { Wetted } \\
\text { area } \\
\text { Awet } \\
\left(\mathrm{ft}^{2}\right)\end{array}$ & $\begin{array}{c}\text { Tank Heat } \\
\text { load } \\
\mathrm{H}_{\mathrm{L}}{ }^{\beta / r} \\
\text { (watt } / \mathrm{kg} \text { ) }\end{array}$ & $\begin{array}{c}\text { Tank } \\
\text { heat load } \\
\mathrm{H}_{\mathrm{L}}^{\text {alpha }} \\
\text { (watt/kg) }\end{array}$ & $\begin{array}{c}\mathrm{NO}_{3} \\
\text { in liquid } \\
\left|\mathrm{NO}_{3}\right| \\
(\text { mole } / \mathrm{L})\end{array}$ & $\begin{array}{c}\mathrm{NO}_{2} \\
\text { in liquid } \\
\left|\mathrm{NO}_{2}\right| \\
(\mathrm{mole} / \mathrm{L})\end{array}$ & $\begin{array}{c}\text { Excess } \mathrm{Na} \\
\text { in liquid } \\
|\mathrm{Na}|_{\mathrm{ex}} \\
(\mathrm{mole} / \mathrm{L})\end{array}$ & $\begin{array}{c}\mathrm{OH} \\
\text { in liquid } \\
|\mathrm{OH}| \\
(\text { mole/L) }\end{array}$ & $\begin{array}{c}\text { TOC } \\
\text { in liquid } \\
\text { [TOC. } \% \\
\text { (wt\%) }\end{array}$ & $\begin{array}{c}\text { Al } \\
\text { in liquid } \\
{[A \mid] \%} \\
(w 1 \%)\end{array}$ & $\mid \begin{array}{c}\mathrm{E}_{\mathrm{H} 2} \text { Efficiency } \\
\text { of } \mathrm{H}_{2} \text { by } \\
\text { corrosion }\end{array}$ & $\begin{array}{c}\text { Total } \\
\text { G values } \\
\mathbf{G}_{\text {TOт }}{ }^{\mathbf{z} / \gamma} \\
\left(\mathbf{H}_{2}, 100 \mathrm{eV}\right)\end{array}$ & $\begin{array}{c}\text { Total } \\
\text { G values } \\
G_{\text {Tor }}^{\text {alsh }} \\
\left(\mathrm{H}_{2} / 100 \mathrm{eV}\right)\end{array}$ \\
\hline 241-BY-107 & 106.2 & $1.74 \mathrm{E} \div 06$ & $49 \%$ & 6503 & $8.30 \mathrm{E}-04$ & $6.81 \mathrm{E}-07$ & 2.11 & 2.54 & 5.90 & 2.25 & 0.16 & 3.51 & 0.20 & 0.020 & 0.138 \\
\hline Supernatant & na & na & na & na & na & na & na & na & na & па & na & na & 0.20 & na & na \\
\hline Solid Layer & 106.2 & $1.74 \mathrm{E}+06$ & $49 \%$ & 6503 & $8.30 \mathrm{E}-04$ & $6.81 \mathrm{E} \cdot 07$ & 2.11 & 2.54 & 5.90 & 2.25 & 0.16 & 3.51 & 0.20 & 0.020 & 0.138 \\
\hline $241-B Y-108$ & 88.2 & $1.25 \mathrm{E}+06$ & $51 \%$ & 6151 & $8.91 \mathrm{E}-04$ & $1.81 \mathrm{E}-06$ & 4.74 & 1.71 & 5.79 & 2.25 & 0.18 & 2.73 & 0.20 & 0.016 & 0.079 \\
\hline Supernatant & na & na & na & na & na & na & naa & na & па & na & na & na & 0.20 & na & na \\
\hline Solid Layer & 88.2 & $1.25 \mathrm{E}+06$ & $51 \%$ & 6151 & $8.91 \mathrm{E}-04$ & $1.81 \mathrm{E}-06$ & 4.74 & 1.71 & 5.79 & 2.25 & 0.18 & 2.73 & 0.20 & 0.016 & 0.079 \\
\hline $24 \mathrm{I}-\mathrm{B} Y-109$ & 111.8 & $1.85 \mathrm{E}+06$ & $34 \%$ & 6613 & $2.88 \mathrm{E}-04$ & $1.92 \mathrm{E}-06$ & 3.07 & 1.80 & 4.24 & 2.25 & 0.13 & 2.58 & 0.20 & 0.022 & 0.111 \\
\hline Supernatant & na & na & na & na & na & na & na & na & na & na & na & na & 0.20 & na & na \\
\hline Solid Layer & 111.8 & $1.85 E+06$ & $34 \%$ & 6613 & $2.88 \mathrm{E}-04$ & $1.92 \mathrm{E}-06$ & 3.07 & 1.80 & 4.24 & 2.25 & 0.13 & 2.58 & 0.20 & 0.022 & 0.111 \\
\hline $241-B Y-110$ & 140.4 & $2.17 \mathrm{E}+06$ & $28 \%$ & 7175 & $9.01 \mathrm{E}-04$ & $1.71 \mathrm{E}-06$ & 2.00 & 2.70 & 6.32 & 2.25 & 1.35 & 4.57 & 0.20 & 0.039 & 0.151 \\
\hline Supernatant & na & na & na & na & na. & na & na & na & na & na & na & na & 0.20 & na & na \\
\hline Solid Layer & 140.4 & $2.17 \mathrm{E}+06$ & $28 \%$ & 7175 & $9.01 \mathrm{E}-04$ & $1.71 \mathrm{E}-06$ & 2.00 & 2.70 & 6.32 & 2.25 & 1.35 & 4.57 & 0.20 & 0.039 & 0.151 \\
\hline $241-B Y-111$ & 153.8 & $2.55 \mathrm{E}+06$ & $9 \%$ & 7437 & $3.51 \mathrm{E}-04$ & $3.04 \mathrm{E}-06$ & 3.24 & 1.19 & 4.99 & 2.25 & 0,09 & 3.03 & 0.20 & 0.020 & 0.111 \\
\hline Supernatant & na & na & na & na & na & na & na & nа & na & na & na & na & 0.20 & na & na \\
\hline Solid Layer & 153.8 & $2.55 \mathrm{E}+06$ & $9 \%$ & 7437 & $3.51 \mathrm{E}-04$ & $3.04 \mathrm{E}-06$ & 3.24 & 1.19 & 4.99 & 2.25 & 0.09 & 3.03 & 0.20 & 0.020 & 0.111 \\
\hline $241-B Y-112$ & 111.5 & $1.88 E+06$ & $12 \%$ & 6607 & $3.78 \mathrm{E}-04$ & $2.13 \mathrm{E}-06$ & 3.06 & 3.05 & 4.50 & 2.25 & 0.12 & 3.77 & 0.20 & 0.021 & 0.103 \\
\hline Supernatant & na & na & na & na & na & na & na & na & na & na & na & па & 0.20 & na & na \\
\hline Solid Layer & 111.5 & $1.88 \mathrm{E}+06$ & $12 \%$ & 6607 & $3.78 \mathrm{E}-04$ & $2.13 \mathrm{E}-06$ & 3.06 & 3.05 & 4.50 & 2.25 & 0.12 & 3.77 & 0.20 & 0.021 & 0.103 \\
\hline $241-\mathrm{C}-101$ & 39.4 & $5.93 \mathrm{E}+05$ & $32 \%$ & 5192 & $6.69 \mathrm{E}-04$ & $3.33 \mathrm{E}-05$ & 1.28 & 0.78 & 1.55 & 1.00 & 0.26 & 0.42 & 0.20 & 0.065 & $0.23 t$ \\
\hline Supernatant & na & na & na & na & na & na & na & na & па & па & na & na & 0.20 & na & 114 \\
\hline Solid Layer & 39.4 & $5.93 E+05$ & $32 \%$ & 5192 & $6.69 \mathrm{E}-04$ & $3.33 \mathrm{E}-05$ & 1.28 & 0.78 & 1.55 & 1.00 & 0.26 & 0.42 & 0.20 & 0.065 & 0.231 \\
\hline $241-C-102$ & 122.3 & $2.01 E+06$ & $43 \%$ & 6820 & $1.54 \mathrm{E}-04$ & $8.20 \mathrm{E}-05$ & 1.28 & 0.78 & 1.55 & 1.00 & 0.26 & 0.42 & 0.20 & 0.064 & 0.231 \\
\hline Supernatant & na & na & na & na & na & na & na & na & na & na & na & na & 0.20 & na & па \\
\hline Solid Layer & 122.3 & $2.01 \mathrm{E}+06$ & $43 \%$ & 6820 & $1.54 \mathrm{E}-04$ & $8.20 \mathrm{E}-05$ & 1.28 & 0.78 & 1.55 & 1.00 & 0.26 & 0.42 & 0.20 & 0.064 & 0.231 \\
\hline $241-C-103$ & 17.2 & $1.65 \mathrm{E}+05$ & $26 \%$ & 4755 & $3.44 \mathrm{E}-03$ & $5.32 \mathrm{E}-05$ & 0.01 & 0.32 & 1.06 & 1.00 & 0.50 & 0.00 & 0.50 & 0.224 & 0.861 \\
\hline Supernatant & na & na & na & na & па & па & na & na & na & na & na & па & 0.20 & na & na \\
\hline Solid Layer & 17.2 & $1.65 \mathrm{E}+05$ & $26 \%$ & 4755 & $3.44 \mathrm{E}-03$ & $5.32 \mathrm{E}-05$ & 0.01 & 0.32 & 1.06 & 1.00 & 0.50 & 0.00 & 0.50 & 0.224 & 0.861 \\
\hline
\end{tabular}


RPP-5926 REV 6

Table B-2. Derived Data for Hydrogen Generation Rate Model Calculations for 177 Tanks. (20 sheets)

\begin{tabular}{|c|c|c|c|c|c|c|c|c|c|c|c|c|c|c|c|}
\hline Tank & $\begin{array}{l}\text { Waste } \\
\text { level } \\
\text { Dw } \\
\text { (inch) }\end{array}$ & $\begin{array}{c}\text { Total } \\
\text { mass } \\
M \\
(\mathrm{~kg})\end{array}$ & $\begin{array}{c}\text { Liquid } \\
\text { in waste } \\
\text { (wto } \% \text { ) }\end{array}$ & $\begin{array}{c}\text { Wetted } \\
\text { area } \\
\text { Awet } \\
\left(\mathrm{ft}^{2}\right)\end{array}$ & $\begin{array}{c}\text { Tank Heat } \\
\text { load } \\
\mathrm{H}_{\mathrm{L}}{ }^{\beta / r} \\
\text { (watt } / \mathrm{kg} \text { ) }\end{array}$ & $\begin{array}{c}\text { Tank } \\
\text { heat load } \\
\mathbf{H}_{\mathrm{l}}^{\text {2lpha }} \\
\text { (watt } / \mathrm{kg} \text { ) }\end{array}$ & $\begin{array}{c}\mathrm{NO}_{3} \\
\text { in liquid } \\
{\left[\mathrm{NO}_{3} \mid\right.} \\
(\mathrm{mole} / \mathrm{L})\end{array}$ & $\begin{array}{c}\mathrm{NO}_{2} \\
\text { in liquid } \\
{\left[\mathrm{NO}_{2}\right]} \\
(\text { mole } / \mathrm{L})\end{array}$ & $\begin{array}{c}\text { Excess } \mathrm{Na} \\
\text { in liquid } \\
{[\mathrm{Na}]_{\mathrm{ex}}} \\
(\mathrm{mole} / \mathrm{L})\end{array}$ & $\begin{array}{c}\mathrm{OH} \\
\text { in liquid } \\
\text { [OH } \\
(\mathrm{mole} / \mathrm{L})\end{array}$ & $\begin{array}{c}\text { TOC } \\
\text { in liquid } \\
\text { [TOC]"\% } \\
(\mathrm{wt} \%)\end{array}$ & $\begin{array}{c}\text { Al } \\
\text { in liquid } \\
\text { [Al|\% } \\
(w t \%)\end{array}$ & $\begin{array}{c}E_{\mathrm{H}_{2}} \text { Efficiency } \\
\text { of } \mathrm{H}_{2} \text { by } \\
\text { corrosion }\end{array}$ & $\begin{array}{c}\text { Total } \\
\text { G values } \\
\mathrm{G}_{\mathrm{TOT}}{ }^{\mathrm{B} / \gamma} \\
\left(\mathrm{H}_{2} / 100 \mathrm{eV}\right)\end{array}$ & $\begin{array}{c}\text { Total } \\
\text { G values } \\
\mathbf{G}_{\text {TOT }}{ }^{\text {alpha }} \\
\left(\mathrm{H}_{2} / 100 \mathrm{eV}\right)\end{array}$ \\
\hline $241-C-104$ & 101.6 & $1.65 E+06$ & $59 \%$ & 6413 & $2.07 \mathrm{E}-03$ & $2.52 \mathrm{E}-04$ & 1.28 & 0.78 & 1.55 & 1.00 & 0.48 & 0.42 & 0.20 & 0.070 & 0.234 \\
\hline Supernatant & na & na & na & na & na & na & na & na & na & na & na & na & 0.20 & na & na \\
\hline Solid Layer & 101.6 & 1. $65 \mathrm{E}+06$ & $59 \%$ & 6413 & $2.07 \mathrm{E}-03$ & $2.52 \mathrm{E}-04$ & 1.28 & 0.78 & 1.55 & 1.00 & 0.48 & 0.42 & 0.20 & 0.070 & 0.234 \\
\hline $241-C-105$ & 55.5 & $7.75 \mathrm{E}+05$ & $42 \%$ & 5507 & $4.27 \mathrm{E}-03$ & $1.45 \mathrm{E}-04$ & 0.55 & 0.80 & 3.62 & 1.00 & 0.23 & 0.00 & 0.20 & 0.056 & 0.375 \\
\hline Supenatant & na & na & na & na & na & na & na & na & na & па & na & na & 0.20 & nа & na \\
\hline Solid Layer & 55.5 & $7.75 \mathrm{E}+05$ & $42 \%$ & 5507 & 4.27E-03 & $1.45 \mathrm{E}-04$ & 0.55 & 0.80 & 3.62 & 1.00 & 0.23 & 0.00 & 0.20 & 0.056 & 0.375 \\
\hline $241-C-106$ & 8.5 & $1.62 \mathrm{E}+04$ & $44 \%$ & 4584 & $2.77 \mathrm{E}-02$ & $1.76 \mathrm{E}-04$ & 0.00 & 0.00 & 0.42 & 0.41 & 0.03 & 0.01 & 0.50 & 0.411 & 1.113 \\
\hline Supernatant & 0.0 & $3.28 E+02$ & $100 \%$ & 1 & $2.31 E-06$ & $3.03 \mathrm{E}-10$ & 0.00 & 0.00 & 0.42 & 0.41 & 0.03 & 0.01 & 0.50 & 0.411 & 1.114 \\
\hline Solid Layer & 8.4 & $1.59 \mathrm{E}+04$ & $43 \%$ & 4583 & $2.83 \mathrm{E}-02$ & $1.79 \mathrm{E}-04$ & 0.00 & 0.00 & 0.42 & 0.41 & 0.03 & 0.01 & 0.50 & 0.411 & 1.113 \\
\hline $241-C-107$ & 97.3 & $1.45 \mathrm{E}+06$ & $40 \%$ & 6328 & $9.79 \mathrm{E}-03$ & $2.06 \mathrm{E}-04$ & 1.28 & 0.78 & 1.55 & 1.00 & 0.26 & 0.42 & 0.20 & 0.068 & 0.233 \\
\hline Superiatant & na & na & па & na & na & na & na & na & na & na & na & na & 0.20 & na & па \\
\hline Solid Layer & 97.3 & $1.45 \mathrm{E}+06$ & $40 \%$ & 6328 & $9.79 \mathrm{E}-03$ & $2.06 \mathrm{E}-04$ & 1.28 & 0.78 & 1.55 & 1.00 & 0.26 & 0.42 & 0.20 & 0.068 & 0.233 \\
\hline $241-\mathrm{C}-10 \mathrm{~B}$ & 31.5 & $3.70 E+05$ & $51 \%$ & 5036 & $1.14 \mathrm{E}-03$ & $1.24 \mathrm{E}-06$ & 1.28 & 0.78 & 1.55 & 1.00 & 0.26 & 0.42 & 0.20 & 0.063 & 0.231 \\
\hline Supernatant & na & na & na & na & na & na & na & na & na & na & na & na & 0.20 & na & na \\
\hline Solid Layer & 31.5 & $3.70 \mathrm{E}+05$ & $51 \%$ & 5036 & $1.14 \mathrm{E}-03$ & $1.24 \mathrm{E}-06$ & 1.28 & 0.78 & 1.55 & 1.00 & 0.26 & 0.42 & 0.20 & 0.063 & 0.231 \\
\hline $241-C-109$ & 30.5 & $3.71 \mathrm{E}+05$ & $60 \%$ & 5017 & $6.75 \mathrm{E}-03$ & $1.09 \mathrm{E}-05$ & 1.28 & 0.78 & 1.55 & 1.00 & 0.26 & 0.42 & 0.20 & 0.063 & 0.231 \\
\hline Supernatant & na & 11a & na & na & nа & na & na & na & na & na & na & na & 0.20 & na & na \\
\hline Solid Layer & 30.5 & $3.71 \mathrm{E}+05$ & $60 \%$ & 5017 & $6.75 \mathrm{E}-03$ & $1.09 \mathrm{E}-05$ & 1.28 & 0.78 & 1.55 & 1.00 & 0.26 & 0.42 & 0.20 & 0.063 & 0.231 \\
\hline $241-C-110$ & 72.2 & $9.02 \mathrm{E}+05$ & $56 \%$ & 5836 & $9.64 \mathrm{E}-05$ & $3.85 \mathrm{E}-06$ & 0.97 & 0.10 & 0.25 & 1.00 & 0.05 & 0.02 & 0.50 & 0.095 & 0.310 \\
\hline Supenatant & 0.4 & $4.40 \mathrm{E}+03$ & $100 \%$ & 8 & $1.63 \mathrm{E}-05$ & $4.95 \mathrm{E}-08$ & 0.97 & 0.10 & 0.25 & 1.00 & 0.05 & 0.02 & 0.50 & 0.095 & 0.310 \\
\hline Solid Layer & 71.8 & $8.98 \mathrm{E}+05$ & $56 \%$ & 5828 & $9.68 \mathrm{E}-05$ & $3.87 \mathrm{E}-06$ & 0.97 & 0.10 & 0.25 & 1.00 & 0.05 & 0.02 & 0.50 & 0.095 & 0.310 \\
\hline $241 \cdot \mathrm{C}-111$ & 28.3 & $3.36 \mathrm{E}+05$ & $55 \%$ & 4974 & $1.84 \mathrm{E}-02$ & $3.74 \mathrm{E}-05$ & 1.28 & 0.78 & 1.55 & 1.00 & 0.26 & 0.42 & 0.20 & 0.063 & 0.231 \\
\hline Supenatant & na & na & na & na & na & na & na & na & na & na & na & nа & 0.20 & na & na \\
\hline Solid Layer & 28.3 & $3.36 \mathrm{E}+05$ & $55 \%$ & 4974 & $1.84 \mathrm{E}-02$ & $3.74 \mathrm{E}-05$ & 1.28 & 0.78 & 1.55 & 1.00 & 0.26 & 0.42 & 0.20 & 0.063 & 0.231 \\
\hline $241-C-112$ & 45.2 & $6.29 E+05$ & $69 \%$ & 5306 & $8.40 \mathrm{E}-03$ & $2.96 E-05$ & 1.28 & 0.78 & 1.55 & 1.00 & 0.26 & 0.42 & 0.20 & 0.064 & 0.231 \\
\hline Supernatant & na & na & na & na & na & na & na & na & na & na & na & na & 0.20 & nat & na \\
\hline Sotid Layer & 45.2 & $6.29 \mathrm{E}+05$ & $69 \%$ & 5306 & $8.40 \mathrm{E}-03$ & $2.96 \mathrm{E}-05$ & 1.28 & 0.78 & 1.55 & 1.00 & 0.26 & 0.42 & 0.20 & 0.064 & 0.231 \\
\hline
\end{tabular}


RPP-5926 REV 6

Table B-2. Derived Data for Hydrogen Generation Rate Model Calculations for 177 Tanks. (20 sheets)

\begin{tabular}{|c|c|c|c|c|c|c|c|c|c|c|c|c|c|c|c|}
\hline Tank & $\begin{array}{l}\text { Waste } \\
\text { level } \\
\text { Dw } \\
\text { (inch) }\end{array}$ & $\begin{array}{c}\text { Total } \\
\text { mass } \\
\mathbf{M} \\
(\mathrm{kg})\end{array}$ & $\begin{array}{c}\text { Liquid } \\
\text { in waste } \\
\text { (wt\%) }\end{array}$ & $\begin{array}{l}\text { Wetted } \\
\text { area } \\
\text { Awet } \\
\left(\mathrm{ft}^{2}\right)\end{array}$ & $\begin{array}{c}\text { Tank Heat } \\
\text { load } \\
\mathrm{H}_{\mathrm{L}}{ }^{\beta / \mathrm{r}} \\
\text { (watt } / \mathrm{kg} \text { ) }\end{array}$ & $\begin{array}{c}\text { rank } \\
\text { heat toad } \\
H_{\mathrm{L}}{ }^{\text {lapha }} \\
\text { (watt } / \mathrm{kg} \text { ) }\end{array}$ & $\begin{array}{c}\mathrm{NO}_{3} \\
\text { in liquid } \\
{\left[\mathrm{NO}_{3}\right]} \\
\left(\mathrm{mole}_{\mathrm{l}} / \mathrm{L}\right)\end{array}$ & $\begin{array}{c}\mathrm{NO}_{2} \\
\text { in liquid } \\
{\left[\mathrm{NO}_{2}\right]} \\
(\mathrm{mole} / \mathrm{L})\end{array}$ & $\begin{array}{c}\text { Excess } \mathrm{Na} \\
\text { in liquid } \\
|\mathrm{Na}|_{\mathrm{ex}} \\
(\text { mole } / \mathrm{L})\end{array}$ & $\begin{array}{c}\mathrm{OH} \\
\text { in liquid } \\
{[\mathrm{OH}]} \\
(\mathrm{mole} / \mathrm{L})\end{array}$ & $\begin{array}{c}\text { TOC } \\
\text { in liquid } \\
{[\text { TOC] } \%} \\
(w t \%)\end{array}$ & $\begin{array}{c}\text { Al } \\
\text { in liquid } \\
{[A \mid] \%} \\
(w t \%)\end{array}$ & $\begin{array}{c}\mathrm{E}_{\mathrm{H} 2} \text { Efficiency } \\
\text { of } \mathrm{H}_{2} \text { by } \\
\text { corrosion }\end{array}$ & $\begin{array}{c}\text { Total } \\
\text { G values } \\
\text { G }_{\text {Tor }}{ }^{\text {B } \gamma} \\
\left(\mathrm{H}_{2}, 100 \mathrm{eV}\right)\end{array}$ & $\begin{array}{c}\text { Total } \\
G \text { values } \\
\mathrm{G}_{\text {TOT }} \text { alpha } \\
\left(\mathrm{H}_{2} / 100 \mathrm{eV}\right)\end{array}$ \\
\hline $241-C-201$ & 3.7 & $9.47 \mathrm{E}+02$ & $25 \%$ & 334 & $1.83 \mathrm{E}-03$ & $7.20 \mathrm{E}-04$ & 0.00 & 0.00 & 0.00 & 1.00 & 0.00 & 0.00 & 0.50 & 0.450 & 1.400 \\
\hline Supernatant & 0.0 & $7.00 \mathrm{E}+00$ & $100 \%$ & 0 & $0.00 \mathrm{E}+\infty$ & $0.00 \mathrm{E}+00$ & 0.00 & 0.00 & 0.00 & 1.00 & 0.00 & 0.00 & 0.50 & 0.450 & 1.400 \\
\hline Solid Layer & 3.7 & $9.40 \mathrm{E}+02$ & $24 \%$ & 334 & $1.84 \mathrm{E}-03$ & $7.25 \mathrm{E}-04$ & 0.00 & 0.00 & 0.00 & 1.00 & 0.00 & 0.00 & 0.50 & 0.450 & 1.400 \\
\hline $241-C-202$ & 3.7 & $9.68 \mathrm{E}+02$ & $25 \%$ & 334 & $3.41 E-03$ & $6.02 \mathrm{E}-04$ & 0.00 & 0.00 & 0.00 & 1.00 & 0.00 & 0.00 & 0.50 & 0.450 & 1.400 \\
\hline Supernatant & 0.0 & $9.00 \mathrm{E} \div 00$ & $100 \%$ & 0 & $0.00 \mathrm{E}+00$ & $0.00 \mathrm{E}+00$ & 0.00 & 0.00 & 0.00 & 1.00 & 0.00 & 0.00 & 0.50 & 0.450 & 1.400 \\
\hline Solid Layer & 3.7 & $9.59 \mathrm{E}+02$ & $24 \%$ & 334 & $3.44 \mathrm{E}-03$ & $6.08 \mathrm{E}-04$ & 0.00 & 0.00 & 0.00 & 1.00 & 0.00 & 0.00 & 0.50 & 0.450 & 1.400 \\
\hline $241-C-203$ & 3.7 & $9.67 \mathrm{E}+02$ & $39 \%$ & 334 & $8.04 \mathrm{E}-05$ & $2.02 \mathrm{E}-05$ & 0.00 & 0.00 & 0.00 & 1.00 & 0.00 & 0.00 & 0.50 & 0.450 & 1.400 \\
\hline Supematant & 0.1 & $4.80 \mathrm{E}+0 \mathrm{1}$ & $100 \%$ & 0 & $0.00 \mathrm{E}+00$ & $0.00 \mathrm{E}+00$ & 0.00 & 0.00 & 0.00 & 1.00 & 0.00 & 0.00 & 0.50 & 0.450 & 1.400 \\
\hline Solid Layer & 3.6 & $9.19 \mathrm{E}+02$ & $36 \%$ & 333 & $8.46 \mathrm{E}-05$ & $2.12 E-05$ & 0.00 & 0.00 & 0.00 & 1.00 & 0.00 & 0.00 & 0.50 & 0.450 & 1.400 \\
\hline $241-C-204$ & 10.6 & $9.11 \mathrm{E}+03$ & $55 \%$ & 369 & $9.50 \mathrm{E}-05$ & $2.63 \mathrm{E}-07$ & 1.28 & 0.78 & 1.55 & 1.00 & 0.26 & 0.42 & 0.20 & 0.062 & 0.230 \\
\hline Supernatant & na & na & na & па & na & na & na & na & na & na & na & na & 0.20 & na & na \\
\hline Solid Layer & 10.6 & $9.11 \mathrm{E}+03$ & $55 \%$ & 369 & $9.50 \mathrm{E}-05$ & $2.63 E-07$ & 1.28 & 0.78 & 1.55 & 1.00 & 0.26 & 0.42 & 0.20 & 0.062 & 0.230 \\
\hline $241-S-101$ & 135.4 & $2.20 \mathrm{E}+06$ & $77 \%$ & 7077 & $1.91 \mathrm{E}-03$ & $8.72 \mathrm{E}-06$ & 3.45 & 2.45 & 3.75 & 2.80 & 0.12 & 2.04 & 0.20 & 0.024 & 0.099 \\
\hline Supenatant & na & ta & แa & na & na & na & na & na & na & na & na & na & 0.20 & na & na \\
\hline Solid Layer & 135.4 & $2.20 \mathrm{E}+06$ & $77 \%$ & 7077 & $1.91 \mathrm{E}-03$ & $8.72 \mathrm{E}-06$ & 3.45 & 2.45 & 3.75 & 2.80 & 0.12 & 2.04 & 0.20 & 0.024 & 0.099 \\
\hline $241-S-102$ & 81.1 & $1.14 \mathrm{E}+06$ & $71 \%$ & 6011 & $8.91 \mathrm{E}-04$ & $5.37 \mathrm{E}-06$ & 2.50 & 2.77 & 4.91 & 2.21 & 0.26 & 3.85 & 0.20 & 0.025 & 0.122 \\
\hline Supenatant & nа & na & na & na & na & na & na & na & na & na & па & na & 0.20 & na & na \\
\hline Solid Layer & 81.1 & $1.14 E+06$ & $71 \%$ & $601 !$ & $8.91 \mathrm{E}-04$ & $5.37 \mathrm{E}-06$ & 2.50 & 2.77 & 4.91 & 2.21 & 0.26 & 3.85 & 0.20 & 0.025 & 0.122 \\
\hline $241-S-103$ & 93.5 & $1.44 \mathrm{E}+06$ & $72 \%$ & 6254 & $8.32 \mathrm{E}-04$ & $5.53 \mathrm{E}-06$ & 3.49 & 3.19 & 3.23 & 2.14 & 0.32 & 2.21 & 0.20 & 0.025 & 0.094 \\
\hline Supernatant & 0.4 & $5.80 E+03$ & $100 \%$ & 8 & $1.05 \mathrm{E}-03$ & $2.82 \mathrm{E}-08$ & 3.48 & 3.20 & 3.23 & 2.15 & 0.32 & 2.21 & 0.20 & 0.025 & 0.094 \\
\hline Solid Layer & 93.1 & $1.44 \mathrm{E}+06$ & $72 \%$ & 6247 & $8.31 \mathrm{E}-04$ & $5.56 \mathrm{E}-06$ & 3.49 & 3.19 & 3.23 & 2.14 & 0.32 & 2.21 & 0.20 & 0.025 & 0.094 \\
\hline $24 !-S-104$ & 112.2 & $1.82 \mathrm{E}+06$ & $70 \%$ & 6620 & $1.68 E-03$ & $1.09 \mathrm{E}-05$ & 4.33 & 0.83 & 3.44 & 0.30 & 0.03 & 0.87 & 0.20 & 0.021 & 0.088 \\
\hline Supematant & na & na & na & па & na & па & na & na & na & na & na & na & 0.20 & na & na \\
\hline Solid Layer & 112.2 & $1.82 \mathrm{E}-00$ & $70 \%$ & 6620 & $1.68 \mathrm{E}-03$ & $1.09 \mathrm{E}-05$ & 4.33 & 0.83 & 3.44 & 0.30 & 0.03 & 0.87 & 0.20 & 0.021 & 0.088 \\
\hline 24 !-S-105 & 155.0 & $2.55 E+06$ & $5 \%$ & 7461 & $1.79 \mathrm{E}-04$ & $2.36 \mathrm{E}-07$ & 3.06 & 2.66 & 4.55 & 4.81 & 0.28 & 2.60 & 0.20 & 0.022 & 0.106 \\
\hline Supematant & na & na & na & na & na & na & na & na & na & na & na & na & 0.20 & na & na \\
\hline Solid Layer & 155.0 & $2.55 \mathrm{E}+06$ & $5 \%$ & 7461 & $1.79 \mathrm{E}-04$ & $2.36 \mathrm{E}-07$ & 3.06 & 2.66 & 4.55 & 4.81 & 0.28 & 2.60 & 0.20 & 0.022 & 0.106 \\
\hline
\end{tabular}


RPP-5926 REV 6

Table B-2. Derived Data for Hydrogen Generation Rate Model Calculations for 177 Tanks. (20 sheets)

\begin{tabular}{|c|c|c|c|c|c|c|c|c|c|c|c|c|c|c|c|}
\hline Tank & $\begin{array}{c}\text { Waste } \\
\text { level } \\
\text { Dw } \\
\text { (inch) }\end{array}$ & $\begin{array}{c}\text { Total } \\
\text { mass } \\
M \\
(\mathbf{k g})\end{array}$ & $\begin{array}{c}\text { Liquid } \\
\text { in waste } \\
(w 1 \%)\end{array}$ & $\begin{array}{c}\text { Wetted } \\
\text { area } \\
\text { Awet } \\
\left(\mathrm{ft}^{2}\right)\end{array}$ & $\begin{array}{c}\text { Tank Heat } \\
\text { load } \\
\mathrm{H}_{\mathbf{L}}{ }^{\beta / r} \\
\text { (watt/kg) }\end{array}$ & $\begin{array}{c}\text { Tank } \\
\text { heal load } \\
\mathrm{H}_{\mathrm{L}}^{\text {alpta }} \\
\text { (watt } / \mathbf{k g} \text { ) }\end{array}$ & $\begin{array}{c}\mathrm{NO}_{3} \\
\text { in liquid } \\
{\left[\mathrm{NO}_{3}\right]} \\
\text { (mole/L) }\end{array}$ & $\begin{array}{c}\mathrm{NO}_{2} \\
\text { in liquid } \\
{\left[\mathrm{NO}_{2}\right]} \\
(\mathrm{mole} / \mathrm{L})\end{array}$ & $\begin{array}{c}\text { Excess } \mathrm{Na} \\
\text { in liquid } \\
{[\mathrm{Na}]_{e x}} \\
(\mathrm{~mole} / \mathrm{L})\end{array}$ & $\begin{array}{c}\mathrm{OH} \\
\text { in liquid } \\
|\mathrm{OH}| \\
\text { (mole/L) }\end{array}$ & $\begin{array}{c}\text { TOC } \\
\text { in liquid } \\
\text { [TOC] } \% \\
\text { (wt\%) }\end{array}$ & $\begin{array}{c}\text { Al } \\
\text { in liquid } \\
|A| \mid \% \\
(w t \%)\end{array}$ & $\begin{array}{c}\mathbf{E}_{\mathrm{H} 2} \text { Efficiency } \\
\text { of } \mathbf{H}_{2} \text { by } \\
\text { corrosion }\end{array}$ & $\begin{array}{c}\text { Total } \\
\text { G values } \\
G_{\text {rot }}^{B / \gamma} \\
\left(H_{z /} 100 \mathrm{eV}\right)\end{array}$ & $\begin{array}{c}\text { Total } \\
\text { G values } \\
\mathbf{G}_{\text {Tot }} \text { alpha }^{2} \\
\left(\mathrm{H}_{2} / 100 \mathrm{eV}\right)\end{array}$ \\
\hline $241-\mathrm{S}-106$ & 173.0 & $2.97 \mathrm{E}+06$ & $64 \%$ & 7834 & $5.14 \mathrm{E}-04$ & $8.75 \mathrm{E}-07$ & 3.81 & 2.26 & 4.55 & 3.26 & 0.14 & 2.66 & 0.20 & 0.019 & 0.091 \\
\hline Supenxatant & ma & na & na & pa & па & na & na & na & na & na & na & na & 0.20 & na & na \\
\hline Solid Layer & 173.0 & $2.97 \mathrm{E}+06$ & $64 \%$ & 7814 & $5.14 \mathrm{E}-04$ & $8.75 \mathrm{E}-07$ & 3.81 & 2.26 & 4.55 & 3.26 & 0.14 & 2.66 & 0.20 & 0.019 & 0.091 \\
\hline $241-S-107$ & 137.7 & $2.41 \mathrm{E}+06$ & $53 \%$ & 7122 & 1.19E-03 & $2.92 \mathrm{E}-05$ & 1.85 & 1.55 & 3.39 & 1.76 & 0.09 & 0.82 & 0.20 & 0.034 & 0.165 \\
\hline Supertatant & na & na & na & pa & na & na & na & na & па & na & na & na & 0.20 & na & na \\
\hline Solid Layer & 137.7 & $2.41 \mathrm{E}+06$ & $53 \%$ & 7122 & $1.19 \mathrm{E}-03$ & $2.92 \mathrm{E}-05$ & 1.85 & 1.55 & 3.39 & 1.76 & 0.09 & 0.82 & 0.20 & 0.034 & 0.165 \\
\hline $24 !-S-108$ & 207.5 & $3.49 \mathrm{E}+06$ & $3 \%$ & 8491 & $5.94 \mathrm{E}-04$ & $3.59 \mathrm{E}-06$ & 3.06 & 2.65 & 4.55 & 2.83 & 0.28 & 2.60 & 0.20 & 0.023 & 0.106 \\
\hline Supeniatant & na & $\mathrm{na}$ & na & na & na & na & na & na & na & na & na & na & 0.20 & na & na \\
\hline Solid Layer & 207.5 & $3.49 \mathrm{E}+06$ & $3 \%$ & 8491 & $5.94 \mathrm{E}-04$ & $3.59 \mathrm{E}-06$ & 3.06 & 2.65 & 4.55 & 2.83 & 0.28 & 2.60 & 0.20 & 0.023 & 0.106 \\
\hline $241-S-109$ & 201.2 & $3.34 E+06$ & $20 \%$ & 8369 & $1.61 \mathrm{E}-04$ & $9.10 \mathrm{E}-07$ & 2.12 & 1.85 & 5.56 & 4.82 & 0.07 & 3.08 & 0.20 & 0.020 & 0.145 \\
\hline Supernatant & na & na & 11а & na & na & na & na & nаa & па & na & na & na & 0.20 & na & na \\
\hline Solid Layer & 201.2 & $3.34 E+06$ & $20 \%$ & 8369 & $1.61 \mathrm{E}-04$ & $9.10 \mathrm{E}-07$ & 2.12 & 1.85 & 5.56 & 4.82 & 0.07 & 3.08 & 0.20 & 0.020 & 0.145 \\
\hline $241-S-110$ & 149.0 & $2.45 \mathrm{E}+06$ & $24 \%$ & 7343 & $9.73 \mathrm{E}-04$ & $8.75 \mathrm{E}-06$ & 3.51 & 1.87 & 3.82 & 4.81 & 0.16 & 2.16 & 0.20 & 0.025 & 0.101 \\
\hline Supernatant & na & na & na & па & na & na & na & na & na & na & na & na & 0.20 & na & na \\
\hline Solid Layer & 149.0 & $2.45 E+06$ & $24 \%$ & 7343 & $9.73 \mathrm{E}-04$ & $8.75 \mathrm{E}-06$ & 3.51 & 1.87 & 3.82 & 4.81 & 0.16 & 2.16 & 0.20 & 0.025 & 0.101 \\
\hline $241-S-111$ & 153.2 & $2.16 \mathrm{E}+06$ & $57 \%$ & 7426 & $1.71 \mathrm{E}-03$ & $7.47 \mathrm{E}-07$ & 3.11 & 1.82 & 4.75 & 3.00 & 0.10 & 2.51 & 0.20 & 0.021 & 0.110 \\
\hline Supernatant & na & na & na & na & na & na & na & na & na & na & na & na & 0.20 & na & na \\
\hline Solid Layer & 153.2 & $2.16 \mathrm{E}+06$ & $57 \%$ & 7426 & $1.71 E-03$ & $7.47 \mathrm{E}-07$ & 3.11 & 1.82 & 4.75 & 3.00 & 0.10 & 2.51 & 0.20 & 0.021 & 0.110 \\
\hline $241-S-112$ & 9.3 & $3.25 \mathrm{E}+04$ & $70 \%$ & 4600 & $4.28 \mathrm{E}-03$ & $2.89 \mathrm{E}-05$ & 2.71 & 1.35 & 5.89 & 2.86 & 0.06 & 2.54 & 0.20 & 0.017 & 0.126 \\
\hline Supernatant & na & na & na & na & na & na & na & na & na & na & па & na & 0.20 & na & na \\
\hline Solid Layer & 9.3 & $3.25 \mathrm{E}+04$ & $70 \%$ & 4600 & $4.28 E-03$ & $2.89 \mathrm{E}-05$ & 2.71 & 1.35 & 5.89 & 2.86 & 0.06 & 2.54 & 0.20 & 0.017 & 0.126 \\
\hline $24 \mathrm{~J}-\mathrm{S} X-101$ & 160.3 & $2.66 \mathrm{E}+06$ & $54 \%$ & 7566 & $1.23 \mathrm{E}-03$ & $1.25 \mathrm{E}-05$ & 1.74 & 1.63 & 7.16 & 2.87 & 0.02 & 5.41 & 0.20 & 0.016 & 0.170 \\
\hline Supernatant & na & na & na & na & $3 \mathbf{a}$ & na & na & na & na & na & na & na & 0.20 & na & na \\
\hline Solid Layer & 160.3 & $2.66 \mathrm{E}+06$ & $54 \%$ & 7566 & $1.23 \mathrm{E}-03$ & $1.25 \mathrm{E}-05$ & 1.74 & 1.63 & 7.16 & 2.87 & 0.02 & 5.41 & 0.20 & 0.016 & 0.170 \\
\hline $241-S X-102$ & 132.3 & $2.19 \mathrm{E}+06$ & $82 \%$ & 7015 & 1.21E-03 & $6.20 \mathrm{E}-06$ & 2.53 & 3.29 & 4.40 & 2.95 & 0.10 & 3.12 & 0.20 & 0.026 & 0.117 \\
\hline Supernatant & na & $\mathrm{na}$ & na & na & na & na & na & na & na & na & na & na & 0.20 & na & na \\
\hline Solid Layer & 132.3 & $2.19 \mathrm{E}+06$ & $82 \%$ & 7015 & $1.21 \mathrm{E}-03$ & $6.20 \mathrm{E}-06$ & 2.53 & 3.29 & 4.40 & 2.95 & 0.10 & 3.12 & 0.20 & 0.026 & 0.117 \\
\hline
\end{tabular}


RPP-5926 REV 6

Table B-2. Derived Data for Hydrogen Generation Rate Model Calculations for 177 Tanks. (20 sheets)

\begin{tabular}{|c|c|c|c|c|c|c|c|c|c|c|c|c|c|c|c|}
\hline Tank & $\begin{array}{c}\text { Waste } \\
\text { level } \\
\text { Dw } \\
\text { (inch) }\end{array}$ & $\begin{array}{c}\text { Total } \\
\text { mass } \\
M \\
(\mathbf{k g})\end{array}$ & $\begin{array}{c}\text { Liquid } \\
\text { in waste } \\
\text { (wt } \% \text { ) }\end{array}$ & $\begin{array}{l}\text { Wetted } \\
\text { area } \\
\text { Awet } \\
\left(\mathrm{ft}^{2}\right)\end{array}$ & $\begin{array}{c}\text { Tank Heat } \\
\text { load } \\
H_{L}{ }^{\beta / r} \\
\text { (watt } / \mathbf{k g} \text { ) }\end{array}$ & $\begin{array}{c}\text { Tank } \\
\text { heat load } \\
\mathrm{H}_{\mathrm{L}}^{\text {algha }} \\
\text { (watt/kg) }\end{array}$ & $\begin{array}{c}\mathrm{NO}_{3} \\
\text { in liquid } \\
\left|\mathrm{NO}_{3}\right| \\
(\text { mole } / \mathrm{L})\end{array}$ & $\begin{array}{c}\mathrm{NO}_{2} \\
\text { in liquid } \\
{\left[\mathrm{NO}_{2}\right]} \\
(\mathrm{mole} / \mathrm{L})\end{array}$ & $\begin{array}{c}\text { Excess } \mathrm{Na} \\
\text { in liquid } \\
{\left[\left.\mathrm{Na}\right|_{\mathrm{ex}}\right.} \\
(\mathrm{mole} / \mathrm{L})\end{array}$ & $\begin{array}{c}\mathrm{OH} \\
\text { in liquid } \\
{[\mathrm{OH}]} \\
(\text { mole } / \mathrm{L})\end{array}$ & $\begin{array}{c}\text { TOC } \\
\text { in liquid } \\
\text { |TOC|\% } \\
(w t \%)\end{array}$ & $\begin{array}{c}\text { Al } \\
\text { in liquid } \\
\mid \text { Al| } \% \\
(w t \%)\end{array}$ & $\begin{array}{c}E_{\mathrm{H} 2} \text { Efficiency } \\
\text { of } \mathrm{H}_{2} \text { by } \\
\text { corrosion }\end{array}$ & $\begin{array}{c}\text { Total } \\
\text { G values } \\
\mathbf{G}_{\text {Tor }}{ }^{B / \gamma} \\
\left(\mathrm{H}_{2 / 1} 100 \mathrm{eV}\right)\end{array}$ & $\begin{array}{c}\text { Total } \\
\text { G values } \\
\mathrm{G}_{\mathrm{TOT}}{ }^{\text {2lph }} \\
\left(\mathrm{H}_{2} / 100 \mathrm{eV}\right)\end{array}$ \\
\hline $241-S X+103$ & 193.2 & $3.33 E+66$ & $75 \%$ & 8211 & $1.61 E-03$ & $9.95 \mathrm{E}-06$ & 2.52 & 3.42 & 4.50 & 2.66 & 0.23 & 3.02 & 0.20 & 0,038 & 0.122 \\
\hline Supernatant & nа & na & na & na & na & na & na & na & na & na & na & na & 0.20 & na & na \\
\hline Solid Layer & 193.2 & $3.33 E+06$ & $75 \%$ & 8211 & $1.61 \mathrm{E}-03$ & $9.95 \mathrm{E}-06$ & 2.52 & 3.42 & 4.50 & 2.66 & 0.23 & 3.02 & 0.20 & 0.038 & 0.122 \\
\hline $241-S X-104$ & 170.3 & $2.86 \mathrm{E}+06$ & $56 \%$ & 7762 & $1.36 \mathrm{E}-03$ & $1.06 \mathrm{E}-05$ & 4.68 & 2.55 & 3.67 & 1.79 & 0.14 & 2.42 & 0.20 & 0.025 & 0.080 \\
\hline Supematant & na & na & na & na & na & na & па & na & na & na & na & па & 0.20 & na & na \\
\hline Solid Layer & 170.3 & $2.86 \mathrm{E}+06$ & $56 \%$ & 7762 & $1.36 E-03$ & $1.06 \mathrm{E}-05$ & 4.68 & 2.55 & 3.67 & 1.79 & 0.14 & 2.42 & 0.20 & 0.025 & 0.080 \\
\hline $241-S X-105$ & 144.8 & $2.32 \mathrm{E}+06$ & $82 \%$ & 7261 & 2.14E-03 & $1.92 \mathrm{E}-05$ & 2.65 & 3.11 & 4.49 & 2.41 & 0.22 & 3.00 & 0.20 & 0.037 & 0.121 \\
\hline Supematant & na & na & na & na & ma & na & па & na & na & na & na & na & 0.20 & na & na \\
\hline Solid Layer & 144.8 & $2.32 \mathrm{E}+06$ & $82 \%$ & 7261 & $2.14 \mathrm{E}-03$ & $1.92 \mathrm{E}-05$ & 2.65 & 3.11 & 4.49 & 2.41 & 0.22 & 3.00 & 0.20 & 0.037 & 0.121 \\
\hline $241-S X-106$ & 152.4 & $2.37 \mathrm{E}+06$ & $8 ! \%$ & 7410 & $7.02 \mathrm{E}-04$ & $8.15 \mathrm{E}-06$ & 3.95 & 3.24 & 2.25 & 1.88 & 0.24 & 1.75 & 0.20 & 0.028 & 0.086 \\
\hline Supernatant & na & na & na & na & na & na & na & na & na & na & па & na & 0.20 & na & na \\
\hline Solid Layer & 152.4 & $2.37 E+06$ & $81 \%$ & 7410 & $7.02 \mathrm{E}-04$ & 8.15E-06 & 3.95 & 3.24 & 2.25 & 1.88 & 0.24 & 1.75 & 0.20 & 0.028 & 0.086 \\
\hline $241-\mathrm{SX}-107$ & 42.4 & $6.30 \mathrm{E}+05$ & $44 \%$ & 5250 & $1.38 \mathrm{E}-02$ & $4.89 \mathrm{E}-05$ & 3.50 & 2.14 & 5.44 & 3.57 & 0.07 & 3.15 & 0.20 & 0.025 & 0.102 \\
\hline Supernatant & na & na & na & na & na & na & na & na & na & na & na & na & 0.20 & na & na \\
\hline Solid Layer & 42.4 & $6.30 \mathrm{E}+05$ & $44 \%$ & 5250 & $1.38 \mathrm{E}-02$ & $4.89 \mathrm{E}-05$ & 3.50 & 2.14 & 5.44 & 3.57 & 0.07 & 3.15 & 0.20 & 0.025 & 0.102 \\
\hline $241-S X-108$ & 35.1 & $4.96 \mathrm{E}+05$ & $3 \%$ & 5106 & $1.80 \mathrm{E}-02$ & $1.04 \mathrm{E}-04$ & 1.28 & 0.78 & 1.55 & 3.57 & 0.26 & 0.42 & 0.20 & 0.106 & 0.252 \\
\hline Supernatant & na & na & na & na & na & na & na & na & na & na & na & na & 0.20 & na & na \\
\hline Solid Layer & 35.1 & $4.96 \mathrm{E}+05$ & $3 \%$ & 5106 & $1.80 \mathrm{E}-02$ & $1.04 \mathrm{E}-04$ & 1.28 & 0.78 & 1.55 & 3.57 & 0.26 & 0.42 & 0.20 & 0.106 & 0.252 \\
\hline $24 !-S X-109$ & 95.9 & $1.58 \mathrm{E}+06$ & $27 \%$ & 6300 & $4.18 E-03$ & $1.64 \mathrm{E}-05$ & 2.83 & 2.33 & 3.43 & 3.57 & 0.66 & 1.98 & 0.20 & 0.058 & 0.130 \\
\hline Supernatant & na & nad & na & na & na & na & na & na & na & $\mathrm{na}$ & na & na & 0.20 & na & na \\
\hline Solid Layer & 95.9 & $1.58 \mathrm{E}+06$ & $27 \%$ & 6300 & $4.18 \mathrm{E}-03$ & $1.64 \mathrm{E}-05$ & 2.83 & 2.33 & 3.43 & 3.57 & 0.66 & 1.98 & 0.20 & 0.058 & 0.130 \\
\hline $241-5 X-110$ & 28.5 & $3.74 \mathrm{E} \div 05$ & $30 \%$ & 4978 & $3.12 \mathrm{E}-02$ & $8.28 \mathrm{E}-05$ & 1.28 & 0.78 & 1.55 & 3.57 & 0.26 & 0.42 & 0.20 & 0.075 & 0.237 \\
\hline Supernatant & na & na & na & na & na & na & na & па & na & na & na & na & 0.20 & na & na \\
\hline Solid Layer & 28.5 & $3.74 \mathrm{E}+05$ & $30 \%$ & 4978 & 3.12E-02 & $8.28 \mathrm{E}-05$ & 1.28 & 0.78 & 1.55 & 3.57 & 0.26 & 0.42 & 0.20 & 0.075 & 0.237 \\
\hline $241-S X-111$ & 50.1 & $7.68 \mathrm{E}+05$ & $44 \%$ & 5401 & $1.80 \mathrm{E}-02$ & $5.52 \mathrm{E}-05$ & 3.50 & 2.15 & 5.44 & 3.57 & 0.07 & 3.14 & 0.20 & 0.023 & 0.101 \\
\hline Supernatant & na & na & na & na & na & na & na & na & na & na & na & na & 0.20 & na & na \\
\hline Solid Layer & 50.1 & $7.68 \mathrm{E} \div 05$ & $44 \%$ & 5401 & $1.80 \mathrm{E}-02$ & $5.52 \mathrm{E}-05$ & 3.50 & 2.15 & 5.44 & 3.57 & 0.07 & 3.14 & 0.20 & 0.023 & 0.101 \\
\hline
\end{tabular}

\section{B-35}


RPP-5926 REV 6

Table B-2. Derived Data for Hydrogen Generation Rate Model Calculations for 177 Tanks. (20 sheets)

\begin{tabular}{|c|c|c|c|c|c|c|c|c|c|c|c|c|c|c|c|}
\hline Tank & $\begin{array}{c}\text { Waste } \\
\text { level } \\
\text { Dw } \\
\text { (inch) }\end{array}$ & $\begin{array}{c}\text { Total } \\
\text { mass } \\
M \\
(\mathrm{~kg})\end{array}$ & $\begin{array}{c}\text { l.iquid } \\
\text { in waste } \\
(w t \%)\end{array}$ & $\begin{array}{c}\text { Wetted } \\
\text { area } \\
\text { Awet } \\
\left(\mathrm{ft}^{2}\right)\end{array}$ & $\begin{array}{c}\text { Tank Heat } \\
\text { load } \\
\mathrm{H}_{\mathrm{L}}^{\text {plr }} \\
\text { (watt } / \mathrm{kg} \text { ) }\end{array}$ & $\begin{array}{c}\text { Tank } \\
\text { heat load } \\
H_{L}{ }^{\text {alpha }} \\
\text { (watt } / \mathbf{k g} \text { ) }\end{array}$ & $\begin{array}{c}\mathrm{NO}_{3} \\
\text { in liquid } \\
{\left[\mathrm{NO}_{3}\right]} \\
(\text { mole/L) }\end{array}$ & $\begin{array}{c}\mathrm{NO}_{2} \\
\text { in liquid } \\
{\left[\mathrm{NO}_{2} \mid\right.} \\
(\mathrm{mole} / \mathrm{L})\end{array}$ & $\begin{array}{c}\text { Excess } \mathrm{Na} \\
\text { in liquid } \\
|\mathrm{Na}|_{\mathrm{ex}} \\
(\text { mole } / \mathrm{L})\end{array}$ & $\begin{array}{c}\mathrm{OH} \\
\text { in liquid } \\
|\mathrm{OH}| \\
(\mathrm{mole} / \mathrm{L})\end{array}$ & $\begin{array}{c}\text { TOC } \\
\text { in liquid } \\
\text { [TOC]"\% } \\
(w+\%)\end{array}$ & $\begin{array}{c}\text { Al } \\
\text { in liquid } \\
|A| \mid \% \\
(w t \%)\end{array}$ & $\begin{array}{c}\mathrm{E}_{\mathrm{H} 2} \text { Efficiency } \\
\text { of } \mathrm{H}_{2} \mathrm{by} \\
\text { corrosion }\end{array}$ & $\begin{array}{c}\text { Total } \\
\text { G values } \\
\mathrm{G}_{\text {Tот }}{ }^{\beta / \gamma} \\
\left(\mathrm{H}_{2} \text { : } 100 \mathrm{eV}\right)\end{array}$ & $\begin{array}{c}\text { Total } \\
\text { G values } \\
\mathrm{G}_{\mathrm{TOT}}{ }^{\text {alpha }} \\
\left(\mathrm{H}_{2} / 100 \mathrm{eV}\right)\end{array}$ \\
\hline $241-S X-112$ & 35.4 & $5.01 \mathrm{E}+05$ & $44 \%$ & 5112 & $1.91 \mathrm{E}-02$ & $5.99 \mathrm{E}-05$ & 3.50 & 2.15 & 5.44 & 3.57 & 0.07 & 3.15 & 0.20 & 0.021 & 0.100 \\
\hline Supernatant & na & na & na & na & na & na & na & Ila & na & na & na & na & 0.20 & na & na \\
\hline Solid Layer & 35.4 & $5.01 \mathrm{E}+05$ & $44 \%$ & 5112 & $1.91 \mathrm{E}-02$ & $5.99 \mathrm{E}-05$ & 3.50 & 2.15 & 5.44 & 3.57 & 0.07 & 3.15 & 0.20 & 0.021 & 0.100 \\
\hline $241 \cdot S X-113$ & 15.1 & $1.03 \mathrm{E}+05$ & $61 \%$ & 4714 & $2.80 \mathrm{E}-04$ & $5.39 E-06$ & 1.28 & 0.78 & 1.55 & 3.57 & 0.26 & 0.42 & 0.20 & 0.064 & 0.231 \\
\hline Supernatant & na & na & ra & na & na & na & na & na & na & na & na & na & 0.20 & па & na \\
\hline Solid Layer & 15.1 & $1.03 \mathrm{E}+05$ & $61 \%$ & 4714 & $2.80 \mathrm{E}-04$ & $5.39 \mathrm{E}-06$ & 1.28 & 0.78 & 1.55 & 3.57 & 0.26 & 0.42 & 0.20 & 0.064 & 0.231 \\
\hline $241-S X-114$ & 64.7 & $1.03 E+06$ & $46 \%$ & 5687 & $1.27 E-02$ & $4.34 \mathrm{E}-05$ & 3.50 & 2.15 & 5.27 & 3.57 & 0.07 & 3.15 & 0.20 & 0.026 & 0.102 \\
\hline Supematant & na & nа & na & na & na & na & na & na & na & na & na & na & 0.20 & na & na \\
\hline Solid Layer & 64.7 & $1.03 E+06$ & $46 \%$ & 5687 & $1.27 \mathrm{E}-02$ & $4.34 \mathrm{E}-05$ & 3.50 & 2.15 & 5.27 & 3.57 & 0.07 & 3.15 & 0.20 & 0.026 & 0.102 \\
\hline $241-S X-115$ & 9.7 & $2.83 \mathrm{E}+04$ & $13 \%$ & 4609 & $9.06 \mathrm{E}-02$ & $1.11 \mathrm{E}-03$ & 1.28 & 0.78 & 1.55 & 3.57 & 0.26 & 0.42 & 0.20 & 0.063 & 0.231 \\
\hline Supematant & na & na & na & na & na & na & na & па & па & na & na & na & 0.20 & na & na \\
\hline Solid Layer & 9.7 & $2.83 \mathrm{E}+04$ & $13 \%$ & 4609 & $9.06 \mathrm{E}-02$ & $1.11 \mathrm{E}-03$ & 1.28 & 0.78 & 1.55 & 3.57 & 0.26 & 0.42 & 0.20 & 0.063 & 0.231 \\
\hline $241-\mathrm{T}-101$ & 43.6 & $5.81 \mathrm{E}+05$ & $80 \%$ & 5273 & $3.24 \mathrm{E}-04$ & $1.74 \mathrm{E}-05$ & 3.06 & 2.65 & 4.59 & 2.83 & 0.28 & 2.60 & 0.20 & 0.022 & 0.106 \\
\hline Supernatant & nа & na & nа & na & na & na & na & na & na & na & па & па & 0.20 & na & na \\
\hline Solid Layer & 43.6 & $5.81 \mathrm{E}+05$ & $80 \%$ & 5273 & 3.24E-04 & $1.74 \mathrm{E}-05$ & 3.06 & 2.65 & 4.59 & 2.83 & 0.28 & 2.60 & 0.20 & 0.022 & 0.106 \\
\hline $241-T-102$ & 19.0 & $1.84 \mathrm{E}+05$ & $56 \%$ & 4790 & $7.24 \mathrm{E}-04$ & $4.66 \mathrm{E}-06$ & 1.76 & 0.50 & 0.55 & 0.00 & 0.04 & 0.74 & 0.50 & 0.058 & 0.190 \\
\hline Supenatant & 4.6 & $5.47 \mathrm{E} \div 04$ & $100 \%$ & 90 & $2.19 \mathrm{E}-04$ & $2.97 E-07$ & 1.76 & 0.50 & 0.55 & 0.00 & 0.04 & 0.74 & 0.50 & 0.058 & 0.190 \\
\hline Solid Layer & 14.4 & $1.29 \mathrm{E}+05$ & $37 \%$ & 4700 & $9.37 \mathrm{E}-04$ & $6.51 \mathrm{E}-06$ & 1.76 & 0.50 & 0.55 & 0.00 & 0.04 & 0.74 & 0.50 & 0.058 & 0.190 \\
\hline 241-T-103 & 17.3 & $1.67 \mathrm{E}+05$ & $47 \%$ & 4757 & 3.92E-05 & $9.91 \mathrm{E}-07$ & 1.26 & 0.86 & 2.03 & 1.00 & 0.47 & 0.88 & 0.20 & 0.058 & 0.232 \\
\hline Supernatant & 1.4 & $1.79 \mathrm{E}+04$ & $100 \%$ & 28 & $6.86 E-05$ & $5.30 \mathrm{E}-07$ & 1.26 & 0.86 & 2.03 & 1.00 & 0.47 & 0.87 & 0.20 & 0.058 & 0.232 \\
\hline Solid Layer & 15.8 & 1.49E+05 & $41 \%$ & 4728 & $3.57 \mathrm{E}-05$ & $1.05 \mathrm{E}-06$ & 1.26 & 0.86 & 2.03 & 1.00 & 0.47 & 0.88 & 0.20 & 0.058 & 0.232 \\
\hline $241-T-104$ & 122.6 & $1.55 \mathrm{E} \div 06$ & $84 \%$ & 6826 & $1.43 \mathrm{E}-05$ & $4.91 \mathrm{E}-06$ & 1.28 & 0.78 & 1.55 & 1.00 & 0.26 & 0.42 & 0.20 & 0.062 & 0.230 \\
\hline Supernatant & na & na & na & na & na & na & па & na & na & na & na & na & 0.20 & na & na \\
\hline Solid Layer & 122.6 & $1.55 \mathrm{E}+06$ & $84 \%$ & 6826 & 1.43E-05 & $4.91 \mathrm{E}-06$ & 1.28 & 0.78 & 1.55 & 1.00 & 0.26 & 0.42 & 0.20 & 0.062 & 0.230 \\
\hline $24 !-T-105$ & 43.1 & $5.42 \mathrm{E}+05$ & $64 \%$ & 5264 & $3.91 \mathrm{E}-04$ & $1.13 \mathrm{E}-05$ & 0.60 & 1.18 & 2.07 & 1.00 & 0.25 & 0.03 & 0.20 & 0.073 & 0.331 \\
\hline Supenatant & na & na & nа & na & na & na & na & na & na & na & na & na & 0.20 & na & na \\
\hline Solid Layer & 43.1 & $5.42 \mathrm{E}+05$ & $64 \%$ & 5264 & $3.91 \mathrm{E}-04$ & $1.13 \mathrm{E}-05$ & 0.60 & 1.18 & 2.07 & 1.00 & 0.25 & 0.03 & 0.20 & 0.073 & 0.331 \\
\hline
\end{tabular}


RPP-5926 REV 6

Table B-2. Derived Data for Hydrogen Generation Rate Model Calculations for 177 Tanks. (20 sheets)

\begin{tabular}{|c|c|c|c|c|c|c|c|c|c|c|c|c|c|c|c|}
\hline Tank & $\begin{array}{l}\text { Waste } \\
\text { level } \\
\text { Dw } \\
\text { (inch) }\end{array}$ & $\begin{array}{c}\text { Total } \\
\text { mass } \\
M \\
(\mathrm{~kg})\end{array}$ & $\begin{array}{c}\text { Liquid } \\
\text { in waste } \\
(w t \%)\end{array}$ & $\begin{array}{c}\text { Wetted } \\
\text { area } \\
\text { Awet } \\
\left(\text { ft }^{2}\right)\end{array}$ & $\begin{array}{c}\text { Tank Heat } \\
\text { load } \\
\mathrm{H}_{\mathrm{L}}{ }^{\beta / \mathrm{r}} \\
\text { (watt } / \mathbf{k g} \text { ) }\end{array}$ & $\begin{array}{c}\text { Tank } \\
\text { heat load } \\
\mathrm{H}_{\mathrm{L}}^{\text {Llpha }} \\
\text { (watt/kg) }\end{array}$ & $\begin{array}{c}\mathrm{NO}_{3} \\
\text { in liquid } \\
{\left[\mathrm{NO}_{3}\right]} \\
\text { (mole/L) }\end{array}$ & $\begin{array}{c}\mathrm{NO}_{2} \\
\text { in liquid } \\
\left|\mathrm{NO}_{2}\right| \\
(\mathrm{mole} / \mathrm{L})\end{array}$ & $\begin{array}{c}\text { Excess } \mathrm{Na} \\
\text { in liquid } \\
{[\mathrm{Na}]_{\mathrm{ex}}} \\
(\mathrm{mole} / \mathrm{L})\end{array}$ & $\begin{array}{c}\text { OH } \\
\text { in liquid } \\
{[\mathrm{OH}]} \\
\text { (mole/L) }\end{array}$ & $\begin{array}{c}\text { TOC } \\
\text { in liquid } \\
\text { \{TOC|\% } \\
\text { (wt } \% \text { ) }\end{array}$ & $\begin{array}{c}\text { Al } \\
\text { in liquid } \\
|A| \% \\
(w+\%)\end{array}$ & $\left\{\begin{array}{c}E_{\mathrm{H}_{2}} \text { Efficiency } \\
\text { of } \mathrm{H}_{2} \text { by } \\
\text { corrosion }\end{array}\right.$ & $\begin{array}{c}\text { Total } \\
\text { G values } \\
\text { G }_{\text {TOT }}{ }^{\mathrm{B} / \mathrm{\gamma}} \\
\left(\mathrm{H}_{2 / 100 \mathrm{eV})}\right.\end{array}$ & $\begin{array}{c}\text { Total } \\
\text { G values } \\
\mathrm{G}_{\text {Toror }}{ }^{\text {alpha }} \\
\left(\mathrm{H}_{2} / 100 \mathrm{eV}\right)\end{array}$ \\
\hline $241-T-106$ & 15.3 & $1.30 \mathrm{E}+05$ & $22 \%$ & 4719 & $1.06 \mathrm{E}-04$ & $6.15 \mathrm{E}-06$ & 1.28 & 0.78 & 1.55 & 1.00 & 0.26 & 0.42 & 0.20 & 0.062 & 0.230 \\
\hline Supernatant & na & na & na & na & na & na & па & na & na & na & na & na & 0.20 & na & ла \\
\hline Solid Layer & 15.3 & $1.30 \mathrm{E}+05$ & $22 \%$ & 4719 & $1.06 \mathrm{E}-04$ & $6.15 \mathrm{E}-06$ & 1.28 & 0.78 & 1.55 & 1.00 & 0.26 & 0.42 & 0.20 & 0.062 & 0.230 \\
\hline $241-T-107$ & 70.4 & $1.02 \mathrm{E}+06$ & $50 \%$ & 5800 & $7.42 \mathrm{E}-04$ & $1.24 \mathrm{E}-05$ & 1.28 & 0.78 & 1.55 & 1.00 & 0.26 & 0.42 & 0.20 & 0.063 & 0.230 \\
\hline Supernatant & na & na & na & na & na & na & na & na & па & na & na & na & 0.20 & na & na \\
\hline Solid layer & 70.4 & $1.02 \mathrm{E}+06$ & $50 \%$ & 5800 & $7.42 \mathrm{E}-04$ & $1.24 \mathrm{E}-05$ & 1.28 & 0.78 & 1.55 & 1.00 & 0.26 & 0.42 & 0.20 & 0.063 & 0.230 \\
\hline $241-T-108$ & 13.2 & $9.28 \mathrm{E}+04$ & $50 \%$ & 4677 & $4.39 E-05$ & $2.22 \mathrm{E}-06$ & 3.50 & 0.18 & 1.23 & 1.00 & 0.00 & 0.03 & 0.20 & 0.032 & 0.110 \\
\hline Supenatant & na & na & na & na & na & na & na & па & na & na & na & na & 0.20 & па & na \\
\hline Solid Layer & 13.2 & $9.28 \mathrm{E}+04$ & $50 \%$ & 4677 & $4.39 E-05$ & $2.22 \mathrm{E}-06$ & 3.50 & 0.18 & 1.23 & 1.00 & 0.00 & 0.03 & 0.20 & 0.032 & 0.110 \\
\hline $241-T-109$ & 30.0 & $3.87 \mathrm{E}+05$ & $73 \%$ & 5008 & $1.18 \mathrm{E}-05$ & $3.19 \mathrm{E}-07$ & 3.50 & 0.18 & 1.22 & 1.00 & 0,00 & 0.03 & 0.20 & 0.032 & 0.110 \\
\hline Supernatant & na & nà & na & na & na & na & na & na & na & na & na & na & 0.20 & na & na \\
\hline Solid Layer & 30.0 & $3.87 \mathrm{E}+05$ & $73 \%$ & 5008 & $1.18 \mathrm{E}-05$ & $3.19 \mathrm{E}-07$ & 3.50 & 0.18 & 1.22 & 1.00 & 0.00 & 0.03 & 0.20 & 0.032 & 0.110 \\
\hline $241-\mathrm{T}-110$ & 141.9 & $1.75 \mathrm{E}+06$ & $87 \%$ & 7205 & $2.53 \mathrm{E}-07$ & $2.06 \mathrm{E}-06$ & 0.30 & 0.00 & 1.31 & 1.00 & 0.00 & 0.00 & 0.50 & 0.146 & 0.615 \\
\hline Supernatant & 0.3 & $3.15 E+03$ & $100 \%$ & 6 & $1.6 \mathfrak{t E - 0 8}$ & $4.97 \mathrm{E}-09$ & 0.30 & 0.00 & 1.31 & 1.00 & 0.00 & 0.00 & 0.50 & 0.146 & 0.615 \\
\hline Solid Layer & 141.7 & $1.75 E+06$ & $87 \%$ & 7199 & $2.53 \mathrm{E}-07$ & $2.07 \mathrm{E}-06$ & 0.30 & 0.00 & 1.31 & 1.00 & 0.00 & 0.00 & 0.50 & 0.146 & 0.615 \\
\hline $241-\Gamma-111$ & 169.9 & $2.10 \mathrm{E}+06$ & $86 \%$ & 7754 & $2.78 \mathrm{E}-05$ & $5.80 \mathrm{E}-06$ & 1.28 & 0.78 & 1.55 & 1.00 & 0.26 & 0.42 & 0.20 & 0.062 & 0.230 \\
\hline Supematant & na & na & na & na & na & na & na & na & na & na & na & na & 0.20 & na & na \\
\hline Solid Layer & 169.9 & $2.10 \mathrm{E}+06$ & $86 \%$ & 7754 & $2.78 \mathrm{E}-05$ & $5.80 \mathrm{E}-06$ & 1.28 & 0.78 & 1.55 & 1.00 & 0.26 & 0.42 & 0.20 & 0.062 & 0.230 \\
\hline $241-\uparrow-112$ & 31.7 & $3.18 \mathrm{E}+05$ & $88 \%$ & 5039 & $1.97 \mathrm{E}-06$ & $7.18 \mathrm{E}-06$ & 0.38 & 0.84 & 1.27 & 1.00 & 0.15 & 0.00 & 0.20 & 0.112 & 0.432 \\
\hline Supernatant & 2.5 & $2.86 \mathrm{E}+04$ & $100 \%$ & 49 & $2.16 \mathrm{E}-05$ & $6.37 \mathrm{E}-07$ & 0.38 & 0.84 & 1.27 & 1.00 & 0.15 & 0.00 & 0.20 & 0.112 & 0.432 \\
\hline Solid Layer & 29.2 & $2.89 \mathrm{E}+05$ & $87 \%$ & 4991 & $3.16 \mathrm{E}-08$ & $7.82 \mathrm{E}-06$ & 0.38 & 0.84 & 1.27 & 1.00 & 0.15 & 0.00 & 0.20 & 0.112 & 0.432 \\
\hline $241-\mathrm{T}-201$ & 158.0 & $1.49 \mathrm{E}+05$ & $75 \%$ & 1141 & $5.24 \mathrm{E}-07$ & $2.19 \mathrm{E}-05$ & 0.74 & 0.01 & 0.34 & 1.00 & 0.01 & 0.00 & 0.50 & 0.115 & 0.376 \\
\hline Supernatant & 10.8 & $8.48 \mathrm{E}+03$ & $100 \%$ & 56 & $4.29 \mathrm{E}-10$ & $8.15 \mathrm{E}-10$ & 0.74 & 0.01 & 0.34 & 1.00 & 0.01 & 0.00 & 0.50 & 0.115 & 0.376 \\
\hline Solid Layer & 147.2 & $1.40 \mathrm{E}+05$ & $74 \%$ & 1085 & $5.55 \mathrm{E}-07$ & $2.32 \mathrm{E}-05$ & 0.74 & 0.01 & 0.34 & 1.00 & 0.01 & 0.00 & 0.50 & 0.115 & 0.376 \\
\hline $241-\mathrm{T}-202$ & 106.8 & $9.09 \mathrm{E}+04$ & $101 \%$ & 873 & $4.70 \mathrm{E}-08$ & $6.85 \mathrm{E}-06$ & 1.28 & 0.78 & 1.55 & 1.00 & 0.26 & 0.42 & 0.20 & 0.062 & 0.230 \\
\hline Supenratant & na & na & na & na & na & na & na & na & na & na & na & na & 0.20 & na & na \\
\hline Solid Layer & 106.8 & $9.09 \mathrm{E}+04$ & $101 \%$ & 873 & $4.70 \mathrm{E}-08$ & $6.85 E-06$ & 1.28 & 0.78 & 1.55 & 1.00 & 0.26 & 0.42 & 0.20 & 0.062 & 0.230 \\
\hline
\end{tabular}


RPP-5926 REV 6

Table B-2. Derived Data for Hydrogen Generation Rate Model Calculations for 177 Tanks. (20 sheets)

\begin{tabular}{|c|c|c|c|c|c|c|c|c|c|c|c|c|c|c|c|}
\hline Tank & $\begin{array}{c}\text { Waste } \\
\text { level } \\
\text { Dw } \\
\text { (inch) }\end{array}$ & $\begin{array}{c}\text { Tota! } \\
\text { mass } \\
M \\
(\mathrm{~kg})\end{array}$ & $\begin{array}{c}\text { Liquid } \\
\text { in waste } \\
\text { (wt"\%) }\end{array}$ & $\begin{array}{c}\text { Wetted } \\
\text { arca } \\
\text { Awet } \\
\left(\mathrm{fft}^{2}\right)\end{array}$ & $\begin{array}{c}\text { Tank Heat } \\
\text { load } \\
\mathrm{H}_{\mathrm{L}}{ }^{\beta / r} \\
\text { (watt/kg) }\end{array}$ & $\begin{array}{c}\text { Tank } \\
\text { heat load } \\
\mathbf{H}_{\mathrm{L}}{ }^{\text {alpha }} \\
\text { (watt/kg) }\end{array}$ & $\begin{array}{c}\mathrm{NO}_{3} \\
\text { in liquid } \\
{\left[\mathrm{NO}_{3}\right]} \\
(\mathrm{mole} / \mathrm{L})\end{array}$ & $\begin{array}{c}\mathrm{NO}_{2} \\
\text { in liquid } \\
\left|\mathrm{NO}_{2}\right| \\
(\text { mole } / \mathrm{L})\end{array}$ & $\begin{array}{c}\text { Excess } \mathrm{Na} \\
\text { in liquid } \\
|\mathrm{Na}|_{\mathrm{ex}} \\
(\mathrm{mole} / \mathrm{L})\end{array}$ & $\begin{array}{c}\text { OH } \\
\text { in liquid } \\
{[\mathrm{OH}]} \\
\text { (mole/L) }\end{array}$ & $\begin{array}{c}\text { TOC } \\
\text { in liquid } \\
\text { |TOC|\% } \\
\text { (wt } \% \text { (s) }\end{array}$ & $\begin{array}{c}\text { Al } \\
\text { in liquid } \\
|A| \mid \% \\
(w t \%)\end{array}$ & $\mid \begin{array}{c}F_{n 2} \text { Efficiency } \\
\text { of } \mathrm{H}_{2} \text { by } \\
\text { corrosion }\end{array}$ & $\begin{array}{c}\text { Total } \\
\text { G values } \\
\mathrm{G}_{\text {Tor }}{ }^{\mathrm{B} / \gamma} \\
\left(\mathrm{H}_{2 /} / 100 \mathrm{eV}\right)\end{array}$ & $\begin{array}{c}\text { Total } \\
G_{\text {values }} \\
G_{\text {Tot }}{ }^{\text {alpha }} \\
\left(\mathrm{H}_{2} / 100 \mathrm{eV}\right)\end{array}$ \\
\hline $241-T-203$ & 186.3 & $1.66 \mathrm{E}+05$ & $90 \%$ & 1290 & 4.62E-08 & $8.97 \mathrm{E}-06$ & 1.28 & 0.78 & 1.55 & 1.00 & 0.26 & 0.42 & 0.20 & 0.062 & 0.230 \\
\hline Supematant & na & na & na & na & na & ña & na & na & na & na & na & na & 0.20 & na & na \\
\hline Solid layer & 186.3 & $1.66 \mathrm{E}+05$ & $90 \%$ & 1290 & 4.62E- 08 & $8.97 \mathrm{E}-06$ & 1.28 & 0.78 & 1.55 & 1.00 & 0.26 & 0.42 & 0.20 & 0.062 & 0.230 \\
\hline $241-T-204$ & 186.3 & $1.60 \mathrm{E}+05$ & $100 \%$ & 1290 & $5.94 \mathrm{E}-08$ & $7.33 \mathrm{E}-06$ & 1.28 & 0.78 & 1.55 & 1.00 & 0.26 & 0.42 & 0.20 & 0.062 & 0.230 \\
\hline Supenratant & na & na & na & na & na & na & na & na & na & na & na & na & 0.20 & na & na \\
\hline Solid Layer & 186.3 & $1.60 \mathrm{E}+05$ & $100 \%$ & 1290 & $5.94 \mathrm{E}-08$ & $7.33 \mathrm{E}-06$ & 1.28 & 0.78 & 1.55 & 1.00 & 0.26 & 0.42 & 0.20 & 0.062 & 0.230 \\
\hline 24I-TX-101 & 40.5 & $5.99 \mathrm{E}+0.5$ & $80 \%$ & 5213 & $2.48 \mathrm{E}-03$ & $3.63 \mathrm{E}-05$ & 3.06 & 2.65 & 4.54 & 1.00 & 0.28 & 2.61 & 0.20 & 0.022 & 0.106 \\
\hline Supematant & na & na & na & nа & па & na & na & na & па & na & na & na & 0.20 & na & na \\
\hline Solid Layet & 40.5 & $5.99 \mathrm{E}+05$ & $80 \%$ & 5213 & $2.48 \mathrm{E}-03$ & $3.63 \mathrm{E}-05$ & 3.06 & 2.65 & 4.54 & 1.00 & 0.28 & 2.61 & 0.20 & 0.022 & 0.106 \\
\hline $24 !-T \times-102$ & 86.3 & $1.33 \mathrm{E}+06$ & $22 \%$ & 6113 & $4.24 E-04$ & $7.51 \mathrm{E}-06$ & 3.07 & 2.66 & 4.55 & 1.00 & 0.28 & 2.60 & 0.20 & 0.023 & 0.106 \\
\hline Supematant & นа & na & na & na & na & na & na & na & na & na & na & na & 0.20 & na & 11a \\
\hline Solid Layer & 86.3 & $1.33 \mathrm{E}+06$ & $22 \%$ & 6113 & $4.24 \mathrm{E}-04$ & $7.51 \mathrm{E}-06$ & 3.07 & 2.66 & 4.55 & 1.00 & 0.28 & 2.60 & 0.20 & 0.023 & 0.106 \\
\hline $241-\mathrm{TX}-103$ & 60.1 & $8.83 E+05$ & $25 \%$ & 5598 & $4.18 E-04$ & $7.42 \mathrm{E}-06$ & 3.08 & 2.56 & 4.45 & 1.00 & 0.27 & 2.53 & 0.20 & 0.022 & 0.106 \\
\hline Supenaatant & na & na & na & na & na & na & na & na & na & na & па & na & 0.20 & na & na \\
\hline Solid Layer & 60.1 & $8.83 E+05$ & $25 \%$ & 5598 & 4. $18 \mathrm{E}-04$ & $7.42 \mathrm{E}-06$ & 3.08 & 2.56 & 4.45 & 1.00 & 0.27 & 2.53 & 0.20 & 0.022 & 0.106 \\
\hline $241-\mathrm{TX}-104$ & 32.6 & $4.53 \mathrm{E}+05$ & $88 \%$ & 5058 & $1.40 \mathrm{E}-03$ & $9.00 \mathrm{E}-06$ & 5.70 & 2.05 & 1.16 & 1.00 & 0.09 & 0.22 & 0.20 & 0.020 & 0.066 \\
\hline Supeniatant & 0.9 & $1.30 \mathrm{E}+04$ & $100 \%$ & 17 & $1.10 \mathrm{E}-03$ & $3.06 \mathrm{E}-07$ & 5.72 & 2.05 & 1.17 & 1.00 & 0.09 & 0.22 & 0.20 & 0.020 & 0.066 \\
\hline Solid Layer & 31.8 & $4.40 \mathrm{E}+05$ & $88 \%$ & 5041 & $1.41 \mathrm{E}-03$ & $9.25 \mathrm{E}-06$ & 5.70 & 2.05 & 1.16 & 1.00 & 0.09 & 0.22 & 0.20 & 0.020 & 0.066 \\
\hline $241-\mathrm{TX}-105$ & 217.0 & $3.56 \mathrm{E}+06$ & $7 \%$ & 8678 & $3.49 \mathrm{E}-04$ & $8.27 \mathrm{E}-06$ & 3.06 & 2.65 & 4.55 & 1.00 & 0.28 & 2.61 & 0.20 & 0.024 & 0.107 \\
\hline Supernatant & na & na & na & na & na & na & na & na & na & na & na & na & 0.20 & na & na \\
\hline Solid Layer & 217.0 & $3.56 \mathrm{E}+06$ & $7 \%$ & 8678 & $3.49 \mathrm{E}-04$ & $8.27 \mathrm{E}-06$ & 3.06 & 2.65 & 4.55 & 1.00 & 0.28 & 2.61 & 0.20 & 0.024 & 0.107 \\
\hline 241-TX-106 & 134.1 & $2.14 \mathrm{E}+06$ & $28 \%$ & 7050 & $4.32 \mathrm{E}-04$ & $8.07 E-06$ & 3.07 & 2.65 & 4.56 & 1.00 & 0.28 & 2.61 & 0.20 & 0.023 & 0.106 \\
\hline Supernatant & na & na & na & na & na & ina & na & na & na & na & na & na & 0.20 & na & na \\
\hline Solid Layer & 134.1 & $2.14 \mathrm{E}+06$ & $28 \%$ & 7050 & $4.32 \mathrm{E}-04$ & $8.07 \mathrm{E}-06$ & 3.07 & 2.65 & 4.56 & 1.00 & 0.28 & 2.61 & 0.20 & 0.023 & 0.106 \\
\hline $241-T X-107$ & 18.2 & $2.00 \mathrm{E}+05$ & $56 \%$ & 4775 & $5.18 \mathrm{E}-04$ & $8.76 \mathrm{E}-05$ & 3.18 & 2.52 & 4.73 & 1.00 & 0.22 & 2.75 & 0.20 & 0.021 & 0.104 \\
\hline Supernatant & па & na & na & na & na & na & na & na & na & na & na & na & 0.20 & nat & na \\
\hline Solid Layer & 18.2 & $2.00 \mathrm{E}+05$ & $56 \%$ & 4775 & $5.18 \mathrm{E}-04$ & 8.76E-05 & 3.18 & 2.52 & 4.73 & 1.00 & 0.22 & 2.75 & 0.20 & 0.021 & 0.104 \\
\hline
\end{tabular}


RPP-5926 REV 6

Table B-2. Derived Data for Hydrogen Generation Rate Model Calculations for 177 Tanks. (20 sheets)

\begin{tabular}{|c|c|c|c|c|c|c|c|c|c|c|c|c|c|c|c|}
\hline Tank & $\begin{array}{l}\text { Waste } \\
\text { level } \\
\text { Dw } \\
\text { (inch) }\end{array}$ & $\begin{array}{c}\text { Total } \\
\text { mass } \\
\text { M } \\
(\mathrm{kg})\end{array}$ & $\begin{array}{c}\text { Liquid } \\
\text { in waste } \\
(w t \%)\end{array}$ & $\begin{array}{l}\text { Wetted } \\
\text { area } \\
\text { Awet } \\
\left(\mathrm{ft}^{2}\right)\end{array}$ & $\begin{array}{c}\text { Tank Heat } \\
\text { load } \\
{\mathbf{H}_{\mathrm{L}}{ }^{\text {pir }}}_{\text {(watt } / \mathrm{kg} \text { ) }}\end{array}$ & $\begin{array}{c}\text { Tank } \\
\text { heat load } \\
\mathrm{H}_{2}{ }^{\text {alpba }} \\
\text { (watt } / \mathrm{kg} \text { ) }\end{array}$ & $\begin{array}{c}\mathrm{NO}_{3} \\
\text { in liquid } \\
{\left[\mathrm{NO}_{3}\right]} \\
(\mathrm{mole} / \mathrm{L})\end{array}$ & $\begin{array}{c}\mathrm{NO}_{2} \\
\text { in liquid } \\
\left\{\mathrm{NO}_{2} \mid\right. \\
(\text { mole/L) }\end{array}$ & $\begin{array}{l}\text { Excess } \mathrm{Na} \\
\text { in liquid } \\
{\left[\left.\mathrm{Na}\right|_{\text {ex }}\right.} \\
(\text { mole } / \mathrm{L})\end{array}$ & $\begin{array}{c}\text { ОН } \\
\text { in liquid } \\
{[\mathrm{OH}]} \\
(\mathrm{mole} / \mathrm{L})\end{array}$ & $\begin{array}{c}\text { TOC } \\
\text { in liquid } \\
\text { [TOC|\%" } \\
\text { (wt } \%)\end{array}$ & $\begin{array}{c}\text { Al } \\
\text { in liquid } \\
\mid A] \mid \% \\
(w t \%)\end{array}$ & $\mid \begin{array}{c}E_{\mathrm{H}^{2}} \text { Efficiency } \\
\text { of } \mathrm{H}_{2} \text { by } \\
\text { corrosion }\end{array}$ & $\begin{array}{c}\text { Total } \\
\text { Givalues } \\
\text { G }_{\text {Tot }}{ }^{\mathrm{B} / \mathrm{r}} \\
\left(\mathrm{H}_{2 / 1} / 00 \mathrm{eV}\right)\end{array}$ & $\begin{array}{c}\text { Total } \\
\text { G values } \\
G_{\text {TOT }}^{\text {alpha }} \\
\left(\mathrm{H}_{2} / 100 \mathrm{eV}\right)\end{array}$ \\
\hline $241-T X-108$ & 53.4 & $7.75 E+05$ & $17 \%$ & 5466 & $3.90 \mathrm{E}-04$ & $7.75 \mathrm{E}-06$ & 3.06 & 2.65 & 4.54 & 1.00 & 0.28 & 2.60 & 0.20 & 0.022 & 0.106 \\
\hline Supematant & na & na & na & na & na & na & na & na & na & na & na & na & 0.20 & na & na \\
\hline Solid Layer & 53.4 & $7.75 \mathrm{E}+05$ & $17 \%$ & 5466 & $3.90 \mathrm{E}-04$ & $7.75 \mathrm{E}-06$ & 3.06 & 2.65 & 4.54 & 1.00 & 0.28 & 2.60 & 0.20 & 0.022 & 0.106 \\
\hline 241-TX-109 & 139.5 & $1.97 \mathrm{E}+06$ & $65 \%$ & 7158 & $1.07 \mathrm{E}-04$ & $3.29 \mathrm{E}-06$ & 1.28 & 0.78 & 1.55 & 1.00 & 0.26 & 0.42 & 0.20 & 0.065 & 0.232 \\
\hline Supematant & na & na & na & na & na & na & na & na & na & na & na & na & 0.20 & na & na \\
\hline Solid Layer & 139.5 & $1.97 E+06$ & $65 \%$ & 7158 & $1.07 \mathrm{E}-04$ & $3.29 \mathrm{E}-06$ & 1.28 & 0.78 & 1.55 & 1.00 & 0.26 & 0.42 & 0.20 & 0.065 & 0.232 \\
\hline $241-T X-110$ & 177.4 & $2.86 \mathrm{E}+06$ & $9 \%$ & 7901 & 3.15E-04 & $8.17 \mathrm{E}-06$ & 3.06 & 2.65 & 4.58 & 1.00 & 0.28 & 2.60 & 0.20 & 0.023 & 0.106 \\
\hline Supernatant & $\mathrm{na}$ & па & па & na & na & па & na & па & na & na & na & $n a$ & 0.20 & na & nà \\
\hline Solid Layer & 177.4 & $2.86 \mathrm{E}+06$ & $9 \%$ & 7901 & $3.15 \mathrm{E}-04$ & $8.17 \mathrm{E}-06$ & 3.06 & 2.65 & 4.58 & 1.00 & 0.28 & 2.60 & 0.20 & 0.023 & 0.106 \\
\hline $241-\mathrm{TX}-111$ & 140.1 & $2.23 \mathrm{E}+06$ & $11 \%$ & 7169 & $3.02 E-04$ & $8.07 \mathrm{E}-06$ & 3.06 & 2.65 & 4.54 & 1.00 & 0.28 & 2.61 & 0.20 & 0.023 & 0.106 \\
\hline Supematant & na & na & na & na & na & na & na & na & na & na & na & na & 0.20 & na & na \\
\hline Solid Layer & 140.1 & $2.23 \mathrm{E}+06$ & $11 \%$ & 7169 & $3.02 E-04$ & $8.07 \mathrm{E}-06$ & 3.06 & 2.65 & 4.54 & 1.00 & 0.28 & 2.61 & 0.20 & 0.023 & 0.106 \\
\hline $241-\mathrm{TX}-112$ & 237.8 & $3.92 E+06$ & $11 \%$ & 9087 & $3.27 \mathrm{E}-04$ & $8.19 \mathrm{E}-06$ & 3.13 & 2.30 & 4.07 & 2.83 & 0.24 & 2.29 & 0.20 & 0.023 & 0.106 \\
\hline Supernatant & na & na & na & na & na & na & na & na & na & na & na & na & 0.20 & na & na \\
\hline Solid Layer & 237.8 & $3.92 \mathrm{E}+06$ & $11 \%$ & 9087 & $3.27 \mathrm{E}-04$ & $8.19 \mathrm{E}-06$ & 3.13 & 2.30 & 4.07 & 2.83 & 0.24 & 2.29 & 0.20 & 0.023 & 0.106 \\
\hline 241-TX-113 & 239.5 & $3.88 \mathrm{E} \div 06$ & $5 \%$ & 9121 & $4.80 \mathrm{E}-05$ & $6.80 \mathrm{E}-07$ & 3.06 & 2.65 & 4.54 & 2.83 & 0.28 & 2.61 & 0.20 & 0.022 & 0.106 \\
\hline Supenatant & na & na & na & na & na & na & na & na & na & na & na & na & 0.20 & na & ina \\
\hline Solid Layer & 239.5 & $3.88 \mathrm{E}+06$ & $5 \%$ & 9121 & $4.80 \mathrm{E}-05$ & $6.80 \mathrm{E}-07$ & 3.06 & 2.65 & 4.54 & 2.83 & 0.28 & 2.61 & 0.20 & 0.022 & 0.106 \\
\hline 241-TX-114 & 200.7 & $3.29 E+06$ & $6 \%$ & 8359 & $2.86 \mathrm{E}-04$ & $7.80 \mathrm{E}-06$ & 3.33 & 1.12 & 2.48 & 1.00 & 0.11 & 1.09 & 0.20 & 0.029 & 0.109 \\
\hline Supernatant & nа & na & na & na & na & na & na & na & na & na & ná & na & 0.20 & na & na \\
\hline Solid Layer & 200.7 & $3.29 \mathrm{E}+06$ & $6 \%$ & 8359 & $2.86 \mathrm{E}-04$ & $7.80 \mathrm{E}-06$ & 3.33 & 1.12 & 2.48 & 1.00 & 0.11 & 1.09 & 0.20 & 0.029 & 0.109 \\
\hline $241-T X-115$ & 208.5 & $3.41 \mathrm{E}+06$ & $8 \%$ & 8512 & $3.52 \mathrm{E}-04$ & $8.29 \mathrm{E}-06$ & 3.07 & 2.66 & 4.53 & 2.83 & 0.28 & 2.60 & 0.20 & 0.022 & 0.106 \\
\hline Supernatant & na & na & na & na & na & na & na & na & na & na & na & na & 0.20 & na & na \\
\hline Solid Layer & 208.5 & $3.41 E+06$ & $8 \%$ & 8512 & $3.52 \mathrm{E}-04$ & $8.29 E-06$ & 3.07 & 2.66 & 4.53 & 2.83 & 0.28 & 2.60 & 0.20 & 0.022 & 0.106 \\
\hline $241-T X-116$ & 224.9 & $3.75 \mathrm{E}+06$ & $9 \%$ & 8835 & $1.68 \mathrm{E}-04$ & $4.21 \mathrm{E}-06$ & 6.11 & 0.23 & 0.24 & 0.15 & 0.02 & 0.00 & 0.20 & 0.021 & 0.067 \\
\hline Supernatant & na & na & na & na & na & na & na & na & na & na & па & na & 0.20 & na & na \\
\hline Solid Layer & 224.9 & $3.75 \mathrm{E}+06$ & $9 \%$ & 8835 & $1.68 \mathrm{E}-04$ & $4.21 \mathrm{E}-06$ & 6.11 & 0.23 & 0.24 & 0.15 & 0.02 & 0.00 & 0.20 & 0.021 & 0.067 \\
\hline
\end{tabular}


RPP-5926 REV 6

Table B-2. Derived Data for Hydrogen Generation Rate Model Calculations for 177 Tanks. (20 sheets)

\begin{tabular}{|c|c|c|c|c|c|c|c|c|c|c|c|c|c|c|c|}
\hline Tank & $\begin{array}{c}\text { Waste } \\
\text { level } \\
\text { Dw } \\
\text { (inch) }\end{array}$ & $\begin{array}{c}\text { Total } \\
\text { mass } \\
M \\
(\mathbf{k g})\end{array}$ & $\begin{array}{l}\text { Liquid } \\
\text { in waste } \\
\text { (wt"1\%) }\end{array}$ & $\begin{array}{l}\text { Wetted } \\
\text { area } \\
\text { Awet } \\
\left(\mathrm{ft}^{2}\right)\end{array}$ & $\begin{array}{c}\text { Tank Heat } \\
\text { load } \\
\mathrm{H}_{\mathbf{L}}{ }^{\mathrm{A} i r} \\
\text { (watt } / \mathbf{k g} \text { ) }\end{array}$ & $\begin{array}{c}\text { Tank } \\
\text { heat load } \\
\mathrm{H}_{\mathrm{L}}^{\text {alpha }} \\
\text { (watt } / \mathrm{kg} \text { ) }\end{array}$ & $\begin{array}{c}\mathrm{NO}_{3} \\
\text { in liquid } \\
{\left[\mathrm{NO}_{3}\right]} \\
\text { (mole } / \mathrm{L})\end{array}$ & $\begin{array}{c}\mathrm{NO}_{2} \\
\text { in liquid } \\
\left|\mathrm{NO}_{2}\right| \\
(\text { mole/L) }\end{array}$ & $\begin{array}{c}\text { Excess } \mathrm{Na} \\
\text { in liquid } \\
{\left[\left.\mathrm{Na}\right|_{\mathrm{ex}}\right.} \\
(\mathrm{mole} / \mathrm{L})\end{array}$ & $\begin{array}{c}\text { Он } \\
\text { in liquid } \\
{[\mathrm{OH} \mid} \\
\text { (mole/L) }\end{array}$ & $\begin{array}{c}\text { Toc } \\
\text { in liquid } \\
\text { (ToC)\% } \\
\text { (wt\%) }\end{array}$ & $\begin{array}{c}\text { Al } \\
\text { in liquid } \\
{[\text { Al] \% }} \\
(w t \%)\end{array}$ & $\mid \begin{array}{c}E_{\mathrm{H} 2} \text { Efticiency } \\
\text { of } \mathrm{H}_{2} \text { by } \\
\text { corrosion }\end{array}$ & 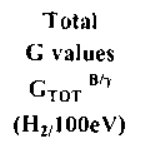 & $\begin{array}{c}\text { Total } \\
\text { G values } \\
\mathrm{G}_{\mathrm{TOT}}{ }^{\text {alpha }} \\
\left(\mathrm{H}_{2} / 100 \mathrm{eV}\right)\end{array}$ \\
\hline 241-TX-117 & 182.0 & $2.87 \mathrm{E}+06$ & $5 \%$ & 7991 & $1.87 E-04$ & $5.65 \mathrm{E}-06$ & 3.49 & 0.18 & 1.23 & 1.00 & 0.00 & 0.03 & 0.20 & 0.032 & 0.117 \\
\hline Supernatant & na & ma & na & na & na & na & na & na & na & $n a$ & na & na & 0.20 & na & na \\
\hline Solid Layer & 182.0 & $2.87 \mathrm{E}+06$ & $5 \%$ & 7991 & $1.87 \mathrm{E}-04$ & $5.65 \mathrm{E}-06$ & 3.49 & 0.18 & 1.23 & 1.00 & 0.00 & 0.03 & 0.20 & 0.032 & 0.111 \\
\hline 241-TX-118 & 97.3 & $1.58 \mathrm{E}+06$ & $33 \%$ & 6328 & $1.06 \mathrm{E}-03$ & $2.08 \mathrm{E}-04$ & 3.06 & 2.66 & 4.54 & 2.83 & 0.28 & 2.60 & 0.20 & 0.022 & 0.106 \\
\hline Supernatant & na & na & na & nа & na & na & na & na & na & na & na & na & 0.20 & ma & na \\
\hline Solid Layer & 97.3 & $1.58 \mathrm{E}+06$ & $33 \%$ & 6328 & $1.06 \mathrm{E}-03$ & $2.08 \mathrm{E}-04$ & 3.06 & 2.66 & 4.54 & 2.83 & 0.28 & 2.60 & 0.20 & 0.022 & 0.106 \\
\hline 241-TY-101 & 50.4 & $7.27 \mathrm{E}+05$ & $14 \%$ & 5407 & $6.57 \mathrm{E}-05$ & $6.12 \mathrm{E}-06$ & 3.49 & 0.18 & 1.22 & 1.00 & 0.00 & 0.03 & 0.20 & 0.032 & 0.111 \\
\hline Supematant & na & na & na & na & na & na & na & na & na & na & na & ва & 0.20 & na & na \\
\hline Solid Layer & 50.4 & $7.27 \mathrm{E}+05$ & $14 \%$ & 5407 & $6.57 \mathrm{E}-0.5$ & $6.32 \mathrm{E}-06$ & 3.49 & 0.18 & 1.22 & 1.00 & 0.00 & 0.03 & 0.20 & 0.032 & 0.111 \\
\hline 241 -TY-102 & 32.6 & $4.60 \mathrm{E}+05$ & $55 \%$ & 5058 & $1.53 \mathrm{E}-04$ & $1.29 \mathrm{E}-07$ & 3.26 & 1.52 & 3.02 & 2.84 & 0.16 & 1.52 & 0.20 & 0.026 & 0.108 \\
\hline Supernalant & na & na & 11a & na & na & na & па & na & na & na & na & na & 0.20 & na & na \\
\hline Solid Layer & 32.6 & $4.60 \mathrm{E}+05$ & $55 \%$ & 5058 & $1.53 \mathrm{E}-04$ & $1.29 \mathrm{E}-07$ & 3.26 & 1.52 & 3.02 & 2.84 & 0.16 & 1.52 & 0.20 & 0.026 & 0.108 \\
\hline $241-T Y-103$ & 63.7 & $9.83 \mathrm{E}+05$ & $91 \%$ & 5668 & $6.00 \mathrm{E}-04$ & $6.35 \mathrm{E}-06$ & 3.06 & 2.65 & 4.52 & 2.82 & 0.28 & 2.61 & 0.20 & 0.022 & 0.106 \\
\hline Supenatant & na & на & na & na & na & na & na & na & na & na & na & na & 0.20 & na & na \\
\hline Solid Layer & 63.7 & $9.83 \mathrm{E}+05$ & $91 \%$ & 5668 & $6.00 \mathrm{E}-04$ & $6.35 \mathrm{E}-06$ & 3.06 & 2.65 & 4.52 & 2.82 & 0.28 & 2.61 & 0.20 & 0.022 & 0.106 \\
\hline 241-TY-104 & 23.6 & $2.75 \mathrm{E}+05$ & $68 \%$ & 4881 & $7.31 \mathrm{E}-04$ & $8.13 \mathrm{E}-06$ & 1.60 & 0.25 & 1.78 & 1.00 & 0.18 & 0.05 & 0.20 & 0.055 & 0.211 \\
\hline Supenatant & 0.5 & $5.90 \mathrm{E}+03$ & $100 \%$ & 9 & 4. $63 \mathrm{E}-05$ & $5.59 \mathrm{E}-08$ & 1.60 & 0.25 & 1.78 & 1.00 & 0.18 & 0.05 & 0.20 & 0.055 & 0.211 \\
\hline Solid Layer & 23.1 & $2.69 \mathrm{E}+05$ & $67 \%$ & 4872 & $7.46 \mathrm{E}-04$ & $8.31 \mathrm{E}-06$ & 1.60 & 0.25 & 1.78 & 1.00 & 0.18 & 0.05 & 0.20 & 0.055 & 0.211 \\
\hline $24 !-T Y-105$ & 91.4 & $1.34 \mathrm{E}+06$ & $39 \%$ & 6213 & $1.02 \mathrm{E}-03$ & $9.70 \mathrm{E}-07$ & 1.28 & 0.78 & 1.55 & 1.00 & 0.26 & 0.42 & 0.20 & 0.063 & 0.231 \\
\hline Supernatant & na & na & na & na & па & na & na & na & na & na & na & na & 0.20 & na & na \\
\hline Solid Layer & 91.4 & $1.34 \mathrm{E}+06$ & $39 \%$ & 6213 & $1.02 \mathrm{E}-03$ & $9.70 \mathrm{E}-07$ & 1.28 & 0.78 & 1.55 & 1.00 & 0.26 & 0.42 & 0.20 & 0.063 & 0.231 \\
\hline $24 !-T Y-106$ & 13.4 & $8.68 \mathrm{E}+04$ & $51 \%$ & 4681 & $1.08 \mathrm{E}-03$ & $1.19 \mathrm{E}-06$ & 1.28 & 0.78 & 1.55 & 1.00 & 0.26 & 0.42 & 0.20 & 0.062 & 0.230 \\
\hline Supernatant & na & na & na & na & na & na & na & na & na & na & na & na & 0.20 & na & na: \\
\hline Solid Layet & 13.4 & $8.68 E+04$ & $51 \%$ & 4681 & $1.08 \mathrm{E}-03$ & $1.19 \mathrm{E}-06$ & 1.28 & 0.78 & 1.55 & 1.00 & 0.26 & 0.42 & 0.20 & 0.062 & 0.230 \\
\hline 241-Li-101 & 15.8 & $1.54 \mathrm{E}+05$ & $39 \%$ & 4728 & $2.76 \mathrm{E}-03$ & $2.18 \mathrm{E}-06$ & 1.28 & 0.78 & 1.55 & 2.89 & 0.26 & 0.42 & 0.20 & 0.063 & 0.231 \\
\hline Supernatant & na & na & na & na & na & na & na & na & na & na & na & na & 0.20 & na & па \\
\hline Solid Layer & 15.8 & $1.54 \mathrm{E} \div 05$ & $39 \%$ & 4728 & $2.76 \mathrm{E}-03$ & $2.18 \mathrm{E}-06$ & 1.28 & 0.78 & 1.55 & 2.89 & 0.26 & 0.42 & 0.20 & 0.063 & 0.231 \\
\hline
\end{tabular}


RPP-5926 REV 6

Table B-2. Derived Data for Hydrogen Generation Rate Model Calculations for 177 Tanks. (20 sheets)

\begin{tabular}{|c|c|c|c|c|c|c|c|c|c|c|c|c|c|c|c|}
\hline Tank & $\begin{array}{c}\text { Waste } \\
\text { level } \\
\text { Dw } \\
\text { (inch) }\end{array}$ & $\begin{array}{c}\text { Total } \\
\text { mass } \\
M \\
(\mathrm{~kg})\end{array}$ & $\begin{array}{c}\text { Liquid } \\
\text { in waste } \\
\text { (wt\%) }\end{array}$ & $\begin{array}{l}\text { Wetted } \\
\text { area } \\
\text { Awet } \\
\left(\mathrm{ft}^{2}\right)\end{array}$ & $\begin{array}{c}\text { Tank Heat } \\
\text { load } \\
\mathrm{H}_{\mathrm{L}}{ }^{3 / r} \\
\text { (watt } / \mathrm{kg} \text { ) }\end{array}$ & $\begin{array}{c}\text { Tank } \\
\text { heat load } \\
H_{\mathrm{L}}^{\text {alphas }} \\
\text { (watt } / \mathrm{kg} \text { ) }\end{array}$ & $\begin{array}{c}\mathrm{NO}_{3} \\
\text { in liquid } \\
{\left[\mathrm{NO}_{3} \mid\right.} \\
(\text { mole } / \mathrm{L})\end{array}$ & $\begin{array}{c}\mathrm{NO}_{2} \\
\text { in liquid } \\
{\left[\mathrm{NO}_{2} \mid\right.} \\
\text { (mole/L) }\end{array}$ & $\begin{array}{c}\text { Excess Na } \\
\text { in liquid } \\
{[\mathrm{Na}]_{\mathrm{ex}}} \\
(\text { mole } / \mathrm{L})\end{array}$ & $\begin{array}{c}\text { OH } \\
\text { in liquid } \\
\text { |OH] } \\
\text { (mole } / \mathrm{L} \text { ) }\end{array}$ & $\begin{array}{c}\text { TOC } \\
\text { in liquid } \\
\text { |TOCl\% } \\
\text { (wt } \% \text { s) }\end{array}$ & $\begin{array}{c}\text { Al } \\
\text { in liquid } \\
|A| \mid \% \\
(w 1 \%)\end{array}$ & $\begin{array}{c}E_{\mathrm{H} 2} \text { Efficiency } \\
\text { of } \mathrm{H}_{2} \text { by } \\
\text { corrosion }\end{array}$ & $\begin{array}{c}\text { Total } \\
\text { G values } \\
\mathbf{G}_{\mathrm{TOT}}^{\mathrm{B} / \gamma} \\
\left(\mathrm{H}_{2 /} 100 \mathrm{eV}\right)\end{array}$ & $\begin{array}{c}\text { Total } \\
\text { G values } \\
\mathbf{G}_{\text {TOT }}{ }^{\text {alph }} \\
\left(\mathbf{H}_{2} / 100 \mathrm{eV}\right)\end{array}$ \\
\hline $24 !-6-102$ & 126.4 & $2.07 \mathrm{E}+06$ & $72 \%$ & 6899 & $1.23 \mathrm{E}-03$ & $8.14 \mathrm{E}-06$ & 3.20 & 3.18 & 3.32 & 1.72 & 0.76 & 2.34 & 0.20 & 0.031 & 0.102 \\
\hline Supernatant & 0.4 & $5.92 \mathrm{E}+03$ & $100 \%$ & 8 & $1.47 \mathrm{E}-03$ & $2.70 \mathrm{E}-07$ & 3.19 & 3.17 & 3.33 & 1.72 & 0.76 & 2.25 & 0.20 & 0.028 & 0.101 \\
\hline Solid Layer & 126.0 & $2.06 \mathrm{E}+06$ & $72 \%$ & 6892 & $1.23 \mathrm{E}-03$ & $8.16 \mathrm{E}-06$ & 3.20 & 3.18 & 3.32 & 1.72 & 0.76 & 2.24 & 0.20 & 0.031 & 0.102 \\
\hline $24 I-U-103$ & 159.0 & $2.40 \mathrm{E}+06$ & $79 \%$ & 7541 & $6.75 \mathrm{E}-04$ & 4.43E-06 & 2.45 & 2.98 & 4.58 & 1.81 & 0.86 & 2.84 & 0.20 & 0.031 & 0.124 \\
\hline Supermatant & 0.2 & $2.88 \mathrm{E}+03$ & $100 \%$ & 4 & $1.51 E-03$ & 4.32E-07 & 2.45 & 2.98 & 4.59 & 1.81 & 0.86 & 2.84 & 0.20 & 0.030 & 0.125 \\
\hline Solid Layer & 158.8 & $2.40 \mathrm{E}+06$ & $79 \%$ & 7537 & $6.74 \mathrm{E}-04$ & $4.44 \mathrm{E}-06$ & 2.45 & 2.98 & 4.58 & 1.81 & 0.86 & 2.84 & 0.20 & 0.031 & 0.124 \\
\hline $24 !-\mathrm{C}-104$ & 27.1 & $2.31 E+05$ & $77 \%$ & 4951 & $1.79 \mathrm{E}-05$ & $2.06 \mathrm{E}-07$ & 3.34 & 2.29 & 3.20 & 2.89 & 0.68 & 2.29 & 0.20 & 0.031 & 0.104 \\
\hline Supenatant & na & na & nа & na & na & na & na & na & na & ta & na & nat & 0.20 & na & na \\
\hline Solid Layer & 27.1 & $2.31 E+05$ & $77 \%$ & 4951 & $1.79 \mathrm{E}-05$ & $2.06 \mathrm{E}-07$ & 3.34 & 2.29 & 3.20 & 2.89 & 0.68 & 2.29 & 0.20 & 0.031 & 0.104 \\
\hline $241-\mathrm{U}-105$ & 135.8 & $3.23 E+06$ & $59 \%$ & 7084 & $7.24 \mathrm{E}-04$ & $2.17 \mathrm{E}-05$ & 2.92 & 2.34 & $4.6 !$ & 1.23 & 1.12 & 2.04 & 0.20 & 0.033 & 0.117 \\
\hline Supernatant & na & na & па & na & па & па & na & na & па & na & na & na & 0.20 & na & na \\
\hline Solid Layer & 135.8 & $2.23 \mathrm{E}-06$ & $59 \%$ & $7084^{\circ}$ & $7.24 \mathrm{E}-04$ & $2.17 \mathrm{E}-05$ & 2.92 & 2.34 & 4.61 & 1.23 & 1.12 & 2.04 & 0.20 & 0.033 & 0.117 \\
\hline $241-\mathrm{J}-106$ & 69.3 & $9.98 \mathrm{E}+05$ & $86 \%$ & 5779 & $1.08 \mathrm{E}-03$ & $2.99 \mathrm{E}-05$ & 4.01 & 2.15 & 3.53 & 0.30 & 2.52 & 0.90 & 0.20 & 0.045 & 0.100 \\
\hline Supernatant & 0.6 & $8.04 \mathrm{E}+03$ & $100 \%$ & 11 & $1.27 \mathrm{E}-03$ & $7.26 \mathrm{E}-06$ & 4.01 & 2.15 & 3.55 & 0.30 & 2.52 & 0.90 & 0.20 & 0.042 & 0.099 \\
\hline Solid Layer & 68.7 & $9.90 \mathrm{E}+05$ & $86 \%$ & 5768 & $1.08 \mathrm{E}-03$ & $3.01 \mathrm{E}-05$ & 4.01 & 2.15 & 3.53 & 0.30 & 2.52 & 0.90 & 0.20 & 0.045 & 0.100 \\
\hline $241-\mathrm{U}-107$ & 114.4 & $1.93 E+06$ & $66 \%$ & 6664 & $3.71 \mathrm{E}-04$ & $1.25 \mathrm{E}-05$ & 2.29 & 2.77 & 3.84 & 1.84 & 0.44 & 2.86 & 0.20 & 0.029 & 0.129 \\
\hline Supetratant & na & na & nа & na & na & nа & na & na & na & na & na & nu & 0.20 & na & na \\
\hline Solid Layer & 114.4 & $1.93 E+06$ & $66 \%$ & 6664 & $3.71 \mathrm{E}-04$ & $1.25 \mathrm{E}-05$ & 2.29 & 2.77 & 3.84 & 1.84 & 0.44 & 2.86 & 0.20 & 0.029 & 0.129 \\
\hline $241-i-108$ & 165.1 & $2.76 \mathrm{E}+06$ & $74 \%$ & 7659 & $6.32 \mathrm{E}-04$ & $3.61 \mathrm{E}-06$ & 2.97 & 2.91 & 4.03 & 2.89 & 0.51 & 2.18 & 0.20 & 0.028 & 0.109 \\
\hline Supernatant & па & na & na & na & na & na & na & na & na & na & na & na & 0.20 & na & na \\
\hline Solid Layer & 165.1 & $2.76 \mathrm{E}+06$ & $74 \%$ & 7659 & $6.32 \mathrm{E}-04$ & $3.61 \mathrm{E}-06$ & 2.97 & 2.91 & 4.03 & 2.89 & 0.51 & 2.18 & 0.20 & 0.028 & 0.109 \\
\hline $24 i-U-109$ & 153.3 & $2.23 \mathrm{E}+06$ & $57 \%$ & 7427 & $5.81 E-04$ & $1.20 \mathrm{E}-06$ & 2.98 & 2.78 & 4.15 & 2.60 & 0.44 & 1.99 & 0.20 & 0.027 & 0.109 \\
\hline Supernatant & na & na & na & na & na & na & na & na & na & na & na & na & 0.20 & па & na \\
\hline Solid Layer & 153.3 & $2.23 \mathrm{E}+06$ & $57 \%$ & 7427 & $5.81 \mathrm{E}-04$ & $1.20 \mathrm{E}-06$ & 2.98 & 2.78 & 4.15 & 2.60 & 0.44 & 1.99 & 0.20 & 0.027 & 0.109 \\
\hline 241-U.110 & 71.3 & $1.14 \mathrm{E}+06$ & $45 \%$ & 5819 & $1.17 \mathrm{E}-03$ & $9.07 \mathrm{E}-06$ & 1.28 & 0.78 & 1.55 & 2.89 & 0.26 & 0.42 & 0.20 & 0.063 & 0.231 \\
\hline Supernatant & na & na & na & na & па & па & na & па & па & na & na & na & 0.20 & na & па \\
\hline Solid Layer & 71.3 & $1.14 \mathrm{E}+06$ & $45 \%$ & 5819 & $1.17 \mathrm{E}-03$ & $9.07 \mathrm{E}-06$ & 1.28 & 0.78 & 1.55 & 2.89 & 0.26 & 0.42 & 0.20 & 0.063 & 0.231 \\
\hline
\end{tabular}


RPP-5926 REV 6

Table B-2. Derived Data for Hydrogen Generation Rate Model Calculations for 177 Tanks. (20 sheets)

\begin{tabular}{|c|c|c|c|c|c|c|c|c|c|c|c|c|c|c|c|}
\hline Tank & $\begin{array}{l}\text { Waste } \\
\text { level } \\
\text { Dw } \\
\text { (inch) }\end{array}$ & $\begin{array}{c}\text { Total } \\
\text { mass } \\
M \\
(\mathrm{~kg})\end{array}$ & $\begin{array}{c}\text { Liquid } \\
\text { in waste } \\
\text { (wt\%) }\end{array}$ & $\begin{array}{l}\text { Wetted } \\
\text { area } \\
\text { Awet } \\
\left(\mathrm{ft}^{2}\right)\end{array}$ & $\begin{array}{c}\text { Tank Heat } \\
\text { load } \\
\mathbf{H}_{\mathrm{L}}{ }^{\beta / \mathrm{r}} \\
\text { (watt/kg) }\end{array}$ & $\begin{array}{c}\text { Tank } \\
\text { heat load } \\
\mathrm{H}_{\mathrm{L}}^{\text {alpha }} \\
\text { (watt } / \mathrm{kg} \text { ) }\end{array}$ & $\begin{array}{c}\mathrm{NO}_{3} \\
\text { in liquid } \\
{\left[\mathrm{NO}_{3}\right]} \\
(\mathrm{mole} / \mathrm{L})\end{array}$ & $\begin{array}{c}\mathrm{NO}_{2} \\
\text { in liquid } \\
{\left[\mathrm{NO}_{2}\right\rfloor} \\
(\text { mole } / \mathrm{L})\end{array}$ & $\begin{array}{c}\text { Excess } \mathrm{Na} \\
\text { in liquid } \\
{[\mathrm{Na}]_{e x}} \\
(\mathrm{~mole} / \mathrm{L})\end{array}$ & $\begin{array}{c}\mathrm{OH} \\
\text { in liquid } \\
\text { lOH } \\
(\mathrm{mole} / \mathrm{L})\end{array}$ & $\begin{array}{c}\text { TOC } \\
\text { in liquid } \\
\text { |roC]\% } \\
\text { (wt \%) }\end{array}$ & $\begin{array}{c}\text { Al } \\
\text { in liquid } \\
\text { |A|j\% } \\
(w t \%)\end{array}$ & $\mid \begin{array}{c}E_{\mathrm{H} 2} \text { Efficiency } \\
\text { of } \mathrm{H}_{2} \text { by } \\
\text { corrosion }\end{array}$ & $\begin{array}{c}\text { Total } \\
\text { G values } \\
\mathrm{G}_{\text {TOT }}^{\mathrm{B} / \gamma} \\
\left(\mathrm{H}_{2 /} 100 \mathrm{c} \mathrm{V}\right)\end{array}$ & $\begin{array}{c}\text { Total } \\
\text { G values } \\
\mathbf{G}_{\text {TOT }}{ }^{\text {alpha }} \\
\left(\mathrm{H}_{2} / 100 \mathrm{eV}\right)\end{array}$ \\
\hline 241-U-111 & 88.1 & $1.35 \mathrm{E}+06$ & $69 \%$ & 6149 & $8.55 \mathrm{E}-04$ & $7.21 \mathrm{E}-06$ & 2.69 & 2.61 & 5.09 & 1.99 & 0.39 & 2.62 & 0.20 & 0.022 & 0.117 \\
\hline Supematant & na & na & na & na & na & na & na & na & na & na & na & na & 0.20 & na & na \\
\hline Solid Layer & 88.1 & $1.35 \mathrm{E}+06$ & $69 \%$ & 6149 & $8.55 \mathrm{E}-04$ & $7.21 \mathrm{E}-06$ & 2.69 & 2.61 & 5.09 & 1.99 & 0.39 & 2.62 & 0.20 & 0.022 & 0.117 \\
\hline $241-\mathrm{E}-112$ & 24.0 & $3.00 \mathrm{E}+05$ & $48 \%$ & 4889 & $1.24 \mathrm{E}-03$ & $7.62 \mathrm{E}-07$ & 1.28 & 0.78 & 1.55 & 2.89 & 0.26 & 0.42 & 0.20 & 0.063 & 0.230 \\
\hline Supernatant & na & na & na & na & na & na & na & na & na & na & na & na & 0.20 & na & na \\
\hline Solid Layer & 24.0 & $3.00 \mathrm{E}+05$ & $48 \%$ & 4889 & $1.24 \mathrm{E}-03$ & $7.62 \mathrm{E}-07$ & 1.28 & 0.78 & 1.55 & 2.89 & 0.26 & 0.42 & 0.20 & 0.063 & 0.230 \\
\hline $241-U-201$ & 23.2 & $2.30 \mathrm{E}+04$ & $52 \%$ & 436 & $4.07 \mathrm{E}-05$ & $9.72 \mathrm{E}-08$ & 2.30 & 0.45 & 2.28 & 0.74 & 0.00 & 0.01 & 0.20 & 0.038 & 0.154 \\
\hline Supenatant & 5.4 & $5.04 \Sigma+03$ & $100 \%$ & 28 & $1.02 E-04$ & $2.09 \mathrm{E}-08$ & 2.30 & 0.45 & 2.28 & 0.74 & 0.00 & 0.01 & 0.20 & 0.038 & 0.154 \\
\hline Solid Layer & 17.8 & $1.79 E+04$ & $39 \%$ & 407 & $2.33 \mathrm{E}-05$ & $1.19 \mathrm{E}-07$ & 2.30 & 0.45 & 2.28 & 0.74 & 0.00 & 0.01 & 0.20 & 0.038 & 0.154 \\
\hline $241-U-202$ & 21.9 & $2.02 \mathrm{E}+04$ & $55 \%$ & 429 & $3.32 \mathrm{E}-05$ & $1.18 \mathrm{E}-07$ & 2.45 & 0.53 & 2.21 & 1.05 & 0.01 & 0.02 & 0.20 & 0.037 & 0.146 \\
\hline Supernatant & 5.4 & $5.12 \mathrm{E}+03$ & $100 \%$ & 28 & $7.23 \mathrm{E}-05$ & $4.06 \mathrm{E}-10$ & 2.45 & 0,53 & 2.21 & 1.05 & 0.01 & 0.02 & 0.20 & 0.037 & 0.146 \\
\hline Solid Layer & 16.5 & $1.51 \mathrm{E}+04$ & $40 \%$ & 400 & $2.00 \mathrm{E}-05$ & $1.58 \mathrm{E}-07$ & 2.45 & 0.53 & 2.21 & 1.05 & 0.01 & 0.02 & 0.20 & 0.037 & 0.146 \\
\hline 24$]-U-203$ & 20.5 & $1.94 \mathrm{E}+04$ & $69 \%$ & 422 & $3.20 \mathrm{E}-05$ & $7.87 \mathrm{E}-08$ & 2.32 & 0.42 & 1.62 & 0.28 & 0.00 & 0.01 & 0.20 & 0.042 & 0.154 \\
\hline Supernatant & 5.4 & $5.12 \mathrm{E}+03$ & $100 \%$ & 28 & $6.78 \mathrm{E}-05$ & $9.45 \mathrm{E}-10$ & 2.32 & 0.42 & 1.62 & 0.28 & 0.00 & 0.01 & 0.20 & 0.042 & 0.154 \\
\hline Solid Layer & 15.1 & $1.43 E+04$ & $59 \%$ & 393 & $1.93 \mathrm{E}-05$ & $1.06 \mathrm{E}-07$ & 2.32 & 0.42 & 1.62 & 0.28 & 0.00 & 0.01 & 0.20 & 0.042 & 0.154 \\
\hline $24 !-U-204$ & 17,8 & $1.47 \mathrm{E}+04$ & $51 \%$ & 407 & $4.30 \mathrm{E}-05$ & $2.14 \mathrm{E}-06$ & 0.89 & 0.15 & 0.66 & 0.09 & 0.01 & 0.00 & 0.50 & 0.096 & 0.325 \\
\hline Supernatant & 5.4 & $4.44 \mathrm{E}+03$ & $100 \%$ & 28 & $2.90 \mathrm{E}-05$ & $4.09 \mathrm{E}-10$ & 0.89 & 0.15 & 0.66 & 0.09 & 0.01 & 0.00 & 0.50 & 0.096 & 0.325 \\
\hline Solid Layer & 12.4 & $1.03 E+04$ & $30 \%$ & 379 & $4.90 \mathrm{E}-05$ & $3.06 \mathrm{E}-06$ & 0.89 & 0.15 & 0.66 & 0.09 & 0.01 & 0.00 & 0.50 & 0.096 & 0.325 \\
\hline AY $102-S-14 U$ & 11.5 & $1.92 \mathrm{E}+05$ & $44 \%$ & 4644 & $8.98 \mathrm{E}+03$ & $2.55 \mathrm{E}-04$ & 0.01 & 0.39 & 3.36 & 0.09 & 0.12 & 0.08 & 0.50 & 0.073 & 0.087 \\
\hline AY102-S-14L & 8.5 & $1.40 \mathrm{E}+05$ & $50 \%$ & 4585 & $8.73 E+03$ & $2.55 \mathrm{E}-04$ & 0.01 & 0.28 & 3.46 & 0.05 & 0.12 & 0.05 & 0.50 & 0.073 & 0.097 \\
\hline AYlo2-S-15U & 3.5 & $5.65 \mathrm{E}+04$ & $44 \%$ & 4487 & $1.63 E+03$ & $2.55 \mathrm{E}-04$ & 0.00 & 0.19 & 3.14 & 0.01 & 0.12 & 0.02 & 0.50 & 0.088 & 0.112 \\
\hline AY'102-S-15]. & 12.0 & $2.00 \mathrm{E}+05$ & $51 \%$ & 4653 & $8.61 E+03$ & $2.55 \mathrm{E}-04$ & 0.00 & 0.08 & 2.91 & 0.02 & 0.15 & 0.02 & 0.50 & 0.110 & 0.141 \\
\hline$A Y-102-S-16$ & 10.5 & $1.53 \mathrm{~F}: 105$ & $50 \%$ & 4624 & $8.08 \mathrm{E}+03$ & $2.55 \mathrm{E}-04$ & 0.00 & 0.01 & 2.34 & 0.01 & 0.16 & 0.00 & 0.50 & 0.190 & 0.233 \\
\hline AY 102-Orig-9" & 9.0 & $1.35 \mathrm{E}+05$ & $51 \%$ & 4595 & $1.28 \mathrm{E}+04$ & $2.55 \mathrm{E}-04$ & 0.00 & 0.00 & 2.18 & 0.01 & 0.10 & 0.01 & 0.50 & 0.227 & 0.256 \\
\hline
\end{tabular}




\section{RPP-5926 REV 6}

Table B-3. Calculated Hydrogen Generation Rate as Mole Per Unit Waste Weight and Volume for 177 Tanks. (20 sheets)

\begin{tabular}{|c|c|c|c|c|c|c|c|c|c|c|}
\hline Tank & $\begin{array}{l}\text { HGR from } \\
\text { radiolysis } \\
\mathrm{RC}_{\mathrm{rad}}^{\mathrm{B} / \mathrm{\gamma}} \\
\text { (mole } / \mathrm{kg} \text {-d) }\end{array}$ & $\begin{array}{l}\text { HGR from } \\
\text { radiolysis } \\
\mathrm{RC}_{\text {rad }}^{\text {alpha }} \\
\text { (mole/kg-d) }\end{array}$ & $\begin{array}{l}\text { HGR from } \\
\text { thermolysis } \\
\mathrm{RC}_{\text {therm }} \\
\text { (mole/kg-d) }\end{array}$ & $\begin{array}{c}\text { HGR from } \\
\text { corrosion } \\
\mathrm{RC}_{\text {rorr }} \\
\text { (mole/kg-d) }\end{array}$ & $\begin{array}{c}\text { Total HGR } \\
\text { from model } \\
\mathbf{R C}_{101} \\
\text { (mole/kg-d) }\end{array}$ & $\begin{array}{l}\text { HGR from } \\
\text { radiolysis } \\
\mathrm{RC}_{\text {rad }} \text { b/r } \\
\left.\text { (mole/m } / \mathrm{m}^{3}-\mathrm{s}\right)\end{array}$ & $\begin{array}{l}\text { HGR from } \\
\text { radiolysis } \\
\mathrm{RC}_{\text {rad }} \text { alpha } \\
\left.\text { (mole/m } / \mathrm{m}^{3}-\mathrm{s}\right)\end{array}$ & $\begin{array}{l}\text { HGR from } \\
\text { thermolysis } \\
\text { RC }_{\text {tberm }} \\
\text { (mole/m } / \text { m }^{3} \text {-s) }\end{array}$ & $\begin{array}{l}\text { HGR from } \\
\text { corrosion } \\
\mathrm{RC}_{\text {rorr }} \\
\left.\text { (mole } / \mathrm{m}^{3}-\mathrm{s}\right)\end{array}$ & $\begin{array}{c}\text { Total HGR } \\
\text { from model } \\
\text { RCtot } \\
\left(\mathrm{mole} / \mathrm{m}^{3}-\mathrm{s}\right)\end{array}$ \\
\hline $241-\mathrm{AN}-101$ & $2.55 \mathrm{E}-07$ & $8.50 \mathrm{E}-11$ & $2.43 \mathrm{E}-07$ & $2.20 \mathrm{E}-07$ & $7.18 \mathrm{E}-07$ & 4.18E-09 & $1.39 \mathrm{E}-12$ & $3.98 \mathrm{E}-09$ & 3.60E-09 & $1.18 \mathrm{E}-08$ \\
\hline Supernatant & $2.58 \mathrm{E}-07$ & $2.61 E-11$ & $2.45 \mathrm{E}-07$ & $2.68 \mathrm{E}-08$ & $5.29 \mathrm{E}-07$ & 4.21E-09 & $4.26 \mathrm{E}-13$ & $3.99 \mathrm{E}-09$ & $4.37 \mathrm{E}-10$ & 8.64E-09 \\
\hline Solid Layer & $1.92 \mathrm{E}-07$ & $1.23 \mathrm{E}-09$ & $1.76 \mathrm{E}-07$ & $5.09 \mathrm{E}-07$ & $8.78 \mathrm{E}-07$ & 3.44E-09 & $2.20 \mathrm{E}-11$ & $3.16 \mathrm{E}-09$ & $9.12 \mathrm{E}-09$ & $1.57 \mathrm{E}-08$ \\
\hline $241-A N-102$ & $1.10 \mathrm{E}-06$ & $7.40 E-09$ & $1.66 \mathrm{E}-06$ & $2.09 \mathrm{E}-07$ & $2.98 \mathrm{E}-06$ & $1.82 \mathrm{E}-08$ & $1.22 \mathrm{E}-10$ & $2.74 \mathrm{E}-08$ & $3.45 \mathrm{E}-09$ & $4.92 \mathrm{E}-08$ \\
\hline Supematant & $1.07 \mathrm{E}-06$ & 4.43E-09 & 1. $69 \mathrm{E}-06$ & $2.68 \mathrm{E}-08$ & $2.79 \mathrm{E}-06$ & $1.75 \mathrm{E}-08$ & $7.24 \mathrm{E}-11$ & $2.75 \mathrm{E}-08$ & $4.37 \mathrm{E}-10$ & $4.55 \mathrm{E}-08$ \\
\hline Solid Layer & $1.26 \mathrm{E}-06$ & $2.25 E-08$ & $1.52 \mathrm{E}-06$ & 1.24E-07 & 2.93E-06 & $2.24 \mathrm{E}-08$ & $3.98 \mathrm{E}-10$ & $2.70 \mathrm{E}-08$ & $2.19 \mathrm{E}-09$ & $5.19 \mathrm{E}-08$ \\
\hline $241-\mathrm{AN}-103$ & 2.65E- -07 & $1.71 E-10$ & $4.56 \mathrm{E}-07$ & 2.07E-07 & $9.29 \mathrm{E}-07$ & 4.90E-09 & $3.16 \mathrm{E}-12$ & $8.42 \mathrm{E}-09$ & 3.83E-09 & $1.72 \mathrm{E}-08$ \\
\hline Supernatam & $4.17 E-07$ & $1.78 E-10$ & $5.37 \mathrm{E}-07$ & $2.55 \mathrm{E}-08$ & $9.80 \mathrm{E}-07$ & $7.15 \mathrm{E}-09$ & $3.05 \mathrm{E}-12$ & $9.20 \mathrm{E}-09$ & $4.37 \mathrm{E}-10$ & $1.68 \mathrm{E}-08$ \\
\hline Solid Layer & $1.51 \mathrm{E}-07$ & $1.60 \mathrm{E}-10$ & $3.84 E-07$ & $5.63 \mathrm{E}-08$ & $5.91 E-07$ & $3.00 E-09$ & $3.19 \mathrm{E}-12$ & 7.64E-09 & $1.12 \mathrm{E}-09$ & $1.18 \mathrm{E}-08$ \\
\hline 241-AN-104 & $3.50 \mathrm{E}-07$ & $3.07 E-10$ & $5.50 \mathrm{E}-07$ & $2.09 \mathrm{E}-07$ & $1.11 \mathrm{E}-06$ & $5.98 \mathrm{E}-09$ & $5.25 \mathrm{E}-12$ & $9.41 \mathrm{E}-09$ & $3.58 \mathrm{E}-09$ & $1,90 \mathrm{E}-08$ \\
\hline Supernatant & 3.6!E-07 & $1.01 \mathrm{E}-11$ & $4.91 \mathrm{E}-07$ & $2.70 \mathrm{E}-08$ & $8.79 \mathrm{E}-07$ & $5.85 \mathrm{E}-09$ & $1.64 \mathrm{E}-13$ & $7.96 \mathrm{E}-09$ & 4. $37 \mathrm{E}-10$ & $1.42 \mathrm{E}-08$ \\
\hline Solid Layer & $3.36 \mathrm{E}-07$ & $6.51 \mathrm{E}-10$ & $6.34 \mathrm{E}-07$ & $6.11 \mathrm{E}-08$ & $1.03 \mathrm{E}-06$ & $6.18 \mathrm{E}-09$ & 1.20E-11 & 1.17E- 08 & $1.12 E-0.9$ & $1.90 \mathrm{E}-08$ \\
\hline $241-\mathrm{AN}-105$ & $2.19 E-07$ & $3.42 E-10$ & $3.12 \mathrm{E}-07$ & $2.02 \mathrm{E}-07$ & $7.33 \mathrm{E}-07$ & $3.77 \mathrm{E}-09$ & $5.89 \mathrm{E}-12$ & $5.37 \mathrm{E}-09$ & $3.47 \mathrm{E}-09$ & $1.26 \mathrm{E}-08$ \\
\hline Supematant & $2.00 \mathrm{E}-07$ & $3.87 E-10$ & $2.90 \mathrm{E}-07$ & $2.66 \mathrm{E}-08$ & $5.18 \mathrm{E}-07$ & $3.29 \mathrm{E}-09$ & $6.36 \mathrm{E}-12$ & 4.77E- 09 & $4.37 E-10$ & $8.51 \mathrm{E}-09$ \\
\hline Solid Layer & $2.36 \mathrm{E}-07$ & $2.97 E-10$ & $3.34 \mathrm{E}-07$ & $5.47 \mathrm{E}-08$ & $6.25 \mathrm{E}-07$ & 4.29E- 09 & $5.40 \mathrm{E}-12$ & $6.07 \mathrm{E}-09$ & $9.94 \mathrm{E}-10$ & $1.14 \mathrm{E}-08$ \\
\hline $241-\mathrm{AN}-106$ & $4.30 \mathrm{E}-06$ & $1.36 \mathrm{E}-07$ & $3.85 \mathrm{E}-0.88$ & $2.90 \mathrm{E}-07$ & 4.77E-06 & $5.74 \mathrm{E}-08$ & $1.82 \mathrm{E}-09$ & $5.14 E-10$ & $3.87 \mathrm{E}-09$ & $6.36 \mathrm{E}-08$ \\
\hline Supematant & $2.49 \mathrm{E}-07$ & $5.04 \mathrm{E}-10$ & 3. $70 \mathrm{E}-08$ & 3.43E- 08 & $3.21 \mathrm{E}-07$ & $3.17 \mathrm{E}-09$ & $6.42 \mathrm{E}-12$ & $4.71 \mathrm{E}-10$ & $4.37 \mathrm{E}-10$ & $4.09 \mathrm{E}-09$ \\
\hline Solid Layer & $1.36 \mathrm{E}-05$ & $4.45 E-07$ & $4.18 \mathrm{E}-08$ & $1,41 E-07$ & 1.42E-05 & 2.19E-07 & $7.18 E-09$ & $6.74 \mathrm{E}-10$ & $2.28 \mathrm{E}-09$ & $2.29 \mathrm{E}-07$ \\
\hline 241-AN-107 & $1.23 \mathrm{E}-06$ & $2.59 \mathrm{E}-08$ & $9.73 \mathrm{E}-07$ & 2.05E-07 & $2.43 \mathrm{E}-06$ & $2.05 \mathrm{E}-08$ & 4.32E-10 & $1.62 \mathrm{E}-08$ & $3.4 ! E-09$ & $4.05 E-08$ \\
\hline Supenatant & $1.10 \mathrm{E}-06$ & $2.26 \mathrm{E}-08$ & $9.74 \mathrm{E}-07$ & $2.64 \mathrm{E}-08$ & $2.12 \mathrm{E}-06$ & $1.82 \mathrm{E}-08$ & $3.75 \mathrm{E}-10$ & $1.61 \mathrm{E}-08$ & $4.37 \mathrm{E}-10$ & $3.51 \mathrm{~F}-08$ \\
\hline Solid Layer & 1. $64 \mathrm{E}-06$ & $3.60 \mathrm{~F}-08$ & $9.59 \mathrm{E}-07$ & $9.41 \mathrm{E}-08$ & $2.73 \mathrm{E}-06$ & $2.81 \mathrm{E}-08$ & $6.17 \mathrm{E}-10$ & $1.64 \mathrm{E}-08$ & $1.61 \mathrm{E}-09$ & 4.68E-08 \\
\hline $24 \mid-A P-101$ & $1.81 E-07$ & $1.26 E-11$ & 3.85E- 08 & $2.26 \mathrm{E}-07$ & $4.45 \mathrm{E}-07$ & $2.72 \mathrm{E}-09$ & 1. $90 \mathrm{E}-13$ & $5.80 \mathrm{E}-10$ & 3.40E-09 & $6.70 \mathrm{E}-09$ \\
\hline Supenaatant & 1.81E-07 & $1.26 \mathrm{E}-11$ & 3.85E-08 & $4.52 E-08$ & $2.65 \mathrm{E}-07$ & $2.72 \mathrm{E}-09$ & $1.90 \mathrm{E}-13$ & $5.80 \mathrm{E}-10$ & $6.80 \mathrm{E}-10$ & $3.98 \mathrm{E}-09$ \\
\hline Solid I ayer & na & na & na & na & na & na & na & na & $\mathrm{na}$ & na \\
\hline 241-AP-102 & $2.20 \mathrm{E}-07$ & $5.25 \mathrm{E}-11$ & $2.77 \mathrm{E}-07$ & $2.12 E-07$ & $7.08 \mathrm{E}-07$ & $3.56 \mathrm{E}-09$ & $8.49 \mathrm{E}-13$ & 4.47E- -09 & 3.42E-09 & $1.15 \mathrm{E}-08$ \\
\hline Supernatant & $2.26 \mathrm{E}-07$ & $5.34 E-11$ & $2.86 \mathrm{E}-07$ & $2.71 \mathrm{E}-08$ & $5.40 \mathrm{E}-07$ & $3.64 E-09$ & $8.59 \mathrm{E}-13$ & 4.60E-09 & $4.37 \mathrm{E}-10$ & $8.68 \mathrm{E}-09$ \\
\hline Solid Layer & $5.30 \mathrm{E}-08$ & $2.29 E-11$ & $6.69 \mathrm{E}-08$ & $5.96 \mathrm{E}-07$ & $7.16 \mathrm{E}-07$ & $1.07 \mathrm{E}-09$ & 4.63E-13 & $1.36 \mathrm{E}-09$ & $1.21 \mathrm{E}-08$ & $1.45 \mathrm{E}-08$ \\
\hline
\end{tabular}




\section{RPP-5926 REV 6}

Table B-3. Calculated Hydrogen Generation Rate as Mole Per Unit Waste Weight and Volume for 177 Tanks. (20 sheets)

\begin{tabular}{|c|c|c|c|c|c|c|c|c|c|c|}
\hline Tank & $\begin{array}{l}\text { HGR from } \\
\text { radiolysis } \\
\text { RC }_{\text {rad }} \text { B/ } \\
\text { (mole/kg-d) }\end{array}$ & $\begin{array}{l}\text { HGR from } \\
\text { radiolysis } \\
R C_{r a d} \text { alpha } \\
\text { (mole/kg-d) }\end{array}$ & $\begin{array}{l}\text { HGR from } \\
\text { thermolysis } \\
\mathrm{RC}_{\text {therm }} \\
\text { (mole/kg-d) }\end{array}$ & $\begin{array}{c}\text { HGR from } \\
\text { corrosion } \\
\mathrm{RC}_{\text {rorr }} \\
\text { (mole/kg-d) }\end{array}$ & $\begin{array}{c}\text { Total HGR } \\
\text { from model } \\
\mathrm{RC}_{\text {tol }} \\
\text { (mole } / \mathrm{kg} \text {-d) }\end{array}$ & $\begin{array}{l}\text { HGR from } \\
\text { radiolysis } \\
\mathrm{RC}_{\text {rad }} \mathrm{a} / \mathrm{r} \\
\left(\mathrm{mole} / \mathrm{m}^{3}-\mathrm{s}\right)\end{array}$ & $\begin{array}{l}\text { HGR from } \\
\text { radiolysis } \\
\mathrm{RC}_{\text {rad }}{ }^{\text {alpha }} \\
\left.\text { (mole/m } \text { m }^{3} \times s\right)\end{array}$ & $\begin{array}{c}\text { HGR from } \\
\text { thermolysis } \\
\mathrm{RC}_{\text {therm }} \\
\left.\text { (mole } / \mathrm{m}^{3}-\mathrm{s}\right)\end{array}$ & $\begin{array}{l}\text { HGR from } \\
\text { corrosion } \\
\text { RC }_{\text {rorr }} \\
\left(\text { mole } / \mathrm{m}^{3}-\mathrm{s}\right)\end{array}$ & $\begin{array}{c}\text { Total HGR } \\
\text { from mode } \\
\text { RCtot } \\
\left(\text { mole } / \mathrm{m}^{3}-\mathrm{s}\right)\end{array}$ \\
\hline 241-AP-103 & $2.49 \mathrm{E}-07$ & $3.53 \mathrm{E}-10$ & $1.69 \mathrm{E}-07$ & $2.35 \mathrm{E}-07$ & $6.54 \mathrm{E}-07$ & $3.92 \mathrm{E}-09$ & $5.54 \mathrm{E}-12$ & $2.66 \mathrm{E}-09$ & $3.70 \mathrm{E}-09$ & $1.03 E-08$ \\
\hline Supematant & $2.53 \mathrm{E}-07$ & $3.65 \mathrm{E}-10$ & $1.69 \mathrm{E}-07$ & $2.80 \mathrm{E}-08$ & $4.51 \mathrm{E}-07$ & $3.96 \mathrm{E}-09$ & $5.70 \mathrm{E}-12$ & 2.64E-09 & 4.37E-10 & $7.04 \mathrm{E}-09$ \\
\hline Solid Layer & $1.24 \mathrm{E}-07$ & $3.84 \mathrm{E}-11$ & $1.76 \mathrm{E}-07$ & $6.81 \mathrm{E}-07$ & $9.81 \mathrm{E}-07$ & $2.42 \mathrm{E}-09$ & $7.47 \mathrm{E}-13$ & $3.42 \mathrm{E}-09$ & $1.32 \mathrm{E}-08$ & $1.91 \mathrm{E}-08$ \\
\hline $241-\mathrm{AP}-104$ & $2.85 \mathrm{E}-07$ & $1.29 \mathrm{E}-10$ & $9.77 \mathrm{E}-08$ & $2.31 \mathrm{E}-07$ & $6.14 E-07$ & 4. $23 \mathrm{E}-09$ & $1.91 \mathrm{E}-12$ & $1.45 \mathrm{E}-09$ & $3.42 \mathrm{E}-09$ & $9.09 \mathrm{E}-09$ \\
\hline Supernatant & $2.85 E-07$ & $1.29 E-10$ & $9.77 \mathrm{E}-08$ & $4.61 \mathrm{E}-08$ & 4.29E- -07 & $4.23 \mathrm{E}-09$ & $1.91 \mathrm{E}-12$ & $1.45 \mathrm{E}-09$ & $6.83 \mathrm{E}-10$ & 6. $36 \mathrm{E}-09$ \\
\hline Solid Layer & not exist & na & па & na & na & na & na & na & na & na \\
\hline 241-AP-105 & $2.01 \mathrm{E}-07$ & $8.60 E-11$ & $1.23 \mathrm{E}-07$ & $2.25 \mathrm{E}-07$ & $5.49 \mathrm{E}-07$ & 3.02E-09 & $1.29 \mathrm{E}-12$ & $1.85 E-09$ & $3.37 \mathrm{E}-09$ & $8.24 \mathrm{E}-09$ \\
\hline Supentatant & $1.85 \mathrm{E}-07$ & $4.32 \mathrm{E}-11$ & $1.46 \mathrm{E}-07$ & $2.97 \mathrm{E}-08$ & $3.60 \mathrm{E}-07$ & $2.72 \mathrm{E}-09$ & $6.36 \mathrm{E}-13$ & $2.14 \mathrm{E}-09$ & $4.37 \mathrm{E}-10$ & $5.30 \mathrm{E}-09$ \\
\hline Solid Layer & 2.75E-07 & $3.4 ! E-10$ & $2.34 \mathrm{E}-08$ & $1.87 \mathrm{E}-07$ & $4.85 \mathrm{E}-07$ & $5.12 \mathrm{E}-09$ & $6.35 \mathrm{E}-12$ & $4.36 \mathrm{E}-10$ & $3.48 \mathrm{E}-09$ & $9.04 \mathrm{E}-09$ \\
\hline 241-AP-106 & $3.56 \mathrm{E}-07$ & $4.13 \mathrm{E}-11$ & $1.19 \mathrm{E}-07$ & $2.41 \mathrm{E}-07$ & $7.15 \mathrm{E}-07$ & 4. $98 \mathrm{E}-09$ & $5.78 \mathrm{E}-13$ & $1.66 \mathrm{E}-09$ & 3.375 .09 & $1.00 \mathrm{E}-08$ \\
\hline Supenatailt & $3.56 \mathrm{E}-07$ & $4.13 \mathrm{E}-11$ & $1.19 \mathrm{E}-07$ & $4.82 \mathrm{E}-08$ & $5.22 \mathrm{E}-07$ & 4.98E-09 & $5.78 \mathrm{E}-13$ & $1.66 \mathrm{E}-09$ & $6.75 \mathrm{E}-10$ & $7.32 \mathrm{~F}-09$ \\
\hline Solid Layer & not exist & na & na & na & กa & $\mathrm{na}$ & na & na & na & na \\
\hline 241-AP-107 & $1.80 \mathrm{E}-07$ & $2.14 \mathrm{E}-11$ & $3.21 \mathrm{E}-08$ & $4.03 \mathrm{E}-07$ & $6.15 \mathrm{E}-07$ & $2.73 \mathrm{E}-09$ & $3.25 \mathrm{E}-13$ & $4.87 \mathrm{E}-10$ & $6.11 \mathrm{E}-09$ & $9.33 \mathrm{E}-09$ \\
\hline Supernatant & $1.80 \mathrm{E}-07$ & $2.14 \mathrm{E}-11$ & $3.21 \mathrm{E}-08$ & $8.05 \mathrm{E}-08$ & $2.93 \mathrm{E}-07$ & $2.73 \mathrm{E}-09$ & $3.25 \mathrm{E}-13$ & $4.87 \mathrm{E}-10$ & $1.22 E-09$ & 4.44E-09 \\
\hline Solid Layer & not exist & na & na & na & na & na & na & 11a & na & na \\
\hline $241-\mathrm{AP}-108$ & $1.62 \mathrm{E}-07$ & $1.80 \mathrm{E}-11$ & $3.27 \mathrm{E}-07$ & 2.11E-07 & $7.00 \mathrm{E}-07$ & $2.69 \mathrm{E}-09$ & $2.98 \mathrm{E}-13$ & $5.41 E-09$ & $3.51 \mathrm{E}-09$ & $1.16 \mathrm{E}-08$ \\
\hline Supernatant & $1.70 \mathrm{E}-07$ & $1.84 \mathrm{E}-11$ & 4.01E-07 & $2.66 \mathrm{E}-08$ & $5.97 \mathrm{E}-07$ & $2.79 \mathrm{E}-09$ & $3.02 \mathrm{E}-13$ & $6.59 \mathrm{E}-09$ & $4.37 \mathrm{E}-10$ & $9.81 \mathrm{E}-09$ \\
\hline Solid Layer & $1.25 \mathrm{E}-07$ & $1.56 \mathrm{E}-11$ & $8.14 \mathrm{E}-08$ & 1. $44 \mathrm{E}-07$ & $3.51 \mathrm{E}-07$ & $2.20 \mathrm{E}-09$ & $2.74 E-13$ & $1.43 \mathrm{E}-09$ & $2.54 \mathrm{E}-09$ & $6.17 \mathrm{E}-09$ \\
\hline $241-\mathrm{AW}-101$ & $2.71 \mathrm{E}-07$ & $1.27 \mathrm{E}-09$ & $2.81 \mathrm{E}-07$ & $1.98 \mathrm{E}-07$ & $7.51 \mathrm{E}-07$ & $4.74 \mathrm{E}-09$ & $2.22 \mathrm{E}-11$ & 4.91E-09 & $3.46 \mathrm{E}-09$ & $1.31 \mathrm{E}-08$ \\
\hline Supernatant & $2.67 \mathrm{E}-07$ & $1.83 \mathrm{E}-11$ & $2.67 E-07$ & $2.57 \mathrm{E}-08$ & $5.60 \mathrm{E}-07$ & $4.55 \mathrm{E}-09$ & $3.11 \mathrm{E}-13$ & $4.55 \mathrm{E}-09$ & 4. $37 \mathrm{E}-10$ & $9.53 \mathrm{E}-09$ \\
\hline Solid Layer & $2.79 \mathrm{E}-07$ & $3.57 E .09$ & $3.06 \mathrm{E}-07$ & $6.50 \mathrm{E}-08$ & $6.54 \mathrm{E}-07$ & $5.14 \mathrm{E}-09$ & $6.56 \mathrm{E}-11$ & 5.64E-09 & $1.20 \mathrm{E}-09$ & $1.20 \mathrm{E}-08$ \\
\hline $241-\mathrm{AW}-102$ & $2.22 \mathrm{E}-07$ & $1.61 \mathrm{E}-09$ & 5.63E- 08 & $3.13 \mathrm{E}-07$ & $5.92 \mathrm{E}-07$ & $3.13 \mathrm{E}-09$ & $2.28 \mathrm{E}-11$ & $7.95 \mathrm{E}-10$ & 4.42E- 09 & $8.37 \mathrm{E}-09$ \\
\hline Supernatant & $2.04 \mathrm{E}-07$ & $2.46 \mathrm{E}-11$ & $5.62 \mathrm{E}-08$ & $3.09 \mathrm{E}-08$ & $2.91 \mathrm{E}-07$ & $2.88 \mathrm{E}-09$ & $3.48 \mathrm{E}-13$ & $7.93 \mathrm{E}-10$ & 4. $37 \mathrm{E}-10$ & $4.11 \mathrm{E}-09$ \\
\hline Solid Layer & $8.69 \mathrm{E}-07$ & $6.39 \mathrm{E}-08$ & $5.07 \mathrm{E}-08$ & $2.71 \mathrm{E}-06$ & $3.69 \mathrm{E}-06$ & $1.33 \mathrm{E}-08$ & $9.76 \mathrm{E}-10$ & $7.75 \mathrm{E}-10$ & $4.14 \mathrm{E}-08$ & $5.64 \mathrm{E}-08$ \\
\hline $241-\mathrm{AW}-103$ & $1.25 \mathrm{E}-07$ & $5.67 \mathrm{E}-09$ & $5.05 \mathrm{E}-08$ & $2.25 \mathrm{E}-07$ & $4.06 \mathrm{E}-07$ & $1.90 \mathrm{E}-09$ & $8.63 \mathrm{E}-11$ & $7.68 \mathrm{E}-10$ & $3.42 \mathrm{E}-09$ & $6.18 \mathrm{E}-09$ \\
\hline Supernatam & $1.97 \mathrm{E}-07$ & $9.46 \mathrm{E}-12$ & $3.02 \mathrm{E}-08$ & $3.04 \mathrm{E}-08$ & $2.58 \mathrm{E}-07$ & $2.83 \mathrm{E}-09$ & $1.36 \mathrm{E}-13$ & 4. $34 \mathrm{E}-10$ & $4.37 \mathrm{E}-10$ & $3.70 \mathrm{E}-09$ \\
\hline Solid Layer & $4.84 \mathrm{E}-08$ & $1.08 \mathrm{E}-08$ & $9.15 \mathrm{E}-08$ & $7.27 \mathrm{E}-08$ & $2.23 \mathrm{E}-07$ & $8.35 \mathrm{E}-10$ & $1.86 \mathrm{E}-10$ & $1.58 \mathrm{E}-09$ & $1.25 \mathrm{E}-09$ & $3.85 \mathrm{E}-09$ \\
\hline
\end{tabular}


RPP-5926 REV 6

Table B-3. Calculated Hydrogen Generation Rate as Mole Per Unit Waste Weight and Volume for 177 Tanks. (20 sheets)

\begin{tabular}{|c|c|c|c|c|c|c|c|c|c|c|}
\hline Tank & $\begin{array}{l}\text { HGR from } \\
\text { radiolysis } \\
\text { RC }_{r 2 d} \text { B/r } \\
\text { (mole/kg-d) }\end{array}$ & $\begin{array}{l}\text { HGR from } \\
\text { radiolysis } \\
\mathrm{RC}_{\text {rat }} \text { apta } \\
\text { (mole/kg-d) }\end{array}$ & $\begin{array}{c}\text { HGR from } \\
\text { thermolysis } \\
\text { RC }_{\text {therm }} \\
\text { (mole } / \mathrm{kg}-\mathrm{d} \text { ) }\end{array}$ & $\begin{array}{l}\text { HGR from } \\
\text { corrosion } \\
\text { RC }_{\text {corr }} \\
\text { (mole/kg-d) }\end{array}$ & $\begin{array}{c}\text { Total HGR } \\
\text { from model } \\
\mathrm{RC}_{\text {tor }} \\
\text { (mole } \mathrm{kg} \text {-d) }\end{array}$ & $\begin{array}{l}\text { HGR from } \\
\text { radiolysis } \\
\mathrm{RC}_{\mathrm{rad}}{ }^{\mathrm{b} / \mathrm{r}} \\
\left(\mathrm{mole} / \mathrm{m}^{3}-\mathrm{s}\right)\end{array}$ & $\begin{array}{c}\text { HGR from } \\
\text { radiolysis } \\
\mathrm{RC}_{\mathrm{rad}}{ }^{\text {alpha }} \\
\left(\mathrm{mole} / \mathrm{m}^{3}-\mathrm{s}\right)\end{array}$ & $\begin{array}{l}\text { HGR from } \\
\text { thermolysis } \\
\mathrm{RC}_{\text {therm }} \\
\left(\text { mole }^{2} \mathbf{m}^{3} \cdot \mathrm{s}\right)\end{array}$ & $\begin{array}{l}\text { HGR from } \\
\text { corrosion } \\
\text { RC }_{\text {rorr }} \\
\left.\text { (mole/m }{ }^{3}-\mathrm{s}\right)\end{array}$ & $\begin{array}{c}\text { Total HGR } \\
\text { from mode } \\
\text { RCtot } \\
\left(\text { mole } / \mathrm{m}^{3}-\mathrm{s}\right)\end{array}$ \\
\hline $241-A W-104$ & $2.29 \mathrm{E}-07$ & $6.28 \mathrm{E}-09$ & $2.08 \mathrm{E}-07$ & $2.16 \mathrm{E}-07$ & $6.60 \mathrm{E}-07$ & $3.66 \mathrm{E}-09$ & $1.00 \mathrm{E}-10$ & $3.32 \mathrm{E}-0.9$ & $3.44 \mathrm{E}-09$ & $1.05 \mathrm{E}-08$ \\
\hline Supematant & $2.66 \mathrm{E}-07$ & $6.32 \mathrm{E}-11$ & $2.12 \mathrm{E}-07$ & $2.80 \mathrm{E}-08$ & $5.06 \mathrm{E}-07$ & 4.16E-09 & $9.87 \mathrm{E}-13$ & 3.31E-09 & $4.37 \mathrm{E}-10$ & $7.91 \mathrm{E}-09$ \\
\hline Solid Layer & $1.38 \mathrm{E}-07$ & $2.46 \mathrm{E}-08$ & 1. $88 \mathrm{E}-07$ & 9.61E-08 & 4.47E-07 & $2.38 \mathrm{E}-09$ & 4.23E-10 & 3.24E-09 & $1.65 \mathrm{E}-09$ & $7.69 \mathrm{E}-09$ \\
\hline $24 \mathrm{I}-\mathrm{AW}-105$ & $1.65 \mathrm{E}-07$ & $5.16 \mathrm{E}-08$ & $2.10 \mathrm{E}-09$ & $3.74 \mathrm{E}-07$ & $5.92 \mathrm{E}-07$ & $2.39 \mathrm{E}-09$ & $7.47 \mathrm{E}-10$ & $3.05 \mathrm{E}-11$ & $5.41 \mathrm{E}-09$ & $8.58 \mathrm{E}-09$ \\
\hline Supernatanı & $6.31 \mathrm{E}-08$ & $5.30 \mathrm{E}-11$ & $1.28 \mathrm{E}-09$ & $8.90 \mathrm{E}-08$ & $1.53 \mathrm{E}-07$ & $7.74 \mathrm{E}-10$ & $6.50 \mathrm{E}-13$ & $1.57 \mathrm{E}-11$ & $1.09 \mathrm{E}-09$ & $1.88 \mathrm{E}-09$ \\
\hline Solid Layer & $1.64 \mathrm{E}-07$ & $5.53 \mathrm{E}-08$ & $2.03 \mathrm{E}-09$ & 9.26E-08 & $3.14 \mathrm{E}-07$ & 2.59E-09 & $8.73 \mathrm{E}-10$ & $3.20 \mathrm{E}-11$ & $1.46 \mathrm{E}-09$ & $4.96 \mathrm{E}-09$ \\
\hline $241-A W-106$ & $2.71 \mathrm{E}-07$ & $6.15 \mathrm{E}-10$ & $8.87 \mathrm{E}-08$ & $2.18 \mathrm{E}-07$ & $5.78 \mathrm{E}-07$ & 4. $20 \mathrm{E}-09$ & $9.54 \mathrm{E}-12$ & $1.38 \mathrm{E}-09$ & $3.38 E-09$ & $8.96 \mathrm{E}-09$ \\
\hline Supcrnatant & $2.04 \mathrm{E}-07$ & $8.96 \mathrm{E}-11$ & $7.49 \mathrm{E}-08$ & 3.02E-08 & $3.09 \mathrm{E}-07$ & $2.95 \mathrm{E}-0.9$ & $1.30 \mathrm{E}-12$ & $1.08 \mathrm{E}-09$ & $4.37 \mathrm{E}-10$ & 4.47E-09 \\
\hline Solid Layer & $3.30 \mathrm{E}-07$ & $1.24 \mathrm{E}-09$ & $1.17 E-07$ & 7.47E- 08 & $5.23 \mathrm{E}-07$ & $6.14 \mathrm{E}-09$ & $2.31 \mathrm{E}-11$ & $2.18 \mathrm{E}-09$ & 1.39E-09 & $9.74 \mathrm{E}-09$ \\
\hline $241-\mathrm{AY}-101$ & $9.05 \mathrm{E}-06$ & $1.71 E-06$ & $4.896-07$ & 5.19E-07 & $1.18 \mathrm{E}-05$ & $1.46 \mathrm{E}-07$ & $2.76 \mathrm{E}-08$ & $7.90 E-09$ & $8.39 \mathrm{E}-09$ & $1.90 \mathrm{E}-07$ \\
\hline Supernatant & $1.11 \mathrm{E}-07$ & $3.01 \mathrm{E}-09$ & $4.03 E-07$ & 8.35E- 08 & $6.01 \mathrm{E}-07$ & 1.45E-09 & $3.93 \mathrm{E}-11$ & $5.27 \mathrm{E}-09$ & $1.09 \mathrm{E}-09$ & $7.86 \mathrm{E}-09$ \\
\hline Solid l.ayer & 1.20E-05 & $1.75 E-06$ & $3.00 \mathrm{E}-07$ & $3.85 \mathrm{E}-07$ & $1.44 \mathrm{E}-05$ & $2.33 \mathrm{E}-07$ & $3.40 \mathrm{E}-08$ & $5.86 \mathrm{E}-09$ & $7.51 \mathrm{E}-09$ & $2.81 \mathrm{E}-07$ \\
\hline $241-\mathrm{A} Y-102$ & $1.29 E-05$ & $3.21 \mathrm{E}-07$ & $4.88 \mathrm{E}-07$ & $2.54 \mathrm{E}-07$ & $1.40 \mathrm{E}-05$ & $1.82 \mathrm{E}-07$ & $4.51 \mathrm{E}-09$ & $6.87 \mathrm{E}-09$ & $3.58 \mathrm{E}-09$ & $1.97 \mathrm{E}-07$ \\
\hline Supernatant & 1.10E-07 & $4.01 \mathrm{E}-10$ & $3.89 \mathrm{E}-07$ & 8.20E-08 & $5.82 \mathrm{E}-07$ & $1.47 \mathrm{E}-09$ & $5.33 \mathrm{E}-12$ & $5.18 \mathrm{E}-09$ & $1.09 \mathrm{E}-09$ & $7.74 \mathrm{E}-09$ \\
\hline Solid Layer & 4.44E- -05 & $1.14 \mathrm{E}-06$ & $6.58 \mathrm{E}-07$ & $3.06 \mathrm{E}-07$ & 4.65E-05 & $8.11 E-07$ & $2.08 \mathrm{e}-08$ & 1.20E.08 & $5.58 \mathrm{E}-09$ & $8.49 \mathrm{E}-07$ \\
\hline $241-A Z-101$ & $7.92 \mathrm{E}-06$ & $4.98 \mathrm{E}-07$ & $1.52 \mathrm{E}-06$ & 2.61E-07 & $1.02 \mathrm{E}-05$ & $1.14 \mathrm{E}-07$ & 7.17E- 09 & $2.18 \mathrm{E}-0.8$ & $3.75 \mathrm{E}-09$ & $1.47 \mathrm{E}-07$ \\
\hline Supernatant & $3.42 \mathrm{E}-06$ & $1.32 \mathrm{E}-10$ & $1.41 \mathrm{E}-06$ & $3.09 \mathrm{E}-08$ & $4.87 \mathrm{E}-06$ & 4.83E- 08 & $1.87 \mathrm{E}-12$ & $2.00 \mathrm{E}-08$ & $4.37 \mathrm{E}-10$ & $6.87 \mathrm{E}-08$ \\
\hline Solid Layer & $3.66 \mathrm{E}-05$ & $3.27 \mathrm{E}-06$ & $2.70 \mathrm{E}-06$ & $3.03 \mathrm{E}-07$ & $4.28 E-05$ & $6.81 E-07$ & $6.10 \mathrm{E}-08$ & $5.03 \mathrm{E}-08$ & 5.64E-09 & $7.98 \mathrm{E}-07$ \\
\hline $241-A Z-102$ & $1.17 \mathrm{E}-05$ & $1.07 \mathrm{E}-06$ & $8.09 \mathrm{E}-07$ & $2.67 \mathrm{E}-07$ & $1.38 \mathrm{E}-05$ & $1.57 \mathrm{E}-07$ & $1.43 \mathrm{E}-08$ & $1.09 \mathrm{E}-08$ & $3.58 \mathrm{E}-09$ & $1.85 \mathrm{E}-07$ \\
\hline Supernatant & $4.84 \mathrm{E}-06$ & $1.41 E-09$ & $7.33 \mathrm{E}-07$ & $3.34 E-08$ & $5.61 \mathrm{E}-06$ & $6.33 \mathrm{E}-08$ & $1.85 E-11$ & $9.59 \mathrm{E}-09$ & $4.37 E-10$ & $7,34 \mathrm{E}-08$ \\
\hline Solid Layer & 4.28E-05 & $5.60 \mathrm{E}-06$ & $1.41 \mathrm{E}-06$ & 1.85E-07 & $5.00 \mathrm{E}-05$ & $6.98 \mathrm{E}-07$ & $9.14 \mathrm{E}-08$ & $2.30 \mathrm{E}-08$ & $3.02 \mathrm{E}-09$ & $8.16 \mathrm{E}-07$ \\
\hline $241-S Y-101$ & $1.20 E-07$ & 3.03E-09 & $2.01 \mathrm{E}-08$ & $2.24 \mathrm{E}-07$ & $3.67 \mathrm{E}-07$ & $1.85 \mathrm{E}-09$ & $4.67 \mathrm{E}-11$ & $3.09 \mathrm{E}-10$ & $3.45 \mathrm{E}-09$ & $5.66 \mathrm{E}-09$ \\
\hline Supernatant & $7.75 \mathrm{E}-08$ & $1.06 E-11$ & $1.81 \mathrm{E}-08$ & $2.95 \mathrm{E}-08$ & $1.25 \mathrm{E}-07$ & $1.15 \mathrm{E}-09$ & $1.57 \mathrm{E}-13$ & $2.68 \mathrm{E}-10$ & $4.37 \mathrm{E}-10$ & $1.85 \mathrm{E}-09$ \\
\hline Solid Layer & $2.13 E-07$ & $9.72 \mathrm{E}-09$ & $2.65 \mathrm{E}-08$ & 9.25E-08 & $3.41 \mathrm{E}-07$ & $3.74 \mathrm{E}-09$ & $1.71 \mathrm{E}-10$ & $4.66 \mathrm{E}-10$ & $1.63 \mathrm{E}-09$ & $6.00 \mathrm{E}-09$ \\
\hline 24 I-SY-102 & $1.44 \mathrm{E}-07$ & $2.09 E-07$ & $3.62 \mathrm{E}-08$ & $2.33 \mathrm{E}-07$ & $6.22 \mathrm{E}-07$ & $2.08 \mathrm{E}-09$ & 3.03E-09 & $5.24 \mathrm{E}-10$ & $3.38 \mathrm{E}-09$ & $9.01 E-09$ \\
\hline Supernatant & $5.63 \mathrm{E}-08$ & $3.49 \mathrm{E}-11$ & 1.95E- 08 & $3.14 \mathrm{E}-08$ & $1.07 \mathrm{E}-07$ & $7.82 \mathrm{E}-10$ & 4. $84 \mathrm{E}-13$ & $2.70 \mathrm{E}-10$ & $4.37 \mathrm{E}-10$ & $1.49 \mathrm{E}-09$ \\
\hline Solid Layer & $4.51 \mathrm{E}-07$ & $9.92 \mathrm{E}-07$ & $1.05 \mathrm{E}-07$ & $1.18 \mathrm{E}-07$ & 1.67E-06 & $8.15 \mathrm{E}-09$ & 1.79E- 08 & $1.90 \mathrm{E}-09$ & $2.13 \mathrm{E}-0.9$ & $3.01 \mathrm{E}-08$ \\
\hline
\end{tabular}

B-45 
RPP-5926 REV 6

Table B-3. Calculated Hydrogen Generation Rate as Mole Per Unit Waste Weight and Volume for 177 Tanks. (20 sheets)

\begin{tabular}{|c|c|c|c|c|c|c|c|c|c|c|}
\hline Tank & $\begin{array}{l}\text { HGR from } \\
\text { radiolysis } \\
\mathrm{RC}_{\mathrm{rad}}^{\mathrm{B} / \mathrm{\gamma}} \\
\text { (mole/kg-d) }\end{array}$ & $\begin{array}{l}\text { HGR from } \\
\text { radiolysis } \\
\text { RC } \text { ras }_{\text {alpha }} \\
\text { (mole } / \mathbf{k g} \text {-d) }\end{array}$ & $\begin{array}{l}\text { HGR from } \\
\text { thermolysis } \\
\text { RC }_{\text {therm }} \\
\text { (mole } / \mathrm{kg}-\mathrm{d})\end{array}$ & $\begin{array}{l}\text { HGR from } \\
\text { corrosion } \\
\text { RC }_{\text {torr }} \\
\text { (mole/kg-d) }\end{array}$ & $\begin{array}{l}\text { Total HGR } \\
\text { from model } \\
\mathbf{R C}_{\text {tol }} \\
\text { (mole/kg-d) }\end{array}$ & $\begin{array}{l}\text { HGR from } \\
\text { radiolysis } \\
\mathrm{RC}_{\mathrm{rad}} \mathrm{b}^{\mathrm{r}} \\
\left.\text { (mole/m } / \mathrm{m}^{3}-\mathrm{s}\right)\end{array}$ & $\begin{array}{l}\text { HGR from } \\
\text { radiolysis } \\
\mathrm{RC}_{\text {rad }} \text { alpha } \\
\left.\text { (mole/m } \mathbf{m}^{3} \cdot \mathrm{s}\right)\end{array}$ & $\begin{array}{l}\text { HGR from } \\
\text { thermolysis } \\
\text { RC }_{\text {therin }} \\
\text { (mole } / \mathrm{m}^{3}-\mathrm{s} \text { ) }\end{array}$ & 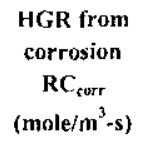 & $\begin{array}{l}\text { Total HGR } \\
\text { from mode } \\
\text { RCtot } \\
\left.\text { (mole } / \mathrm{m}^{3}-\mathrm{s}\right)\end{array}$ \\
\hline $241-5 Y-103$ & $4.28 \mathrm{E}-07$ & $1.06 \mathrm{E}-08$ & $7.41 \mathrm{E}-07$ & $2.34 \mathrm{E}-07$ & $1.4 ! E-06$ & $7.58 \mathrm{E}-09$ & $1.87 \mathrm{E}-10$ & $1.31 \mathrm{JE}-08$ & $4.15 \mathrm{E}-09$ & $2.51 E-08$ \\
\hline Supernatant & $4.31 \mathrm{E}-07$ & $2.34 \mathrm{E}-10$ & $4.18 \mathrm{E}-07$ & $2.57 \mathrm{E}-08$ & $8.76 \mathrm{E}-07$ & $7.34 \mathrm{E}-09$ & $3.99 \mathrm{E}-12$ & $7.12 \mathrm{E}-09$ & $4.37 E-10$ & 1.49E-08 \\
\hline Solid Layer & $4.31 \mathrm{E}-07$ & $2.10 \mathrm{E}-08$ & $1.37 \mathrm{E}-06$ & $7.09 \mathrm{E}-08$ & 1.90E-06 & $8.03 \mathrm{E}-09$ & $3.91 \mathrm{E}-10$ & $2.56 \mathrm{E}-08$ & 1.32E-09 & $3.54 E-08$ \\
\hline $241-\mathrm{A}-10 \mathrm{t}$ & $2.07 \mathrm{E}-07$ & $5.92 E-09$ & $7.17 \mathrm{E}-07$ & $3.05 \mathrm{E}-07$ & $1.23 \mathrm{E}-06$ & $4.07 \mathrm{E}-09$ & $1.16 \mathrm{E}-10$ & $1.41 \mathrm{E}-08$ & $6.00 \mathrm{E}-09$ & $2.43 \mathrm{E}-08$ \\
\hline Supenatant & na & na & na & na & na & na & na & na & па & na \\
\hline Solid Layer & $2.07 \mathrm{E}-07$ & $5.92 E-09$ & $7.17 E-07$ & 3.05E-07 & $1.23 E-06$ & 4.07E- 09 & $1.16 \mathrm{E}-10$ & $1.41 \mathrm{E}-08$ & $6.00 \mathrm{E}-09$ & $2.43 \mathrm{E}-08$ \\
\hline $241-A-102$ & $6.17 \mathrm{E}-07$ & $5.88 \mathrm{E}-08$ & $6.63 \mathrm{E}-08$ & $1.50 \mathrm{E}-06$ & $2.25 \mathrm{E}-06$ & $1.19 \mathrm{E}-08$ & 1.13E-09 & $1.27 \mathrm{E}-09$ & $2.89 E_{-}-08$ & $4.32 \mathrm{E}-08$ \\
\hline Supenlatant & $4.28 \mathrm{E}-07$ & $1.02 \mathrm{E}-10$ & $2.56 \mathrm{E}-07$ & $9.63 \mathrm{E}-08$ & $3.80 \mathrm{E}-07$ & $7.77 E-09$ & $1.86 \mathrm{E}-12$ & $4.64 \mathrm{E}-09$ & $1.75 \mathrm{E}-09$ & $1.42 \mathrm{E}-08$ \\
\hline Solid Layer & $6.26 \mathrm{E}-07$ & $6.23 \mathrm{E}-08$ & $5.91 \mathrm{E}-08$ & 1.62E-06 & $2.37 \mathrm{E}-06$ & 1.21E-08 & $1.20 \mathrm{E}-09$ & $1.14 \mathrm{E}-09$ & 3.13E-08 & 4.57E- 08 \\
\hline $241-A-103$ & $2.60 \mathrm{E}-07$ & $5.68 \mathrm{E}-09$ & $7.16 \mathrm{E}-07$ & $2.91 \mathrm{E}-07$ & $1.27 \mathrm{E}-06$ & $4.11 \mathrm{E}-09$ & $9.00 \mathrm{E}-1 \mathrm{I}$ & 1.13E-08 & $4.61 \mathrm{E}-09$ & $2.01 \mathrm{E}-08$ \\
\hline Supcrnatant & $2.45 \mathrm{E}-07$ & $5.25 \mathrm{E}-10$ & $8.68 \mathrm{E}-07$ & $1.00 \mathrm{E}-07$ & $1.21 \mathrm{E}-06$ & 4.28E-09 & $9.17 E-12$ & $1.52 E-08$ & $1.75 E-09$ & $2.12 \mathrm{E}-08$ \\
\hline Solid Layer & $2.60 \mathrm{E}-07$ & $5.74 \mathrm{E}-09$ & $7.14 \mathrm{E}-07$ & $2.94 \mathrm{E}-07$ & $1.27 \mathrm{E}-06$ & 4.11E-09 & $9.07 \mathrm{E}-11$ & 1.13E- 08 & 4.64E- 09 & $2.01 E-08$ \\
\hline $241-A \cdot 104$ & $0.00 \mathrm{E}+00$ & $0.00 E+00$ & $0.00 \mathrm{E}-00$ & 3.69E-06 & $3.69 \mathrm{E}-06$ & $0.00 \mathrm{E}+00$ & $0.00 \mathrm{E}+00$ & $0.00 \mathrm{E}+00$ & $4.05 \mathrm{E}-08$ & $4.05 \mathrm{E}-08$ \\
\hline Supernatant & na & na & па & na & na & na & na & na & na & $\mathrm{na}$ \\
\hline Solid Layer & $0.00 \mathrm{~F}+00$ & $0.00 \mathrm{t}+00$ & $0.00 \mathrm{E}+00$ & $3.69 \mathrm{E}-06$ & $3.69 \mathrm{E}-06$ & $0.00 \mathrm{E}-00$ & $0.00 \mathrm{E}+00$ & $0.00 \mathrm{E}+00$ & 4.05E-08 & $4.05 \mathrm{E}-08$ \\
\hline $241-A-105$ & $0.00 \mathrm{E}+00$ & $0.00 \mathrm{E}+00$ & $0.00 \mathrm{E}+00$ & $1.75 \mathrm{E}-06$ & $1.75 \mathrm{E}-06$ & $0.00 \mathrm{E}+00$ & $0.00 \mathrm{E}+00$ & $0.00 \mathrm{E}+00$ & 3.13E-08 & 3.13E-08 \\
\hline Supernatant & na & nat & na & na & na & na & na & na & na & na \\
\hline Solid Layer & $0.00 \mathrm{~F}+00$ & $0.00 \mathrm{E}+00$ & $0.00 \mathrm{E}+00$ & $1.75 \mathrm{E}-06$ & $1.75 \mathrm{E}-06$ & $0.00 \mathrm{E}+00$ & $0.00 \mathrm{E}+00$ & $0.00 \mathrm{E} r 00$ & 3.13E-08 & $3.13 \mathrm{E}-08$ \\
\hline $241-\mathrm{A}-106$ & $3.11 \mathrm{E}-06$ & $1.21 E-07$ & $1.57 \mathrm{E}-06$ & $7.86 \mathrm{E}-07$ & $5.58 \mathrm{E}-06$ & $6.11 \mathrm{E}-08$ & $2.37 \mathrm{E}-09$ & $3.08 \mathrm{E}-08$ & $1.55 \mathrm{E}-08$ & $1.10 \mathrm{E}-07$ \\
\hline Supenatant & na & na & na & na & na & na & na & na & na & na \\
\hline Solid Layer & $3.11 \mathrm{E}-06$ & $1.21 \mathrm{E}-07$ & $1.57 \mathrm{E}-06$ & $7.86 \mathrm{E}-07$ & $5.58 \mathrm{E}-06$ & 6.11E-08 & 2.37E-09 & $3.08 \mathrm{E}-08$ & $1.55 \mathrm{E}-08$ & $1.10 \mathrm{E}-07$ \\
\hline $241-A X-101$ & $2.24 \mathrm{E}-07$ & 4.15E-09 & $2.51 E-07$ & $2.43 \mathrm{E}-07$ & $7.22 \mathrm{E}-07$ & 4. $42 E-09$ & $8.18 E-11$ & $4.94 \mathrm{E}-09$ & $4.78 \mathrm{E}-09$ & $1.42 \mathrm{E}-08$ \\
\hline Supernatant & na & na & na & na & na & na & na & na & na & na \\
\hline Solid Layer & $2.24 \mathrm{E}-07$ & 4.15E-09 & $2.51 \mathrm{E}-07$ & $2.43 \mathrm{E}-07$ & $7.22 \mathrm{E}-07$ & 4.42E-09 & 8.18E-11 & $4.94 \mathrm{E}-09$ & $4.78 E-09$ & $1.42 \mathrm{E}-08$ \\
\hline $241-\mathrm{AX}-102$ & $2.29 \mathrm{E}-06$ & $3.26 \mathrm{E}-07$ & $3.07 \mathrm{E}-07$ & $2.08 \mathrm{E}-06$ & $5.00 \mathrm{E}-06$ & $4.18 \mathrm{E}-08$ & $5.95 \mathrm{E}-09$ & $5.60 E-09$ & $3.8 \mathrm{IE}-08$ & $9.14 \mathrm{E}-08$ \\
\hline Supernatant & na & na & nа & па & na & па & na & na & na & na \\
\hline Solid Layer & $2.29 \mathrm{~F}-06$ & $3.26 \mathrm{E}-07$ & $3.07 \mathrm{E}-07$ & $2.08 \mathrm{E}-06$ & $5.00 \mathrm{E}-06$ & $4.18 E-08$ & $5.95 \mathrm{E}-09$ & $5.60 \mathrm{E}-09$ & $3.81 \mathrm{E}-08$ & $9.14 \mathrm{E}-08$ \\
\hline
\end{tabular}




\section{RPP-5926 REV 6}

Table B-3. Calculated Hydrogen Generation Rate as Mole Per Unit Waste Weight and Volume for 177 Tanks. (20 sheets)

\begin{tabular}{|c|c|c|c|c|c|c|c|c|c|c|}
\hline Tank & $\begin{array}{c}\text { HGR from } \\
\text { radiolysis } \\
\mathrm{RC}_{\text {rad }}^{\mathrm{B} / \gamma} \\
\text { (mole } / \mathrm{kg}-\mathbf{d} \text { ) }\end{array}$ & $\begin{array}{l}\text { HGR from } \\
\text { radiolysis } \\
\mathrm{RC}_{\text {rad }} \text { Jpha } \\
\text { (mole/kg-d) }\end{array}$ & $\begin{array}{l}\text { HGR from } \\
\text { thermolysis } \\
R_{\text {iherm }} \\
\text { (mole } / \mathrm{kg}-\mathrm{d} \text { ) }\end{array}$ & $\begin{array}{l}\text { HGR from } \\
\text { corrosion } \\
\text { RC }_{\text {corr }} \\
\text { (mole/kg-d) }\end{array}$ & $\begin{array}{l}\text { Total HGR } \\
\text { from model } \\
\text { RC }_{\text {tot }} \\
\text { (mole } / \mathbf{k g}-\mathrm{d})\end{array}$ & $\begin{array}{l}\text { HGR from } \\
\text { radiolysis } \\
\mathrm{RC}_{r \mathrm{dud}}^{\mathrm{b} / \mathrm{r}} \\
\left.\text { (mole } / \mathrm{m}^{3}-\mathrm{s}\right)\end{array}$ & $\begin{array}{l}\text { HGR from } \\
\text { radiolysis } \\
\mathrm{RC}_{\text {rad }} \text { alpha } \\
\left.\text { (mole/m } / \mathrm{m}^{3}-\mathrm{s}\right)\end{array}$ & $\begin{array}{l}\text { HGR from } \\
\text { thermolysis } \\
\mathrm{RC}_{\text {therm }} \\
\left(\mathrm{mole} / \mathrm{m}^{3}-\mathrm{s}\right)\end{array}$ & $\begin{array}{c}\text { HGR from } \\
\text { corrosion } \\
\text { RC }_{\text {corr }} \\
\left(\mathrm{mole}^{3} \mathrm{~m}^{3} \mathrm{~s}\right)\end{array}$ & $\begin{array}{l}\text { Total HGR } \\
\text { from model } \\
\text { RCtot } \\
\left.\text { (mole } / \mathrm{m}^{3}-\mathrm{s}\right)\end{array}$ \\
\hline $241-A X-103$ & $1.14 \mathrm{E}-06$ & $3.75 \mathrm{E}-08$ & $3.71 \mathrm{E}-07$ & $6.52 \mathrm{E}-07$ & $2.20 \mathrm{E}-06$ & $2.08 \mathrm{E}-08$ & $6.86 \mathrm{E}-10$ & $6.79 E-09$ & $1.19 \mathrm{E}-08$ & $4.02 \mathrm{E}-08$ \\
\hline Supernatant & na & па & na & na & na & $n a$ & na & na & na & na \\
\hline Solid Layer & $1.14 \mathrm{E}-06$ & $3.75 \mathrm{E}-08$ & $3.71 \mathrm{E}-07$ & $6.52 \mathrm{E}-07$ & $2.20 \mathrm{E}-06$ & $2.08 \mathrm{E}-08$ & $6.86 \mathrm{E}-10$ & $6.79 \mathrm{E}-09$ & $1.19 \mathrm{E}-08$ & $4.02 \mathrm{E}-08$ \\
\hline $24 \mathrm{I}-\mathrm{AX}-104$ & $1.75 \mathrm{E}-05$ & $1.88 \mathrm{E}-07$ & $1.01 \mathrm{E}-08$ & $7.12 \mathrm{E}-06$ & $2.48 \mathrm{E}-05$ & 3.65E-07 & $3.92 \mathrm{E}-09$ & $2.10 \mathrm{E}-10$ & $1.48 \mathrm{E}-07$ & $5.18 \mathrm{E}-07$ \\
\hline Supernatant & 119 & na & na & na & na & па & na & na & na & na \\
\hline Solid Layer & $1.75 \mathrm{E}-05$ & $1.88 \mathrm{E}-07$ & $1.01 \mathrm{E}-08$ & $7.12 \mathrm{E}-06$ & $2.48 \mathrm{E}-05$ & $3.65 \mathrm{E}-07$ & $3.92 \mathrm{E}-09$ & $2.10 \mathrm{E}-10$ & 2.48E-07 & $5.18 \mathrm{E}-07$ \\
\hline 241-B-10I & $3.01 \mathrm{E}-07$ & 4. $40 \mathrm{E}-08$ & $1.14 \mathrm{E}-10$ & $6.89 \mathrm{E}-07$ & $1.03 \mathrm{E}-06$ & $5.27 \mathrm{E}-09$ & $7.70 \mathrm{E}-10$ & $200 \mathrm{E}-12$ & $1.21 \mathrm{E}-08$ & $1.81 \mathrm{E}-08$ \\
\hline Supernatant & па & na & na & na & na & n9 & na & na & na & na \\
\hline Solid Layer & $3.01 E-07$ & $4.40 \mathrm{E}-08$ & 1.14 E-10 & $6.89 \mathrm{E}-07$ & J.03E-06 & $5.27 \mathrm{E}-09$ & $7.70 \mathrm{E}-10$ & $2.00 \mathrm{E}-12$ & $1.21 \mathrm{E}-08$ & $1.81 \mathrm{E}-08$ \\
\hline $241-\mathrm{B}-102$ & 3.82E-09 & $6.31 \mathrm{E}-10$ & $4.39 \mathrm{E}-12$ & $2.03 \mathrm{E}-06$ & $2.03 \mathrm{E}-06$ & $6.93 \mathrm{E}-11$ & $1.15 \mathrm{E}-11$ & $7.96 \mathrm{E}-14$ & 3. $68 \mathrm{E}-08$ & $3.69 \mathrm{E}-08$ \\
\hline Supernatant & $1.30 \mathrm{E}-08$ & $1.80 \mathrm{E}-10$ & $6.41 \mathrm{E}-12$ & $1.20 \mathrm{E}-07$ & $1.33 E-07$ & $1.89 \mathrm{E}-10$ & $2.63 \mathrm{E}-12$ & $9.35 \mathrm{E}-14$ & $1.75 \mathrm{E}-09$ & $1.94 \mathrm{E}-09$ \\
\hline Solid Layer & $3.09 \mathrm{E}-09$ & $6.52 \mathrm{E}-10$ & $4.16 E-12$ & $2.24 \mathrm{E}-06$ & $2.24 \mathrm{E}-06$ & $5.77 \mathrm{E}-11$ & $1.22 \mathrm{E}-11$ & $7.77 \mathrm{E}-14$ & $4.17 \mathrm{E}-08$ & $4.18 \mathrm{E}-08$ \\
\hline $241-8-103$ & 4.49E- 09 & $3.63 \mathrm{E}-09$ & $4.13 \mathrm{E}-12$ & $1.17 \mathrm{E}-06$ & $1.18 \mathrm{E}-06$ & $8.38 \mathrm{E}-11$ & $6.77 \mathrm{E}-11$ & $7.71 E-14$ & $2.18 \mathrm{E}-08$ & $2.20 \mathrm{E}-08$ \\
\hline Supernatant & na & na & na & na & na & $\mathrm{na}$ & na & na & na & na \\
\hline Solid Layer & $4.49 \mathrm{E}-09$ & 3.63E-09 & $4.13 \mathrm{E}-12$ & $1.17 \mathrm{E}-06$ & $1.18 \mathrm{E}-06$ & $8.38 \mathrm{E}-11$ & $6.77 \mathrm{E}-11$ & $7.71 \mathrm{E}-14$ & $2.18 \mathrm{E}-08$ & $2.20 \mathrm{E}-08$ \\
\hline $24 \mid-B-104$ & $8.82 \mathrm{E}-09$ & $1.07 \mathrm{E}-09$ & $3.63 \mathrm{E}-12$ & $2.96 \mathrm{E}-07$ & $3.06 \mathrm{E}-07$ & $1.41 \mathrm{E}-10$ & $1.71 \mathrm{E}-11$ & $5.82 \mathrm{E}-14$ & $4.74 \mathrm{~F}-09$ & $4.90 \mathrm{E}-09$ \\
\hline Supenatant & na & na & nа & na & na & na & $\mathrm{na}$ & na & na & na \\
\hline Solid Layer & $8.82 \mathrm{E}-09$ & $1.07 \mathrm{E}-09$ & $3.63 \mathrm{E}-12$ & $2.96 \mathrm{E}-07$ & $3.06 \mathrm{E}-07$ & $1.41 \mathrm{E}-10$ & $1.71 \mathrm{E}-11$ & $5.82 \mathrm{E}-14$ & $4.74 \mathrm{E}-09$ & $4.90 \mathrm{E}-09$ \\
\hline 241-B-105 & $2.66 \mathrm{E}-09$ & $7.37 \mathrm{E}-10$ & $4.25 \mathrm{E}-12$ & $2.93 \mathrm{E}-07$ & $2.97 E-07$ & $5.10 E-11$ & $1.41 \mathrm{E}-11$ & $8.13 \mathrm{E}-14$ & $5.6 ! \mathrm{E}-09$ & $5.68 \mathrm{E}-09$ \\
\hline Supenatant & na & $\mathrm{na}$ & na & na & na & na & na & na & na & na \\
\hline Solio Layer & $2.66 \mathrm{E}-09$ & $7.37 \mathrm{E}-10$ & $4.25 \mathrm{E}-12$ & $2.93 \mathrm{E}-07$ & $2.97 \mathrm{E}-07$ & $5.10 \mathrm{E}-11$ & $1.41 \mathrm{E}-11$ & $8.13 \mathrm{E}-14$ & $5.61 \mathrm{E}-09$ & $5.68 \mathrm{E}-09$ \\
\hline $241-B-106$ & $1.01 \mathrm{E}-07$ & $1.13 \mathrm{E}-09$ & $1.10 \mathrm{E}-11$ & $6.81 \mathrm{E}-07$ & 7.84E-07 & $1.61 \mathrm{E}-09$ & $1.81 \mathrm{E}-11$ & $1.76 \mathrm{E}-13$ & $1.09 \mathrm{E}-08$ & $1.25 \mathrm{E}-08$ \\
\hline Supernatant & $1.30 \mathrm{E}-08$ & $1.80 \mathrm{E}-10$ & $6.01 \mathrm{E}-12$ & $1.20 \mathrm{E}-07$ & $1.33 \mathrm{E}-07$ & 1.89E-10 & 2.63E-12 & $8.76 \mathrm{E}-14$ & $1.75 \mathrm{E}-09$ & $1.94 \mathrm{E}-09$ \\
\hline Solid Layer & $1.02 \mathrm{E}-07$ & $1.14 \mathrm{E}-09$ & $1.10 \mathrm{E}-11$ & $6.86 \mathrm{E}-07$ & $7.89 \mathrm{E}-07$ & $1.62 \mathrm{E}-09$ & $1.82 \mathrm{E}-11$ & $1.77 \mathrm{E}-13$ & $1.10 \mathrm{E}-08$ & $1.26 \mathrm{E}-08$ \\
\hline $241-B-107$ & $1.05 \mathrm{E}-08$ & $7.24 \mathrm{E}-10$ & $1.48 \mathrm{E}-12$ & $4.62 \mathrm{E}-07$ & $4.73 \mathrm{E}-07$ & $1.976-10$ & $1.36 \mathrm{E}-11$ & $2.78 \mathrm{E}-14$ & $8.69 \mathrm{E}-09$ & $8.90 \mathrm{E}-09$ \\
\hline Supernatant & na & na & na & na & na & na & na & na & na & na \\
\hline Solid Layer & $1.05 \mathrm{E}-08$ & $7.24 \mathrm{E}-10$ & $1.48 \mathrm{E}-12$ & 4.62E-07 & $4.73 \mathrm{E}-07$ & $1.97 \mathrm{E}-10$ & $1.36 \mathrm{E}-11$ & $2.78 E-14$ & $8.69 \mathrm{E}-09$ & $8.90 \mathrm{E}-09$ \\
\hline
\end{tabular}




\section{RPP-5926 REV 6}

Table B-3. Calculated Hydrogen Generation Rate as Mole Per Unit Waste Weight and Volume for 177 Tanks. (20 sheets)

\begin{tabular}{|c|c|c|c|c|c|c|c|c|c|c|}
\hline Tank & $\begin{array}{l}\text { HGR from } \\
\text { radiolysis } \\
\mathrm{RC}_{\mathrm{rad}}^{\mathrm{B} / \mathrm{\gamma}} \\
\text { (mole } / \mathrm{kg}-\mathrm{d})\end{array}$ & $\begin{array}{l}\text { HGR from } \\
\text { radiolysis } \\
\text { RC }_{\text {rad }} \text { alpha } \\
\text { (mole/kg- } 1 \text { ) }\end{array}$ & $\begin{array}{l}\text { HGR from } \\
\text { thermolysis } \\
R_{\text {therm }} \\
\text { (mole } / \mathrm{kg}-\mathrm{d} \text { ) }\end{array}$ & $\begin{array}{l}\text { HGR from } \\
\text { corrosion } \\
R_{\text {corr }} \\
\text { (moie/kg-d) }\end{array}$ & $\begin{array}{c}\text { Total HGR } \\
\text { from model } \\
\mathrm{RC}_{\text {lot }} \\
\text { (mole } / \mathrm{kg} \text {-d) }\end{array}$ & $\begin{array}{l}\text { HGR from } \\
\text { radiolysis } \\
\mathrm{RC}_{\mathrm{rad}} \mathrm{b} / \mathrm{r} \\
\left(\mathrm{mole} / \mathrm{m}^{3}-\mathrm{s}\right)\end{array}$ & $\begin{array}{l}\text { HGR from } \\
\text { radiolysis } \\
\mathrm{RC}_{\text {rad }} \text { alpha } \\
\left.\text { (mole } / \mathrm{m}^{3}-\mathrm{s}\right)\end{array}$ & $\begin{array}{l}\text { HGR from } \\
\text { thermolysis } \\
\text { RC }_{\text {therm }} \\
\left.\text { (mole/ } \mathrm{m}^{3}-\mathrm{s}\right)\end{array}$ & $\begin{array}{l}\text { HGR from } \\
\text { corrosion } \\
\mathrm{RC}_{\text {corr }} \\
\left.\text { (mole } / \mathrm{m}^{3}-\mathrm{s}\right)\end{array}$ & $\begin{array}{c}\text { Total HGR } \\
\text { from model } \\
\text { RCtot } \\
\left.\text { (mole } / \mathrm{m}^{3}-\mathrm{s}\right)\end{array}$ \\
\hline $241-B-108$ & $6.16 \mathrm{E}-09$ & $3.50 \mathrm{E}-11$ & $6.50 \mathrm{E}-12$ & $7.03 \mathrm{E}-07$ & $7.09 \mathrm{E}-07$ & $1.21 \mathrm{E}-10$ & $6.89 \mathrm{E}-13$ & $1.28 \mathrm{E}-13$ & 1. $39 \mathrm{E}-08$ & $1.40 \mathrm{E}-08$ \\
\hline Supernatant & ла & na & na & na & па & $\mathrm{na}$ & na & na & na & na \\
\hline Solid Layer & $6.16 \mathrm{E}-09$ & $3.50 \mathrm{E}-11$ & $6.50 \mathrm{E}-12$ & 7.03E-07 & $7,09 \mathrm{E}-07$ & $1.21 \mathrm{E}-10$ & $6.89 \mathrm{E}-13$ & $1.28 \mathrm{E}-13$ & 1.39E-08 & $1.40 \mathrm{E}-08$ \\
\hline $24 !-B-109$ & $1.63 E-09$ & $4.97 \mathrm{E}-10$ & $4.60 \mathrm{t}-12$ & $5.07 \mathrm{E}-07$ & $5.095-07$ & $3.44 \mathrm{E}-11$ & $1.05 \mathrm{E}-11$ & $9.69 \mathrm{E}-14$ & $1.07 \mathrm{E}-08$ & $1.07 \mathrm{E}-08$ \\
\hline Supenatant & na & na & na & na & па & na & na & na & na & na \\
\hline Solid Layer & $1.63 E-09$ & $4.97 \mathrm{E}-10$ & $4.60 \mathrm{E}-12$ & $5.07 \mathrm{E}-07$ & $5.09 \mathrm{E}-07$ & $3.44 \mathrm{E}-11$ & $1.05 E-11$ & $9.69 \mathrm{E}-14$ & $1.07 \mathrm{E}-08$ & $1.07 \mathrm{E}-08$ \\
\hline $241-B-110$ & $2.31 \mathrm{E}-07$ & $9.33 \mathrm{E}-09$ & $6.37 \mathrm{E}-08$ & $4.01 \mathrm{E}-07$ & $7.06 \mathrm{E}-07$ & $3.64 \mathrm{E}-09$ & $1.47 \mathrm{k}-10$ & $1.00 \mathrm{E}-09$ & $6.31 \mathrm{E}-09$ & $1.1 \pm E-08$ \\
\hline Supematant & $3.62 \mathrm{E}-08$ & $7.32 \mathrm{E}-10$ & $6.65 \mathrm{E}-08$ & $1.27 \mathrm{E}-07$ & $2.30 \mathrm{E}-07$ & $4.98 \mathrm{E}-10$ & $1.01 \mathrm{E}-11$ & $9.16 \mathrm{E}-10$ & $1.75 \mathrm{E}-09$ & $3.17 E-09$ \\
\hline Solid Layer & $2.32 \mathrm{E}-07$ & $9.35 \mathrm{E}-09$ & $6.37 \mathrm{E} \cdot 08$ & $4.02 E-07$ & $7.07 \mathrm{E}-07$ & $3.65 \mathrm{E}-09$ & $1.47 \mathrm{E}-10$ & $1.00 \mathrm{E}-09$ & $6.33 \mathrm{E}-09$ & $1.11 \mathrm{E}-08$ \\
\hline $241-B-111$ & $7.65 \mathrm{E}-07$ & $9.25 E-09$ & $9.93 \mathrm{E}-08$ & $4.35 \mathrm{E}-07$ & $1.31 \mathrm{E}-06$ & 1.12E-08 & $1.36 \mathrm{E}-10$ & $1.46 \mathrm{E}-09$ & $6.39 \mathrm{E}-09$ & $1.92 E-08$ \\
\hline Supernatant & $3.65 \mathrm{E}-08$ & $7.33 \mathrm{E}-10$ & $8.68 \mathrm{E}-08$ & $1.27 \mathrm{E}-07$ & $2.51 \mathrm{E}-07$ & $5.03 E-10$ & $1.01 \mathrm{E}-11$ & $1.20 \mathrm{E}-09$ & $1.75 \mathrm{E}-09$ & $3.46 \mathrm{E}-09$ \\
\hline Solid Layer & $7.67 \mathrm{E}-07$ & $9.27 E-09$ & $9.93 \mathrm{E}-08$ & $4.36 \mathrm{E}-07$ & $1,31 \mathrm{E}-06$ & $1.13 \mathrm{E}-08$ & $1.36 \mathrm{E}-10$ & $1.46 \mathrm{E}-09$ & $6.41 \mathrm{E}-09$ & $1.93 E-08$ \\
\hline $241-B-112$ & $4.48 E-08$ & $1.12 \mathrm{E}-10$ & $2.48 E-07$ & $1.96 \mathrm{E}-06$ & $2.25 \mathrm{E}-06$ & $7.75 E-10$ & $1.94 \mathrm{E}-12$ & $4.29 \mathrm{E}-09$ & $3.39 \mathrm{E}-08$ & $3.89 \mathrm{E}-08$ \\
\hline Supernatant & $1.13 \mathrm{E}-07$ & $2.47 \mathrm{E}-10$ & $2.76 \mathrm{E}-07$ & $1.00 E-07$ & $4.90 \mathrm{E}-07$ & $1.98 \mathrm{E}-09$ & $4.32 \mathrm{E}-12$ & $4.83 \mathrm{E}-09$ & $1.75 \mathrm{E}-09$ & $8.56 \mathrm{E}-09$ \\
\hline Solid Layer & $3.92 \mathrm{E}-08$ & $1.01 \mathrm{E}-10$ & $2.45 \mathrm{E}-07$ & $2.13 \mathrm{E}-06$ & $2.41 \mathrm{E}-06$ & $6.78 E-10$ & $1.74 \mathrm{E}-12$ & $4.24 \mathrm{E}-09$ & $3.68 \mathrm{E}-08$ & $4.17 \mathrm{E}-08$ \\
\hline $241-B+201$ & $5.76 \mathrm{E}-09$ & $4.48 \mathrm{E}-08$ & $1.32 \mathrm{E}-08$ & $6.39 \mathrm{E}-07$ & $7.02 \mathrm{E}-07$ & $8.40 \mathrm{E}-11$ & $6.53 \mathrm{E}-10$ & $1.93 \mathrm{E}-10$ & $9.31 \mathrm{E}-0.9$ & $1.02 \mathrm{E}-08$ \\
\hline Supematarit & $n a$ & na & ná & na & па & na & na & na & na & na \\
\hline Solid Layer & $5.76 \mathrm{E}-09$ & $4.48 \mathrm{E}-08$ & $1.32 \mathrm{E}-08$ & $6.39 \mathrm{E}-07$ & $7.02 \mathrm{E}-07$ & $8.40 \mathrm{E}-11$ & $6.53 \mathrm{E}-10$ & 1.93E-10 & $9.31 \mathrm{E}-09$ & $1.02 \mathrm{E}-08$ \\
\hline $241-B-202$ & $1.04 \mathrm{E}-08$ & $1.29 \mathrm{E}-08$ & $1.46 \mathrm{E}-08$ & $6.65 \mathrm{E}-07$ & $7.03 E-07$ & 1.47E-10 & $1.82 \mathrm{E}-10$ & $2.06 \mathrm{E}-10$ & $9.39 \mathrm{E}-09$ & $9.92 \mathrm{E}-09$ \\
\hline Supernatant & na & na & na & na & na & na & na & na & na & na \\
\hline Solid Layer & I. $04 \mathrm{E}-08$ & $1.29 \mathrm{E}-08$ & $1.46 \mathrm{E}-08$ & $6.65 \mathrm{E}-07$ & 7.03E-07 & $1.47 \mathrm{E}-10$ & $1.82 \mathrm{E}-10$ & $2.06 \mathrm{E}-10$ & $9.39 \mathrm{E}-09$ & $9.92 \mathrm{E}-09$ \\
\hline $241-B-203$ & $3.70 \mathrm{E}-10$ & $2.27 \mathrm{E}-08$ & $0.00 E \div 00$ & 1.48E-06 & $1.51 \mathrm{E}-06$ & $5.08 \mathrm{E}-12$ & $3.12 \mathrm{E}-10$ & $0.00 \mathrm{E}+00$ & $2.04 E-08$ & $2.07 \mathrm{E}-08$ \\
\hline Supernatant & $8.40 \mathrm{E}-13$ & $3.93 \mathrm{E}-10$ & $0.00 \mathrm{E}+00$ & $1.35 \mathrm{E}-06$ & $1.35 \mathrm{E}-06$ & $1.02 \mathrm{E}-14$ & $4.78 \mathrm{E}-12$ & $0.00 \mathrm{E}+00$ & $1.64 \mathrm{E}-08$ & $1.64 \mathrm{E}-08$ \\
\hline Solid Layer & $3.72 \mathrm{E}-10$ & $2.29 \mathrm{E}-08$ & $0.00 E+00$ & $1.49 \mathrm{E}-06$ & $1.5 t E-06$ & $5.13 E-12$ & $3.15 \mathrm{E}-10$ & $0.00 E+00$ & 3.05E-08 & $2.08 \mathrm{E}-08$ \\
\hline $241-\mathrm{B}-204$ & $1.38 \mathrm{E}-10$ & $2.72 \mathrm{E}-08$ & $0.00 \mathrm{E}+00$ & $1.49 \mathrm{E}-06$ & $1.52 \mathrm{E}-06$ & $1.90 \mathrm{E}-12$ & $3.74 \mathrm{E}-10$ & $0.00 \mathrm{E}+00$ & $2.05 \mathrm{E}-08$ & $2.09 \mathrm{E}-08$ \\
\hline Supernatant & $4.91 \mathrm{E}-13$ & $9.12 \mathrm{E}-12$ & $0.00 E+00$ & $1.35 \mathrm{E}-06$ & $1.35 \mathrm{E}-06$ & $5.96 \mathrm{E}-15$ & $1.11 E-13$ & $0.00 \mathrm{E}+00$ & $1.64 \mathrm{E}-08$ & $1.64 \mathrm{E}-08$ \\
\hline Solid Layer & $1.40 \mathrm{E}-10$ & $2.75 \mathrm{E}-08$ & $0.00 \mathrm{E}+00$ & $1.49 \mathrm{E}-06$ & 1. $52 \mathrm{E}-06$ & $1.93 \mathrm{E}-12$ & $3.79 \mathrm{E}-10$ & $0.00 \mathrm{E}+00$ & $2.05 \mathrm{E}-08$ & $2.09 \mathrm{E}-08$ \\
\hline
\end{tabular}




\section{RPP-5926 REV 6}

Table B-3. Calculated Hydrogen Generation Rate as Mole Per Unit Waste Weight and Volume for 177 Tanks. (20 sheets)

\begin{tabular}{|c|c|c|c|c|c|c|c|c|c|c|}
\hline Tank & $\begin{array}{l}\text { HGR from } \\
\text { radiolysis } \\
\mathrm{RC}_{\mathrm{rad} / \gamma} \\
\text { (mole/kg-d) }\end{array}$ & $\begin{array}{l}\text { HGR from } \\
\text { radiolysis } \\
\text { RC }_{\text {rad pha }} \\
\text { (mole/kg-d) }\end{array}$ & $\begin{array}{l}\text { HGR from } \\
\text { thermolysis } \\
R_{\text {therm }} \\
\text { (mole/kg-d) }\end{array}$ & $\begin{array}{l}\text { HGR from } \\
\text { corrosion } \\
\mathrm{RC}_{\text {rorr }} \\
\text { (mole/kg-d) }\end{array}$ & $\begin{array}{c}\text { Total HGR } \\
\text { from model } \\
\mathbf{R C}_{\text {tol }} \\
\text { (mole } / \mathbf{k g - d} \text { ) }\end{array}$ & $\begin{array}{l}\text { HGR from } \\
\text { radiolysis } \\
\mathrm{RC}_{\mathrm{rad}}^{\mathrm{b} / \mathrm{r}} \\
\left(\mathrm{mole}^{\mathrm{m}} \mathrm{m}^{3}-\mathrm{s}\right)\end{array}$ & $\begin{array}{l}\text { HGR from } \\
\text { radiolysis } \\
\text { RC }_{\text {rad }} \text { alpha } \\
\left.\text { (mole } / \mathrm{m}^{3}-\mathrm{s}\right)\end{array}$ & $\begin{array}{l}\text { HGR from } \\
\text { thermolysis } \\
\text { RC }_{\text {therm }} \\
\left(\mathrm{mole} / \mathrm{m}^{3}-\mathrm{s}\right)\end{array}$ & $\begin{array}{l}\text { HGR from } \\
\text { corrosion } \\
\text { RC }_{\text {corr }} \\
\text { (mole/m } \text { m }^{3} \text { s) }\end{array}$ & $\begin{array}{c}\text { Total HGR } \\
\text { from model } \\
\text { RCtot } \\
\left.\text { (mole } / \mathrm{m}^{3}-\mathrm{s}\right)\end{array}$ \\
\hline $241-B X-101$ & $1.54 \mathrm{E}-07$ & $8.59 \mathrm{E}-09$ & $9.56 \mathrm{E}-09$ & $1.30 \mathrm{E}-06$ & $1.47 \mathrm{E}-06$ & $2.99 \mathrm{E}-09$ & $1.67 \mathrm{E}-10$ & $1.86 \mathrm{E}-10$ & $2.53 \mathrm{E}-08$ & $2.86 \mathrm{E}-08$ \\
\hline Supenatant & na & na & na & na & na & na & na & na & na & na \\
\hline Solid Layer & $1.54 \mathrm{E}-07$ & $8.59 E-09$ & $9.56 \mathrm{E}-09$ & $1.30 \mathrm{E}-06$ & $1.47 \mathrm{E}-06$ & $2.99 \mathrm{E}-09$ & $1.67 \mathrm{E}-10$ & $1.86 \mathrm{E}-10$ & $2.53 \mathrm{E}-08$ & $2.86 \mathrm{E}-08$ \\
\hline $241-B X-102$ & $7.54 \mathrm{E}-08$ & $8.42 \mathrm{E}-10$ & 1. $10 \mathrm{E}-08$ & $1.39 \mathrm{E}-06$ & $1.48 \mathrm{E}-06$ & $8.65 E-10$ & $9.66 \mathrm{E}-12$ & $1.26 \mathrm{E}-10$ & $1.60 \mathrm{E}-08$ & $1.70 \mathrm{E}-08$ \\
\hline Supernatant & na & na & na & na & na & na & na & па & na & na \\
\hline Solid Layer & $7.54 \mathrm{E}-08$ & $8.42 \mathrm{E}-10$ & $1.10 \mathrm{E}-08$ & $1.39 \mathrm{E}-06$ & $1.48 \mathrm{E}-06$ & $8.65 E-10$ & $9.66 \mathrm{E}-12$ & $1.26 \mathrm{E}-10$ & 1.60E-08 & $1.70 \mathrm{E}-08$ \\
\hline $241-\mathrm{BX}-103$ & $3.84 \mathrm{E}-08$ & $1.78 \mathrm{E}-07$ & $4.17 \mathrm{E}-08$ & $9.26 \mathrm{E}-07$ & $1.18 \mathrm{E}-06$ & $6.94 \mathrm{E}-10$ & $3.22 \mathrm{E}-09$ & $7.53 \mathrm{E}-10$ & $1.67 \mathrm{E}-08$ & $2.14 \mathrm{E}-08$ \\
\hline Supcrnatant & $5.90 \mathrm{E}-09$ & $1.21 \mathrm{E}-09$ & $6.35 \mathrm{E}-08$ & $1.41 \mathrm{E}-07$ & $2.12 \mathrm{E}-07$ & $7.30 \mathrm{E}-11$ & $1.50 \mathrm{E}-11$ & $7.86 \mathrm{E}-10$ & $1.75 E-09$ & $2.62 \mathrm{E}-09$ \\
\hline Solid Layer & 4.03E-08 & $1.89 \mathrm{E}-07$ & $3.89 \mathrm{E}-08$ & $1.03 \mathrm{E}-06$ & $1,30 \mathrm{E}-06$ & $7.75 E-10$ & $3.64 \mathrm{E}-09$ & $7.48 E-10$ & $1.98 \mathrm{E}-08$ & $2.50 \mathrm{E}-08$ \\
\hline $241-\mathrm{BX}-104$ & $2.53 \mathrm{E}-07$ & $1.83 \mathrm{E}-08$ & $2.54 \mathrm{E}-08$ & $6.68 \mathrm{E}-07$ & $9.66 \mathrm{E}-07$ & $4.89 \mathrm{E}-09$ & $3.54 \mathrm{E}-10$ & $4.91 \mathrm{E}-10$ & $1.29 \mathrm{E}-08$ & $1.86 \mathrm{E}-08$ \\
\hline Supernatant & $1.62 E-07$ & $1.76 \mathrm{E}-11$ & $5.44 \mathrm{E}-08$ & $1.18 \mathrm{E}-07$ & $3.35 \mathrm{E}-07$ & $2.40 \mathrm{E}-09$ & $2.61 \mathrm{E}-13$ & $8.06 \mathrm{E}-10$ & $1.75 \mathrm{E}-09$ & $4.96 \mathrm{E}-09$ \\
\hline Solid Layer & $2.51 \mathrm{E}-07$ & $1.83 \mathrm{E}-08$ & $2.48 \mathrm{E}-08$ & $6.81 \mathrm{E}-07$ & $9.75 \mathrm{E}-07$ & $4.88 \mathrm{E}-09$ & $3.55 \mathrm{E}-10$ & $4.82 \mathrm{E}-10$ & $1.32 \mathrm{E}-08$ & $1.90 \mathrm{E}-08$ \\
\hline $241 \cdot \mathrm{BX}-105$ & $1.45 \mathrm{E}-07$ & $2.01 \mathrm{~F}-09$ & $1.67 \mathrm{E}-08$ & $8.98 \mathrm{E}-07$ & $1.06 \mathrm{E}-06$ & $2.80 \mathrm{E}-09$ & $3.87 E-11$ & $3.22 \mathrm{E}-10$ & $1.73 \mathrm{E}-08$ & $2.05 \mathrm{E}-08$ \\
\hline Supernatant & $1.89 \mathrm{E}-07$ & 1.63E-09 & $7.00 \mathrm{E}-08$ & $1.17 \mathrm{E}-07$ & $3.77 \mathrm{E}-07$ & $2.81 E-09$ & $2.44 \mathrm{E}-11$ & $1.05 E-09$ & $1.75 \mathrm{E}-09$ & $5.63 \mathrm{E}-09$ \\
\hline Solid Layer & $1.25 \mathrm{E}-07$ & $1.74 \mathrm{E}-09$ & $1.39 \mathrm{E}-08$ & $9.41 \mathrm{E}-07$ & $1.08 \mathrm{E}-06$ & $2.45 \mathrm{E}-09$ & $3.4 ! \mathrm{E}-11$ & $2.72 \mathrm{E}-10$ & $1.84 \mathrm{E}-08$ & $2.12 \mathrm{E}-08$ \\
\hline $241-B X-106$ & $7.87 \mathrm{E}-08$ & $1.20 \mathrm{E}-08$ & $2.17 \mathrm{E}-07$ & 1. $68 \mathrm{E}-06$ & $1.98 \mathrm{E}-06$ & $1.47 \mathrm{E}-09$ & $2.24 \mathrm{E}-10$ & $4.06 \mathrm{E}-09$ & $3.14 \mathrm{E}-08$ & $3.72 E-08$ \\
\hline Supernatant & na & па & na & na & na & na & na & na & na & na \\
\hline Solid Layer & $7.87 E-08$ & $1.20 \mathrm{E}-08$ & $2.17 \mathrm{E}-07$ & $1.68 \mathrm{E}-06$ & $1,98 E-06$ & $1.47 E-09$ & $2.24 \mathrm{E}-10$ & 4.06E-09 & $3.14 \mathrm{E}-08$ & $3.72 \mathrm{E}-08$ \\
\hline $241-B X-107$ & $4.28 \mathrm{E}-08$ & $3.03 \mathrm{E}-09$ & $1.35 \mathrm{E}-08$ & $2.99 \mathrm{E}-07$ & $3.58 \mathrm{E}-07$ & $7.14 E-10$ & $5.05 E-11$ & $2.26 \mathrm{E}-10$ & $4.98 \mathrm{E}-09$ & $5.97 \mathrm{E}-09$ \\
\hline Supernatant & na & ла & nа & na & na & $\mathrm{na}$ & na & na & na & na \\
\hline Solid Layer & $4.28 \mathrm{E}-08$ & $3.03 E-09$ & $1.35 \mathrm{E}-08$ & $2.99 \mathrm{E}-07$ & $3.58 \mathrm{E}-07$ & $7.14 E-10$ & $5.05 \mathrm{E}-11$ & $2.26 \mathrm{E}-10$ & $4.98 \mathrm{E}-09$ & $5.97 \mathrm{E}-09$ \\
\hline $241-\mathrm{BX}-108$ & $1.25 \mathrm{E}-07$ & $1.06 \mathrm{E}-09$ & $5.14 \mathrm{E}-09$ & $2.22 \mathrm{E}-06$ & $2.35 \mathrm{~F}-06$ & $2.10 \mathrm{E}-09$ & $1.79 \mathrm{E}-11$ & $8.66 \mathrm{E}-11$ & $3.74 \mathrm{E}-08$ & $3.96 \mathrm{E}-08$ \\
\hline Supernatant & na & na & na & na & na & na & na & na & na & na \\
\hline Solid Layer & $1.25 \mathrm{E}-07$ & $1.06 \mathrm{E}-09$ & $5.14 \mathrm{E}-09$ & $2.22 E-06$ & $2.35 \mathrm{E}-06$ & $2.10 E-09$ & $1.79 \mathrm{E}-11$ & $8.66 \mathrm{E}-11$ & $3.74 \mathrm{E}-08$ & $3.96 \mathrm{E}-08$ \\
\hline $24 !-8 \times-109$ & $3.03 E-07$ & $7.36 \mathrm{E}-10$ & $1.90 \mathrm{E}-08$ & $4.30 \mathrm{E}-07$ & $7,53 \mathrm{E}-07$ & $5.34 E-09$ & $1.29 \mathrm{E}-11$ & 3.35E-10 & $7.56 \mathrm{E}-09$ & $1.32 \mathrm{E}-08$ \\
\hline Supernatant & na & па & na & na & na & na & na & na & na & na \\
\hline Solid Layer & $3.03 \mathrm{E}-07$ & $7.36 \mathrm{E}-10$ & $1.90 \mathrm{E}-08$ & $4.30 \mathrm{E}-07$ & $7,53 \mathrm{E}-07$ & $5.34 \mathrm{E}-09$ & $1.29 E-11$ & $3.35 \mathrm{E}-10$ & $7.56 \mathrm{E}-09$ & $1.32 \mathrm{E}-08$ \\
\hline
\end{tabular}


Table B-3. Calculated Hydrogen Generation Rate as Mole Per Unit Waste Weight and Volume for 177 Tanks. (20 sheets)

\begin{tabular}{|c|c|c|c|c|c|c|c|c|c|c|}
\hline Tank & $\begin{array}{l}\text { HGR from } \\
\text { radiolysis } \\
\mathrm{RC}_{\mathrm{rad}}^{\mathrm{B} / \gamma} \\
\text { (mole } / \mathbf{k g - d} \text { ) }\end{array}$ & $\begin{array}{l}\text { HGR from } \\
\text { radiolysis } \\
\operatorname{RC}_{\text {rad }}^{\text {alpha }} \\
\text { (mole/kg-d) }\end{array}$ & $\begin{array}{c}\text { HGR from } \\
\text { thermolysis } \\
\text { RC }_{\text {therm }} \\
\text { (mole/kg-d) }\end{array}$ & $\begin{array}{c}\text { HGR from } \\
\text { corrosion } \\
\mathrm{RC}_{\text {corr }} \\
\text { (mole/kg-d) }\end{array}$ & $\begin{array}{c}\text { Total HGR } \\
\text { from model } \\
R_{\text {tol }} \\
\text { (mole } / \mathbf{k g} \cdot \mathbf{d})\end{array}$ & $\begin{array}{l}\text { HGR from } \\
\text { radiolysis } \\
\mathrm{RC}_{\mathrm{rad}} \mathrm{b} / \mathrm{r} \\
\text { (mole } / \mathrm{m}^{3}-\mathrm{s} \text { ) }\end{array}$ & $\begin{array}{l}\text { HGR from } \\
\text { radiolysis } \\
\mathrm{RC}_{\text {rad }} \text { alpha } \\
\left.\text { (mole } / \mathrm{m}^{3}-\mathrm{s}\right)\end{array}$ & $\begin{array}{l}\text { HGR from } \\
\text { thermolysis } \\
\text { RC }_{\text {therm }} \\
\left.\text { (mole/m }{ }^{3}-\mathrm{s}\right)\end{array}$ & $\begin{array}{l}\text { HGR from } \\
\text { corrosion } \\
\text { RC }_{\text {curr }} \\
\text { (mole } / \mathrm{m}^{3}-\mathrm{s} \text { ) }\end{array}$ & $\begin{array}{c}\text { Total HGR } \\
\text { from model } \\
\text { RCtot } \\
\left(\mathrm{mole} / \mathrm{m}^{3}-\mathrm{s}\right)\end{array}$ \\
\hline $241-B X-110$ & $4.78 E-08$ & $2.95 \mathrm{E}-10$ & $6.44 E-08$ & $3.62 \mathrm{E}-07$ & $4.74 E-07$ & $9.22 \mathrm{E}-10$ & $5.69 \mathrm{E}-12$ & $1.24 \mathrm{E}-09$ & $6.97 \mathrm{E}-09$ & $9.14 \mathrm{E}-09$ \\
\hline Supenalant & $9.20 \mathrm{E}-08$ & $1.36 \mathrm{E}-10$ & $8.13 \mathrm{E}-08$ & $1.05 \mathrm{E}-07$ & $2.78 \mathrm{E}-07$ & $1.53 \mathrm{E}-09$ & $2.27 \mathrm{E}-12$ & $1.35 \mathrm{E}-09$ & $1.75 \mathrm{E}-09$ & $4,64 \mathrm{E}-09$ \\
\hline Solid Layer & $4.76 \mathrm{E}-08$ & $2.96 \mathrm{E}-10$ & $6.44 \mathrm{E}-08$ & $3.63 \mathrm{E}-07$ & $4.75 \mathrm{E}-07$ & $9.18 \mathrm{E}-10$ & $5.70 \mathrm{E}-12$ & $1.24 \mathrm{E}-09$ & $7.00 \mathrm{E}-09$ & $9.17 \mathrm{E}-09$ \\
\hline 241-BX-111 & $7.08 \mathrm{E}-09$ & $5.84 \mathrm{E}-11$ & $2.39 \mathrm{E}-09$ & $4.60 \mathrm{E}-07$ & 4.69E-07 & $1.18 \mathrm{E}-10$ & $9.77 \mathrm{E}-13$ & $4.01 \mathrm{E}-11$ & $7.70 \mathrm{E}-09$ & $7.86 \mathrm{E}-09$ \\
\hline Supernatant & na & na & na & па & na & na & na & ta & na & na \\
\hline Solid Layer & $7.08 \mathrm{E}-09$ & $5.84 \mathrm{E}-11$ & $2.39 \mathrm{E}-09$ & $4.60 \mathrm{E}-07$ & $4.69 \mathrm{E}-07$ & $1.18 \mathrm{E}-10$ & $9.77 \mathrm{E}-13$ & $4.01 \mathrm{E}-11$ & $7.70 \mathrm{E}-09$ & $7.86 \mathrm{E}-09$ \\
\hline $24 !-B X-112$ & $1.11 \mathrm{E}-07$ & $9.45 \mathrm{E}-09$ & $0.00 \mathrm{E}+00$ & $5.65 \mathrm{E}-07$ & $6.85 \mathrm{E}-07$ & $1.67 \mathrm{E}-09$ & 1. $43 \mathrm{E}-10$ & $0.00 \mathrm{E}+00$ & $8.57 \mathrm{E}-09$ & $1.04 \mathrm{E}-08$ \\
\hline Supernatant & $3.92 E \cdot 09$ & $9.22 \mathrm{E}-11$ & $0.00 \mathrm{E}+00$ & 1. $28 \mathrm{E}-07$ & $1.32 \mathrm{E}-07$ & $5.35 \mathrm{E}-11$ & $1.26 \mathrm{E}-12$ & $0.00 E+00$ & $1.75 \mathrm{E}-09$ & $1.80 \mathrm{E}-09$ \\
\hline Solid Layer & $1.11 \mathrm{E}-07$ & $9.52 E-09$ & $0.00 \mathrm{E}+00$ & $5.69 \mathrm{E}-07$ & $6.89 \mathrm{E}-07$ & $1.69 \mathrm{E}-09$ & 1.44E-10 & $0.00 \mathrm{E}+00$ & $8.62 \mathrm{E}-09$ & $1.05 \mathrm{E}-08$ \\
\hline $241-B Y-101$ & 8.23E-08 & $4.99 \mathrm{E}-10$ & 1.32E-07 & $2.26 \mathrm{~F}-07$ & $4.41 \mathrm{E}-07$ & $1.74 \mathrm{E}-09$ & $1.06 \mathrm{E}-11$ & $2.80 \mathrm{E}-09$ & $4.78 \mathrm{E}-09$ & $9.33 \mathrm{E}-09$ \\
\hline Supernatant & na & na & na & na & na & na & na & na & na & na \\
\hline Solid Layer & $8.23 \mathrm{E}-08$ & $4.99 \mathrm{E}-10$ & $1.32 \mathrm{~F}-07$ & $2.26 \mathrm{E}-07$ & $4.41 \mathrm{E}-07$ & $1.74 \mathrm{E}-09$ & $1.06 \mathrm{E}-11$ & $2.80 \mathrm{E}-09$ & $4.78 E-09$ & $9.33 \mathrm{E}-09$ \\
\hline $241-\mathrm{BY}-102$ & $2.10 \mathrm{E}-08$ & $1.01 \mathrm{E}-09$ & 9.79E-09 & $3.18 E-07$ & $3.49 \mathrm{E}-07$ & $3.82 \mathrm{E}-10$ & $1.84 \mathrm{E}-11$ & $1.78 \mathrm{E}-10$ & $5.77 \mathrm{E}-09$ & $6.35 \mathrm{E}-09$ \\
\hline Supernatant & na & na & па & na & na & na & na & na & na & na \\
\hline Solid l ayer & $2.10 \mathrm{E}-08$ & $1.01 \mathrm{E}-09$ & $9.79 \mathrm{E}-09$ & $3.18 \mathrm{E}-07$ & $3.49 \mathrm{E}-07$ & $3.82 \mathrm{E}-10$ & $1.84 E-11$ & $1.78 E \cdot 10$ & $5.77 \mathrm{E}-09$ & $6.35 \mathrm{E}-09$ \\
\hline 241-BY-103 & $3.00 \mathrm{E}-08$ & $2.73 \mathrm{E}-10$ & $1.18 \mathrm{E}-08$ & $2.32 \mathrm{E}-07$ & $2.74 \mathrm{E}-07$ & $5.77 \mathrm{E}-10$ & $5.25 \mathrm{E}-12$ & $2.27 \mathrm{E}-10$ & $4.46 \mathrm{E}-09$ & $5.27 \mathrm{E}-09$ \\
\hline Supemalant & na & na & na & na & па & na & na & na & na & па \\
\hline Solid laycr & $3.00 \mathrm{E}-08$ & $2.73 \mathrm{E}-10$ & $1.18 \mathrm{E}-08$ & $2.32 \mathrm{E}-07$ & $2.74 \mathrm{E}-07$ & $5.77 \mathrm{E}-10$ & $5.25 \mathrm{E}-12$ & 2.27 E. 10 & $4.46 \mathrm{E}-09$ & $5.27 \mathrm{E}-09$ \\
\hline $241-B Y \cdot 104$ & $7.45 \mathrm{E}-08$ & $7.68 \mathrm{E}-10$ & $1.19 \mathrm{E}-07$ & $2.28 \mathrm{E}-07$ & 4.22E-07 & I. $48 E-09$ & $1.52 \mathrm{E}-11$ & $2.35 \mathrm{E}-09$ & $4.52 E-09$ & $8.36 \mathrm{E}-09$ \\
\hline Supernatant & па & na & na & na & na & na & na & na & na & na \\
\hline Solid Layer & $7.45 \mathrm{E}-08$ & $7.68 \mathrm{E}-10$ & $1.19 \mathrm{E}-07$ & $2.28 \mathrm{E}-07$ & $4.22 \mathrm{E}-07$ & $1.48 \mathrm{E}-09$ & $1.52 \mathrm{E}-11$ & $2.35 \mathrm{E}-09$ & $4.52 \mathrm{E}-09$ & $8.36 \mathrm{E}-09$ \\
\hline 24l-BY-105 & $3.74 \mathrm{E}-08$ & $7.03 \mathrm{E}-10$ & $5,52 \mathrm{E}-08$ & $1.96 \mathrm{E}-07$ & $2,89 \mathrm{E}-07$ & $7.79 \mathrm{E}-10$ & $1.46 \mathrm{E}-11$ & $1.15 E-09$ & $4.08 \mathrm{E}-09$ & $6.02 \mathrm{E}-09$ \\
\hline Superratant & nа & na & na & na & па & na & na & na & na & na \\
\hline Solid Layer & $3.74 \mathrm{E}-08$ & $7.03 \mathrm{E}-10$ & $5.52 E-08$ & $1.96 \mathrm{E}-07$ & $2.89 \mathrm{E}-07$ & $7.79 \mathrm{E}-10$ & $1.46 \mathrm{E}-11$ & $1.15 \mathrm{E}-09$ & $4.08 \mathrm{E}-09$ & $6.02 \mathrm{E}-0.9$ \\
\hline $241-\mathrm{BY}-106$ & $1.21 \mathrm{E}-07$ & $5.27 \mathrm{E}-10$ & $9.84 \mathrm{E}-08$ & $2.29 \mathrm{E}-07$ & $4.48 E-07$ & $2.30 \mathrm{E}-09$ & $1.01 \mathrm{E}-1 !$ & $1.88 \mathrm{E}-09$ & $4.36 \mathrm{E}-09$ & $8.55 \mathrm{E}-09$ \\
\hline Supenatant & na & na & na & na & па & na & na & na & na & na \\
\hline Solid Layer & $1.21 E_{-0} .07$ & $5.27 \mathrm{E}-10$ & $9.84 \mathrm{E}-08$ & $2.29 \mathrm{E}-07$ & $4.48 \mathrm{E}-07$ & $2.30 \mathrm{E}-09$ & $1.01 \mathrm{E}-11$ & $1.88 \mathrm{E}-09$ & $4.36 \mathrm{E}-09$ & $8.55 \mathrm{E}-09$ \\
\hline
\end{tabular}




\section{RPP-5926 REV 6}

Table B-3. Calculated Hydrogen Generation Rate as Mole Per Unit Waste Weight and Volume for 177 Tanks. (20 sheets)

\begin{tabular}{|c|c|c|c|c|c|c|c|c|c|c|}
\hline Tank & $\begin{array}{l}\text { HGR from } \\
\text { radiolysis } \\
\mathbf{R C}_{\text {rad }} \mathbf{B} / \gamma \\
(\text { mole } / \mathrm{kg}-\mathrm{d})\end{array}$ & $\begin{array}{l}\text { HGR from } \\
\text { radiolysis } \\
\mathrm{RC}_{\text {rad }}^{\text {alpha }} \\
\text { (mole/kg-d) }\end{array}$ & $\begin{array}{l}\text { HGR from } \\
\text { thermolysis } \\
\text { RC }_{\text {therm }} \\
\text { (mole/kg-d) }\end{array}$ & $\begin{array}{l}\text { HGR from } \\
\text { corrosion } \\
\text { RC }_{\text {rorr }} \\
\text { (mole/kg-d) }\end{array}$ & $\begin{array}{c}\text { Total HGR } \\
\text { from model } \\
R_{\text {lot }} \\
\text { (mole/kg-d) }\end{array}$ & $\begin{array}{l}\text { HGR from } \\
\text { radiolysis } \\
\mathrm{RC}_{\mathrm{rad}}^{\mathrm{b} / \mathrm{r}} \\
\left.\text { (mole } / \mathrm{m}^{3}-\mathrm{s}\right)\end{array}$ & $\begin{array}{l}\text { HGR from } \\
\text { radiolysis } \\
\mathrm{RC}_{\text {rad }} \text { alpha } \\
\text { (mole/m } \text { m }^{3} \text { ) }\end{array}$ & $\begin{array}{c}\text { HGR from } \\
\text { thermolysis } \\
\mathrm{RC}_{\text {therm }} \\
\left.\text { (mole } / \mathrm{m}^{3}-\mathrm{s}\right)\end{array}$ & $\begin{array}{c}\text { HGR from } \\
\text { corrosion } \\
\text { RC }_{\text {corr }} \\
\text { (mole/m } \text { m }^{3} \text {-s) }\end{array}$ & $\begin{array}{c}\text { Total HGR } \\
\text { from model } \\
\text { RCtot } \\
\left.\text { (mole } / \mathrm{m}^{3}-\mathrm{s}\right)\end{array}$ \\
\hline 241-BY-107 & $7.29 \mathrm{E}-08$ & 4.16E-10 & 6.14E-08 & $3.00 E-07$ & $4.35 \mathrm{E}-07$ & 1.43E- 09 & $8.13 \mathrm{E}-12$ & $1.20 \mathrm{E}-09$ & $5.87 \mathrm{E}-09$ & $8.51 \mathrm{E}-09$ \\
\hline Supernatant & na & na & na & na & ua & nas & na & na & na & na \\
\hline Solid Layer & $7.29 \mathrm{E}-08$ & $4.16 \mathrm{E}-10$ & $6.14 \mathrm{E}-08$ & $3.00 \mathrm{E}-07$ & $4.35 \mathrm{E}-07$ & 1.43E-09 & $8.13 E-12$ & $1.20 \mathrm{E}-09$ & $5.87 \mathrm{E}-09$ & $8.51 \mathrm{E}-09$ \\
\hline 241-BY-108 & $6.58 \mathrm{E}-08$ & $6.59 \mathrm{E}-10$ & $6.57 \mathrm{E}-08$ & $3.95 \mathrm{E}-07$ & $5.27 \mathrm{E}-07$ & 1. $13 E-09$ & $1.13 \mathrm{E}-11$ & $1.13 \mathrm{E}-09$ & $6.79 \mathrm{E}-09$ & $9.06 E-09$ \\
\hline Supematant & na & na & nа & na & na & na & na & na & na & na \\
\hline Solid Layer & $6.58 \mathrm{E}-08$ & $6.59 \mathrm{E}-10$ & $6.57 \mathrm{E}-08$ & $3.95 \mathrm{E}-07$ & $5.27 \mathrm{E}-07$ & $1.13 \mathrm{E}-09$ & $1.13 \mathrm{E}-11$ & $1.13 E-09$ & $6.79 \mathrm{E}-09$ & $9.06 \mathrm{E}-09$ \\
\hline $241-\mathrm{BY}-109$ & $1.96 \mathrm{E}-08$ & $6.53 E-10$ & $6.52 \mathrm{E}-09$ & $2.86 \mathrm{E}-07$ & $3.13 E-07$ & $3.87 E-10$ & $1.29 \mathrm{E}-11$ & $1.29 \mathrm{E}-10$ & $5.65 \mathrm{E}-09$ & $6.18 \mathrm{E}-09$ \\
\hline Supernatant & na & na & na & na & na & na & na & nà & na & na \\
\hline Solid Layer & $1.96 \mathrm{E}-08$ & $6.53 E-10$ & $6.52 E-09$ & $2.86 \mathrm{E}-07$ & $3.13 E-07$ & $3.87 \mathrm{E}-10$ & $1.29 \mathrm{E}-11$ & $1.29 \mathrm{E}-10$ & $5.65 \mathrm{E}-09$ & 6.18E-09 \\
\hline $241-B Y-110$ & $8.81 \mathrm{E}-08$ & $6.5 \mathrm{JE}-10$ & $4.74 \mathrm{E}-07$ & $2.66 \mathrm{E}-07$ & $8.28 \mathrm{E}-07$ & $1.60 \mathrm{E}-09$ & $1.18 E-11$ & $8.58 \mathrm{E}-09$ & $4.81 E-0.9$ & $1.50 \mathrm{E}-08$ \\
\hline Supernatant & na & na & na & na & na & na & na & na & na & na \\
\hline Solid Layer & $8.81 \mathrm{E}-08$ & $6.51 \mathrm{E}-10$ & $4.74 \mathrm{E} \cdot 07$ & $2.66 \mathrm{E}-07$ & $8.28 \mathrm{E}-07$ & $1.60 \mathrm{E}-09$ & $1.18 \mathrm{E}-11$ & $8.58 \mathrm{E}-09$ & 4.81E-09 & 1.50E-08 \\
\hline $241-B Y-111$ & $5.52 E-09$ & $2.70 \mathrm{E}-10$ & $2.98 \mathrm{E}-09$ & $2.34 \mathrm{E}-07$ & $2.43 \mathrm{E}-07$ & $1.07 t-10$ & $5.22 \mathrm{E}-12$ & $5.78 E-11$ & $4.53 \mathrm{E}-09$ & 4. $70 \mathrm{E}-09$ \\
\hline Supernatant & na & na & na & па & na & na & na & na & na & na \\
\hline Solid Layer & $5.52 \mathrm{E}-09$ & $2.70 \mathrm{E}-10$ & $2.98 \mathrm{E}-09$ & $2.34 \mathrm{E}-07$ & $2.43 \mathrm{E}-07$ & $1.07 \mathrm{E}-10$ & $5.22 \mathrm{E}-12$ & $5.78 \mathrm{E}-11$ & 4.53E-09 & $4.70 \mathrm{E}-09$ \\
\hline $241-\mathrm{BY}-112$ & 8.65E-09 & $2.40 \mathrm{E}-10$ & 7.95E-09 & $2.81 \mathrm{E}-07$ & $2.98 \mathrm{E}-07$ & $1.74 \mathrm{E}-10$ & $4.83 \mathrm{E}-12$ & $1.60 \mathrm{E}-10$ & $5.67 \mathrm{E}-09$ & $6.00 \mathrm{E}-09$ \\
\hline Supernatant & na & na & na & na & na & na & na & na & na & na \\
\hline Solid Layer & 8.65E-09 & $2.40 \mathrm{E}-10$ & $7.95 \mathrm{E}-09$ & $2.81 \mathrm{E}-07$ & $2.98 \mathrm{E}-07$ & $1.74 \mathrm{E}-10$ & 4. $83 \mathrm{E}-12$ & $1.60 \mathrm{E}-10$ & $5.67 \mathrm{E}-09$ & $6.00 \mathrm{E}-09$ \\
\hline $241-C-101$ & $1.22 E-07$ & $2.18 \mathrm{E}-08$ & 3.05 E- -08 & $7.03 \mathrm{E}-07$ & $8.77 \mathrm{E}-07$ & $2.52 E-09$ & $4.50 \mathrm{E}-10$ & $6.28 \mathrm{E}-10$ & 1.45 $=08$ & $1.83 \mathrm{E}-08$ \\
\hline Supematant & nа & na & na & na & na & na & na & na & na & 11a \\
\hline Solid Layer & $1.22 \mathrm{E}-07$ & $2.18 \mathrm{E}-08$ & $3.05 E-08$ & $7.03 \mathrm{E}-07$ & $8.77 \mathrm{E}-07$ & $2.52 \mathrm{E}-09$ & $4.50 \mathrm{E}-10$ & $6.28 \mathrm{E}-10$ & $1.45 \mathrm{E}-08$ & 1.81E-08 \\
\hline $241-\mathrm{C}-102$ & $3.80 \mathrm{E}-08$ & $7.32 E-08$ & $2.82 \mathrm{E}-08$ & $2.72 \mathrm{E}-07$ & 4.11E-07 & $7.39 \mathrm{E}-10$ & $1.42 E-09$ & $5.49 \mathrm{E}-10$ & $5.30 \mathrm{E}-09$ & $8.01 \mathrm{E}-0.9$ \\
\hline Supernatant & na & na & na & na & na & na & na & na & na & na \\
\hline Solid Layer & $3.80 \mathrm{E}-08$ & 7.32E-08 & $2.82 \mathrm{E}-08$ & $2.72 \mathrm{E}-07$ & $4.11 \mathrm{E} \cdot 07$ & $7.39 \mathrm{E}-10$ & $1.42 E-09$ & $5.49 \mathrm{E}-10$ & $5.30 \mathrm{E}-09$ & $8.01 \mathrm{E}-09$ \\
\hline $241-C-103$ & $1.80 \mathrm{E}-06$ & $1.07 \mathrm{E}-07$ & $0.00 E+00$ & $5.79 \mathrm{E}-06$ & $7.70 \mathrm{E}-06$ & $3.39 \mathrm{E}-08$ & $2.02 \mathrm{E}-09$ & $0.00 \mathrm{E}+00$ & 1.09E-07 & 1.45E-07 \\
\hline Supenatant & na & na & na & na & na & na & na & na & па & na \\
\hline Solid Layer & $1.80 \mathrm{E}-06$ & $1.07 \mathrm{E}-07$ & $0.00 \mathrm{E}+00$ & $5.79 \mathrm{E}-06$ & $7.70 \mathrm{E}-06$ & 3.39E-08 & $2.02 E-09$ & $0.00 \mathrm{E}+00$ & $1.09 \mathrm{E}-07$ & $1.45 \mathrm{E}-07$ \\
\hline
\end{tabular}


Table B-3. Calculated Hydrogen Generation Rate as Mole Per Unit Waste Weight and Volume for 177 Tanks. (20 sheets)

\begin{tabular}{|c|c|c|c|c|c|c|c|c|c|c|}
\hline Tank & $\begin{array}{l}\text { HGR from } \\
\text { radiolysis } \\
\mathrm{RC}_{\mathrm{rad}} \mathrm{B}^{\prime} / \mathrm{j} \\
\text { (mole } / \mathrm{kg}-\mathrm{d})\end{array}$ & $\begin{array}{l}\text { HGR from } \\
\text { radiolysis } \\
\text { RC }_{\text {rad }} \text { alpha } \\
\text { (mole } / \mathbf{k g - d} \text { ) }\end{array}$ & $\begin{array}{l}\text { HGR from } \\
\text { thermolysis } \\
\text { RC }_{\text {tberm }} \\
\text { (mole } / \mathbf{k g}^{-\mathrm{d})}\end{array}$ & $\begin{array}{l}\text { HGR from } \\
\text { corrosion } \\
\text { RC corr } \\
\text { (mole } / \text { kg-d) }\end{array}$ & $\begin{array}{c}\text { Total HGR } \\
\text { from model } \\
\mathbf{R C}_{\text {tol }} \\
\text { (mole/kg-d) }\end{array}$ & $\begin{array}{l}\text { HGR from } \\
\text { radiolysis } \\
\mathrm{RC}_{\mathrm{rad}}^{\mathrm{bir}} \\
\left(\mathrm{mole} / \mathrm{m}^{3}-\mathrm{s}\right)\end{array}$ & $\begin{array}{l}\text { HGR from } \\
\text { radiolysis } \\
\mathbf{R C}_{r \text { ad }}^{\text {alpha }} \\
\left.\text { (mole } / \mathrm{m}^{3}-\mathrm{s}\right)\end{array}$ & $\begin{array}{l}\text { HGR from } \\
\text { thermolysis } \\
\mathrm{RC}_{\text {therm }} \\
\left(\mathrm{mole} / \mathrm{m}^{3} \cdot \mathrm{s}\right)\end{array}$ & $\begin{array}{l}\text { HGR from } \\
\text { corrosion } \\
\text { RC }_{\text {corr }} \\
\left.\text { (mole } / \mathrm{m}^{3}-\mathbf{s}\right)\end{array}$ & $\begin{array}{c}\text { Total HGR } \\
\text { from model } \\
\text { RCtot } \\
\left.\text { (mole/m } \text { m }^{3}-s\right)\end{array}$ \\
\hline $24 !-C-104$ & $7.66 \mathrm{E}-07$ & $3.12 \mathrm{E}-07$ & $1.71 \mathrm{E}-07$ & $3.13 \mathrm{E}-07$ & $1.56 \mathrm{E}-06$ & $1.49 \mathrm{E}-08$ & $6.07 \mathrm{E}-09$ & $3.32 \mathrm{E}-09$ & $6.08 \mathrm{E}-09$ & $3.04 \mathrm{E}-08$ \\
\hline Supematant & na & na & na & na & nа & па & na & na & na & na \\
\hline Solid Layer & $7.66 \mathrm{E}-07$ & 3.12E-07 & $1.71 \mathrm{E}-07$ & $3.13 \mathrm{E}-07$ & $1.56 \mathrm{E}-06$ & $1.49 \mathrm{E}-08$ & $6.07 \mathrm{E}-09$ & 3.32E-09 & $6.08 \mathrm{E}-09$ & $3.04 \mathrm{E}-08$ \\
\hline $241-C \cdot 105$ & $9.04 \mathrm{E}-07$ & $2.06 E-07$ & $1.54 \mathrm{E}-08$ & $5.70 \mathrm{E}-07$ & $1.70 \mathrm{E}-06$ & 1.62E-08 & $3.69 \mathrm{E}-09$ & $2.77 \mathrm{E}-10$ & $1.02 E-08$ & $3.04 \mathrm{E}-08$ \\
\hline Supematant & na & na & na & $\mathrm{na}$ & na & na & na & $\mathrm{na}$ & na & na \\
\hline Solid Layer & $9.04 \mathrm{E}-07$ & $2.06 \mathrm{E}-07$ & $1.54 E-08$ & $5.70 \mathrm{E}-07$ & $1.70 \mathrm{E}-06$ & $1.62 \mathrm{E}-08$ & $3.69 \mathrm{E}-09$ & $2.77 \mathrm{E} \cdot 10$ & $1.02 \mathrm{E}-08$ & $3.04 \mathrm{E}-08$ \\
\hline $241 \cdot C \cdot 106$ & $4.50 \mathrm{E}-05$ & $7.71 \mathrm{E}-07$ & $7.64 \mathrm{E}-10$ & $5.68 \mathrm{E}-05$ & $1.03 E-04$ & $8.03 \mathrm{E}-07$ & $1.38 \mathrm{E}-08$ & $1.36 \mathrm{E}-11$ & $1.01 \mathrm{E}-06$ & $1.83 \mathrm{E}-06$ \\
\hline Supernatant & $8.49 \mathrm{E}-09$ & $3.03 \mathrm{E}-12$ & $2.37 \mathrm{E}-09$ & $3.70 \mathrm{E}-07$ & $3.81 \mathrm{E}-07$ & $1.00 \mathrm{E}-10$ & $3.57 \mathrm{E}-14$ & $2.80 \mathrm{E}-11$ & $4.37 \mathrm{E}-09$ & $4.50 \mathrm{E}-09$ \\
\hline Solid Layer & $4.47 \mathrm{E}-05$ & $7.67 \mathrm{E}-07$ & $7.38 \mathrm{E}-10$ & $5.80 \mathrm{E}-05$ & $1.03 \mathrm{E}-04$ & $8.07 \mathrm{E}-07$ & $1.38 \mathrm{E}-08$ & $1.33 \mathrm{E}-11$ & $1.05 \mathrm{E}-06$ & $1.87 \mathrm{E}-06$ \\
\hline $241-C-107$ & $2.41 \mathrm{E}-06$ & $1.74 \mathrm{E}-07$ & $1.30 \mathrm{E}-07$ & $3.50 \mathrm{E}-07$ & 3.06E-06 & $4.32 \mathrm{E}-08$ & $3.12 \mathrm{E}-09$ & $2.34 \mathrm{E}-09$ & $6.28 \mathrm{~F}-09$ & $5.50 \mathrm{E}-08$ \\
\hline Supernatant & na & ta & na & na & na & na & na. & na & na & na \\
\hline Solid Layer & $2.41 \mathrm{E}-06$ & $1.74 \mathrm{E}-07$ & $1.30 \mathrm{E}-07$ & $3.50 \mathrm{E}-07$ & $3.06 \mathrm{E}-06$ & $4.32 \mathrm{E}-08$ & $3.12 \mathrm{E}-09$ & $2.34 \mathrm{E}-09$ & $6.28 \mathrm{E}-09$ & $5.50 \mathrm{E}-08$ \\
\hline $24 !-C-108$ & $3.29 \mathrm{E}-07$ & $1.31 \mathrm{E}-09$ & $2.55 \mathrm{E}-08$ & $1.09 \mathrm{E}-06$ & $1.45 \mathrm{E}-06$ & $5.64 \mathrm{E}-09$ & $2.24 E-11$ & $4.36 \mathrm{E}-10$ & $1.87 \mathrm{E}-08$ & $2.48 \mathrm{E}-08$ \\
\hline Supernatant & na & na & na & na & na & na & па & па & na & na \\
\hline Solid Layer & $3.29 \mathrm{E}-07$ & 1.31E-09 & $2.55 E-08$ & $1.09 E-06$ & $1.45 \mathrm{E}-06$ & $5.64 E-09$ & $2.24 E-11$ & $4.36 \mathrm{E}-10$ & $1.87 \mathrm{E}-08$ & $2.48 \mathrm{E}-08$ \\
\hline $241-C-109$ & $2.31 \mathrm{E}-06$ & $1.36 \mathrm{E}-08$ & $2.73 \mathrm{E}-08$ & $1.08 \mathrm{E}-06$ & $3.43 \mathrm{E}-06$ & 4.14E-08 & $2.43 \mathrm{E}-10$ & $4.89 \mathrm{E}-10$ & $1.94 \mathrm{E}-08$ & $6.15 \mathrm{E}-08$ \\
\hline Supenatant & na & na & na & na & na & na & na & na & na & па \\
\hline Solid Layer & $2.31 E-06$ & $1.36 \mathrm{E}-08$ & $2.73 \mathrm{E} \cdot 08$ & $1.08 \mathrm{E}-06$ & $3.43 \mathrm{E}-06$ & 4.14E- 08 & $2.43 \mathrm{E} \cdot 10$ & $4.89 \mathrm{E}-10$ & $1.94 \mathrm{E}-08$ & $6.15 \mathrm{E}-08$ \\
\hline $241-\mathrm{C}-110$ & $4.58 \mathrm{E}-08$ & $5.96 \mathrm{E}-09$ & $8.09 \mathrm{E}-10$ & $1.30 \mathrm{E}-06$ & 1.35E-06 & $7.10 \mathrm{E}-10$ & $9.24 \mathrm{E}-11$ & $1.25 \mathrm{E}-11$ & $2.01 \mathrm{E}-08$ & $2.09 \mathrm{E}-08$ \\
\hline Supernatant & $1.38 \mathrm{E}-08$ & $1.37 \mathrm{E}-10$ & $1.50 \mathrm{E}-09$ & $3.43 \mathrm{E}-07$ & $3.59 \mathrm{E}-07$ & $1.76 \mathrm{E}-10$ & $1.75 \mathrm{E}-12$ & $1.91 \mathrm{E}-11$ & $4.37 \mathrm{E}-09$ & $4.57 \mathrm{E}-09$ \\
\hline Solid Layer & $4.58 \mathrm{E}-08$ & $5.97 \mathrm{E}-09$ & $8.06 \mathrm{E}-10$ & $1.30 \mathrm{E}-06$ & 1. $35 \mathrm{E}-06$ & $7.11 E-10$ & $9.26 \mathrm{E}-11$ & $1.25 E-11$ & $2.02 \mathrm{E}-08$ & $2.10 \mathrm{E}-08$ \\
\hline 241-C-111 & $5.70 \mathrm{E}-06$ & $4.24 \mathrm{E}-08$ & $2.32 \mathrm{E}-08$ & 1.19E-06 & $6.96 E-06$ & $1.02 \mathrm{E}-07$ & $7.58 \mathrm{E}-10$ & 4.15E-10 & $2.13 E-08$ & $1.24 \mathrm{E}-07$ \\
\hline Supentatant & па & nia & na & na & na & na & па & па & na & na \\
\hline Solid Layer & $5.70 \mathrm{E}-06$ & $4.24 \mathrm{E}-08$ & $2.32 \mathrm{E}-0.0$ & $1.19 \mathrm{E}-06$ & 6.96E-06 & $1.02 \mathrm{E}-07$ & $7.58 \mathrm{E}-10$ & $4.15 \mathrm{E}-10$ & $2.13 \mathrm{E}-08$ & $1.24 \mathrm{E}-07$ \\
\hline $241-C \cdot 112$ & $3.28 \mathrm{E}-06$ & $4.20 E-08$ & $3.84 \mathrm{E}-08$ & $6.77 \mathrm{E}-07$ & $4.04 \mathrm{E}-06$ & $6.08 \mathrm{E}-08$ & $7.79 \mathrm{E}-10$ & $7.11 \mathrm{E}-10$ & $1.25 \mathrm{E}-08$ & $7.48 \mathrm{E}-08$ \\
\hline Supernatant & na & na & na & na & แа & na & na & na & na & na \\
\hline Solid Layer & $3.28 \mathrm{E}-06$ & $4.20 \mathrm{E}-08$ & $3.84 \mathrm{E}-08$ & $6.77 \mathrm{E}-07$ & $4.04 \mathrm{E}-06$ & $6.08 \mathrm{E}-08$ & $7.79 \mathrm{E}-10$ & $7.11 \mathrm{E}-10$ & $1.25 \mathrm{E}-08$ & $7.48 \mathrm{E}-08$ \\
\hline
\end{tabular}


RPP-5926 REV 6

Table B-3. Calculated Hydrogen Generation Rate as Mole Per Unit Waste Weight and Volume for 177 Tanks. (20 sheets)

\begin{tabular}{|c|c|c|c|c|c|c|c|c|c|c|}
\hline Tank & $\begin{array}{l}\text { HGR from } \\
\text { radiolysis } \\
R_{\text {rad }} / 2 \\
\text { (mole/kg-d) }\end{array}$ & $\begin{array}{l}\text { HGR from } \\
\text { radiolysis } \\
R_{\text {rad }} \text { glpha } \\
\text { (mole/kg-d) }\end{array}$ & $\begin{array}{l}\text { HGR from } \\
\text { thermolysis } \\
\text { RC }_{\text {therm }} \\
\text { (mole/kg-d) }\end{array}$ & $\begin{array}{l}\text { HGR from } \\
\text { corrosion } \\
\text { RC }_{\text {corr }} \\
\text { (mole } / \mathbf{k g - d})\end{array}$ & $\begin{array}{l}\text { Total HGR } \\
\text { from model } \\
\mathbf{R C}_{\text {tox }} \\
\text { (mole } / \mathrm{kg} \text {-d) }\end{array}$ & $\begin{array}{l}\text { HGR from } \\
\text { radiolysis } \\
\mathrm{RC}_{\mathrm{rad}}{ }^{\mathrm{s} / \mathrm{r}} \\
\left(\mathrm{mole} / \mathrm{m}^{3}-\mathrm{s}\right)\end{array}$ & $\begin{array}{l}\text { HGR from } \\
\text { radiolysis } \\
\mathrm{RC}_{\text {rat }} \text { alpha } \\
\left(\mathrm{mole} / \mathrm{m}^{3}-\mathrm{s}\right)\end{array}$ & $\begin{array}{l}\text { HGR from } \\
\text { thermolysis } \\
\text { RC }_{\text {therm }} \\
\left.\text { (mole } / \mathrm{m}^{3}-\mathrm{s}\right)\end{array}$ & $\begin{array}{l}\text { HGR from } \\
\text { corrosion } \\
R_{\text {corr }} \\
\left.\text { (mole } / \mathrm{m}^{3}-\mathrm{s}\right)\end{array}$ & $\begin{array}{c}\text { Total HGR } \\
\text { from model } \\
\text { RCtot } \\
\left.\text { (mole/m } / \mathrm{m}^{3}-\mathrm{s}\right)\end{array}$ \\
\hline $241-C-201$ & $1.84 \mathrm{E}-06$ & $2.25 \mathrm{E}-06$ & $0.00 E+00$ & $7.07 E-05$ & $7.48 \mathrm{E}-05$ & $3.71 \mathrm{E}-08$ & $4.54 \mathrm{E}-08$ & $0.00 \mathrm{E}+00$ & 1. $42 \mathrm{E}-06$ & $1.51 \mathrm{E} \cdot 06$ \\
\hline Supernatant & $0.00 \mathrm{E}+00$ & $0.00 \mathrm{E}+00$ & $0.00 \mathrm{E}+00$ & 1.42E-06 & $1.42 E-06$ & $0.00 \mathrm{E}+00$ & $0.00 \mathrm{E}+00$ & $0.00 \mathrm{E}+00$ & 1. $64 E-08$ & $1.64 \mathrm{E}-08$ \\
\hline Solid Layer & $1.81 \mathrm{E}-06$ & $2.22 \mathrm{E}-06$ & $0.00 \mathrm{E}+00$ & $7.12 \mathrm{E}-05$ & $7.52 \mathrm{E}-05$ & $3.68 \mathrm{E}-08$ & 4. $50 \mathrm{E}-08$ & $0.00 \mathrm{E}+00$ & $1.44 \mathrm{E}-06$ & $1.52 \mathrm{E}-06$ \\
\hline $241-C-202$ & $3.45 \mathrm{E}-06$ & $1.90 \mathrm{E}-06$ & $0.00 \mathrm{E}+00$ & $6.92 E-05$ & $7.45 \mathrm{E}-0.05$ & $6.94 \mathrm{E}-08$ & $3.81 E-08$ & $0.00 \mathrm{E}+00$ & $1.39 \mathrm{H}-06$ & $1.50 \mathrm{E}-06$ \\
\hline Supernatant & $0.00 \mathrm{E}+00$ & $0.00 \mathrm{E}+00$ & $0.00 \mathrm{E}+00$ & $1.42 \mathrm{E}-06$ & $1.42 \mathrm{E}-06$ & $0.00 \mathrm{E}+00$ & $0.00 \mathrm{E}+00$ & $0.00 \mathrm{E}+00$ & $1.64 \mathrm{E}-08$ & $1.64 \mathrm{E}-08$ \\
\hline Solid Layer & $3.38 \mathrm{E}-06$ & $1.86 \mathrm{E}-06$ & $0.00 \mathrm{E}+00$ & $6.98 \mathrm{E}-05$ & $7.50 \mathrm{E}-05$ & $6.86 \mathrm{E}-08$ & 3. $77 \mathrm{E}-08$ & $0.00 \mathrm{E}+00$ & $1.4 ! \mathrm{E}-06$ & $1.52 \mathrm{E}-06$ \\
\hline $241-C-203$ & $1.25 \mathrm{E}-07$ & $9.79 \mathrm{E}-08$ & $0.00 \mathrm{E}+00$ & $6.92 \mathrm{E}-05$ & $6.94 \mathrm{E}-05$ & $2.68 E .09$ & $2.09 \mathrm{E}-09$ & $0.00 \mathrm{E}+00$ & $1.48 \mathrm{E}-06$ & $1.48 \mathrm{E}-06$ \\
\hline Supernatant & $0.00 \mathrm{E}+00$ & $0.00 \mathrm{E}+00$ & $0.00 \mathrm{E}+00$ & 1.42E-06 & $1.42 \mathrm{E}-06$ & $0.00 \mathrm{E}+00$ & $0.00 \mathrm{E}+00$ & $0,00 \mathrm{E}+00$ & $1.64 E-08$ & $1.64 \mathrm{E}-08$ \\
\hline Solid Layer & $1.21 \mathrm{E}-07$ & $9.45 E-08$ & $0.00 \mathrm{E}+00$ & $7.27 \mathrm{E}-05$ & 7.30E-05 & $2.71 \mathrm{E}-09$ & $2.11 \mathrm{E}-09$ & $0.00 \mathrm{E}+00$ & $1.62 \mathrm{E}-06$ & $1.63 \mathrm{E}-06$ \\
\hline $241-C-204$ & $2.91 \mathrm{E}-08$ & $2.98 \mathrm{E}-10$ & $9.03 \mathrm{E}-09$ & $3.25 \mathrm{E}-06$ & $3.29 \mathrm{E}-06$ & $5.45 \mathrm{E}-10$ & $5.59 \mathrm{E}-12$ & $1.69 \mathrm{E}-10$ & $6.10 \mathrm{E}-08$ & $6.17 \mathrm{E}-08$ \\
\hline Supernatant & na & па & na & na & na & na & na & na & na & na \\
\hline Solid Layer & $2.91 \mathrm{E}-08$ & $2.98 \mathrm{E}-10$ & $9.03 \mathrm{E}-09$ & $3.25 \mathrm{E}-06$ & $3.29 \mathrm{E}-06$ & $5.45 \mathrm{E} \times 10$ & $5.59 \mathrm{E}-12$ & $1.69 \mathrm{E}-30$ & $6.10 \mathrm{E}-08$ & $6.17 \mathrm{~F}-08$ \\
\hline $241-S-101$ & $3.18 \mathrm{E}-07$ & $5.95 \mathrm{E}-09$ & 1.52E-07 & $2.58 E-07$ & $7.34 \mathrm{E}-07$ & $6.08 \mathrm{E}-09$ & $1.14 \mathrm{E}-10$ & $2.91 \mathrm{E}-09$ & $4.93 \mathrm{E}-09$ & 1.40E-08 \\
\hline Supernatant & па & na & ua & na & na & na & na & na & na & na \\
\hline Solid Layer & $3.18 \mathrm{E}-07$ & $5.95 \mathrm{E}-09$ & $1.52 \mathrm{E}-07$ & $2.58 \mathrm{E} \cdot 07$ & $7.34 \mathrm{E}-07$ & $6.08 \mathrm{E}-09$ & $1.14 E-10$ & $2.91 \mathrm{E}-09$ & 4. $93 \mathrm{E}-09$ & $1.40 \mathrm{E}-08$ \\
\hline 24$]-S-102$ & $1.40 \mathrm{E}-07$ & 4.19E- 09 & $2.47 \mathrm{E}-07$ & $4.25 \mathrm{E}-07$ & $8.16 \mathrm{E}-07$ & $2.78 \mathrm{E}-09$ & $8.29 \mathrm{E}-11$ & $4.89 \mathrm{E}-09$ & $8.41 \mathrm{E}-0.9$ & $1.62 E-08$ \\
\hline Supernatant & na & na & na & na & na & па & $\mathrm{na}$ & na & na & na \\
\hline Solid Layer & $1.40 \mathrm{E}-07$ & 4.19E-09 & $2.47 \mathrm{E}-07$ & $4.25 \mathrm{E}-07$ & $8.16 \mathrm{E}-07$ & $2.78 \mathrm{E}-09$ & $8.29 \mathrm{E}-11$ & $4.89 \mathrm{E}-09$ & $8.41 E-09$ & $1.62 \mathrm{E}-08$ \\
\hline $241-S-103$ & 1.36E- 07 & $3.34 \mathrm{E}-09$ & $9.00 \mathrm{E}-08$ & $3.48 \mathrm{E}-07$ & $5.77 \mathrm{E}-07$ & $2.54 \mathrm{E}-09$ & $6,23 \mathrm{E}-11$ & $1.68 \mathrm{E}-09$ & $6.48 \mathrm{E}-09$ & $1.08 \mathrm{E}-08$ \\
\hline Supernatant & $2.40 \mathrm{E}-07$ & $2.37 \mathrm{E}-11$ & $1.25 \mathrm{E}-07$ & $1.04 \mathrm{E}-07$ & $4.70 \mathrm{E}-07$ & $4.03 E-09$ & $3.98 \mathrm{E}-13$ & $2.10 \mathrm{E}-09$ & $1.75 \mathrm{E}-09$ & $7.89 \mathrm{E}-09$ \\
\hline Solid Layer & $1.36 \mathrm{E}-07$ & $3.35 \mathrm{E}-09$ & $8.98 \mathrm{E}-0 \mathrm{~B}$ & $3.49 E-07$ & $5.77 \mathrm{E}-07$ & $2.53 \mathrm{E}-09$ & $6.25 \mathrm{E}-11$ & $1.68 \mathrm{E}-09$ & $6.50 \mathrm{E}-09$ & $1.08 \mathrm{E}-08$ \\
\hline $241-S-104$ & $2.25 \mathrm{E}-07$ & $6.02 \mathrm{E}-09$ & $1.98 \mathrm{E}-08$ & 2.92E-07 & $5.43 \mathrm{E}-07$ & $4.34 \mathrm{E}-09$ & 1. $16 \mathrm{E}-10$ & $3.83 \mathrm{E}-10$ & $5.64 \mathrm{~L}-09$ & $1.05 \mathrm{E}-08$ \\
\hline Supematant & na & na & па & na & па & na & na & na & na & na \\
\hline Solid Layer & $2.25 \mathrm{E}-07$ & $6.02 \mathrm{E}-09$ & $1.98 \mathrm{E}-08$ & $2.92 \mathrm{E}-07$ & $5.43 \mathrm{E}-07$ & 4.34E-09 & 1.16E-10 & 3.83E-10 & $5.64 \mathrm{E}-09$ & $1.05 \mathrm{E}-08$ \\
\hline 241-S- 105 & $1.77 \mathrm{E}-09$ & $1.11 \mathrm{E}-11$ & $4.69 \mathrm{E}-09$ & $2.35 \mathrm{E}-07$ & $2.42 \mathrm{E}-07$ & $3.40 \mathrm{E}-11$ & $2.13 \mathrm{E}-13$ & $9.00 \mathrm{E}-11$ & $4.51 \mathrm{E}-09$ & $4.64 \mathrm{E}-09$ \\
\hline Supernatant & na & na & na & na & na & na & na & na & na & na \\
\hline Solid Layer & $1.77 \mathrm{E}-09$ & $1.11 \mathrm{E}-11$ & $4.69 \mathrm{E}-09$ & $2.35 \mathrm{E}-07$ & $2.42 \mathrm{E}-07$ & $3.40 \mathrm{E}-11$ & $2.13 \mathrm{E}-13$ & $9.00 \mathrm{E}-11$ & $4.51 \mathrm{E}-09$ & $4.64 \mathrm{E}-0.9$ \\
\hline
\end{tabular}




\section{RPP-5926 REV 6}

Table B-3. Calculated Hydrogen Generation Rate as Mole Per Unit Waste Weight and Volume for 177 Tanks. (20 sheets)

\begin{tabular}{|c|c|c|c|c|c|c|c|c|c|c|}
\hline Tank & $\begin{array}{l}\text { HGR from } \\
\text { radiolysis } \\
\mathrm{RC}_{\mathrm{rad}}^{\mathrm{B} / \mathrm{\gamma}} \\
\text { (mole/kg-d) }\end{array}$ & $\begin{array}{l}\text { HGR from } \\
\text { radiolysis } \\
\mathrm{RC}_{\text {rad pha }} \\
\text { (mole } / \mathrm{kg}-\mathrm{d} \text { ) }\end{array}$ & $\begin{array}{l}\text { HGR from } \\
\text { thermolysis } \\
R_{\text {therm }} \\
\text { (mole/kg-d) }\end{array}$ & $\begin{array}{l}\text { HGR from } \\
\text { corrosion } \\
\text { RC }_{\text {iorr }} \\
\text { (mole } / \mathbf{k g - d} \text { ) }\end{array}$ & $\begin{array}{c}\text { Total HGR } \\
\text { from model } \\
\text { RC }_{\text {lot }} \\
\text { (mole } / \mathbf{k g - d} \text { ) }\end{array}$ & $\begin{array}{l}\text { HGR from } \\
\text { radiolysis } \\
R_{\text {rad }} \text { b/r } \\
\left.\text { (mole/m } \text { m }^{3}-s\right)\end{array}$ & $\begin{array}{l}\text { HGR from } \\
\text { radiolysis } \\
\mathrm{RC}_{\text {rad }} \text { alpha } \\
\left(\mathrm{mole} / \mathrm{m}^{3}-\mathrm{s}\right)\end{array}$ & $\begin{array}{l}\text { HGR from } \\
\text { thermolysis } \\
\text { RC }_{\text {therm }} \\
\left.\text { (mole/ } / \mathrm{m}^{3}-\mathrm{s}\right)\end{array}$ & $\begin{array}{l}\text { HGR from } \\
\text { corrosion } \\
\mathrm{RC}_{\text {corr }} \\
\left(\mathrm{mole} / \mathrm{m}^{3}-\mathrm{s}\right)\end{array}$ & $\begin{array}{c}\text { Total HGR } \\
\text { from model } \\
\text { RCtot } \\
\left.\text { (mole } / \mathrm{m}^{3}-\mathrm{s}\right)\end{array}$ \\
\hline $241-S-106$ & $5.61 \mathrm{E}-08$ & $4.56 \mathrm{E}-10$ & $2.46 \mathrm{E}-08$ & 2.11E-07 & $2.92 \mathrm{E}-07$ & 1.12E-09 & $9.08 \mathrm{E}-12$ & $4.90 \mathrm{E}-10$ & $4.21 \mathrm{E}-09$ & $5.83 \mathrm{E}-09$ \\
\hline Supematant & na & na & па & na & na & na & na & nа & na & na \\
\hline Solid Layer & $5.61 \mathrm{E}-0.8$ & $4,56 \mathrm{E}-10$ & $2.46 \mathrm{E}-08$ & $2.11 E-07$ & $2.92 \mathrm{E}-07$ & $1.12 \mathrm{E}-09$ & $9.08 \mathrm{E}-12$ & $4.90 \mathrm{E}-10$ & $4.21 \mathrm{E}-09$ & $5.83 \mathrm{E}-09$ \\
\hline $241-5-107$ & $1.93 \mathrm{E}-07$ & $2.30 \mathrm{E}-08$ & $2.71 \mathrm{E}-08$ & $2.37 \mathrm{~F}-07$ & $4.80 \mathrm{E}-07$ & $3.96 \mathrm{E}-09$ & $4.72 \mathrm{E}-10$ & $5.56 \mathrm{E}-10$ & $4.88 \mathrm{E}-09$ & $9.86 \mathrm{E}-09$ \\
\hline Supernatant & na & na & na & $\mathrm{na}$ & $\mathrm{na}$ & na & na & na & na & na \\
\hline Solid Layer & $1.93 \mathrm{E}-07$ & $2.30 \mathrm{E}-08$ & $2.71 \mathrm{E}-08$ & $2.37 \mathrm{E}-07$ & $4.80 \mathrm{E}-07$ & $3.96 \mathrm{E}-09$ & $4.72 \mathrm{E}-10$ & $5.56 \mathrm{E}-10$ & $4.88 \mathrm{E}-09$ & $9.86 \mathrm{E}-09$ \\
\hline $241-S-108$ & $3.44 \mathrm{E}-09$ & $9.79 \mathrm{E}-11$ & 3.35E-09 & $1.95 \mathrm{E}-07$ & $2.02 \mathrm{E}-07$ & $6.68 \mathrm{E}-11$ & $1.90 \mathrm{E}-12$ & $6.50 \mathrm{E}-11$ & $3.79 \mathrm{E}-09$ & $3.92 \mathrm{E}-09$ \\
\hline Supenatam & na & na & na & $\mathrm{na}$ & nа & na & na & na & па & na \\
\hline Solid Layer & 3.44E- 09 & $9.79 \mathrm{E}-\mathrm{J} 1$ & 3.35E-09 & $1.95 E-07$ & $2.02 \mathrm{E}-07$ & $6.68 \mathrm{E}-11$ & $1.90 \mathrm{E}-12$ & $6.50 \mathrm{E}-11$ & $3.79 \mathrm{E}-09$ & $3.92 \mathrm{E}-09$ \\
\hline $241-S-109$ & $5.83 \mathrm{E}-09$ & $2.38 \mathrm{E}-10$ & $6.14 \mathrm{E}-09$ & $2.01 \mathrm{E}-07$ & $2.13 \mathrm{E}-07$ & $1.12 \mathrm{E}-10$ & $4.57 \mathrm{E}-12$ & $1.18 \mathrm{E}-10$ & $3.85 \mathrm{E}-09$ & $4.09 \mathrm{E}-09$ \\
\hline Supernatant & na & na & na & na & па & na & па & na & na & na \\
\hline Solid Layer & $5.83 \mathrm{E}-09$ & $2.38 \mathrm{E}-10$ & $6.14 \mathrm{E}-09$ & $2.0\{E-07$ & $2.13 \mathrm{E}-07$ & $1.12 \mathrm{E}-10$ & $4.57 \mathrm{E}-12$ & $1.185-10$ & 3.85E-09 & $4.09 \mathrm{E}-09$ \\
\hline $241-S-110$ & $5.33 \mathrm{E}-08$ & $1.92 \mathrm{E}-09$ & $6.77 \mathrm{E}-08$ & 2.41E-07 & $3.63 \mathrm{E}-07$ & $1.02 \mathrm{E}-09$ & $3.70 \mathrm{E}-11$ & $1,30 E-09$ & 4.63E-09 & $6.99 \mathrm{E}-09$ \\
\hline Supernatant & иа & na & na & na & na & na & na & na & nа & na \\
\hline Solid Layer & $5.33 \mathrm{E}-08$ & 1,92E-09 & $6.77 \mathrm{E}-08$ & $2.41 \mathrm{E}-07$ & $3.63 \mathrm{E}-07$ & $1.02 \mathrm{E}-09$ & $3.70 \mathrm{E}-11$ & 1.30E-09 & $4.63 E-09$ & $6.99 \mathrm{E}-09$ \\
\hline $24 \mid-S-111$ & $1.80 \mathrm{E}-07$ & $4.18 \mathrm{E}-10$ & $2.71 E-08$ & $2.76 \mathrm{E}-07$ & $4.83 \mathrm{E}-07$ & $3.23 \mathrm{E}-09$ & $7.49 \mathrm{E}-12$ & $4.85 \mathrm{E}-10$ & $4.94 \mathrm{E}-09$ & $8.66 \mathrm{E}-09$ \\
\hline Supernatant & wa & na & na & na & na & na & na & na & na & na \\
\hline Solid Layer & $1.80 \mathrm{E}-07$ & $4.18 E-10$ & $2.71 \mathrm{E}-08$ & $2.76 \mathrm{E}-07$ & $4.83 \mathrm{E}-07$ & $3.23 \mathrm{E}-09$ & $7.49 \mathrm{E}-1 ?$ & $4.85 E-10$ & $4.94 \mathrm{E}-09$ & $8.66 \mathrm{E}-09$ \\
\hline $241-S-112$ & $4.67 \mathrm{E}-07$ & $2.27 \mathrm{E}-08$ & $9.71 \mathrm{E}-09$ & 1.14E- -05 & $1.19 \mathrm{E}-05$ & $9.26 \mathrm{E}-09$ & $4.50 \mathrm{E}-10$ & $1.92 \mathrm{E}-10$ & $2.25 \mathrm{E}-07$ & $2.35 \mathrm{E}-07$ \\
\hline Supernatan & na & na & na & na & na & na & na & na & na & na \\
\hline Solid Layer & $4.67 \mathrm{E}-07$ & $2,27 \mathrm{E}-08$ & $9.71 E-09$ & 1.14E-05 & $1.19 \mathrm{E}-05$ & $9.26 \mathrm{E}-09$ & $4.50 \mathrm{E}-10$ & $1.92 E-10$ & $2.25 \mathrm{E}-07$ & $2.35 \mathrm{E}-07$ \\
\hline 241-SX-101 & $9.44 \mathrm{E}-08$ & $1.03 \mathrm{E}-08$ & $8.09 \mathrm{E}-08$ & $2.28 E-07$ & $4.14 E-07$ & $1.83 \mathrm{E}-09$ & $2.01 \mathrm{E}-10$ & $1.57 \mathrm{E}-09$ & 4.44E-09 & $8.04 \mathrm{E}-09$ \\
\hline Superratant & na & na & na & nhat & na & na & na & па & na & na \\
\hline Solid Layer & $9.44 \mathrm{E}-08$ & $1.03 E-08$ & $8.09 \mathrm{E}-08$ & $2.28 \mathrm{E}-07$ & $4.14 \mathrm{E}-07$ & $1.83 \mathrm{E}-09$ & $2.01 E-10$ & $1.57 E-09$ & $4.44 \mathrm{E}-09$ & $8.04 \mathrm{E}-09$ \\
\hline $241-S X-102$ & $2.34 \mathrm{E}-07$ & $5.31 \mathrm{E}-09$ & $6.43 \mathrm{E}-07$ & $2.57 E-07$ & $1.14 \mathrm{E}-06$ & $4.59 \mathrm{E}-09$ & $1.04 \mathrm{E}-10$ & $1.26 \mathrm{E}-08$ & $5.04 E-09$ & $2.24 \mathrm{E}-08$ \\
\hline Supernatant & na & па & na & na & na & nа & na & na & na & na \\
\hline Solid Layer & $2.34 \mathrm{E}-07$ & $5.31 \mathrm{E}-09$ & $6.43 \mathrm{E}-07$ & $2.57 \mathrm{E}-07$ & $1 .\{4 \mathrm{E}-06$ & $4.59 \mathrm{E}-09$ & $1.04 \mathrm{E}-10$ & $1.26 \mathrm{E}-08$ & $5.04 \mathrm{E}-09$ & $2.24 \mathrm{E}-08$ \\
\hline
\end{tabular}




\section{RPP-5926 REV 6}

Table B-3. Calculated Hydrogen Generation Rate as Mole Per Unit Waste Weight and Volume for 177 Tanks. (20 sheets)

\begin{tabular}{|c|c|c|c|c|c|c|c|c|c|c|}
\hline Tank & $\begin{array}{l}\text { HGR from } \\
\text { radiolysis } \\
\mathrm{RC}_{\mathrm{rad}}^{\mathbf{B} / \mathrm{\gamma}} \\
\text { (mole/kg-d) }\end{array}$ & $\begin{array}{l}\text { HGR from } \\
\text { radiolysis } \\
\mathrm{RC}_{\text {rad }}^{\text {alpha }} \\
\text { (mole/kg-d) }\end{array}$ & $\begin{array}{l}\text { HGR from } \\
\text { thermolysis } \\
\text { RC }_{\text {therm }} \\
\text { (mole } / \mathbf{k g - d} \text { ) }\end{array}$ & $\begin{array}{l}\text { HGR from } \\
\text { corrosion } \\
\mathrm{RC}_{\text {corr }} \\
\text { (mole } / \mathrm{kg}-\mathrm{d} \text { ) }\end{array}$ & $\begin{array}{c}\text { Total HGR } \\
\text { from model } \\
\text { RC }_{\text {tol }} \\
\text { (mole } / \mathrm{kg}-\mathrm{d} \text { ) }\end{array}$ & $\begin{array}{l}\text { HGR from } \\
\text { radiolysis } \\
\mathrm{RC}_{\mathrm{rzd}} \mathrm{b} / \mathrm{r} \\
\left(\mathrm{mole} / \mathrm{m}^{3}-\mathrm{s}\right)\end{array}$ & $\begin{array}{l}\text { HGR from } \\
\text { radiolysis } \\
\text { RC }_{\text {rad }}^{\text {slpha }} \\
\left.\text { (mole/m } \text { m }^{3} \cdot \mathrm{s}\right)\end{array}$ & $\begin{array}{l}\text { HGR from } \\
\text { thermolysis } \\
\text { RC }_{\text {itherm }} \\
\left.\text { (mole/m } \text { m }^{3}-\mathrm{s}\right)\end{array}$ & $\begin{array}{l}\text { HGR from } \\
\text { corrosion } \\
\text { RC }_{\text {corr }} \\
\left.\text { (mole } / \mathrm{m}^{3}-\mathrm{s}\right)\end{array}$ & $\begin{array}{c}\text { Total HGR } \\
\text { from model } \\
\text { RCtot } \\
\left(\text { mole } / \mathbf{m}^{3}-s\right)\end{array}$ \\
\hline $241-S X-103$ & $4.11 \mathrm{E}-07$ & $8.19 \mathrm{E}-09$ & $2.80 \mathrm{E}-06$ & 1.98E-07 & $3.41 \mathrm{E}-06$ & $8.22 \mathrm{E}-09$ & 1.64E-10 & $5.60 \mathrm{H}-08$ & $3.96 \mathrm{E}-09$ & $6.83 \mathrm{E}-08$ \\
\hline Supernatant & nа & ra & na & na & na & ta & na & na & na & na \\
\hline Solid Layer & $4.11 \mathrm{E}-07$ & $8.19 \mathrm{E}-09$ & $2.80 E-06$ & $1.98 \mathrm{E}-07$ & $3.41 \mathrm{E}-06$ & $8.22 \mathrm{E}-09$ & $1.64 \mathrm{E}-10$ & $5.60 \mathrm{E}-08$ & $3.96 \mathrm{E}-09$ & $6.83 E-08$ \\
\hline 241-SX-104 & $1.73 \mathrm{E}-07$ & $4.21 \mathrm{E}-09$ & $6.33 \mathrm{E}-07$ & $2.18 E-07$ & $1.03 \mathrm{E}-06$ & $3.38 \mathrm{E}-09$ & $8.25 \mathrm{E}-11$ & $1.24 \mathrm{E}-08$ & $4.27 \mathrm{E}-09$ & $2.02 \mathrm{E}-08$ \\
\hline Supernatant & na & 1ra & na & na & na & na & na & na & na & $\mathrm{na}$ \\
\hline Solid t.ayer & $1.73 \mathrm{E}-07$ & $4.21 \mathrm{E}-09$ & $6.33 \mathrm{E}-07$ & $2.18 \mathrm{E}-07$ & $1.03 \mathrm{E}-06$ & $3.38 \mathrm{E}-09$ & $8.25 \mathrm{E}-11$ & $1.24 \mathrm{E}-08$ & $4.27 \mathrm{E}-09$ & $2.02 \mathrm{E}-08$ \\
\hline $241-S X-105$ & $5.73 E-07$ & $1.70 \mathrm{E}-08$ & $2.76 \mathrm{E}-06$ & 2.5 IE-07 & $3.60 \mathrm{E}-06$ & $1.08 \mathrm{E}-08$ & $3.20 \mathrm{E}-10$ & $5.20 \mathrm{E}-08$ & 4.74E-09 & $6.79 \mathrm{E}-08$ \\
\hline Supernatant & па & na & na & na & na & na & $\mathrm{nla}$ & na & na & na \\
\hline Solid Layer & $5.73 \mathrm{E}-07$ & $1.70 \mathrm{E}-08$ & $2.76 \mathrm{E}-06$ & $2.51 \mathrm{E}-07$ & $3.60 \mathrm{E}-06$ & $1.08 \mathrm{E}-08$ & $3.20 \mathrm{E}-10$ & $5.20 \mathrm{E}-08$ & 4. $74 \mathrm{E}-09$ & $6.79 \mathrm{E}-08$ \\
\hline 241-SX-106 & $1.42 \mathrm{E}-07$ & $5.08 \mathrm{E}-09$ & $2.46 \mathrm{E}-07$ & $2.51 \mathrm{E}-07$ & $6.43 \mathrm{E}-07$ & $2.59 \mathrm{E}-09$ & $9.28 \mathrm{E}-11$ & 4.49E-09 & $4.58 \mathrm{E}-09$ & $1.18 \mathrm{t}-08$ \\
\hline Supenatant & na & na & па & na & na & na & na & na & na & na \\
\hline Solid Layer & $1.42 \mathrm{E}-07$ & $5.08 \mathrm{E}-09$ & $2.46 \mathrm{E}-07$ & $2.51 \mathrm{E}-07$ & $6.43 \mathrm{E}-07$ & $2.59 \mathrm{E}-09$ & $9.28 E-11$ & 4. $49 \mathrm{E}-09$ & 4.58E-09 & $1.18 \mathrm{E}-08$ \\
\hline 241-SX-107 & $1.33 E-06$ & $1.95 \mathrm{E}-08$ & $1.24 \mathrm{E}-06$ & $6.68 \mathrm{~F}-07$ & 3.25E-06 & $2.72 \mathrm{E}-08$ & $3.99 \mathrm{E}-10$ & $2.54 \mathrm{E}-08$ & 1.37E-08 & $6.67 \mathrm{E}-08$ \\
\hline Supenatant & na & na & na & na & na & na & na & na & na & na \\
\hline Solid l. ayer & $1.33 \mathrm{E}-06$ & $1.95 E-08$ & $1.24 \mathrm{E}-06$ & $6.68 \mathrm{E}-07$ & $3.25 \mathrm{E}-06$ & $2.72 \mathrm{E}-08$ & $3.99 \mathrm{E}-10$ & $2.54 \mathrm{E}-08$ & 1.37E-08 & $6.67 \mathrm{E}-08$ \\
\hline $241-5 X-108$ & $4.61 \mathrm{E}-07$ & $6.32 \mathrm{E}-09$ & $2.53 \mathrm{E}-07$ & $8.27 \mathrm{E}-07$ & $1.55 \mathrm{E}-06$ & $9.45 E-09$ & 5. $30 \mathrm{E}-10$ & $5.19 \mathrm{E}-09$ & $1.69 \mathrm{E}-08$ & $3.17 \mathrm{E}-08$ \\
\hline Supernatant & па & na & па & na & na & na & na & na & na & na \\
\hline Solid Layer & $4.61 \mathrm{E}-07$ & $6.32 \mathrm{E}-09$ & $2.53 \mathrm{E}-07$ & $8.27 E-07$ & $1.55 \mathrm{E}-06$ & $9.45 \mathrm{E}-09$ & $1.30 \mathrm{E}-10$ & $5.19 \mathrm{E}-09$ & $1.69 \mathrm{E}-08$ & $3.17 \mathrm{E}-08$ \\
\hline $241-S X-109$ & $6.02 \mathrm{E}-07$ & $5.25 \mathrm{E}-09$ & $1.24 \mathrm{E}-06$ & $3.19 \mathrm{E}-07$ & $2.17 \mathrm{E}-06$ & $1.21 \mathrm{E}-08$ & $1.05 \mathrm{E}-10$ & $2.50 \mathrm{E}-08$ & $6.41 \mathrm{E}-09$ & 4.35E-08 \\
\hline Supematant & na & na & na & na & na & na & na & па & na & na \\
\hline Solid Layer & $6.02 \mathrm{E}-07$ & $5.25 \mathrm{E}-09$ & $1.24 \mathrm{E}-06$ & $3.19 \mathrm{E}-07$ & $2.17 \mathrm{E}-06$ & 1.21E-08 & $1.05 \mathrm{E}-10$ & $2.50 E-08$ & $6.41 \mathrm{E}-09$ & $4.35 \mathrm{E}-08$ \\
\hline $241-S X-110^{\circ}$ & $6.40 \mathrm{E}-06$ & $5.34 \mathrm{E}-08$ & $3.62 \mathrm{E}-07$ & $1.07 \mathrm{E}-06$ & $7.88 \mathrm{E}-06$ & $1.31 \mathrm{E}-07$ & $1.09 \mathrm{E}-09$ & $7.40 \mathrm{E}-09$ & $2.18 \mathrm{E}-08$ & $1.61 \mathrm{E}-07$ \\
\hline Supernatant & па & па & na & na & na & na & na & na & na & na \\
\hline Solid Layer & $6.40 \mathrm{E}-06$ & $5.34 \mathrm{E}-08$ & $3.62 \mathrm{E}-07$ & $1.07 \mathrm{E}-06$ & $7.88 \mathrm{E}-06$ & $1.31 \mathrm{E}-07$ & $1.09 \mathrm{E}-09$ & $7.40 \mathrm{E}-09$ & $2.18 \mathrm{E}-08$ & $1.61 \mathrm{E}-07$ \\
\hline $241-\mathrm{SX}-111$ & $1.60 \mathrm{E}-06$ & $2.19 \mathrm{E}-08$ & $7.55 \mathrm{E}-07$ & $5.64 \mathrm{E}-07$ & $2.94 \mathrm{E}-06$ & $3.27 \mathrm{E}-08$ & $4.47 \mathrm{E}-10$ & 1.54E-08 & 1.15E-08 & $6.01 \mathrm{E}-08$ \\
\hline Supematant & na & na & na & na & na & na & na & na & na & na \\
\hline Solid Layer & $1.60 \mathrm{E}-06$ & $2.19 \mathrm{E}-08$ & $7.55 \mathrm{E}-07$ & $5.64 \mathrm{~F}-07$ & $2.94 \mathrm{E}-06$ & $3.27 \mathrm{E}-08$ & $4.47 \mathrm{E}-10$ & $1.54 \mathrm{E}-08$ & $1.15 \mathrm{E}-08$ & $6.01 \mathrm{E}-08$ \\
\hline
\end{tabular}




\section{RPP-5926 REV 6}

Table B-3. Calculated Hydrogen Generation Rate as Mole Per Unit Waste Weight and Volume for 177 Tanks. (20 sheets)

\begin{tabular}{|c|c|c|c|c|c|c|c|c|c|c|}
\hline Tank & $\begin{array}{l}\text { HGR from } \\
\text { radiolysis } \\
\mathrm{RC}_{\mathrm{rad}}^{\mathrm{B} / \mathrm{r}} \\
\text { (mole/kg-d) }\end{array}$ & $\begin{array}{l}\text { HGR from } \\
\text { radiolysis } \\
\text { RC }_{\text {rad }} \text { alpha } \\
\text { (mole } / \mathrm{kg}-\mathrm{d})\end{array}$ & $\begin{array}{l}\text { HGR from } \\
\text { thermolysis } \\
\text { RC }_{\text {therm }} \\
\text { (mole/kg-d) }\end{array}$ & $\begin{array}{l}\text { HGR from } \\
\text { corrosion } \\
\text { RC }_{\text {corr }} \\
\text { (mole/kg-d) }\end{array}$ & $\begin{array}{l}\text { Total HGR } \\
\text { from model } \\
\mathbf{R C}_{\text {tol }} \\
\text { (mole } / \mathbf{k g}-\mathrm{d})\end{array}$ & $\begin{array}{l}\text { HGR from } \\
\text { radiolysis } \\
\mathrm{RC}_{\mathrm{rud}} \mathrm{b} / \mathrm{r} \\
\left.\text { (mole } / \mathrm{m}^{3}-\mathrm{s}\right)\end{array}$ & $\begin{array}{l}\text { HGR from } \\
\text { radiolysis } \\
\mathrm{RC}_{\text {rad }} \mathrm{atphrz} \\
\left(\mathrm{mole} / \mathrm{m}^{3} \cdot \mathrm{s}\right)\end{array}$ & $\begin{array}{l}\text { HGR from } \\
\text { thermolysis } \\
\text { RC }_{\text {theran }} \\
\left(\mathrm{mole}^{\prime} \mathbf{m}^{3}-\mathrm{s}\right)\end{array}$ & $\begin{array}{l}\text { HGR from } \\
\text { corrosion } \\
\mathrm{RC}_{\text {sorr }} \\
\left.\text { (mole/m } \mathbf{m}^{3}-\mathbf{s}\right)\end{array}$ & $\begin{array}{c}\text { Total HGR } \\
\text { from model } \\
\text { RCtot } \\
\left.\text { (mole } / \mathrm{m}^{3}-\mathrm{s}\right)\end{array}$ \\
\hline $241-S X-112$ & $1.59 \mathrm{E}-06$ & $2.35 \mathrm{E}-08$ & $4.97 \mathrm{E}-07$ & $8.19 \mathrm{E}-07$ & $2.93 \mathrm{E}-06$ & $3.26 \mathrm{E}-08$ & $4.81 E-10$ & $1.02 \mathrm{E}-08$ & 1. $68 \mathrm{E}-08$ & $6.00 \mathrm{E}-08$ \\
\hline Supernatant & na & na & na & na & 11 & na & na & na & na & na \\
\hline Solid Layer & $1.59 \mathrm{E}-06$ & 2.35E-08 & $4.97 \mathrm{E}-07$ & $8.19 \mathrm{E}-07$ & $2.93 \mathrm{E}-06$ & $3.26 \mathrm{E}-08$ & $4.81 \mathrm{E}-10$ & $1.02 \mathrm{E}-08$ & I. $68 \mathrm{E}-08$ & $6.00 \mathrm{E}-08$ \\
\hline $241-\mathrm{SX}-113$ & $9.79 \mathrm{E}-08$ & $6.84 \mathrm{E}-09$ & $3.70 \mathrm{E}-08$ & $3.67 \mathrm{E}-06$ & $3.82 \mathrm{E}-06$ & $1.62 \mathrm{E}-09$ & $1.33 E-10$ & $6.12 \mathrm{E}-30$ & $6.08 \mathrm{E}-08$ & $6.31 \mathrm{E}-08$ \\
\hline Supernatant & na & na & na & na & na & na & па & na & na & na \\
\hline Solid Layer & $9.79 \mathrm{E}-08$ & $6.84 \mathrm{E}-09$ & 3.70E-08 & $3.67 \mathrm{E}-06$ & $3.82 \mathrm{E}-06$ & 1.62E-09 & $1.13 \mathrm{E}-10$ & $6.12 \mathrm{E}-10$ & $6.08 \mathrm{E}-08$ & $6.31 \mathrm{E}-08$ \\
\hline $241-S X-114$ & $1.36 \mathrm{E}-06$ & $1.83 \mathrm{E}-08$ & $1.58 \mathrm{E}-06$ & 4.43E- -07 & $3.40 \mathrm{E}-06$ & 2.75E- 08 & $3.71 \mathrm{E}-10$ & $3.20 \mathrm{~F}-08$ & $8.98 \mathrm{E}-09$ & $6.89 \mathrm{E}-08$ \\
\hline Supernatant & na & na & na & na & na & na & na & na & na & nа \\
\hline Solid Layer & $1.36 \mathrm{E}-06$ & $1.83 \mathrm{E}-08$ & $1.58 E-06$ & 4.43E- 07 & $3.40 E-06$ & $2.75 \mathrm{E}-08$ & $3.71 \mathrm{E}-10$ & 3.20E-08 & $8.98 E-09$ & $6.89 \mathrm{~F}-08$ \\
\hline $241-S X-115$ & $6.91 \mathrm{E}-06$ & $3.08 \mathrm{E}-07$ & $5.92 \mathrm{E}-09$ & 1.31E-05 & $2.03 \mathrm{E}-05$ & $1.42 \mathrm{E}-07$ & $6.31 \mathrm{E}-09$ & $1.21 \mathrm{E}-10$ & $2.67 E-07$ & $4.15 \mathrm{E}-07$ \\
\hline Supematant & na & t1a & na & na & na & ma & na & na & na & na \\
\hline Solid Layer & $6.91 \mathrm{E}-06$ & $3.08 \mathrm{E}-07$ & $5.92 \mathrm{E}-09$ & $1.31 \mathrm{E}-05$ & $2.03 \mathrm{E}-05$ & $1.42 \mathrm{E}-07$ & $6.31 \mathrm{E}-09$ & $1.21 \mathrm{E}-10$ & $2.67 \mathrm{E}-07$ & $4.15 \mathrm{E}-07$ \\
\hline $241-T-101$ & $5.05 \mathrm{E}-08$ & $1.33 \mathrm{E}-08$ & $4.85 \mathrm{E}-08$ & $7.29 \mathrm{E}-07$ & $8.41 \mathrm{E}-07$ & $9.02 \mathrm{E}-10$ & $2.37 \mathrm{E}-10$ & $8.66 \mathrm{E}-10$ & $1.30 \mathrm{E}-08$ & $1.50 \mathrm{E}-08$ \\
\hline Supernatant & na & na & na & na & na & na & na & na & na & na \\
\hline Solid Layer & $5.05 \mathrm{E}-08$ & $1.33 \mathrm{E}-08$ & 4. $85 \mathrm{E}-08$ & $7.29 \mathrm{E}-07$ & $8.41 \mathrm{E}-07$ & $9.02 \mathrm{E}-10$ & $2.37 \mathrm{E}-10$ & $8.66 \mathrm{E}-10$ & $1.30 \mathrm{E}-08$ & $1.50 \mathrm{E}-08$ \\
\hline $241-\mathrm{T}-102$ & $2.08 \mathrm{E}-07$ & $4.43 \mathrm{E}-09$ & $1.86 \mathrm{E}-09$ & $5.22 \mathrm{E}-06$ & $5.43 \overline{E-06}$ & $3.70 \mathrm{E}-09$ & $7.86 E-11$ & $3.30 \mathrm{E}-11$ & $9.27 \mathrm{E}-08$ & 9.65E-08 \\
\hline Supernatant & $1.13 \mathrm{E}-07$ & $5.06 \mathrm{E}-10$ & $3.33 \mathrm{E}-09$ & $3.31 \mathrm{E}-07$ & $4.48 \mathrm{E}-07$ & $1.49 E-09$ & $6.68 \mathrm{E}-12$ & $4.40 \mathrm{E}-11$ & $4.37 \mathrm{E}-09$ & $5.92 \mathrm{E}-09$ \\
\hline Solid Layer & $1.79 \mathrm{E}-07$ & 4.11E-09 & $1.24 \mathrm{E}-09$ & $7.29 \mathrm{E}-06$ & 7.47E-06 & $3.73 E-09$ & $8.55 \mathrm{E}-11$ & $2.57 \mathrm{E}-11$ & $1.52 \mathrm{E}-07$ & $1.55 \mathrm{E}-07$ \\
\hline 241-T-103 & $9.64 \mathrm{E}-09$ & $9.67 E-10$ & $2.33 \mathrm{E}-08$ & $2.29 \mathrm{E}-06$ & $2.32 \mathrm{E}-06$ & $1.83 \mathrm{E}-10$ & $1.83 \mathrm{E}-11$ & $4.42 \mathrm{E}-10$ & 4.33E- 08 & 4.39E-08 \\
\hline Supernatant & $3.59 \mathrm{E}-08$ & $1.10 \mathrm{E}-09$ & $5.15 \mathrm{E}-08$ & $1.27 \mathrm{E}-07$ & $2.16 \mathrm{E}-07$ & 4.95E-10 & $1.52 \mathrm{E}-11$ & $7.09 \mathrm{E}-10$ & $1.75 \mathrm{E}-09$ & $2.97 \mathrm{E}-09$ \\
\hline Solid Layer & $7.59 \mathrm{E}-09$ & $8.83 \mathrm{E}-10$ & $2.01 \mathrm{E}-08$ & $2.54 \mathrm{E}-06$ & $2.57 \mathrm{E}-06$ & $1.51 \mathrm{E}-10$ & $1.75 \mathrm{E}-11$ & $3.996-10$ & $5.05 \mathrm{E}-08$ & $5.10 \mathrm{E}-08$ \\
\hline $241-\mathrm{T}-104$ & $6.72 \mathrm{E}-09$ & $8.51 \mathrm{E}-09$ & $1.77 \mathrm{E}-08$ & $3.54 E-07$ & $3.87 \mathrm{E}-07$ & $1.00 \mathrm{E}-10$ & $1.27 \mathrm{E}-10$ & $2.64 \mathrm{E}-10$ & $5.29 \mathrm{E}-09$ & $5.78 \mathrm{E}-09$ \\
\hline Supernatant & na & na & na & na & na & na & na & na & na & na \\
\hline Solid Layer & $6.72 \mathrm{E}-09$ & $8.51 \mathrm{E}-09$ & $1.77 \mathrm{E}-08$ & $3.54 \mathrm{E}-07$ & $3.87 \mathrm{E}-07$ & $1.00 \mathrm{E}-10$ & 1.27E-10 & $2.64 \mathrm{E}-10$ & $5.29 \mathrm{E}-09$ & $5.78 \mathrm{E}-09$ \\
\hline $241-T \cdot 105$ & 1.65E-07 & $2.16 \mathrm{E}-08$ & $3.30 \mathrm{E}-09$ & $7.80 \mathrm{E}-07$ & $9.70 \mathrm{E}-07$ & $2.80 \mathrm{E}-09$ & $3.64 \mathrm{E}-10$ & $5.58 \mathrm{E}-11$ & 1.32E-08 & $1.64 \mathrm{E}-08$ \\
\hline Supernatant & na & па & na & na & 1ra & na & na & na & na & na \\
\hline Solid Layer & $1.65 E-07$ & $2.16 \mathrm{E}-08$ & $3.30 \mathrm{E}-09$ & $7.80 \mathrm{E}-07$ & $9.70 \mathrm{E}-07$ & $2.80 \mathrm{E}-09$ & $3.64 \mathrm{E}-10$ & $5.58 \mathrm{E}-11$ & $1.32 \mathrm{E}-08$ & $1.64 \mathrm{E}-08$ \\
\hline
\end{tabular}


RPP-5926 REV 6

Table B-3. Calculated Hydrogen Generation Rate as Mole Per Unit Waste Weight and Volume for 177 Tanks. (20 sheets)

\begin{tabular}{|c|c|c|c|c|c|c|c|c|c|c|}
\hline Tank & $\begin{array}{l}\text { HGR from } \\
\text { radiolysis } \\
\mathrm{RC}_{\mathrm{rad}} \mathrm{B} / \gamma \\
\text { (mole } / \mathrm{kg}-\mathrm{d})\end{array}$ & $\begin{array}{l}\text { HGR from } \\
\text { radiolysis } \\
\text { RC }_{\text {rad }}^{\text {dipha }} \\
\text { (mole/kg-d) }\end{array}$ & $\begin{array}{l}\text { HGR from } \\
\text { thermolysis } \\
\text { RC }_{\text {therm }} \\
\text { (mole } / \text { kg-d) }\end{array}$ & $\begin{array}{l}\text { HGR from } \\
\text { corrosion } \\
\text { RC }_{\text {uurr }} \\
\text { (mole/kg-d) }\end{array}$ & $\begin{array}{l}\text { Total HGR } \\
\text { from model } \\
\text { RC }_{\text {tot }} \\
\text { (mole } / \mathrm{kg} \text {-d) }\end{array}$ & $\begin{array}{l}\text { HGR from } \\
\text { radiolysis } \\
\text { RC }_{\text {rad }}^{\mathrm{b} / \mathrm{r}} \\
\text { (mole/m }^{3} \text {-s) }\end{array}$ & $\begin{array}{l}\text { HGR from } \\
\text { radiolysis } \\
\mathrm{RC}_{\text {rad }}^{\text {alpha }} \\
\left.\text { (mole/m } / \mathrm{m}^{3}-\mathrm{s}\right)\end{array}$ & $\begin{array}{l}\text { HGR from } \\
\text { thermolysis } \\
\text { RC }_{\text {therm }} \\
\left(m o l e / m^{3}-s\right)\end{array}$ & $\begin{array}{l}\text { HGR from } \\
\text { corrosion } \\
\mathrm{RC}_{\text {corr }} \\
\text { (mole/m }{ }^{3} \text {-s) }\end{array}$ & $\begin{array}{c}\text { Total HGR } \\
\text { from model } \\
\text { RCtot } \\
\left.\text { (mole/m } / \mathrm{m}^{3}-\mathrm{s}\right)\end{array}$ \\
\hline 241-T-106 & 1.32E-08 & $2.83 \mathrm{E}-09$ & $4.80 \mathrm{E}-09$ & $2.91 \mathrm{E}-06$ & $2.93 \mathrm{E}-06$ & $2.43 \mathrm{E}-10$ & $5.19 \mathrm{E}-11$ & $8.82 \mathrm{E}-11$ & $5.34 \mathrm{E}-08$ & $5.38 \mathrm{E}-08$ \\
\hline Supematant & na & na & na & na & na & na & na & na & na & na \\
\hline Solid layer & 1.32E-08 & $2.83 \mathrm{E}-09$ & $4.80 \mathrm{E}-09$ & $2.91 \mathrm{E}-06$ & $2.93 \mathrm{E}-06$ & $2.43 E-10$ & $5.19 E-11$ & $8.82 \mathrm{E}-11$ & $5.34 \mathrm{E}-08$ & $5.38 \mathrm{E} \cdot 08$ \\
\hline $241-T-107$ & $2.09 \mathrm{E}-07$ & $1.28 \mathrm{E}-08$ & $1.15 \mathrm{E}-08$ & $4.55 \mathrm{E}-07$ & $6.89 \mathrm{E}-07$ & $3.78 \mathrm{E}-09$ & $2.32 \mathrm{E}-10$ & $2.08 \mathrm{E}-10$ & $8.22 \mathrm{E}-09$ & $1.24 \mathrm{E}-08$ \\
\hline Supematant & na & na & na & na & na & na & па & na & na & na \\
\hline Solid Layer & $2.09 \mathrm{E}-07$ & $1.28 \mathrm{E}-08$ & $1.15 \mathrm{E}-08$ & $4.55 \mathrm{E}-07$ & $6.89 \mathrm{E}-07$ & $3.78 \mathrm{E}-09$ & $2.32 \mathrm{E}-10$ & $2.08 \mathrm{E}-10$ & $8.22 \mathrm{E}-09$ & $1.24 E-08$ \\
\hline $241-T-108$ & $6.30 \mathrm{E}-09$ & $1.09 \mathrm{E}-09$ & $2.49 \mathrm{E}-12$ & $4.04 \mathrm{E}-06$ & $4.05 \mathrm{E}-06$ & $1.13 E-10$ & $1.95 \mathrm{~F}-11$ & $4.45 \mathrm{E}-14$ & $7.24 \mathrm{~K}-08$ & $7.25 \mathrm{E}-08$ \\
\hline Supematant & па & na & na & na & na & па & na & na & na & na \\
\hline Solid Layer & $6.30 \mathrm{E}-09$ & $1.09 \mathrm{E}-09$ & $2.49 \mathrm{E}-12$ & $4.04 E-06$ & $4.05 \mathrm{E}-06$ & 1.13E-10 & $1.95 \mathrm{E}-11$ & $4.45 E-14$ & $7.24 \mathrm{E}-08$ & $7.25 \mathrm{E}-08$ \\
\hline $241-T-109$ & $2.49 \mathrm{E}-09$ & $2.29 \mathrm{E}-10$ & $5.19 \mathrm{E}-12$ & $1.04 \mathrm{E} .06$ & $1.04 \mathrm{E}-06$ & $4.74 E-11$ & $4.36 \mathrm{E}-12$ & $9.89 \mathrm{E}-14$ & $1.98 \mathrm{E}-08$ & $1.98 \mathrm{E}-08$ \\
\hline Supernatan1 & na & па & na & na & na & na & na & na & na & na \\
\hline Solid Layer & $2.49 \mathrm{E}-09$ & $2.29 \mathrm{E}-10$ & $5.19 \mathrm{E}-12$ & $1.04 \mathrm{E}-06$ & $1.04 \mathrm{E}-06$ & $4.74 \mathrm{E}-11$ & $4.36 \mathrm{E}-12$ & $9.89 \mathrm{E}-14$ & $1.98 \mathrm{E}-08$ & $1.98 \mathrm{E}-08$ \\
\hline $241-T-110$ & $2.87 E-10$ & $9.87 \mathrm{E}-09$ & $0.00 E+00$ & $8.26 \mathrm{E}-07$ & $8.36 \mathrm{E}-07$ & 4.15E-12 & $1.43 \mathrm{E}-10$ & $0.00 \mathrm{E}+00$ & $1.19 \mathrm{E}-08$ & $1.21 \mathrm{E}-08$ \\
\hline Supernatant & $2.10 \mathrm{E}-11$ & $2.74 \mathrm{E}-11$ & $0.00 \mathrm{E}+00$ & $3.60 \mathrm{E}-07$ & $3.60 \mathrm{E}-07$ & $2.55 \mathrm{E}-13$ & $3.33 \mathrm{E}-13$ & $0.00 \mathrm{E}+00$ & $4.37 \mathrm{E}-09$ & $4.37 \mathrm{E}-09$ \\
\hline Solid Layer & $2.87 \mathrm{E}-10$ & $9.89 \mathrm{E}-09$ & $0.00 \mathrm{E}+00$ & $8.27 \mathrm{E}-07$ & $8.37 \mathrm{E}-07$ & $4.16 \mathrm{E}-12$ & $1.43 \mathrm{E}-10$ & $0.00 \mathrm{E}+00$ & $1.20 \mathrm{E}-08$ & $1.21 \mathrm{E}-08$ \\
\hline $24 I-T-111$ & $1.33 \mathrm{E}-08$ & $1.03 \mathrm{E}-08$ & $1.61 \mathrm{E}-08$ & $2.97 \mathrm{E}-07$ & $3.36 \mathrm{E}-07$ & $1.91 \mathrm{E}-10$ & $1.47 \mathrm{E}-10$ & $2.30 E-10$ & $4.26 \mathrm{E}-09$ & $4.835-09$ \\
\hline Supernatant & na & na & $\mathrm{na}$ & na & na & na & na & na & na & nа \\
\hline Solid Layer & $1.33 \mathrm{E}-08$ & $1.03 E-08$ & $1.61 \mathrm{E}-08$ & $2.97 \mathrm{E}-07$ & $3.36 \mathrm{E}-07$ & $1.91 E-10$ & $1.47 E-10$ & $2.30 \mathrm{E}-10$ & $4.26 \mathrm{E}-09$ & $4.83 \mathrm{E}-09$ \\
\hline $241-\mathrm{T}-112$ & $1.74 \mathrm{E}-09$ & $2.45 \mathrm{E}-08$ & $6.59 \mathrm{E}-10$ & $1.27 E-06$ & $1.30 \mathrm{E}-06$ & $2.54 \mathrm{E}-11$ & $3.57 \mathrm{E}-10$ & $9.62 \mathrm{E}-12$ & $1.86 \mathrm{E}-08$ & $1.90 \mathrm{E}-08$ \\
\hline Supernatant & $2.16 \mathrm{E}-08$ & $2.46 \mathrm{E}-09$ & $7.40 \mathrm{E}-10$ & $1.37 \mathrm{E}-07$ & $1.62 \mathrm{E}-07$ & $2.75 \mathrm{E}-10$ & $3.14 \mathrm{E}-11$ & $9.42 \mathrm{E}-12$ & $1.75 \mathrm{E}-09$ & $2.06 \mathrm{~F}-09$ \\
\hline Solid Layer & $2.75 \mathrm{E}-11$ & $2.63 E-08$ & $6.51 \mathrm{E}-10$ & $1.38 \mathrm{E}-06$ & $1.41 \mathrm{E}-06$ & $4.07 \mathrm{E}-13$ & $3.90 \mathrm{E}-10$ & $9.64 \mathrm{E}-12$ & $2.05 \mathrm{E}-08$ & $2.09 \mathrm{E}-08$ \\
\hline $241-\mathrm{T}-201$ & $4.05 E-10$ & $5.57 \mathrm{E}-08$ & $0.00 \mathrm{E}+00$ & $1.54 \mathrm{E}-06$ & $1.60 \mathrm{E}-06$ & $6.07 \mathrm{E}-12$ & $8.34 E-10$ & $0.00 \mathrm{E}+00$ & $2.30 \mathrm{E}-08$ & $2.39 \mathrm{E}-08$ \\
\hline Supenatant & $4.40 E-13$ & $2.75 \mathrm{E}-12$ & $0.00 E+00$ & $1.34 \mathrm{E}-06$ & $1.34 \mathrm{E}-06$ & $5.40 \mathrm{E}-15$ & $3.37 \mathrm{E}-14$ & $0.00 \mathrm{E}+00$ & 1. $64 \mathrm{E}-08$ & 1. $64 \mathrm{E}-08$ \\
\hline Solid Layer & $4.21 \mathrm{E}-10$ & $5.79 \mathrm{E}-08$ & $0.00 \mathrm{E}+00$ & $1.55 \mathrm{E}-06$ & $1.61 \mathrm{E}-06$ & $6.39 \mathrm{E}-12$ & $8.78 \mathrm{E}-10$ & $0.00 \mathrm{E}+00$ & $2.35 \mathrm{E}-08$ & $2.44 \mathrm{E}-08$ \\
\hline $241-T-202$ & $2.64 \mathrm{E}-11$ & $1.43 \mathrm{E}-08$ & $1.55 \mathrm{E}-08$ & $7.71 \mathrm{E}-07$ & $8.01 \mathrm{E}-07$ & $3.61 \mathrm{E}-13$ & $1.95 \mathrm{~F}-10$ & $2.11 \mathrm{E}-10$ & $1.05 \mathrm{E}-08$ & $1.09 \mathrm{E}-08$ \\
\hline Supernatant & nа & na & na & na & $\mathrm{na}$ & иа & nа & $\pi a ̀$ & na & na \\
\hline Solid Layer & $2.64 \mathrm{E}-11$ & $1.43 \mathrm{E}-08$ & $1.55 \mathrm{E}-08$ & $7.71 \mathrm{E}-07$ & $8.01 \mathrm{E}-07$ & $3.61 \mathrm{E}-13$ & $1.95 \mathrm{E}-10$ & $2.11 \mathrm{E}-10$ & $1.05 \mathrm{E}-08$ & $1.09 \mathrm{E}-08$ \\
\hline
\end{tabular}


Table B-3. Calculated Hydrogen Generation Rate as Mole Per Unit Waste Weight and Volume for 177 Tanks. (20 sheets)

\begin{tabular}{|c|c|c|c|c|c|c|c|c|c|c|}
\hline Tank & $\begin{array}{l}\text { HGR from } \\
\text { radiolysis } \\
\mathrm{RC}_{\text {rad }} \mathrm{B/ \gamma} \\
\text { (mole } / \mathrm{kg}-\mathrm{d} \text { ) }\end{array}$ & $\begin{array}{l}\text { HGR from } \\
\text { radiolysis } \\
\mathrm{RC}_{\mathrm{rad}} \text { alpha } \\
\text { (mole/kg-d) }\end{array}$ & $\begin{array}{l}\text { HGR from } \\
\text { thermolysis } \\
\text { RC }_{\text {therm }} \\
\text { (mole } / \mathrm{kg-d} \text { ) }\end{array}$ & $\begin{array}{l}\text { HGR from } \\
\text { corrosion } \\
\text { RC }_{\text {sors }} \\
\text { (mole/kg-d) }\end{array}$ & $\begin{array}{c}\text { Total HGR } \\
\text { from model } \\
\text { RC }_{101} \\
\text { (mole } / \mathbf{k g} \text {-d) }\end{array}$ & $\begin{array}{l}\text { HGR from } \\
\text { radiolysis } \\
\mathrm{RC}_{\mathrm{rad}}^{\mathrm{b} / \mathrm{t}} \\
\left.\text { (mole/m } / \mathrm{m}^{3}-\mathrm{s}\right)\end{array}$ & $\begin{array}{l}\text { HGR from } \\
\text { radiolysis } \\
\mathrm{RC}_{\text {rad }}^{\text {atpha }} \\
\text { (mole } / \mathrm{m}^{3}-\mathrm{s} \text { ) }\end{array}$ & $\begin{array}{l}\text { HGR from } \\
\text { thermolysis } \\
\text { RC }_{\text {merm }} \\
\left.\text { (mole/m } \mathbf{m}^{3}-\mathrm{s}\right)\end{array}$ & $\begin{array}{l}\text { HGR from } \\
\text { corrosion } \\
\mathrm{RC}_{\text {corr }} \\
\text { (mole/m } / \mathrm{m}^{3} \text {-s) }\end{array}$ & $\begin{array}{c}\text { Total HGR } \\
\text { from model } \\
\text { RCtot } \\
\left.\text { (mole/m } \mathrm{m}^{3}-\mathrm{s}\right)\end{array}$ \\
\hline $241-T-203$ & $2.31 \mathrm{E}-11$ & $1.66 \mathrm{E}-08$ & $1.46 \mathrm{E}-08$ & $6.24 \mathrm{E}-07$ & $6.55 \mathrm{E}-07$ & $3.26 \mathrm{E}-13$ & $2.34 \mathrm{E}-10$ & $2.06 \mathrm{E}-10$ & $8.81 \mathrm{E}-09$ & $9.25 \mathrm{E}-09$ \\
\hline Supernatant & na & па & na & na & na & na & na & na & na & na \\
\hline Solid Layer & $2.31 E-11$ & 1.66E-08 & $1.46 \mathrm{E}-08$ & $6.24 \mathrm{E}-07$ & $6.55 \mathrm{E}-07$ & $3.26 \mathrm{E}-13$ & $2.34 \mathrm{E}-10$ & $2.06 \mathrm{E}-10$ & $8.81 E-09$ & $9.25 \mathrm{E}-09$ \\
\hline $241-\mathrm{T}-204$ & $3.31 \mathrm{E}-11$ & $1.51 E-98$ & $1.60 \mathrm{E}-08$ & $6.45 \mathrm{E}-07$ & $6.76 \mathrm{E}-07$ & $4.52 E-13$ & $2.06 \mathrm{E}-10$ & $2.18 \mathrm{E}-10$ & $8.81 E-09$ & $9.23 \mathrm{E}-09$ \\
\hline Supernatant & na & na & na & na & na & na & na & na & na & na \\
\hline Solid Layer & $3.31 \mathrm{E}-11$ & $1.51 \mathrm{E}-08$ & $1.60 \mathrm{E}-08$ & $6.45 \mathrm{E}-07$ & $6.76 \mathrm{E}-07$ & $4.52 \mathrm{E}-13$ & $2.06 \mathrm{E}-10$ & $2.18 \mathrm{E}-10$ & 8.81 E-09 & $9.23 \mathrm{E}-09$ \\
\hline $241-\mathrm{TX}-101$ & $3.96 \mathrm{E}-07$ & $2.75 E-08$ & $7.80 \mathrm{E}-08$ & $6.99 \mathrm{E}-07$ & $1.20 \mathrm{E}-06$ & 7.98E-09 & $5.53 E-10$ & $1.57 \mathrm{E}-09$ & 1.41E-08 & $2.42 \mathrm{E}-08$ \\
\hline Supernatant & na & na & na & na & na & na & na & na & na & na \\
\hline Solid Layer & $3.96 \mathrm{E}-07$ & $2.75 \mathrm{E}-0.08$ & $7.80 \mathrm{E}-08$ & $6.99 \mathrm{E}-07$ & $1.20 \mathrm{E}-06$ & $7.98 \mathrm{E}-09$ & $5.53 \mathrm{E}-10$ & $1.57 \mathrm{E}-09$ & $1.41 \mathrm{E}-08$ & $2.42 \mathrm{E}-08$ \\
\hline $241-\mathrm{TX}-102$ & $1.87 \mathrm{E}-08$ & $1.56 \mathrm{E}-09$ & $2.45 \mathrm{E}-08$ & $3.70 \mathrm{E}-07$ & $4.15 \mathrm{E}-07$ & $3.49 \mathrm{E}-10$ & $2.91 \mathrm{E}-11$ & $4.58 \mathrm{E}-10$ & $6.91 \mathrm{E}-09$ & $7.75 \mathrm{E}-09$ \\
\hline Supernatant & na & na & na & na & na & na & na & na & na & $n a$ \\
\hline Solid Layer & $1.87 \mathrm{E}-08$ & $1.56 \mathrm{E}-09$ & $2.45 \mathrm{E}-08$ & $3.70 \mathrm{E}-07$ & 4.15E-07 & $3.49 E-10$ & $2.91 \mathrm{E}-11$ & $4.58 \mathrm{E}-10$ & $6.91 \mathrm{E}-09$ & $7.75 \mathrm{E}-09$ \\
\hline $241-\mathrm{TX}-103$ & $2.06 \mathrm{~F}-08$ & $1.75 \mathrm{E}-09$ & $1.62 \mathrm{E}-08$ & $5.09 \mathrm{E}-07$ & $5.47 \mathrm{E}-07$ & $3.83 E-10$ & $3.25 \mathrm{E}-11$ & $3.01 \mathrm{E}-10$ & $9.49 \mathrm{E}-09$ & $1.02 \mathrm{E}-08$ \\
\hline Supernatant & na & na & na & na & na & na & na & na & na & na \\
\hline Solid Layer & $2.06 \mathrm{E}-08$ & $1.75 \mathrm{E}-09$ & 1. $62 \mathrm{E}-08$ & $5.09 E-07$ & $5.47 \mathrm{E}-07$ & $3.83 E-10$ & $3.25 \mathrm{E}-11$ & $3.01 \mathrm{E}-10$ & $9.49 \mathrm{E}-09$ & $1.02 \mathrm{E}-08$ \\
\hline $241-\Upsilon X-104$ & $2.24 \mathrm{~F}-07$ & $4.69 \mathrm{E}-09$ & $6.15 \mathrm{E}-09$ & $8.97 \mathrm{E}-07$ & $3.13 E-06$ & $4.48 \mathrm{E}-09$ & $9.38 \mathrm{E}-11$ & $1.23 \mathrm{E}-10$ & $1.79 \Xi-08$ & $2.26 \mathrm{E}-08$ \\
\hline Supernatant & $2.00 \mathrm{E}-07$ & $1.81 E-10$ & $6.97 \mathrm{E}-09$ & $1.05 \mathrm{E}-07$ & $3.12 \mathrm{E}-07$ & 3. $33 \mathrm{E}-09$ & $3.01 \mathrm{E}-12$ & $1.16 \mathrm{E}-10$ & $1.75 \mathrm{E}-09$ & $5.20 \mathrm{E}-09$ \\
\hline Solid Layer & $2.25 \mathrm{E}-07$ & $4.81 \mathrm{E}-09$ & $6.12 E-09$ & $9.20 \mathrm{E}-07$ & $1.16 \mathrm{E}-06$ & $4.52 \mathrm{E}-09$ & $9.66 \mathrm{E}-11$ & 1. $23 \mathrm{E}-10$ & $1.85 \mathrm{E}-08$ & $2.32 \mathrm{E}-08$ \\
\hline $241-T X-105$ & $5.17 \mathrm{E}-09$ & $5.42 \mathrm{E}-10$ & $1.83 \mathrm{E}-08$ & $1.95 \mathrm{E}-07$ & $2.19 \mathrm{E}-07$ & $9.78 \mathrm{E}-11$ & $1.02 \mathrm{E}-11$ & $3.46 \mathrm{E}-10$ & $3.69 \mathrm{E}-09$ & $4.15 \mathrm{E}-09$ \\
\hline Supematant & па & na & na & na & na & na & na & na & na & na \\
\hline Solid Layer & $5.17 \mathrm{E}-09$ & $5.42 \mathrm{E}-10$ & $1.83 E-08$ & $1.95 \mathrm{E}-07$ & $2.19 \mathrm{E}-07$ & $9.78 \mathrm{E}-11$ & $1.02 \mathrm{E}-11$ & $3.46 \mathrm{E}-10$ & $3.69 \mathrm{E}-09$ & $4.15 \mathrm{E}-09$ \\
\hline $241-\mathrm{TX}-106$ & $2.41 \mathrm{E}-08$ & $2.13 \mathrm{E}-09$ & $3.07 \mathrm{E}-08$ & $2.65 \mathrm{E}-07$ & $3.22 \mathrm{E}-07$ & $4.53 \mathrm{E}-10$ & $3.99 \mathrm{E}-11$ & $5.76 \mathrm{E}-10$ & $4.97 \mathrm{E}-09$ & $6.04 \mathrm{E}-09$ \\
\hline Supenatant & nа & na & na & na & $\mathrm{na}$ & na & na & na & na & na \\
\hline Solid Layer & $2.41 \mathrm{E}-08$ & $2.13 \mathrm{E}-09$ & $3.07 E-08$ & $2.65 \mathrm{E}-07$ & $3.22 \mathrm{E}-07$ & $4.53 E-10$ & $3.99 \mathrm{E}-11$ & $5.76 \mathrm{E}-10$ & $4.97 \mathrm{E}-09$ & $6.04 \mathrm{E}-09$ \\
\hline $241-T X-107$ & $5.31 \mathrm{E}-08$ & $4.52 \mathrm{E}-08$ & $3.07 \mathrm{E}-08$ & $1.92 \mathrm{E}-06$ & $2.05 \mathrm{E}-06$ & $1.10 \mathrm{E}-09$ & $9.32 E-10$ & $6.33 \mathrm{E}-10$ & $3.96 \mathrm{E}-08$ & 4.23E- -08 \\
\hline Supernatant & na & $11 \mathrm{a}$ & na & na & na & na & na & $\mathrm{na}$ & na & na \\
\hline Solid Layer & $5.31 \mathrm{E}-08$ & $4.52 \mathrm{E}-08$ & $3.07 \mathrm{E}-08$ & $1.92 \mathrm{E}-06$ & $2.05 \mathrm{E}-06$ & $1.10 \mathrm{E}-09$ & $9.32 \mathrm{E}-10$ & $6.33 \mathrm{E}-10$ & 3.96E-08 & $4.23 \mathrm{E}-08$ \\
\hline
\end{tabular}


RPP-5926 REV 6

Table B-3. Calculated Hydrogen Generation Rate as Mole Per Unit Waste Weight and Volume for 177 Tanks. (20 sheets)

\begin{tabular}{|c|c|c|c|c|c|c|c|c|c|c|}
\hline Tank & $\begin{array}{l}\text { HGR from } \\
\text { radiolysis } \\
\mathrm{RC}_{\mathrm{rad}}{ }^{\mathrm{B} / \gamma} \\
\text { (mole/kg-d) }\end{array}$ & $\begin{array}{l}\text { HGR from } \\
\text { radiolysis } \\
\mathrm{RC}_{\text {rad }}^{\text {alpha }} \\
\text { (mole/kg-d) }\end{array}$ & $\begin{array}{l}\text { HGR from } \\
\text { thermolysis } \\
\mathbf{R C}_{\text {therm }} \\
\text { (mole } / \mathrm{kg}-\mathrm{d})\end{array}$ & $\begin{array}{l}\text { HGR from } \\
\text { corrosion } \\
\text { RC }_{\text {corr }} \\
\text { (mole/kg-d) }\end{array}$ & $\begin{array}{c}\text { Total HGR } \\
\text { from model } \\
R C_{\text {lot }} \\
\text { (mole } / \mathrm{kg}-\mathrm{d} \text { ) }\end{array}$ & $\begin{array}{l}\text { HGR from } \\
\text { radiolysis } \\
\text { RC }_{\text {rad }}{ }^{\text {r }} \\
\left.\text { (mole } / \mathrm{m}^{3}-\mathrm{s}\right)\end{array}$ & $\begin{array}{l}\text { HGR from } \\
\text { radiolysis } \\
\text { RC }_{\text {rut }}^{\text {alpha }} \\
\left.\text { (mole/m } / \mathrm{m}^{3}-\mathbf{s}\right)\end{array}$ & $\begin{array}{l}\text { HGR from } \\
\text { thermolysis } \\
\mathrm{RC}_{\text {therm }} \\
\left(\mathrm{mole} / \mathrm{m}^{3}-\mathrm{s}\right)\end{array}$ & $\begin{array}{l}\text { HGR from } \\
\text { corrosion } \\
\text { RC }_{\text {corr }} \\
\text { (mole } / \mathrm{m}^{3} \text {-s) }\end{array}$ & $\begin{array}{c}\text { Total HGR } \\
\text { from model } \\
\text { RCtot } \\
\left(\mathrm{mole}^{2} \mathrm{~m}^{3}-\mathrm{s}\right)\end{array}$ \\
\hline $241-T X-108$ & $1.26 \mathrm{E}-08$ & $1.22 \mathrm{E}-09$ & $9.31 \mathrm{E}-09$ & $5.66 \mathrm{E}-07$ & $5.89 \mathrm{E}-07$ & $2.36 \mathrm{E}-10$ & $2.28 \mathrm{E}-11$ & $1.75 \mathrm{E}-10$ & $1.06 \mathrm{E}-08$ & $1.11 \mathrm{E}-08$ \\
\hline Supeniatant & na & na & na & na & na & na & na & na & na & na \\
\hline Solid Layer & 1.26E- 08 & $1.22 \mathrm{E}-09$ & $9.31 \mathrm{E}-0.9$ & $5.66 \mathrm{E}-07$ & $5.89 \mathrm{E}-07$ & $2.36 \mathrm{E}-10$ & $2.28 \mathrm{E}-11$ & $1.75 E-10$ & $1.06 \mathrm{E}-08$ & $1.11 \mathrm{E}-08$ \\
\hline $241-\Upsilon X-109$ & $4.04 \mathrm{E}-08$ & $4.42 \mathrm{E}-09$ & $7.59 \mathrm{E}-08$ & $2.92 \mathrm{E}-07$ & $4.13 \mathrm{E}-07$ & $6.69 \mathrm{E}-10$ & $7.32 \mathrm{E}-11$ & $1.26 \mathrm{E}-09$ & $4.83 \mathrm{E}-09$ & $6.83 \mathrm{E}-09$ \\
\hline Supentatant & na & na & na & na & na & na & na & na & na & na \\
\hline Solid Layer & $4.04 \mathrm{E}-08$ & 4. $42 \mathrm{E}-09$ & $7.59 \mathrm{E}-08$ & 2.92E-07 & 4.13E-07 & $6.69 \mathrm{E}-10$ & $7.32 \mathrm{E}-11$ & $1.26 \mathrm{E}-09$ & $4.83 \mathrm{E}-09$ & $6.83 \mathrm{E}-09$ \\
\hline 24I-TX-110 & $6.01 \mathrm{E}-09$ & $7.30 E-10$ & $1.20 \mathrm{E}-08$ & $2.21 \mathrm{E}-07$ & $2.40 \mathrm{E}-07$ & 1. $12 \mathrm{E}-10$ & $1.37 \mathrm{E}-11$ & $2.25 \mathrm{E}-10$ & $4.15 E-09$ & $4.50 \mathrm{E}-09$ \\
\hline Supernatant & na & na & na & na & па & na & naia & na & na & na \\
\hline Solid layer & $6.01 \mathrm{E}-09$ & $7.30 \mathrm{E}-10$ & I. $20 \mathrm{E}-08$ & $2.21 \mathrm{E}-07$ & $2.40 \mathrm{E}-07$ & $1.12 \mathrm{E}-10$ & $1.37 \mathrm{E}-11$ & $2.25 \mathrm{E}-10$ & $4.15 \mathrm{E}-099$ & $4.50 \mathrm{E}-09$ \\
\hline 241-TX-111 & $6.61 E-09$ & $8.33 \mathrm{E}-10$ & $1.17 \mathrm{E}-08$ & $2.58 \mathrm{E}-07$ & $2.78 \mathrm{E}-07$ & $1.23 \mathrm{E}-10$ & $1.55 \mathrm{E}-11$ & $2.19 \mathrm{E}-10$ & $4.82 \mathrm{E}-09$ & $5.18 \mathrm{E}-09$ \\
\hline Supernatant & na & na & na & na & па & na & $\mathrm{na}$ & na & nа & na \\
\hline Solid Layer & $6.61 \mathrm{E}-09$ & $8.33 \mathrm{E}-10$ & $1.17 E-08$ & $2.58 \mathrm{E}-07$ & $2.78 \mathrm{E}-07$ & $1.23 \mathrm{E}-10$ & $1.55 \mathrm{E}-11$ & $2.19 \mathrm{E}-10$ & 4.82E- 09 & $5.18 \mathrm{E}-09$ \\
\hline $241-T X-112$ & $7.85 \mathrm{E}-09$ & $8.96 \mathrm{E}-10$ & $6.60 \mathrm{E}-09$ & $1.86 \mathrm{E}-07$ & $2.02 \mathrm{E}-07$ & 1. $48 \mathrm{E}-10$ & $1.69 \mathrm{E}-11$ & $1.25 \mathrm{E}-10$ & $3.52 \mathrm{E}-09$ & 3.81 E-09 \\
\hline Supernatant & na & na & na & na & na & na & na & na & na & na \\
\hline Solid Layer & $7.85 \mathrm{E}-09$ & $8.96 \mathrm{E}-10$ & $6.60 \mathrm{E}-09$ & $1.86 \mathrm{E}-07$ & $2.02 \mathrm{E}-07$ & $1.48 \mathrm{E}-10$ & $1.69 \mathrm{E}-11$ & $1.25 \mathrm{E}-10$ & $3.52 \mathrm{E}-09$ & $3.81 \mathrm{E}-09$ \\
\hline $241 \cdot \mathrm{TX}-113$ & $5.04 \mathrm{E}-10$ & $3.46 \mathrm{E}-11$ & $3.49 \mathrm{E}-09$ & $1.88 \mathrm{E}-07$ & $1.92 \mathrm{E}-07$ & $9.39 \mathrm{E}-12$ & $6.44 \mathrm{E}-13$ & $6.49 \mathrm{E}-11$ & $3.51 \mathrm{E}-09$ & $3.58 \mathrm{E}-09$ \\
\hline Supernatant & na & na & па & na & ria & па & па & na & na & na \\
\hline Solid Layer & $5.04 \mathrm{E}-10$ & $3.46 \mathrm{E}-11$ & $3.49 \mathrm{E}-09$ & $1.88 \mathrm{E}-07$ & $1.92 \mathrm{E}-07$ & $9.39 \mathrm{E}-12$ & $6.44 \mathrm{E}-13$ & $6.49 \mathrm{E}-1]$ & $3.51 \mathrm{E}-09$ & $3.58 \mathrm{E}-09$ \\
\hline $241 \cdot T X-114$ & $4.59 \mathrm{E}-09$ & $4.75 \mathrm{E}-10$ & $1.05 \mathrm{E}-09$ & $2.04 \mathrm{E}-07$ & $2.10 \mathrm{E}-07$ & $8.67 \mathrm{E}-11$ & $8.98 E-12$ & $1.98 \mathrm{E}-11$ & $3.86 \mathrm{E}-09$ & $3.97 \mathrm{E}-09$ \\
\hline Supcrnatant & na & na & na & na & па & na & na & na & na & na \\
\hline Solid Layer & $4.59 \mathrm{E}-09$ & $4.75 \mathrm{E}-10$ & $1.05 \mathrm{E}-09$ & $2.04 \mathrm{E}-07$ & $2.10 \mathrm{E}-07$ & $8.67 \mathrm{E}-11$ & $8.98 \mathrm{E}-12$ & $1.98 \mathrm{E}-11$ & $3.86 \mathrm{E}-09$ & $3.97 \mathrm{E}-09$ \\
\hline $241-\mathrm{rX}-115$ & $5.75 \mathrm{E}-09$ & $6.56 \mathrm{E}-10$ & $5.28 \mathrm{E}-09$ & $2.00 \mathrm{E}-07$ & $2.12 \mathrm{E}-07$ & $1.08 E-10$ & $1.24 E-11$ & $9.96 \mathrm{E}-11$ & $3.78 \mathrm{E}-09$ & $4.00 \mathrm{E}-09$ \\
\hline Supernalant & па & na & na & na & na & na & na & na & na & na \\
\hline Solid Layer & $5.75 E-09$ & $6.56 \mathrm{E}-10$ & $5.28 E-09$ & $2.00 \mathrm{E}-07$ & $2.12 \mathrm{E}-07$ & $1.08 \mathrm{E}-10$ & $1.24 \mathrm{E}-11$ & $9.96 \mathrm{E}-11$ & $3.78 \mathrm{E}-09$ & $4.00 \mathrm{E}-09$ \\
\hline $241-T X-116$ & $2.65 \mathrm{E}-09$ & $2.15 \mathrm{E}-10$ & 1. $48 \mathrm{E}-11$ & $1.89 \mathrm{E}-07$ & $1.92 \mathrm{E}-07$ & $5.09 \mathrm{E}-11$ & $4.12 \mathrm{E}-12$ & $2.84 \mathrm{E}-13$ & $3.62 \mathrm{E}-09$ & $3.68 \mathrm{E}-09$ \\
\hline Supernatant & nà & na & na & na & na & na & na & na & na & na \\
\hline Solid Layer & $2.65 \mathrm{E}-09$ & $2.15 \mathrm{E}-10$ & $1.48 E-11$ & $1.89 \mathrm{E}-07$ & $1.92 \mathrm{E}-07$ & $5.09 \mathrm{E}-11$ & 4.12E-12 & $2.84 \mathrm{E}-13$ & $3.62 \mathrm{E}-09$ & $3.68 \mathrm{E}-09$ \\
\hline
\end{tabular}


RPP-5926 REV 6

Table B-3. Calculated Hydrogen Generation Rate as Mole Per Unit Waste Weight and Volume for 177 Tanks. (20 sheets)

\begin{tabular}{|c|c|c|c|c|c|c|c|c|c|c|}
\hline Tank & $\begin{array}{c}\text { HGR from } \\
\text { radiolysis } \\
\mathrm{RC}_{\mathrm{rad}}^{\mathrm{B} / \gamma} \\
\text { (mole } / \mathrm{kg-d} \text { ) }\end{array}$ & $\begin{array}{l}\text { HGR from } \\
\text { radiolysis } \\
R_{\text {rad }} \text { alpha } \\
\text { (mole/kg-d) }\end{array}$ & $\begin{array}{l}\text { HGR from } \\
\text { thermolysis } \\
\text { RC }_{\text {therm }} \\
\text { (mole/kg-d) }\end{array}$ & $\begin{array}{l}\text { HGR from } \\
\text { corrosion } \\
\text { RC }_{\text {corr }} \\
\text { (mole/kg-d) }\end{array}$ & $\begin{array}{c}\text { Total HGR } \\
\text { from model } \\
\mathrm{RC}_{\text {tot }} \\
\text { (mole } / \mathrm{kg}-\mathrm{d} \text { ) }\end{array}$ & $\begin{array}{l}\text { HGR from } \\
\text { radiolysis } \\
\mathrm{RC}_{\mathrm{rad}} \mathrm{b} / \mathrm{r} \\
\left(\mathrm{mole} / \mathrm{m}^{3}-\mathrm{s}\right)\end{array}$ & $\begin{array}{l}\text { HGR from } \\
\text { radiolysis } \\
\text { RC }_{\text {rad }} \text { alpha } \\
\left.\text { (mole } / \mathrm{m}^{3}-\mathrm{s}\right)\end{array}$ & $\begin{array}{l}\text { HGR from } \\
\text { thermolysis } \\
\mathrm{RC}_{\text {thern }} \\
\left.\text { (mole } / \mathrm{m}^{3}-\mathrm{s}\right)\end{array}$ & $\begin{array}{l}\text { HGR from } \\
\text { corrosion } \\
\mathrm{RC}_{\text {curr }} \\
\left.\text { (mole } / \mathrm{m}^{3}-\mathrm{s}\right)\end{array}$ & $\begin{array}{l}\text { Total HGR } \\
\text { from model } \\
\text { RCtot } \\
\left.\text { (mole } / \mathrm{m}^{3}-\mathrm{s}\right)\end{array}$ \\
\hline $241-T X-117$ & $2.72 \mathrm{E}-09$ & $2.80 \mathrm{E}-10$ & $4.65 \mathrm{E}-13$ & $2.23 \mathrm{E}-07$ & $2.26 \mathrm{E}-07$ & $4.98 \mathrm{E}-11$ & $5.12 \mathrm{E}-12$ & $8.51 \mathrm{E}-15$ & $4.08 \mathrm{E}-09$ & $4.14 \mathrm{E}-09$ \\
\hline Superratant & na & na & na & na & na & na & na & na & na & na \\
\hline Solid Layer & $2.72 \mathrm{E}-09$ & $2.80 \mathrm{E}-10$ & $4.65 \mathrm{E}-13$ & $2.23 \mathrm{E}-07$ & $2.26 \mathrm{E}-07$ & $4.98 E-11$ & $5.12 \mathrm{E}-12$ & $8.51 \mathrm{E}-15$ & $4.08 \mathrm{E}-09$ & 4. $14 \mathrm{E}-09$ \\
\hline $241-T X-118$ & $6.95 \mathrm{E}-08$ & $6.46 \mathrm{E}-08$ & $2.95 \mathrm{E}-08$ & $3.21 \mathrm{E}-07$ & $4.85 \mathrm{E}-07$ & $1.36 \mathrm{E}-09$ & $1.26 \mathrm{E}-09$ & $5.77 \mathrm{E}-10$ & $6.28 \mathrm{E}-09$ & $9.49 \mathrm{E}-09$ \\
\hline Supernatant & na & na & na & na & na & na & na & na & na & na \\
\hline Solid Layer & $6.95 \mathrm{E}-08$ & $6.46 \mathrm{E}-08$ & $2.95 \mathrm{E}-08$ & $3.21 \mathrm{E}-07$ & $4.85 \mathrm{E}-07$ & $1.36 \mathrm{E}-09$ & $1.26 \mathrm{E}-09$ & $5.77 \mathrm{E}-10$ & $6.28 \mathrm{E}-09$ & $9.49 \mathrm{E}-09$ \\
\hline $241-\mathrm{TY}-101$ & $2.69 \mathrm{E}-09$ & $8.56 \mathrm{E}-10$ & $9.04 \mathrm{E}-13$ & $5.96 \mathrm{E}-07$ & $6.00 \mathrm{E}-07$ & $5.07 \mathrm{E}-11$ & $1.61 \mathrm{E}-11$ & $1.70 \mathrm{E}-14$ & $1.12 \mathrm{E}-0.8$ & $1.13 \mathrm{E}-08$ \\
\hline Supernatant & na & na & na & па & na & na & na & na & na & na \\
\hline Solid Layer & $2.69 \mathrm{E}-09$ & $8.56 \mathrm{E}-10$ & $9.04 \mathrm{E}-13$ & $5.96 \mathrm{E}-07$ & $6.00 \mathrm{E}-07$ & $5.07 E-11$ & $1.61 \mathrm{E}-11$ & $1.70 \mathrm{E}-14$ & 3.12E-08 & 1.13E-08 \\
\hline $24 !-T Y-102$ & $1.99 \mathrm{E}-08$ & $6.84 \mathrm{E}-11$ & $7.75 \mathrm{E}-09$ & $8.82 \mathrm{E}-07$ & $9.10 \mathrm{E}-07$ & $4.04 \mathrm{E}-10$ & $1.39 \mathrm{E}-12$ & $1.57 \mathrm{E}-10$ & $1.79 \mathrm{E}-08$ & $1.85 \mathrm{E}-08$ \\
\hline Supernatant & nia & ла & na & na & na & na & na & na & na & na \\
\hline Solid Layer & $1.99 \mathrm{E}-08$ & $6.84 \mathrm{E}-11$ & $7.75 \mathrm{E}-09$ & $8.82 \mathrm{E}-07$ & $9.10 \mathrm{E}-07$ & $4.04 \mathrm{E}-10$ & $1.39 \mathrm{E}-12$ & $1.57 \mathrm{E}-10$ & 1.79E-08 & $1.85 \mathrm{E}-08$ \\
\hline $241-T Y-103$ & $1.07 E-07$ & $5.48 \mathrm{~F}-09$ & $6.11 \mathrm{E}-08$ & 4.62E-07 & $6.36 \mathrm{E}-07$ & $2.09 \mathrm{E}-0.9$ & $1.07 \mathrm{E}-10$ & $1.19 \mathrm{E}-09$ & $9.00 \mathrm{E}-09$ & $1.24 \mathrm{E}-08$ \\
\hline Supcrnatant & na & na & na & na & na & na & na & na & na & na \\
\hline Solid layer & $1.07 \mathrm{E}-07$ & $5.48 \mathrm{E}-09$ & $6.11 \mathrm{E}-08$ & $4.62 \mathrm{E}-07$ & $6.36 \mathrm{E}-07$ & $2.09 \mathrm{E}-09$ & $1.07 \mathrm{E}-10$ & $1.19 \mathrm{E}-09$ & $9.00 \mathrm{E}-09$ & $1.24 \mathrm{E}-08$ \\
\hline 241-TY-104 & $2.45 \mathrm{E}-07$ & $1.04 \mathrm{E}-08$ & $4.37 \mathrm{E}-09$ & $1.42 \mathrm{E}-06$ & $1.68 \mathrm{E}-06$ & 4. $64 \mathrm{E}-09$ & 1.97E-10 & $8.28 \mathrm{E}-11$ & $2.70 \mathrm{E}-08$ & $3.19 \mathrm{E}-08$ \\
\hline Supenatant & $2.29 \mathrm{E}-08$ & $1.06 \mathrm{E}-10$ & $6.71 \mathrm{E}-09$ & $1.28 \mathrm{E}-07$ & $1.58 \mathrm{E}-07$ & $3.13 E-10$ & 1.44E-12 & $9.17 E-11$ & $1.75 \mathrm{E}-09$ & $2.16 \mathrm{E}-09$ \\
\hline Solid Layer & $2.48 \mathrm{E}-07$ & $1.05 \mathrm{E}-08$ & $4.32 \mathrm{E}-09$ & $1.45 \mathrm{E}-06$ & $1.72 \mathrm{E}-06$ & $4.73 E-09$ & $2.01 \mathrm{E}-10$ & $8.25 \mathrm{E}-11$ & $2.78 \mathrm{E}-08$ & $3.28 \mathrm{E}-08$ \\
\hline 241-TY-105 & $2.26 \mathrm{E}-07$ & $7.86 \mathrm{E}-10$ & $1.67 \mathrm{E}-08$ & $3.73 \mathrm{E}-07$ & $6.17 \mathrm{E}-07$ & $4.01 \mathrm{E}-09$ & $1.39 \mathrm{E}-11$ & $2.95 \mathrm{E}-10$ & $6.60 \mathrm{E}-09$ & $1.09 \mathrm{E}-08$ \\
\hline Supernatant & na & na & na & na & na & na & ina & na & na & na \\
\hline Solid Layer & $2.26 \mathrm{E}-07$ & $7.86 \mathrm{E}-10$ & $1.67 \mathrm{E}-08$ & $3.73 \mathrm{E}-07$ & $6.17 \mathrm{E}-07$ & $4.01 \mathrm{E}-09$ & $1.39 \mathrm{E}-11$ & $2.95 \mathrm{E}-10$ & $6.60 \mathrm{E}-09$ & $1.09 \mathrm{E}-08$ \\
\hline $241-$ TY-106 & $3.05 \mathrm{E}-07$ & $1.25 \mathrm{E}-09$ & $6.95 \mathrm{E}-09$ & $4.33 \mathrm{E}-06$ & 4. $64 E-06$ & 4.93E-09 & $2.02 \mathrm{E}-11$ & $1.13 E-10$ & 7.01E-08 & $7.52 \mathrm{E}-08$ \\
\hline Supernatant & na & na & na & na & na & na & na & na & na & na \\
\hline Solid Layer & $3.05 \mathrm{E}-07$ & $1.25 E-09$ & $6.95 \mathrm{E}-09$ & 4.33E-06 & 4. $64 \mathrm{E}-06$ & $4.93 \mathrm{E}-09$ & $2.02 E-11$ & t. $13 \mathrm{E}-10$ & 7.01E-08 & $7.52 \mathrm{E}-08$ \\
\hline $241-U-101$ & $6.07 \mathrm{E}-07$ & $1.76 \mathrm{E}-09$ & $1.09 \mathrm{E}-08$ & $2.46 \mathrm{E}-06$ & $3.08 \mathrm{E}-06$ & $1.24 \mathrm{E}-08$ & $3.61 \mathrm{E}-11$ & $2.22 \mathrm{E}-10$ & $5.05 \mathrm{E}-08$ & $6.32 \mathrm{E}-08$ \\
\hline Supernatant & na & na & na & na & na & na & na & na & na & na \\
\hline Solid Layer & $6.07 \mathrm{E}-07$ & $1.76 \mathrm{E}-09$ & $1.09 \mathrm{E}-08$ & $2.46 \mathrm{E}-06$ & $3.08 \mathrm{E}-06$ & $1.24 \mathrm{E}-08$ & $3.61 \mathrm{E}-11$ & $2.22 \mathrm{E}-10$ & $5.05 \mathrm{E}-08$ & $6.32 \mathrm{E}-08$ \\
\hline
\end{tabular}


Table B-3. Calculated Hydrogen Generation Rate as Mole Per Unit Waste Weight and Volume for 177 Tanks. (20 sheets)

\begin{tabular}{|c|c|c|c|c|c|c|c|c|c|c|}
\hline Tank & $\begin{array}{l}\text { HGR from } \\
\text { radiolysis } \\
\mathrm{RC}_{\mathrm{rad} / \gamma}^{\mathrm{B} / \gamma} \\
\text { (mole/kg-d) }\end{array}$ & $\begin{array}{l}\text { HGR from } \\
\text { radiolysis } \\
\text { RC }_{\text {rad }}^{\text {atpho }} \\
\text { (mole/kg-d) }\end{array}$ & $\begin{array}{l}\text { HGR from } \\
\text { thermolysis } \\
\text { RC }_{\text {therin }} \\
\text { (mole } / \mathrm{kg}-\mathrm{d} \text { ) }\end{array}$ & $\begin{array}{l}\text { HGR from } \\
\text { corrosion } \\
\text { RC }_{\text {corr }} \\
\text { (mole } / \mathrm{kg} \text {-d) }\end{array}$ & $\begin{array}{c}\text { Total HGR } \\
\text { from model } \\
\mathrm{RC}_{\mathrm{tot}} \\
\text { (mole } / \mathrm{kg}-\mathrm{d})\end{array}$ & $\begin{array}{l}\text { HGR from } \\
\text { radiolysis } \\
\mathrm{RC}_{\mathrm{rad}} \mathrm{b} / \mathrm{r} \\
\left.\text { (mole/m } \mathrm{m}^{3}-\mathrm{s}\right)\end{array}$ & $\begin{array}{l}\text { HGR from } \\
\text { radiolysis } \\
\mathrm{RC}_{\text {rad }} \text { alpha } \\
\left.\text { (mole/m } \mathbf{m}^{3}-\mathrm{s}\right)\end{array}$ & $\begin{array}{l}\text { HGR from } \\
\text { thermolysis } \\
\text { RC }_{\text {therm }} \\
\text { (mole/m } \text { m }^{3} \text {-s) }\end{array}$ & $\begin{array}{l}\text { HGR from } \\
\text { corrosion } \\
\text { RC }_{\text {rorr }} \\
\left(\mathrm{mole} / \mathrm{m}^{3}-\mathrm{s}\right)\end{array}$ & $\begin{array}{c}\text { Total HGR } \\
\text { from model } \\
\text { RCtot } \\
\text { (mole/m } \mathrm{m}^{3}-\mathrm{s} \text { ) }\end{array}$ \\
\hline $241-U-102$ & $2.42 \mathrm{E}-07$ & $5.35 \mathrm{E}-09$ & $2.05 E-07$ & $2.67 \mathrm{E}-07$ & $7.20 \mathrm{E}-07$ & $4.68 \mathrm{E}-09$ & $1.04 \mathrm{E}-10$ & $3.97 \mathrm{E}-09$ & $5.18 E-09$ & $1.39 \mathrm{E}-08$ \\
\hline Supematant & $3.75 E-07$ & $2.45 \mathrm{E}-10$ & $1.56 \mathrm{E}-07$ & $1,02 E-07$ & $6.33 \mathrm{E}-07$ & $6.42 \mathrm{E}-09$ & 4. $19 \mathrm{E}-12$ & $2.68 \mathrm{E}-09$ & $1.75 \mathrm{E}-09$ & $1.09 \mathrm{E}-08$ \\
\hline Solid Layer & $2.42 \mathrm{E}-07$ & $5.36 \mathrm{E}-09$ & 2.05E-07 & $2.68 \mathrm{E}-07$ & $7.20 \mathrm{E}-07$ & $4.68 \mathrm{E}-09$ & $1.04 \mathrm{E}-10$ & $3.97 \mathrm{E}-09$ & $5.19 \mathrm{E}-09$ & $1.39 \mathrm{E}-08$ \\
\hline $241-U-103$ & $1.46 \mathrm{E}-07$ & $3.92 \mathrm{E}-09$ & $3.16 \mathrm{E}-07$ & $2.52 \mathrm{E}-07$ & $7.18 \mathrm{E}-07$ & $2.88 \mathrm{E}-09$ & $7.71 E-11$ & $6.22 E-09$ & $4.97 \mathrm{E}-09$ & $1.41 \mathrm{E}-08$ \\
\hline Supernatant & $4.11 \mathrm{E}-07$ & $4.82 \mathrm{E}-10$ & $3.98 \mathrm{E}-07$ & $1.05 E-07$ & $9.14 \mathrm{E}-07$ & $6.85 \mathrm{E}-09$ & $8.03 \mathrm{E}-12$ & $6.63 \mathrm{E}-09$ & $1.75 \mathrm{E}-09$ & $1.52 \mathrm{E}-08$ \\
\hline Solid Layer & 1.46E:-07 & $3.92 \mathrm{E}-09$ & $3.16 \mathrm{E}-07$ & $2.52 \mathrm{E}-07$ & $7.18 \mathrm{E}-07$ & $2.87 \mathrm{E}-09$ & $7.72 \mathrm{E}-11$ & $6.22 \mathrm{E}-09$ & $4.97 E-09$ & $1.41 \mathrm{E}-0.8$ \\
\hline $241-0-104$ & 3.89E-09 & $1.48 \mathrm{E}-10$ & $2.44 \mathrm{E}-07$ & 1.72E-06 & $1.97 \mathrm{E}-06$ & $5.07 \mathrm{E}-11$ & $1.93 E-12$ & $3.18 \mathrm{E}-09$ & $2.24 \mathrm{E}-08$ & $2.57 \mathrm{E}-08$ \\
\hline Supernatant & na & na & na & na & па & na & na & na & na & na \\
\hline Solid Layer & $3.89 \mathrm{E}-09$ & $1.48 \mathrm{E}-10$ & $2.44 \mathrm{E}-07$ & $1.72 \mathrm{E}-06$ & $1.97 \mathrm{E}-06$ & $5.07 E-11$ & 1.93E-12 & $3.18 \mathrm{E}-09$ & $2.24 \mathrm{E}-08$ & $2.57 \mathrm{E}-08$ \\
\hline $24 t-U-105$ & $1.25 \mathrm{E}-07$ & $1.34 \mathrm{E}-08$ & $2.93 \mathrm{E}-07$ & $2.55 \mathrm{E}-07$ & $6.86 \mathrm{E}-07$ & $2.42 \mathrm{E}-09$ & $2.59 \mathrm{E}-10$ & $5.67 \mathrm{E}-09$ & $4.92 \mathrm{E}-09$ & $1.33 E-08$ \\
\hline Supeniatant & na & па & na & na & na & na & na & naa & na & na \\
\hline Solid Layer & $1.25 \mathrm{E}-07$ & $1.34 \mathrm{E}-08$ & 2.93E-07 & $2.55 \mathrm{E}-07$ & $6.86 \mathrm{E}-07$ & $2.42 \mathrm{E}-09$ & $2.59 \mathrm{E}-10$ & $5.67 \mathrm{E}-09$ & $4.92 \mathrm{E}-09$ & $1.33 \mathrm{E}-08$ \\
\hline $241-\mathrm{U}-106$ & $3.74 E-07$ & $2.30 \mathrm{E}-0.8$ & $5.24 \mathrm{E}-07$ & $4.64 \mathrm{E}-07$ & $1.38 \mathrm{E}-06$ & $6.71 E-09$ & 4.12E-10 & $9.40 \mathrm{E}-09$ & $8.33 \mathrm{E}-0.9$ & $2.48 \mathrm{E}-08$ \\
\hline Supenaatant & $4.82 \mathrm{E}-07$ & $6.42 \mathrm{E}-09$ & $5.05 \mathrm{E}-07$ & $1.13 \mathrm{E}-07$ & $3.11 \mathrm{E}-06$ & $7.48 \mathrm{E}-09$ & $9.96 \mathrm{E}-11$ & $7.84 \mathrm{E}-09$ & $1.75 \mathrm{E}-09$ & $1.72 \mathrm{E}-08$ \\
\hline Solid Layer & $3.73 \mathrm{E}-07$ & $2.31 \mathrm{E}-08$ & $5.24 \mathrm{E}-07$ & $4.67 \mathrm{E}-07$ & 1. $39 \mathrm{E}-06$ & $6.70 \mathrm{E}-09$ & 4. $15 E-10$ & $9.41 \mathrm{E}-09$ & $8.40 \mathrm{E}-09$ & $2.49 \mathrm{E}-08$ \\
\hline $241-\cup-107$ & $6.30 \mathrm{E}-08$ & $9.50 \mathrm{E}-09$ & $7.86 E-08$ & $2.76 \mathrm{E}-07$ & $4.27 \mathrm{E}-07$ & $1.27 E-09$ & $1.91 E-10$ & $1.58 \mathrm{E}-09$ & $5.56 \mathrm{E}-09$ & $8.60 \mathrm{E}-09$ \\
\hline Supernatant & na & na & na & na & na & na & na & na & na & na \\
\hline Solid Layer & $6.30 \mathrm{E}-08$ & $9.50 \mathrm{E}-09$ & $7.86 \mathrm{E}-08$ & $2.76 \mathrm{E}-07$ & $4.27 \mathrm{E}-07$ & $1.27 \mathrm{E}-09$ & $1.91 \mathrm{E}-10$ & $1.58 \mathrm{E}-09$ & $5.56 \mathrm{E}-09$ & $8.60 \mathrm{E}-09$ \\
\hline $24 I-U-108$ & $1.18 \mathrm{E}-07$ & $2.60 \mathrm{E}-09$ & $2.17 E-07$ & $2.23 \mathrm{E}-07$ & $5.60 \mathrm{~F}-07$ & $2.29 \mathrm{E}-09$ & $5.06 E-11$ & 4.22E-09 & $4.33 \mathrm{E}-09$ & $1.09 \mathrm{E}-08$ \\
\hline Supernatant & na & na & na & na & na & na & na & na & na & na \\
\hline Solid Layer & $1.18 \mathrm{E}-07$ & $2.60 \mathrm{E}-09$ & $2.17 \mathrm{E}-07$ & $2.23 \mathrm{E}-07$ & $5.60 \mathrm{E}-07$ & $2.29 \mathrm{E}-09$ & $5.06 \mathrm{E}-11$ & $4.22 \mathrm{E}-09$ & 4.33E-09 & $1.09 \mathrm{E}-08$ \\
\hline $24 !-U-109$ & $7.87 \mathrm{E}-08$ & $6.63 \mathrm{E}-10$ & $1.25 \mathrm{E}-07$ & $2.67 \mathrm{E}-07$ & $4.71 \mathrm{E}-07$ & $1.51 \mathrm{E}-09$ & $1.27 \mathrm{E}-11$ & $2.40 \mathrm{E}-09$ & $5.10 \mathrm{E}-09$ & $9.02 \mathrm{E}-09$ \\
\hline Supernatant & na & па & na & па & na & na & na & na & na & na \\
\hline Solid Layer & $7.87 \mathrm{E}-08$ & $6.63 \mathrm{E}-10$ & $1.25 \mathrm{E}-07$ & $2.67 \mathrm{E}-07$ & $4.71 \mathrm{E}-07$ & $1.51 \mathrm{E}-09$ & $1.27 \mathrm{E}-11$ & $2.40 \mathrm{E}-09$ & $5.10 \mathrm{E}-09$ & $9.02 E-09$ \\
\hline $241-\mathrm{U}-110$ & $3.00 \mathrm{E}-07$ & $8.50 \mathrm{E}-09$ & $1.88 \mathrm{E}-08$ & $4.09 E-07$ & $7.37 E-07$ & $5.97 \mathrm{E}-09$ & $1.69 \mathrm{E}-10$ & $3.74 \mathrm{E}-10$ & $8.13 \mathrm{E}-09$ & $1.46 \mathrm{E}-08$ \\
\hline Supernatant & na & na & na & па & 11a & na & na & na & na & na \\
\hline Solid Layer & $3.00 \mathrm{E}-07$ & $8.50 \mathrm{E}-09$ & $1.88 \mathrm{E}-08$ & $4.09 \mathrm{E}-07$ & $7.37 \mathrm{E}-07$ & $5.97 \mathrm{E}-09$ & $1.69 E-10$ & $3.74 \mathrm{E}-10$ & $8.13 \mathrm{E}-09$ & $1.46 \mathrm{E}-08$ \\
\hline
\end{tabular}


RPP-5926 REV 6

Table B-3. Calculated Hydrogen Generation Rate as Mole Per Unit Waste Weight and Volume for 177 Tanks. (20 sheets)

\begin{tabular}{|c|c|c|c|c|c|c|c|c|c|c|}
\hline Tank & $\begin{array}{l}\text { HGR from } \\
\text { radiolysis } \\
\mathrm{RC}_{\mathrm{rad} / \mathrm{\gamma}} \\
\text { (mole/kg-d) }\end{array}$ & $\begin{array}{l}\text { HGR from } \\
\text { radiolysis } \\
\text { RC }_{\text {rad }}^{\text {alpaz }} \\
\text { (mole/kg-d) }\end{array}$ & $\begin{array}{l}\text { HGR from } \\
\text { thermolysis } \\
\text { RC }_{\text {therm }} \\
\text { (mole/kg-d) }\end{array}$ & $\begin{array}{l}\text { HGR from } \\
\text { corrosion } \\
\mathrm{RC}_{\text {carr }} \\
\text { (mole } / \mathbf{k g - d} \text { ) }\end{array}$ & $\begin{array}{c}\text { Total HGR } \\
\text { from model } \\
\mathrm{RC}_{\text {tot }} \\
\text { (mole } / \mathrm{kg} \text {-d) }\end{array}$ & $\begin{array}{l}\text { HGR from } \\
\text { radiolysis } \\
\text { RC }_{\mathrm{rad}} \mathrm{b} / \mathrm{r} \\
\left(\mathrm{mole} / \mathrm{m}^{3}-\mathrm{s}\right)\end{array}$ & $\begin{array}{l}\text { HGR from } \\
\text { radiolysis } \\
\mathrm{RC}_{\text {rad }} \text { alpha } \\
\left.\text { (mole/m } \mathrm{m}^{3}-\mathrm{s}\right)\end{array}$ & $\begin{array}{l}\text { HGR from } \\
\text { thermolysis } \\
\mathrm{RC}_{\text {therm }} \\
\left.\text { (mole } / \mathrm{m}^{3}-\mathrm{s}\right)\end{array}$ & $\begin{array}{c}\text { HGR from } \\
\text { corrosion } \\
\mathrm{RC}_{\text {corr }} \\
\left(\mathrm{mole}^{\mathrm{m}} / \mathrm{m}^{3} \cdot \mathrm{s}\right)\end{array}$ & $\begin{array}{c}\text { Total HGR } \\
\text { from model } \\
\text { RCtot } \\
\left(\mathrm{mole} / \mathrm{m}^{3}-\mathrm{s}\right)\end{array}$ \\
\hline $241-U-111$ & $1.18 \mathrm{E}-07$ & $5.21 \mathrm{E}-09$ & $8.03 E-08$ & $3.65 \mathrm{E}-07$ & $5.68 \mathrm{E}-07$ & $2.19 \mathrm{E}-09$ & $9.71 \mathrm{E}-11$ & $1.50 \mathrm{E}-09$ & $6.80 \mathrm{E}-09$ & $1.06 \mathrm{E}-08$ \\
\hline Supernatant & па & nа & na & na & na & na & na & na & na & na \\
\hline Solid Layer & $1.18 \mathrm{E}-07$ & $5.21 \mathrm{E}-09$ & $8.03 \mathrm{E}-08$ & $3.65 \mathrm{E}-07$ & $5.68 \mathrm{E}-07$ & $2.19 \mathrm{E}-09$ & $9.71 \mathrm{E}-11$ & $1.50 \mathrm{E}-09$ & $6.80 \mathrm{E}-09$ & $1.06 \mathrm{E}-08$ \\
\hline $241-U \cdot 112$ & $3.36 \mathrm{E}-07$ & $7.57 \mathrm{E}-10$ & $1.21 \mathrm{E}-08$ & $1.31 \mathrm{E}-06$ & $1.66 \mathrm{E}-06$ & $6.77 \mathrm{E}-09$ & $1.53 \mathrm{E}-11$ & $2.43 E-10$ & $2.64 \mathrm{E}-08$ & $3.34 \mathrm{E}-08$ \\
\hline Supenatant & ла & na & na & na & na & na & na & na & na & na \\
\hline Solid Layer & $3.36 \mathrm{E}-07$ & $7.57 \mathrm{E}-10$ & $1.21 \mathrm{E}-08$ & $1.31 \mathrm{E}-06$ & $1.66 \mathrm{E}-06$ & $6.77 \mathrm{E}-09$ & $1.53 \mathrm{E}-11$ & $2.43 \mathrm{E}-10$ & $2.64 \mathrm{E}-0.8$ & $3.34 \mathrm{E}-08$ \\
\hline $24 !-U-201$ & $7.34 \mathrm{E}-09$ & $7.05 E-11$ & $0.00 \mathrm{E}+00$ & $1.52 \mathrm{E}-06$ & $1.53 \mathrm{E}-06$ & $5.92 E-11$ & $1.25 E-12$ & $0.00 \mathrm{E}+00$ & $2.70 E-08$ & $2.70 \mathrm{E}-08$ \\
\hline Supeniatant & $3.52 \mathrm{~F}-08$ & $2.88 \mathrm{E}-11$ & $0.00 \mathrm{E}+00$ & $4.49 \mathrm{E}-07$ & $4.85 \mathrm{E}-07$ & $5.13 \mathrm{E}-10$ & $4.21 \mathrm{E}-13$ & $0.00 \mathrm{E}+00$ & $6.55 \mathrm{E}-09$ & $7.07 \mathrm{E}-09$ \\
\hline Solid Layer & $3.14 \mathrm{E}-09$ & $6.41 \mathrm{E}-11$ & $0.00 \mathrm{E}+00$ & $1.82 \mathrm{E}-06$ & $1.83 \mathrm{E}-06$ & $5.92 \mathrm{E}-11$ & $1.21 \mathrm{E}-12$ & $0.00 \mathrm{E}+00$ & $3.44 \mathrm{E}-08$ & $3.45 \mathrm{E}-08$ \\
\hline $241-\mathrm{L}-202$ & $6.11 \mathrm{E}-09$ & $8.53 \mathrm{E}-11$ & $1.34 \mathrm{E}-10$ & $1.70 \mathrm{E}-06$ & $1.71 \mathrm{E}-06$ & $4.66 E-11$ & $1.43 \mathrm{E}-12$ & $2.24 \mathrm{E}-12$ & $2.84 E-08$ & $2.85 \mathrm{E}-08$ \\
\hline Supernatant & $2.40 \mathrm{E}-08$ & $5.30 \mathrm{E}-13$ & $2.24 \mathrm{E}-10$ & $4.42 \mathrm{E}-07$ & $4.67 \mathrm{E}-07$ & $3.56 \mathrm{E}-10$ & $7.85 E-15$ & 3.32E-12 & $6.55 \mathrm{E}-09$ & $6.91 \mathrm{E}-09$ \\
\hline Solid Layer & $2.67 \mathrm{E}-09$ & $8.29 \mathrm{~F}-11$ & $1.00 \mathrm{E}-10$ & $2.13 \mathrm{E}-06$ & $2.13 \mathrm{E}-06$ & $4.66 \mathrm{E}-11$ & $1.45 \mathrm{E}-12$ & $1.75 \mathrm{E}-12$ & $3.72 \mathrm{E}-08$ & $3.72 \mathrm{E}-08$ \\
\hline $241-\mathrm{L}-203$ & 8.42E-09 & $7.52 \mathrm{E}-11$ & $0.00 \mathrm{E}+00$ & $1.74 \mathrm{E}-06$ & $1.75 \mathrm{E}-06$ & $7.84 \mathrm{E}-11$ & $1.30 \mathrm{E}-12$ & $0.00 \mathrm{E}+00$ & $3.01 \mathrm{E}-08$ & $3.02 \mathrm{E}-08$ \\
\hline Supernatant & $2.57 \mathrm{E}-08$ & $1.30 \mathrm{E}-12$ & $0.00 \mathrm{E}+00$ & $4.42 \mathrm{E}-07$ & $4.68 \mathrm{E}-07$ & $3.80 E-10$ & $1.93 \mathrm{E}-14$ & $0.00 E+00$ & $6.55 \mathrm{E}-09$ & $6.93 \mathrm{E}-09$ \\
\hline Solid Layer & $4.26 \mathrm{E}-09$ & $8.57 \mathrm{E}-11$ & $0.00 \mathrm{E}+00$ & $2.21 \mathrm{E}-06$ & $2.21 \mathrm{E}-06$ & $7.84 \mathrm{E}-11$ & $1.58 E-12$ & $0.00 \mathrm{E}+00$ & $4.06 \mathrm{E}-08$ & $4.07 \mathrm{E}-08$ \\
\hline $241-t-204$ & $1.89 \mathrm{E}-08$ & $3.18 \mathrm{E}-09$ & $2.48 \mathrm{~F}-11$ & $5.55 \mathrm{E}-06$ & $5.57 \mathrm{E}-06$ & $2.16 \mathrm{E}-10$ & $4.93 \mathrm{E}-11$ & $3.84 \mathrm{E}-13$ & $1.26 \mathrm{E}-07$ & $1.26 \mathrm{E}-07$ \\
\hline Supematant & $2.49 \mathrm{E}-08$ & $1.19 \mathrm{E}-12$ & $5.01 E-11$ & $1.28 \mathrm{E}-06$ & $1.30 \mathrm{E}-06$ & $3.20 \mathrm{E}-10$ & 1. $53 \mathrm{E}-14$ & $6.44 \mathrm{E}-13$ & $1.64 \mathrm{E}-08$ & $1.67 \mathrm{E}-08$ \\
\hline Solid Layer & $1.27 \mathrm{E}-08$ & $2.68 \mathrm{E}-09$ & $1.44 \mathrm{E}-1 \mathrm{I}$ & $7.39 \mathrm{E}-06$ & $7.41 \mathrm{E}-06$ & $2.16 E-10$ & $4.56 \mathrm{E}-11$ & $2.44 \mathrm{E}-13$ & $1.26 \mathrm{E}-07$ & $1.26 \mathrm{E}-07$ \\
\hline AY 102-S-14U & 1.62E-05 & $8.55 \mathrm{E}-07$ & $2.60 \mathrm{E}-07$ & $1.21 \mathrm{E}-06$ & $1.85 \mathrm{E}-05$ & $3.00 \mathrm{E}-07$ & $1.59 E-08$ & $4.84 E-09$ & $2.25 \mathrm{E}-0.08$ & $3.44 \mathrm{E}-07$ \\
\hline AY102-S-14L & $2.72 \mathrm{E}-05$ & $1.03 \mathrm{E}-06$ & $6.87 \mathrm{E}-07$ & $1.64 \mathrm{E}-06$ & $3.06 \mathrm{E}-05$ & $4.98 \mathrm{E}-07$ & $1.89 \mathrm{E}-08$ & $1.26 \mathrm{E}-08$ & $3.00 \mathrm{E}-08$ & $5.60 \mathrm{E}-07$ \\
\hline AY 102-S-15U & 1.27E-05 & $9.51 \mathrm{E}-07$ & $4.14 \mathrm{E}-07$ & $3.98 \mathrm{E}-06$ & $1.81 \mathrm{E}-05$ & $2.28 \mathrm{E}-07$ & $1.71 \mathrm{E}-08$ & $7.43 \mathrm{E}-09$ & 7.13E- 08 & $3.24 \mathrm{E}-07$ \\
\hline AY102-S-15L & $2.78 \mathrm{E}-05$ & $1,18 \mathrm{E}-06$ & $6.54 \mathrm{E}-07$ & $1.17 \mathrm{E}-06$ & $3.08 \mathrm{E}-05$ & 5.14E-07 & $2.19 \mathrm{E}-08$ & $1.21 \mathrm{E}-08$ & $2.16 \mathrm{E}-08$ & $5.70 \mathrm{E}-07$ \\
\hline$A Y-102-S-16$ & $5.52 \mathrm{E}-05$ & 1. $24 \mathrm{E}-06$ & $5.48 \mathrm{E}-07$ & $1.51 \mathrm{E}-06$ & $5.85 \mathrm{E}-05$ & $8.93 \mathrm{E}-07$ & $2.01 \mathrm{E}-08$ & $8.86 \mathrm{E}-09$ & $2.45 \mathrm{E}-08$ & $9.46 \mathrm{E}-07$ \\
\hline AY102-Orig-9" & $1.10 \mathrm{E}-04$ & $1.27 \mathrm{E}-06$ & $4.30 \mathrm{E}-07$ & $1.71 \mathrm{E}-06$ & $1.14 \mathrm{E}-04$ & $1.83 \mathrm{E}-06$ & $2.11 \mathrm{E}-08$ & $7.15 \mathrm{E}-09$ & $2.84 \mathrm{E}-08$ & $1.89 \mathrm{E}-06$ \\
\hline
\end{tabular}


RPP-5926 REV 6

Table B-4. Calculated Total Hydrogen Generation Rate In Dome Space for 177 Tanks. (20 sheets)

\begin{tabular}{|c|c|c|c|c|c|c|c|c|c|c|}
\hline Tank & $\begin{array}{c}\text { HGR from } \\
\text { radiolysis } \\
\text { HGR }_{\text {rad }}{ }^{\text {r }} \\
\text { (cfm) }\end{array}$ & $\begin{array}{l}\text { HGR from } \\
\text { radiolysis } \\
\text { HGR }_{\text {rad }}^{\text {alpha }} \\
\text { (cfm) }\end{array}$ & $\begin{array}{c}\text { HGR from } \\
\text { thermolysis } \\
\text { HGR }_{\text {therm }} \\
\text { (cfm) }\end{array}$ & $\begin{array}{c}\text { HGR from } \\
\text { corrosion } \\
\text { HGR }_{\text {carr }} \\
\text { (cfm) }\end{array}$ & $\begin{array}{l}\text { Percent of } \\
\text { HGR from } \\
\text { radiolysis } \\
\mathrm{HGR}_{\text {rad }} \text { b/r }\end{array}$ & $\begin{array}{l}\text { Percent of } \\
\text { HGR from } \\
\text { radiolysis } \\
\text { HGR }_{\text {rad }} \text { alpha }\end{array}$ & $\begin{array}{l}\text { Percent of } \\
\text { HGR from } \\
\text { thermolysis } \\
\text { HGR }_{\text {therm }}\end{array}$ & $\begin{array}{l}\text { Percent of } \\
\text { HGR fram } \\
\text { corrosion } \\
\text { HGR }_{\text {sorr }}\end{array}$ & $\begin{array}{l}\text { Total HGR } \\
\text { from model } \\
\text { HGR }_{\text {nwel }} \\
\text { (cfm) }\end{array}$ & $\begin{array}{c}\text { Total HGR } \\
\text { from model } \\
H^{\prime} G R_{\text {mod }} \\
\text { (L/day) }\end{array}$ \\
\hline 241-AN-101 & $7.88 \mathrm{E}-04$ & $2.12 E-07$ & $7.46 \mathrm{E}-04$ & $1.35 \mathrm{E}-04$ & $47.2 \%$ & $1.27 \mathrm{E}-04$ & $44.7 \%$ & $8.1 \%$ & $1.67 \mathrm{E}-03$ & 68 \\
\hline Supernatant & $7.67 \mathrm{E}-04$ & $7.76 \mathrm{E}-08$ & $7.27 \mathrm{E}-04$ & 7.95E-05 & $48.7 \%$ & 4.93E-05 & $46.2 \%$ & $5.1 \%$ & $1.57 E-03$ & 64 \\
\hline Solid Layer & $2.11 \mathrm{E}-05$ & $1.35 \mathrm{E}-0.7$ & $1.94 \mathrm{E}-05$ & $5.59 \mathrm{E}-05$ & $21.8 \%$ & 1.40F-03 & $20.1 \%$ & $57.9 \%$ & $9.65 \mathrm{E}-05$ & 4 \\
\hline $241-\mathrm{AN}-102$ & $3.88 \mathrm{E}-03$ & $2.55 E-05$ & $5.86 \mathrm{E}-03$ & 1. $47 \mathrm{E}-04$ & $39.1 \%$ & $2.57 \mathrm{E}-03$ & $59.1 \%$ & $1.5 \%$ & $9.92 \mathrm{E}-03$ & 404 \\
\hline Supernatant & $3.19 \mathrm{E}-03$ & $1.32 \mathrm{E}-05$ & $5.03 \mathrm{E}-03$ & $7.99 \mathrm{~F}-05$ & $38.4 \%$ & $1.59 \mathrm{E}-03$ & $60.5 \%$ & $1.0 \%$ & $8.32 \mathrm{E}-03$ & 339 \\
\hline Solid Layer & $6.89 E-04$ & $1.22 \mathrm{E}-05$ & $8.30 \mathrm{E}-04$ & $6.75 E-05$ & $43.1 \%$ & $7.66 \mathrm{E}-03$ & $51.9 \%$ & $4.2 \%$ & $1.60 \mathrm{E}-03$ & 65 \\
\hline $241-\mathrm{AN}-103$ & $9.16 \mathrm{E}-04$ & $5.54 \mathrm{E}-07$ & $1.50 \mathrm{E}-03$ & $1.36 \mathrm{E}-04$ & $35.9 \%$ & $2.17 \mathrm{E}-04$ & $58.8 \%$ & $5.3 \%$ & $2.56 \mathrm{E}-03$ & 104 \\
\hline Supcriatant & $6.60 \mathrm{E}-04$ & $2.82 \mathrm{E}-07$ & $8.49 \mathrm{E}-04$ & $4.03 \mathrm{E}-05$ & $42.6 \%$ & 1. $82 \mathrm{E}-04$ & $54.8 \%$ & $2.6 \%$ & $1.55 \mathrm{E}-03$ & 63 \\
\hline Solid Layer & $2.57 \mathrm{E}-04$ & $2.73 \mathrm{E}-07$ & $6.53 \mathrm{E}-04$ & $9.58 \mathrm{E}-05$ & $25.5 \%$ & $2.71 \mathrm{E}-04$ & $64.9 \%$ & $9.5 \%$ & $1.01 \mathrm{E}-03$ & 41 \\
\hline 241-AN-104 & $1.21 \mathrm{E}-03$ & $1.00 \mathrm{E}-06$ & $3.91 \mathrm{E}-03$ & $1.45 \mathrm{E}-04$ & $37.0 \%$ & $3.06 \mathrm{E}-04$ & $58.5 \%$ & $4.4 \%$ & $3.27 \mathrm{E}-03$ & 133 \\
\hline Supenatant & $7.04 \mathrm{E}-04$ & $1.97 \mathrm{E}-0.8$ & $9.57 \mathrm{E}-04$ & $5.26 \mathrm{E}-05$ & $41.1 \%$ & 1.15E-05 & $55.9 \%$ & $3.1 \%$ & $1.71 \mathrm{E}-03$ & 70 \\
\hline Solid Layer & $5.07 \mathrm{E}-04$ & $9.82 \mathrm{E}-07$ & $9.57 \mathrm{E}-04$ & $9.22 \mathrm{E}-05$ & $32.5 \%$ & $6.31 \mathrm{E}-04$ & $61.5 \%$ & $5.9 \%$ & $1.56 \mathrm{E}-03$ & 63 \\
\hline $241-A \times-105$ & $8.10 \mathrm{E}-04$ & $1.28 \mathrm{E}-06$ & $1.16 \mathrm{E}-03$ & $1.50 \mathrm{E}-04$ & $38.2 \%$ & $6.02 E-04$ & $54.7 \%$ & $7.1 \%$ & $2.12 \mathrm{E}-0.3$ & 86 \\
\hline Supernatant & $3.81 \mathrm{E}-04$ & $7.37 \mathrm{E}-0.7$ & $5.52 \mathrm{E}-04$ & $5.05 \mathrm{E}-05$ & $38.7 \%$ & $7.48 \mathrm{E}-04$ & $56.1 \%$ & $5.1 \%$ & $9.85 \mathrm{E}-04$ & 40 \\
\hline Solid Laycr & $4.29 \mathrm{E}-04$ & $5.40 \mathrm{E}-07$ & $6.08 E-04$ & $9.94 \mathrm{E}-05$ & $37.7 \%$ & 4.75E-04 & $53.5 \%$ & $8.7 \%$ & $1.14 \mathrm{E}-03$ & 46 \\
\hline 241-AN-106 & $6.61 \mathrm{E}-03$ & $2.04 \mathrm{E}-04$ & $7.875-05$ & $1.20 \mathrm{E}-04$ & $94.3 \%$ & $2.91 E-02$ & $1.1 \%$ & $1.7 \%$ & $7.01 \mathrm{E}-03$ & 286 \\
\hline Supernatant & $4.01 \mathrm{E}-04$ & $8.13 \mathrm{E}-07$ & $5.96 \mathrm{E}-05$ & $5.53 \mathrm{E}-05$ & $77.6 \%$ & $1.57 \mathrm{E}-03$ & $11.5 \%$ & $10.7 \%$ & $5.17 \mathrm{E}-04$ & 21 \\
\hline Solid Layer & $0.20 \mathrm{E}-03$ & $2.03 \mathrm{E}-04$ & $1.91 \mathrm{E}-05$ & $6.46 \mathrm{E}-05$ & $95.6 \%$ & $3.13 \mathrm{E}-02$ & $0.3 \%$ & $1.0 \%$ & $6.49 \mathrm{E}-03$ & 265 \\
\hline $241-\mathrm{AN}-107$ & $4.45 E-03$ & $9.35 \mathrm{E}-0.5$ & $3.56 \mathrm{E}-03$ & $1.50 \mathrm{E}-04$ & $53.9 \%$ & $1.13 E-02$ & $43.1 \%$ & $1.8 \%$ & $8.25 \mathrm{E}-03$ & 336 \\
\hline Supenatant & $3.16 \mathrm{E}-03$ & $6.51 \mathrm{E}-0.5$ & $2.80 \mathrm{E}-03$ & $7.59 \mathrm{E}-05$ & $51.8 \%$ & $1.07 \mathrm{E}-02$ & $45.9 \%$ & $1.2 \%$ & $6.10 \mathrm{E}-03$ & 249 \\
\hline Solid Layer & $1.29 \mathrm{E}-03$ & $2.83 \mathrm{E}-05$ & $7.55 \mathrm{E}-04$ & $7.41 \mathrm{E}-05$ & $60.1 \%$ & $1.32 \mathrm{E}-02$ & $35.1 \%$ & $3.4 \%$ & $2.15 \mathrm{E}-03$ & 88 \\
\hline $241-\mathrm{AP}-101$ & $5.90 \mathrm{E}-04$ & 4.13E-08 & $1.26 \mathrm{E}-04$ & 1. $47 \mathrm{E}-04$ & $68.4 \%$ & $4.78 \mathrm{E}-05$ & $14.6 \%$ & $17.1 \%$ & $8.64 \mathrm{E}-04$ & 35 \\
\hline Supernatant & $5.90 \mathrm{E}-04$ & $4.13 \mathrm{E}-08$ & $1.26 \mathrm{E}-04$ & $1.47 \mathrm{E}-04$ & $68.4 \%$ & $4.78 \mathrm{E}-05$ & $14.6 \%$ & $17.1 \%$ & $8.64 \mathrm{~F}-04$ & 35 \\
\hline Solid Layer & na & na & na & na & na & na & na & $\mathrm{na}$ & na & na \\
\hline $241-A P-102$ & $7.85 \mathrm{E}-04$ & $1.86 \mathrm{E}-07$ & $9.91 \mathrm{E}-04$ & $1.50 \mathrm{E}-04$ & $40.7 \%$ & $9.66 \mathrm{E}-05$ & $51.5 \%$ & $7.8 \%$ & $1.93 \mathrm{E}-03$ & 79 \\
\hline Supernatant & $7.80 \mathrm{E}-04$ & $1.84 \mathrm{E}-07$ & $9.85 \mathrm{E}-04$ & $9.35 \mathrm{E}-05$ & $41.9 \%$ & $9.90 \mathrm{E}-05$ & $53.0 \%$ & $5.0 \%$ & $1.86 \mathrm{E}-03$ & 76 \\
\hline Solid Layer & $4.99 \mathrm{E}-06$ & $2.16 \mathrm{E}-09$ & $6.31 \mathrm{E}-06$ & 5.62E-05 & $7.4 \%$ & $3.19 \mathrm{E}-05$ & $9.3 \%$ & $83.3 \%$ & $6.75 \mathrm{E}-05$ & 3 \\
\hline
\end{tabular}


RPP-5926 REV 6

Table B-4. Calculated Total Hydrogen Generation Rate In Dome Space for 177 Tanks. (20 sheets)

\begin{tabular}{|c|c|c|c|c|c|c|c|c|c|c|}
\hline Tank & $\begin{array}{c}\text { HGR from } \\
\text { radiolysis } \\
\text { HGR }_{\text {rad }}{ }^{\prime} \text { r } \\
\text { (cfm) }\end{array}$ & $\begin{array}{l}\text { HGR from } \\
\text { radiolysis } \\
\text { HGR }_{\text {red }}^{2 \text { lpha }} \\
\text { (cfm) }\end{array}$ & $\begin{array}{l}\text { HGR from } \\
\text { thermolysis } \\
\text { HGR }_{\text {therm }} \\
\text { (cfm) }\end{array}$ & $\begin{array}{c}\text { HGR from } \\
\text { corrosion } \\
\text { HGR }_{\text {corr }} \\
\text { (cfm) }\end{array}$ & $\begin{array}{l}\text { Percent of } \\
\text { HGR from } \\
\text { radiolysis } \\
\text { HGR }_{\text {rad }}\end{array}$ & $\begin{array}{l}\text { Percent of } \\
\text { HGR from } \\
\text { radiolysis } \\
\text { HGR }_{\mathrm{rad}} \text { alpha }\end{array}$ & $\begin{array}{l}\text { Percent of } \\
\text { HGR from } \\
\text { thermolysis } \\
\text { HGR }_{\text {therm }}\end{array}$ & $\begin{array}{l}\text { Percent of } \\
\text { HGR from } \\
\text { corrosion } \\
\text { HGR }_{\text {rorr }}\end{array}$ & $\begin{array}{c}\text { Total HGR } \\
\text { from model } \\
\text { HGR }_{\text {mod }} \\
\text { (cfm) }\end{array}$ & $\begin{array}{c}\text { Total HGR } \\
\text { from model } \\
\text { HGR }_{\text {mod }} \\
(\mathrm{L} / \text { day })\end{array}$ \\
\hline 241-AP-103 & $6.81 \mathrm{E}-04$ & $9.69 \mathrm{E}-07$ & $4.61 \mathrm{E}-04$ & $1.28 \mathrm{E}-04$ & $53.6 \%$ & 7.62E-04 & $36.3 \%$ & $10.1 \%$ & $1.27 E-03$ & 52 \\
\hline Supernatant & $6.71 \mathrm{E}-04$ & $9.65 \mathrm{E}-07$ & $4.47 \mathrm{E}-04$ & $7.40 \mathrm{E}-05$ & $56.3 \%$ & $8.10 \mathrm{E}-04$ & $37.5 \%$ & $6.2 \%$ & $1.19 \mathrm{E}-03$ & 49 \\
\hline Solid 1.ayer & $9.93 \mathrm{E}-06$ & $3.07 \mathrm{E}-09$ & $1.40 \mathrm{E}-05$ & $5.44 \mathrm{E}-05$ & $12.7 \%$ & 3.91E-05 & $17.9 \%$ & $69.4 \%$ & 7.84E-05 & 3 \\
\hline $241-\mathrm{AP}-104$ & $9.00 \mathrm{E}-04$ & $4.06 \mathrm{E}-07$ & $3.08 \mathrm{E}-04$ & $1.45 \mathrm{E}-04$ & $66.5 \%$ & $3.00 \mathrm{E}-04$ & $22.8 \%$ & $10.7 \%$ & $1.35 \mathrm{E}-03$ & 55 \\
\hline Supernatant & $9.00 \mathrm{E}-04$ & $4.06 \mathrm{E}-07$ & $3.08 \mathrm{E}-04$ & $1.45 \mathrm{E}-04$ & $66.5 \%$ & $3.00 \mathrm{E}-04$ & $22.8 \%$ & $10.7 \%$ & $1.35 E-03$ & 55 \\
\hline Solid Layer & na & naa & na & na & na & na & $\mathrm{n}$ & na & na & na \\
\hline $24 \mathrm{I}-\mathrm{AP}-105$ & $6.63 \mathrm{E}-04$ & $2.47 \mathrm{E}-07$ & $4.58 \mathrm{E}-04$ & $1.54 \mathrm{E}-04$ & $52.0 \%$ & $1.94 \mathrm{E}-04$ & $35.9 \%$ & $12.1 \%$ & $1.27 \mathrm{E}-03$ & 52 \\
\hline Supcrnatant & $5.71 \mathrm{E}-04$ & $1.34 E-07$ & $4.50 \mathrm{E}-04$ & $9.18 \mathrm{E}-05$ & $51.3 \%$ & $1.20 \mathrm{E}-04$ & $40.4 \%$ & $8.2 \%$ & $1.11 \mathrm{E}-03$ & 45 \\
\hline Solid Layer & $9.15 \mathrm{E}-05$ & $1.13 \mathrm{E}_{-}-07$ & $7.78 \mathrm{E}-06$ & $6.21 \mathrm{E}-05$ & $56.7 \%$ & $7.02 \mathrm{E}-04$ & $4.8 \%$ & $38.5 \%$ & $1.61 E-04$ & 7 \\
\hline $241-\mathrm{AP}^{\prime}-106$ & $1.09 E-03$ & $1.26 \mathrm{E}-07$ & 3.63E-04 & $1.48 \mathrm{E}-04$ & $68.1 \%$ & $7.90 \mathrm{E}-05$ & $22.7 \%$ & $9.2 \%$ & $1.60 \mathrm{E}-03$ & 65 \\
\hline Supernatant & $1.09 \mathrm{E}-03$ & $1.26 \mathrm{~F}-07$ & $3.63 \mathrm{E}-04$ & $1.48 E-04$ & $68.1 \%$ & $7.90 \mathrm{E}-05$ & $22.7 \%$ & $9.2 \%$ & $1.60 \mathrm{E}-03$ & 65 \\
\hline Solid Layer & Ha & pa & na & na & na & na & na & na & na & na \\
\hline $241-\mathrm{AP}-107$ & $1.78 \mathrm{E}-04$ & $2.12 \mathrm{E}-08$ & $3.18 \mathrm{E}-05$ & $7.96 \mathrm{E}-05$ & $61.5 \%$ & $7.31 \mathrm{E}-05$ & $11.0 \%$ & $27.5 \%$ & $2.90 E-04$ & 12 \\
\hline Supenatant & $1.78 \mathrm{E}-04$ & $2.12 \mathrm{E}-08$ & $3.18 \mathrm{E}-05$ & $7.96 \mathrm{E}-05$ & $61.5 \%$ & $7.31 \mathrm{E}-05$ & $11.0 \%$ & $27.5 \%$ & $2.90 \mathrm{E}-04$ & 12 \\
\hline Solid Layer & na & па & na & na & na & nа & na & na & na & na \\
\hline $241-\mathrm{AP}-108$ & $5.64 \mathrm{E}-04$ & $6.20 \mathrm{E}-08$ & $1.23 \mathrm{E}-03$ & $1.46 \mathrm{E}-04$ & $29.0 \%$ & $3.19 \mathrm{E}-05$ & $63.5 \%$ & $7.5 \%$ & $1.94 \mathrm{E}-03$ & 79 \\
\hline Supernatant & $5.07 \mathrm{E}-04$ & $5.48 \mathrm{E}-08$ & $1.20 \mathrm{E}-03$ & $7.94 \mathrm{E}-05$ & $28.4 \%$ & $3.07 \mathrm{E}-05$ & $67.1 \%$ & $4.5 \%$ & $1.78 \mathrm{E}-03$ & 73 \\
\hline Solid Layer & $5.76 \mathrm{E}-05$ & $7.17 \mathrm{E}-09$ & $3.75 \mathrm{E}-05$ & $6.64 \mathrm{E}-05$ & $35.7 \%$ & $4.44 \mathrm{E}-05$ & $23.2 \%$ & $41.1 \%$ & $1.61 \mathrm{E}-04$ & 7 \\
\hline $24 !-A W-101$ & $1.02 \mathrm{E}-03$ & $4.76 \mathrm{E}-06$ & $1.05 \mathrm{E}-03$ & $1.48 \mathrm{E}-04$ & $45.7 \%$ & $2.15 \mathrm{E}-03$ & $47.4 \%$ & $6.7 \%$ & $2.22 \mathrm{E}-03$ & 91 \\
\hline Supernatant & $6.45 \mathrm{E}-04$ & $4.42 \mathrm{E}-08$ & $6.46 \mathrm{E}-04$ & $6.20 \mathrm{E}-05$ & $47.7 \%$ & $3.26 \mathrm{E}-05$ & $47.7 \%$ & $4.6 \%$ & $1.35 \mathrm{E}-03$ & 55 \\
\hline Solid Layer & $3.70 \mathrm{E}-04$ & 4.72E-06 & $4.06 \mathrm{E}-04$ & $8.60 \mathrm{E}-05$ & $42.7 \%$ & $5.45 \mathrm{E}-03$ & $46.8 \%$ & $9.9 \%$ & $8.66 \mathrm{E}-04$ & 35 \\
\hline $241-\mathrm{AW}-102$ & $3.52 \mathrm{E}-04$ & $1.29 \mathrm{E}-06$ & $9.30 \mathrm{E}-05$ & $1.04 \mathrm{E}-04$ & $64.0 \%$ & $2.34 \mathrm{E}-03$ & $16.9 \%$ & $18.9 \%$ & $5.50 \mathrm{E}-04$ & 22 \\
\hline Supernatant & $3.35 \mathrm{E}-04$ & $4.03 \mathrm{E}-08$ & 9. $20 \mathrm{E}-05$ & $5.07 \mathrm{E}-05$ & $70.1 \%$ & $8.45 \mathrm{E}-05$ & $19.3 \%$ & $10.6 \%$ & $4.77 \mathrm{E}-04$ & 19 \\
\hline Solid Layer & $1.70 \mathrm{E}-05$ & 1.25E-06 & $9.92 \mathrm{E}-07$ & $5.30 \mathrm{E}-05$ & $23.5 \%$ & $1.73 \mathrm{E}-02$ & $1.4 \%$ & $73.4 \%$ & $7.22 \mathrm{E}-05$ & 3 \\
\hline 241-AW-103 & $4.73 \mathrm{E}-04$ & $1.20 \mathrm{E}-05$ & $1.66 \mathrm{E}-04$ & $1.45 \mathrm{E}-04$ & $59.4 \%$ & $1.51 \mathrm{E}-02$ & $20.8 \%$ & $18.2 \%$ & $7.95 \mathrm{E}-04$ & 32 \\
\hline Supernatant & 4.19E-04 & $2.01 \mathrm{E}-08$ & $6.42 E-05$ & $6.47 \mathrm{E}-05$ & $76.5 \%$ & $3.67 \mathrm{E}-05$ & $11.7 \%$ & $11.8 \%$ & $5.48 \mathrm{E}-04$ & 22 \\
\hline Solid Layer & $5.37 \mathrm{E}-05$ & $1.20 \mathrm{E}-05$ & $1.01 \mathrm{E}-04$ & $8.05 E-05$ & $21.7 \%$ & $4.84 \mathrm{E}-02$ & $41.0 \%$ & $32.5 \%$ & $2.47 \mathrm{E}-04$ & 10 \\
\hline
\end{tabular}


RPP-5926 REV 6

Table B-4. Calculated Total Hydrogen Generation Rate In Dome Space for 177 Tanks. (20 sheets)

\begin{tabular}{|c|c|c|c|c|c|c|c|c|c|c|}
\hline Tank & $\begin{array}{c}\text { HGR from } \\
\text { radiolysis } \\
\text { HGR }_{\text {rad }} \text { bir } \\
\text { (cfm) }\end{array}$ & $\begin{array}{l}\text { HGR from } \\
\text { radiolysis } \\
\text { HGR }_{\text {rad }}^{\text {alphz }} \\
\text { (cfm) }\end{array}$ & $\begin{array}{l}\text { HGR from } \\
\text { thermolysis } \\
\text { HGR }_{\text {therm }} \\
\text { (cfm) }\end{array}$ & $\begin{array}{c}\text { HGR from } \\
\text { corrosion } \\
\text { HGR }_{\text {corr }} \\
\text { (cfm) }\end{array}$ & $\begin{array}{l}\text { Percent of } \\
\text { HGR from } \\
\text { radiolysis } \\
\text { HGR }_{r \text { as }} \text { bir }\end{array}$ & $\begin{array}{l}\text { Percent of } \\
\text { HGR from } \\
\text { radiolysis } \\
\text { HGR }_{\text {rad }} 2 \text { apha }\end{array}$ & $\begin{array}{l}\text { Percent of } \\
\text { HGR from } \\
\text { thermolysis } \\
\text { HGR }_{\text {therm }}\end{array}$ & $\begin{array}{l}\text { Percent of } \\
\text { HGR from } \\
\text { corrosion } \\
\text { HGR }_{\text {corr }}\end{array}$ & $\begin{array}{c}\text { Total HGR } \\
\text { from model } \\
\text { HGR }_{\text {mod }} \\
\text { (cfm) }\end{array}$ & $\begin{array}{c}\text { Total HGR } \\
\text { from model } \\
\mathrm{HGR}_{\text {nod }} \\
\text { (L/day) }\end{array}$ \\
\hline $241-\mathrm{AW}-104$ & $7.99 \mathrm{E}-04$ & $1.87 \mathrm{E}-05$ & $6.94 \mathrm{E}-04$ & $1.45 \mathrm{E}-04$ & $48.2 \%$ & $1.13 \mathrm{E}-02$ & $41.9 \%$ & $8.8 \%$ & $1.66 \mathrm{E}-03$ & 68 \\
\hline Supernatant & $6.95 \mathrm{E}-04$ & $1.65 \mathrm{E}-07$ & $5.52 \mathrm{E}-04$ & $7.29 \mathrm{E}-05$ & $52.6 \%$ & $1.25 \mathrm{E}-04$ & $41.8 \%$ & $5.5 \%$ & 1.32E-03 & 54 \\
\hline Solid Layer & $1.04 \mathrm{E}-04$ & $1.85 \mathrm{E}-0 \mathrm{~S}$ & $1.41 \mathrm{E}-04$ & $7.22 \mathrm{E}-05$ & $30.9 \%$ & $5.51 \mathrm{E}-02$ & $42.1 \%$ & $21.5 \%$ & $3.36 \mathrm{E}-04$ & 14 \\
\hline $24 \mathrm{I}-\mathrm{AW}-105$ & $1.55 \mathrm{E}-04$ & $4.44 \mathrm{E}-05$ & $2.10 \mathrm{E}-06$ & $1.07 \mathrm{E}-04$ & $50.2 \%$ & $1.44 \mathrm{E}-0 \mathrm{I}$ & $0.7 \%$ & $34.7 \%$ & $3.08 \mathrm{E}-04$ & 13 \\
\hline Supematant & $2.31 \mathrm{E}-05$ & $1.94 \mathrm{E}-08$ & $4.70 E-07$ & $3.26 \mathrm{E}-05$ & $41.1 \%$ & $3.45 \mathrm{E}-04$ & $0.8 \%$ & $58.0 \%$ & $5.62 \mathrm{E}-05$ & 2 \\
\hline Solid Layer & 1.32E-04 & 4.44E-05 & $1.63 \mathrm{E}-06$ & $7.43 \mathrm{E}-05$ & $52.3 \%$ & $1.76 \mathrm{E}-01$ & $0.6 \%$ & $29.5 \%$ & $2.52 \mathrm{E}-04$ & 10 \\
\hline $241-A W-106$ & $8.30 \mathrm{E}-04$ & $1.50 \mathrm{E}-06$ & $3.01 \mathrm{E}-04$ & $1.50 \mathrm{E}-04$ & $64.7 \%$ & $1.17 \mathrm{E}-03$ & $23.5 \%$ & $11.7 \%$ & $1.28 \mathrm{E}-03$ & 52 \\
\hline Supernatant & $4.90 \mathrm{E}-04$ & $2.16 \mathrm{E}-07$ & $1.80 \mathrm{E}-04$ & $7.27 \mathrm{E}-05$ & $65.9 \%$ & $2.90 \mathrm{E}-04$ & $24.3 \%$ & $9.8 \%$ & 7.44E-04 & 30 \\
\hline Solid Layer & $3.40 \mathrm{E}-04$ & $1.28 \mathrm{E}-06$ & $1.21 \mathrm{E}-04$ & $7.71 \mathrm{E}-05$ & $63.1 \%$ & $2.37 \mathrm{E}-03$ & $22.4 \%$ & $14.3 \%$ & $5.39 \mathrm{E}-04$ & 22 \\
\hline $24 \mid-A Y-101$ & $5.13 F-03$ & $7.44 E-04$ & $2.51 \mathrm{E}-04$ & $1.89 \mathrm{E}-04$ & $81.2 \%$ & $1.18 \mathrm{E}-01$ & $4.0 \%$ & $3.0 \%$ & $6.31 \mathrm{E}-03$ & 257 \\
\hline Supernatant & $3.38 \mathrm{~F}-05$ & $9.16 \mathrm{E}-07$ & $1.23 \mathrm{E}-04$ & $2.54 \mathrm{E}-05$ & $18.5 \%$ & $5.00 \mathrm{E}-03$ & $67.1 \%$ & $13.9 \%$ & $1.835-04$ & 7 \\
\hline Solid Layer & $5.09 \mathrm{E}-03$ & $7.43 \mathrm{E}-04$ & $1.28 \mathrm{E}-04$ & $1.64 \mathrm{E}-04$ & $83.1 \%$ & $1.21 \mathrm{E}-01$ & $2.1 \%$ & $2.7 \%$ & $6.13 E-0.3$ & 250 \\
\hline $241-\mathrm{AY}-102$ & $2.54 \mathrm{E}-02$ & 6.47E-04 & $1.24 \mathrm{E}-03$ & $3.57 \mathrm{E}-04$ & $91.9 \%$ & $2.34 \mathrm{E}-02$ & $4.5 \%$ & $1.3 \%$ & $2.77 \mathrm{E}-02$ & 1128 \\
\hline Supenatant & $2.47 \mathrm{E}-04$ & $8.96 \mathrm{E}-07$ & $8.70 \mathrm{E}-04$ & $1.84 \mathrm{E}-04$ & $19.0 \%$ & $6.89 \mathrm{E}-04$ & $66.9 \%$ & $14.1 \%$ & $1.30 \mathrm{E}-03$ & 53 \\
\hline Solid Layer & $2.52 \mathrm{E}-02$ & $6.46 \mathrm{E}-04$ & $3.73 \mathrm{E}-04$ & $1.73 \mathrm{E}-04$ & $95.5 \%$ & $2.45 \mathrm{E}-02$ & $1.4 \%$ & $0.7 \%$ & $2.64 \mathrm{E}-02$ & 1075 \\
\hline $24 !-A Z-101$ & $1.67 \mathrm{E}-02$ & 7.11E-04 & $4.22 \mathrm{E}-03$ & $1.45 \mathrm{E}-04$ & $76.7 \%$ & $3.26 \mathrm{E}-02$ & $19.3 \%$ & $0.7 \%$ & $2.18 \mathrm{E}-02$ & 889 \\
\hline Supernatant & $8.79 \mathrm{~F}-03$ & $3.40 \mathrm{E}-07$ & $3.63 E-03$ & $7.94 \mathrm{E}-05$ & $70.3 \%$ & $2.72 \mathrm{E}-05$ & $29.0 \%$ & $0.6 \%$ & $1.25 \mathrm{t}-02$ & 510 \\
\hline Solid Layer & $7.94 \mathrm{E}-03$ & $7.11 \mathrm{E}-04$ & $5.86 \mathrm{E}-04$ & $6.57 \mathrm{E}-05$ & $85.4 \%$ & $7.65 E-02$ & $6.3 \%$ & $0.7 \%$ & $9.30 \mathrm{E}-03$ & 379 \\
\hline $241-A Z-102$ & $2.82 \mathrm{E}-02$ & $2.11 E-03$ & $2.36 \mathrm{E}-03$ & I. $53 \mathrm{E}-04$ & $85.9 \%$ & $6.44 \mathrm{E}-02$ & $7.2 \%$ & $0.5 \%$ & $3.28 \mathrm{~F}-02$ & 1338 \\
\hline Supernatant & 1.21E-02 & 3.52E-06 & $1.83 \mathrm{E}-03$ & $8.32 \mathrm{E}-05$ & $86.3 \%$ & $2.52 \mathrm{E}-04$ & $13.1 \%$ & $0.6 \%$ & $1.40 \mathrm{E}-02$ & 570 \\
\hline Solid Layer & $1.61 \mathrm{E}-02$ & $2.11 \mathrm{E}-03$ & $5.30 \mathrm{E}-04$ & $6.98 \mathrm{E}-05$ & $85.6 \%$ & $1.12 \mathrm{E}-01$ & $2.8 \%$ & $0.4 \%$ & $1.88 \mathrm{E}-02$ & 768 \\
\hline $24 \mathrm{I}-\mathrm{SY}-101$ & $3.62 E-04$ & $7.78 E-06$ & $6.61 \mathrm{E}-05$ & $1.47 \mathrm{E}-04$ & $62.1 \%$ & $1.34 \mathrm{E}-02$ & $11.3 \%$ & $25.2 \%$ & $5.83 \mathrm{E}-04$ & 24 \\
\hline Supernatant & $1.92 \mathrm{E}-04$ & $2.63 \mathrm{E}-08$ & $4.50 \mathrm{E}-05$ & $7.32 \mathrm{E}-05$ & $61.9 \%$ & $8.46 \mathrm{E}-05$ & $14.5 \%$ & $23.6 \%$ & $3.11 \mathrm{E}-04$ & 13 \\
\hline Solid Layer & $1.70 \mathrm{E}-04$ & $7.76 \mathrm{E}-06$ & $2.11 \mathrm{E}-05$ & $7.38 \mathrm{E}-05$ & $62.3 \%$ & $2.85 \mathrm{E}-02$ & $7.8 \%$ & $27.1 \%$ & $2.72 \mathrm{E}-04$ & 11 \\
\hline $241-S Y-102$ & $4.06 E-04$ & $5.63 E-04$ & $1.11 \mathrm{E}-04$ & $1.51 E-04$ & $33.0 \%$ & $4.57 \mathrm{E}-01$ & $9.0 \%$ & $12.2 \%$ & $1.23 \mathrm{E}-03$ & 50 \\
\hline Supernatant & $1.50 \mathrm{E}-04$ & $9.27 \mathrm{E}-08$ & $5.18 \mathrm{E}-05$ & $8.36 \mathrm{E}-05$ & $52.5 \%$ & $3.25 \mathrm{E}-04$ & $18.1 \%$ & $29.3 \%$ & $2.85 \mathrm{E} \cdot 04$ & 12 \\
\hline Solid Layer & $2.56 \mathrm{E}-04$ & $5.63 E-04$ & 5.95E-05 & $6.69 \mathrm{E}-05$ & $27.1 \%$ & $5.95 \mathrm{E}-01$ & $6.3 \%$ & $7.1 \%$ & $9.45 \mathrm{E}-04$ & 39 \\
\hline
\end{tabular}




\section{RPP-5926 REV 6}

Table B-4. Calculated Total Hydrogen Generation Rate In Dome Space for 177 Tanks. (20 sheets)

\begin{tabular}{|c|c|c|c|c|c|c|c|c|c|c|}
\hline Tank & $\begin{array}{c}\text { HGR from } \\
\text { radiolysis } \\
\text { HGR }_{\mathrm{rad}} \\
\text { (cfm) }\end{array}$ & $\begin{array}{l}\text { HGR from } \\
\text { radiolysis } \\
\text { HGR }_{\text {rad }}^{\text {atphi }} \\
\text { (cfm) }\end{array}$ & $\begin{array}{l}\text { HGR from } \\
\text { thermolysis } \\
\text { HGR }_{\text {therm }} \\
\text { (cfm) }\end{array}$ & $\begin{array}{c}\text { HGR from } \\
\text { corrosion } \\
\text { HGR }_{\text {corr }} \\
\text { (cfm) }\end{array}$ & $\begin{array}{l}\text { Percent of } \\
\text { HGR from } \\
\text { radiolysis } \\
\text { HGR }_{\text {rad }} \mathrm{b} / \mathrm{r}\end{array}$ & $\begin{array}{l}\text { Percent of } \\
\text { HGR from } \\
\text { radiolysis } \\
\text { HGR }_{\text {rad }}\end{array}$ & $\begin{array}{l}\text { Percent of } \\
\text { HGR from } \\
\text { thermolysis } \\
\text { HGR }_{\text {therm }}\end{array}$ & $\begin{array}{l}\text { Percent of } \\
\text { HGR from } \\
\text { corrosion } \\
\text { HGR }_{\text {corr }}\end{array}$ & $\begin{array}{c}\text { Total HGR } \\
\text { from model } \\
\text { HGR }_{\text {mod }} \\
\text { (cfm) }\end{array}$ & $\begin{array}{c}\text { Total HGR } \\
\text { from model } \\
\text { HGR }_{\text {mod }} \\
\text { (L/day) }\end{array}$ \\
\hline $241-S Y-103$ & $1.06 \mathrm{E}-03$ & 2.43E-05 & $2.12 \mathrm{E}-03$ & $1.15 \mathrm{E}-04$ & $31.9 \%$ & $7.33 \mathrm{E}-03$ & $63.9 \%$ & $3.5 \%$ & $3.31 \mathrm{t}-03$ & 135 \\
\hline Supernatant & $5.63 \mathrm{E}-04$ & $3.06 \mathrm{E}-07$ & $5.46 \mathrm{E}-04$ & $3.35 \mathrm{E}-0.5$ & $49.3 \%$ & $2.68 \mathrm{E}-0.04$ & $47.8 \%$ & $2.9 \%$ & $1.14 \mathrm{E}-03$ & 47 \\
\hline Solid Layer & 4.93E-04 & $2.40 \mathrm{E}-05$ & $1.57 E-03$ & $8.12 E-05$ & $22.7 \%$ & $1.11 E-02$ & $72.5 \%$ & $3.7 \%$ & $2.17 \mathrm{E}-03$ & 89 \\
\hline $241-A-101$ & $2.22 \mathrm{E}-04$ & $6.35 \mathrm{E}-06$ & $7.69 \mathrm{E}-04$ & $3.27 \mathrm{E}-04$ & $16.7 \%$ & $4.79 \mathrm{E}-03$ & $58.1 \%$ & $24.7 \%$ & $1.32 \mathrm{E}-03$ & 54 \\
\hline Supernatant & na & na & na & na & na & na & па & na & na & па \\
\hline Solid Layer & $2.22 \mathrm{E}-04$ & $6.35 \mathrm{E}-06$ & $7.69 \mathrm{E}-04$ & $3.27 \mathrm{E}-04$ & $16.7 \%$ & $4.79 \mathrm{E}-03$ & $58.1 \%$ & $24.7 \%$ & $1.32 \mathrm{E}-03$ & 54 \\
\hline $241-\mathrm{A}-102$ & $9.44 \mathrm{E}-05$ & $8.91 \mathrm{E}-06$ & $1.14 \mathrm{E}-05$ & $2.32 \mathrm{E}-04$ & $27.2 \%$ & $2.57 \mathrm{E}-02$ & $3.3 \%$ & $66.9 \%$ & $3.47 \mathrm{E}-04$ & 14 \\
\hline Supernatant & $4.96 \mathrm{E}-06$ & 1. $19 \mathrm{E}-09$ & $2.97 \mathrm{E}-06$ & $1.12 \mathrm{E}-06$ & $54.9 \%$ & $1.31 \mathrm{E}-04$ & $32.8 \%$ & $12.3 \%$ & $9.05 \mathrm{E}-06$ & 0 \\
\hline Solid Layer & $8.95 \mathrm{E}-05$ & 8.91 E-06 & 8.44E-06 & $2.31 \mathrm{E}-04$ & $26.5 \%$ & $2.63 \mathrm{E}-02$ & $2.5 \%$ & $68.4 \%$ & $3.38 \mathrm{E}-04$ & 14 \\
\hline $241-\mathrm{A}-103$ & $3.10 \mathrm{E}-04$ & $6.76 \mathrm{E}-06$ & $8.54 \mathrm{E}-04$ & $3.47 \mathrm{E}-04$ & $20.4 \%$ & $4.45 \mathrm{E}-03$ & $56.3 \%$ & $22.9 \%$ & $1.52 \mathrm{E}-03$ & 62 \\
\hline Supernatant & $3.83 \mathrm{E}-06$ & $8.19 \mathrm{E}-09$ & $1.36 \mathrm{E}-05$ & $1.56 \mathrm{E}-06$ & $20.2 \%$ & 4.32E-04 & $71.5 \%$ & $8.2 \%$ & $1.90 \mathrm{E}-05$ & 1 \\
\hline Solid Layer & $3.06 \mathrm{E}-04$ & $6.75 E-06$ & $8.40 \mathrm{E}-04$ & $3.46 \mathrm{E}-04$ & $20.4 \%$ & $4.51 \mathrm{E}-03$ & $56.1 \%$ & $23.1 \%$ & $1.50 \mathrm{E}-03$ & 61 \\
\hline $24 !-A-104$ & $0.00 \mathrm{E} \div 00$ & $0.00 \mathrm{E}+00$ & $0.00 \mathrm{E} \times 00$ & $2.29 \mathrm{E}-04$ & $0.0 \%$ & $0.00 \mathrm{E}+00$ & $0.0 \%$ & $100.0 \%$ & $2.29 \mathrm{E}-04$ & 9 \\
\hline Supernatant & na & na & na & na & na & na & na & na & na & na \\
\hline Solid Layer & $0.00 \mathrm{E}+00$ & $0.00 \mathrm{E}+00$ & $0.00 \mathrm{E}+00$ & $2.29 \mathrm{E}-04$ & $0.0 \%$ & $0.00 \mathrm{E}+00$ & $0.0 \%$ & $100.0 \%$ & $2.29 \mathrm{E}-04$ & 9 \\
\hline $241-\mathrm{A}-105$ & $0.00 \mathrm{E}+00$ & $0.00 \mathrm{E}+00$ & $0.00 \mathrm{E}+00$ & $2.06 \mathrm{E}-04$ & $0.0 \%$ & $0.00 \mathrm{E}+00$ & $0.0 \%$ & $100.0 \%$ & $2.06 \mathrm{E}-04$ & 8 \\
\hline Supernatant & па & na & na & na & na & na & na & na & na & na \\
\hline Solid Layer & $0.00 \mathrm{E}+00$ & $0.00 \mathrm{E}^{+} 00$ & $0.00 \mathrm{E}+00$ & $2.06 \mathrm{E}-04$ & $0.0 \%$ & $0.00 \mathrm{E}+00$ & $0.0 \%$ & $100.0 \%$ & $2.06 \mathrm{E}-04$ & 8 \\
\hline $241-\mathrm{A}-106$ & $9.91 E-04$ & $3.84 \mathrm{E}-05$ & $4.99 \mathrm{E}-04$ & $2.51 \mathrm{E}-04$ & $55.7 \%$ & $2.16 \mathrm{E}-02$ & $28.1 \%$ & $14.1 \%$ & $1.78 \mathrm{E}-03$ & 73 \\
\hline Supernatant & na & na & na & na & na & na & na & na & na & па \\
\hline Solid Layer & 9.91F-04 & $3.84 \mathrm{~F}-05$ & $4.99 \mathrm{~F}-04$ & $2.51 \mathrm{E}-04$ & $55.7 \%$ & $2.16 \mathrm{E}-02$ & $28.1 \%$ & $14.1 \%$ & $1.78 \mathrm{E}-03$ & 73 \\
\hline $241-A X-101$ & 3.13E-04 & $5.80 \mathrm{E}-06$ & $3.50 E-04$ & $3.39 E-04$ & $31.1 \%$ & $5.75 \mathrm{E}-03$ & $34.7 \%$ & $33.6 \%$ & $1.01 \mathrm{E}-03$ & 41 \\
\hline Supenratan & na & na & na & na & na & na & na & na & na & na \\
\hline Solid Layer & $3.13 \mathrm{E}-04$ & $5.80 E-06$ & $3.50 \mathrm{E}-04$ & $3.39 \mathrm{E}-04$ & $31.1 \%$ & $5.75 \mathrm{E}-03$ & $34.7 \%$ & $33.6 \%$ & $1.01 \mathrm{E}-03$ & 41 \\
\hline 241-AX-102 & $2.44 E-04$ & $3.47 \mathrm{E}-05$ & $3.27 \mathrm{E}-05$ & $2.22 \mathrm{E}-04$ & $45.7 \%$ & $6.51 \mathrm{IE}-02$ & $6.1 \%$ & $41.6 \%$ & $5.34 \mathrm{E}-04$ & 22 \\
\hline Supernatant & na & na & na & па & na & na & na & na & na & na \\
\hline Solid Layer & $2.44 \mathrm{E}-04$ & $3.47 E-05$ & $3.27 \mathrm{E}-05$ & $2.22 \mathrm{E}-04$ & $45.7 \%$ & $6.51 E-02$ & $6.1 \%$ & $41.6 \%$ & $5.34 \mathrm{E}-04$ & 22 \\
\hline
\end{tabular}


RPP-5926 REV 6

Table B-4. Calculated Total Hydrogen Generation Rate In Dome Space for 177 Tanks. (20 sheets)

\begin{tabular}{|c|c|c|c|c|c|c|c|c|c|c|}
\hline Tank & $\begin{array}{c}\text { HGR from } \\
\text { radiolysis } \\
\text { HGR }_{\text {rad }}{ }^{\circ} \\
\text { (cfm) }\end{array}$ & $\begin{array}{l}\text { HGR from } \\
\text { radiolysis } \\
\text { HGR }_{\text {ratlphx }}^{\text {alph }} \\
\text { (cfm) }\end{array}$ & $\begin{array}{l}\text { HGR from } \\
\text { thermolysis } \\
\text { HGR }_{\text {therm }} \\
\text { (cfm) }\end{array}$ & $\begin{array}{c}\text { HGR from } \\
\text { corrosion } \\
\text { HGR }_{\text {corr }} \\
\text { (cfm) }\end{array}$ & $\begin{array}{l}\text { Percent of } \\
\text { HGR from } \\
\text { radiolysis } \\
\text { HGR }_{\mathrm{rad}} \mathrm{b} / \mathrm{r}\end{array}$ & $\begin{array}{c}\text { Percent of } \\
\text { HGR from } \\
\text { radiolysis } \\
\text { HGR }_{\text {rad }} \text { Ilpha }\end{array}$ & $\begin{array}{l}\text { Percent of } \\
\text { HGR from } \\
\text { thermolysis } \\
\text { HGR }_{\text {therm }}\end{array}$ & $\begin{array}{c}\text { Percent of } \\
\text { HGR from } \\
\text { corrosion } \\
\text { HGR }_{\text {corr }}\end{array}$ & $\begin{array}{c}\text { Total HGR } \\
\text { from model } \\
\text { HGR }_{\text {mod }} \\
\text { (cfm) }\end{array}$ & $\begin{array}{c}\text { Total HGR } \\
\text { from model } \\
\text { HGR }_{\text {mod }} \\
\text { (L/day) }\end{array}$ \\
\hline 241-AX-103 & $4.43 \mathrm{E}-04$ & $1.46 \mathrm{E}-05$ & 1.45E-04 & $2.54 \mathrm{E}-04$ & $51.8 \%$ & $1.71 \mathrm{E}-02$ & $16.9 \%$ & $29.7 \%$ & $8.57 \mathrm{E}-04$ & 35 \\
\hline Supenatant & na & na & na & $\mathrm{na}$ & na & na & na & na & $\mathrm{na}$ & na \\
\hline Solid Layer & $4.43 \mathrm{E}-04$ & $1.46 \mathrm{E}-05$ & $1.45 \mathrm{E}-04$ & $2.54 \mathrm{E}-04$ & $51.8 \%$ & $1.71 \mathrm{E}-02$ & $16.9 \%$ & $29.7 \%$ & $8.57 \mathrm{E}-04$ & 35 \\
\hline $241-A X-104$ & $5.40 \mathrm{E}-04$ & $5.80 \mathrm{E}-06$ & $3.11 \mathrm{E}-07$ & 2.19E-04 & $70.6 \%$ & $7.58 \mathrm{E}-03$ & $0.0 \%$ & $28.6 \%$ & $7.65 \mathrm{E}-04$ & 31 \\
\hline Supernatant & na & na & na & na & na & na & na & na & na & na \\
\hline Solid Layer & $5.40 \mathrm{E}-04$ & $3.80 \mathrm{E}-06$ & $3.11 \mathrm{E}-07$ & $2.19 \mathrm{E}-04$ & $70.6 \%$ & $7.58 \mathrm{E}-03$ & $0.0 \%$ & $28.6 \%$ & $7.65 \mathrm{E}-04$ & 31 \\
\hline $241-B-10\}$ & $1.15 E-04$ & $1.68 E-05$ & $4.37 \mathrm{E}-08$ & $2.64 \mathrm{E}-04$ & $29.1 \%$ & 4. $25 \mathrm{E}-02$ & $0.0 \%$ & $66.6 \%$ & $3.96 \mathrm{E}-04$ & 16 \\
\hline Supematant & na & na & na & na & na & na & na & na & na & na \\
\hline Solid layer & $1.15 \mathrm{E}-04$ & $1.68 \mathrm{E}-05$ & $4.37 \mathrm{E}-08$ & $2.64 \mathrm{E}-04$ & $29.1 \%$ & $4.25 E-02$ & $0.0 \%$ & $66.6 \%$ & $3.96 \mathrm{E}-04$ & 16 \\
\hline $241-\mathrm{B}-102$ & $4.59 \mathrm{E}-07$ & $6.81 \mathrm{E}-08$ & $4.94 \mathrm{E}-10$ & $2.28 \mathrm{E}-04$ & $0.2 \%$ & $2.98 \mathrm{E}-04$ & $0.0 \%$ & $99.8 \%$ & $2.29 \mathrm{E}-04$ & 9 \\
\hline Supernatant & $1.46 \mathrm{E} \cdot 07$ & $2.02 \mathrm{E}-09$ & $7.19 \mathrm{E}-11$ & $1.34 \mathrm{E}-06$ & $9.8 \%$ & $1.35 \mathrm{E}-03$ & $0.0 \%$ & $90.1 \%$ & $1.49 \mathrm{E}-06$ & 0 \\
\hline Solid Layer & $3.13 \mathrm{E}-07$ & $6.61 \mathrm{E}-0.88$ & $4.22 \mathrm{E}-10$ & $2.27 \mathrm{E}-04$ & $0.1 \%$ & $2.91 \mathrm{E}-04$ & $0.0 \%$ & $99.8 \%$ & $2.27 F-04$ & 9 \\
\hline $241-8-103$ & $9.09 \mathrm{E}-07$ & $7.34 E-07$ & $8.37 \mathrm{E}-10$ & $2.37 \mathrm{E}-04$ & $0.4 \%$ & $3.08 \mathrm{E}-03$ & $0.0 \%$ & $99.3 \%$ & $2.39 \mathrm{E}-04$ & 10 \\
\hline Supenatan & na & na & ma & na & na & na & na & na & na & na \\
\hline Solid Layer & $9.09 \mathrm{E}-07$ & $7.34 \mathrm{E}-07$ & $8.37 E-10$ & $2.37 \mathrm{E}-04$ & $0.4 \%$ & $3.08 \mathrm{E}-03$ & $0.0 \%$ & $99.3 \%$ & $2.39 \mathrm{E}-04$ & 10 \\
\hline $241-B-104$ & $1.02 \mathrm{E}-05$ & $1.23 E-06$ & 4.20F- -09 & $3.42 E-04$ & $2.9 \%$ & $3.49 \mathrm{E}-03$ & $0.0 \%$ & $96.8 \%$ & $3.54 \mathrm{E}-04$ & 14 \\
\hline Supernatant & na & na & na & ná & na & па & nad & na & naa & па \\
\hline Solid Layer & $1.02 \mathrm{E}-05$ & 1.23E-06 & 4.20E-09 & $3.42 \mathrm{E}-04$ & $2.9 \%$ & $3.49 \mathrm{E}-03$ & $0.0 \%$ & $96.8 \%$ & $3.54 \mathrm{E}-04$ & 14 \\
\hline $241-B-105$ & $2.84 \mathrm{E}-06$ & $7.86 \mathrm{E}-07$ & $4.53 \mathrm{E}-09$ & 3.13E-04 & $0.9 \%$ & $2.49 \mathrm{E}-03$ & $0.0 \%$ & $98.9 \%$ & $3.16 \mathrm{E}-04$ & 13 \\
\hline Supernatant & na & na & na & na & na & na & na & na & na & na \\
\hline Solid Layer & $2.84 \mathrm{E}-06$ & $7.86 \mathrm{E}-07$ & 4.53E-09 & $3.13 \mathrm{E}-04$ & $0.9 \%$ & $2.49 \mathrm{E}-03$ & $0.0 \%$ & $98.9 \%$ & $3.16 \mathrm{E}-04$ & 13 \\
\hline $241-B-106$ & $3.82 \mathrm{E}-05$ & $4.28 \mathrm{E}-07$ & 4.17E-09 & $2.58 \mathrm{E}-04$ & $12.9 \%$ & $1.44 \mathrm{E}-03$ & $0.0 \%$ & $87.0 \%$ & $2.97 \mathrm{E}-04$ & 12 \\
\hline Supenatant & $3.87 \mathrm{E}-08$ & $5.37 \mathrm{E}-10$ & $1.79 \mathrm{E}-11$ & $3.58 \mathrm{E}-07$ & $9.7 \%$ & 1.35E-03 & $0.0 \%$ & $90.1 \%$ & $3.97 \mathrm{E}-07$ & 0 \\
\hline Solid Layer & $3.82 \mathrm{E}-05$ & 4. $28 \mathrm{E}-07$ & 4.15E-09 & $2.58 \mathrm{E}-04$ & $12.9 \%$ & $1.44 \mathrm{E}-03$ & $0.0 \%$ & $87.0 \%$ & $2.97 \mathrm{E}-04$ & 12 \\
\hline $241-B-107$ & $6.12 \mathrm{E}-06$ & $4.24 E-07$ & $8.64 \mathrm{E}-10$ & $2.70 \mathrm{E}-04$ & $2.2 \%$ & $1.53 \mathrm{E}-03$ & $0.0 \%$ & $97.6 \%$ & $2.77 \mathrm{E}-04$ & 11 \\
\hline Supernatant & na & na & nа & na & na & na & ma & na & na & na \\
\hline Solid Layer & $6.12 \mathrm{E}-06$ & $4.24 \mathrm{E}-07$ & $8.64 E-10$ & $2.70 \mathrm{E}-04$ & $2.2 \%$ & $1.53 \mathrm{E}-03$ & $0.0 \%$ & $97.6 \%$ & $2.77 \mathrm{E}-04$ & 11 \\
\hline
\end{tabular}


RPP-5926 REV 6

Table B-4. Calculated Total Hydrogen Generation Rate In Dome Space for 177 Tanks. (20 sheets)

\begin{tabular}{|c|c|c|c|c|c|c|c|c|c|c|}
\hline Tank & $\begin{array}{c}\text { HGR from } \\
\text { radiolysis } \\
\text { HGR }_{\text {rad }} \text { b/r } \\
\text { (cfm) }\end{array}$ & $\begin{array}{l}\text { HGR from } \\
\text { radiolysis } \\
\text { HGR }_{\text {rad }}^{\text {alpha }} \\
\text { (cfm) }\end{array}$ & $\begin{array}{l}\text { HGR from } \\
\text { thermolysis } \\
\text { HGR }_{\text {therm }} \\
\text { (cfm) }\end{array}$ & $\begin{array}{c}\text { HGR from } \\
\text { corrosion } \\
\text { HGR }_{\text {rorr }} \\
\text { (cfm) }\end{array}$ & $\begin{array}{c}\text { Percent of } \\
\text { HGR from } \\
\text { radiolysis } \\
\mathrm{HGR}_{\mathrm{rad}} \mathrm{b} / \mathrm{r}\end{array}$ & $\begin{array}{l}\text { Percent of } \\
\text { HGR from } \\
\text { radiolysis } \\
\text { HGR }_{\text {Yad }} \text { alphz }\end{array}$ & $\begin{array}{c}\text { Percent of } \\
\text { HGR from } \\
\text { thermolysis } \\
\text { HGR }_{\text {therm }}\end{array}$ & $\begin{array}{l}\text { Percent of } \\
\text { HGR from } \\
\text { corrosion } \\
\text { HGR }_{\text {rorr }}\end{array}$ & $\begin{array}{c}\text { Total HGR } \\
\text { from model } \\
\text { HGR }_{\operatorname{mxx}} \\
\text { (cfm) }\end{array}$ & $\begin{array}{c}\text { Total HGR } \\
\text { from mode } \\
\text { HGR }_{\text {taosd }} \\
\text { (L/day) }\end{array}$ \\
\hline $241-B-108$ & 2.17E-06 & $1.23 \mathrm{E}-08$ & $2.30 \mathrm{E}-09$ & $2.48 \mathrm{~F}-04$ & $0.9 \%$ & $4.93 E-05$ & $0.0 \%$ & $99.1 \%$ & $2.51 \mathrm{E}-04$ & 10 \\
\hline Supenatant & na & nа & na & na & na & na & na & na & na & па \\
\hline Solid Layer & $2.17 \mathrm{E}-06$ & $1.23 \mathrm{E}-98$ & $2.30 \mathrm{E}-09$ & $2.48 \mathrm{E}-04$ & $0.9 \%$ & 4.93E-05 & $0.0 \%$ & $99.1 \%$ & $2.51 \mathrm{E}-04$ & 10 \\
\hline 241-B-109 & $8.35 \mathrm{E}-07$ & $2.54 \mathrm{E}-07$ & 2.35E-09 & $2.59 \mathrm{E}-04$ & $0.3 \%$ & $9.76 \mathrm{E}-04$ & $0.0 \%$ & $99.6 \%$ & 2.60E- -04 & 11 \\
\hline Supernatant & na & na & na & na & na & $\mathrm{na}$ & na & па & na & na \\
\hline Solid Layer & 8.35E-07 & $2.54 E-07$ & $2.35 E-09$ & $2.59 \mathrm{E}-04$ & $0.3 \%$ & $9.76 \mathrm{E}-04$ & $0.0 \%$ & $99.6 \%$ & $2.60 \mathrm{E}-04$ & 11 \\
\hline $241 \cdot B-110$ & $1.73 \mathrm{E}-04$ & $6.97 \mathrm{E}-06$ & 4. $76 \mathrm{E}-05$ & $3.00 \mathrm{E}-04$ & $32.7 \%$ & 1.32E-02 & $9.0 \%$ & $56.9 \%$ & $5.27 \mathrm{E}-04$ & 22 \\
\hline Supernatant & $1.02 \mathrm{E}-07$ & $2.06 \mathrm{E}-09$ & $1.87 F-07$ & $3.58 \mathrm{E}-07$ & $15.7 \%$ & $3.18 \mathrm{E}-03$ & $28.9 \%$ & $55.1 \%$ & $6.49 \mathrm{E}-07$ & 0 \\
\hline Solid Layer & $1.73 \mathrm{E}-04$ & $6.97 \mathrm{E}-06$ & 4.74E-05 & $3.00 E-04$ & $32.8 \%$ & $1.32 \mathrm{E}-02$ & $9.0 \%$ & $56.9 \%$ & $5.27 \mathrm{E}-04$ & 21 \\
\hline 241-B-111 & $5.33 \mathrm{E}-04$ & $6.44 \mathrm{E}-06$ & $6.92 \mathrm{E}-05$ & $3.03 E-04$ & $58.5 \%$ & $7.06 \mathrm{E}-03$ & $7.6 \%$ & $33.3 \%$ & $9,11 \mathrm{E}-04$ & 37 \\
\hline Supeniatanı & $1.04 \mathrm{~F}-07$ & 2.09ㅌ-09 & $2.48 \mathrm{E}-07$ & $3.63 \mathrm{E}-07$ & $14.5 \%$ & $2.92 \mathrm{~F}-03$ & $34.6 \%$ & $50.6 \%$ & $7.18 \mathrm{E}-07$ & 0 \\
\hline Solid Layer & $5.33 \mathrm{E}-04$ & 6.43E- -06 & $6.89 \mathrm{E}-05$ & 3.03E-04 & $58.5 \%$ & 7.07E-03 & $7.6 \%$ & $33.2 \%$ & $9.10 \mathrm{E}-04$ & 37 \\
\hline $241-B-112$ & $5.32 \mathrm{E}-06$ & $1.33 \mathrm{E}-08$ & $2.90 \mathrm{E}-05$ & $2.29 \mathrm{E}-04$ & $2.0 \%$ & $5.03 \mathrm{E}-05$ & $11.0 \%$ & $87.0 \%$ & $2.64 \mathrm{E}-04$ & 11 \\
\hline Supernatant & $1.12 \mathrm{E}-06$ & $2.44 \mathrm{E}-09$ & 2.72E-06 & $9.87 E-07$ & $23.1 \%$ & $5.05 \mathrm{E}-04$ & $56.4 \%$ & $20.4 \%$ & 4. $83 \mathrm{E}-06$ & 0 \\
\hline Solid Layer & $4.21 E-06$ & $1.08 E-08$ & $2.63 E-05$ & $2.28 \mathrm{E}-04$ & $1.6 \%$ & 4. $18 \mathrm{E}-05$ & $10.2 \%$ & $88.2 \%$ & $2.59 \mathrm{E}-04$ & 11 \\
\hline $241-\mathrm{B}-201$ & $4.74 \mathrm{E}-07$ & $3.68 \mathrm{E}-06$ & $1.09 \mathrm{E}-06$ & $5.26 \mathrm{E}-05$ & $0.8 \%$ & $6.37 \mathrm{E}-02$ & $1.9 \%$ & $90.9 \%$ & $5.78 \mathrm{E}-05$ & 2 \\
\hline Supenatant & na & na & na & na & na & па & na & $\mathfrak{n a}$ & na & na \\
\hline Solid Layer & 4.74E-07 & $3.68 \mathrm{E}-06$ & $1.09 \mathrm{E}-06$ & $5.26 \mathrm{E}-05$ & $0.8 \%$ & $6.37 \mathrm{E}-02$ & $1.9 \%$ & $90.9 \%$ & $5.78 \mathrm{E}-05$ & 2 \\
\hline 241-B-202 & $8.07 \mathrm{E}-07$ & $9.99 \mathrm{E}-07$ & 1. $13 E-06$ & $5.15 \mathrm{E}-05$ & $1.5 \%$ & J.83E-02 & $2.1 \%$ & $94.6 \%$ & $5.45 \mathrm{E}-05$ & 2 \\
\hline Supernatant & na & na & na & na & $\mathrm{ma}$ & ma & na & na & na & па \\
\hline Solid Layer & $8.07 E-07$ & $9.99 \mathrm{E}-07$ & $1.13 \mathrm{E}-06$ & $5.15 \mathrm{E}-05$ & $1.5 \%$ & 1.83E- 02 & $2.1 \%$ & $94.6 \%$ & $5.45 \mathrm{E}-05$ & 2 \\
\hline $241-B-203$ & $4.78 \mathrm{E}-08$ & $2.94 \mathrm{E}-06$ & $0.00 \mathrm{E}+00$ & 1.92E-04 & $0.0 \%$ & $1.50 \mathrm{E}-02$ & $0.0 \%$ & $98.5 \%$ & $1.95 \mathrm{E}-04$ & 8 \\
\hline Supenatant & $1.01 \mathrm{E}-12$ & 4. $74 \mathrm{E}-10$ & $0.00 \mathrm{E}+00$ & $1.62 \mathrm{E}-06$ & $0.0 \%$ & $2.92 \mathrm{E}-04$ & $0.0 \%$ & $100.0 \%$ & $1.63 \mathrm{E}-06$ & 0 \\
\hline Solid Layer & $4.78 \mathrm{E}-08$ & $2.94 \mathrm{E}-06$ & $0.00 \mathrm{E}+00$ & $1.91 \mathrm{E}-04$ & $0.0 \%$ & $1.52 \mathrm{E}-02$ & $0.0 \%$ & $98.5 \%$ & $1.94 \mathrm{E}-04$ & 8 \\
\hline 241-B-204 & $1.82 \mathrm{E}-08$ & 3.57E-06 & $0.00 \mathrm{E} \div 00$ & $1.96 \mathrm{E}-04$ & $0.0 \%$ & $1.79 \mathrm{E}-02$ & $0.0 \%$ & $98.2 \%$ & $2.00 \mathrm{E}-04$ & 8 \\
\hline Supernatant & $9.17 \mathrm{E}-13$ & $1.70 \mathrm{E}-11$ & $0.00 \mathrm{E}+00$ & $2.52 \mathrm{E}-06$ & $0.0 \%$ & $6.76 \mathrm{E}-06$ & $0.0 \%$ & $100.0 \%$ & $2,52 \mathrm{E}-06$ & 0 \\
\hline Solid Layer & $1.82 \mathrm{E}-08$ & 3.57E-06 & $0.00 \mathrm{E}+00$ & $1.94 \mathrm{E}-04$ & $0.0 \%$ & 1.81E-02 & $0.0 \%$ & $98.2 \%$ & $1.97 \mathrm{E}-04$ & 8 \\
\hline
\end{tabular}


RPP-5926 REV 6

Table B-4. Calculated Total Hydrogen Generation Rate In Dome Space for 177 Tanks. (20 sheets)

\begin{tabular}{|c|c|c|c|c|c|c|c|c|c|c|}
\hline Tank & $\begin{array}{c}\text { HGR from } \\
\text { radiolysis } \\
\text { HGR }_{\text {rad }} \text { bir } \\
\text { (cfm) }\end{array}$ & $\begin{array}{l}\text { HGR from } \\
\text { radiolysis } \\
\text { HGR }_{\text {rad alpha }} \\
\text { (cfm) }\end{array}$ & $\begin{array}{c}\text { HGR from } \\
\text { thermolysis } \\
\text { HGR }_{\text {therm }} \\
\text { (cfm) }\end{array}$ & $\begin{array}{c}\text { HGR from } \\
\text { corrosion } \\
\text { HGR }_{\text {corr }} \\
\text { (cfm) }\end{array}$ & $\begin{array}{l}\text { Percent of } \\
\text { HGR from } \\
\text { radiolysis } \\
\text { HGR }_{\text {rat }} \text { bir }\end{array}$ & $\begin{array}{l}\text { Percent of } \\
\text { HGR from } \\
\text { radiolysis } \\
\text { HGR }_{\text {rad }} \text { aiphs }\end{array}$ & $\begin{array}{l}\text { Percent of } \\
\text { HGR from } \\
\text { thermolysis } \\
\text { HGR }_{\text {therm }}\end{array}$ & $\begin{array}{c}\text { Percent of } \\
\text { HGR from } \\
\text { corrosion } \\
\text { HGR }_{\text {corr }}\end{array}$ & $\begin{array}{c}\text { Total HGR } \\
\text { from model } \\
\text { HGR }_{\text {mod }} \\
\text { (cfm) }\end{array}$ & $\begin{array}{c}\text { Total HGR } \\
\text { from model } \\
\text { HGR }_{\text {mod }} \\
\text { ( } \mathrm{L} / \mathrm{d} \text { ay) }\end{array}$ \\
\hline $241-\mathrm{BX}-101$ & $2.76 \mathrm{E}-05$ & 1.54E-06 & $1.72 \mathrm{E}-06$ & $2.34 \mathrm{E}-04$ & $10.4 \%$ & $5.83 E-03$ & $0.6 \%$ & $88.3 \%$ & $2.65 \mathrm{E}-04$ & 11 \\
\hline Supernatant & na & na & na & na & na & na & na & na & na & na \\
\hline Solid Layer & $2.76 \mathrm{E}-05$ & $1.54 \mathrm{E}-06$ & 1.72E-06 & $2.34 \mathrm{E}-04$ & $10.4 \%$ & $5.83 \mathrm{E}-03$ & $0.6 \%$ & $88.3 \%$ & $2.65 \mathrm{E}-04$ & 11 \\
\hline $241-\mathrm{BX}-102$ & $1.32 \mathrm{E}-05$ & $1.47 \mathrm{E}-07$ & $1.92 \mathrm{E}-06$ & 2.43E-04 & $5.1 \%$ & $5.69 \mathrm{E}-04$ & $0.7 \%$ & $94.1 \%$ & $2.58 \mathrm{E}-04$ & 11 \\
\hline Supernatant & na & na & na & па & na & na & na & $\mathrm{na}$ & na & na \\
\hline Solid Layer & $1.32 \mathrm{E}-05$ & $1.47 \mathrm{E}-07$ & $1.92 \mathrm{E}-06$ & $2.43 E-04$ & $5.1 \%$ & $5.69 \mathrm{E}-04$ & $0.7 \%$ & $94.1 \%$ & $2.58 \mathrm{E}-04$ & 11 \\
\hline $241-\mathrm{BX}-103$ & $9.49 E-06$ & 4. $38 \mathrm{E}-05$ & $1.09 \mathrm{E}-05$ & $2.42 \mathrm{E}-04$ & $3.1 \%$ & $1.43 \mathrm{E}-01$ & $3.6 \%$ & $79.0 \%$ & 3.06E-04 & 12 \\
\hline Supematant & $1.79 \mathrm{E}-07$ & $3.68 \mathrm{E}-08$ & $1.93 \mathrm{E}-06$ & $4.30 \mathrm{E}-06$ & $2.8 \%$ & $5.71 \mathrm{E}-03$ & $30.0 \%$ & $66.7 \%$ & $6.44 \mathrm{E}-06$ & 0 \\
\hline Solid Layer & $9.31 \mathrm{E}-06$ & $4.38 \mathrm{E}-05$ & $9.00 \mathrm{E}-06$ & $2.38 \mathrm{E}-04$ & $3.1 \%$ & 1.46E- -01 & $3.0 \%$ & $79.3 \%$ & $3.00 \mathrm{E}-04$ & 12 \\
\hline $241-\mathrm{BX}-104$ & $9.45 \mathrm{E}-05$ & $6.78 E-06$ & $9.65 \mathrm{E}-06$ & $2.54 \mathrm{E}-04$ & $25.9 \%$ & $1.86 E-02$ & $2.6 \%$ & $69.6 \%$ & $3.65 \mathrm{E}-04$ & 15 \\
\hline Superratant & $1.37 \mathrm{E}-06$ & $1.49 \mathrm{E}-10$ & $4.59 \mathrm{E}-07$ & $9.95 \mathrm{E}-07$ & $48.4 \%$ & $5.27 \mathrm{E}-05$ & $16.3 \%$ & $35.3 \%$ & $2.82 \mathrm{E}-06$ & 0 \\
\hline Solid Layer & $9.31 \mathrm{E}-05$ & $6.78 \mathrm{E}-06$ & $9.19 \mathrm{E}-06$ & $2.53 E-04$ & $25.7 \%$ & $1.87 \mathrm{E}-02$ & $2.5 \%$ & $69.8 \%$ & $3.62 \mathrm{E}-04$ & 15 \\
\hline $241-8 X-105$ & $3.43 \mathrm{E}-05$ & $4.64 E-07$ & $4.48 E-06$ & $2.41 \mathrm{E}-04$ & $12.3 \%$ & $1.66 \mathrm{E}-03$ & $1.6 \%$ & $86.0 \%$ & $2.80 \mathrm{E}-04$ & 11 \\
\hline Supeniatant & $2.59 \mathrm{E}-06$ & $2.24 \mathrm{E}-08$ & $9.61 \mathrm{E}-07$ & $1.61 \mathrm{E}-06$ & $50.0 \%$ & $4.33 \mathrm{E}-03$ & $18.6 \%$ & $31.0 \%$ & $5.18 \mathrm{E}-06$ & 0 \\
\hline Solid Layer & $3.17 \mathrm{E}-05$ & 4. $42 \mathrm{E}-07$ & 3. $52 \mathrm{E}-06$ & $2.39 \mathrm{E}-04$ & $11.6 \%$ & 1.61E- 03 & $1.3 \%$ & $87.0 \%$ & $2.75 \mathrm{E}-04$ & 11 \\
\hline $24 !-3 X-106$ & $1.07 \mathrm{E}-05$ & $1.64 E-06$ & $2.96 \mathrm{E}-05$ & $2.29 \mathrm{E}-04$ & $4.0 \%$ & $6.04 \mathrm{E}-03$ & $10.9 \%$ & $84.5 \%$ & $2.71 \mathrm{E}-04$ & 11 \\
\hline Supernatant & na & na & na & na & na & nа & na & na & na & na \\
\hline Solid Layer & $1.07 \mathrm{E}-05$ & $1.64 \mathrm{E}-06$ & $2.96 \mathrm{E}-05$ & $2.29 \mathrm{E}-04$ & $4.0 \%$ & $6.04 \mathrm{E}-03$ & $10.9 \%$ & $84.5 \%$ & $2.71 \mathrm{E}-04$ & 11 \\
\hline $241-\mathrm{BX}-107$ & $4.78 \mathrm{E}-05$ & 3.39E-06 & 1.51E-05 & $3.34 \mathrm{E}-04$ & $12.0 \%$ & $8.46 \mathrm{E}-03$ & $3.8 \%$ & $83.4 \%$ & $4.00 \mathrm{E} \cdot 04$ & 16 \\
\hline Supernatunt & wa & na & na & na & na & na & na & $\mathrm{na}$ & na & na \\
\hline Solid Layer & 4.78E-05 & $3.39 \mathrm{E}-06$ & $1.51 \mathrm{E}-05$ & $3.34 \mathrm{E}-04$ & $12.0 \%$ & $8.46 \mathrm{E}-03$ & $3.8 \%$ & $83.4 \%$ & $4.00 \mathrm{E}-04$ & 16 \\
\hline $241-\mathrm{BX}-108$ & 1.27E-05 & $1.09 \mathrm{E}-07$ & $5.25 \mathrm{E}-07$ & $2.27 \mathrm{E}-04$ & $5.3 \%$ & $4.53 \mathrm{E}-04$ & $0.2 \%$ & $94.4 \%$ & $2.40 \mathrm{H} \cdot 04$ & 10 \\
\hline Supernatant & na & na & na & na & na & na & $\mathrm{na}$ & $\mathrm{n}_{a}$ & na & na \\
\hline Solid Layer & $1.27 \mathrm{E}-05$ & $1.09 \mathrm{E}-07$ & $5.25 \mathrm{E}-07$ & $2.27 \mathrm{E}-04$ & $5.3 \%$ & $4.53 \mathrm{E}-04$ & $0.2 \%$ & $94.4 \%$ & $2.40 \mathrm{E}-04$ & 10 \\
\hline $241-B X-109$ & $2.00 \mathrm{E}-04$ & 4.84E-07 & $1.25 \mathrm{E}-05$ & $2.83 \mathrm{E}-04$ & $40.3 \%$ & $9.77 \mathrm{E}-04$ & $2.5 \%$ & $57.1 \%$ & $4.95 \mathrm{E}-04$ & 20 \\
\hline Supernatant & na & na & na & na & na & na & na & na & nа & na \\
\hline Solid Layer & $2.00 \mathrm{E}-04$ & $4.84 \mathrm{E}-07$ & $1.25 \mathrm{E}-05$ & $2.83 \mathrm{E}-04$ & $40.3 \%$ & $9.77 \mathrm{E}-04$ & $2.5 \%$ & $57.1 \%$ & $4.95 \mathrm{E}-04$ & 20 \\
\hline
\end{tabular}


RPP-5926 REV 6

Table B-4. Calculated Total Hydrogen Generation Rate In Dome Space for 177 Tanks. (20 sheets)

\begin{tabular}{|c|c|c|c|c|c|c|c|c|c|c|}
\hline Tank & $\begin{array}{l}\text { HGR from } \\
\text { radiulysis } \\
\text { HGR }_{\mathrm{rad} / \mathrm{r}} \\
\text { (cfm) }\end{array}$ & $\begin{array}{c}\text { HGR from } \\
\text { radioly sis } \\
\text { HGR }_{\text {rad }}^{\text {alpta }} \\
\text { (cfm) }\end{array}$ & $\begin{array}{l}\text { HGR from } \\
\text { thermolysìs } \\
\text { HGR }_{\text {therm }} \\
\text { (cfm) }\end{array}$ & $\begin{array}{c}\text { HGR from } \\
\text { corrosion } \\
\text { HGR }_{\text {corr }} \\
\text { (cfm) }\end{array}$ & $\begin{array}{l}\text { Percent of } \\
\text { HGR from } \\
\text { radiolysis } \\
\text { HGR }_{\text {ratl }} \text { bir }\end{array}$ & $\begin{array}{l}\text { Percent of } \\
\text { HGR from } \\
\text { radiolysis } \\
\text { HGR }_{\text {rad }} \text { alp১a }\end{array}$ & $\begin{array}{l}\text { Percent of } \\
\text { HGR from } \\
\text { thermolysis } \\
\text { HGR }_{\text {therm }}\end{array}$ & $\begin{array}{l}\text { Percent of } \\
\text { HGR from } \\
\text { corrosion } \\
\text { HGR }_{\text {corr }}\end{array}$ & $\begin{array}{c}\text { Total HGR } \\
\text { from model } \\
\text { HGR }_{\text {mod }} \\
\text { (cfm) }\end{array}$ & $\begin{array}{c}\text { Total HGR } \\
\text { from model } \\
\text { HGR }_{\text {morl }} \\
(\mathrm{L} / \mathrm{day})\end{array}$ \\
\hline $241-\mathrm{BX}-110$ & $3.82 \mathrm{E}-05$ & $2.35 \mathrm{E}-07$ & $5.14 E-05$ & $2.89 \mathrm{E}-04$ & $10.1 \%$ & $6.22 \mathrm{E}-04$ & $13.6 \%$ & $76.3 \%$ & $3.79 \mathrm{E}-04$ & 15 \\
\hline Supernatant & $3.91 \mathrm{E}-07$ & $5.80 \mathrm{E}-10$ & $3.45 \mathrm{E}-07$ & $4.46 \mathrm{E}-07$ & $33.1 \%$ & $4.90 \mathrm{E}-04$ & $29.2 \%$ & $37.7 \%$ & $1.18 \mathrm{E}-06$ & 0 \\
\hline Solid Layer & $3.78 \mathrm{E}-05$ & $2.35 \mathrm{E}-07$ & $5.11 \mathrm{E}-05$ & $2.88 \mathrm{E}-04$ & $10.0 \%$ & $6.22 \mathrm{E}-04$ & $13.5 \%$ & $76.4 \%$ & $3.77 \mathrm{E}-04$ & 15 \\
\hline $24 I-B X-111$ & $4.29 \mathrm{E}-06$ & $3.54 \mathrm{E}-08$ & $1.45 \mathrm{E}-06$ & $2.79 E-04$ & $1.5 \%$ & $1.24 \mathrm{E}-04$ & $0.5 \%$ & $98.0 \%$ & $2.85 \mathrm{E}-0.4$ & 12 \\
\hline Supernatant & na & na & na & na & na & na & na & na & na & na \\
\hline Solid Layer & $4.29 \mathrm{E}-06$ & $3.54 \mathrm{E}-08$ & $1.45 \mathrm{E}-06$ & $2.79 \mathrm{~F}-04$ & $1.5 \%$ & $1.24 \mathrm{E}-04$ & $0.5 \%$ & $98.0 \%$ & $2.85 \mathrm{E}-04$ & 12 \\
\hline $241-B X-112$ & $5.29 \mathrm{E}-05$ & $4.53 \mathrm{E}-06$ & $0.00 \mathrm{E}-00$ & $2.71 \mathrm{E}-04$ & $16.1 \%$ & $1.38 E-02$ & $0.0 \%$ & $82.5 \%$ & $3.28 E-04$ & 13 \\
\hline Supenatant & 1.36E- 08 & $3.20 \mathrm{E}-10$ & $0.00 \mathrm{E}+00$ & $4.45 \mathrm{E}-07$ & $3.0 \%$ & $6.98 \mathrm{E}-04$ & $0.0 \%$ & $97.0 \%$ & $4.59 \mathrm{E}-07$ & 0 \\
\hline Solid Layer & $5.29 \mathrm{E}-05$ & $4.53 \mathrm{E}-06$ & $0.00 \mathrm{E}+00$ & $2.70 \mathrm{E}-04$ & $16.1 \%$ & 1.38E-02 & $0.0 \%$ & $82.5 \%$ & $3.28 \mathrm{E}-04$ & 13 \\
\hline $241-B Y-101$ & $1.26 \mathrm{E}-04$ & $7.63 \mathrm{E}-07$ & $2.02 \mathrm{E}-04$ & $3.45 \mathrm{E}-04$ & $18.7 \%$ & $1.13 E-03$ & $30.0 \%$ & $51.2 \%$ & $6.74 \mathrm{E}-04$ & 27 \\
\hline Supernatant & na & na & nа & na & па & na & na & na & na & na \\
\hline Solid Layer & $1.26 \mathrm{E}-04$ & $7.63 \mathrm{E}-07$ & $2.02 \mathrm{E}-04$ & $3.45 \mathrm{E}-04$ & $18.7 \%$ & $1.13 E-03$ & $30.0 \%$ & $51.2 \%$ & $6.74 \mathrm{E}-04$ & 27 \\
\hline $24 I-B Y-102$ & $2.07 \mathrm{E}-05$ & $9.98 \mathrm{E}-07$ & $9.63 \mathrm{E}-06$ & $3.12 \mathrm{E}-04$ & $6.0 \%$ & $2.90 E-03$ & $2.8 \%$ & $90.9 \%$ & $3.44 \mathrm{E}-04$ & 14 \\
\hline Supematant & na & na & na & na & na & na & na & na & na & na \\
\hline Solid Layer & $2.07 \mathrm{E}-05$ & $9.98 \mathrm{E}-07$ & $9.63 \mathrm{E}-06$ & 3.12E-04 & $6.0 \%$ & $2.90 \mathrm{E}-03$ & $2.8 \%$ & $90.9 \%$ & $3.44 \mathrm{E}-04$ & 14 \\
\hline $241-B Y-103$ & $4.60 \mathrm{E}-05$ & $4.19 \mathrm{E}-07$ & $1.81 \mathrm{E}-05$ & $3.56 \mathrm{E}-04$ & $10.9 \%$ & $9.96 \mathrm{E}-04$ & $4.3 \%$ & $84.7 \%$ & $4.20 \mathrm{E}-04$ & 17 \\
\hline Supernatant & na & na & na & na & na & na & na & na & na & na \\
\hline Solid Layer & $4.60 \mathrm{E}-05$ & $4.19 \mathrm{E}-07$ & $1.81 \mathrm{~F}-05$ & $3.56 \mathrm{E}-04$ & $10.9 \%$ & $9.96 \mathrm{E}-04$ & $4.3 \%$ & $84.7 \%$ & $4.20 \mathrm{E}-04$ & 17 \\
\hline $241-B Y-104$ & $1.18 \mathrm{E}-04$ & 1. $22 \mathrm{E}-06$ & $1.88 \mathrm{E}-04$ & $3.61 \mathrm{E}-04$ & $17.7 \%$ & $1.82 \mathrm{E}-03$ & $28.1 \%$ & $54.0 \%$ & $6.69 \mathrm{E}-04$ & 27 \\
\hline Superratant & na & na & na & na & nla & na & na & na & na & na \\
\hline Solid L.ayer & $1.18 \mathrm{E}-04$ & $1.22 \mathrm{E}-06$ & i. $88 \mathrm{E}-04$ & $3.61 \mathrm{E}-04$ & $17.7 \%$ & $1.82 \mathrm{E}-03$ & $28.1 \%$ & $54.0 \%$ & $6.69 \mathrm{E}-04$ & 27 \\
\hline 241-BY-105 & $7.49 \mathrm{E}-05$ & $1.41 \mathrm{E}-06$ & 1.1:E-04 & $3.92 \mathrm{E}-04$ & $12.9 \%$ & $2.43 \mathrm{E}-03$ & $19.1 \%$ & $67.7 \%$ & $5.79 \mathrm{E}-04$ & 24 \\
\hline Supernatant & na & na & na & na & na & na & na & na & na & na \\
\hline Solid Layer & $7.49 \mathrm{E}-05$ & $1.41 \mathrm{E}-06$ & $1.11 \mathrm{E}-04$ & $3.92 \mathrm{E}-04$ & $12.9 \%$ & $2.43 \mathrm{E}-03$ & $19.1 \%$ & $67.7 \%$ & $5.79 \mathrm{E}-04$ & 24 \\
\hline $24 I-B Y-106$ & $2.00 \mathrm{E}-04$ & $8.72 \mathrm{E} \cdot 07$ & $1.63 \mathrm{E}-04$ & $3.78 \mathrm{E}-04$ & $26.9 \%$ & $1.18 \mathrm{E}-03$ & $21.9 \%$ & $51.0 \%$ & $7.41 \mathrm{E}-04$ & 30 \\
\hline Supernatant & na & na & na & na & na & na & na & na & na & na \\
\hline Solid Layer & $2.00 \mathrm{E}-04$ & $8.72 E-07$ & $1.63 \mathrm{E}-04$ & $3.78 \mathrm{E}-04$ & $26.9 \%$ & $1.18 \mathrm{E}-03$ & $21.9 \%$ & $51.0 \%$ & $7.41 \mathrm{E}-04$ & 30 \\
\hline
\end{tabular}


RPP-5926 REV 6

Table B-4. Calculated Total Hydrogen Generation Rate In Dome Space for 177 Tanks. (20 sheets)

\begin{tabular}{|c|c|c|c|c|c|c|c|c|c|c|}
\hline Tank & $\begin{array}{l}\text { HGR from } \\
\text { radiolysis } \\
\text { HGR }_{r a d} \mathrm{~b} / \mathrm{r} \\
\text { (cfm) }\end{array}$ & $\begin{array}{l}\text { HGR from } \\
\text { radiolysis } \\
\text { HGR }_{\text {rat }} \text { ilpha } \\
\text { (cfm) }\end{array}$ & $\begin{array}{l}\text { HGR from } \\
\text { thermolysis } \\
\text { HGR }_{\text {therm }} \\
\text { (cfm) }\end{array}$ & $\begin{array}{c}\text { HGR from } \\
\text { corrosion } \\
\text { HGR }_{\text {rorr }} \\
\text { (cfm) }\end{array}$ & $\begin{array}{l}\text { Percent of } \\
\text { HGR from } \\
\text { radiolysis } \\
\text { HGR }_{\text {rad }}{ }^{\text {brr }}\end{array}$ & $\begin{array}{l}\text { Percent of } \\
\text { HGR from } \\
\text { radiolysis } \\
\text { HGR }_{\text {rat }} \text { alpha }\end{array}$ & $\begin{array}{l}\text { Percent of } \\
\text { HGR from } \\
\text { thermolysis } \\
\text { HGR }_{\text {therm }}\end{array}$ & $\begin{array}{l}\text { Percent of } \\
\text { HGR from } \\
\text { corrosion } \\
\text { HGR }_{\text {corr }}\end{array}$ & $\begin{array}{c}\text { Total HGR } \\
\text { from model } \\
\text { HGR }_{\text {mod }} \\
\text { (efm) }\end{array}$ & $\begin{array}{c}\text { Total HGR } \\
\text { from model } \\
\text { HGR }_{\text {mod }} \\
\text { (L/day) }\end{array}$ \\
\hline $241-\mathrm{BY}-107$ & $7.62 \mathrm{E}-05$ & $4.34 \mathrm{E}-07$ & $6.42 \mathrm{E}-05$ & $3.14 E-04$ & $16.7 \%$ & $9.55 \mathrm{E}-04$ & $14.1 \%$ & $69.0 \%$ & $4.55 \mathrm{E}-04$ & 19 \\
\hline Supernatant & na & na & nа & nа & na & na & na & na & na & na \\
\hline Solid Layer & $7.62 \mathrm{E}-05$ & $4.34 \mathrm{E}-07$ & $6.42 \mathrm{E}-05$ & $3.14 \mathrm{E}-04$ & $16.7 \%$ & $9.55 \mathrm{E}-04$ & $14.1 \%$ & $69.0 \%$ & $4.55 \mathrm{E}-04$ & 19 \\
\hline $241-8 Y-108$ & $4.95 \mathrm{E}-05$ & $4.95 \mathrm{E}-07$ & $4.93 \mathrm{E}-05$ & $2.97 \mathrm{E}-04$ & $12.5 \%$ & $1.25 \mathrm{E}-03$ & $12.4 \%$ & $74.9 \%$ & $3.96 \mathrm{E}-04$ & 16 \\
\hline Supernatant & nа & na & na & na & na & na & na & na & na & na \\
\hline Solid Layer & $4.95 \mathrm{E}-05$ & $4.95 \mathrm{E}-07$ & 4.93E-05 & $2.97 E-04$ & $12.5 \%$ & $1.25 \mathrm{E}-03$ & $12.4 \%$ & $74.9 \%$ & $3.96 \mathrm{E}-04$ & 16 \\
\hline 241-BY-109 & $2.12 E-05$ & $7.06 E-07$ & $7.05 \mathrm{E}-06$ & $3.10 \mathrm{E}-04$ & $6.3 \%$ & $2.08 E-03$ & $2.1 \%$ & $91.5 \%$ & $3.39 \mathrm{E}-04$ & 14 \\
\hline Supernatant & na & na & na & na & na & na & na & na & na & na \\
\hline Sulid Layer & $2.12 \mathrm{E}-05$ & $7.06 \mathrm{E}-07$ & $7.05 \mathrm{E}-06$ & $3.10 \mathrm{E}-04$ & $6.3 \%$ & $2.08 \mathrm{E}-03$ & $2.1 \%$ & $91.5 \%$ & $3.39 \mathrm{E}-04$ & 14 \\
\hline $241-B Y-110$ & 1.13E-04 & $8.35 E_{-}-07$ & $6.07 \mathrm{E}-04$ & $3.41 \mathrm{E}-04$ & $10.6 \%$ & $7.87 E-04$ & $57.2 \%$ & $32.1 \%$ & $1.06 \mathrm{E}-03$ & 43 \\
\hline Supernatant & na & na & na & na & na & па & na & na & na & na \\
\hline Solid Layer & 1.13E-04 & $8.35 E-07$ & $6.07 \mathrm{E}-04$ & 3.41E-04 & $10.6 \%$ & $7.87 \mathrm{E}-04$ & $57.2 \%$ & $32.1 \%$ & $1.06 \mathrm{E}-03$ & 43 \\
\hline $241-\mathrm{BY}-111$ & $8.30 \mathrm{E}-06$ & $4.06 \mathrm{E}-07$ & 4. $49 \mathrm{E}-06$ & $3.52 \mathrm{E}-04$ & $2.3 \%$ & 1.11E-03 & $1.2 \%$ & $96.4 \%$ & $3.65 \mathrm{E}-04$ & 15 \\
\hline Suppernatant & na & na & na & na & na & na & na & na & na & na \\
\hline Solid Layer & $8.30 \mathrm{E}-06$ & $4.06 \mathrm{E}-07$ & 4.49E-06 & $3.52 \mathrm{E}-04$ & $2.3 \%$ & $1.11 E-03$ & $1.2 \%$ & $96.4 \%$ & $3.65 \mathrm{E}-04$ & 15 \\
\hline $241-B Y-112$ & $9.64 \mathrm{E}-06$ & $2.68 \mathrm{E}-07$ & $8.86 \mathrm{E}-06$ & $3.14 \mathrm{E}-04$ & $2.9 \%$ & $8.05 \mathrm{E}-04$ & $2.7 \%$ & $94.4 \%$ & $3.32 \mathrm{E}-04$ & 14 \\
\hline Supernatant & na & na & na & па & па & na & na & na & nаa & na \\
\hline Solid Layer & $9.64 \mathrm{E}-06$ & $2.68 \mathrm{E}-07$ & $8.86 \mathrm{E}-06$ & $3.14 \mathrm{E}-04$ & $2.9 \%$ & $8.05 E-04$ & $2.7 \%$ & $94.4 \%$ & $3.32 \mathrm{E}-04$ & 14 \\
\hline $241-C-101$ & $4.45 E-05$ & $7.96 \mathrm{E}-06$ & $1.11 \mathrm{E}-05$ & $2.56 \mathrm{E}-04$ & $13.9 \%$ & $2.49 \mathrm{E}-02$ & $3.5 \%$ & $80.1 \%$ & $3.20 \mathrm{E}-04$ & 13 \\
\hline Supernatant & na & $\mathrm{na}$ & na & na & na & na & na & na & na & na \\
\hline Solid Layer & 4.45E-05 & $7.96 \mathrm{E}-06$ & $1.11 \mathrm{E}-05$ & $2.56 \mathrm{E}-04$ & $13.9 \%$ & $2.49 \mathrm{E}-02$ & $3.5 \%$ & $80.1 \%$ & $3.20 \mathrm{E}-04$ & 13 \\
\hline $241-C-102$ & $4.59 \mathrm{E}-05$ & $8.85 E-05$ & $3.41 \mathrm{E}-05$ & $3.29 \mathrm{E}-04$ & $9.2 \%$ & $1.78 \mathrm{E}-01$ & $6.9 \%$ & $66.1 \%$ & $4.98 \mathrm{E}-04$ & 20 \\
\hline Supernatant & na & na & na & na & na & na & na & na & na & na \\
\hline Solid Layer & 4.59E-05 & $8.85 \mathrm{E}-05$ & 3.41E-05 & $3.29 \mathrm{E}-04$ & $9.2 \%$ & $1.78 \mathrm{E}-01$ & $6.9 \%$ & $66.1 \%$ & $4.98 \mathrm{E}-04$ & 20 \\
\hline $241-C-103$ & $1.85 \mathrm{E}-04$ & $1.10 \mathrm{E}-05$ & $0.00 \mathrm{E}+00$ & $5.95 \mathrm{E}-04$ & $23.4 \%$ & $1.39 \mathrm{E}-02$ & $0.0 \%$ & $75.2 \%$ & $7.91 \mathrm{E}-04$ & 32 \\
\hline Supernatant & па & na & na & na & na & na & na & na & na & na \\
\hline Solid Layer & $1.85 \mathrm{E}-04$ & $1.10 \mathrm{E}-0 \mathrm{~S}$ & $0.00 \mathrm{E}+00$ & $5.95 \mathrm{E}-04$ & $23.4 \%$ & 1.39E-02 & $0.0 \%$ & $75.2 \%$ & $7.91 \mathrm{E}-04$ & 32 \\
\hline
\end{tabular}




\section{RPP-5926 REV 6}

Table B-4. Calculated Total Hydrogen Generation Rate In Dome Space for 177 Tanks. (20 sheets)

\begin{tabular}{|c|c|c|c|c|c|c|c|c|c|c|}
\hline Tank & $\begin{array}{l}\text { HGR from } \\
\text { radiolysis } \\
\text { HGR }_{\mathrm{rad}} \mathrm{b} / \mathrm{r} \\
\text { (cfm) }^{\text {cfm }}\end{array}$ & $\begin{array}{l}\text { HGR from } \\
\text { radiolysis } \\
\text { HGR }_{\text {rad }}^{\text {alpha }} \\
\text { (cfm) }\end{array}$ & $\begin{array}{c}\text { HGR from } \\
\text { thermolysis } \\
\text { HGR }_{\text {therm }} \\
\text { (cfm) }\end{array}$ & $\begin{array}{c}\text { HGR from } \\
\text { corrosion } \\
\text { HGR }_{\text {corr }} \\
\text { (cfm) }\end{array}$ & $\begin{array}{l}\text { Percent of } \\
\text { HGR from } \\
\text { radiolysis } \\
\text { HGR }_{\text {rad }} \text { b/r }\end{array}$ & $\begin{array}{l}\text { Percent of } \\
\text { HGR from } \\
\text { radiolysis } \\
\text { HGR }_{\text {rag }}\end{array}$ & $\begin{array}{c}\text { Percent of } \\
\text { HGR from } \\
\text { thermolysis } \\
\text { HGR }_{\text {therm }}\end{array}$ & $\begin{array}{l}\text { Percent of } \\
\text { HGR from } \\
\text { corrosion } \\
\text { HGR }_{\text {corr }}\end{array}$ & $\begin{array}{c}\text { Total HGR } \\
\text { from model } \\
\text { HGR }_{\text {mod }} \\
\text { (cfm) }\end{array}$ & $\begin{array}{c}\text { Total HGR } \\
\text { from model } \\
H G R_{\text {mad }} \\
\text { (L.day) }\end{array}$ \\
\hline $241-C-104$ & $7.78 \mathrm{E}-04$ & $3.17 \mathrm{E}-04$ & $1.74 \mathrm{E}-04$ & $3.18 \mathrm{E}-04$ & $49.1 \%$ & $2.00 \mathrm{E}-01$ & $11.0 \%$ & $20.0 \%$ & $1.59 \mathrm{E}-03$ & 65 \\
\hline Supernatant & na & na & na & na & na & na & na & na & na & na \\
\hline Solid Layer & $7.78 \mathrm{E}-04$ & 3.17E-04 & $1.74 \mathrm{E}-04$ & $3.18 \mathrm{E}-04$ & $49.1 \%$ & $2.00 \mathrm{E}-01$ & $11.0 \%$ & $20.0 \%$ & $1.59 \mathrm{E}-03$ & 65 \\
\hline $241-C \cdot 105$ & $4.43 E-04$ & $1.01 \mathrm{E}-04$ & $7.56 \mathrm{E}-106$ & $2.79 \mathrm{E}-04$ & $53.3 \%$ & $1.21 \mathrm{E}-01$ & $0.9 \%$ & $33.6 \%$ & $8.31 \mathrm{E}-04$ & 34 \\
\hline Supernatant & na & na & na & na & na & na & na & na & na & na \\
\hline Solid Layer & $4.43 \mathrm{E}-04$ & $1.01 \mathrm{E}-04$ & $7.56 \mathrm{E}-06$ & $2.79 \mathrm{E}-04$ & $53.3 \%$ & $1.21 \mathrm{E} \cdot 01$ & $0.9 \%$ & $33.6 \%$ & $8.31 \mathrm{E}-04$ & 34 \\
\hline $24 !-\mathrm{C}-106$ & $4.25 E-04$ & 7.29E-06 & $7.48 \mathrm{E}-09$ & $5.51 \mathrm{E}-04$ & $43.2 \%$ & $7.41 \mathrm{E}-03$ & $0.0 \%$ & $56.1 \%$ & $9.83 E-04$ & 40 \\
\hline Supenatant & $1.67 \mathrm{E}-09$ & $5.95 \mathrm{E}-13$ & $4.67 \mathrm{E}-10$ & $7.29 \mathrm{E}-08$ & $2.2 \%$ & $7.94 \mathrm{E}-06$ & $0.6 \%$ & $97.1 \%$ & $7.50 \mathrm{E}-08$ & 0 \\
\hline Solid Layer & $4.25 \mathrm{E}-04$ & $7.29 \mathrm{E}-06$ & $7.02 \mathrm{E}-09$ & $5.51 \mathrm{E}-04$ & $43.2 \%$ & $7.41 \mathrm{E} .03$ & $0.0 \%$ & $56.1 \%$ & $9.83 \mathrm{E}-04$ & 40 \\
\hline $241-\mathrm{C}-107$ & $2.20 \mathrm{E}-03$ & $1.59 \mathrm{E}-04$ & $1.19 \mathrm{E}-04$ & $3.21 \mathrm{E}-04$ & $78.6 \%$ & $5.67 \mathrm{E}-02$ & $4.3 \%$ & $11.4 \%$ & $2.80 \mathrm{E}-03$ & 114 \\
\hline Supernatant & na & na & wa & na & na & ma & na & na & na & na \\
\hline Solid Layer & $2.20 \mathrm{E}-03$ & $1.59 \mathrm{E}-04$ & $1.19 \mathrm{E}-04$ & $3.21 E-04$ & $78.6 \%$ & $5.67 \mathrm{E}-02$ & $4.3 \%$ & $11.4 \%$ & $2.80 \mathrm{E}-03$ & 114 \\
\hline $241-\mathrm{C}-108$ & $7.34 \mathrm{E}-05$ & $2.91 \mathrm{E}-07$ & $5.68 \mathrm{E}-06$ & $2.44 \mathrm{E}-04$ & $22.7 \%$ & $9.02 \mathrm{E}-04$ & $1.8 \%$ & $75.4 \%$ & 3.23E-04 & 13 \\
\hline Supernatant & na & na & па & па & na & na & na & na & na & na \\
\hline Solid Layer & $7.34 \mathrm{E}-05$ & $2.91 \mathrm{E}-07$ & $5.68 \mathrm{E}-06$ & $2.44 \mathrm{E}-04$ & $22.7 \%$ & $9.02 \mathrm{E}-04$ & $1.8 \%$ & $75.4 \%$ & $3.23 \mathrm{E}-04$ & 13 \\
\hline $241-C-109$ & $5.15 \mathrm{E}-04$ & $3.03 \mathrm{E}-06$ & $6.09 \mathrm{E}-06$ & $2.42 \mathrm{E}-04$ & $67.3 \%$ & $3.95 \mathrm{E}-03$ & $0.8 \%$ & $31.6 \%$ & $7.66 \mathrm{E}-04$ & 31 \\
\hline Supernatant & na & na & na & na & na & na & na & na & na & na \\
\hline Solid Layer & $5.15 E-04$ & $3.03 \mathrm{E}-06$ & $6.09 \mathrm{E}-06$ & $2.42 \mathrm{E}-04$ & $67.3 \%$ & $3.95 \mathrm{E}-03$ & $0.8 \%$ & $31.6 \%$ & $7.66 \mathrm{E}-04$ & 31 \\
\hline $241-\mathrm{C}-110$ & $2.45 \mathrm{E}-05$ & $3.18 \mathrm{E}-06$ & $4.34 \mathrm{E}-07$ & $6.96 \mathrm{E}-04$ & $3.4 \%$ & $4.40 \mathrm{E}-03$ & $0.1 \%$ & $96.1 \%$ & $7.24 \mathrm{E}-04$ & 30 \\
\hline Supenatant & $3.62 \mathrm{E}-08$ & $3.59 \mathrm{E}-10$ & $3.92 \mathrm{E}-09$ & $8.98 \mathrm{E}-07$ & $3.9 \%$ & 3.82E-04 & $0.4 \%$ & $95.7 \%$ & $9.38 \mathrm{E}-07$ & 0 \\
\hline Solid Layer & $2.45 \mathrm{E}-05$ & $3.18 \mathrm{E}-06$ & $4.30 \mathrm{E}-07$ & $6.95 \mathrm{E}-04$ & $3.4 \%$ & $4.40 \mathrm{E}-03$ & $0.1 \%$ & $96.1 \%$ & $7.23 \mathrm{E}-04$ & 29 \\
\hline $241-C-111$ & $1.15 \mathrm{E}-03$ & $8.52 \mathrm{E}-06$ & $4.67 \mathrm{E}-06$ & $2.39 E-04$ & $82.0 \%$ & $6.09 \mathrm{E}-03$ & $0.3 \%$ & $17.1 \%$ & $1.40 \mathrm{E}-03$ & 57 \\
\hline Supernatant & na & na & na & na & na & na & na & na & na & na \\
\hline Solid Layer & $1.15 \mathrm{E}-03$ & $8.52 \mathrm{E}-06$ & $4.67 \mathrm{E}-06$ & $2.39 \mathrm{E}-04$ & $82.0 \%$ & $6.09 \mathrm{E}-03$ & $0.3 \%$ & $17.1 \%$ & $1.40 \mathrm{E}-03$ & 57 \\
\hline $241-C-112$ & $1.25 \mathrm{E}-03$ & $1.60 \mathrm{E}-05$ & $1.46 \mathrm{E}-05$ & $2.58 \mathrm{E}-04$ & $81.3 \%$ & $1.04 \mathrm{~F} .02$ & $0.9 \%$ & $16.8 \%$ & $1.54 \mathrm{E}-03$ & 63 \\
\hline Supernatant & na & na & na & na & na & na & na & na & na & na \\
\hline Solid Layer & $1,25 \mathrm{E}-03$ & $1.60 \mathrm{E}-05$ & $1.46 \mathrm{E}-05$ & $2.58 \mathrm{E}-04$ & $81.3 \%$ & $1.04 \mathrm{E}-02$ & $0.9 \%$ & $16.8 \%$ & $1.54 \mathrm{E}-0.3$ & 63 \\
\hline
\end{tabular}


RPP-5926 REV 6

Table B-4. Calculated Total Hydrogen Generation Rate In Dome Space for 177 Tanks. (20 sheets)

\begin{tabular}{|c|c|c|c|c|c|c|c|c|c|c|}
\hline Tank & $\begin{array}{l}\text { HGR from } \\
\text { radiolysis } \\
\text { HCR }_{\mathrm{rad} / \mathrm{r}} \\
\text { (efm) }^{\text {(efm }}\end{array}$ & $\begin{array}{l}\text { HGR from } \\
\text { radiolysis } \\
\text { HGR }_{\text {rad }}^{\text {alpha }} \\
\text { (cfm) }\end{array}$ & $\begin{array}{l}\text { HGR from } \\
\text { thermolysis } \\
\text { HGR }_{\text {therm }} \\
\text { (cfm) }\end{array}$ & $\begin{array}{c}\text { HGR from } \\
\text { corrosion } \\
\text { HGR }_{\text {torr }} \\
\text { (cfm) }\end{array}$ & $\begin{array}{l}\text { Percent of } \\
\text { HGR from } \\
\text { radiolysis } \\
\text { HGR }_{\text {rad }}\end{array}$ & $\begin{array}{l}\text { Percent of } \\
\text { HGR from } \\
\text { radiolysis } \\
\text { HGR }_{\text {rad }} \text { alpha }\end{array}$ & $\begin{array}{l}\text { Percent of } \\
\text { HGR from } \\
\text { thermolysis } \\
\text { HGR }_{\text {therm }}\end{array}$ & $\begin{array}{l}\text { Percent of } \\
\text { HGR from } \\
\text { corrosion } \\
\text { HGR }_{\text {corr }}\end{array}$ & $\begin{array}{c}\text { Total HGR } \\
\text { from model } \\
\text { HGR }_{\text {trood }} \\
\text { (cfm) }\end{array}$ & $\begin{array}{c}\text { Total HGR } \\
\text { from model } \\
\text { HGR }_{\text {mod }} \\
(\mathrm{L} / \text { day) }\end{array}$ \\
\hline $24 !-C-201$ & $1.00 \mathrm{E}-06$ & $1.23 \mathrm{E}-06$ & $0.00 \mathrm{E}+00$ & $3.94 \mathrm{E}-05$ & $2.4 \%$ & $2.95 \mathrm{E}-02$ & $0.0 \%$ & $94.6 \%$ & $4.17 \mathrm{E}-05$ & 2 \\
\hline Supematant & $0.00 \mathrm{E}+00$ & $0.00 \mathrm{E}+00$ & $0.00 \mathrm{E}+00$ & $5.84 \mathrm{E}-09$ & $0.0 \%$ & $0.00 E+00$ & $0.0 \%$ & $100.0 \%$ & $5.84 \mathrm{E}-09$ & 0 \\
\hline Solid Layer & $1.00 \mathrm{E}-06$ & $1.23 \mathrm{E}-06$ & $0.00 \mathrm{E}+00$ & $3.94 \mathrm{E}-05$ & $2.4 \%$ & $2.95 \mathrm{E}-02$ & $0.0 \%$ & $94.6 \%$ & $4.16 \mathrm{E}-05$ & 2 \\
\hline $24 i-C-202$ & $1.91 \mathrm{E}-06$ & $1.05 \mathrm{E}-06$ & $0.00 \mathrm{E}+00$ & $3.93 \mathrm{E}-05$ & $4.5 \%$ & $2.48 \mathrm{E}-02$ & $0.0 \%$ & $93.0 \%$ & $4.23 \mathrm{E}-05$ & 2 \\
\hline Supenatanı & $0.00 \mathrm{E}+00$ & $0.00 E+00$ & $0.00 \mathrm{E} \vdash 00$ & $7.48 \mathrm{E}-09$ & $0.0 \%$ & $0.00 \mathrm{E}+00$ & $0.0 \%$ & $100.0 \%$ & $7.48 \mathrm{E}-09$ & 0 \\
\hline Solid Layer & $1.91 \mathrm{E}-06$ & $1.05 E .06$ & $0.00 \mathrm{E}+00$ & $3.93 \mathrm{E}-05$ & $4.5 \%$ & $2.48 \mathrm{E}-02$ & $0.0 \%$ & $93.0 \%$ & 4.23E-05 & 2 \\
\hline $241-C-203$ & $6.54 \mathrm{E}-08$ & $5.10 \mathrm{E}-08$ & $0.00 \mathrm{E}+00$ & $3.93 \mathrm{~F}-05$ & $0.2 \%$ & $1.29 \mathrm{E}-03$ & $0.0 \%$ & $99.7 \%$ & 3.94E-05 & 2 \\
\hline Supernatant & $0.00 E+00$ & $0.00 E+00$ & $0.00 \mathrm{E}+00$ & $3.99 \mathrm{E}-08$ & $0.0 \%$ & $0.00 \mathrm{E}+00$ & $0.0 \%$ & $100.0 \%$ & $3.99 E-08$ & 0 \\
\hline Solid Layer & $6.54 \mathrm{E}-08$ & $5.10 \mathrm{E}-08$ & $0.00 \mathrm{E}+00$ & $3.93 \mathrm{E}-05$ & $0.2 \%$ & $1.29 \mathrm{E}-03$ & $0.0 \%$ & $99.7 \%$ & $3.94 \mathrm{E}-05$ & 2 \\
\hline $241-C-204$ & $1.54 \mathrm{E}-07$ & $1.58 \mathrm{E}-09$ & 4. $78 \mathrm{E}-08$ & $1.72 \mathrm{E}-05$ & $0.9 \%$ & $9.05 \mathrm{E}-05$ & $0.3 \%$ & $98.8 \%$ & $1.74 \mathrm{E}-05$ & 1 \\
\hline Supernacant & па & na & na & na & na & na & na & na & $\mathrm{na}$ & ra \\
\hline Solid Layer & $1.54 \mathrm{E}-07$ & $1.58 \mathrm{E}-09$ & 4.78E-08 & $1.72 E-05$ & $0.9 \%$ & $9.05 \mathrm{E}-05$ & $0.3 \%$ & $98.8 \%$ & $1.74 \mathrm{E}-05$ & 1 \\
\hline $241-S-101$ & $4.28 \mathrm{E}-04$ & $8.00 \mathrm{E}-06$ & 2.05E-04 & $3.47 \mathrm{E}-04$ & $43.3 \%$ & $8.10 \mathrm{E}-03$ & $20.7 \%$ & $35.1 \%$ & $9.88 \mathrm{E}-04$ & 40 \\
\hline Supematant & na & na & na & na & na & na & na & na & na & na \\
\hline Solid Layer & $4.28 \mathrm{E}-04$ & $8.00 \mathrm{E}-06$ & $2.05 \mathrm{E}-04$ & $3.47 \mathrm{E}-04$ & $43.3 \%$ & $8.10 \mathrm{~F}-03$ & $20.7 \%$ & $35.1 \%$ & $9.88 E-04$ & 40 \\
\hline $241-S-102$ & $9.80 \mathrm{E}-05$ & $2.93 \mathrm{E}-06$ & $1.73 \mathrm{E}-04$ & $2.97 \mathrm{E}-04$ & $17.2 \%$ & $5.13 E-03$ & $30.3 \%$ & $52.0 \%$ & $5.71 \mathrm{E}-04$ & 23 \\
\hline Supernatant & na & na & na & na & na & na & na & na & na & na \\
\hline Solid Layer & $9.80 \mathrm{E}-05$ & $2.93 \mathrm{E}-06$ & $1.73 E-04$ & $2.97 \mathrm{E}-04$ & $17.2 \%$ & $5.13 \mathrm{E}-03$ & $30.3 \%$ & $52,0 \%$ & $5.71 \mathrm{E}-04$ & 23 \\
\hline $241-S-103$ & $1.18 \mathrm{E}-04$ & $2.89 \mathrm{E}-06$ & $7.79 \mathrm{E}-05$ & $3.01 \mathrm{E}-04$ & $23.6 \%$ & $5.78 \mathrm{E}-03$ & $15.6 \%$ & $60.2 \%$ & $4.99 \mathrm{E}-04$ & 20 \\
\hline Supernatant & $8.36 \mathrm{E}-07$ & $8.26 \mathrm{E}-1 \mathrm{~J}$ & $4.36 \mathrm{E}-07$ & $3.62 \mathrm{E}-07$ & $51.1 \%$ & $5.05 \mathrm{E}-05$ & $26.7 \%$ & $22.2 \%$ & $1.63 \mathrm{E}-06$ & 0 \\
\hline Solid Layer & $1.17 \mathrm{E}-04$ & $2.89 \mathrm{E}-06$ & $7.75 E-05$ & $3.00 \mathrm{E}-04$ & $23.5 \%$ & $5.80 \mathrm{E}-03$ & $15.6 \%$ & $60.4 \%$ & $4.98 \mathrm{E}-04$ & 20 \\
\hline $241-S-104$ & $2.51 \mathrm{E}-04$ & $6.71 \mathrm{E}-06$ & $2.21 \mathrm{E}-05$ & $3.26 \mathrm{E}-04$ & $41.4 \%$ & $1.11 E-02$ & $3.7 \%$ & $53.8 \%$ & $6.06 \mathrm{E}-04$ & 25 \\
\hline Supennatant & na & na & na & na & na & na & nа & na & na & na \\
\hline Solid Layer & $2.51 \mathrm{E}-04$ & $6.71 \mathrm{E}-06$ & 2.21E-05 & $3.26 \mathrm{E}-04$ & $41.4 \%$ & $1.11 \mathrm{E}-02$ & $3.7 \%$ & $53.8 \%$ & $6.06 \mathrm{E}-04$ & 25 \\
\hline $241-S \cdot 105$ & $2.69 \mathrm{E}-06$ & $1.68 \mathrm{E}-08$ & $7.12 \mathrm{E}-06$ & $3.57 \mathrm{E}-04$ & $0.7 \%$ & $4.60 \mathrm{E}-05$ & $1.9 \%$ & $97.3 \%$ & $3.66 \mathrm{E}-04$ & 15 \\
\hline Supernatant & na & na & na & na & na & na & na & na & na & 11a \\
\hline Solid Layer & $2.69 E-06$ & $1.68 \mathrm{E}-08$ & $7.12 E-06$ & $3.57 \mathrm{E}-04$ & $0.7 \%$ & 4. $60 \mathrm{E}-05$ & $1.9 \%$ & $97.3 \%$ & $3.66 \mathrm{E}-04$ & 15 \\
\hline
\end{tabular}




\section{RPP -5926 REV 6}

Table B-4. Calculated Total Hydrogen Generation Rate In Dome Space for 177 Tanks. (20 sheets)

\begin{tabular}{|c|c|c|c|c|c|c|c|c|c|c|}
\hline Tank & $\begin{array}{c}\text { HGR from } \\
\text { radiolysis } \\
\text { HGR }_{\text {rad }} \text { b/r } \\
\text { (cfm) }\end{array}$ & $\begin{array}{l}\text { HGR from } \\
\text { radiolysis } \\
\text { HGR }_{\text {rad }}^{\text {alpha }} \\
\text { (cfm) }\end{array}$ & $\begin{array}{c}\text { HGR from } \\
\text { thermolysis } \\
\text { HGR }_{\text {therm }} \\
\text { (cfm) }\end{array}$ & $\begin{array}{l}\text { HGR from } \\
\text { corrosion } \\
\text { HGR }_{\text {corr }} \\
\text { (cfm) }\end{array}$ & $\begin{array}{l}\text { Percent of } \\
\text { HGR from } \\
\text { radiolysis } \\
\text { HGR }_{\text {rad }} \text { b/r }\end{array}$ & $\begin{array}{l}\text { Percent of } \\
\text { HGR from } \\
\text { radiolysis } \\
\text { HGR }_{\text {rad }} \text { alph2 }\end{array}$ & $\begin{array}{l}\text { Percent of } \\
\text { HGR from } \\
\text { thermolysis } \\
\text { HGR }_{\text {therm }}\end{array}$ & $\begin{array}{c}\text { Percent of } \\
\text { HGR from } \\
\text { corrosion } \\
\text { HGR }_{\text {torr }}\end{array}$ & $\begin{array}{c}\text { Total HGR } \\
\text { from model } \\
\text { HGR }_{\text {mod }} \\
\text { (cfm) }\end{array}$ & $\begin{array}{c}\text { Total HGR } \\
\text { fram model } \\
\text { HGR }_{\text {mod }} \\
\text { (L/day) }\end{array}$ \\
\hline $241-S-106$ & $9.93 \mathrm{E}-05$ & $8.06 E-07$ & $4.35 \mathrm{E}-05$ & $3.74 \mathrm{E}-04$ & $19.2 \%$ & $1.56 \mathrm{E}-03$ & $8.4 \%$ & $72.3 \%$ & $5.17 \mathrm{E}-04$ & 21 \\
\hline Supernatant & na & na & na & nа & na & na & па & nа & na & na \\
\hline Solid Layer & $9.93 E-05$ & $8.06 \mathrm{E}-07$ & 4.35E-05 & $3.74 \mathrm{E}-04$ & $19.2 \%$ & $1.56 \mathrm{E}-03$ & $8.4 \%$ & $72.3 \%$ & $5.17 \mathrm{E}-04$ & 21 \\
\hline 24 I-S-107 & $2.78 \mathrm{E}-04$ & $3.31 \mathrm{E}-05$ & $3.91 \mathrm{E}-05$ & $3.43 \mathrm{E}-04$ & $40.1 \%$ & $4.78 \mathrm{E}-02$ & $5.6 \%$ & $49.5 \%$ & $6.93 \mathrm{E}-04$ & 28 \\
\hline Supernatant & na & na & na & na & na & nа & na & па & na & na \\
\hline Solid Layer & $2.78 \mathrm{E}-04$ & 3.31E-05 & $3.91 \mathrm{E}-05$ & $3.43 \mathrm{E}-04$ & $40.1 \%$ & $4.78 \mathrm{E}-02$ & $5.6 \%$ & $49.5 \%$ & $6.93 \mathrm{E}-04$ & 28 \\
\hline $241-S \cdot 108$ & $7.19 \mathrm{E}-06$ & $2.04 \mathrm{E}-07$ & $6.99 \mathrm{E}-06$ & $4.07 \mathrm{E}-04$ & $1.7 \%$ & $4.85 \mathrm{E}-04$ & $1.7 \%$ & $96.6 \%$ & $4.22 \mathrm{E} \cdot 04$ & 17 \\
\hline Supenaatant & na & na & иа & na & na & na & na & na & na & na \\
\hline Solid Layer & $7.19 \mathrm{E}-06$ & $2.04 \mathrm{E}-07$ & $6.99 \mathrm{E}-06$ & $4.07 \mathrm{E}-04$ & $1.7 \%$ & $4.85 \mathrm{E}-04$ & $1.7 \%$ & $96.6 \%$ & $4.22 \mathrm{E}-04$ & 17 \\
\hline $241-\$ .109$ & $1.16 \mathrm{E}-05$ & $4.74 \mathrm{E}-07$ & $1.22 \mathrm{E}-05$ & $4.00 \mathrm{E}-04$ & $2.7 \%$ & 1. $\{2 E-03$ & $2.9 \%$ & $94.3 \%$ & $4.24 \mathrm{E}-04$ & 17 \\
\hline Supernatant & na & na & na & na & па & па & na & na & na & na \\
\hline Solid Layer & $1.16 \mathrm{E}-05$ & $4.74 \mathrm{E}-07$ & 1.22E-05 & $4.00 \mathrm{E}-04$ & $2.7 \%$ & $1.12 \mathrm{E}-03$ & $2.9 \%$ & $94.3 \%$ & $4.24 \mathrm{E}-04$ & 17 \\
\hline $241-S-110$ & $7.80 \mathrm{E}-05$ & $2.81 \mathrm{E}-06$ & $9.90 \mathrm{~b}-05$ & $3.52 \mathrm{E}-04$ & $14.7 \%$ & $5.29 E-03$ & $18.6 \%$ & $66.2 \%$ & $5.32 \mathrm{E}-04$ & 22 \\
\hline Supernatant & na & na & na & na & na & na & na & па & na & na \\
\hline Solid L.ayer & $7.80 \mathrm{E}-05$ & $2.81 \mathrm{E}-06$ & $9.90 \mathrm{E}-05$ & $3.52 \mathrm{E}-04$ & $14.7 \%$ & $5.29 \mathrm{E}-03$ & $18.6 \%$ & $66.2 \%$ & $5.32 \mathrm{E}-04$ & 22 \\
\hline $241-S-111$ & $2.33 \mathrm{E}-04$ & $5.40 \mathrm{E}-07$ & $3.50 \mathrm{E}-05$ & 3.56E-04 & $37.3 \%$ & $8.65 \mathrm{E}-04$ & $5.6 \%$ & $57.0 \%$ & $6.24 \mathrm{E}-04$ & 25 \\
\hline Supernatant & na & na & па & na & na & na & na & na & na & na \\
\hline Solid L.ayer & $2.33 \mathrm{E}-04$ & $5.40 \mathrm{E}-07$ & $3.50 \mathrm{E}-05$ & $3.56 \mathrm{E}-04$ & $37.3 \%$ & $8.65 \mathrm{E}-04$ & $5.6 \%$ & $57.0 \%$ & $6.24 \mathrm{E}-04$ & 25 \\
\hline $241-5-112$ & $9.15 \mathrm{E}-06$ & $4.45 \mathrm{E}-07$ & $1.90 \mathrm{k}-07$ & 2.22E-04 & $3.9 \%$ & $1.92 \mathrm{E}-03$ & $0.1 \%$ & $95.8 \%$ & $2.32 \mathrm{E}-04$ & 9 \\
\hline Supernatant & па & na & na & na & na & na & na & na & па & $11 \mathrm{a}$ \\
\hline Solid Layer & $9.15 \mathrm{E}-06$ & $4.45 \mathrm{E}-07$ & $1.90 \mathrm{E}-07$ & $2.22 \mathrm{E}-04$ & $3.9 \%$ & 1.92E-03 & $0.1 \%$ & $95.8 \%$ & $2.32 \mathrm{E}-04$ & 9 \\
\hline $241-S X-101$ & $1.58 \mathrm{E}-04$ & $1.73 \mathrm{E}-05$ & $1.35 \mathrm{E}-04$ & $3.81 \mathrm{E}-04$ & $22.8 \%$ & $2.50 \mathrm{E}-02$ & $19.5 \%$ & $55.1 \%$ & $6.91 \mathrm{E}-04$ & 28 \\
\hline Supenatant & na & na & na & na & na & na & na & na & na & na \\
\hline Solid Layer & $1.58 \mathrm{E}-04$ & $1.73 \mathrm{E}-05$ & 1.35E-04 & $3.81 E-04$ & $22.8 \%$ & $2.50 \mathrm{E}-02$ & $19.5 \%$ & $55.1 \%$ & $6.91 \mathrm{E}-04$ & 28 \\
\hline $241-S X-102$ & $3.15 E-04$ & $7.16 \mathrm{E}-06$ & $8.66 E-04$ & $3.46 \mathrm{E}-04$ & $20.5 \%$ & $4.66 \mathrm{E}-03$ & $56.5 \%$ & $22.5 \%$ & $1.53 \mathrm{E}-03$ & 63 \\
\hline Supenatant & na & na & na & na & na & na & na & na & na & na \\
\hline Solid Layer & 3.15E-04 & $7.16 \mathrm{E}-06$ & $8.66 \mathrm{E}-04$ & $3.46 \mathrm{E}-04$ & $20.5 \%$ & $4.66 \mathrm{E}-03$ & $56.5 \%$ & $22.5 \%$ & $1.53 \mathrm{E}-03$ & 63 \\
\hline
\end{tabular}


RPP-5926 REV 6

Table B-4. Calculated Total Hydrogen Generation Rate In Dome Space for 177 Tanks. (20 sheets)

\begin{tabular}{|c|c|c|c|c|c|c|c|c|c|c|}
\hline Tank & $\begin{array}{l}\text { HGR from } \\
\text { radiolysis } \\
\text { HGR }_{\text {rad }}{ }^{\prime} / r \\
\text { (cfm) }\end{array}$ & $\begin{array}{l}\text { HGR from } \\
\text { radiolysis } \\
\text { HGR }_{\text {rad }}^{\text {atpha }} \\
\text { (cfm) }\end{array}$ & $\begin{array}{l}\text { HGR from } \\
\text { thermolysis } \\
\text { HGR }_{\text {therm }} \\
\text { (cfm) }\end{array}$ & $\begin{array}{l}\text { HGR from } \\
\text { corrosion } \\
\text { HGR }_{\text {carr }} \\
\text { (cfm) }\end{array}$ & $\begin{array}{l}\text { Percent of } \\
\text { HGR from } \\
\text { radiolysis } \\
\text { HGR }_{\text {rad }} \text { b/r }\end{array}$ & $\begin{array}{l}\text { Percent of } \\
\text { HGR from } \\
\text { radiolysis } \\
\text { HGR }_{\text {rad }} \text { apha }\end{array}$ & $\begin{array}{l}\text { Percent of } \\
\text { HGR from } \\
\text { thermolysis } \\
\text { HGR }_{\text {therm }}\end{array}$ & $\begin{array}{l}\text { Percent of } \\
\text { HGR from } \\
\text { corrosion } \\
\text { HGR }_{\text {carr }}\end{array}$ & $\begin{array}{c}\text { Total HGR } \\
\text { from model } \\
\text { HCR }_{\text {mod }} \\
\text { (cfm) }\end{array}$ & $\begin{array}{c}\text { Total HGR } \\
\text { from model } \\
\text { HGR }_{\text {mods }} \\
(\mathrm{L} / \text { day) }\end{array}$ \\
\hline $241-S X-103$ & $8.26 \mathrm{E}-04$ & $1.65 E .05$ & $5.63 E-03$ & $3.98 \mathrm{E}-04$ & $12.0 \%$ & $2.40 \mathrm{E}-03$ & $81.9 \%$ & $5.8 \%$ & $6.87 E-03$ & 280 \\
\hline Supenatant & па & na & na & na & па & na & na & na & nа & na \\
\hline Solid Layer & $8.26 \mathrm{E}-04$ & $1.65 \mathrm{E}-05$ & $5.63 \mathrm{E}-03$ & $3.98 \mathrm{E}-04$ & $12.0 \%$ & $2.40 \mathrm{E}-03$ & $81.9 \%$ & $5.8 \%$ & $6.87 \mathrm{E}-03$ & 280 \\
\hline 24$\}-S X-104$ & $3.06 \mathrm{E}-04$ & $7.45 \mathrm{E}-06$ & $1.12 \mathrm{E}-03$ & $3.86 \mathrm{E}-04$ & $16.8 \%$ & $4.09 \mathrm{E}-03$ & $61.6 \%$ & $21.2 \%$ & $1.82 \mathrm{E}-03$ & 74 \\
\hline Supernatant & na & na & na & na & na & na & na & na & na & na \\
\hline Solid Layer & $3.06 \mathrm{E}-04$ & $7.45 \mathrm{E}-06$ & $1.12 \mathrm{E}-03$ & $3.86 \mathrm{E}-04$ & $16.8 \%$ & $4.09 \mathrm{E}-03$ & $61.6 \%$ & $21.2 \%$ & $1.82 \mathrm{E}-03$ & 74 \\
\hline $241 \cdot S X-105$ & $8.20 \mathrm{E}-04$ & $2.43 \mathrm{E}-05$ & $3.95 \mathrm{E}-03$ & $3.60 \mathrm{E}-04$ & $15.9 \%$ & $4.72 \mathrm{E}-03$ & $76.6 \%$ & $7.0 \%$ & $5.15 \mathrm{E}-03$ & 210 \\
\hline Supernatant & па & na & na & na & па & na & na & na & na & na \\
\hline Solid Layer & $8.20 \mathrm{E}-04$ & $2.43 E-05$ & $3.95 \mathrm{E}-03$ & $3.60 \mathrm{E}-04$ & $15.9 \%$ & $4.72 \mathrm{E}-03$ & $76.6 \%$ & $7.0 \%$ & $5.15 \mathrm{E}-03$ & 210 \\
\hline $241-S X-106$ & $2.02 \mathrm{E}-04$ & $7.24 \mathrm{E}-06$ & $3.50 \mathrm{E}-04$ & $3.58 \mathrm{E}-04$ & $22.0 \%$ & $7.90 \mathrm{E}-03$ & $38.2 \%$ & $39.0 \%$ & $9.17 \mathrm{E}-04$ & 37 \\
\hline Supernatant & па & na & na & na & na & na & na & $\pi \mathrm{a}$ & na & na \\
\hline Solid Layer & $2.02 \mathrm{E}-04$ & $7.24 \mathrm{E}-06$ & $3.50 \mathrm{~F}-04$ & $3.58 \mathrm{E}-04$ & $22.0 \%$ & $7.90 \mathrm{E}-0.3$ & $38.2 \%$ & $39.0 \%$ & $9.17 \mathrm{E}-04$ & 37 \\
\hline $241-\$ X-107$ & $5.49 \mathrm{E}-04$ & $8.06 \mathrm{E}-06$ & $5.13 E-04$ & $2.77 \mathrm{E}-04$ & $40.7 \%$ & $5.99 \mathrm{E}-03$ & $38.1 \%$ & $20.5 \%$ & $1.35 \mathrm{E}-0.3$ & 55 \\
\hline Supernatant & na & na & na & na & na & na & na & na & nа & na \\
\hline Solid Layer & $5.49 \mathrm{E}-04$ & $8.06 \mathrm{E} .06$ & $5.13 \mathrm{E}-04$ & $2.77 \mathrm{E}-04$ & $40.7 \%$ & $5.99 \mathrm{E}-03$ & $38.1 \%$ & $20.5 \%$ & 1.35E-03 & 55 \\
\hline 241-SX-108 & $1.53 \mathrm{E}-04$ & $2.10 \mathrm{E}-06$ & $8.40 \mathrm{E}-05$ & $2.74 \mathrm{E}-04$ & $29.8 \%$ & $4.09 E-03$ & $16.4 \%$ & $53.4 \%$ & $5.13 \mathrm{E}-04$ & 21 \\
\hline Supernatant & nat & na & na & na & na & na & na & na & na & na \\
\hline Solid Layer & $1.53 \mathrm{E}-04$ & $2.10 \mathrm{E}-06$ & $8.40 \mathrm{E}-05$ & $2.74 \mathrm{E}-04$ & $29.8 \%$ & $4.09 \mathrm{E}-03$ & $16.4 \%$ & $53.4 \%$ & $5.13 E-04$ & 21 \\
\hline $241-S X-109$ & $6.16 \mathrm{E}-04$ & $5.38 \mathrm{E}-06$ & $1.27 \mathrm{E}-03$ & $3.27 \mathrm{E}-04$ & $27.7 \%$ & $2.42 \mathrm{E}-03$ & $57.3 \%$ & $14.7 \%$ & $2.22 \mathrm{E}-03$ & 91 \\
\hline Supernatant & na & na & na & na & na & na & na & na & na & па \\
\hline Solid Layer & $6.16 \mathrm{E}-04$ & $5.38 \mathrm{E}-06$ & $1.27 E-03$ & $3.27 \mathrm{E}-04$ & $27.7 \%$ & $2.42 \mathrm{E}-03$ & $57.3 \%$ & $14.7 \%$ & $2.22 \mathrm{E}-03$ & 91 \\
\hline $241-S X-110$ & $1.63 \mathrm{E}-03$ & $1.36 \mathrm{E}-05$ & $9.21 \mathrm{E}-05$ & $2.72 \mathrm{E}-04$ & $81.2 \%$ & $6.77 E-03$ & $4.6 \%$ & $13.6 \%$ & $2.00 \mathrm{~F}-03$ & 82 \\
\hline Supernatant & na & na & na & na & na & na & na & na & $\pi \mathrm{a}$ & na \\
\hline Solid Layer & $1.63 \mathrm{E}-03$ & $1.36 \mathrm{E}-05$ & $9.21 \mathrm{E}-05$ & $2.72 \mathrm{E}-04$ & $81.2 \%$ & $6.77 \mathrm{E}-03$ & $4.6 \%$ & $13.6 \%$ & $2.00 \mathrm{~F}-03$ & 82 \\
\hline 241-SX-111 & $8.30 \mathrm{E}-04$ & $1.13 \mathrm{E}-05$ & $3.91 \mathrm{E}-04$ & $2.92 \mathrm{E}-04$ & $54.5 \%$ & $7.44 \mathrm{E}-03$ & $25.6 \%$ & $19.2 \%$ & $1.52 \mathrm{E}-03$ & 62 \\
\hline Supematant & nа & na & na & na & na & na & па & $n a$ & na & na \\
\hline Solid Layer & $8.30 \mathrm{E}-04$ & $1.13 \mathrm{E}-05$ & $3.91 \mathrm{E}-04$ & $2.92 \mathrm{E}-04$ & $54.5 \%$ & 7.44E-03 & $25.6 \%$ & $19.2 \%$ & $1.52 \mathrm{E}-03$ & 62 \\
\hline
\end{tabular}


Table B-4. Calculated Total Hydrogen Generation Rate In Dome Space for 177 Tanks. (20 sheets)

\begin{tabular}{|c|c|c|c|c|c|c|c|c|c|c|}
\hline Tank & $\begin{array}{c}\text { HGR from } \\
\text { radiolysis } \\
\text { HGR }_{\mathrm{rad}} \mathrm{b} / \mathrm{r} \\
\text { (cfm) }\end{array}$ & $\begin{array}{l}\text { HGR from } \\
\text { radioly sis } \\
\text { HGR }_{\text {rad }}^{\text {alpha }} \\
\text { (cfm) }\end{array}$ & $\begin{array}{c}\text { HGR from } \\
\text { thermolysis } \\
\text { HGR theru } \\
\text { (cfm) }\end{array}$ & $\begin{array}{c}\text { HGR from } \\
\text { corrosion } \\
\text { HGR }_{\text {sorr }} \\
\text { (cfm) }\end{array}$ & $\begin{array}{l}\text { Percent of } \\
\text { HGR from } \\
\text { radiolysis } \\
\text { HGR }_{\text {rati }} \mathrm{b} / \mathrm{s}\end{array}$ & $\begin{array}{l}\text { Percent of } \\
\text { HGR from } \\
\text { radiolysis } \\
\text { HGR }_{\mathrm{rad}}^{2 \text { pha }}\end{array}$ & $\begin{array}{l}\text { Percent of } \\
\text { HGR from } \\
\text { thermolysis } \\
\text { HGR }_{\text {therm }}\end{array}$ & $\begin{array}{l}\text { Percent of } \\
\text { HGR from } \\
\text { corrosion } \\
\text { HGR }_{\text {corr }}\end{array}$ & $\begin{array}{c}\text { Total HGR } \\
\text { from model } \\
\text { HGR }_{\text {mod }} \\
\text { (cfm) }\end{array}$ & $\begin{array}{c}\text { Total HGR } \\
\text { from model } \\
\text { HGR }_{\text {max }} \\
\text { (L/day) }\end{array}$ \\
\hline $241-\mathrm{SX}-112$ & $5.20 \mathrm{E}-04$ & $7.68 \mathrm{E}-06$ & $1.63 \mathrm{E}-04$ & $2.68 \mathrm{E}-04$ & $54.3 \%$ & $8.02 \mathrm{E}-03$ & $17.0 \%$ & $27.9 \%$ & $9.58 \mathrm{E}-04$ & 39 \\
\hline Supernatant & na & na & na & na & na & na & иа & ra & ma & na \\
\hline Solid Layer & $5.20 \mathrm{E}-04$ & 7.68E-06 & $1.63 \mathrm{E}-04$ & $2.68 \mathrm{E}-04$ & $54.3 \%$ & $8.02 \mathrm{E}-03$ & $17.0 \%$ & $27.9 \%$ & $9.58 \mathrm{E}-04$ & 39 \\
\hline $241-5 X-113$ & $6.10 \mathrm{E}-06$ & $4.26 \mathrm{E}-07$ & $2.30 \mathrm{E}-06$ & $2.29 \mathrm{E}-04$ & $2.6 \%$ & $1.79 \mathrm{E}-03$ & $1.0 \%$ & $96.3 \%$ & $2.38 \mathrm{E}-04$ & 10 \\
\hline Supernatant & na & na & na & na & na & na & па & na & na & na \\
\hline Solid Layer & $6.10 \mathrm{E}-06$ & $4.26 \mathrm{E}-07$ & $2.30 \mathrm{E}-06$ & $2.29 \mathrm{E}-04$ & $2.6 \%$ & $1.79 \mathrm{E}-03$ & $1.0 \%$ & $96.3 \%$ & $2.38 \mathrm{E}-04$ & 10 \\
\hline $241-S X-114$ & $9.14 \mathrm{E}-04$ & $1.23 \mathrm{E}-05$ & $1.06 \mathrm{E}-03$ & $2.99 \mathrm{E}-04$ & $39.9 \%$ & $5.39 \mathrm{E}-03$ & $46.5 \%$ & $13.0 \%$ & 2.29E-03 & 93 \\
\hline Supernatant & na & na & na & na & na & na & nа & nа & $\mathrm{na}$ & na \\
\hline Solid Layer & $9.14 \mathrm{E}-04$ & $1.23 \mathrm{E}-05$ & $1.06 \mathrm{E}-03$ & $2.99 \mathrm{E}-04$ & $39.9 \%$ & $5.39 \mathrm{E}-03$ & $46.5 \%$ & $13.0 \%$ & $2.29 \mathrm{E}-03$ & 93 \\
\hline $241-\mathrm{SX}-115$ & $1.17 \mathrm{E}-04$ & $5.24 \mathrm{E}-06$ & $1.01 \mathrm{E}-07$ & $2.22 \mathrm{E}-04$ & $34.1 \%$ & $1.52 \mathrm{E}-02$ & $0.0 \%$ & $64.4 \%$ & 3.45E-04 & 14 \\
\hline Supenaatant & na & na & na & na & na & na & nа & na & na & na \\
\hline Solid Layer & $1.17 \mathrm{E}-04$ & $5.24 \mathrm{E}-06$ & $1.01 \mathrm{E}-07$ & $2.22 \mathrm{E}-04$ & $34.1 \%$ & $1.52 \mathrm{E}-02$ & $0.0 \%$ & $64,4 \%$ & $3.45 \mathrm{E}-04$ & 14 \\
\hline $241-\mathrm{T}-101$ & $1.74 \mathrm{E}-05$ & $4.57 \mathrm{E}-06$ & $1.67 \mathrm{E}-05$ & $2.51 \mathrm{E}-04$ & $6.0 \%$ & $1.58 \mathrm{E}-02$ & $5.8 \%$ & $86.7 \%$ & $2.89 \mathrm{E}-04$ & 12 \\
\hline Supernatant & па & na & na & na & na & na & na & na & na & na \\
\hline Solid Layer & $1.74 \mathrm{E}-05$ & $4.57 \mathrm{E}-06$ & $1.67 \mathrm{E}-05$ & $2.51 \mathrm{E}-04$ & $6.0 \%$ & $1.58 \mathrm{E}-02$ & $5.8 \%$ & $86.7 \%$ & $2.89 \mathrm{E}-04$ & 12 \\
\hline $241-T-102$ & $1.72 \mathrm{~b}-0 \mathrm{~S}$ & $3.28 \mathrm{E}-07$ & $2.01 \mathrm{E}-07$ & $5.63 \mathrm{E}-04$ & $3.0 \%$ & $5.64 E-04$ & $0.0 \%$ & $96.9 \%$ & $5.81 \mathrm{E}-04$ & 24 \\
\hline Supernatant & $3.63 \mathrm{E}-06$ & $1.62 \mathrm{E}-08$ & $1.07 \mathrm{E}-07$ & $1.06 \mathrm{E}-05$ & $25.2 \%$ & $1.13 \mathrm{E}-03$ & $0.7 \%$ & $73.9 \%$ & $1.44 \mathrm{E}-05$ & 1 \\
\hline Solid Layer & 1.36F-05 & $3.12 \mathrm{E}-07$ & $9.37 \mathrm{E}-08$ & $5.52 \mathrm{E}-04$ & $2.4 \%$ & $5.50 \mathrm{E}-04$ & $0.0 \%$ & $97.5 \%$ & $5.66 \mathrm{E}-04$ & 23 \\
\hline $241-\mathrm{T}-103$ & $1.04 \mathrm{E}-06$ & $8.91 \mathrm{E}-08$ & $2.31 \mathrm{E}-06$ & $2.25 \mathrm{E}-04$ & $0.5 \%$ & $3.90 \mathrm{E}-04$ & $1.0 \%$ & $98.5 \%$ & $2.28 \mathrm{E}-04$ & 9 \\
\hline Supernatant & $3.78 \mathrm{E}-07$ & $1.16 \mathrm{E}-08$ & $5.42 \mathrm{E}-07$ & $1.34 \mathrm{E}-06$ & $16.7 \%$ & $5.11 \mathrm{E}-03$ & $23.9 \%$ & $58.9 \%$ & $2.27 \mathrm{E}-06$ & 0 \\
\hline Solid Layer & $6.67 \mathrm{E}-07$ & 7.76E- 08 & $1.77 \mathrm{E}-06$ & $2.24 \mathrm{E}-04$ & $0.3 \%$ & $3.43 \mathrm{E}-04$ & $0.8 \%$ & $98.9 \%$ & $2.26 \mathrm{E}-04$ & 9 \\
\hline $241-T-104$ & 6.18E-06 & $7.82 \mathrm{E}-06$ & $1.63 \mathrm{E}-05$ & $3.25 \mathrm{E}-04$ & $1.7 \%$ & $2.20 \mathrm{E}-02$ & $4.6 \%$ & $91.5 \%$ & $3.56 \mathrm{E}-04$ & 15 \\
\hline Supernatant & na & tra & na & na & na & na & na & na & na & na \\
\hline Solid Layer & $6.18 \mathrm{~F}-06$ & 7.82E-06 & $1.63 E-05$ & $3.25 \mathrm{E}-04$ & $1.7 \%$ & $2.20 \mathrm{E}-02$ & $4.6 \%$ & $91.5 \%$ & $3.56 \mathrm{E}-04$ & 15 \\
\hline $241+T-105$ & $5.21 E-05$ & $6.79 \mathrm{E}-06$ & $1.04 \mathrm{E}-06$ & $2.46 \mathrm{E}-04$ & $17.1 \%$ & $2.22 \mathrm{E}-02$ & $0.3 \%$ & $80.4 \%$ & $3.05 \mathrm{E}-04$ & 12 \\
\hline Supernatant & na & na & na & na & na & na & na & na & na & na \\
\hline Solid Layer & $5.21 \mathrm{E}-05$ & $6.79 \mathrm{E}-06$ & $1.04 \mathrm{E}-06$ & $2.46 \mathrm{E}-04$ & $17.1 \%$ & $2.22 \mathrm{E}-02$ & $0.3 \%$ & $80.4 \%$ & $3.05 \mathrm{E}-04$ & 12 \\
\hline
\end{tabular}


RPP-5926 REV 6

Table B-4. Calculated Total Hydrogen Generation Rate In Dome Space for 177 Tanks. (20 sheets)

\begin{tabular}{|c|c|c|c|c|c|c|c|c|c|c|}
\hline Tank & $\begin{array}{c}\text { HGR from } \\
\text { radiolysis } \\
\text { HGR }_{\mathrm{ran}} \mathrm{b} \\
\text { (cfm) }\end{array}$ & $\begin{array}{l}\text { HGR from } \\
\text { radiolysis } \\
\text { HGR }_{\text {rad pha }} \\
\text { (cfm) }\end{array}$ & $\begin{array}{c}\text { HGR from } \\
\text { thermolysis } \\
\text { HGR }_{\text {therm }} \\
\text { (cfm) }\end{array}$ & $\begin{array}{c}\text { HGR from } \\
\text { corrosion } \\
\text { HGR }_{\text {corr }} \\
(\mathrm{cfm})\end{array}$ & $\begin{array}{l}\text { Percent of } \\
\text { HGR from } \\
\text { radiolysis } \\
\text { HGR }_{\mathrm{rad}} \mathrm{btr}\end{array}$ & $\begin{array}{l}\text { Percent of } \\
\text { HGR from } \\
\text { radiolysis } \\
\text { HGR }_{\text {rad }} \text { alpha }\end{array}$ & $\begin{array}{l}\text { Percent of } \\
\text { HGR from } \\
\text { thermolysis } \\
\text { HGR }_{\text {therm }}\end{array}$ & $\begin{array}{l}\text { Percent of } \\
\text { HGR from } \\
\text { corrosion } \\
\text { HGR }_{\text {corr }}\end{array}$ & $\begin{array}{c}\text { Total HGR } \\
\text { from model } \\
\text { HGR }_{\text {mod }} \\
(\mathrm{cfm})\end{array}$ & $\begin{array}{c}\text { Total HGR } \\
\text { from model } \\
\text { HGR }_{\text {mort }} \\
\text { (l./day) }\end{array}$ \\
\hline $241-\mathrm{T}-106$ & $1.02 \mathrm{E}-06$ & $2.17 \mathrm{E}-07$ & $3.69 \mathrm{E}-07$ & $2.24 \mathrm{E}-04$ & $0.5 \%$ & $9.64 \mathrm{E}-04$ & $0.2 \%$ & $99.3 \%$ & $2.25 \mathrm{E}-04$ & 9 \\
\hline Supernatant & na & na & na & na & na & na & na & na & na & na \\
\hline Solid Layer & $1.02 \mathrm{E}-06$ & $2.17 \mathrm{E}-07$ & $3.69 \mathrm{E}-07$ & $2.24 \mathrm{E}-04$ & $0.5 \%$ & $9.64 \mathrm{E}-04$ & $0.2 \%$ & $99.3 \%$ & $2.25 \mathrm{E}-04$ & 9 \\
\hline $241-T-107$ & $1.27 \mathrm{E}-04$ & 7.79E-06 & $6.98 E-06$ & $2.76 \mathrm{E}-04$ & $30.4 \%$ & $1.86 \mathrm{E}-02$ & $1.7 \%$ & $66.1 \%$ & $4.18 \mathrm{E}-04$ & 17 \\
\hline Supernatant & na & na & na & na & na & na & na & na & na & na \\
\hline Solid Layer & $1.27 \mathrm{E}-04$ & $7.79 \mathrm{E}-06$ & $6.98 \mathrm{E}-06$ & $2.76 \mathrm{E}-04$ & $30.4 \%$ & $1.86 \mathrm{E}-02$ & $1.7 \%$ & $66.1 \%$ & $4.18 \mathrm{E}-04$ & 17 \\
\hline $241-T-108$ & $3.48 \mathrm{E}-07$ & $6.01 \mathrm{E}-08$ & $1.37 E-10$ & $2.23 \mathrm{E}-04$ & $0.2 \%$ & $2.68 \mathrm{E}-04$ & $0.0 \%$ & $99.8 \%$ & $2.24 \mathrm{E}-04$ & 9 \\
\hline Supenatant & na & na & nа & na & na & na & na & na & na & na \\
\hline Solid Layer & $3.48 E-07$ & $6.01 \mathrm{E}-08$ & $1.37 \mathrm{E}-10$ & 2.23E-04 & $0.2 \%$ & $2.68 \mathrm{E}-04$ & $0.0 \%$ & $99.8 \%$ & $2.24 \mathrm{E}-04$ & 9 \\
\hline $241-T-109$ & $5.67 \mathrm{E}-07$ & $5.23 \mathrm{E}-08$ & $1.18 E-09$ & $2.37 \mathrm{E}-04$ & $0.2 \%$ & $2.20 \mathrm{E}-04$ & $0.0 \%$ & $99.7 \%$ & $2.38 \mathrm{E}-04$ & 10 \\
\hline Supenatant & na & na & na & na & na & na & na & na & na & na \\
\hline Solid Layer & $5.67 \mathrm{E}-07$ & $5.23 \mathrm{E}-08$ & $1.18 \mathrm{E}-09$ & $2.37 \mathrm{E}-04$ & $0.2 \%$ & $2.20 \mathrm{E}-04$ & $0.0 \%$ & $99.7 \%$ & $2.38 E-04$ & 10 \\
\hline $241-\mathrm{T}-110$ & $2.96 \mathrm{E}-07$ & $1.02 \mathrm{E}-05$ & $0.00 \mathrm{E}-00$ & $8.54 \mathrm{E}-04$ & $0.0 \%$ & $1.18 \mathrm{E}-02$ & $0.0 \%$ & $98.8 \%$ & $8.64 \mathrm{E}-04$ & 35 \\
\hline Supernatant & $3.90 \mathrm{E}-11$ & $5.09 \mathrm{E}-11$ & $0.00 \mathrm{E}+00$ & $6.69 \mathrm{E}-07$ & $0.0 \%$ & $7.61 \mathrm{E}-05$ & $0.0 \%$ & $100.0 \%$ & $6.69 \mathrm{E}-07$ & 0 \\
\hline Solid Layer & $2.96 \mathrm{E}-07$ & $1.02 \mathrm{E}-05$ & $0.00 \mathrm{E}+00$ & 8. $53 \mathrm{E}-04$ & $0.0 \%$ & $1.18 \mathrm{E}-02$ & $0.0 \%$ & $98.8 \%$ & $8.63 \mathrm{E}-04$ & 35 \\
\hline $241-\mathrm{T}-111$ & $1.65 \mathrm{E}-05$ & $1.28 \mathrm{E}-05$ & $2.00 \mathrm{E}-05$ & $3.69 \mathrm{E}-04$ & $4.0 \%$ & $3.05 \mathrm{E}-02$ & $4.8 \%$ & $88.2 \%$ & $4.18 \mathrm{E}-04$ & 17 \\
\hline Supernatant & na & na & $11 \mathrm{a}$ & na & na & na & na & na & na & na \\
\hline Solid Layer & $1.65 \mathrm{E}-05$ & $1.28 \mathrm{E}-05$ & $2.00 \mathrm{E}-05$ & $3.69 \mathrm{E}-04$ & $4.0 \%$ & $3.05 \mathrm{E}-02$ & $4.8 \%$ & $88.2 \%$ & 4.18E-04 & 17 \\
\hline $241-\mathrm{T}-112$ & $3.69 \mathrm{E}-07$ & $4.53 \mathrm{~L}-06$ & $1.24 E-07$ & $2.39 \mathrm{E}-04$ & $0.2 \%$ & $1.86 \mathrm{E}-02$ & $0.1 \%$ & $97.9 \%$ & $2.44 \mathrm{E}-04$ & 10 \\
\hline Supenratant & $3.65 \mathrm{E}-07$ & $4.16 \mathrm{E}-08$ & $1.25 \mathrm{E}-08$ & $2.32 \mathrm{E}-06$ & $13.3 \%$ & $1.52 \mathrm{E}-02$ & $0.5 \%$ & $84.7 \%$ & $2.74 \mathrm{E}-06$ & 0 \\
\hline Solid Layer & $4.69 \mathrm{E}-09$ & $4.49 \mathrm{E}-06$ & $1.11 \mathrm{E}-07$ & $2.36 \mathrm{E}-04$ & $0.0 \%$ & $1.86 \mathrm{E}-02$ & $0.0 \%$ & $98.1 \%$ & $2.41 \mathrm{E}-04$ & 10 \\
\hline $241-\mathrm{T}-201$ & $3.48 \mathrm{E}-08$ & $4.79 \mathrm{E}-06$ & $0.00 \mathrm{E}+00$ & $1.35 \mathrm{E}-04$ & $0.0 \%$ & $3.42 \mathrm{E}-02$ & $0.0 \%$ & $96.6 \%$ & $1.40 E-04$ & 6 \\
\hline Supernatant & $2.20 \mathrm{E}-12$ & $1.37 E-1]$ & $0.00 \mathrm{E}+00$ & $6.67 \mathrm{E}-06$ & $0.0 \%$ & $2.06 E-06$ & $0.0 \%$ & $100.0 \%$ & $6.67 \mathrm{E}-06$ & 0 \\
\hline Solid Layer & $3.48 \mathrm{E}-08$ & $4.79 \mathrm{E}-06$ & $0.00 \mathrm{E}+00$ & $1.28 \mathrm{E}-04$ & $0.0 \%$ & $3.60 \mathrm{E}-02$ & $0.0 \%$ & $96.4 \%$ & $1.33 \mathrm{E}-04$ & 5 \\
\hline $241-T-202$ & $1.41 \mathrm{E}-09$ & $7.64 \mathrm{E}-07$ & $8.27 \mathrm{E}-07$ & 4.12E-05 & $0.0 \%$ & $1.78 \mathrm{E}-02$ & $1.9 \%$ & $96.3 \%$ & $4.28 \mathrm{E}-05$ & 2 \\
\hline Supernatant & na & na & па & na & nа & na & na & na & na & na \\
\hline Solid Layer & $1.41 \mathrm{E}-09$ & $7.64 \mathrm{E}-07$ & $8.27 \mathrm{E}-07$ & 4.12E-05 & $0.0 \%$ & $1.78 \mathrm{E}-02$ & $1.9 \%$ & $96.3 \%$ & $4.28 \mathrm{E}-0.5$ & 2 \\
\hline
\end{tabular}




\section{RPP-5926 REV 6}

Table B-4. Calculated Total Hydrogen Generation Rate In Dome Space for 177 Tanks. (20 sheets)

\begin{tabular}{|c|c|c|c|c|c|c|c|c|c|c|}
\hline Tank & $\begin{array}{c}\text { HGR from } \\
\text { radiolysis } \\
\text { HGR }_{\text {rad }} \text { br } \\
\text { (cfm) }\end{array}$ & $\begin{array}{c}\text { HGR from } \\
\text { radiolysis } \\
\text { HGR }_{\text {rad }}^{\text {alpha }} \\
\text { (cfm) }^{\text {afm }}\end{array}$ & $\begin{array}{c}\text { HGR from } \\
\text { thermolysis } \\
\text { HGR }_{\text {therm }} \\
\text { (cfm) }\end{array}$ & $\begin{array}{l}\text { HGR from } \\
\text { corrosion } \\
\text { HGR }_{\text {corr }} \\
\text { (cfm) }\end{array}$ & $\begin{array}{l}\text { Percent of } \\
\text { HGR from } \\
\text { radiolysis } \\
\text { HGR }_{\text {rad }} \text { br }\end{array}$ & $\begin{array}{l}\text { Percent of } \\
\text { HGR from } \\
\text { radiolysis } \\
\text { HGR }_{\text {rad }} \text { alpha }\end{array}$ & $\begin{array}{l}\text { Percent of } \\
\text { HGR from } \\
\text { thermolysis } \\
\text { HGR }_{\text {therm }}\end{array}$ & $\begin{array}{l}\text { Percent of } \\
\text { HGR from } \\
\text { corrosion } \\
\text { HGR }_{\text {carr }}\end{array}$ & $\begin{array}{c}\text { Total HGR } \\
\text { from model } \\
\text { HGR }_{\text {rnod }} \\
\text { (cfm) }\end{array}$ & $\begin{array}{c}\text { Total HGR } \\
\text { from model } \\
\text { HGR }_{\text {mod }} \\
\text { (L/day) }\end{array}$ \\
\hline $241-T-203$ & $2.26 \mathrm{E}-09$ & $1.62 E-06$ & 1. $42 \mathrm{E}-06$ & $6.10 \mathrm{E}-05$ & $0.0 \%$ & $2.53 \mathrm{E}-02$ & $2.2 \%$ & $95.2 \%$ & $6.41 E-05$ & 3 \\
\hline Supernatant & na & na & na & na & na & na & na & na & na & nat \\
\hline Solid Layer & $2.26 \varepsilon-09$ & $1.62 \mathrm{E}-06$ & 1.42E-06 & 6.10E-05 & $0.0 \%$ & $2.53 \mathrm{E}-02$ & $2.2 \%$ & $95.2 \%$ & $6.41 E-05$ & 3 \\
\hline $241-T-204$ & $3.13 \mathrm{E}-09$ & $1.43 \mathrm{E}-06$ & $1.51 \mathrm{E}-06$ & $6.10 \mathrm{E}-05$ & $0.0 \%$ & $2.24 \mathrm{E}-02$ & $2.4 \%$ & $95.4 \%$ & $6.40 \mathrm{E}-05$ & 3 \\
\hline Supematant & na & nа & na & na & na & па & na & па & na & na \\
\hline Solid Layer & 3.13E-09 & $1.43 \mathrm{E}-06$ & $1.51 E-06$ & $6.10 \mathrm{E}-05$ & $0.0 \%$ & $2.24 \mathrm{E}-02$ & $2.4 \%$ & $95.4 \%$ & $6.40 \mathrm{E}-05$ & 3 \\
\hline $241-T X-101$ & $1.41 E-04$ & $9.80 \mathrm{E}-06$ & $2.78 \mathrm{E}-05$ & $2.50 \mathrm{E}-04$ & $33.0 \%$ & $2.29 \mathrm{E}-02$ & $6.5 \%$ & $58.2 \%$ & $4.29 \mathrm{E}-04$ & 17 \\
\hline Supenatant & naa & na & na & na & na & na & na & na & na & na \\
\hline Solid Layer & 1.41E-04 & $9.80 \mathrm{E}-06$ & $2.78 \mathrm{E}-05$ & $2.50 \mathrm{E}-04$ & $33.0 \%$ & $2.29 \mathrm{E}-02$ & $6.5 \%$ & $58.2 \%$ & $4.29 \mathrm{E}-04$ & 17 \\
\hline $241-\mathrm{TX}-102$ & $1.48 \mathrm{E}-05$ & $1.24 \mathrm{E}-06$ & $1.95 \mathrm{E}-05$ & $2.94 \mathrm{E}-04$ & $4.5 \%$ & $3.75 \mathrm{E}-03$ & $5.9 \%$ & $89.2 \%$ & $3.30 \mathrm{E}-04$ & 13 \\
\hline Supernatant & na & na & na & па & na & na & na & na & na & na \\
\hline Solid Layer & $1.48 \mathrm{E}-05$ & 1. $24 \mathrm{E}-06$ & 1.95E-05 & $2.94 \mathrm{E}-04$ & $4.5 \%$ & $3.75 \mathrm{E}-03$ & $5.9 \%$ & $89.2 \%$ & $3.30 \mathrm{E}-04$ & 13 \\
\hline $241-\mathrm{TX}-103$ & $1.07 \mathrm{E}-05$ & $9.12 E-07$ & $8.45 \mathrm{E}-06$ & $2.66 \mathrm{E}-04$ & $3.8 \%$ & $3.19 \mathrm{E}-03$ & $3.0 \%$ & $93.0 \%$ & $2.86 \mathrm{t}-04$ & 12 \\
\hline Supernatant & na & na & na & na & па & na & na & na & na & na \\
\hline Solid Layer & $1.07 \mathrm{E}-05$ & $9.12 \mathrm{E}-07$ & 8.45E-06 & $2.66 \mathrm{E}-04$ & $3.8 \%$ & $3.19 \mathrm{E}-03$ & $3.0 \%$ & $93.0 \%$ & $2.86 \mathrm{E}-04$ & 12 \\
\hline 24I-TX-104 & $6.00 \mathrm{E}-05$ & $1.25 \mathrm{E}-06$ & $1.65 \mathrm{E}-06$ & $2.40 \mathrm{E}-04$ & $19.8 \%$ & $4.13 \mathrm{E}-03$ & $0.5 \%$ & $79.3 \%$ & $3.03 \mathrm{E}-04$ & 12 \\
\hline Supernatant & $1.53 \mathrm{E}-06$ & $1.38 \mathrm{E}-09$ & $5.35 \mathrm{E}-08$ & $8.05 \mathrm{E}-07$ & $64.1 \%$ & $5.79 \mathrm{E}-04$ & $2.2 \%$ & $33.7 \%$ & $2.39 \mathrm{E}-06$ & 0 \\
\hline Solid Layer & $5.84 \mathrm{E}-05$ & $1.25 \mathrm{E}-06$ & $1.59 \mathrm{E}-06$ & $2.39 \mathrm{E}-04$ & $19.4 \%$ & 4.16E-03 & $0.5 \%$ & $79.6 \%$ & $3.01 \mathrm{E}-04$ & 12 \\
\hline 241-TX-105 & 1.10E-05 & $1.15 E-06$ & $3.88 E-05$ & $4.15 \mathrm{E}-04$ & $2.4 \%$ & $2.47 \mathrm{E}-03$ & $8.3 \%$ & $89.1 \%$ & $4.66 \mathrm{E}-04$ & 19 \\
\hline Supernatant & na & na & na & na & $\mathrm{na}$ & na & na & na & na & па \\
\hline Solid layer & 1.10E-05 & $1.15 \mathrm{E}-06$ & $3.88 \mathrm{E}-05$ & 4.15E-04 & $2.4 \%$ & $2.47 E-03$ & $8.3 \%$ & $89.1 \%$ & 4. $66 \mathrm{E}-04$ & 19 \\
\hline $241-\mathrm{TX}-106$ & 3.08E-05 & $2.72 \mathrm{E}-06$ & 3.93E-05 & $3.38 \mathrm{E}-04$ & $7.5 \%$ & $6.61 \mathrm{IE}-03$ & $9.5 \%$ & $82.3 \%$ & 4.11E-04 & 17 \\
\hline Supernatant & nat & na & na & na & na & naa & na & na & na & na \\
\hline Solid Layer & $3.08 \mathrm{E}-05$ & 2.72E-06 & 3.93E-05 & $3.38 \mathrm{E}-04$ & $7.5 \%$ & $6.61 \mathrm{E}-03$ & $9.5 \%$ & $82.3 \%$ & 4.11E-04 & 17 \\
\hline $241-T X-107$ & $6.25 E-06$ & $5.32 \mathrm{E}-06$ & $3.61 \mathrm{E}-06$ & $2.26 \mathrm{E}-04$ & $2.6 \%$ & $2.21 \mathrm{E}-02$ & $1.5 \%$ & $93.7 \%$ & $2.41 \mathrm{E}-04$ & 10 \\
\hline Supernatant & $\mathrm{na}$ & па & na & na & "ma & na & na & na & na & па \\
\hline Solid Layer & $6.25 \mathrm{E}-06$ & $5.32 \mathrm{E}-06$ & $3.61 E-06$ & $2.26 \mathrm{E}-04$ & $2.6 \%$ & $2.2 \mid \mathrm{E}-02$ & $1.5 \%$ & $93.7 \%$ & $2.4 ! E-04$ & 10 \\
\hline
\end{tabular}


RPP-5926 REV 6

Table B-4. Calculated Total Hydrogen Generation Rate In Dome Space for 177 Tanks. (20 sheets)

\begin{tabular}{|c|c|c|c|c|c|c|c|c|c|c|}
\hline Tank & $\begin{array}{l}\text { HGR from } \\
\text { radiolysis } \\
\text { HGR }_{\text {rad }}{ }^{\circ / r} \\
\text { (cfm) }\end{array}$ & $\begin{array}{l}\text { HGR from } \\
\text { radiolysis } \\
\text { HGR }_{\text {rad }} \text { dpha } \\
\text { (cfrn) }\end{array}$ & $\begin{array}{l}\text { HGR from } \\
\text { thermolysis } \\
\text { HGR }_{\text {therm }} \\
\text { (cfm) }\end{array}$ & $\begin{array}{c}\text { HGR from } \\
\text { corrosion } \\
\text { HGR }_{\text {corr }} \\
\text { (cfm) }\end{array}$ & $\begin{array}{l}\text { Percent of } \\
\text { HGR from } \\
\text { radiolysis } \\
\text { HGR }_{\text {rad }} \text { b/r }\end{array}$ & $\begin{array}{l}\text { Percent of } \\
\text { HGR from } \\
\text { radiolysis } \\
\text { HGR }_{\text {rad }}^{\text {alpha }}\end{array}$ & $\begin{array}{l}\text { Percent of } \\
\text { HGR from } \\
\text { thermolysis } \\
\text { HGR }_{\text {therm }}\end{array}$ & $\begin{array}{l}\text { Percent of } \\
\text { HGR from } \\
\text { corrosion } \\
\text { HGR }_{\text {corr }}\end{array}$ & $\begin{array}{c}\text { Total HGR } \\
\text { from model } \\
\text { HGR }_{\text {moll }} \\
\text { (cfm) }\end{array}$ & $\begin{array}{c}\text { Total HGR } \\
\text { from model } \\
\text { HGR }_{\text {mod }} \\
(\mathrm{L} / \mathrm{day})\end{array}$ \\
\hline $241-T X-108$ & $5.76 \mathrm{E}-06$ & $5.58 E-07$ & $4.27 \mathrm{E}-06$ & $2.59 \mathrm{E}-04$ & $2.1 \%$ & $2.07 \mathrm{E}-03$ & $1.6 \%$ & $96.1 \%$ & $2.70 \mathrm{E}-04$ & 11 \\
\hline Supematant & nat & na & na & па & na & na & na & na & na & na \\
\hline Solid Layer & $5.76 \mathrm{E}-06$ & $5.58 \mathrm{E}-07$ & $4.27 \mathrm{E}-06$ & $2.59 \mathrm{E}-04$ & $2.1 \%$ & $2.07 \mathrm{E}-03$ & $1.6 \%$ & $96.1 \%$ & $2.70 \mathrm{E}-04$ & 11 \\
\hline $241-\mathrm{TX}-109$ & $4.99 \mathrm{E}-05$ & $5.46 \mathrm{E}-06$ & 9.37E-05 & $3.60 \mathrm{E}-04$ & $9.8 \%$ & $1.07 \mathrm{E}-02$ & $18.4 \%$ & $70.7 \%$ & $5.09 \mathrm{E}-04$ & 21 \\
\hline Supematant & na & na & na & na & na & na & na & na & na & na \\
\hline Solid Laver & 4.99E-05 & $5.46 \mathrm{E}-06$ & $9.37 \mathrm{E}-05$ & $3.60 \mathrm{E}-04$ & $9.8 \%$ & $1.07 \mathrm{E}-02$ & $18.4 \%$ & $70.7 \%$ & $5.09 \mathrm{t}-04$ & 21 \\
\hline $241-\int X-110$ & $1.02 E-05$ & $1.24 \mathrm{E}-06$ & $2.04 \mathrm{E}-05$ & $3.76 \mathrm{E}-04$ & $2.5 \%$ & $3.04 \mathrm{E}-03$ & $5.0 \%$ & $92.2 \%$ & $4.08 \mathrm{E}-04$ & 17 \\
\hline Supernatant & na & ná & na & na & na & na & na & $\mathrm{na}$ & na & na \\
\hline Solid Layer & $1.02 \mathrm{E}-05$ & $1.24 \mathrm{E}-06$ & $2.04 \mathrm{E}-05$ & $3.76 \mathrm{E}-04$ & $2.5 \%$ & $3.04 \mathrm{E}-03$ & $5.0 \%$ & $92.2 \%$ & $4.08 \mathrm{E}-04$ & 17 \\
\hline $241-\mathrm{TX}-111$ & $8.74 \mathrm{E}-06$ & $1.10 \mathrm{E}-06$ & $1.55 \mathrm{E}-05$ & $3.42 \mathrm{E}-04$ & $2.4 \%$ & $3.00 \mathrm{E}-03$ & $4.2 \%$ & $93.1 \%$ & $3.67 \mathrm{E}-04$ & 15 \\
\hline Supernatant & na & na & na & na & na & na & na & na & na & na \\
\hline Solid Layer & $8.74 E-06$ & 1.10E-06 & $1.55 \mathrm{E}-05$ & 3.42E-04 & $2.4 \%$ & $3.00 \mathrm{E}-03$ & $4.2 \%$ & $93.1 \%$ & $3.67 \mathrm{E}-04$ & 15 \\
\hline $241-T X-112$ & $1.81 \mathrm{E}-05$ & $2.06 \mathrm{E}-06$ & $1.52 E-05$ & $4.29 \mathrm{E}-04$ & $3.9 \%$ & $4.45 \mathrm{E}-03$ & $3.3 \%$ & $92.4 \%$ & $4.64 \mathrm{E}-04$ & 19 \\
\hline Supernatant & na & na & na & na & na & na & na & na & na & na \\
\hline Solid Layer & 1.81E-05 & $2.06 \mathrm{E}-06$ & $1.52 \mathrm{E}-05$ & $4.29 \mathrm{E}-04$ & $3.9 \%$ & $4.45 \mathrm{E}-03$ & $3.3 \%$ & $92.4 \%$ & 4. $64 \mathrm{E}-04$ & 19 \\
\hline $241-[X-1] 3$ & $1.16 \mathrm{E}-06$ & $7.94 \mathrm{E}-08$ & $8.00 \mathrm{E}-06$ & $4.32 \mathrm{E}-04$ & $0.3 \%$ & $1.80 \mathrm{E}-04$ & $1.8 \%$ & $97.9 \%$ & $4.41 E-04$ & 18 \\
\hline Supematant & na & na & ma & na & na & na & na & na & na & na \\
\hline Solid Layer & $1.16 \mathrm{E}-06$ & 7.94E-08 & $8.00 \mathrm{E}-06$ & $4.32 E-04$ & $0.3 \%$ & $1.80 \mathrm{E}-04$ & $1.8 \%$ & $97.9 \%$ & 4. 4 !E- 04 & 18 \\
\hline $241-T X-114$ & $8.83 \mathrm{E}-06$ & $9.13 E-07$ & $2.01 \mathrm{E}-06$ & $3.93 \mathrm{E}-04$ & $2.2 \%$ & $2.26 \mathrm{E}-03$ & $0.5 \%$ & $97.1 \%$ & $4.04 \mathrm{E}-04$ & 16 \\
\hline Supernatant & na & na & na & na & na & na & na & na & na & na \\
\hline Solid Layer & $8.83 \mathrm{E}-06$ & $9.13 \mathrm{E}-07$ & $2.01 \mathrm{E}-06$ & $3.93 \mathrm{E}-04$ & $2.2 \%$ & $2.26 \mathrm{E}-03$ & $0.5 \%$ & $97.1 \%$ & 4.04E-04 & 16 \\
\hline 241-TX-115 & 1.16E-05 & $1.32 E-06$ & $1.07 E-05$ & $4.05 \mathrm{E}-04$ & $2.7 \%$ & $3.09 \mathrm{E}-03$ & $2.5 \%$ & $94.5 \%$ & 4. $28 \mathrm{E}-04$ & 17 \\
\hline Supernatant & na & na & na & na & na & na & na & na & na & na \\
\hline Solid Layer & $1.16 \mathrm{E}-05$ & 1.32E-06 & $1.07 E-05$ & $4.05 \mathrm{E}-04$ & $2.7 \%$ & $3.09 \mathrm{E}-03$ & $2.5 \%$ & $94.5 \%$ & $4.28 \mathrm{E}-04$ & 17 \\
\hline $241-T X-116$ & $5.86 \mathrm{E}-06$ & $4.75 \mathrm{E}-07$ & $3.27 \mathrm{E}-08$ & $4.17 \mathrm{E}-04$ & $1.4 \%$ & $1.12 \mathrm{E}-03$ & $0.0 \%$ & $98.5 \%$ & $4.24 \mathrm{E}-04$ & 17 \\
\hline Supernatant & na & ma & na & na & na & na & na & $\mathrm{na}$ & na & na \\
\hline Solid Layer & $5.86 \mathrm{E}-06$ & $4.75 \mathrm{E}-07$ & $3.27 \mathrm{E}-08$ & $4.17 \mathrm{E}-04$ & $1.4 \%$ & $1.12 \mathrm{E}-03$ & $0.0 \%$ & $98.5 \%$ & $4.24 \mathrm{E}-04$ & 17 \\
\hline
\end{tabular}


RPP-5926 REV 6

Table B-4. Calculated Total Hydrogen Generation Rate In Dome Space for 177 Tanks. (20 sheets)

\begin{tabular}{|c|c|c|c|c|c|c|c|c|c|c|}
\hline Tank & $\begin{array}{c}\text { HGR from } \\
\text { radiolysis } \\
\mathrm{HGR}_{\mathrm{rat}} \mathrm{b} / \mathrm{r} \\
\text { (cfm) }\end{array}$ & $\begin{array}{c}\text { HGR from } \\
\text { radiolysis } \\
\text { HGR }_{\text {ras }} \text { agha } \\
\text { (cfm) }\end{array}$ & $\begin{array}{l}\text { HGR from } \\
\text { thermolysis } \\
\text { HGR }_{\text {therm }} \\
\text { (cfm) }\end{array}$ & $\begin{array}{c}\text { HGR from } \\
\text { corrosion } \\
\text { HGR }_{\text {corr }} \\
\text { (cfm) }\end{array}$ & $\begin{array}{l}\text { Percent of } \\
\text { HGR from } \\
\text { radiolysis } \\
\text { HGR }_{\text {rad }} \mathrm{b} r\end{array}$ & $\begin{array}{l}\text { Percent of } \\
\text { HGR from } \\
\text { radiolysis } \\
\text {HGR }_{\text {rad }} \text { alpha }\end{array}$ & $\begin{array}{c}\text { Percent of } \\
\text { HGR from } \\
\text { thermolysis } \\
\text { HGR }_{\text {therm }}\end{array}$ & $\begin{array}{c}\text { Percent of } \\
\text { HGR from } \\
\text { corrosion } \\
\text { HGR }_{\text {corr }}\end{array}$ & $\begin{array}{c}\text { Total HGR } \\
\text { from model } \\
\text { HGR }_{\text {mod }} \\
\text { (cfm) }\end{array}$ & $\begin{array}{c}\text { Total HGR } \\
\text { from model } \\
\text { HGR }_{\text {mand }} \\
\text { (L/day) }\end{array}$ \\
\hline $241-T X-117$ & $4.60 \mathrm{E}-06$ & $4.73 E-07$ & $7.86 \mathrm{E}-10$ & $3.77 \mathrm{E}-04$ & $1.2 \%$ & $1.24 \mathrm{E}-03$ & $0.0 \%$ & $98.7 \%$ & $3.82 \mathrm{E}-04$ & 16 \\
\hline Supernatant & na & na & na & na & na & na & na & na & na & na \\
\hline Solid Layer & $4.60 \mathrm{E}-06$ & 4. $73 \mathrm{E}-07$ & $7.86 \mathrm{E}-10$ & $3.77 \mathrm{E}-04$ & $1.2 \%$ & $1.24 \mathrm{E}-03$ & $0.0 \%$ & $98.7 \%$ & 3. $82 \mathrm{E}-04$ & 16 \\
\hline $241-7 X-118$ & $6.58 \mathrm{E}-05$ & 6.11F-05 & $2.79 \mathrm{E}-05$ & $3.04 \mathrm{E}-04$ & $14.3 \%$ & $1.33 \mathrm{E}-01$ & $6.1 \%$ & $66.2 \%$ & $4.59 \mathrm{E}-04$ & 19 \\
\hline Supernatant & na & na & na & na & na & na & na & na & na & na \\
\hline Solid Layer & $6.58 \mathrm{E}-05$ & $6.11 \mathrm{E}-05$ & $2.79 \mathrm{E}-0.5$ & $3.04 \mathrm{E}-04$ & $14.3 \%$ & $1.33 \mathrm{E}-01$ & $6.1 \%$ & $66.2 \%$ & $4.59 \mathrm{E}-04$ & 19 \\
\hline $241-T Y-101$ & $1.14 \mathrm{E}-06$ & 3.63E-07 & $3.83 \mathrm{E}-10$ & $2.53 \mathrm{E}-04$ & $0.4 \%$ & 1. $43 \mathrm{E}-03$ & $0.0 \%$ & $99.4 \%$ & $2.55 \mathrm{E}-04$ & 10 \\
\hline Supernatant & na & na & na & na & na & wa & $\mathrm{na}$ & na & na & na \\
\hline Solid Layer & $1.14 \mathrm{E}-06$ & $3.63 \mathrm{E}-07$ & 3. $83 \mathrm{E}-10$ & $2.53 E-04$ & $0.4 \%$ & $1.43 \mathrm{E}-03$ & $0.0 \%$ & $99.4 \%$ & $2.55 \mathrm{E}-04$ & 10 \\
\hline $241-T Y-102$ & $5.38 \mathrm{E}-06$ & $1.85 \mathrm{E}-08$ & $2.10 \mathrm{E}-06$ & $2.39 \mathrm{E}-04$ & $2.2 \%$ & $7.52 \mathrm{E}-05$ & $0.9 \%$ & $97.0 \%$ & $2.46 \mathrm{E}-04$ & 10 \\
\hline Supernatant & na & na & na & na & na & na & na & na & na & $\mathrm{na}$ \\
\hline Solid Layer & $5.38 \mathrm{E}-06$ & $1.85 \mathrm{E}-08$ & $2.10 E-06$ & $2.39 \mathrm{E}-04$ & $2.2 \%$ & $7.52 \mathrm{E}-0.5$ & $0.9 \%$ & $97.0 \%$ & $2.46 \mathrm{E}-04$ & 10 \\
\hline 241-TY-103 & $6.26 \mathrm{E}-05$ & $3.20 \mathrm{E}-06$ & $3.56 \mathrm{E}-05$ & $2.70 \mathrm{E}-04$ & $16.9 \%$ & $8.61 \mathrm{E}-03$ & $9.6 \%$ & $72.7 \%$ & $3.71 \mathrm{E}-04$ & 15 \\
\hline Supernatant & na & na & na & na & na & na & na & na & na & na \\
\hline Solid Layer & $6.26 \mathrm{E}-05$ & $3.20 \mathrm{~F}-\mathrm{OOG}$ & $3.56 \mathrm{E}-05$ & $2.70 E-04$ & $16.9 \%$ & $8.61 E-03$ & $9.6 \%$ & $72.7 \%$ & $3.71 \mathrm{E}-04$ & 15 \\
\hline $241-T Y-104$ & $3.95 \mathrm{E}-05$ & $1.68 \mathrm{E}-06$ & 7. $12 \mathrm{E}-07$ & $2.32 \mathrm{E}-04$ & $14.4 \%$ & $6.12 \mathrm{E}-03$ & $0.3 \%$ & $84.7 \%$ & $2.74 \mathrm{E}-04$ & 11 \\
\hline Supernatant & $8.02 \mathrm{E}-08$ & $3.70 \mathrm{E}-10$ & $2.35 \mathrm{E}-08$ & $4.48 \mathrm{E}-07$ & $14.5 \%$ & $6.70 \mathrm{E}-04$ & $4.3 \%$ & $81.1 \%$ & $5.52 \mathrm{E}-07$ & 0 \\
\hline Solid Layer & $3.94 \mathrm{E}-05$ & $1.68 \mathrm{E}-06$ & $6.88 \mathrm{~F}-07$ & $2.32 \mathrm{E}-04$ & $14.4 \%$ & $6.14 \mathrm{E}-03$ & $0.3 \%$ & $84.7 \%$ & $2.73 E-04$ & 11 \\
\hline 241-TY-105 & $1.80 \mathrm{E}-04$ & $6.27 \mathrm{E}-07$ & $1.33 \mathrm{E}-05$ & $2.97 \mathrm{E}-04$ & $36.7 \%$ & $1.27 \mathrm{E}-03$ & $2.7 \%$ & $60.5 \%$ & 4. $92 \mathrm{E}-04$ & 20 \\
\hline Supernatant & na & na & na & na & na & na & na & na & na & na \\
\hline Solid Layer & 1.80E-04 & $6.27 \mathrm{E}-07$ & 1.33E-05 & $2.97 \mathrm{E}-04$ & $36.7 \%$ & $1.27 E-03$ & $2.7 \%$ & $60.5 \%$ & 4.92E-04 & 20 \\
\hline $241-T Y-106$ & $1.56 \mathrm{E}-05$ & $6.37 \mathrm{E}-08$ & $3.55 \mathrm{E}-07$ & $2.21 \mathrm{E}-04$ & $6.6 \%$ & $2.69 \mathrm{E}-04$ & $0.1 \%$ & $93.3 \%$ & 2.37E-04 & 10 \\
\hline Supernatant & na & na & na & na & na & na & na & na & na & na \\
\hline Solid Layer & t. $56 \mathrm{E}-05$ & $6.37 E-08$ & $3.55 \mathrm{E}-07$ & $2.21 E-04$ & $6.6 \%$ & $2.69 \mathrm{E}-04$ & $0.1 \%$ & $93.3 \%$ & $2.37 \mathrm{E}-04$ & 10 \\
\hline 241-U-101 & $5.53 \mathrm{E}-05$ & $1.60 \mathrm{E}-07$ & $9.89 \mathrm{E}-07$ & $2.24 \mathrm{E}-04$ & $19.7 \%$ & $5.71 \mathrm{E}-04$ & $0.4 \%$ & $79.9 \%$ & $2.81 \mathrm{E}-04$ & 11 \\
\hline Supernatant & na & na & na & na & na & 11a & na & na & na & na \\
\hline Solid Layer & $5.53 \mathrm{E}-05$ & $1.60 \mathrm{E}-07$ & $9.89 E-07$ & $2.24 \mathrm{E}-04$ & $19.7 \%$ & $5.71 \mathrm{E}-04$ & $0.4 \%$ & $79.9 \%$ & $2.81 \mathrm{E}-04$ & 11 \\
\hline
\end{tabular}




\section{RPP-5926 REV 6}

Table B-4. Calculated Total Hydrogen Generation Rate In Dome Space for 177 Tanks. (20 sheets)

\begin{tabular}{|c|c|c|c|c|c|c|c|c|c|c|}
\hline Tank & $\begin{array}{c}\text { HGR from } \\
\text { radiolysis } \\
\text { HGR }_{\text {rad }} b / r \\
\text { (cfm) }\end{array}$ & $\begin{array}{l}\text { HGR from } \\
\text { radiolysis } \\
\text { HGR }_{\text {rad }} \text { ilphr } \\
\text { (cfm) }\end{array}$ & $\begin{array}{l}\text { HGR from } \\
\text { thermolysis } \\
\text { HGR }_{\text {thern }} \\
\text { (cfm) }\end{array}$ & $\begin{array}{l}\text { HGR from } \\
\text { corrosion } \\
\text { HGR }_{\text {cosr }} \\
\text { (cfm) }\end{array}$ & $\begin{array}{l}\text { Percent of } \\
\text { HGR from } \\
\text { radiolysis } \\
\text { HGR }_{\text {rad }} b / r\end{array}$ & $\begin{array}{l}\text { Percent of } \\
\text { HGR from } \\
\text { radiolysis } \\
\text { HGR }_{\text {rad }} \text { algnz }\end{array}$ & $\begin{array}{l}\text { Percent of } \\
\text { HGR from } \\
\text { thermolysis } \\
\text { HGR }_{\text {therm }}\end{array}$ & $\begin{array}{l}\text { Percent of } \\
\text { HGR from } \\
\text { corrosion } \\
\text { HGR }_{\text {corr }}\end{array}$ & $\begin{array}{c}\text { Total HGR } \\
\text { from model } \\
\text { HGR } \\
\text { (cfmod }\end{array}$ & $\begin{array}{c}\text { Total HGR } \\
\text { from mode } \\
\text { HGR }_{\text {rnod }} \\
\text { (L/day) }\end{array}$ \\
\hline $241-U-102$ & $3.03 \mathrm{E}-04$ & $6.70 \mathrm{E}-06$ & $2.57 \mathrm{E}-04$ & 3.35F-.04 & $33.6 \%$ & 7.43E-03 & $28.5 \%$ & $37.2 \%$ & $9.01 \mathrm{E}-04$ & 37 \\
\hline Supernatant & $1.34 \mathrm{E}-06$ & $8.75 \mathrm{E}-10$ & $5.60 \mathrm{E}-07$ & $3.66 \mathrm{E}-07$ & $59.1 \%$ & $3.865-0.04$ & $24.7 \%$ & $16.1 \%$ & $2.27 \mathrm{E}-06$ & 0 \\
\hline Solid Layer & $3.02 \mathrm{E}-04$ & $6.70 \mathrm{E}-06$ & $2.56 \mathrm{E}-04$ & $3.34 \mathrm{E}-04$ & $33.6 \%$ & 7.45E-03 & $28.5 \%$ & $37.2 \%$ & $8.99 \mathrm{E}-04$ & 37 \\
\hline $241-\mathrm{L}-103$ & $2.10 \mathrm{E}-04$ & $5.62 \mathrm{E}-06$ & $4.54 \mathrm{E}-04$ & $3.62 \mathrm{E}-04$ & $20.3 \%$ & $5.45 \mathrm{E}-03$ & $44.0 \%$ & $35.1 \%$ & $1.03 E-03$ & 42 \\
\hline Supernatan & $7.095-07$ & $8.31 \mathrm{E}-10$ & $6.86 E-07$ & $1.81 \mathrm{E}-07$ & $45.0 \%$ & $5.27 E-04$ & $43.5 \%$ & $11.5 \%$ & $1.58 \mathrm{E}-06$ & 0 \\
\hline Solid Layer & $2.09 \mathrm{E}-04$ & $5.62 \mathrm{E}-06$ & 4. $53 \mathrm{E}-04$ & $3.62 \mathrm{E}-04$ & $20.3 \%$ & $5.46 \mathrm{E}-03$ & $44.0 \%$ & $35.1 \%$ & $1.03 \mathrm{E}-03$ & 42 \\
\hline 241-U-104 & $5.37 \mathrm{E}-07$ & $2.05 E-08$ & $3.37 \mathrm{E}-05$ & $2.38 \mathrm{E}-04$ & $0.2 \%$ & $7.53 \mathrm{E}-05$ & $12.4 \%$ & $87.4 \%$ & $2.72 E-04$ & 11 \\
\hline Supenatant & na & na & па & na & na & na & na & na & na & ná \\
\hline Solid Layer & $5.37 \mathrm{E}-07$ & $2.05 \mathrm{E}-08$ & 3.37E-05 & $2.38 \mathrm{E}-04$ & $0.2 \%$ & $7.53 \mathrm{E}-05$ & $12.4 \%$ & $87.4 \%$ & $2.72 \mathrm{E}-04$ & 11 \\
\hline $241-U-105$ & $1.68 \mathrm{E}-04$ & $1.80 \varepsilon-05$ & $3.94 \mathrm{E}-04$ & $3.42 \mathrm{E}-04$ & $18.3 \%$ & $1.95 \mathrm{E}-02$ & $42.7 \%$ & $37.1 \%$ & $9.23 \mathrm{E}-04$ & 38 \\
\hline Supernatant & na & na & иа & na & na & na & na & na & na & na \\
\hline Solid l.ayer & $1.68 \mathrm{E}-04$ & $1.80 \mathrm{E}-05$ & 3.94E-04 & $3.42 \mathrm{E}-04$ & $18.3 \%$ & 1.95E-02 & $42.7 \%$ & $37.1 \%$ & $9.23 E-04$ & 38 \\
\hline $241-1-106$ & $2.24 \mathrm{E}-0.04$ & $1.37 \mathrm{E}-05$ & 3.13E-04 & $2.78 \mathrm{E}-04$ & $27.0 \%$ & $1.66 \mathrm{E}-02$ & $37.8 \%$ & $33.5 \%$ & $8.29 \mathrm{E}-04$ & 34 \\
\hline Supemalant & $2.32 E-06$ & $3.10 \mathrm{E}-08$ & $2.43 E-06$ & $5.43 \mathrm{E}-07$ & $43.6 \%$ & $5.80 \pi-03$ & $45.7 \%$ & $10.2 \%$ & $5.33 \mathrm{E}-06$ & 0 \\
\hline Solid Layer & $2.21 \mathrm{E}-04$ & $1.37 \mathrm{E}-05$ & $3.11 \mathrm{E}-04$ & $2.77 \mathrm{E}-04$ & $26.9 \%$ & 1.67E- 02 & $37.8 \%$ & $33.7 \%$ & $8.23 \mathrm{E}-04$ & 34 \\
\hline $24 !-U-107$ & $7.26 \mathrm{E}-05$ & 1.10E-05 & $9.06 \mathrm{E}-05$ & $3.19 \mathrm{E}-04$ & $14.7 \%$ & $2.22 \mathrm{E}-02$ & $18.4 \%$ & $64.7 \%$ & 4.93E-04 & 20 \\
\hline Superiatant & $\mathfrak{n a}$ & na & na & na & na & na & na & na & na & na \\
\hline Solid Layer & $7.26 \mathrm{E}-05$ & $1.10 \mathrm{E}-05$ & $9.06 \mathrm{E}-05$ & $3.19 \mathrm{E}-04$ & $14.7 \%$ & $2.22 \mathrm{E}-02$ & $18.4 \%$ & $64.7 \%$ & 4.93E-04 & 20 \\
\hline $24 !-U-108$ & $1.96 \mathrm{E}-04$ & 4.33E-06 & $3.61 \mathrm{E}-04$ & 3.7JE-04 & $21.0 \%$ & $4.64 \mathrm{~F}-03$ & $38.7 \%$ & $39.8 \%$ & $9.33 \mathrm{E}-04$ & 38 \\
\hline Supematan & ma & na & na & na & па & na & na & na & na & na \\
\hline Solid Layer & $1.96 \mathrm{E}-04$ & $4.33 \mathrm{E}-06$ & $3.61 \mathrm{E}-04$ & $3.71 \mathrm{E}-04$ & $21.0 \%$ & $4.64 \mathrm{E}-03$ & $38.7 \%$ & $39.8 \%$ & $9.33 \mathrm{E}-04$ & 38 \\
\hline $241-1\}-109$ & $1.06 \mathrm{~F}-04$ & $8.88 \mathrm{E}-07$ & $1.68 \mathrm{E}-04$ & $3.58 \mathrm{E}-04$ & $16.7 \%$ & $1.41 \mathrm{E}-03$ & $26.6 \%$ & $56.6 \%$ & $6.32 \mathrm{E}-04$ & 26 \\
\hline Supermatant & na & na & na & tha & na & na & na & na & na & na \\
\hline Solid Layer & $1.06 E-04$ & $8.88 \mathrm{E}-07$ & $1.68 \mathrm{E}-04$ & $3.58 \mathrm{E}-04$ & $16.7 \%$ & $1.41 \mathrm{E}-03$ & $26.6 \%$ & $56.6 \%$ & $6.32 \mathrm{E}-04$ & 26 \\
\hline $241-\mathrm{U}-110$ & $2.05 \mathrm{E}-04$ & $5.80 \mathrm{E}-06$ & $1.28 \mathrm{E}-05$ & $2.79 \mathrm{E}-04$ & $40.8 \%$ & $1.15 \mathrm{E}-02$ & $2.6 \%$ & $55.5 \%$ & $5.03 \mathrm{E}-04$ & 21 \\
\hline Supenatant & na & na & na & na & na & $n a$ & na & na & na & na \\
\hline Solid Layer & $2.05 \mathrm{E}-04$ & $5.80 \mathrm{E}-06$ & $1.28 \mathrm{E}-05$ & $2.79 \mathrm{E}-04$ & $40.8 \%$ & $1.15 \mathrm{E}-02$ & $2.6 \%$ & $55.5 \%$ & $5.03 E-04$ & 21 \\
\hline
\end{tabular}


RPP-5926 REV 6

Table B-4. Calculated Total Hydrogen Generation Rate In Dome Space for 177 Tanks. (20 sheets)

\begin{tabular}{|c|c|c|c|c|c|c|c|c|c|c|}
\hline Tank & $\begin{array}{l}\text { HGR from } \\
\text { radiolysis } \\
\text { HGR }_{\text {rad }} \text { b/r } \\
\text { (cfm) }\end{array}$ & $\begin{array}{l}\text { HGR from } \\
\text { radiolysis } \\
\text { HGR }_{\text {rad }} \text { alpha } \\
\text { (cfm) }\end{array}$ & $\begin{array}{c}\text { HGR from } \\
\text { thermolysis } \\
\text { HGR }_{\text {therm }} \\
\text { (cfm) }\end{array}$ & $\begin{array}{l}\text { HGR from } \\
\text { corrosion } \\
\text { HGR }_{\text {corr }} \\
\text { (cfm) }\end{array}$ & $\begin{array}{l}\text { Percent of } \\
\text { HGK from } \\
\text { radiolysis } \\
\text { HGR }_{\text {rad }}\end{array}$ & $\begin{array}{l}\text { Percent of } \\
\text { HGR from } \\
\text { radiolysis } \\
H_{G} R_{\text {rad }} \text { glpha }\end{array}$ & $\begin{array}{c}\text { Percent of } \\
\text { HGR from } \\
\text { thermolysis } \\
\text { HGR }_{\text {therm }}\end{array}$ & $\begin{array}{l}\text { Percent of } \\
\text { HGR from } \\
\text { corrosion } \\
\text { HGR }_{\text {sorr }}\end{array}$ & $\begin{array}{c}\text { Total HGR } \\
\text { from model } \\
\text { HGR }_{\text {mod }} \\
\text { (cfm) }\end{array}$ & $\begin{array}{c}\text { Total HGR } \\
\text { from model } \\
\text { HGR }_{\text {mod }} \\
\text { (L/day) }\end{array}$ \\
\hline $241-\mathrm{L}-111$ & $9.54 \mathrm{E}-05$ & $4.22 \mathrm{E}-06$ & $6.51 \mathrm{E}-05$ & $2.96 \mathrm{E}-04$ & $20.7 \%$ & $9.17 \mathrm{E}-03$ & $14.1 \%$ & $64.2 \%$ & 4. $6 ! \mathrm{E}-04$ & 19 \\
\hline Supernatant & na & $\mathrm{na}$ & na & na & $\mathrm{na}$ & na & na & na & па & na \\
\hline Solid Layer & $9.54 \mathrm{~F}-05$ & 4.22E-06 & $6.51 \mathrm{E}-05$ & $2.96 \mathrm{E}-04$ & $20.7 \%$ & $9.17 E-03$ & $14.1 \%$ & $64.2 \%$ & $4.61 \mathrm{E}-04$ & 19 \\
\hline 241-U-1 12 & $5.95 E-05$ & $1.34 \mathrm{E}-07$ & $2.14 \mathrm{E}-06$ & $2.32 \mathrm{E}-04$ & $20.3 \%$ & $4.57 \mathrm{E}-04$ & $0.7 \%$ & $79.0 \%$ & $2.94 \mathrm{E}-04$ & 12 \\
\hline Supernatant & na & na & na & na & na & па & na & na & na & na \\
\hline Solid Layer & $5.95 \mathrm{E}-05$ & $1.34 E-07$ & $2.14 \mathrm{E}-06$ & $2.32 \mathrm{E}-04$ & $20.3 \%$ & $4.57 E-04$ & $0.7 \%$ & $79.0 \%$ & $2.94 \mathrm{E}-04$ & 12 \\
\hline 24$\}-U-201$ & $1.38 \mathrm{E}-07$ & $7.66 \mathrm{E}-10$ & $0.00 \mathrm{E}+00$ & $2.07 \mathrm{E}-05$ & $0.7 \%$ & $3.68 \mathrm{E}-05$ & $0.0 \%$ & $99.3 \%$ & $2.08 \mathrm{E}-05$ & 1 \\
\hline Supenatant & $1.05 \mathrm{E}-07$ & $8.60 \mathrm{E}-11$ & $0.00 E+00$ & $1.34 \mathrm{E}-06$ & $7.3 \%$ & $5.95 \mathrm{E}-05$ & $0.0 \%$ & $92.7 \%$ & $1.44 \mathrm{E}-06$ & 0 \\
\hline Solid Layer & $3.32 \mathrm{E}-0.8$ & $6.80 \mathrm{E}-10$ & $0.00 \mathrm{E}+00$ & $1.93 \mathrm{E}-0.5$ & $0.2 \%$ & $3.51 \mathrm{E}-05$ & $0.0 \%$ & $99.8 \%$ & $1.94 E-05$ & 1 \\
\hline $241-U-202$ & $9.61 \mathrm{E}-08$ & $7.38 \mathrm{E}-10$ & $1.56 \mathrm{E}-09$ & $2.02 \mathrm{E}-05$ & $0.5 \%$ & $3.63 \mathrm{E}-05$ & $0.0 \%$ & $99.5 \%$ & 2.03E-0.5 & 1 \\
\hline Supernatant & $7.24 \mathrm{E}-08$ & $1.60 \mathrm{E}-12$ & $6.75 \mathrm{E}-10$ & $1.33 \mathrm{E}-06$ & $5.2 \%$ & $3.14 \mathrm{E}-06$ & $0.0 \%$ & $94.8 \%$ & $1.41 \mathrm{E}-06$ & 0 \\
\hline Solid Layer & $2.37 \mathrm{E}-08$ & $7.36 \mathrm{E}-10$ & $8.89 \mathrm{E}-10$ & $1.89 \mathrm{E}-05$ & $0.1 \%$ & $3.89 \mathrm{E}-05$ & $0.0 \%$ & $99.9 \%$ & 1.89E-05 & 1 \\
\hline $24 i-i-203$ & $1.13 \mathrm{E}-07$ & $7.25 \mathrm{E}-10$ & $0.00 \mathrm{E}+00$ & $1.99 \mathrm{E}-05$ & $0.6 \%$ & 3.63E- 05 & $0.0 \%$ & $99.4 \%$ & $2,00 \mathrm{E}-05$ & 1 \\
\hline Supernatant & 7.72E-08 & $3.91 \mathrm{E}-12$ & $0.00 \mathrm{t}: 00$ & $1.33 \mathrm{E}-06$ & $5.5 \%$ & $2.78 \mathrm{E}-06$ & $0.0 \%$ & $94.5 \%$ & $1.41 E-06$ & 0 \\
\hline Solid Layer & $3.58 \mathrm{E}-08$ & $7.21 \mathrm{E}-10$ & $0.00 \mathrm{E}+00$ & 1.85E-05 & $0.2 \%$ & $3.88 \mathrm{E}-05$ & $0.0 \%$ & $99.8 \%$ & $1.86 \mathrm{E}-05$ & 1 \\
\hline $241-L ;-204$ & $1.41 \mathrm{E}-07$ & 1.62E-08 & $2.17 \mathrm{E}-10$ & $4.79 \mathrm{E}-05$ & $0.3 \%$ & $3.36 \mathrm{E}-04$ & $0.0 \%$ & $99.7 \%$ & $4.81 \mathrm{E}-05$ & 2 \\
\hline Supernatant & $6.48 \mathrm{E}-08$ & 3.09F. 12 & $1.31 \mathrm{E}-10$ & $3.32 \mathrm{E}-06$ & $1.9 \%$ & $9.14 \mathrm{E}-07$ & $0.0 \%$ & $98.1 \%$ & 3.38E-06 & 0 \\
\hline Solid Layer & $7.66 \mathrm{E}-08$ & $1.62 \mathrm{E}-08$ & $8.66 \mathrm{E}-11$ & 4. $46 \mathrm{E}-05$ & $0.2 \%$ & 3.62E-04 & $0.0 \%$ & $99.8 \%$ & $4.47 \mathrm{E}-05$ & 2 \\
\hline AY 102-S-14U & $1.99 \mathrm{E}-03$ & $1.05 E-04$ & $3.20 \mathrm{E}-05$ & 1.49E-04 & $87.4 \%$ & $4.62 \%$ & $1.4 \%$ & $6.5 \%$ & 2.27E-03 & 93 \\
\hline AY 102-S-14L & $2.44 \mathrm{E}-03$ & $9.23 \mathrm{E}-05$ & $6.15 E-05$ & $1.47 \mathrm{E}-04$ & $89.0 \%$ & $3.37 \%$ & $2.2 \%$ & $5.4 \%$ & $2.74 \mathrm{E}-03$ & 112 \\
\hline$A Y 102-S-15 U$ & $4.60 \mathrm{E}-04$ & 3.43E-05 & $1.50 \mathrm{E}-05$ & $1.44 \mathrm{E}-04$ & $70.4 \%$ & $5.26 \%$ & $2.3 \%$ & $22.0 \%$ & $6.52 \mathrm{E}-04$ & 27 \\
\hline AY 102-S-15L & $3.55 \mathrm{~F}-03$ & $1.51 E-0.4$ & $8.35 E-05$ & 1.49E-04 & $90.2 \%$ & $3.85 \%$ & $2.1 \%$ & $3.8 \%$ & $3.93 \mathrm{E}-03$ & 160 \\
\hline$A Y-102-S-16$ & $5.39 \mathrm{E} .03$ & $1.22 \mathrm{E}-04$ & $5.35 \mathrm{E}-05$ & 1. $48 \mathrm{E}-04$ & $94.3 \%$ & $2.13 \%$ & $0.9 \%$ & $2.6 \%$ & $5.72 \mathrm{E}-03$ & 233 \\
\hline AY 102-Orig-9" & $9.49 \mathrm{E}-03$ & $1.09 \mathrm{E}-04$ & $3.70 \mathrm{E}-05$ & $1.47 E-04$ & $97.0 \%$ & $1.12 \%$ & $0.4 \%$ & $1.5 \%$ & $9.78 \mathrm{E}-03$ & 399 \\
\hline
\end{tabular}




\section{RPP-5926 REV 6}

Table B-5. Input Data With Water Addition for Hydrogen Generation Rate Model Calculations for 177 Tanks. (20 sheets)

\begin{tabular}{|c|c|c|c|c|c|c|c|c|c|c|c|c|c|c|c|c|c|c|c|}
\hline Tank & $\begin{array}{c}\mathrm{OH} \\
\text { in liquid } \\
|\mathrm{OH}| \\
(\mu \mathrm{g} / \mathrm{mL})\end{array}$ & $\begin{array}{c}\text { TOC } \\
\text { in liquid } \\
{[\mathrm{TOC}]} \\
(\mu \mathrm{g} / \mathrm{mL})\end{array}$ & $\begin{array}{c}\mathrm{NO}_{2} \\
\text { in liquid } \\
\left|\mathrm{NO} \mathrm{O}_{2}\right| \\
(\mu \mathrm{g} / \mathrm{mL})\end{array}$ & $\begin{array}{c}\mathrm{NO}_{3} \\
\text { in liquid } \\
{\left[\mathrm{NO}_{3} \mid\right.} \\
(\mu \mathrm{g} / \mathrm{mL})\end{array}$ & $\begin{array}{c}\mathrm{Na} \\
\text { in liquid } \\
|\mathrm{Na}| \\
(\mathrm{ug} / \mathrm{mL})\end{array}$ & $\begin{array}{c}\mathrm{Al} \\
\text { in liquid } \\
{[\mathrm{Al}]} \\
(\mu \mathrm{g} / \mathrm{mL})\end{array}$ & $\begin{array}{c}90 \mathrm{Sr} \\
\text { in waste } \\
|\mathrm{Sr}| \\
(\mu \mathrm{Ci} / \mathrm{g})\end{array}$ & $\begin{array}{c}{ }^{241} \mathrm{Am} \\
\text { in waste } \\
\text { |Am241| } \\
(\mu \mathrm{Cl} / \mathrm{g})\end{array}$ & $\begin{array}{c}{ }^{240} \mathrm{Pu} \\
\text { in waste } \\
{[\mathrm{Pu} 240 \mid} \\
(\mu \mathrm{Ci} / \mathrm{g})\end{array}$ & $\begin{array}{c}{ }^{239} \mathrm{Pu} \\
\text { in waste } \\
|\mathrm{Pu} 240| \\
(\mu \mathrm{Ci} / \mathrm{g})\end{array}$ & $\begin{array}{c}{ }^{238} \mathrm{Pu} \\
\text { in waste } \\
|\mathrm{Pu} 238| \\
(\mu \mathrm{Ci} / \mathrm{g})\end{array}$ & $\begin{array}{c}{ }^{137} \mathrm{Cs} \\
\text { in waste } \\
|\mathrm{Cs}| \\
(\mu \mathrm{Ci} / \mathrm{g})\end{array}$ & $\begin{array}{c}\text { Bulk } \\
\text { density } \\
D \\
(\mathrm{~g} / \mathrm{mL})\end{array}$ & $\begin{array}{c}\text { Liquid } \\
\text { density } D_{L} \\
(\mathrm{~g} / \mathrm{ml})\end{array}$ & $\begin{array}{c}\text { Non-RGS } \\
\text { waste } \\
\text { volume } \\
(\mathrm{kL})\end{array}$ & $\begin{array}{c}\text { Bulk } \\
\text { water } \\
{\left[\mathrm{H}_{2} \mathrm{O} \mid\right.} \\
(\mathrm{wt} / \mathrm{w})\end{array}$ & $\begin{array}{c}\text { Liquid } \\
\text { water } \\
\left|\mathrm{H}_{2} \mathrm{O}\right| \\
(\mathrm{wt} \%)\end{array}$ & $\begin{array}{c}\text { Waste } \\
\text { temp. } \\
\mathrm{Tw} \\
\left({ }^{\circ} \mathrm{C}\right)\end{array}$ & $\begin{array}{l}\text { Dome } \\
\text { temp. } \\
\mathrm{Td} \\
\left(^{\circ} \mathrm{C}\right)\end{array}$ \\
\hline 24I-AN-10I & $7 E+04$ & $2 \mathrm{E}+03$ & $1.15 \mathrm{E}+05$ & $1.61 E \div 05$ & $3 E+05$ & $93 \mathrm{E}+04$ & $09 \mathrm{E}+00$ & $89 \mathrm{E}-03$ & $7 E-05$ & $13 \mathrm{E}-04$ & $1.60 E-05$ & $1.96 \mathrm{E}+02$ & 1.41 & 1.41 & 3662 & $15 \mathrm{~F}-01$ & $18 \mathrm{E}-01$ & 32 & 31 \\
\hline Supernatant & $3.47 E+04$ & $5.05 \mathrm{E}-03$ & $1.15 \mathrm{E}+05$ & $1.60 \mathrm{E}+05$ & $2.12 \mathrm{E}+05$ & $1.87 E-04$ & $1.26 \mathrm{E}+00$ & $6.52 \mathrm{E}-04$ & $1.02 \mathrm{E}-05$ & $6.16 \mathrm{E}-05$ & $1.53 \mathrm{E}-06$ & $1.97 \mathrm{E}+02$ & $1.4 !$ & 1.41 & 3544 & $5.19 \mathrm{E}-01$ & $5.19 \mathrm{E}-01$ & 32 & 31 \\
\hline Solid Layer & $3.51 \mathrm{E}+04$ & $4.04 E+03$ & $1.22 E+05$ & $1.90 \mathrm{E}+05$ & $2.36 \mathrm{E}+05$ & $.79 \mathrm{E} \div 04$ & $2.46 \mathrm{E}+01$ & $3.55 \mathrm{E}-02$ & $2.31 \mathrm{E}-03$ & $9.97 E-03$ & $10 \mathrm{E}-04$ & $1.84 \mathrm{E}+02$ & 1.55 & 1.45 & 118 & $03 \mathrm{E}-01$ & $4.91 \mathrm{E}-01$ & 31 & 31 \\
\hline $241-$ AN- 102 & $8.77 \mathrm{E}+03$ & $2.39 \mathrm{E}+04$ & $8.63 \mathrm{E}+04$ & $2.07 E+05$ & $2.05 \mathrm{E} \div 05$ & $1.30 \mathrm{E}+04$ & $6.80 E+01$ & $89 \mathrm{E}-01$ & $2.35 \mathrm{E}-03$ & $9.04 \mathrm{E}-03$ & $6.56 \mathrm{E}-04$ & $2.74 \mathrm{E}+02$ & 1.42 & 1.41 & 4090 & $4.26 \mathrm{E}-01$ & $4.31 \mathrm{E}-01$ & 37 & 35 \\
\hline Supernatant & $8.76 \mathrm{E}+03$ & $2.39 \mathrm{E}+04$ & $8.61 \mathrm{E}+04$ & $2.07 \mathrm{E}+05$ & $.05 \mathrm{E}+05$ & $1.30 \mathrm{E}+04$ & $56 \mathrm{E}+01$ & $14 \mathrm{E}-01$ & $.71 \mathrm{E}-04$ & $.35 \mathrm{E}-03$ & 2.44E-04 & $2.76 \mathrm{E}+02$ & 1.41 & 1.41 & 3506 & $4.31 \mathrm{E}-01$ & $4.31 \mathrm{E}-01$ & 37 & 35 \\
\hline Solid Layer & $8.85 \mathrm{E}+03$ & $2.4 ! E+04$ & $8.71 E+04$ & $2.09 \mathrm{E}+05$ & $2.07 \mathrm{E}+05$ & $1.31 \mathrm{E}+04$ & $1.37 \mathrm{E} \div 02$ & $5.99 \mathrm{E}-01$ & $1.05 \mathrm{E}-02$ & $4.04 \mathrm{E}-02$ & $2.93 \mathrm{E}-03$ & $2.64 \mathrm{E}+02$ & 1.53 & 1.41 & 584 & $3.99 \mathrm{E}-01$ & $4.27 \mathrm{E}-01$ & 36 & 35 \\
\hline 241-AN-103 & $6.64 \mathrm{E}+04$ & $3.01 E+03$ & $1.28 \mathrm{E}+05$ & $1.27 \mathrm{E}+0.5$ & $2.67 \mathrm{E}+05$ & $96 \mathrm{E} \cdot 04$ & $1.15 \mathrm{E}+00$ & $78 \mathrm{E}-03$ & $2.06 \mathrm{E}-04$ & $.93 \mathrm{E}-04$ & $5.75 \mathrm{E}-05$ & $3.10 \mathrm{E}+02$ & 1.59 & 1.47 & 3445 & $4.10 \mathrm{E}-01$ & $4.71 E-01$ & 42 & 32 \\
\hline Supernatant & $6.58 \mathrm{E}+04$ & $2.98 \mathrm{E}+03$ & $1.27 E+05$ & $1.26 E+05$ & $65 E+05$ & $93 E+04$ & $18 \mathrm{E}-02$ & 07E-03 & 41E-05 & $.70 \mathrm{E}-04$ & $1.23 \mathrm{E}-05$ & $4.22 \mathrm{E}+$ & 1.47 & 1.47 & 1807 & 4.75E-01 & $75 \mathrm{E}-01$ & 42 & 32 \\
\hline Solid Layer & $6.72 E+04$ & $3.05 \mathrm{E}+03$ & $1.30 \mathrm{E}+05$ & $1.28 \mathrm{E}+05$ & $2.70 \mathrm{E}+05$ & $3.00 \mathrm{E}+04$ & $2.22 \mathrm{E}+00$ & $3.51 \mathrm{E}-03$ & $3.58 \mathrm{E}-04$ & $1.38 \mathrm{E}-03$ & $1.00 \mathrm{E}-04$ & $2.06 \mathrm{E}+02$ & 1.72 & 1.48 & 1638 & $3.49 \mathrm{E}-01$ & $4.67 \mathrm{E}-01$ & 42 & 32 \\
\hline $241 \cdot \mathrm{AN}-104$ & $6.46 \mathrm{E}+04$ & $11 E-03$ & $1.181+05$ & $1.87 \mathrm{E}-05$ & $.56 \mathrm{E}+05$ & $86 \mathrm{E}+04$ & $1.25 \mathrm{E}-01$ & $34 \mathrm{E}-03$ & $30 \mathrm{E}-04$ & $27 \mathrm{E}-03$ & $9.19 \mathrm{E}-05$ & $3.27 \mathrm{E}+02$ & 1.47 & 1.40 & 3902 & $4.91 \mathrm{E}-01$ & $5.14 E-01$ & $4 !$ & 33 \\
\hline Supernatant & $6.42 \mathrm{E}+04$ & $09 \mathrm{E}+03$ & $1.17 \mathrm{E}+05$ & $1.86 \mathrm{E} \div 05$ & $54 \mathrm{E}+05$ & $84 \mathrm{E}-04$ & $78 \mathrm{E}-02$ & $48 E-04$ & $33 \mathrm{E}$ & $10 \mathrm{E}-05$ & $3.69 \mathrm{E}-06$ & 3 & 1 & 1.39 & 2336 & 5.171 & $7 \mathrm{E}-01$ & 40 & 33 \\
\hline Solid Layer & $6.53 E+04$ & $3.14 \mathrm{E}+03$ & $1.19 E \div 05$ & $1.89 E+05$ & $2.58 \mathrm{E}+05$ & $3.90 \mathrm{E}+04$ & $2.88 \mathrm{E}+01$ & $1.89 \mathrm{E}-02$ & $7.43 E-04$ & $2.86 \mathrm{E}-03$ & $2.07 \mathrm{E}-04$ & $3.00 \mathrm{E}+02$ & 1.59 & 1.40 & 1566 & 4.57E-01 & $5.11 \mathrm{E}-01$ & 43 & 33 \\
\hline $24 \mathrm{I} \cdot \mathrm{AN}-105$ & $5.92 \mathrm{E}+04$ & $74 E+03$ & $17 \mathrm{E}+05$ & $1.55 \mathrm{E}+05$ & $2.45 \mathrm{E} \div 05$ & $13 \mathrm{E}+04$ & $62 E+00$ & $50 E-03$ & $2.83 \mathrm{E}-04$ & $1.08 \mathrm{E}-03$ & $7.86 \mathrm{E}-05$ & $2.26 \mathrm{E}+02$ & 1.49 & 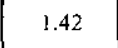 & 4190 & 4. $75 \mathrm{E}-01$ & $5.02 \mathrm{E}-01$ & 37 & 31 \\
\hline Supernatant & $5.87 \mathrm{E}+04$ & $2.72 \mathrm{E}+03$ & $1.17 \mathrm{E}+05$ & $1.54 \mathrm{E}+05$ & $435+6$ & $4.10 \mathrm{E}+04$ & $42 \mathrm{E}-02$ & $.00 \mathrm{E}-02$ & $0.9 \mathrm{~L}-$ & $4.19 E-04$ & $3.04 \mathrm{E}-05$ & $2.04 \mathrm{E}+02$ & 1.41 & 1. & 22 & $=$ & 01 & 36 & 31 \\
\hline Solid Layer & $5.97 \mathrm{E}+04$ & $2.76 \mathrm{E}+03$ & $1.19 \mathrm{E}+05$ & $1.56 \mathrm{E}+05$ & $2.47 E+05$ & $4.17 \mathrm{E} \div 04$ & $9.50 \mathrm{E}+00$ & $6.92 \mathrm{E}-03$ & $4.67 \mathrm{E}-04$ & $1.79 \mathrm{E}-03$ & $1.30 \mathrm{E}-04$ & $2.50 \mathrm{E}+02$ & 1.57 & 1.42 & 1925 & 4.44E-01 & $4.99 \mathrm{E}-01$ & 38 & 31 \\
\hline 241-AN-106 & $5.02 \mathrm{E}+03$ & $1.93 \mathrm{E}+03$ & $1.85 E+04$ & $2 E+04$ & $13 E+04$ & $30 \mathrm{E}+03$ & +02 & $E-01$ & $5 \mathrm{E}-02$ & $9 \mathrm{E}-0 !$ & $5 \mathrm{E}-02$ & $\mathrm{E}+0 !$ & 1 & 1.10 & 079 & $7.73 \mathrm{E}-01$ & $8.33 \mathrm{E}-01$ & 28 & 25 \\
\hline Supernatant & $5.01 \mathrm{E}+03$ & $1.93 \mathrm{E}+03$ & $1.84 \mathrm{E}+04$ & $2.31 \mathrm{E}+04$ & 4. $12 \mathrm{E} \div 04$ & $3.79 \mathrm{E}+03$ & $5.30 \mathrm{E}-01$ & $3.90 \mathrm{E}-04$ & $4.40 \mathrm{E}-04$ & $2.84 \mathrm{E}-03$ & $4.51 \mathrm{E}-05$ & $4.29 \mathrm{E}-$ & 1.10 & 1.10 & 25 & 1 & 01 & 7 & 5 \\
\hline Solid Layer & $5.09 \mathrm{E}+03$ & $1.96 \mathrm{E}+03$ & $1.87 \mathrm{E}+04$ & $2.35 \mathrm{E}+04$ & 4. $19 \mathrm{E}+04$ & $3.85 \mathrm{E}+03$ & $2.46 \mathrm{E}+03$ & $3.05 \mathrm{E}+00$ & $2.94 \mathrm{E}-01$ & $1.36 \mathrm{E}+00$ & $4.80 \mathrm{E}-02$ & $9.79 \mathrm{E}+01$ & 1.39 & 1.10 & 556 & $5.58 \mathrm{E}-01$ & 8.31E-01 & 31 & 25 \\
\hline $241-A N-107$ & 7 & 04 & 04 & 5 & $2.06 \mathrm{E}+05$ & $1.11 \mathrm{E}+03$ & $7.73 \mathrm{E}-01$ & 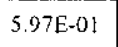 & $\mathrm{E}-03$ & $\mathrm{EE}-02$ & $\therefore$ & $2.13 \mathrm{E}+02$ & 1.44 & 1.43 & 4207 & 1 & $18 \mathrm{E}-01$ & $\&$ & is \\
\hline Supernatant & $1.78 \mathrm{E}+04$ & $3.21 \mathrm{E}+04$ & $6.60 \mathrm{E}+04$ & $2.07 \mathrm{E}+05$ & $2.05 \mathrm{E}+05$ & $1.11 \mathrm{E} \div 03$ & $5.32 \mathrm{E} \div 01$ & $5.07 \mathrm{E}-01$ & $8.14 \mathrm{E}-03$ & $3.14 \mathrm{E}-02$ & $2.27 \mathrm{E}-03$ & $2.07 \mathrm{E}+02$ & 1.43 & 1.43 & 33 & $5.19 \mathrm{E}-01$ & $5.19 \mathrm{E}-01$ & 38 & 35 \\
\hline Solid Layer & $1.80 \mathrm{E}+04$ & $3.25 \mathrm{E}+04$ & $6.67 E+04$ & $2.09 \mathrm{E}+05$ & $2.08 \mathrm{E}+05$ & $1.12 \mathrm{E}+03$ & $1.66 \mathrm{E}+02$ & $9.30 \mathrm{E}-01$ & $1.43 \mathrm{E}-02$ & $5.49 \mathrm{E}-02$ & $3.98 \mathrm{E}-03$ & $2.33 \mathrm{E}+02$ & 1.48 & 1.43 & 872 & $4.43 \mathrm{E}-0 \mathrm{I}$ & $5.15 \mathrm{E}-0 \mathrm{I}$ & 39 & 35 \\
\hline $241-\mathrm{A}$ & $3.95 \mathrm{E}+04$ & $8 \mathrm{E}+03$ & $4 E+04$ & 5 & $1.28 \mathrm{E}+05$ & $4 \mathrm{E}+03$ & $8.00 \mathrm{E}-05$ & $\mathrm{E}-$ & $8.00 \mathrm{E}-05$ & 05 & 06 & 65 & 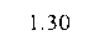 & 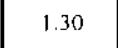 & 4257 & $38 \mathrm{E}-01$ & $38 \mathrm{E}-01$ & 27 & 28 \\
\hline Supernatant & $3.95 E+04$ & $1.88 \mathrm{E}+03$ & $4.04 \mathrm{E}: 04$ & $1.17 \mathrm{E}+05$ & $1.28 \mathrm{E}+05$ & $7.24 \mathrm{E}+03$ & $7.46 \mathrm{E}-02$ & $1.47 \mathrm{E}-04$ & $2.08 \mathrm{E}-05$ & $8.00 \mathrm{~F}-05$ & $5.81 \mathrm{E}-06$ & $1.06 \mathrm{E} \div 02$ & 1.30 & 1.30 & 4257 & $6.38 \mathrm{E}-0 \mathrm{I}$ & $6.38 \mathrm{E}-01$ & 27 & 28 \\
\hline Solid Layer & $0.00 \mathrm{E}+00$ & $0.00 \mathrm{E}+00$ & $0.00 \mathrm{E}+00$ & $0.00 E+00$ & $0.00 \mathrm{E}+00$ & $0.00 \mathrm{E}+00$ & $0.00 \mathrm{E}+00$ & $0.00 \mathrm{E}+00$ & $0.00 \mathrm{~F}+00$ & $0.00 \mathrm{E}+00$ & $0.00 \mathrm{E}+00$ & $0.00 \mathrm{E}+00$ & 0.00 & 0.00 & 0 & $0.00 \mathrm{E}+00$ & $0.00 \mathrm{E} r 00$ & 5 & 5 \\
\hline $241-\mathrm{A}$ & $3.18 \mathrm{E}+04$ & 3 & 04 & 1.66 & 05 & 14 & $2.78 \mathrm{E}-01$ & 03 & 05 & 04 & 36 & 2 & ${ }^{y}$ & 1.39 & 4175 & & 01 & 36 & 6 \\
\hline Supenratant & $3.18 \mathrm{E}+04$ & $2.99 \mathrm{E}+03$ & $9.74 \mathrm{E}+04$ & $1.66 \mathrm{E} \div 05$ & $1.93 \mathrm{E}+05$ & $3.06 \mathrm{E}+04$ & $2.75 \mathrm{E}-01$ & $1.24 \mathrm{E}-03$ & $3.72 \mathrm{E}-05$ & $2.21 \mathrm{E}-04$ & $5.72 \mathrm{E}-06$ & $1.71 E+02$ & 1.39 & 1.39 & 4087 & $5.90 \mathrm{E}-01$ & $5.90 \mathrm{E}-01$ & 36 & 36 \\
\hline Solid Layer & $3.21 \mathrm{E}+04$ & $01 \mathrm{E}+03$ & $9.83 \mathrm{E}+04$ & $1.67 \mathrm{k}, 05$ & $1.95 \mathrm{E}+05$ & $3.09 E+04$ & $3.68 \mathrm{E}-01$ & $6.95 \mathrm{E}-04$ & $7.92 \mathrm{E}-05$ & $4.68 \mathrm{E}-04$ & $1.20 \mathrm{E}-05$ & $8.12 \mathrm{E}+01$ & 1.75 & 1.39 & 88 & $3.1 ! \mathrm{E}-01$ & 5.87E-01 & 29 & 36 \\
\hline
\end{tabular}


RPP-5926 REV 6

Table B-5. Input Data With Water Addition for Hydrogen Generation Rate Model Calculations for 177 Tanks. (20 sheets)

\begin{tabular}{|c|c|c|c|c|c|c|c|c|c|c|c|c|c|c|c|c|c|c|c|}
\hline Tank & $\begin{array}{c}\mathrm{OH} \\
\text { in liquid } \\
|\mathrm{OH}| \\
(\mu \mathrm{g} / \mathrm{mL})\end{array}$ & $\begin{array}{c}\text { TOC } \\
\text { in liquid } \\
\text { [TOC] } \\
(\mu \mathrm{g} / \mathrm{mL})\end{array}$ & $\begin{array}{c}\mathrm{NO}_{2} \\
\text { in liquid } \\
{\left[\mathrm{NO}_{2} \mid\right.} \\
(\mu \mathrm{g} / \mathrm{mL})\end{array}$ & $\begin{array}{c}\mathrm{NO}_{3} \\
\text { in liquid } \\
\left|\mathrm{NO}_{3}\right| \\
(\mu \mathrm{g} / \mathrm{mL})\end{array}$ & $\begin{array}{c}\text { ra } \\
\text { in liquid } \\
\text { |Nal } \\
(\mathrm{ug} / \mathrm{mL} \text { ) }\end{array}$ & $\begin{array}{c}\text { Al } \\
\text { in liquid } \\
\text { |A|| } \\
(\mu \mathrm{g} / \mathrm{mL})\end{array}$ & $\begin{array}{c}90 \mathrm{Sr} \\
\text { in waste } \\
{[\mathrm{Sr}]} \\
(\mu \mathrm{Ci} / \mathrm{g})\end{array}$ & $\begin{array}{c}{ }^{241} \mathrm{Am} \\
\text { in waste } \\
{[\mathrm{Am} 241]} \\
(\mu \mathrm{Cl} / \mathrm{g})\end{array}$ & $\begin{array}{c}{ }^{2 \mathrm{~s} 0} \mathrm{Pu} \\
\text { in waste } \\
|\mathrm{Pu} 40| \\
(\mu \mathrm{Ci} / \mathrm{g})\end{array}$ & $\begin{array}{c}{ }^{239} \mathrm{Pu} \\
\text { in waste } \\
{[\mathrm{Pu} 240]} \\
(\mu \mathrm{Ci} / \mathrm{g})\end{array}$ & $\begin{array}{c}{ }^{238} \mathrm{Pu} \\
\text { in waste } \\
{[\mathrm{Pu238}]} \\
(\mu \mathrm{Cig} / \mathrm{g})\end{array}$ & $\begin{array}{c}{ }^{137} \mathrm{Cs} \\
\text { in waste } \\
\mid \mathrm{Cs}] \\
(\mu \mathrm{Ci} / \mathrm{g})\end{array}$ & $\begin{array}{c}\text { Bulk } \\
\text { density } \\
\text { D } \\
(\mathrm{g} / \mathrm{mL})\end{array}$ & $\begin{array}{c}\text { Liquid } \\
\text { density } \mathrm{D}_{\mathrm{L}} \\
(\mathrm{g} / \mathrm{ml})\end{array}$ & $\begin{array}{c}\text { Non-RGS } \\
\text { waste } \\
\text { volume } \\
(\mathbf{k L})\end{array}$ & $\begin{array}{c}\text { Bulk } \\
\text { water } \\
\left|\mathrm{H}_{2} \mathrm{O}\right| \\
\left(\mathrm{wt}^{\circ} \%\right)\end{array}$ & $\begin{array}{c}\text { Liquid } \\
\text { water } \\
\left|\mathrm{H}_{2} \mathrm{O}\right| \\
(w 1 \%)\end{array}$ & $\begin{array}{l}\text { Waste } \\
\text { temp. } \\
\mathrm{Tw} \\
\left(^{\circ} \mathrm{C}\right)\end{array}$ & $\begin{array}{c}\text { Dome } \\
\text { temp. } \\
\text { Td } \\
\left({ }^{\circ} \mathrm{C}\right)\end{array}$ \\
\hline $241-\mathrm{AP}-103$ & $5 E \div 04$ & $9 E+03$ & $5 E+04$ & $9 E+05$ & $7 \mathrm{E}+05$ & $4 \mathrm{E}+04$ & $6 \mathrm{E}+00$ & $10-0$ & $3 \mathrm{E}-\mathrm{C}$ & $9 \mathrm{E}-04$ & 09E-05 & $.67 \mathrm{E}^{+}$ & 1.35 & 1.35 & 3416 & $.66 \mathrm{E}-01$ & $71 \mathrm{E}-01$ & 26 & 27 \\
\hline Supernatant & $1.65 \mathrm{E}+04$ & $28 \mathrm{E}+03$ & $7.94 \mathrm{E}+04$ & $1.49 \mathrm{E} \cdot 05$ & $77 \mathrm{E}+05$ & $34 E+04$ & $1.80 \mathrm{E}+00$ & $8.31 \mathrm{E}-03$ & $1.12 \mathrm{E}-04$ & $70 \mathrm{E}-04$ & $2.06 \mathrm{E}-05$ & $1.69 \mathrm{E}+02$ & 1.35 & 1.35 & 3336 & $5.71 \mathrm{E}-01$ & $5.71 E-01$ & 26 & 27 \\
\hline Solid Layer & $1.67 E+04$ & $7.37 E+03$ & $8.04 \mathrm{E}: 04$ & $1.51 \mathrm{E}+05$ & $1.79 E+05$ & $1.86 \mathrm{E}: 04$ & $2.44 \mathrm{E}-01$ & $2.49 \mathrm{E}-04$ & $1.62 E-04$ & $9.60 E-04$ & $3.17 \mathrm{E}-05$ & $1.12 E+02$ & 1.68 & 1.35 & 80 & $4.09 \mathrm{E}-0 \mathrm{l}$ & $5.67 E-01$ & 29 & 27 \\
\hline $241-\mathrm{AP}_{-104}$ & $1.95 E+0.4$ & $4.13 \mathrm{E}+03$ & $6.34 \mathrm{~F}+04$ & $9.99 \mathrm{E}+04$ & $1.27 \mathrm{E}-05$ & $1.66 \mathrm{E}: 04$ & $1.37 \mathrm{E}-04$ & $1.37 E-04$ & $1.37 \mathrm{E}-04$ & $1.37 \mathrm{E}-04$ & $3.08 \mathrm{E}-05$ & 1. $44 \mathrm{E}+02$ & 1.28 & 1.28 & 4193 & $6.66 \mathrm{E}-01$ & $6.66 \mathrm{E}-01$ & 26 & 27 \\
\hline Supernatant & $1.95 \mathrm{E} \cdot 04$ & $4.13 E+03$ & $34 \mathrm{E}+04$ & $9.99 \mathrm{E}+04$ & $27 \mathrm{E}+05$ & $66 \mathrm{E}+04$ & $1.30 \mathrm{E} \div 00$ & $2.18 \mathrm{E}-03$ & $2.33 \mathrm{E}-05$ & $1.37 \mathrm{E}-04$ & $3.08 \mathrm{E}-05$ & $1.44 E+02$ & 1.28 & 1.28 & 4193 & $6.66 \mathrm{E}-0 \mathrm{I}$ & $.66 \mathrm{E}-01$ & 26 & 27 \\
\hline Solid Layer & $0.00 \mathrm{E}+00$ & $0.00 \mathrm{E}+00$ & $0.00 \mathrm{E}+00$ & $0.00 \mathrm{E} \div 00$ & $0.00 E+00$ & $0.00 \mathrm{E}+00$ & $0.00 \mathrm{E}+00$ & $0.00 \mathrm{E}+00$ & $0.00 E+00$ & $0.00 \mathrm{E}+00$ & $0.00 \mathrm{E}+00$ & $0.00 \mathrm{E}+00$ & 0.00 & 0.00 & 0 & $0.00 \mathrm{E}+00$ & $0.00 \mathrm{E}+00$ & 5 & 5 \\
\hline $241 \cdot \mathrm{AP}-105$ & $7 \mathrm{E} \div 04$ & $1.48 \mathrm{E}+03$ & $4.70 \mathrm{E}+04$ & $1.05 \mathbf{E}+05$ & $1.33 \mathrm{E}+05$ & $91 \mathrm{E}+04$ & $1.92 \mathrm{E}+00$ & $9.94 \mathrm{E}-04$ & 1.19E-04 & $5.31 E-04$ & $3.54 \mathrm{E}-05$ & $1.18 \mathrm{E}+02$ & 1.29 & 1.27 & 4340 & $6.93 \mathrm{E}-01$ & $7.16 \mathrm{E}-01$ & 36 & 37 \\
\hline Supcriatant & $2.57 \mathrm{E}+0.4$ & $48 E+03$ & $70 \mathrm{E}+04$ & $1.05 E+05$ & $33 E+05$ & $91 E+04$ & $2.13 \mathrm{~F}-01$ & $42 \mathrm{E}-04$ & $63 \mathrm{E}-05$ & $21 \mathrm{E}-04$ & $1.26 \mathrm{E}-05$ & $1.06 \mathrm{E}+02$ & 1.27 & 1.27 & 4003 & $7.16 \mathrm{E}-0 \mathrm{I}$ & $16 \mathrm{E}-01$ & 37 & 37 \\
\hline Solid Layer & $2.59 \mathrm{E}+04$ & $1.50 \mathrm{E}+03$ & $4.74 \mathrm{E}+04$ & $1.06 \mathrm{E}=05$ & $1.34 \mathrm{E}+05$ & $1.92 \mathrm{E}+04$ & $1.79 \mathrm{E}+01$ & $5.22 \mathrm{E}-03$ & $8.90 \mathrm{E}-04$ & $3.43 \mathrm{E}-03$ & $2.49 \mathrm{E}-04$ & $2.25 \mathrm{E}-02$ & 1.61 & 1.27 & 337 & $4.77 \mathrm{E}-01$ & $14 \mathrm{E}-0 \mathrm{I}$ & 25 & 37 \\
\hline $241-\mathrm{AP}-106$ & $1 E+03$ & $3.43 \mathrm{E} \div 03$ & $4.10 E+04$ & $7.01 \mathbf{E}+04$ & $1.03 \mathrm{E}+05$ & $34 \Sigma+04$ & $6.07 \mathrm{E}-05$ & $6.07 \mathrm{E}-05$ & $6.07 \mathrm{E}-05$ & $6.07 \mathrm{E}-05$ & $1.70 \mathrm{E}-06$ & $1.44 \mathrm{E}+02$ & 1.21 & 1.21 & 4339 & $7.26 \mathrm{E}-01$ & $7.26 \mathrm{E}-01$ & 29 & 24 \\
\hline Supeniatant & $7.31 \mathrm{E}+03$ & $3 \mathrm{~F}+03$ & $10 \mathrm{E}+04$ & $7.01 \mathrm{E}+04$ & $03 \mathrm{E} \cdot 05$ & $34 \mathrm{E}-04$ & 77E-01 & 985 & 450,0 & 07E-05 & $.70 \mathrm{E}-06$ & $1.44 E+02$ & 1.21 & 1.2 & 4339 & $7,26 \mathrm{E}-01$ & 7.261 & 20 & 24 \\
\hline Solid Layer & $0.00 \mathrm{E}+00$ & $0.00 E+00$ & $0.00 \mathrm{E}+00$ & $0.00 \mathrm{E}+00$ & $0.00 \mathrm{E}+00$ & $0.00 \mathrm{E}+00$ & $0.00 E+00$ & $0.00 \mathrm{E}^{-100}$ & $0.00 \mathrm{E}+00$ & $0.00 \mathrm{E} \div 00$ & $0.00 \mathrm{E}+00$ & $0.00 \mathrm{E}+00$ & 0.00 & 0.00 & 0 & $0.00 \mathrm{E}+00$ & $0.00 \mathrm{E}+00$ & 5 & 5 \\
\hline $241-\mathrm{AP}-107$ & $6 \mathrm{E}+04$ & $70 E \cdot 03$ & $60 \mathrm{E} \div 04$ & $1.22 \mathrm{E}+05$ & $1.35 \mathrm{E}+05$ & $59 E+04$ & $14 E-04$ & $1.14 E-04$ & $1.14 \mathrm{E}-04$ & $1.14 \mathrm{E}-04$ & $6.63 \mathrm{E}-06$ & $1.09 \mathrm{E}+02$ & 1.30 & 1.30 & 1344 & $6.39 \mathrm{E}-01$ & $6.39 \mathrm{E}-01$ & 20 & 19 \\
\hline Superratant & 1. & $E+03$ & +04 & $2 \mathbf{E}+05$ & 5 & $1.59 \mathrm{E}+04$ & $6.29 \mathrm{E}-01$ & $3.16 \mathrm{E}-04$ & $2.16 \mathrm{E}-05$ & $1.14 \mathrm{E}-04$ & $6.63 \mathrm{E}-06$ & 1 & 1 & ( & 134 & 6.39 & 630 & 20 & 19 \\
\hline Solid Layer & $0.00 \mathrm{E}+00$ & $0.00 E+00$ & $0.00 \mathrm{E}+00$ & $0.00 \mathrm{E}+00$ & $0.00 \mathrm{E} \div 00$ & $0.00 \mathrm{E}+00$ & $0.00 \mathrm{E}+00$ & $0.00 E+00$ & $0.00 \mathrm{E}+00$ & $0.00 \mathrm{E}+00$ & $0.00 \mathrm{E}+00$ & $0.00 \mathrm{E}+00$ & 0.00 & 0.00 & 0 & $0.00 \mathrm{E}+00$ & $0.00 \mathrm{E}+00$ & 5 & 5 \\
\hline 241-AP-108 & $1 E+04$ & $E+03$ & $4 \mathrm{E}+04$ & $6 \mathrm{E}-05$ & $1 \mathrm{E}+05$ & $7 \mathrm{E}+04$ & $02 \mathrm{E}-01$ & $5 E-04$ & $4.65 \mathrm{E}-05$ & $29 \mathrm{E}-04$ & $1.72 \mathrm{E}-05$ & $1.40 \mathrm{E}+02$ & 1.43 & 1.42 & 3910 & $4.96 \mathrm{E}-01$ & $5.00 \mathrm{E}-01$ & 39 & 41 \\
\hline Supernatant & $3.91 \mathrm{E}+04$ & $2.77 \mathrm{E}+03$ & $7.83 E+04$ & $E+05$ & $E+05$ & $E+$ & $5.06 \mathrm{E}-01$ & $2.37 \mathrm{E}-04$ & $4.69 \mathrm{E}-05$ & $2.31 \mathrm{E}-04$ & 5 & 1 & 1.42 & 1 & 3423 & 01 & 1 & 41 & 41 \\
\hline Solid Layer & $3.95 \mathrm{E}+04$ & $2.80 \mathrm{E}+03$ & $7.92 E+04$ & $1.78 E+05$ & $2.13 E+05$ & $2.40 \mathrm{E}+04$ & 4.77E-01 & $2.23 \mathrm{E}-04$ & $4.42 \mathrm{E}-05$ & $2.17 \mathrm{E}-04$ & $1.63 \mathrm{E}-05$ & $1.33 \mathrm{E}+02$ & 1.52 & 1.42 & 487 & $4.60 \mathrm{E}-01$ & $4.97 \mathrm{E}-01$ & 28 & 41 \\
\hline-101 & 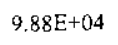 & $59 \mathrm{E}-103$ & $04 \mathrm{E}+05$ & $0 \mathrm{E}+05$ & $2.31 \mathrm{E}+05$ & $2.92 \mathrm{E} \cdot 04$ & $1.31 \mathrm{E}+01$ & $1.65 \mathrm{E}-02$ & $4.12 \mathrm{E}-03$ & $1.58 \mathrm{E}-02$ & $1.15 \mathrm{E}-03$ & $2.41 \mathrm{E}+02$ & 1.51 & 1.47 & 4211 & $4.42 \mathrm{E}-01$ & $4.41 \mathrm{E}-01$ & 38 & 27 \\
\hline Supematant & $9.83 \mathrm{E}+04$ & $2.58 \mathrm{E}+03$ & $1.04 \mathrm{E}+05$ & $1.69 \mathrm{E}+05$ & $2.30 E+05$ & $2.91 \mathrm{E}+04$ & $2.53 \mathrm{E}-01$ & 04 & $7.20 \mathrm{E}$ & 4 & $2.01 \mathrm{E}-05$ & $2.55 \mathrm{E}+02$ & 1.4 & 1. & 2808 & $4.43 \mathrm{E}-01$ & $4.43 \mathrm{E}-01$ & 38 & 27 \\
\hline Solid Layer & $9.96 \mathrm{E}+04$ & $2.61 E \div 03$ & $1.05 E+05$ & $1.71 E+05$ & $2.33 \mathrm{E}+05$ & $2.95 E+04$ & $3.68 \mathrm{E}-01$ & $4.66 \mathrm{E}-02$ & $1.16 \mathrm{E}-02$ & $4.45 \mathrm{E}-02$ & $3.23 \mathrm{E}-03$ & $2.13 \mathrm{E}+02$ & 1.59 & 1.47 & 1403 & $4.39 \mathrm{E}-01$ & $4.38 \mathrm{E}-01$ & 39 & 27 \\
\hline $241-4 W-102$ & $1.82 \mathrm{E}+04$ & $03 \mathrm{E}: 03$ & $38 E \div 04$ & 104 & 05 & $\because 04$ & $6.95 \mathrm{E}+00$ & $2.16 \mathrm{E}-02$ & $1.09 \mathrm{E}-03$ & $5.25 \mathrm{E}-03$ & $9.69 \mathrm{E}-05$ & $9.21 \mathrm{E}+01$ & 1.22 & 1.22 & 2331 & $7.10 \mathrm{E}-01$ & $7.15 \mathrm{E}-01$ & 27 & 26 \\
\hline Supcrnatant & $1.82 \mathrm{E}: 04$ & $2.03 \mathrm{E}-03$ & $4.38 \mathrm{~F} \cdot 04$ & $9.54 \mathrm{E}-04$ & $1.04 E \div 05$ & $1.19 \mathrm{E}+04$ & $6.49 \mathrm{E}-01$ & 3.33E-04 & 1.25E-05 & 7.17F-05 & $7.88 \mathrm{E}-06$ & $9.23 \mathrm{E}+01$ & 1.22 & 1.22 & 2306 & 7.1SE-01 & 7.15 & 27 & 26 \\
\hline Solid Layer & $1.85 E+04$ & $2.06 \mathrm{E}+03$ & $4.45 \mathrm{E}+04$ & $9.70 E+04$ & $1.06 \mathrm{E}+05$ & $1.21 \mathrm{E}+04$ & $5.42 \mathrm{E}+02$ & $1.83 \mathrm{E}+00$ & $9.24 \mathrm{E}-02$ & 4.45E-01 & 7.67E-03 & $7.12 \mathrm{E}+01$ & 1.32 & 1.22 & 25 & $3.34 \mathrm{E}-0 \mathrm{I}$ & $7.11 E-01$ & 33 & 26 \\
\hline $241-A W-103$ & $2.62 \mathrm{E}+04$ & $4 E+03$ & $5.27 \mathrm{E}+04$ & $1.45 \mathrm{E}+05$ & $1.33 \mathrm{E} \div 05$ & $02 \mathrm{E}+03$ & $2.43 \mathrm{E} \div 00$ & $05 \mathrm{E}-02$ & $E-02$ & $\mathrm{E}-02$ & $\mathrm{E}-03$ & 6.85 & 31 & 1.30 & 187 & $44 \mathrm{E}-01$ & $30 \mathrm{E}-01$ & 25 & 26 \\
\hline Supenatant & $1.55 \mathrm{E}+04$ & $1.85 \mathrm{E}+03$ & $3.31 E+04$ & $9.52 \mathrm{E} \div 04$ & $9.96 \mathrm{E}+04$ & $7.98 \mathrm{E} \div 03$ & $1.76 \mathrm{E}-01$ & $4.08 \mathrm{E}-05$ & $2.02 \mathrm{E}-05$ & $9.52 \mathrm{E}-05$ & $8.61 E-06$ & $8.97 \mathrm{E}+01$ & 1.24 & 1.24 & 2931 & $6.95 \mathrm{E}-01$ & $6.95 \mathrm{E}-01$ & 25 & 26 \\
\hline Solid Layer & $4.81 \mathrm{E} \div 04$ & $11 E+03$ & $9.31 \mathrm{E}+04$ & $2.47 \mathrm{E}+05$ & $2.01 E+05$ & $8.08 \mathrm{E}+03$ & $6.81 E+00$ & $8.94 \mathrm{E}-02$ & $6.13 \mathrm{E}-02$ & $2.19 \mathrm{E}-01$ & $2.16 \mathrm{E}-02$ & $2.75 \mathrm{E}+01$ & 1.49 & 1.42 & 1256 & $5.46 \mathrm{E}-01$ & $5.05 \mathrm{E}-01$ & 25 & 26 \\
\hline
\end{tabular}


RPP-5926 REV 6

Table B-5. Input Data With Water Addition for Hydrogen Generation Rate Model Calculations for 177 Tanks. (20 sheets)

\begin{tabular}{|c|c|c|c|c|c|c|c|c|c|c|c|c|c|c|c|c|c|c|c|}
\hline Tank & $\begin{array}{c}\mathrm{OH} \\
\text { in liquid } \\
|\mathrm{OH}| \\
(\mu \mathrm{g} / \mathrm{mL})\end{array}$ & $\begin{array}{c}\text { rOC } \\
\text { in liquid } \\
|\mathrm{TOC}| \\
(\mu \mathrm{g} / \mathrm{mL})\end{array}$ & $\begin{array}{c}\mathrm{NO}_{2} \\
\text { in liquid } \\
{\left[\mathrm{NO}_{2} \mid\right.} \\
(\mu \mathrm{g} / \mathrm{mL})\end{array}$ & $\begin{array}{c}\mathrm{NO}_{3} \\
\text { in licquid } \\
\left(\mathrm{NO}_{3} \mid\right. \\
(\mu \mathrm{g} / \mathrm{mL})\end{array}$ & $\begin{array}{c}\mathrm{Na} \\
\text { in liquid } \\
|\mathrm{Na}| \\
\text { (ug/mL) }\end{array}$ & $\begin{array}{c}\text { Al } \\
\text { in liquid } \\
|\mathrm{A}|\} \\
(\mu \mathrm{g} / \mathrm{mL})\end{array}$ & $\begin{array}{c}90 \mathrm{Sr} \\
\text { in waste } \\
{[\mathrm{Sr}]} \\
(\mu \mathrm{Ci} / \mathrm{g})\end{array}$ & $\begin{array}{c}{ }^{2 \mathrm{~N}} \mathrm{Am} \\
\text { in waste } \\
{[\mathrm{Am} 241]} \\
(\mu \mathrm{Ci} / \mathrm{g})\end{array}$ & $\begin{array}{c}{ }^{240} \mathrm{Pu} \\
\text { in waste } \\
\text { [Puz240| } \\
(\mu \mathrm{Ci} / \mathrm{g})\end{array}$ & $\begin{array}{c}{ }^{239} \mathrm{Pu} \\
\text { in waste } \\
{[\mathrm{Pu} 240]} \\
(\mu \mathrm{Ci} / \mathrm{g})\end{array}$ & $\begin{array}{c}{ }^{238} \mathrm{Pu} \\
\text { in waste } \\
\text { |Pu238| } \\
(\mu \mathrm{Ci} / \mathrm{g})\end{array}$ & $\begin{array}{c}{ }^{137} \mathrm{Cs} \\
\text { in waste } \\
|\mathrm{Cs}| \\
(\mu \mathrm{Ci} / \mathrm{g})\end{array}$ & $\begin{array}{c}\text { Bulk } \\
\text { density } \\
D \\
(\mathrm{~g} / \mathrm{mL})\end{array}$ & $\mid \begin{array}{c}\text { Liquid } \\
\text { density } D_{t} \\
(\mathrm{~g} / \mathrm{ml})\end{array}$ & $\begin{array}{c}\text { Non-RGS } \\
\text { waste } \\
\text { volume } \\
(\mathrm{kL})\end{array}$ & $\begin{array}{l}\text { Bulk } \\
\text { water } \\
\left|\mathrm{H}_{2} \mathrm{O}\right| \\
\left(\mathrm{wt}^{\prime} \%\right)\end{array}$ & $\begin{array}{l}\text { Liquid } \\
\text { water } \\
\left|\mathrm{H}_{2} \mathrm{O}\right| \\
(\mathrm{wt} \%)\end{array}$ & $\begin{array}{l}\text { Waste } \\
\text { temp. } \\
\mathrm{Tw} \\
\left({ }^{\circ} \mathrm{C}\right)\end{array}$ & $\begin{array}{c}\text { Dome } \\
\text { temp. } \\
\text { Td } \\
\left({ }^{\circ} \mathrm{C}\right)\end{array}$ \\
\hline 241-AW-104 & $0 E+04$ & $0 \mathrm{E}+03$ & $7.50 \mathrm{E}+04$ & $2 \mathrm{E}+05$ & $1 \mathrm{E}+05$ & $66 \mathrm{E} \div 04$ & $6 \mathrm{E} \div 00$ & $1.20 \mathrm{E}-02$ & $2.58 \mathrm{E}-02$ & $20 \mathrm{E}-02$ & $9.15 \mathrm{E}-03$ & $1.74 \mathrm{E}+02$ & 1.37 & 1.36 & 4102 & $.45 \mathrm{E}-01$ & $58 \mathrm{E}-01$ & 31 & 30 \\
\hline Supematant & $2.50 \mathrm{E}+04$ & $27 \mathrm{E}+03$ & $7.36 \mathrm{E}+04$ & $1.11 \mathrm{E}+05$ & $1.68 \mathrm{E}+05$ & $2.51 \mathrm{E}+04$ & $1.19 \mathrm{E}+00$ & $1.15 \mathrm{E}-03$ & $1.86 \mathrm{E}-05$ & $1.04 \mathrm{E}-04$ & 3.33E-06 & $1.75 \mathrm{E}+02$ & 1.35 & 1.35 & 3259 & $5.59 \mathrm{E}-01$ & $5.59 \mathrm{E}-01$ & 31 & 30 \\
\hline Solid Layer & $4.01 \mathrm{E}+04$ & $3.42 \mathrm{E}+03$ & $8.04 \mathrm{E}+04$ & $1.18 \mathrm{E}+05$ & $2.36 \mathrm{E}+05$ & $3.29 \mathrm{E}+04$ & $5.88 \mathrm{E}-01$ & $5.02 \mathrm{E}-02$ & $1.16 \mathrm{E}-01$ & $4.14 \mathrm{E}-01$ & 4.12E-02 & $1.69 \mathrm{E}+02$ & 1.48 & 1.41 & 843 & $4.96 \mathrm{E}-01$ & $5.55 \mathrm{E}-01$ & 32 & 30 \\
\hline $241-A W-105$ & $4 \mathrm{E}+03$ & $0 \mathrm{E}+02$ & $09 \mathrm{E}+04$ & $19 E+04$ & $85 E+04$ & $72 E+02$ & $1.55 \mathrm{E}+01$ & $1.91 \mathrm{E}-01$ & $9.09 \mathrm{E}-02$ & $3.22 \mathrm{E}-01$ & $3.20 \mathrm{E}-02$ & $2.48 \mathrm{E}+01$ & 1.25 & 1.08 & 1624 & $6.77 \mathrm{E}-01$ & $8.61 \mathrm{E}-01$ & 22 & 25 \\
\hline Supernatant & 4.12E+03 & $3.87 \mathrm{E}-02$ & $2.42 \mathrm{E}+03$ & $2.34 \mathrm{E}+04$ & $2.14 \mathrm{E}+04$ & 1. $36 \mathrm{E}+02$ & $1.98 \mathrm{E}-02$ & $5.73 \mathrm{E}-05$ & $5.80 \mathrm{E}-05$ & $2.06 \mathrm{E}-04$ & $2.05 \mathrm{E}-05$ & $8.97 \mathrm{E}-00$ & 1.06 & 1.06 & 625 & $9.05 \mathrm{E}-01$ & $9.05 \mathrm{E}-01$ & 23 & 25 \\
\hline Solid Layer & $8.70 \mathrm{E}+03$ & $4.09 \mathrm{E}: 02$ & $1.73 E+04$ & $5.58 \mathrm{E}-04$ & $5.14 \mathrm{E}+04$ & $1.60 \mathrm{E}+03$ & $2.30 \mathrm{E}+01$ & $2.84 \mathrm{E}-01$ & $1.35 \mathrm{E}-01$ & $4.78 \mathrm{E}-01$ & $4.75 \mathrm{E}-02$ & $3.25 \mathrm{E}+01$ & 1.36 & 1.10 & 999 & $5.67 \mathrm{E}-0 \mathrm{~J}$ & $8.39 \mathrm{E}-01$ & 22 & 25 \\
\hline $241-\mathrm{AW} \cdot 106$ & $0 \mathrm{~F}+04$ & $3 E \div 03$ & $: 04$ & $2 \mathrm{E}-04$ & $5 \mathrm{E}+05$ & +04 & 0 & 3 & $1.07 \mathrm{E}-03$ & 3 & 04 & $F+0$ & 1. & 1. & 33 & 1 & 1 & 32 & 29 \\
\hline Supenlatant & $2.69 \mathrm{E}-04$ & $1.81 E-03$ & $4.45 E-04$ & $8.80 E+04$ & $1.15 \mathrm{E}+05$ & $1.61 \mathrm{E}+04$ & $3.98 \mathrm{E}-01$ & $5.29 \mathrm{E}-04$ & $1.31 \mathrm{E}-04$ & 8.41E-04 & $1.41 \mathrm{E}-05$ & $9.96 \mathrm{E}+01$ & 1.25 & 1.25 & 3262 & $7.48 E-01$ & $7.48 \mathrm{E}-01$ & 30 & 29 \\
\hline Solid Layer & $2.72 \mathrm{~F}+04$ & $1.83 E+03$ & $4.50 \mathrm{E}+04$ & $8.90 \mathrm{E}+04$ & $1.16 E+05$ & $1.63 \mathrm{E}+04$ & $1.95 \mathrm{E} \div 01$ & $1.92 \mathrm{E}-02$ & $3.28 \mathrm{E}-03$ & $1.26 \mathrm{E}-02$ & $9.15 \mathrm{E}-04$ & $2.45 \mathrm{E}+02$ & 1.61 & 1.25 & 1072 & $4.31 \mathrm{E}-01$ & 7.46E-01 & 38 & 29 \\
\hline 2 & $1.95 \mathrm{E}+04$ & 03 & $=04$ & 33 & +04 & 03 & +03 & IE: & $1.97 \mathrm{E}-01$ & 1 & $E-02$ & $E+0$ & 1.38 & 3.13 & 863 & 01 & 01 & 56 & 47 \\
\hline Supernatant & $3.16 E+04$ & $8.52 \mathrm{E} \div 02$ & $2.10 \mathrm{E}+04$ & $2.89 E+03$ & $7.24 \mathrm{E}-04$ & $2.98 \mathrm{E}+03$ & $5.18 \mathrm{E}-0 \mathrm{I}$ & $1.49 \mathrm{E}-03$ & $2.23 E-03$ & $9.57 \mathrm{E}-03$ & $4.10 \mathrm{E}-04$ & $2.60 \mathrm{E}+01$ & 1.12 & 1.12 & 464 & $8.62 \mathrm{E}-01$ & $8.62 \mathrm{E}-01$ & 56 & 47 \\
\hline Solid Layer & $8.95 E-01$ & $5 \mathrm{E}+03$ & $6.53 E+04$ & $3.38 \mathbf{E}+03$ & $7.80 \mathrm{E}+04$ & $30 \mathrm{E}+02$ & $3.17 \mathrm{E}+03$ & $2.14 \mathrm{E}+\mathrm{OJ}$ & $3.47 \mathrm{E}-01$ & $1.13 \mathrm{E}+00$ & $9.67 \mathrm{E}-02$ & $1.06 \mathrm{E}+02$ & 1.68 & 1.14 & 399 & $3.98 \mathrm{E}-01$ & $8.14 \mathrm{E}-01$ & 56 & 47 \\
\hline $241-A Y-102$ & $37 \mathrm{E}+03$ & $8.28 \mathrm{E}+02$ & $2.45 E+04$ & $3 E+02$ & $49 \mathrm{E}+04$ & $72 \mathrm{E}+02$ & $1.65 \mathrm{E}+03$ & $1.11 \mathrm{E}+00$ & $\mathrm{E}-02$ & $3.52 \mathrm{E}-01$ & $1.36 \mathrm{E}-02$ & $6.82 \mathrm{E}+01$ & 1.21 & 1. & $3701^{\circ}$ & $41 \mathrm{E}-01$ & $27 \mathrm{E}-01$ & 64 & 45 \\
\hline Supernatant & $7.65 E+03$ & $7.44 \mathrm{E}+02$ & $2.74 \mathrm{E}+04$ & $3.13 E+02$ & $5.30 \mathrm{E}+04$ & $1.05 E+03$ & $8.93 \mathrm{E}-01$ & $1.66 \mathrm{E}-04$ & $2.70 \mathrm{E}-04$ & $1.25 \mathrm{E}-03$ & $2.00 \mathrm{E}-04$ & $1.58 \mathrm{E}+01$ & 1.15 & 1.15 & 3130 & $8.29 \mathrm{E}-01$ & $8.29 \mathrm{E}-01$ & 62 & 45 \\
\hline Solid Layer & $3.69 \mathrm{E} \div 02$ & $3 E+03$ & $25 \mathrm{E}+01$ & $3.10 E+02$ & $7.03 \mathrm{E} \div 04$ & $3.41 \mathrm{E}+02$ & $8.23 E+03$ & $5.52 \mathrm{E}+00$ & $4.04 \mathrm{E}-0 \mathrm{]}$ & $1.75 \mathrm{~F}+00$ & $6.72 \mathrm{E}-02$ & $2.77 \mathrm{E}+02$ & 1.58 & 1.15 & 57 & $3.93 \mathrm{E}-0 \mathrm{I}$ & $8.20 \mathrm{E}-01$ & 72 & 45 \\
\hline $241-A Z-101$ & $1.14 \mathrm{E}+04$ & $5.34 E+02$ & $6.40 E+04$ & $5.50 \mathrm{E}+04$ & $1.16 \mathrm{E}+05$ & $30 \mathrm{E}+03$ & $1.35 E+03$ & $6.23 E+00$ & $10 \mathrm{E}-02$ & $2.86 \mathrm{E}-0 \mathrm{I}$ & 2.82E-02 & $1.29 \mathrm{E}+03$ & 1.24 & 1.22 & 309 & $6.72 \mathrm{E}-01$ & $00 \mathrm{E}-01$ & 73 & 72 \\
\hline Supernatant & $1.13 E+04$ & $5.33 \mathrm{E}+02$ & $6.39 \mathrm{E}+04$ & $5.50 \mathrm{E} \div 04$ & $1.16 E+05$ & $6.30 \mathrm{E}: 03$ & $7.42 E-01$ & $1.23 \mathrm{E}-04$ & $3.43 \mathrm{E}-04$ & $1.20 \mathrm{E}-03$ & $1.24 \mathrm{E}-04$ & $1.34 \mathrm{E}+03$ & 1.22 & 1.22 & 3112 & $6.99 \mathrm{E}-01$ & $6.99 \mathrm{E}-0 \mathrm{I}$ & 72 & 72 \\
\hline Solid Layer & $1.15 E+04$ & $5.40 \mathrm{E}+02$ & $0.47 \mathrm{~L}+1.4$ & $5.56 \mathrm{E}+04$ & tactos & $6.38 \mathrm{E}+03$ & $1.74 \mathrm{E}+04$ & $0.07 \mathrm{~L}-0$. & $1.04 \mathrm{E}+00$ & $3.69 \mathrm{E}+00$ & $3.63 \mathrm{E}-01$ & $7.66 \mathrm{E} \div 02$ & Not & $1.2=$ & . & 3.43E-01 & $7.10 \mathrm{E}-01$ & 88 & 72 \\
\hline $241-A Z-102$ & $2.43 E+03$ & $1.04 \mathrm{E}+03$ & $3.36 E+04$ & $1.33 \mathrm{E}+04$ & $5.72 \mathrm{E}+04$ & $5.54 \mathrm{E}+02$ & $9.12 \mathrm{E}+02$ & $6.61 \mathrm{E}+00$ & $8.18 \mathrm{E}-02$ & $3.11 \mathrm{E}-01$ & $4.59 \mathrm{E}-02$ & $8.16 \mathrm{E}+02$ & 1.16 & 1.13 & 3701 & $8.04 \mathrm{E}-01$ & $8.42 \mathrm{E}-01$ & 69 & 67 \\
\hline Supernatant & $2.42 \mathrm{E}+03$ & $1.04 \mathrm{E}+03$ & $3.36 \mathrm{E}+04$ & $1.33 \mathrm{E}+04$ & $5.72 \mathrm{E}+04$ & $5.54 \mathrm{E}+02$ & $1.75 \mathrm{E}+00$ & $5.55 \mathrm{E}-04$ & $1.77 \mathrm{E}-03$ & $6.49 \mathrm{E}-03$ & $6.27 \mathrm{E}-04$ & $8.39 \Xi+02$ & 1.13 & 1.13 & 3305 & $8.43 \mathrm{E}-01$ & $8.43 \mathrm{E}-0 \mathrm{l}$ & 67 & 67 \\
\hline Solid Layer & $2.45 \mathrm{E}+03$ & $1.05 \mathrm{E}-03$ & $3.40 E+04$ & $1.35 \mathrm{E}+04$ & 3. 170704 & $5.60 \mathrm{E}+02$ & $6.99 \mathrm{E}+03$ & $5.08 \mathrm{E}+01$ & $6.16 \mathrm{E}-01$ & $2.34 \mathrm{E}+00$ & $3.48 \mathrm{E} \cdot 01$ & $6.63 \mathrm{E}+02$ & 1.41 & 1.1 & 20 & $5.48 \mathrm{E}-01$ & $8.41 \mathrm{E}-0 \mathrm{I}$ & 79 & 67 \\
\hline $241-S Y \cdot 101$ & $3.71 E+04$ & $1.10 \mathrm{E}+03$ & $2.13 E+04$ & $1.67 \mathrm{E}+05$ & $1.21 \mathrm{E}+05$ & $6.00 \mathrm{E}+03$ & $9.56 \mathrm{E}+00$ & $7.36 \mathrm{E}-02$ & $1.39 \mathrm{E}-03$ & $6.47 \mathrm{E}-03$ & $2.26 \mathrm{E}-04$ & $6.96 \mathrm{E}+01$ & 1.33 & 1.28 & 4231 & $6.48 \mathrm{E}-01$ & $6.93 \mathrm{E}-01$ & 27 & 24 \\
\hline Supernatant & $3.70 E+04$ & $1.10 \mathrm{E}+03$ & $2.12 E+04$ & $1.67 \mathrm{E}+05$ & $1.21 \mathrm{E}+05$ & $5.99 E+03$ & $3.61 \mathrm{E}-01$ & $1.89 \mathrm{E}-04$ & $1.39 \mathrm{E}-05$ & $6.48 \mathrm{E}-05$ & $2.25 \mathrm{E}-06$ & $4.95 \mathrm{E}+01$ & 1.28 & 1.28 & 3338 & $6.94 \mathrm{E}-01$ & $6.94 \mathrm{E}-01$ & 26 & 24 \\
\hline Solid Layer & $3.74 E \cdot 04$ & $1.12 \mathrm{E}+03$ & $2.15 \mathrm{E}+04$ & $1.68 \mathrm{~F}+05$ & $1.22 \mathrm{E}+05$ & $6.06 \mathrm{E} \div 03$ & $3.85 \mathrm{E} \div 01$ & $3.04 \mathrm{E}-01$ & $5.70 \mathrm{E}-03$ & $2.66 \mathrm{E}-02$ & $9.28 \mathrm{E}-04$ & $1.33 E+02$ & 1.52 & 1.28 & (1) & $5.04 \mathrm{E}-01$ & $6.91 \mathrm{~F}-01$ & 32 & 24 \\
\hline $241-S Y-102$ & $1.10 E-04$ & $1.17 \mathrm{E}+03$ & $2.14 E+04$ & $1.60 E+05$ & $1.13 \mathrm{E}+05$ & $4.51 \mathrm{E}+03$ & $3.04 \mathrm{E}+01$ & $4.13 \mathrm{E}+00$ & $2.20 \mathrm{E}-01$ & $9.58 \mathrm{E}-01$ & $4.49 \mathrm{E}-02$ & $4.71 \mathrm{E}+01$ & 1.25 & 1.22 & 4331 & $6.36 \mathrm{E}-01$ & $6.64 \mathrm{E}-01$ & 31 & 31 \\
\hline Supernatant & $9.54 \mathrm{E}+03$ & $6.84 \mathrm{E}+02$ & $1.43 E+04$ & $1.64 \mathrm{E}+05$ & $1.08 \mathrm{E}+05$ & $4.08 \mathrm{E}+03$ & $5.83 \mathrm{E}-01$ & $5.13 \mathrm{E}-04$ & 5.33 & -04 & $6.88 \mathrm{E}-06$ & 3.3 & 1.20 & 1.20 & 26 & 1 & 01 & 31 & 1 \\
\hline Solid Layer & $2.14 \mathrm{E}+04$ & $4.65 \mathrm{E}+03$ & $7.31 \mathrm{E} \div 04$ & $1.34 \mathrm{E} \cdot 05$ & $1.52 E+05$ & $7.58 \mathrm{E}+03$ & $1.71 E+02$ & $2.37 \mathrm{E}+01$ & $1.26 \mathrm{E}-00$ & $5.49 \mathrm{E}+00$ & $2.57 \mathrm{E}-01$ & $1.33 E+02$ & 1.56 & 1.36 & 605 & $4.72 \mathrm{E}-01$ & $6.32 \mathrm{E}-01$ & 31 & 31 \\
\hline
\end{tabular}


Table B-5. Input Data With Water Addition for Hydrogen Generation Rate Model Calculations for 177 Tanks. (20 sheets)

\begin{tabular}{|c|c|c|c|c|c|c|c|c|c|c|c|c|c|c|c|c|c|c|c|}
\hline Tank & $\begin{array}{c}\mathrm{OH} \\
\text { in liquid } \\
{[\mathrm{OH}]} \\
(\mu \mathrm{g} / \mathrm{mL})\end{array}$ & $\begin{array}{c}\text { TOC } \\
\text { in liquid } \\
|\mathrm{TOC}| \\
(\mu \mathrm{g} / \mathrm{mL})\end{array}$ & $\begin{array}{c}\mathrm{NO}_{2} \\
\text { in liquid } \\
{\left[\mathrm{NO}_{2}\right]} \\
(\mu \mathrm{g} / \mathrm{mL})\end{array}$ & $\begin{array}{c}\mathrm{NO}_{3} \\
\text { in liquid } \\
{\left[\mathrm{NO}_{3}\right]} \\
(\mu \mathrm{g} / \mathrm{mL})\end{array}$ & $\begin{array}{c}\mathrm{Na} \\
\text { in liquid } \\
|\mathrm{Na}| \\
(\mathrm{ug} / \mathrm{mL})\end{array}$ & $\begin{array}{c}\text { AI } \\
\text { in liquid } \\
{[\mathrm{AI}]} \\
(\mu \mathrm{g} / \mathrm{mL})\end{array}$ & $\begin{array}{c}90 \mathrm{Sr} \\
\text { in waste } \\
|\mathrm{Sr}| \\
(\mu \mathrm{Ci} / \mathrm{g})\end{array}$ & $\begin{array}{c}{ }^{241} \mathrm{Am} \\
\text { in waste } \\
{\left[\begin{array}{c}\text { Am241| } \\
(\mu \mathrm{Ci} / \mathrm{g})\end{array}\right.}\end{array}$ & $\begin{array}{c}{ }^{240} \mathrm{Pu} \\
\text { in waste } \\
\{\mathrm{Pu} 240 \mid \\
(\mu \mathrm{Ci} / \mathrm{g})\end{array}$ & $\begin{array}{c}{ }^{239} \mathrm{Pu} \\
\text { in waste } \\
\{\mathrm{Pu} 240] \\
(\mu \mathrm{Ci} / \mathrm{g})\end{array}$ & $\begin{array}{c}{ }^{239} \mathrm{Pu} \\
\text { in waste } \\
{\left[\begin{array}{l}\mathbf{P u} 238] \\
(\mu \mathrm{Ci} / \mathrm{g})\end{array}\right.}\end{array}$ & $\begin{array}{c}{ }^{137} \mathrm{Cs} \\
\text { in waste } \\
{[\mathrm{Cs} \mid} \\
(\mu \mathrm{Ci} / \mathrm{g})\end{array}$ & $\begin{array}{c}\text { Bulk } \\
\text { density } \\
\text { D } \\
(\mathrm{g} / \mathrm{mL})\end{array}$ & $\mid \begin{array}{c}\text { Liquid } \\
\text { density } D_{L} \\
(\mathrm{~g} / \mathrm{ml})\end{array}$ & $\begin{array}{c}\text { Non-RGS } \\
\text { waste } \\
\text { volume } \\
(\mathrm{kL})\end{array}$ & $\begin{array}{c}\text { Bulk } \\
\text { water } \\
{\left[\mathrm{H}_{2} \mathrm{O} \mid\right.} \\
(\mathrm{wt} \%)\end{array}$ & $\begin{array}{c}\text { Liquid } \\
\text { water } \\
\left|\mathrm{H}_{2} \mathrm{O}\right| \\
\left(\mathrm{wt}^{\mathrm{m}} \%\right)\end{array}$ & $\begin{array}{c}\text { Waste } \\
\text { temp. } \\
\text { Tw } \\
\left({ }^{\circ} \mathrm{C}\right)\end{array}$ & $\begin{array}{l}\text { Dome } \\
\text { temp. } \\
\mathrm{Td} \\
\left({ }^{\circ} \mathrm{C}\right)\end{array}$ \\
\hline 241-SY-103 & $4 \mathrm{E}+04$ & $13 E+03$ & $50 \mathrm{E}+05$ & $65 \mathrm{E}+05$ & $2 \mathrm{E}+05$ & 34 & $3 \mathrm{~S}$ & $.98 \mathrm{E}-01$ & $.84 \mathrm{E}-03$ & $.73 \mathrm{E}-02$ & $50 \mathrm{E}-04$ & 2.56 & 1.52 & 1.46 & 27 & $.06 \mathrm{E}-01$ & $36 \mathrm{E}-01$ & 39 & 26 \\
\hline Supernatant & $2.91 \mathrm{E}+04$ & $6.07 E+03$ & $1.49 \mathrm{E}+05$ & $1.64 \mathrm{E}+05$ & $10 \mathrm{E}+05$ & $3.67 \mathrm{E} \div 04$ & $.02 \mathrm{E}+00$ & $6.81 E-03$ & $.83 \mathrm{E}-06$ & 3.65E-05 & $.28 \mathrm{E}-06$ & $2.87 \mathrm{E}+02$ & 1.46 & 1.46 & $154 !$ & $4.41 \mathrm{E}-01$ & $4.4 ! \mathrm{E}-01$ & 33 & 26 \\
\hline Solid L.ayer & $2.99 E+04$ & $6.22 \mathrm{E}+03$ & $1.52 \mathrm{E}+05$ & $1.68 \mathrm{~F}+05$ & $2.15 \mathrm{E}+05$ & $3.76 \mathrm{E}+04$ & $3.28 \mathrm{E}+01$ & $6.35 \mathrm{E}-01$ & $1.26 \mathrm{E}-02$ & $5.89 \mathrm{E}-02$ & $2.05 \mathrm{E}-03$ & $2.20 \mathrm{E}+02$ & 1.61 & 1.47 & 1203 & $3.65 \mathrm{E}-01$ & $4.31 \mathrm{E}-01$ & 45 & 26 \\
\hline $241-\mathrm{A}-101$ & $4.27 \mathrm{E}+04$ & $3.74 \mathrm{E}+03$ & $1.65 \mathrm{E}+05$ & $2.33 \mathrm{E}+05$ & $2.78 \mathrm{E}+05$ & $5.18 \mathrm{E}+04$ & $8.11 \mathrm{E}+01$ & $2.70 \mathrm{E}-01$ & $1.33 \mathrm{E}-02$ & $5.53 \mathrm{E}-02$ & $2.49 \mathrm{E}-03$ & $1.72 \mathrm{E}+02$ & 1.69 & 1.49 & 1075 & $3.17 \mathrm{E}-01$ & 4.69E- -01 & 46 & 29 \\
\hline Supernatant & $4.27 \mathrm{E}+04$ & $3.74 E+03$ & $1.65 \mathrm{~F}+05$ & $2.33 \mathrm{E}+05$ & $2.78 \mathrm{E}+05$ & $5.18 \mathrm{E}+04$ & $8.11 \mathrm{E}+0 \mathrm{l}$ & $2.70 \mathrm{E}-01$ & 1.33E-02 & $5.53 \mathrm{E}-02$ & $2.49 \mathrm{E}-03$ & $1.72 \mathrm{E}+02$ & 1.49 & 1.49 & 38 & $4.69 \mathrm{E}-01$ & 4. $69 \mathrm{E}-01$ & 0 & 0 \\
\hline Solid Layer & $4.27 E+04$ & $3.74 \mathrm{E}: 03$ & $1.65 \mathrm{E}+05$ & $2.33 \mathrm{~F}+0.5$ & $.78 E+05$ & $5.18 \mathrm{E}+04$ & $8.11 \mathrm{E}+01$ & $2.70 \mathrm{E}-0 \mathrm{I}$ & 1. $33 \mathrm{E}-02$ & $5.53 \mathrm{E}-02$ & $2.49 \mathrm{E}-03$ & $1.72 \mathrm{E}+02$ & 1.70 & 1.49 & 1037 & $3.12 \mathrm{E}-01$ & 4.69E-01 & 46 & 29 \\
\hline $241 \cdot \mathrm{A} \cdot 102$ & $1.34 \mathrm{E}+04$ & $3.43 \mathrm{E}+03$ & $1.34 \mathrm{E} \cdot 05$ & $1.47 E+05$ & $.55 \mathrm{E}+05$ & $3.17 \mathrm{E} \div 04$ & $2.69 \mathrm{E}+02$ & $8.59 \mathrm{E}-0 \mathrm{I}$ & $2.51 \mathrm{E}-01$ & $1.08 \mathrm{E}+00$ & 4. $44 \mathrm{E}-02$ & $1.41 \mathrm{E}+02$ & 1.53 & 1.44 & 189 & $4.58 \mathrm{E}-01$ & $5.33 \mathrm{E}-01$ & 24 & 33 \\
\hline Supematant & $4.17 \mathrm{~F}+03$ & $1.06 \mathrm{E}+03$ & $4.15 E+04$ & $4.55 \mathrm{E}+04$ & $81 E-04$ & $9.87 \mathrm{E}+0.3$ & $2.35 \mathrm{E}-01$ & $2.28 \mathrm{E}-04$ & $1.80 \mathrm{E}-04$ & $7.78 \mathrm{E}-04$ & $.19 \mathrm{E}-05$ & $1.05 \mathrm{E}+02$ & 1.14 & 1.14 & 50 & $8.21 \mathrm{E}-01$ & $8.21 \mathrm{E}-01$ & 33 & 33 \\
\hline Solid L.ayer & $1.73 \mathrm{E}+04$ & $4.42 E-03$ & $1.72 \mathrm{~F} .05$ & $1.89 \mathrm{E}+05$ & $2.00 \mathrm{E}+0.5$ & $4.09 E+04$ & $3.34 E+02$ & $1.07 \mathrm{E}+00$ & $3.12 \mathrm{E}-01$ & $1.35 \mathrm{E}+00$ & $5.52 \mathrm{E}-02$ & $1.50 \mathrm{E}+02$ & 1.67 & 1.57 & 139 & $3.70 \mathrm{E}-01$ & $4.62 \mathrm{E}-01$ & 23 & 33 \\
\hline $241-\mathrm{A}-103$ & $4.71 \mathrm{E}+04$ & $8.03 \mathrm{E}+03$ & $1.20 \mathrm{E}+05$ & $2.34 E+05$ & $27 k+05$ & $3.43 \mathrm{E}+04$ & $6.18 \mathrm{E}+01$ & $1.18 \mathrm{E}-01$ & $2.53 \mathrm{E}-02$ & $1.10 \mathrm{E}-01$ & $4.38 \mathrm{E}-03$ & $1.41 \mathrm{E}+02$ & 1.36 & 1.49 & 1472 & 4.25E-01 & $5.12 \mathrm{E}-01$ & 39 & 29 \\
\hline Supernatant & $1.52 \mathrm{E}+04$ & $57 \mathrm{E}+03$ & $3.85 E+04$ & $7.49 E+04$ & $29 E+04$ & $1.10 \mathrm{E}+04$ & 5.18E- & $8.36 \mathrm{E}-\mathrm{C}$ & $5 \mathrm{E}-$ & $5.82 \mathrm{E}-$ & $39 \mathrm{E}-04$ & $7.23 \mathrm{E}+0$ & 1.16 & 16 & 55 & $7.99 \mathrm{E}-01$ & $99 \mathrm{E}-01$ & 39 & 29 \\
\hline Solid Layer & $4.88 \mathrm{E}+04$ & $8.31 E+03$ & $1.24 \mathrm{E}+05$ & $2.42 \mathrm{E}+05$ & $2.35 \mathrm{E}-05$ & $3.55 \mathrm{E}+04$ & $6.39 E+01$ & $1.21 \mathrm{E}-01$ & $2.61 \mathrm{E}-02$ & $1.14 \mathrm{E}-01$ & $4.52 \mathrm{E}-03$ & $1.43 \mathrm{E}+02$ & 1.37 & 1.51 & 1417 & 4.13E-01 & $5.03 \mathrm{E}-01$ & 39 & 29 \\
\hline $24 \mathrm{I}-\mathrm{A} \cdot 104$ & $7 \varepsilon \div 04$ & $2.98 \mathrm{E}+03$ & $60 \mathrm{E}+04$ & $96 \mathrm{E}+04$ & $2 \mathrm{E}+04$ & $94 E+03$ & $29 \mathrm{E}+04$ & 4 & $49 \mathrm{E}+00$ & $6.79 E+00$ & $25 \mathrm{E}-01$ & $6.18 E+02$ & 1.00 & 17 & 144 & $2.30 \mathrm{E}-01$ & $7.50 \mathrm{E}-01$ & 74 & 34 \\
\hline Supernatant & $4.27 \mathrm{E}+04$ & $2.98 \mathrm{E}+03$ & $3.60 \mathrm{E}+04$ & $7.96 \mathrm{E}+04$ & $32 \mathrm{E}+04$ & $4.94 \mathrm{E}+03$ & $2.29 \mathrm{E}+04$ & $8.04 \mathrm{E}+00$ & $1.49 \mathrm{E}+00$ & $6.79 \mathrm{E}+00$ & $2.25 \mathrm{E}-01$ & 6 & 1.17 & 7 & 38 & 1 & 01 & 0 & 0 \\
\hline Solid Layer & $4.27 \mathrm{E}+04$ & $2.98 \mathrm{E}+03$ & $3.60 E+04$ & $7.96 \mathrm{E}+04$ & $8.32 E \div 04$ & $4.94 \mathrm{E} \div 03$ & $2.29 \mathrm{E}+04$ & $8.04 \mathrm{E}+00$ & $1.49 E+00$ & $6.79 \mathrm{E}-00$ & $2.25 \mathrm{E}-01$ & $6.18 \mathrm{E}+02$ & 0.95 & 1.17 & 106 & $0.00 \mathrm{E}+00$ & $7.50 \mathrm{E}-01$ & 74 & 34 \\
\hline $241-A-105$ & 01 & 4 & ol & \#DIV/o! & \#DIV/0! & $/ 0 !$ & 04 & . & $6.35 \mathrm{E}-01$ & $50 \mathrm{E}+00$ & $22 \mathrm{E}-01$ & $5.00 \mathrm{E}+02$ & 1.46 & \#DIV/0! & 177 & $0.00 \mathrm{E}+00$ & $0.00 \mathrm{E}+00$ & 0 & 0 \\
\hline Supernatant & $4.27 \mathrm{E}+04$ & $2.98 \mathrm{E}+03$ & $3.60 \mathrm{E}+04$ & $7.96 \mathrm{E}+04$ & $32 \mathrm{E}+04$ & $E+03$ & 1 & 2. & $6.35 \mathrm{E}$ & $2.60 \mathrm{E}+00$ & $1.22 \mathrm{E}-01$ & $5.00 \mathrm{E}+02$ & 1.17 & 1.17 & 3 & $0.00 \mathrm{E}+00$ & $0.00 E+00$ & 0 & 0 \\
\hline Solid Layer & $4.27 \mathrm{E}+04$ & $2.98 E+03$ & $3.60 E+04$ & $7.96 \mathrm{E}+04$ & $8.32 E+04$ & $4.94 \mathrm{E}+03$ & $1.35 \mathrm{E} \div 04$ & $2.63 E+01$ & $6.35 \mathrm{E}-0 \mathrm{]}$ & $2.60 \mathrm{E}+00$ & 1. $22 \mathrm{E}-01$ & $5.00 \mathrm{E}+02$ & 1.54 & 1.17 & 139 & $0.00 \mathrm{E}+00$ & $0.00 E+00$ & 0 & 0 \\
\hline $241-A-106$ & 4 & 4 & -04 & 4 & 04 & 3 & 02 & $1.14 \mathrm{E}+00$ & $E 0$ & $E+00$ & $93 \mathrm{E}-02$ & $1.26 \mathrm{E}+02$ & 16 & 1.17 & 337 & $76 \mathrm{E}-01$ & $50 \mathrm{E}-01$ & 53 & 39 \\
\hline Supernatant & $4.27 \mathrm{E} \div 04$ & $1.10 \mathrm{E}-04$ & $3.60 \mathrm{~F}: 104$ & $7.96 \mathrm{E}+04$ & $8.32 \mathrm{E}+04$ & $4.94 \mathrm{E}+03$ & $9.62 \mathrm{E}+02$ & $1.14 E+00$ & 4.64E-01 & $2.03 \mathrm{E}+00$ & 7.93E-02 & $1.26 \mathrm{E}+02$ & 1.17 & 1.17 & 3 & 1 & $E-01$ & 0 & 0 \\
\hline Solid Layer & $4.27 \mathrm{E}+04$ & $1.10 \mathrm{E}+04$ & $3.60 \mathrm{E}+04$ & $7.96 \mathrm{E}+04$ & $8.32 \mathrm{E}+04$ & $4.94 E+03$ & $9.62 \mathrm{E}+02$ & $1.14 \mathrm{E}+00$ & $4.64 \mathrm{E}-01$ & $2.03 \mathrm{E}+00$ & $7.93 \mathrm{E}-02$ & $1.26 \mathrm{E}+02$ & 1.70 & 1.17 & 299 & $3.43 \mathrm{E}-01$ & $7.50 \mathrm{E}-01$ & 53 & 39 \\
\hline $241-A X-101$ & $0 \mathrm{E}+04$ & $8 E+03$ & $3 E+05$ & $2 E+05$ & 95 & 04 & I & 1 & $1.17 \mathrm{E}-02$ & 2 & 03 & 02 & 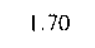 & 1.53 & 1 & 01 & $.31 \mathrm{E}-01$ & 35 & 28 \\
\hline Supernatant & $3.90 E+04$ & $3.98 \mathrm{E}+03$ & $1.73 \mathrm{E}+05$ & $2.72 \mathrm{E}+05$ & $2.82 \mathrm{E}+05$ & $5.03 \mathrm{E}+04$ & $9.59 \mathrm{E}+01$ & $1.71 \mathrm{E}-01$ & $1.17 \mathrm{E}-02$ & $5.02 \mathrm{E}-02$ & $2.08 \mathrm{E}-03$ & $1.76 \mathrm{E}+02$ & 1.53 & 1.53 & 38 & $4.31 \mathrm{E}-01$ & $4.31 \mathrm{E}-01$ & 0 & 0 \\
\hline Solid Layer & $3.90 E+04$ & $3.98 \mathrm{E}+03$ & $1.73 \mathrm{E}+05$ & $2.72 \mathrm{E}+05$ & $2.82 \mathrm{E}+05$ & $5.03 \mathrm{E}+04$ & $9.59 \mathrm{E}+01$ & $1.71 \mathrm{E}-01$ & $1.17 \mathrm{E}-02$ & $5.02 \mathrm{E}-02$ & $2.08 \mathrm{E}-03$ & $1.76 \mathrm{E}+02$ & 1.70 & 1.53 & 1353 & 3.40E-01 & 4.31E-01 & 35 & 28 \\
\hline 241-AX-102 & $3.89 \mathrm{E}+04$ & $.30 \mathrm{E}+04$ & $1.07 \mathrm{E}+05$ & $1.76 \mathrm{E}+05$ & .05 & 84 & 3 & 01 & 01 & 01 & -02 & 02 & 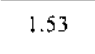 & 9 & 151 & 1 & 01 & 24 & 24 \\
\hline Supernatant & $3.89 \mathrm{E}+04$ & $2.30 \mathrm{E}+04$ & $1.07 \mathrm{E}+05$ & $1.76 \mathrm{E}+05$ & $1.98 \mathrm{E}+05$ & $2.75 \mathrm{E}+04$ & $1.29 \mathrm{E} \cdot 03$ & $1.20 E+01$ & $2.73 \mathrm{E}-01$ & $8.55 F_{-}-01$ & $7.90 \mathrm{E}-02$ & $1.38 \mathrm{E}+02$ & 1.39 & 1.39 & 38 & $5.10 \mathrm{E}-01$ & $5.10 \mathrm{E}-01$ & 0 & 0 \\
\hline Solid Layer & $3.89 \mathrm{E}+04$ & $2.30 \mathrm{E}+04$ & $1.07 \mathrm{E}+05$ & $1.76 \mathrm{E} \cdot 05$ & $98 \mathrm{E}+05$ & $2.75 \mathrm{E}+04$ & $1.29 \mathrm{E}+03$ & $1.20 \mathrm{E}+01$ & $2.73 \mathrm{E}-01$ & $8.55 \mathrm{E}-01$ & $7.90 \mathrm{E}-02$ & $1.38 E+02$ & 1.58 & 1.39 & 113 & $3.56 \mathrm{E}-01$ & $5.10 \mathrm{E}-01$ & 24 & 24 \\
\hline
\end{tabular}


RPP-5926 REV 6

Table B-5. Input Data With Water Addition for Hydrogen Generation Rate Model Calculations for 177 Tanks. (20 sheets)

\begin{tabular}{|c|c|c|c|c|c|c|c|c|c|c|c|c|c|c|c|c|c|c|c|}
\hline Tank & $\begin{array}{c}\text { OH } \\
\text { in liquid } \\
{[\mathrm{OH} \mid} \\
(\mu \mathrm{g} / \mathrm{mL})\end{array}$ & $\begin{array}{c}\text { TOC } \\
\text { in liquid } \\
\text { [TOC) } \\
(\mu \mathrm{g} / \mathrm{mL})\end{array}$ & $\begin{array}{c}\mathrm{NO}_{2} \\
\text { in liquid } \\
{\left[\mathrm{NO}_{2}\right]} \\
(\mu \mathrm{g} / \mathrm{mL})\end{array}$ & $\begin{array}{c}\mathrm{NO}_{3} \\
\text { in liquid } \\
{\left[\mathrm{NO}_{3}\right]} \\
(\mu \mathrm{g} / \mathrm{mL})\end{array}$ & $\begin{array}{c}\mathrm{Na} \\
\text { in liquid } \\
|\mathrm{Na}| \\
(\mathrm{ug} / \mathrm{mL})\end{array}$ & $\begin{array}{c}\text { Al } \\
\text { in liquid } \\
{[\mathrm{Al}]} \\
(\mu \mathrm{g} / \mathrm{mL})\end{array}$ & $\begin{array}{c}90 \mathrm{Sr} \\
\text { in waste } \\
\text { [Sr] } \\
(\mu \mathrm{Ci} / \mathrm{g})\end{array}$ & $\begin{array}{c}{ }^{241} \mathrm{Am} \\
\text { in waste } \\
{[\text { Am241] }} \\
(\mu \mathrm{Ci} / \mathrm{g})\end{array}$ & $\begin{array}{c}{ }^{240} \mathrm{Pu} \\
\text { in waste } \\
{[\mathrm{Pu} 240]} \\
(\mu \mathrm{Ci} / \mathrm{g})\end{array}$ & $\begin{array}{c}{ }^{239} \mathrm{Pu} \\
\text { in waste } \\
{[\mathrm{Pu} 240 \mid} \\
(\mu \mathrm{Ci} / \mathrm{g})\end{array}$ & $\begin{array}{c}{ }^{238} \mathrm{Pu}_{\mathrm{u}} \\
\text { in waste } \\
{\left[\mathrm{P}_{\mathrm{u} 238]}\right.} \\
(\mu \mathrm{Ci} / \mathrm{g})\end{array}$ & $\begin{array}{c}{ }^{137} \mathrm{Cs} \\
\text { in waste } \\
{[\mathrm{Cs} \mid} \\
(\mu \mathrm{Ci} / \mathrm{g})\end{array}$ & $\begin{array}{c}\text { Bulk } \\
\text { density } \\
D \\
(\mathrm{~g} / \mathrm{mL})\end{array}$ & $\mid \begin{array}{c}\text { Liquid } \\
\text { density } \mathrm{D}_{\mathrm{L}} \\
(\mathrm{g} / \mathrm{ml})\end{array}$ & $\begin{array}{c}\text { Non-RGS } \\
\text { waste } \\
\text { volume } \\
(\mathrm{kL})\end{array}$ & $\begin{array}{l}\text { Bulk } \\
\text { water } \\
{\left[\mathrm{H}_{2} \mathrm{O} \mid\right.} \\
\left(\mathrm{wt}^{4} \%\right)\end{array}$ & $\begin{array}{c}\text { Liquid } \\
\text { water } \\
{\left[\mathrm{H}_{2} \mathrm{O}\right]} \\
(w t \%)\end{array}$ & $\begin{array}{c}\text { Waste } \\
\text { temp. } \\
\mathrm{Tw} \\
\left(^{\circ} \mathrm{C}\right)\end{array}$ & $\begin{array}{c}\text { Dome } \\
\text { temp. } \\
\text { Td } \\
\text { ("C) }\end{array}$ \\
\hline $241-A X-103$ & $39 E+04$ & $4.03 \mathrm{E}+03$ & $22 \mathrm{E}+05$ & $1.91 \mathrm{E}+05$ & $36 \mathrm{E}+05$ & $3.79 \mathrm{E} \div(04$ & $6.98 \mathrm{E}+02$ & $.14 E+00$ & 3.66E-02 & $1.45 \mathrm{E}-01$ & $6.80 \mathrm{E}-03$ & $1.81 \mathrm{E}+02$ & 1.57 & 1.45 & 441 & 4.47E-01 & 4.91E-01 & 38 & 31 \\
\hline Supernatant & $3.89 \mathrm{E}+04$ & $4.03 \mathrm{E}+03$ & $1.22 \mathrm{E}+05$ & $1.91 E+05$ & $2.36 \mathrm{E}+105$ & $3.79 \mathrm{E}+04$ & $6.98 \mathrm{E}+02$ & $1.14 \mathrm{E}+00$ & $3.66 \mathrm{E}-02$ & $1.45 \mathrm{E}-01$ & $6.80 \mathrm{E}-03$ & $1.81 \mathrm{E} \div 02$ & 1.45 & 1.45 & 38 & $4.91 \mathrm{E}-01$ & 4.91E-01 & 0 & 0 \\
\hline Solid Layer & $3.89 \mathrm{E}+04$ & $4.03 \mathrm{E}+03$ & $1.22 \mathrm{E}+05$ & $1.91 E+05$ & $2.36 \mathrm{E}+05$ & $3.79 E+04$ & $6.98 \mathrm{E}+02$ & $1.14 E+00$ & $3.66 \mathrm{E}-02$ & $1.45 \mathrm{E}-01$ & $6.80 \mathrm{E}-03$ & $1.81 \mathrm{E}+02$ & 1.58 & 1.45 & 403 & 4.43E-01 & $4.91 \mathrm{E}-01$ & 38 & 31 \\
\hline $241-\mathrm{AX}-104$ & $3.891+04$ & $2.98 \mathrm{E} \cdot 03$ & $3.60 E+04$ & $7.96 \mathrm{E}+04$ & $8.32 \mathrm{E}-04$ & $4.94 \Sigma+03$ & $4.07 \mathrm{E}+04$ & $1.88 \mathrm{E}+01$ & $1.31 E+00$ & $5.34 \mathrm{E}+00$ & $2.52 \mathrm{E}-01$ & $9.86 \mathrm{E}+02$ & 1.44 & 1.17 & 66 & $3.95 \mathrm{E}-01$ & $7.50 \mathrm{E}-0 \mathrm{I}$ & 32 & 31 \\
\hline Supcriatant & $3.89 \mathrm{E}-04$ & $2.98 \mathrm{E}+03$ & $3.60 \mathrm{E}+04$ & $7.96 \mathrm{E}+04$ & $8.32 F+04$ & $4.94 \mathrm{E}+03$ & $4.07 \mathrm{E}+04$ & $1.88 \mathrm{E}+01$ & $1.31 E+00$ & $5.34 \mathrm{E}+00$ & $2.52 \mathrm{E}-01$ & $9.86 E+02$ & 1.17 & 1.17 & 38 & $7.50 \mathrm{E}-01$ & $7.50 \mathrm{E}-0 \mathrm{~J}$ & 0 & 0 \\
\hline Solid I.ayer & $3.89 \mathrm{E} \div 04$ & $2.98 E+03$ & $3.60 \mathrm{E}+04$ & $7.96 \mathrm{E}+04$ & $8.32 \mathrm{E}+04$ & $4.94 \mathrm{E}+03$ & $4.07 \mathrm{E}+04$ & $1.88 \mathrm{E}+01$ & $1.31 \mathrm{E}+00$ & $5.34 \mathrm{E}+00$ & $2.52 \mathrm{E}-01$ & $9.86 \mathrm{E}+02$ & 1.80 & 1.17 & 28 & 8. $23 \mathrm{E}-02$ & 7.50E-01 & 32 & 31 \\
\hline $241-\mathrm{B}-101$ & $1.70 \mathrm{E}+04$ & $6.77 \mathrm{E}+00$ & $1.32 E+05$ & $3.56 \mathrm{E}+05$ & $2.26 \mathrm{E}+05$ & $7.23 \mathrm{E}+02$ & $3.26 \mathrm{E}+02$ & $2.28 E+00$ & $1.55 E-01$ & $5.02 \mathrm{E}-01$ & $4.39 \mathrm{E}-02$ & $3.48 \mathrm{E}-00$ & 1.51 & 1.53 & 449 & 4. $11 \mathrm{E}-01$ & $5.00 \mathrm{E}-01$ & 38 & 33 \\
\hline Supernatant & $3.70 \mathrm{E}+04$ & $6.77 \mathrm{E}+00$ & $1.32 E+05$ & $3.56 \mathrm{E}+05$ & $2.26 \mathrm{E}+05$ & $7.23 E+02$ & $3.26 \mathrm{E}+02$ & $2.28 \mathrm{E}+00$ & $1.55 \mathrm{E}-01$ & $5.02 \mathrm{E}-01$ & $4.39 \mathrm{E}-02$ & $3.48 \mathrm{E} \div 00$ & 1.53 & 1.53 & 38 & $5.00 \mathrm{E}-01$ & $5.00 \mathrm{E}-01$ & 0 & 0 \\
\hline Solid Layer & $1.70 E+04$ & $6.77 \mathrm{E}+00$ & $1.32 \mathrm{E}: 05$ & $3.56 \mathrm{E} \div 05$ & $2.26 \mathrm{E}+05$ & $7.23 \mathrm{E}+02$ & $3.26 \mathrm{E}+02$ & $2.28 \mathrm{E}+00$ & $1.55 \mathrm{E}-01$ & $5.02 \mathrm{E}-01$ & $4.39 \mathrm{E}-02$ & $3.48 \mathrm{E}+00$ & 1.51 & 1.53 & 411 & 4.03E-01 & $5.00 \mathrm{E}-01$ & 38 & 33 \\
\hline 24!-B-102 & $24 E+04$ & $2.47 \mathrm{E}+00$ & $23 E+03$ & $1.59 \mathrm{E}+05$ & $8.25 E+04$ & $2.64 \mathrm{E}+02$ & $5.84 \mathrm{E}-0 \mathrm{l}$ & $4.15 E-04$ & $2.53 \mathrm{E}-03$ & $2.24 \mathrm{E}-02$ & $1.66 \mathrm{E}-04$ & $2.57 \mathrm{E}+00$ & 1.43 & 1.19 & 159 & $5.64 \mathrm{E}-01$ & 47E-0I & 18 & 22 \\
\hline Supenatant & $4.82 \mathrm{E}+03$ & $9.59 \mathrm{E}-01$ & $2.40 E+03$ & $6.15 \mathrm{E}+04$ & $3.20 \mathrm{E}+04$ & $1.03 \mathrm{E}+02$ & $5.60 \mathrm{E}-01$ & $4.09 \mathrm{E}-04$ & $1.56 \mathrm{E}-04$ & $1.38 \mathrm{E}-03$ & $1.03 \mathrm{E}-05$ & $2.36 \mathrm{E}+00$ & 1.07 & 1.07 & 53 & $8.99 \mathrm{E}-01$ & $8.99 \mathrm{E}-01$ & 18 & 22 \\
\hline Solid Layer & $1.70 E+04$ & $3.38 \mathrm{E}+00$ & $8.52 \mathrm{E} .03$ & $2.17 \mathrm{E}+05$ & $1.13 \mathrm{~F}-05$ & $3.61 E+02$ & $5.91 \mathrm{E}-01$ & $4.17 \mathrm{E}-04$ & $3.32 \mathrm{E}-03$ & $2.93 \mathrm{E} \cdot 02$ & $2.18 \mathrm{E}-04$ & $2.64 \mathrm{E}+00$ & 1.61 & 1.26 & 106 & 4.52E-01 & $6.96 \mathrm{E}-01$ & 18 & 22 \\
\hline $241-B-103$ & +04 & $3.38 \mathrm{E}+00$ & $8.50 \mathrm{E}+03$ & $18 \mathrm{E}-05$ & $13 E+05$ & $3.62 \mathrm{E} \div 02$ & $1.40 \mathrm{E}+00$ & 23E-03 & $1.70 \mathrm{E}-02$ & $1.50 \mathrm{E}-01$ & $12 \mathrm{E}-03$ & $2.65 \mathrm{E}+00$ & 1.56 & 26 & 249 & $5.19 \mathrm{E}-01$ & $6,96 \mathrm{E}-01$ & 17 & 23 \\
\hline Supernatant & $1.70 \mathrm{E}+04$ & $3.38 \mathrm{E}+00$ & $8.50 \mathrm{E}+03$ & $2.18 \mathrm{E} \cdot 05$ & $1.13 E+05$ & $3.62 \mathrm{E}+02$ & $1.40 \mathrm{E}+00$ & $1.23 \mathrm{E}-03$ & $1.70 \mathrm{E}-02$ & $1.50 \mathrm{E}-01$ & $1.12 \mathrm{E}-03$ & $2.65 E+00$ & 1.26 & 1.26 & 38 & $6.96 \mathrm{E}-01$ & $6.96 \mathrm{E}-01$ & 0 & 0 \\
\hline Solid Layer & $1.70 \mathrm{E}+04$ & $3.38 \mathrm{~F}+00$ & $8.50 \mathrm{E}+03$ & $2.18 \mathrm{E}+05$ & $1.13 \mathrm{E}+05$ & $3.62 \mathrm{E}+02$ & $1.40 \mathrm{E}+00$ & $1.23 \mathrm{E}-03$ & $1.70 \mathrm{E}-02$ & $1.50 \mathrm{E}-01$ & $1.12 \mathrm{E}-03$ & $2.65 \mathrm{E}+00$ & 1.61 & 1.26 & 211 & 4.95E-01 & $6.96 \mathrm{E}-01$ & 17 & 23 \\
\hline 241-B-104 & $1.70 \mathrm{E} \cdot 04$ & $38 E+00$ & $8.49 \mathrm{E}+03$ & $2.16 \mathrm{E}+05$ & $1.13 E+05$ & $3.62 \mathrm{E}+02$ & $1.98 \mathrm{E}+00$ & $2.44 \mathrm{E}-03$ & $4.71 \mathrm{E}-03$ & $4.40 \mathrm{E}-02$ & $3.03 \mathrm{E}-04$ & $6.62 \mathrm{E}+00$ & 1.38 & 1.26 & 14 & 4. $80 \mathrm{E}-01$ & $6.96 \mathrm{E}-01$ & 17 & 20 \\
\hline Supenatant & $1.70 \mathrm{E}+04$ & $3.38 \mathrm{E}+00$ & $8.49 \mathrm{E}+03$ & $2.16 \mathrm{E}+05$ & $1.13 E+05$ & $3.62 \mathrm{E}+02$ & $1.98 \mathrm{E}+00$ & $2.44 \mathrm{E}-03$ & $4.71 \mathrm{E}-03$ & $4.40 \mathrm{E}-02$ & $3.03 \mathrm{E}-04$ & $6.62 \mathrm{E}+00$ & 1.26 & 1.26 & 38 & $6.96 \mathrm{E}-01$ & $6.96 \mathrm{E}-01$ & 0 & 0 \\
\hline Solid Layer & $1.70 \mathrm{E}+04$ & $3.38 \mathrm{E}+00$ & $8.49 \mathrm{E}+03$ & $2.16 \mathrm{E}+05$ & $1.13 \mathrm{E}+05$ & $3.62 \mathrm{E}+02$ & $1.98 \mathrm{E}+00$ & $2.44 \mathrm{E}-03$ & $4.71 \mathrm{E}-03$ & $4.40 \mathrm{E}-02$ & $3.03 \mathrm{E}-04$ & $6.62 \mathrm{E}+00$ & 1.38 & 1.26 & 1417 & $4.75 \mathrm{E}-01$ & $6.96 \mathrm{E}-01$ & 17 & 20 \\
\hline $241-B-105$ & $1.70 \mathrm{E}+04$ & $3.38 \mathrm{E}+00$ & $8.51 \mathrm{E} \div 03$ & $16 \mathrm{E}+05$ & $13 \mathrm{E} \div 05$ & 3.62 & $5.56 \mathrm{E}-01$ & $7.57 \mathrm{E}-04$ & $3.87 \mathrm{E}-03$ & $3.43 \mathrm{E}-02$ & 53E-04 & 00 & 1 & 1.26 & 1136 & 4.39E-01 & $6.96 \mathrm{E}-01$ & 19 & 19 \\
\hline Supenatant & $1.70 \mathrm{E}+04$ & $3.38 E+00$ & $8.51 \mathrm{E}+03$ & $2.16 \mathrm{E}+05$ & $1.13 E+05$ & $3.62 \mathrm{E}+02$ & $5.56 \mathrm{E}-01$ & $7.57 \mathrm{E}-04$ & $3.87 \mathrm{E}-03$ & $3.43 \mathrm{E}-02$ & $2.53 \mathrm{E}-04$ & $2.34 \mathrm{E}+00$ & 1.26 & 1.26 & 38 & $6.96 \mathrm{E}-01$ & $6.96 \mathrm{E}-01$ & 0 & 0 \\
\hline Solid Layer & $1.70 \mathrm{E}+04$ & $3.38 \mathrm{E}+00$ & $8.51 \mathrm{E}+03$ & $2.16 \mathrm{E}+05$ & $1.13 \mathrm{E}+05$ & $3.62 \mathrm{E} \cdot 02$ & $5.56 \mathrm{E}-01$ & $7.57 \mathrm{E}-04$ & $3.87 \mathrm{E}-03$ & $3.43 \mathrm{E}-02$ & $2.53 \mathrm{E}-04$ & $2.34 \mathrm{E}+00$ & 1.65 & 1.26 & 1098 & 4. $32 \mathrm{E}-01$ & $6.96 \mathrm{E}-01$ & 19 & 19 \\
\hline $24 !-13-106$ & $1.57 \mathrm{E}+04$ & $3.12 \mathrm{E}+\infty 0$ & $7.87 E+03$ & $2.01 E+05$ & $1.05 E+05$ & $3.33 \mathrm{E}+02$ & $4.6 \mathrm{JE}+01$ & $9.33 \mathrm{E}-03$ & $2.95 \mathrm{E}-03$ & $2.74 \mathrm{E}-02$ & $1.91 \mathrm{E}-04$ & $1.46 \mathrm{E}+01$ & 1.35 & 1.10 & 502 & $6.09 \mathrm{E}-01$ & $6.90 巨-01$ & 23 & 21 \\
\hline Supernatant & $1.62 \mathrm{E}+03$ & $3.23 \mathrm{E}-01$ & $8.12 \mathrm{E}+02$ & $2.07 E+04$ & $1.08 \mathrm{E} \div 04$ & $3.44 \mathrm{E}+0 \mathrm{I}$ & $1.98 \mathrm{E}-01$ & $1.44 \mathrm{E}-04$ & $5.50 \mathrm{E}-05$ & $4.87 \mathrm{E}-04$ & 3.61E-06 & $8.32 \mathrm{E}-01$ & 1.02 & 1.02 & 42 & $9.61 E-01$ & $9.61 \mathrm{E}-01$ & 18 & 21 \\
\hline Solid Layer & $1.70 \mathrm{E}+04$ & $3.38 \mathrm{E}+00$ & $8.50 \mathrm{E}+03$ & $2.17 \mathrm{E}+05$ & $1.13 \mathrm{E}+05$ & $3.60 \mathrm{E}+02$ & $4.92 \mathrm{E}+01$ & $9.95 \mathrm{E}-03$ & $3.14 \mathrm{E}-03$ & $2.92 \mathrm{E}-02$ & 2.04E-04 & $1.55 \mathrm{E}+0 \mathrm{I}$ & 1.38 & 1.19 & 460 & -01 & $6.72 \mathrm{E}-01$ & 23 & 21 \\
\hline $241-B \cdot 107$ & $1.70 \mathrm{E}+04$ & $5.04 \mathrm{E}+00$ & $6.51 \mathrm{E}+03$ & $3.31 E+05$ & $1.46 \mathrm{E}+05$ & $2.01 \mathrm{E}+01$ & $3.22 \mathrm{E} \div 00$ & $4.59 \mathrm{E}-03$ & $4.74 \mathrm{E}-03$ & $4.03 \mathrm{E}-02$ & $3.27 \mathrm{E}-04$ & $1.10 \mathrm{E}-0 \mathrm{I}$ & 1.61 & 1.34 & 649 & $4.09 \mathrm{E}-01$ & $5.75 \mathrm{E}-01$ & 16 & 20 \\
\hline Supernatant & $1.70 \mathrm{E}+04$ & $5.04 \mathrm{E} \div 00$ & $6.51 E+03$ & $3.31 E+05$ & $1.46 \mathrm{E}+05$ & $2.01 \mathrm{E}+01$ & $3.22 \mathrm{E}+00$ & $4.59 \mathrm{E}-03$ & $4.74 \mathrm{E}-03$ & 4.03E-02 & $3.27 \mathrm{E}-04$ & $1.10 \mathrm{E}+01$ & 1.34 & 1.34 & 38 & $5.75 \mathrm{E}-01$ & $5.75 \mathrm{E}-01$ & 0 & 0 \\
\hline Solid Layer & $1.70 \mathrm{E}+04$ & $5.04 \mathrm{E}+00$ & $6.51 \mathrm{E}+03$ & $3.31 \mathrm{E}+05$ & $1.46 \mathrm{E}+05$ & $2.01 \mathrm{E}+01$ & $3.22 \mathrm{E}+00$ & $4.59 \mathrm{E}-03$ & $4.74 \mathrm{E}-03$ & $4.03 \mathrm{E}-02$ & $3.27 \mathrm{E}-04$ & $1.10 \mathrm{E}+01$ & 1.63 & 1.34 & 611 & $4.01 \mathrm{E}-01$ & $5.75 \mathrm{E}-01$ & 16 & 20 \\
\hline
\end{tabular}


RPP-5926 REV 6

Table B-5. Input Data With Water Addition for Hydrogen Generation Rate Model Calculations for 177 Tanks. (20 sheets)

\begin{tabular}{|c|c|c|c|c|c|c|c|c|c|c|c|c|c|c|c|c|c|c|c|}
\hline Tank & $\begin{array}{c}\mathrm{OH} \\
\text { in liquid } \\
|\mathrm{OH}| \\
(\mu \mathrm{g} / \mathrm{mL})\end{array}$ & $\begin{array}{c}\text { TOC } \\
\text { in liquid } \\
\text { [TOC] } \\
(\mu \mathrm{g} / \mathrm{mL})\end{array}$ & $\begin{array}{c}\mathrm{NO}_{2} \\
\text { in liquid } \\
\left|\mathrm{NO}_{2}\right| \\
(\mu \mathrm{g} / \mathrm{mL})\end{array}$ & $\begin{array}{c}\mathrm{NO}_{3} \\
\text { in liquid } \\
\left|\mathrm{NO} \mathrm{O}_{3}\right| \\
(\mu \mathrm{g} / \mathbf{m L})\end{array}$ & $\begin{array}{c}\mathrm{Na} \\
\text { in tiquid } \\
|\mathrm{Na}| \\
(\mathrm{ug} / \mathrm{mL})\end{array}$ & $\begin{array}{c}\mathrm{A}] \\
\text { in liquid } \\
{[\mathrm{A} \mid} \\
(\mu \mathrm{g} / \mathrm{mL})\end{array}$ & $\begin{array}{c}90 \mathrm{Sr} \\
\text { in waste } \\
{[\mathrm{Sr} \mid} \\
(\mu \mathrm{Ci} / \mathrm{g})\end{array}$ & $\begin{array}{c}{ }^{241} \mathrm{Am} \\
\text { in waste } \\
\text { \{Am241\} } \\
(\mu \mathrm{Ci} / \mathrm{g})\end{array}$ & $\begin{array}{c}{ }^{240} \mathrm{Pu} \\
\text { in waste } \\
\mid \mathrm{Pu240|} \\
(\mu \mathrm{Ci} / \mathrm{g})\end{array}$ & $\begin{array}{c}{ }^{239} \mathrm{Pu} \\
\text { in waste } \\
\mid \mathrm{Pu240|} \\
(\mu \mathrm{Ci} / \mathrm{g})\end{array}$ & $\begin{array}{c}{ }^{238} \mathrm{Pu}_{\mathrm{u}} \\
\text { in waste } \\
|\mathrm{Pu} \mathbf{\mathrm { P }} 38| \\
(\mu \mathrm{Ci} / \mathrm{g})\end{array}$ & $\begin{array}{c}{ }^{137} \mathrm{Cs} \\
\text { in waste } \\
{[\mathrm{Cs}]} \\
(\mu \mathrm{Ci} / \mathrm{g})\end{array}$ & $\begin{array}{c}\text { Bulk } \\
\text { density } \\
\text { D } \\
(\mathrm{g} / \mathrm{mL})\end{array}$ & $\begin{array}{c}\text { Liquid } \\
\text { density } D_{L} \\
(g / m l)\end{array}$ & $\mid \begin{array}{c}\text { Non-RGS } \\
\text { waste } \\
\text { volume } \\
(\mathrm{kL} .)\end{array}$ & $\begin{array}{l}\text { Bulk } \\
\text { water } \\
{\left[\mathrm{H}_{2} \mathrm{O} \mid\right.} \\
(\mathrm{wt} \%)\end{array}$ & $\begin{array}{c}\text { Liquid } \\
\text { water } \\
\left|\mathrm{H}_{2} \mathrm{O}\right| \\
(w t \%)\end{array}$ & $\begin{array}{l}\text { Waste } \\
\text { temp. } \\
T_{w} \\
\text { ("C) }\end{array}$ & $\begin{array}{c}\text { Dome } \\
\text { temp. } \\
\text { Td } \\
\text { ("C) }\end{array}$ \\
\hline $241-B-108$ & $0 E+04$ & $1 \mathrm{E}+00$ & $5.38 \mathrm{E}+04$ & $6 \mathrm{E}+05$ & $4 E+05$ & $8 \mathrm{E}+02$ & $56 \mathrm{E}+00$ & $80 \mathrm{E}-04$ & $83 \mathrm{E}-04$ & 29E-03 & $3.91 \mathrm{E}-05$ & $1.26 \mathrm{E}+01$ & 1.67 & 1.38 & 388 & $3.73 \mathrm{E}-01$ & $6.96 \mathrm{E}-01$ & 21 & 21 \\
\hline Supernatant & $1.70 E+04$ & $3.71 E+00$ & $5.38 \mathrm{E}+04$ & $3.76 \mathrm{E}+05$ & $1.74 E+05$ & $8.98 \mathrm{E}+02$ & $1.56 \mathrm{E}+00$ & $1.80 \mathrm{E}-04$ & 4. $83 \mathrm{E}-04$ & $3.29 \mathrm{E}-03$ & $3.91 \mathrm{E}-05$ & $1.26 E+01$ & 1.38 & 1.38 & 38 & $6.96 \mathrm{E}-01$ & $6.96 \mathrm{E}-01$ & 0 & 0 \\
\hline Solid Layer & $1.70 \mathrm{E}+04$ & $3.71 \mathrm{E}+00$ & $5.38 E+04$ & $3.76 E+05$ & $1.74 \mathrm{E}+05$ & $8.98 \mathrm{E}+02$ & $1.56 \mathrm{E}+00$ & $1.80 \mathrm{E}-04$ & 4.83E-04 & $3.29 \mathrm{E}-03$ & $3.91 \mathrm{E}-05$ & $1.26 \mathrm{E}+01$ & 1.70 & 1.38 & 350 & $3.45 \mathrm{E}-01$ & $6.96 \mathrm{E}-01$ & 21 & 21 \\
\hline $241-B-109$ & 1. $70 \mathrm{E}+04$ & $3.39 \mathrm{E}+00$ & $8.51 \mathrm{E}+03$ & $2.17 \mathrm{E}+05$ & $1.13 \mathrm{E} \times 05$ & $3.61 \mathrm{E}+02$ & 4. $34 \mathrm{E}-01$ & $7.25 \mathrm{E}-04$ & $3.33 \mathrm{E}-03$ & 2.47E-02 & $2.54 \mathrm{E}-04$ & $1.49 \mathrm{E}+00$ & 1.78 & 1.26 & 513 & 4.10E-01 & $6.96 \mathrm{E}-01$ & 20 & 21 \\
\hline Supernatant & $1.70 \mathrm{E}+04$ & $3.39 \mathrm{E}+00$ & $8.51 \mathrm{~J}+03$ & $2.17 \mathrm{E}+05$ & $1.13 E+0.5$ & $3.61 E+02$ & 4. $34 \mathrm{E}-01$ & $7.25 \mathrm{E}-04$ & $3.33 E-03$ & $2.47 \mathrm{E}-02$ & $2.54 \mathrm{E}-04$ & $1.49 \mathrm{E}+00$ & 1.26 & 1.26 & 38 & $6.96 \mathrm{t}-01$ & $6.96 \mathrm{E}-01$ & 0 & 0 \\
\hline Solid Layer & $1.70 \mathrm{E}-04$ & $3.395+100$ & $8.51 E+03$ & $2.17 \mathrm{E}+05$ & $1.13 E+05$ & $3.61 \mathrm{E}+02$ & $4.34 \mathrm{E}-01$ & $7.25 \mathrm{E}-04$ & $3.33 \mathrm{E}-03$ & $2.47 \mathrm{E}-02$ & $2.54 \mathrm{E}-04$ & $1.49 \mathrm{E}+00$ & 1.82 & 1.26 & 475 & $3.94 \mathrm{E}-01$ & $6.96 \mathrm{E}-01$ & 20 & 21 \\
\hline $241-B-110$ & $4 E+04$ & $34 E-03$ & $3.80 厄+04$ & $7.48 E+04$ & $9.18 \mathrm{E}+04$ & $1.00 \mathrm{E}+04$ & $52 \mathrm{E}+01$ & $7.00 \mathrm{E}-02$ & $.04 \mathrm{E}-02$ & $9.78 \mathrm{E}-02$ & $3.03 \mathrm{E}-03$ & $1.05 \mathrm{E}+01$ & 1.35 & 1.01 & 967 & $5.96 \mathrm{E}-01$ & 7. $69 \mathrm{E}-01$ & 21 & $2 !$ \\
\hline Supernatant & $1.62 \mathrm{E}+03$ & $5.30 \mathrm{E}+02$ & $3.78 \mathrm{E} \cdot 03$ & $7.43 \mathrm{E} \div 03$ & $9.13 E+03$ & $9.94 \mathrm{E}+02$ & $2.17 E-01$ & 6. $.34 \mathrm{E}-04$ & 1. $10 \mathrm{E}-04$ & $4.74 \mathrm{E}-04$ & $1.98 \mathrm{E}-05$ & $1.31 \mathrm{E}-00$ & 1.02 & 1.02 & 42 & $9.73 \mathrm{E}-01$ & $9.73 \mathrm{E}-01$ & 21 & 21 \\
\hline Solid Layer & $1.70 E+04$ & $5.55 \mathrm{E}+03$ & $3.95 \mathrm{E}+04$ & $7.78 \mathrm{E}+04$ & $9.55 \mathrm{E}+04$ & $1.04 \mathrm{E}+04$ & $.77 E+0 !$ & $24 \mathrm{E}-02$ & $1.08 \mathrm{E}-02$ & $1.01 \mathrm{E}-01$ & 3.13E-03 & $1.09 \mathrm{E}+01$ & 1.36 & 1.01 & 925 & $5.83 \mathrm{E}-01$ & 7.62E-01 & 21 & 21 \\
\hline 241-B-111 & $1.63 \mathrm{E}+04$ & $32 E+03$ & $3.79 E+04$ & $7.45 \mathrm{E}+04$ & $9.15 E+04$ & $9.97 \mathrm{E} \div 03$ & $85 \mathrm{E} \div 02$ & $8.21 \mathrm{E}-02$ & $.06 \mathrm{E}-02$ & $8.33 \mathrm{E}-02$ & $2.85 \mathrm{E}-03$ & $1.19 \mathrm{E}+02$ & 1.26 & 1.01 & 952 & $6.43 \mathrm{E}-01$ & $.70 \mathrm{E}-01$ & 25 & 25 \\
\hline Supematant & $1.62 \mathrm{E}+03$ & $5.30 \mathrm{E}+02$ & $3.78 \mathrm{E}+03$ & $7.43 \mathrm{E}+03$ & $9.13 E \div .03$ & $9.94 \mathrm{E}+02$ & $2.17 E-01$ & $6.34 \mathrm{E}-04$ & $1.10 \mathrm{E}-04$ & $4.74 \mathrm{E}-04$ & $1.98 E-05$ & $1.31 E+00$ & 1.02 & 1.02 & 42 & $9.73 \mathrm{E}-01$ & $9.73 \mathrm{E}-01$ & 24 & 25 \\
\hline Solid Layer & $1.70 \mathrm{E}+04$ & $5.55 \mathrm{E}+03$ & $3.95 E+04$ & $7.78 \mathrm{E}+04$ & $9.55 \mathrm{E}+04$ & $1.04 \mathrm{E}+04$ & $92 \mathrm{E}+02$ & $8.51 \mathrm{E}-02$ & $1.09 \mathrm{E}-02$ & $8.64 \mathrm{E}-02$ & $2.96 \mathrm{E}-03$ & $1.24 \mathrm{E}+02$ & 3.27 & 1.01 & 910 & $6.31 E-01$ & $7.62 \mathrm{E}$ & 25 & 25 \\
\hline $241 \cdot B-112$ & $1.28 \mathrm{E}+04$ & $1.37 E+04$ & $8.84 E+04$ & $1.64 \mathrm{E}+05$ & $1.93 E+05$ & $3.58 \mathrm{E} \div 04$ & $3.35 \mathrm{E}+00$ & 1.21E-03 & $3.30 E-04$ & $2.17 \mathrm{E}-03$ & $3.88 \mathrm{E}-05$ & $3.36 \mathrm{E}+01$ & 1.38 & 1.39 & 170 & $5.09 \mathrm{E}-01$ & $5.49 \mathrm{E}-01$ & 21 & 22 \\
\hline Supernatant & $3.83 \mathrm{E}+03$ & 4.095 .03 & $2.64 \mathrm{E}+04$ & $4.89 \mathrm{E}+04$ & $5.73 \mathrm{E}+04$ & $1.06 \mathrm{E}+04$ & $4.66 \mathrm{E}-01$ & 1. $36 \mathrm{E}-03$ & $2.44 \mathrm{E}-04$ & $1.02 \mathrm{E}-03$ & $3.93 \mathrm{E}-05$ & $3.08 \mathrm{E}+01$ & 1.11 & 1.11 & 49 & $8.36 \mathrm{E}-01$ & $8.36 \mathrm{E}-01$ & 21 & 22 \\
\hline Solid Layer & $1.70 \mathrm{E}+04$ & $1.82 \mathrm{E}+04$ & $1.17 \mathrm{~F} .05$ & $2.17 \mathrm{E}-05$ & $2.55 \mathrm{E}: 05$ & $4.73 \mathrm{E}+04$ & $4.23 \mathrm{k}+00$ & $.16 \mathrm{E}-03$ & $56 \mathrm{E}-04$ & $2.52 \mathrm{E}-03$ & $3.86 \mathrm{E}-05$ & $3.44 \mathrm{E} 101$ & 1.49 & 1.51 & 121 & 4.10E-01 & $4.62 \mathrm{E}$ & 21 & 22 \\
\hline $241 \cdot \mathrm{B}-20 \mathrm{I}$ & $1.70 E+04$ & $2.98 \mathrm{E}+03$ & $3.60 \mathrm{E}+04$ & $7.96 \mathrm{E}+04$ & $8.32 \mathrm{E}+04$ & $4.94 \mathrm{E}+03$ & $1.71 \mathrm{E}+00$ & $2.84 \mathrm{E}-02$ & 4. $33 \mathrm{E}-02$ & $7.51 \mathrm{E}-01$ & $6.28 \mathrm{E}-03$ & $1.36 \mathrm{E}-01$ & 1.26 & 1.17 & 115 & $6.45 \mathrm{E}-01$ & $7.50 \mathrm{~F}-01$ & 17 & 19 \\
\hline Supernatant & $1.70 E+04$ & $2.98 E+03$ & $3.60 \mathrm{E}+04$ & $7.96 \mathrm{E}+04$ & $8.32 \mathrm{E}+04$ & $4.94 \mathrm{E}+03$ & $1.71 \mathrm{E}+00$ & $2.84 \mathrm{E}-02$ & $4.33 \mathrm{E}-02$ & $7.51 \mathrm{E}-01$ & $6.28 \mathrm{E}-03$ & $1.36 E-01$ & 1.17 & 1.17 & 4 & $7.50 \mathrm{E}-01$ & $7.50 \mathrm{E}-01$ & 0 & 0 \\
\hline Solid Layer & $1.70 E+04$ & $2.98 \mathrm{E}+03$ & $3.60 \mathrm{E}+04$ & $7.96 \mathrm{E}+04$ & $8.32 \mathrm{E}+04$ & $4.94 \mathrm{E}+03$ & $1.71 \mathrm{E}+00$ & $2.84 \mathrm{E}-02$ & $33 \mathrm{E}-02$ & $7.51 \mathrm{E}-01$ & $6.28 \mathrm{E}-03$ & $1.36 \mathrm{E}-01$ & 1.26 & 1.17 & 11 & 6.42 & $7.50 \mathrm{E}-0 !$ & 17 & 19 \\
\hline $241-B-202$ & $1.70 \mathrm{E}+04$ & $2.98 \mathrm{E}+0.3$ & $3.60 \mathrm{E}+04$ & $7.96 \mathrm{E}+04$ & 8. $32 \mathrm{E}+04$ & $4.94 \mathrm{E}+03$ & $3.00 \mathrm{E}+00$ & $6.67 \mathrm{E}-02$ & $2.17 \mathrm{E}-02$ & $1.28 \mathrm{E}-01$ & 2.03E-03 & $7.67 \mathrm{E}-02$ & 1.22 & 1.17 & 112 & $7.61 \mathrm{E}-0 \mathrm{~J}$ & $8.30 \mathrm{E}-01$ & 17 & 19 \\
\hline Supernatant & $1.70 \mathrm{E}+04$ & $2.98 \mathrm{E}+03$ & $3.60 \mathrm{E}+04$ & $7.96 \mathrm{E}+04$ & $8.32 E+04$ & $4.94 \mathrm{E}+03$ & $3.00 \mathrm{E}+00$ & $6.67 \mathrm{E}-02$ & $2.17 \mathrm{E}-02$ & $1.28 \mathrm{E}-01$ & $2.03 \mathrm{E}-03$ & 7.67E-02 & 1.17 & 1.17 & 4 & $8.30 \mathrm{E}-01$ & $8.30 \mathrm{E}-01$ & 0 & 0 \\
\hline Solid Layer & $1.70 \mathrm{E}+04$ & $2.98 \mathrm{E}+03$ & $3.60 \mathrm{E}+04$ & $7.96 \mathrm{E}+04$ & $8.32 \mathrm{E}+04$ & $4.94 \mathrm{E}+03$ & $3.00 \mathrm{E}+00$ & $6.67 \mathrm{E}-02$ & $2.17 \mathrm{E}-02$ & $1.28 \mathrm{E}-01$ & 2.03E-03 & $7.67 \mathrm{E}-02$ & 1.22 & 1.17 & 108 & $7.59 \mathrm{E}-01$ & $8.30 \mathrm{E}-01$ & 17 & 19 \\
\hline 241-B-203 & $1.65 \mathrm{E}+04$ & $9.40 \mathrm{E}+01$ & $1.54 \mathrm{E}+03$ & $5.54 \mathrm{E}+04$ & $3.05 \mathrm{E}+04$ & $0.00 E+00$ & $6.75 \mathrm{E}-02$ & $3.37 \mathrm{E}-02$ & $2.87 \mathrm{E}-02$ & $2.27 \mathrm{E}-01$ & $1.97 \mathrm{E}-03$ & $6.45 \mathrm{E}-03$ & 1.18 & 1.05 & 194 & $7.62 \mathrm{E}-01$ & $8.96 \mathrm{E}-01$ & 18 & 12 \\
\hline Supenatant & $8.53 E+02$ & $3.32 \mathrm{E}+01$ & $5.43 \mathrm{E}+02$ & $1.95 \mathrm{E}+04$ & $1.08 \mathrm{E}+04$ & $0.00 \mathrm{E}+00$ & $2.77 \mathrm{E}-05$ & $1.45 \mathrm{E}-05$ & $1.73 \mathrm{E}-04$ & $1.37 \mathrm{E}-03$ & 1.18E-05 & $3.21 \mathrm{E}-05$ & 1.02 & 1.02 & 6 & $9.62 \mathrm{E}-01$ & $9.62 \mathrm{E}-01$ & 22 & 12 \\
\hline Solid Layer & $1.70 \mathrm{E}+04$ & $9.60 \mathrm{E}+01$ & $1.57 \mathrm{E}+03$ & $5.65 \mathrm{E}+04$ & $3.12 E+04$ & $0.00 E+00$ & $6.93 \mathrm{E}-02$ & $3.46 \mathrm{E}-02$ & $2.95 \mathrm{E}-02$ & $2.33 \mathrm{E}-01$ & $2.02 \mathrm{E}-03$ & $6.62 \mathrm{E}-03$ & 1.19 & 1.05 & 188 & $7.57 \mathrm{E}-01$ & $8.94 E-01$ & 18 & 12 \\
\hline 241. & $1.64 \mathrm{E}+04$ & $7.38 \mathrm{E} r 01$ & $1.34 \mathrm{E}+03$ & $3.40 \mathrm{E}+04$ & $2.73 \mathrm{E}+04$ & $0.00 \mathrm{E}+00$ & $2.54 \mathrm{E}-03$ & 3.72E- 02 & $2.43 \mathrm{E}-02$ & $1.93 \mathrm{E}-01$ & 1.67E-03 & $2.54 \mathrm{E}-02$ & 1.18 & 1.05 & 191 & $7.79 \mathrm{E}-01$ & $9.15 \mathrm{E}-01$ & 18 & 22 \\
\hline Supenatant & $1.25 \mathrm{E}+03$ & $3.33 \mathrm{E}+01$ & $6.03 \mathrm{E} \div 02$ & $1.53 E+04$ & 1. $23 \mathrm{E}-04$ & $0.00 \mathrm{E}+00$ & $1.57 \mathrm{~F}-05$ & $7.09 \mathrm{E}-08$ & $2.13 E-06$ & $3.17 \mathrm{E}-05$ & $1.07 \mathrm{E}-07$ & $1.80 \mathrm{E}-05$ & 1.02 & 1.02 & 7 & $9.61 \mathrm{E}-0 \mathrm{]}$ & $9.61 \mathrm{E}-01$ & 19 & 22 \\
\hline Solid Layer & $1.70 E+04$ & $7.53 \mathrm{E}+01$ & $1.36 \mathrm{E}+03$ & $3.47 \mathrm{E}+04$ & $2.78 \mathrm{E}+04$ & $0.00 \mathrm{E}+00$ & $2.62 \mathrm{E}-03$ & $3.84 \mathrm{E}-02$ & $2.51 \mathrm{E}-02$ & $1.99 E-01$ & $1.72 \mathrm{E}-03$ & $2.62 \mathrm{E}-02$ & 1.19 & 1.05 & 184 & $7.73 E-01$ & $9.14 \mathrm{E}-01$ & 18 & 22 \\
\hline
\end{tabular}


Table B-5. Input Data With Water Addition for Hydrogen Generation Rate Model Calculations for 177 Tanks. (20 sheets)

\begin{tabular}{|c|c|c|c|c|c|c|c|c|c|c|c|c|c|c|c|c|c|c|c|}
\hline Tank & $\begin{array}{c}\text { Он } \\
\text { in liquid } \\
{[\mathrm{OH}]} \\
(\mu \mathrm{g} / \mathrm{mL})\end{array}$ & $\begin{array}{c}\text { TOC } \\
\text { in liquid } \\
{[\mathrm{TOC}]} \\
(\mu \mathrm{g} / \mathrm{mL})\end{array}$ & $\begin{array}{c}\mathrm{NO}_{2} \\
\text { in liquid } \\
{\left[\mathrm{NO}_{2} \mid\right.} \\
(\mu \mathrm{g} / \mathrm{mL})\end{array}$ & $\begin{array}{c}\mathrm{NO}_{3} \\
\text { in liquid } \\
{\left[\mathrm{NO}_{3} \mid\right.} \\
(\mu \mathrm{g} / \mathrm{mL})\end{array}$ & $\begin{array}{c}\mathrm{Na} \\
\text { in liquuid } \\
{[\mathrm{Na}]} \\
(\mathrm{ug} / \mathrm{mL})\end{array}$ & $\begin{array}{c}\mathrm{Al} \\
\text { in lìquid } \\
\text { |A|| } \\
(\mu \mathrm{g} / \mathrm{mL})\end{array}$ & $\begin{array}{c}90 \mathrm{Sr} \\
\text { in waste } \\
{[\mathrm{Sr}]} \\
(\mu \mathrm{Ci} / \mathrm{g})\end{array}$ & $\begin{array}{c}{ }^{241} \mathrm{Am} \\
\text { in waste } \\
\text { [Am241] } \\
(\mu \mathrm{Ci} / \mathrm{g})\end{array}$ & $\begin{array}{c}{ }^{240} \mathrm{P}_{u} \\
\text { in waste } \\
\text { [Pu240| } \\
(\mu \mathrm{Ci} / \mathrm{g})\end{array}$ & $\begin{array}{c}{ }^{239} \mathrm{Pu} \\
\text { in waste } \\
\mathrm{jPu} \mathrm{Pu} 40 \mid \\
(\mu \mathrm{Ci} / \mathrm{g})\end{array}$ & $\begin{array}{c}{ }^{238} \mathrm{Pu} \\
\text { in waste } \\
{[\mathrm{Pu238]}} \\
(\mu \mathrm{Ci} / \mathrm{g})\end{array}$ & $\begin{array}{c}{ }^{137} \mathrm{Cs} \\
\text { in waste } \\
{[\mathrm{Cs}]} \\
(\mu \mathrm{Ci} / \mathrm{g})\end{array}$ & $\begin{array}{c}\text { Bulk } \\
\text { density } \\
\text { D } \\
(\mathrm{g} / \mathrm{ml})\end{array}$ & $\begin{array}{c}\text { Liquid } \\
\text { density } \mathrm{D}_{1} \\
(\mathrm{~g} / \mathrm{ml})\end{array}$ & $\begin{array}{c}\text { Non-RGS } \\
\text { waste } \\
\text { volume } \\
(\mathrm{kL})\end{array}$ & $\begin{array}{l}\text { Bulk } \\
\text { water } \\
{\left[\mathrm{H}_{2} \mathrm{O}\right]} \\
\left(w^{4} \%\right)\end{array}$ & $\begin{array}{c}\text { Liquid } \\
\text { water } \\
\left|\mathrm{H}_{2} \mathrm{O}\right| \\
\left(\mathrm{wt}^{2} \%\right)\end{array}$ & $\begin{array}{c}\text { Waste } \\
\text { temp. } \\
\text { Tw } \\
\left({ }^{\circ} \mathrm{C}\right)\end{array}$ & $\begin{array}{c}\text { Dome } \\
\text { temp. } \\
\text { Td } \\
\left({ }^{\circ} \mathrm{C}\right)\end{array}$ \\
\hline $24 !-B X-101$ & $0 \mathrm{E}+04$ & $98 \mathrm{E}+03$ & $6.43 \mathrm{E}+04$ & $1.21 \mathrm{~F}+05$ & $42 E+05$ & $17 \mathrm{E}+03$ & $2.55 \mathrm{E}+02$ & $1.37 \mathrm{E}-01$ & $1.32 \mathrm{E}-01$ & $6.39 \mathrm{E}-01$ & $11 \mathrm{E}-02$ & $9.08 \mathrm{E}+01$ & 1.61 & 1.28 & 218 & $2.37 t-01$ & $7.50 \mathrm{E}-01$ & 23 & 22 \\
\hline Supermatant & $1.70 \mathrm{E}-04$ & $4.98 \mathrm{k}+03$ & $6.43 \mathrm{E} \div 04$ & $1.21 E+05$ & $1.42 \mathrm{E}+0.05$ & $5.17 \mathrm{E}+03$ & $2.55 \mathrm{E}+02$ & $1.37 \mathrm{E}-01$ & $1.32 \mathrm{E}-01$ & 6.39E-01 & $2.11 \mathrm{E}-02$ & $9.08 \mathrm{E}+01$ & 1.28 & 1.28 & 38 & $7.50 \mathrm{E}-0 \mathrm{I}$ & $7.50 \mathrm{E}-01$ & 0 & 0 \\
\hline Solid Layer & $1.70 \mathrm{E}+04$ & $4.98 \mathrm{E}+03$ & $6.43 E \div 04$ & $1.21 \mathrm{E}-05$ & $1.42 \mathrm{E}+05$ & $17 E+03$ & $2.55 \mathrm{E}+02$ & $1.37 \mathrm{E}-01$ & 1.32E-0 & $6.39 \mathrm{E}-01$ & $2.11 \mathrm{E}-02$ & $9.08 \mathrm{E}+01$ & 1.68 & 1.28 & 180 & $1.55 \mathrm{E}-01$ & $7.50 \mathrm{E}-01$ & 23 & 22 \\
\hline $241-\mathrm{BX}-102$ & 1. $70 E+04$ & $2.98 \mathrm{E}+03$ & $3.60 E+04$ & $7.96 \mathrm{E}+04$ & $8.32 \mathrm{E}+04$ & $4.94 \mathrm{E}+03$ & $3.84 \mathrm{E}+01$ & $3.93 \mathrm{E}-03$ & $3.97 \mathrm{E}-03$ & $1.89 \mathrm{E}-02$ & $3.83 E-04$ & $4.31 \mathrm{E}+00$ & 1.01 & 1.17 & 336 & $4.15 \mathrm{E}-01$ & $7.50 \mathrm{E}-01$ & 20 & 21 \\
\hline Supernatant & $1.70 E+04$ & $98 \mathrm{E}+03$ & $60 \mathrm{E}+04$ & $7.96 \mathrm{E}+04$ & $32 E+04$ & $4.94 \mathrm{E}+03$ & $84 \mathrm{E}+01$ & $3.93 \mathrm{E}-03$ & $3.97 \mathrm{E}-03$ & $1.89 \mathrm{E}-02$ & 3.83E-04 & $4.31 \mathrm{E}+00$ & 1.17 & 1.17 & 38 & $7.50 \mathrm{E}-01$ & $7.50 \mathrm{E}-01$ & 0 & 0 \\
\hline Solid Layer & $1.70 \mathrm{E}+04$ & $2.98 \mathrm{E}+03$ & $3.60 \mathrm{E}+04$ & $7.96 \mathrm{E}+04$ & $8.32 \mathrm{E}+04$ & $4.94 \mathrm{E}+03$ & $3.84 \mathrm{E}+01$ & $3.93 \mathrm{E}-03$ & $3.97 \mathrm{E}-03$ & $1.89 \mathrm{E}-02$ & 3.83E-04 & $4.31 \mathrm{E}+00$ & 0.99 & 1.17 & 298 & 3.64E-01 & $7.50 \mathrm{E}-01$ & 20 & 21 \\
\hline 24I-BX-103 & $1.49 \mathrm{E}+04$ & $3.33 E+03$ & $1.25 \mathrm{E}+04$ & $4.06 \mathrm{E}+04$ & $3.55 \mathrm{E}-04$ & $62 \mathrm{E}+04$ & $7.46 \mathrm{E}-00$ & $2.68 \mathrm{E}-01$ & $4.02 \mathrm{E}-01$ & $1.77 \mathrm{E}+00$ & $3.97 \mathrm{E}-02$ & $1.09 \mathrm{E}+00$ & 1.49 & 1.09 & 321 & $5.66 \mathrm{E}-01$ & $7.96 \mathrm{E}-01$ & 21 & 21 \\
\hline Supernatant & $9.50 E+03$ & $12 \mathrm{E}+03$ & $7.94 E+03$ & $2.59 \mathrm{E}+04$ & $26 \mathrm{E}+04$ & $03 \mathrm{E}+04$ & 2.93E-01 & $3.25 \mathrm{E}-03$ & $6.95 \mathrm{E}-04$ & $2.95 \mathrm{~F}-03$ & $.95 \mathrm{E}-05$ & $3.44 \mathrm{E}-01$ & 1.04 & 1.04 & 86 & $8.72 \mathrm{E}-01$ & 8.72E-01 & 21 & 21 \\
\hline Solid Layer & $1.70 \mathrm{E}+04$ & $3.79 E+03$ & $1.42 \mathrm{E}+04$ & $4.63 E+04$ & $4.04 \mathrm{E}+04$ & $1.84 \mathrm{E}+04$ & $9.10 \mathrm{E}+00$ & $3.29 \mathrm{E}-01$ & $4.94 \mathrm{E}-01$ & $2.18 \mathrm{E}+00$ & $4.88 \mathrm{E}-02$ & $1.26 \mathrm{E}+00$ & 1.66 & 1.11 & 235 & $4.96 \mathrm{E}-01$ & $7.78 \mathrm{E}-01$ & 21 & 21 \\
\hline $241 \cdot B X-104$ & $6 \mathrm{~F}: 04$ & $74 \mathrm{E}-03$ & 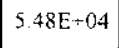 & $1.15 \mathrm{E}+05$ & $1205-0=$ & $445+03$ & $1.79 \mathrm{E}+02$ & $4.95 \mathrm{E}-01$ & 4. $16 E-02$ & $3.22 \mathrm{E}-01$ & $3.19 \mathrm{E}-03$ & $5.80 \mathrm{E}+0 \mathrm{I}$ & 1.61 & 1.24 & 418 & $3.17 \mathrm{E}-01$ & $6.14 \mathrm{~F}-01$ & 30 & 25 \\
\hline Supernatant & $3.83 E+03$ & $18 \mathrm{~F}+02$ & $1.43 \mathrm{E}+04$ & $3.01 \mathrm{E}+04$ & $15 \mathrm{E} \div 04$ & $39 \mathrm{E}+02$ & $3.58 \mathrm{E}-01$ & $5.70 \mathrm{E}-05$ & $8.65 E-06$ & 4.54E-05 & $.07 \mathrm{E}-07$ & $2.62 \mathrm{~F}$ & 1.06 & 1.06 & 49 & $8.89 \mathrm{E}-01$ & $39 E-01$ & 30 & 25 \\
\hline Solid Layer & $1.70 \mathrm{E} \div 04$ & $3.19 \mathrm{E}+03$ & $6.37 \mathrm{E}+04$ & $1.34 \mathrm{E}+05$ & $1.40 \mathrm{E}+05$ & $2.84 \mathrm{E}+03$ & $1.94 \mathrm{E}+02$ & $5.37 \mathrm{E}-01$ & $4.51 \mathrm{E}-02$ & 3.49E-01 & $3.46 \mathrm{E}-03$ & $6.07 \mathrm{E}+01$ & 1.68 & 1.28 & 369 & $2.69 \mathrm{E}-01$ & $5.91 \mathrm{E}-01$ & 30 & 25 \\
\hline 241-B & +04 & $E+03$ & .04 & $E+04$ & $\bar{E}+04$ & 13 & +02 & $5.25 E-02$ & $70 \mathrm{E}-02$ & $9 \mathrm{E}-0$ & $81 \mathrm{E}-03$ & $6.85 \mathrm{E}+01$ & 1.59 & 1.20 & 310 & $2.30 \mathrm{E}-01$ & 7.11E-01 & 20 & 21 \\
\hline Supernatant & $5.48 \mathrm{E}+03$ & $3.04 \mathrm{E}+03$ & $1.29 \mathrm{E}+04$ & $3.78 E+04$ & $3.99 \mathrm{E}+04$ & $1.92 \mathrm{E} \div 03$ & $2.21 \mathrm{E}+00$ & 7.27E-03 & $9.63 \mathrm{E}-\mathrm{C}$ & $4.09 \mathrm{E}-03$ & $9.64 \mathrm{E}-05$ & $3.50 \mathrm{E}+01$ & 1.09 & 1.09 & 56 & $8.81 \mathrm{E}-01$ & $8.81 \mathrm{E}-01$ & 20 & 21 \\
\hline Solid Layer & $1.70 E+04$ & $9.44 \mathrm{E}+03$ & $4.00 \mathrm{E}+04$ & $1.17 \mathrm{E}+05$ & $1.24 \mathrm{E}+05$ & $5.94 \mathrm{E}+03$ & $1.83 \mathrm{E} \div 02$ & $5.90 \mathrm{E}-02$ & $1.92 \mathrm{E}-02$ & $9.64 \mathrm{E}-02$ & $2.05 \mathrm{E}-03$ & $7.32 \mathrm{E}+01$ & 1.69 & 1.29 & 254 & $1.38 \mathrm{~F}-01$ & $6.87 \mathrm{E}-01$ & 20 & 21 \\
\hline $24 \mid-B X-106$ & $0 \mathrm{E}+04$ & +04 & 05 & $17 \mathrm{E}+05$ & $2.55 \mathrm{E}+05$ & $72 \mathrm{E}+04$ & $2.54 \mathrm{E}+01$ & $7=5001$ & $401 \mathrm{~F}_{0} 02$ & $196 \mathrm{E}_{-}$ & $5.18 E-03$ & $4.87 \mathrm{E}+01$ & 1.59 & 51 & 181 & $4.12 \mathrm{E}-01$ & $4.62 \mathrm{E}-0 !$ & 20 & 20 \\
\hline Supernatant & $1.70 \mathrm{E}+04$ & $1.82 \mathrm{E}+04$ & $1.17 \mathrm{E}+05$ & $2.17 \mathrm{E}+05$ & $2.55 \mathrm{E}+05$ & $4.72 E+04$ & $2.54 \mathrm{E}+01$ & $2.50 \mathrm{E}-01$ & $4.01 \mathrm{E}-02$ & $1.96 \mathrm{E}-01$ & $5.18 \mathrm{E}-03$ & $4.87 \mathrm{E}+01$ & 1.51 & 1.51 & 38 & $4.62 \mathrm{E}-01$ & $4.62 \mathrm{E}-0 \mathrm{r}$ & 0 & 0 \\
\hline Solid Layer & $1.70 E+04$ & $1.82 \mathrm{E}+04$ & $1.17 \mathrm{E}+05$ & $2.17 \mathrm{E}+05$ & $2.55 \mathrm{E} \sim 05$ & $4.72 \mathrm{E}+04$ & $2.54 \mathrm{E}+0 \mathrm{I}$ & $2.50 \mathrm{E}-01$ & $4.01 \mathrm{E}-02$ & $1.96 \mathrm{E}-01$ & $5.18 \mathrm{E}-03$ & $4.87 \mathrm{E}+0 \mathrm{I}$ & 1.62 & 1.51 & 143 & $3.99 \mathrm{E}-01$ & 4. $62 \mathrm{E}-01$ & 20 & 20 \\
\hline $241-13 X-107$ & $1.70 \mathrm{E}+04$ & $88 \mathrm{~F}+03$ & $3.60 \mathrm{E}+04$ & $7.96 \mathrm{E}+04$ & $32 \mathrm{E}+04$ & $4.94 \mathrm{E}+03$ & $7.51 \mathrm{E}+00$ & $1{ }^{\prime}$ & $=61503$ & $5.16 \mathrm{E}-02$ & $3.63 \mathrm{E}-04$ & $1.34 E+01$ & 1.43 & 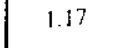 & 1351 & $5.11 \mathrm{E}-01$ & $7.50 \mathrm{E}-0 \mathrm{~J}$ & 19 & 21 \\
\hline Supernatant & $1.70 \mathrm{E}+04$ & $2.98 \Sigma+03$ & $3.60 \mathrm{E} \div 04$ & $7.96 \mathrm{E}+04$ & $8.32 E+04$ & $4.94 E+03$ & $7.51 \mathrm{E}+00$ & 1.30k-02 & $5.61 \mathrm{E}-03$ & $5.16 \mathrm{E}-0 ?$ & 3.63E-04 & $1.34 \mathrm{E}+01$ & 1.17 & 1.17 & 38 & $7.50 \mathrm{E}-01$ & 01 & 0 & 0 \\
\hline Solid Layer & $1.70 \mathrm{E}+04$ & $2.98 \mathrm{E}+03$ & $3.60 \mathrm{~F}+04$ & $7.96 \mathrm{E}+04$ & $8.32 E+04$ & $4.94 \mathrm{E}+03$ & $7.51 \mathrm{E}+00$ & $1.30 \mathrm{~F}-02$ & $5.61 \mathrm{E}-03$ & $5.16 \mathrm{E}-02$ & $3.63 \mathrm{E}-04$ & $1.34 \mathrm{E}+01$ & 1.44 & 1.17 & 1313 & $5.05 \mathrm{E}-01$ & $7.50 \mathrm{E}-01$ & 19 & 21 \\
\hline 241-BX-108 & $1.70 E+04$ & $8 E+03$ & $3.60 \mathrm{E}+04$ & $7.96 \mathrm{E}+04$ & $32 \mathrm{E}+04$ & $94 \mathrm{E}+03$ & $1.29 \mathrm{E}+02$ & $1.87 \mathrm{E}-02$ & E-03 & $4.79 \mathrm{E}-02$ & $.34 \mathrm{E}-04$ & 01 & 1.39 & 1.17 & 157 & $2.90 \mathrm{E}-01$ & $7.50 \mathrm{E}-01$ & 20 & 20 \\
\hline Supernatant & $1.70 \mathrm{E}+04$ & $2.98 \mathrm{E} \cdot 03$ & $3.60 \mathrm{E}+04$ & $7.96 \mathrm{E}+04$ & $8.32 E+04$ & $4.94 \mathrm{E}+03$ & $1.29 \mathrm{E}+02$ & $1.87 \mathrm{E}-02$ & $5.16 \mathrm{E}-03$ & $4.79 \mathrm{E}-02$ & 3.34E- 04 & $2.26 \mathrm{E}+01$ & 1.17 & 1.17 & 38 & $7.50 \mathrm{E}-01$ & 7.50E-01 & 0 & 0 \\
\hline Solid Layer & $1.70 \mathrm{E}+04$ & $2.98 \mathrm{E}+03$ & $3.60 \mathrm{E}+04$ & $7.96 \mathrm{E}+04$ & $8.32 \mathrm{E}+04$ & $4.94 E+03$ & $1.29 \mathrm{E}+02$ & $1.87 \mathrm{E}-02$ & $5.16 \mathrm{E}-03$ & $4.79 \mathrm{E}-02$ & $3.34 \mathrm{E}-04$ & $2.26 \mathrm{E}+01$ & 1.46 & 1.17 & $\$ 19$ & $1.72 \mathrm{E}-01$ & $7.50 \mathrm{E}-01$ & 20 & 20 \\
\hline 241-BX-109 & $1.70 E+04$ & $2.98 \mathrm{E}+03$ & $3.60 E+04$ & $7.96 \mathrm{E} \div 04$ & $32 \mathrm{E} \cdot 04$ & $4.94 \mathrm{E}+03$ & $1.07 \mathrm{E}+02$ & $5.36 \mathrm{E}-03$ & $5 E-03$ & $9.73 \mathrm{E}-03$ & $79 \mathrm{E}-05$ & $1.08 \mathrm{E}+0 \mathrm{~J}$ & 1.50 & 1.17 & 768 & $5.14 \mathrm{E}-01$ & $7.20 \mathrm{E}-01$ & 21 & 22 \\
\hline Supernatant & $1.70 \mathrm{E}+04$ & $2.98 \mathrm{E}+03$ & $3.60 \mathrm{E}+04$ & $7.96 \mathrm{E}+04$ & $8.32 \mathrm{E}+04$ & $4.94 \mathrm{E}+03$ & $1.07 \mathrm{E}+02$ & $5.36 \mathrm{E}-03$ & $1.05 \mathrm{E}-03$ & $9.73 \mathrm{E}-03$ & $6.79 \mathrm{E}-05$ & $1.08 \mathrm{E}+01$ & 1.17 & 1.17 & 38 & $7.20 \mathrm{E}-01$ & $7.20 \mathrm{E}-01$ & 0 & 0 \\
\hline Solid Layer & $1.70 \mathrm{E}+04$ & $2.98 \mathrm{E}+03$ & $3.60 \mathrm{E}+04$ & $7.96 E+04$ & $8.32 \mathrm{E}+04$ & $4.94 \mathrm{E}+03$ & $1.07 \mathrm{E}+02$ & $5.36 \mathrm{E}-03$ & $1.05 E-03$ & $9.73 \mathrm{E}-03$ & $6.79 \mathrm{E}-05$ & $1.08 \mathrm{E}+01$ & 1.52 & 1.17 & 730 & $5.06 \mathrm{t}-01$ & $7.20 \mathrm{E}-01$ & 21 & 22 \\
\hline
\end{tabular}


RPP-5926 REV 6

Table B-5. Input Data With Water Addition for Hydrogen Generation Rate Model Calculations for 177 Tanks. (20 sheets)

\begin{tabular}{|c|c|c|c|c|c|c|c|c|c|c|c|c|c|c|c|c|c|c|c|}
\hline Tank & $\begin{array}{c}\mathrm{OH} \\
\text { in liquid } \\
{[\mathrm{OH}]} \\
(\mu \mathrm{g} / \mathrm{mL})\end{array}$ & $\begin{array}{c}\text { TOC } \\
\text { in liquid } \\
\text { [TOC] } \\
(\mu \mathrm{g} / \mathrm{mL})\end{array}$ & $\begin{array}{c}\mathrm{NO}_{2} \\
\text { in liquid } \\
{\left[\mathrm{NO}_{2}\right\rfloor} \\
(\mu \mathrm{g} / \mathrm{mL})\end{array}$ & $\begin{array}{c}\mathrm{NO}_{3} \\
\text { in liquid } \\
\left|\mathrm{NO}_{3}\right| \\
(\mu \mathrm{g} / \mathrm{mL})\end{array}$ & $\begin{array}{c}\mathrm{Na} \\
\text { in liquid } \\
|\mathrm{Na}| \\
(\mathrm{ug} / \mathrm{mL})\end{array}$ & $\begin{array}{c}\text { Al } \\
\text { in liquid } \\
\text { |A|| } \\
(\mu \mathrm{g} / \mathrm{mL})\end{array}$ & $\begin{array}{c}90 \mathrm{Sr} \\
\text { in waste } \\
{[\mathrm{Sr}]} \\
(\mu \mathrm{Ci} / \mathrm{g})\end{array}$ & $\begin{array}{c}{ }^{2+1} \mathrm{Am} \\
\text { in waste } \\
{[\mathrm{Am241]}} \\
(\mu \mathrm{Ci} / \mathrm{g})\end{array}$ & $\begin{array}{c}{ }^{2+0} \mathrm{Pu} \\
\text { in waste } \\
{[\mathrm{Pu} 240]} \\
(\mu \mathrm{Ci} / \mathrm{g})\end{array}$ & $\begin{array}{c}{ }^{239} \mathrm{Pu} \\
\text { in waste } \\
|\mathrm{Pu} 240| \\
(\mu \mathrm{Ci} / \mathrm{g})\end{array}$ & \begin{tabular}{|c|}
${ }^{238} \mathrm{Pu}$ \\
in waste \\
{$[$ Pu238] } \\
$(\mu \mathrm{Ci} / \mathrm{g})$
\end{tabular} & $\begin{array}{c}{ }^{137} \mathrm{Cs} \\
\text { in waste } \\
{[\mathrm{Cs}]} \\
(\mu \mathrm{Ci} / g)\end{array}$ & $\begin{array}{c}\text { Bulk } \\
\text { density } \\
\text { D } \\
(\mathrm{g} / \mathrm{mL})\end{array}$ & $\begin{array}{c}\text { Liquid } \\
\text { density } D_{L} \\
(\mathrm{~g} / \mathrm{ml})\end{array}$ & $\begin{array}{c}\text { Non-RGS } \\
\text { waste } \\
\text { volume } \\
(\mathrm{kL})\end{array}$ & $\begin{array}{c}\text { Bulk } \\
\text { water } \\
{\left[\mathrm{H}_{2} \mathrm{O} \mid\right.} \\
(\mathrm{wt} \%)\end{array}$ & $\begin{array}{c}\text { Liquid } \\
\text { water } \\
\left|\mathrm{H}_{2} \mathrm{O}\right| \\
(\mathrm{wt} \%)\end{array}$ & $\begin{array}{c}\text { Waste } \\
\text { temp. } \\
\text { Tw } \\
\left({ }^{\circ} \mathrm{C}\right)\end{array}$ & $\begin{array}{c}\text { Dome } \\
\text { temp. } \\
\text { Td } \\
\left({ }^{\circ} \mathrm{C}\right)\end{array}$ \\
\hline $241-B X-110$ & $2 \mathrm{E}+04$ & $14 E+04$ & $95 \mathrm{E} \div 04$ & $2 E+05$ & $12 \mathrm{E}+05$ & $18 \mathrm{E}+03$ & $1 \mathrm{E}+00$ & $4.60 \mathrm{E}-03$ & $75 \mathrm{E}-03$ & $1.47 \mathrm{E}-02$ & 4IE-04 & $4.79 E+0 t$ & 1.64 & 1.42 & 850 & $4.27 \mathrm{E}-01$ & $.44 E-01$ & 20 & 20 \\
\hline Supcrnatant & $1.98 \mathrm{E}+03$ & $1.77 \mathrm{E}+03$ & $6.09 \mathrm{E}+03$ & $4.81 E+04$ & $2.61 \mathrm{E}+04$ & $5.13 \mathrm{E}+02$ & $2.13 \mathrm{E}-01$ & $6.24 \mathrm{E}-04$ & 1.12E-04 & $4.66 \mathrm{E}-04$ & $1.80 \mathrm{E}-05$ & $1.41 \mathrm{E}+01$ & 1.05 & 1.05 & 43 & $9.25 \mathrm{E}-01$ & $9.25 \mathrm{E}-01$ & 20 & 20 \\
\hline Solid Layer & $1.70 \mathrm{E}+04$ & $1.52 \mathrm{E}+04$ & $5.21 \mathrm{E}+04$ & 4.12E+05 & $2.23 \mathrm{E}+05$ & $4.40 \mathrm{E}+03$ & $7.86 \mathrm{E}-00$ & 4.73E- 03 & $1.81 \mathrm{E}-03$ & $1.52 \mathrm{E}-02$ & $1.45 \mathrm{E}-04$ & $4.90 \mathrm{E}+01$ & 1.67 & 1.44 & 807 & $4.11 E-01$ & $5.31 \mathrm{E}-01$ & 20 & 20 \\
\hline $241-\beta X-111$ & $\mathrm{OEE}+04$ & $1.70 \mathrm{E}+03$ & $06 \mathrm{E}-05$ & $1.85 E+05$ & $2.26 \mathrm{E}+05$ & $4.87 \mathrm{E} \div 04$ & $15 E+01$ & $3.43 \mathrm{E}-03$ & $1.64 \mathrm{E}-03$ & $1.45 \mathrm{E}-02$ & $17 \mathrm{E}-04$ & $6.47 \mathrm{E}+01$ & 1.45 & 1.45 & 751 & 2.61E-01 & $5.12 \mathrm{E}-01$ & 20 & 19 \\
\hline Supernatant & 1. $70 \mathrm{E}-04$ & $1.70 \mathrm{E} \div 03$ & $1.06 \mathrm{E}+05$ & 1.85E:05 & $2.26 \mathrm{E}+05$ & $4.87 \mathrm{E}+04$ & $1.15 E+01$ & 3.43E-03 & $1.64 \mathrm{E}-03$ & 1.45E-02 & $1.17 \mathrm{E}-04$ & $6.47 \mathrm{E}+01$ & 1.45 & 1.45 & 38 & $5.12 \mathrm{E}-01$ & $5.12 E-01$ & 0 & 0 \\
\hline Solid Layer & $1.70 \mathrm{E}+04$ & $1.70 \mathrm{E}+03$ & $1.06 \mathrm{E} \div 05$ & $1.85 E+05$ & $2.26 \mathrm{E}+05$ & $4.87 E+04$ & $1.15 \mathrm{E}+01$ & $3.43 \mathrm{E}-03$ & $1.64 \mathrm{E}-03$ & 1. $45 \mathrm{E}-02$ & $1.17 \mathrm{E}-04$ & $6.47 \mathrm{E}+01$ & 1.45 & 1.45 & 713 & $2.48 \mathrm{E}-01$ & $5.12 \mathrm{E}-01$ & 20 & 19 \\
\hline $241-\mathrm{BX}-112$ & $1.61 E+04$ & $0.00 \mathrm{E}+00$ & $3.33 \mathrm{E}+04$ & $9.31 E+0.4$ & $7.35 \mathrm{E}+04$ & $4.52 \mathrm{E}+01$ & $5.52 \mathrm{E}+00$ & $2.29 \mathrm{E}-02$ & $1.50 E-02$ & 37E-01 & $70 E-04$ & $3.85 \mathrm{E}+4$ & 1.29 & 1.17 & 660 & $6.50 \varepsilon-01$ & $90 \mathrm{E}-01$ & 20 & 19 \\
\hline Supernatant & $1.98 \mathrm{E}+03$ & $0.00 \mathrm{E}+00$ & $4.11 E+03$ & $1.15 \mathrm{E}+04$ & $9.08 \mathrm{E}+03$ & $5.58 \mathrm{E}+00$ & $8.39 \mathrm{E}-02$ & $1.84 \mathrm{E}-05$ & $2.58 \mathrm{E}-05$ & $1.82 \mathrm{E}-04$ & $1.81 E-06$ & $9.85 \mathrm{E}-02$ & 1.02 & 1.02 & 43 & $9.56 \mathrm{E}-01$ & $9.56 \mathrm{E}-01$ & 20 & 19 \\
\hline Solid Layer & 1. $70 \mathrm{E}+04$ & $0.00 \mathrm{E}+00$ & $3.52 \mathrm{E}+04$ & $9.86 \mathrm{E}+04$ & $78 \mathrm{E}+04$ & $4.78 \mathrm{E}+0$ & $5.81 \mathrm{E}+00$ & $2.41 E-02$ & $1.58 \mathrm{E}-02$ & 1.45E-01 & $1.02 \mathrm{E}-03$ & $4.06 \mathrm{E}+01$ & 1.31 & 1.18 & 617 & $6.33 \mathrm{E}-01$ & $6.76 \mathrm{E}-01$ & 20 & 19 \\
\hline $241-B Y-101$ & $3.83 E+04$ & $1.82 E+04$ & $1.17 \mathrm{E}+05$ & $17 E+05$ & $2.56 \mathrm{E}-05$ & $4.73 \mathrm{E}+04$ & $7.38 \mathrm{E}+01$ & $82 \mathrm{E}-02$ & $7.14 \mathrm{E}-04$ & $5.69 \mathrm{E}-03$ & $88 \mathrm{E}-05$ & $1.19 \mathrm{E}+02$ & 1.82 & 1.51 & 1438 & $2.40 \mathrm{E}-01$ & $62 \mathrm{E}-01$ & 25 & 24 \\
\hline Supernatant & $3.83 \mathrm{E}+0.04$ & $1.82 \mathrm{E}+04$ & $1.17 \mathrm{E}+05$ & $2.17 \mathrm{E}+05$ & $2.56 \mathrm{E}+05$ & $4.73 \mathrm{E}+04$ & $7.38 \mathrm{E}+01$ & $4.82 \mathrm{E}-02$ & $7.14 \mathrm{E}-04$ & $5.69 \mathrm{E}-03$ & $6.08 \mathrm{E}-05$ & $1.19 E+02$ & 1.51 & 1.51 & 38 & $4.62 \mathrm{E} \cdot 01$ & $4.62 \mathrm{E}-0 \mathrm{I}$ & 0 & 0 \\
\hline Solid Layer & $3.83 \mathrm{E}+04$ & $1.82 E+04$ & $1.17 \mathrm{E}+05$ & $2.17 \mathrm{E}+05$ & $56 \mathrm{E}+05$ & $4.73 E+04$ & $7.38 \mathrm{E}+\mathrm{C}$ & 4.82F-02 & $7.14 \mathrm{E}-04$ & $5.69 \mathrm{E}-03$ & $6.08 \mathrm{E}-05$ & $1.19 \mathrm{E}+02$ & 1.83 & 1.51 & 1400 & $2.35 \mathrm{E}-01$ & $62 E-01$ & 25 & 24 \\
\hline $241-B Y-102$ & $3.83 \mathrm{E} \div 04$ & $1.55 \mathrm{E}: 03$ & $8.41 \mathrm{E}+04$ & $1.48 E+05$ & $2.34 \mathrm{E}-05$ & $5.66 巨 \div 04$ & $1.05 \mathrm{E}-01$ & $5.05 \mathrm{E}-02$ & $5.15 \mathrm{E}-0.3$ & $2.16 \mathrm{E}-02$ & $8.32 \mathrm{E}-04$ & $6.65 \mathrm{E}+01$ & 1.57 & 1.46 & 1092 & $3.17 \mathrm{E}-01$ & $5.08 \mathrm{E}-01$ & 22 & 22 \\
\hline Supernatant & $3.83 \mathrm{E} \times 04$ & $1.55 \mathrm{E}+03$ & $8.41 E+04$ & $1.48 E+05$ & $2.34 E+05$ & $5.665,04$ & $1.05 \mathrm{E}+01$ & $5.05 \mathrm{E}-02$ & $5.15 \mathrm{E}-03$ & $2.16 \mathrm{E}-02$ & $8.32 \mathrm{E}-04$ & $6.65 E+01$ & 1.46 & 1.46 & 38 & $5.08 E-01$ & $5.08 \mathrm{~F}-01$ & 0 & 0 \\
\hline Solid Layer & $3.83 \mathrm{E}+04$ & $1.55 \mathrm{E}+03$ & $8.41 E+04$ & $1.48 \mathrm{E}+05$ & $34 \mathrm{E}+05$ & $5.66 \mathrm{E}+04$ & $1.05 E+01$ & $5.05 \mathrm{E}-02$ & $5.15 \mathrm{E}-03$ & $2.16 \mathrm{E}-02$ & $8.32 \mathrm{E}-04$ & $6.65 E+01$ & 1.57 & 1.46 & 1054 & $3.11 \mathrm{E}-01$ & $5.08 \mathrm{E}-01$ & 22 & 22 \\
\hline $241-B Y-103$ & $3.15 E+04$ & $1.59 \mathrm{E} \div 03$ & $4.49 \mathrm{E}+04$ & $41 E+05$ & $1.80 \mathrm{E}+05$ & $3.46 E+04$ & $1.13 \mathrm{E}+01$ & $1.16 \mathrm{E}-02$ & $1.34 \mathrm{E}-03$ & $5.61 \mathrm{E}-03$ & $2.05 \mathrm{E}-04$ & $6.69 \mathrm{E}+01$ & 1.65 & 1.29 & 1602 & $2.84 \mathrm{E}-01$ & $5.90 \mathrm{E}-01$ & 23 & 20 \\
\hline Supernatant & $3.15 \mathrm{E}+04$ & $1.59 \mathrm{E}+03$ & $4.49 \mathrm{E}+04$ & $1.41 E+05$ & $1.80 \mathrm{E}+05$ & $3.46 \mathrm{E}+04$ & $1.13 \mathrm{E}+01$ & $1.16 \mathrm{E}-02$ & $1.34 \mathrm{E}-03$ & $5.61 \mathrm{E}-03$ & $2.05 \mathrm{E}-04$ & $6.69 \mathrm{E}+01$ & 1.29 & 1.29 & 38 & $5.90 \mathrm{E}-01$ & $5.90 \mathrm{E}-01$ & 0 & 0 \\
\hline Solid Layer & $3.15 E+04$ & $1.59 \mathrm{E}+03$ & $4.49 \mathrm{E}+04$ & $1.41 \mathrm{E}+05$ & $1.80 \mathrm{E}+05$ & $3.46 \mathrm{E}+04$ & $1.13 \mathrm{E}+01$ & 1.16E-02 & $1.34 \mathrm{E}-03$ & $5.61 \mathrm{E}-03$ & $2.05 \mathrm{E}-04$ & $6.69 \mathrm{E}+01$ & 1.66 & 1.29 & 1564 & $2.78 \mathrm{E}-01$ & $5.90 \mathrm{E}-01$ & 23 & 20 \\
\hline $241-B Y \cdot 104$ & $3.83 \mathrm{E}+04$ & $2.37 \mathrm{E}-03$ & $1.11 \mathrm{E}+05$ & $2.07 E+05$ & $2.48 \mathrm{E}+05$ & $7.19 \mathrm{E}+04$ & $1.38 \mathrm{E}+02$ & $5.52 \mathrm{E}-02$ & $5.83 \mathrm{E}-03$ & $2.94 \mathrm{E}-02$ & $8.46 \mathrm{E}-04$ & $8.65 \mathrm{E}+01$ & 1.71 & 1.51 & 1571 & $2.64 \mathrm{E}-01$ & $4.89 \mathrm{E}-01$ & 41 & 27 \\
\hline Supernatant & $3.83 \mathrm{E} \div 04$ & $2.37 \mathrm{E}+03$ & $1.11 \mathrm{E}+05$ & $2.07 \mathrm{E}+05$ & $2.48 \mathrm{E}+05$ & $7.19 \mathrm{E} \div 04$ & $1.38 \mathrm{E}+\mathrm{C}$ & $5.52 E-02$ & $5.83 \mathrm{E}-03$ & $2.94 \mathrm{E}-02$ & 46E-04 & $8.65 \mathrm{E}+0$ & 1.51 & 1.51 & 38 & $4.89 \mathrm{E}-01$ & $4.89 \mathrm{E}-01$ & 0 & 0 \\
\hline Solid Layer & $3.83 E+04$ & $2.37 \mathrm{E}+03$ & $1.11 E \div 05$ & $2.07 E+05$ & $2.48 E+05$ & $7.19 \mathrm{E}+04$ & $1.38 \mathrm{E}+02$ & $5.52 \mathrm{E}-02$ & $5.83 E-03$ & $2.94 \mathrm{E}-02$ & $8.46 \mathrm{E}-04$ & $8.65 \mathrm{E}+01$ & 1.71 & 1.51 & 1533 & $2.59 \mathrm{E}-01$ & $4.89 \mathrm{E}-01$ & 41 & 27 \\
\hline $241-B Y-105$ & $3.82 \mathrm{E}+04$ & $2.43 \mathrm{~F} \div 03$ & $6.86 \mathrm{E}+04$ & $1.99 \mathrm{E}+05$ & $2.08 \mathrm{E}+05$ & $3.92 E+04$ & $4.26 \mathrm{E}+0 \mathrm{~J}$ & $3.61 \mathrm{E}-02$ & $3.91 \mathrm{E}-03$ & $2.38 \mathrm{E}-02$ & $4.88 \mathrm{E}-04$ & $4.65 \mathrm{E}+0 \mathrm{I}$ & 1.79 & 1.44 & 1859 & 1. $84 \mathrm{E}-01$ & $5.13 \mathrm{E}-01$ & 33 & 31 \\
\hline Supematant & $3.82 E \cdot 04$ & $2.43 \mathrm{E}+03$ & $6.86 \mathrm{E}+04$ & $1.99 \mathrm{E}+05$ & $2.08 \mathrm{E}+05$ & $3.92 E+04$ & $4.26 \mathrm{E}+01$ & 02 & $3.91 \mathrm{E}-03$ & $2.38 E-02$ & $4.88 \mathrm{E}-04$ & $4.65 \mathrm{E} \multimap 0 \mathrm{I}$ & 1.44 & 1.44 & 38 & $5.13 \mathrm{E}-0 \mathrm{l}$ & $5.13 \mathrm{E}-01$ & 0 & 0 \\
\hline Solid Layer & $3.82 \mathrm{E}+04$ & $2.43 \mathrm{E}+03$ & $6.86 \mathrm{E} \div 04$ & $1.99 \mathrm{E}-05$ & $2.08 \mathrm{E}+05$ & $3.92 \mathrm{E}+04$ & $4.26 \mathrm{E}+01$ & $3.61 \mathrm{E}-02$ & $3.91 \mathrm{E}-03$ & $2.38 \mathrm{E}-02$ & $4.88 \mathrm{E}-04$ & $4.65 \mathrm{E}+01$ & 1.80 & 1.44 & 3821 & $1.79 \mathrm{E}-01$ & $5.13 \mathrm{E}-01$ & 33 & 31 \\
\hline $241-B Y-106$ & $3.58 \mathrm{E}+04$ & $2.67 \mathrm{E}+03$ & $83 \mathrm{E}+04$ & 1.101 & 05 & 3 & 1 & 02 & 03 & 03 & $23 \mathrm{E}-04$ & $1.12 \mathrm{E}+02$ & 1.64 & 1.31 & 1661 & $2.74 \mathrm{E}-01$ & $5.94 \mathrm{E}-0 \mathrm{~J}$ & 34 & 34 \\
\hline Supematant & $3.58 E+04$ & $2.67 \mathrm{E}+03$ & $7.83 \mathrm{E}+04$ & $1.10 \mathrm{E}+05$ & $1.64 \mathrm{E}+05$ & $3.98 \mathrm{E}+04$ & $5.09 \mathrm{E}+01$ & $1.44 \mathrm{E}-02$ & $1.20 \mathrm{E}-03$ & $8.72 E-03$ & $1.23 \mathrm{E}-04$ & $1.12 \mathrm{E}+02$ & 1.31 & 1.31 & 38 & $5.94 \mathrm{E}-01$ & $5.94 \mathrm{E}-01$ & 0 & 0 \\
\hline Solid Layer & $3.58 \mathrm{E}+04$ & $2.67 \mathrm{E}+03$ & $7.83 \mathrm{E}+04$ & $1.10 \mathrm{E} \div 05$ & $1.64 \mathrm{E}+05$ & $3.98 \mathrm{E} \cdots 04$ & $5.09 \mathrm{E}+01$ & $1.44 \mathrm{E}-02$ & $1.20 \mathrm{E}-03$ & $8.72 \mathrm{E}-03$ & $1.23 \mathrm{E}-04$ & $1.12 \mathrm{E}+02$ & 1.65 & 1.31 & 1623 & $2.68 \mathrm{E}-01$ & $5.94 \mathrm{E}-01$ & 34 & 34 \\
\hline
\end{tabular}


RPP-5926 REV 6

Table B-5. Input Data With Water Addition for Hydrogen Generation Rate Model Calculations for 177 Tanks. (20 sheets)

\begin{tabular}{|c|c|c|c|c|c|c|c|c|c|c|c|c|c|c|c|c|c|c|c|}
\hline Tank & $\begin{array}{c}\mathrm{OH} \\
\text { in liquid } \\
{[\mathrm{OH} \mid} \\
(\mu \mathrm{g} / \mathrm{mL})\end{array}$ & $\begin{array}{c}\text { TOC } \\
\text { in liquid } \\
\text { |TOC| } \\
(\mu \mathrm{g} / \mathrm{mL})\end{array}$ & $\begin{array}{c}\mathrm{YO}_{2} \\
\text { in liquid } \\
{\left[\mathrm{NO}_{2} \mid\right.} \\
(\mu \mathrm{g} / \mathrm{mL})\end{array}$ & $\begin{array}{c}\mathrm{NO}_{3} \\
\text { in liquid } \\
\left|\mathrm{NO}_{3}\right| \\
(\mu \mathrm{g} / \mathrm{mL})\end{array}$ & $\begin{array}{c}\mathrm{Na} \\
\text { in liquid } \\
|\mathrm{Na}| \\
(\mathrm{ug} / \mathrm{mL})\end{array}$ & $\begin{array}{c}\text { Al } \\
\text { in liquid } \\
{[\mathrm{Al}]} \\
(\mu \mathrm{g} / \mathrm{mL})\end{array}$ & $\begin{array}{c}90 \mathrm{Sr} \\
\text { in waste } \\
{[\mathrm{Sr}]} \\
(\mu \mathrm{Ci} / \mathrm{g})\end{array}$ & $\begin{array}{c}{ }^{241} \mathrm{Am} \\
\text { in waste } \\
{[\mathrm{Am241]}} \\
(\mu \mathrm{Ci} / \mathrm{g})\end{array}$ & $\begin{array}{c}{ }^{240} \mathrm{Pu} \\
\text { in waste } \\
|\mathrm{Pu} 240| \\
(\mu \mathrm{Ci} / \mathrm{g})\end{array}$ & $\begin{array}{c}{ }^{239} \mathrm{Pu} \\
\text { in waste } \\
{[\mathrm{Pu} 240 \mid} \\
(\mu \mathrm{Ci} / \mathrm{g})\end{array}$ & $\begin{array}{c}{ }^{238} \mathrm{Pu} \\
\text { in waste } \\
|\mathrm{Pu} 238| \\
(\mu \mathrm{Ci} / \mathrm{g})\end{array}$ & $\begin{array}{c}{ }^{137} \mathrm{Cs} \\
\text { in waste } \\
{[\mathrm{Cs}]} \\
(\mu \mathrm{Ci} / \mathrm{g})\end{array}$ & $\begin{array}{c}\text { Bulk } \\
\text { density } \\
\text { D } \\
(\mathrm{g} / \mathrm{mL})\end{array}$ & $\mid \begin{array}{c}\text { Liquid } \\
\text { density } D_{L} \\
(\mathrm{~g} / \mathrm{ml})\end{array}$ & $\begin{array}{c}\text { Non-RGS } \\
\text { waste } \\
\text { volume } \\
(\mathrm{kL})\end{array}$ & $\begin{array}{l}\text { Bulk } \\
\text { water } \\
\left\{\mathrm{H}_{2} \mathrm{O} \mid\right. \\
(w t \%)\end{array}$ & $\begin{array}{c}\text { Liquid } \\
\text { water } \\
\left|\mathrm{H}_{2} \mathrm{O}\right| \\
(\mathrm{wt} \%)\end{array}$ & $\begin{array}{c}\text { Waste } \\
\text { temp. } \\
\mathrm{Tw} \\
\left({ }^{\circ} \mathrm{C}\right)\end{array}$ & $\begin{array}{c}\text { Dome } \\
\text { temp. } \\
\text { Td } \\
\left({ }^{\circ} \mathrm{C}\right)\end{array}$ \\
\hline $241-B Y-107$ & $3 \mathrm{E}+04$ & $40 \mathrm{E}+03$ & $1.17 \mathrm{E}+05$ & $31 \mathrm{E}+05$ & $.43 E+05$ & $12 \mathrm{E}+04$ & $4.29 E+01$ & $3.18 \mathrm{E}-02$ & $1.33 \mathrm{E}-03$ & $8.10 \mathrm{E}-03$ & $.66 \mathrm{E}-04$ & $1.15 \mathrm{E}+02$ & 1.68 & 1.46 & 1066 & $3.81 E-01$ & $4.86 \mathrm{E}-01$ & 31 & 26 \\
\hline Supernatant & $3.83 E+04$ & $2.40 \mathrm{E}+03$ & $1.17 \mathrm{E}+05$ & $1.31 \mathrm{E}+05$ & $2.43 E+05$ & $5.12 \mathrm{E}+04$ & $4.29 E+01$ & $1.18 \mathrm{E}-02$ & 1. $33 \mathrm{E}-03$ & $8.10 \mathrm{E}-03$ & $1.66 \mathrm{E}-04$ & $1.15 \mathrm{E}+02$ & 1.46 & 1.46 & 38 & $4.86 \mathrm{E}-0 \mathrm{I}$ & $4.86 \mathrm{E}-01$ & 0 & 0 \\
\hline Solid Layer & $3.83 \mathrm{E}+04$ & $40 \mathrm{E}+03$ & $1.17 \mathrm{E}+05$ & $1.39 E+05$ & $2.43 \mathrm{E}+05$ & $12 \mathrm{E}+04$ & $4.29 \mathrm{E}+01$ & $1.18 \mathrm{E}-02$ & $1.33 \mathrm{E}-03$ & 8. $10 \mathrm{E}-03$ & $1.66 \mathrm{E}-04$ & $1.15 \mathrm{E}+02$ & 1.69 & 1.46 & 1028 & $3.78 \mathrm{E}-01$ & $4.86 \mathrm{E}-01$ & 31 & 26 \\
\hline $241-B Y-108$ & $3.83 \mathrm{E}+04$ & $2.37 \mathrm{E}+03$ & $7.85 \mathrm{E}+04$ & $2.94 \mathrm{E}+05$ & $2.82 \mathrm{E}+05$ & $3.63 \mathrm{E}+04$ & $1.03 \mathrm{E}+02$ & 3.05E-02 & $3.43 \mathrm{E}-03$ & $2.26 \mathrm{E}-02$ & $3.96 \mathrm{E}-04$ & $4.2 ! E+01$ & 1.48 & 1.33 & 879 & $2.75 \mathrm{E}-01$ & $3.27 \mathrm{E}-01$ & 31 & 26 \\
\hline Supernatant & $3.83 E \div 04$ & $2.37 \mathrm{E}+03$ & $7.85 \mathrm{E}+04$ & $2.94 \mathrm{E}+05$ & $.82 \mathrm{E}+05$ & $63 E+04$ & $1.03 E+02$ & $3.05 \mathrm{E}-02$ & $3.43 \mathrm{E}-03$ & $2.26 \mathrm{E}-02$ & $.96 \mathrm{E}-04$ & $4.21 \mathrm{E}-01$ & 1.33 & 1.33 & 38 & $3.27 \mathrm{E}-01$ & $3.27 \mathrm{E}-01$ & 0 & 0 \\
\hline Solid Layer & $3.83 \mathrm{E}+04$ & $2.37 E+03$ & $7.85 \mathrm{E}+04$ & $2.94 \mathrm{E}+05$ & $2.82 \mathrm{E}+05$ & $3.63 \mathrm{E}^{+04}$ & $1.03 \mathrm{E}+02$ & $3.05 \mathrm{E}-02$ & $3.43 \mathrm{E}-03$ & $2.26 \mathrm{E}-02$ & $3.96 \mathrm{E}-04$ & $4.21 \mathrm{E}+01$ & 1.48 & 1.33 & 841 & $2.73 \mathrm{E}-01$ & $3.27 \mathrm{E}-0 \mathrm{I}$ & 31 & 26 \\
\hline $241-B Y-109$ & $3.83 \mathrm{E}+04$ & $1.88 \mathrm{E}=03$ & $8.29 E+04$ & $1.90 \mathrm{~F}+05$ & $2.10 \mathrm{E}-05$ & $88 E+04$ & $7.75 \mathrm{E}-00$ & $3.69 \mathrm{~F}-02$ & $4.35 \mathrm{E}-03$ & 1.82E-02 & $6.67 \mathrm{E}-04$ & $4.98 \mathrm{E}+01$ & 1.70 & 1.50 & 1124 & 4.23E-01 & $5.30 \mathrm{E}-01$ & 18 & 17 \\
\hline Supematant & $3.83 E \div 04$ & $1.88 \mathrm{~F}+03$ & $.29 \mathrm{E}+04$ & $1.90 \mathrm{E}+05$ & $.10 E+05$ & $88 \mathrm{E}+04$ & $7.75 \mathrm{E}+00$ & $3.69 \mathrm{E}-02$ & 4.35F-03 & 1.82E-02 & $6.67 \mathrm{E}-04$ & $4.98 \mathrm{E}+0 \mathrm{I}$ & 1.50 & 1.50 & 38 & $5.30 \mathrm{E}-01$ & $5.30 \mathrm{E}-01$ & 0 & 0 \\
\hline Solid Layer & $3.83 \mathrm{E}+04$ & $1.88 \mathrm{E}+03$ & $8.29 E+04$ & $1.90 \mathrm{E}+05$ & $2.10 E+05$ & $3.88 E+04$ & $7.75 \mathrm{E}+00$ & $3.69 \mathrm{E}-02$ & $4.35 \mathrm{E}-03$ & $1.82 \mathrm{E}-02$ & $6.67 \mathrm{E}-04$ & $4.98 \mathrm{E} \div 01$ & 1.71 & 1.50 & 1086 & 4.20E-01 & $5.30 \mathrm{E}-01$ & 18 & 17 \\
\hline $241-B Y-110$ & $3.83 \mathrm{E}+04$ & $94 \mathrm{E}+04$ & $.24 E+05$ & $1.24 E+05$ & $254 \mathrm{~F}+05$ & $.58 \mathrm{E}: 04$ & $7.28 \mathrm{E}+01$ & $2.76 \mathrm{E}-02$ & $3.82 \mathrm{E}-03$ & $2.22 \mathrm{E}-02$ & $4.97 \mathrm{E}-04$ & $8.75 \mathrm{E}+01$ & 1.56 & 1.44 & 1422 & $2.86 \mathrm{E}-01$ & $3.99 \mathrm{E}-01$ & 34 & 21 \\
\hline Supematant & $3.83 E+04$ & $1.94 \mathrm{E}+04$ & $1.24 \mathrm{E} \div 05$ & $1.24 \mathrm{E}+05$ & $54 \mathrm{E}+05$ & $.58 \mathrm{E}+04$ & $7.28 \mathrm{E}+01$ & $.76 E-02$ & $82 \mathrm{E}-\mathrm{C}$ & $2.22 \mathrm{E}-02$ & $.97 \mathrm{E}-04$ & $8.75 \mathrm{E}+$ & 1.44 & 1.44 & 38 & $3.99 \mathrm{E}-01$ & $.99 \mathrm{E}-01$ & 0 & 0 \\
\hline Solid Layer & $3.83 E+0.4$ & $1.94 \mathrm{E}+04$ & $1.24 \mathrm{E}+05$ & $1.24 \mathrm{E}+05$ & $2.54 E+05$ & $6.58 \mathrm{E}+04$ & $7.28 \mathrm{E}+01$ & $2.76 \mathrm{E}-02$ & $3.82 \mathrm{E}-03$ & $2.22 \mathrm{E}-02$ & 4.97E-04 & $8.75 E+01$ & 1.57 & 1.44 & 1384 & $2.83 \mathrm{E}-01$ & $3.99 \mathrm{E}-01$ & 34 & 21 \\
\hline $241-\mathrm{B}$ & 4 & 03 & 4 & 35 & 15 & $4.30 \mathrm{E}+04$ & 1 & $6.14 \mathrm{E}-02$ & 886.03 & $\mathrm{E}-02$ & $1 E-03$ & $5.96 \mathrm{E}+01$ & 1.67 & 1.42 & 1561 & $3.56 \mathrm{E}-01$ & $5.38 \mathrm{E}-01$ & 25 & 20 \\
\hline Supemratant & $3.83 \mathrm{E}+04$ & $1.28 \mathrm{E}+03$ & $5.46 \mathrm{E}+04$ & $2.01 \mathrm{E}+05$ & $2.17 \mathrm{E}-0 \mathrm{~S}$ & $4.30 \mathrm{E}+04$ & $1.02 \mathrm{E}+01$ & 6. $14 \mathrm{~F}-02$ & $6.28 \mathrm{E}-03$ & $2.63 \mathrm{E}-02$ & $1.01 \mathrm{E}-03$ & $5.96 \mathrm{E}+01$ & 1.42 & 1.42 & 38 & $5.38 \mathrm{E}-01$ & $5.38 \mathrm{E}-01$ & 0 & 0 \\
\hline Solid Layer & $3.83 E \div 04$ & $1.28 \mathrm{E}+03$ & $5.46 \mathrm{E} \div 04$ & $2.01 E-05$ & $2.17 \mathrm{E}+0.5$ & 4. $30 \mathrm{E} \div 04$ & $1.02 \mathrm{E}+01$ & $6.14 \mathrm{E}-02$ & $6.28 \mathrm{E}-03$ & 2.63E-02 & $1.01 \mathrm{E}-03$ & $5.96 \mathrm{E}+01$ & 1.67 & 1.42 & 1523 & $3.52 \mathrm{E}-01$ & $5.38 \mathrm{E}-01$ & 25 & 20 \\
\hline $241-B Y-112$ & 3 & -03 & 05 & +05 & 05 & $50+0$ & $1.18 \mathrm{E}+01$ & 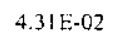 & $440 E_{0} 03$ & 1.84E-02 & $7.09 \mathrm{E}-04$ & $6.33 \mathrm{E}+01$ & 1.73 & 147 & 1121 & $3.02 \mathrm{E}-01$ & 4.85E-01 & 28 & 21 \\
\hline Supernatant & $3.83 \mathrm{E}+04$ & 1. $72 \mathrm{E}+03$ & $1.41 F+05$ & $1.90 E+05$ & $2.44 \mathrm{E}+05$ & $5.54 \mathrm{E}+04$ & $1.18 \mathrm{E}+01$ & $4.31 \mathrm{E}-02$ & $4.40 \mathrm{E}-03$ & $1.84 \mathrm{E}-02$ & $7.09 \mathrm{E}-04$ & $6.33 E+01$ & 1.47 & 1.47 & 38 & $4.85 \mathrm{E}-01$ & 01 & 0 & 0 \\
\hline Solid Layer & $3.83 \mathrm{E}+04$ & $1.72 \mathrm{E}+03$ & $1.41 E+05$ & $1.90 \mathrm{E}+05$ & $2.44 \mathrm{E}+05$ & $5.54 \mathrm{E} \div 04$ & $1.18 \mathrm{E}+01$ & $4.31 \mathrm{E}-02$ & $4.40 \mathrm{E}-03$ & $1.84 \mathrm{E}-02$ & $7.09 \mathrm{E}-04$ & $6.33 \mathrm{E}+01$ & 1.74 & 1.47 & 1083 & $2.96 \mathrm{E}-01$ & $4.85 \mathrm{E}-01$ & 28 & 21 \\
\hline $241-C-101$ & $1.70 \mathrm{E}-04$ & $8 \mathrm{E}+03$ & 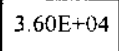 & 04 & $2 E+04$ & 3 & 1 & $1.43 \mathrm{E}-01$ & 1.40E-01 & 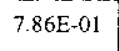 & -02 & $5.06 \mathrm{E}+0$ & 172 & 17 & 371 & $2.69 \mathrm{E}-01$ & $.40 \mathrm{E}-01$ & 32 & 32 \\
\hline Supenratant & $1.70 E+04$ & $2.98 E+03$ & $3.60 \mathrm{E}+04$ & $7.96 \mathrm{E}+04$ & $8.32 \mathrm{E}+04$ & $4.94 \mathrm{E}+03$ & $6.42 \mathrm{E}+01$ & $1.43 \mathrm{E}-01$ & $1.40 \mathrm{E}-01$ & $7.86 \mathrm{E}-01$ & $1.11 \mathrm{E}-02$ & $5.06 \mathrm{E}+01$ & 1. & 1 & 38 & $7.40 \mathrm{E}-01$ & $7.40 \mathrm{E}-01$ & 0 & 0 \\
\hline Solid Layer & $1.70 \mathrm{E}+04$ & $2.98 \mathrm{E}+03$ & $3.60 E+04$ & $7.96 \mathrm{E}+04$ & $8.32 \mathrm{E}+04$ & $4.94 E+03$ & $6.42 \mathrm{E}+01$ & $1.43 \mathrm{E}-01$ & $1.40 \mathrm{E}-01$ & $7.86 \mathrm{E}-01$ & $1.11 \mathrm{E}-02$ & $5.06 \mathrm{E}+(0)$ & 1.78 & 1.17 & 333 & $2.34 \mathrm{E}-01$ & $7.40 \mathrm{E}-01$ & 32 & 32 \\
\hline $241-C-102$ & $1.70 \mathrm{E}+04$ & $2.98 \mathrm{E}+03$ & $3.00 t+04$ & $7.96 \mathrm{E}+04$ & $2 \mathrm{E} \div 04$ & $94 \mathrm{E}+03$ & $1.39 \mathrm{E}+01$ & $.08 \mathrm{E}-01$ & $4.05 \mathrm{E}-01$ & 100 & -02 & $1.29 \mathrm{E}+01$ & 1.67 & 1.17 & 1234 & $01 \mathrm{E}-01$ & $7.50 \mathrm{E}-01$ & 29 & 26 \\
\hline Supernatant & $1.70 E+04$ & $2.98 E+03$ & $3.60 \mathrm{E}+04$ & $7.96 \mathrm{E}+04$ & $8.32 \mathrm{E}+04$ & $4.94 \mathrm{E}+03$ & $1.39 \mathrm{E}+01$ & $5.08 \mathrm{E}-01$ & $4.05 \mathrm{E}-01$ & $1.69 \mathrm{E}+00$ & $4.38 \mathrm{E}-02$ & $1.29 \mathrm{E}+01$ & 1.17 & 1.17 & 38 & $7.50 \mathrm{E}-01$ & 7.50E-01 & 0 & 0 \\
\hline Solid Layer & $1.70 \mathrm{E}+04$ & $2.98 \mathrm{E}+03$ & $3.60 \mathrm{E}+04$ & $7.96 \mathrm{E}+04$ & $8.32 \mathrm{E}+04$ & $4.94 \mathrm{E}+03$ & $1.39 \mathrm{E}+01$ & $5.08 \mathrm{E}-01$ & $4.05 \mathrm{E}-01$ & $1.69 \mathrm{E} \div 00$ & 4.38E-02 & $1.29 E+01$ & 1.68 & 1.17 & 1196 & $4.95 \mathrm{E}-01$ & $7.50 \mathrm{E}-01$ & 29 & 26 \\
\hline $241-C-103$ & $1.70 \mathrm{E}+04$ & $5.60 \mathrm{E}+0.3$ & $1.47 \mathrm{E} \div 04$ & $8.20 \mathrm{E}+02$ & $3.21 \mathrm{E}+04$ & $0.00 \mathrm{E}+00$ & $4.82 \mathrm{E}+02$ & 2.64E-02 & $E-U 1$ & $1.40 \mathrm{E}+00$ & $41 E-02$ & $4.67 E+01$ & 1.49 & 1.11 & 139 & -01 & 01 & 43 & 37 \\
\hline Supe & $1.70 \mathrm{E}+04$ & $5.60 \mathrm{E}+03$ & $1.47 \mathrm{E}+04$ & $8.20 \mathrm{E}+02$ & $3.21 \mathrm{E}-04$ & $0.00 \mathrm{E}-00$ & $4.82 \mathrm{E}+02$ & $2.64 \mathrm{E}-02$ & $2.9 \mid$ E- 01 & $1.40 E+00$ & $2.41 \mathrm{E}-02$ & $4.67 \mathrm{E}-01$ & 1.11 & 1.11 & 38 & $8.89 \mathrm{E}-01$ & $8.89 \mathrm{E}-01$ & 0 & 0 \\
\hline Solid Layer & $1.70 \mathrm{E}+04$ & $5.60 \mathrm{E}+03$ & $1.47 \mathrm{E}+04$ & $8.20 \mathrm{E}+02$ & $3.21 E+04$ & $0.00 \mathrm{E}+00$ & $4.82 \mathrm{E}-02$ & $2.64 \mathrm{E}-02$ & $2.91 \mathrm{E}-01$ & $1.40 \mathrm{E}+00$ & $2.41 \mathrm{E}-02$ & $4.67 \mathrm{E}+01$ & 1.63 & 1.31 & 101 & $2.32 \mathrm{E}-01$ & $8.89 \mathrm{E}-01$ & 43 & 37 \\
\hline
\end{tabular}


RPP-5926 REV 6

Table B-5. Input Data With Water Addition for Hydrogen Generation Rate Model Calculations for 177 Tanks. (20 sheets)

\begin{tabular}{|c|c|c|c|c|c|c|c|c|c|c|c|c|c|c|c|c|c|c|c|}
\hline Tank & $\begin{array}{c}\mathrm{OH} \\
\text { in liquid } \\
|\mathrm{OH}| \\
(\mu \mathrm{g} / \mathrm{mL})\end{array}$ & $\begin{array}{c}\text { TOC } \\
\text { in liquid } \\
{[\mathrm{TOC}]} \\
(\mu \mathrm{g} / \mathrm{mL})\end{array}$ & $\begin{array}{c}\mathrm{NO}_{2} \\
\text { in liquid } \\
\left|\mathrm{NO}_{2}\right| \\
(\mu \mathrm{g} / \mathrm{mL})\end{array}$ & $\begin{array}{c}\mathrm{NO}_{3} \\
\text { in liquid } \\
\left\{\mathrm{NO} \mathrm{O}_{3} \mid\right. \\
(\mu \mathrm{g} / \mathrm{mL})\end{array}$ & $\begin{array}{c}\mathrm{Na} \\
\text { in liquid } \\
{[\mathrm{Na}]} \\
\text { (ug/mL) }\end{array}$ & $\begin{array}{c}\text { Al } \\
\text { in liquid } \\
{[\mathrm{Al}]} \\
(\mu \mathrm{g} / \mathrm{mL})\end{array}$ & $\begin{array}{c}90 \mathrm{Sr} \\
\text { in waste } \\
{[\mathrm{Sr} \mid} \\
(\mu \mathrm{Ci} / \mathrm{g})\end{array}$ & $\begin{array}{c}{ }^{24 \mathrm{I}} \mathrm{Am} \\
\text { in waste } \\
\text { (Am241) } \\
(\mu \mathrm{Ci} / \mathrm{g})\end{array}$ & $\begin{array}{c}{ }^{240} \mathrm{Pu} \\
\text { in waste } \\
{[\mathrm{Pu} 240\}} \\
(\mu \mathrm{Ci} / \mathrm{g})\end{array}$ & $\begin{array}{c}{ }^{239} \mathrm{Pu} \\
\text { in waste } \\
\{\mathrm{Pu} 240\} \\
(\mu \mathrm{Ci} / \mathrm{g})\end{array}$ & $\begin{array}{c}{ }^{238} \mathrm{Pu} \\
\text { in waste } \\
{[\mathrm{Pu} 238]} \\
(\mu \mathrm{Ci} / \mathrm{g})\end{array}$ & $\begin{array}{c}{ }^{137} \mathrm{Cs} \\
\text { in waste } \\
{[\mathrm{Cs}]} \\
(\mu \mathrm{Ci} / \mathrm{g})\end{array}$ & $\begin{array}{c}\text { Bulk } \\
\text { density } \\
\text { D } \\
(\mathrm{g} / \mathrm{mL})\end{array}$ & $\begin{array}{c}\text { Liquid } \\
\text { density } D_{2} \\
(g / m l)\end{array}$ & $\left|\begin{array}{c}\text { Non-RGS } \\
\text { waste } \\
\text { volume } \\
\text { (kL) }\end{array}\right|$ & $\begin{array}{l}\text { Bulk } \\
\text { water } \\
{\left[\mathrm{H}_{2} \mathrm{O}\right]} \\
(w t \%)\end{array}$ & $\begin{array}{c}\text { Liquid } \\
\text { water } \\
\left|\mathrm{H}_{2} \mathrm{O}\right| \\
(\mathrm{wt} \%)\end{array}$ & $\begin{array}{c}\text { Waste } \\
\text { temp. } \\
\text { Tw } \\
\left({ }^{\circ} \mathrm{C}\right)\end{array}$ & $\begin{array}{c}\text { Dome } \\
\text { temp. } \\
\text { Td } \\
\left({ }^{\circ} \mathrm{C}\right)\end{array}$ \\
\hline $241-C-104$ & $E+04$ & $\mathrm{OE}+03$ & $60 E+04$ & $7.96 E+04$ & $32 E+04$ & $4.94 E+03$ & $71 \mathrm{E}+02$ & $3.85 \mathrm{E}+00$ & 8. $10 \mathrm{E}-01$ & $3.14 E+00$ & 1.39E-01 & $5.38 \mathrm{E}+01$ & 1.66 & 1.17 & 1018 & $4.88 \mathrm{E}-01$ & $8.10 \mathrm{E}-01$ & 36 & 34 \\
\hline Supernatant & $1.70 \mathrm{E}+04$ & $5.60 \mathrm{E}+03$ & $3.60 \mathrm{E}+04$ & $7.96 \mathrm{E}+04$ & $8.32 \mathrm{E}+04$ & $4.94 \mathrm{E} \div 03$ & $2.71 \mathrm{E}+02$ & $3.85 \mathrm{E}+00$ & $8.10 \mathrm{E}-01$ & $3.14 \mathrm{E} 00$ & $1.39 \mathrm{E}-01$ & $5.38 \mathrm{E} \div 01$ & 1.17 & 1.17 & 38 & $8.10 \mathrm{E}-01$ & $8.10 \mathrm{E}-01$ & 0 & 0 \\
\hline Solid Layer & $1.70 \mathrm{E}+04$ & $5.60 \mathrm{E}+03$ & $3.60 \mathrm{E}+04$ & $7.96 \mathrm{E}+04$ & $8.32 \mathrm{E}+04$ & $4.94 \mathrm{E}+03$ & $2.71 E+02$ & $3.85 \mathrm{E}+00$ & $8.10 \mathrm{E}-01$ & $3.14 \mathrm{E}+00$ & $1.39 \mathrm{E}-01$ & $5.38 \mathrm{E}+01$ & 1.68 & 1.17 & 980 & $4.79 \mathrm{E}-01$ & $8.10 \mathrm{E}-01$ & 36 & 34 \\
\hline $241-C-105$ & $0 E+04$ & $2.87 \mathrm{E}+03$ & $70 E+04$ & $3.43 E+04$ & $1.14 \mathrm{E}+05$ & $02 \mathrm{E}+00$ & $5.69 \mathrm{E}+02$ & $1.54 \mathrm{E}+00$ & $5.03 \mathrm{E}-01$ & $2.56 \mathrm{E}+00$ & $11 \mathrm{E}-02$ & $9.85 \mathrm{E}+0 \mathrm{t}$ & 1.53 & 1.23 & 538 & $3.05 \mathrm{E}-01$ & $6.70 \mathrm{E}-01$ & 47 & 41 \\
\hline Supernatant & $1.70 \mathrm{E}+04$ & $2.87 \mathrm{E} \div 03$ & $3.70 \mathrm{E}+04$ & $3.43 \mathrm{E} \div 04$ & $1.14 \mathrm{E}+05$ & $9.02 \mathrm{E}+00$ & $5.69 \mathrm{E}+02$ & $1.54 \mathrm{E}+00$ & $5.03 \mathrm{E}-01$ & $2.56 \mathrm{E}+00$ & $4.11 \mathrm{~F}-02$ & $9.85 \mathrm{E}+01$ & 1.23 & 1.23 & 38 & $6.70 \mathrm{E}-01$ & $6.70 \mathrm{E}-01$ & 0 & 0 \\
\hline Solid l.ayer & $1.70 \mathrm{E}+04$ & $2.87 \mathrm{E}+03$ & $3.70 \mathrm{E}+04$ & $3.43 \mathrm{E}+04$ & $1.14 \mathrm{E}+05$ & $9.02 \mathrm{E}+00$ & $5.69 \mathrm{E}+02$ & $1.54 \mathrm{E}+00$ & $5.03 \mathrm{E}-01$ & $2.56 \mathrm{E}+00$ & $4.11 \mathrm{E}-02$ & $9.85 \mathrm{E}+01$ & 1.55 & 1.23 & 500 & $2.83 \mathrm{E}-01$ & $6.70 \mathrm{E}-01$ & 47 & 41 \\
\hline $24 !-C-106$ & $9 \mathrm{E}+02$ & $4.73 \mathrm{E}+01$ & $7.74 \mathrm{E}+00$ & $8.58 \mathrm{E}+00$ & $39 \mathrm{E}+03$ & $1.33 \mathrm{E}+0 !$ & $1.22 \mathrm{E}+03$ & $1.2 ! \mathrm{E}+00$ & $6.61 \mathrm{E}-02$ & $3.09 \mathrm{E}-01$ & $5.00 \mathrm{E}-02$ & $2.68 \mathrm{E}+01$ & 1.12 & 1.02 & 48 & $8.29 \mathrm{E}-01$ & $9.93 \mathrm{E}-0 \mathrm{l}$ & 30 & 25 \\
\hline Supernatant & $5.92 \mathrm{E}+01$ & $2.80 \mathrm{E} \div 00$ & $4.58 \mathrm{E}-01$ & $5.08 \mathrm{E}-01$ & $8.25 \mathrm{E}+01$ & $7.91 \mathrm{E}-01$ & $3.72 \mathrm{E}-04$ & $3.54 \mathrm{E}-08$ & $7.31 \mathrm{E}-09$ & $3.43 \mathrm{E}-08$ & $5.50 \mathrm{E}-09$ & $3.67 \mathrm{E}-03$ & 1.00 & 1.00 & 38 & $1.00 \mathrm{E}+00$ & $1.00 \mathrm{E}+00$ & 31 & 25 \\
\hline Solid Layer & $7.02 \mathrm{E}+03$ & $3.32 \mathrm{E}+02$ & $5.43 \mathrm{E}+01$ & $6.02 \mathrm{E}+01$ & $9.78 \mathrm{E}+03$ & $9.38 \mathrm{E}+01$ & $4.17 \mathrm{E}+03$ & $4.11 \mathrm{E}+00$ & 2.25E-01 & $1.05 E+00$ & $1.70 \mathrm{E}-01$ & $9.14 \mathrm{E}+01$ & 1.56 & 1.14 & 10 & $4.19 \mathrm{E}-0 \mathrm{t}$ & $9.78 \mathrm{E}-01$ & 30 & 25 \\
\hline $241-C-107$ & $O F \cdot: 04$ & $2.98 \mathrm{E}+03$ & $3.60 \mathrm{E}+04$ & $7.96 \mathrm{E}+04$ & $32 E+04$ & $4.94 \mathrm{E}+03$ & $1.44 E+03$ & $62 E+00$ & $2.44 \mathrm{E}-01$ & $1.51 E+00$ & $05 \mathrm{E}-02$ & $4.07 \mathrm{E}+01$ & 1.54 & 1.17 & 973 & $4.84 \mathrm{E}-01$ & $90 \mathrm{E}-01$ & 43 & 41 \\
\hline Supernatant & $1.70 \mathrm{E}+04$ & $2.98 \mathrm{E}-03$ & $3.60 \mathrm{E} \cdot 04$ & $7.96 \mathrm{E}+04$ & $8.32 \mathrm{E} \cdot 04$ & $4.94 \mathrm{E} \div 03$ & $1.44 E+03$ & $4.62 \mathrm{E}+00$ & $2.44 \mathrm{E}-01$ & $1.51 \mathrm{E}+00$ & $3.05 \mathrm{E}-02$ & $4.07 \mathrm{E}+01$ & 1.17 & 1.17 & 38 & $7.90 \mathrm{E}-01$ & $7.90 \mathrm{E}-01$ & 0 & 0 \\
\hline Solid Layer & $1.70 \mathrm{E}+04$ & $2.98 \mathrm{E}+03$ & $3.60 \mathrm{E}+04$ & $7.96 \mathrm{E}+04$ & $32 \mathrm{E}+04$ & $4.94 \mathrm{E}+03$ & $1.44 \mathrm{E}+03$ & $4.62 \mathrm{E}+00$ & $2.44 \mathrm{E}-01$ & $1.51 \mathrm{E}+00$ & $3.05 \mathrm{E}-02$ & $4.07 E+01$ & 1.55 & 1.17 & 935 & $4.75 \mathrm{E}-01$ & $7.90 \mathrm{E}-01$ & 43 & 41 \\
\hline $241 \cdot C-108$ & $1.70 \mathrm{E}+04$ & $2.98 \mathrm{E}+03$ & $3.60 \mathrm{E}+04$ & $7.96 \mathrm{E}+04$ & $8.32 \mathrm{E}+04$ & $4.94 \mathrm{E}+03$ & $2.16 \mathrm{E}+01$ & $2.90 \mathrm{E}-02$ & $9.18 \mathrm{E}-04$ & $8.43 \mathrm{E}-03$ & $5.95 \mathrm{E}-05$ & $2.10 \mathrm{E}+02$ & 1.44 & 1.17 & 288 & $4.21 \mathrm{E}-01$ & $50 \mathrm{E}-01$ & 26 & 27 \\
\hline Supenatant & $1.70 \mathrm{E}+04$ & $2.98 E+03$ & $3.60 \mathrm{E}+04$ & $7.96 \mathrm{E}+04$ & $8.32 \mathrm{E}+04$ & $4.94 \mathrm{E}+03$ & $2.16 \mathrm{E}+01$ & $2.90 \mathrm{E}-02$ & $9.18 \mathrm{E}-04$ & $8.43 \mathrm{E}-0.3$ & $5.95 E-05$ & $2.10 \mathrm{E}+02$ & 1.17 & 1.17 & 38 & $7.50 \mathrm{E}-01$ & $7.50 E-01$ & 0 & 0 \\
\hline Solid Layer & $1.70 \mathrm{E}+04$ & $2.98 \mathrm{E}+03$ & $3.60 \mathrm{E}+04$ & $7.96 \mathrm{E}+04$ & $32 \mathrm{E}+04$ & $4.94 \mathrm{E}+03$ & $2.16 \mathrm{E} \div 0 \mathrm{I}$ & $2.90 \mathrm{E}-02$ & $9.18 \mathrm{E}-04$ & $8.43 \mathrm{E}-03$ & $5.95 \mathrm{E}-05$ & $2.10 \mathrm{~F}+02$ & 1.48 & 1.17 & 250 & 3.82E-01 & $7.50 \mathrm{E}-01$ & 26 & 27 \\
\hline $241-C-109$ & $1.70 \mathrm{E}+04$ & $2.98 \mathrm{E}+03$ & $3.60 \mathrm{E}+04$ & $7.96 \mathrm{E}+04$ & $8.32 \mathrm{E} \vdash 04$ & $4.94 \mathrm{E}+03$ & $6.80 \mathrm{E}+02$ & $1.35 \mathrm{E}-0 \mathrm{t}$ & $3.02 \mathrm{~F}-02$ & $1.76 \mathrm{E}-01$ & $4.44 \mathrm{E}-03$ & $4.65 \mathrm{E}+02$ & 1.50 & 1.17 & 278 & 4. $85 \mathrm{E}-01$ & $7.50 \mathrm{E}-01$ & 26 & 26 \\
\hline Supernatant & $1.70 \mathrm{E} \div 04$ & $2.98 \mathrm{E}+03$ & $3.60 \mathrm{E}+04$ & $7.96 \mathrm{E}+04$ & $8.32 E+04$ & $4.94 \mathrm{E}+03$ & $6.80 \mathrm{E}+02$ & $1.35 \mathrm{E}-0 \mathrm{I}$ & $3.02 \mathrm{E}-02$ & $1.76 \mathrm{E}-0 \mathrm{I}$ & $4.44 \mathrm{E}-03$ & $4.65 \mathrm{E}-02$ & 1.17 & 1.17 & 38 & $7,50 E-01$ & $7.50 \mathrm{E}-01$ & 0 & 0 \\
\hline Solid Layer & $1.70 E+04$ & $2.98 \mathrm{E}-03$ & $3.60 \mathrm{E}+04$ & $7.96 \mathrm{E} \cdot 04$ & $8.32 \mathrm{E}-04$ & $4.94 \mathrm{E}+03$ & $6.80 \mathrm{E}+02$ & $1.35 \mathrm{E}-01$ & $3.02 \mathrm{E}-02$ & J.76E-01 & $4.44 \mathrm{E}-03$ & $4.65 \mathrm{E}+02$ & 1.55 & 1.17 & 240 & $4.53 \mathrm{E}-01$ & $7.50 \mathrm{E}-01$ & 26 & 26 \\
\hline $241-C-\$ 10$ & $1.57 \mathrm{E}+04$ & $5.36 \mathrm{E}+02$ & $4.25 \mathrm{E}+03$ & $5.57 \mathrm{E}+04$ & $2.82 \mathrm{E}+04$ & $1.93 \mathrm{E}+02$ & $3.67 \mathrm{E}=00$ & $4.11 \mathrm{E}-02$ & $7.50 \mathrm{E}-03$ & $6.89 \mathrm{E}-02$ & $4.85 \mathrm{E}-04$ & $1.44 E+01$ & 1.32 & 1.09 & 712 & $6.19 \mathrm{E}-01$ & $8.36 \mathrm{E}-01$ & 21 & 22 \\
\hline Supernatant & $1.62 \mathrm{E}+03$ & $5.54 \mathrm{E}+01$ & $4.40 \mathrm{E}+02$ & $5.76 \mathrm{~F}+03$ & $2.91 E+03$ & $1.99 \mathrm{E} .01$ & $2.00 \mathrm{E}-03$ & $1.79 \mathrm{E}-06$ & $1.05 E-05$ & $1.56 \mathrm{E}-04$ & $5.28 \mathrm{E}-07$ & $3.55 E-01$ & 1.01 & 1.01 & 42 & $9.82 \mathrm{E}-01$ & $9.82 \mathrm{E}-01$ & 21 & 22 \\
\hline Solid Layer & $1.70 E+04$ & $5.80 \mathrm{E}-02$ & $4.60 \mathrm{E}+03$ & $6.03 \mathrm{E}+04$ & $3.05 \mathrm{E}+04$ & $2.09 \mathrm{E}+02$ & $3.84 \mathrm{E}+00$ & $4.30 \mathrm{E}-02$ & $7.85 \mathrm{E}-03$ & $7.22 \mathrm{E}-02$ & $5.08 \mathrm{E}-04$ & $1.50 \mathrm{E}+01$ & 1.34 & 1.10 & 670 & $6.02 \mathrm{~F}-01$ & $8.29 \mathrm{E}-01$ & 21 & 22 \\
\hline $241-C-111$ & $1.70 \mathrm{E}+04$ & $2.98 \mathrm{E}+03$ & $3.60 \mathrm{E}+04$ & $7.96 \mathrm{E}+04$ & $8.32 \mathrm{E}+04$ & $4.94 \mathrm{E}+03$ & $2.70 E+03$ & $5.67 \mathrm{E} .01$ & $9.85 \mathrm{E}-02$ & $5.06 \mathrm{H}-01$ & $9.30 \mathrm{E}-03$ & $5.89 \mathrm{E}+01$ & 1.49 & 1.17 & 255 & $4.51 \mathrm{E}-01$ & $7.50 \mathrm{E}-01$ & 25 & 25 \\
\hline Supernatant & $1.70 \mathrm{E}+04$ & $2.98 \mathrm{E}+03$ & $3.60 \mathrm{E}+04$ & $7.96 \mathrm{E}+04$ & $8.32 \mathrm{E}+04$ & $4.94 E \div 03$ & $2.70 \mathrm{E} \div 03$ & $5.67 \mathrm{E}-01$ & $9.85 \mathrm{E}-02$ & $5.06 \mathrm{E}-01$ & $9.30 \mathrm{E}-03$ & $5.89 E+01$ & 1.17 & 1.17 & 38 & $7.50 \mathrm{E}-01$ & $7.50 \mathrm{E}-01$ & 0 & 0 \\
\hline Solid Layer & $1.70 \mathrm{E}+04$ & $2.98 \mathrm{E}+03$ & $3.60 \mathrm{E}+04$ & $7.96 \mathrm{E}+04$ & $8.32 \mathrm{E}+04$ & $4.94 \mathrm{E}+03$ & $2.70 \mathrm{E}+03$ & $5.67 \mathrm{E}-0 \mathrm{I}$ & $9.85 E-02$ & $5.06 \mathrm{E}-01$ & $9.30 \mathrm{E}-03$ & $5.89 \mathrm{E}+01$ & 1.55 & 1.17 & 217 & $4.11 E-01$ & $7.50 \mathrm{E}-0 \mathrm{I}$ & 25 & 25 \\
\hline $241-C-112$ & $1.70 \mathrm{E}+04$ & $2.98 \mathrm{E} \div 03$ & $3.60 \mathrm{E}+04$ & $7.96 \mathrm{E}+04$ & $8.32 \mathrm{E} \div 04$ & $4.94 \mathrm{E}+03$ & $9.56 \mathrm{E}+02$ & $7.49 \mathrm{E}-01$ & $1.69 \mathrm{E}-02$ & $1.34 \mathrm{E}-01$ & $1.25 \mathrm{E}-02$ & $4.23 \mathrm{E}+02$ & 1.56 & 1.17 & 431 & $5.30 \mathrm{E}-01$ & $7.50 \mathrm{E}-01$ & 27 & 28 \\
\hline Supernatant & $1.70 \mathrm{E}+04$ & $2.98 \mathrm{E}+03$ & $3.60 \mathrm{E}+04$ & $7.96 \mathrm{E}+04$ & $8.32 \mathrm{E}+04$ & $4.94 \mathrm{E}+03$ & $9.56 \mathrm{E}+02$ & $7.49 \mathrm{E}-01$ & $1.69 \mathrm{E}-02$ & $1.34 \mathrm{E}-01$ & $1.25 \mathrm{E}-02$ & $A 235+0$ & 1.17 & 1.17 & 38 & $7.50 \mathrm{E}-01$ & $7.50 \mathrm{E}-01$ & 0 & 0 \\
\hline Solid Layer & $1.70 E+04$ & $2.98 E+03$ & $3.60 \mathrm{E}+04$ & $7.96 E+04$ & $8.32 \mathrm{E}+04$ & $4.94 \mathrm{E} \div 03$ & $9.56 \mathrm{E}+02$ & $7.49 \mathrm{E}-01$ & $1.69 \mathrm{E}-02$ & $1.34 \mathrm{E}-01$ & $1.25 \mathrm{E}-02$ & $4.23 \mathrm{E}+02$ & 1.60 & 1.17 & 393 & $5.15 \mathrm{E}-01$ & $7.50 \mathrm{E}-01$ & 27 & 28 \\
\hline
\end{tabular}




\section{RPP-5926 REV 6}

Table B-5. Input Data With Water Addition for Hydrogen Generation Rate Model Calculations for 177 Tanks. (20 sheets)

\begin{tabular}{|c|c|c|c|c|c|c|c|c|c|c|c|c|c|c|c|c|c|c|c|}
\hline Tank & $\begin{array}{c}\mathrm{OH} \\
\text { in liquid } \\
|\mathrm{OH}| \\
(\mu \mathrm{g} / \mathrm{mL})\end{array}$ & $\begin{array}{c}\text { TOC } \\
\text { in liquid } \\
\text { [TOC } \mid \\
(\mu \mathrm{g} / \mathrm{mL})\end{array}$ & $\begin{array}{c}\mathrm{NO}_{z} \\
\text { in liquid } \\
{\left[\mathrm{NO}_{2} \ddagger\right.} \\
(\mu \mathrm{g} / \mathbf{m L})\end{array}$ & $\begin{array}{c}\mathrm{NO}_{3} \\
\text { in liquid } \\
{\left[\mathrm{NO}_{3}\right]} \\
(\mu \mathrm{g} / \mathrm{mL})\end{array}$ & $\begin{array}{c}\mathrm{Na} \\
\text { in liquid } \\
{[\mathrm{Na}]} \\
\text { (ug/mL) }\end{array}$ & $\begin{array}{c}\text { Al } \\
\text { in liquid } \\
{[\mathrm{Al}]} \\
(\mu \mathrm{g} / \mathrm{mL})\end{array}$ & $\begin{array}{c}90 \mathrm{Sr} \\
\text { in waste } \\
{[\mathrm{Sr}]} \\
(\mu \mathrm{Ci} / \mathrm{g})\end{array}$ & $\begin{array}{c}{ }^{241} \mathrm{Am} \\
\text { in waste } \\
|\mathrm{Am} 241| \\
(\mu \mathrm{Ci} / \mathrm{g})\end{array}$ & $\begin{array}{c}{ }^{2+10} \mathrm{Pu} \\
\text { in waste } \\
{\left[\mathrm{Pu}^{240]}\right.} \\
(\mu \mathrm{Ci} / \mathrm{g})\end{array}$ & $\begin{array}{c}{ }^{239} \mathrm{Pu} \\
\text { in waste } \\
|\mathrm{Pu} 240| \\
(\mu \mathrm{Ci} / \mathrm{g})\end{array}$ & $\begin{array}{c}{ }^{238} \mathrm{Pu} \\
\text { in waste } \\
\text { |Puz38| } \\
(\mu \mathrm{Ci} / \mathrm{g})\end{array}$ & $\begin{array}{c}{ }^{137} \mathrm{Cs} \\
\text { in waste } \\
{[\mathrm{Cs} \mid} \\
(\mu \mathrm{Ci} / \mathrm{g})\end{array}$ & $\begin{array}{c}\text { Bulk } \\
\text { density } \\
\text { D } \\
(\mathrm{g} / \mathrm{mL})\end{array}$ & $\begin{array}{c}\text { Liquid } \\
\text { density } D_{L} \\
(\mathrm{~g} / \mathrm{ml})\end{array}$ & $\begin{array}{c}\text { Non-RGS } \\
\text { waste } \\
\text { volume } \\
(\mathrm{kL})\end{array}$ & $\begin{array}{c}\text { Bulk } \\
\text { water } \\
\left|\mathrm{H}_{2} \mathrm{O}\right| \\
(\mathrm{wt} \%)\end{array}$ & $\begin{array}{l}\text { I.iquid } \\
\text { water } \\
\left\{\mathrm{H}_{2} \mathrm{O}\right\} \\
(\mathrm{wt} \%)\end{array}$ & $\begin{array}{c}\text { Waste } \\
\text { temp. } \\
\text { Tw } \\
\left({ }^{\circ} \mathrm{C}\right)\end{array}$ & $\begin{array}{c}\text { Dome } \\
\text { temp. } \\
\text { Td } \\
\left({ }^{\circ} \mathrm{C}\right)\end{array}$ \\
\hline $241-C-201$ & $2 E+02$ & $00 \mathrm{E}+00$ & $0.00 E+00$ & $0.00 E+00$ & $.00 \mathrm{E}+00$ & $00 \mathrm{E}+00$ & $33 E+01$ & $5.11 \mathrm{E}-01$ & $18 \mathrm{E}-01$ & $34 \mathrm{E}+00$ & $.06 \mathrm{E}-01$ & $13 E+00$ & 1.09 & 1.00 & 4 & $8.50 \mathrm{E}-01$ & $1.00 \mathrm{E}+00$ & 16 & 20 \\
\hline Superuatant & $3.14 \mathrm{E}+00$ & $0.00 \mathrm{E}+00$ & $0.00 \mathrm{~F}-00$ & $0.00 \mathrm{E}+00$ & $0.00 \mathrm{E}+00$ & $0.00 \mathrm{E}+00$ & $0.00 \mathrm{E}+00$ & $0.00 \mathrm{E}+00$ & $0.00 \mathrm{E}+00$ & $0.00 \mathrm{E}+00$ & $0.00 \mathrm{E}+00$ & $0.00 E+00$ & 1.00 & 1.00 & 4 & $1.00 \mathrm{E} \div 00$ & $1.00 \mathrm{E}-00$ & 16 & 20 \\
\hline Solid Layer & $1.70 \mathrm{E}+04$ & $0.00 \mathrm{k} \cdot 00$ & $0.00 \mathrm{E}+00$ & $0.00 \mathrm{E}+00$ & $0.00 E: 00$ & $0.00 \mathrm{E}+00$ & $2.68 \mathrm{E}+02$ & $2.58 \mathrm{E}+00$ & $3.62 \mathrm{E}+00$ & $1.68 \mathrm{E}+01$ & $5.33 \mathrm{E}-01$ & $1.07 \mathrm{E}+01$ & 1.75 & 1.00 & 1 & $2.44 \mathrm{E}-01$ & $1.00 \mathrm{E}+00$ & 16 & 20 \\
\hline $241-C-202$ & $9.91 E+02$ & $0.00 \mathrm{E}+00$ & $0.00 \mathrm{E}+00$ & $0.00 E+00$ & $0.00 \mathrm{E}-00$ & $0.00 \mathrm{E}+00$ & $1.02 E+02$ & $2.42 \mathrm{E}-01$ & $6.485-01$ & $3.01 E+00$ & $9.55 \mathrm{E}-02$ & $1.88 E+00$ & 1.09 & 1.00 & 4 & 8.47E-01 & $1.00 \mathrm{E}+00$ & 16 & 19 \\
\hline Supernatant & $4.04 \mathrm{E} \div 00$ & $0.00 \mathrm{E}+00$ & $0.00 E+00$ & $0.00 \mathrm{E}+00$ & $0.00 \mathrm{E}+00$ & $0.00 \mathrm{E}+00$ & $0.00 \mathrm{E}+00$ & $0.00 \mathrm{E}+00$ & $0.00 \mathrm{E}+00$ & $0.00 \mathrm{E}+00$ & $0.00 \mathrm{E}+00$ & $0.00 \mathrm{E}+00$ & 1.00 & 1.00 & 4 & $1.00 \mathrm{E}+00$ & $1.00 E+00$ & 16 & 19 \\
\hline Solid Layer & $1.70 \mathrm{E}+04$ & $0.005: 00$ & $0.00 \mathrm{E}+00$ & $0.00 \mathrm{E}+00$ & $0.00 \mathrm{E}+00$ & $0.00 \mathrm{E}+00$ & $5.08 \mathrm{E}+02$ & $1.20 \mathrm{E}+00$ & $3.21 \mathrm{E}+00$ & $1,49 \mathrm{E}+01$ & $4.73 \mathrm{E}-01$ & $9.31 \mathrm{E}+00$ & 1.75 & 1.00 & 1 & $2.44 \mathrm{E}-01$ & $1.00 \mathrm{E}+00$ & 16 & 19 \\
\hline $241-\mathrm{C}-203$ & $1.35 E+03$ & $0.00 \mathrm{E}+00$ & $0.00 \mathrm{E}+00$ & $0.00 \mathrm{E}+00$ & $0.00 \mathrm{E}+00$ & $0.00 E+00$ & $4.97 \mathrm{E}-01$ & $6.12 \mathrm{E}-03$ & $2.21 \mathrm{E}-02$ & $1.02 \mathrm{E}-0 !$ & $3.26 \mathrm{E}-03$ & $2.76 \mathrm{E}+00$ & 1.10 & 1.00 & 4 & $8.75 \mathrm{E}-01$ & $1.00 \mathrm{E}+00$ & 15 & 19 \\
\hline Supernatant & $2.15 \mathrm{E}+01$ & $0.00 \mathrm{E}+00$ & $0.00 \mathrm{E}+00$ & $0.00 \mathrm{E}+00$ & $0.00 \mathrm{E}+00$ & $.00 \mathrm{E}+00$ & $0.00 \mathrm{E}+00$ & $0.00 E+00$ & $0.00 \mathrm{E}+00$ & $0.00 \mathrm{E}+00$ & $0.00 \mathrm{E}+00$ & $0.00 \mathrm{E}+00$ & 1.00 & 1.00 & 4 & $1.00 \mathrm{E}+00$ & $1.00 \mathrm{E}+00$ & 19 & 19 \\
\hline Solid Layer & $1.70 \mathrm{E} \div 04$ & $0.00 \mathrm{E}+00$ & $0.00 \mathrm{E}+00$ & $0.00 \mathrm{E}+00$ & $0.00 \mathrm{E}+00$ & $0.00 \mathrm{E}+00$ & $2.57 \mathrm{E}-00$ & $3.17 \mathrm{E}-02$ & $1.14 \mathrm{E}-01$ & $5.29 \mathrm{E}-0 \mathrm{I}$ & 1.69E-02 & $1.43 \mathrm{E}+01$ & 1.93 & 1.00 & 0 & $3.55 \mathrm{E}-01$ & $1.00 \mathrm{E}+00$ & 15 & 19 \\
\hline $241-\mathrm{C}-204$ & $170 E+04$ & $98 \mathrm{E}+03$ & $60 \mathrm{~F}+\mathrm{CH}^{-1}$ & $7.96 \mathrm{E}+04$ & $8.32 \mathrm{E}+04$ & $4.94 E+03$ & $1.09 \mathrm{E}+01$ & $1.39 \mathrm{E}-03$ & $1.23 \mathrm{E}-03$ & $5.70 \mathrm{E}-03$ & $1.81 \mathrm{E}-04$ & $4.72 \mathrm{E}+00$ & 1.44 & 1.17 & 9 & $5.23 \mathrm{E}-01$ & $7.50 \mathrm{E}-01$ & 17 & 16 \\
\hline Supenatant & $1.70 E-04$ & $98 \mathrm{~F}+03$ & $3.60 \mathrm{E} \div 04$ & $7.96 E+04$ & 8. $32 E \div 04$ & $.94 E+03$ & $1.09 \mathrm{E}+01$ & $1.39 \mathrm{E}$ & $23 E-03$ & $5.70 \mathrm{E}-$ & $1.81 \mathrm{E}-04$ & $4.72 \mathrm{E}+00$ & 1.17 & 1.17 & 4 & $7.50 \mathrm{E}-01$ & $7.50 \mathrm{E}-0 \mathrm{]}$ & 0 & 0 \\
\hline Solid Layer & $1.70 \mathrm{~F} i 04$ & $2.98 E-03$ & $3.60 \mathrm{E}-04$ & $7.96 \mathrm{E} \div 04$ & $8.32 \mathrm{E}+04$ & $4.94 \mathrm{E} r 03$ & $1.09 \mathrm{E}+01$ & $1.39 \mathrm{E}-03$ & $1.23 \mathrm{E}-03$ & $5.70 \mathrm{E}-03$ & $1.81 \mathrm{E}-04$ & $4.72 \mathrm{E}+00$ & 1.62 & 1.17 & 6 & $4.12 \mathrm{E}-01$ & $7.50 \mathrm{E}-01$ & 17 & 16 \\
\hline 2. & 4 & -03 & $1.13 E+05$ & 5 & $2.22 \mathrm{E}+05$ & 4 & 22 & 1 & $2.87 E-02$ & $1.46 \mathrm{E}-01$ & 03 & $12 E+02$ & 1 & 147 & 1370 & $.95 E-01$ & $5.07 \mathrm{E}-01$ & 39 & 31 \\
\hline Supernatant & $4.76 \mathrm{E}-0.4$ & $1.79 \mathrm{E}+03$ & $1.13 E+05$ & $2.14 \mathrm{E}+05$ & $2.22 \mathrm{E}+05$ & $2.99 \mathrm{E}+04$ & $2.06 \mathrm{E}+02$ & $1.00 \mathrm{E}-01$ & $2.87 \mathrm{E}-02$ & $1.46 \mathrm{E}-01$ & $3.05 \mathrm{E}-03$ & $1.12 E+02$ & 1.47 & 1.47 & 38 & $5.07 \mathrm{E}-01$ & $5.07 \mathrm{E}-01$ & 0 & 0 \\
\hline Solid Layer & $4.76 \mathrm{E} 104$ & $1.79 E+03$ & $1.13 \mathrm{E}+05$ & $2.14 \mathrm{E}-05$ & $2.22 \mathrm{E}+05$ & $2.99 \mathrm{E} \sim 04$ & $2.06 \mathrm{E}+02$ & $1.00 \mathrm{E}-01$ & $2.87 \mathrm{E}-02$ & $1.46 \mathrm{E}-01$ & $3.05 \mathrm{E}-03$ & $1.12 \mathrm{E} \cdot 02$ & 1.65 & 1.47 & 1332 & $3.92 \mathrm{E}-01$ & 5.07E-01 & 39 & 31 \\
\hline $241-S-102$ & $3.76 \mathrm{E}+04$ & 03 & 55 & 55 & $2.34 \mathrm{E}+05$ & 4 & 01 & 2 & $1.19 \mathrm{E}-02$ & 2 & E-03 & 01 & 1. & 1.46 & 70 & 01 & E-01 & 35 & 33 \\
\hline Supernatant & $3.76 \mathrm{E}+04$ & $3.76 \mathrm{E}+03$ & $1.27 \mathrm{E}+05$ & $1.55 E+05$ & $2.34 \mathrm{E} \div 05$ & $5.62 E+04$ & $6.57 \mathrm{E}+01$ & $9.88 \mathrm{E}-02$ & $1.19 \mathrm{E}-02$ & $5.59 \mathrm{E}-02$ & $1.83 \mathrm{E}-03$ & $9.55 \mathrm{E}+01$ & 1.46 & 1.46 & 38 & 4.85E-01 & $4.85 \mathrm{E}-01$ & 0 & 0 \\
\hline Solid Layer & $3.76 \mathrm{E}+04$ & $3.76 \mathrm{E}+03$ & $1.27 \mathrm{E}+05$ & $1.55 \mathrm{E}+05$ & $2.34 \mathrm{E}+05$ & $5.62 \mathrm{E}+04$ & $6.57 \mathrm{E}+01$ & $9.88 \mathrm{E}-02$ & $1.19 \mathrm{E}-02$ & $5.59 \mathrm{E}-02$ & $1.83 \mathrm{E}-03$ & $9.55 \mathrm{E}+01$ & 1.71 & 1.46 & 664 & $3.46 \mathrm{E}-01$ & $4.85 \mathrm{E}-01$ & 35 & 33 \\
\hline 24I-S-103 & $3.46 \mathrm{E}+04$ & $4.44 \mathrm{E}+03$ & $1.40 \mathrm{E}+05$ & $2.05 \mathrm{E}+05$ & $2.16 \mathrm{E}+05$ & $3.04 \mathrm{E}+04$ & $2.80 \mathrm{E}+01$ & בסקים & $1.32 \mathrm{E}-02$ & 6.34E-02 & $1.89 \mathrm{E}-03$ & $1.32 \mathrm{E}+02$ & 1.59 & 143 & 934 & $3.74 \mathrm{E}-01$ & 5.11E-01 & 7 & 25 \\
\hline Supematant & $3.49 \mathrm{E}+03$ & $4.47 \mathrm{E}+02$ & $1.40 \mathrm{E}+04$ & $2.06 \mathrm{E}+04$ & $2.18 \mathrm{E}+04$ & $3.06 \mathrm{E}+03$ & $3.39 \mathrm{E}-02$ & $1.08 \mathrm{E}-04$ & $1.12 E-06$ & $5.25 \mathrm{E}-06$ & $1.83 \mathrm{E}-07$ & $2.96 \mathrm{E}+01$ & 1.04 & 1.04 & 42 & $9.33 \mathrm{E}-01$ & $9.33 \mathrm{E}-01$ & 27 & 25 \\
\hline Solid Layer & $3.64 \mathrm{E} \div 04$ & $4.68 \mathrm{E}+03$ & $1.47 E+05$ & $2.16 E+05$ & $2.28 \mathrm{E}-05$ & $3.20 \mathrm{E}+04$ & $2.88 \mathrm{E}+01$ & $9.40 \mathrm{E}-02$ & $1.36 \mathrm{E}-02$ & 6.54E-02 & $1.95 \mathrm{E}-03$ & $1.35 \mathrm{E}+02$ & 1.61 & 1.45 & 892 & $3.57 \mathrm{E}-01$ & $4.98 \mathrm{E}-01$ & 27 & 25 \\
\hline $241-S-104$ & $5.10 \mathrm{~F}+03$ & $3.96 \mathrm{E}+02$ & $3.82 \mathrm{E}+04$ & $2.68 \mathrm{E}-05$ & $1.98 \mathrm{E}+05$ & $1.19 \mathrm{E}+04$ & $2.21 E+02$ & $1.11 \mathrm{E}-01$ & $3.90 \mathrm{E}-02$ & 1.92E-01 & $5.50 \mathrm{E}-03$ & $4.26 \mathrm{E}+01$ & 1.66 & 1,77 & 1128 & $3.93 \mathrm{E}-01$ & $5.51 \mathrm{E}-01$ & 38 & 32 \\
\hline Superiatant & $5.10 E+03$ & $3.96 \mathrm{E}+02$ & $3.82 \mathrm{E}+04$ & $2.68 \mathrm{E}+05$ & $1.98 \mathrm{~F}+05$ & $1.19 \mathrm{E}+04$ & $2.21 \mathrm{E}+02$ & $1.1 \mathrm{IE}-01$ & $3.90 \mathrm{E}-02$ & $1.92 \mathrm{E}-01$ & $5.50 \mathrm{E}-03$ & $4.26 \mathrm{E}+01$ & 1.37 & 1.37 & 38 & $5.51 \mathrm{~F}-01$ & $5.51 \mathrm{E}-01$ & 0 & 0 \\
\hline Solid Layer & $5.10 E \div 03$ & $3.96 \mathrm{E}+02$ & $3.82 E+04$ & $2.68 E+05$ & $1.98 \mathrm{E}+05$ & $1.19 E+04$ & $2.21 \mathrm{E}+02$ & $1.11 \mathrm{E}-01$ & $3.90 \mathrm{E}-02$ & $1.92 E-01$ & -03 & $4.26 \mathrm{E}+0 \mathrm{I}$ & 1.67 & 1.37 & 1090 & $3.88 \mathrm{E}-01$ & $5.51 E-01$ & 38 & 32 \\
\hline $241-S-105$ & $8.18 \mathrm{E}+04$ & $4.03 E+03$ & $1.22 \mathrm{E}+05$ & $1.90 E+05$ & $2.36 \mathrm{E}+05$ & $3.78 \mathrm{E}+04$ & $2.56 \mathrm{E}+00$ & $3.05 \mathrm{E}-03$ & $7.25 \mathrm{E}-04$ & $3.63 \mathrm{E}-03$ & $8.19 \mathrm{E}-05$ & $3.42 \mathrm{E}+01$ & 1.65 & 1.45 & 1574 & 8.62E-02 & $4.91 \mathrm{E}-01$ & 25 & 23 \\
\hline Supernatant & $8.18 \mathrm{E}+04$ & -03 & $1.22 \mathrm{E}+05$ & $1.90 \mathrm{E}+05$ & $2.36 \mathrm{E}+05$ & $3.78 \mathrm{E}+04$ & $2.56 \mathrm{E}+00$ & $3.05 \mathrm{E}-03$ & 7.25E-04 & $3.63 \mathrm{E}-03$ & $8.19 \mathrm{E}-05$ & $3.42 \mathrm{E} \div 01$ & 1.45 & 1.45 & 38 & $4.91 \mathrm{E}-01$ & $4.91 \mathrm{E}-01$ & 0 & 0 \\
\hline Solid Layer & $8.18 E+04$ & $4.03 \mathrm{E}+03$ & $1.22 \mathrm{E}+05$ & $1.90 \mathrm{E}+05$ & $2.36 \mathrm{E}+05$ & $3.78 \mathrm{E}+04$ & $2.56 \mathrm{E}+00$ & $3.05 \mathrm{E}-03$ & $7.25 \mathrm{E}-04$ & $3.63 \mathrm{E}-03$ & $8.19 \mathrm{E}-05$ & $3.42 \mathrm{E}+0 \mathrm{~J}$ & 1.66 & 1.45 & 1536 & $7.75 \mathrm{E}-02$ & $4.91 \mathrm{E}-01$ & 25 & 23 \\
\hline
\end{tabular}


RPP-5926 REV 6

Table B-5. Input Data With Water Addition for Hydrogen Generation Rate Model Calculations for 177 Tanks. (20 sheets)

\begin{tabular}{|c|c|c|c|c|c|c|c|c|c|c|c|c|c|c|c|c|c|c|c|}
\hline Tank & $\begin{array}{c}\mathrm{OH} \\
\text { in liquid } \\
|\mathrm{OH}| \\
(\mu \mathrm{g} / \mathrm{mL})\end{array}$ & $\begin{array}{c}\text { TOC } \\
\text { in liquid } \\
\text { [TOC] } \\
(\mu \mathrm{g} / \mathrm{mL})\end{array}$ & $\begin{array}{c}\mathrm{NO}_{2} \\
\text { in liquid } \\
\left|\mathrm{NO}_{2}\right| \\
(\mu \mathrm{g} / \mathrm{mL})\end{array}$ & $\begin{array}{c}\mathrm{NO}_{3} \\
\text { in liquid } \\
\left|\mathrm{NO}_{3}\right| \\
(\mu \mathrm{g} / \mathrm{mL})\end{array}$ & $\begin{array}{c}\text { Ya } \\
\text { in liquid } \\
|\mathrm{Na}| \\
\text { (ug/mL) }\end{array}$ & $\begin{array}{c}\text { Al } \\
\text { in liquid } \\
|\mathrm{A}| \mid \\
(\mu \mathrm{g} / \mathrm{mL})\end{array}$ & $\begin{array}{c}90 \mathrm{Sr} \\
\text { in waste } \\
|\mathrm{Sr}| \\
(\mu \mathrm{Ci} / \mathrm{g})\end{array}$ & $\begin{array}{c}{ }^{241} \mathrm{Am} \\
\text { in waste } \\
\text { |Am241| } \\
(\mu \mathrm{Ci} / \mathrm{g})\end{array}$ & $\begin{array}{c}{ }^{240} \mathrm{Pu} \\
\text { in waste } \\
|\mathrm{Pu} 240| \\
(\mu \mathrm{Ci} / \mathrm{g})\end{array}$ & $\begin{array}{c}{ }^{239} \mathrm{Pu}_{\mathrm{u}} \\
\text { in waste } \\
{[\mathrm{Pu} 240]} \\
(\mu \mathrm{Ci} / \mathrm{g})\end{array}$ & $\begin{array}{c}{ }^{238} \mathrm{Pu} \\
\text { in waste } \\
|\mathrm{Pu238}| \\
(\mu \mathrm{Ci} / \mathrm{g})\end{array}$ & $\begin{array}{c}{ }^{137} \mathrm{Cs} \\
\text { in waste } \\
{[\mathrm{Cs} \mid} \\
(\mu \mathrm{Ci} / \mathrm{g})\end{array}$ & $\begin{array}{c}\text { Bulk } \\
\text { density } \\
\mathrm{D} \\
(\mathrm{g} / \mathrm{mL})\end{array}$ & $\begin{array}{c}\text { Liquid } \\
\text { density } D_{L} \\
(g / m l)\end{array}$ & $\begin{array}{c}\text { von-RGS } \\
\text { waste } \\
\text { volume } \\
\text { (kL) }\end{array}$ & $\begin{array}{l}\text { Bulk } \\
\text { water } \\
\left\{\mathrm{H}_{2} \mathrm{O} \mid\right. \\
(\mathrm{wt} \%)\end{array}$ & $\begin{array}{l}\text { Liquid } \\
\text { water } \\
\left|\mathrm{H}_{2} \mathrm{O}\right| \\
(w t \%)\end{array}$ & $\begin{array}{l}\text { Waste } \\
\text { temp. } \\
\mathrm{Tw} \\
\left({ }^{\circ} \mathrm{C}\right)\end{array}$ & $\begin{array}{c}\text { Dome } \\
\text { temp. } \\
\text { Td } \\
\left({ }^{\circ} \mathrm{C}\right)\end{array}$ \\
\hline $241-S-106$ & $5 \mathrm{E}+04$ & $8 \mathrm{E}+03$ & $1.04 E+05$ & $2.36 E+05$ & $44 \mathrm{E}+05$ & $80 E+04$ & $1.23 \mathrm{E} \div 01$ & $1.96 \mathrm{E}-02$ & 1.32E-03 & $6.04 \mathrm{E}-03$ & $.13 \mathrm{E}-04$ & $9.13 E+01$ & 1.72 & 1.43 & 1761 & $3.48 \mathrm{E}-0 \mathrm{~J}$ & $5.42 \mathrm{E}-01$ & 23 & 23 \\
\hline Supernatant & $5.55 E+04$ & $1.98 \mathrm{~F}+03$ & $1.04 \mathrm{E} \div 05$ & $2.36 \mathrm{E}+05$ & $2.44 \mathrm{E}+05$ & $3.80 \mathrm{E}+04$ & $1.23 \mathrm{E}+0 \mathrm{l}$ & $1.96 \mathrm{E}-02$ & $1.32 \mathrm{E}-03$ & $6.04 \mathrm{E}-03$ & $2.13 \mathrm{E}-04$ & $9.13 \mathrm{E}+01$ & 1.43 & 1.43 & 38 & $5.42 \mathrm{E}-01$ & $5.42 \mathrm{E}-01$ & 0 & 0 \\
\hline Solid Layer & $5.55 \mathrm{E}+04$ & $1.98 \mathrm{E}+03$ & $1.04 \mathrm{E}+05$ & $2.36 \mathrm{E}-05$ & $2.44 \mathrm{E}+05$ & $3.80 \mathrm{E}+04$ & $1.23 \mathrm{E}+01$ & $1.96 \mathrm{E}-02$ & $1.32 \mathrm{E}-03$ & $6.04 \mathrm{E}-03$ & 2.1.3E-04 & $9.13 \mathrm{E}+01$ & 1.72 & 1.43 & 1723 & 3.45E-01 & $5.42 \mathrm{E}-01$ & 23 & 23 \\
\hline $241-S-107$ & $2.99 \mathrm{E}+04$ & $1.18 \mathrm{E}+03$ & $7.11 E+04$ & $14 \mathrm{E}+05$ & $.56 \mathrm{E} \div 05$ & $1.07 \mathrm{E}+04$ & $1.30 E+02$ & $3.10 \mathrm{E}-01$ & $1.06 \mathrm{E}-01$ & $5.04 \mathrm{E}-01$ & $1.22 \mathrm{E}-02$ & $6.77 \mathrm{E}+01$ & 1.76 & 1.31 & 1394 & $3.47 \mathrm{E}-01$ & $6.40 E-01$ & 33 & 25 \\
\hline Supernatant & $2.99 \mathrm{E}+04$ & $1.18 \mathrm{E}+03$ & $7.11 \mathrm{E}-04$ & $1.14 E+05$ & $1.56 \mathrm{E}+05$ & $1.07 \mathrm{E}+04$ & $1.30 \mathrm{E} \div 02$ & $3.10 \mathrm{E}-01$ & $1.06 \mathrm{E}-01$ & $5.04 \mathrm{E}-01$ & $1.22 \mathrm{E}-02$ & $6.77 \mathrm{E}+01$ & 1.31 & 1.31 & 38 & $6.40 \mathrm{E}-01$ & 6. $40 \mathrm{E}-01$ & 0 & 0 \\
\hline Solid Layer & $2.99 \mathrm{E}+04$ & $1.18 E \div 03$ & $7.11 \mathrm{E}+04$ & $1.14 \mathrm{E}-05$ & $1.56 \mathrm{E} \div 05$ & $1.07 \mathrm{E} \sim 04$ & $1.30 \mathrm{E}+02$ & $3.10 \mathrm{E}-01$ & $1.06 \mathrm{E}-01$ & $5.04 \mathrm{E}-01$ & $1.22 \mathrm{E}-02$ & $6.77 \mathrm{E}+01$ & 1.78 & 1.31 & 1356 & $3.41 \mathrm{E}-01$ & $6.40 \mathrm{E}-01$ & 33 & 25 \\
\hline $241-\mathrm{S}-108$ & $81 E 104$ & $03 E+03$ & $1.22 E+05$ & $1.90 \mathrm{E} \div 05$ & $2.36 \mathrm{E}-05$ & $.77 E+04$ & $2.10 E+01$ & $72 \mathrm{E}-02$ & $5.96 \mathrm{~F}-03$ & $2.79 \mathrm{E}-02$ & $9.05 \mathrm{E}-04$ & $9.60 \mathrm{E}+01$ & 1.67 & 1.45 & 2120 & $3.07 \mathrm{E}-01$ & $4.91 E-01$ & 27 & 24 \\
\hline Supernatant & $4.81 E+04$ & $4.03 E+03$ & $1.22 \mathrm{E} \cdot 05$ & $1.90 \mathrm{E}+05$ & $2.36 \mathrm{E}+05$ & $3.77 \mathrm{E}+04$ & $2.10 \mathrm{E} \div 01$ & $7.72 \mathrm{E}-02$ & $5.96 \mathrm{E}-03$ & $2.79 \mathrm{E}-02$ & $9.05 \mathrm{E}-04$ & $9.60 \mathrm{E}+01$ & 1.45 & 1.45 & 38 & $4.91 \mathrm{E}-01$ & $4.91 E-0\}$ & 0 & 0 \\
\hline Solid Layer & $4.81 E+04$ & $4.03 E+03$ & $1.22 \mathrm{E}+05$ & $1.90 \mathrm{E}+05$ & $2.36 \mathrm{E}+05$ & $3.77 \mathrm{E}+04$ & $2.10 \mathrm{E}+01$ & $7.72 \mathrm{E}-02$ & $5.96 \mathrm{E}-03$ & $2.79 \mathrm{E}-02$ & $9.05 E-04$ & $9.60 \mathrm{E}+01$ & 1.68 & 1.45 & 2082 & $3.04 \mathrm{E}-01$ & 4.91E-01 & 27 & 24 \\
\hline $24 !-S \cdot 109$ & $9 \mathrm{E}+04$ & $1 E-03$ & $8.49 \mathrm{E}+04$ & $1.32 \mathrm{E}+05$ & $19 \mathrm{E}-05$ & $59 \mathrm{E}+04$ & $1.48 \mathrm{E}+0 \mathrm{I}$ & $5 E-02$ & $2.83 \mathrm{E}-03$ & $44 E-02$ & $.92 \mathrm{E}-04$ & $1.30 \mathrm{E}+01$ & 1.65 & 1.49 & 2055 & $1.14 \mathrm{E}-01$ & $5.31 \mathrm{E}-01$ & 26 & 23 \\
\hline Supenatant & $8.19 E+04$ & $1.13 E+03$ & $8.49 E+04$ & $1.32 E+05$ & $2.19 E+05$ & $4.59 \mathrm{E}+04$ & $1.48 E+01$ & $1.15 E-02$ & $2.83 \mathrm{~F}-03$ & $1.44 E-02$ & $2.92 \mathrm{E}-04$ & $1.30 \mathrm{E}+01$ & 1.49 & 1.49 & 38 & $5.31 \mathrm{E}-01$ & $5.31 \mathrm{E}-01$ & 0 & 0 \\
\hline Solid Layer & $8.19 \mathrm{E}+04$ & $1.11 \mathrm{E}+03$ & $8.49 \mathrm{E}+04$ & $1.32 \mathrm{E}+05$ & $2.19 \mathrm{E}+05$ & $4.59 \mathrm{E}+04$ & $1.48 \mathrm{E}+01$ & $1.15 \mathrm{E}-02$ & $2.83 \mathrm{E}-03$ & 1.44E-02 & $2.92 E-04$ & $1.30 \mathrm{E}+01$ & 1.66 & 1.49 & 2017 & $1.07 \mathrm{E}-01$ & $5.31 \mathrm{E}-01$ & 26 & 23 \\
\hline $241-\mathrm{S}-110$ & $8.18 \mathrm{E}+04$ & $2.28 \mathrm{E}-03$ & $8.58 \mathrm{E}+04$ & $2.17 \mathrm{E}-05$ & $2.11 E+05$ & $3.10 \mathrm{t}+04$ & $9.40 \mathrm{E}+01$ & $1.23 \mathrm{E}-01$ & $2.50 \mathrm{E}-02$ & $1.26 \mathrm{E}-01$ & $2.63 \mathrm{E}-03$ & $7.27 \mathrm{E}+01$ & 1.66 & 1.43 & 1511 & $2.06 \mathrm{E}-01$ & $4.93 \mathrm{E}-01$ & 40 & 24 \\
\hline Supenatant & $8.18 E+04$ & $2.28 \mathrm{E}: 03$ & $8.58 \mathrm{E}+04$ & $2.17 E+05$ & $2.11 \mathrm{E}-05$ & $3.10 \mathrm{E}+04$ & $9.40 \mathrm{E}+01$ & $1.23 E-01$ & $2.50 \mathrm{~F}-02$ & $1.26 \mathrm{E}-01$ & $2.63 \mathrm{E}-03$ & $7.27 \mathrm{E}+01$ & 1.43 & 1.43 & 38 & $4.93 \mathrm{E}-01$ & 4. $93 \mathrm{~F}-01$ & 0 & 0 \\
\hline Solid & $8.18 E+04$ & $2.28 \mathrm{E}+03$ & $8.58 \mathrm{E} \cdot 04$ & $2.17 \mathrm{E}+05$ & $2.11 \mathrm{E}+05$ & $3.10 \mathrm{E}+04$ & $9.40 \mathrm{E}-01$ & I.23E-01 & $2.50 \mathrm{E}-02$ & $1.26 \mathrm{E}-01$ & $2.63 \mathrm{E}-03$ & $7.27 \mathrm{E}+0 \mathrm{I}$ & 1.66 & 1.43 & 1473 & $2.00 \mathrm{E}-01$ & $4.93 \mathrm{E}-01$ & 40 & 24 \\
\hline $241-S-111$ & $5.10 \mathrm{E}+04$ & $1.495: 03$ & $8.39 \mathrm{E}+04$ & $1.93 \mathrm{E}-05$ & $2.23 E+05$ & $3.64 \mathrm{E} \div 04$ & $1.83 \mathrm{E}+02$ & $1.48 \mathrm{E}-02$ & $1.44 \mathrm{E}-03$ & $6.90 \mathrm{E}-03$ & $1.96 \mathrm{E}-04$ & $1.03 E+02$ & 1.54 & 1.45 & 1435 & $2.96 \mathrm{E}-01$ & $5.10 \mathrm{E}-01$ & 28 & 24 \\
\hline Supernatant & $5.10 \mathrm{E}+04$ & $1.49 \mathrm{E}+03$ & $8.39 E+04$ & $1.93 \mathrm{E}+05$ & $2.23 \mathrm{E}-05$ & $3.64 \mathrm{E} \div 04$ & $1.83 \mathrm{E}+02$ & $1.48 \mathrm{E}-02$ & $1.44 \mathrm{E}-03$ & $6.90 \mathrm{E}-03$ & $1.96 \mathrm{E}-04$ & $1.03 \mathrm{E}+02$ & 1.45 & 1.45 & 38 & $5.10 \mathrm{E}-01$ & 5. $10 \mathrm{E}-01$ & 0 & 0 \\
\hline Solid layer & 5.10E-04 & $1.49 \mathrm{E}+03$ & $8.39 \mathrm{E}-04$ & $1.93 E+05$ & $2.23 E+05$ & 4 & 1 & 1 & 3 & 33 & $1.96 \mathrm{E}-04$ & 92 & 1.5 & 1.45 & 1397 & $2.90 \mathrm{E}-01$ & $5.10 \mathrm{E}-01$ & 28 & 24 \\
\hline $241-5-112$ & $4.86 \mathrm{E}+04$ & $8.31 E+02$ & $6.21 \mathrm{E}+04$ & $1.68 \mathrm{E}+05$ & $2.29 \mathrm{E}+05$ & $3.69 \mathrm{E}+04$ & $6.28 \mathrm{E}-02$ & $5.24 \mathrm{E}-01$ & $5.68 E-02$ & $3.20 \mathrm{E}-01$ & 7.23E-03 & $1.72 \mathrm{E}+01$ & 1.54 & 1.45 & 57 & $4.61 \mathrm{E}-01$ & $5.20 \mathrm{E}-01$ & 22 & 26 \\
\hline Supematant & $4.86 \mathrm{E}+04$ & $8.31 \mathrm{E}+02$ & $6.21 \mathrm{E}+04$ & $1.68 \mathrm{E}+05$ & $2.29 \mathrm{E}+05$ & $3.69 \mathrm{E}+04$ & $6.28 \mathrm{E}+02$ & $5.24 \mathrm{E}-01$ & $5.68 \mathrm{E}-02$ & $3.20 \mathrm{E}-01$ & $7.23 \mathrm{E}-03$ & $1.72 E+01$ & 1.45 & 1.45 & 38 & $5.20 \mathrm{E}-01$ & $5.20 \mathrm{E}-01$ & 0 & 0 \\
\hline Solid Layer & $4.86 \mathrm{E}+04$ & $8.31 \mathrm{E}+02$ & $6.21 E+04$ & $1.68 E+05$ & $2.29 \mathrm{E}+05$ & $3.69 \mathrm{E}+04$ & $E+02$ & $5.24 \mathrm{E}-\mathrm{C}$ & $5.68 \mathrm{E}-02$ & $3.20 \mathrm{E}-01$ & $7.23 \mathrm{E}-03$ & 01 & 1.7 & 1.45 & 19 & 01 & 01 & 22 & 26 \\
\hline $241-S X-101$ & $4.88 \mathrm{E}+04$ & $3.35 \mathrm{E}+02$ & $7.49 \mathrm{E}+04$ & $1.08 \mathrm{E}+05$ & $2.42 E+05$ & $8.00 \mathrm{E}+04$ & $1.13 E+02$ & $1.91 \mathrm{E}-01$ & $3.39 \mathrm{E}-02$ & $1.66 \mathrm{E}-01$ & $4.59 \mathrm{E}-03$ & $1.00 \mathrm{E}+02$ & 1.67 & 1.48 & 1622 & $2.71 \mathrm{E}-01$ & $4.91 \mathrm{E}-01$ & 49 & 39 \\
\hline Supematant & $4.88 \mathrm{E}+04$ & $3.35 \mathrm{E}+02$ & $7.49 \mathrm{E}+04$ & $1.08 \mathrm{E}+05$ & $2.42 \mathrm{E}+05$ & $8.00 \mathrm{E}+04$ & $1.13 \mathrm{E}+02$ & $1.91 \mathrm{E}-01$ & $3.39 \mathrm{E}-02$ & $1.66 \mathrm{E}-01$ & $4.59 \mathrm{E}-03$ & $1.00 \mathrm{E}+02$ & 1.48 & 1.48 & 38 & $4.91 \mathrm{E}-01$ & $4.91 \mathrm{E}-01$ & 0 & 0 \\
\hline Solid Layer & $4.88 \mathrm{E}-04$ & $3.35 \mathrm{E}+02$ & $7.49 \mathrm{E}+04$ & $1.08 E+05$ & $2.42 \mathrm{E}-05$ & $8.00 \mathrm{E} \div 04$ & $1.13 \mathrm{E}+02$ & $1.91 \mathrm{E}-01$ & $3.39 \mathrm{E}-02$ & $1.66 \mathrm{E}-01$ & $4.59 \mathrm{E}-03$ & $1.00 E+02$ & 1.68 & .48 & 1584 & $2.66 \mathrm{E}-01$ & $4.91 \mathrm{E}-01$ & 49 & 39 \\
\hline $241-S X-102$ & $5.01 \mathrm{E}+04$ & $1.51 \mathrm{E}+03$ & $1.51 \mathrm{E}+05$ & $1.57 \mathrm{E}+05$ & $2.35 \mathrm{E}+05$ & $4.56 \mathrm{E}+04$ & $8.21 \mathrm{E}+01$ & $1.22 \mathrm{E}-01$ & $1.21 \mathrm{E}-02$ & $5.77 \mathrm{E}-02$ & $1.69 \mathrm{E}-03$ & $1.40 \mathrm{E}+02$ & 1.69 & 1.46 & 1330 & $3.95 \mathrm{E}-01$ & $4.81 E-01$ & 52 & 32 \\
\hline Supernatant & $5.01 \mathrm{E}+04$ & $1.51 E \div 03$ & $1.51 \mathrm{E}+05$ & $1.57 \mathrm{E}-05$ & $2.35 E+05$ & $4.56 \mathrm{E}+04$ & $8.21 \mathrm{E}$ & 1.221 & $1.21 \mathrm{E}-02$ & $5.77 \mathrm{E}-02$ & $1.69 \mathrm{E}-03$ & $1, \mathrm{SOF}^{2}$ & 1.46 & 1.46 & 38 & $4.81 E-01$ & $4.81 \mathrm{E}-01$ & 0 & 0 \\
\hline Solid Layer & $5.01 \mathrm{E}+04$ & $1.51 E+03$ & $1.51 \mathrm{E}+05$ & $1.57 \mathrm{E}+05$ & $2.35 \mathrm{E}+05$ & $4.56 E+04$ & $8.21 \mathrm{E}+01$ & $1.22 \mathrm{E}-01$ & $1.21 \mathrm{E}-02$ & $5.77 \mathrm{E}-02$ & $1.69 \mathrm{E}-03$ & $1.40 \mathrm{E}+02$ & 1.70 & 1.46 & 1292 & $3.93 \mathrm{E}-01$ & $4.81 \mathrm{E}-01$ & 52 & 32 \\
\hline
\end{tabular}


RPP-5926 REV 6

Table B-5. Input Data With Water Addition for Hydrogen Generation Rate Model Calculations for 177 Tanks. (20 sheets)

\begin{tabular}{|c|c|c|c|c|c|c|c|c|c|c|c|c|c|c|c|c|c|c|c|}
\hline Tank & $\begin{array}{c}\mathrm{OH} \\
\text { in liquid } \\
{[\mathrm{OH}]} \\
(\mu \mathrm{g} / \mathrm{mL})\end{array}$ & $\begin{array}{c}\text { TOC } \\
\text { in liquid } \\
\mid \text { TOC| } \\
(\mu \mathrm{g} / \mathrm{mL})\end{array}$ & $\begin{array}{c}\mathrm{NO}_{2} \\
\text { in liquid } \\
\left|\mathrm{NO}_{2}\right| \\
(\mu \mathrm{g} / \mathrm{mL})\end{array}$ & $\begin{array}{c}\mathrm{NO}_{3} \\
\text { in liquid } \\
\left|\mathrm{NO}_{3}\right| \\
(\mu \mathrm{g} / \mathrm{mL})\end{array}$ & $\begin{array}{c}\mathrm{Na} \\
\text { in liquid } \\
{[\mathrm{Na} \mid} \\
(\mathrm{ug} / \mathrm{mL})\end{array}$ & $\begin{array}{c}\text { Al } \\
\text { in liquid } \\
|\mathrm{A}| \mid \\
(\mu \mathrm{g} / \mathrm{mL})\end{array}$ & $\begin{array}{c}90 \mathrm{Sr} \\
\text { in waste } \\
|\mathrm{Sr}| \\
(\mu \mathrm{Ci} / \mathrm{g})\end{array}$ & $\begin{array}{c}{ }^{241} \mathrm{Am} \\
\text { in waste } \\
|\mathrm{Am} 241| \\
(\mu \mathrm{Ci} / \mathrm{g})\end{array}$ & $\begin{array}{c}{ }^{240} \mathrm{Pu} \mathbf{u} \\
\text { in waste } \\
\text { |Pu240| } \\
(\mu \mathrm{Ci} / \mathrm{g})\end{array}$ & $\begin{array}{c}{ }^{239} \mathrm{Pu} \\
\text { in waste } \\
{[\mathrm{Pu} 240 \mid} \\
(\mu \mathrm{Ci} / \mathrm{g})\end{array}$ & $\begin{array}{c}{ }^{2339} \mathrm{Pu} \\
\text { in waste } \\
|\mathrm{Pu238}| \\
(\mu \mathrm{Ci} / \mathrm{g})\end{array}$ & $\begin{array}{c}{ }^{137} \mathrm{Cs} \\
\text { in waste } \\
{[\mathrm{Cs}]} \\
(\mu \mathrm{Ci} / \mathrm{g})\end{array}$ & $\begin{array}{c}\text { Bulk } \\
\text { density } \\
D \\
(\mathrm{~g} / \mathrm{mL})\end{array}$ & $\begin{array}{c}\text { Liquid } \\
\text { density } D_{L} \\
(\mathrm{~g} / \mathrm{ml})\end{array}$ & $\begin{array}{c}\text { Non-RGS } \\
\text { waste } \\
\text { volume } \\
(\mathrm{kL})\end{array}$ & $\begin{array}{l}\text { Bulk } \\
\text { water } \\
\left|\mathrm{H}_{2} \mathrm{O}\right| \\
\left(\mathrm{wt}^{\prime \prime} / 6\right)\end{array}$ & $\begin{array}{l}\text { Liquid } \\
\text { water } \\
\left|\mathrm{H}_{2} \mathrm{O}\right| \\
\left(\mathrm{wt}^{\mathrm{m}} \% \mathrm{w}\right)\end{array}$ & $\begin{array}{l}\text { Waste } \\
\text { temp. } \\
\text { Tw } \\
\left({ }^{\circ} \mathrm{C}\right)\end{array}$ & $\begin{array}{c}\text { Dome } \\
\text { temp. } \\
\text { Td } \\
\left.{ }^{\circ} \mathrm{C}\right)\end{array}$ \\
\hline 241-SX-103 & $2 \mathrm{E}+04$ & $8 E \div 03$ & $1.57 \mathrm{E}+05$ & $56 \mathrm{E}+05$ & $2.40 \mathrm{E}+05$ & $44 \mathrm{E}+04$ & $54 \mathrm{E}+02$ & $16 E-01$ & $56 \mathrm{~F}-02$ & $61 \mathrm{E}-02$ & $2.05 \mathrm{E}-03$ & $1.23 \mathrm{E}+02$ & 1.72 & 1.47 & 1964 & $3.52 \mathrm{E}-01$ & $4.66 \mathrm{E}-01$ & 60 & 27 \\
\hline Supernatant & $4.52 E+04$ & $3.38 \mathrm{t}+03$ & $1.57 \mathrm{E}+05$ & $1.56 \mathrm{E}+05$ & $2.40 \mathrm{E}-05$ & $4.44 \mathrm{E}+04$ & $1.54 \mathrm{E} \div 02$ & $2.16 \mathrm{E}-01$ & $1.56 \mathrm{E}-02$ & $7.61 \mathrm{E}-02$ & $2.05 \mathrm{E}-03$ & $1.23 \mathrm{E}+02$ & 1.47 & 1.47 & 38 & $4.66 \mathrm{E}-01$ & $4.66 \mathrm{E}-01$ & 0 & 0 \\
\hline Solid Layer & $4.52 \mathrm{E}+04$ & $3.38 \mathrm{E}+03$ & $1.57 \mathrm{E}+05$ & $1.56 \mathrm{E}-05$ & $2.40 \mathrm{E}+05$ & $4.44 \mathrm{E} \sim 04$ & $1.54 \mathrm{E}+02$ & $2.16 \mathrm{E}-01$ & $1.56 \mathrm{E}-02$ & $7.61 \mathrm{E}-02$ & $2.05 \mathrm{E}-03$ & $1.23 \mathrm{E}+02$ & 1.73 & 1.47 & 1926 & $3.50 \mathrm{E}-01$ & 4.66F-01 & 60 & 27 \\
\hline 241-SX-104 & $55 \mathrm{E}+04$ & $13 E+03$ & $1.17 \mathrm{E} \div 05$ & $90 \mathrm{E}+05$ & $2.50 \mathrm{E}+05$ & $3.56 \mathrm{E}+04$ & $1.42 \mathrm{E}+02$ & $1.26 \mathrm{E}-01$ & $3.42 \mathrm{E}-02$ & $1.74 \mathrm{E}-01$ & $3.52 \mathrm{E}-03$ & $8.70 \mathrm{E}+01$ & 1.69 & 1.47 & 1726 & $2.56 \mathrm{E}-01$ & $4.53 \mathrm{E}-01$ & 54 & 35 \\
\hline Supernatant & $3.05 E+04$ & $2.13 \mathrm{E}+03$ & $1.17 \mathrm{E}+05$ & $2.90 \mathrm{E}+05$ & $2.50 \mathrm{E}+05$ & $3.56 \mathrm{E}+04$ & $1.42 \mathrm{E}+02$ & $1.26 \mathrm{E}-01$ & $3.42 \mathrm{E}-02$ & $1.74 \mathrm{E}-01$ & $3.52 \mathrm{E}-03$ & $8.70 \mathrm{E}+01$ & 1.47 & 1.47 & 38 & $4.53 \mathrm{E}-01$ & $4.53 \mathrm{E}-01$ & 0 & 0 \\
\hline Solid Layer & $3.05 \mathrm{E}+04$ & $2.13 E+03$ & $1.17 \mathrm{E}+05$ & $2.90 \mathrm{E}+05$ & $2.50 \mathrm{E}+05$ & $3.56 \mathrm{E}^{\circ}-04$ & $1.42 \mathrm{E}+02$ & $1.26 \mathrm{E}-0$ & $3.42 \mathrm{E}-02$ & $1.74 \mathrm{E}-01$ & $3.52 \mathrm{E}-03$ & $8.70 \mathrm{E}+01$ & 1.69 & 1.47 & 1688 & $2.52 \mathrm{E}-01$ & $4.53 \mathrm{E}-01$ & 54 & 35 \\
\hline $241-S X-105$ & $0 \mathrm{E}+04$ & $26 \mathrm{E}+03$ & $1.43 \mathrm{E}-05$ & $64 \mathrm{E}+05$ & $2.36 \mathrm{E}+05$ & $41 \mathrm{E}+04$ & $52 \mathrm{E}+02$ & $2.57 \mathrm{E}-01$ & $.74 \mathrm{E}-02$ & $89 \mathrm{E}-01$ & $.66 \mathrm{E}-03$ & $9.47 \mathrm{E}+01$ & 1.63 & 1.47 & 1460 & $.53 \mathrm{E}-01$ & $29 \mathrm{E}-01$ & 59 & 34 \\
\hline Supernatant & 4. $10 \mathrm{E}-04$ & $3.26 \mathrm{E}+03$ & $1.43 \mathrm{E}+05$ & $1.64 \mathrm{E}+05$ & $2.36 \mathrm{E} \div 05$ & $4.41 E+04$ & $2.52 \mathrm{E}+02$ & $2.57 \mathrm{E}-0 \mathrm{]}$ & $5.74 \mathrm{E}-02$ & $2.89 \mathrm{E}-01$ & $6.66 \mathrm{E}-03$ & $9.47 \mathrm{E}+01$ & 1.47 & 1.47 & 38 & $4.29 \mathrm{E}-01$ & $4.29 \mathrm{E}-01$ & 0 & 0 \\
\hline Solid Layer & $4.10 \mathrm{E}+04$ & $3.26 \mathrm{E}+03$ & $1.43 E+05$ & $1.64 \mathrm{E}+05$ & $2.36 \mathrm{E}+05$ & $4.41 E \div 04$ & $2.52 \mathrm{E}+02$ & $2.57 \mathrm{E}-01$ & $5.74 \mathrm{E}-02$ & $2.89 \mathrm{E}-01$ & $6.66 \mathrm{E}-03$ & $9.47 \mathrm{E}+01$ & 1.63 & I.47 & 1422 & $3.51 \mathrm{E}-01$ & $4.29 \mathrm{E}-01$ & 59 & 34 \\
\hline 241 & $E+04$ & $4 E+03$ & $1.49 \mathrm{E}+05$ & $5 \mathrm{E}+05$ & $17 E+05$ & +04 & 1.01 & $7 \mathrm{E}-$ & $\mathrm{E}-02$ & $\mathrm{E}-02$ & $03 \mathrm{E}-03$ & $E+02$ & 1. & 1.2 & 1539 & $00 \mathrm{E}-01$ & $94 \mathrm{E}-01$ & 38 & 26 \\
\hline Supernatant & $3.20 \mathrm{E}-04$ & $3.14 \mathrm{E}+03$ & $1.49 \mathrm{E}+05$ & $2.45 \mathrm{H}+05$ & $2.17 \mathrm{E}+05$ & $2.26 E+04$ & $1.21 E+01$ & $1.47 \mathrm{E}-01$ & $1.86 \mathrm{E}-02$ & $8.68 \mathrm{E}-02$ & $3.03 \mathrm{E}-03$ & $1.31 E+02$ & 1.29 & 1.29 & 38 & $4.94 \mathrm{E}-01$ & $4.94 \mathrm{E}-01$ & 0 & 0 \\
\hline Solid Layer & $3.20 \mathrm{E}+04$ & $3.14 \mathrm{E} \cdot 03$ & $1.49 E+05$ & $2.45 \mathrm{E}+05$ & $2.17 \mathrm{E}+05$ & $2.26 \mathrm{E}+04$ & $1.21 \mathrm{E}+0 \mathrm{I}$ & $1.47 \mathrm{E}-01$ & $1.86 \mathrm{E}-02$ & 8.68E-02 & $3.03 E-03$ & $1.31 \mathrm{E}+02$ & 1.58 & 1.29 & 1501 & $3.98 \mathrm{E}-01$ & $4.94 \mathrm{E}-01$ & 38 & 26 \\
\hline $241-S X-107$ & $6.07 E+04$ & $1 E+03$ & $9.86 \mathrm{E}^{-04}$ & $17 \mathrm{E}+05$ & $2.55 \mathrm{E}+05$ & $.72 \mathrm{E}+04$ & $00 \mathrm{E}+03$ & 87E-01 & $08 E-01$ & $19 \mathrm{E}-01$ & $87 \mathrm{E}-02$ & $E+01$ & 1.74 & 1. & 394 & $51 \mathrm{E}-01$ & E- 01 & 70 & 3 \\
\hline Supernatant & $6.07 E+04$ & $1.01 \mathrm{E}+03$ & $9.86 \mathrm{E}+04$ & $2.17 \mathrm{E}+05$ & $2.55 \mathrm{E}+05$ & $4.72 \mathrm{E}+04$ & $2.00 \mathrm{E}+03$ & 8.87E-01 & $1.08 \mathrm{E}-01$ & $5.19 \mathrm{E}-01$ & $1.87 \mathrm{E}-02$ & $8.20 \mathrm{E}+01$ & 1.50 & 1.50 & 38 & $5.19 E-01$ & $5.19 \mathrm{E}-01$ & 0 & 0 \\
\hline Solid l.ayer & $6.07 \mathrm{E}+04$ & $1.01 E+03$ & $9.86 \mathrm{E}+04$ & $2.17 \mathrm{E}+05$ & $2.55 \mathrm{E}+05$ & $4.72 E+04$ & $00 E+03$ & $87 E-01$ & $1.08 \mathrm{E}-0 \mathrm{I}$ & $5.19 \mathrm{E}-01$ & $1.87 \mathrm{E}-02$ & $8.20 \mathrm{E}+01$ & 1.77 & 1.50 & 356 & $2.27 \mathrm{E}-01$ & $5.19 \mathrm{E}-01$ & 70 & 53 \\
\hline $241 \cdot S X-108$ & $6.07 \mathrm{E}+04$ & $2.98 \mathrm{E}+03$ & $3.60 \mathrm{E}+04$ & $7.96 \mathrm{E}+04$ & $8.32 \mathrm{E}+04$ & $4.94 \mathrm{E}+03$ & $2.57 \mathrm{E}+03$ & $1.46 \mathrm{E}+00$ & $2.96 \mathrm{E}-0 \mathrm{I}$ & $1.49 \mathrm{E}+00$ & $3.78 \mathrm{E}-02$ & $E \div 02$ & 1.70 & 1.17 & 318 & $8.02 \mathrm{E}-02$ & OE-01 & 77 & 59 \\
\hline Supenatant & $6.07 E+04$ & $2.98 \mathrm{E}+03$ & $3.60 \mathrm{E}+04$ & $7,96 \mathrm{E}+04$ & $8.32 \mathrm{E}+04$ & $4.94 \mathrm{E}+03$ & $2.57 \mathrm{E}+03$ & $1.46 \mathrm{E}+00$ & $2.96 \mathrm{E}-01$ & $1.49 \mathrm{E}+00$ & $3.78 \mathrm{E}-02$ & $1.65 \mathrm{E}+02$ & 1.17 & 1.17 & 38 & $7.50 \mathrm{E}-0 \mathrm{I}$ & $7.50 \mathrm{E}-01$ & 0 & 0 \\
\hline Solid Laycr & 6. & $2.98 \mathrm{E}+03$ & 3. & 4 & 8 & $4.94 \mathrm{E}+03$ & $2.57 E+03$ & 1.46 & 1 & 00 & $E-02$ & $1.65 \mathrm{E}+02$ & 3.7 & 1.17 & 280 & $2.03 E-02$ & $7.50 \mathrm{E}-01$ & 77 & 59 \\
\hline $241-5 X-109$ & $0.07 \mathrm{E}+04$ & $0.20 \mathrm{~F}+03$ & $1.07 E+05$ & $1.76 \mathrm{E}-05$ & $1.98 \mathrm{E}+05$ & $2.75 \mathrm{E}-04$ & $5.70 \mathrm{E} \div 02$ & $2.92 \mathrm{E}-01$ & $3.75 \mathrm{E}-02$ & $1.79 \mathrm{E}-01$ & $6.46 \mathrm{E}-03$ & $7.91 \mathrm{E}+01$ & 1.72 & 1.39 & 951 & $2.41 \mathrm{E}-0 !$ & $5.10 \mathrm{E}-01$ & 53 & 48 \\
\hline Supematant & $6.07 \mathrm{E}-04$ & $9.20 \mathrm{E}+03$ & $1.07 E+05$ & $1.76 \mathrm{E} \div 05$ & $1.98 \mathrm{E}+05$ & $2.75 \mathrm{E}^{-0} 04$ & $5.70 \mathrm{E}+02$ & $2.92 \mathrm{E}-01$ & $3.75 \mathrm{E}-02$ & $1.79 \mathrm{E}-01$ & $6.46 \mathrm{E}-03$ & $7.91 E+01$ & 1.39 & 1.39 & 38 & $5.10 \mathrm{E}-01$ & $5.10 \mathrm{E}-01$ & 0 & 0 \\
\hline Solid Layer & $6.07 \mathrm{E}+04$ & $9.20 E+03$ & $E+05$ & $1.76 \mathrm{E}+05$ & +05 & $\div+04$ & $E+02$ & $2.92 \mathrm{E}-01$ & $\mathrm{E}-02$ & $79 \mathrm{E}-01$ & $6.46 \mathrm{E}-03$ & 01 & 13 & 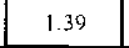 & 913 & -01 & 01 & 53 & 48 \\
\hline $241-S X-110$ & $6.07 \mathrm{E}+04$ & $2.98 \mathrm{E}: 03$ & $3.60 \mathrm{E} \div 04$ & $7.96 \mathrm{E}-04$ & $8.32 \mathrm{E}+04$ & $4.94 \mathrm{E}+03$ & $4.58 \mathrm{E} \div 03$ & $1.91 E+00$ & $1.18 \mathrm{E}-01$ & $5.04 \mathrm{E}-01$ & $3.34 \mathrm{E}-02$ & $1.15 \mathrm{E}+02$ & 1.67 & 1.17 & 250 & $2.83 \mathrm{E}-01$ & $7.50 E-01$ & 55 & 65 \\
\hline Supernatant & $6.07 E+04$ & $2.98 \mathrm{~F}+0.3$ & $3.60 \mathrm{E}+04$ & $7.96 \mathrm{E}+04$ & $8.32 E+04$ & $4.94 \mathrm{E}+03$ & $4.58 \mathrm{E}+03$ & $1.91 \mathrm{E}+00$ & $1.18 \mathrm{E}-01$ & $5.04 \mathrm{E}-01$ & $3.34 \mathrm{E}-02$ & $1.15 \mathrm{E}+02$ & 1.17 & 1.17 & 38 & $7.50 \mathrm{E}-01$ & $7.50 \mathrm{E}-01$ & 0 & 0 \\
\hline Solid Layer & $6.07 \mathrm{E}+04$ & $2.98 E+03$ & $3.60 \mathrm{E}+04$ & $7.96 \mathrm{E}+04$ & $8.32 E+04$ & $4.94 \mathrm{E}+03$ & $4.58 \mathrm{E}+03$ & $1.91 \mathrm{E}+00$ & $1.18 \mathrm{E}-01$ & $5.04 \mathrm{E}-01$ & $3.34 \mathrm{E}-02$ & $1.15 \mathrm{E}+02$ & 1.76 & 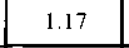 & 212 & $2.28 \mathrm{E}-0 \mathrm{I}$ & $7.50 \mathrm{E}-0 \mathrm{I}$ & 55 & 65 \\
\hline $241-S X-111$ & $6.07 \mathrm{E}+04$ & $1.01 \mathrm{E} \div 03$ & $9.89 \mathrm{E}+04$ & $2.17 \mathrm{E}+05$ & $2.55 \mathrm{E}+05$ & $4.71 E+04$ & $2.63 \mathrm{E}+03$ & $1.14 E+00$ & $1.01 \mathrm{E}-01$ & $4.64 \mathrm{E}-01$ & $2.17 \mathrm{E}-02$ & $9.25 E+01$ & 1.74 & 1.50 & 474 & $2.48 \mathrm{E}-01$ & $5.19 \mathrm{E}-01$ & 65 & 62 \\
\hline Supematant & 6.071 & $1.01 E+03$ & $9.89 \mathrm{E}+04$ & $2.17 \mathrm{E}+05$ & $2.55 \mathrm{E}+05$ & $4.71 \mathrm{E}+04$ & $2.63 \mathrm{E} \div 03$ & $1.14 \mathrm{E}+00$ & $1.05 \mathrm{E}-01$ & 4.64E-01 & $2.17 \mathrm{E}-02$ & $9.25 \mathrm{E}+01$ & 1.50 & 15 & 38 & $5.19 \mathrm{E}-01$ & $5.19 \mathrm{E}-01$ & 0 & 0 \\
\hline Solid Layer & $6.07 E+0.4$ & $1.01 E+03$ & $9.89 \mathrm{E}+04$ & $2.17 \mathrm{E}+05$ & $2.55 \mathrm{E}+05$ & $4.71 \mathrm{E}+04$ & $2.63 \mathrm{E}+03$ & $1.14 \mathrm{E}+00$ & $1.01 E-01$ & $4.64 \mathrm{E}-01$ & $2.17 \mathrm{E}-02$ & $9.25 \mathrm{E}+01$ & 1.76 & 1.50 & 436 & $2.28 \mathrm{E}-0 \mathrm{I}$ & $5.19 \mathrm{E}-01$ & 65 & 62 \\
\hline
\end{tabular}


RPP-5926 REV 6

Table B-5. Input Data With Water Addition for Hydrogen Generation Rate Model Calculations for 177 Tanks. (20 sheets)

\begin{tabular}{|c|c|c|c|c|c|c|c|c|c|c|c|c|c|c|c|c|c|c|c|}
\hline Tank & $\begin{array}{c}\text { OH } \\
\text { in liquid } \\
{[\mathrm{OH}]} \\
(\mu \mathrm{g} / \mathrm{mL})\end{array}$ & $\begin{array}{c}\text { TOC } \\
\text { in liquid } \\
\text { [TOC] } \\
(\mu \mathrm{g} / \mathrm{mL})\end{array}$ & $\begin{array}{c}\mathrm{NO}_{2} \\
\text { in liquid } \\
{\left[\mathrm{NO}_{2}\right]} \\
(\mu \mathrm{g} / \mathrm{mL})\end{array}$ & $\begin{array}{c}\mathrm{NO}_{3} \\
\text { in liquid } \\
\left|\mathrm{NO}_{3}\right| \\
(\mu \mathrm{g} / \mathrm{mL})\end{array}$ & $\begin{array}{c}\mathrm{Na} \\
\text { in liquid } \\
|\mathrm{Na}| \\
(\mathrm{ug} / \mathrm{mL})\end{array}$ & $\begin{array}{c}\mathrm{Al} \\
\text { in liquid } \\
{[\mathrm{Al}]} \\
(\mu \mathrm{g} / \mathrm{mL})\end{array}$ & $\begin{array}{c}90 \mathrm{Sr} \\
\text { in waste } \\
{[\mathrm{Sr}]} \\
(\mu \mathrm{Ci} / \mathrm{g})\end{array}$ & $\begin{array}{c}{ }^{241} \mathrm{Am} \\
\text { in waste } \\
{[\mathrm{Am} 241]} \\
(\mu \mathrm{Ci} / \mathrm{g})\end{array}$ & $\begin{array}{c}{ }^{240} \mathrm{Pu} \\
\text { in waste } \\
{[\mathrm{Pu} 240]} \\
(\mu \mathrm{Ci} / \mathrm{g})\end{array}$ & $\begin{array}{c}{ }^{2319} \mathrm{Pu} \\
\text { in waste } \\
{[\mathrm{Pu} 240\}} \\
(\mu \mathrm{Ci} / \mathrm{g})\end{array}$ & $\begin{array}{c}{ }^{238} \mathrm{Pu} \\
\text { in waste } \\
{\left[\mathrm{Pu}^{238]}\right.} \\
(\mu \mathrm{Ci} / \mathrm{g})\end{array}$ & $\begin{array}{c}{ }^{137} \mathrm{Cs} \\
\text { in waste } \\
|\mathrm{Cs}| \\
(\mu \mathrm{Ci} / \mathrm{g})\end{array}$ & $\begin{array}{c}\text { Bulk } \\
\text { density } \\
\text { D } \\
(\mathrm{g} / \mathrm{mL})\end{array}$ & $\begin{array}{c}\text { Liquid } \\
\text { density } \mathrm{D}_{\mathrm{L}} \\
(\mathrm{g} / \mathrm{ml})\end{array}$ & $\begin{array}{c}\text { Non-RGS } \\
\text { waste } \\
\text { volume } \\
\text { (kL) }\end{array}$ & $\begin{array}{l}\text { Bulk } \\
\text { water } \\
{\left[\mathrm{H}_{2} \mathrm{O}\right]} \\
\left(\mathrm{wt}^{2} / 1 \mathrm{u}\right)\end{array}$ & $\begin{array}{l}\text { Liquid } \\
\text { water } \\
\left|\mathrm{H}_{2} \mathrm{O}\right| \\
(w t " \%)\end{array}$ & $\begin{array}{c}\text { Waste } \\
\text { temp. } \\
T w \\
\left({ }^{\circ} \mathrm{C}\right)\end{array}$ & $\begin{array}{c}\text { Dome } \\
\text { temp. } \\
\text { Td } \\
\left({ }^{\circ} \mathrm{C}\right)\end{array}$ \\
\hline $241-S X-112$ & $7 \mathrm{E}+04$ & $1 \mathrm{~B}+03$ & $9.88 E+04$ & $17 \mathrm{E}+05$ & $.55 E+05$ & $72 \mathrm{E}-04$ & $2.78 \mathrm{E}+03$ & $20 \mathrm{E}+00$ & $1.15 \mathrm{E}-01$ & $5.31 \mathrm{E}-01$ & $2.34 \mathrm{E}-02$ & $9.14 \mathrm{E}+01$ & 1.74 & 1.50 & 321 & $2.57 \mathrm{E}-01$ & $5.19 \mathrm{E}-01$ & 60 & 52 \\
\hline Supernatant & $6.07 E+04$ & $1.01 E+03$ & $9.88 \mathrm{E}+04$ & $2.17 \mathrm{E}+05$ & $2.55 \mathrm{E} \div 05$ & $4.72 \mathrm{E}+04$ & $2.78 \mathrm{E}+03$ & $1.20 \mathrm{E}+00$ & $1.15 \mathrm{E}-0 !$ & $5.31 E-01$ & $2.34 \mathrm{E}-02$ & $9.14 \mathrm{E}+01$ & 1.50 & 1.50 & 38 & 5.19E-01 & $5.19 E-01$ & 0 & 0 \\
\hline Solid Layer & $6.07 \mathrm{E} \div 04$ & $1.01 E \div 03$ & $9.88 \mathrm{E}+04$ & $2.17 \mathrm{E}+05$ & $2.55 E+05$ & $4.72 E+04$ & $2.78 \mathrm{E}+03$ & $1.20 E+00$ & $1.15 \mathrm{E}-01$ & $5.3 \mid \mathrm{E}-0 \mathrm{t}$ & $2.34 \mathrm{E}-02$ & $9.14 \mathrm{E}+01$ & 1.77 & 1.50 & 283 & $2.27 \mathrm{E}-01$ & $5.19 \mathrm{E}-01$ & 60 & 52 \\
\hline $241-5 X-113$ & $07 E+04$ & $98 \mathrm{E}+03$ & $60 \mathrm{E}+04$ & $7.96 \mathrm{E}+04$ & $8.32 E+04$ & $4.94 E+03$ & $2.75 \mathrm{E} \div 01$ & $1.09 \mathrm{E}-01$ & $9.47 \mathrm{E}-03$ & $4.88 \mathrm{E}-02$ & $9.06 \mathrm{E}-04$ & $2.02 \mathrm{t} \div 01$ & 1.34 & 1.17 & 110 & $5.47 E-01$ & $7.50 \mathrm{E}-01$ & 28 & 28 \\
\hline Supernatant & $6.07 E+04$ & $2.98 \mathrm{E}+03$ & $3.60 \mathrm{E}+04$ & $7.96 E+04$ & $8.32 \mathrm{E}-04$ & $4.94 \mathrm{E}+03$ & $2.75 \mathrm{E}+0 \mathrm{I}$ & $1.09 \mathrm{E}-01$ & $9.47 E-03$ & $4.88 \mathrm{E}-02$ & 9.06E-04 & $2.02 \mathrm{E}+01$ & 1.17 & 1.17 & 38 & $7.50 \mathrm{E}-01$ & $7.50 \mathrm{E}-0 \mathrm{I}$ & 0 & 0 \\
\hline Solid Layer & $6.07 \mathrm{E}+04$ & $2.98 \mathrm{E}+03$ & $3.60 \mathrm{E} \cdot 04$ & $7.96 \mathrm{E} \div 04$ & $8.32 E+04$ & $4.94 E+03$ & $2.75 E+0$ & $1.09 \mathrm{E}-01$ & $9.47 \mathrm{E}-03$ & $4.88 \mathrm{E}-02$ & $9.06 \mathrm{E}-04$ & $2.02 \mathrm{E}+01$ & 1.43 & 1.17 & 72 & 4. $60 \mathrm{E}-01$ & $7.50 \mathrm{E}-01$ & 28 & 28 \\
\hline $11-S X-114$ & $7 E+04$ & $01 \mathrm{E}+03$ & $88 E+04$ & $2.17 \mathrm{E}+05$ & $5 ! E+05$ & $73 E+04$ & $1.84 \mathrm{E}+03$ & $15 E-0$ & $20 \mathrm{E}-02$ & $37 \mathrm{E}-01$ & $.67 \mathrm{E}-02$ & $8.77 E+0$ & 1.7 & 1.50 & 626 & $2.54 \mathrm{E}-01$ & $5.19 E-01$ & 72 & 52 \\
\hline Supernatant & $6.07 \mathrm{E}+04$ & $1.01 \mathrm{E}-03$ & $9.88 \mathrm{E}-04$ & $2.17 E+05$ & $2.51 \mathrm{E}+05$ & $4.73 E+04$ & $1.84 \mathrm{E}+03$ & $8.15 \mathrm{E}-01$ & $9.20 \mathrm{E}-02$ & $4.37 \mathrm{E}-01$ & $1.67 \mathrm{E}-02$ & $8.77 \mathrm{E}+01$ & 1.50 & 1.50 & 38 & $5.19 \mathrm{E}-01$ & $5.19 \mathrm{E}-01$ & 0 & 0 \\
\hline Solid Layer & $6.07 \mathrm{E}+04$ & $1.01 E+03$ & $9.88 \mathrm{E}+04$ & $2.17 \mathrm{E}+05$ & $2.51 \mathrm{E}+05$ & $4.73 \mathrm{E}+04$ & $1.84 \mathrm{E}+03$ & $8.15 \mathrm{E}-01$ & $9.20 \mathrm{E}-02$ & $4.37 \mathrm{E}-01$ & $1.67 \mathrm{E}-02$ & $8.77 \mathrm{E}+0 \mathrm{~J}$ & 1.75 & 1.50 & 588 & $2.39 \mathrm{E}-01$ & $5.19 \mathrm{E}-01$ & 72 & 52 \\
\hline-115 & +04 & $8 \mathrm{E}+03$ & $60 E+04$ & $E+04$ & $2 E+04$ & $34 \mathrm{E}+03$ & $t+04$ & $E+$ & $E+00$ & $E+01$ & $1.07 \mathrm{E}+00$ & $2.05 \mathrm{E}+01$ & 1.3 & 17 & 54 & 4.97E-01 & OE-01 & 25 & 25 \\
\hline Supematant & $6.07 \mathrm{~F}-04$ & $2.98 \mathrm{E}-03$ & $3.60 \mathrm{E}+04$ & $7.96 \mathrm{E}+04$ & $8.32 \mathrm{E} \cdot 04$ & $4.94 \mathrm{E}+03$ & $1.35 \mathrm{E}+04$ & 1. $42 \mathrm{E}-0 \mathrm{I}$ & $3.78 \mathrm{E} \div 00$ & $1.61 \mathrm{E}+01$ & $1.07 E+00$ & $2.05 \mathrm{E}+01$ & 1.17 & 1.17 & 38 & $7.50 \mathrm{E}-01$ & $7.50 \mathrm{E}-0 \mathrm{t}$ & 0 & 0 \\
\hline Solid Layer & $6.07 \mathrm{E}+04$ & $2.98 \mathrm{E}+03$ & $3.60 E+04$ & $7.96 \mathrm{E}+04$ & $8.32 \mathrm{E}+04$ & $4.94 \mathrm{~F}+03$ & $1.35 \mathrm{E}+04$ & $1.42 \mathrm{E}+01$ & $3.78 \mathrm{E}+00$ & $1.61 \mathrm{E}+01$ & $1.07 \mathrm{E}+00$ & $2.05 \mathrm{E}+01$ & 1.77 & 1.17 & 16 & $1.01 \mathrm{E}-01$ & $7.50 \mathrm{E}-01$ & 25 & 25 \\
\hline $241-\mathrm{T}-101$ & $1 E+04$ & $04 \mathrm{E}+03$ & $1.22 E+05$ & $1.89 \mathrm{E}+05$ & $2.37 E+05$ & $77 E+04$ & $6.63 E-01$ & $1.15 \mathrm{E}-01$ & $7.84 \mathrm{E}-02$ & $3.58 \mathrm{E}-01$ & $9.37 \mathrm{E}-03$ & $6.76 \mathrm{E}+01$ & 1.54 & 1.45 & 414 & $4.03 \mathrm{E}-01$ & $4.91 \mathrm{E}-01$ & 21 & 22 \\
\hline Supernatant & $4.81 E+04$ & $4.04 \mathrm{E} \cdot 03$ & $1.22 \mathrm{E}-05$ & $1.89 \mathrm{E}+05$ & $2.37 E+05$ & $3.77 \mathrm{E}-04$ & t.63E-01 & $1.15 E-01$ & $7.84 \mathrm{E}-02$ & $3.58 \mathrm{E}-0 \mathrm{I}$ & $9.37 \mathrm{E}-03$ & $6.76 E+01$ & 1.45 & 1.45 & 38 & 4.91 E-01 & $4.91 E-01$ & 0 & 0 \\
\hline Solid Layer & $4.81 \mathrm{E}+04$ & $\mathrm{E}+03$ & $1.22 \mathrm{E}+0$ & +05 & 2 & $E+04$ & 6 & 1 & $7.84 \mathrm{E}$ & $3.58 \mathrm{E}-01$ & 9.37E-03 & 6 & 1.54 & 145 & 376 & $3.95 \mathrm{E}-01$ & $4.91 \mathrm{E}-01$ & 21 & 22 \\
\hline $241-\mathrm{T}-102$ & $4.40 \mathrm{E}+01$ & $3.17 \mathrm{E}+02$ & $1.61 E+04$ & $7.67 \mathrm{E}+04$ & $4.55 \mathrm{t}+04$ & $5.96 \mathrm{E}+03$ & $7.51 E+01$ & $9.65 \mathrm{E}-02$ & $4.27 \mathrm{E}-03$ & $1.83 \mathrm{E}-02$ & $4.46 \mathrm{E}-04$ & $2.07 \mathrm{E}+01$ & 1.41 & 1.10 & 158 & $5.40 \mathrm{E}-01$ & $32 \mathrm{E}-01$ & 18 & 18 \\
\hline Supernatant & $3.49 \mathrm{E} \div 01$ & $2.52 \mathrm{E} \div 02$ & $1.28 \mathrm{E}+04$ & $6.09 \mathrm{E}+04$ & $3.61 E+04$ & $4.73 E \cdot 03$ & $5.06 E-01$ & $2.2 ! \mathrm{E}-03$ & $6.14 \mathrm{E}-04$ & $2.65 \mathrm{E}-03$ & $1.11 \mathrm{E}-04$ & $2.67 \mathrm{E}+01$ & 1.08 & 1.08 & 86 & $8.80 \mathrm{E}-01$ & $8.80 \mathrm{E}-0 \mathrm{I}$ & 18 & 18 \\
\hline Solid Layer & $6.25 \mathrm{E}+01$ & $4.50 \mathrm{E}+02$ & $2.29 \mathrm{E}+04$ & $1.09 \mathrm{E}+05$ & $6.46 \mathrm{E} \div 04$ & $46 \mathrm{E}+03$ & $E+02$ & 1 & 6 & 2 & 94 & 1 & 0 & 1.14 & 72 & 01 & $8 E .01$ & 18 & 18 \\
\hline $24 !-T-103$ & $1.08 \mathrm{E}+04$ & $3.54 \mathrm{E}+03$ & $2.52 \mathrm{E}+04$ & $4.97 \mathrm{E}+04$ & $6.07 \mathrm{E}+04$ & $6.62 \mathrm{E}+03$ & $3.40 \mathrm{E}+00$ & $3.12 \mathrm{E}-03$ & 4.29E-03 & $1.84 \mathrm{E}-02$ & $4.37 \mathrm{E}-04$ & $1.95 \mathrm{E}+00$ & 1.46 & 1.12 & 140 & $4.59 \mathrm{E}-01$ & $7.68 \mathrm{E}-01$ & 19 & 20 \\
\hline Supematant & $4.82 \mathrm{E} \div 03$ & $1.58 \mathrm{E}-03$ & $1.12 \mathrm{E}+04$ & $2.21 \mathrm{E}+04$ & $2.71 E+04$ & $2.95 E+03$ & $6.21 E-01$ & $2,75 \mathrm{~F}-03$ & $4.76 \mathrm{E}-04$ & 2.05E-03 & $8.58 \mathrm{E}-05$ & $3.77 \mathrm{E}+00$ & 1.05 & 1.05 & 53 & $9.09 \mathrm{E}-01$ & $9.09 \mathrm{E}-01$ & 19 & 20 \\
\hline Solid Layer & $1.70 E+04$ & $5.55 \mathrm{E}+03$ & $3.96 \mathrm{E}+04$ & $7.80 \mathrm{~F}+04$ & $9.53 \mathrm{E}-04$ & $1.04 \mathrm{E} \div 04$ & $4.43 \mathrm{E}+00$ & $3.26 \mathrm{E}-03$ & $5.72 \mathrm{E}-03$ & $2.44 \mathrm{E}-02$ & $5.68 \mathrm{E}-04$ & $1.27 \mathrm{E}+00$ & 1.71 & 1.18 & 87 & $2.91 \mathrm{E}-01$ & $7.16 \mathrm{E}-01$ & 19 & 20 \\
\hline $24 !-T \cdot 104$ & $1.70 \mathrm{E}+04$ & $2.98 \mathrm{E}+03$ & $3.60 \mathrm{E}+04$ & $7.96 \mathrm{E}+04$ & $8.32 E+04$ & $4.94 \mathrm{E}+03$ & $2.03 \mathrm{E}+00$ & $1.81 \mathrm{E}-02$ & $1.51 \mathrm{E}-02$ & $1.25 \mathrm{E}-01$ & $1.62 \mathrm{E}-03$ & 1.55E-0I & 1.29 & 1.17 & 1237 & $7.09 \mathrm{E}-01$ & $8.40 \mathrm{E}-0 \mathrm{I}$ & 19 & 22 \\
\hline Supernatant & $1.70 E-04$ & $2.98 \mathrm{r} \cdot 03$ & $3.60 \mathrm{E}-04$ & $7.96 \mathrm{E}+04$ & $8.32 E+04$ & $4.94 \mathrm{E}+03$ & $2.03 E+00$ & $1.81 \mathrm{E}-02$ & $1.51 \mathrm{E}-02$ & $1.25 E-01$ & $1.62 \mathrm{E}-03$ & .55501 & 1.17 & 1.17 & 38 & $8.40 \mathrm{E}-01$ & $8.40 \mathrm{E}-01$ & 0 & 0 \\
\hline Solid Layer & $1.70 \mathrm{E}+04$ & $2.98 \mathrm{E}+03$ & $3.60 \mathrm{E}+04$ & $7.96 \mathrm{E}+04$ & $8.32 E \div 04$ & $4.94 \mathrm{E} \div 03$ & $2.03 \mathrm{E}-00$ & $1.81 \mathrm{E}-02$ & $1.51 \mathrm{E}-02$ & $1.25 \mathrm{E}-01$ & 1.62E-03 & $1.55 \mathrm{E}-0 \mathrm{]}$ & 1.29 & 1.17 & 1199 & $7.05 \mathrm{E}-01$ & $8.40 \mathrm{E}-01$ & 19 & 22 \\
\hline $241-T-105$ & $1.70 \mathrm{E}+04$ & $2.98 \mathrm{E}+03$ & $5.42 E+04$ & $3.71 \mathrm{E}-04$ & $8.84 \mathrm{E}+04$ & $3.62 \mathrm{E}+02$ & $5.19 \mathrm{E}+01$ & $5.81 \mathrm{E}-02$ & $2.93 \mathrm{E}-02$ & $2.77 \mathrm{E}-01$ & $2.08 \mathrm{E}-03$ & $9.23 \mathrm{E}+00$ & 1.43 & 1.18 & 409 & $5.36 \mathrm{E}-01$ & $8.00 \mathrm{E}-0 \mathrm{I}$ & 17 & 16 \\
\hline Supernatant & $1.70 \mathrm{E}=04$ & $2.98 \mathrm{E} \div 03$ & $5.42 \mathrm{E}+04$ & $3.71 E+04$ & $8.84 \mathrm{E}+04$ & $3.62 E+02$ & $5.19 E+01$ & $5.81 \mathrm{E}-02$ & .02 & $E-01$ & 2.08 & $9.23 \mathrm{E}+00$ & 1.18 & 18 & 38 & $8.00 \mathrm{E}-01$ & $8.00 \mathrm{E}-01$ & 0 & 0 \\
\hline Solid Layer & $1.70 \mathrm{E}+04$ & $2.98 \mathrm{E}+03$ & $5.42 \mathrm{E}+04$ & $3.71 E+04$ & $8.84 \mathrm{E}+04$ & $3.62 \mathrm{E}+02$ & $5.19 \mathrm{E}+01$ & $5.81 \mathrm{E}-02$ & $2.93 \mathrm{E}-02$ & $2.77 E-01$ & $2.08 \mathrm{E}-03$ & $9.23 \mathrm{E}+00$ & 1.46 & 1.18 & 371 & $5.14 \mathrm{E}-01$ & $8.00 \mathrm{E}-01$ & 17 & 16 \\
\hline
\end{tabular}


RPP-5926 REV 6

Table B-5. Input Data With Water Addition for Hydrogen Generation Rate Model Calculations for 177 Tanks. (20 sheets)

\begin{tabular}{|c|c|c|c|c|c|c|c|c|c|c|c|c|c|c|c|c|c|c|c|}
\hline Tank & $\begin{array}{c}\text { OH } \\
\text { in liquid } \\
{[\mathrm{OH}]} \\
(\mu \mathrm{g} / \mathrm{ml} .)\end{array}$ & $\begin{array}{c}\text { TOC } \\
\text { in liquid } \\
\text { |roC) } \\
(\mu \mathrm{gg} / \mathrm{mL})\end{array}$ & $\begin{array}{c}\mathrm{NO}_{2} \\
\text { in liquid } \\
{\left[\mathrm{NO}_{2}\right\rfloor} \\
(\mu \mathrm{g} / \mathrm{mL})\end{array}$ & $\begin{array}{c}\mathrm{NO}_{3} \\
\text { in liquid } \\
\left|\mathrm{NO}_{3}\right| \\
(\mu \mathrm{g} / \mathrm{mL})\end{array}$ & $\begin{array}{c}\mathrm{Na} \\
\text { in liquid } \\
|\mathrm{Na}| \\
(\mathrm{ug} / \mathrm{mL})\end{array}$ & $\begin{array}{c}\mathrm{Al} \\
\text { in liquid } \\
\text { [Al| } \\
(\mu \mathrm{g} / \mathrm{mL})\end{array}$ & $\begin{array}{c}90 \mathrm{Sr} \\
\text { in waste } \\
{[\mathrm{Sr} \mid} \\
(\mu \mathrm{Ci} / \mathrm{g})\end{array}$ & $\begin{array}{c}{ }^{241} \mathrm{Am} \\
\text { in waste } \\
{\left[\begin{array}{c}\mathrm{Am} 241] \\
(\mu \mathrm{Ci} / \mathrm{g})\end{array}\right.}\end{array}$ & $\begin{array}{c}{ }^{240} \mathrm{P}^{\mathrm{P} u} \\
\text { in waste } \\
{[\mathrm{Pu} 240]} \\
(\mu \mathrm{Ci} / \mathrm{g})\end{array}$ & $\begin{array}{c}{ }^{239} \mathrm{Pu} \\
\text { in waste } \\
{[\mathrm{Pu} 240\}} \\
(\mu \mathrm{Ci} / \mathrm{g})\end{array}$ & $\begin{array}{c}{ }^{238} \mathrm{Pu} \\
\text { in waste } \\
{\left[\begin{array}{l}\mathrm{Pu} z 38] \\
(\mu \mathrm{Ci} / \mathrm{g})\end{array}\right.}\end{array}$ & $\begin{array}{c}1.37 \mathrm{Cs} \\
\text { in waste } \\
|\mathrm{Cs}| \\
(\mu \mathrm{Ci} / \mathrm{g})\end{array}$ & $\begin{array}{c}\text { Bulk } \\
\text { density } \\
D \\
(\mathrm{~g} / \mathrm{mL})\end{array}$ & $\begin{array}{c}\text { Liquid } \\
\text { density } \mathrm{D}_{L} \\
(\mathrm{~g} / \mathrm{ml})\end{array}$ & $\begin{array}{c}\text { Non-RGS } \\
\text { waste } \\
\text { volume } \\
\text { (kL) }\end{array}$ & $\begin{array}{l}\text { Bulk } \\
\text { water } \\
\left|\mathrm{H}_{2} \mathrm{O}\right| \\
(w t \%)\end{array}$ & $\begin{array}{l}\text { L.jquid } \\
\text { water } \\
\left|\mathrm{H}_{2} \mathrm{O}\right| \\
(\mathrm{wt} \%(1)\end{array}$ & $\begin{array}{c}\text { Waste } \\
\text { temp. } \\
\text { Tw } \\
\left({ }^{\circ} \mathrm{C}\right)\end{array}$ & $\begin{array}{l}\text { Dome } \\
\text { temp. } \\
\text { Td } \\
\left({ }^{\circ} \mathrm{C}\right)\end{array}$ \\
\hline $241-\mathrm{T}-106$ & $0 巨+04$ & $8 E+03$ & $3.60 \mathrm{E}+04$ & $36 \mathrm{E}+04$ & 34 & 4.9415 & $.02 \mathrm{E}+00$ & 27E- 02 & $0 \mathrm{E}-02$ & $1.34 \mathrm{E}-01$ & $1.61 \mathrm{E}-03$ & $1.53 \mathrm{E}+01$ & 1.46 & 1.17 & 120 & $3.15 \mathrm{E}-01$ & $.50 \mathrm{E}-01$ & 20 & 21 \\
\hline Supernatant & 1.70E+04 & $2.98 \mathrm{E} \div 03$ & $3.60 \mathrm{E}+04$ & $7.96 \mathrm{E}+04$ & $8.32 \mathrm{E}+04$ & $4.94 \mathrm{E}+03$ & $5.02 \mathrm{E}+00$ & $4.27 \mathrm{E}-02$ & $2.00 \mathrm{E}-02$ & $1.34 \mathrm{E}-01$ & $1.61 \mathrm{E}-03$ & $1.53 \mathrm{E}+01$ & 1.17 & 1.17 & 38 & $7.50 \mathrm{E}-01$ & $7.50 \mathrm{E}-01$ & 0 & 0 \\
\hline Solid Layer & $1.70 \mathrm{E}+04$ & $2.98 \mathrm{E}+03$ & $3.60 \mathrm{E}+04$ & $7.96 \mathrm{E}+04$ & $8.32 \mathrm{E}+04$ & $4.94 \mathrm{E}+03$ & $5.02 \mathrm{E}+00$ & $4.27 \mathrm{E}-02$ & $2.00 \mathrm{E}-02$ & $1.34 \mathrm{E}-01$ & $1.61 \mathrm{E}-03$ & $1.53 \mathrm{E}+0\}$ & 1.59 & 1.17 & 82 & $1.67 \mathrm{E}-01$ & $7.50 \mathrm{E}-01$ & 20 & 21 \\
\hline $241-T-107$ & $1.70 \mathrm{E}+04$ & $2.98 \mathrm{E}+03$ & $3.60 \mathrm{E}+04$ & $7.96 \mathrm{E}+04$ & $8.32 \mathrm{E}+04$ & $4.94 \mathrm{E}+03$ & $1.0\rfloor E+02$ & $5.89 \mathrm{E}-02$ & $3.48 \mathrm{E}-02$ & $3.04 \mathrm{E}-01$ & $2.37 \mathrm{E}-03$ & $1.47 \mathrm{E}+01$ & 1.54 & 1.17 & 693 & $4.73 \mathrm{E}-01$ & $8.40 \mathrm{E}-01$ & 20 & 22 \\
\hline Supernatant & $1.70 E+04$ & $2.98 \mathrm{E}+03$ & $3.60 \mathrm{E}+04$ & $7.96 \mathrm{E}-04$ & $8.32 E+04$ & $4.94 \mathrm{E}+03$ & $1.01 \mathrm{E}-02$ & $5.89 \mathrm{E}-02$ & $3.48 \mathrm{E}-02$ & $3.04 \mathrm{E}-01$ & $2.37 \mathrm{E}-03$ & $1.47 \mathrm{E}+01$ & 1.17 & 1.17 & 38 & $8.40 \mathrm{E}-01$ & $8.40 \mathrm{E}-0 \mathrm{I}$ & 0 & 0 \\
\hline Solid Layer & $1.70 \mathrm{E}+04$ & $2.98 \mathrm{E}+03$ & $3.60 \mathrm{E}+04$ & $7.96 \mathrm{E}+04$ & $8.32 \mathrm{E} r 04$ & $4.94 \mathrm{E}+03$ & $1.01 \mathrm{E}+02$ & $5.89 \mathrm{E}-02$ & $3.48 \mathrm{E}-02$ & $3.04 \mathrm{E}-01$ & 2.37E-03 & $1.47 \mathrm{E}+01$ & 1.56 & 1.17 & 655 & 4.57E-01 & $8.40 E-01$ & 20 & 22 \\
\hline $241-T-108$ & $1.70 E+04$ & $3.38 \mathrm{~F}-00$ & $8.50 \mathrm{E}+03$ & $2.17 \mathrm{E}+05$ & $1.13 \mathrm{E}+05$ & $3.61 \mathrm{E}+02$ & $1.77 \mathrm{E}+00$ & $4.65 E-03$ & $6.74 \mathrm{~F}-03$ & $6.05 \mathrm{E}-02$ & 4.41E-04 & $6.77 E+00$ & 1.44 & 1.26 & 98 & $4.64 \mathrm{E}-01$ & $6.96 \mathrm{E}-01$ & 16 & 23 \\
\hline Superiatant & $1.70 E-04$ & $3.38 \mathrm{E}+00$ & $8.50 \mathrm{E} \cdot 03$ & $2.17 \mathrm{E}-0.05$ & 1.13E+05 & $61 E+012$ & $1.77 \mathrm{E}-00$ & $65 \mathrm{E}-$ & $74 E-03$ & $35 \mathrm{E}-02$ & $4.41 E-04$ & $0.77 \mathrm{E}-00$ & 1.26 & 1.26 & 38 & $6.96 \mathrm{E}-01$ & $96 \mathrm{E}-$ & 0 & 0 \\
\hline Solid Layer & $1.70 \mathrm{E}+04$ & $3.38 \mathrm{E}+00$ & $8.50 \mathrm{E}: 03$ & $2.17 \mathrm{E}+05$ & $1.13 \mathrm{E}+05$ & $3.61 \mathrm{E}+02$ & $1.77 \mathrm{E}+00$ & $4.65 \mathrm{E}-03$ & $6.74 \mathrm{E}-03$ & $6.05 E-02$ & 4.41E- 04 & $6.77 \mathrm{E}+00$ & 1.55 & 1.26 & 60 & $3.45 \mathrm{E}-0 \mathrm{]}$ & $6.96 \mathrm{E}-01$ & 16 & 23 \\
\hline $241+\mathrm{T}-109$ & $70 \mathrm{E}+04$ & $3.37 \mathrm{E}+00$ & $8.50 \mathrm{E}+03$ & $2.17 E+05$ & $1.13 E+05$ & $61 E+02$ & $2.62 \mathrm{E}-01$ & 2.10E-04 & $1.04 \mathrm{E}-03$ & $9.12 \mathrm{E}-03$ & $6.79 \mathrm{~F}-05$ & $2.13 E+00$ & 1.59 & 1.26 & 273 & $5.27 \mathrm{E}-01$ & $6.96 \mathrm{E}-0 \mathrm{I}$ & 19 & 20 \\
\hline Supernatant & $1.70 \mathrm{E}+04$ & $3.37 \mathrm{E}+00$ & $8.50 \mathrm{E}+03$ & $2.17 \mathrm{E}+05$ & $1.13 E+05$ & $.61 \mathrm{E}+02$ & $2.62 \mathrm{E}-0 \mathrm{I}$ & 10E-04 & 04E-03 & $9.12 \mathrm{E}-03$ & $6.79 \mathrm{E}-05$ & $2.13 \mathrm{E}+00$ & 1.26 & 1.26 & 38 & $6.96 \mathrm{E}-01$ & $6.96 \mathrm{E}-01$ & 0 & 0 \\
\hline Solid Layer & $1.70 \mathrm{E}+04$ & $3.37 \mathrm{E}+00$ & $8.50 \mathrm{E}+03$ & $2.17 \mathrm{E}+05$ & $1.13 \mathrm{E}+05$ & $3.61 \mathrm{E} \div 02$ & $2.62 \mathrm{E}-01$ & $2.10 \mathrm{E}-04$ & $1.04 \mathrm{E}-03$ & $9.12 \mathrm{E}-03$ & $6.79 \mathrm{E}-05$ & $2.13 \mathrm{E}+00$ & 1.65 & 1.26 & 235 & $5.06 \mathrm{E}-01$ & $6.96 \mathrm{E}-01$ & 19 & 20 \\
\hline $241-\mathrm{T}-110$ & +04 & .01 & 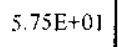 & 04 & 4 & $00 \mathrm{E}+00$ & $2.67 \mathrm{E}-02$ & 13 & $5.75 \mathrm{E}-03$ & 2 & -04 & $1.45 \mathrm{E}-02$ & 17 & 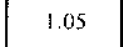 & 14 & $.60 \mathrm{E}-01$ & $.71 \mathrm{E}-01$ & 17 & 21 \\
\hline Supernatant & $1.25 \mathrm{E}+03$ & $3.30 \mathrm{E}+00$ & $4.33 \mathrm{E}+00$ & $1.34 \mathrm{E}+03$ & $2.72 E+03$ & $0.00 \mathrm{E}+00$ & $3.29 \mathrm{E}-05$ & $2.19 \mathrm{E}-07$ & $7.66 \mathrm{E}-07$ & $1.14 \mathrm{E}-05$ & $1.46 \mathrm{E}-07$ & $2.14 \mathrm{E}-04$ & 1.00 & 1.00 & 41 & $9.90 \mathrm{E}-01$ & $9.90 \mathrm{E}-01$ & 19 & 21 \\
\hline Solid Layer & $1.70 \mathrm{E}+04$ & $4.50 \mathrm{E}+01$ & $5.90 \mathrm{E}+01$ & $1.83 \mathrm{E}+04$ & $3.70 \mathrm{E}-04$ & $0.00 \mathrm{E}+00$ & $2.73 \mathrm{E}-02$ & $4.94 \mathrm{E}-03$ & $5.88 \mathrm{E}-03$ & $5.61 \mathrm{E}-02$ & $3.75 \mathrm{E}-04$ & $1.49 \mathrm{E}-02$ & 1.25 & 1.05 & 1397 & $7.55 \mathrm{E}-01$ & $8.68 \mathrm{E}-01$ & 17 & 21 \\
\hline 241 & 4 & -03 & +04 & 4 & $E+04$ & $4.94 \mathrm{E} \div 03$ & $4.08 \mathrm{E}+00$ & 12 & $1.44 E-02$ & 1 & 04 & 01 & 1.24 & 1.17 & 1 & 01 & -01 & 18 & 22 \\
\hline Supernatant & $1.70 E: 04$ & $2.98 \mathrm{E}+03$ & $3.60 E+04$ & $7.96 \mathrm{E}+04$ & 8. $32 \mathrm{E}+04$ & $4.94 \mathrm{~F}+03$ & $4.08 \mathrm{E}+00$ & $4.70 F-02$ & $1.44 \mathrm{E}-02$ & $1.24 \mathrm{E}-01$ & $6.19 \mathrm{E}-04$ & $1.02 \mathrm{E}-01$ & 1.17 & 1.17 & 38 & $7.50 E-01$ & $7.50 \mathrm{E}-01$ & 0 & 0 \\
\hline Solid Layer & $1.70 \mathrm{E}+04$ & $2.98 \mathrm{E}+03$ & $3.60 \mathrm{E}-04$ & $7.96 \mathrm{E}: 04$ & $8.32 \mathrm{E}+04$ & $4.94 E+03$ & $4.08 \mathrm{E}+00$ & $4.70 \mathrm{E}-02$ & 1.44E-02 & $1.24 \mathrm{E}-01$ & $6.19 \mathrm{E}-04$ & $1.02 \mathrm{E}-01$ & 1.24 & 1.17 & 1691 & $7.53 \mathrm{E}-01$ & $7.50 \mathrm{E}-01$ & 18 & 22 \\
\hline $24 !-T-112$ & $1.48 E+04$ & $1.40 E+03$ & $E+04$ & $2.03 E+04$ & 194 & 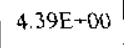 & $5.05 E-02$ & $1.32 \mathrm{E}-02$ & $E-02$ & $5 E-01$ & 03 & 300 & 3 & s. & 290 & $7.76 \mathrm{E}-01$ & 01 & 10 & 20 \\
\hline Supernatant & $6.92 \mathrm{E}+03$ & $6.56 \mathrm{E}+02$ & $1.58 \mathrm{E}+04$ & $9.49 \mathrm{E}+03$ & $2.33 \mathrm{E}+04$ & $2.05 \mathrm{E}+00$ & $2.62 \mathrm{E}-01$ & $4.42 \mathrm{E}-03$ & $7.67 \mathrm{E}-04$ & $3.31 E-03$ & $1.38 \mathrm{E}-04$ & $1.60 \mathrm{E}+00$ & 1.04 & 1.04 & 64 & $9.36 \mathrm{E}-01$ & $9.36 \mathrm{E}-01$ & 19 & 20 \\
\hline Solid Layer & $1.70 \mathrm{E}+04$ & $1.61 \mathrm{E}+03$ & $3.88 \mathrm{E} \sim 04$ & $2.33 \mathrm{E}+04$ & $5.72 \mathrm{E}^{2} 04$ & $5.04 \mathrm{E}+00$ & $2.00 \mathrm{E}-03$ & $1.52 \mathrm{E}-02$ & $2.41 \mathrm{E}-02$ & $2.14 \mathrm{E}-01$ & $\begin{array}{r}1.58 \mathrm{E}-03 \\
\end{array}$ & $3.85 \mathrm{E}-03$ & 1.28 & 1.10 & 226 & $7.39 \mathrm{E}-0 !$ & $8.51 \mathrm{E}-01$ & 19 & 20 \\
\hline $241-\mathrm{T}-201$ & $1.55 \mathrm{E}+04$ & $7.51 \mathrm{E}+01$ & $3.23 \mathrm{E}+02$ & $4.45 \mathrm{E}+04$ & $2.43 \mathrm{E}+04$ & $0.00 E+00$ & $6.19 \mathrm{E}-0$ & $.65 E-02$ & 4.16E-02 & $.17 \mathrm{E}-01$ & $2.09 \mathrm{E}-03$ & $2.03 E-02$ & 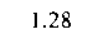 & 1.0 & 11 & $6.96 \mathrm{E}-01$ & $9.14 \mathrm{E}-01$ & 17 & 20 \\
\hline Supernatant & $2.97 \mathrm{E}+03$ & $5.28 \mathrm{E} \div 01$ & $2.27 E+02$ & $3.13 E+04$ & $1.71 E+04$ & $0.00 \mathrm{E}+00$ & $2.45 \mathrm{E}-05$ & $3.85 \mathrm{E}-08$ & $1.16 \mathrm{E}-06$ & $1.72 \mathrm{E}-05$ & $5.81 \mathrm{E}-08$ & $2.81 \mathrm{E}-05$ & 1.04 & 1.04 & 12 & $9.39 \mathrm{E}-0 \mathrm{I}$ & $9.39 \mathrm{E}-01$ & 19 & 20 \\
\hline Solid Layer & $1.70 \mathrm{E}+04$ & $7.78 E+01$ & $3.35 \mathrm{E}+02$ & $4.61 \mathrm{I}+04$ & $2.51 \mathrm{E}+04$ & $0.00 \mathrm{E}+00$ & $6.73 \mathrm{E}-02$ & $3.97 \mathrm{~F}-02$ & $4.52 \mathrm{E}-02$ & $6.71 \mathrm{E}-01$ & 2.27E- 03 & $2.21 \mathrm{E}-02$ & 1.31 & 1.06 & 107 & $6.75 \mathrm{E}-01$ & $9.12 \mathrm{E}-01$ & 17 & 20 \\
\hline $241-\mathrm{T}-202$ & $1.70 \mathrm{E}+04$ & $2.98 E+03$ & $3.60 \mathrm{E} \div 04$ & $7.96 \mathrm{E}+04$ & $8.32 \mathrm{E} \div 04$ & $4.94 \mathrm{E}+03$ & $2.23 \mathrm{E}-03$ & $3.22 E-02$ & $2.11 \mathrm{E}-02$ & $1.67 \mathrm{E}-01$ & $1.45 \mathrm{E}-03$ & $6.77 \mathrm{E}-03$ & 1.18 & 1.17 & 81 & $7.58 \mathrm{E}-01$ & $7.50 \mathrm{E}-01$ & 17 & 19 \\
\hline Supernatant & $1.70 E+04$ & $2.98 \mathrm{E}+03$ & $3.60 \mathrm{E}+04$ & $7.96 \mathrm{E}+04$ & $8.32 \mathrm{E}+04$ & $4.94 \mathrm{E}+03$ & $2.23 \mathrm{E}-03$ & $3.22 \mathrm{E}-02$ & $2.11 \mathrm{E}-02$ & $1.67 \mathrm{E}-01$ & $1.45 \mathrm{E}-03$ & $6.77 \mathrm{E}-03$ & 1.17 & 1.17 & 4 & $7.50 \mathrm{E}-0 \mathrm{l}$ & $7.50 \mathrm{E}-01$ & 0 & 0 \\
\hline Solid Layer & $1.70 \mathrm{E}+04$ & $2.98 \mathrm{E}+03$ & $3.60 E-04$ & $7.96 \mathrm{E}+04$ & $8.32 E+04$ & $4.94 \mathrm{E}+03$ & $2.23 \mathrm{E}-03$ & $3.22 \mathrm{E}-02$ & $2.11 \mathrm{E}-02$ & $1.67 \mathrm{E}-01$ & $1.45 \mathrm{E}-03$ & $6.77 \mathrm{E}-03$ & 1.18 & 1.17 & 77 & $7.58 \mathrm{E}-01$ & $7.50 \mathrm{E}-01$ & 17 & 19 \\
\hline
\end{tabular}


RPP-5926 REV 6

Table B-5. Input Data With Water Addition for Hydrogen Generation Rate Model Calculations for 177 Tanks. (20 sheets)

\begin{tabular}{|c|c|c|c|c|c|c|c|c|c|c|c|c|c|c|c|c|c|c|c|}
\hline Tank & $\begin{array}{c}\mathrm{OH} \\
\text { in liquid } \\
|\mathrm{OH}| \\
(\mu \mathrm{g} / \mathrm{mL})\end{array}$ & $\begin{array}{c}\text { TOC } \\
\text { in liquid } \\
|\mathrm{TOC}| \\
(\mu \mathrm{g} / \mathrm{mL})\end{array}$ & $\begin{array}{c}\mathrm{NO}_{2} \\
\text { in liquidd } \\
\mid \mathrm{NO}_{2} ! \\
(\mu \mathrm{g} / \mathrm{mL})\end{array}$ & $\begin{array}{c}\mathrm{NO}_{3} \\
\text { in liquid } \\
\left|\mathrm{NO}_{3}\right| \\
(\mu \mathrm{g} / \mathrm{mL})\end{array}$ & $\begin{array}{c}\mathrm{Na} \\
\text { in liquid } \\
{[\mathrm{Na}]} \\
\text { (ug/mL) }\end{array}$ & $\begin{array}{c}\text { Al } \\
\text { in liquid } \\
|A|] \\
(\mu \mathrm{g} / \mathrm{mL})\end{array}$ & $\begin{array}{c}90 \mathrm{Sr} \\
\text { in waste } \\
{[\mathrm{Sr}]} \\
(\mu \mathrm{Ci} / \mathrm{g})\end{array}$ & $\begin{array}{c}{ }^{241} \mathrm{Am} \\
\text { in waste } \\
\mid \mathrm{Am} 241] \\
(\mu \mathrm{Ci} / \mathrm{g})\end{array}$ & $\begin{array}{c}{ }^{240} \mathrm{Pu} \\
\text { in waste } \\
\mid \mathrm{Pu} 240] \\
(\mu \mathrm{Ci} / \mathrm{g})\end{array}$ & $\begin{array}{c}{ }^{239} \mathrm{Pu} \\
\text { in waste } \\
{[\mathrm{Pu} 240]} \\
(\mu \mathrm{Ci} / \mathrm{g})\end{array}$ & $\begin{array}{c}{ }^{238} \mathrm{Pu} \\
\text { in waste } \\
\mid \mathrm{Pu} 238] \\
(\mu \mathrm{Ci} / \mathrm{g})\end{array}$ & $\begin{array}{c}{ }^{137} \mathrm{Cs} \\
\text { in waste } \\
|\mathrm{Cs}| \\
(\mu \mathrm{Ci} / \mathrm{g})\end{array}$ & $\begin{array}{c}\text { Bulk } \\
\text { density } \\
\text { D } \\
(\mathrm{g} / \mathrm{ml})\end{array}$ & $\mid \begin{array}{c}\text { Liquid } \\
\text { density } \mathrm{D}_{\mathrm{L}} \\
\text { (g/ml) }\end{array}$ & $\begin{array}{c}\text { Non-RGS } \\
\text { waste } \\
\text { volume } \\
(\mathrm{kL})\end{array}$ & $\begin{array}{l}\text { Bulk } \\
\text { water } \\
\left\{\mathrm{H}_{2} \mathrm{O}\right\} \\
(\mathrm{wt} " 10)\end{array}$ & $\begin{array}{l}\text { Liquid } \\
\text { water } \\
\left|\mathrm{H}_{2} \mathrm{O}\right| \\
\left(\mathrm{wt}^{\prime} \%\right)\end{array}$ & $\begin{array}{c}\text { Waste } \\
\text { temp. } \\
\text { Tw } \\
\left.{ }^{\circ} \mathrm{C}\right)\end{array}$ & $\begin{array}{c}\text { Dome } \\
\text { temp. } \\
\text { Td } \\
\left({ }^{0} \mathrm{C}\right)\end{array}$ \\
\hline $241-\mathrm{T}-203$ & $O E+04$ & $88 \mathrm{~F}+03$ & $3.60 \mathrm{E}+04$ & $6 \mathrm{E}+04$ & $32 t \div 04$ & $94 \mathrm{E}+03$ & $2.21 \mathrm{E}-03$ & $75 \mathrm{E}-02$ & $2.81 \mathrm{E}-02$ & $2.24 \mathrm{E}-0$ & $.93 \mathrm{E}-03$ & $6.63 \mathrm{E}-03$ & 1.22 & 1.17 & 140 & $7.64 \mathrm{E}-01$ & $50 \mathrm{E}-01$ & 17 & 20 \\
\hline Supernatanı & $1.70 \mathrm{E} \div 04$ & $2.98 E+03$ & $3.60 \mathrm{E}+04$ & $7.96 \mathrm{E}+04$ & $8.32 \mathrm{E}+04$ & $4.94 \mathrm{E}+03$ & $2.21 \mathrm{E}-03$ & $3.75 \mathrm{E}-02$ & $2.81 \mathrm{E}-02$ & $2.24 \mathrm{E}-01$ & $1.93 \mathrm{E}-03$ & $6.63 \mathrm{E}-03$ & 1.17 & 1.17 & 4 & $8.50 \mathrm{E}-01$ & $8.50 \mathrm{E}-01$ & 0 & 0 \\
\hline Solid Layer & $1.70 \mathrm{E}+04$ & $2.98 \mathrm{E}+03$ & $3.60 \mathrm{E}-04$ & $7.96 \mathrm{E}+04$ & $8.32 \mathrm{E}+04$ & $4.94 E+03$ & $2.21 \mathrm{E}-03$ & $3.75 \mathrm{E}-02$ & $2.81 \mathrm{E}-02$ & $2.24 \mathrm{E}-0 \mathrm{I}$ & $1.93 \mathrm{E}-03$ & $6.63 \mathrm{E}-03$ & 1.22 & 1.17 & 136 & 7.62E-01 & $8.50 \mathrm{E}-0 \mathrm{I}$ & 17 & 20 \\
\hline $24 !-\uparrow-204$ & $0 E+04$ & $2.98 \mathrm{E} \div 03$ & $3.60 \mathrm{E}+04$ & $7.96 \mathrm{E}+04$ & $8.32 E: 04$ & $4.94 \mathrm{E}+03$ & 4.03E-03 & $27 \mathrm{E}-02$ & $2.39 \mathrm{E}-02$ & $1.90 \mathrm{E}-01$ & j. $64 \mathrm{E}-03$ & $6.85 \mathrm{E}-03$ & 1.18 & 1.17 & 140 & $7.50 \mathrm{E}-01$ & $7.50 \mathrm{E}-0 \mathrm{~J}$ & 17 & 20 \\
\hline Supernatant & $1.70 \mathrm{E}+04$ & $2.98 E+03$ & $3.60 \mathrm{E}+04$ & $7.96 \mathrm{E}+04$ & $8.32 \mathrm{E}+04$ & $4.94 \mathrm{E} \cdot 03$ & $4.03 \mathrm{E}-03$ & $2.27 \mathrm{E}-02$ & $2.39 \mathrm{E}-02$ & $1.90 \mathrm{E}-01$ & $1.64 \mathrm{E}-03$ & $6.85 \mathrm{E}-03$ & 1.17 & 1.17 & 4 & $7.50 \mathrm{E}-01$ & $7.50 \mathrm{E}-01$ & 0 & 0 \\
\hline Solid Layer & $1.70 \mathrm{E}+04$ & $2.98 \mathrm{E} \div 03$ & $3.60 \mathrm{E}+04$ & $7.96 \mathrm{E}+04$ & $8.32 E+04$ & $4.94 \mathrm{E}+03$ & 4.03E-03 & $2.27 \mathrm{E}-02$ & $2.39 \mathrm{E}-02$ & $1.90 \mathrm{E}-01$ & $1.64 \mathrm{E}-03$ & $6.85 \mathrm{E}-03$ & 1.18 & 1.17 & 136 & $7.50 \mathrm{E}-01$ & $7.50 \mathrm{E}-01$ & 17 & 20 \\
\hline $241-\Upsilon X-101$ & $0 E+04$ & $03 \mathrm{E}+03$ & $1.22 \mathrm{E}+05$ & $1.90 E+05$ & $2.36 \mathrm{E}+05$ & $78 \mathrm{E}+04$ & $23 E+02$ & $20 \mathrm{E}-01$ & 1.26E-01 & $5.90 \mathrm{E}-01$ & $1.95 \mathrm{E}-02$ & $6.70 \mathrm{E}+01$ & 1.71 & 1.45 & 382 & $.00 \mathrm{E}-01$ & $91 \mathrm{E}-01$ & 25 & 23 \\
\hline Supernatant & $1.70 E+04$ & $4.03 \mathrm{E}+03$ & $1.22 E+05$ & $1.90 \mathrm{E} \div 05$ & $2.36 \mathrm{E}+05$ & $3.78 \mathrm{E}+04$ & $3.23 E+02$ & $4.20 \mathrm{E}-01$ & $1.26 \mathrm{E}-0 \mathrm{~J}$ & $5.90 \mathrm{E}-01$ & $1.95 \mathrm{E}-02$ & $6.70 \mathrm{E}+01$ & 1.45 & 1.45 & 38 & 4.91E-01 & $4.91 \mathrm{E}-01$ & 0 & 0 \\
\hline Solid Layer & $1.70 \mathrm{E}+04$ & $4.03 E \div 03$ & $1.22 \mathrm{E}+05$ & $1.90 \mathrm{E}+05$ & $2.36 \mathrm{E}+05$ & $.78 \mathrm{E}+04$ & $23 \mathrm{E} ; 02$ & $4.20 \mathrm{E}-01$ & $1.26 \mathrm{E}-01$ & $5.90 \mathrm{E}-01$ & $1.95 \mathrm{E}-02$ & $6.70 \mathrm{E}+01$ & 1.74 & 1.45 & 344 & 3.91E-01 & $4.91 \mathrm{E}-01$ & 25 & 23 \\
\hline $241-T X-102$ & $1.70 E-04$ & $03 \mathrm{E}+03$ & $1.22 \mathrm{E}+05$ & $1.90 E+05$ & $2.36 \mathrm{E}+05$ & $3.78 \mathrm{E}+04$ & $1.57 \mathrm{E}+00$ & 4SE-01 & $1.52 \mathrm{E}-02$ & $7.23 \mathrm{E}-02$ & $2.54 \mathrm{E}-03$ & $8.75 \mathrm{E}+01$ & 1.61 & 1.45 & 859 & $3.19 \mathrm{E}-01$ & $1.91 \mathrm{E}-01$ & 26 & 25 \\
\hline Supernatant & $1.70 \mathrm{E}+04$ & $4.03 E+0.03$ & $1.22 E+05$ & $1.90 E-05$ & $2.36 \mathrm{E}+05$ & $3.78 E+04$ & $1.57 \mathrm{E}+00$ & $1.45 \mathrm{E}-01$ & $1.52 \mathrm{E}-02$ & $7.23 \mathrm{E}-02$ & $2.54 \mathrm{E}-03$ & $8.75 \mathrm{E}+01$ & 1.45 & 1.45 & 38 & $4.91 \mathrm{E}-01$ & $4.91 \mathrm{~F}-01$ & 0 & 0 \\
\hline Solid Layer & $1.70 \mathrm{E}+04$ & $4.03 \mathrm{E} \div 03$ & $1.22 \mathrm{E}+05$ & $90 \mathrm{E}+05$ & $2.36 \mathrm{E}+05$ & $78 \mathrm{E}+04$ & $1.57 \mathrm{E}+00$ & $1.45 \mathrm{E}$ & $1.52 \mathrm{E}-02$ & $7.23 \mathrm{E}-02$ & $2.54 \mathrm{E}-03$ & $8.75 \mathrm{E}^{+}$ & 1.61 & 1.45 & 821 & 3.12E-01 & 01 & 26 & 25 \\
\hline 24 J-TX-103 & $1.70 \mathrm{E} \div 04$ & $3.88 \mathrm{E}+03$ & $1.18 \mathrm{E}+05$ & $1.91 \mathrm{E}+05$ & $2.32 \mathrm{E}+05$ & $3.65 \mathrm{E}+04$ & $7.29 \mathrm{E}-01$ & $1.43 \mathrm{E}-01$ & $1.50 \mathrm{E}-02$ & $7.18 \mathrm{E}-02$ & $2.51 \mathrm{E}-03$ & $8.73 E+01$ & 1.60 & 1.44 & 586 & $3.59 \mathrm{E}-01$ & $6.90 \mathrm{E}-01$ & 22 & 21 \\
\hline Supematant & $1.70 \mathrm{E} \vdash 04$ & $3.88 \mathrm{E}+03$ & $1.18 E+05$ & $1.91 \mathrm{E}+05$ & $2.32 \mathrm{E}+05$ & $3.65 \mathrm{E}+04$ & $7.29 \mathrm{E}-01$ & $1.43 \mathrm{E}-01$ & 1. $50 \mathrm{E}-02$ & $7.18 \mathrm{E}-02$ & $2.51 \mathrm{E}-03$ & $8.73 \mathrm{E}+01$ & 1.44 & 1.44 & 38 & $6.90 \mathrm{E}-0 \mathrm{I}$ & $6.90 \mathrm{E}-01$ & 0 & 0 \\
\hline Solid Layer & $1.70 \mathrm{E}+04$ & $3.88 E+03$ & $1.18 \mathrm{E}+05$ & $1.91 \mathrm{E}+05$ & $2.32 \mathrm{E}+05$ & $3.65 \mathrm{E}+04$ & $7.29 \mathrm{E}-01$ & $1.43 \mathrm{E}-01$ & $1.50 \mathrm{E}-02$ & $7.18 \mathrm{E}-02$ & $2.51 \mathrm{E}-03$ & 更 & 1.61 & 1.44 & 548 & $3.39 \mathrm{E}-01$ & $6.90 \mathrm{E}-01$ & 22 & 21 \\
\hline $241 \cdot \mathrm{TX}-104$ & $1.50 \mathrm{E}+04$ & $1.19 \mathrm{E}+03$ & $8.30 \mathrm{E}+04$ & $3.11 \mathrm{E}+05$ & $1.80 E+05$ & $2.81 \mathrm{E} \div 03$ & $1.47 \mathrm{E}+02$ & $1.44 \mathrm{E}-01$ & $1.95 \mathrm{E}-02$ & $9.53 \mathrm{E}-02$ & $2.81 \mathrm{E}-03$ & $6.42 \mathrm{E}+01$ & 1.64 & 1.39 & 300 & $4.99 \mathrm{~F}-01$ & $5.56 \mathrm{E}-01$ & 21 & 21 \\
\hline Supernatant & $3.27 \mathrm{E}-03$ & $2.60 \mathrm{E}+02$ & $1.81 E+04$ & $6.81 E+04$ & $3.95 \mathrm{E}-04$ & $6.13 \mathrm{E}+02$ & $1.65 \mathrm{E}-01$ & $2.26 \mathrm{E}-03$ & $2.07 \mathrm{E}-05$ & 9.84 & 3.48E-06 & 1 & 1.08 & 08 & 47 & -01 & -01 & 21 & 21 \\
\hline Solid Layer & $1.70 \mathrm{E}+04$ & $1.36 \mathrm{E}+03$ & $9.43 \mathrm{E} \div 04$ & $3.53 \mathrm{E}+05$ & $2.05 \mathrm{E}+05$ & TEV & $1.045+02$ & $3.00 \mathrm{~L}-01$ & $2.17 \mathrm{E}-02$ & $1.06 \mathrm{E}-01$ & $3.13 \mathrm{E}-03$ & $0.40 \mathrm{~L}+01$ & 1.74 & 1.47 & 5 & $4.55 \mathrm{E}-01$ & $5.19 \mathrm{E}-01$ & 21 & 21 \\
\hline $241-\mathrm{TX}-105$ & $1.70 \mathrm{E} \cdot 04$ & $4.03 E+03$ & $1.22 \mathrm{E}+05$ & $1.90 \mathrm{E}+05$ & $2.36 \mathrm{E}+05$ & $3.78 \mathrm{E}+04$ & $1.95 \mathrm{E}^{+} 00$ & $1.60 \mathrm{E}-0 \mathrm{I}$ & $1.67 \mathrm{E}-02$ & $7.95 \mathrm{E}-02$ & $2.79 \mathrm{E}-03$ & $7.10 \mathrm{E}+0 \mathrm{~J}$ & 1.63 & 1.45 & 2219 & $2.97 \mathrm{E}-01$ & $4.91 \mathrm{E}-01$ & 34 & 23 \\
\hline Supernatant & $1.70 E+04$ & $4.03 \mathrm{~F}+03$ & $1.22 \mathrm{E}+05$ & $1.90 \mathrm{E}+05$ & $2.36 \mathrm{E}+05$ & $3.78 \mathrm{E}+04$ & $1.95 \mathrm{E}+00$ & $1.60 \mathrm{E}-01$ & $1.67 \mathrm{E}-02$ & $7.95 \mathrm{E}-02$ & $2.79 \mathrm{E}-03$ & $7.10 \mathrm{E}+01$ & 1.45 & 1.45 & 38 & $4.91 \mathrm{E}-01$ & $4.91 \mathrm{E}-01$ & 0 & 0 \\
\hline Solid Layer & $1.70 \mathrm{E}+04$ & $4.03 \mathrm{E}+03$ & 1.22E+05 & $1.90 \mathrm{E}-05$ & $2.36 \mathrm{E} \div 05$ & $3.78 \mathrm{E}+04$ & $1.95 \mathrm{E}+00$ & $1.60 \mathrm{E}-01$ & $1.67 \mathrm{E}-02$ & $7.95 \mathrm{E}-02$ & $2.79 E-03$ & $7.10 \mathrm{E}+01$ & 1.63 & 1.43 & 2181 & $2.94 \mathrm{E}-01$ & $4.91 \mathrm{E}-01$ & 34 & 23 \\
\hline 241-TX-106 & $1.70 \mathrm{E}+04$ & $4.03 E+03$ & $1.22 \mathrm{E}+05$ & $1.90 \mathrm{E}+05$ & $2.36 \mathrm{E}+05$ & $3.78 \mathrm{E}+04$ & $6.09 \mathrm{E}+00$ & $1.53 \mathrm{E}-01$ & $1.68 \mathrm{E}-02$ & $8.06 \mathrm{E}-02$ & $2.73 E_{-}-03$ & $8.27 \mathrm{E}+0 \mathrm{I}$ & $1.6 ?$ & 45 & 1356 & $3.09 \mathrm{E}-01$ & $4.91 \mathrm{E}-01$ & 26 & 24 \\
\hline Superuatant & $1.70 E+04$ & $4.03 E+03$ & $1.22 \mathrm{E}+05$ & 1.90E +05 & $2.36 \mathrm{E}+05$ & $3.78 \mathrm{E}+04$ & $6.09 \mathrm{E}+00$ & $1.53 \mathrm{E}-0 \mathrm{I}$ & $1.68 \mathrm{E}-02$ & $8.06 \mathrm{E}-02$ & $2.73 \mathrm{E}-03$ & $8.27 \mathrm{E}+01$ & 1.45 & 1.45 & 38 & -01 & -01 & 0 & 0 \\
\hline Solid layer & $1.70 \mathrm{E}+04$ & $4.03 \mathrm{E}+03$ & $1.22 E+05$ & $1.90 \mathrm{E}+05$ & $2.36 \mathrm{E}+05$ & $3.78 \mathrm{E}+04$ & $6.09 \mathrm{E}+00$ & $1.53 \mathrm{E}-01$ & $1.68 \mathrm{E}-02$ & $8.06 \mathrm{E}-02$ & $2.73 \mathrm{E}-03$ & $8.27 \mathrm{E}+01$ & 1.62 & 1.45 & 1318 & $3.04 \mathrm{E}-01$ & $4.91 \mathrm{E}-01$ & 26 & 24 \\
\hline 17 & +04 & 03 & $1.16 \mathrm{E}+05$ & $1.97 \mathrm{E}+05$ & $2.40 \mathrm{E}+05$ & $4.03 E+04$ & $5.47 \mathrm{E}+00$ & $1.69 E+00$ & $1.77 \mathrm{E}-01$ & $8.41 \mathrm{E}-01$ & $2.97 \mathrm{E}-02$ & $1.02 \mathrm{E}+02$ & 1.70 & 1.46 & 150 & $3.34 \mathrm{E}-01$ & $5.11 \mathrm{E}-01$ & 22 & 20 \\
\hline Supernatant & $1.70 \mathrm{E}+04$ & $3.22 \mathrm{E}+03$ & $1.16 \mathrm{E}+05$ & $1.97 \mathrm{E}+05$ & $2.40 \mathrm{E}+05$ & $4.03 \mathrm{E}+04$ & $5.47 \mathrm{E}+00$ & $1.69 \mathrm{E}+00$ & $1.77 \mathrm{E}-01$ & $8.41 \mathrm{E}-01$ & $2.97 \mathrm{E}-02$ & $1.02 \mathrm{E}+02$ & 1.46 & 1.46 & 38 & $5.11 E-01$ & $5.11 \mathrm{E}-01$ & 0 & 0 \\
\hline Solid Layer & $1.70 \mathrm{E}-04$ & $3.22 \mathrm{E}+03$ & $1.16 \mathrm{E}+05$ & $1.97 \mathrm{E}+05$ & $2.40 \mathrm{E}+05$ & $4.03 \mathrm{E}+04$ & $5.47 \mathrm{E}+00$ & $1.69 \mathrm{E} \div 00$ & $1.77 E-01$ & $8.41 \mathrm{E}-01$ & $2.97 \mathrm{E}-02$ & $1.02 E+02$ & 1.78 & 1.46 & 112 & $2.84 \mathrm{E}-01$ & $5.11 \mathrm{E}-01$ & 22 & 20 \\
\hline
\end{tabular}




\section{RPP-5926 REV 6}

Table B-5. Input Data With Water Addition for Hydrogen Generation Rate Model Calculations for 177 Tanks. (20 sheets)

\begin{tabular}{|c|c|c|c|c|c|c|c|c|c|c|c|c|c|c|c|c|c|c|c|}
\hline Tank & $\begin{array}{c}\mathrm{OH} \\
\text { in liquid } \\
{[\mathrm{OH}]} \\
(\mu \mathrm{g} / \mathrm{mL})\end{array}$ & $\begin{array}{c}\text { TOC } \\
\text { in liquid } \\
\text { ['TOC] } \\
(\mu \mathrm{g} / \mathrm{mL})\end{array}$ & $\begin{array}{c}\mathrm{NO}_{2} \\
\text { in liquid } \\
{\left[\mathrm{NO}_{2}\right]} \\
(\mu \mathrm{g} / \mathrm{mL})\end{array}$ & $\begin{array}{c}\mathrm{NO}_{3} \\
\text { in liquid } \\
{\left[\mathrm{NO}_{3}\right]} \\
(\mu \mathrm{g} / \mathrm{mL})\end{array}$ & $\begin{array}{c}\mathrm{Na} \\
\text { in liquid } \\
|\mathrm{Na}| \\
(\mathrm{ug} / \mathbf{m L})\end{array}$ & $\begin{array}{c}\text { Al } \\
\text { in liquid } \\
[\mathrm{A}]] \\
(\mu \mathrm{g} / \mathrm{mL})\end{array}$ & $\begin{array}{c}90 \mathrm{Sr} \\
\text { in waste } \\
{[\mathrm{Sr}]} \\
(\mu \mathrm{Ci} / \mathrm{g})\end{array}$ & $\begin{array}{c}{ }^{24 !} \mathrm{Am} \\
\text { in waste } \\
{[\mathrm{Am} 241]} \\
(\mu \mathrm{Ci} / \mathrm{g})\end{array}$ & $\begin{array}{c}{ }^{240} \mathrm{Pu} \\
\text { in waste } \\
{[\mathrm{Pu} 240]} \\
(\mu \mathrm{Ci} / \mathrm{g})\end{array}$ & $\begin{array}{c}{ }^{239} \mathrm{Pu} \\
\text { in waste } \\
{\left[\begin{array}{l}|\mathrm{Pu2} 40| \\
(\mu \mathrm{Cig} / \mathrm{g})\end{array}\right.}\end{array}$ & $\begin{array}{c}{ }^{238} \mathrm{Pu} \\
\text { in waste } \\
\left|\mathbf{P}_{\text {u238 }}\right| \\
(\mu \mathrm{Ci} / \mathrm{g})\end{array}$ & $\begin{array}{c}{ }^{137} \mathrm{Cs} \\
\text { in waste } \\
{[\mathrm{Cs}]} \\
(\mu \mathrm{Ci} / \mathrm{g})\end{array}$ & $\begin{array}{c}\text { Bulk } \\
\text { density } \\
\text { D } \\
(\mathrm{g} / \mathrm{mL})\end{array}$ & $\begin{array}{c}\text { Liquid } \\
\text { density } \mathrm{D}_{\mathrm{t}} \\
(\mathrm{g} / \mathrm{ml})\end{array}$ & $\begin{array}{c}\text { Non-RGS } \\
\text { waste } \\
\text { volume } \\
\text { (kL) }\end{array}$ & $\begin{array}{c}\text { Bulk } \\
\text { water } \\
\left|\mathrm{H}_{2} \mathrm{O}\right| \\
\left(\mathrm{wt}^{\circ} \%\right)\end{array}$ & $\begin{array}{l}\text { Liquid } \\
\text { water } \\
\left|\mathrm{H}_{2} \mathrm{O}\right| \\
(w t \%)\end{array}$ & $\begin{array}{l}\text { Waste } \\
\text { temp. } \\
\mathrm{Tw} \\
\left({ }^{\circ} \mathrm{C}\right)\end{array}$ & $\begin{array}{c}\text { Dome } \\
\text { temp. } \\
\text { Td } \\
\left({ }^{\circ} \mathrm{C}\right)\end{array}$ \\
\hline 24$]-T X-108$ & $0 \mathrm{E}+04$ & $0.3 E+03$ & $1.22 \mathrm{~F}+05$ & $1.90 \mathrm{E}+05$ & $36 \mathrm{E}+05$ & $3.78 \mathrm{E}+04$ & $5.27 \mathrm{E}+00$ & $1.50 \mathrm{E}-01$ & $1.55 \mathrm{E}-02$ & 7.45E-02 & $2.61 \mathrm{E}-03$ & $7.50 \mathrm{E}+01$ & 1.61 & 1.45 & 516 & $3.20 \mathrm{E}-01$ & $4.91 \mathrm{E}-01$ & 21 & 21 \\
\hline Supematant & $1.70 \mathrm{E}+04$ & $4.03 \mathrm{E}+03$ & $1.22 \mathrm{E}+05$ & $1.90 \mathrm{E}+05$ & $2.36 \mathrm{E} \times 05$ & $3.78 \mathrm{E}+04$ & $5.27 \mathrm{E}+00$ & $1.50 \mathrm{E}-01$ & $1.55 \mathrm{E}-02$ & 7.45E- 02 & $2.6 \mathrm{JE}-03$ & $7.50 \mathrm{E}+0 \mathrm{~J}$ & 1.45 & 1.45 & 38 & $4.91 \mathrm{~F}-01$ & $4.91 \mathrm{E}-01$ & 0 & 0 \\
\hline Solid Layer & $1.70 \mathrm{E}-04$ & $4.03 E+03$ & $1.22 \mathrm{~F}+05$ & $1.90 \mathrm{E}+05$ & $2.36 \mathrm{E}+05$ & $3.78 \mathrm{E}+04$ & $5.27 E+00$ & $1.50 \mathrm{E}-0 \mathrm{H}$ & $1.55 \mathrm{E}-02$ & $7.45 \mathrm{E}-02$ & $2.61 E-03$ & $7.50 \mathrm{E}+01$ & 1.62 & 1.45 & 478 & $3.07 \mathrm{E}-01$ & $4.91 \mathrm{E}-01$ & 21 & 21 \\
\hline $241-I X-109$ & $1.70 \mathrm{~F}+04$ & $2.98 \mathrm{E}=03$ & $3.60 \mathrm{E} \div 04$ & $7.96 E+04$ & $8.32 E+04$ & $4.94 \mathrm{E}+03$ & $4.82 \mathrm{E}+00$ & $1.38 \mathrm{E}-02$ & $9.05 \mathrm{E}-03$ & 8. 34E-02 & $5.85 \mathrm{E}-04$ & $1.59 \mathrm{E}+01$ & 1.42 & 1.17 & 1413 & $5.46 \mathrm{E}-01$ & $7.50 \mathrm{E}-01$ & 34 & 39 \\
\hline Supeniatant & $1.70 E+04$ & $2.98 \mathrm{E}+03$ & $3.60 \mathrm{E} \cdot 04$ & $7.96 \mathrm{E}+04$ & $8.32 \mathrm{E}+04$ & $4.94 \mathrm{E}-03$ & $4.82 \mathrm{E}+00$ & $1.38 \mathrm{E}-02$ & $9.05 \mathrm{E}-03$ & $8.34 \mathrm{E}-02$ & $5.85 \mathrm{E}-04$ & $1.59 \mathrm{E}-01$ & 1.17 & 1.17 & 38 & $7.50 E-01$ & $7.50 \mathrm{E}-01$ & 0 & 0 \\
\hline Solid Layer & $1.70 \mathrm{E} \div 04$ & $2.98 \mathrm{E}+03$ & $3.60 \mathrm{E}+04$ & $7.96 \mathrm{E}+04$ & $8.32 \mathrm{E} \div 04$ & $4.94 \mathrm{E}+03$ & $4.82 \mathrm{E}+00$ & $1.38 \mathrm{E}-02$ & $9.05 \mathrm{E}-03$ & $8.34 \mathrm{E}-02$ & $5.85 \mathrm{E}-04$ & $1.59 \mathrm{E}+01$ & 1.43 & 1.17 & 1375 & $5.41 E-01$ & $7.50 \mathrm{E}-01$ & 34 & 39 \\
\hline $241-T X-110$ & $10 \mathrm{E}+04$ & $4.02 \mathrm{E}-03$ & $1.22 \mathrm{E}+05$ & $1.90 \mathrm{E}+05$ & $2.37 \mathrm{E}+05$ & $3.78 \mathrm{E}+04$ & $1.03 E+00$ & $1.55 \mathrm{E}-01$ & $1.66 \mathrm{E}-02$ & $8.20 \mathrm{E}-02$ & $2.73 \mathrm{E}-03$ & $6.52 E+01$ & 1.61 & 1.45 & 1807 & $3.10 \mathrm{E}-01$ & $4.91 \mathrm{E}-01$ & 28 & 22 \\
\hline Supernatant & $1.70 \mathrm{~F}+04$ & $4.02 \mathrm{E}+03$ & $1.22 \mathrm{E}+05$ & $1.906+05$ & $2.37 \mathrm{E}+05$ & $3.78 E+04$ & $1.03 \mathrm{E}+00$ & $1.55 \mathrm{E}-01$ & $1.666 .-02$ & $8.20 \mathrm{E}-02$ & $2.73 \mathrm{E}-03$ & $6.52 \mathrm{E} \div$ & 1.45 & 1.45 & 38 & $4.91 \mathrm{E}-01$ & $4.91 \mathrm{~F}-01$ & 0 & 0 \\
\hline Solid Layer & $1.70 \mathrm{E}+04$ & $4.02 \mathrm{E}+03$ & $1.22 E+05$ & $1.90 \mathrm{E}+05$ & $2.37 \mathrm{E}+05$ & $3.78 \mathrm{E}-04$ & $1.03 E+00$ & $1.55 \mathrm{E}-01$ & $1.66 \mathrm{E}-02$ & $8.20 \mathrm{E}-02$ & $2.73 \mathrm{E}-03$ & $6.52 \mathrm{E}+01$ & 1.62 & 1.45 & 1769 & $3.06 \mathrm{E}-01$ & $4.91 \mathrm{E}-01$ & 28 & 22 \\
\hline $241 \cdot T X-111$ & $0 \mathrm{E}+04$ & $4.03 \mathrm{E}+0.3$ & $1.22 \mathrm{E}+05$ & $0 E+05$ & $36 \mathrm{E}+05$ & $3.78 \mathrm{E}+04$ & $1.17 \mathrm{E}+00$ & $51 \mathrm{E}-01$ & $S E-02$ & $8.28 \mathrm{E}-02$ & 67E-03 & $6.21 E+01$ & 1.61 & 1.45 & 1419 & 3.18E-01 & $91 \mathrm{~F}-01$ & 26 & 22 \\
\hline Supernatant & $1.70 \mathrm{E}+04$ & $4.03 \mathrm{E}+03$ & $1.22 \mathrm{E} \div 05$ & $1.90 \mathrm{E}+05$ & $2.36 \mathrm{E}+05$ & $3.78 E+04$ & $1.17 \mathrm{E}+00$ & $1.51 \mathrm{E}-01$ & 1.65E-02 & $8.28 \mathrm{E}-02$ & $2.67 \mathrm{E}-03$ & $6.21 \mathrm{E}+01$ & 1.45 & 1.45 & 38 & $4.91 \mathrm{E}-01$ & $4.91 \mathrm{E}-01$ & 0 & 0 \\
\hline Solid Layer & $1.70 E+04$ & $4.03 E+03$ & $1.22 \mathrm{E}+05$ & $1.90 \mathrm{E}-05$ & $2.36 \mathrm{E} \cdot 05$ & $3.78 \mathrm{E}+04$ & $1.17 \mathrm{E}+00$ & $1.51 \mathrm{E}-0 \mathrm{~J}$ & $1.65 \mathrm{E}-02$ & $8.28 \mathrm{E}-02$ & $2.67 \mathrm{E}-03$ & $6.21 \mathrm{E} \div 01$ & 1.61 & 1.45 & 1381 & 3.13F-01 & 4.91E-01 & 26 & 22 \\
\hline $241-T X-112$ & $4.81 t+04$ & $3.47 \mathrm{E}-03$ & $1.06 \mathrm{E}+05$ & $1.94 \mathrm{E}+05$ & $2.18 \mathrm{E}+05$ & $3.26 \mathrm{E}+04$ & $7.24 \mathrm{E}-01$ & $1.58 \mathrm{E}-01$ & $1.66 \mathrm{E}-02$ & $7.94 \mathrm{E}-02$ & $77 \mathrm{E}-03$ & $6.81 E+01$ & 1.63 & 1.42 & 2436 & $2.51 \mathrm{E}-01$ & $6 \mathrm{E}-01$ & 22 & 19 \\
\hline Supernatant & $4.81 E+04$ & $3.47 \mathrm{E}-103$ & $1.06 \mathrm{E}+05$ & $1.94 \mathrm{~F}+05$ & $2.18 \mathrm{E}+05$ & $3.26 \mathrm{E}+04$ & $7.24 \mathrm{E}-01$ & $1.58 \mathrm{E}-01$ & $1.66 \mathrm{E}-02$ & $7.94 \mathrm{E}-02$ & 2.77E-03 & $6.81 E+01$ & 1.42 & 1.42 & 38 & $5.16 \mathrm{E}-01$ & $5.16 \mathrm{E}-0 \mathrm{I}$ & 0 & 0 \\
\hline Solid Layer & $4.81 E \div 04$ & $3.47 \mathrm{E}+03$ & $1.06 \mathrm{E}+05$ & $1.94 \mathrm{E}+05$ & $2.18 \mathrm{E}+05$ & $3.26 \mathrm{E}+04$ & $7.24 \mathrm{E}-01$ & $1.58 \mathrm{E}-01$ & $1.66 \mathrm{E}-02$ & 7.94E- 02 & $2.77 \mathrm{E}-03$ & $6.81 \mathrm{E} \div 01$ & 1.63 & 1.42 & 2398 & $2.48 \mathrm{E}-01$ & $5.16 \mathrm{E}-01$ & 22 & 19 \\
\hline $24|-| X-113$ & $4.81 \mathrm{E}+04$ & $4.03 E+03$ & $1.22 \mathrm{E} 105$ & 1.90E+05 & $2.36 \mathrm{E} \div 05$ & $3.78 \mathrm{E} \div 04$ & $1.40 \mathrm{~F}+00$ & $6.72 \mathrm{E}-03$ & $1.68 \mathrm{E}-03$ & $1.32 \mathrm{E}-02$ & $1.62 \mathrm{E}-04$ & $8.18 \mathrm{E}+00$ & 1.61 & 1.45 & 2454 & $1.60 \mathrm{E}-01$ & $91 \mathrm{E}-01$ & 22 & 20 \\
\hline Supernatant & $4.81 \mathrm{E}+04$ & $4.03 \mathrm{E}+03$ & $1.22 \mathrm{E}+05$ & $1.90 \mathrm{E}+05$ & $2.36 \mathrm{E}+05$ & $3.78 \mathrm{E}+04$ & $1.40 E+00$ & $6.72 \mathrm{E}-03$ & $1.68 \mathrm{E}-03$ & $1.32 \mathrm{E}-02$ & $1.62 \mathrm{E}-04$ & $8.18 E+00$ & 1.45 & 1.45 & 38 & $4.91 \mathrm{E}-01$ & $4.91 \mathrm{E}-01$ & 0 & 0 \\
\hline Solid Layer & 4.8 & $E+03$ & 1. & 1. & $2.36 \mathrm{E}+05$ & 04 & 1. & 6. & $1.68 \mathrm{E}-03$ & 1.32E-02 & $1.62 \mathrm{E}-04$ & $8.18 E+00$ & 1.61 & 1.45 & 2416 & $1.55 \mathrm{E}-01$ & $4.91 \mathrm{E}-01$ & 22 & 20 \\
\hline $241-5 X-114$ & $1.70 \mathrm{E}+04$ & $1.53 E+03$ & $5.15 \mathrm{E}+04$ & $2.07 \mathrm{E}+05$ & $1.60 \mathrm{E}+05$ & $1.45 E+04$ & $7.29 \mathrm{E}-01$ & $1.48 \mathrm{E}-01$ & $1.59 \mathrm{E}-02$ & $7.75 \mathrm{E}-02$ & $2.62 \mathrm{E}-03$ & $5.94 \mathrm{E}+01$ & 1.63 & 1.33 & 2050 & $2.58 \mathrm{E}-01$ & $6.12 \mathrm{E}-01$ & 21 & 18 \\
\hline Supernatant & $1.70 \mathrm{E}+04$ & $1.53 \mathrm{E}+03$ & $5.15 \mathrm{E}+04$ & $2.07 \mathrm{E}+05$ & $1.60 \mathrm{E}+05$ & $1.45 \mathrm{E}+04$ & $7.29 \mathrm{E}-01$ & $1.48 \mathrm{E}-0 \mathrm{l}$ & $1.59 \mathrm{E}-02$ & 7.75E-02 & $2.62 \mathrm{E}-03$ & $5.94 \mathrm{E}+01$ & 1.33 & 1.33 & 38 & $6.12 \mathrm{E}-01$ & $6.12 \mathrm{E}-01$ & 0 & 0 \\
\hline Solid Layer & $1.70 \mathrm{E}+04$ & $1.53 E+03$ & $5.15 \mathrm{E}+04$ & $2.07 E+05$ & $1.60 \mathrm{E}+05$ & $1.45 \mathrm{E}+04$ & $7.29 \mathrm{E}-01$ & $1.48 \mathrm{E}-01$ & $1.59 \mathrm{E}-02$ & $7.75 \mathrm{E}-02$ & $2.62 \mathrm{E}-03$ & $5.94 \mathrm{E}+01$ & 1.63 & 33 & 2012 & 01 & $6.12 \mathrm{E}-01$ & 21 & 18 \\
\hline $241-\Upsilon X-115$ & $4.81 E-04$ & $4.03 \mathrm{E}+03$ & $1.22 \mathrm{E}+05$ & I. $90 \mathrm{E} \div 05$ & $2.36 \mathrm{E}+05$ & $3.78 \mathrm{E}+04$ & $2.15 E+00$ & $1.60 \mathrm{E}-01$ & $1.67 \mathrm{E}-02$ & $7.97 \mathrm{E}-02$ & $2.80 \mathrm{E}-03$ & $7.13 \mathrm{E}+01$ & 1.63 & 1.45 & 2131 & $2.98 \mathrm{E}-01$ & $4.91 \mathrm{E}-01$ & 22 & 21 \\
\hline Supenlatant & $4.81 \mathrm{E}+04$ & $4.03 E-03$ & $1.22 \mathrm{E}+05$ & $1.90 \mathrm{E}+05$ & $2.36 \mathrm{E}-05$ & $3.78 \mathrm{E}+04$ & $2.15 \mathrm{E}+00$ & $1.60 \mathrm{E}-01$ & $1.67 \mathrm{E}-02$ & $7.97 \mathrm{E}-02$ & $2.80 \mathrm{E}-03$ & $7.13 \mathrm{E}+01$ & 1.45 & 1.45 & 38 & 4.91E-01 & $4.91 \mathrm{E}-01$ & 0 & 0 \\
\hline Solid Layer & $4.81 \mathrm{E}+04$ & $4.03 E+03$ & $1.22 E+05$ & $1.90 \mathrm{~F}+05$ & $2.36 \mathrm{E}+05$ & $3.78 \mathrm{E}+04$ & $2.15 \mathrm{E}+00$ & $1.60 \mathrm{E}-01$ & $1.67 \mathrm{E}-02$ & 7.97E-02 & $2.80 \mathrm{E}-03$ & $7.13 \mathrm{E}+01$ & 1.63 & 1.45 & 2093 & $2.95 \mathrm{E}-01$ & $4.91 \mathrm{E}-01$ & 22 & 21 \\
\hline $24 !-T X-116$ & $2.54 \mathrm{E}+03$ & $3.19 \mathrm{E}+02$ & $1.05 \mathrm{E} \cdots 04$ & $3.79 \mathrm{E}+05$ & $1.51 E+05$ & $8.79 \mathrm{E}+00$ & $3.42 \mathrm{E}-0 \mathrm{l}$ & $6.88 \mathrm{E}-02$ & $9.33 \mathrm{E}-03$ & $5.34 \mathrm{E}-02$ & $1.34 \mathrm{E}-03$ & $3.51 E+01$ & 1.65 & 1.39 & 2302 & $2.25 \mathrm{E}-01$ & $5.30 \mathrm{E}-01$ & 21 & 20 \\
\hline Supernatant & $2.54 \mathrm{E}+03$ & $E+02$ & $1.05 \mathrm{E} \cdot 04$ & $3.79 \mathrm{E}+05$ & 1.511 & $E+00$ & 3. & 6. & $9.33 \mathrm{E}-03$ & 5.3 & $1.34 \mathrm{E}-03$ & $3.51 E \div 01$ & 1.39 & 1.39 & 38 & $5.30 \mathrm{E}-01$ & $5.30 \mathrm{E}-01$ & 0 & 0 \\
\hline Solid Layer & $2.54 \mathrm{E}+03$ & $3.19 \mathrm{E}+02$ & $1.05 E+04$ & $3.79 \mathrm{E}+05$ & $1.5 \mathrm{JE}+05$ & $8.79 \mathrm{E}+00$ & $3.42 \mathrm{E}-01$ & $6.88 \mathrm{E}-02$ & $9.33 \mathrm{E}-03$ & $5.34 E-02$ & $1.34 \mathrm{E}-03$ & $3.51 \mathrm{E}+01$ & 1.66 & 1.39 & 2264 & $2.21 \mathrm{E}-01$ & $5.30 E-01$ & 21 & 20 \\
\hline
\end{tabular}


Table B-5. Input Data With Water Addition for Hydrogen Generation Rate Model Calculations for 177 Tanks. (20 sheets)

\begin{tabular}{|c|c|c|c|c|c|c|c|c|c|c|c|c|c|c|c|c|c|c|c|}
\hline Tank & $\begin{array}{c}\mathrm{OH} \\
\text { in liquid } \\
|\mathrm{OH}| \\
(\mu \mathrm{g} / \mathrm{mL})\end{array}$ & $\begin{array}{c}\text { TOC } \\
\text { in liquid } \\
\text { [TOC.] } \\
(\mu \mathrm{g} / \mathrm{mL})\end{array}$ & $\begin{array}{c}\mathrm{NO}_{2} \\
\text { in liquid } \\
{\left[\mathrm{NO}_{2}\right]} \\
(\mu \mathrm{g} / \mathrm{mL})\end{array}$ & $\begin{array}{c}\mathrm{NO}_{3} \\
\text { in liquid } \\
\left|\mathrm{NO}_{3}\right| \\
(\mu \mathrm{g} / \mathrm{mL})\end{array}$ & $\begin{array}{c}\mathrm{Na} \\
\text { in liquid } \\
|\mathrm{Na}| \\
\text { (ug/mL) }\end{array}$ & $\begin{array}{c}A \mid \\
\text { in liquid } \\
|A| \mid \\
(\mu \mathrm{g} / \mathrm{mL})\end{array}$ & $\begin{array}{c}90 \mathrm{Sr} \\
\text { in waste } \\
|\mathrm{Sr}| \\
(\mu \mathrm{Ci} / \mathrm{g})\end{array}$ & $\begin{array}{c}{ }^{241} \mathrm{Am} \\
\text { in waste } \\
|\mathrm{Am} 241| \\
(\mu \mathrm{Ci} / \mathrm{g})\end{array}$ & $\begin{array}{c}{ }^{240} \mathrm{Pu} \\
\text { in waste } \\
{[\mathrm{Pu} 240 \mid} \\
(\mu \mathrm{C} / \mathrm{g})\end{array}$ & $\begin{array}{c}{ }^{23.9} \mathrm{Pu} \\
\text { in waste } \\
|\mathrm{Pu} 240| \\
(\mu \mathrm{C} i \mathrm{~g})\end{array}$ & $\begin{array}{c}{ }^{238} \mathrm{Pu} \\
\text { in waste } \\
{[\mathrm{Pu} 238 \mid} \\
(\mu \mathrm{Ci} / \mathrm{g})\end{array}$ & $\begin{array}{c}{ }^{137} \mathrm{Cs} \\
\text { in waste } \\
{[\mathrm{Cs}]} \\
(\mu \mathrm{Ci} / \mathrm{g})\end{array}$ & $\begin{array}{c}\text { Bulk } \\
\text { density } \\
\text { D } \\
(\mathrm{g} / \mathrm{mL})\end{array}$ & $\mid \begin{array}{c}\text { Liquid } \\
\text { density } D_{\perp} \\
\text { (g/ml) }\end{array}$ & $\begin{array}{c}\text { Non-RGS } \\
\text { waste } \\
\text { volume } \\
(\mathrm{kL})\end{array}$ & $\begin{array}{l}\text { Bulk } \\
\text { water } \\
\left\{\mathrm{H}_{2} \mathrm{O} \mid\right. \\
(\mathrm{wt} \%(1)\end{array}$ & $\begin{array}{l}\text { Liquid } \\
\text { water } \\
\left\{\mathrm{H}_{2} \mathrm{O} \mid\right. \\
(\mathbf{w t} \% \text { u }\end{array}$ & $\begin{array}{c}\text { Waste } \\
\text { temp. } \\
\mathrm{Tw} \\
\left({ }^{\mathrm{a}} \mathrm{C}\right)\end{array}$ & $\begin{array}{c}\text { Dome } \\
\text { temp. } \\
\text { Td } \\
\left({ }^{\circ} \mathrm{C}\right)\end{array}$ \\
\hline $241-\mathrm{TX}-117$ & $0 \mathrm{E}+04$ & $3.38 \mathrm{E}+00$ & $8.50 \mathrm{E}+03$ & $2.17 \mathrm{E}+05$ & $1.13 \mathrm{E}+05$ & $3.60 \mathrm{E}+02$ & $5.08 \mathrm{E}-01$ & $1.00 \mathrm{E}-01$ & $1.20 \mathrm{E}-02$ & $6.32 \mathrm{E}-02$ & $1.85 \mathrm{E}-03$ & $3.89 \mathrm{E}+01$ & 1.57 & 1.26 & 1855 & $2.77 \mathrm{E}-01$ & $6.96 \mathrm{E}-01$ & 21 & 20 \\
\hline Supernatant & $1.70 \mathrm{E} \div 04$ & $38 \mathrm{E}+00$ & $8.50 \mathrm{E}+03$ & $2.17 \mathrm{E}+05$ & $1.13 E+05$ & $3.60 \mathrm{E}+02$ & $5.08 \mathrm{E}-01$ & $1.00 \mathrm{E}-01$ & $1.20 \mathrm{E}-02$ & $6.32 \mathrm{E}-02$ & $1.85 \mathrm{E}-03$ & $3.89 \mathrm{E}+01$ & 1.26 & 1.26 & 38 & $6.96 \mathrm{E}-01$ & $6.96 \mathrm{E}-0 \mathrm{I}$ & 0 & 0 \\
\hline Solid Layer & $1.70 \mathrm{E}+04$ & $3.38 \mathrm{E}+00$ & $8.50 \mathrm{E}+03$ & $2.17 \mathrm{E}-05$ & $1.13 E \div 05$ & $3.60 \mathrm{E}+02$ & $5.08 \mathrm{E}-01$ & $1.00 \mathrm{E}-01$ & $1.20 \mathrm{E}-02$ & $6.32 \mathrm{E}-02$ & $1.85 \mathrm{E}-03$ & $3.89 \mathrm{E}+01$ & 1.58 & 1.26 & 1817 & $2.70 \mathrm{E}-01$ & $6.96 \mathrm{E}-01$ & 21 & 20 \\
\hline 241-TX-118 & $81 \mathrm{E}+04$ & $4.03 \mathrm{~F} \div .03$ & $1.22 \mathrm{E}+05$ & $1.90 \mathrm{E}+05$ & $2.36 \mathrm{E}+05$ & $3.78 \mathrm{E}+0.4$ & $1.21 \mathrm{E}+02$ & $3.62 \mathrm{E}+00$ & $4.93 \mathrm{E}-01$ & $2.34 \mathrm{E}+00$ & $8.26 \mathrm{E}-02$ & $5.39 \mathrm{E}+01$ & 1.68 & 1.45 & 973 & $4.48 \mathrm{E}-01$ & 4.91E-01 & 25 & 24 \\
\hline Supernatant & $4.81 E+04$ & $4.03 \mathrm{E}+03$ & $1.22 \mathrm{E}+05$ & $1.90 \mathrm{E}+05$ & $2.36 \mathrm{E}+05$ & $3.78 \mathrm{E}+04$ & $1.21 \mathrm{E}+02$ & $3.62 \mathrm{E}+00$ & $4.93 E-01$ & $2.34 \mathrm{E}+00$ & $8.26 \mathrm{E}-02$ & $5.39 \mathrm{E}+01$ & 1.45 & 1.45 & 38 & $4.91 \mathrm{E}-01$ & $4.91 \mathrm{E}-01$ & 0 & 0 \\
\hline Solid Layer & $4.81 \mathrm{E}+04$ & $4.03 E+03$ & $1.22 \mathrm{E} \cdot 05$ & $1.90 \mathrm{E}+05$ & $2.36 \mathrm{E}+05$ & $3.78 \mathrm{E}+04$ & $1.21 E+02$ & $3.62 \mathrm{E}+00$ & $4.93 \mathrm{E}-01$ & $2.34 \mathrm{E}+00$ & $8.26 \mathrm{E}-02$ & $5.39 \mathrm{E}+01$ & 1.69 & 1.45 & 935 & $4.47 \mathrm{E}-01$ & $4.91 \mathrm{E}-01$ & 25 & 24 \\
\hline $241-\mathrm{TY}-101$ & $1.70 \mathrm{E}+04$ & $3.38 \mathrm{E}+00$ & $8.47 \mathrm{E} \div 03$ & $2.17 \mathrm{E}+05$ & $1.13 \mathrm{E}+05$ & $3.61 \mathrm{E} \div 02$ & $9.53 \mathrm{E}-00$ & $1.26 \mathrm{E}-02$ & $.44 \mathrm{E}-02$ & $1.72 E-01$ & $8.48 \mathrm{E}-04$ & $4.04 \mathrm{E}-01$ & 1.60 & 1.26 & 485 & $4.57 \mathrm{E}-01$ & $6.96 \mathrm{E}-01$ & 18 & 17 \\
\hline Supernatant & $1.70 \mathrm{E}+04$ & $3.38 \mathrm{E}+00$ & $8.47 E+03$ & $2.17 \mathrm{E}+05$ & $1.13 \mathrm{E}+05$ & $3.61 \mathrm{E}+02$ & $9.53 \mathrm{E}+00$ & $1.26 \mathrm{E}-02$ & $1.44 \mathrm{E}-02$ & $1.72 \mathrm{E}-01$ & $8.48 \mathrm{E}-04$ & 4.04E-01 & 1.26 & 1.26 & 38 & $6.96 \mathrm{E}-01$ & $6.96 \mathrm{E}-01$ & 0 & 0 \\
\hline Solid Layer & $1.70 \mathrm{E}+04$ & $3.38 \mathrm{E}+00$ & $8.47 \mathrm{E}+03$ & $2.17 E+05$ & $1.13 \mathrm{E}+05$ & $3.61 E+02$ & $9.53 \mathrm{E}+00$ & $1.26 \mathrm{E}-02$ & $1.44 \mathrm{E}-02$ & $1.72 \mathrm{E}-01$ & $8.48 \mathrm{E}-04$ & $4.04 \mathrm{E}-01$ & 1.63 & 1.26 & 447 & $4.42 \mathrm{E}-01$ & $6.96 \mathrm{E}-01$ & 18 & 17 \\
\hline $24 \mathrm{t}-\mathrm{TY}-102$ & $4.82 \mathrm{E}+04$ & $2.18 \mathrm{E} \div 03$ & $6.98 \mathrm{E}+04$ & $2.02 \mathrm{E}+05$ & $1.79 E+05$ & $2.06 \mathrm{E}+04$ & $2.62 E+00$ & $2.50 \mathrm{E}-03$ & $2.02 \mathrm{E}-04$ & $1.33 \mathrm{E}-03$ & 2.46E-05 & $2.87 \mathrm{E}+01$ & 1.71 & 1.36 & 300 & $3.43 \mathrm{E}-01$ & $5.78 \mathrm{E}-01$ & 16 & 19 \\
\hline Supernatan & $4.82 E+04$ & $2.18 E+03$ & $6.98 \mathrm{E}+0.4$ & $2.02 \mathrm{E}+05$ & $1.79 \mathrm{E}+05$ & $2.06 \mathrm{E}-04$ & $2.62 \mathrm{E}+00$ & $2.50 \mathrm{E}-03$ & $2.02 \mathrm{E}-04$ & $1.33 E-03$ & $2.46 \mathrm{E}-05$ & $2.87 E+01$ & 1.36 & 1.36 & 38 & $5.78 \mathrm{E}-0 \mathrm{I}$ & $5.78 \mathrm{E}-01$ & 0 & 0 \\
\hline Solid Layer & $4.82 \mathrm{E} \div 04$ & $2.18 \mathrm{E}+03$ & $6.98 E+04$ & $2.02 E+05$ & $1.79 \mathrm{E}+05$ & $2.06 \mathrm{E}+04$ & $2.62 \mathrm{E}+00$ & $2.50 \mathrm{E}-03$ & $2.02 \mathrm{E}-04$ & $1.33 \mathrm{E}-03$ & $2.46 \mathrm{~F}-05$ & $2.87 \mathrm{E}+01$ & 1.76 & 1.36 & 262 & $3.17 \mathrm{E}-01$ & $5.78 \mathrm{E}-01$ & 16 & 19 \\
\hline $241-T Y-103$ & $4.80 \mathrm{E}+04$ & $4.02 \mathrm{E}+03$ & $1.22 \mathrm{E}+05$ & $1.90 \mathrm{E}+05$ & $2.36 \mathrm{E}+05$ & $3.78 \mathrm{E}+04$ & $7.00 \mathrm{E}+01$ & $2.42 \mathrm{E}-02$ & $1.93 E-02$ & $1.60 \mathrm{E}-01$ & $2.10 \mathrm{E}-03$ & $2.78 \mathrm{E}+01$ & 1.67 & 1.45 & 623 & $5.16 \mathrm{E}-01$ & 4.91E-01 & 22 & 22 \\
\hline Supernatant & $4.80 E+04$ & $4.02 \mathrm{E}+03$ & $1.22 \mathrm{E}+05$ & $1.90 \mathrm{E}+05$ & $2.36 \mathrm{E}+05$ & $3.78 \mathrm{E} \div 04$ & $7.00 \mathrm{E}+01$ & $2.42 \mathrm{E}-02$ & $1.93 \mathrm{E}-02$ & 1.60F-01 & $2.10 \mathrm{E}-03$ & $2.78 E+01$ & 1.45 & 1.45 & 38 & $4.91 E-01$ & 4.91E-01 & 0 & 0 \\
\hline Solid Layer & $4.80 \mathrm{E}+04$ & $4.02 E+03$ & $1.22 \mathrm{E}+05$ & $1.90 \mathrm{E}+05$ & $2.36 \mathrm{E}+05$ & $3.78 \mathrm{E}+04$ & $7.00 \mathrm{E}+0 \mathrm{I}$ & $2.42 \mathrm{E}-02$ & 1.93F-02 & $1.60 \mathrm{E}-01$ & $2.10 \mathrm{E}-03$ & $2.78 \mathrm{E}+01$ & 1.68 & 1.45 & 585 & $5.17 E-01$ & $4.91 \mathrm{E}-01$ & 22 & 22 \\
\hline $241-T Y-104$ & $1.37 \mathrm{E}-04$ & $1.71 \mathrm{E}+03$ & $9.11 E+03$ & $7.98 \mathrm{E}+04$ & $6.73 E+04$ & $4.60 \mathrm{E}+02$ & $7.46 \mathrm{E}+01$ & $6.86 \mathrm{~F}-02$ & $1.17 \mathrm{E}-02$ & {$[.48 \mathrm{E}-01$} & $6.74 \mathrm{E}-04$ & $3.04 \mathrm{E}+01$ & 1.52 & 1.15 & 206 & $5.82 \mathrm{E}-01$ & $8.02 \mathrm{E}-01$ & 20 & 21 \\
\hline Supernatant & $1.98 \mathrm{E}+03$ & $2.47 \mathrm{E}+02$ & $1.32 \mathrm{E}+03$ & $1.16 \mathrm{E}+04$ & $9.73 E \div 03$ & $6.65 \mathrm{E}+01$ & $6.90 \mathrm{E}-03$ & $4.94 \mathrm{E}-05$ & $3.57 \mathrm{E}-0.5$ & $1.49 \mathrm{E}-04$ & 8.46E-06 & $1.3 \mathrm{JE}+00$ & 1.02 & 1.02 & 43 & $9.70 \mathrm{E}-01$ & $9.70 \mathrm{E}-01$ & 20 & 21 \\
\hline Solid Layer & $1.70 \mathrm{E}+04$ & $2.12 E+03$ & $1.13 E+04$ & $9.90 \mathrm{E}+04$ & $8.34 \mathrm{E}+04$ & $5.70 \mathrm{~F}+02$ & $8.67 \mathrm{E} \div 01$ & $7.98 \mathrm{E}-02$ & $1.36 \mathrm{E}-02$ & $1.72 \mathrm{E}-01$ & $7.83 \mathrm{E}-04$ & $3.51 E+01$ & 1.65 & 1.18 & 163 & $5.19 E-01$ & $7.75 \mathrm{~F}-01$ & 20 & 21 \\
\hline 24\}-TY-105 & $1.70 \mathrm{E}-04$ & $2.98 \mathrm{E}+03$ & $3.60 \mathrm{E} \cdot 04$ & $7.96 E+04$ & $8.32 \mathrm{E}+04$ & $4.94 E+03$ & $1.48 \mathrm{E}+02$ & 7.55E-03 & $2.27 \mathrm{E}-03$ & $2.12 \mathrm{E}-02$ & $1.47 \mathrm{E}-04$ & $6.24 \mathrm{E}+00$ & 1.52 & 1.17 & 912 & 4.05E-01 & $7.50 \mathrm{E}-0 \mathrm{~J}$ & 25 & 23 \\
\hline Supeniatant & $1.70 \mathrm{E}+04$ & $2.98 \mathrm{E} \div 03$ & $3.60 \mathrm{E}+04$ & $7.96 \mathrm{E}+04$ & $8.32 E+04$ & $4.94 \mathrm{E}+03$ & $1.48 \mathrm{E}+02$ & $7.55 \mathrm{E}-03$ & $2.27 \mathrm{E}-03$ & $2.12 \mathrm{E}-02$ & 1.47E-04 & $6.24 \mathrm{E}+00$ & 1.17 & 1.17 & 38 & $7.50 \mathrm{E}-01$ & $7.50 \mathrm{E}-01$ & 0 & 0 \\
\hline Solid Layer & $1.70 \mathrm{E}+04$ & $2.98 \mathrm{E}+03$ & $3.60 \mathrm{E}+04$ & $7.96 \mathrm{E}+04$ & $8.32 \mathrm{E}+04$ & $4.94 \mathrm{E}^{+0} 03$ & $1.48 E+02$ & $7.55 \mathrm{E}-03$ & $2.27 \mathrm{E}-03$ & 2.12E-02 & $1.47 \mathrm{E}-04$ & $6.24 \mathrm{E}+00$ & 1.53 & 1.17 & 874 & $3.94 \mathrm{E}-01$ & $7.50 \mathrm{E}-01$ & 25 & 23 \\
\hline 241-TY-106 & $1.70 \mathrm{E}+04$ & $2.98 \mathrm{E}+03$ & $3.60 E+04$ & $7.96 \mathrm{E}+04$ & $8.32 \mathrm{E}+04$ & $4.94 \mathrm{E}+03$ & $1.21 \mathrm{E}+02$ & 1.66F-02 & $2.04 \mathrm{E}-03$ & $1.90 \mathrm{E}-02$ & $1.32 \mathrm{E}-04$ & $5.66 \mathrm{E}+01$ & 1.31 & 1.17 & 100 & $5.06 \mathrm{E}-01$ & $7.50 \mathrm{E}-01$ & 16 & 20 \\
\hline Supernatant & $1.70 \mathrm{E}+04$ & $2.98 E+03$ & $3.60 \mathrm{E}-04$ & $7.96 \mathrm{E} \div 04$ & $8.32 E+04$ & $4.94 \mathrm{E}+03$ & $1.21 \mathrm{E}+02$ & $1.66 \mathrm{E}-02$ & $2.04 \mathrm{E}-03$ & $1.90 \mathrm{E}-02$ & $1.32 \mathrm{E}-04$ & $5.66 \mathrm{E}+01$ & 1.17 & 1.17 & 38 & $7.50 \mathrm{E}-01$ & $7.50 \mathrm{E}-01$ & 0 & 0 \\
\hline Solid Layer & $1.70 \mathrm{E}+04$ & $2.98 \mathrm{E}-03$ & $3.60 \mathrm{E}+04$ & $7.96 \mathrm{E}+04$ & $8.32 E-04$ & $4.94 \mathrm{E}+03$ & $1.21 \mathrm{E}+02$ & $1.66 \mathrm{E}-02$ & $2.04 \mathrm{E}-03$ & $1.90 \mathrm{E}-02$ & 1.32E-04 & $5.66 \mathrm{E}+01$ & 1.40 & 1.17 & 62 & $3.81 \mathrm{E}-0 \mathrm{~J}$ & $7.50 \mathrm{E}-01$ & 16 & 20 \\
\hline $241-\mathrm{U}-101$ & $4.91 E+04$ & $2.98 E+03$ & $3.60 \mathrm{E}+04$ & $7.96 \mathrm{E}+04$ & $8.32 \mathrm{E}+04$ & $4.94 \mathrm{E}+03$ & $3.71 \mathrm{E}, 02$ & $2.05 \mathrm{E}-02$ & $7.86 \mathrm{E}-03$ & $4.06 \mathrm{E}-02$ & $7.53 \mathrm{E}-04$ & $5.73 E+01$ & 1.59 & 1.17 & 125 & $4.01 \mathrm{E}-01$ & $7.60 \mathrm{E}-01$ & 22 & $2 !$ \\
\hline Supeniatant & $4.91 \mathrm{E}-04$ & $2.98 \mathrm{E}+03$ & $3.60 \mathrm{E}-04$ & $7.96 \mathrm{E} \div 04$ & $8.32 \mathrm{E}+04$ & $4.94 \mathrm{E}+03$ & $3.71 \mathrm{E}+02$ & 2.05E-02 & $7.86 \mathrm{E}-03$ & $4.06 \mathrm{E}-02$ & $7.53 E-04$ & $5.73 E+01$ & 1.17 & 1.17 & 38 & $7.60 \mathrm{E}-01$ & $7.60 \mathrm{E}-01$ & 0 & 0 \\
\hline Solid Layef & $4.91 \mathrm{E}+04$ & $2.98 \mathrm{E}+03$ & $3.60 \mathrm{E}+04$ & $7.96 \mathrm{E}+04$ & $8.32 E+04$ & $4.94 E+03$ & $3.71 E+02$ & $2.05 \mathrm{E}-02$ & $7.86 \mathrm{E}-03$ & $4.06 \mathrm{E}-02$ & $7.53 \mathrm{E}-04$ & $5.73 \mathrm{E}+01$ & 1.77 & 1.17 & 87 & $2.98 \mathrm{E}-01$ & $7.60 \mathrm{E}-0 \mathrm{~J}$ & 22 & 21 \\
\hline
\end{tabular}

\section{B-100}




\section{RPP-5926 REV 6}

Table B-5. Input Data With Water Addition for Hydrogen Generation Rate Model Calculations for 177 Tanks. (20 sheets)

\begin{tabular}{|c|c|c|c|c|c|c|c|c|c|c|c|c|c|c|c|c|c|c|c|}
\hline Tank & $\begin{array}{c}\mathrm{OH} \\
\text { in liquid } \\
|\mathrm{OH}| \\
(\mu \mathrm{g} / \mathrm{mL})\end{array}$ & $\begin{array}{c}\text { TOC } \\
\text { in liquid } \\
\text { |TOC] } \\
(\mu \mathrm{g} / \mathrm{mL})\end{array}$ & $\begin{array}{c}\mathrm{NO}_{2} \\
\text { in liquid } \\
{\left[\mathrm{NO}_{2}\right]} \\
(\mu \mathrm{g} / \mathrm{mL})\end{array}$ & $\begin{array}{c}\mathrm{NO}_{3} \\
\text { in liquid } \\
\left|\mathrm{NO} O_{3}\right| \\
(\mu \mathrm{g} / \mathrm{mL})\end{array}$ & $\begin{array}{c}\mathrm{Na} \\
\text { in liquid } \\
\text { [Na] } \\
\text { (ug/ml })\end{array}$ & $\begin{array}{c}\mathrm{Al} \\
\text { in liquid } \\
\mid \mathrm{Al}] \\
(\mu \mathrm{g} / \mathrm{mL})\end{array}$ & $\begin{array}{c}90 \mathrm{Sr} \\
\text { in waste } \\
{[\mathrm{Sr}]} \\
(\mu \mathrm{Ci} / \mathrm{g})\end{array}$ & $\begin{array}{c}{ }^{241} \mathrm{Am} \\
\text { in waste } \\
\text { |Am241] } \\
(\mu \mathrm{Ci} / \mathrm{g})\end{array}$ & $\begin{array}{c}{ }^{240} \mathrm{Pu} \\
\text { in waste } \\
{[\mathrm{Pu} 240]} \\
(\mu \mathrm{Ci} / \mathrm{g})\end{array}$ & $\begin{array}{c}{ }^{239} \mathrm{Pu} \\
\text { in waste } \\
|\mathrm{Pu} 240| \\
(\mu \mathrm{Ci} / \mathrm{g})\end{array}$ & $\begin{array}{c}{ }_{238}^{23 u} \\
\text { in waste } \\
{[\mathrm{Pu} 238]} \\
(\mu \mathrm{Ci} / \mathrm{g})\end{array}$ & $\begin{array}{c}{ }^{137} \mathrm{Cs} \\
\text { in waste } \\
|\mathrm{Cs}| \\
(\mu \mathrm{Ci} / \mathrm{g})\end{array}$ & $\begin{array}{c}\text { Bulk } \\
\text { density } \\
\text { D } \\
(\mathrm{g} / \mathrm{mL})\end{array}$ & $\mid \begin{array}{c}\text { Liquid } \\
\text { density } \mathrm{D}_{\ell} \\
\text { (g/ml) }\end{array}$ & $\begin{array}{c}\text { Non-RGS } \\
\text { waste } \\
\text { volume } \\
(\mathrm{kL})\end{array}$ & $\begin{array}{l}\text { Bulk } \\
\text { water } \\
{\left[\mathrm{H}_{2} \mathrm{O}\right]} \\
(\mathrm{wt} \% \text { ) }\end{array}$ & $\begin{array}{l}\text { Liquid } \\
\text { water } \\
\left\{\mathrm{H}_{2} \mathrm{O} \mid\right. \\
(\mathrm{wt} \%)\end{array}$ & $\begin{array}{c}\text { Waste } \\
\text { temp. } \\
\mathrm{Tw} \\
\left.{ }^{\circ} \mathrm{C}\right)\end{array}$ & $\begin{array}{l}\text { Dome } \\
\text { temp. } \\
\text { Td } \\
\left({ }^{\circ} \mathrm{C}\right)\end{array}$ \\
\hline $241-L-102$ & $33 E+04$ & $08 \mathrm{E}+04$ & $1.41 E+05$ & $1.91 E+05$ & 2.15E+05 & $3.20 \mathrm{E} \div 04$ & $8.28 \mathrm{E} \div 01$ & $1.16 \mathrm{E}-01$ & $2.25 \mathrm{E}-02$ & 1.12E-01 & $2.81 \mathrm{E}-03$ & $1.38 \mathrm{E}+02$ & 1.65 & 1.46 & 1276 & $3.53 \mathrm{E}-01$ & $4.84 \mathrm{E}-01$ & 26 & 28 \\
\hline Supematant & $2.80 \mathrm{E}+03$ & $1.07 \mathrm{E}: 03$ & $1.40 E+04$ & $1.89 \mathrm{E}+04$ & $2.13 \mathrm{E}+04$ & $3.18 \mathrm{E}+03$ & $6.15 \mathrm{E}-01$ & $1.06 E-03$ & $8.91 \mathrm{E}-06$ & 4.25E-05 & $1.50 \mathrm{E}-06$ & $4.11 \mathrm{E}+01$ & 1.05 & 1.05 & 42 & $9.29 \mathrm{E}-01$ & $9.29 \mathrm{E}-01$ & 21 & 28 \\
\hline Solid Layer & $2.93 \mathrm{E}+04$ & $1.12 \mathrm{E}+04$ & $1.46 \mathrm{E}-05$ & $1.98 \mathrm{E}+05$ & $2.23 \mathrm{E}+05$ & $3.32 \mathrm{E}+04$ & $8.45 E+01$ & $1.18 \mathrm{E}-01$ & $2.30 \mathrm{E}-02$ & $1.14 \mathrm{E}-01$ & $2.87 \mathrm{E}-03$ & $1.40 \mathrm{E}+02$ & 1.67 & 1.48 & 1234 & $3.41 \mathrm{E}-01$ & $4.75 \mathrm{E}-01$ & 26 & 28 \\
\hline $241-\mathrm{L}-103$ & $3.00 \mathrm{E}+04$ & $1.21 \mathrm{E}+04$ & $1.33 E+05$ & $1.48 E+05$ & $2.24 E+05$ & $3.98 \mathrm{E}+04$ & $2.25 \mathrm{E}+01$ & $8.40 \mathrm{E}-02$ & $8.91 E-03$ & $4.24 \mathrm{~F}-02$ & $1.29 \mathrm{E}-03$ & $1.09 E+02$ & 1.68 & 1.43 & 1448 & $4.05 \mathrm{E}-01$ & $5.07 \mathrm{E}-01$ & 27 & 25 \\
\hline Supernatant & $1.55 \mathrm{E}+03$ & $6.22 \mathrm{E} \div 02$ & $6.88 \mathrm{E}+03$ & $7.63 \mathrm{E}+03$ & $1.16 \mathrm{E}+04$ & $2.05 E+03$ & $4.59 \mathrm{E}-01$ & $9.08 \mathrm{E}-04$ & $4.22 \mathrm{E}-06$ & $1.93 \mathrm{E}-05$ & $6.82 \mathrm{E}-07$ & $2.19 \mathrm{E}+01$ & 1.02 & 1.02 & 40 & $9.65 E-01$ & $9.65 \mathrm{E}-01$ & 27 & 25 \\
\hline Solid Layer & $3.08 \mathrm{E}+04$ & $1.24 \mathrm{E}+04$ & $1.37 \mathrm{E}+05$ & $1.52 \mathrm{E}+05$ & $2.30 \mathrm{E}+05$ & $4.09 \mathrm{E}+04$ & $2.28 \mathrm{E}+01$ & $8.54 \mathrm{E}-02$ & $9.07 \mathrm{E}-03$ & $4.31 \mathrm{E}-02$ & $1.31 \mathrm{E}-03$ & $1.10 \mathrm{E}+02$ & 1.70 & 1.44 & 1408 & $3.95 \mathrm{E}-0\}$ & $4.99 \mathrm{E}-01$ & 27 & 25 \\
\hline $241-U-104$ & $4.91 \mathrm{E}+04$ & $9.58 \mathrm{E}+03$ & $1.05 E+05$ & $2.07 E+05$ & $2.03 E-05$ & $3.21 \mathrm{E}: 04$ & $2.52 \mathrm{E}+00$ & $1.27 \mathrm{E}-03$ & $6.00 \mathrm{E}-04$ & $4.74 \mathrm{E}-03$ & $4.10 \mathrm{E}-05$ & $2.21 \mathrm{E}-01$ & 1.17 & 1.40 & 243 & $4.48 E-01$ & $5.50 \mathrm{E}-01$ & 28 & 24 \\
\hline Supernatant & $4.91 \mathrm{E}+04$ & $9.58 \mathrm{E}+03$ & $1.05 \mathrm{E}+05$ & $2.07 \mathrm{E}+05$ & $2.03 \mathrm{E}+05$ & $3.21 \mathrm{E}+04$ & $2.52 \mathrm{E}+00$ & $1: 27 E-03$ & $6.00 \mathrm{E}-04$ & $4.74 \mathrm{E}-03$ & 4. $30 \mathrm{E}-05$ & $2.21 \mathrm{~F}-01$ & 1.40 & 1.40 & 38 & $5.50 \mathrm{E}-01$ & $5.50 \mathrm{E}-0 \mathrm{1}$ & 0 & 0 \\
\hline Solid Layer & $4.91 E \div 04$ & $9.58 \mathrm{E}+03$ & $1.05 \mathrm{E}+05$ & $2.07 \mathrm{E}+05$ & $2.03 E+05$ & $3.21 E+04$ & $2.52 \mathrm{E}+00$ & $1.27 \mathrm{E}-03$ & $6.00 \mathrm{E}-04$ & $4.74 \mathrm{E}-03$ & 4.10E-05 & $2.21 \mathrm{E}-0 \mathrm{I}$ & 1.12 & 1.40 & 205 & 4.24E-01 & $5.50 \mathrm{E}-01$ & 28 & 24 \\
\hline $241-U \cdot 105$ & $2.09 \mathrm{E} \div 04$ & $64 E+04$ & $1.08 \mathrm{E}+05$ & $1.81 E+05$ & $2.27 \mathrm{E}+05$ & $2.985 \div 04$ & $1.14 E+0 !$ & $3.63 \mathrm{E}-01$ & $5.43 \mathrm{E}-02$ & $2.59 \mathrm{E}-01$ & $7.94 \mathrm{E}-03$ & $1.37 \mathrm{E}+02$ & 1.66 & 1.46 & 1374 & $3.09 E-01$ & $5.16 \mathrm{E}-01$ & 28 & 26 \\
\hline Supernatant & $2.09 \mathrm{E} \div 04$ & $1.64 \mathrm{E} \div 04$ & $1.08 \mathrm{E}+0.5$ & $1.81 \mathrm{E} \cdot 05$ & $2.27 \mathrm{E}+05$ & $2.98 \mathrm{E}+04$ & $1.14 \mathrm{E}+01$ & $63 E-$ & $5.41 \mathrm{E}-02$ & $2.59 \mathrm{E}-\mathrm{C}$ & $7.94 \mathrm{E}-03$ & $1.37 \mathrm{E}+02$ & 1.46 & 1.46 & 38 & $5.16 \mathrm{E}-0 \mathrm{~J}$ & $5.16 E-01$ & 0 & 0 \\
\hline Solid Layer & $2.09 E \div 04$ & $1.64 \mathrm{E}+04$ & $1.08 \mathrm{E}+05$ & $1.81 E+05$ & $2.27 \mathrm{E}+05$ & $2.98 \mathrm{E}+04$ & $1.14 \mathrm{E} \vdash 01$ & $3.63 \mathrm{E}-01$ & $5.41 \mathrm{E}-02$ & $2.59 \mathrm{~F}-01$ & $7.94 \mathrm{E}-03$ & $1.37 \mathrm{E}+02$ & 1.67 & 1.46 & 1336 & 3.04E-01 & $5.16 \mathrm{E}-01$ & 28 & 26 \\
\hline $241-\mathrm{U}-106$ & $4.82 \mathrm{E}+03$ & $9 E+04$ & $9.32 E+04$ & $2.35 E+05$ & $2.10 \mathrm{E}+05$ & $1.13 E+04$ & $5.71 E+01$ & $6.56 \mathrm{E}-01$ & $4.16 \mathrm{E}-02$ & $1.91 \mathrm{E}-01$ & $6.72 \mathrm{E}-03$ & $1.40 \mathrm{E}+02$ & 1.52 & 1.32 & 682 & $4.38 \mathrm{E}-01$ & $5.05 \mathrm{E}-01$ & 26 & 25 \\
\hline Supematant & $6.99 \mathrm{E}+02$ & $4.63 \mathrm{E}+03$ & $1.35 \mathrm{E}=04$ & $3.40 \mathrm{E}+04$ & $3.06 \mathrm{E}+04$ & $1.65 \mathrm{E}+03$ & $7.19 \mathrm{E} \div 00$ & $3.79 \mathrm{E}-02$ & $1.61 \mathrm{E}-04$ & $7.39 \mathrm{E}-04$ & $2.59 \mathrm{E}-0.5$ & $3.68 \mathrm{E}+01$ & 1.05 & 1.05 & 44 & $9.10 \mathrm{E}-01$ & $9.10 E-01$ & 24 & 25 \\
\hline Solid layer & $5.11 E+03$ & $3.38 \mathrm{E}+04$ & $9.87 \mathrm{E}+04$ & $2.49 \mathrm{E}+05$ & $2.23 \mathrm{E}+05$ & $1.20 \mathrm{E}+04$ & $5.94 \mathrm{E}+01$ & $6.85 \mathrm{E}-01$ & 4.35E-02 & $2.00 \mathrm{E}-01$ & $7.03 \mathrm{E}-03$ & $1.45 \mathrm{E}+02$ & 1.55 & 1.34 & 638 & 4.16E- 01 & $4.86 \mathrm{E}-01$ & 26 & 25 \\
\hline $24 i-U-107$ & $3.12 \mathrm{E}+04$ & $6.28 \mathrm{E}+03$ & $1.28 \mathrm{E}+05$ & $1.42 E+05$ & $2.05 \mathrm{E}+05$ & $4.10 E+04$ & $2.09 E+00$ & $2.15 \mathrm{E}-01$ & $3.04 \mathrm{E}-02$ & $1.42 \mathrm{E}-01$ & $4.96 \mathrm{E}-03$ & $7.55 \mathrm{E}+01$ & 1.7 & 1.43 & 1151 & $3.31 \mathrm{E}-01$ & $4.96 \mathrm{E}-01$ & 23 & 23 \\
\hline Supernatant & $3.12 \mathrm{E}+04$ & $6.28 \mathrm{E}+03$ & $1.28 \mathrm{E}+05$ & $1.42 E+05$ & $2.05 \mathrm{E}+05$ & $4.10 E-04$ & $2.09 \mathrm{E}+00$ & $2.15 \mathrm{E}-01$ & $3.04 \mathrm{E}-02$ & $1.42 \mathrm{E}-01$ & $4.96 \mathrm{E}-03$ & $7.55 E+01$ & 1.43 & 1.43 & 38 & $4.96 \mathrm{E}-01$ & $4.96 E-01$ & 0 & 0 \\
\hline Solid Layer & 3.12E+04 & $6.28 E+03$ & $1.28 \mathrm{E}+05$ & $1.42 \mathrm{E}+05$ & $2.05 \mathrm{E}+05$ & $4.10 \mathrm{E}+04$ & $2.09 \mathrm{E}: 00$ & $2.15 E-01$ & $3.04 \mathrm{E}-02$ & $1.42 \mathrm{E}-01$ & $4.96 \mathrm{E}-03$ & $7.55 \mathrm{E} .01$ & 1.74 & 1.43 & 1113 & 3.26E-01 & $4.96 \mathrm{E}-01$ & 23 & 23 \\
\hline $241-\mathrm{U}-108$ & $4.91 \mathrm{E}+04$ & $7.07 \mathrm{E}+03$ & $1.34 \mathrm{E}+05$ & $1.84 E+05$ & $2.28 E+05$ & 404 & 7. & $3.43 \mathrm{E}-02$ & $1.43 \mathrm{E}-02$ & $6.53 E-02$ & $1.76 \mathrm{E}-03$ & 22 & 1.6 & 40 & 1679 & $3.71 \mathrm{E}-01$ & 4.99 & 30 & 27 \\
\hline Supernatant & $4.91 \mathrm{E}+04$ & $7.07 \mathrm{E}+03$ & $1.34 \mathrm{E}-05$ & $1.84 E+05$ & $2.28 \mathrm{E}+05$ & $3.05 \mathrm{E} \times 04$ & $7.54 \mathrm{E}+00$ & $3.43 \mathrm{E}-02$ & $1.43 \mathrm{E}-02$ & $6.53 \mathrm{E}-02$ & $1.76 \mathrm{E}-03$ & $1.23 \mathrm{E}+02$ & 1.40 & 1.40 & 38 & $4.99 \mathrm{E}-01$ & $4.995 .-01$ & 0 & 0 \\
\hline Solid !-ayer & $4.91 E+04$ & $7.07 \mathrm{E}+03$ & $1.34 \mathrm{E}+05$ & $1.84 \mathrm{E}+05$ & $2.28 \mathrm{E}+05$ & $3.05 E+04$ & $7.54 \mathrm{E}+00$ & $3.43 \mathrm{E}-02$ & $1.43 E-02$ & $6.53 \mathrm{E}-02$ & $1.76 \mathrm{E}-03$ & $1.23 E+02$ & 1.68 & 1.40 & 1641 & $3.69 \mathrm{E}-01$ & $4.99 \mathrm{E}-01$ & 30 & 27 \\
\hline $241-U-109$ & $4.42 \mathrm{E} \cdot 04$ & $6.53 \mathrm{E}+03$ & $1.28 E+05$ & $1.85 \mathrm{E}+05$ & $2.28 \mathrm{E}+05$ & $2.92 \mathrm{E}+04$ & $6.95 E+00$ & $2.36 \mathrm{E}-02$ & $2.37 \mathrm{E}-03$ & $1.11 \mathrm{E}-02$ & $3.67 \mathrm{E}-04$ & $1.13 \mathrm{E}+02$ & 1.6 & 1.47 & 1390 & $2.93 \mathrm{E}-01$ & $5.07 \mathrm{E}-01$ & 29 & 25 \\
\hline Supernatant & $4.42 \mathrm{E}+04$ & $6.53 \mathrm{E}+03$ & $1.28 \mathrm{E}+05$ & $1.85 E^{*} 05$ & $2.28 \mathrm{E}+05$ & $2.92 \mathrm{E} \cdot 04$ & $6.95 E+00$ & $2.36 \mathrm{E}-02$ & $2.37 \mathrm{E}-03$ & $1.11 \mathrm{E}-02$ & $3.67 \mathrm{E}-04$ & $1.13 \mathrm{E}+02$ & 1.47 & 1.47 & 38 & $5.07 \mathrm{E}-01$ & $5.07 \mathrm{E}-01$ & 0 & 0 \\
\hline Solid Layer & $4.42 E+04$ & $6.53 \mathrm{E}+0.3$ & $1.28 \mathrm{E}+05$ & $1.85 \mathrm{E}+05$ & $2.28 \mathrm{E}+05$ & $2.92 \mathrm{E}+04$ & $6.95 \mathrm{E}+00$ & $2.36 \mathrm{E}-02$ & $2.37 \mathrm{E}-03$ & $1.11 \mathrm{E}-02$ & $3.67 \mathrm{E}-04$ & $1.13 \mathrm{E}+02$ & 1.65 & 1.47 & 1352 & $2.88 \mathrm{~F}-01$ & $5.07 \mathrm{E}-01$ & 29 & 25 \\
\hline $241-\mathrm{U}-110$ & $4.91 E+04$ & $2.98 \mathrm{E}+03$ & $3.60 \mathrm{E}+04$ & $7.96 \mathrm{E}+04$ & $8.32 E+04$ & $4.94 \mathrm{E}+03$ & $1.62 \mathrm{E}+02$ & $7.35 \mathrm{E}-02$ & $3.16 \mathrm{E}-02$ & $1.84 \mathrm{E}-01$ & $2.84 \mathrm{E}-03$ & $1.81 \mathrm{E}+01$ & 1.69 & 1.17 & 703 & $3.55 \mathrm{E}-01$ & $7.50 \mathrm{E}-01$ & 25 & 24 \\
\hline Supematant & $4.91 E+04$ & $2.98 \mathrm{E}+03$ & $3.60 \mathrm{E} \div 04$ & $7.96 \mathrm{t}+04$ & $8.32 \mathrm{E}+04$ & $4.94 \mathrm{E}+03$ & $1.62 E+02$ & $7.35 \mathrm{E}-02$ & $3.16 \mathrm{E}-02$ & $1.84 \mathrm{E}-01$ & $2.84 \mathrm{E}-03$ & $1.81 \mathrm{E}+01$ & 1.17 & 1.17 & 38 & $7.50 \mathrm{E}-01$ & $7.50 \mathrm{E}-01$ & 0 & 0 \\
\hline Solid Layer & $4.91 \mathrm{E}+04$ & $2.98 E+03$ & $3.60 \mathrm{E}+04$ & $7.96 \mathrm{E} \div 04$ & $8.32 \mathrm{E}, 04$ & $4.94 \mathrm{E}+03$ & $1.62 \mathrm{E} \cdot 02$ & $7.35 \mathrm{E} 02$ & $3.16 \mathrm{E}-02$ & $1.84 \mathrm{E}-01$ & $2.84 \mathrm{E}-03$ & $1.81 E \div 01$ & $\$ .72$ & 1.17 & 665 & $3.40 \mathrm{E}-01$ & $7.50 \mathrm{E}-01$ & 25 & 24 \\
\hline
\end{tabular}


RPP-5926 REV 6

Table B-5. Input Data With Water Addition for Hydrogen Generation Rate Model Calculations for 177 Tanks. (20 sheets)

\begin{tabular}{|c|c|c|c|c|c|c|c|c|c|c|c|c|c|c|c|c|c|c|c|}
\hline Tank & $\begin{array}{c}\text { OH } \\
\text { in liquid } \\
\text { |OH| } \\
(\mu \mathrm{g} / \mathrm{mL})\end{array}$ & $\begin{array}{c}\text { TOC } \\
\text { in liquid } \\
\text { [TOC] } \\
(\mu \mathrm{g} / \mathrm{mL})\end{array}$ & $\begin{array}{c}\mathrm{NO}_{2} \\
\text { in liquid } \\
{\left[\mathrm{NO}_{2}\right\rfloor} \\
(\mu \mathrm{g} / \mathrm{mL})\end{array}$ & $\begin{array}{c}\mathrm{NO}_{3} \\
\text { in liquid } \\
{\left[\mathrm{NO}_{3}\right]} \\
(\mu \mathrm{g} / \mathrm{ml} .)\end{array}$ & $\begin{array}{c}\text { Na } \\
\text { in liquid } \\
|\mathrm{Na}| \\
(\mathrm{ug} / \mathrm{mL})\end{array}$ & $\begin{array}{c}\text { Al } \\
\text { in liquid } \\
{[\mathrm{Al}]} \\
(\mu \mathrm{g} / \mathrm{mL})\end{array}$ & $\begin{array}{c}90 \mathrm{Sr} \\
\text { in waste } \\
{[\mathrm{Sr}]} \\
(\mu \mathrm{Ci} / \mathrm{g})\end{array}$ & $\begin{array}{c}{ }^{241} \mathrm{Am} \\
\text { in waste } \\
{[\mathrm{Am241]}} \\
(\mu \mathrm{Ci} / \mathrm{g})\end{array}$ & $\begin{array}{c}{ }^{240} \mathrm{Pu} \\
\text { in waste } \\
{[\mathrm{Pu} 240]} \\
(\mu \mathrm{Ci} / \mathrm{g})\end{array}$ & $\begin{array}{c}{ }^{239} \mathrm{Pu} \\
\text { in waste } \\
\mid \mathrm{Pu240|} \\
(\mu \mathrm{Ci} / \mathrm{g})\end{array}$ & $\begin{array}{c}{ }^{238} \mathrm{Pu} \\
\text { in waste } \\
{[\mathrm{Pu} 238]} \\
(\mu \mathrm{Ci} / \mathrm{g})\end{array}$ & $\begin{array}{c}{ }^{137} \mathrm{Cs} \\
\text { in waste } \\
|\mathrm{Cs}| \\
(\mu \mathrm{Ci} / \mathrm{g})\end{array}$ & $\begin{array}{c}\text { Bulk } \\
\text { density } \\
\text { D } \\
(\mathrm{g} / \mathrm{mL})\end{array}$ & $\begin{array}{c}\text { Liquid } \\
\text { density } D_{4} \\
(\mathrm{~g} / \mathrm{ml})\end{array}$ & $\begin{array}{c}\text { Non-RGS } \\
\text { waste } \\
\text { volume } \\
(\mathrm{kL})\end{array}$ & $\begin{array}{l}\text { Bulk } \\
\text { water } \\
{\left[\mathrm{H}_{2} \mathrm{O}\right]} \\
(w t \%)\end{array}$ & $\begin{array}{l}\text { Liquid } \\
\text { water } \\
{\left[\mathrm{H}_{2} \mathrm{O} \mid\right.} \\
(\mathrm{wt} \%)\end{array}$ & $\begin{array}{l}\text { Waste } \\
\text { temp. } \\
\mathrm{Tw} \\
\left({ }^{\circ} \mathrm{C}\right)\end{array}$ & $\begin{array}{c}\text { Dome } \\
\text { temp. } \\
\text { Td } \\
\left({ }^{\circ} \mathrm{C}\right)\end{array}$ \\
\hline 24I-U-111 & $39 \mathrm{E}+04$ & $41 \mathrm{E}+03$ & $1.20 \mathrm{E}+05$ & $1.67 \mathrm{E}+05$ & $39 E+05$ & $3.66 \mathrm{E}+04$ & $3.82 E+01$ & $1.14 \mathrm{E}-01$ & $.88 E-02$ & $9.26 \mathrm{E}-02$ & $2.59 \mathrm{E}-03$ & $1.27 \mathrm{E}+02$ & 1.60 & 1.40 & 878 & $3.69 \mathrm{E}-01$ & $5.26 \mathrm{E}-01$ & 24 & 25 \\
\hline Supeniatant & $3.39 \mathrm{E}+04$ & $5.41 E-03$ & $1.20 E+05$ & $1.67 E+05$ & $2.39 \mathrm{E}+05$ & $3.66 \mathrm{E}+04$ & $3.82 \mathrm{E}+0$ & 1. $14 \mathrm{E}-01$ & $1.88 \mathrm{E}-02$ & $9.26 \mathrm{E}-02$ & $2.59 \mathrm{E}-03$ & $1.27 E+02$ & 1.40 & 1.40 & 38 & $5.26 \mathrm{E}-01$ & $5.26 \mathrm{E}-01$ & 0 & 0 \\
\hline Solid Layer & $3.39 \varepsilon+04$ & $5.41 \mathrm{E}+03$ & $1.20 \mathrm{E}+05$ & $1.67 \mathrm{k}+05$ & $2.39 \mathrm{E} \div 05$ & $3.66 \mathrm{E}+04$ & $3.82 \mathrm{E}+01$ & $1.14 \mathrm{t}-01$ & $1.88 \mathrm{E}-02$ & $9.26 \mathrm{E}-02$ & $2.59 \mathrm{E}-03$ & $1.27 \mathrm{E}+02$ & 1.61 & 1.40 & 840 & $3.62 \mathrm{E}-01$ & $5.26 \mathrm{E}-01$ & 24 & 25 \\
\hline $241-\mathrm{L}-112$ & $4.91 \mathrm{E}-04$ & $2.98 \mathrm{E}+03$ & $3.60 \mathrm{E}+04$ & $7.96 \mathrm{f}+04$ & $8.32 \mathrm{E}+04$ & $4.94 E+03$ & $1.65 \mathrm{E}+02$ & 3.36E-03 & $2.11 \mathrm{E}-03$ & $1.91 E-02$ & $1.38 \mathrm{E}-04$ & $3.01 \mathrm{E}+01$ & 1.64 & 1.17 & 210 & 4.IIE- -01 & $7.50 \mathrm{E}-01$ & 21 & 21 \\
\hline Supernatant & $4.91 \mathrm{l}+04$ & $2.98 \mathrm{E} \cdot 0.03$ & $3.60 \mathrm{E}+04$ & $7.96 \mathrm{E}+04$ & $8.32 E+04$ & $4.94 \mathrm{E} \div 03$ & $1.65 \mathrm{E}+02$ & 3.36E-03 & $11 \mathrm{E}-03$ & $1.91 \mathrm{E}-02$ & $1.38 \mathrm{E}-04$ & $3.01 \mathrm{E}+$ & 1.17 & 1.17 & 38 & $7.50 \mathrm{E}-01$ & $7.50 \mathrm{E}-01$ & 0 & 0 \\
\hline Solid Layer & $4.91 \mathrm{E}-04$ & $2.98 \mathrm{E}+03$ & $3.60 \mathrm{E}-04$ & $7.96 \mathrm{E}+04$ & $8.32 \mathrm{E}+04$ & $4.94 \mathrm{E}+03$ & $1.65 E+02$ & $3.36 \mathrm{E}-03$ & $2.11 \mathrm{E}-03$ & 1.91E-02 & $1.38 \mathrm{E}-04$ & $3.01 E+0 \mathrm{H}$ & 1.74 & 1.17 & 172 & $3.61 \mathrm{E}-01$ & $7.50 \mathrm{E}-01$ & 21 & 21 \\
\hline $24 I-U-201$ & $6.07 E+03$ & $0.00 \mathrm{E} \cdot 00$ & $1.49 \mathrm{E}+04$ & $1.03 E+05$ & $8.34 E+04$ & $1.02 E+02$ & $9.87 \mathrm{E}-02$ & 1.11E-03 & $2.43 \mathrm{E}-04$ & $1.28 \mathrm{E}-03$ & $1.99 \mathrm{E}-05$ & $7.24 \mathrm{E}-00$ & 1.42 & 1.17 & 19 & $4.54 \mathrm{E}-01$ & $7.36 \mathrm{E}-01$ & 20 & 21 \\
\hline Supernatant & $1.21 \mathrm{E}+03$ & $0.00 \mathrm{E}+00$ & $1.06 E+04$ & $7.33 \mathrm{E}+04$ & $5.95 \mathrm{E}+04$ & $7.26 \mathrm{E}+01$ & $5.78 \mathrm{E}-03$ & $2.47 \mathrm{E}-04$ & $1.97 \mathrm{E}-05$ & $1.04 \mathrm{E}-04$ & $1.61 \mathrm{E}-06$ & $1.24 \mathrm{E}+01$ & 1.13 & 1.13 & 8 & $8.25 \mathrm{E}-01$ & $8.25 E-01$ & 21 & 21 \\
\hline Solid Layer & $1.27 \mathrm{E}+04$ & $0.00 E+00$ & $2.07 \mathrm{E}+04$ & $1.43 E+05$ & $1.16 \mathrm{E}+05$ & $1.41 \mathrm{E}+02$ & $1.44 \mathrm{~F}-01$ & $1.53 \mathrm{E}-03$ & $3.52 \mathrm{E}-04$ & $1.86 \mathrm{E}-03$ & $2.89 \mathrm{E}-05$ & $4.72 \mathrm{E}+00$ & 1.63 & 1.22 & $1 !$ & $2.71 E-01$ & $6.93 \mathrm{E}-01$ & 20 & 21 \\
\hline $241-11-202$ & $8.00 \mathrm{E}+03$ & $9.92 \mathrm{E}-01$ & $1.73 E+04$ & $1.07 E+05$ & $8.41 \mathrm{E} 104$ & $1.68 \mathrm{E}+02$ & $3.89 E-02$ & $1.20 \mathrm{E}-03$ & $3.10 \mathrm{E}-04$ & $1.63 \mathrm{E}-03$ & $2.54 \mathrm{E}-05$ & $5.86 \mathrm{E}+100$ & 1.35 & 1.17 & 18 & $4.80 \mathrm{E}-01$ & $7.40 \mathrm{E}-01$ & 18 & 19 \\
\hline Supematant & $1.71 E+03$ & $7.24 \mathrm{~F}+01$ & $1.26 \mathrm{E} \div 04$ & $7.80 \mathrm{E}^{-}-04$ & $6.14 \mathrm{E}+04$ & $1.23 \mathrm{E}+02$ & $2.77 \mathrm{E}-04$ & $4.15 \mathrm{E}-06$ & $5.02 \mathrm{E}-07$ & $2.64 \mathrm{E}-06$ & $4.11 \mathrm{E}-08$ & $8.79 \mathrm{E}+00$ & 1.14 & 1.14 & 8 & $8.22 \mathrm{E}-01$ & 8.22 & 18 & 19 \\
\hline Solid Layer & $1.79 \mathrm{t}+04$ & $1.41 E+02$ & $2.45 \mathrm{E}+04$ & $1.52 \mathrm{E}+05$ & $1.20 \mathrm{E}+05$ & $2.39 \mathrm{E}+02$ & $6.17 \mathrm{E}-02$ & $1.90 \mathrm{E}-03$ & $4.93 \mathrm{E}-04$ & $2.60 \mathrm{E}-03$ & $4.04 \mathrm{E}-05$ & $4.13 E+00$ & 1.51 & 1.22 & 10 & $2.78 \mathrm{E}-01$ & $6.91 \mathrm{E}-01$ & 19 & 19 \\
\hline $241-0.203$ & $2.42 \mathrm{E}+03$ & $0.00 \mathrm{E}+00$ & $1.42 E+04$ & $1.06 \mathrm{E}+05$ & $7.38 E+04$ & $1.38 E+02$ & $7.76 \mathrm{E}-02$ & $9.53 \mathrm{E}-04$ & $.78 \mathrm{E}-04$ & $9.39 \mathrm{E}-04$ & $1.46 \mathrm{E}-05$ & $5.560+00$ & 1.38 & 1.2 & 17 & $5.62 \mathrm{E}-01$ & $7.38 \mathrm{E}-01$ & 19 & 19 \\
\hline Supernatant & $4.54 E \div 02$ & $0.00 \mathrm{~F}+00$ & $9.94 \mathrm{E}+03$ & $7.40 E+04$ & $5.15 \mathrm{E}+04$ & $9.63 \mathrm{E} \div 01$ & $2.44 \mathrm{E}-04$ & $3.99 \mathrm{E}-06$ & $2.13 \mathrm{E}-06$ & 1.12E-05 & $1.74 \mathrm{E}-07$ & $8.24 \mathrm{E}+00$ & 1.14 & 1.14 & 8 & $8.20 \mathrm{E}-01$ & $8.20 \mathrm{E}-01$ & 19 & 19 \\
\hline Solid Layer & $4.75 E 1.03$ & $0.00 \mathrm{E}+00$ & $1.94 \mathrm{E}+04$ & $1.44 \mathrm{E} \cdot 05$ & $1.00 \mathrm{E}+05$ & $1.88 \mathrm{E}+02$ & $1.26 \mathrm{E}-0 \mathrm{I}$ & $1.54 E-03$ & $2.88 \mathrm{E}-04$ & $1.52 \mathrm{E}-03$ & $2.35 \mathrm{E}-05$ & $3.89 \mathrm{E}+00$ & 1.59 & 1.28 & 9 & $4.02 \mathrm{E}-01$ & $6.87 \mathrm{E}-01$ & 19 & 19 \\
\hline $24 !-(\mathrm{J}-204$ & $5.38 \mathrm{E}+02$ & $5.20 E+01$ & $4.51 \mathrm{E}+03$ & $3.55 \mathrm{E}+04$ & $2.52 \mathrm{E}+04$ & $9.39 E+00$ & $2.12 \mathrm{E}+00$ & $1.33 \mathrm{E}-02$ & $6.70 \mathrm{E}-03$ & $3.53 \mathrm{E}-02$ & 5.51E-04 & $4.23 E+00$ & 1.25 & 1.07 & 15 & $5.59 \mathrm{E}-01$ & $8.97 E-01$ & 18 & 18 \\
\hline Supernatant & $1.54 \mathrm{E}+02$ & $4.16 \mathrm{E}+01$ & $3.61 E+03$ & $2.84 E+04$ & $2.02 \mathrm{E}+04$ & $7.51 \mathrm{E}+00$ & $9.56 \mathrm{E}-05$ & $4.58 \mathrm{E}-06$ & $3.62 \mathrm{E}-07$ & $1.91 \mathrm{E}-06$ & $2.95 \mathrm{E}-08$ & $3.31 \mathrm{E}+00$ & 1.06 & 1.06 & 8 & $9.30 \mathrm{E}-01$ & $9.30 \mathrm{E}-01$ & 18 & 18 \\
\hline Solid Layer & $1.61 \mathrm{E}+03$ & $8.10 E+01$ & $7.03 \mathrm{E}+03$ & $5.53 \mathrm{E}+04$ & $3.93 \mathrm{E}+04$ & $1.46 \mathrm{E}+0 \mathrm{~J}$ & $3.81 \mathrm{E}+00$ & $2.2 \mathrm{lE}-02$ & $1.21 \mathrm{E}-02$ & $6.35 \mathrm{E}-02$ & $9.91 \mathrm{E}-04$ & $4.98 \mathrm{E}+00$ & 1.47 & 1.11 & 7 & $2.62 \mathrm{E}-01$ & $8.70 \mathrm{E}-01$ & 18 & 18 \\
\hline
\end{tabular}


RPP-5926 REV 6

APPENDIX C

CALCULATIONS OF THE HENRY'S LAW CONSTANTS AND THE MASS TRANPORT PRODUCTS OF h AND A 
RPP-5926 REV 6

This page intentionally left blank.

C-ii 
RPP-5926 REV 6

\section{CONTENTS}

Table C-1 Input Ion Concentration for $\mathrm{NH}_{3}$ Calculation ......................................................... -3

Table C-2 Calculations of Henry's Law Constant by Schumpe Model ...................................... 6

Table C-3 Calculation of Mass Transport Properties of $h$ and A from Normal Ventilation Condition and Prediction for Off-Normal Condition.................................-9 
RPP-5926 REV 6

This page intentionally left blank.

C-2 
RPP-5926 REV 6

Table C-1. Input Ion Concentration for $\mathrm{NH}_{3}$ Calculation. ( 3 sheets)

\begin{tabular}{|c|c|c|c|c|c|c|c|c|c|c|c|c|c|c|}
\hline Tanks & $\begin{array}{c}\mathrm{Na}+ \\
\text { in liquid } \\
(\mu \mathrm{g} / \mathrm{mL})\end{array}$ & $\begin{array}{c}\mathrm{Al}+3 \\
\text { in liquid } \\
(\mu \mathrm{g} / \mathrm{mL})\end{array}$ & $\begin{array}{c}\mathrm{Fe}+3 \\
\text { in liquid } \\
(\mu \mathrm{g} / \mathrm{mL})\end{array}$ & $\begin{array}{c}\mathrm{Cr}+3 \\
\text { in liquid } \\
(\mu \mathrm{g} / \mathrm{mL})\end{array}$ & $\begin{array}{c}\mathrm{Ni}+2 \\
\text { in liquid } \\
(\mu \mathrm{g} / \mathrm{mL})\end{array}$ & $\begin{array}{c}\mathrm{K}+1 \\
\text { in liquid } \\
(\mu \mathrm{g} / \mathrm{mL})\end{array}$ & $\begin{array}{c}\text { OH-l } \\
\text { in liquid } \\
(\mu \mathrm{g} / \mathrm{mL})\end{array}$ & $\begin{array}{c}\text { NO3-1 } \\
\text { in liquid } \\
(\mu \mathrm{g} / \mathrm{mL})\end{array}$ & $\begin{array}{l}\mathrm{NO} 2-1 \\
\text { in liquid } \\
(\mu \mathrm{g} / \mathrm{ml})\end{array}$ & $\begin{array}{c}\text { CO3-2 } \\
\text { in liquid } \\
(\mu \mathrm{g} / \mathrm{mL})\end{array}$ & $\begin{array}{c}\text { PO4-3 } \\
\text { in liquid } \\
(\mu \mathrm{g} / \mathrm{mL})\end{array}$ & $\begin{array}{c}\text { SO4-2 } \\
\text { in liquid } \\
(\mu \mathrm{g} / \mathrm{mL})\end{array}$ & $\begin{array}{c}F-1 \\
\text { in liquid } \\
(\mu \mathrm{g} / \mathrm{mL})\end{array}$ & $\begin{array}{c}\text { C.l-l } \\
\text { in liquid } \\
(\mu \mathrm{g} / \mathrm{mL})\end{array}$ \\
\hline $241-\mathrm{AN}-101$ & $29 \mathrm{E}+04$ & $1.09 \mathrm{E}+04$ & $32 \mathrm{E}+00$ & $1.21 \mathrm{E}+02$ & $61 \mathrm{E}+00$ & $2.68 \mathrm{E}+03$ & $3.08 \mathrm{E}+04$ & $.18 \mathrm{E}+04$ & $4.07 \mathrm{E}+04$ & $9.65 \mathrm{E}+03$ & $1.59 E+03$ & $1.36 \mathrm{E}+03$ & $2.89 E+02$ & $2.33 E+03$ \\
\hline $241-\mathrm{AN}-102$ & $2.27 \mathrm{E}+05$ & $1.54 \mathrm{E}+04$ & $4.90 \mathrm{E}+01$ & $2.97 \mathrm{E}+02$ & 4. $17 \mathrm{E}+02$ & $2.88 \mathrm{E}+03$ & $3.23 \mathrm{E}+03$ & $2.36 \mathrm{E}+05$ & $9.04 E+04$ & $7.00 \mathrm{E}+04$ & $5.52 \mathrm{E}+03$ & $1.53 E+04$ & $1.91 \mathrm{E}+03$ & $4.11 \mathrm{E}+03$ \\
\hline $241-\mathrm{AN}-103$ & $2.85 \mathrm{E}+0.5$ & $3.02 \mathrm{E}+04$ & $3.01 \mathrm{E}+01$ & $5.66 \mathrm{E}+02$ & $1.20 \mathrm{E}+01$ & $1.68 \mathrm{E}+04$ & $9.93 E+04$ & $1.68 \mathrm{E}+05$ & $1.65 \mathrm{E}+05$ & $5.80 \mathrm{E}+03$ & $1.65 E+03$ & $1.45 E+03$ & $6.60 \mathrm{E}+02$ & $9.76 \mathrm{E}+03$ \\
\hline 241-AN-104 & $2.56 \mathrm{E}+05$ & $3.86 \mathrm{E}+04$ & $3.01 \mathrm{E}+0 \mathrm{I}$ & $3.36 \mathrm{E}+02$ & $1.20 \mathrm{E}+01$ & $6.69 \mathrm{E}+03$ & $7.09 \mathrm{E}+04$ & $1.70 \mathrm{E}+05$ & $1.25 \mathrm{E}+05$ & $1.07 \mathrm{E}+04$ & $2.71 E+03$ & $3.54 \mathrm{E}+03$ & $8.19 \bar{E}+01$ & $8.09 E+03$ \\
\hline $241-\wedge N-105$ & $2.46 \mathrm{E}+05$ & $4.11 \mathrm{E}+04$ & $2.47 \mathrm{E}+01$ & $2.22 \mathrm{E}+02$ & $9.95 E+00$ & $6.50 \mathrm{E}+03$ & $5.86 \mathrm{E}+04$ & $1.62 \mathrm{E}+05$ & $1.21 E+05$ & $1.18 \mathrm{E}+04$ & $1.22 \mathrm{E}+03$ & $2.85 E+03$ & $3.06 E+02$ & $9.90 \mathrm{E}+03$ \\
\hline $24 !-\mathrm{AN}-106$ & $7.56 \mathrm{E}+04$ & $3.22 \mathrm{E}+03$ & $1.77 \mathrm{E}+00$ & $9.91 \mathrm{E}+01$ & $2.79 \mathrm{E}+02$ & $5.96 \mathrm{E}+03$ & $9.93 E+04$ & $6.58 \mathrm{E}+04$ & $1.76 \mathrm{E}+04$ & $2.18 \mathrm{~F}+04$ & $2.02 \mathrm{E}+03$ & $5.50 \mathrm{E}+03$ & $5.11 E+03$ & $9.60 \mathrm{E}+02$ \\
\hline $241-\mathrm{AN}-107$ & $2.03 E+05$ & $2.93 \mathrm{E}+02$ & $1.67 \mathrm{E}+03$ & $1.72 \mathrm{E}+02$ & $5.51 \mathrm{~F}+02$ & $1.81 \mathrm{E}+03$ & $9.93 E+04$ & $2.38 E+05$ & $6.59 \mathrm{E}+04$ & $7.55 \mathrm{E}+04$ & $2.94 \mathrm{E}+03$ & $8.98 E+03$ & 4. $18 \mathrm{E}+03$ & $2.02 \mathrm{E}+03$ \\
\hline $241-\mathrm{AP}-101$ & $1.29 \mathrm{E}+05$ & $6.94 \mathrm{E}+03$ & $2.50 \mathrm{~F}+00$ & $1.43 \mathrm{E}+02$ & $7.90 \mathrm{E}+00$ & $3.12 \mathrm{E}+04$ & $4.94 \mathrm{E}+04$ & $1.32 \mathrm{E}+05$ & $4.19 \mathrm{E}+04$ & $3.23 \mathrm{E}+04$ & $1.02 \mathrm{E}+03$ & $4.03 E+03$ & $2.90 \mathrm{E}+03$ & $1.98 \mathrm{E}+03$ \\
\hline 241-AP-102 & $1.02 \mathrm{E}+05$ & $1.15 \mathrm{E}+04$ & $3.82 \mathrm{E}+00$ & $6.19 \Sigma+02$ & $2.66 \mathrm{E}+01$ & $1.29 E+03$ & $9.09 \mathrm{E}+03$ & $7.60 \mathrm{E}+04$ & $3.59 \mathrm{E}+04$ & $2.66 \mathrm{E}+04$ & $1.16 \mathrm{E}+04$ & $4.52 E+03$ & $1.68 \mathrm{E}+02$ & $2.73 E+03$ \\
\hline 241-AP-103 & $1.81 E+05$ & $8.43 E+03$ & $3.01 \mathrm{E}+01$ & $3.72 \mathrm{~F}+02$ & $1.22 \mathrm{E}+02$ & $1.33 \mathrm{E}+04$ & $9.51 \mathrm{E}+03$ & $1.35 \mathrm{E}+05$ & $1.06 \mathrm{E}+05$ & $7.55 \mathrm{E}+04$ & $3.46 \mathrm{E}+03$ & $4.50 \mathrm{E}+03$ & $2.09 \mathrm{E}+03$ & $2.82 \mathrm{E}+03$ \\
\hline 241-AP-104 & $1.36 \mathrm{E}+05$ & $1.97 \mathrm{E}+04$ & $1.04 \mathrm{E}+02$ & 1.37E+03 & $7.85 \mathrm{E}+01$ & $2.11 \mathrm{E}+03$ & $2.26 \mathrm{E}+03$ & $1.13 E+05$ & $E+04$ & $1.93 E+04$ & $3.86 E+03$ & $E+03$ & $\therefore+02$ & +03 \\
\hline 241-AP-105 & $1.90 \mathrm{E}+05$ & $2.07 \mathrm{E}+04$ & $3.01 \mathrm{~F}+01$ & $5.13 \mathrm{E}+02$ & $2.54 \mathrm{E}+01$ & $1.23 E+04$ & $3.35 E+04$ & $2.32 \mathrm{E}+05$ & $1.01 \mathrm{E}+05$ & $1.12 \mathrm{E}+05$ & $3.16 \mathrm{E}+03$ & $6.78 \mathrm{E}+03$ & $4.74 \mathrm{E}+03$ & $5.45 E+03$ \\
\hline 241-AP-106 & 1.22E+05 & 1. $83 E+04$ & 1.13E+02 & $1.57 \mathrm{E}+03$ & $7.56 \mathrm{E}+01$ & $3.12 \mathrm{E}+03$ & $9.01 E+03$ & $9.03 \mathrm{~F}+04$ & $6.14 \mathrm{E}+04$ & $1.88 \mathrm{E}+04$ & $2.76 \mathrm{E}+03$ & $3.20 \mathrm{E}+03$ & $6.71 E+02$ & $4.92 \mathrm{E}+03$ \\
\hline 241-AP-107 & $7.97 \mathrm{~F}+04$ & $8.69 \mathrm{E}+03$ & $1.01 \mathrm{E}+01$ & $6.55 \mathrm{E}+02$ & $2.36 \mathrm{E}+00$ & $1.50 \mathrm{CT}$ & $7.28 \mathrm{E}-$ & 9.5 & 2 & 4.5 & 4.8 & 03 & 02 & $2.29 \mathrm{E}+03$ \\
\hline $241-A P-108$ & $9.03 E+04$ & $1.09 E+04$ & $1.01 E+01$ & $8.21 E+02$ & $4.02 \mathrm{E}+00$ & $2.85 \mathrm{E}+03$ & $1.39 \mathrm{E}+04$ & $9.10 \mathrm{E}+04$ & $3.27 \mathrm{E}+04$ & $1.27 \mathrm{E}+04$ & $3.40 \mathrm{E}+03$ & $2.72 \mathrm{E}+03$ & $9.70 \mathrm{E}+02$ & $2.86 \mathrm{E}+03$ \\
\hline 241. & $3 E+05$ & $3.07 \mathrm{E}+04$ & $30 E+01$ & $2 \mathrm{E}+02$ & $6.10 \mathrm{E}+00$ & $4.12 \mathrm{~F}+04$ & $8.63 \mathrm{~F}+04$ & $1.70 \mathrm{E}+05$ & $1.20 \mathrm{E}+05$ & $9.20 \mathrm{E}+03$ & $9.96 \mathrm{E}+02$ & $1.26 \mathrm{E}+03$ & $9.62 \mathrm{E}+02$ & $7.30 \mathrm{E}+03$ \\
\hline 241-AW-102 & $5.68 \mathrm{E}+04$ & $6.39 \mathrm{E}+03$ & $6.95 \mathrm{E}+00$ & $4.81 \mathrm{E}+02$ & $1.90 \mathrm{E}+00$ & $1.32 E+03$ & $7.45 E+03$ & $6.50 \mathrm{E}$ & 2 & $2.3+3$ & 02 & 03 & 02 & $1.68 \mathrm{E}+03$ \\
\hline $241-A W-103$ & $2.46 \mathrm{E}+04$ & $6.46 \mathrm{E}+01$ & $5.57 \mathrm{E}+00$ & $2.53 \mathrm{E}+01$ & $5.08 \mathrm{E}+00$ & $1.43 \mathrm{E}+04$ & $9.62 E+03$ & $4.22 \mathrm{E}+03$ & $1.78 \mathrm{E}+03$ & $4.70 \mathrm{E}+03$ & $5.92 \mathrm{E}+01$ & $6.72 \mathrm{E}+02$ & $1.68 \mathrm{E}+04$ & $1.38 \mathrm{E}+02$ \\
\hline $241-\mathrm{AW}-104$ & $1.74 \mathrm{E}+04$ & $7.11 \mathrm{E}+02$ & +00 & $E+00$ & -01 & $7 E+03$ & $5 \mathrm{E}+03$ & +04 & $5.33 \mathrm{E}+03$ & $7.25 E+03$ & $2.93 \mathrm{E}+02$ & $6.54 \mathrm{E}+02$ & 1.32E+02 & $2.39 \mathrm{E}+02$ \\
\hline $24 !-A W-105$ & 1. $49 \mathrm{E}+04$ & $4.34 \mathrm{E}+01$ & $4.61 \mathrm{E}+00$ & $2.12 \mathrm{E}+00$ & $9.91 \mathrm{E}-01$ & $1.99 E+03$ & $6.12 \mathrm{E}+03$ & $2.70 \mathrm{E}+04$ & $1.28 \mathrm{E}+03$ & $1.62 \mathrm{E}+03$ & $1.85 \mathrm{E}+02$ & $3.01 \mathrm{E}+02$ & $4.81 \mathrm{E}+02$ & $2.45 E+02$ \\
\hline 241-AW-106 & $1.77 \mathrm{E}+05$ & $1.99 \mathrm{E}+04$ & $2.18 \mathrm{E}+01$ & $1.47 \mathrm{E}+03$ & $8.39 \mathrm{E}+00$ & $5.65 \mathrm{E}+03$ & $9.80 \mathrm{E}+03$ & $1.92 \mathrm{E}+05$ & $6.35 \mathrm{E}+04$ & $2.84 \mathrm{E}+04$ & $1.47 \mathrm{E}+03$ & $5.14 \mathrm{E}+03$ & 1.23E+03 & $5.17 \mathrm{E}+03$ \\
\hline 241-AY-101 & $5.19 E+04$ & $1.21 E+01$ & $1.20 \mathrm{E}+01$ & tot & $5.90 \mathrm{E}+01$ & $E+02$ & +02 & $2.60 \mathrm{E}+04$ & 3.531 & $3.44 \mathrm{E}+04$ & +03 & $5.87 \mathrm{E}+03$ & 1. $74 \mathrm{E}+02$ & $6.36 \mathrm{E}+02$ \\
\hline $241-\mathrm{A} Y-102$ & $5.40 \mathrm{E}+04$ & $1.97 \mathrm{H}+03$ & $1.01 \mathrm{E}+01$ & $2.91 \mathrm{E}+0 \mathrm{l}$ & $4.02 \mathrm{E}+00$ & $3.88 \mathrm{E}+02$ & $1.44 E+03$ & $4.86 \mathrm{E}+02$ & $7.29 \mathrm{E}+03$ & $4.87 \mathrm{E}+04$ & $3.01 \mathrm{E}+03$ & $2.32 \mathrm{E}+03$ & $1.72 \mathrm{E}+02$ & $1.60 \mathrm{E}+02$ \\
\hline $241-\mathrm{AZ}-101$ & $1.11 \mathrm{E}+05$ & $8.56 \mathrm{E}+03$ & $2.24 \mathrm{E}+01$ & $7.23 \mathrm{E}+02$ & $8.02 \mathrm{E}+00$ & $4.76 \mathrm{E}+03$ & $1.15 \mathrm{E}+04$ & $7.12 \mathrm{E}+04$ & $7.26 \mathrm{E}+04$ & $3.42 \mathrm{~F}+04$ & $1.34 \mathrm{E}+03$ & $1.50 \mathrm{E}+04$ & $1.75 \mathrm{~F} \div 03$ & $1.51 E+02$ \\
\hline $241-\mathrm{AZ}-102$ & $5.59 \mathrm{E}+04$ & $6.68 \mathrm{E}+02$ & $1.00 \mathrm{E}+00$ & $7.37 \mathrm{E}+02$ & $1.75 E+00$ & $3.20 E+03$ & $1.21 \mathrm{E}+03$ & $1.85 E+04$ & $3.55 E+04$ & $3.50 E+04$ & $4.97 E+02$ & $1.86 E+04$ & $1.14 E+03$ & $7.66 \mathrm{E}+01$ \\
\hline $241-S Y-101$ & $1.63 \mathrm{~F}+05$ & $1.34 \mathrm{E}+04$ & $3.97 \mathrm{E}+02$ & $5.18 \mathrm{E}+03$ & $2.02 E+02$ & $4.14 \mathrm{E}+03$ & $2.63 \mathrm{E}+04$ & $1.52 \mathrm{E}+0.5$ & $9.56 E+04$ & $3.44 \mathrm{E}+04$ & $4.94 \mathrm{E}+03$ & 4.19E+03 & $5.50 \mathrm{E}+02$ & $1.11 E+04$ \\
\hline $241-S Y-102$ & $9.84 E+04$ & $1.44 \mathrm{E}+04$ & $1.41 \mathrm{E}+01$ & $2.41 E+02$ & $5.04 \mathrm{E}+01$ & $1.53 \mathrm{E}+03$ & $1.97 \mathrm{~F}+04$ & $9.20 \mathrm{E}+04$ & $5.89 \mathrm{E}+04$ & $1.31 E+04$ & $3.48 \mathrm{E}+03$ & $2.91 E+03$ & $1.51 E+02$ & $4.64 E+03$ \\
\hline
\end{tabular}




\section{RPP-5926 REV 6}

Table C-1. Input Ion Concentration for $\mathrm{NH}_{3}$ Calculation. ( 3 sheets)

\begin{tabular}{|c|c|c|c|c|c|c|c|c|c|c|c|c|c|c|}
\hline Tanks & $\begin{array}{c}\mathrm{Na}^{+} \\
\text {in liquid } \\
(\mu \mathrm{g} / \mathrm{mL})\end{array}$ & $\begin{array}{c}\mathrm{Al}+3 \\
\text { in liquid } \\
(\mu \mathrm{g} / \mathrm{mL})\end{array}$ & $\begin{array}{c}\mathrm{Fe}+3 \\
\text { in tiquid } \\
(\mu \mathrm{g} / \mathrm{mL})\end{array}$ & $\begin{array}{c}C \mathrm{r}+3 \\
\text { in liquid } \\
(\mu \mathrm{g} / \mathrm{mL})\end{array}$ & $\begin{array}{c}\mathrm{Ni}+2 \\
\text { in liquid } \\
(\mu \mathrm{g} / \mathrm{mL})\end{array}$ & $\begin{array}{c}\mathrm{K}+1 \\
\text { in liquid } \\
(\mu \mathrm{g} / \mathrm{mL})\end{array}$ & $\begin{array}{c}\text { OH-l } \\
\text { in liquid } \\
(\mu \mathrm{g} / \mathrm{mL})\end{array}$ & $\begin{array}{l}\text { NO3-1 } \\
\text { in liquid } \\
(\mu \mathrm{g} / \mathrm{mL})\end{array}$ & $\begin{array}{l}\text { NO2-1 } \\
\text { in liquid } \\
(\mu \mathrm{g} / \mathrm{ml} .)\end{array}$ & $\begin{array}{c}\text { CO3-2 } \\
\text { in liquid } \\
(\mu \mathrm{g} / \mathrm{mL})\end{array}$ & $\begin{array}{l}\text { PO4-3 } \\
\text { in liquid } \\
(\mu \mathrm{g} / \mathrm{mL})\end{array}$ & $\begin{array}{l}\text { SO4-2 } \\
\text { in liquid } \\
(\mu \mathrm{g} / \mathrm{mL})\end{array}$ & $\begin{array}{c}F-1 \\
\text { in liquid } \\
(\mu \mathrm{g} / \mathrm{ml})\end{array}$ & $\begin{array}{c}\text { Cl-l } \\
\text { in liquid } \\
(\mu \mathrm{g} / \mathrm{mL})\end{array}$ \\
\hline $24 \mathrm{I}-\mathrm{SY}-103$ & $12 \mathrm{E}+05$ & $4.14 \mathrm{E}+04$ & $2.10 \mathrm{E}+01$ & $3.30 E+01$ & $4.90 E+01$ & $3.91 E+03$ & $2.89 E+04$ & $1.79 E+05$ & $1.43 E+05$ & $3.44 E+04$ & $3.12 E+03$ & $4.08 \mathrm{E}+03$ & $3.51 \mathrm{E}+02$ & $1.16 \mathrm{E}+04$ \\
\hline $241-A-101$ & $2.78 \mathrm{E}+05$ & $5.18 \mathrm{E}+04$ & $4.29 \mathrm{E}+01$ & $5.73 \mathrm{E}+0 \mathrm{l}$ & $1.85 \mathrm{E}+01$ & $7.42 \mathrm{E}+03$ & $4.48 \mathrm{E}+04$ & $2.33 \mathrm{E}+05$ & $1.65 \mathrm{E}+05$ & $1.33 \mathrm{E}+04$ & $5.89 \mathrm{E}+03$ & $1.40 \mathrm{~F}+03$ & $2.17 \mathrm{E}+02$ & $9.01 \mathrm{E}+03$ \\
\hline $241-\mathrm{A}-103$ & $2.20 \mathrm{E}+05$ & $3.56 \mathrm{E}+04$ & $1.81 \mathrm{E}+01$ & $2.96 \mathrm{E}+02$ & $9.46 \mathrm{E}+01$ & $5.44 E+03$ & $4.48 \mathrm{E}+04$ & $1.33 \mathrm{E}+05$ & $1.65 \mathrm{E}+05$ & $1.33 \mathrm{E}+04$ & $3.67 \mathrm{E}+03$ & $1.40 \mathrm{E}+03$ & $2.17 E+02$ & $9.01 E+03$ \\
\hline $241-A \times-101$ & $2.82 E+05$ & $5.03 E+04$ & $3.01 E+01$ & $1.75 \mathrm{E}+02$ & $1.20 E+01$ & $7.42 E+03$ & $3.90 \mathrm{E}+04$ & $2.72 \mathrm{E}+05$ & $1.73 E+05$ & $1.10 \mathrm{E}+04$ & $6.59 \bar{E}+03$ & $1.54 \mathrm{E}+03$ & $5.78 \mathrm{E}+01$ & $8.82 \mathrm{E}+03$ \\
\hline $241-B-203$ & $3.12 E+04$ & $1.00 \mathrm{E}+01$ & $1.00 \mathrm{E}+01$ & $1.62 \mathrm{E}+02$ & $4.02 \mathrm{E}+00$ & $5.44 \mathrm{E}+03$ & $3.90 \mathrm{E}+04$ & $5.67 \mathrm{E}+04$ & $1.57 \mathrm{E}+03$ & $1.09 \mathrm{E}+04$ & $1.90 E+03$ & $5.62 \mathrm{E}+02$ & $6.04 \mathrm{E}+03$ & $6.77 \mathrm{E}+02$ \\
\hline $241-\mathrm{B}-204$ & $2.94 \mathrm{~F}+04$ & $8.35 \mathrm{E}+00$ & $8.35 \mathrm{E}+00$ & $1.52 \mathrm{E}+02$ & $3.35 \mathrm{E}+00$ & $6.42 \mathrm{E}+03$ & $3.90 \mathrm{E}+04$ & $4.82 \mathrm{E}+04$ & $1.82 \mathrm{E}+03$ & $1.09 \mathrm{E}+04$ & $7.14 \mathrm{E}+02$ & $8.24 \mathrm{E}+02$ & $6.55 \mathrm{E}+03$ & $6.22 \mathrm{E}+02$ \\
\hline $241-B Y-106$ & $65 E+05$ & $98 \mathrm{E}+04$ & $3.20 \mathrm{E}+01$ & $3.54 \mathrm{E}+02$ & $2.80 \mathrm{E}+01$ & $8.93 E+03$ & $3.58 \mathrm{E}+04$ & $10 \mathrm{E}+05$ & $7.81 \mathrm{E}+04$ & $6.60 \mathrm{E}+03$ & $1.99 \mathrm{E}+03$ & $2.02 \mathrm{E}+03$ & $93 \mathrm{E}+02$ & $4.65 F+03$ \\
\hline $241-C-103$ & $3.53 \mathrm{E}+04$ & $1.16 \mathrm{~F}+01$ & $5.40 \mathrm{E}+00$ & $7.86 \mathrm{E}+01$ & $7.85 E+01$ & $3.10 \mathrm{E}+02$ & $4.49 \mathrm{E}+04$ & $4.30 \mathrm{E}+03$ & $2.43 E+04$ & $2.94 \mathrm{E}+04$ & $4.59 E+03$ & $3.22 \mathrm{E}+03$ & $1.16 \mathrm{E}+03$ & $4.05 \mathrm{E}+02$ \\
\hline $241-S+101$ & $2.15 E+05$ & $2.45 \mathrm{E}+04$ & $2.01 E+01$ & $8.13 E+02$ & $8.02 \mathrm{E}+00$ & $2.36 \mathrm{E}+03$ & $4.49 \mathrm{E}+04$ & $1.81 \mathrm{E}+05$ & $9.40 \mathrm{E}+04$ & $5.30 \mathrm{E}+03$ & $3.86 \mathrm{E}+03$ & $5.65 E+03$ & $1.06 E+02$ & $7.45 \mathrm{E}+03$ \\
\hline $241-S-102$ & $2.36 \mathrm{E}+05$ & $4.19 E+04$ & $4.01 E+01$ & $1.08 \mathrm{E}+02$ & $1.64 \mathrm{E}+01$ & $3.25 \mathrm{E}+03$ & $4.10 \mathrm{E}+04$ & $1.59 \mathrm{E}+05$ & $1.14 \mathrm{E}+05$ & $2.01 E+04$ & $4.93 E+03$ & $4.63 \mathrm{E}+03$ & $3.50 \mathrm{E}+02$ & $1.04 \mathrm{E}+04$ \\
\hline $241-S-103$ & $2.28 \mathrm{E}+05$ & $3.20 \mathrm{E}+04$ & $3.01 E+01$ & $2.69 \mathrm{E}+02$ & $1.20 \mathrm{E}+0 \mathrm{I}$ & $3.90 \mathrm{E}+03$ & $3.65 \mathrm{E}+04$ & $2.16 \mathrm{E}+05$ & $1.47 \mathrm{E}+05$ & $2.21 \mathrm{E}+04$ & $3.57 \mathrm{E}+03$ & $5.10 \mathrm{E}+03$ & $2.56 \mathrm{E}+02$ & $1.33 \mathrm{E}+04$ \\
\hline $241-S-106$ & $2.44 E+05$ & $3.85 \mathrm{E}+04$ & $3.01 \mathrm{E}+01$ & $9.41 \mathrm{E}+03$ & $1.20 \mathrm{E}+01$ & $1.84 \mathrm{E}+03$ & $5.49 \mathrm{E}+04$ & $2.40 \mathrm{E}+05$ & $1.09 \mathrm{E}+05$ & $1.58 \mathrm{E}+04$ & $6.38 \mathrm{E}+03$ & $5.73 E+03$ & $1.40 \mathrm{E}+02$ & $1.11 \mathrm{E}+04$ \\
\hline $241-S-111$ & $2.19 \mathrm{~F}+05$ & $2.91 \mathrm{E}+04$ & $2.01 \mathrm{E}+01$ & $4.06 \mathrm{H}+03$ & $8.02 \mathrm{E}+00$ & $1.64 \mathrm{E}+03$ & $4.73 E+04$ & $1.90 \mathrm{E}+05$ & $7.05 \mathrm{E}+04$ & $1.28 \mathrm{E}+04$ & $1.02 E+04$ & $4.32 \mathrm{E}+03$ & $1.05 E+02$ & $6.39 \mathrm{E}+03$ \\
\hline $241-S X-101$ & $2.42 \mathrm{E}+05$ & $7.56 \mathrm{E}+04$ & $3.56 \mathrm{E}+01$ & $1.21 \mathrm{E}+04$ & $3.44 \mathrm{E}+04$ & $2.52 E+03$ & $4.87 \mathrm{E}+04$ & $1.05 \mathrm{E}+05$ & $7.80 \mathrm{E}+04$ & $8.05 \mathrm{E}+03$ & $5.18 \mathrm{E}+03$ & $1.83 \mathrm{E}+03$ & $7.14 \mathrm{E}+01$ & $1.67 \mathrm{E}+04$ \\
\hline $241-S X-102$ & $2.35 E+05$ & $4.56 \mathrm{E}+04$ & $3.65 \mathrm{E}+01$ & $2.77 \mathrm{E}+03$ & $1.45 \mathrm{E}+01$ & $3.55 \mathrm{E}+03$ & $5.01 \mathrm{E}+04$ & $1.58 \mathrm{E}+05$ & $1.51 \mathrm{E}+05$ & $8.80 \mathrm{E}+03$ & $3.49 \mathrm{E}+03$ & $3.33 E+03$ & $7.80 \mathrm{E}+01$ & $1.02 \mathrm{E}+04$ \\
\hline $24 !-5 X-103$ & $2.37 E+05$ & $4.41 E+04$ & $3.01 \mathrm{E}+01$ & $8.36 \mathrm{E}+01$ & $2.05 \mathrm{E}+04$ & $4.24 \mathrm{E}+03$ & $4.15 \mathrm{E}+04$ & $1.59 E+05$ & $1.58 \mathrm{E}+05$ & $1.28 \mathrm{E}+04$ & $3.33 \mathrm{E}+03$ & $2.01 \mathrm{E}+03$ & $1.01 \mathrm{E}+02$ & $1.10 \mathrm{E}+04$ \\
\hline $241-S X-104$ & $2.50 \mathrm{E}+05$ & $3.56 \mathrm{E}+04$ & $3.38 \mathrm{~F}+0 \mathrm{l}$ & $9.39 \mathrm{E}+02$ & $1.35 \mathrm{E}+01$ & $2.88 \mathrm{E}+03$ & $3.42 \mathrm{E}+04$ & $2.91 \mathrm{E}+05$ & $1.17 \mathrm{E}+05$ & $2.48 \mathrm{E}+04$ & $1.27 \mathrm{E}+04$ & $3.90 \mathrm{E}+03$ & $1.87 E+02$ & $8.23 E+03$ \\
\hline 241-SX-105 & $2.36 \mathrm{E}+05$ & $4.4 ! E+04$ & $3.01 \mathrm{E}+01$ & $3.38 \mathrm{E}+02$ & $1.20 E+01$ & $3.38 \mathrm{E}+03$ & $4.09 E+04$ & $1.65 E+05$ & $1.43 \mathrm{E}+05$ & $1.41 E+04$ & $3.27 \mathrm{E}+03$ & $4.20 \mathrm{E}+03$ & $1.04 E+02$ & $9.01 E+03$ \\
\hline $241-S X-106$ & $2.45 E+05$ & $2.59 E+04$ & $3.01 E+01$ & $1.30 \mathrm{E}+02$ & $1.20 \mathrm{E}+01$ & $3.89 \mathrm{E}+03$ & $3.31 \mathrm{E}+04$ & $2.10 \mathrm{E}+05$ & $1.32 \mathrm{E}+05$ & $2.56 \mathrm{E}+04$ & $3.52 E+03$ & $7.20 \mathrm{E}+03$ & $3.11 \mathrm{E}+02$ & $1.17 E+04$ \\
\hline $241-S X-108$ & $2.27 E+05$ & $1.54 \mathrm{E}+04$ & $4.90 \mathrm{E}+0 \mathrm{l}$ & $2.97 \mathrm{E}+02$ & $4.17 \mathrm{E}+02$ & $2.88 \mathrm{E}+03$ & 4. $14 \mathrm{E}+04$ & $2.36 \mathrm{E}+05$ & $9.04 E+04$ & $7.00 \mathrm{E}+04$ & $5.66 \mathrm{E}+03$ & $1.53 \mathrm{E}+04$ & $1.91 E+03$ & 4. $11 \mathrm{E}+03$ \\
\hline $241-\mathrm{T}-110$ & $3.70 \mathrm{E}+04$ & $5.00 \mathrm{E}+00$ & $5.00 \mathrm{E}+00$ & $3.46 \mathrm{E}+01$ & $2.00 \mathrm{E}+00$ & $3.27 \mathrm{E}+02$ & $5.11 \mathrm{E}+03$ & $1.83 E+04$ & $5.90 \mathrm{E}+01$ & $7.00 \mathrm{E}+04$ & $1.49 \mathrm{E}+03$ & $4.36 \mathrm{E}+03$ & $4.22 \mathrm{E}+03$ & $9.58 \mathrm{E}+02$ \\
\hline $241-T-203$ & $4.12 E+04$ & $5.05 \mathrm{E}+00$ & $5.05 \mathrm{E}+00$ & $4.26 \mathrm{E}+0 \mathrm{l}$ & $2.02 \mathrm{E}+00$ & $8.39 \mathrm{E}+03$ & $5.11 \mathrm{E}+03$ & $7.37 \mathrm{E}+04$ & $3.53 \mathrm{E}+02$ & $7.00 \mathrm{E}+04$ & $1.90 \mathrm{E}+03$ & $4.69 \mathrm{E}+02$ & $7.02 \mathrm{E}+03$ & $7.07 \mathrm{E}+02$ \\
\hline $241-U-102$ & $2.43 E+05$ & 1. $80 \mathrm{E}+04$ & $2.01 \mathrm{E}+01$ & $2.79 \mathrm{E}+02$ & $1.26 \mathrm{E}+02$ & $3.77 \mathrm{E}+03$ & $2.93 \mathrm{E}+04$ & $2.45 \mathrm{E}+05$ & $1.05 E+05$ & $4.68 \mathrm{E}+04$ & $3.80 \mathrm{E}+03$ & $9.84 E+03$ & $1.33 \mathrm{E}+02$ & $8.82 \mathrm{E}+03$ \\
\hline $241-U-103$ & $2.31 E+05$ & $4.09 E+04$ & $3.01 \mathrm{E}+01$ & $1.08 \mathrm{E}+02$ & $1.69 \mathrm{E}+02$ & $4.83 \mathrm{E}+03$ & $3.08 E+04$ & $1.52 \mathrm{E}+05$ & $1.37 \mathrm{E}+05$ & $1.56 \mathrm{E}+04$ & $7.64 \mathrm{E}+02$ & $2.68 \mathrm{E}+03$ & $1.09 \mathrm{E}+03$ & $1.14 \mathrm{E}+04$ \\
\hline
\end{tabular}




\section{RPP-5926 REV 6}

Table C-1. Input Ion Concentration for $\mathrm{NH}_{3}$ Calculation. (3 sheets)

\begin{tabular}{|c|c|c|c|c|c|c|c|c|c|c|c|c|c|c|}
\hline Tanks & $\begin{array}{c}\mathrm{Na}+ \\
\text { in liquid } \\
(\mu \mathrm{g} / \mathrm{ml})\end{array}$ & $\begin{array}{c}\mathrm{A} l+3 \\
\text { in liquid } \\
(\mu \mathrm{g} / \mathrm{mL})\end{array}$ & $\begin{array}{c}\text { Fe+3 } \\
\text { in liquid } \\
(\mu \mathrm{g} / \mathrm{mL})\end{array}$ & $\begin{array}{c}\mathrm{Cr}+3 \\
\text { in liquid } \\
(\mu \mathrm{g} / \mathrm{mL})\end{array}$ & $\begin{array}{c}\mathrm{Ni}+2 \\
\text { in liquid } \\
(\mu \mathrm{g} / \mathrm{mL})\end{array}$ & $\begin{array}{c}\mathrm{K}+\mathrm{l} \\
\text { in liquid } \\
(\mu \mathrm{g} / \mathrm{mL})\end{array}$ & $\begin{array}{c}\mathrm{OH}-1 \\
\text { in liquid } \\
(\mu \mathrm{g} / \mathrm{mL})\end{array}$ & $\begin{array}{l}\mathrm{NO} 3-1 \\
\text { in liquid } \\
(\mu \mathrm{g} / \mathrm{ml} \text { ) }\end{array}$ & $\begin{array}{c}\mathrm{NO} 2-1 \\
\text { in liquid } \\
(\mu \mathrm{g} / \mathrm{ml} \text { ) }\end{array}$ & $\begin{array}{c}\text { CO3-2 } \\
\text { in liquid } \\
(\mu \mathrm{g} / \mathrm{mL})\end{array}$ & $\begin{array}{c}\text { PO4-3 } \\
\text { in liquid } \\
\text { ( } \mathrm{Hg} / \mathrm{mL})\end{array}$ & $\begin{array}{c}\mathrm{SO} 4-2 \\
\text { in liquid } \\
(\mu \mathrm{g} / \mathrm{mL})\end{array}$ & $\begin{array}{c}\text { F-l } \\
\text { in liquid } \\
(\mu \mathrm{g} / \mathrm{mL})\end{array}$ & $\begin{array}{c}\mathrm{Cl}-\mathrm{l} \\
\text { in liquid } \\
(\mu \mathrm{g} / \mathrm{mL})\end{array}$ \\
\hline $241-U-105$ & $2.27 \mathrm{E}+05$ & $2.98 \mathrm{E}+04$ & $3.10 \mathrm{E}+01$ & $2.01 \mathrm{E}+02$ & $2.48 \mathrm{E}+02$ & $3.98 \mathrm{E}+03$ & $2.09 E+04$ & $1.81 E+05$ & $1.08 \mathrm{E}+05$ & $2.78 \mathrm{E}+04$ & $3.92 E+03$ & $8.01 E+03$ & $1.31 E+03$ & $8.92 \mathrm{E}+03$ \\
\hline $24 \mathrm{I}-\mathrm{U}-106$ & $2.23 \mathrm{E}+05$ & $1.20 \mathrm{E}+04$ & $3.93 \mathrm{E}+01$ & $4.96 E+02$ & $5.22 \mathrm{E}+02$ & $1.89 \mathrm{E}+03$ & $5.11 \mathrm{E}+03$ & $2.52 \mathrm{E}+05$ & $9.90 \mathrm{E}+04$ & $4.73 \mathrm{E}+04$ & $3.84 \mathrm{E}+03$ & $8.64 \mathrm{E}+03$ & $3.32 \mathrm{E}+02$ & $5.46 \mathrm{E}+03$ \\
\hline $241-()-107$ & $2.21 E+05$ & $2.64 \mathrm{E}+04$ & $3.77 \mathrm{E}+01$ & $5.75 E+02$ & $1.72 \mathrm{E}+01$ & $3.27 \mathrm{E}+03$ & $3.61 \mathrm{E}+04$ & $2.27 \mathrm{E}+05$ & $1.11 \mathrm{E}+05$ & $2.65 E+04$ & $3.44 \mathrm{E}+03$ & $6.63 \mathrm{~F}+03$ & $1.67 \mathrm{~F}+02$ & $8.99 \mathrm{E}+03$ \\
\hline $241-U-108$ & $2.43 \mathrm{k}+05$ & $3.29 \mathrm{E}+04$ & $3.33 E+01$ & $1.28 \mathrm{E}+03$ & $3.13 \mathrm{E}+01$ & $4.015+03$ & $4.92 E+04$ & $1.81 E+05$ & $1.30 \mathrm{E}+05$ & $2.20 \mathrm{E}+04$ & $3.13 \mathrm{E}+03$ & $4.23 \mathrm{E}+03$ & $7.90 \mathrm{E}+02$ & $8.94 E+03$ \\
\hline $241-U-109$ & $2.28 \mathrm{E}+05$ & $2.92 \mathrm{E}+04$ & $1.00 E+02$ & $2.61 \mathrm{E}+03$ & $4.55 E+01$ & $3.33 \mathrm{E}+03$ & $4.42 E+04$ & $1.85 \mathrm{E}+05$ & $1.28 E+05$ & $1.87 E+04$ & $3.37 \mathrm{E}+03$ & $5.17 \mathrm{E}+03$ & $3.96 \mathrm{E}+02$ & $8.69 E+03$ \\
\hline
\end{tabular}


RPP-5926 REV 6

Table C-2. Calculations of Henry's Law Constant by Schumpe Model. ( 3 sheets)

\begin{tabular}{|c|c|c|c|c|c|c|c|c|c|c|c|c|}
\hline Tanks & $\begin{array}{c}\left(\mathrm{NH}_{3}\right) \mathrm{ss} \\
\text { in dome } \\
(\mathrm{ppm})\end{array}$ & $\begin{array}{c}\text { Liquid } \\
\mathrm{NH}_{3} \\
(\mu \mathrm{g} / \mathrm{mL})\end{array}$ & $\begin{array}{c}\mathrm{w} \% \% \mathrm{H}_{2} \mathrm{O} \\
\text { in liquid } \\
(\%)\end{array}$ & $\begin{array}{c}\text { Spg } \\
\text { in Liquid } \\
(\mathrm{g} / \mathrm{ml})\end{array}$ & $\begin{array}{c}\text { Waste } \\
\text { Volume } \\
\text { (kL) }\end{array}$ & $\begin{array}{c}\text { Head space } \\
\quad T\left({ }^{\circ} \mathrm{C}\right)\end{array}$ & $\begin{array}{c}\text { Waste T } \\
\left({ }^{\circ} \mathrm{C}\right)\end{array}$ & $\begin{array}{c}\text { Sum of } \\
\text { ion conc. } \\
\text { Sum(Ci) } \\
\text { (mole/L) }\end{array}$ & $\begin{array}{l}\text { Sum of ion conc. } \\
\text { times ionic cocff. } \\
\text { Sum }\left(\mathrm{C}^{*} \mathrm{Hi}\right) \\
(\text { mole } / \mathrm{l})\end{array}$ & $\begin{array}{c}\text { KHo } \\
\text { in water } \\
(\text { mole } / \text { L-atm })\end{array}$ & $\begin{array}{l}\text { KH in solns' } \\
\text { correction } \\
\text { factor }\end{array}$ & $\begin{array}{c}\mathrm{KH} \\
\text { in solns' } \\
\text { (mole/L-atm) }\end{array}$ \\
\hline 241-AN-101 & 11 & $4.00 \mathrm{E}+01$ & 70 & 1.15 & 606 & 18 & 18 & 8.64 & 0.82 & 86.11 & 1.25 & 41.96 \\
\hline $241-\mathrm{AN}-102$ & 300 & $2.10 \mathrm{E}+02$ & 48 & 1.43 & 4013 & 29 & 31 & 18.10 & 1.69 & 46.00 & 2.03 & 14.07 \\
\hline 241-AN-103 & 7 & $3.45 \mathrm{E}+02$ & 49 & 1.49 & 3627 & 28 & 38 & 26.53 & 2.54 & 34.01 & 2.30 & 4.25 \\
\hline $24 !-A N-104$ & 21 & $2.46 \mathrm{E}+02$ & 51 & 1.40 & 3986 & 29 & 36 & 22.85 & 2.24 & 37.50 & 2.30 & 6.17 \\
\hline $241-\mathrm{AN}-105$ & 15 & $2.10 \mathrm{~F}+02$ & 50 & 1.42 & 4263 & 28 & 34 & 21.62 & 2.15 & 40.39 & 2.26 & 7.15 \\
\hline $241-\mathrm{AN}-106$ & 1 & $2.10 \mathrm{E}+02$ & 76 & 1.20 & 148 & 18 & 17 & 11.59 & 1.04 & $88.5 \mathrm{I}$ & 1.38 & 40.30 \\
\hline 24l-AN-107 & 400 & $2.10 \mathrm{E}+02$ & 50 & 1.37 & 3952 & 26 & 29 & 21.70 & 1.89 & 50.41 & 2.26 & 16.15 \\
\hline $241-\mathrm{AP}-10 \mathrm{I}$ & 125 & $4.39 \mathrm{E}+02$ & 58 & 1.31 & 4221 & 19 & 19 & 13.43 & 1.22 & 81.52 & 1.41 & 30.97 \\
\hline $241-\mathrm{AP}-102$ & 75 & $4.39 \mathrm{~F}+02$ & 75 & 1.20 & 4134 & 20 & 20 & 8.15 & 0.82 & 77.21 & 1.23 & 35.13 \\
\hline 241-AP-103 & $i$ & $2.50 \mathrm{E}+02$ & 80 & 1.31 & 1079 & 16 & 16 & 15.11 & 1.46 & 96.18 & 1.40 & 24.73 \\
\hline 241-AP-104 & 3 & $1.33 \mathrm{E}+02$ & 97 & 1.29 & 91 & 15 & 15 & 10.73 & 1.06 & 98.90 & 1.33 & 37.70 \\
\hline $241-A P-105$ & 7 & $3.24 \mathrm{E}+02$ & 62 & 1.35 & 2896 & 26 & 19 & 19.62 & 1.84 & 79.34 & 1.95 & 19.84 \\
\hline 241-AP-106 & 5 & $6.75 \mathrm{E}+01$ & 94 & 1.25 & 352 & 19 & 18 & 9.95 & 0.99 & 86.11 & 1.31 & 34.54 \\
\hline $241-\mathrm{AP}-107$ & 5 & $1.06 E+03$ & 87 & 1.21 & 3695 & 17 & 14 & 6.67 & 0.60 & 101.71 & 1.17 & 61.92 \\
\hline 241-AP-108 & 27 & $8.00 E+02$ & 81 & 1.25 & 1397 & 19 & 16 & 7.83 & 0.74 & 93.55 & 1.21 & 49.38 \\
\hline $241-\mathrm{AW}-101$ & 5 & $1.00 \mathrm{~F}+01$ & 49 & 1.47 & 4263 & 16 & 34 & 24.06 & 2.31 & 40.39 & 2.26 & 6.46 \\
\hline $24 \mathrm{I}-\mathrm{AW}-\mathrm{I} 02$ & 3 & $7.43 \mathrm{E}+01$ & 88 & 1.15 & 303 & 26 & 21 & 4.81 & 0.44 & 75.16 & 1.10 & 51.68 \\
\hline $241-\mathrm{AW}-103$ & 138 & $5.12 \mathrm{E}+02$ & 93 & 1.02 & 1931 & 27 & 17 & 3.09 & 0.30 & 88.51 & 1.10 & 68.43 \\
\hline $241-\mathrm{AW}-104$ & 6 & $1.69 \Sigma+02$ & 82 & 1.06 & 4236 & 25 & 21 & 1.56 & 0.15 & 73.16 & 1.10 & 68.40 \\
\hline $241 \cdot A W-105$ & 43 & $1.01 \mathrm{E}+03$ & 93 & 1.02 & 1624 & 33 & 16 & 1.59 & 0.12 & 93.55 & 1.10 & 92.16 \\
\hline $241-\mathrm{AW}-106$ & 28 & $3.55 \mathrm{E}+02$ & 59 & 1.38 & 1791 & 33 & 32 & 14.42 & 1.34 & 44.63 & 1.60 & 16.10 \\
\hline $241-\mathrm{AY}-101$ & 5 & $1.13 E+02$ & 84 & 1.09 & 583 & 30 & 38 & 4.15 & 0.42 & 34.01 & 1.00 & 20.48 \\
\hline $241-\mathrm{AY}-102$ & 4 & $7.37 \mathrm{E}+01$ & 92 & 1.12 & 2355 & 21 & 37 & 3.56 & 0.43 & 35.19 & 1.00 & 19.36 \\
\hline $241-A Z-101$ & 1 & $1.25 \mathrm{E}+02$ & 72 & 1.22 & 3199 & 27 & 51 & 9.52 & 0.94 & 19.52 & 1.67 & 10.74 \\
\hline $241-\mathrm{AZ}-102$ & 6 & $4.38 \mathrm{E}+02$ & 84 & 1.14 & 3524 & 19 & 46 & 4.54 & 0.47 & 23.96 & 1.00 & 13.29 \\
\hline $241-S Y-101$ & 400 & $1.04 \mathrm{E}+03$ & 61 & 1.33 & 3685 & 30 & 32 & 14.89 & 1.37 & 43.96 & 1.63 & 15.79 \\
\hline $24 \mathrm{I}-\mathrm{SY}-102$ & 12 & $1.15 \mathrm{E}+03$ & 75 & 1.23 & 2317 & 15 & 22 & 9.20 & 0.87 & 69.34 & 1.28 & 32.86 \\
\hline
\end{tabular}




\section{RPP-5926 REV 6}

Table C-2. Calculations of Henry's Law Constant by Schumpe Model. (3 sheets)

\begin{tabular}{|c|c|c|c|c|c|c|c|c|c|c|c|c|}
\hline Tanks & $\begin{array}{c}\left(\mathrm{NH}_{3}\right) \text { ss } \\
\text { in dome } \\
(\mathrm{ppm})\end{array}$ & $\begin{array}{c}\text { I.iquid } \\
\mathrm{NH}_{3} \\
(\mu \mathrm{g} / \mathrm{nI} . .)\end{array}$ & $\begin{array}{c}\text { wt } \% \mathrm{H}_{2} \mathrm{O} \\
\text { in liquid } \\
(\%)\end{array}$ & $\begin{array}{c}\text { Spg } \\
\text { in Liquid } \\
(\mathrm{g} / \mathrm{ml})\end{array}$ & $\begin{array}{c}\text { Waste } \\
\text { Volume } \\
\text { (kL.) }\end{array}$ & $\begin{array}{c}\text { Head space } \\
\mathrm{T}\left({ }^{\circ} \mathrm{C}\right)\end{array}$ & $\begin{array}{c}\text { Waste } \mathrm{T} \\
\left({ }^{\circ} \mathrm{C}\right)\end{array}$ & $\begin{array}{c}\text { Sum of } \\
\text { ion conc. } \\
\text { Sum(Ci) } \\
\text { (mole/L) }\end{array}$ & $\begin{array}{l}\text { Sum of ion conc. } \\
\text { times ionic coeff. } \\
\text { Sum }\left(\mathrm{Ci}^{*} \mathrm{Hi}\right) \\
(\text { mole } / \mathrm{L})\end{array}$ & $\begin{array}{c}\text { KHo } \\
\text { in water } \\
\text { (mole } / \text { L-atm) }\end{array}$ & $\begin{array}{l}\text { KH in solns' } \\
\text { correction } \\
\text { factor }\end{array}$ & $\begin{array}{c}\mathrm{KH} \\
\text { in solns' } \\
\text { (mole/L-atm) }\end{array}$ \\
\hline $24 !-S Y-103$ & 59 & $1.29 E+03$ & 49 & 1.47 & 2817 & 16 & 31 & 19.55 & 1.93 & 45.77 & 2.25 & 10.57 \\
\hline $241-\mathrm{A}-101$ & 394 & $5.31 t+02$ & 55 & 1.49 & 3608 & 36 & 60 & 24.74 & 2.42 & 13.69 & 3.28 & 2.61 \\
\hline 24$]-A-103$ & 261 & $5.31 \mathrm{E}+02$ & 75 & 1.48 & 1404 & 36 & 58 & 19.95 & 1.98 & 14.96 & 3.28 & 4.70 \\
\hline $241-A X-101$ & 41 & $5.41 E+02$ & 48 & 1.49 & 2832 & 29 & 49 & 25.28 & 2.42 & 20.79 & 2.57 & 3.32 \\
\hline 241-B-203 & 5 & $1.00 \mathrm{E}+02$ & 89 & 1.17 & 193 & 12 & 12 & 5.28 & 0.44 & 115.89 & 1.00 & 76.38 \\
\hline $241-B-204$ & 5 & $1.00 \mathrm{E}+02$ & 89 & 1.05 & 189 & 14 & 13 & 5.11 & 0.43 & 107.29 & 1.00 & 70.65 \\
\hline $241-B Y-106$ & 79 & $1.03 E+03$ & 59 & 1.31 & 2127 & 25 & 49 & 14.76 & 1.52 & 21.17 & 2.50 & 8.12 \\
\hline $241-\mathrm{C}-103$ & 324 & $1.27 \mathrm{E}+03$ & 86 & 1.08 & 750 & 38 & 43 & 5.43 & 0.53 & 27.01 & 1.17 & 16.99 \\
\hline $241-5-101$ & 765 & $1.21 \mathrm{E}+03$ & 63 & 1.36 & 1616 & 31 & 43 & 18.34 & 1.73 & 26.82 & 2.27 & 8.66 \\
\hline $241-S-102$ & 588 & $1.70 \mathrm{E}+03$ & 50 & 139 & 1946 & 27 & 39 & 20.10 & 2.03 & 32.41 & $2.3 !$ & 6.54 \\
\hline $241-S-103$ & 149 & $1.41 \mathrm{E}+03$ & 50 & 1.45 & 939 & 23 & 28 & 20.88 & 1.96 & 53.20 & 2.26 & 13.35 \\
\hline $241-5-106$ & 14 & $2.40 \mathrm{E}+02$ & 51 & 1.46 & 1613 & 20 & 25 & 22.44 & 2.12 & 62.01 & 2.26 & 12.86 \\
\hline $241-5-111$ & 120 & $1.50 \mathrm{E}+03$ & 52 & 1.40 & 2044 & 23 & 28 & 18.67 & 1.79 & 51.99 & 2.24 & 14.83 \\
\hline $24 t-S X-101$ & 4 & 1. $32 \mathrm{E}+02$ & 49 & 1.46 & 1696 & 38 & 52 & 21.14 & 2.37 & 19.00 & 2.57 & 2.14 \\
\hline $241-\mathrm{SX}-102$ & 5 & $1.37 \mathrm{E}+03$ & 48 & 1.45 & 1946 & 33 & 55 & 21.34 & 2.13 & 16.63 & 3.28 & 4.30 \\
\hline $241-S X-103$ & 31 & $1.80 \mathrm{E}+03$ & 47 & 1.47 & 2400 & 31 & 62 & 21.45 & 2.17 & 12.84 & 3.28 & 3.10 \\
\hline $241-S X-104$ & 5 & $7.93 \mathrm{E}+02$ & 51 & 1.39 & 1768 & 29 & 46 & 22.36 & 2.07 & 23.85 & 2.57 & 6.23 \\
\hline 241-SX-105 & 27 & $1.21 \mathrm{E}+03$ & 43 & 1.47 & 2411 & 34 & 63 & 20.74 & 2.07 & 12.06 & 3.28 & 3.31 \\
\hline $241-S X-106$ & 177 & $2.50 \mathrm{E}+03$ & 46 & 1.42 & 1601 & 26 & 37 & 20.81 & 1.96 & 35.71 & 2.31 & 9.06 \\
\hline 241-SX-108 & 5 & $1.30 \mathrm{~F}+03$ & 47 & 1.43 & 329 & 53 & 72 & 20.35 & 1.88 & 8.86 & 4.04 & 4.48 \\
\hline $241-T-110$ & 107 & $1.50 \mathrm{E}+03$ & 87 & 1.17 & 1465 & 16 & 17 & 3.69 & 0.41 & 90.99 & 1.00 & 53.35 \\
\hline $241-T-203$ & 5 & $1.00 \mathrm{E}+02$ & 85 & 1.17 & 132 & 15 & 17 & 5.08 & 0.47 & 89.83 & 1.00 & 53.34 \\
\hline $24 !-U-102$ & 651 & $7.45 \mathrm{E}+02$ & 48 & 1.38 & 1420 & 24 & 28 & 20.47 & 1.88 & 53.34 & 2.26 & 15.39 \\
\hline 241-U-103 & 722 & $1.40 \mathrm{E}+03$ & 49 & 1.41 & 1772 & 25 & 28 & 19.59 & 1.97 & 51.99 & 2.26 & 11.13 \\
\hline
\end{tabular}


RPP-5926 REV 6

Table C-2. Calculations of Henry's Law Constant by Schumpe Model. (3 sheets)

\begin{tabular}{|c|c|c|c|c|c|c|c|c|c|c|c|c|}
\hline Tanks & $\begin{array}{c}\left(\mathrm{NH}_{3}\right) \mathrm{ss} \\
\text { in dome } \\
(\mathrm{ppm})\end{array}$ & $\begin{array}{c}\text { Liquid } \\
\mathrm{NH}_{3} \\
(\mu \mathrm{g} / \mathrm{mL})\end{array}$ & $\begin{array}{c}\text { wt } \% \mathrm{H}_{2} \mathrm{O} \\
\text { in liquid } \\
(\%)\end{array}$ & $\begin{array}{c}\text { Spg } \\
\text { in Liquid } \\
(\mathrm{g} / \mathrm{ml})\end{array}$ & $\begin{array}{c}\text { Waste } \\
\text { Volume } \\
(\mathrm{kL})\end{array}$ & $\begin{array}{c}\text { Head space } \\
\mathrm{T}\left({ }^{\circ} \mathrm{C}\right)\end{array}$ & $\begin{array}{c}\text { Waste } \mathrm{T} \\
\left({ }^{\circ} \mathrm{C}\right)\end{array}$ & $\begin{array}{l}\text { Sum of } \\
\text { ion conc. } \\
\text { Sum }(\mathrm{Ci}) \\
(\mathrm{mole} / \mathrm{L})\end{array}$ & $\begin{array}{l}\text { Surn of ion conc. } \\
\text { times ionic coeff. } \\
\text { Sum }\left(\mathrm{Ci}^{*} \mathrm{Hi}\right) \\
(\text { mole } / \mathrm{L})\end{array}$ & $\begin{array}{c}\text { KHo } \\
\text { in water } \\
(\text { mole } / \text { L-atm) }\end{array}$ & $\begin{array}{c}\text { KH in solns' } \\
\text { correction } \\
\text { factor }\end{array}$ & $\begin{array}{c}\text { Kll } \\
\text { in solns' } \\
\text { (mole/L-atm) }\end{array}$ \\
\hline $241-U-105$ & 1936 & $1.60 \mathrm{E}+03$ & 69 & 1.46 & 1582 & 25 & 30 & 18.50 & 1.80 & 48.15 & 2.10 & 12.29 \\
\hline $241-U-106$ & 940 & $1.05 \mathrm{E}+03$ & 49 & 1.36 & 856 & 24 & 26 & 17.81 & 1.60 & 57.66 & 2.01 & 21.04 \\
\hline $241+U-107$ & 448 & $4.03 \mathrm{E}+02$ & 58 & 1.41 & 1545 & 23 & 24 & 19.69 & 1.82 & 62.37 & 2.00 & 16.58 \\
\hline $241-U-108$ & 684 & $1.05 \mathrm{E}+03$ & 50 & 1.40 & 1772 & 24 & 28 & 21.29 & 2.06 & 51.99 & 2.26 & 10.70 \\
\hline $241-U-109$ & 5 & $1.10 \mathrm{E}+03$ & 52 & 1.47 & 1760 & 23 & 26 & 20.17 & 1.93 & 57.66 & 2.26 & 14.45 \\
\hline
\end{tabular}




\section{RPP-5926 REV 6}

Table C-3. Calculation of Mass Transport Product of $\mathrm{h}$ and A from Normal Ventilation Condition and Prediction for Off-Normal Condition. (3 sheets)

\begin{tabular}{|c|c|c|c|c|c|c|c|c|c|c|c|c|c|c|c|c|}
\hline Tanks & $\begin{array}{c}\text { Liquid } \\
\mathrm{NH}_{3} \mathrm{Cl} \\
(\mathrm{M} / \mathrm{L})\end{array}$ & $\begin{array}{c}\text { Ammonia } \\
\text { Partial } P_{\mathrm{NH} 3} \\
\text { (atm1) }\end{array}$ & $\begin{array}{c}\text { Steady } \\
\text { State } \\
\mathrm{NH}_{3} \mathrm{Co} \\
\text { (M) }\end{array}$ & $\begin{array}{c}\text { Molar } \\
\text { Volume } \\
\text { (Limole) }\end{array}$ & $\begin{array}{c}\text { Normal } \\
\text { Vent Q } \\
\text { (cfm) }\end{array}$ & $\begin{array}{c}\text { Ileadspace } \\
\text { Volume } V \\
\left(\mathrm{fl}^{3}\right)\end{array}$ & $\begin{array}{c}\text { Equiv }\left(\mathrm{NH}_{3}\right) \text { ss } \\
\text { in Liquid } \\
\text { (mole/L) }\end{array}$ & $\begin{array}{c}\text { Product hA } \\
\text { from Normal } \\
\text { Condition } \\
(\mathrm{cfm})\end{array}$ & $\begin{array}{c}\text { Normal } \\
\text { Steady-State } \\
\text { Release Rate } \\
\text { (cfm) }\end{array}$ & $\begin{array}{c}\text { Off-Normal } \\
\text { Barometric } \\
\text { Vent Q } \\
\text { (cfm) }\end{array}$ & $\begin{array}{c}\text { Off-normal } \\
\text { const. } \\
k l \text { (min-1) }\end{array}$ & $\begin{array}{c}\text { Off-normal } \\
\text { const. } k 2 \\
\text { (mole/L- } \\
\text { min) }\end{array}$ & $\begin{array}{c}\text { Off-Normal } \\
\left(\mathrm{NH}_{3}\right) \mathrm{ss} \\
(\mathrm{atm})\end{array}$ & $\begin{array}{c}\text { (NH3)ss } \\
\text { at off- } \\
\text { Normal } \\
\text { Vent } \\
\text { (ppm) }\end{array}$ & $\begin{array}{c}\text { Off-Norma! } \\
\text { Steady-State } \\
\text { Release Rate } \\
\text { (cfm) }\end{array}$ & $\begin{array}{c}\text { Time t to } \\
\text { reach } \\
\text { steady } \\
\text { state (day) }\end{array}$ \\
\hline $241-\mathrm{AN}-101$ & 0.002 & 0.000056 & $4.73 \mathrm{E}-07$ & 23.85 & 113 & 166,700 & 0.00047 & $2.85 \mathrm{E}-02$ & $1.28 \mathrm{E}-03$ & 0.52 & $1.74 \mathrm{E}-04$ & $4.02 \mathrm{E}-10$ & $5.50 \mathrm{E}-05$ & 55 & $2.86 \mathrm{E}-05$ & 49 \\
\hline 241-AN-102 & 0.012 & 0.000876 & $1.21 \mathrm{E}-05$ & 24.78 & 119 & 46,387 & 0.00422 & $1.78 \mathrm{E}-01$ & $3.57 \mathrm{E}-02$ & 0.14 & 1.34E-03 & $4.72 \mathrm{E}-08$ & $8.74 \mathrm{E}-04$ & 874 & 1.27E-04 & 5 \\
\hline 241-AN-103 & 0.020 & 0.004763 & $2.69 \mathrm{E}-07$ & 24.69 & 100 & 60,023 & 0.00003 & $1.33 \mathrm{E}-03$ & $6.65 \mathrm{E}-04$ & 0.19 & $5.46 \mathrm{E}-06$ & $4.49 \mathrm{E}-10$ & $2.03 \mathrm{E}-03$ & 2034 & $3.82 \mathrm{E}-04$ & 976 \\
\hline $24 !-A N-104$ & 0.014 & 0.002339 & $8.47 \mathrm{E}-07$ & 24.79 & 100 & 47,323 & 0.00013 & $5.92 \mathrm{E}-03$ & $2.10 \mathrm{E}-03$ & 0.15 & 2.23E-05 & $1.81 \mathrm{E}-09$ & $2.01 \mathrm{E}-03$ & 2011 & $2.97 \mathrm{E}-04$ & 250 \\
\hline $241-\mathrm{AN}-105$ & 0.012 & 0.001725 & 6.14E-07 & 24.66 & 100 & $3756430 \%$ & 0.00011 & $5.02 \mathrm{E}-03$ & $1.51 \mathrm{E}-03$ & 0.12 & $2.67 E-05$ & $1.65 E-09$ & $1.52 \mathrm{E}-03$ & 1523 & J.79E-04 & 271 \\
\hline $241-\mathrm{AN}-106$ & 0.012 & 0.000306 & $4.19[-08$ & 23.84 & 117 & 182,875 & 0.00004 & $3.99 \mathrm{E}-04$ & $1.17 \mathrm{E}-04$ & 0.57 & $5.22 \mathrm{E}-06$ & $2.69 \mathrm{E}-11$ & 1.23E-04 & 123 & $7.02 \mathrm{E}-05$ & 200 \\
\hline $241-A N-107$ & 0.012 & 0.000763 & $1.63 \mathrm{E}-05$ & 24.53 & 129 & 48,526 & 0.00646 & $3.58 \mathrm{E}-0 \mathrm{l}$ & $5.16 \mathrm{E}-02$ & 0.15 & $2.93 \mathrm{E}-03$ & $11 \mathrm{E}-08$ & $7.63 \mathrm{E}-04$ & 763 & $1.16 \mathrm{E}-04$ & 1 \\
\hline $241-\mathrm{AP}-101$ & 0.026 & 0.000832 & $5.21 \mathrm{E}-06$ & 23.98 & 108 & 39,035 & 0.00387 & $2.57 \mathrm{E}-02$ & $1.35 \mathrm{E}-02$ & 0.12 & $4.92 \mathrm{E}-04$ & $1.70 \mathrm{E}-08$ & $8.27 \mathrm{E}-04$ & 827 & $1.01 \mathrm{E}-04$ & 13 \\
\hline 241-AP-102 & 0.026 & 0.000734 & $3.13 \mathrm{E}-06$ & 24.02 & 135 & 42,109 & 0.00264 & $1.83 E-02$ & $1.02 \mathrm{E}-02$ & 0.13 & $3.69 \mathrm{E}-04$ & $1.12 \mathrm{E}-08$ & $7.28 \mathrm{E}-04$ & 728 & $9.57 \mathrm{E}-05$ & 13 \\
\hline 241-AP-103 & 0.015 & 0.000594 & 4.22E-08 & 23.68 & 200 & 149,990 & 0.00002 & $5.76 \mathrm{E}-04$ & $2.00 \mathrm{E}-04$ & 0.47 & $5.38 \mathrm{E}-06$ & $5.64 \mathrm{E}-11$ & $2.48 \mathrm{E}-04$ & 248 & $1.16 \mathrm{~F}-04$ & 822 \\
\hline $241-\mathrm{AP}-104$ & 0.008 & 0.000207 & $1.33 \mathrm{E}-07$ & 23.64 & 71 & 184,881 & 0.00012 & $1.23 \mathrm{E}-03$ & $2.23 E-04$ & 0.58 & $9.04 \mathrm{E}-06$ & $5.19 \mathrm{E}-11$ & $1.36 \mathrm{E}-04$ & 136 & $7.83 \mathrm{E}-05$ & 433 \\
\hline $241-A P=105$ & 0.019 & 0.000959 & $2.99 \mathrm{E}-07$ & 24.52 & 176 & 85,823 & 0.00015 & $2.79 \mathrm{E}-03$ & $1.29 \mathrm{E}-03$ & 0.27 & $1.89 \mathrm{E}-05$ & $6.18 \mathrm{E}-10$ & $8.00 \mathrm{E}-04$ & 800 & $2.15 \mathrm{E}-04$ & 281 \\
\hline 241-AP-106 & 0.004 & 0.000115 & $2.23 \mathrm{E}-07$ & 23.94 & 111 & 175,657 & 0.00018 & $6.54 \mathrm{E}-03$ & $5.92 \mathrm{E}-04$ & 0.55 & $3.39 \mathrm{E}-05$ & $1.48 \mathrm{E}-10$ & 1.04E-04 & 104 & $5.72 \mathrm{E}-0.5$ & 96 \\
\hline $241-\wedge P-107$ & 0.062 & 0.001005 & $2.10 \mathrm{E}-07$ & 23.81 & 342 & 57,616 & 0.00031 & $1.16 \mathrm{E}-03$ & $1.71 \mathrm{E}-03$ & 0.18 & $3.28 \mathrm{E}-05$ & $1.25 \mathrm{E}-09$ & $9.09 \mathrm{E}-04$ & 909 & $1.64 \mathrm{E}-04$ & 151 \\
\hline 241-AP-108 & 0.047 & 0.000951 & $1.11 \mathrm{E}-06$ & 23.96 & 129 & 138,761 & 0.00131 & $3.14 \mathrm{E}-03$ & $3.43 \mathrm{E}-03$ & 0.43 & $2.99 \mathrm{E}-05$ & $1.06 \mathrm{E}-09$ & $8.52 \mathrm{E}-04$ & 852 & $3.69 \mathrm{E}-04$ & 188 \\
\hline $241-\mathrm{AW}-10 \mathrm{I}$ & $0.00 !$ & 0.000091 & $2.17 \mathrm{E}-07$ & 23.70 & 100 & 37,564 & 0.00003 & $3.92 \mathrm{E}-02$ & $5.15 \mathrm{E}-04$ & 0.12 & $1.63 \mathrm{E}-04$ & $6.13 \mathrm{E}-10$ & $8.92 \mathrm{E}-05$ & 89 & $1.05 E-05$ & 22 \\
\hline $241-A W-102$ & 0.004 & 0.000084 & $1.04 \mathrm{~F}: 07$ & 24.55 & 292 & 177,394 & 0.00013 & $7.16 \mathrm{E}-03$ & 7.44E-04 & 0.55 & $5.44 \mathrm{E}-05$ & $1.76 \mathrm{E}-10$ & $7.96 \mathrm{E}-05$ & 80 & $4.41 \mathrm{E}-05$ & 95 \\
\hline 241-AW-103 & 0.030 & 0.000439 & $5.59 \mathrm{E}-06$ & 24.62 & 134 & 119,912 & 0.00942 & $3.63 \mathrm{E}-02$ & $1.85 \mathrm{E}-02$ & 0.37 & $5.13 \mathrm{E}-04$ & $9.10 \mathrm{E}-09$ & $4.37 \mathrm{E}-04$ & 437 & $1.64 \mathrm{E}-04$ & 13 \\
\hline $241-A W \cdot 104$ & 0.010 & 0.000145 & $2.45 \mathrm{E}-07$ & 24.46 & 141 & 38,500 & 0.00041 & $3.64 \mathrm{E}-03$ & $8.46 \mathrm{E}-04$ & 0.12 & $1.61 E-04$ & $9.37 \mathrm{E}-10$ & $1.42 \mathrm{E}-04$ & 142 & $1.71 \mathrm{E}-05$ & 25 \\
\hline $241-\mathrm{AW}-105$ & 0.059 & 0.000643 & $1.70 \mathrm{E}-06$ & 25.10 & 194 & 130,740 & 0.00394 & $5.97 \mathrm{E}-03$ & $8.30 \mathrm{E}-03$ & 0.41 & $1.09 \mathrm{E}-04$ & $2.71 \mathrm{E}-09$ & $6.25 \mathrm{E}-04$ & 625 & $2.55 \mathrm{E}-04$ & 43 \\
\hline $241-\wedge W-106$ & 0.021 & 0.001295 & $1.11 \mathrm{E}-06$ & 25.10 & 158 & 124,858 & 0.00045 & $8.59 \sqsubseteq-03$ & $4.40 \mathrm{E}-03$ & 0.39 & $3.09 \mathrm{~L}: 05$ & $1.43 \mathrm{E}-09$ & $1.16 \mathrm{E}-03$ & 1164 & 4.54E-04 & 184 \\
\hline $241-A Y-101$ & 0.007 & 0.000324 & $2.01 \mathrm{E}-07$ & 24.87 & 250 & 167,502 & 0.00010 & $7.69 \mathrm{E}-03$ & $1.25 \mathrm{E}-03$ & 0.52 & $2.65 \mathrm{E}-05$ & $3.05 \mathrm{E}-10$ & $2.86 \mathrm{E}-04$ & 286 & $1.50 \mathrm{E}-04$ & 188 \\
\hline $241-\mathrm{AY}-102$ & 0.004 & 0.000224 & $1.78 \mathrm{E}-07$ & 24.12 & 250 & 104,939 & 0.00008 & $1.05 \mathrm{E}-02$ & $1.07 \mathrm{E}-03$ & 0.33 & $4.97 \mathrm{E}-05$ & $4.32 \mathrm{E}-10$ & $2.09 E-04$ & 209 & $6.87 \mathrm{E}-05$ & 129 \\
\hline $241-\mathrm{A} Z-101$ & 0.007 & 0.000683 & $4.06 \mathrm{E}-08$ & $24.6 !$ & 150 & 75,129 & 0.00001 & $8.32 \mathrm{E}-04$ & $1.50 \mathrm{E}-04$ & 0.23 & $6.05 \mathrm{E}-06$ & $8.12 \mathrm{E}-1 \mathrm{~J}$ & $3.30 \mathrm{E}-04$ & 330 & $7.76 \mathrm{E}-05$ & 680 \\
\hline $241-A Z-102$ & 0.026 & 0.001936 & $2.31 \mathrm{E}-07$ & 23.96 & 112 & 63,632 & 0.00007 & $1.01 \mathrm{E}-03$ & $6.19 \mathrm{E}-04$ & 0.20 & $8.16 \mathrm{E}-06$ & $4.07 \mathrm{E}-10$ & $1.19 \mathrm{E}-03$ & 1195 & $2.38 \mathrm{~F}-04$ & 681 \\
\hline $241-S Y-101$ & 0.061 & 0.003883 & $1.61 \mathrm{E}-05$ & 24.86 & 486 & 57,834 & 0.00632 & $1.42 E-01$ & $1.94 \mathrm{E}-01$ & 0.18 & $9.68 \mathrm{E}-04$ & $1.51 \mathrm{E}-07$ & $3.87 \mathrm{E}-03$ & 3870 & $6.99 \mathrm{E}-04$ & 8 \\
\hline $241-S Y-102$ & 0.068 & 0.002055 & $4.95 \mathrm{E}-07$ & 23.59 & 363 & 106,276 & 0.00038 & $2.67 \mathrm{E}-03$ & $4.24 \mathrm{E}-03$ & 0.33 & $2.26 \mathrm{E}-05$ & $1.70 \mathrm{~F}-09$ & $1.77 \mathrm{E}-03$ & $177 !$ & $5.88 \mathrm{~F}, 04$ & 256 \\
\hline
\end{tabular}


RPP-5926 REV 6

Table C-3. Calculation of Mass Transport Product of $\mathrm{h}$ and A from Normal Ventilation Condition and Prediction for Off-Normal Condition. (3 sheets)

\begin{tabular}{|c|c|c|c|c|c|c|c|c|c|c|c|c|c|c|c|c|}
\hline Tanks & $\begin{array}{c}\text { Liquid } \\
\mathrm{NH}_{3} \mathrm{Cl} \\
(\mathrm{M} / \mathrm{L})\end{array}$ & $\begin{array}{c}\text { Ammonia } \\
\text { Partial } \mathrm{P}_{\mathrm{NH} 3} \\
(\mathrm{~atm})\end{array}$ & $\begin{array}{l}\text { Steady } \\
\text { State } \\
\mathrm{NH}_{3} \mathrm{Co} \\
\text { (M) }\end{array}$ & $\begin{array}{c}\text { Molar } \\
\text { Volume } \\
\text { (L/mole) }\end{array}$ & $\begin{array}{c}\text { Normal } \\
\text { Vent Q } \\
(\mathrm{cfm})\end{array}$ & $\begin{array}{c}\text { Headspace } \\
\text { Volume V } \\
\left(\mathrm{ft}^{3}\right)\end{array}$ & $\begin{array}{c}\text { Equiv }\left(\mathrm{NH}_{3}\right) \mathrm{ss} \\
\text { in Liquid } \\
\text { (mole/L) }\end{array}$ & $\begin{array}{l}\text { Product hA } \\
\text { from Normal } \\
\text { Condition } \\
\text { (cim) }\end{array}$ & $\begin{array}{c}\text { Normal } \\
\text { Steady-State } \\
\text { Release Rate } \\
\text { (cfm) }\end{array}$ & $\begin{array}{c}\text { Off-Normal } \\
\text { Barometric } \\
\text { Vent Q } \\
\text { (cfm) }\end{array}$ & $\begin{array}{l}\text { Off-normal } \\
\text { const. } \\
\text { kl }(\min -1)\end{array}$ & $\begin{array}{c}\text { Off-normal } \\
\text { const. k2 } \\
\text { (mole/L- } \\
\text { min) }\end{array}$ & $\begin{array}{c}\text { Off-Normal } \\
\left(\mathrm{NH}_{3}\right) \mathrm{ss} \\
(\mathrm{atm})\end{array}$ & $\begin{array}{c}\mathrm{NH} 3) \mathrm{ss} \\
\text { at off- } \\
\text { Normal } \\
\text { Vent } \\
(\mathrm{ppm})\end{array}$ & $\begin{array}{c}\text { Off-Normal } \\
\text { Steady-State } \\
\text { Release Rate } \\
\text { (cfm) }\end{array}$ & $\begin{array}{l}\text { Time t to } \\
\text { reach } \\
\text { steady } \\
\text { state (day) }\end{array}$ \\
\hline $241-S Y-103$ & 0.076 & 0.007169 & $2.50 \mathrm{E}-06$ & 23.74 & 116 & 88,630 & 0.00063 & $3.88 \mathrm{E}-03$ & $6.92 \mathrm{E}-03$ & 0.28 & $1.41 \mathrm{~F}-05$ & 3.32E-09 & $5.58 \mathrm{E}-03$ & 5581 & $1.55 \mathrm{E}-03$ & 487 \\
\hline $241-\mathrm{A}-101$ & 0.031 & 0.011934 & $1.56 \mathrm{E}-05$ & 25.32 & 10 & 48,793 & 0.00103 & $5.17 \mathrm{E}-03$ & $3.94 \mathrm{E}-03$ & 0.15 & $1.01 \mathrm{E}-05$ & $3.30 \mathrm{E}-09$ & $8.25 \mathrm{E}-03$ & 8253 & $1.26 \mathrm{E}-03$ & 709 \\
\hline $24 \mathrm{~J}-\mathrm{A}-103$ & 0.031 & 0.006640 & $1.03 \mathrm{E}-05$ & 25.32 & 8 & 126,596 & 0.00122 & $2.61 \mathrm{E}-03$ & $1.98 \mathrm{E}-03$ & 0.40 & $5.58 \mathrm{E}-06$ & $6.44 E-10$ & $2.92 \mathrm{E}-03$ & 2922 & $1.16 \mathrm{E}-03$ & 1058 \\
\hline 241-AX-101 & 0.032 & 0.009580 & $1.67 \mathrm{E}-06$ & 24.77 & 10 & 80,208 & 0.00014 & $5.29 \mathrm{E}-04$ & 4. $15 \mathrm{E}-04$ & 0.25 & $3.67 \mathrm{E}-06$ & $2.10 \mathrm{E}-10$ & $1.42 \mathrm{E}-03$ & 1416 & $3.55 \mathrm{E}-04$ & 1668 \\
\hline $241-B-203$ & 0.006 & 0.000077 & $2.14 \mathrm{E}-07$ & 23.40 & 0 & 1,069 & 0.00038 & $1.10 \mathrm{E}-05$ & $1.41 \mathrm{E}-06$ & 0.00 & $2.15 \mathrm{E}-05$ & $6.03 \mathrm{E}-11$ & $6.57 \mathrm{~F}-05$ & 66 & $2.20 \mathrm{E}-07$ & 187 \\
\hline $241-B-204$ & 0.006 & 0.000083 & $2.12 \mathrm{E}-07$ & 23.54 & 0 & 1,203 & 0.00035 & $1.05 \mathrm{E}-05$ & $1.36 \mathrm{E}-06$ & 0.00 & $1.76 \mathrm{E}-05$ & $5.10 \mathrm{E}-11$ & $6.83 \mathrm{E}-05$ & 68 & $2.57 \mathrm{E}-07$ & 171 \\
\hline $241-B Y-106$ & 0.060 & 0.007449 & $3.23 \mathrm{E}-06$ & 24.46 & 6 & 64,969 & 0.00064 & $3.18 E-04$ & $4.66 \mathrm{E}-04$ & 0.20 & $4.10 \mathrm{E}-06$ & $2.96 \mathrm{E}-10$ & $1.77 \mathrm{E}-03$ & 1769 & $3.59 \mathrm{E}-04$ & 470 \\
\hline $241-C-103$ & 0.071 & 0.004182 & $1.27 \mathrm{E}-05$ & 25.48 & 10 & 87,026 & 0.00550 & $1.94 \mathrm{E}-03$ & $3.24 \mathrm{E}-03$ & 0.27 & $1.28 \mathrm{E}-05$ & $1.58 \mathrm{E}-09$ & $3.16 \mathrm{E}-03$ & 3159 & $8.59 E-04$ & 470 \\
\hline $241-S+101$ & 0.071 & 0.008203 & $3.07 \mathrm{E}-05$ & 24.93 & 6 & 83,283 & 0.00662 & $2.94 \mathrm{E}-03$ & $4.72 \mathrm{E}-03$ & 0.26 & $1.07 \mathrm{E}-05$ & $2.51 \mathrm{E}-09$ & $5.82 \mathrm{E}-03$ & 5817 & $1.51 \mathrm{E}-03$ & 720 \\
\hline $241-S+102$ & 0.100 & 0.015269 & $2.39 \mathrm{E}-05$ & 24.64 & 2 & 71,653 & 0.00384 & $5.47 E-04$ & $1.29 \mathrm{E}-03$ & 0.22 & $4.35 \mathrm{E}-06$ & $7.62 \mathrm{E}-10$ & $4.31 \mathrm{E}-03$ & 4312 & $9.66 \mathrm{E}-04$ & 1450 \\
\hline $241-S-103$ & 0.083 & 0.006203 & $6.11 \mathrm{E}-06$ & 24.32 & 15 & 107,212 & 0.00198 & $1.16 \mathrm{E}-03$ & $2.28 \mathrm{E}-03$ & 0.34 & $6.63 \mathrm{E}-06$ & $8.95 \mathrm{E}-10$ & $3.28 \mathrm{E}-03$ & 3281 & $1.10 \mathrm{E}-03$ & 921 \\
\hline $241-S-106$ & 0.014 & 0.001096 & $5.72 \mathrm{E}-07$ & 24.08 & 9 & 83,417 & 0.00018 & $3.78 \mathrm{E}-04$ & $1.27 \mathrm{~F}-04$ & 0.26 & $4.53 \mathrm{E}-06$ & $6.38 \mathrm{E}-11$ & $3.40 \mathrm{E}-04$ & 340 & $8.85 \mathrm{E}-05$ & 1549 \\
\hline $241-\mathrm{S}-111$ & 0.088 & 0.005938 & 4.94E-06 & 24.30 & 5 & 68,177 & 0.00178 & $3.09 \mathrm{E}-04$ & $6.49 \mathrm{E}-04$ & 0.21 & $4.76 \mathrm{E}-06$ & $4.00 \mathrm{E}-10$ & $2.04 \mathrm{E}-03$ & 2041 & $4.35 \mathrm{~F}-04$ & 1316 \\
\hline 241-SX-101 & 0.008 & 0.003619 & $1.48 \mathrm{E}-07$ & 25.53 & 40 & 113,228 & 0.00001 & $7.62 \mathrm{E}-04$ & $1.51 \mathrm{E}-04$ & 0.35 & $3.49 \mathrm{E}-06$ & $5.22 \mathrm{E}-11$ & $3.81 \mathrm{E}-04$ & 381 & $1.35 \mathrm{E}-04$ & 1206 \\
\hline $241-S X-102$ & 0.080 & 0.018726 & $1.99 \mathrm{E}-07$ & 25.10 & 40 & 104,405 & 0.00002 & $9.91 \mathrm{E}-05$ & $2.00 \mathrm{E}-04$ & 0.33 & $3.23 \mathrm{E}-06$ & $7.63 \mathrm{E}-11$ & 5.94F-04 & 594 & $1.94 \mathrm{E}-04$ & 1696 \\
\hline $241-S X-103$ & 0.106 & 0.034140 & $1.23 \mathrm{E}-06$ & 24.93 & 53 & 88,363 & 0.00009 & $6.11 E-04$ & $1.6 \mathrm{~J}-03$ & 0.28 & $3.66 \mathrm{E}-06$ & $7.30 \mathrm{E}-10$ & $4.98 \mathrm{E}-03$ & 4977 & $1.37 \mathrm{E}-03$ & 1660 \\
\hline $241-S X-104$ & 0.047 & 0.007472 & $2.02 \mathrm{E}-07$ & 24.75 & 30 & 110,688 & 0.00003 & 1.30E-04 & $1.50 \mathrm{E}-04$ & 0.35 & -06 & 5.48 & $4.10 \mathrm{E}-04$ & 410 & 1.42 & 1360 \\
\hline 241-SX-105 & 0.071 & 0.021446 & $1.07 \mathrm{~F}-06$ & 25.19 & 40 & 87,962 & 0.00009 & $6.11 \mathrm{E}-04$ & $1.09 \mathrm{E}-03$ & 0.27 & $3.71 E-06$ & $4.94 \mathrm{E}-10$ & $3.36 \mathrm{E}-03$ & 3358 & $9.23 \mathrm{E}-04$ & 1789 \\
\hline $241-S X-106$ & 0.147 & 0.016198 & $7.22 \mathrm{E}-06$ & 24.53 & 39 & 116,570 & 0.00160 & $1.93 \mathrm{E}-03$ & $6.86 \mathrm{E}-03$ & 0.36 & $6.80 \mathrm{~F}-06$ & $2.43 \mathrm{E}-09$ & $8.75 \mathrm{E}-03$ & 8752 & $3.198-03$ & 962 \\
\hline 241-SX-108 & 0.076 & 0.017030 & $1.87 \mathrm{E}-07$ & 26.76 & 455 & 161,486 & 0.00002 & $1.11 \mathrm{E}-03$ & $2.28 \mathrm{E}-03$ & 0.50 & $3.95 E-06$ & $5.27 \mathrm{E}-10$ & 3.57E-03 & 3565 & $1.80 \mathrm{E}-03$ & 1463 \\
\hline $241-T-110$ & 0.088 & 0.001651 & 4.51E-06 & 23.69 & 7 & 61,761 & 0.00570 & $3.83 \mathrm{E}-04$ & $7.48 \mathrm{E}-04$ & 0.19 & $1.10 \mathrm{E}-05$ & $5.47 \mathrm{E}-10$ & 1.18E-03 & 1181 & $2.28 \mathrm{E}-04$ & 573 \\
\hline $241-\mathrm{T}-203$ & 0.006 & 0.000110 & $2.12 \mathrm{E}-07$ & 23.59 & 6 & 3,208 & 0.00027 & $2.28 \mathrm{E}-04$ & $3.02 \mathrm{E}-05$ & 0.01 & $9.27 \mathrm{E}-05$ & $4.18 \mathrm{E}-10$ & $1.06 \mathrm{E}-04$ & 106 & $1.07 \mathrm{E}-06$ & 36 \\
\hline $24 !-U-102$ & 0.044 & 0.002842 & $2.67 \mathrm{E}-05$ & 24.36 & 2 & 63,365 & 0.01002 & $1.66 \mathrm{E}-03$ & $1.37 \mathrm{E}-03$ & 0.20 & $1.30 \mathrm{E}-05$ & $1.15 \mathrm{E}-09$ & $2.16 \mathrm{E}-03$ & 2157 & 4.27Е-04 & 432 \\
\hline $241-U-103$ & 0.082 & 0.007383 & 2.95E-05 & 24.45 & 2 & 50,932 & 0.00804 & $9.56 \mathrm{E}-04$ & $1.73 \mathrm{E}-03$ & 0.16 & $8.24 \mathrm{E}-06$ & $1.54 \mathrm{E}-09$ & $4.58 \mathrm{E}-03$ & 4582 & $7.29 \mathrm{E}-04$ & 838 \\
\hline
\end{tabular}


RPP-5926 REV 6

Table C-3. Calculation of Mass Transport Product of h and A from Normal Ventilation Condition and Prediction for Off-Normal Condition. (3 sheets)

\begin{tabular}{|c|c|c|c|c|c|c|c|c|c|c|c|c|c|c|c|c|}
\hline Tanks & $\begin{array}{c}\text { Liquid } \\
\mathrm{NH}_{3} \mathrm{Cl} \\
(\mathrm{M} / \mathrm{L})\end{array}$ & $\begin{array}{c}\text { Amnonia } \\
\text { Partial } \mathrm{P}_{\mathrm{NH} 3} \\
\text { (atm) }\end{array}$ & $\begin{array}{l}\text { Steady } \\
\text { State } \\
\mathrm{NH}_{3} \mathrm{Co} \\
(\mathrm{M})\end{array}$ & $\begin{array}{c}\text { Molar } \\
\text { Volume } \\
\text { ( } \mathrm{L} \text { /mole) }\end{array}$ & $\begin{array}{c}\text { Normal } \\
\text { Vent Q } \\
(\mathrm{cfm})\end{array}$ & $\begin{array}{c}\text { Weadspace } \\
\text { Volume V } \\
\left(\mathrm{ft}^{3}\right)\end{array}$ & $\begin{array}{c}\text { Equiv }\left(\mathrm{NH}_{3}\right) \text { ss } \\
\text { in Liquid } \\
\text { (mole/I })\end{array}$ & $\begin{array}{c}\text { Product ha } \\
\text { from Normal } \\
\text { Condition } \\
\text { (cfm) }\end{array}$ & $\begin{array}{c}\text { Normal } \\
\text { Steady-State } \\
\text { Release Rate } \\
\text { (cfm) }\end{array}$ & $\begin{array}{c}\text { Off-Normal } \\
\text { Barometric } \\
\text { Vent Q } \\
\text { (cfm) }\end{array}$ & $\begin{array}{c}\text { Off-normal } \\
\text { const. } \\
\mathrm{kl} \text { (min-1) }\end{array}$ & $\begin{array}{c}\text { Off-normal } \\
\text { const. k2 } \\
\text { (mole/L- } \\
\text { min) }\end{array}$ & $\begin{array}{c}\text { Off-Normal } \\
\left(\mathrm{NH}_{3}\right) \mathrm{ss} \\
(\mathrm{atm})\end{array}$ & $\begin{array}{c}\text { (NH3)ss } \\
\text { at off- } \\
\text { Normal } \\
\text { Vent } \\
\text { (ppm) }\end{array}$ & $\begin{array}{c}\text { Otf-Normal } \\
\text { Steady-State } \\
\text { Release Rate } \\
\text { (cfm) }\end{array}$ & $\begin{array}{c}\text { Time t to } \\
\text { reach } \\
\text { steady } \\
\text { state (day) }\end{array}$ \\
\hline $241-U-105$ & 0.094 & 0.007642 & $7.91 \mathrm{E}-05$ & 24.47 & 5 & 57,616 & 379 & & $9.68 \mathrm{E}-03$ & 0.18 & $3.26 \mathrm{E}-05$ & $9.19 \mathrm{E}-09$ & $91 \mathrm{E}-03$ & 6909 & -03 & 199 \\
\hline $241-U \cdot 106$ & 0.062 & 0.002931 & $3.86 \mathrm{E}-05$ & 24.33 & 1 & 83,283 & 0.01977 & $1.20 \mathrm{E}-03$ & $1.22 \mathrm{E}-03$ & 0.26 & $1.05 \mathrm{~F}-05$ & $8.88 \mathrm{E}-10$ & $2.06 \mathrm{E}-03$ & 2058 & $5.36 \mathrm{E}-04$ & 521 \\
\hline $241 \cdot U-107$ & 0.024 & 0.001428 & $1.85 \mathrm{E}-05$ & 24.26 & 12 & 58,953 & 0.00743 & 1. $34 \mathrm{E}-02$ & $5.27 \mathrm{E}-03$ & 0.18 & $9.45 \mathrm{E}-05$ & $5.37 \mathrm{E}-09$ & 1.38E-03 & 1380 & $2.54 \mathrm{E}-04$ & 52 \\
\hline $241-U-108$ & 0.062 & 0.005764 & $2.81 \mathrm{E}-05$ & 24.36 & 4 & 50,932 & 0.00732 & $1.87 \mathrm{E} .03$ & $2.47 E-03$ & 0.16 & $1.27 \mathrm{E}-05$ & $2.26 \mathrm{E}-09$ & $4.34 \mathrm{E}-03$ & 4345 & $6.92 E-04$ & 538 \\
\hline $24 !-U-109$ & 0.065 & 0.004469 & $2.06 \mathrm{E}-07$ & 24.28 & 4 & 51,333 & 0.00007 & J.37E-05 & $2.14 \mathrm{E}-05$ & 0.16 & $3.22 \mathrm{E}-06$ & $1.72 \mathrm{E}-11$ & $1.30 \mathrm{E}-04$ & 130 & $2.08 \mathrm{E}-05$ & 1423 \\
\hline
\end{tabular}


RPP-5926 REV 6

This page intentionally left blank. 


\section{RPP-5926 REV 6}

APPENDIX D

\section{CALCULATION RESULTS OF STEADY-STATE FLAMMABILITY EVALUATIONS IN TANK DOME SPACES FOR 177 HANFORD TANKS}


RPP-5926 REV 6

This page intentionally left blank.

D-ii 


\section{RPP-5926 REV 6}

\section{CONTENTS}

Table D-1 Steady-State Flammability Calculation Under Normal Ventilation Conditions for Current Waste at Documented Safety Analysis Conditions.

Table D-2 Steady-State Flammability Calculations With Barometric Breathing Rate for Current Waste at Documented Safety Analysis Conditions.

Table D-3 Time to Reach $25 \%$ of the Lower Flammability Limit With Barometric Breathing Rate for Current Waste at Documented Safety Analysis Conditions. ... D-19

Table D-4 Time to Reach $100 \%$ of the Lower Flammability Limit Under Barometric Breathing Rate for Current Waste at Documented Safety Analysis Conditions. ... D-27

Table D-5 Time to Reach $25 \%$ and $100 \%$ of the Lower Flammability Limit Under Zero Vent Rate at Documented Safety Analysis Conditions D-35 
RPP-5926 REV 6

This page intentionally left blank.

D-2 
RPP-5926 REV 6

Table D-1. Steady-State Flammability Calculation Under Normal Ventilation Conditions for Current Waste at Documented Safety Analysis Conditions. (8 sheets)

\begin{tabular}{|c|c|c|c|c|c|c|c|c|c|c|c|}
\hline Tank & $\begin{array}{c}\text { Ammonia } \\
\text { headspace } \\
\text { concentration } \\
{\left[\mathrm{NH}_{3}\right]} \\
(\%) \\
\end{array}$ & $\begin{array}{c}\text { Methane } \\
\text { headspace } \\
\text { concentration } \\
{\left[\mathrm{CH}_{4}\right]} \\
(\%) \\
\end{array}$ & $\begin{array}{c}\mathrm{N}_{2} \mathrm{O} \text { headspace } \\
\text { concentration } \\
{\left[\mathrm{N}_{2} \mathrm{O}\right]} \\
(\%)\end{array}$ & $\begin{array}{c}\text { Waste } \\
\text { volume } \\
\mathrm{V}_{\mathrm{w}} \\
\text { (kgal) }\end{array}$ & $\begin{array}{c}\text { Headspace } \\
\text { volume } \\
V_{h} \\
\left(\mathrm{ft}^{3}\right)\end{array}$ & $\begin{array}{c}\text { Normal } \\
\text { ventilation } \\
\text { rate } \\
(\mathrm{Vr})_{\text {norm }} \\
(\mathrm{cfm}) \\
\end{array}$ & $\begin{array}{c}\text { Steady-state } \\
\text { flammability } \\
\text { at normal } \\
\text { condition } \\
\text { (\% LFL) }\end{array}$ & $\begin{array}{l}\text { Ammonia } \\
\text { release rate } \\
\left.\text { Rr(NH})_{3}\right) \\
(\mathrm{cfm})\end{array}$ & $\begin{array}{c}\mathrm{N}_{2} \mathrm{O} \\
\text { release rate } \\
\operatorname{Rr}\left(\mathrm{N}_{2} \mathrm{O}\right) \\
(\mathrm{cfm})\end{array}$ & 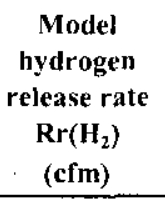 & $\begin{array}{c}\text { Methane } \\
\text { release rate } \\
\operatorname{Rr}\left(\mathrm{CH}_{4}\right) \\
(\mathrm{cfm})\end{array}$ \\
\hline $241-\mathrm{AN}-101$ & 0.0011 & 0.0002 & 0.0011 & 967 & 58,777 & 113.0 & $0.06 \%$ & $1.28 \mathrm{E}-03$ & $1.20 \mathrm{E}-03$ & $2.38 \mathrm{~F}-03$ & $1.92 \mathrm{E}-04$ \\
\hline 241-AN- 102 & 0.0300 & 0.0003 & 0.0053 & 1,080 & 43,663 & 119.0 & $0.53 \%$ & $3.57 \mathrm{E}-02$ & $6.31 \mathrm{E}-03$ & $1.56 \mathrm{E}-02$ & $3.19 \mathrm{E} \cdot 04$ \\
\hline $241-\mathrm{AN}-103$ & 0.0007 & 0.0002 & 0.0007 & 968 & 58,671 & 100.0 & $0.10 \%$ & $6.65 E-04$ & $6.50 \mathrm{E}-04$ & $3.84 \mathrm{E}-03$ & $2.05 \mathrm{E}-04$ \\
\hline 241-AN-104 & 0.0021 & 0.0002 & 0.0007 & 1,062 & 46,100 & 100.0 & $0.14 \%$ & $2.10 \mathrm{E}-03$ & $7.09 \mathrm{E}-04$ & 4.91E-03 & $1.95 \mathrm{E}-04$ \\
\hline 241-AN-105 & 0.0015 & 0.0002 & 0.0013 & 1,136 & 36,283 & 100.0 & $0.09 \%$ & $1.51 \mathrm{E}-03$ & $1.28 \mathrm{E}-03$ & $3.14 \mathrm{E}-03$ & $2.20 \mathrm{E}-04$ \\
\hline $241-\mathrm{AN}-106$ & 0.0000 & 0.0000 & 0.0002 & 813 & 79,364 & 117.0 & $0.16 \%$ & $0.00 \mathrm{E}+00$ & $2.32 \mathrm{E}-04$ & $7.25 \mathrm{E}-03$ & $7.25 \mathrm{E}-04$ \\
\hline 241-AN-107 & 0.0400 & 0.0005 & 0.0053 & 1,111 & 39,532 & 129.0 & $0.52 \%$ & $5.16 \mathrm{E}-02$ & $6.84 \mathrm{E}-03$ & 1.24E-02 & $6.29 \mathrm{E}-04$ \\
\hline 241-AP-301 & 0.0125 & 0.0003 & 0.0061 & 1,124 & 37,766 & 108.0 & $0.11 \%$ & $1.35 \mathrm{E}-02$ & $6.63 \mathrm{E}-03$ & $1.00 \mathrm{E}-03$ & $2.01 E-04$ \\
\hline 241-AP-102 & 0.0075 & 0.0002 & 0.0048 & 1,103 & 40,662 & 135.0 & $0.11 \%$ & $1.02 \mathrm{E}-02$ & $6.50 \mathrm{E}-03$ & $2.80 \mathrm{E}-03$ & $3.29 \mathrm{E}-04$ \\
\hline $241-\mathrm{AP}-103$ & 0.0000 & 0.0000 & 0.0008 & 902 & 67,464 & 200.0 & $0.02 \%$ & $0.00 \mathrm{E}+00$ & $1.70 \mathrm{E}-03$ & $1.75 \mathrm{E}-03$ & $8.58 \mathrm{E}-05$ \\
\hline 241-AP-104 & 0.0003 & 0.00001 & 0.0002 & 1,108 & 40,026 & 71.0 & $0.06 \%$ & $2.23 \mathrm{E}-04$ & $1.43 \mathrm{E}-04$ & $1.68 \mathrm{E}-03$ & $7.23 \mathrm{E}-06$ \\
\hline $241-\wedge P-105$ & 0.0007 & 0.0001 & 0.0026 & 1,146 & 34,835 & 176.0 & $0.03 \%$ & $1.29 \mathrm{E}-03$ & $4.55 \mathrm{E}-03$ & $1.68 \mathrm{E}-03$ & $2.30 \mathrm{E}-04$ \\
\hline 241-AP-106 & 0.0005 & 0.00003 & 0.0005 & 1,146 & 34,871 & 111.0 & $0.05 \%$ & $5.92 \mathrm{E}-04$ & $5.53 \mathrm{E}-04$ & $1.98 \mathrm{E}-03$ & $2.80 \mathrm{E}-05$ \\
\hline $241-\mathrm{AP}-107$ & 0.0005 & 0.0001 & 0.0017 & 355 & 140,632 & 342.0 & $0.01 \%$ & $1.71 \mathrm{E}-03$ & $5.81 \mathrm{E}-03$ & $3.33 \mathrm{E}-04$ & $6.67 \mathrm{E}-05$ \\
\hline 241-AP-108 & 0.0027 & 0.0000 & 0.0017 & 1,033 & 50,020 & 129.0 & $0.08 \%$ & $3.43 \mathrm{E}-03$ & $2.20 \mathrm{E}-03$ & $2.96 \mathrm{E}-03$ & $1.11 \mathrm{E}-05$ \\
\hline 24l-AW-101 & 0.0005 & 0.0002 & 0.0007 & 1,137 & 36,036 & 100.0 & $0.09 \%$ & $5.15 \mathrm{E}-04$ & $7.29 \mathrm{E}-04$ & $3.16 \mathrm{E}-03$ & $1.56 \mathrm{E}-04$ \\
\hline $241-A W-102$ & 0.0003 & 0.0000 & 0.0002 & 616 & 105,778 & 292.0 & $0.01 \%$ & $7.44 \mathrm{E}-04$ & $4.76 \mathrm{E}-04$ & $6.50 \mathrm{E}-04$ & $2.41 E-05$ \\
\hline $241-\mathrm{AW}-103$ & 0.0138 & 0.0004 & 0.0009 & 1,106 & 40,238 & 134.0 & $0.12 \%$ & $1.85 \mathrm{E}-02$ & 1.23E-03 & $9.70 \mathrm{E}-04$ & $1.94 \mathrm{E}-04$ \\
\hline $24 \mathrm{l}-\mathrm{AW}-104$ & 0.0006 & 0.0007 & 0.0009 & 1,084 & 43,240 & 141.0 & $0.06 \%$ & $8.46 \mathrm{E}-04$ & $1.30 \mathrm{E}-03$ & $2.32 \mathrm{E}-03$ & $4.63 E-04$ \\
\hline $241-A W-105$ & 0.0043 & 0.0002 & 0.0009 & 429 & 130,744 & 194.0 & $0.04 \%$ & $8.30 \mathrm{E}-03$ & $1.78 \mathrm{E}-03$ & 3.19E-04 & $6.37 \mathrm{E}-05$ \\
\hline 241-AW-106 & 0.0028 & 0.0001 & 0.0018 & 1,145 & 35,047 & 158.0 & $0.05 \%$ & $4.40 \mathrm{E}-03$ & $2.81 \mathrm{E}-03$ & $1.58 \mathrm{E}-03$ & $1.42 \mathrm{E}-04$ \\
\hline $241-A Y-101$ & 0.0005 & 0.0001 & 0.0009 & 228 & 157,617 & 250.0 & $0.07 \%$ & $1.25 \mathrm{E}-03$ & $2.21 \mathrm{E}-03$ & $6.88 \mathrm{E}-03$ & $1.42 \mathrm{E}-04$ \\
\hline $241-\mathrm{AY}-102$ & 0.0004 & 0.0001 & 0.0010 & 978 & 57,400 & 250.0 & $0.31 \%$ & $1.07 \mathrm{E}-03$ & $2.50 \mathrm{E}-03$ & $3.00 \mathrm{E}-02$ & $3.61 \mathrm{E}-04$ \\
\hline $241-A Z-101$ & 0.0001 & 0.0005 & 0.0010 & 874 & 71,242 & 150.0 & $0.44 \%$ & $1.88 \mathrm{E}-04$ & $1.50 \mathrm{E}-03$ & $2.56 \mathrm{E}-02$ & $7.79 \mathrm{E}-04$ \\
\hline
\end{tabular}




\section{RPP-5926 REV 6}

Table D-1. Steady-State Flammability Calculation Under Normal Ventilation Conditions for Current Waste at Documented Safety Analysis Conditions. (8 sheets)

\begin{tabular}{|c|c|c|c|c|c|c|c|c|c|c|c|}
\hline Tank & $\begin{array}{c}\text { Ammonia } \\
\text { headspace } \\
\text { concentration } \\
{\left[\mathrm{NH}_{3}\right]} \\
(\%)\end{array}$ & $\begin{array}{c}\text { Methane } \\
\text { headspace } \\
\text { concentration } \\
{\left[\mathrm{CH}_{4}\right]} \\
(\%)\end{array}$ & $\begin{array}{c}\mathrm{N}_{2} \mathrm{O} \text { headspace } \\
\text { concentration } \\
{\left[\mathrm{N}_{2} \mathrm{O}\right]} \\
(\%)\end{array}$ & $\begin{array}{c}\text { Waste } \\
\text { volume } \\
V_{w} \\
\text { (kgal) }\end{array}$ & $\begin{array}{c}\text { Headspace } \\
\text { volume } \\
\mathrm{V}_{\mathrm{h}} \\
\left(\mathrm{ft}^{3}\right)\end{array}$ & $\begin{array}{l}\text { Normal } \\
\text { ventilation } \\
\text { rate } \\
(V r)_{\text {norm }} \\
(\mathrm{cfm})\end{array}$ & $\begin{array}{l}\text { Steady-state } \\
\text { flammability } \\
\text { at normal } \\
\text { condition } \\
\text { ( } \% \text { LFL) }\end{array}$ & $\begin{array}{c}\text { Ammonia } \\
\text { release rate } \\
\operatorname{Rr}\left(\mathrm{NH}_{3}\right) \\
(\mathrm{cfm})\end{array}$ & $\begin{array}{c}\mathrm{N}_{2} \mathrm{O} \\
\text { release rate } \\
\mathrm{Rr}\left(\mathrm{N}_{2} \mathrm{O}\right) \\
(\mathrm{cfm})\end{array}$ & $\begin{array}{c}\text { Model } \\
\text { hydrogen } \\
\text { release rate } \\
\operatorname{Rr}\left(\mathrm{H}_{2}\right) \\
(\mathrm{cfm})\end{array}$ & $\begin{array}{l}\text { Methane } \\
\text { release rate } \\
\left.\operatorname{Rr}_{(\mathrm{CH}}\right) \\
(\mathrm{cfm})\end{array}$ \\
\hline 241-AZ-102 & 0.0006 & 0.0008 & 0.0010 & 978 & 57,400 & 112.0 & $0.82 \%$ & $6.19 \mathrm{E}-04$ & $1.12 \mathrm{E}-03$ & $3.6 ! \mathrm{E}-02$ & $8.58 \mathrm{E}-04$ \\
\hline $241-S Y-101$ & 0.0200 & 0.0001 & 0.0005 & 1,141 & 35,612 & 461.0 & $0.14 \%$ & $9.22 \mathrm{E}-02$ & $2.46 \mathrm{E}-03$ & $6.57 \mathrm{E}-04$ & $1.31 \mathrm{E}-04$ \\
\hline 241-SY-102 & 0.0012 & 0.0004 & 0.0005 & 1,144 & 35,153 & 363.0 & $0.03 \%$ & $4.24 \mathrm{E}-03$ & $1.95 \mathrm{E}-03$ & $1.37 \mathrm{E}-03$ & $2.74 \mathrm{E}-04$ \\
\hline $241 \cdot S Y-103$ & 0.0059 & 0.0003 & 0.0016 & 749 & 87,981 & 116.4 & $0.15 \%$ & $6.92 \mathrm{E}-03$ & $1.87 \mathrm{E}-03$ & $5.10 \mathrm{E}-03$ & $3.15 \mathrm{E}-04$ \\
\hline $241-\mathrm{A}-101^{*}$ & 0.0394 & 0.0006 & 0.0134 & 335 & 131,294 & 10.0 & $0.66 \%$ & $3.94 \mathrm{E}-03$ & $1.34 \mathrm{E}-03$ & $1.53 \mathrm{E}-03$ & $5.51 \mathrm{E}-05$ \\
\hline $241-\Lambda-102$ & 0.0254 & 0.0002 & 0.0224 & 50 & 169,386 & 7.6 & $0.29 \%$ & $1.93 \mathrm{E}-03$ & $1.71 \mathrm{E}-03$ & $3.60 \mathrm{E}-04$ & $1.31 \mathrm{E}-05$ \\
\hline $241-\mathrm{A}-103 *$ & 0.0261 & 0.0004 & 0.0220 & 389 & 124,080 & 10.0 & $0.56 \%$ & $2.61 \mathrm{E}-03$ & $2.20 \mathrm{E}-03$ & $1.52 \mathrm{E}-03$ & $3.92 \mathrm{E}-05$ \\
\hline $241-A-104$ & 0.0005 & 0.0003 & 0.0040 & 38 & 170,975 & 10.0 & $0.07 \%$ & $5.00 \mathrm{E}-05$ & $4.02 \mathrm{E}-04$ & $2.32 \mathrm{E}-04$ & $2.58 \mathrm{E}-05$ \\
\hline $241-A-105$ & na & na & $\mathrm{na}$ & na & na & na & na & na & na & na & na \\
\hline $241-\mathrm{A}-106$ & 0.0275 & 0.0012 & 0.0198 & 89 & 164,160 & 10.0 & $0.68 \%$ & $2.75 \mathrm{E}-03$ & $1.98 \mathrm{E}-03$ & $1.88 \mathrm{E}-03$ & $1.15 \mathrm{E}-04$ \\
\hline 241-AX-101 & 0.0041 & 0.0002 & 0.0006 & 367 & 129,213 & 28.3 & $0.12 \%$ & $1.17 \mathrm{E}-03$ & $1.81 \mathrm{E}-04$ & $1.02 \mathrm{E}-03$ & $5.95 \mathrm{E}-05$ \\
\hline 241-AX-102 & 0.0034 & 0.0001 & 0.0050 & 40 & 173,001 & 16.5 & $0.12 \%$ & $5.55 \mathrm{E}-04$ & $8.23 \mathrm{E}-04$ & $6.17 \mathrm{E}-04$ & $9.04 \mathrm{E}-06$ \\
\hline 241-AX-103 & 0.0041 & 0.0002 & 0.0024 & 116 & 162,760 & 24.7 & $0.12 \%$ & $1.02 \mathrm{E}-03$ & $5.85 \mathrm{E}-04$ & $8.92 \mathrm{E}-04$ & $4.94 \mathrm{E}-05$ \\
\hline $241-\mathrm{AX}-104$ & 0.0007 & 0.0001 & 0.0016 & 17 & 176,002 & 7.0 & $1.62 \%$ & $5.18 \mathrm{E}-05$ & $1.15 \mathrm{E}-04$ & $4.50 \mathrm{E}-03$ & $7.37 \mathrm{E}-06$ \\
\hline $241-\mathrm{B}-101$ & 0.0013 & 0.0004 & 0.0086 & 119 & 97,777 & 7.8 & $0.15 \%$ & $9.81 \mathrm{E}-05$ & $6.72 \mathrm{E}-04$ & $4.13 \mathrm{E}-04$ & $3.40 \mathrm{E}-05$ \\
\hline $241-\mathrm{B}-102$ & 0.0005 & 0.0001 & 0.0063 & 42 & 108,018 & 7.0 & $0.09 \%$ & $3.28 \mathrm{E}-05$ & $4.40 \mathrm{E}-04$ & $2.39 \mathrm{E}-04$ & $9.64 \mathrm{E}-06$ \\
\hline $241-B-103$ & 0.0008 & 0.0003 & 0.0061 & 66 & 104,839 & 7.0 & $0.10 \%$ & $5.82 \mathrm{E}-05$ & $4.26 \mathrm{E}-04$ & $2.42 \mathrm{E}-04$ & $1.78 \mathrm{E}-05$ \\
\hline 241-B-104 & 0.0005 & 0.0049 & 0.0076 & 384 & 62,253 & 7.0 & $0.23 \%$ & $3.50 \mathrm{E}-05$ & $5.31 \mathrm{E} .04$ & $3.57 \mathrm{E}-04$ & 7.15E-05 \\
\hline $241-B-105$ & 0.0012 & 0.0005 & 0.0092 & 300 & 73,517 & 7.0 & $0.13 \%$ & $8.40 \mathrm{E}-05$ & $6.45 \mathrm{E}-04$ & $3.19 \mathrm{E}-04$ & $3.27 \mathrm{E} \sim 05$ \\
\hline $241-B-106$ & 0.0005 & 0.0015 & 0.0240 & 133 & 95,905 & 7.0 & $0.14 \%$ & $3.50 \mathrm{E}-05$ & $1.68 \mathrm{E}-03$ & $3.06 \mathrm{E}-04$ & $6.12 \mathrm{E}-05$ \\
\hline $241 \cdot B \cdot 107$ & 0.0021 & 0.0007 & 0.0017 & 171 & 90,714 & 7.0 & $0.13 \%$ & 1.47E.04 & $1.19 \mathrm{E}-04$ & $2.80 \mathrm{E}-04$ & $4.95 \mathrm{E}-05$ \\
\hline $241-\mathrm{B}-108$ & 0.0034 & 0.0004 & 0.0087 & 102 & 99,931 & 7.0 & $0.12 \%$ & $2.40 \mathrm{E}-04$ & $6.08 \mathrm{E}-04$ & $2.54 \mathrm{E}-04$ & $3.00 \mathrm{E}-05$ \\
\hline $241-B-109$ & 0.0042 & 0.0006 & 0.0117 & 135 & 95,517 & 7.0 & $0.13 \%$ & $2.92 \mathrm{E}-04$ & $8.21 \mathrm{E}-04$ & $2.64 \mathrm{E}-04$ & $4.05 \mathrm{E}-05$ \\
\hline $241-B-110$ & 0.0050 & 0.0032 & 0.0050 & 255 & 79,485 & 7.0 & $0.29 \%$ & $3.50 \mathrm{E}-04$ & $3.49 \mathrm{E}-04$ & $5.37 \mathrm{E}-04$ & $1.07 E-04$ \\
\hline
\end{tabular}


RPP-5926 REV 6

Table D-1. Steady-State Flammability Calculation Under Normal Ventilation Conditions for Current Waste at Documented Safety Analysis Conditions. (8 sheets)

\begin{tabular}{|c|c|c|c|c|c|c|c|c|c|c|c|}
\hline Tank & $\begin{array}{c}\text { Ammonia } \\
\text { headspace } \\
\text { concentration } \\
{\left[\mathrm{NH}_{3}\right]} \\
(\%)\end{array}$ & $\begin{array}{c}\text { Methane } \\
\text { headspace } \\
\text { concentration } \\
{\left[\mathrm{CH}_{4}\right]} \\
(\%)\end{array}$ & $\begin{array}{c}\mathrm{N}_{2} \mathrm{O} \text { headspace } \\
\text { concentration } \\
{\left[\mathrm{N}_{2} \mathrm{O}\right]} \\
(\%)\end{array}$ & $\begin{array}{c}\text { Waste } \\
\text { volume } \\
\mathrm{V}_{\mathrm{w}} \\
\text { (kgal) }\end{array}$ & $\begin{array}{c}\text { Headspace } \\
\text { volume } \\
V_{h} \\
\left(\mathrm{ft}^{3}\right)\end{array}$ & $\begin{array}{l}\text { Normal } \\
\text { ventilation } \\
\text { rate } \\
(\mathrm{Vr})_{\text {norm }} \\
(\mathrm{cfm})\end{array}$ & $\begin{array}{c}\text { Steady-state } \\
\text { flammability } \\
\text { at normal } \\
\text { condition } \\
\text { (\% LFL) }\end{array}$ & $\begin{array}{c}\text { Ammonia } \\
\text { release rate } \\
\operatorname{Rr}\left(\mathrm{NH}_{3}\right) \\
(\mathrm{cfm})\end{array}$ & $\begin{array}{c}\mathrm{N}_{2} \mathrm{O} \\
\text { release rate } \\
\operatorname{Rr}\left(\mathrm{N}_{2} \mathrm{O}\right) \\
(\mathrm{cfm})\end{array}$ & $\begin{array}{c}\text { Model } \\
\text { hydrogen } \\
\text { release rate } \\
\mathrm{Rr}\left(\mathrm{H}_{2}\right) \\
(\mathrm{cfm})\end{array}$ & $\begin{array}{c}\text { Methane } \\
\text { release rate } \\
\operatorname{Rr}\left(\mathrm{CH}_{4}\right) \\
(\mathrm{cfm})\end{array}$ \\
\hline $241-B-111$ & 0.0025 & 0.0058 & 0.0090 & 251 & 80,015 & 3.5 & $0.79 \%$ & $8.79 \mathrm{E}-05$ & $3.15 \mathrm{E}-04$ & $9.21 \mathrm{E}-04$ & $1.84 \mathrm{E}-04$ \\
\hline $241-B-112$ & 0.0031 & 0.0004 & 0.0068 & 45 & 107,629 & 7.0 & $0.13 \%$ & 2. $14 \mathrm{E}-04$ & 4.741E-04 & 2.69E-04 & $3.04 \mathrm{E}-05$ \\
\hline $241-B-201$ & 0.0005 & 0.0004 & 0.0006 & 30 & 3,887 & 7.0 & $0.03 \%$ & $3.50 \mathrm{E}-05$ & 4.16E-05 & $5.91 E-05$ & $1.18 \mathrm{E}-05$ \\
\hline $241-B-202$ & 0.0003 & 0.0003 & 0.0017 & 30 & 3,993 & 7.2 & $0.03 \%$ & $1.92 \mathrm{E}-05$ & $1.23 E-04$ & $5.57 区-05$ & $1.11 \mathrm{E}-05$ \\
\hline $241-B-203$ & 0.0005 & 0.0007 & 0.0105 & 51 & 1,097 & 0.3 & $1.77 \%$ & $1.4 ! \mathrm{E}-06$ & $2.95 E-05$ & $1.98 \mathrm{E}-04$ & $1.89 \mathrm{E}-06$ \\
\hline $241-B-204$ & 0.0005 & 0.0007 & 0.0103 & 50 & 1,203 & 0.3 & $1.88 \%$ & $1.36 \mathrm{E}-06$ & $2.79 \mathrm{E}-05$ & $2.03 E-04$ & $1.79 \mathrm{E}-06$ \\
\hline $241-B X+101$ & 0.0050 & 0.0006 & 0.0088 & 58 & 105,800 & 7.0 & $0.15 \%$ & $3.50 \mathrm{E}-04$ & $6.17 \mathrm{E}-04$ & $2.88 \mathrm{E}-04$ & $3.96 \mathrm{E}-05$ \\
\hline $241-B X-102$ & 0.0003 & 0.0013 & 0.0017 & 89 & 101,634 & 7.0 & $0.12 \%$ & $1.79 \mathrm{E}-05$ & 1.191-04 & $2.65 \mathrm{E}-04$ & $5.31 \mathrm{E}-05$ \\
\hline $241-B X-103$ & 0.0090 & 0.0009 & 0.0079 & 85 & 102,163 & 7.0 & $0.19 \%$ & $6.27 \mathrm{E}-04$ & $5.52 \mathrm{E}-04$ & $3.10 \mathrm{E}-04$ & $6.19 \mathrm{E}-05$ \\
\hline $241-B X-104$ & 0.0212 & 0.0010 & 0.0130 & 110 & 98,738 & 8.7 & $0.27 \%$ & $1.85 \mathrm{E}-03$ & $1.14 \mathrm{E}-03$ & $3.71 \mathrm{E}-04$ & $7.42 \mathrm{E}-05$ \\
\hline $241-B X-105$ & 0.0145 & 0.0006 & 0.0116 & 82 & 102,552 & 8.5 & $0.19 \%$ & $1.23 \mathrm{E}-03$ & $9.76 \mathrm{E}-04$ & $2.87 \mathrm{E}-04$ & 4.70E-05 \\
\hline 241-BX-106 & 0.0047 & 0.0005 & 0.0043 & 48 & 107,107 & 7.0 & $0.14 \%$ & $3.26 \mathrm{E}-04$ & $3.02 \mathrm{E}-04$ & $2.77 \mathrm{E}-04$ & $3.50 \mathrm{E}-05$ \\
\hline $24 !-B X-107$ & 0.0082 & 0.0004 & 0.0012 & 357 & 65,791 & 7.0 & $0.21 \%$ & $5.74 \mathrm{E}-04$ & $8.34 \mathrm{E}-05$ & $4.05 \mathrm{E}-04$ & $2.75 \mathrm{E}-05$ \\
\hline $241-B X-108$ & 0.0024 & 0.0003 & 0.0053 & 41 & 107,955 & 7.0 & $0.11 \%$ & $1.68 \mathrm{E}-04$ & $3.73 \mathrm{E}-04$ & $2.56 \mathrm{E}-04$ & $2.40 \mathrm{E}-05$ \\
\hline $241-\mathrm{BX}-109$ & 0.0020 & 0.0025 & 0.0396 & 203 & 86,379 & 7.0 & $0.25 \%$ & $1.40 \mathrm{E}-04$ & $2.77 \mathrm{E}-03$ & $5.09 \mathrm{E}-04$ & $1.02 E-04$ \\
\hline $241-B X-110$ & 0.0063 & 0.0008 & 0.0017 & 224 & 83,483 & 7.0 & $0.19 \%$ & $4.40 \mathrm{E}-04$ & $1.19 \mathrm{E}-04$ & $3.83 \mathrm{E}-04$ & $5.57 \mathrm{E}-05$ \\
\hline $241-B X-111$ & 0.0062 & 0.0002 & 0.0017 & 198 & 86,979 & 7.0 & $0.15 \%$ & $4.35 E-04$ & 1.19E-04 & $2.90 \mathrm{E}-04$ & 1.15E-05 \\
\hline $241-B X-112$ & 0.0122 & 0.0022 & 0.0339 & 174 & 90,192 & 7.0 & $0.25 \%$ & $8.51 \mathrm{E}-04$ & $2.37 \mathrm{E}-03$ & 3.37E-04 & $6.75 \mathrm{E}-05$ \\
\hline 241-BY-101 & 0.0059 & 0.0010 & 0.0056 & 380 & 89,455 & 7.0 & $0.30 \%$ & 4.15E-04 & $3.91 \mathrm{E}-04$ & $6.83 \mathrm{E}-04$ & $6.80 \mathrm{E}-05$ \\
\hline 241-BY-102 & 0.0173 & 0.0004 & 0.0017 & 288.4 & 101,673 & 7.0 & $0.25 \%$ & $1.21 \mathrm{E}-03$ & 1.22E-04 & $3.49 \mathrm{E}-04$ & $2.75 \mathrm{E}-05$ \\
\hline $241-B Y-103$ & 0.0029 & 0.0002 & 0.0017 & 423 & 83,664 & 14.4 & $0.10 \%$ & 4.12E-04 & $2.43 \mathrm{E}-04$ & $4.26 \mathrm{E}-04$ & $2.75 \mathrm{E}-05$ \\
\hline $241-B Y-104$ & 0.0259 & 0.0007 & 0.0250 & 415 & 84,758 & 7.6 & $0.41 \%$ & $1.98 \mathrm{E}-03$ & $1.92 \mathrm{E}-03$ & $6.79 \mathrm{E}-04$ & $5.41 \mathrm{E}-05$ \\
\hline $241-B Y-105$ & 0.0083 & 0.0004 & 0.0076 & 491 & 74,588 & 15.9 & $0.16 \%$ & $1.32 \mathrm{E}-03$ & $1.21 \mathrm{E}-03$ & $5.85 \mathrm{E}-04$ & $6.93 \mathrm{E}-05$ \\
\hline $241-B Y-106$ & 0.0079 & 0.0005 & 0.0132 & 439 & 81,580 & 5.9 & $0.38 \%$ & $4.64 E-04$ & $7.75 \mathrm{E}-04$ & $7.51 \mathrm{E}-04$ & $2.89 \mathrm{E}-05$ \\
\hline
\end{tabular}


RPP-5926 REV 6

Table D-1. Steady-State Flammability Calculation Under Normal Ventilation Conditions for Current Waste at Documented Safety Analysis Conditions. (8 sheets)

\begin{tabular}{|c|c|c|c|c|c|c|c|c|c|c|c|}
\hline Tank & $\begin{array}{c}\text { Ammonia } \\
\text { headspace } \\
\text { concentration } \\
{\left[\mathrm{NH}_{3}\right]} \\
(\%) \\
\end{array}$ & $\begin{array}{c}\text { Methane } \\
\text { headspace } \\
\text { concentration } \\
{\left[\mathrm{CH}_{4}\right]} \\
(\%) \\
\end{array}$ & $\begin{array}{c}\mathrm{N}_{2} \mathrm{O} \text { headspace } \\
\text { concentration } \\
{\left[\mathrm{N}_{2} \mathrm{O}\right]} \\
(\%)\end{array}$ & $\begin{array}{c}\text { Waste } \\
\text { volume } \\
V_{w} \\
\text { (kgal) }\end{array}$ & $\begin{array}{c}\text { Headspace } \\
\text { volume } \\
V_{h} \\
\left(\mathrm{ft}^{3}\right)\end{array}$ & $\begin{array}{c}\text { Normal } \\
\text { ventilation } \\
\text { rate } \\
(\mathrm{Vr})_{\mathrm{nnrm}} \\
(\mathrm{cfm}) \\
\end{array}$ & $\begin{array}{l}\text { Steady-state } \\
\text { flammability } \\
\text { at normal } \\
\text { condition } \\
(\% \text { LFL }) \\
\end{array}$ & $\begin{array}{c}\text { Ammonia } \\
\text { release rate } \\
\operatorname{Rr}\left(\mathrm{NH}_{3}\right) \\
(\mathbf{c f m})\end{array}$ & $\begin{array}{c}\mathrm{N}_{2} \mathrm{O} \\
\text { release rate } \\
\operatorname{Rr}\left(\mathrm{N}_{2} \mathrm{O}\right) \\
(\mathrm{cfm})\end{array}$ & $\begin{array}{c}\text { Model } \\
\text { hydrogen } \\
\text { release rate } \\
\operatorname{Rr}\left(\mathrm{H}_{2}\right) \\
(\mathrm{cfm}) \\
\end{array}$ & $\begin{array}{c}\text { Methane } \\
\text { release rate } \\
\operatorname{Rr}\left(\mathrm{CH}_{4}\right) \\
(\mathrm{cfm})\end{array}$ \\
\hline 241-BY-107 & 0.0962 & 0.0010 & 0.0704 & 282 & 102,591 & 5.2 & $0.88 \%$ & $5.03 \mathrm{E}-03$ & $3.68 \mathrm{E}-03$ & $4.62 \mathrm{E}-04$ & $5.20 \mathrm{E}-05$ \\
\hline 241-BY-108 & 0.0870 & 0.0008 & 0.0553 & 232 & 109,195 & 6.8 & $0.74 \%$ & $5.88 \mathrm{E}-03$ & $3.74 \mathrm{E}-03$ & $4.03 \mathrm{E}-04$ & $5.16 \mathrm{E}-05$ \\
\hline 241-BY-109 & 0.0045 & 0.0002 & 0.0011 & 296.9 & 100,543 & 14.7 & $0.09 \%$ & $6.57 \mathrm{E}-04$ & $1.57 \mathrm{E}-04$ & $3.44 \mathrm{E}-04$ & $3.11 \mathrm{E}-05$ \\
\hline $241-B Y \cdot 110$ & 0.0397 & 0.0003 & 0.0102 & 376 & 90,020 & 13.3 & $0.47 \%$ & $5.30 \mathrm{E}-03$ & $1.37 \mathrm{E}-03$ & $1.07 \mathrm{E}-03$ & 4.36E-05 \\
\hline 241-BY-111 & 0.0059 & 0.0003 & 0.0082 & 412 & 85,112 & 6.0 & $0.20 \%$ & $3.51 \mathrm{E}-04$ & $4.93 \mathrm{E}-04$ & $3.70 E-04$ & $1.65 \mathrm{E}-05$ \\
\hline $241-B Y-112$ & 0.0063 & 0.0001 & 0.0039 & 296 & 100,649 & 13.6 & $0.11 \%$ & $8.55 \mathrm{E}-04$ & $5.34 \mathrm{E}-04$ & $3.38 \mathrm{E}-04$ & $1.72 \mathrm{E}-05$ \\
\hline $241 \cdot C-101$ & 0.0098 & 0.0012 & 0.0636 & 98 & 100,531 & 3.6 & $0.32 \%$ & $3.52 \mathrm{E}-04$ & $2.29 \mathrm{E}-03$ & $3.33 \mathrm{E}-04$ & $4.30 \mathrm{E}-05$ \\
\hline $241-\mathrm{C}-102$ & 0.0187 & 0.0008 & 0.0141 & 326 & 70,057 & 11.8 & $0.25 \%$ & $2.20 \mathrm{E}-03$ & $1.66 \mathrm{E}-03$ & $5.07 \mathrm{E}-04$ & $9.81 \mathrm{E}-05$ \\
\hline $241-\mathrm{C}-103^{*}$ & 0.0324 & 0.0016 & 0.0737 & 37 & 108,724 & 10.0 & $0.49 \%$ & $3.24 \mathrm{E}-03$ & $7.37 \mathrm{E}-03$ & $9.57 \mathrm{E}-04$ & $1.58 \mathrm{E}-04$ \\
\hline $241-\mathrm{C}-104$ & 0.0044 & 0.0004 & 0.0063 & 269 & 77,684 & 67.1 & $0.10 \%$ & $2.96 \mathrm{E}-03$ & 4.24E-03 & $1.63 \mathrm{E}-03$ & $2.72 \mathrm{E}-04$ \\
\hline 24$)-C-105$ & 0.0002 & 0.0000 & 0.0005 & 142 & 94,634 & 535.0 & $0.01 \%$ & $1.29 \mathrm{E}-03$ & $2.81 \mathrm{E}-03$ & $8.94 \mathrm{E}-04$ & $1.24 \mathrm{E}-04$ \\
\hline $241-\mathrm{C}-106$ & 0.0002 & 0.0001 & 0.0004 & 13 & 111,920 & 176.1 & $0.02 \%$ & $4.38 \mathrm{E}-04$ & $6.49 \mathrm{E}-04$ & $9.91 \mathrm{E}-04$ & $9.86 \mathrm{E}-05$ \\
\hline $241-\mathrm{C}-107$ & 0.0066 & 0.0010 & 0.0096 & 257 & 79,273 & 1.1 & $6.76 \%$ & $7.24 \mathrm{E}-05$ & $1.05 \mathrm{E}-04$ & $2.95 \mathrm{E}-03$ & $1.08 \mathrm{E}-05$ \\
\hline $241-C-108$ & 0.0003 & 0.0000 & 0.0178 & 76 & 103,462 & 7.0 & $0.12 \%$ & $1.84 \mathrm{E}-05$ & $1.25 \mathrm{E}-03$ & $3.41 \mathrm{E}-04$ & $2.17 \mathrm{E}-06$ \\
\hline $241-C-109$ & 0.0010 & 0.0001 & 0.0365 & 73 & 103,815 & 13.0 & $0.17 \%$ & $1.30 \mathrm{E}-04$ & $4.76 \mathrm{E}-03$ & $8.60 \mathrm{E}-04$ & $1.19 \mathrm{E}-05$ \\
\hline $241-\mathrm{C}-110$ & 0.0123 & 0.0031 & 0.0014 & 188 & 88,490 & 7.9 & $0.38 \%$ & $9.69 \mathrm{E}-04$ & $1.08 \mathrm{E}-04$ & $7.32 \mathrm{E}-04$ & $1.46 \mathrm{E}-04$ \\
\hline $241-\mathrm{C}-111$ & 0.0004 & $0.000 !$ & 0.0053 & 67 & 104,628 & 7.0 & $0.59 \%$ & $2.74 \mathrm{E}-05$ & $3.73 \mathrm{E}-04$ & $1.65 \mathrm{E}-03$ & $3.54 \mathrm{E}-06$ \\
\hline $241 \cdot \mathrm{C}-112$ & 0.0023 & 0.0001 & 0.0538 & 114 & 98,413 & 11.2 & $0.39 \%$ & $2.52 \mathrm{E}-04$ & $6.02 \mathrm{E}-03$ & $1.65 \mathrm{E}-03$ & $1.12 \mathrm{E}-05$ \\
\hline $241-C-201$ & 0.0001 & 0.0000 & 0.0007 & 1 & 7,788 & 3.9 & $0.03 \%$ & 4.02E-06 & $2.87 \mathrm{E}-05$ & $4.48 \mathrm{E}-05$ & $1.84 \mathrm{E}-06$ \\
\hline $241-\mathrm{C}-202$ & 0.0001 & 0.0000 & 0.0004 & 1 & 7,787 & 3.6 & $0.03 \%$ & $2.48 \mathrm{E}-06$ & $1.44 \mathrm{E}-05$ & 4.54E-05 & $9.22 \mathrm{E}-07$ \\
\hline $241-C-203$ & 0.0005 & 0.0001 & 0.0010 & 1 & 7,788 & 7.0 & $0.02 \%$ & $3.23 \mathrm{E}-05$ & $7.18 \mathrm{E}-05$ & $4.26 \mathrm{E}-05$ & 4.61E-06 \\
\hline $241-\mathrm{C}-204$ & 0.0001 & 0.0001 & 0.0014 & 2 & 7,608 & 3.0 & $0.02 \%$ & $2.18 \mathrm{E}-06$ & $4.31 \mathrm{E}-05$ & $1.87 \mathrm{E}-05$ & $2.76 \mathrm{E}-06$ \\
\hline $241-S-10]^{*}$ & 0.0765 & 0.0007 & 0.0197 & 362 & 91,990 & 6.2 & $0.93 \%$ & $4.72 \mathrm{E}-03$ & $1.21 \mathrm{E}-03$ & $1.00 \mathrm{E}-03$ & $4.36 \mathrm{E}-05$ \\
\hline $241-S-102^{*}$ & 0.0588 & 0.0008 & 0.0394 & 213 & 111,942 & 2.2 & $1.07 \%$ & $1.29 \mathrm{E}-03$ & $8.68 \mathrm{E}-04$ & $5.79 \mathrm{E}-04$ & $1.83 \mathrm{E}-05$ \\
\hline
\end{tabular}


Table D-1. Steady-State Flammability Calculation Under Normal Ventilation Conditions for Current Waste at Documented Safety Analysis Conditions. (8 sheets)

\begin{tabular}{|c|c|c|c|c|c|c|c|c|c|c|c|}
\hline Tank & $\begin{array}{c}\text { Ammonia } \\
\text { headspace } \\
\text { concentration } \\
{\left[\mathrm{NH}_{3}\right]} \\
(\%)\end{array}$ & $\begin{array}{c}\text { Methane } \\
\text { headspace } \\
\text { concentration } \\
{\left[\mathrm{CH}_{4}\right]} \\
(\%)\end{array}$ & $\begin{array}{c}\mathrm{N}_{2} \mathrm{O} \text { headspace } \\
\text { concentration } \\
{\left[\mathrm{N}_{2} \mathrm{O}\right]} \\
(\%)\end{array}$ & $\begin{array}{c}\text { Waste } \\
\text { volume } \\
V_{\mathrm{w}} \\
\text { (kgal) }\end{array}$ & $\begin{array}{c}\text { Headspace } \\
\text { volume } \\
V_{n} \\
\left(\mathrm{ft}^{3}\right)\end{array}$ & $\begin{array}{l}\text { Normal } \\
\text { ventilation } \\
\text { rate } \\
(\mathrm{Vr})_{\text {norm }} \\
(\mathrm{cfm})\end{array}$ & $\begin{array}{l}\text { Steady-state } \\
\text { flammability } \\
\text { at normal } \\
\text { condition } \\
\text { ( } \% \text { LFL) }\end{array}$ & 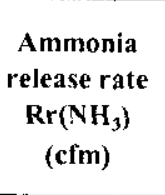 & $\begin{array}{c}\mathrm{N}_{2} \mathrm{O} \\
\text { release rate } \\
\operatorname{Rr}\left(\mathrm{N}_{2} \mathrm{O}\right) \\
(\mathbf{c f m})\end{array}$ & $\begin{array}{c}\text { Model } \\
\text { hydrogen } \\
\text { release rate } \\
\operatorname{Rr}\left(\mathrm{H}_{2}\right) \\
(\mathrm{cfm})\end{array}$ & $\begin{array}{c}\text { Methane } \\
\text { release rate } \\
\operatorname{Rr}\left(\mathrm{CH}_{4}\right) \\
(\mathrm{cfm})\end{array}$ \\
\hline $24 !-S-103$ & 0.0149 & 0.0005 & 0.0135 & 247 & 107,386 & 15.3 & $0.19 \%$ & $2.28 \mathrm{E}-03$ & $2.06 \mathrm{E}-03$ & $5.07 \mathrm{E}-04$ & $6.96 \mathrm{E}-05$ \\
\hline $241-S-104$ & 0.0025 & 0.0047 & 0.0073 & 298 & 100,536 & 5.6 & $0.39 \%$ & $1.40 \mathrm{E}-04$ & 4. $10 \mathrm{E}-04$ & $6.18 \mathrm{E}-04$ & $1.24 \mathrm{E}-04$ \\
\hline $241-S-105$ & 0.0036 & 0.0002 & 0.0008 & 416 & 84,786 & 16.7 & $0.08 \%$ & $6.00 \mathrm{E}-04$ & $1.25 \mathrm{E}-04$ & $3.71 \mathrm{E}-04$ & $3.84 \mathrm{E}-05$ \\
\hline $241-S-106$ & 0.0014 & 0.0016 & 0.0006 & 465 & 78,183 & 9.2 & $0.18 \%$ & $1.27 \mathrm{E}-04$ & $5.11 \mathrm{E}-05$ & $5.23 \mathrm{E}-04$ & $1.05 \mathrm{E}-04$ \\
\hline $241-S-107$ & 0.0181 & 0.0010 & 0.0184 & 368 & 91,142 & 4.4 & $0.54 \%$ & $7.93 \mathrm{E}-04$ & $8.08 \mathrm{E}-04$ & $7.07 \mathrm{E}-04$ & $4.45 \mathrm{E}-05$ \\
\hline $241-S-108$ & 0.0025 & 0.0001 & 0.0003 & 560 & 65,506 & 7.0 & $0.17 \%$ & $1.77 \mathrm{E}-04$ & $2.08 \mathrm{E}-05$ & $4.28 \mathrm{E}-04$ & $7.66 \mathrm{E}-06$ \\
\hline $241-S-109$ & 0.0025 & 0.0196 & 0.0021 & 543 & 67,801 & 7.0 & $0.56 \%$ & $1.74 \mathrm{E}-04$ & $1.48 \mathrm{E}-04$ & $4.28 \mathrm{E}-04$ & $8.56 \mathrm{E}-05$ \\
\hline $241-S-110$ & 0.0146 & 0.0005 & 0.0039 & 399 & 87,011 & 10.4 & $0.24 \%$ & $1.52 \mathrm{E}-03$ & $4.07 \mathrm{E}-04$ & $5.41 \mathrm{E}-04$ & 5.19E-05 \\
\hline $241-S-111$ & 0.0120 & 0.0003 & 0.0021 & 411 & 85,457 & 5.4 & $0.38 \%$ & $6.49 \mathrm{E}-04$ & $1.14 \mathrm{E}-04$ & $6.36 \mathrm{E}-04$ & $1.50 \mathrm{E}-05$ \\
\hline $241-S-112$ & 0.0088 & 0.0003 & 0.0006 & 15 & 138,355 & 4.0 & $0.22 \%$ & $3.52 \mathrm{E}-04$ & $2.53 \mathrm{E}-05$ & $2.56 \mathrm{E}-04$ & $1.05 \mathrm{E}-05$ \\
\hline 241-SX-101 & 0.0004 & 0.0002 & 0.0007 & 428 & 116,110 & 40.0 & $0.05 \%$ & $1.51 \mathrm{E}-04$ & $2.74 \mathrm{E}-04$ & $7.00 \mathrm{E}-04$ & $6.57 \mathrm{E}-05$ \\
\hline $241-S X-102$ & 0.0000 & 0.0002 & 0.0006 & 351 & 126,421 & 40.0 & $0.10 \%$ & $0.00 \mathrm{E}+00$ & $2.40 \mathrm{E}-04$ & $1.55 \mathrm{E}-03$ & $8.00 \mathrm{E}-05$ \\
\hline $241-S X-103^{*}$ & 0.0031 & 0.0005 & 0.0010 & 519 & 104,033 & 52.5 & $0.36 \%$ & $1.61 \mathrm{E}-03$ & $5.41 \mathrm{E}-04$ & $6.89 \mathrm{E}-03$ & $2.38 \mathrm{E}-04$ \\
\hline $241-S X-104$ & 0.0005 & 0.0002 & 0.0007 & 456 & 112,437 & 30.0 & $0.16 \%$ & $1.50 \mathrm{E}-04$ & $2.00 \mathrm{E}-04$ & $1.83 \mathrm{E}-03$ & $6.53 \mathrm{E}-05$ \\
\hline $241-S X-105$ & 0.0027 & 0.0002 & 0.0008 & 386 & 121,831 & 40.5 & $0.34 \%$ & $1.09 \mathrm{E}-03$ & $3.38 \mathrm{E}-04$ & $5.17 \mathrm{E}-03$ & $7.38 \mathrm{E}-05$ \\
\hline 241-SX-106 & 0.0177 & 0.0003 & 0.0023 & 407 & 119,041 & 38.8 & $0.18 \%$ & $6.86 \mathrm{E}-03$ & $9.10 \mathrm{E}-04$ & $9.24 \mathrm{E}-04$ & $1.07 \mathrm{E}-04$ \\
\hline 24$]-S X-107$ & 0.0002 & 0.0000 & 0.0004 & 104 & 159,474 & 387.0 & $0.01 \%$ & $6.73 \mathrm{E}-04$ & $1.49 E-03$ & $1.41 \mathrm{E} \cdot 03$ & $9.58 \mathrm{E}-05$ \\
\hline 241-SX-108 & $0.000 !$ & 0.0000 & 0.0003 & 84 & 162,157 & 455.0 & $0.01 \%$ & $5.63 \mathrm{E}-04$ & 1.25E-03 & $7.61 \mathrm{E}-04$ & $8.02 \mathrm{E}-05$ \\
\hline $241-S X-109$ & 0.0017 & 0.0001 & 0.0009 & 251 & 139,805 & 30.0 & $0.20 \%$ & $5.05 \mathrm{E}-04$ & $2.72 \mathrm{E}-04$ & $2.25 \mathrm{E}-03$ & $3.75 \mathrm{E}-05$ \\
\hline $241-\mathrm{SX}-110$ & 0.0001 & 0.0000 & 0.0003 & 66 & 164,559 & 293.0 & $0.02 \%$ & $4.01 \mathrm{E}-04$ & $8.90 \mathrm{E}-04$ & $2.43 \mathrm{E}-03$ & $5.71 \mathrm{E}-05$ \\
\hline 241-SX-111 & 0.0003 & 0.0000 & 0.0007 & 125 & 156,649 & 264.0 & $0.02 \%$ & $7.89 \mathrm{E}-04$ & $1.75 \mathrm{E}-03$ & $1.61 \mathrm{E}-03$ & $1.12 \mathrm{E}-04$ \\
\hline $24 I-\$ X-112$ & 0.0003 & 0.0000 & 0.0007 & 85 & 162,052 & 225.0 & $0.01 \%$ & $6.99 \mathrm{E}-04$ & $1.55 \mathrm{E}-03$ & $1.05 \mathrm{E}-03$ & $9.95 \mathrm{E}-05$ \\
\hline $241-S X-113$ & 0.0029 & 0.0004 & 0.0064 & 29 & 169,502 & 7.0 & $0.11 \%$ & $2.01 \mathrm{E}-04$ & $4.45 \mathrm{E}-04$ & $2.45 \mathrm{E}-04$ & $2.86 \mathrm{E}-05$ \\
\hline $241-\mathrm{SX}-114$ & 0.0004 & 0.0001 & 0.0008 & 165 & 151,281 & 325.0 & $0.02 \%$ & $1.17 \mathrm{E}-03$ & $2.60 \mathrm{E}-03$ & $2.35 \mathrm{E}-03$ & $1.67 \mathrm{E}-04$ \\
\hline
\end{tabular}


RPP-5926 REV 6

Table D-1. Steady-State Flammability Calculation Under Normal Ventilation Conditions for Current Waste at

Documented Safety Analysis Conditions. (8 sheets)

\begin{tabular}{|c|c|c|c|c|c|c|c|c|c|c|c|}
\hline Tank & $\begin{array}{c}\text { Ammonia } \\
\text { headspace } \\
\text { concentration } \\
{\left[\mathrm{NH}_{3}\right]} \\
(\%)\end{array}$ & $\begin{array}{c}\text { Methane } \\
\text { headspace } \\
\text { concentration } \\
{\left[\mathrm{CH}_{4}\right]} \\
(\%)\end{array}$ & $\begin{array}{c}\mathrm{N}_{2} \mathrm{O} \text { headspace } \\
\text { concentration } \\
{\left[\mathrm{N}_{2} \mathrm{O}\right]} \\
(\%)\end{array}$ & $\begin{array}{c}\text { Waste } \\
\text { volume } \\
V_{w} \\
\text { (kgal) }\end{array}$ & $\begin{array}{c}\text { Headspace } \\
\text { volume } \\
\mathrm{V}_{\mathrm{h}} \\
\left(\mathrm{ft}^{3}\right)\end{array}$ & $\begin{array}{l}\text { Normal } \\
\text { ventilation } \\
\text { rate } \\
(\mathrm{Vr})_{\text {norm }} \\
(\mathrm{cfm})\end{array}$ & $\begin{array}{l}\text { Steady-state } \\
\text { flammability } \\
\text { at normal } \\
\text { condition } \\
\text { (\% LFL) }\end{array}$ & $\begin{array}{c}\text { Ammonia } \\
\text { release rate } \\
\operatorname{Rr}\left(\mathrm{NH}_{3}\right) \\
(\mathrm{cfm})\end{array}$ & $\begin{array}{c}\mathrm{N}_{2} \mathrm{O} \\
\text { release rate } \\
\operatorname{Rr}\left(\mathrm{N}_{2} \mathrm{O}\right) \\
(\mathrm{cfm})\end{array}$ & $\begin{array}{c}\text { Model } \\
\text { hydrogen } \\
\text { release rate } \\
\operatorname{Rr}\left(\mathbf{H}_{2}\right) \\
(\mathbf{c f m})\end{array}$ & $\begin{array}{c}\text { Methane } \\
\text { release rate } \\
\operatorname{Rr}\left(\mathrm{CH}_{4}\right) \\
(\mathrm{cfm})\end{array}$ \\
\hline $241-\mathrm{SX}-115$ & 0.0003 & 0.0002 & 0.0025 & 14 & 171,480 & 7.0 & $0.58 \%$ & $1.75 \mathrm{E}-05$ & $1.72 \mathrm{E}-04$ & $1.61 \mathrm{E}-03$ & $1.11 \mathrm{E}-05$ \\
\hline $241-\mathrm{T}-101$ & 0.0022 & 0.0004 & 0.0050 & 109 & 99,013 & 7.0 & $0.13 \%$ & $1.52 E-04$ & $3.47 \mathrm{E}-04$ & 2.95E-04 & $2.76 \mathrm{E}-05$ \\
\hline $241-\mathrm{T}-102$ & 0.0005 & 0.0004 & 0.0066 & 42 & 108,053 & 7.0 & $0.22 \%$ & $3.50 \mathrm{E}-05$ & $4.59 \mathrm{E}-04$ & $5.91 \mathrm{E}-04$ & $2.95 \mathrm{E}-05$ \\
\hline $24 \mid-T-103$ & 0.0005 & 0.0004 & 0.0055 & 37 & 108,689 & 7.0 & $0.09 \%$ & $3.50 \mathrm{E}-05$ & $3.88 \mathrm{E}-04$ & $2.32 \mathrm{E}-04$ & $2.49 \mathrm{E}-05$ \\
\hline $241-\mathrm{T}-\mathrm{I} 04$ & 0.0104 & 0.0004 & 0.0008 & 327 & 69,951 & 7.0 & $0.21 \%$ & $7.30 \mathrm{E}-04$ & $5.26 \mathrm{E}-05$ & $3.59 \mathrm{E}-04$ & $2.75 \mathrm{E}-05$ \\
\hline $241-\mathrm{T}-105$ & 0.0094 & 0.0013 & 0.0201 & 108 & 99,189 & 7.0 & $0.20 \%$ & $6.60 \mathrm{E}-04$ & $1.41 \mathrm{E}-03$ & $3.16 \mathrm{E}-04$ & $6.31 \mathrm{E}-05$ \\
\hline $241-T-106$ & 0.0100 & 0.0003 & 0.0043 & 32 & 109,395 & 7.0 & $0.15 \%$ & $7.00 \mathrm{E}-04$ & $3.01 \mathrm{E}-04$ & $2.30 \mathrm{E}-04$ & $1.94 \mathrm{E}-05$ \\
\hline $24 !-T-107$ & 0.0123 & 0.0023 & 0.0041 & 183 & 89,161 & 6.4 & $0.30 \%$ & $7.85 \mathrm{E}-04$ & $2.62 \mathrm{E}-04$ & $4.32 E-04$ & 8.64E-05 \\
\hline $241-\mathrm{T}-108$ & 0.0020 & 0.0002 & 0.0041 & 26 & 110,172 & 7.0 & $0.10 \%$ & $1.40 \mathrm{E}-04$ & $2.85 \mathrm{E}-04$ & $2.27 \mathrm{E}-04$ & 1.40E-05 \\
\hline $241-T-109$ & 0.0005 & 0.0002 & 0.0049 & 72 & 103,992 & 7.0 & $0.09 \%$ & $3.50 \mathrm{E}-05$ & $3.45 \mathrm{E}-04$ & $2.41 \mathrm{E}-04$ & $1.75 \mathrm{E}-05$ \\
\hline $241-T-110$ & 0.0107 & 0.0002 & 0.0015 & 380 & 62,853 & 7.0 & $0.39 \%$ & $7.48 \mathrm{E}-04$ & 1.02E-04 & $8.72 \mathrm{E}-04$ & $1.70 \mathrm{E}-05$ \\
\hline 241-T-111 & 0.0223 & 0.0058 & 0.0013 & 457 & 52,577 & 6.0 & $0.44 \%$ & $1.35 \mathrm{E}-03$ & $7.64 \mathrm{E}-05$ & $4.22 \mathrm{E}-04$ & $8.44 \mathrm{E}-05$ \\
\hline $241-\mathrm{T}-112$ & 0.0029 & 0.0009 & 0.0137 & 77 & 103,392 & 7.0 & $0.13 \%$ & 2.01 E-04 & $9.62 \mathrm{E}-04$ & $2.47 \mathrm{E}-04$ & $4.95 \mathrm{E}-05$ \\
\hline $241-\mathrm{T}-201$ & 0.0001 & 0.0004 & 0.0059 & 31 & 3,746 & 7.0 & $0.06 \%$ & $7.00 \mathrm{E}-06$ & 4. $16 \mathrm{E}-04$ & I. $43 \mathrm{E}-04$ & $2.67 \mathrm{E}-05$ \\
\hline $241-T-202$ & 0.0002 & 0.0003 & 0.0043 & 21 & 5,088 & 7.0 & $0.02 \%$ & $1.60 \mathrm{E}-05$ & $3.01 \mathrm{E}-04$ & $4.40 \mathrm{E}-05$ & $8.81 \mathrm{E}-06$ \\
\hline $241-\mathrm{T}-203$ & 0.0000 & 0.0085 & 0.1318 & 37 & 3,004 & 0.4 & $0.60 \%$ & $0.00 \mathrm{E}+00$ & $5.02 \mathrm{E}-04$ & $6.53 \mathrm{E}-05$ & 1.31E-05 \\
\hline $241-\mathrm{T}-204$ & 0.0001 & 0.0005 & 0.0078 & 37 & 3,004 & 7.0 & $0.03 \%$ & $8.75 \mathrm{E}-06$ & $5.45 \mathrm{E}-04$ & $6.52 \mathrm{E}-05$ & $1.30 \mathrm{E}-05$ \\
\hline $241-\mathrm{TX}-101$ & 0.0014 & 0.0011 & 0.0050 & 101 & 126,745 & 7.0 & $0.19 \%$ & $9.65 \mathrm{E}-05$ & $3.47 \mathrm{E}-04$ & $4.46 \mathrm{E}-04$ & $8.02 \mathrm{E}-05$ \\
\hline 241-TX-102 & 0.0168 & 0.0002 & 0.0054 & 227 & 109,901 & 11.5 & $0.19 \%$ & $1.93 \mathrm{E}-03$ & $6.28 \mathrm{E}-04$ & $3.35 \mathrm{E}-04$ & $2.24 \mathrm{E}-05$ \\
\hline $24 !-T X-103$ & 0.0107 & 0.0003 & 0.0086 & 155 & 119,541 & 7.0 & $0.18 \%$ & $7.49 \mathrm{E}-04$ & $6.05 \mathrm{E}-04$ & $2.92 \mathrm{E}-04$ & $2.20 \mathrm{E}-05$ \\
\hline $241-\mathrm{TX}-104$ & 0.0028 & 0.0006 & 0.0050 & 79 & 129,641 & 3.5 & $0.25 \%$ & $9.83 \mathrm{E}-05$ & $1.73 \mathrm{E}-04$ & $3.11 \mathrm{E}-04$ & $2.07 \mathrm{E}-05$ \\
\hline 241-TX-105 & 0.0020 & 0.0001 & 0.0013 & 586 & 61,876 & 14.4 & $0.10 \%$ & $2.83 \mathrm{E}-04$ & $1.82 \mathrm{E}-04$ & $4.72 \mathrm{E}-(04$ & $1.98 \mathrm{E}-05$ \\
\hline $241-T X-106$ & 0.0252 & 0.0007 & 0.0050 & 358 & 92,351 & 7.0 & $0.33 \%$ & $1.77 \mathrm{E}-03$ & $3.47 \mathrm{E}-04$ & $4.17 \mathrm{E}-04$ & $4.69 \mathrm{E}-05$ \\
\hline 24I-TX-107 & 0.0204 & 0.0002 & 0.0031 & 40 & 134,938 & 7.0 & $0.23 \%$ & $1.43 \mathrm{E}-03$ & $2.14 \mathrm{E}-04$ & $2.49 \mathrm{E}-04$ & $1.08 \mathrm{E}-05$ \\
\hline
\end{tabular}


RPP-5926 REV 6

Table D-1. Steady-State Flammability Calculation Under Normal Ventilation Conditions for Current Waste at Documented Safety Analysis Conditions. (8 sheets)

\begin{tabular}{|c|c|c|c|c|c|c|c|c|c|c|c|}
\hline Tank & $\begin{array}{c}\text { Ammonia } \\
\text { headspace } \\
\text { concentration } \\
{\left[\mathrm{NH}_{3}\right]} \\
(\%) \\
\end{array}$ & $\begin{array}{c}\text { Methane } \\
\text { headspace } \\
\text { concentration } \\
{\left[\mathrm{CH}_{4}\right]} \\
(\%) \\
\end{array}$ & $\begin{array}{c}\mathrm{N}_{2} \mathrm{O} \text { headspace } \\
\text { concentration } \\
{\left[\mathrm{N}_{2} \mathrm{O}\right]} \\
(\%)\end{array}$ & $\begin{array}{c}\text { Waste } \\
\text { volume } \\
V_{w} \\
\text { (kgal) }\end{array}$ & $\begin{array}{c}\text { Headspace } \\
\text { volume } \\
\mathrm{V}_{\mathrm{h}} \\
\left(\mathrm{ft}^{3}\right)\end{array}$ & $\begin{array}{c}\text { Normal } \\
\text { ventilation } \\
\text { rate } \\
(\mathrm{Vr})_{\text {norm }} \\
(\mathrm{cfm}) \\
\end{array}$ & $\begin{array}{l}\text { Steady-state } \\
\text { flammability } \\
\text { at normal } \\
\text { condition } \\
(\% \text { LFL) } \\
\end{array}$ & $\begin{array}{c}\text { Ammonia } \\
\text { release rate } \\
\operatorname{Rr}\left(\mathrm{NH}_{3}\right) \\
\text { (cfm) }\end{array}$ & $\begin{array}{c}\mathrm{N}_{2} \mathrm{O} \\
\text { release rate } \\
\operatorname{Rr}\left(\mathrm{N}_{2} \mathrm{O}\right) \\
(\mathrm{cfm})\end{array}$ & $\begin{array}{l}\text { Model } \\
\text { hydrogen } \\
\text { release rate } \\
\operatorname{Rr}\left(\mathrm{H}_{2}\right) \\
(\mathrm{cfm}) \\
\end{array}$ & $\begin{array}{l}\text { Methane } \\
\text { release rate } \\
\left.\operatorname{Rr}_{(\mathrm{CH}}\right) \\
(\mathrm{cfm})\end{array}$ \\
\hline $241-\mathrm{TX}-108$ & 0.0025 & 0.0001 & 0.0022 & 136 & 122,013 & 9.0 & $0.09 \%$ & $2.2 ! \mathrm{E}-04$ & $1.99 \mathrm{E}-04$ & $2.76 \mathrm{E}-04$ & $1.01 \mathrm{E}-05$ \\
\hline 241-TX-109 & 0.0400 & 0.0043 & 0.0668 & 373 & 90,338 & 7.0 & $0.54 \%$ & $2.80 \mathrm{E}-03$ & $4.68 \mathrm{E}-03$ & 5. 14E-04 & $1.03 \mathrm{E}-04$ \\
\hline 241-TX-110 & 0.0974 & 0.0003 & 0.0129 & 477 & 76,425 & 7.0 & $0.80 \%$ & $6.82 \mathrm{E}-03$ & $9.01 \mathrm{E}-04$ & $4.13 \mathrm{E}-04$ & $2.12 \mathrm{E}-05$ \\
\hline $24 !-T X-111$ & 0.0605 & 0.0002 & 0.0175 & 375 & 90,126 & 9.7 & $0.50 \%$ & $5.88 \mathrm{E}-03$ & $1.70 \mathrm{E}-03$ & $3.72 \mathrm{E}-04$ & $1.87 \mathrm{E}-05$ \\
\hline 24I-TX-112 & 0.0112 & 0.0007 & 0.0050 & 643 & 54,213 & 7.0 & $0.26 \%$ & $7.84 \mathrm{E}-04$ & $3.47 \mathrm{E}-04$ & $4.70 \mathrm{E}-04$ & $4.57 \mathrm{E}-05$ \\
\hline 241-TX-113 & 0.0021 & 0.0004 & 0.0050 & 648 & 53,578 & 9.3 & $0.14 \%$ & $1.99 \mathrm{E}-04$ & $4.61 \mathrm{~F}-04$ & $4.45 \mathrm{E}-04$ & $3.46 \mathrm{E}-05$ \\
\hline 241-TX-114 & 0.0160 & 0.0002 & 0.0040 & 541 & 67,844 & 12.0 & $0.20 \%$ & $1.93 \mathrm{E}-03$ & $4.79 \mathrm{E}-04$ & $4.10 \mathrm{E}-04$ & $2.43 \mathrm{E}-05$ \\
\hline 24J-TX-115 & 0.0014 & 0.0002 & 0.0046 & 563 & 64,983 & 10.0 & $0.12 \%$ & $1.38 \mathrm{E}-04$ & $4.58 \mathrm{E}-04$ & $4.34 \mathrm{E}-04$ & $2.32 \mathrm{E}-05$ \\
\hline $241-\mathrm{TX}-116$ & 0.0014 & 0.0012 & 0.0050 & 608 & 58,945 & 3.1 & $0.38 \%$ & 4.34E-05 & $1.55 \mathrm{E}-04$ & $4.28 \mathrm{E}-04$ & $3.69 \mathrm{E}-05$ \\
\hline 241-TX-117 & 0.0014 & 0.0003 & 0.0050 & 490 & 74,730 & 6.5 & $0.16 \%$ & $8.97 \mathrm{E}-05$ & $3.22 \mathrm{E}-04$ & $3.87 \mathrm{E}-04$ & $1.87 \mathrm{E}-05$ \\
\hline $241-\mathrm{TX}-118$ & 0.0036 & 0.0022 & 0.0018 & 257 & 105,875 & 10.0 & $0.19 \%$ & $3.57 \mathrm{E}-04$ & $1.80 \mathrm{E}-04$ & $4.74 \mathrm{E}-04$ & $9.47 E-05$ \\
\hline 24l-TY-10l & 0.0016 & 0.0003 & 0.0097 & 128 & 123,242 & 3.2 & $0.21 \%$ & $5.23 \mathrm{E}-05$ & $3.15 \mathrm{E}-04$ & $2.58 \mathrm{E}-04$ & $8.38 \mathrm{E}-06$ \\
\hline $241-T Y-102$ & 0.0004 & 0.0003 & 0.0066 & 79 & 129,774 & 7.0 & $0.10 \%$ & $2.84 \mathrm{E}-05$ & $4.65 \mathrm{E}-04$ & $2.50 \mathrm{E}-04$ & $1.93 \mathrm{E}-05$ \\
\hline $241-\mathrm{T} Y-103$ & 0.0045 & 0.0015 & 0.0198 & 165 & 118,368 & 8.2 & $0.17 \%$ & $3.73 \mathrm{E}-04$ & $1.63 \mathrm{E}-03$ & $3.78 \mathrm{E}-04$ & $7.56 \mathrm{E}-05$ \\
\hline $241-\mathrm{TY}-104$ & 0.0059 & 0.0003 & 0.0097 & 54 & 133,094 & 13.4 & $0.10 \%$ & $7.87 \mathrm{E}-04$ & $1.30 \mathrm{E}-03$ & $2.83 \mathrm{E}-04$ & $4.24 \mathrm{E}-05$ \\
\hline 241-ТY-105 & 0.0003 & 0.0023 & 0.0051 & 241 & 108,163 & 7.0 & $0.23 \%$ & $1.75 \mathrm{E}-05$ & $3.54 \mathrm{E}-04$ & $5.08 \mathrm{E}-04$ & $1.02 \mathrm{E}-04$ \\
\hline 241-TY-106 & 0.0019 & 0.0003 & 0.0043 & 26 & 136,837 & 7.0 & $0.11 \%$ & $1.36 \mathrm{E}-04$ & $3.01 \mathrm{E}-04$ & $2.55 \mathrm{E}-04$ & $1.94 \mathrm{E}-05$ \\
\hline $241-U \cdot 101$ & 0.0100 & 0.0003 & 0.0051 & 33 & 109,218 & 7.0 & $0.19 \%$ & $7.00 \mathrm{E}-04$ & $3.59 \mathrm{E}-04$ & $3.21 \mathrm{E}-04$ & $2.30 \mathrm{E}-05$ \\
\hline $241-U-102^{*}$ & 0.0651 & 0.0012 & 0.0805 & 337 & 68,574 & 2.1 & $1.54 \%$ & $1.37 \mathrm{E}-03$ & $1.69 \mathrm{E}-03$ & $9.1 ! \mathrm{E}-04$ & $2.47 \mathrm{E}-05$ \\
\hline $241+U-103^{*}$ & 0.0722 & 0.0014 & $0.076 !$ & 427 & 56,567 & 2.4 & $1.60 \%$ & 1.73E-03 & $1.83 \mathrm{E}-03$ & $1.04 \mathrm{E}-03$ & $3.37 \mathrm{E}-05$ \\
\hline $241-U-104$ & 0.0001 & 0.0006 & 0.0085 & 64 & 105,051 & 7.0 & $0.11 \%$ & $4.88 \mathrm{E}-06$ & $5.96 \mathrm{E}-04$ & $2.75 \mathrm{E}-04$ & $3.89 \mathrm{E}-05$ \\
\hline $241-U-105^{*}$ & 0.1936 & 0.0039 & 0.1324 & 363 & 65,113 & 5.0 & $1.83 \%$ & $9.68 \mathrm{E}-03$ & $6.62 \mathrm{E}-03$ & $9.31 \mathrm{E}-04$ & $1.86 \mathrm{E}-04$ \\
\hline $241-U-106$ & 0.0940 & 0.0052 & 0.0554 & 180 & 89,549 & 1.3 & $2.35 \%$ & $1.22 \mathrm{E}-03$ & $7.20 \mathrm{E}-04$ & $8.42 \mathrm{E}-04$ & $6.81 \mathrm{E}-05$ \\
\hline $241-U-107^{*}$ & 0.0448 & 0.0010 & 0.0445 & 304 & 72,988 & 11.8 & $0.42 \%$ & $5.27 \mathrm{E}-03$ & $5.23 \mathrm{E}-03$ & 4.99E-04 & $9.98 \mathrm{E}-05$ \\
\hline
\end{tabular}




\section{RPP-5926 REV 6}

Table D-1. Steady-State Flammability Calculation Under Normal Ventilation Conditions for Current Waste at Documented Safety Analysis Conditions. (8 sheets)

\begin{tabular}{|c|c|c|c|c|c|c|c|c|c|c|c|}
\hline Tank & $\begin{array}{c}\text { Ammonia } \\
\text { headspace } \\
\text { concentration } \\
{\left[\mathrm{NH}_{3}\right]} \\
(\%)\end{array}$ & $\begin{array}{c}\text { Methane } \\
\text { headspace } \\
\text { concentration } \\
{\left[\mathrm{CH}_{4}\right]} \\
(\%)\end{array}$ & $\begin{array}{c}\mathrm{N}_{2} \mathrm{O} \text { headspace } \\
\text { concentration } \\
{\left[\mathrm{N}_{2} \mathrm{O}\right]} \\
(\%)\end{array}$ & $\begin{array}{c}\text { Waste } \\
\text { volume } \\
V_{\mathrm{w}} \\
\text { (kgal) }\end{array}$ & $\begin{array}{c}\text { Headspace } \\
\text { volume } \\
V_{\mathrm{h}} \\
\left(\mathrm{ft}^{3}\right)\end{array}$ & $\begin{array}{l}\text { Normal } \\
\text { ventilation } \\
\text { rate } \\
(V r)_{\text {aarm }} \\
(\mathrm{cfm})\end{array}$ & $\begin{array}{l}\text { Steady-state } \\
\text { flammability } \\
\text { at normal } \\
\text { condition } \\
(\% \text { LFL })\end{array}$ & $\begin{array}{c}\text { Ammonia } \\
\text { release rate } \\
\operatorname{Rr}\left(\mathrm{NH}_{3}\right) \\
(\mathrm{cfm})\end{array}$ & $\begin{array}{c}\mathrm{N}_{2} \mathrm{O} \\
\text { release rate } \\
\operatorname{Rr}\left(\mathrm{N}_{2} \mathrm{O}\right) \\
(\mathrm{cfm})\end{array}$ & $\begin{array}{c}\text { Model } \\
\text { hydrogen } \\
\text { release rate } \\
\operatorname{Rr}\left(\mathbf{H}_{2}\right) \\
(\mathrm{cfm})\end{array}$ & $\begin{array}{l}\text { Methane } \\
\text { release rate } \\
\operatorname{Rr}\left(\mathrm{CH}_{4}\right) \\
(\mathrm{cfm})\end{array}$ \\
\hline 241-U-108* & 0.0684 & 0.0011 & 0.0485 & 443 & 54,343 & 3.6 & $1.13 \%$ & $2.47 \mathrm{E}-03$ & $1.75 \mathrm{E}-03$ & $9.40 \mathrm{E}-04$ & $3.94 \mathrm{E}-05$ \\
\hline $241-U-109$ & 0.0005 & 0.0010 & 0.0499 & 411 & 58,686 & 4.3 & $0.40 \%$ & 2.14E-05 & $2.14 \mathrm{E}-03$ & $6.39 \mathrm{E}-04$ & 4.45E-05 \\
\hline 24$]-(J-1) 10$ & 0.0225 & 0.0091 & 0.1418 & 186 & 88,808 & 1.9 & $1.03 \%$ & $4.24 \mathrm{E}-04$ & $2.67 \mathrm{E}-03$ & 5.22E-04 & $1.04 \mathrm{E}-04$ \\
\hline $24 !-U-11 !$ & 0,0669 & 0.0012 & 0.0324 & 232 & 82,628 & 1.9 & $1.09 \%$ & 1.27E-03 & $6.15 \mathrm{E}-04$ & $4.68 \mathrm{E}-04$ & $2.27 \mathrm{E}-05$ \\
\hline $241-U-112$ & 0.0301 & 0.0035 & 0.0203 & 55 & 106,217 & 1.3 & $0.88 \%$ & $3.85 \mathrm{E}-04$ & $2.60 \mathrm{E}-04$ & $3.14 \mathrm{E}-04$ & $4.52 \mathrm{E}-05$ \\
\hline $241-U-201$ & 0.0005 & 0.0001 & 0.0010 & 5 & 7,277 & 7.0 & $0.01 \%$ & $3.23 \mathrm{E}-05$ & $7.18 \mathrm{E}-05$ & $2.61 \mathrm{E}-05$ & $4.6 ! \mathrm{E}-06$ \\
\hline $241-U-202$ & 0.0005 & 0.0001 & 0.0010 & 5 & 7,312 & 7.0 & $0.01 \%$ & $3.23 \mathrm{E}-05$ & $7.18 \mathrm{E}-05$ & $2.17 \mathrm{E}-05$ & $4.33 \mathrm{E}-06$ \\
\hline $24 !-U-203$ & 0.0001 & 0.0001 & 0.0021 & 4 & 7,348 & 2.1 & $0.03 \%$ & $1.77 \mathrm{E}-06$ & $4.31 \mathrm{E}-05$ & $2.52 \mathrm{E}-05$ & $2.76 \mathrm{E}-06$ \\
\hline $241-U-204$ & 0.0000 & 0.0000 & 0.0004 & 4 & 7,418 & 10.2 & $0.01 \%$ & $1.35 \mathrm{E}-06$ & $4.31 \mathrm{E}-05$ & $5.12 \mathrm{E}-05$ & $2.76 \mathrm{E}-06$ \\
\hline
\end{tabular}

Note: 'DSA conditions are the conditions with current waste plus water additions of $10 \mathrm{kgal}$ and $1 \mathrm{kgal}$ for 100 - and 200 - series, respectively and $5{ }^{\circ} \mathrm{C}$ increase for DSTs

"For all DSTs and SSTs with "*", the ammonia was analyzed with equilibrium model. 


\section{RPP-5926 REV 6}

Table D-2. Steady-State Flammability Calculations With Barometric Breathing Rate for Current Waste at

Documented Safety Analysis Conditions. (8 sheets)

\begin{tabular}{|c|c|c|c|c|c|c|c|c|c|}
\hline Tank & $\begin{array}{c}\text { Off-normal } \\
\text { ventilation } \\
\text { rate } \\
(\mathrm{Vr})_{\mathrm{bb}} \\
(\mathrm{cfm}) \\
\end{array}$ & $\begin{array}{c}\text { Steady-state } \\
\text { hydrogen } \\
\text { concentration } \\
{\left[\mathrm{H}_{2}\right]} \\
(\%) \\
\end{array}$ & $\begin{array}{c}\text { Steady-state } \\
\text { ammonia } \\
\text { concentration } \\
{\left[\mathrm{NH}_{3}\right]} \\
(\%) \\
\end{array}$ & $\begin{array}{c}\text { Steady-state } \\
\text { methane } \\
\text { concentration } \\
{\left[\mathrm{CH}_{4}\right]} \\
(\%) \\
\end{array}$ & $\begin{array}{c}\text { Steady-state } \\
\mathrm{N}_{2} \mathrm{O} \\
\text { concentration } \\
{\left[\mathrm{N}_{2} \mathrm{O}\right]} \\
(\%) \\
\end{array}$ & $\begin{array}{c}\text { Steady-state } \\
\text { hydrogen } \\
\text { flammability } \\
(\% \text { LFL })\end{array}$ & $\begin{array}{c}\text { Steady-state } \\
\text { ammonia } \\
\text { flammability } \\
\text { (\% LFL) }\end{array}$ & $\begin{array}{c}\text { Steady-state } \\
\text { methane } \\
\text { flammability } \\
(\% \text { LFL) }\end{array}$ & $\begin{array}{c}\begin{array}{c}\text { Steady-state } \\
\text { total }\end{array} \\
\text { flammability } \\
(\% \text { LFL) }\end{array}$ \\
\hline $241-A N-101$ & 0.18 & 1.30 & 0.01 & 0.10 & 0.65 & 32 & 0.04 & 2.09 & 34.6 \\
\hline $241-\mathrm{AN}-102$ & 0.14 & 11.46 & 0.09 & 0.23 & 4.62 & 286 & 0.58 & 4.68 & 291.7 \\
\hline 241-AN-103 & 0.18 & 2.09 & 0.20 & 0.11 & 0.35 & 52 & 1.36 & 2.23 & 56.0 \\
\hline $241-\mathrm{AN}-104$ & 0.14 & 3.41 & 0.20 & 0.14 & 0.49 & 85 & 1.34 & 2.71 & 89.3 \\
\hline $241-\mathrm{AN}-105$ & 0.11 & 2.77 & 0.15 & 0.19 & 1.13 & 69 & 1.02 & 3.88 & 74.0 \\
\hline 241-AN-106 & 0.25 & 2.92 & 0.01 & 0.29 & 0.09 & 73 & 0.08 & 5.84 & 79.0 \\
\hline $241-\mathrm{AN}-107$ & 0.12 & 10.02 & 0.08 & 0.51 & 5.53 & 250 & 0.51 & 10.18 & 261.2 \\
\hline 24 I-AP-101 & 0.12 & 0.85 & 0.08 & 0.17 & 5.62 & 21 & 0.55 & 3.40 & 25.2 \\
\hline 241-AP-102 & 0.13 & 2.20 & 0.07 & 0.26 & 5.11 & 55 & 0.49 & 5.18 & 60.7 \\
\hline $241-\Lambda^{P}-103$ & 0.21 & 0.83 & 0.02 & 0.04 & 0.80 & 21 & 0.17 & 0.81 & 21.8 \\
\hline 24l-AP-104 & 0.13 & 1.35 & 0.01 & 0.01 & 0.11 & 34 & 0.09 & 0.12 & 33.9 \\
\hline 241-AP-105 & 0.11 & 1.54 & 0.08 & 0.21 & 4.18 & 39 & 0.53 & 4.23 & 43.3 \\
\hline 241-AP-106 & 0.11 & 1.81 & $0.0 \mathrm{I}$ & 0.03 & 0.51 & 45 & 0.07 & 0.51 & 45.9 \\
\hline 241-AP-107 & 0.44 & 0.08 & 0.09 & 0.02 & 1.32 & 2 & 0.61 & 0.30 & 2.8 \\
\hline 24!-AP-108 & 0.16 & 1.89 & 0.09 & 0.01 & 1.40 & 47 & 0.57 & 0.14 & 48.1 \\
\hline 241-AW-101 & 0.11 & 2.81 & 0.01 & 0.14 & 0.65 & 70 & 0.06 & 2.76 & 73.0 \\
\hline $241-A W-102$ & 0.33 & 0.20 & 0.01 & 0.01 & 0.14 & 5 & 0.05 & 0.15 & 5.1 \\
\hline $241-A W-103$ & 0.13 & 0.77 & 0.04 & 0.15 & 0.98 & 19 & 0.29 & 3.09 & 22.7 \\
\hline $241-\mathrm{AW}-104$ & 0.14 & 1.71 & 0.01 & 0.34 & 0.96 & 43 & 0.09 & 6.86 & 49.8 \\
\hline 24l-AW-105 & $0.4 !$ & 0.08 & 0.06 & 0.02 & 0.44 & 2 & 0.42 & 0.31 & 2.7 \\
\hline $241-\mathrm{AW}-106$ & 0.11 & 1.44 & 0.12 & 0.13 & 2.57 & 36 & 0.78 & 2.60 & 39.3 \\
\hline $241-\mathrm{AY}-101$ & 0.49 & 1.40 & 0.03 & 0.03 & 0.45 & 35 & 0.19 & 0.58 & 35.7 \\
\hline $241-\mathrm{AY}-102$ & 0.18 & 16.73 & 0.02 & 0.20 & 1.39 & 418 & 0.14 & 4.03 & 422.5 \\
\hline $241-\mathrm{AZ} \cdot 101$ & 0.22 & 11.52 & 0.03 & 0.35 & 0.67 & 288 & 0.22 & 7.00 & 295.1 \\
\hline
\end{tabular}


RPP-5926 REV 6

Table D-2. Steady-State Flammability Calculations With Barometric Breathing Rate for Current Waste at

Documented Safety Analysis Conditions. (8 sheets)

\begin{tabular}{|c|c|c|c|c|c|c|c|c|c|}
\hline Tank & $\begin{array}{c}\text { Off-normal } \\
\text { ventilation } \\
\text { rate } \\
(\mathrm{Vr})_{\mathrm{bb}} \\
(\mathrm{cfm}) \\
\end{array}$ & $\begin{array}{c}\text { Steady-state } \\
\text { hydrogen } \\
\text { concentration } \\
{\left[\mathrm{H}_{2}\right]} \\
(\%) \\
\end{array}$ & $\begin{array}{c}\text { Steady-state } \\
\text { ammonia } \\
\text { concentration } \\
{\left[\mathrm{NH}_{3}\right]} \\
(\%) \\
\end{array}$ & $\begin{array}{c}\text { Steady-state } \\
\text { methane } \\
\text { concentration } \\
{\left[\mathrm{CH}_{4}\right]} \\
(\%) \\
\end{array}$ & $\begin{array}{c}\text { Steady-state } \\
\mathrm{N}_{2} \mathrm{O} \\
\text { concentration } \\
{\left[\mathrm{N}_{2} \mathrm{O}\right]} \\
(\%) \\
\end{array}$ & $\begin{array}{c}\text { Steady-state } \\
\text { hydrogen } \\
\text { flammability } \\
\text { (\% LFL) }\end{array}$ & $\begin{array}{c}\text { Steady-state } \\
\text { ammonia } \\
\text { flammability } \\
(\% \text { LFL) }\end{array}$ & $\begin{array}{c}\text { Steady-state } \\
\text { methane } \\
\text { flammability } \\
(\% \text { LFL) }\end{array}$ & $\begin{array}{c}\text { Steady-state } \\
\text { total } \\
\text { flammability } \\
(\% \text { LFL) }\end{array}$ \\
\hline $241-A Z-102$ & 0.18 & 20.11 & 0.12 & 0.48 & 0.62 & 503 & 0.80 & 9.57 & 513.2 \\
\hline 241-SY-101 & 0.11 & 0.59 & 0.39 & 0.12 & 2.21 & 15 & 2.58 & 2.36 & 19.7 \\
\hline $241-S Y-102$ & 0.11 & 1.25 & 0.18 & 0.25 & 1.77 & 31 & 1.18 & 4.98 & 37.3 \\
\hline 241-SY-103 & 0.27 & 1.86 & 0.56 & 0.11 & 0.68 & 46 & 3.72 & 2.29 & 52.4 \\
\hline $241-A-101^{*}$ & 0.41 & 0.37 & 0.83 & 0.01 & 0.33 & 9 & 5.50 & 0.27 & 15.1 \\
\hline $241-\mathrm{A}-102$ & 0.53 & 0.07 & 0.36 & 0.00 & 0.32 & 2 & 2.43 & 0.05 & 4.2 \\
\hline $241-\Lambda-103^{*}$ & 0.39 & 0.39 & 0.29 & 0.01 & 0.57 & 10 & 1.95 & 0.20 & 12.0 \\
\hline $24 !-A-104$ & 0.53 & 0.04 & 0.01 & 0.00 & 0.08 & 1 & 0.06 & 0.10 & 1.2 \\
\hline $241-A-105$ & na & na & na & na & na & na & na & na & na \\
\hline $241-A-106$ & 0.51 & 0.37 & 0.54 & 0.02 & 0.39 & 9 & 3.57 & 0.45 & 13.2 \\
\hline $241-\mathrm{AX}-10\}^{*}$ & 0.40 & 0.25 & 0.14 & 0.01 & 0.04 & 6 & 0.94 & 0.29 & 7.5 \\
\hline $241-\wedge X-102$ & 0.54 & 0.11 & 0.10 & 0.00 & 0.15 & 3 & 0.68 & 0.03 & 3.6 \\
\hline $241-A X-103$ & 0.51 & 0.18 & 0.20 & 0.01 & 0.11 & 4 & 1.33 & 0.19 & 5.9 \\
\hline 241-AX-104 & 0.55 & 0.82 & 0.01 & 0.00 & 0.02 & 20 & 0.06 & 0.03 & 20.6 \\
\hline $241-B-101$ & 0.31 & 0.14 & 0.03 & 0.01 & 0.22 & 3 & 0.21 & 0.22 & 3.8 \\
\hline $241-\mathrm{B}-102$ & 0.34 & 0.07 & 0.01 & 0.00 & 0.13 & 2 & 0.06 & 0.06 & 1.9 \\
\hline $241-B-103$ & 0.33 & 0.07 & 0.02 & 0.01 & 0.13 & 2 & 0.12 & 0.11 & 2.1 \\
\hline $241-B-104$ & 0.19 & 0.18 & 0.02 & 0.04 & 0.27 & 5 & 0.12 & 0.73 & 5.4 \\
\hline $241-B-105$ & 0.23 & 0.14 & 0.04 & 0.01 & 0.28 & 3 & 0.24 & 0.28 & 4.0 \\
\hline 241-B-106 & 0.30 & 0.10 & 0.01 & 0.02 & 0.56 & 3 & 0.08 & 0.41 & 3.0 \\
\hline $241-B-107$ & 0.28 & 0.10 & 0.05 & 0.02 & 0.04 & 2 & 0.35 & 0.35 & 3.2 \\
\hline $241-B-108$ & 0.31 & 0.08 & 0.08 & 0.01 & 0.19 & 2 & 0.51 & 0.19 & 2.7 \\
\hline $241-B-109$ & 0.30 & 0.09 & 0.10 & 0.01 & 0.28 & 2 & 0.65 & 0.27 & 3.1 \\
\hline $241-B-110$ & 0.25 & 0.22 & 0.14 & 0.04 & 0.14 & 5 & 0.94 & 0.86 & 7.2 \\
\hline
\end{tabular}




\section{RPP-5926 REV 6}

Table D-2. Steady-State Flammability Calculations With Barometric Breathing Rate for Current Waste at

Documented Safety Analysis Conditions. (8 sheets)

\begin{tabular}{|c|c|c|c|c|c|c|c|c|c|}
\hline Tank & $\begin{array}{c}\text { Off-normal } \\
\text { ventilation } \\
\text { rate } \\
(\mathrm{Vr})_{\mathrm{bb}} \\
(\mathrm{cfm}) \\
\end{array}$ & $\begin{array}{c}\text { Steady-state } \\
\text { hydrogen } \\
\text { concentration } \\
\left\{\mathrm{H}_{2}\right\} \\
(\%) \\
\end{array}$ & $\begin{array}{c}\text { Steady-state } \\
\text { ammonia } \\
\text { concentration } \\
{\left[\mathrm{NH}_{3}\right]} \\
(\%) \\
\end{array}$ & $\begin{array}{c}\text { Steady-state } \\
\text { methane } \\
\text { concentration } \\
{\left[\mathrm{CH}_{4}\right]} \\
(\%) \\
\end{array}$ & $\begin{array}{c}\text { Steady-state } \\
\mathrm{N}_{2} \mathrm{O} \\
\text { concentration } \\
{\left[\mathrm{N}_{2} \mathrm{O}\right]} \\
(\%) \\
\end{array}$ & $\begin{array}{c}\text { Steady-state } \\
\text { hydrogen } \\
\text { flammability } \\
(\% \text { LFL })\end{array}$ & $\begin{array}{c}\text { Steady-state } \\
\text { ammonia } \\
\text { flammability } \\
\text { (\% LFL) }\end{array}$ & $\begin{array}{c}\text { Steady-state } \\
\text { methane } \\
\text { flammability } \\
(\% \text { LFL) }\end{array}$ & $\begin{array}{c}\text { Steady-state } \\
\text { total } \\
\text { flammability } \\
(\% \mathrm{LFL})\end{array}$ \\
\hline $241-B-111$ & 0.25 & 0.37 & 0.04 & 0.07 & 0.13 & 9 & 0.23 & 1.47 & 10.9 \\
\hline $241-B-112$ & 0.34 & 0.08 & 0.06 & 0.01 & 0.14 & 2 & 0.42 & 0.18 & 2.6 \\
\hline $241-B-201$ & 0.01 & 0.49 & 0.29 & 0.10 & 0.34 & 12 & 1.92 & 1.95 & 16.0 \\
\hline $241-B-202$ & 0.01 & 0.45 & 0.15 & 0.09 & 0.98 & 11 & 1.02 & 1.79 & 14.0 \\
\hline $241-\mathrm{B}-203^{*}$ & 0.003 & 5.78 & 0.01 & 0.06 & 0.86 & 145 & 0.04 & 1.10 & 145.8 \\
\hline $241-B-204^{*}$ & 0.004 & 5.40 & 0.01 & 0.05 & 0.74 & 135 & 0.05 & 0.95 & 136.0 \\
\hline $241-\mathrm{BX}-101$ & 0.33 & 0.09 & 0.11 & 0.01 & 0.19 & 2 & 0.71 & 0.24 & 3.1 \\
\hline $241-\mathrm{BX}-102$ & 0.32 & 0.08 & 0.01 & 0.02 & 0.04 & 2 & 0.04 & 0.33 & 2.5 \\
\hline $241-\mathrm{BX}-103$ & 0.32 & 0.10 & 0.20 & 0.02 & 0.17 & 2 & 1.31 & 0.39 & 4.1 \\
\hline $241-B X-104$ & 0.31 & 0.12 & 0.60 & 0.02 & 0.37 & 3 & 3.99 & 0.48 & 7.5 \\
\hline $241-B X-105$ & 0.32 & 0.09 & 0.38 & 0.01 & 0.30 & 2 & 2.55 & 0.29 & 5.1 \\
\hline $24 !-B X-106$ & 0.33 & 0.08 & 0.10 & 0.01 & 0.09 & 2 & 0.65 & 0.21 & 2.9 \\
\hline $241-B X-107$ & 0.21 & 0.20 & 0.28 & 0.01 & 0.04 & 5 & 1.86 & 0.27 & 7.1 \\
\hline 241-BX-108 & 0.34 & 0.08 & 0.05 & 0.01 & 0.11 & 2 & 0.33 & 0.14 & 2.4 \\
\hline $241-B X-109$ & 0.27 & 0.19 & 0.05 & 0.04 & 1.03 & 5 & 0.35 & 0.75 & 5.8 \\
\hline $241-B X-110$ & 0.26 & 0.15 & 0.17 & 0.02 & 0.05 & 4 & 1.13 & 0.43 & 5.2 \\
\hline $241-B X-111$ & 0.27 & 0.11 & 0.16 & 0.00 & 0.04 & 3 & 1.07 & 0.08 & 3.8 \\
\hline $241-B X-112$ & 0.28 & 0.12 & 0.30 & 0.02 & 0.84 & 3 & 2.01 & 0.48 & 5.5 \\
\hline $241-B Y-101$ & 0.28 & 0.24 & 0.15 & 0.02 & 0.14 & 6 & 0.99 & 0.49 & 7.6 \\
\hline $241-B Y-102$ & 0.32 & 0.11 & 0.38 & 0.01 & 0.04 & 3 & 2.54 & 0.17 & 5.5 \\
\hline $241-B Y-103$ & 0.26 & 0.16 & 0.16 & 0.01 & 0.09 & 4 & 1.05 & 0.21 & 5.3 \\
\hline $241-B Y-104$ & 0.26 & 0.26 & 0.75 & 0.02 & 0.72 & 6 & 4.98 & 0.41 & 11.8 \\
\hline 241-BY-105 & 0.23 & 0.25 & 0.57 & 0.03 & 0.52 & 6 & 3.78 & 0.59 & 10.6 \\
\hline $241-B Y-106^{*}$ & 0.25 & 0.29 & 0.18 & 0.01 & 0.30 & 7 & 1.18 & 0.23 & 8.8 \\
\hline
\end{tabular}




\section{RPP-5926 REV 6}

Table D-2. Steady-State Flammability Calculations With Barometric Breathing Rate for Current Waste at

Documented Safety Analysis Conditions. (8 sheets)

\begin{tabular}{|c|c|c|c|c|c|c|c|c|c|}
\hline Tank & $\begin{array}{c}\text { Off-normal } \\
\text { ventilation } \\
\text { rate } \\
(\mathrm{Vr})_{\mathrm{bb}} \\
(\mathrm{cfm}) \\
\end{array}$ & $\begin{array}{c}\text { Steady-state } \\
\text { hydrogen } \\
\text { concentration } \\
{\left[\mathrm{H}_{2}\right]} \\
(\%) \\
\end{array}$ & $\begin{array}{c}\text { Steady-state } \\
\text { ammonia } \\
\text { concentration } \\
{\left[\mathrm{NH}_{3} \mid\right.} \\
(\%) \\
\end{array}$ & $\begin{array}{c}\text { Steady-state } \\
\text { methane } \\
\text { concentration } \\
{\left[\mathrm{CH}_{4}\right]} \\
(\%)\end{array}$ & $\begin{array}{c}\text { Steady-state } \\
\mathrm{N}_{2} \mathrm{O} \\
\text { concentration } \\
{\left[\mathrm{N}_{2} \mathrm{O}\right]} \\
(\%) \\
\end{array}$ & $\begin{array}{c}\text { Steady-state } \\
\text { hydrogen } \\
\text { flammability } \\
(\% \text { LFL })\end{array}$ & $\begin{array}{c}\text { Steady-state } \\
\text { ammonia } \\
\text { flammability } \\
\text { (\% LFL) }\end{array}$ & $\begin{array}{c}\text { Steady-state } \\
\text { methane } \\
\text { flammability } \\
\text { (\% LFL) }\end{array}$ & $\begin{array}{c}\text { Steady-state } \\
\text { total } \\
\text { flammability } \\
(\% \mathrm{LF})\end{array}$ \\
\hline $241-\mathrm{BY}-107$ & 0.32 & 0.14 & 1.00 & 0.02 & 1.15 & 4 & 6.67 & 0.32 & 10.6 \\
\hline $241-B Y-108$ & 0.34 & 0.12 & 1.00 & 0.02 & 1.10 & 3 & 6.67 & 0.30 & 9.9 \\
\hline $241-\mathrm{BY}-109$ & 0.31 & 0.11 & 0.21 & 0.01 & 0.05 & 3 & 1.39 & 0.20 & 4.3 \\
\hline $241-B Y-110$ & 0.28 & 0.38 & 1.00 & 0.02 & 0.49 & 10 & 6.67 & 0.31 & 16.5 \\
\hline 241-BY-111 & 0.27 & 0.14 & 0.13 & 0.01 & 0.19 & 3 & 0.88 & 0.12 & 4.5 \\
\hline $241-\mathrm{BY}-112$ & 0.31 & 0.11 & 0.27 & 0.01 & 0.17 & 3 & 1.81 & 0.11 & 4.6 \\
\hline $241-\mathrm{C}-101$ & 0.31 & 0.11 & 0.11 & 0.01 & 0.73 & 3 & 0.75 & 0.27 & 3.7 \\
\hline $241-C \cdot 102$ & 0.22 & 0.23 & 1.00 & 0.04 & 0.76 & 6 & 6.67 & 0.90 & 13.4 \\
\hline $241-C \cdot 103^{*}$ & 0.34 & 0.28 & 0.32 & 0.05 & 2.17 & 7 & 2.11 & 0.93 & 10.1 \\
\hline $241-C-104$ & 0.24 & 0.67 & 1.00 & 0.11 & 1.75 & 17 & 6.67 & 2.24 & 25.7 \\
\hline $24 i-C-105$ & 0.30 & 0.30 & 0.44 & 0.04 & 0.95 & 8 & $2.9 !$ & 0.84 & 11.3 \\
\hline $241-\mathrm{C}-106$ & 0.35 & 0.28 & 0.13 & 0.03 & 0.19 & 7 & 0.83 & 0.56 & 8.5 \\
\hline $241-C-107$ & 0.25 & 1.19 & 0.03 & 0.00 & 0.04 & 30 & 0.19 & 0.09 & 30.0 \\
\hline $241-C-108$ & 0.32 & 0.11 & 0.01 & 0.00 & 0.39 & 3 & 0.04 & 0.01 & 2.7 \\
\hline $241-\mathrm{C}-109$ & 0.32 & 0.27 & 0.04 & 0.00 & 1.47 & 7 & 0.27 & 0.07 & 7.0 \\
\hline $241-C-110$ & 0.28 & 0.26 & 0.35 & 0.05 & 0.04 & 7 & 2.34 & 1.06 & 10.0 \\
\hline $241-C-111$ & 0.33 & 0.50 & 0.01 & 0.00 & 0.11 & 13 & 0.06 & 0.02 & 12.7 \\
\hline $241-\mathrm{C}-112$ & 0.31 & 0.54 & 0.08 & 0.00 & 1.96 & 13 & 0.55 & 0.07 & 14.1 \\
\hline $241-\mathrm{C}-201$ & 0.02 & 0.18 & 0.02 & 0.01 & 0.12 & 5 & 0.11 & 0.15 & 4.9 \\
\hline $241-\mathrm{C}-202$ & 0.02 & 0.19 & 0.01 & 0.00 & 0.06 & 5 & 0.07 & 0.08 & 4.8 \\
\hline 241-C-203 & 0.02 & 0.17 & 0.13 & 0.02 & 0.29 & 4 & 0.89 & 0.38 & 5.6 \\
\hline $241-C-204$ & 0.02 & 0.08 & 0.01 & 0.01 & 0.18 & 2 & 0.06 & 0.23 & 2.3 \\
\hline 241 -S-101* & 0.29 & 0.35 & 0.58 & 0.02 & 0.42 & 9 & 3.88 & 0.30 & 12.9 \\
\hline $241-S-102^{*}$ & 0.35 & 0.17 & 0.43 & 0.01 & 0.25 & 4 & 2.87 & 0.10 & 7.1 \\
\hline
\end{tabular}


RPP-5926 REV 6

Table D-2. Steady-State Flammability Calculations With Barometric Breathing Rate for Current Waste at

Documented Safety Analysis Conditions. (8 sheets)

\begin{tabular}{|c|c|c|c|c|c|c|c|c|c|}
\hline Tank & $\begin{array}{c}\text { Off-normal } \\
\text { ventilation } \\
\text { rate } \\
(\mathrm{Vr})_{\mathrm{bb}} \\
(\mathrm{cfm}) \\
\end{array}$ & $\begin{array}{c}\text { Steady-state } \\
\text { hydrogen } \\
\text { concentration } \\
\left\{\mathrm{H}_{2}\right] \\
(\%) \\
\end{array}$ & $\begin{array}{c}\text { Steady-state } \\
\text { ammonia } \\
\text { concentration } \\
{\left[\mathrm{NH}_{3}\right]} \\
(\%) \\
\end{array}$ & $\begin{array}{c}\text { Steady-state } \\
\text { methane } \\
\text { concentration } \\
{\left[\mathrm{CH}_{4}\right]} \\
(\%) \\
\end{array}$ & $\begin{array}{c}\text { Steady-state } \\
\mathrm{N}_{2} \mathrm{O} \\
\text { concentration } \\
{\left[\mathrm{N}_{2} \mathrm{O}\right]} \\
(\%) \\
\end{array}$ & $\begin{array}{c}\text { Steady-state } \\
\text { hydrogen } \\
\text { flammability } \\
(\% \text { LFL })\end{array}$ & $\begin{array}{c}\text { Steady-state } \\
\text { ammonia } \\
\text { flammability } \\
(\% \text { LFL })\end{array}$ & $\begin{array}{c}\text { Steady-state } \\
\text { methane } \\
\text { flammability } \\
(\% \text { LFL) }\end{array}$ & $\begin{array}{c}\text { Steady-state } \\
\text { total } \\
\text { flammability } \\
(\% \text { LFL })\end{array}$ \\
\hline $241-S-103^{*}$ & 0.34 & 0.15 & 0.33 & 0.02 & 0.61 & 4 & 2.19 & 0,41 & 6.4 \\
\hline 24$]-S-104$ & 0.31 & 0.20 & 0.04 & 0.04 & 0.13 & 5 & 0.30 & 0.79 & 6.0 \\
\hline $241-S-105$ & 0.26 & 0.14 & 0.23 & 0.01 & 0.05 & 3 & 1.51 & 0.29 & 5.3 \\
\hline 241 -S-106* & 0.24 & 0.21 & 0.03 & 0.04 & 0.02 & 5 & 0.23 & 0.86 & 6.4 \\
\hline $241-S-107$ & 0.28 & 0.25 & 0.28 & 0.02 & 0.28 & 6 & 1.86 & 0.31 & 8.4 \\
\hline $241-S-108$ & 0.20 & 0.21 & 0.09 & 0.00 & 0.01 & 5 & 0.58 & 0.07 & 5.9 \\
\hline $241-S-109$ & 0.21 & 0.20 & 0.08 & 0.04 & 0.07 & 5 & 0.55 & 0.81 & 6.4 \\
\hline $241-S-110$ & 0.27 & 0.20 & 0.56 & 0.02 & 0.15 & 5 & 3.71 & 0.38 & 9.1 \\
\hline $241-S-111^{*}$ & 0.27 & 0.24 & 0.20 & 0.01 & 0.04 & 6 & 1.36 & 0.11 & 7.4 \\
\hline $241-S-112$ & 0.43 & 0.06 & 0.08 & 0.00 & 0.01 & 1 & 0.54 & 0.05 & 2.1 \\
\hline $241-S X-101 *$ & 0.36 & 0.19 & 0.04 & 0.02 & 0.08 & 5 & 0.25 & 0.36 & 5.4 \\
\hline $241-S X-102^{*}$ & 0.40 & 0.39 & 0.06 & 0.02 & 0.06 & 10 & 0.40 & 0.40 & 10.6 \\
\hline $241-\mathrm{SX}-103^{*}$ & 0.33 & 2.12 & 0.50 & 0.07 & 0.17 & 53 & 3.32 & 1.46 & 57.7 \\
\hline $24 !-S X-104^{*}$ & 0.35 & 0.52 & 0.04 & 0.02 & 0.06 & 13 & 0.27 & 0.37 & 13.7 \\
\hline 241-SX-105* & 0.38 & 1.36 & 0.34 & 0.02 & 0.09 & 34 & 2.24 & 0.39 & 36.6 \\
\hline $241-S X-106^{*}$ & 0.37 & 0.25 & 0.88 & 0.03 & 0.24 & 6 & 5.83 & 0.57 & 12.6 \\
\hline 241-SX-107 & 0.50 & 0.28 & 0.14 & 0.02 & 0.30 & 7 & 0.90 & 0.38 & 8.4 \\
\hline $24 \mathrm{I}-\mathrm{SX}-108^{*}$ & 0.51 & 0.15 & 0.36 & 0.02 & 0.25 & 4 & 2.38 & 0.32 & 6.4 \\
\hline 24]-SX-109 & 0.44 & 0.52 & 0.12 & 0.01 & 0.06 & 13 & 0.77 & 0.17 & 13.8 \\
\hline $241-S X-110$ & 0.51 & 0.47 & 0.08 & 0.01 & 0.17 & 12 & 0.52 & 0.22 & 12.5 \\
\hline 241-SX-111 & 0.49 & 0.33 & 0.16 & 0.02 & 0.36 & 8 & 1.07 & 0.46 & 9.8 \\
\hline $241-S X-112$ & 0.51 & 0.21 & 0.14 & 0.02 & 0.31 & 5 & 0.92 & 0.39 & 6.5 \\
\hline $24 J-S X-113$ & 0.53 & 0.05 & 0.04 & 0.01 & 0.08 & 1 & 0.25 & 0.11 & 1.5 \\
\hline 241-SX-114 & 0.47 & 0.50 & 0.25 & 0.04 & 0.55 & 12 & 1.65 & 0.71 & 14.8 \\
\hline
\end{tabular}


RPP-5926 REV 6

Table D-2. Steady-State Flammability Calculations With Barometric Breathing Rate for Current Waste at

Documented Safety Analysis Conditions. (8 sheets)

\begin{tabular}{|c|c|c|c|c|c|c|c|c|c|}
\hline Tank & $\begin{array}{c}\text { Off-normal } \\
\text { ventilation } \\
\text { rate } \\
(\mathrm{Vr})_{\mathrm{bb}} \\
(\mathrm{cfm})\end{array}$ & $\begin{array}{c}\text { Steady-state } \\
\text { hydrogen } \\
\text { concentration } \\
{\left[\mathrm{H}_{2}\right]} \\
(\%)\end{array}$ & $\begin{array}{c}\text { Steady-state } \\
\text { ammonia } \\
\text { concentration } \\
{\left[\mathrm{NH}_{3}\right]} \\
(\%)\end{array}$ & $\begin{array}{c}\text { Steady-state } \\
\text { methane } \\
\text { concentration } \\
{\left[\mathrm{CH}_{4}\right]} \\
(\%)\end{array}$ & $\begin{array}{c}\text { Steady-state } \\
\mathrm{N}_{2} \mathrm{O} \\
\text { concentration } \\
{\left[\mathrm{N}_{2} \mathrm{O}\right]} \\
(\%)\end{array}$ & $\begin{array}{c}\text { Steady-state } \\
\text { hydrogen } \\
\text { flammability } \\
(\% \text { LFL })\end{array}$ & $\begin{array}{c}\text { Steady-state } \\
\text { ammonia } \\
\text { flammability } \\
(\% \text { LFL })\end{array}$ & $\begin{array}{c}\text { Steady-state } \\
\text { methane } \\
\text { flammability } \\
(\% \text { LFL) }\end{array}$ & $\begin{array}{c}\text { Steady-state } \\
\text { total } \\
\text { flammability } \\
(\% \text { LFL })\end{array}$ \\
\hline $241-S X-115$ & 0.54 & 0.30 & 0.00 & 0.00 & 0.03 & 8 & 0.02 & 0.04 & 7.6 \\
\hline $241-T-101$ & 0.31 & 0.10 & 0.05 & 0.01 & 0.11 & 2 & 0.33 & 0.18 & 2.9 \\
\hline $241-T-102$ & 0.34 & 0.18 & 0.01 & 0.01 & 0.14 & 4 & 0.07 & 0.17 & 4.6 \\
\hline $241-\Upsilon-103$ & 0.34 & 0.07 & 0.01 & 0.01 & 0.11 & 2 & 0.07 & 0.15 & 1.9 \\
\hline $241-T-104$ & 0.22 & 0.16 & 0.33 & 0.01 & 0.02 & 4 & 2.23 & 0.25 & 6.6 \\
\hline $241-T-105$ & 0.31 & 0.10 & 0.21 & 0.02 & 0.45 & 3 & 1.42 & 0.41 & 4.4 \\
\hline $241-T-106$ & 0.34 & 0.07 & 0.20 & 0.01 & 0.09 & 2 & 1.37 & 0.11 & 3.2 \\
\hline 24!-T-107 & 0.28 & 0.15 & 0.28 & 0.03 & 0.09 & 4 & 1.88 & 0.62 & 6.4 \\
\hline $241-T-108$ & 0.34 & 0.07 & 0.04 & 0.00 & 0.08 & 2 & 0.27 & 0.08 & 2.0 \\
\hline $241-\mathrm{T}-109$ & 0.32 & 0.07 & 0.01 & 0.01 & 0.11 & 2 & 0.07 & 0.11 & 2.0 \\
\hline $241-T-110^{*}$ & 0.20 & 0.44 & 0.12 & 0.01 & 0.05 & 11 & 0.79 & 0.17 & 12.1 \\
\hline $241-\mathrm{T}-111$ & 0.16 & 0.26 & 0.82 & 0.05 & 0.05 & 6 & 5.48 & 1.03 & 12.9 \\
\hline $241-T-112$ & 0.32 & 0.08 & 0.06 & 0.01 & 0.30 & 2 & 0.42 & 0.31 & 2.6 \\
\hline $24 !-T-201$ & 0.01 & 1.22 & 0.06 & 0.23 & 3.56 & 31 & 0.40 & 4.57 & 35.5 \\
\hline $241-\mathrm{T}-202$ & 0.02 & 0.28 & 0.10 & 0.05 & 1.90 & 7 & 0.67 & 1.11 & 8.7 \\
\hline 241-T-203* & 0.01 & 0.70 & 0.01 & 0.14 & 5.35 & 17 & 0.07 & 2.78 & 20.2 \\
\hline $241-T-204$ & 0.01 & 0.69 & 0.09 & 0.14 & 5.81 & 17 & 0.62 & 2.78 & 20.8 \\
\hline 241-TX-101 & 0.40 & 0.11 & 0.02 & 0.02 & 0.09 & 3 & 0.16 & 0.40 & 3.4 \\
\hline 24I-TX-102 & 0.34 & 0.10 & 0.56 & 0.01 & 0.18 & 2 & 3.75 & 0.13 & 6.3 \\
\hline $241-T X-103$ & 0.37 & 0.08 & 0.20 & 0.01 & 0.16 & 2 & 1.34 & 0.12 & 3.4 \\
\hline $241-T X-104$ & 0.41 & 0.08 & 0.02 & 0.01 & 0.04 & 2 & 0.16 & 0.10 & 2.2 \\
\hline $241-T X-105$ & 0.19 & 0.24 & 0.15 & 0.01 & 0.09 & 6 & 0.98 & 0.20 & 7.3 \\
\hline $241-T X+106$ & 0.29 & 0.14 & 0.61 & 0.02 & 0.12 & 4 & 4.08 & 0.32 & 8.0 \\
\hline 241-TX-107 & 0.42 & 0.06 & 0.34 & 0.00 & 0.05 & 1 & 2.25 & 0.05 & 3.8 \\
\hline
\end{tabular}


RPP-5926 REV 6

Table D-2. Steady-State Flammability Calculations With Barometric Breathing Rate for Current Waste at

Documented Safety Analysis Conditions. (8 sheets)

\begin{tabular}{|c|c|c|c|c|c|c|c|c|c|}
\hline Tank & $\begin{array}{c}\text { Off-normal } \\
\text { ventilation } \\
\text { rate } \\
(\mathrm{Vr})_{b \mathrm{~b}} \\
(\mathrm{cfm}) \\
\end{array}$ & $\begin{array}{c}\text { Steady-state } \\
\text { hydrogen } \\
\text { concentration } \\
{\left[\mathrm{H}_{2} \mid\right.} \\
(\%) \\
\end{array}$ & $\begin{array}{c}\text { Steady-state } \\
\text { ammonia } \\
\text { concentration } \\
{\left[\mathrm{NH}_{3}\right]} \\
(\%) \\
\end{array}$ & $\begin{array}{c}\text { Steady-state } \\
\text { methane } \\
\text { concentration } \\
{\left[\mathrm{CH}_{4}\right]} \\
(\%)\end{array}$ & $\begin{array}{c}\text { Steady-state } \\
\mathrm{N}_{2} \mathrm{O} \\
\text { concentration } \\
{\left[\mathrm{N}_{2} \mathrm{O}\right]} \\
(\%) \\
\end{array}$ & $\begin{array}{c}\text { Steady-state } \\
\text { hydrogen } \\
\text { flammability } \\
(\% \text { LFL })\end{array}$ & $\begin{array}{l}\text { Steady-state } \\
\text { ammonia } \\
\text { flammability } \\
(\% \text { LFL })\end{array}$ & $\begin{array}{c}\text { Steady-state } \\
\text { methane } \\
\text { flammability } \\
(\% \text { LFL) }\end{array}$ & $\begin{array}{c}\text { Steady-state } \\
\text { total } \\
\text { flammability } \\
(\% \text { LFL) }\end{array}$ \\
\hline 24!-TX-108 & 0.38 & 0.07 & 0.06 & 0.00 & 0.05 & 2 & 0.39 & 0.05 & 2.2 \\
\hline $241-T X-109$ & 0.28 & 0.18 & 0.99 & 0.04 & 1.66 & 5 & 6.61 & 0.73 & 11.9 \\
\hline $24 !+T X-110$ & 0.24 & 0.17 & 1.00 & 0.01 & 0.38 & 4 & 6.67 & 0.18 & 11.2 \\
\hline $241-T X-111$ & 0.28 & 0.13 & 1.00 & 0.01 & 0.60 & 3 & 6.67 & 0.13 & 10.1 \\
\hline 241-TX-112 & 0.17 & 0.28 & 0.46 & 0.03 & 0.20 & 7 & 3.08 & 0.54 & 10.6 \\
\hline $241-\mathrm{TX}-113$ & 0.17 & 0.27 & 0.12 & 0.02 & 0.28 & 7 & 0.79 & $0.4 !$ & 7.8 \\
\hline $241-\mathrm{TX}-114$ & 0.21 & 0.19 & 0.91 & 0.01 & 0.23 & 5 & 6.06 & 0.23 & 11.1 \\
\hline 241-TX-115 & 0.20 & 0.21 & 0.07 & 0.01 & 0.23 & 5 & 0.45 & 0.23 & 6.0 \\
\hline $241-7 X-116$ & 0.18 & 0.23 & 0.02 & 0.02 & 0.08 & 6 & 0.16 & 0.40 & 6.4 \\
\hline 241-TX-117 & 0.23 & 0.17 & 0.04 & 0.01 & 0.14 & 4 & 0.26 & 0.16 & 4.6 \\
\hline 241-TX-118 & 0.33 & 0.14 & 0.11 & 0.03 & 0.05 & 4 & 0.72 & 0.57 & 4.9 \\
\hline 241-TY-101 & 0.39 & 0.07 & 0.01 & 0.00 & 0.08 & 2 & 0.09 & 0.04 & 1.8 \\
\hline 241-TY-102 & 0.41 & 0.06 & 0.01 & 0.00 & 0.11 & 2 & 0.05 & 0.10 & 1.7 \\
\hline $241-\mathrm{TY}-103$ & 0.37 & 0.10 & 0.10 & 0.02 & 0.44 & 3 & 0.67 & 0.41 & 3.6 \\
\hline $241-T Y-104$ & 0.42 & 0.07 & 0.19 & 0.01 & 0.31 & 2 & 1.26 & 0.20 & 3.2 \\
\hline 241-TY-105 & 0.34 & 0.15 & 0.01 & 0.03 & 0.10 & 4 & 0.03 & 0.60 & 4.4 \\
\hline $241-\mathrm{TY}-106$ & 0.43 & 0.06 & 0.03 & 0.00 & 0.07 & 1 & 0.21 & 0.09 & 1.8 \\
\hline $24 \mathrm{l}-\mathrm{U}-101$ & 0.34 & 0.09 & 0.21 & 0.01 & 0.11 & 2 & 1.37 & 0.13 & 3.9 \\
\hline $241-U-102 *$ & 0.21 & 0.43 & 0.22 & 0.01 & 0.79 & 11 & 1.44 & 0.23 & 12.3 \\
\hline $241-U-103^{*}$ & 0.18 & 0.59 & 0.46 & 0.02 & 1.03 & 15 & 3.05 & 0.38 & 18.2 \\
\hline $241-U-104$ & 0.33 & 0.08 & 0.00 & 0.01 & 0.18 & 2 & 0.01 & 0.24 & 2.3 \\
\hline $241-U-105^{*}$ & 0.20 & 0.46 & 0.69 & 0.09 & 3.25 & 11 & 4.61 & 1.83 & 17.9 \\
\hline 241-U-106* & 0.28 & 0.30 & 0.21 & 0.02 & 0.26 & 8 & 1.37 & 0.49 & 9.4 \\
\hline $241-U-107^{*}$ & 0.23 & 0.22 & 0.14 & 0.04 & 2.29 & 5 & 0.92 & 0.88 & 7.3 \\
\hline
\end{tabular}




\section{RPP-5926 REV 6}

Table D-2. Steady-State Flammability Calculations With Barometric Breathing Rate for Current Waste at

Documented Safety Analysis Conditions. (8 sheets)

\begin{tabular}{|c|c|c|c|c|c|c|c|c|c|}
\hline Tank & $\begin{array}{c}\text { Off-normal } \\
\text { ventilation } \\
\text { rate } \\
(\mathrm{Vr})_{b \mathrm{~b}} \\
(\mathrm{cfm})\end{array}$ & $\begin{array}{c}\text { Steady-state } \\
\text { hydrogen } \\
\text { concentration } \\
{\left[\mathrm{H}_{2}\right]} \\
(\%)\end{array}$ & $\begin{array}{c}\text { Steady-state } \\
\text { ammonia } \\
\text { concentration } \\
{\left[\mathrm{NH}_{3}\right]} \\
(\%) \\
\end{array}$ & $\begin{array}{c}\text { Steady-state } \\
\text { methane } \\
\text { concentration } \\
{\left[\mathrm{CH}_{4}\right]} \\
(\%)\end{array}$ & $\begin{array}{c}\text { Steady-state } \\
\mathrm{N}_{2} \mathrm{O} \\
\text { concentration } \\
{\left[\mathrm{N}_{2} \mathrm{O}\right]} \\
(\%)\end{array}$ & $\begin{array}{c}\text { Steady-state } \\
\text { hydrogen } \\
\text { flammability } \\
(\% \text { LFL })\end{array}$ & $\begin{array}{c}\text { Steady-state } \\
\text { ammonia } \\
\text { flammability } \\
\text { (\% LFL) }\end{array}$ & $\begin{array}{c}\text { Steady-state } \\
\text { methane } \\
\text { flammability } \\
(\% \text { LFL })\end{array}$ & $\begin{array}{c}\text { Steady-state } \\
\text { total } \\
\text { flammability } \\
\text { (\% LFL) }\end{array}$ \\
\hline $241-\mathrm{U}-108^{*}$ & 0.17 & 0.55 & 0.43 & 0.02 & 1.03 & 14 & 2.90 & 0.46 & 17.2 \\
\hline $241-U-109^{*}$ & 0.18 & 0.35 & 0.01 & 0.02 & 1.17 & 9 & 0.09 & 0.49 & 9.3 \\
\hline $241-U-110$ & 0.28 & 0.19 & 0.15 & 0.04 & 0.96 & 5 & 1.02 & 0.75 & 6.5 \\
\hline 241-U-111 & 0.26 & 0.18 & 0.49 & 0.01 & 0.24 & 5 & 3.28 & 0.18 & 8.0 \\
\hline $241-\mathrm{U}-112$ & 0.33 & 0.09 & 0.12 & 0.01 & 0.08 & 2 & 0.77 & 0.27 & 3.4 \\
\hline $241-U-201$ & 0.023 & 0.11 & 0.14 & 0.02 & 0.32 & 3 & 0.95 & 0.41 & 4.2 \\
\hline 241-U-202 & 0.023 & 0.09 & 0.14 & 0.02 & 0.31 & 2 & 0.94 & 0.38 & 3.7 \\
\hline $241 \cdot U-203$ & 0.023 & 0.11 & 0.01 & 0.01 & 0.19 & 3 & 0.05 & 0.24 & 3.0 \\
\hline $241-U-204$ & 0.023 & 0.22 & 0.01 & 0.01 & 0.19 & 6 & 0.04 & 0.24 & 5.8 \\
\hline
\end{tabular}

Note: ${ }^{2}$ DSA conditions are the conditions with current waste plus water additions of $10 \mathrm{kgal}$ and $1 \mathrm{kgal}$ for 100 - and 200 - scries, respectively and $5{ }^{\circ} \mathrm{C}$ increase for DSTs.

"For all DSTs and SSTs with "*", the ammonia was analyzed with equilibrium model. 


\section{RPP-5926 REV 6}

Table D-3. Time to Reach $25 \%$ of the Lower Flammability Limit Under Barometric Breathing Rate for Current Waste at

\begin{tabular}{|c|c|c|c|c|c|c|c|c|c|}
\hline Tank & $\begin{array}{c}\text { Time to } \\
\text { reach } \\
25 \% \mathrm{LFL} \\
\mathrm{t}_{25 \%} \\
\text { (day) } \\
\end{array}$ & $\begin{array}{l}\text { Minimum } \\
\text { vent rate } \\
\text { to prevent } \\
25 \% \mathrm{LFL} \\
\text { (cfm) }\end{array}$ & $\begin{array}{c}\text { Hydrogen } \\
\text { concentration } \\
\text { at time } \mathrm{t}_{25 \%} \\
{\left[\mathrm{H}_{2}\right](\mathbf{t})} \\
(\%) \\
\end{array}$ & $\begin{array}{c}\text { Ammonia } \\
\text { concentration } \\
\text { at time } \mathrm{t}_{25 \%} \\
{\left[\mathrm{NH}_{3}\right](\mathrm{t})} \\
(\%)\end{array}$ & $\begin{array}{c}\text { Methane } \\
\text { concentration } \\
\text { at time } \mathrm{t}_{25 \%} \\
{\left[\mathrm{CH}_{4} \mid(\mathrm{t})\right.} \\
(\%) \\
\end{array}$ & $\begin{array}{c}\mathrm{N}_{2} \mathrm{O} \\
\text { concentration } \\
\text { at time } t_{25 \%} \\
{\left[\mathrm{~N}_{2} \mathrm{O}\right](\mathrm{t})} \\
(\%) \\
\end{array}$ & $\begin{array}{c}\text { Hydrogen } \\
\text { flammability } \\
\text { at time } t_{25 \%} \\
(\%)\end{array}$ & $\begin{array}{c}\text { Ammonia } \\
\text { flammability } \\
\text { at time } t_{25 \%} \\
(\%)\end{array}$ & $\begin{array}{c}\text { Methane } \\
\text { flammability } \\
\text { at time } t_{25 \%} \\
(\%)\end{array}$ \\
\hline $241-\mathrm{AN}-101$ & 284 & 0.26 & 0.94 & 0.01 & 0.08 & 0.47 & 23.42 & 0.04 & 1.51 \\
\hline $241-\mathrm{AN}-102$ & 19 & 1.66 & 0.94 & 0.09 & 0.02 & 0.38 & 23.56 & 0.58 & 0.39 \\
\hline 241-AN-103 & 129 & 0.42 & 0.92 & 0.13 & 0.05 & 0.16 & 23.10 & 0.87 & 0.99 \\
\hline $24 \mathrm{I}-\mathrm{AN}-104$ & 69 & 0.54 & 0.91 & 0.18 & 0.04 & 0.13 & 22.80 & 1.20 & 0.73 \\
\hline $241 \cdot \mathrm{AN}-105$ & 88 & 0.35 & 0.91 & 0.15 & 0.06 & 0.37 & 22.70 & 0.98 & 1.27 \\
\hline 241-AN-106 & 84 & 0.80 & 0.92 & 0.01 & 0.09 & 0.03 & 23.00 & 0.04 & 1.84 \\
\hline 241-AN-107 & 21 & 1.34 & 0.91 & 0.08 & 0.05 & 0.50 & 22.64 & 0.51 & 0.93 \\
\hline $241-\mathrm{AP}-10 \mathrm{I}$ & 1038 & 0.12 & 0.84 & 0.08 & 0.17 & 5.57 & 21.08 & 0.55 & 3.37 \\
\hline $241-\mathrm{AP}-102$ & 116 & 0.32 & 0.89 & 0.07 & 0.10 & 2.07 & 22.28 & 0.49 & 2.09 \\
\hline 241-AP- 103 & not occur & not occur & not occur & not occur & not occur & not occur & not occur & not occur & not occur \\
\hline $241-A P-104$ & 296 & 0.17 & 0.99 & 0.01 & 0.00 & 0.08 & 24.79 & 0.09 & 0.09 \\
\hline 241-AP- 105 & 188 & 0.19 & 0.88 & 0.08 & 0.12 & 2.39 & 22.02 & 0.53 & 2.42 \\
\hline $241-\mathrm{AP}-106$ & 174 & 0.20 & 0.99 & 0.01 & 0.01 & 0.28 & 24.63 & 0.07 & 0.28 \\
\hline 241-AP- 107 & not occur & not occur & not occur & not occur & nol occur & not occur & not occur & not occur & not occur \\
\hline 24l-AP-108 & 160 & 0.31 & 0.97 & 0.09 & 0.00 & 0.72 & 24.33 & 0.57 & 0.07 \\
\hline 241-AW-101 & 92 & 0.34 & 0.96 & 0.01 & 0.05 & 0.22 & 23.97 & 0.06 & 0.94 \\
\hline $241-A W-102$ & not occur & not occur & not occus & not occur & not occur & not occur & not occur & not occur & not occur \\
\hline $241-A W-103$ & not occur & not occur & not occur & not occur & not occur & not occur & not occur & not occur & not occur \\
\hline 241-AW-104 & 154 & 0.28 & 0.86 & 0.01 & 0.17 & 0.48 & 21.45 & 0.09 & 3.42 \\
\hline $241-A W-105$ & not occur & not occur & not occur & not occur & not occur & not occur & not occur & not occur & not occur \\
\hline $241-\mathrm{AW}-106$ & 219 & 0.18 & 0.90 & 0.12 & 0.08 & 1.61 & 22.55 & 0.78 & 1.63 \\
\hline $241-A Y-101$ & 266 & 0.72 & 0.98 & 0.03 & 0.02 & 0.31 & 24.38 & 0.19 & 0.40 \\
\hline $241-A Y-102$ & 13 & 3.10 & 0.95 & 0.01 & 0.01 & 0.08 & 23.82 & 0.09 & 0.23 \\
\hline 241-AZ-101 & 19 & 2.68 & 0.95 & 0.01 & 0.03 & 0.06 & 23.69 & 0.03 & 0.58 \\
\hline
\end{tabular}




\section{RPP-5926 REV 6}

Table D-3. Time to Reach $25 \%$ of the Lower Flammability Limit Under Barometric Breathing Rate for Current Waste at

Documented Safety Analysis Conditions. (8 sheets)

\begin{tabular}{|c|c|c|c|c|c|c|c|c|c|}
\hline Tank & $\begin{array}{c}\text { Time to } \\
\text { reach } \\
25 \% \text { LFL } \\
t_{25 \%} \\
\text { (day) }\end{array}$ & $\begin{array}{l}\text { Minimum } \\
\text { vent rate } \\
\text { to prevent } \\
25 \% \mathrm{LFL} \\
(\mathrm{cfm})\end{array}$ & $\begin{array}{c}\text { Hydrogen } \\
\text { concentration } \\
\text { at time } \mathrm{t}_{25 \%} \\
{\left[\mathrm{H}_{2} \mid(\mathrm{t})\right.} \\
(\%)\end{array}$ & $\begin{array}{l}\text { Ammonia } \\
\text { concentration } \\
\text { at time } \mathrm{t}_{25 \%} \\
{\left[\mathrm{NH}_{3}\right](\mathrm{t})} \\
(\%)\end{array}$ & $\begin{array}{c}\text { Methane } \\
\text { concentration } \\
\text { at cime } \mathrm{t}_{25 \%} \\
{\left[\mathrm{CH}_{4}\right](\mathrm{t})} \\
(\%)\end{array}$ & $\begin{array}{c}\mathrm{N}_{2} \mathrm{O} \\
\text { concentration } \\
\text { at time } \mathrm{t}_{25 \%} \\
{\left[\mathrm{~N}_{2} \mathrm{O}\right](\mathrm{t})} \\
(\%)\end{array}$ & $\begin{array}{c}\text { Hydrogen } \\
\text { 17ammability } \\
\text { at time } t_{25 \%} \\
(\%)\end{array}$ & $\begin{array}{c}\text { Ammonia } \\
\text { flammability } \\
\text { at time } t_{25 \%} \\
(\%)\end{array}$ & $\begin{array}{c}\text { Methane } \\
\text { flammability } \\
\text { at time } t_{25 \%} \\
(\%)\end{array}$ \\
\hline 241-AZ-102 & 10 & 3.77 & 0.89 & 0.01 & 0.02 & 0.03 & 22.33 & 0.09 & 0.44 \\
\hline $24 \mid-S Y-10 !$ & not occur & notoccur & not occur & not occur & not occur & not occur & not occur & not occur & not occur \\
\hline $241-S Y-102$ & 239 & 0.17 & 0.82 & 0.18 & 0.16 & 1.17 & 20.52 & 1.18 & 3.29 \\
\hline $241-S Y-103$ & 129 & 0.62 & 0.82 & 0.52 & 0.05 & 0.30 & 20.46 & 3.45 & 1.01 \\
\hline $24 !-A-101^{*}$ & not occur & not occur & not occur & not occur & not occur & not occur & not occur & not occur & not occur \\
\hline $241-\mathrm{A}-102$ & not occur & not occur & not occur & not occur & not occur & not occur & not occur & not occur & not occur \\
\hline $241-A-103^{*}$ & not occur & not occur & not occur & not occur & not occur & not occur & not occur & not occur & not occur \\
\hline $24 !-A-104$ & not occur & not occur & not occur & not occur & not occur & not occur & not occur & not occur & not occur \\
\hline $241-A-105$ & na & na & na & na & na & na & na & na & na \\
\hline $241-A-106$ & not occur & not occur & not occur & not occur & not occur & not occur & not occur & not occur & not occur \\
\hline $241-\mathrm{AX}-101^{*}$ & not occur & not occur & not occur & not occur & not occur & not occur & not occur & not occur & not occur \\
\hline $24 !-A X-102$ & not occur & not occur & not occur & not occur & not occur & not occur & not oceur & not occur & not occur \\
\hline $241-A X-103$ & not occur & not occur & not occur & not occur & not occur & not occur & nol occur & not occur & not occur \\
\hline $241-\mathrm{AX}-104$ & not occur & not occur & not occur & not occur & not occur & not occur & not occur & not occur & not occur \\
\hline $241-B-10 !$ & not occur & not occur & not occur & not occur & not occur & not occur & not occur & not occur & not occur \\
\hline $241-B-102$ & not occur & not occur & not occur & not occur & not occur & not occur & not occur & not occur & not occur \\
\hline $241-B-103$ & not occur & not occur & not occur & not occur & not occur & not occur & not occur & not occur & not occur \\
\hline $241-B-104$ & not occur & not occur & not occur & not occur & not occur & not occur & not occur & not occur & not occur \\
\hline $241-B-105$ & not occur & not occur & not occur & not occur & not occur & not occur & not occur & not occur & not occur \\
\hline $241-B-106$ & not occur & not occur & not occur & not occur & not occur & not occur & not occur & not occur & not occur \\
\hline $241-B-107$ & not occur & not occur & not occur & not occur & not occur & nol occur & not occur & not occur & not occur \\
\hline $241-B-108$ & not occur & not occur & not occur & not occur & not occur & not occur & not occur & not occur & not occur \\
\hline $24 !-B * 109$ & not occur & not occur & not occur & not occur & not occur & not occur & not occur & not occur & not occur \\
\hline $241-B-110$ & not occur & not occur & not occur & not occur & not occur & not occur & not occur & not occur & not occur \\
\hline
\end{tabular}




\section{RPP-5926 REV 6}

Table D-3. Time to Reach $25 \%$ of the Lower Flammability Limit Under Barometric Breathing Rate for Current Waste at

Documented Safety Analysis Conditions. (8 sheets)

\begin{tabular}{|c|c|c|c|c|c|c|c|c|c|}
\hline Tank & $\begin{array}{c}\text { Time to } \\
\text { reach } \\
25 \% \mathrm{LFL} \\
\mathrm{t}_{25 \%} \\
\text { (day) } \\
\end{array}$ & $\begin{array}{l}\text { Minimum } \\
\text { vent rate } \\
\text { to prevent } \\
25 \% \text { LFL } \\
\text { (cfm) }\end{array}$ & $\begin{array}{c}\text { Hydrogen } \\
\text { concentration } \\
\text { at time } \mathrm{t}_{25 \%} \\
{\left[\mathrm{H}_{2}\right](\mathrm{t})} \\
(\%) \\
\end{array}$ & $\begin{array}{c}\text { Ammonia } \\
\text { concentration } \\
\text { at time } \mathrm{t}_{25 \%} \\
{\left[\mathrm{NH}_{3}\right](\mathrm{t})} \\
(\%)\end{array}$ & $\begin{array}{c}\text { Methane } \\
\text { concentration } \\
\text { at time } \mathrm{t}_{25 \%} \\
{\left[\mathrm{CH}_{4}\right](\mathrm{t})} \\
(\%) \\
\end{array}$ & $\begin{array}{c}\mathrm{N}_{2} \mathrm{O} \\
\text { concentration } \\
\text { at time } \mathrm{t}_{25 \%} \\
{\left[\mathrm{~N}_{2} \mathrm{O} \mid(\mathrm{t})\right.} \\
(\%) \\
\end{array}$ & $\begin{array}{c}\text { Hydrogen } \\
\text { flammability } \\
\text { at time } t_{25 \%} \\
(\%)\end{array}$ & $\begin{array}{c}\text { Ammonia } \\
\text { flammability } \\
\text { at time } t_{25 \%} \\
(\%)\end{array}$ & $\begin{array}{c}\text { Methane } \\
\text { flammability } \\
\text { at time } t_{25 \%} \\
(\%)\end{array}$ \\
\hline $241-B-111$ & not occur & not occur & not occur & not occur & not occur & not occur & not occur & not occur & not occur \\
\hline $241-B-112$ & not occur & not occur & not occur & not occur & not occur & not occur & not occur & not occur & not occur \\
\hline $241-B-201$ & not occur & not occur & not occur & not occur & not occur & not occur & not occur & not occur & not occur \\
\hline $241-B-202$ & not occur & not occur & not occur & not occur & not occur & not occur & not occur & not occur & not occur \\
\hline $24 \mid-B-203^{*}$ & 39 & 0.02 & 0.99 & 0.01 & 0.01 & 0.15 & 24.75 & 0.05 & 0.20 \\
\hline $241-\mathrm{B}-204^{*}$ & 42 & 0.02 & 0.99 & 0.01 & 0.01 & 0.14 & 24.77 & 0.05 & 0.18 \\
\hline $241-B X-101$ & not occur & not occur & not occur & not occur & not occur & not occur & not occur & not occur & not occur \\
\hline $241-B X-102$ & not occur & not occur & not occur & not occur & not occur & not occur & not occur & not occur & not occur \\
\hline $241-B X-103$ & not occur & not occur & not occur & not occur & not occur & not occur & not occur & not oceur & not occur \\
\hline 241-BX-104 & not occur & not occur & not occur & not occur & not occur & not occur & not occur & not occur & not occur \\
\hline $241-\mathrm{BX}-105$ & not occur & not occur & not occur & not occur & not occur & not occur & not occur & not occur & not occur \\
\hline $241-\mathrm{BX}-106$ & not occur & not occur & not occur & not occur & not occur & not occur & not occur & not occur & not occur \\
\hline 241-BX-107 & not occur & not occur & not occur & not occur & not occur & not occur & not occur & not occur & not occur \\
\hline $241-B X-108$ & not occur & not occur & not occur & not occur & not occur & not occur & not occur & not occur & not occur \\
\hline $241-B X-109$ & not occur & not occur & not occur & not occur & not occur & not occur & not occur & not occur & not occur \\
\hline $241-B X-110$ & not occur & not occur & not occur & not occur & not occur & not occur & not occur & not occur & not occur \\
\hline 241-BX-111 & not occur & not occur & not occur & not occur & not occur & not occur & not occur & not occur & not occur \\
\hline $241-\mathrm{BX}-1 \mathrm{I} 2$ & not occur & not occur & not occur & not occur & not occur & not occur & not occur & not occur & not occur \\
\hline 241-BY-101 & not occur & not occur & not occur & not occur & not occur & not occur & not occur & not occur & not occur \\
\hline $241-B Y \cdot 102$ & not occur & not occur & not occur & not occur & not occur & not occur & not occur & not occur & not occur \\
\hline $241-B Y-103$ & not occur & not occur & not occur & not occur & not occur & not occur & not occur & not occur & not occur \\
\hline 24!-BY-104 & not occur & not occur & nol occur & not occur & not occur & not occur & not occur & not occur & not occur \\
\hline $241-B Y-105$ & not occur & not occur & not occur & not occur & not occur & not occur & not occur & not occur & not occur \\
\hline $241-B Y-106^{*}$ & not occur & not occur & not occur & not occur & not occur & not occur & not occur & not occur & not occur \\
\hline
\end{tabular}


RPP-5926 REV 6

Table D-3. Time to Reach $25 \%$ of the Lower Flammability Limit Under Barometric Breathing Rate for Current Waste at

Documented Safety Analysis Conditions. (8 sheets)

\begin{tabular}{|c|c|c|c|c|c|c|c|c|c|}
\hline Tank & $\begin{array}{l}\text { Time to } \\
\text { reach } \\
25 \% \text { LFL } \\
t_{25 \%} \\
\text { (day) }\end{array}$ & $\begin{array}{l}\text { Minimum } \\
\text { vent rate } \\
\text { to prevent } \\
25 \% \text { LFL } \\
\text { (cfm) }\end{array}$ & $\begin{array}{c}\text { Hydrogen } \\
\text { concentration } \\
\text { at time } \mathrm{t}_{25 \%} \\
{\left[\mathrm{H}_{2}\right](\mathrm{t})} \\
(\%)\end{array}$ & $\begin{array}{c}\text { Ammonia } \\
\text { concentration } \\
\text { at time } \mathrm{t}_{25 \%} \\
{\left[\mathrm{NH}_{3}\right](\mathrm{t})} \\
(\%)\end{array}$ & $\begin{array}{c}\text { Methane } \\
\text { concentration } \\
\text { at time } \mathrm{t}_{25 \%} \\
{\left[\mathrm{CH}_{4} \mid(\mathrm{t})\right.} \\
(\%)\end{array}$ & $\begin{array}{c}\mathrm{N}_{2} \mathrm{O} \\
\text { concentration } \\
\text { at time } \mathrm{t}_{25 \%} \\
{\left[\mathrm{~N}_{2} \mathrm{O}\right](\mathrm{t})} \\
(\%)\end{array}$ & $\begin{array}{c}\text { Hydrogen } \\
\text { flammability } \\
\text { at time } t_{25 \%} \\
(\%)\end{array}$ & $\begin{array}{c}\text { Ammonia } \\
\text { flammability } \\
\text { at time } t_{25 \%} \\
(\%)\end{array}$ & $\begin{array}{c}\text { Methane } \\
\text { flammability } \\
\text { at time } t_{25 \%} \\
(\%)\end{array}$ \\
\hline $241-\mathrm{BY}-107$ & not occur & not occur & not occur & not occur & not occur & not occur & nol occur & not occur & not occur \\
\hline $24 t-8 Y-108$ & not occur & not occur & not occur & not occur & notoccur & not occur & not occur & not occur & not occur \\
\hline 241-BY-109 & not occur & not occur & not occur & not occur & not occur & not occur & not occur & not occur & not occur \\
\hline $241-B Y-110$ & not occur & nol occur & not occur & not occur & not occur & not occur & not occur & not occur & not occur \\
\hline 241-BY-11! & not occur & not occur & not occur & not occur & not occur & not occur & not occur & not occur & not occur \\
\hline $241-B Y-112$ & not occur & not occur & not occur & not occur & not occur & not occur & not occur & not occur & not occur \\
\hline $241-\mathrm{C}-101$ & not occur & not occur & not occur & not occur & not occur & not occur & not occur & not occur & not occur \\
\hline $24 !-C-102$ & not occur & not occur & not occur & not occur & not occur & not oceur & not occur & not occur & not occur \\
\hline $241-\mathrm{C}-103^{*}$ & not occur & not occur & not occur & not occur & not occur & not occur & not occur & not occur & not occur \\
\hline $241-C-104$ & 736 & 0.27 & 0.65 & 1.00 & 0.11 & 1.68 & 16.18 & 6.67 & 2.18 \\
\hline $241-C-105$ & not occur & not occur & not occur & not occur & not occur & not occur & not occur & not occur & not occur \\
\hline $241-\mathrm{C}-106$ & not occur & not occur & not occur & not occur & nol occur & not occur & not occur & not occur & not occur \\
\hline $241-\mathrm{C}-107$ & 341 & 0.30 & 0.99 & 0.03 & 0.00 & 0.04 & 24.76 & 0.17 & 0.08 \\
\hline $241 \cdot C-108$ & not occur & not occur & not occur & not occur & not occur & not occus & not occur & not occur & not occur \\
\hline $241-C-109$ & not occur & not occur & not occur & not occur & not occur & not occur & not occur & not occur & not occur \\
\hline $241-C-110$ & not occur & not occur & not occur & not occur & not occur & not occur & not occur & not occur & not occur \\
\hline $24 I-C-111$ & not occur & not occur & not occur & not occur & not occur & not occur & not occur & not occur & not occur \\
\hline $241-C-112$ & not occur & not occur & not occur & not occur & not occur & not occur & not occur & not occur & not occur \\
\hline $241-C-201$ & not occur & not occur & not occur & not occur & not occur & not occur & not occur & not occur & not occur \\
\hline $24 !-C-202$ & not occur & not occur & not occur & not occur & not occur & not occur & not occur & not occur & not occur \\
\hline $241-C \cdot 203$ & not occur & not occur & not occur & not occur & not occur & not occur & not occur & not occur & not occur \\
\hline $241-\mathrm{C}-204$ & not occur & not occur & not occur & not occur & not occur & not occur & not occur & not occur & not occur \\
\hline $241-S-101^{*}$ & not occur & not occur & not occur & not occur & not occur & not occur & not occur & not occur & not occur \\
\hline $241-S-102 *$ & not occur & not occur & not occur & not occur & not occur & not occur & not occur & not occur & not occur \\
\hline
\end{tabular}




\section{RPP-5926 REV 6}

Table D-3. Time to Reach 25\% of the Lower Flammability Limit Under Barometric Breathing Rate for Current Waste at

Documented Safety Analysis Conditions. (8 sheets)

\begin{tabular}{|c|c|c|c|c|c|c|c|c|c|}
\hline Tank & $\begin{array}{c}\text { Time to } \\
\text { reach } \\
25 \% \text { LFL } \\
t_{25 \%} \\
\text { (day) } \\
\end{array}$ & $\begin{array}{l}\text { Minimum } \\
\text { vent rate } \\
\text { to prevent } \\
25 \% \text { LFL } \\
(\mathrm{cfm})\end{array}$ & $\begin{array}{c}\text { Hydrogen } \\
\text { concentration } \\
\text { at time } \mathrm{t}_{25 \%} \\
{\left[\mathrm{H}_{2}\right](\mathrm{t})} \\
(\%) \\
\end{array}$ & $\begin{array}{c}\text { Ammonia } \\
\text { concentration } \\
\text { at time } \mathrm{t}_{25 \%} \\
{\left[\mathrm{NH}_{3}\right](\mathrm{t})} \\
(\%) \\
\end{array}$ & $\begin{array}{c}\text { Methane } \\
\text { concentration } \\
\text { at time } \mathrm{t}_{25 \%} \\
{\left[\mathrm{CH}_{4} \mid(\mathrm{t})\right.} \\
(\%)\end{array}$ & $\begin{array}{c}\mathrm{N}_{2} \mathrm{O} \\
\text { concentration } \\
\text { at time } \mathrm{t}_{25 \%} \\
{\left[\mathrm{~N}_{2} \mathrm{O}(\mathrm{t})\right.} \\
(\%) \\
\end{array}$ & $\begin{array}{c}\text { Hydrogen } \\
\text { flammability } \\
\text { at time } t_{25 \%} \\
(\%)\end{array}$ & $\begin{array}{c}\text { Ammonia } \\
\text { flammability } \\
\text { at time } \mathrm{t}_{25 \%} \\
(\%)\end{array}$ & $\begin{array}{c}\text { Methane } \\
\text { flammability } \\
\text { at time } t_{25 \%} \\
(1 \%)\end{array}$ \\
\hline 241-S-103* & not occur & not occur & not occur & not occur & not occur & not occur & not occur & not occur & not occur \\
\hline $24 !-S-104$ & not occur & not occur & not occur & not occur & not occur & not occur & not occur & not occur & not occur \\
\hline $241-S-105$ & not occur & not occur & not occur & not occur & not occur & not occur & not occus & not occur & not occur \\
\hline $241-S-106^{*}$ & not occur & not occur & not occur & not occur & not occur & not occur & not occur & not occur & not occur \\
\hline $241-S-107$ & not occur & not occur & not occur & not occur & not occur & not occur & not occur & not occur & not occur \\
\hline $241-S-108$ & not occur & not occur & not occur & not occur & not occur & not occur & not occur & not occur & not occur \\
\hline $241-S-109$ & not occur & not occur & not occur & not occur & not occur & not occur & not occur & not occur & not occur \\
\hline 24$]-S-110$ & not occur & not occur & not occur & not occur & not occur & not occur & not occur & not occur & not occur \\
\hline $241-S-111^{*}$ & not occur & not occur & not occur & not occur & not occur & not occur & not occur & not occur & not occur \\
\hline $241-S \cdot 112$ & not occur & not occur & not occur & not occur & not occur & not occur & not occur & not occur & not occur \\
\hline $241-S X-101 *$ & not occur & not occur & not occur & not occur & not occur & not occur & not occur & not occur & not occur \\
\hline $241-S X-102^{*}$ & not occur & not occur & not occur & not occur & not occur & not occur & not occur & not occur & not occur \\
\hline $241-S X-103^{*}$ & 123 & 0.75 & 0.91 & 0.24 & 0.03 & 0.07 & 22.66 & 1.60 & 0.63 \\
\hline $241-S X-104^{*}$ & not occur & not occur & not occur & nol accur & not occur & not occur & not occur & not occur & not occur \\
\hline $241-\mathrm{SX}-105^{*}$ & $25 !$ & 0.56 & 0.94 & 0.20 & 0.01 & 0.06 & 23.41 & 1.32 & 0.27 \\
\hline $241-S X-106^{*}$ & not occur & not occur & not occur & not occur & not occur & not occur & not occur & not occur & not occur \\
\hline $241-\mathrm{SX}-107$ & not occur & not occur & not occur & not occur & not occur & not occur & not occur & not occur & not occur \\
\hline $24 \mid-S X-108^{*}$ & not occur & notoccur & not occur & not occur & not occur & not occur & not occur & not occur & not occur \\
\hline $241 \cdot S X-109$ & not occur & not occur & not occur & not occur & not occur & not occur & not occur & not occur & not occur \\
\hline $241-\mathrm{SX}-110$ & not occur & not occur & not occur & not occur & not occur & not occur & not occur & not occur & not occur \\
\hline 241-SX-111 & not occur & not occur & not occur & not occur & not occur & not occur & not occur & not occur & not occur \\
\hline $24 !-\mathrm{SX}-112$ & not occur & not occur & not occur & not occur & not occur & not occur & not occur & not occur & not occur \\
\hline 241-SX-113 & not occur & not occur & not occur & not occur & not occur & not occur & not occur & not occur & not occur \\
\hline $241-S X-114$ & not occur & not occur & not occur & not occur & not occur & not occur & not occur & not occur & not occur \\
\hline
\end{tabular}


RPP-5926 REV 6

Table D-3. Time to Reach $25 \%$ of the Lower Flammability Limit Under Barometric Breathing Rate for Current Waste at

Documented Safety Analysis Conditions. (8 sheets)

\begin{tabular}{|c|c|c|c|c|c|c|c|c|c|}
\hline Tank & $\begin{array}{c}\text { Time to } \\
\text { reach } \\
25 \% \mathrm{LFL} \\
\mathrm{t}_{25 \%} \\
\text { (day) } \\
\end{array}$ & $\begin{array}{l}\text { Minimum } \\
\text { vent rate } \\
\text { to prevent } \\
25 \% \mathrm{LFL} \\
(\mathrm{cfm}) \\
\end{array}$ & $\begin{array}{c}\text { Hydrogen } \\
\text { concentration } \\
\text { at time } t_{25 \%} \\
{\left[\mathrm{H}_{2}\right](\mathrm{t})} \\
(\%)\end{array}$ & $\begin{array}{c}\text { Ammonia } \\
\text { concentration } \\
\text { at time } \mathrm{t}_{25 \%} \\
{\left[\mathrm{NH}_{3} \mid(\mathrm{t})\right.} \\
(\%)\end{array}$ & $\begin{array}{c}\text { Methane } \\
\text { concentration } \\
\text { at time } \mathrm{t}_{25 \%} \\
{\left[\mathrm{CH}_{4} \mid(\mathrm{t})\right.} \\
(\%) \\
\end{array}$ & $\begin{array}{c}\mathrm{N}_{2} \mathrm{O} \\
\text { concentration } \\
\text { at time } \mathrm{t}_{25 \%} \\
{\left[\mathrm{~N}_{2} \mathrm{O}\right](\mathrm{t})} \\
(\%) \\
\end{array}$ & $\begin{array}{c}\text { Hydrogen } \\
\text { flammability } \\
\text { at time } t_{25 \%} \\
(\%)\end{array}$ & $\begin{array}{c}\text { Ammonia } \\
\text { fammability } \\
\text { at time } t_{25 \%} \\
(\%)\end{array}$ & $\begin{array}{c}\text { Methane } \\
\text { flammability } \\
\text { at time } t_{25 \%} \\
(\%)\end{array}$ \\
\hline 24I-SX-115 & not occur & not occur & not occur & not occur & not occur & not occur & not occur & not occur & not occur \\
\hline $241-\mathrm{T} \cdot 101$ & not occur & not occur & not occur & not occur & not occur & not occur & not occur & not occur & not occur \\
\hline $241 \cdot T-102$ & not occur & not occur & not occur & not occur & not occur & not occur & not occur & not occur & not occur \\
\hline $241-T-103$ & not occur & not occur & not occur & not occur & not occur & not occur & not occur & not occur & not occur \\
\hline $241-T-104$ & not occur & not occur & not occur & not occur & not occur & not occur & not occur & notoccur & not occur \\
\hline $241-T-105$ & not occur & not occur & not occur & not occur & not occur & not occur & nol occur & not occur & not occur \\
\hline $241-T-106$ & not occur & not occur & not occur & not occur & not occur & not occur & not occur & not occur & not occur \\
\hline $241-T-107$ & not occur & not occur & not occur & not occur & not occur & not occur & not occur & not occur & not occur \\
\hline $241-T-108$ & not occur & not occur & not occur & not occur & not occur & not occur & not occur & not occur & not occur \\
\hline $241-T-109$ & not occur & not occur & not occur & not occur & not occur & not occur & not occur & not occur & not occur \\
\hline $241-T-110^{*}$ & not occur & not occur & not occur & not occur & not occur & not occur & not occur & not occur & not occur \\
\hline 241-T-111 & not occur & not occur & not occur & not occur & not occur & not occur & not occur & not occur & not occur \\
\hline $241-1-112$ & not occur & not occur & not occur & not occur & not occur & not occur & not occur & not occur & not occur \\
\hline $241-T-201$ & 271 & 0.02 & 0.86 & 0.04 & 0.16 & 2.50 & 21.50 & 0.28 & 3.24 \\
\hline $241-\mathrm{T}-202$ & not occur & nol occur & not occur & not occur & not occur & not occur & not occur & not occur & not occur \\
\hline $241-\mathrm{T}-203^{*}$ & not occur & not occur & not occur & not occur & not occur & not occur & not occur & not occur & not occur \\
\hline $241-T-204$ & not occur & not occur & not occur & not occur & not occur & not occur & not occur & not occur & not occur \\
\hline $241-T X-101$ & not occur & not occur & not occur & not occur & not occur & not occur & not occur & not occur & not occur \\
\hline $241-\mathrm{TX}-102$ & not occur & not occur & not occur & not occur & not occur & not occur & not occur & not occur & not oecur \\
\hline 241-TX-103 & not occur & not occur & not occur & not occur & not occur & not occur & not occur & not occur & not occur \\
\hline 241-TX-104 & not occur & not occur & not occur & not occur & not occur & not occur & not occur & not occur & not occur \\
\hline $241-1 X-105$ & not occur & not occur & not occur & not occur & not occur & not occur & not occur & not occur & not occur \\
\hline 241-TX-106 & not occur & not occur & not occur & not occur & not occur & not occur & not occur & not occur & not occur \\
\hline $241-T X-107$ & not occur & not occur & not occur & not occur & not occur & not occur & not occur & not occur & not occur \\
\hline
\end{tabular}


Table D-3. Time to Reach $25 \%$ of the Lower Flammability Limit Under Barometric Breathing Rate for Current Waste at

Documented Safety Analysis Conditions. (8 sheets)

\begin{tabular}{|c|c|c|c|c|c|c|c|c|c|}
\hline Tank & $\begin{array}{c}\text { Time to } \\
\text { reach } \\
25 \% \mathrm{IJL} \\
\mathrm{t}_{25 \%} \\
\text { (day) } \\
\end{array}$ & $\begin{array}{l}\text { Minimum } \\
\text { vent rate } \\
\text { to prevent } \\
25 \% \text { LFL } \\
\text { (cfm) } \\
\end{array}$ & $\begin{array}{c}\text { Hydrogen } \\
\text { concentration } \\
\text { at time } \mathrm{t}_{25 \%} \\
{\left[\mathrm{H}_{2}\right](\mathrm{t})} \\
(\%) \\
\end{array}$ & $\begin{array}{c}\text { Ammonia } \\
\text { concentration } \\
\text { at time } \mathrm{t}_{25 \%} \\
{\left[\mathrm{NH}_{3}\right](\mathrm{t})} \\
(\%) \\
\end{array}$ & $\begin{array}{c}\text { Methane } \\
\text { concentration } \\
\text { at time } \mathrm{t}_{25 \%} \\
{\left[\mathrm{CH}_{4}\right](\mathbf{t})} \\
(\%) \\
\end{array}$ & $\begin{array}{c}\mathrm{N}_{2} \mathrm{O} \\
\text { concentration } \\
\text { at time } \mathrm{t}_{25 \%} \\
{\left[\mathrm{~N}_{2} \mathrm{O}\right](\mathrm{t})} \\
(\%) \\
\end{array}$ & $\begin{array}{c}\text { Hydrogen } \\
\text { flammability } \\
\text { at time } t_{25 \%} \\
(\%)\end{array}$ & $\begin{array}{c}\text { Ammonia } \\
\text { flammability } \\
\text { at time } t_{25 \%} \\
(\%)\end{array}$ & $\begin{array}{c}\text { Methane } \\
\text { flammability } \\
\text { at time } t_{25 \%} \\
(\%)\end{array}$ \\
\hline $24 !-\mathrm{TX}-108$ & not occur & not occur & not occur & not occur & not occur & not occur & not occur & not occur & not occur \\
\hline $241-\mathrm{TX}-109$ & not occur & not occur & not occur & not occur & not occur & not occur & not occur & not occur & not occur \\
\hline $241-\mathrm{TX}-110$ & not occur & not occur & not occur & not occur & not occur & not occur & not occur & not occur & not occur \\
\hline 241-TX-111 & not occur & not occur & not occur & not occur & not occur & not occur & not occur & not occur & not occur \\
\hline $241-T X-112$ & not occur & not occur & not occur & not occur & not occur & not occur & not occur & not occur & not occur \\
\hline $241-T X-113$ & not occur & not occur & not occur & not occur & not occur & not occur & not occur & not occur & not occur \\
\hline 241-TX-114 & not occur & not occur & not occur & not occur & not occur & not occur & not occur & not occur & not occur \\
\hline $241-\mathrm{TX}-115$ & not occur & not occur & not occur & not occur & not occur & not occur & not occur & not occur & not occur \\
\hline $241-T X-116$ & not occur & not occur & not occur & not occur & not occur & not occur & not occur & not occur & not occur \\
\hline $241-\mathrm{TX}-117$ & not occur & not occur & not occur & not occur & not occur & not occur & not occur & not occur & not occur \\
\hline $241-\mathrm{TX}-118$ & not occur & not occur & not occur & not occur & not occur & not occur & not occur & not occur & not occur \\
\hline $241-\mathrm{TY}-101$ & not occur & not occur & not occur & not occur & not occur & not occur & not occur & not occur & not occur \\
\hline 241-TY-102 & not occur & not occur & not occur & not occur & not occur & not occur & not occur & not occur & not occur \\
\hline $241-T Y-103$ & not occur & not occur & not occur & not occur & not occur & not occur & not occur & not occur & not oecur \\
\hline $241-T Y-104$ & nol occur & not occur & not occur & not occur & not occur & not occur & not occur & not occur & not occur \\
\hline 241-TY-105 & not occut & not occur & not occur & not occur & not occur & not occur & not occur & not occur & not occur \\
\hline $24 !-Г Y-106$ & not occur & not occur & not occur & not occur & not occur & not occur & not occur & not occur & not occur \\
\hline 241-U-101 & not occur & not occur & not occur & not occur & not occur & not occur & not occur & not occur & not occur \\
\hline $241-U-102^{*}$ & not occur & not occur & not occur & not occur & not occur & not occur & not occur & not occur & not occur \\
\hline $241-\mathrm{U}-103^{*}$ & not occur & not occur & not occur & not occur & not occur & not occur & not occur & not occur & not occur \\
\hline $241 \cdot \mathrm{U}-104$ & not occur & not occur & not occur & not occur & not occur & not occur & not occur & not occur & not occur \\
\hline $241-\mathrm{U}-105^{*}$ & not occur & not occur & not occur & not occur & not occur & not occur & not occur & not occur & not occur \\
\hline 241-U-106* & not occur & not occur & not occur & not occur & not occur & not occur & not occur & not occur & not occur \\
\hline $241-U \cdot 107^{*}$ & not occur & not occur & not occur & not occur & not occur & not occur & not occur & not occur & not occur \\
\hline
\end{tabular}


RPP-5926 REV 6

Table D-3. Time to Reach $25 \%$ of the Lower Flammability Limit Under Barometric Breathing Rate for Current Waste at

\begin{tabular}{|c|c|c|c|c|c|c|c|c|c|}
\hline Tank & $\begin{array}{c}\text { Tíme to } \\
\text { reach } \\
25 \% \text { L.FL } \\
t_{25 \%} \\
\text { (day) } \\
\end{array}$ & $\begin{array}{l}\text { Minimum } \\
\text { vent rate } \\
\text { to prevent } \\
25 \% \text { LFL } \\
\text { (cfm) }\end{array}$ & $\begin{array}{c}\text { Hydrogen } \\
\text { concentration } \\
\text { at time } \mathfrak{f}_{25 \%} \\
{\left[\mathrm{H}_{2}\right](\mathrm{t})} \\
(\%) \\
\end{array}$ & $\begin{array}{c}\text { Ammonia } \\
\text { concentration } \\
\text { at time } \mathrm{t}_{25 \%} \\
{\left[\mathrm{NH}_{3} \mid(\mathrm{t})\right.} \\
(\%) \\
\end{array}$ & $\begin{array}{c}\text { Methane } \\
\text { concentration } \\
\text { at time } \mathrm{t}_{25 \%} \\
{\left[\mathrm{CH}_{4}\right](\mathrm{t})} \\
(\%) \\
\end{array}$ & $\begin{array}{c}\mathrm{N}_{2} \mathrm{O} \\
\text { concentration } \\
\text { at time } \mathrm{t}_{25 \%} \\
{\left[\mathrm{~N}_{2} \mathrm{O}\right](\mathrm{t})} \\
(\%) \\
\end{array}$ & $\begin{array}{l}\text { Hydrogen } \\
\text { flammability } \\
\text { at time } t_{25 \%} \\
(\%)\end{array}$ & $\begin{array}{c}\text { Ammonia } \\
\text { flammability } \\
\text { at time } t_{25 \%} \\
(\%)\end{array}$ & $\begin{array}{c}\text { Methane } \\
\text { farmmability } \\
\text { at time } t_{25 \%} \\
(\%)\end{array}$ \\
\hline $241-U-108^{*}$ & not occur & not occur & not occur & not occur & not occur & not occur & not occur & not occur & not occur \\
\hline $241-U-109^{*}$ & not occur & not occur & not occur & not occur & not occur & not occur & not occur & not occur & not occur \\
\hline $241-U-110$ & not occur & not occur & not occur & not occur & not occur & not occur & not occur & not occur & not occur \\
\hline $241-U-111$ & not occur & not occur & not occur & not occur & not occur & not occur & not occur & not occur & not occur \\
\hline $241-\mathrm{U}-112$ & not occur & not occur & not occur & not occur & not occur & not occur & not occur & not occur & not occur \\
\hline $241-U-201$ & not occur & not occur & not occur & not occur & not occur & not occur & not occur & not occur & not occur \\
\hline $241-U-202$ & not occur & not occur & not occur & not occur & not occur & not occur & not occur & not occur & not occur \\
\hline $241-y-203$ & not occur & not occur & not occur & not occur & not occur & not occur & not occur & not occur & not occur \\
\hline $241-U-204$ & not occur & not occur & not occur & not occur & not occur & not occur & not occur & not occur & not occur \\
\hline
\end{tabular}

Note: 'DSA conditions are the conditions with current waste plus water additions of $10 \mathrm{kgal}$ and $1 \mathrm{kgal}$ for 100 - and 200 - series, respectively and $5{ }^{\circ} \mathrm{C}$ increase for DSTs.

${ }^{2}$ For all DSTs and SSTs with "*", the ammonia was analyzed with equilibrium model. 
RPP-5926 REV 6

Table D-4. Time to Reach 100\% of the Lower Flammability Limit Under Barometric Breathing Rate for Current Waste at

Documented Safety Analysis Conditions. (8 sheets)

\begin{tabular}{|c|c|c|c|c|c|c|c|c|c|}
\hline Tank & $\begin{array}{c}\text { Time to reach } \\
100 \% \text { LFL } \\
t_{100 \%} \\
\text { (day) }\end{array}$ & $\begin{array}{c}\text { Minimum } \\
\text { vent rate } \\
\text { to prevent } \\
100 \% \mathrm{LFL} \\
(\mathrm{cfm}) \\
\end{array}$ & $\begin{array}{c}\text { Hydrogen } \\
\text { concentration } \\
\text { at time } \mathbf{t}_{100 \%} \\
{\left[\mathbf{H}_{2}\right](\mathrm{t})} \\
(\%) \\
\end{array}$ & $\begin{array}{c}\text { Ammonia } \\
\text { concentration } \\
\text { at time } \mathrm{t}_{100 \%} \\
{\left[\mathrm{NH}_{3}\right](\mathrm{t})} \\
(\%) \\
\end{array}$ & $\begin{array}{c}\text { Methane } \\
\text { concentration } \\
\text { at time } \mathrm{t}_{100 \%} \\
{\left[\mathrm{CH}_{\mathbf{4}} \mid(\mathrm{t})\right.} \\
(\%)\end{array}$ & $\begin{array}{c}\mathrm{N}_{2} \mathrm{O} \\
\text { concentration } \\
\text { at time } \mathrm{t}_{100 \%} \\
{\left[\mathrm{~N}_{2} \mathrm{O}\right](\mathfrak{t})} \\
(\%) \\
\end{array}$ & $\begin{array}{c}\text { Hydrogen } \\
\text { flammability at } \\
\text { time } \mathrm{t}_{1(0) \%} \\
(\%)\end{array}$ & $\begin{array}{c}\text { Ammonia } \\
\text { flammability } \\
\text { at time } t_{100 \%} \\
(\%)\end{array}$ & $\begin{array}{c}\text { Methane } \\
\text { flammability } \\
\text { at time } t_{100 \%} \\
(\%)\end{array}$ \\
\hline $24 \mathrm{I}-\mathrm{AN}-10 \mathrm{I}$ & not occur & not occur & not occur & not occur & not occur & not occur & not occur & not occur & not occur \\
\hline $24 !-A N-102$ & 92 & 0.40 & 3.89 & 0.09 & 0.08 & 1.57 & 97.17 & 0.58 & 1.59 \\
\hline $241-\mathrm{AN}-103$ & not occur & not occur & not occur & not occur & not occur & not occur & not occur & not occur & not occur \\
\hline 241-AN-104 & not occur & not occur & not occur & not occur & not occur & not occur & not occur & not occur & not occur \\
\hline 241-AN-105 & not occur & not occur & not occur & not occur & not occur & not occur & not occur & not occur & not occur \\
\hline $241-\mathrm{AN}-106$ & not occur & not occur & not occur & not occur & not occur & not occur & not occur & not occur & not occur \\
\hline 241-AN-107 & 106 & 0.32 & 3.80 & 0.08 & 0.19 & 2.10 & 95.06 & 0.51 & 3.87 \\
\hline 241-AP-101 & not occur & not occur & not occur & not occur & not occur & not occur & not occur & not occur & not occur \\
\hline $241-\mathrm{AP}-102$ & not occur & not occur & not occur & not occur & not occur & not occur & not occur & not occur & not occur \\
\hline $241-\mathrm{AP}-103$ & not occur & not occur & nol occur & not occur & not occur & not occur & not occur & not occur & not occur \\
\hline $241-\mathrm{AP}-104$ & not occur & not occur & not occur & not occur & not occur & not occur & not occur & not occur & not occur \\
\hline 241-AP-105 & not occur & not occur & not occur & not occur & not occur & not occur & not occur & not occur & not occur \\
\hline 241-AP-106 & not occur & not occur & not occur & not occur & not occur & not occur & not occur & not occur & not occur \\
\hline $241-A P \cdot 107$ & not occur & not occur & not occur & not occur & not occur & not occur & not occur & not occur & not occur \\
\hline 241-AP-108 & not occur & not occur & not occur & not occur & not occur & not occur & not occur & not occur & not occur \\
\hline $241-A W-101$ & not occur & not occur & not occur & not occur & not occur & not occur & not occur & not occur & not occur \\
\hline 241-AW-102 & not occur & not occur & not occur & not occur & not occur & not occur & not occur & not occur & not occur \\
\hline $241-A W-103$ & not occur & not occur & not occur & not occur & not occur & not occur & not occur & not occur & not occur \\
\hline $241-\mathrm{AW}-104$ & not occur & not occur & not occur & not occur & not occur & not occur & not occur & not occur & not occur \\
\hline $241-A W-105$ & not occur & not occur & not occur & not occur & not occur & not occur & not occur & not occur & not occur \\
\hline $241-A W-106$ & not occur & not occur & not occur & not occur & not occur & not occur & not occur & not occur & not occur \\
\hline 241-AY-101 & not occur & not occur & not occur & not occur & not occur & not occur & not occur & not occur & not occur \\
\hline $241-\mathrm{AY}-102$ & 59 & 0.76 & 3.90 & 0.02 & 0.05 & 0.33 & 97.60 & 0.14 & 0.94 \\
\hline $241-A Z-101$ & 91 & 0.66 & 3.87 & 0.02 & 0.12 & 0.23 & 96.82 & 0.12 & 2.36 \\
\hline
\end{tabular}




\section{RPP-5926 REV 6}

Table D-4. Time to Reach 100\% of the Lower Flammability Limit Under Barometric Breathing Rate for Current Waste at

Documented Safety Analysis Conditions. (8 sheets)

\begin{tabular}{|c|c|c|c|c|c|c|c|c|c|}
\hline Tank & $\begin{array}{c}\text { Time to reach } \\
100 \% \mathrm{LFL} \\
t_{100 \%} \\
\text { (day) }\end{array}$ & $\begin{array}{c}\text { Minimum } \\
\text { vent rate } \\
\text { to prevent } \\
100 \% \mathrm{LFL} \\
\text { (cfm) }\end{array}$ & $\begin{array}{l}\text { Hydrogen } \\
\text { concentration } \\
\text { at time } t_{100 \%} \\
{\left[\mathrm{H}_{2}\right](\mathrm{t})} \\
(\%)\end{array}$ & $\begin{array}{c}\text { Ammonia } \\
\text { concentration } \\
\text { at time } \mathrm{t}_{100 \%} \\
{\left[\mathrm{NH}_{3}\right](\mathrm{t})} \\
(\%)\end{array}$ & $\begin{array}{c}\text { Methane } \\
\text { concentration } \\
\text { at time } \mathrm{t}_{100 \%} \\
{\left[\mathrm{CH}_{4}\right](\mathrm{t})} \\
(\%)\end{array}$ & $\begin{array}{c}\mathrm{N}_{2} \mathrm{O} \\
\text { concentration } \\
\text { at time } \mathrm{t}_{100 \%} \\
{\left[\mathrm{~N}_{2} \mathrm{O}\right](\mathrm{t})} \\
(\%)\end{array}$ & $\begin{array}{c}\text { Hydrogen } \\
\text { flammability at } \\
\text { time } \mathrm{t}_{\mathbf{i} 00 \%} \\
(\%)\end{array}$ & $\begin{array}{c}\text { Ammonia } \\
\text { flammability } \\
\text { at time } t_{100 \%} \\
(\%)\end{array}$ & $\begin{array}{c}\text { Methane } \\
\text { flammability } \\
\text { at time } \mathrm{t}_{\mathrm{t} 00 \%} \\
(\%)\end{array}$ \\
\hline 241-AZ-102 & 47 & 0.92 & 3.84 & 0.05 & 0.09 & 0.12 & 96.03 & 0.34 & 1.84 \\
\hline 241-SY-101 & not occur & not occur & not occur & not occur & not occur & not occur & not occur & not occur & not occur \\
\hline $241-S Y-102$ & not occur & not occur & not occur & not occur & not occur & not occur & not occur & not occur & not occur \\
\hline 241-SY-103 & not occur & not occur & not occur & not occur & not occur & not occur & not occur & not occur & not occur \\
\hline $241-A \cdot 101^{*}$ & not occur & not occur & not occur & not occur & not occur & not occur & not occur & not occur & not occur \\
\hline $241-\Lambda-102$ & not occur & not occur & not occur & not occur & not occur & not occur & not occur & not occur & not occur \\
\hline $241-\mathrm{A}-103^{*}$ & not occur & not occur & not occur & not occur & not occur & not occur & not occur & not occur & not occur \\
\hline $241-A-104$ & not occur & not occur & not occur & not occur & not occur & not occur & not occur & not occur & not occur \\
\hline $241-A-105$ & na & na & na & na & na & na & na & na & na \\
\hline $241-\mathrm{A}-106$ & not occur & not occur & not occur & not occur & not occur & not occur & not occur & not occur & not occur \\
\hline $241-A X-101 *$ & not occur & not occur & not occur & not occur & not occur & not occur & not occur & not occur & not occur \\
\hline $241-A X-102$ & not occur & not occur & not occur & not occur & not occur & not occur & not occur & not occur & not occur \\
\hline $241-\mathrm{AX}-103$ & not occur & not occur & not occur & not occur & not occur & not oceus & not oecur & not occur & not occus \\
\hline $241-\Lambda X-104$ & not occur & not occur & not occur & not occur & not occur & not occur & not occur & not occur & not occur \\
\hline $24 \mid-B-101$ & not occur & not occur & not occur & not occur & not occur & not occur & not occur & not occur & not occur \\
\hline $24 !-B-102$ & not occur & not occur & not occur & not occur & not occur & not occur & not occur & not occur & not occur \\
\hline $241-B-103$ & not occur & not occur & not occur & not occur & not occur & not occur & not occur & not occur & not occur \\
\hline $24 !-B-104$ & not occur & not occur & not occur & not occur & not occur & not occur & not occur & not occur & not occur \\
\hline $241-B-105$ & not occur & not occur & not occur & not occur & not occur & not occur & not occur & not occur & not occur \\
\hline $241-B-106$ & not occur & not occur & not occur & not occur & not occur & not occur & not occur & not occur & not occur \\
\hline $241-B-107$ & not occur & not occur & not occur & not occur & not occur & not occur & not occur & not occur & not occur \\
\hline $241-B-108$ & not occur & not occur & not occur & not occur & not occur & not occur & not occur & not occur & not occur \\
\hline $241-B-109$ & not occur & not occur & not occur & not occur & not occur & not occur & not occur & not occur & not occur \\
\hline $241-B-110$ & not occur & not occur & not occur & not occur & not occur & not occur & not occur & not occur & not occur \\
\hline
\end{tabular}




\section{RPP-5926 REV 6}

Table D-4. Time to Reach 100\% of the Lower Flammability Limit Under Barometric Breathing Rate for Current Waste at

Documented Safety Analysis Conditions. (8 sheets)

\begin{tabular}{|c|c|c|c|c|c|c|c|c|c|}
\hline Tank & $\begin{array}{c}\text { Time to reach } \\
100 \% \text { LFL } \\
t_{100 \%} \\
\text { (day) }\end{array}$ & $\begin{array}{l}\text { Minimum } \\
\text { vent rate } \\
\text { to prevent } \\
100 \% \text { LFL } \\
\text { (cfm) }\end{array}$ & $\begin{array}{c}\text { Hydrogen } \\
\text { concentration } \\
\text { at time } \mathbf{t}_{100 \%} \\
{\left[\mathrm{H}_{2}\right](\mathrm{t})} \\
(\%)\end{array}$ & $\begin{array}{c}\text { Ammonia } \\
\text { concentration } \\
\text { at time } \mathrm{t}_{10 \mathrm{H} \%} \\
{\left[\mathrm{NH}_{3}\right](\mathrm{t})} \\
(\%)\end{array}$ & $\begin{array}{c}\text { Methane } \\
\text { concentration } \\
\text { at time } \mathrm{t}_{100 \%} \\
{\left[\mathrm{CH}_{4}\right](\mathrm{t})} \\
(\%)\end{array}$ & $\begin{array}{c}\mathrm{N}_{2} \mathrm{O} \\
\text { concentration } \\
\text { at time } \mathrm{t}_{100 \%} \\
{\left[\mathrm{~N}_{2} \mathrm{O} \mid(\mathrm{t})\right.} \\
(\% / \%)\end{array}$ & $\begin{array}{c}\text { Hydrogen } \\
\text { flammability at } \\
\text { time } \mathrm{t}_{1(\mathfrak{m} \%} \\
(\%)\end{array}$ & $\begin{array}{c}\text { Ammonia } \\
\text { flammability } \\
\text { at time } t_{100 \%} \\
(\%)\end{array}$ & $\begin{array}{c}\text { Methane } \\
\text { flammability } \\
\text { at time } t_{100 \%} \\
(\%)\end{array}$ \\
\hline 241-B-111 & not occur & not occur & not occur & not occur & not occur & not occur & not occur & not occur & not occur \\
\hline $241-B-112$ & not occur & not occur & not occur & not occur & not occur & not occur & not occur & not occur & not occur \\
\hline $241-B-201$ & not occur & not occur & not occur & not occur & not occur & not occur & not occur & not occur & not occur \\
\hline $241 \cdot B-202$ & not occur & not occur & not occur & not occur & not occur & not occur & not occur & not occur & not occur \\
\hline $241-B-203^{*}$ & 254 & 0.005 & 3.96 & 0.03 & 0.04 & 0.59 & 99.05 & 0.19 & 0.78 \\
\hline $241-B-204^{*}$ & 292 & 0.005 & 3.96 & 0.03 & 0.04 & 0.55 & 99.12 & 0.18 & 0.73 \\
\hline 24l-BX-101 & nor occur & not occur & not occur & not occur & not occur & not occur & not occur & not occur & not occur \\
\hline $241-8 \times-102$ & not occur & not occur & not occur & not oceut & not occur & not occur & not occur & not occur & not occur \\
\hline $241-\mathrm{BX}-103$ & not occur & not occur & not occur & not occur & not occur & not occur & not occur & not occur & not occur \\
\hline $241-B X-104$ & not occur & not occur & not occur & not occur & not occur & not occur & not occur & not occur & not occur \\
\hline $241-B X-105$ & not occur & not occur & not occur & not occur & not occur & not occur & not occur & not occur & not occur \\
\hline $241-B X-106$ & not occur & not occur & not occur & not occur & not occur & not occur & not occur & not occur & not occur \\
\hline $241-B X-107$ & not occur & not occur & not occur & not occur & not occur & not occur & not occur & not occur & not occur \\
\hline $241+B X-108$ & not occur & not occur & not occur & not occur & not occur & not occur & not occur & not occur & not occur \\
\hline $241-B X-109$ & not occur & not occur & not occur & not occur & not occur & not occur & not occur & not occur & not occur \\
\hline $241-B X-110$ & not occur & not occur & not occur & not occur & not occur & not occur & not occur & not occur & not occur \\
\hline $241-B X-111$ & not occur & not occur & not occur & not occur & not occur & not occur & not occur & not occur & not occur \\
\hline $241-B X-112$ & not occur & not occur & not occur & not occur & not occur & not occur & not occur & notoccur & not occus \\
\hline $241-B Y-101$ & not oceur & not occur & not occur & not occur & not occur & not occur & not occur & not occur & not occur \\
\hline $241-B Y-102$ & not occur & not occur & not occur & not occur & not occur & not occur & not occur & not occur & not occur \\
\hline $241-B Y-103$ & not occur & not occur & not occur & not occur & not occur & not occur & not occur & not occur & not occur \\
\hline $241-B Y+104$ & not occur & not occur & not occur & not occur & not occur & not occur & not occur & not occur & not occur \\
\hline $24 !-B Y-105$ & not occur & not occur & not occur & not occur & not occur & not occur & not occur & not occur & not occur \\
\hline $241-B Y-106^{*}$ & not occur & not occur & not occur & not occur & not occur & not occur & not occur & not occur & not occur \\
\hline
\end{tabular}




\section{RPP-5926 REV 6}

Table D-4. Time to Reach 100\% of the Lower Flammability Limit Under Barometric Breathing Rate for Current Waste at

Documented Safety Analysis Conditions. (8 sheets)

\begin{tabular}{|c|c|c|c|c|c|c|c|c|c|}
\hline Tank & $\begin{array}{l}\text { Time to reach } \\
\begin{array}{c}100 \% \mathrm{LFL} \\
\mathbf{t}_{10 \% \%} \\
\text { (day) }\end{array}\end{array}$ & $\begin{array}{l}\text { Minimum } \\
\text { vent rate } \\
\text { to prevent } \\
100 \% \mathrm{LFL} \\
(\mathrm{cfm}) \\
\end{array}$ & $\begin{array}{c}\text { Hydrogen } \\
\text { concentration } \\
\text { at time } \mathrm{t}_{100 \%} \\
{\left[\mathrm{H}_{2}\right](\mathrm{t})} \\
(\%) \\
\end{array}$ & $\begin{array}{l}\text { Ammonia } \\
\text { concentration } \\
\text { at time } \mathrm{t}_{100 \%} \\
{\left[\mathrm{NH}_{3}\right](\mathrm{t})} \\
(\%)\end{array}$ & $\begin{array}{c}\text { Methane } \\
\text { concentration } \\
\text { at time } \mathrm{t}_{100 \%} \\
{\left[\mathrm{CH}_{4}\right](\mathrm{t})} \\
(\%)\end{array}$ & $\begin{array}{c}\mathrm{N}_{2} \mathrm{O} \\
\text { concentration } \\
\text { at time } \mathrm{t}_{100 \%} \\
{\left[\mathrm{~N}_{2} \mathrm{O}(\mathrm{t})\right.} \\
(\%) \\
\end{array}$ & $\begin{array}{c}\text { Hydrogen } \\
\text { flammability at } \\
\text { time } \mathrm{t}_{100 \%} \\
(\%)\end{array}$ & $\begin{array}{c}\text { Ammonia } \\
\text { flammability } \\
\text { at time } \mathrm{t}_{100 \%} \\
(\%)\end{array}$ & $\begin{array}{c}\text { Methane } \\
\text { flammability } \\
\text { at time } t_{10) \%} \\
(\%)\end{array}$ \\
\hline $241-B Y-107$ & not occur & not occur & not occur & not occur & not occur & not occur & not occur & not occur & not occur \\
\hline $241-B Y-108$ & not occur & not occur & not occur & not occur & not occur & not occur & not occur & not occur & not occur \\
\hline $241-B Y-109$ & not occur & not occur & not occur & not occur & not occur & not occur & not occur & not occur & not occur \\
\hline $241-B Y-110$ & not occur & not occur & not occur & not occur & not occur & not occur & not occur & not occur & not occur \\
\hline $241-B Y-111$ & not occur & not occur & not occur & not occur & not occur & not occur & not occur & not occur & not occur \\
\hline $241-B Y-112$ & not occur & not occur & not occur & not occur & not occur & not occur & not occur & not occur & not occur \\
\hline $241-C-101$ & not occur & not occur & not occur & not occur & not occur & not occur & not occur & not occur & not occur \\
\hline $241-C-102$ & not occur & not occur & not occur & not occur & not occur & not occur & not occur & not occur & not occur \\
\hline $241-\left(-103^{*}\right.$ & not occur & not occur & not occur & not occur & not occur & not occur & not occur & not occur & not occur \\
\hline $241-C-104$ & not occur & not occur & not occur & not occur & not occur & not occur & not occur & not occur & not occur \\
\hline $241-C-105$ & not occur & not occur & not occur & not occur & not occur & not occur & not occur & not occur & not occur \\
\hline $241-C-106$ & not occur & not occur & not occur & not occur & not occur & not occur & not occur & not occur & not occur \\
\hline $241-C-107$ & not occur & not occur & not occur & not occur & not occur & not occur & not occur & not occur & not occur \\
\hline 24$]-C-108$ & not occur & not occur & not occur & not occur & not occur & not occur & not occur & not occur & not occur \\
\hline $241-C-109$ & not occur & not occur & not occur & not occur & not occur & not occur & not occur & not occur & not occur \\
\hline $241-C-110$ & not occur & not occur & not occur & not occur & not occur & not occur & not occur & not occur & not occur \\
\hline $241-C+111$ & not occur & not occur & not occur & not occur & not occur & not occur & not occur & not occur & not occur \\
\hline $241-C-112$ & not occur & not occur & not occur & not occur & not occur & not occur & not occur & not occur & not occur \\
\hline $241-C-201$ & not occur & not occur & not occur & not occur & not occur & not occur & not occur & not occur & not occur \\
\hline $241-\mathrm{C}-202$ & not occur & not occur & not occur & not occur & not occur & not occur & not occur & not occur & not occur \\
\hline $241-C-203$ & not occur & not occur & not occur & not occur & not occur & not occur & not occur & not occur & not occur \\
\hline $241-C-204$ & not occur & not occur & not occur & not occur & not occur & not occur & not occur & not occur & not occur \\
\hline $241-S-101^{*}$ & not occur & not occur & not occur & not occur & not occur & not occur & not occur & not occur & not occur \\
\hline $241-S-102^{*}$ & not occur & not occur & not occur & not occur & not occur & not occur & not occur & not occur & not occur \\
\hline
\end{tabular}


RPP-5926 REV 6

Table D-4. Time to Reach 100\% of the Lower Flammability Limit Under Barometric Breathing Rate for Current Waste at

Documented Safety Analysis Conditions. (8 sheets)

\begin{tabular}{|c|c|c|c|c|c|c|c|c|c|}
\hline Tank & $\begin{array}{c}\text { Time to reach } \\
100 \% \text { LFL } \\
t_{100 \%} \\
\text { (day) }\end{array}$ & $\begin{array}{c}\text { Minimum } \\
\text { vent rate } \\
\text { to prevent } \\
100 \% \mathrm{LFL} \\
\text { (cfm) } \\
\end{array}$ & $\begin{array}{c}\text { Hydrogen } \\
\text { concentration } \\
\text { at time } \mathrm{t}_{100 \%} \\
{\left[\mathrm{H}_{2}\right\}(\mathrm{t})} \\
(\%)\end{array}$ & $\begin{array}{c}\text { Ammonia } \\
\text { concentration } \\
\text { at time } \mathrm{t}_{100 \%} \\
{\left[\mathrm{NH}_{3}\right](\mathrm{t})} \\
(\%)\end{array}$ & $\begin{array}{c}\text { Methane } \\
\text { concentration } \\
\text { at time } \mathrm{t}_{100 \%} \\
{\left[\mathrm{CH}_{4}\right](\mathrm{t})} \\
(\%)\end{array}$ & $\begin{array}{c}\mathrm{N}_{2} \mathrm{O} \\
\text { concentration } \\
\text { at time } \mathrm{t}_{100 \%} \\
{\left[\mathrm{~N}_{2} \mathrm{O}\right](\mathrm{t})} \\
(\%)\end{array}$ & $\begin{array}{c}\text { Hydrogen } \\
\text { flammability at } \\
\text { time } \mathrm{t}_{100 \%} \\
(1 \%)\end{array}$ & $\begin{array}{c}\text { Ammonia } \\
\text { flammability } \\
\text { at time } \mathrm{t}_{100 \%} \\
(\%)\end{array}$ & $\begin{array}{c}\text { Methane } \\
\text { flammability } \\
\text { at time } t_{100 \%} \\
(\%)\end{array}$ \\
\hline $241-S-103^{*}$ & not occur & not occur & not occur & not occur & not occur & not occur & not occur & not occur & not occur \\
\hline 24$]-S-104$ & not occur & not occur & not occur & not occur & not occur & not occur & not occur & not occur & not occur \\
\hline $241-S-105$ & not occur & not occur & not occur & not occur & not occur & not occur & not occur & not occur & not occur \\
\hline $241-S-106^{*}$ & not occur & not occur & not occur & not occur & not occur & not occur & not occur & not occur & not occur \\
\hline $241-S-107$ & not occur & not occur & not occur & not occur & not occur & not occur & not occur & not occur & not occur \\
\hline $241-S-108$ & not occur & not occur & not occur & not occur & not occur & not occur & not occur & not occur & not occur \\
\hline $241-S-109$ & not occur & not occur & not occur & not occur & not occur & not occur & not occur & not occur & not occur \\
\hline $241-S-110$ & not occur & not occur & not occur & not occur & not occur & not occur & not occur & not occur & not occur \\
\hline $241-S-111^{*}$ & not occur & not occur & not occur & not occur & not occur & not occur & not occur & not occur & not occur \\
\hline $241-S-112$ & not occur & not occur & not occur & not occur & not occur & not occur & not occur & not occur & not occur \\
\hline 241-SX-101* & not occur & not occur & not occur & not occur & not occur & not occur & not occur & not occur & not occur \\
\hline $24 !-S X-102^{*}$ & not occur & not occur & not occur & not occur & not occur & not occur & not occur & not occur & not occur \\
\hline $241-S X-103^{*}$ & not occur & not occur & not occur & not occur & not occur & not occur & not occur & not occur & not occur \\
\hline 241-SX-104* & not occur & not occur & not occur & not occur & not occur & not occur & not occur & not occur & not occur \\
\hline 241-SX-105* & not occur & not occur & not occur & not occur & not occur & not occur & not occur & not occur & not occur \\
\hline $241-\mathrm{SX}-106^{*}$ & not occur & not occur & not occur & not occur & not occur & not occur & not occur & not occur & not occur \\
\hline $241-S X-107$ & not occur & not occur & not occur & not occur & not occur & not occur & not occur & not occur & not oceur \\
\hline $241-S X-108^{*}$ & not occur & not occur & not occur & not occur & not occur & not occur & not occur & not occur & not occur \\
\hline $241-S X-109$ & not occur & not occur & not occur & not occur & not occur & not occur & not occur & not occur & not occur \\
\hline $241-S X-110$ & not occur & not occur & not occur & not occur & not occur & not occur & not occur & not occur & not occur \\
\hline 24J-SX-111 & not occur & not occur & not occur & not occur & not occur & not occur & not occur & not occur & not occur \\
\hline $241-S X-112$ & not occur & not occur & not occur & not occur & not occur & nol occur & not occur & not occur & not occur \\
\hline $241-\mathrm{SX}-113$ & not occur & not occur & nol occur & not occur & not occur & not occur & not occur & not occur & not occur \\
\hline 241-SX-114 & not occur & not occur & not occur & not occur & not occur & not occur & not occur & not occur & not occur \\
\hline
\end{tabular}


RPP-5926 REV 6

Table D-4. Time to Reach 100\% of the Lower Flammability Limit Under Barometric Breathing Rate for Current Waste at

Documented Safety Analysis Conditions. (8 sheets)

\begin{tabular}{|c|c|c|c|c|c|c|c|c|c|}
\hline Tank & $\begin{array}{l}\text { Time to reach } \\
100 \% \text { LFL } \\
t_{100 \%} \\
\text { (day) }\end{array}$ & $\begin{array}{c}\text { Minimum } \\
\text { vent rate } \\
\text { to prevent } \\
100 \% \mathrm{LFL} \\
(\mathrm{cfm}) \\
\end{array}$ & $\begin{array}{c}\text { Hydrogen } \\
\text { concentration } \\
\text { at time } t_{100 \%} \\
{\left[\mathrm{H}_{2}\right](\mathrm{t})} \\
(\%) \\
\end{array}$ & $\begin{array}{c}\text { Ammonia } \\
\text { concentration } \\
\text { at time } \mathrm{t}_{100 \%} \\
{\left[\mathrm{NH}_{3}\right](\mathrm{t})} \\
(\%)\end{array}$ & $\begin{array}{c}\text { Methane } \\
\text { concentration } \\
\text { at time } \mathfrak{t}_{100 \%} \\
{\left[\mathrm{CH}_{4}\right](\mathrm{t})} \\
(\%)\end{array}$ & $\begin{array}{c}\mathrm{N}_{2} \mathrm{O} \\
\text { concentration } \\
\text { at time } \mathrm{t}_{100 \%} \\
{\left[\mathrm{~N}_{2} \mathrm{O}\right](\mathrm{t})} \\
(\%)\end{array}$ & $\begin{array}{c}\text { Hydrogen } \\
\text { flammability at } \\
\text { time } t_{100 \%} \\
(\%)\end{array}$ & $\begin{array}{c}\text { Ammonia } \\
\text { flammability } \\
\text { at time } \mathrm{t}_{100 \%} \\
(\%)\end{array}$ & $\begin{array}{c}\text { Methane } \\
\text { flammability } \\
\text { at time } t_{100 \%} \\
(\%)\end{array}$ \\
\hline $241-S X-115$ & not occur & not occur & not occur & not occur & not occur & not occur & not occur & not occur & not occur \\
\hline $241-T-101$ & not $\alpha c$ cur & not occur & not occur & not occur & not occur & not occur & not occur & not occur & not occur \\
\hline $241-T-102$ & not occur & not occur & not occur & not occur & not occur & not occur & not occur & not occur & not occur \\
\hline $24 !-T-103$ & not occur & not occur & not occur & not occur & not occur & not occur & not occur & not occur & not occur \\
\hline $241-T-104$ & not occur & not occur & not occur & not occur & not occur & not occur & not occur & not occur & not occur \\
\hline $241-T-105$ & not occur & not occur & not occur & not occur & not occur & not occur & not occur & not occur & not occur \\
\hline $241-T-106$ & not occur & not occur & not occur & not occur & not occur & not occur & not occur & not occur & not occur \\
\hline $241-T-107$ & not occur & not occur & not occur & not occur & not occur & not occur & not occur & not occur & not occur \\
\hline $241-\top-108$ & not occur & not occur & not occur & not occur & not occur & not occur & not occur & not occur & not occur \\
\hline $241-T-109$ & not occur & not occur & not occur & not occur & not occur & not occur & not occur & not occur & not occur \\
\hline $241-T-110^{*}$ & not occur & not occur & not occur & not occur & not occur & nol occur & not occur & not occur & not occur \\
\hline $241-T-111$ & not occur & not occur & not occur & not occur & not occur & not occur & not occur & not occur & not occur \\
\hline $241-T-112$ & not occur & not occur & not occur & not occur & not occur & not occur & not occur & not occur & not occur \\
\hline $241-T-201$ & not occur & not occur & not occur & not occur & not occur & not occur & not occur & not occur & not occur \\
\hline $241-\gamma-202$ & not occur & not occur & not occur & not occur & not occur & not occur & not occur & not occur & not occur \\
\hline 24 I-T-203* & not occur & not occur & not occur & not occur & not occur & not occur & not occur & not occur & not occur \\
\hline $241-T-204$ & not occur & not occur & not occur & not occur & not occur & not occur & not occur & not occur & not occur \\
\hline $241-T X-101$ & not occur & not occur & not occur & not occur & not occur & not occur & not occur & not occur & not occur \\
\hline $24 !-T X-102$ & not occur & not occur & not occur & not occur & not occur & not occur & not occur & not occur & not occur \\
\hline 241-TX-103 & not occur & not occur & not occur & not occur & not occur & not occur & not occur & not occur & not occur \\
\hline $241-\Gamma X-104$ & not occur & not occur & not occur & not occur & not occur & not occur & not occur & not occur & not occur \\
\hline 241-TX-105 & not occur & not occur & not occur & not occur & not occur & not occur & not occur & not occur & not occur \\
\hline 241-TX-106 & not occur & not occur & not occur & not occur & not occur & not occur & not occur & not occur & not occur \\
\hline $24 !-T X-107$ & not occur & not occur & not occur & not occur & not occur & not occur & not occur & not occur & not occur \\
\hline
\end{tabular}


RPP-5926 REV 6

Table D-4. Time to Reach $100 \%$ of the Lower Flammability Limit Under Barometric Breathing Rate for Current Waste at

Documented Safety Analysis Conditions. (8 sheets)

\begin{tabular}{|c|c|c|c|c|c|c|c|c|c|}
\hline Tank & $\begin{array}{c}\text { Time to reach } \\
100 \% \text { LFL } \\
\mathbf{t}_{100 \%} \\
\text { (day) }\end{array}$ & $\begin{array}{c}\text { Minimum } \\
\text { vent rate } \\
\text { to prevent } \\
100 \% \mathrm{LFL} \\
(\mathrm{cfm}) \\
\end{array}$ & $\begin{array}{c}\text { Hydrogen } \\
\text { concentration } \\
\text { at time } \mathrm{t}_{100 \%} \\
{\left[\mathrm{H}_{2}\right](\mathrm{t})} \\
(\%) \\
\end{array}$ & $\begin{array}{c}\text { Ammonia } \\
\text { concentration } \\
\text { at time } \mathrm{t}_{100 \%} \\
{\left[\mathrm{NH}_{3}\right](\mathrm{t})} \\
(\%) \\
\end{array}$ & $\begin{array}{c}\text { Methane } \\
\text { concentration } \\
\text { at time } t_{100 \%} \\
{\left[\mathrm{CH}_{4}\right](\mathrm{t})} \\
(\%) \\
\end{array}$ & $\begin{array}{c}\mathrm{N}_{2} \mathrm{O} \\
\text { concentration } \\
\text { at time } \mathrm{t}_{100 \%} \\
{\left[\mathrm{~N}_{2} \mathrm{O}\right](\mathrm{t})} \\
(\%) \\
\end{array}$ & $\begin{array}{c}\text { Hydrogen } \\
\text { flammability at } \\
\text { time } \mathrm{t}_{100 \%} \\
(\%)\end{array}$ & $\begin{array}{c}\text { Ammonia } \\
\text { flammability } \\
\text { at time } \mathrm{t}_{100 \%} \\
(\%)\end{array}$ & $\begin{array}{c}\text { Methane } \\
\text { flammability } \\
\text { at time } t_{100 \%} \\
(\%)\end{array}$ \\
\hline 24$\}-T X-108$ & not occur & not occur & not occur & not occur & not occur & not occur & not occur & not occur & not occur \\
\hline 241-TX-109 & not occur & not occur & not occur & not occur & not occur & not occur & not occur & not occur & not occur \\
\hline 241-TX-110 & not occur & not occur & not occur & not occur & not occur & not occur & not occur & not occur & nor occur \\
\hline $241-\mathrm{TX}-111$ & not occur & not occur & not occur & not occur & not occur & not occur & not occur & not occur & not occur \\
\hline 241-TX-112 & not occur & not occur & not occur & not occur & not occur & not occur & not occur & not occur & not occur \\
\hline 241-TX-113 & not occur & not occur & not occur & not occur & not occur & not occur & not occur & not occur & not occur \\
\hline $24 \mathrm{I}-\mathrm{TX}-114$ & not occur & not occur & not occur & not occur & not occur & not occur & thot occur & not occur & not occur \\
\hline 241-TX-115 & not occur & not occur & not occur & not occur & not occur & not occur & not occur & not occur & not occur \\
\hline $241-\mathrm{TX}-116$ & not occur & not occur & not occur & not occur & not occur & not occur & not occur & not occur & not occur \\
\hline $241-\mathrm{TX}-117$ & not occur & not occur & not occur & not occur & not occur & not occur & not occur & not occur & not occur \\
\hline 241-TX-118 & not occur & not occur & not occur & not occur & not occur & not occur & not occur & not occur & not occur \\
\hline $241-\mathrm{TY}-101$ & not occur & not occur & not occur & not occur & not occur & not occur & not occur & not occur & not occur \\
\hline $241-\mathrm{TY}-102$ & not occur & not occur & not occur & not occur & not occur & not occur & not occur & not occur & not occur \\
\hline 24)-TY-103 & not occur & not occur & not occur & not occur & not occur & not occur & not occur & not occur & not occur \\
\hline $241-\mathrm{TY}-104$ & not occur & not occur & not occur & not occur & not occur & not occur & not occur & not occur & not occur \\
\hline 241-TY-105 & not occur & not occur & not occur & not occur & not occur & not occur & not occur & not occur & not occur \\
\hline 241-TY-106 & not occur & not occur & not occur & not occur & not occur & not occur & not occur & not occur & not occur \\
\hline $241-U-101$ & not occur & not occur & not occur & not occur & not occur & not occur & not occur & not occur & not occur \\
\hline $24 !-U-102^{*}$ & not occur & not accur & not occur & not occus & not occur & not occus & not occur & not occur & not occur \\
\hline 241-U-103* & not occur & not occur & not occur & not occur & not occur & not occur & not occur & not occur & not occur \\
\hline $241-U-104$ & not occur & not occur & not occur & not occur & not occur & not occur & not occur & not occur & not occur \\
\hline $241-\mathrm{U}-105^{*}$ & not occur & not occur & not occur & not occur & not occur & not occur & not occur & not occur & not occur \\
\hline $241-\mathrm{U}-106^{*}$ & not occur & not occur & not occur & not occur & not occur & not occur & not occur & not occur & not occur \\
\hline $241-U-107^{*}$ & not occur & not occur & not occur & not occur & not occur & not occur & not occur & not occur & not occur \\
\hline
\end{tabular}




\section{RPP-5926 REV 6}

Table D-4. Time to Reach $100 \%$ of the Lower Flammability Limit Under Barometric Breathing Rate for Current Waste at Documented Safety Analysis Conditions. (8 sheets)

\begin{tabular}{|c|c|c|c|c|c|c|c|c|c|}
\hline Taṇk & $\begin{array}{c}\text { Time to reach } \\
100 \% \text { LFL } \\
t_{100 \%} \\
\text { (day) }\end{array}$ & $\begin{array}{c}\text { Minimum } \\
\text { vent rate } \\
\text { to prevent } \\
100 \% \mathrm{LFL} \\
\text { (cfm) }\end{array}$ & $\begin{array}{c}\text { Hydrogen } \\
\text { concentration } \\
\text { at time } \mathbf{t}_{100 \%} \\
{\left[\mathbf{H}_{2}\right](t)} \\
(\%) \\
\end{array}$ & $\begin{array}{c}\text { Ammonia } \\
\text { concentration } \\
\text { at time } \mathrm{t}_{100 \%} \\
{\left[\mathrm{NH}_{3}\right](\mathbf{t})} \\
(\%) \\
\end{array}$ & $\begin{array}{c}\text { Methane } \\
\text { concentration } \\
\text { at time } \mathrm{t}_{100 \%} \\
{\left[\mathrm{CH}_{4}\right](\mathrm{t})} \\
(\%) \\
\end{array}$ & $\begin{array}{c}\mathrm{N}_{2} \mathrm{O} \\
\text { concentration } \\
\text { at time } \mathrm{t}_{100 \%} \\
{\left[\mathrm{~N}_{2} \mathrm{O}\right](\mathrm{t})} \\
(\%) \\
\end{array}$ & $\begin{array}{c}\text { Hydrogen } \\
\text { flammability at } \\
\text { time } \mathrm{t}_{100 \%} \\
(\%)\end{array}$ & $\begin{array}{c}\text { Ammonia } \\
\text { flammability } \\
\text { at time } t_{100 \%} \\
(\%)\end{array}$ & $\begin{array}{c}\text { Methane } \\
\text { flammability } \\
\text { at time } t_{100 \%} \\
(\%)\end{array}$ \\
\hline $241-\mathrm{U}-108^{*}$ & not occur & not occur & not occur & not occur & not occur & not occur & not occur & not occur & not occur \\
\hline $241-U-109^{*}$ & not occur & notoccur & not occur & not occur & not occur & not occur & not occur & not occur & not occur \\
\hline $241-U-110$ & not occur & not occur & not occur & not occur & not occur & not occur & not occur & not occur & not occur \\
\hline $24 !-U-111$ & not occur & not occur & not occur & not occur & not occur & not occur & not occur & not occur & not occur \\
\hline $241-\mathrm{U}-112$ & not occur & not occur & not occur & not occur & not occur & not occur & not occur & not occur & not occur \\
\hline $241-1)-201$ & not occur & not occur & not occur & not occur & not occur & not occur & not occur & not occur & not occur \\
\hline $241-U-202$ & not occur & not occur & not occur & not occur & not occur & not occur & not occur & not occur & not occur \\
\hline $241-U-203$ & not occur & not occur & not occur & not occur & not occur & not occur & not occur & not occur & not occur \\
\hline 241-U-204 & not occur & not occur & not occur & not occur & not occur & not occur & not occur & not occur & not occur \\
\hline
\end{tabular}

Note: ${ }^{1}$ DSA conditions are the conditions with current waste plus water additions of $10 \mathrm{kgal}$ and $1 \mathrm{kgal}$ for 100 - and 200 - series, respectively and $5{ }^{\circ} \mathrm{C}$ increase for DSTs.

${ }^{2}$ For alt DSTs and SSTs with "*", the ammonia was analyzed with equilibrium model. 
RPP-5926 REV 6

Table D-5. Time to Reach $25 \%$ and $100 \%$ of the Lower Flammability Limit Under Zero Vent Rate at Documented Safety Analysis Conditions. (8 sheets)

\begin{tabular}{|c|c|c|c|c|c|c|c|}
\hline Tank & $\begin{array}{c}\text { Hydrogen } \\
\text { diffusion } \\
\text { rate (Dr) } \\
\text { (cfm }^{1}\end{array}$ & $\begin{array}{l}\text { Methane } \\
\text { diffusion } \\
\text { rate (Dr) }{ }_{\mathrm{CH} 4} \\
\quad(\mathrm{cfm})^{l}\end{array}$ & $\begin{array}{c}\text { Steady state } \\
\text { total } \\
\text { flammability } \\
(\%)^{2}\end{array}$ & $\begin{array}{l}\text { Time to } \\
\text { reach } 25 \% \\
\text { of the } L F L \\
\text { (days) }\end{array}$ & $\begin{array}{l}\text { Time to } \\
\text { reach } 100 \% \\
\text { of the } L F L \\
\text { (days) }\end{array}$ & $\begin{array}{l}\text { Minimum } \\
\text { vent rate to } \\
\text { keep below } \\
25 \% \text { LFL } \\
\text { (cfm) }\end{array}$ & $\begin{array}{l}\text { Minimum } \\
\text { vent rate to } \\
\text { keep below } \\
100 \% \text { LFL } \\
\text { (cfm) }\end{array}$ \\
\hline $241-\mathrm{AN}-101$ & na & na & na & 151 & 522 & 0.25 & 0.06 \\
\hline 24l-AN-102 & na & na & $\mathrm{na}$ & 15 & 47 & 1.63 & 0.40 \\
\hline $24 \mathrm{l}-\mathrm{AN}-103$ & na & na & na & 91 & 313 & 0.41 & 0.10 \\
\hline 241-AN-104 & na & na & na & 56 & 201 & 0.53 & 0.13 \\
\hline 241-AN-105 & na & na & na & 69 & 259 & 0.34 & 0.08 \\
\hline 241-AN. 106 & na & na & na & 68 & 248 & 0.78 & 0.20 \\
\hline 241-AN-107 & na & na & na & 20 & 78 & 1.31 & 0.32 \\
\hline 241-AP-101 & na & na & na & 208 & 316 & 0.12 & 0.03 \\
\hline 241-AP-102 & na & na & na & 88 & 340 & 0.31 & 0.08 \\
\hline $241-\mathrm{AP}-103$ & na & na & na & 234 & 798 & 0.18 & 0.05 \\
\hline 241-AP- 104 & na & na & na & 151 & 490 & 0.17 & 0.04 \\
\hline 241-AP-105 & na & na & na & 127 & 425 & 0.19 & 0.05 \\
\hline 241-AP-106 & na & na & na & 114 & 390 & 0.20 & 0.05 \\
\hline $241-\mathrm{AP}-107$ & na & na & na & 1304 & 1345 & 0.04 & 0.01 \\
\hline 241-AP-108 & na & na & na & 114 & 467 & 0.30 & 0.07 \\
\hline 241-AW-101 & na & na & na & 72 & 255 & 0.33 & 0.08 \\
\hline 241-AW-102 & na & na & na & 1094 & 1500 & 0.07 & 0.02 \\
\hline $241-\mathrm{AW}-103$ & na & na & na & 245 & 993 & 0.11 & 0.03 \\
\hline 241-AW-104 & na & na & na & 107 & 393 & 0.27 & 0.07 \\
\hline 241-AW-105 & na & na & na & 1500 & 1500 & 0.04 & 0.01 \\
\hline 241-AW-106 & na & na & na & 136 & 534 & 0.17 & 0.04 \\
\hline 241-AY-101 & $\overline{\mathrm{na}}$ & na & na & 115 & 240 & 0.70 & 0.18 \\
\hline $241-\mathrm{AY}-102$ & $\mathrm{na}$ & $\mathrm{na}$ & $\mathrm{na}$ & 12 & 46 & 3.04 & 0.76 \\
\hline $241-A Z-101$ & na & na & na & 16 & 50 & 2.63 & 0.66 \\
\hline
\end{tabular}


RPP-5926 REV 6

Table D-5. Time to Reach $25 \%$ and $100 \%$ of the Lower Flammability Limit Under Zero Vent Rate at Documented Safety Analysis Conditions. (8 sheets)

\begin{tabular}{|c|c|c|c|c|c|c|c|}
\hline Tank & $\begin{array}{l}\text { Hydrogen } \\
\text { diffusion } \\
\text { rate }(\mathrm{Dr})_{\mathrm{H} 2} \\
(\mathrm{cfm})^{1}\end{array}$ & $\begin{array}{c}\text { Methane } \\
\text { diffusion } \\
\text { rate }(\mathrm{Dr})_{\mathrm{CH} 4} \\
\text { (cfm) }^{1}\end{array}$ & $\begin{array}{c}\text { Steady state } \\
\text { total } \\
\text { flammability } \\
(\%)^{2}\end{array}$ & $\begin{array}{l}\text { Time to } \\
\text { reach } 25 \% \\
\text { of the } L F L \\
\text { (days) }\end{array}$ & $\begin{array}{c}\text { Time to } \\
\text { reach } 100 \% \\
\text { of the } L F L \\
\text { (days) }\end{array}$ & $\begin{array}{l}\text { Minimum } \\
\text { vent rate to } \\
\text { keep below } \\
25 \% \mathrm{LFL} \\
(\mathrm{cfm})\end{array}$ & $\begin{array}{c}\text { Minimum } \\
\text { vent rate to } \\
\text { keep below } \\
100 \% \text { LFL } \\
\text { (cfm) }\end{array}$ \\
\hline 241-AZ-102 & na & na & na & 10 & 38 & 3.69 & 0.92 \\
\hline $241-S Y-101$ & na & na & na & 290 & 804 & 0.09 & 0.02 \\
\hline $241-S Y-102$ & na & na & na & 146 & 615 & 0.17 & 0.04 \\
\hline $241 \cdot S Y-103$ & na & na & na & 93 & 361 & 0.61 & 0.14 \\
\hline $241-A-101^{*}$ & $7.25 \mathrm{E}-03$ & $7.09 \mathrm{E}-03$ & 548 & 451 & 1500 & 0.18 & 0.04 \\
\hline $241-A-102$ & $7.25 \mathrm{E}-03$ & $7.09 \mathrm{E}-03$ & 130 & 1500 & 1500 & 0.09 & 0.02 \\
\hline $241-A-103^{*}$ & $7.25 \mathrm{E}-03$ & $7.09 \mathrm{E}-03$ & 538 & 515 & 1500 & 0.18 & 0.04 \\
\hline $241-A-104$ & $7.25 \mathrm{E}-03$ & $7.09 \mathrm{E}-03$ & 87 & 1500 & not occur & 0.03 & 0.01 \\
\hline $241-A-105$ & $7.25 \mathrm{E}-03$ & $7.09 \mathrm{E}-03$ & na & na & na & na & na \\
\hline $241-\mathrm{A}-106$ & $7.25 \mathrm{E}-03$ & $7.09 \mathrm{E}-03$ & 686 & 417 & 1500 & 0.27 & 0.07 \\
\hline $241-\mathrm{AX}-101^{*}$ & $7.11 \mathrm{E}-01$ & $2.23 \mathrm{E}-01$ & 5 & not occur & not occur & 0.11 & 0.03 \\
\hline $241-\mathrm{AX}-102$ & 7.11E-01 & $2.23 \mathrm{E}-01$ & 3 & not occur & not occur & 0.08 & 0.02 \\
\hline $241-\mathrm{AX}-103$ & $7.11 \mathrm{E}-01$ & $2.23 \mathrm{E}-01$ & 5 & not occur & not occur & 0.12 & 0.03 \\
\hline $241-\mathrm{AX}-104$ & $7.11 \mathrm{E}-01$ & $2.23 \mathrm{E}-01$ & 16 & not occur & not occur & 0.45 & 0.11 \\
\hline $241-B-101$ & $4.89 \mathrm{E}-03$ & $4.82 \mathrm{E}-03$ & 225 & 1500 & 1500 & 0.05 & 0.01 \\
\hline $241-B-102$ & $4.89 \mathrm{E}-03$ & 4.82E-03 & 126 & 1500 & 1500 & 0.03 & 0.01 \\
\hline $241-\mathrm{B}-103$ & $4.89 \mathrm{E}-03$ & $4.82 \mathrm{E}-03$ & 131 & 1500 & 1500 & 0.03 & 0.01 \\
\hline $241-\mathrm{B}-104$ & $4.89 \mathrm{E}-03$ & $4.82 \mathrm{E}-03$ & 212 & 1074 & 1500 & 0.04 & 0.01 \\
\hline $241-B-105$ & $4.89 \mathrm{E}-03$ & $4.82 \mathrm{E}-03$ & 177 & 1481 & 1500 & 0.04 & 0.01 \\
\hline $241-B-106$ & $4.89 \mathrm{E}-03$ & $4.82 \mathrm{E}-03$ & 182 & 1500 & 1500 & 0.04 & 0.01 \\
\hline $241-B-107$ & $4.89 \mathrm{E}-03$ & $4.82 \mathrm{E}-03$ & 164 & 1500 & 1500 & 0.04 & 0.01 \\
\hline $241-B-108$ & $4.89 \mathrm{E}-03$ & $4.82 \mathrm{E}-03$ & 143 & 1500 & 1500 & 0.03 & 0.01 \\
\hline $241-B-109$ & $4.89 \mathrm{E}-03$ & $4.82 \mathrm{E}-03$ & 152 & 1500 & 1500 & 0.04 & 0.01 \\
\hline 241-B-110 & $4.89 \mathrm{E}-03$ & $4.82 \mathrm{E}-03$ & 320 & 790 & 1500 & 0.07 & 0.02 \\
\hline
\end{tabular}


RPP-5926 REV 6

Table D-5. Time to Reach $25 \%$ and $100 \%$ of the Lower Flammability Limit Under Zero Vent Rate at Documented Safety Analysis Conditions. ( 8 sheets)

\begin{tabular}{|c|c|c|c|c|c|c|c|}
\hline Tank & $\begin{array}{c}\text { Hydrogen } \\
\text { diffusion } \\
\text { rate }(\mathrm{Dr})_{\mathrm{H} 2} \\
\text { (cfm) }^{\prime}\end{array}$ & $\begin{array}{c}\text { Methane } \\
\text { diffusion } \\
\text { rate (Dr) })_{\text {CHA }} \\
(\mathrm{cfm})^{\prime}\end{array}$ & $\begin{array}{c}\text { Steady state } \\
\text { total } \\
\text { flammability } \\
(\%)^{2}\end{array}$ & $\begin{array}{c}\text { Time to } \\
\text { reach } 25 \% \\
\text { of the LFL } \\
\text { (days) }\end{array}$ & $\begin{array}{l}\text { Time to } \\
\text { reach } 100 \% \\
\text { of the LFL } \\
\text { (days) }\end{array}$ & $\begin{array}{c}\text { Minimum } \\
\text { vent rate to } \\
\text { keep below } \\
25 \% \mathrm{LFL} \\
(\mathrm{cfm})\end{array}$ & $\begin{array}{l}\text { Minimum } \\
\text { vent rate to } \\
\text { keep below } \\
100 \% \text { LFL } \\
\text { (cfm) }\end{array}$ \\
\hline $241-B-111$ & $4.89 \mathrm{E}-03$ & $4.82 \mathrm{E}-03$ & 547 & 505 & 1500 & 0.11 & 0.03 \\
\hline $24 !-B-112$ & $4.89 \mathrm{E}-03$ & $4.82 \mathrm{E}-03$ & 150 & 1500 & 1500 & 0.04 & 0.01 \\
\hline $241-B-201$ & $1.61 \mathrm{E}-04$ & $1.60 \mathrm{E}-04$ & 1066 & 350 & 1500 & 0.01 & 0.002 \\
\hline $241-\mathrm{B}-202$ & $1.61 \mathrm{E}-04$ & $1.60 \mathrm{E}-04$ & 1003 & 402 & 1500 & 0.01 & 0.00 \\
\hline $241-B-203^{*}$ & $1.61 \mathrm{E}-04$ & $1.60 \mathrm{E}-04$ & 3095 & 35 & 154 & 0.02 & 0.005 \\
\hline 241 -B-204* & $1.61 \mathrm{E}-04$ & $1.60 \mathrm{E}-04$ & 3166 & 37 & 165 & 0.02 & 0.005 \\
\hline $241-B X-101$ & $4.89 \mathrm{E}-03$ & $4.82 \mathrm{E}-03$ & 164 & 1500 & 1500 & 0.04 & 0.01 \\
\hline $241-\mathrm{BX}-102$ & $4.89 \mathrm{E}-03$ & $4.82 \mathrm{E}-03$ & 158 & 1500 & 1500 & 0.03 & 0.01 \\
\hline $241-B X-103$ & $4.89 \mathrm{E}-03$ & $4.82 \mathrm{E}-03$ & 185 & 1500 & 1500 & 0.05 & 0.01 \\
\hline 241-BX-104 & $4.89 \mathrm{E}-03$ & $4.82 \mathrm{E}-03$ & 224 & 1211 & 1500 & 0.09 & 0.02 \\
\hline $24 \mathrm{~J}-\mathrm{BX}-105$ & $4.89 \mathrm{E}-03$ & $4.82 \mathrm{E}-03$ & 169 & 1500 & 1500 & 0.07 & 0.02 \\
\hline $241-B X-106$ & $4.89 \mathrm{E}-03$ & $4.82 \mathrm{E}-03$ & 157 & 1500 & 1500 & 0.04 & 0.01 \\
\hline $24 \mathrm{I}-\mathrm{BX}+107$ & $4.89 \mathrm{E}-03$ & $4.82 \mathrm{E}-03$ & 220 & 817 & 1500 & 0.06 & 0.01 \\
\hline $241-B X-108$ & $4.89 \mathrm{E}-03$ & $4.82 \mathrm{E}-03$ & 141 & 1500 & 1500 & 0.03 & 0.01 \\
\hline $241-B X-109$ & $4.89 \mathrm{E}-03$ & $4.82 \mathrm{E}-03$ & 303 & 986 & 1500 & 0.06 & 0.02 \\
\hline $241-B \times-110$ & $4.89 \mathrm{E}-03$ & $4.82 \mathrm{E}-03$ & 220 & 1106 & 1500 & 0.05 & 0.01 \\
\hline $241-\mathrm{BX}-111$ & $4.89 E+03$ & $4.82 \mathrm{E}-03$ & 154 & 1500 & 1500 & 0.04 & 0.01 \\
\hline $241-\mathrm{BX}-112$ & $4.89 \mathrm{E}-03$ & $4.82 \mathrm{E}-03$ & 203 & 1220 & 1500 & 0.06 & 0.02 \\
\hline $241-\mathrm{BY}-101$ & $4.84 \mathrm{E}-03$ & $4.76 \mathrm{E}-03$ & 383 & 745 & 1500 & 0.08 & 0.02 \\
\hline $241-\mathrm{BY}-102$ & $4.84 \mathrm{E}-03$ & $4.76 \mathrm{E}-03$ & 194 & 1458 & 1500 & 0.07 & 0.02 \\
\hline $241-\mathrm{BY}-103$ & $4.84 \mathrm{E}-03$ & $4.76 \mathrm{E}-03$ & 233 & 1086 & 1500 & 0.06 & 0.01 \\
\hline $241-B Y-104$ & $4.84 \mathrm{E}-03$ & $4.76 \mathrm{E}-03$ & 379 & 605 & 1500 & 0.12 & 0.03 \\
\hline $241-\mathrm{BY}-105$ & $4.84 \mathrm{E}-03$ & $4.76 \mathrm{E}-03$ & 335 & 607 & 1500 & 0.10 & 0.02 \\
\hline 241-BY-106* & 4.84E-03 & $4.76 \mathrm{E}-03$ & 402 & 709 & 1500 & 0.08 & 0.02 \\
\hline
\end{tabular}




\section{RPP-5926 REV 6}

Table D-5. Time to Reach $25 \%$ and $100 \%$ of the Lower Flammability Limit Under Zero Vent Rate at Documented Safety Analysis Conditions. ( 8 sheets)

\begin{tabular}{|c|c|c|c|c|c|c|c|}
\hline Tank & $\begin{array}{c}\text { Hydrogen } \\
\text { diffusion } \\
\text { rate }(\mathrm{Dr})_{\mathrm{Hz}} \\
{(\mathrm{cfm})^{3}}^{1}\end{array}$ & $\begin{array}{c}\text { Methane } \\
\text { diffusion } \\
\text { rate (Dr) }{ }_{\mathrm{CH} 4} \\
(\mathrm{cfm})^{1}\end{array}$ & $\begin{array}{c}\text { Steady state } \\
\text { total } \\
\text { flammability } \\
(\%)^{2}\end{array}$ & $\begin{array}{l}\text { Time to } \\
\text { reach } 25 \% \\
\text { of the LFL } \\
\text { (days) }\end{array}$ & $\begin{array}{l}\text { Time to } \\
\text { reach } 100 \% \\
\text { of the } L F L \\
\text { (days) }\end{array}$ & $\begin{array}{l}\text { Minimum } \\
\text { vent rate to } \\
\text { keep below } \\
25 \% \text { LFL } \\
\text { (cfm) }\end{array}$ & $\begin{array}{c}\text { Minimum } \\
\text { vent rate to } \\
\text { keep below } \\
100 \% \mathrm{LFL} \\
\text { (cfm) }\end{array}$ \\
\hline $24 !-B Y-107$ & $4.84 \mathrm{E}-03$ & $4.76 \mathrm{E}-03$ & 267 & 1062 & 1500 & 0.18 & 0.05 \\
\hline $241-B Y-108$ & $4.84 \mathrm{E}-03$ & $4.76 \mathrm{E}-03$ & 236 & 1295 & 1500 & 0.20 & 0.05 \\
\hline $241-B Y-109$ & $4.84 \mathrm{E}-03$ & $4.76 \mathrm{E}-03$ & 192 & 1455 & 1500 & 0.05 & 0.01 \\
\hline $24 !-B Y-110$ & $4.84 \mathrm{E}-03$ & $4.76 \mathrm{E}-03$ & 578 & 417 & 1500 & 0.25 & 0.06 \\
\hline 241-BY-111 & $4.84 \mathrm{E}-03$ & $4.76 \mathrm{E}-03$ & 199 & 1296 & 1500 & 0.05 & 0.01 \\
\hline $241-B Y-112$ & $4.84 \mathrm{E}-03$ & $4.76 \mathrm{E}-03$ & 184 & 1500 & 1500 & 0.06 & 0.01 \\
\hline $241-\mathrm{C}-101$ & $4.89 \mathrm{E}-03$ & $4.82 \mathrm{E}-03$ & 189 & 1500 & 1500 & 0.05 & 0.01 \\
\hline $241-C-102$ & $4.89 \mathrm{E}-03$ & $4.82 \mathrm{E}-03$ & 306 & 625 & 1500 & 0.12 & 0.03 \\
\hline $241-C-103 *$ & $4.89 \mathrm{E}-03$ & $4.82 \mathrm{E}-03$ & 557 & 644 & 835 & 0.12 & 0.03 \\
\hline $241 \cdot \mathrm{C}-104$ & $4.89 \mathrm{E}-03$ & $4.82 \mathrm{E}-03$ & 953 & 215 & 1068 & 0.26 & 0.07 \\
\hline $241-C-105$ & $4.89 \mathrm{E}-03$ & $4.82 \mathrm{E}-03$ & 511 & 500 & 1500 & 0.13 & 0.03 \\
\hline $241-C-106$ & $4.89 \mathrm{E}-03$ & $4.82 \mathrm{E}-03$ & 548 & 668 & 1500 & 0.12 & 0.03 \\
\hline $241-C-107$ & $4.89 \mathrm{E}-03$ & $4.82 \mathrm{E}-03$ & 1511 & 136 & 716 & 0.30 & 0.07 \\
\hline $24 !-C-108$ & $4.89 \mathrm{E} \cdot 03$ & 4.82E-03 & 175 & 1500 & 1500 & 0.03 & 0.01 \\
\hline $241-C-109$ & $4.89 \mathrm{E}-03$ & $4.82 \mathrm{E}-03$ & 445 & 814 & 1259 & 0.09 & 0.02 \\
\hline $241-\mathrm{C}-110$ & $4.89 \mathrm{E}-03$ & $4.82 \mathrm{E}-03$ & 437 & 559 & 1500 & 0.11 & 0.03 \\
\hline $241-\mathrm{C}-111$ & $4.89 \mathrm{E}-03$ & $4.82 \mathrm{E}-03$ & 845 & 434 & 1500 & 0.17 & 0.04 \\
\hline $241-\mathrm{C}-112$ & $4.89 \mathrm{E}-03$ & $4.82 \mathrm{E}-03$ & 851 & 395 & 934 & 0.17 & 0.04 \\
\hline $241-\mathrm{C}-201$ & $1.61 \mathrm{E}-04$ & $1.60 \mathrm{E}-04$ & 717 & 1161 & 1500 & 0.005 & 0.001 \\
\hline $241-C-202$ & $1.61 \mathrm{E}-04$ & $1.60 \mathrm{E}-04$ & 715 & 1174 & 1500 & 0.005 & 0.001 \\
\hline $241 \cdot \mathrm{C}-203$ & $1.61 \mathrm{E}-04$ & $1.60 \mathrm{E}-04$ & 718 & 1000 & 1500 & 0.005 & 0.001 \\
\hline $241-C-204$ & $1.61 \mathrm{E}-04$ & $1.60 \mathrm{E}-04$ & 325 & 1500 & 1500 & 0.00 & 0.001 \\
\hline $241-S-101 *$ & $4.84 \mathrm{E}-03$ & $4.76 \mathrm{E}-03$ & 541 & 520 & 1500 & 0.13 & 0.03 \\
\hline 241 -S- $102^{*}$ & $4.84 \mathrm{E}-03$ & $4.76 \mathrm{E}-03$ & 310 & 1167 & 1500 & 0.08 & 0.02 \\
\hline
\end{tabular}


RPP-5926 REV 6

Table D-5. Time to Reach $25 \%$ and $100 \%$ of the Lower Flammability Limit Under Zero Vent Rate at Documented Safety Analysis Conditions. (8 sheets)

\begin{tabular}{|c|c|c|c|c|c|c|c|}
\hline Tank & $\begin{array}{l}\text { Hydrogen } \\
\text { diffusion } \\
\text { rate }(\mathrm{Dr})_{\mathrm{H} 2} \\
(\mathrm{cfm})^{1}\end{array}$ & $\begin{array}{c}\text { Methane } \\
\text { diffusion } \\
\text { rate }(\mathrm{Dr})_{\mathrm{CH} 4} \\
(\mathrm{cfm})^{1}\end{array}$ & $\begin{array}{c}\text { Steady state } \\
\text { total } \\
\text { flammability } \\
(\%)^{2}\end{array}$ & $\begin{array}{l}\text { Time to } \\
\text { reach } 25 \% \\
\text { of the } \mathrm{LFL} \\
\text { (days) }\end{array}$ & $\begin{array}{c}\text { Time to } \\
\text { reach } 100 \% \\
\text { of the LFL } \\
\text { (days) }\end{array}$ & $\begin{array}{c}\text { Minimum } \\
\text { vent rate to } \\
\text { keep below } \\
25 \% \text { LFL } \\
\text { (cfm) }\end{array}$ & $\begin{array}{c}\text { Minimum } \\
\text { vent rate to } \\
\text { keep below } \\
100 \% \mathrm{LFL} \\
\text { (cfm) }\end{array}$ \\
\hline $241-S-103^{*}$ & $4.84 \mathrm{E}-03$ & $4.76 \mathrm{E}-03$ & 294 & 1254 & 1500 & 0.06 & 0.01 \\
\hline $241-S-104$ & $4.84 \mathrm{E}-03$ & $4.76 \mathrm{E}-03$ & 372 & 942 & 1500 & 0.08 & 0.02 \\
\hline $241-S-105$ & $4.84 \mathrm{E}-03$ & $4.76 \mathrm{E}-03$ & 209 & 1123 & 1500 & 0.06 & 0.01 \\
\hline $241-S-106^{*}$ & $4.84 \mathrm{E}-03$ & $4.76 \mathrm{E}-03$ & 315 & 917 & 1500 & 0.06 & 0.02 \\
\hline $241 \cdot S-107$ & $4.84 \mathrm{E}-03$ & $4.76 \mathrm{E}-03$ & 386 & 666 & 1500 & 0.10 & 0.02 \\
\hline $241-S-108$ & $4.84 \mathrm{E}-03$ & $4.76 \mathrm{E}-03$ & 225 & 989 & 1500 & 0.05 & 0.01 \\
\hline 241-S- 109 & $4.84 \mathrm{E}-03$ & $4.76 \mathrm{E}-03$ & 258 & 889 & 1500 & 0.05 & 0.01 \\
\hline $241-S-110$ & $4.84 \mathrm{E}-03$ & $4.76 \mathrm{E}-03$ & 305 & 779 & 1500 & 0.10 & 0.02 \\
\hline $241-S-111^{*}$ & 4.84E-03 & $4.76 \mathrm{E}-03$ & 337 & 886 & 1500 & 0.06 & 0.02 \\
\hline $24 \mid-S-112$ & $4.84 \mathrm{E}-03$ & $4.76 \mathrm{E}-03$ & 137 & 1500 & 1500 & 0.04 & 0.01 \\
\hline $241-\mathrm{SX}-101^{*}$ & $7.11 \mathrm{E}-01$ & $2.23 \mathrm{E}-01$ & 3 & not occur & not occur & 0.08 & 0.02 \\
\hline $24 \mathrm{I}-\mathrm{SX}-102^{*}$ & $7.11 \mathrm{E}-01$ & $2.23 \mathrm{E}-01$ & 7 & not occur & not occur & 0.16 & 0.04 \\
\hline $241-S X-103^{*}$ & $7.11 \mathrm{E}-01$ & $2.23 \mathrm{E}-01$ & 30 & 241 & not occur & 0.73 & 0.18 \\
\hline 241-SX-104* & $7.11 \mathrm{E}-01$ & $2.23 \mathrm{E}-01$ & 7 & not occur & not occur & 0.19 & 0.05 \\
\hline $241-S X-105^{*}$ & $7.11 \mathrm{E}-01$ & $2.23 \mathrm{E}-01$ & 21 & not occur & not occur & 0.52 & 0.13 \\
\hline $241-\mathrm{SX}-106^{*}$ & $7.11 \mathrm{E}-0 \mathrm{l}$ & $2.23 \mathrm{E}-01$ & 10 & not occur & not occur & 0.10 & 0.03 \\
\hline 241-SX-107 & $7.11 \mathrm{E}-01$ & $2.23 \mathrm{E}-01$ & 7 & not occur & not occur & 0.17 & 0.04 \\
\hline $241-S X-108^{*}$ & 7.11E-01 & $2.23 \mathrm{E}-01$ & 6 & not occur & not occur & 0.08 & 0.02 \\
\hline 241-SX-109 & $7.11 \mathrm{E}-01$ & $2.23 \mathrm{E}-01$ & 9 & not occur & not occur & 0.24 & 0.06 \\
\hline $241-S X-110$ & $7.11 \mathrm{E}-01$ & $2.23 \mathrm{E}-01$ & 10 & not occur & not occur & 0.26 & 0.06 \\
\hline 241-SX-111 & $7.11 \mathrm{E}-01$ & $2.23 \mathrm{E}-01$ & 8 & not occur & not occur & 0.19 & 0.05 \\
\hline $241-S X-112$ & $7.11 \mathrm{E}-01$ & $2.23 \mathrm{E}-01$ & 6 & not occur & not occur & 0.13 & 0.03 \\
\hline $241-S X+113$ & 7.11E-01 & $2.23 \mathrm{E}-01$ & 1 & not occur & not occur & 0.03 & 0.01 \\
\hline $241 \cdot S X-114$ & $7.11 \mathrm{E}-01$ & $2.23 \mathrm{E}-0 \mathrm{~J}$ & 11 & not occur & not occur & 0.28 & 0.07 \\
\hline
\end{tabular}


RPP-5926 REV 6

Table D-5. Time to Reach $25 \%$ and $100 \%$ of the Lower Flammability Limit Under Zero Vent Rate at Documented Safety Analysis Conditions. (8 sheets)

\begin{tabular}{|c|c|c|c|c|c|c|c|}
\hline Tank & $\begin{array}{l}\text { Hydrogen } \\
\text { diffusion } \\
\text { rate }(\mathrm{Dr})_{\mathrm{H} 2} \\
(\mathrm{cfm})^{1}\end{array}$ & $\begin{array}{c}\text { Methane } \\
\text { diffusion } \\
\text { rate }(\mathrm{Dr})_{\mathrm{CH} 4} \\
(\mathrm{cfm})^{1}\end{array}$ & $\begin{array}{c}\text { Steady state } \\
\text { total } \\
\text { flammability } \\
(\%)^{2}\end{array}$ & $\begin{array}{c}\text { Time to } \\
\text { reach } 25 \% \\
\text { of the } \mathrm{LFL} \\
\text { (days) }\end{array}$ & $\begin{array}{l}\text { Time to } \\
\text { reach } 100 \% \\
\text { of the } I . F L \\
\text { (days) }\end{array}$ & $\begin{array}{l}\text { Minimum } \\
\text { vent rate to } \\
\text { keep below } \\
25 \% \text { LFL } \\
\text { (cfm) }\end{array}$ & $\begin{array}{c}\text { Minimum } \\
\text { vent rate to } \\
\text { keep below } \\
100 \% \text { LFL } \\
\text { (cfm) }\end{array}$ \\
\hline $241-S X-115$ & $7.11 \mathrm{E}-01$ & $2.23 \mathrm{E}-01$ & 6 & not occur & not occur & 0.16 & 0.04 \\
\hline $241-T-101$ & $4.89 \mathrm{E}-03$ & $4.82 \mathrm{E}-03$ & 163 & 1500 & 1500 & 0.04 & 0.01 \\
\hline $241-\mathrm{T}-102$ & $4.89 \mathrm{E}-03$ & $4.82 \mathrm{E}-03$ & 315 & 1241 & 1500 & 0.06 & 0.02 \\
\hline $241-1-103$ & $4.89 \mathrm{E}-03$ & $4.82 \mathrm{E}-03$ & 129 & 1500 & 1500 & 0.03 & 0.01 \\
\hline $241-T-104$ & $4.89 \mathrm{E}-03$ & $4.82 \mathrm{E}-03$ & 197 & 974 & 1500 & 0.06 & 0.01 \\
\hline $241-\mathrm{T}-105$ & $4.89 \mathrm{E}-03$ & $4.82 \mathrm{E}-03$ & 189 & 1441 & 1500 & 0.05 & 0.01 \\
\hline $241-\mathrm{T}-106$ & $4.89 \mathrm{E}-03$ & $4.82 \mathrm{E}-03$ & 127 & 1500 & 1500 & 0.04 & 0.01 \\
\hline $241-T-107$ & $4.89 \mathrm{E}-03$ & $4.82 \mathrm{E}-03$ & 258 & 930 & 1500 & 0.07 & 0.02 \\
\hline $241-\mathrm{T}-108$ & $4.89 \mathrm{E}-03$ & $4.82 \mathrm{E}-03$ & 122 & 1500 & 1500 & 0.03 & 0.01 \\
\hline $24 !-T-109$ & $4.89 \mathrm{E}-03$ & $4.82 \mathrm{E}-03$ & 130 & 1500 & 1500 & 0.03 & 0.01 \\
\hline $24 \div-T-110^{*}$ & $4.89 \mathrm{E}-03$ & $4.82 \mathrm{E}-03$ & 454 & 484 & 1500 & 0.09 & 0.02 \\
\hline $241-T+111$ & $4.89 \mathrm{E}-03$ & $4.82 \mathrm{E}-03$ & 256 & 560 & 1500 & 0.08 & 0.02 \\
\hline $241-T-112$ & 4.89E-03 & $4.82 \mathrm{E}-03$ & 147 & 1500 & 1500 & 0.03 & 0.01 \\
\hline $241-\Gamma-201$ & 1.61E-04 & $1.60 \mathrm{E}-04$ & 2548 & 157 & 508 & 0.02 & 0.004 \\
\hline $241-\mathrm{T}-202$ & $1.6 \mathrm{IE}-04$ & $1.60 \mathrm{E}-04$ & 793 & 647 & 958 & 0.01 & 0.001 \\
\hline $241 . T-203^{*}$ & $1.61 \mathrm{E}-04$ & 1.60E-04 & 1174 & 271 & $33 !$ & 0.01 & 0.002 \\
\hline $241-\Upsilon-204$ & $1.61 \mathrm{E}-04$ & $1.60 \mathrm{E}-04$ & 1174 & 270 & 309 & 0.01 & 0.002 \\
\hline 241-TX-101 & $4.84 \mathrm{E}-03$ & $4.76 \mathrm{E}-03$ & 265 & 1500 & 1500 & 0.05 & 0.01 \\
\hline $241-T X-102$ & $4.84 \mathrm{E}-03$ & $4.76 \mathrm{E}-03$ & 187 & 1500 & 1500 & 0.09 & 0.02 \\
\hline $24 !-\mathrm{TX}-103$ & 4.84E-03 & $4.76 \mathrm{E}-03$ & 161 & 1500 & 1500 & 0.05 & 0.01 \\
\hline $241-\mathrm{TX}-104$ & $4.84 \mathrm{E}-03$ & $4.76 \mathrm{E}-03$ & 170 & 1500 & 1500 & 0.04 & 0.01 \\
\hline 24]-TX-105 & 4.84E-03 & $4.76 \mathrm{E}-03$ & 253 & 795 & 1500 & 0.06 & 0.01 \\
\hline $241-\mathrm{TX}-106$ & 4.84E-03 & $4.76 \mathrm{E}-03$ & 239 & 1068 & 1500 & 0.09 & 0.02 \\
\hline $241 \cdot T X-107$ & $4.84 \mathrm{E}-03$ & $4.76 \mathrm{E}-03$ & 136 & 1500 & 1500 & 0.06 & 0.02 \\
\hline
\end{tabular}




\section{RPP-5926 REV 6}

Table D-5. Time to Reach $25 \%$ and $100 \%$ of the Lower Flammability Limit Under Zero Vent Rate at Documented Safety Analysis Conditions. (8 sheets)

\begin{tabular}{|c|c|c|c|c|c|c|c|}
\hline Tank & $\begin{array}{l}\text { Hydrogen } \\
\text { diffusion } \\
\text { rate }(\mathrm{Dr})_{\mathrm{H} 2} \\
\quad(\mathrm{cfm})^{1}\end{array}$ & $\begin{array}{c}\text { Methane } \\
\text { diffusion } \\
\text { rate }(\mathrm{Dr})_{\mathrm{CH} 4} \\
\quad(\mathrm{cfm})^{1}\end{array}$ & $\begin{array}{c}\text { Steady state } \\
\text { total } \\
\text { flammability } \\
(\% / \%)^{2}\end{array}$ & $\begin{array}{c}\text { Time to } \\
\text { reach } 25 \% \\
\text { of the } \mathrm{LFL} \\
\text { (days) }\end{array}$ & $\begin{array}{l}\text { Time to } \\
\text { reach } 100 \% \\
\text { of the LFL } \\
\text { (days) }\end{array}$ & $\begin{array}{l}\text { Minimum } \\
\text { vent rate to } \\
\text { keep below } \\
25 \% \text { LFL } \\
\text { (cfm) }\end{array}$ & $\begin{array}{c}\text { Minimum } \\
\text { vent rate to } \\
\text { keep below } \\
100 \% \text { LFL } \\
\text { (cfm) }\end{array}$ \\
\hline $241-\mathrm{TX}-108$ & $4.84 \mathrm{~F}-03$ & $4.76 \mathrm{E}-03$ & 147 & 1500 & 1500 & 0.03 & 0.01 \\
\hline 24!-TX-109 & $4.84 \mathrm{E}-03$ & $4.76 \mathrm{E}-03$ & 316 & 784 & 1111 & 0.13 & 0.03 \\
\hline $241-\mathrm{TX}-110$ & $4.84 \mathrm{E}-03$ & $4.76 \mathrm{E}-03$ & 229 & 937 & 1500 & 0.22 & 0.06 \\
\hline 241-TX-11! & $4.84 \mathrm{E}-03$ & $4.76 \mathrm{E}-03$ & 207 & 1237 & 1500 & 0.20 & 0.05 \\
\hline $241-T X-112$ & $4.84 \mathrm{E}-03$ & $4.76 \mathrm{E}-03$ & 265 & 560 & 1500 & 0.07 & 0.02 \\
\hline $241-T X-113$ & $4.84 \mathrm{E}-03$ & $4.76 \mathrm{E}-03$ & 245 & 739 & 1500 & 0.05 & 0.01 \\
\hline $241-T X-114$ & $4.84 \mathrm{E}-03$ & $4.76 \mathrm{E}-03$ & 228 & 837 & 1500 & 0.09 & 0.02 \\
\hline $241-\mathrm{TX}-115$ & $4.84 \mathrm{E}-03$ & $4.76 \mathrm{E}-03$ & 234 & 967 & 1500 & 0.05 & 0.01 \\
\hline 24$]-T X-116$ & $4.84 \mathrm{E}-03$ & $4.76 \mathrm{~F}-03$ & 237 & 908 & 1500 & 0.05 & 0.01 \\
\hline $241-T X-117$ & $4.84 \mathrm{E}-03$ & $4.76 \mathrm{E}-03$ & 208 & 1285 & 1500 & 0.04 & 0.01 \\
\hline $241-T X-118$ & $4.84 \mathrm{E}-03$ & $4.76 E-03$ & 285 & 1177 & 1500 & 0.06 & 0.02 \\
\hline $241-\mathrm{TY}-101$ & $4.84 \mathrm{E}-03$ & $4.76 \mathrm{E}-03$ & 137 & 1500 & 1500 & 0.03 & 0.01 \\
\hline 241-TY-102 & $4.84 \mathrm{E}-03$ & $4.76 \mathrm{E}-03$ & 138 & 1500 & 1500 & 0.03 & 0.01 \\
\hline $241-\mathrm{TY}-103$ & $4.84 \mathrm{E}-03$ & $4.76 \mathrm{E}-03$ & 228 & 1500 & 1500 & 0.05 & 0.01 \\
\hline 241.TY-104 & $4.84 \mathrm{E}-03$ & $4.76 \mathrm{E}-03$ & 166 & 1500 & 1500 & 0.05 & 0.01 \\
\hline $241-T Y-105$ & $4.84 \mathrm{E}-03$ & $4.76 \mathrm{E}-03$ & 306 & 1306 & 1500 & 0.06 & 0.01 \\
\hline $241 . T Y-106$ & $4.84 \mathrm{E}-03$ & $4.76 \mathrm{E}-03$ & 140 & 1500 & 1500 & 0.03 & 0.01 \\
\hline $241-$ U-101 & $4.89 \mathrm{E}-03$ & $4.82 \mathrm{E}-03$ & 175 & 1500 & 1500 & 0.05 & 0.01 \\
\hline $241-U-102^{*}$ & $4.89 \mathrm{E}-03$ & $4.82 \mathrm{E}-03$ & 477 & 471 & 1500 & 0.10 & 0.02 \\
\hline $241-\mathrm{U}-103^{*}$ & 4.89E-03 & $4.82 \mathrm{E}-03$ & 551 & 312 & 1500 & 0.12 & 0.03 \\
\hline $241-U-104$ & $4.89 \mathrm{E}-03$ & $4.82 \mathrm{E}-03$ & 157 & 1500 & 1500 & 0.03 & 0.01 \\
\hline $241-$ U- $105^{*}$ & $4.89 \mathrm{E}-03$ & $4.82 \mathrm{E}-03$ & 558 & 338 & 554 & 0.13 & 0.03 \\
\hline $241-U-106^{*}$ & $4.89 \mathrm{E}-03$ & $4.82 \mathrm{E}-03$ & 460 & 625 & 1500 & 0.09 & 0.02 \\
\hline $241 . \mathrm{U}-107^{*}$ & $4.89 \mathrm{E}-03$ & $4.82 \mathrm{E}-03$ & 297 & 801 & 801 & 0.06 & 0.01 \\
\hline
\end{tabular}




\section{RPP-5926 REV 6}

Table D-5. Time to Reach $25 \%$ and $100 \%$ of the Lower Flammability Limit Under Zero Vent Rate at Documented Safety Analysis Conditions. (8 sheets)

\begin{tabular}{|c|c|c|c|c|c|c|c|}
\hline Tank & $\begin{array}{l}\text { Hydrogen } \\
\text { diffusion } \\
\text { rate }(\mathrm{Dr})_{\mathrm{H} 2} \\
(\mathrm{cfm})^{1}\end{array}$ & $\begin{array}{l}\text { Methane. } \\
\text { diffusion } \\
\text { rate }(\mathrm{Dr})_{\mathrm{CH} 4} \\
\quad(\mathrm{cfm})^{1}\end{array}$ & $\begin{array}{c}\text { Steady state } \\
\text { total } \\
\text { flammability } \\
(\%)^{2}\end{array}$ & $\begin{array}{l}\text { Time to } \\
\text { reach } 25 \% \\
\text { of the } L F L \\
\text { (days) }\end{array}$ & $\begin{array}{l}\text { Time to } \\
\text { reach } 100 \% \\
\text { of the } L F L \\
\text { (days) }\end{array}$ & $\begin{array}{l}\text { Minimum } \\
\text { vent rate to } \\
\text { keep below } \\
25 \% \mathrm{LFL} \\
\quad(\mathrm{cfm})\end{array}$ & $\begin{array}{c}\text { Minimum } \\
\text { vent rate to } \\
\text { keep below } \\
100 \% \mathrm{LFL} \\
\text { (cfm) }\end{array}$ \\
\hline $241-U-108^{*}$ & $4.89 \mathrm{E}-03$ & $4.82 \mathrm{E}-03$ & 500 & 340 & 1500 & 0.11 & 0.03 \\
\hline $241-U-109 *$ & $4.89 \mathrm{E}-03$ & $4.82 \mathrm{E}-03$ & 345 & 615 & 1500 & 0.07 & 0.02 \\
\hline $241+U-110$ & $4.89 \mathrm{E}-03$ & $4.82 \mathrm{E}-03$ & 311 & 853 & 1500 & 0.07 & 0.02 \\
\hline $24 \mathrm{I}-\mathrm{U}-111$ & $4.89 \mathrm{E}-03$ & $4.82 \mathrm{E}-03$ & 252 & 868 & 1500 & 0.08 & 0.02 \\
\hline $241-U-112$ & $4.89 \mathrm{E}-03$ & $4.82 \mathrm{E}-03$ & 180 & 1500 & 1500 & 0.05 & 0.01 \\
\hline $241-U-201$ & $1.61 \mathrm{E}-04$ & $1.60 \mathrm{E}-04$ & 463 & 1344 & 1500 & 0.00 & $0.00 \mathrm{~J}$ \\
\hline $241-U-202$ & $1.61 \mathrm{E}-04$ & $1.60 \mathrm{E}-04$ & 390 & 1500 & 1500 & 0.00 & 0.001 \\
\hline $241-U-203$ & $1.61 \mathrm{E}-04$ & $1.60 \mathrm{E}-04$ & 425 & 1500 & 1500 & 0.00 & 0.001 \\
\hline $241-U-204$ & $1.61 \mathrm{E}-04$ & $1.60 \mathrm{E}-04$ & 828 & 971 & 1500 & 0.005 & 0.001 \\
\hline
\end{tabular}

Note: 1 . The diffusion mechanism only apply to single-shell tanks.

2. The double-shell tank is treat as closed system under zero ventiatlion condtion. 
RPP-5926 REV 6

APPENDIX E

EQUATIONS TO CALCULATE THE MASS TRANSPORT COEFFICIENT h FOR SOLUBLE GAS

E-i 
RPP-5926 REV 6

This page intentionally left blank.

E-ii 


\section{APPENDIX E}

\section{EQUATIONS TO CALCULATE THE MASS TRANSPORT COEFFICIENT h FOR SOLUBLE GAS}

As described in RPP-4941, Methodology for Predicting Flammable Gas Mixtures in Double-Contained Receiver Tanks, the overall mass transport coefficient for steady state can be written in terms of the mass transport coefficient in vapor phase, $h_{g}$, and in liquid, $h_{1}$, as:

$$
h=\frac{1}{\frac{1}{h_{l}}+\frac{K_{H} R T}{h_{g}}}
$$

with

$$
\begin{aligned}
& h_{g}=0.15 \cdot \frac{D_{g}}{L_{c}} \cdot\left(S_{c} \cdot G_{r}\right)^{1 / 3} \\
& h_{l}=0.15 \cdot D_{L} \cdot\left(\frac{\mu_{L}}{D_{L} \cdot \rho_{L}}\right)^{1 / 3} \cdot\left(\frac{g \cdot \beta_{L} \cdot \rho_{L}{ }^{2} \cdot \Delta T}{\mu_{L}{ }^{2}}\right)^{1 / 3}
\end{aligned}
$$

and

$$
S_{c}=\frac{\mu_{g}}{D_{g} \cdot \rho_{g}} \quad G_{r}=\frac{g \cdot \beta_{g} \cdot \rho_{g}{ }^{2} \cdot L_{c}{ }^{3} \cdot \Delta T}{\mu_{g}{ }^{2}}
$$

where

$$
\begin{aligned}
& \rho_{\mathrm{g}}=\text { density of gas } \\
& \mathrm{kg} / \mathrm{m}^{3} \quad 1.17 \\
& \mathrm{D}_{\mathrm{g}}=\text { diffusion coefficient in gas phase } \\
& \mathrm{m}^{2} / \mathrm{s} \\
& 2.50 \mathrm{E}-05 \\
& \mu_{\mathrm{g}}=\text { viscosity of gas } \\
& \mathrm{kg} / \mathrm{m}-\mathrm{s} \quad 2.0 \mathrm{E}-05 \\
& \mathrm{~S}_{\mathrm{c}}=\text { Schmidt number } \\
& \text { (none) } \\
& 6.84 \mathrm{E}-01 \\
& \mathrm{G}=\text { gravitational constant } \\
& \mathrm{m} / \mathrm{s}^{2} \quad 9.8 \\
& \Delta \mathrm{T}=\text { delta temperature between waste surface and gas } \\
& \mathrm{K} \\
& (\mathrm{K})^{-1} \\
& 1.00 \\
& \beta_{\mathrm{g}}=\text { thermal expansion coeffficient of gas } \\
& \mathrm{m} \\
& 3.30 \mathrm{E}-03 \\
& \mathrm{~L}_{\mathrm{c}}=\text { characteristic length ( } 1 / 4 \text { of diameter) } \\
& 5.7825 \\
& G_{r}=\text { Grashoff number } \\
& \text { (none) } \\
& 2.14 \mathrm{E}+10 \\
& h_{g}=\text { vapor film coefficient } \\
& \mathrm{m} / \mathrm{s} \\
& \mathrm{D}_{\mathrm{L}}=\text { diffusion coefficient in liquid phase } \\
& \mathrm{m}^{2} / \mathrm{s} \\
& 0.001586 \\
& \rho_{\mathrm{L}}=\text { density of liquid } \\
& \mathrm{kg} / \mathrm{m}^{3} \quad 1,300 \\
& \mu_{\mathrm{L}}=\text { viscosity of liquid } \\
& \beta_{\mathrm{L}}=\text { thermal expansion coefficient of liquid } \\
& \mathrm{kg} / \mathrm{m}-\mathrm{s} \quad 6.82 \mathrm{E}-04 \\
& (\mathrm{~K})^{-1} \quad 3.02 \mathrm{E}-04
\end{aligned}
$$




\section{RPP-5926 REV 6}

$\mathrm{h}_{\mathrm{L}}=$ liquid film coefficient. $\quad \mathrm{m} / \mathrm{s} \quad 5.3047 \mathrm{E}-06$

The last column lists the typical values used in the calculation of $h$.

Reference

RPP-4941, 2000, Methodology for Predicting Flammable Gas Mixtures in Double-Contained Receiver Tanks, Rev 0B, CH2M HILL Hanford Group, Inc., Richland, Washington. 
RPP-5926 REV 6

APPENDIX F

CALCULATIONS OF THE GAS DIFFUSION COEFFICIENTS FOR THE CONCRETE DOME OF SINGLE-SHELL TANKS

PREPARED BY JOE MEACHAM

F-i 
RPP-5926 REV 6

This page intentionally left blank.

F-ii 


\section{RPP-5926 REV 6}

\section{CONTENTS}

Table F-1 The Thickness of Dome Coating for 149 Single-Shell Tanks and Diffusion Coefficients of Hydrogen and Methane ...............................................................F-3

Table F-2 The Diffusion Resistance and Gas Diffusion Rate of Hydrogen and Methane for

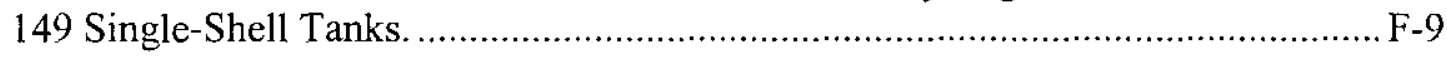


RPP-5926 REV 6

This page intentionally left blank.

F-2 
RPP-5926 REV 6

Table F-1. The Thickness of Dome Coating for 149 Single-Shell Tanks and Diffusion Coefficients of Hydrogen and Methane. (6 sheets)

\begin{tabular}{|c|c|c|c|c|c|c|c|c|c|c|c|c|c|c|}
\hline Tank & $\begin{array}{l}\text { Dome } \\
\text { Area } \\
\left(\mathrm{cm}^{2}\right)\end{array}$ & Coatings of the Tank Dome & $\begin{array}{c}\text { Concrete } \\
\text { Thick } \\
(\mathrm{cm})\end{array}$ & $\begin{array}{c}\text { Asphatic } \\
\text { Thick } \\
(\mathrm{cm})\end{array}$ & $\begin{array}{c}\text { Gunite } \\
\text { Thick } \\
(\mathrm{cm})\end{array}$ & \begin{tabular}{|} 
Soil \\
Thick \\
$(\mathrm{cm})$
\end{tabular} & $\begin{array}{l}\text { Concrete } \\
\text { De of } \mathrm{H}_{2} \\
\left(\mathrm{~cm}^{2} / \mathrm{sec}\right)\end{array}$ & $\begin{array}{l}\text { Asphatic } \\
\text { De of } \mathrm{H}_{2} \\
\left(\mathrm{~cm}^{2} / \mathrm{sec}\right)\end{array}$ & $\begin{array}{c}\text { Gunite De } \\
\text { of } \mathrm{H}_{2} \\
\left(\mathrm{~cm}^{2} / \mathrm{sec}\right)\end{array}$ & $\begin{array}{c}\text { Soil } \\
\text { De of } \mathrm{H}_{2} \\
\left(\mathrm{~cm}^{2} / \mathrm{sec}\right)\end{array}$ & $\begin{array}{c}\text { Concrete De } \\
\text { of } \mathrm{CH}_{4} \\
\left(\mathrm{~cm}^{2} / \mathrm{sec}\right)\end{array}$ & $\begin{array}{c}\text { Asphatic De } \\
\text { of } \mathrm{CH}_{4} \\
\left(\mathrm{~cm}^{2} / \mathrm{sec}\right)\end{array}$ & $\begin{array}{c}\text { Gunite De } \\
\text { of } \mathrm{CH}_{4} \\
\left(\mathrm{~cm}^{2} / \mathrm{sec}\right)\end{array}$ & $\begin{array}{c}\text { Soil } \\
\text { De of } \mathrm{CH}_{4} \\
\left(\mathrm{~cm}^{2} / \mathrm{sec}\right)\end{array}$ \\
\hline A-101 & $4.39 E+06$ & 2-Ply & 38.1 & 0.6350 & 0.000 & 366 & $3.80 \mathrm{E}-03$ & $5.00 \mathrm{E}-07$ & $3.80 \mathrm{E}-03$ & 0.12 & $1.20 \mathrm{E}-03$ & $5.00 \mathrm{E}-07$ & $1.20 \mathrm{E}-03$ & 0.037 \\
\hline A-102 & $4.39 \mathrm{E}+06$ & 2.Ply & 38.1 & 0.6350 & 0.000 & 366 & $3.80 \mathrm{E}-03$ & $5.00 \mathrm{E}-07$ & $3.80 \mathrm{E}-03$ & 0.12 & $1.20 \mathrm{E}-03$ & $5.00 \mathrm{E}-07$ & $1.20 \mathrm{E}-03$ & 0.037 \\
\hline A-103 & $4.39 \mathrm{E}+06$ & 2-Ply & 38.1 & 0.6350 & 0.000 & 366 & $3.80 \mathrm{E}-03$ & $5.00 \mathrm{E}-07$ & $3.80 \mathrm{E}-03$ & 0.12 & $1.20 \mathrm{E}-03$ & $5.00 \mathrm{E}-07$ & $1.20 \mathrm{E}-03$ & 0.037 \\
\hline A-104 & $4.39 \mathrm{E}+06$ & 2-Ply & 38.1 & 0.6350 & 0.000 & 366 & $3.80 \mathrm{E}-03$ & $5.00 \mathrm{E}-07$ & $3.80 \mathrm{E}-03$ & 0.12 & $1.20 \mathrm{E}-03$ & $5.00 \mathrm{E}-07$ & $1.20 \mathrm{E}-03$ & 0.037 \\
\hline $\mathrm{A}-105$ & $4.39 \mathrm{E}+06$ & 2-Ply & 38.1 & 0.6350 & 0.000 & 366 & $3.80 \mathrm{E}-03$ & $5.00 \mathrm{E}-07$ & $3.80 \mathrm{E}-03$ & 0.12 & $1.20 \mathrm{E}-03$ & $5.00 \mathrm{E}-07$ & $1.20 \mathrm{E}-03$ & 0.037 \\
\hline $\mathrm{A}-106$ & $4.39 \mathrm{E}+06$ & 2-Ply & 38.1 & 0.6350 & 0.000 & 366 & $3.80 \mathrm{E}-03$ & $5.00 \mathrm{E} \cdot 07$ & $3.80 \mathrm{E}-03$ & 0.12 & $1.20 \mathrm{E}-03$ & $5.00 \mathrm{E}-07$ & $1.20 \mathrm{E}-03$ & 0.037 \\
\hline $\mathrm{AX}-101$ & $4.39 \mathrm{E}+06$ & No Coatings & 38.1 & 0.0000 & 0.000 & 366 & $3.80 \mathrm{E}-03$ & $5.00 \mathrm{E}-07$ & $3.80 \mathrm{E}-03$ & 0.12 & $1.20 \mathrm{E}-03$ & $5.00 \mathrm{E}-07$ & $1.20 \mathrm{E}-03$ & 0.037 \\
\hline$\Lambda X-102$ & 4.39E+06 & No Coatings & 38.1 & 0.0000 & 0.000 & 366 & $3.80 \mathrm{E}-03$ & $5.00 \mathrm{E}-07$ & $3.80 \mathrm{E}-03$ & 0.12 & $1.20 \mathrm{E}-03$ & $5.00 \mathrm{E}-07$ & $1.20 \mathrm{E}-03$ & 0.037 \\
\hline $\mathrm{AX}-103$ & $4.39 \mathrm{E}+06$ & No Coatings & 38.1 & 0.0000 & 0.000 & 366 & $3.80 \mathrm{E}-03$ & $5.00 \mathrm{E}-07$ & $3.80 \mathrm{E}-03$ & 0.12 & $1.20 \mathrm{E}-03$ & $5.00 \mathrm{E}-07$ & $1.20 \mathrm{E}-03$ & 0.037 \\
\hline AX-104 & $4.39 \mathrm{E}+06$ & No Coatings & 38.1 & 0.0000 & 0.000 & 366 & $3.80 \mathrm{E}-03$ & $5.00 \mathrm{E}-07$ & $3.80 \mathrm{E}-03$ & 0.12 & $1.20 \mathrm{E}-03$ & $5.00 \mathrm{E}-07$ & $1.20 \mathrm{E}-03$ & 0.037 \\
\hline$B-101$ & $4.43 \mathrm{E}+06$ & 3-Ply $+3 / 4$ Mortar & 38.1 & 0.9525 & 1.905 & 404 & $3.80 \mathrm{E}-03$ & $5.00 \mathrm{E}-07$ & $3.80 \mathrm{E}-03$ & 0.12 & $1.20 \mathrm{E}-03$ & $5.00 \mathrm{E}-07$ & $1.20 \mathrm{E}-03$ & 0.037 \\
\hline B-102 & $4.43 \mathrm{E}+06$ & 3-Ply + 3/4 Morlar & 38.1 & 0.9525 & 1.905 & 404 & $3.80 \mathrm{E}-03$ & $5.00 \mathrm{E}-07$ & $3.80 \mathrm{E}-03$ & 0.12 & $1.20 \mathrm{E}-03$ & $5.00 \mathrm{E}-07$ & $1.20 \mathrm{E}-03$ & 0.037 \\
\hline B- 103 & $4.43 \mathrm{E}+06$ & 3-Ply $+3 / 4$ Mortar & 38.1 & 0.9525 & 1.905 & 404 & $3.80 \mathrm{E}-03$ & $5.00 \mathrm{E}-07$ & $3.80 \mathrm{E}-03$ & 0.12 & $1.20 \mathrm{E}-03$ & $5.00 \mathrm{E}-07$ & $1.20 \mathrm{E}-03$ & 0.037 \\
\hline B-104 & $4.43 \mathrm{~F}+06$ & 3-Ply + 3/4 Mortar & 38.1 & 0.9525 & 1.905 & 404 & $3.80 \mathrm{E}-03$ & $5.00 \mathrm{E}-07$ & $3.80 \mathrm{E}-03$ & 0.12 & $1.20 \mathrm{E}-03$ & $5.00 \mathrm{E}-07$ & $1.20 \mathrm{E}-03$ & 0.037 \\
\hline B-105 & $4.43 \mathrm{E}+06$ & 3-Ply $+3 / 4$ Mortar & 38.1 & 0.9525 & 1.905 & 404 & $3.80 \mathrm{E}-03$ & $5.00 \mathrm{E}-07$ & $3.80 \mathrm{E}-03$ & 0.12 & $1.20 \mathrm{E}-03$ & $5.00 \mathrm{E}-07$ & $1.20 \mathrm{E}-03$ & 0.037 \\
\hline B-106 & $4.43 \mathrm{E}+06$ & 3-Ply + 3/4 Mortar & 38.1 & 0.9525 & 1.905 & 404 & $3.80 \mathrm{E}-03$ & $5.00 \mathrm{E}-07$ & $3.80 \mathrm{E}-03$ & 0.12 & $1.20 \mathrm{E}-03$ & $5.00 \mathrm{E}-07$ & $1.20 \mathrm{E}-03$ & 0.037 \\
\hline B-107 & $4.43 \mathrm{~F}+06$ & 3-Ply $+3 / 4$ Mortar & 38.1 & 0.9525 & 1.905 & 404 & $3.80 \mathrm{E}-03$ & $5.00 \mathrm{E}-07$ & $3.80 \mathrm{E}-03$ & 0.12 & $1.20 \mathrm{E}-03$ & $5.00 \mathrm{E}-07$ & $1.20 \mathrm{E}-03$ & 0.037 \\
\hline B-108 & $4.43 \mathrm{E}+06$ & 3-Ply $+3 / 4$ Mortar & 38.1 & 0.9525 & 1.905 & 404 & $3.80 \mathrm{E}-03$ & $5.00 \mathrm{E}-07$ & $3.80 \mathrm{E}-03$ & 0.12 & $1.20 \mathrm{E}-03$ & $5.00 \mathrm{E}-07$ & $1.20 \mathrm{E}-03$ & 0.037 \\
\hline B-109 & $4.43 \mathrm{E}+06$ & 3-Ply + 3/4 Mortar & 38.1 & 0.9525 & 1.905 & 404 & $3.80 \mathrm{E}-03$ & $5.00 \mathrm{E}-07$ & $3.80 \mathrm{E}-03$ & 0.12 & $1.20 \mathrm{E}-03$ & $5.00 \mathrm{E}-07$ & $1.20 \mathrm{E}-03$ & 0.037 \\
\hline $\mathrm{B}-110$ & $4.43 \mathrm{E}+06$ & 3-Ply + 3/4 Mortar & 38.1 & 0.9525 & 1.905 & 404 & $3.80 \mathrm{E}-03$ & $5.00 \mathrm{E}-07$ & $3.80 \mathrm{E}-03$ & 0.12 & $1.20 \mathrm{E}-03$ & $5.00 \mathrm{E}-07$ & $1.20 \mathrm{E}-03$ & 0.037 \\
\hline$[3-11]$ & $4.43 \mathrm{E}+06$ & 3-Ply $+3 / 4$ Mortar & 38.1 & 0.9525 & 1.905 & 404 & $3.80 \mathrm{E}-03$ & $5.00 \mathrm{E}-07$ & $3.80 \mathrm{E}-03$ & 0.12 & $1.20 \mathrm{E}-03$ & $5.00 \mathrm{E}-07$ & $1.20 \mathrm{E}-03$ & 0.037 \\
\hline B-112 & $4.43 \mathrm{E}+06$ & 3-Ply $+3 / 4$ Mortar & 38.1 & 0.9525 & 1.905 & 404 & $3.80 \mathrm{E}-03$ & $5.00 \mathrm{E}-07$ & $3.80 \mathrm{E}-03$ & 0.12 & $1.20 \mathrm{E}-03$ & $5.00 \mathrm{E}-07$ & $1.20 \mathrm{E}-03$ & 0.037 \\
\hline B-201 & $1.46 \mathrm{E}+05$ & Hardner + 3-Ply & 30.48 & 0.9525 & 0.000 & 335 & $3.80 \mathrm{E}-03$ & $5.00 \mathrm{E}-07$ & $3.80 \mathrm{E}-03$ & 0.12 & $1.20 \mathrm{E}-03$ & $5.00 \mathrm{E}-07$ & $1.20 \mathrm{E}-03$ & 0.037 \\
\hline B-202 & $1.46 \mathrm{E}+05$ & Hardner + 3-Ply & 30.48 & 0.9525 & 0.000 & 335 & $3.80 \mathrm{E}-03$ & $5.00 \mathrm{E}-07$ & $3.80 \mathrm{E}-03$ & 0.12 & $1.20 \mathrm{E}-03$ & $5.00 \mathrm{E}-07$ & $1.20 \mathrm{E}-03$ & 0.037 \\
\hline $\mathrm{B}-203$ & $1.46 \mathrm{E}+05$ & Hardner + 3-Ply & 30.48 & 0.9525 & 0.000 & 335 & $3.80 \mathrm{E}-03$ & $5.00 \mathrm{E}-07$ & $3.80 \mathrm{E}-03$ & 0.12 & $1.20 \mathrm{E}-03$ & $5.00 \mathrm{E}-07$ & $1.20 \mathrm{E}-03$ & 0.037 \\
\hline $13-204$ & $1.46 \mathrm{E}+05$ & Hardner + 3-Ply & 30.48 & 0.9525 & 0.000 & 335 & $3.80 \mathrm{E}-03$ & $5.00 \mathrm{E}-07$ & $3.80[-03$ & 0.12 & $1.20 \mathrm{E}-03$ & $5.00 \mathrm{E}-07$ & $1.20 \mathrm{E}-03$ & 0.037 \\
\hline BX-101 & $4.43 \mathrm{E}+06$ & 3-Ply $+3 / 4$ Mortar & 38.1 & 0.9525 & 1.905 & 404 & $3.80 \mathrm{E}-03$ & $5.00 \mathrm{E}-07$ & $3.80 \mathrm{E}-03$ & 0.12 & $1.20 \mathrm{E}-03$ & $5.00 \mathrm{E}-07$ & $1.20 \mathrm{E}-03$ & 0.037 \\
\hline
\end{tabular}

F-3 
Table F-1. The Thickness of Dome Coating for 149 Single-Shell Tanks and Diffusion Coefficients of Hydrogen and Methane. (6 sheets)

\begin{tabular}{|c|c|c|c|c|c|c|c|c|c|c|c|c|c|c|}
\hline Tank & $\begin{array}{c}\text { Dome } \\
\text { Area } \\
\left(\mathrm{cm}^{2}\right)\end{array}$ & Coatings of the Tank Dome & $\begin{array}{c}\text { Concrete } \\
\text { Thick } \\
\text { (cm) }\end{array}$ & $\begin{array}{c}\text { Asphatic } \\
\text { Thick } \\
(\mathrm{cm})\end{array}$ & $\begin{array}{c}\text { Gunite } \\
\text { Thick } \\
(\mathrm{cm})\end{array}$ & $\begin{array}{c}\text { Soil } \\
\text { Thick } \\
(\mathrm{cm})\end{array}$ & $\begin{array}{l}\text { Concrete } \\
\text { De of } \mathrm{H}_{2} \\
\left(\mathrm{~cm}^{2} / \mathrm{sec}\right)\end{array}$ & $\begin{array}{l}\text { Asphatic } \\
\text { De of } \mathrm{H}_{2} \\
\left(\mathrm{~cm}^{2} / \mathrm{sec}\right)\end{array}$ & $\begin{array}{c}\text { Gunite De } \\
\text { of } \mathrm{H}_{2} \\
\left(\mathrm{~cm}^{2} / \mathrm{sec}\right)\end{array}$ & $\begin{array}{c}\text { Soil } \\
\text { De of } \mathrm{H}_{2} \\
\left(\mathrm{~cm}^{2} / \mathrm{sec}\right)\end{array}$ & $\begin{array}{c}\text { Concrete De } \\
\text { of } \mathrm{CH}_{4} \\
\left(\mathrm{~cm}^{2} / \mathrm{sec}\right)\end{array}$ & $\begin{array}{c}\text { Asphatic De } \\
\text { of } \mathrm{CH}_{4} \\
\left(\mathrm{~cm}^{2} / \mathrm{sec}\right)\end{array}$ & $\begin{array}{c}\text { Gunite De } \\
\text { of } \mathrm{CH}_{4} \\
\left(\mathrm{~cm}^{2} / \mathrm{sec}\right)\end{array}$ & $\begin{array}{c}\text { Soil } \\
\text { De of } \mathrm{CH}_{4} \\
\left(\mathrm{~cm}^{2} / \mathrm{sec}\right)\end{array}$ \\
\hline $\mathrm{BX}-102$ & $4.43 \mathrm{~F}+06$ & 3-Ply $+3 / 4$ Mortar & 38.1 & 0.9525 & 1.905 & 404 & $3.80 \mathrm{E}-03$ & $5.00 \mathrm{E}-07$ & $3.80 \mathrm{E}-03$ & 0.12 & $1.20 \mathrm{E}-03$ & $5.00 \mathrm{E}-07$ & $1.20 \mathrm{E}-03$ & 0.037 \\
\hline BX-103 & 4.43E+06 & 3-Ply +3/4 Mortar & 38.1 & 0.9525 & 1.905 & 404 & $3.80 \mathrm{E}-03$ & $5.00 \mathrm{E}-07$ & $3.80 \mathrm{E}-03$ & 0.12 & $1.20 \mathrm{E}-03$ & $5.00 \mathrm{E}-07$ & $1.20 \mathrm{E}-03$ & 0.037 \\
\hline $\mathrm{BX}-104$ & $4.43 \mathrm{E}+06$ & 3-Ply $+3 / 4$ Mortar & 38.1 & 0.9525 & 1.905 & 404 & $3.80 \mathrm{E}-03$ & $5.00 \mathrm{E}-07$ & $3.80 \mathrm{E}-03$ & 0.12 & $1.20 \mathrm{E}-03$ & $5.00 \mathrm{E}-07$ & $1.20 \mathrm{E}-03$ & 0.037 \\
\hline $\mathrm{BX}-105$ & $4.43 \mathrm{E}+06$ & 3-Ply + 3/4 Mortar & 38.1 & 0.9525 & 1.905 & 404 & $3.80 \mathrm{E}-03$ & $5.00 \mathrm{E}-07$ & $3.80 \mathrm{E}-03$ & 0.12 & $1.20 \mathrm{E}-03$ & $5.00 \mathrm{E}-07$ & $1.20 \mathrm{E}-03$ & 0.037 \\
\hline $\mathrm{BX}-106$ & $4.43 \mathrm{E}+06$ & $3-\mathrm{Ply}+3 / 4$ Mortar & 38.1 & 0.9525 & 1.905 & 404 & $3.80 \mathrm{E}-03$ & $5.00 \mathrm{E} \cdot 07$ & $3.80 \mathrm{E}-03$ & 0.12 & $1.20 \mathrm{E}-03$ & $5.00 \mathrm{E}-07$ & $1.20 \mathrm{E}-03$ & 0.037 \\
\hline $\mathrm{BX}-107$ & $4.43 \mathrm{E}+06$ & 3-Ply $+3 / 4$ Mortar & 38.1 & 0.9525 & 1.905 & 404 & $3.80 \mathrm{E}-03$ & $5.00 \mathrm{E}-07$ & $3.80 \mathrm{E}-03$ & 0.12 & $1.20 \mathrm{E}-03$ & $5.00 \mathrm{E}-07$ & $1.20 \mathrm{E}-03$ & 0.037 \\
\hline BX-108 & $4.43 E+06$ & 3-Ply + 3/4 Mortar & 38.1 & 0.9525 & 1.905 & 404 & $3.80 \mathrm{E}-03$ & $5.00 \mathrm{E}-07$ & $3.80 \mathrm{E}-03$ & 0.12 & $1.20 \mathrm{E}-03$ & $5.00 \mathrm{E}-07$ & $1.20 \mathrm{E}-03$ & 0.037 \\
\hline $\mathrm{BX}-109$ & $4.43 \mathrm{E}+06$ & 3-Ply $+3 / 4$ Mortar & 38.1 & 0.9525 & 1.905 & 404 & $3.80 \mathrm{E}-03$ & $5.00 \mathrm{E}-07$ & $3.80 \mathrm{E}-03$ & 0.12 & $1.20 \mathrm{E}-03$ & $5.00 \mathrm{E}-07$ & $1.20 \mathrm{E}-03$ & 0.037 \\
\hline$B X-110$ & $4.43 E+06$ & 3-Ply +3/4 Mortar & 38.1 & 0.9525 & 1.905 & 404 & $3.80 \mathrm{E}-03$ & $5.00 \mathrm{E}-07$ & $3.80 \mathrm{E}-03$ & 0.12 & $1.20 \mathrm{E}-03$ & $5.00 \mathrm{E}-07$ & $1.20 \mathrm{E}-03$ & 0.037 \\
\hline$B X-111$ & $4.43 \mathrm{E}+06$ & 3-Ply $+3 / 4$ Mortar & 38.1 & 0.9525 & 1.905 & 404 & $3.80 \mathrm{E}-03$ & $5.00 \mathrm{E}-07$ & $3.80 \mathrm{E}-03$ & 0.12 & $1.20 E-03$ & $5.00 \mathrm{E}-07$ & $1.20 \mathrm{E}-03$ & 0.037 \\
\hline $\mathrm{BX}-112$ & $4.43 \mathrm{E}+06$ & 3-Ply $+3 / 4$ Mortar & 38.1 & 0.9525 & 1.905 & 404 & $3.80 \mathrm{E}-03$ & $5.00 \mathrm{E}-07$ & $3.80 \mathrm{E}-03$ & 0.12 & $1.20 \mathrm{E}-03$ & $5.00 \mathrm{E}-07$ & $1.20 \mathrm{E}-03$ & 0.037 \\
\hline$B Y-101$ & $4.38 \mathrm{E}+06$ & Hardner $+3-P l y+3 / 4$ Gunite & 38.1 & 0.9525 & 1.905 & 427 & $3.80 \mathrm{E}-03$ & $5.00 \mathrm{E}-07$ & $3.80 \mathrm{E}-03$ & 0.12 & $1.20 \mathrm{E}-03$ & $5.00 \mathrm{E}-07$ & $1.20 \mathrm{E}-03$ & 0.037 \\
\hline BY-102 & $4.38 \mathrm{E}+06$ & Hardner $+3-\mathrm{Ply}+3 / 4$ Gunite & 38.1 & 0.9525 & 1.905 & 427 & $3.80 \mathrm{E}-03$ & $5.00 \mathrm{E}-07$ & $3.80 \mathrm{E}-03$ & 0.12 & $1.20 \mathrm{E}-03$ & $5.00 \mathrm{E}-07$ & $1.20 \mathrm{E}-03$ & 0.037 \\
\hline BY-103 & $4.38 \mathrm{E}+06$ & Jardner $+3-P l y+3 / 4$ Gunite & 38.1 & 0.9525 & 1.905 & 427 & $3.80 \mathrm{E}-03$ & $5.00 \mathrm{E}-07$ & $3.80 \mathrm{E}-03$ & 0.12 & $1.20 \mathrm{E}-03$ & $5.00 \mathrm{E}-07$ & $1.20 \mathrm{E}-03$ & 0.037 \\
\hline$B Y-104$ & $4.38 \mathrm{E}+06$ & Hardner $+3-\mathrm{Ply}+3 / 4$ Gunite & 38.1 & 0.9525 & 1.905 & 427 & $3.80 \mathrm{E}-03$ & $5.00 \mathrm{E}-07$ & $3.80 \mathrm{E}-03$ & 0.12 & $1.20 \mathrm{E}-03$ & $5.00 \mathrm{E}-07$ & $1.20 \mathrm{E}-03$ & 0.037 \\
\hline BY-105 & $4.38 \mathrm{E}+06$ & Hardner $+3-\mathrm{Ply}+3 / 4$ Gunite & 38.1 & 0.9525 & 1.905 & 427 & $3.80 \mathrm{E}-03$ & $5.00 \mathrm{E}-07$ & $3.80 \mathrm{E}-03$ & 0.12 & $1.20 \mathrm{E}-03$ & $5.00 \mathrm{E}-07$ & $1.20 \mathrm{E}-03$ & 0.037 \\
\hline BY-106 & $4.38 \mathrm{E}+06$ & Hardner $+3-\mathrm{Ply}+3 / 4$ Gunite & 38.1 & 0.9525 & 1.905 & 427 & $3.80 \mathrm{E}-03$ & $5.00 \mathrm{E}-07$ & $3.80 \mathrm{E}-03$ & 0.12 & $1.20 \mathrm{E}-03$ & $5.00 \mathrm{E}-07$ & $1.20 \mathrm{E}-03$ & 0.037 \\
\hline BY -107 & $4.38 \mathrm{E}+06$ & Hardner $+3-\mathrm{Ply}+3 / 4$ Gunite & 38.1 & 0.9525 & 1.905 & 427 & $3.80 \mathrm{E}-03$ & $5.00 \mathrm{E}-07$ & $3.80 \mathrm{E}-03$ & 0.12 & $1.20 \mathrm{E}-03$ & 5.00E-07 & $1.20 \mathrm{E}-03$ & 0.037 \\
\hline$B Y-108$ & $4.38 \mathrm{E}+06$ & Hardner $+3-\mathrm{Ply}+3 / 4$ Gunite & 38.1 & 0.9525 & 1.905 & 427 & $3.80 \mathrm{E}-03$ & $5.00 \mathrm{E}-07$ & $3.80 \mathrm{E}-03$ & 0.12 & $1.20 \mathrm{E}-03$ & $5.00 \mathrm{E}-07$ & $1.20 \mathrm{E}-03$ & 0.037 \\
\hline BY-109 & $4.38 \mathrm{E}+06$ & Hardner + 3-Ply + 3/4 Gunite & 38.1 & 0.9525 & 1.905 & 427 & $3.80 \mathrm{E}-03$ & $5.00 \mathrm{E}-07$ & $3.80 \mathrm{E}-03$ & 0.12 & $1.20 \mathrm{E}-03$ & $5.00 \mathrm{E}-07$ & $1.20 \mathrm{E}-03$ & 0.037 \\
\hline BY-110 & $4.38 \mathrm{E}+06$ & Hardner $+3-P l y+3 / 4$ Gunite & 38.1 & 0.9525 & 1.905 & 427 & $3.80 \mathrm{E}-03$ & $5.00 \mathrm{E}-07$ & $3.80 \mathrm{E}-03$ & 0.12 & $1.20 \mathrm{E}-03$ & $5.00 \mathrm{E}-07$ & $1.20 \mathrm{E}-03$ & 0.037 \\
\hline BY -111 & $4.38 \mathrm{E}+06$ & Hardner + 3-Ply + 3/4 Gunite & 38.1 & 0.9525 & 1.905 & 427 & $3.80 \mathrm{E}-03$ & $5.00 \mathrm{E}-07$ & $3.80 \mathrm{E}-03$ & 0.12 & $1.20 \mathrm{E}-03$ & $5.00 \mathrm{E}-07$ & $1.20 \mathrm{E}-03$ & 0.037 \\
\hline BY-112 & $4.38 \mathrm{E}+06$ & Hardner + 3-Ply + 3/4 Gunite & 38.1 & 0.9525 & 1.905 & 427 & $3.80 \mathrm{E}-03$ & $5.00 \mathrm{E}-07$ & $3.80 \mathrm{E}-03$ & 0.12 & $1.20 \mathrm{E}-03$ & $5.00 \mathrm{E}-07$ & $1.20 \mathrm{E}-03$ & 0.037 \\
\hline $\mathrm{C}-101$ & $4.43 \mathrm{E}+06$ & 3-Ply $+3 / 4$ Mortar & 38.1 & 0.9525 & 1.905 & 404 & $3.80 \mathrm{E}-03$ & $5.00 \mathrm{E}-07$ & $3.80 \mathrm{E}-03$ & 0.12 & $1.20 \mathrm{E}-03$ & $5.00 \mathrm{E}-07$ & $1.20 \mathrm{E}-03$ & 0.037 \\
\hline C. -102 & $4.43 \mathrm{E}+06$ & 3-Ply $+3 / 4$ Mortar & 38.1 & 0.9525 & 1.905 & 404 & $3.80 \mathrm{E}-03$ & $5.00 \mathrm{E}-07$ & $3.80 \mathrm{E}-03$ & 0.12 & $1.20 \mathrm{E}-03$ & $5.00 \mathrm{E}-07$ & $1.20 \mathrm{E}-03$ & 0.037 \\
\hline $\mathrm{C}+103$ & $4.43 \mathrm{E}+06$ & 3-Ply $+3 / 4$ Mortar & 38.1 & 0.9525 & 1.905 & 404 & $3.80 \mathrm{E}-03$ & $5.00 \mathrm{E}-07$ & $3.80 \mathrm{E}-03$ & 0.12 & $1.20 \mathrm{E}-03$ & $5.00 \mathrm{E}-07$ & $1.20 \mathrm{E}-03$ & 0.037 \\
\hline C-104 & $4.43 E+06$ & 3-Ply $+3 / 4$ Mortar & 38.1 & 0.9525 & 1.905 & 404 & $3.80 \mathrm{E}-03$ & $5.00 \mathrm{E}-07$ & $3.80 \mathrm{E}-03$ & 0.12 & $1.20 \mathrm{E}-03$ & $5.00 \mathrm{E}-07$ & $1.20 \mathrm{E}-03$ & 0.037 \\
\hline
\end{tabular}


RPP-5926 REV 6

Table F-1. The Thickness of Dome Coating for 149 Single-Shell Tanks and Diffusion Coefficients of Hydrogen and Methane. (6 sheets)

\begin{tabular}{|c|c|c|c|c|c|c|c|c|c|c|c|c|c|c|}
\hline Tank & $\begin{array}{l}\text { Dome } \\
\text { Area } \\
\left(\mathrm{cm}^{2}\right)\end{array}$ & Coatings of the Tank Dome & $\begin{array}{c}\text { Concrete } \\
\text { Thick } \\
(\mathrm{cm})\end{array}$ & $\begin{array}{c}\text { Asphatic } \\
\text { Thick } \\
(\mathrm{cm})\end{array}$ & $\begin{array}{c}\text { Gunite } \\
\text { Thick } \\
(\mathrm{cm})\end{array}$ & $\begin{array}{c}\text { Soil } \\
\text { Thick } \\
(\mathrm{cm})\end{array}$ & $\begin{array}{l}\text { Concrete } \\
\text { De of } \mathrm{H}_{2} \\
\left(\mathrm{~cm}^{2} / \mathrm{sec}\right)\end{array}$ & $\begin{array}{l}\text { Asphatic } \\
\text { De of } \mathrm{H}_{2} \\
\left(\mathrm{~cm}^{2} / \mathrm{sec}\right)\end{array}$ & $\begin{array}{c}\text { Gunite De } \\
\text { of } \mathrm{H}_{2} \\
\left(\mathrm{~cm}^{2} / \mathrm{sec}\right)\end{array} \mid$ & $\begin{array}{c}\text { Soil } \\
\text { De of } \mathrm{H}_{2} \\
\left(\mathrm{~cm}^{2} / \mathrm{sec}\right)\end{array}$ & $\begin{array}{c}\text { Concrete De } \\
\text { of } \mathrm{CH}_{4} \\
\left(\mathrm{~cm}^{2} / \mathrm{sec}\right)\end{array}$ & $\begin{array}{c}\text { Asphatic De } \\
\text { of } \mathrm{CH}_{4} \\
\left(\mathrm{~cm}^{2} / \mathrm{sec}\right)\end{array}$ & $\begin{array}{c}\text { Gunite De } \\
\text { of } \mathrm{CH}_{4} \\
\left(\mathrm{~cm}^{2} / \mathrm{sec}\right)\end{array}$ & $\begin{array}{c}\text { Soil } \\
\text { De of } \mathrm{CH}_{4} \\
\left(\mathrm{~cm}^{2} / \mathrm{sec}\right)\end{array}$ \\
\hline$C-105$ & $4.43 \mathrm{E}+06$ & 3-Ply $+3 / 4$ Mortar & 38.1 & 0.9525 & 1.905 & 404 & $3.80 \mathrm{E}-03$ & $5.00 \mathrm{E}-07$ & $3.80 \mathrm{E}-03$ & 0.12 & $1.20 \mathrm{E}-03$ & $5.00 \mathrm{E}-07$ & $1.20 \mathrm{E}-03$ & 0.037 \\
\hline $\mathrm{C}-106$ & $4.43 E+06$ & 3-Ply $+3 / 4$ Mortar & 38.1 & 0.9525 & 1.905 & 404 & $3.80 \mathrm{E}-03$ & $5.00 \mathrm{E}-07$ & $3.80 \mathrm{E}-03$ & 0.12 & $1.20 \mathrm{E}-03$ & $5.00 \mathrm{E}-07$ & $1.20 \mathrm{E}-03$ & 0.037 \\
\hline $\mathrm{C}-107$ & $4.43 \mathrm{E}+06$ & 3-Ply $+3 / 4$ Mortar & 38.1 & 0.9525 & 1.905 & 404 & $3.80 \mathrm{E}-03$ & $5.00 \mathrm{E}-07$ & $3.80 \mathrm{E}-03$ & 0.12 & $1.20 \mathrm{E}-03$ & $5.00 \mathrm{E}-07$ & $1.20 \mathrm{E}-03$ & 0.037 \\
\hline C-108 & $4.43 E+06$ & 3-Ply $+3 / 4$ Mortar & 38.1 & 0.9525 & 1.905 & 404 & $3.80 \mathrm{E}-03$ & $5.00 \mathrm{E}-07$ & $3.80 \mathrm{E}-03$ & 0.12 & $1.20 \mathrm{E}-03$ & $5.00 \mathrm{E}-07$ & $1.20 \mathrm{E}-03$ & 0.037 \\
\hline $\mathrm{C}-109$ & $4.43 \mathrm{E}+06$ & $3-\mathrm{Ply}+3 / 4$ Mortar & 38.1 & 0.9525 & 1.905 & 404 & $3.80 \mathrm{E}-03$ & $5.00 \mathrm{E}-07$ & $3.80 \mathrm{E}-03$ & 0.12 & $1.20 \mathrm{E}-03$ & $5.00 \mathrm{E}-07$ & $1.20 \mathrm{E}-03$ & 0.037 \\
\hline $\mathrm{C}-110$ & $4.43 \mathrm{E}+06$ & 3-Ply $+3 / 4$ Mortar & 38.1 & 0.9525 & 1.905 & 404 & $3.80 \mathrm{E}-03$ & $5.00 \mathrm{E}-07$ & $3.80 \mathrm{E}-03$ & 0.12 & $1.20 \mathrm{E}-03$ & $5.00 \mathrm{E}-07$ & $1.20 \mathrm{E}-03$ & 0.037 \\
\hline $\mathrm{C}-111$ & $4.43 E+06$ & 3-Ply $+3 / 4$ Mortar & 38.1 & 0.9525 & 1.905 & 404 & $3.80 \mathrm{E}-03$ & $5.00 \mathrm{E}-07$ & $3.80 \mathrm{E}-03$ & 0.12 & $1.20 \mathrm{E}-03$ & $5.00 \mathrm{E}-07$ & $1.20 \mathrm{E}-03$ & 0.037 \\
\hline $\mathrm{C}-112$ & $4.43 E+06$ & 3-Ply $+3 / 4$ Mortar & 38.1 & 0.9525 & 1.905 & 404 & $3.80 \mathrm{E}-03$ & $5.00 \mathrm{E}-07$ & $3.80 \mathrm{E}-03$ & 0.12 & $1.20 \mathrm{E}-03$ & $5.00 \mathrm{E}-07$ & $1.20 \mathrm{E}-03$ & 0.037 \\
\hline C. -201 & $1.46 E+05$ & Hardner + 3-Ply & 30.48 & 0.9525 & 0.000 & 335 & $3.80 \mathrm{E}-03$ & $5.00 \mathrm{E}-07$ & $3.80 \mathrm{E}-03$ & 0.12 & $1.20 \mathrm{E}-03$ & $5.00 \mathrm{E}-07$ & $1.20 \mathrm{E}-03$ & 0.037 \\
\hline $\mathrm{C}-202$ & $1.46 \mathrm{E}+05$ & Hardner + 3-Ply & 30.48 & 0.9525 & 0.000 & 335 & $3.80 \mathrm{E}-03$ & $5.00 \mathrm{E}-07$ & $3.80 \mathrm{E}-03$ & 0.12 & $1.20 \mathrm{E}-03$ & $5.00 \mathrm{E}-07$ & $1.20 \mathrm{E}-03$ & 0.037 \\
\hline $\mathrm{C}-203$ & $1.46 \mathrm{E}+05$ & Hardner + 3-Ply & 30.48 & 0.9525 & 0.000 & 335 & $3.80 \mathrm{E}-03$ & $5.00 \mathrm{E}-07$ & $3.80 \mathrm{E}-03$ & 0.12 & $1.20 \mathrm{E}-03$ & $5.00 \mathrm{~F}-07$ & $1.20 \mathrm{E}-03$ & 0.037 \\
\hline C. 204 & $1.46 \mathrm{E}+05$ & Hardner + 3-Ply & 30.48 & 0.9525 & 0.000 & 335 & $3.80 \mathrm{E}-03$ & $5.00 \mathrm{E}-07$ & $3.80 \mathrm{E}-03$ & 0.12 & $1.20 \mathrm{E}-03$ & $5.00 \mathrm{E}-07$ & $1.20 \mathrm{E}-03$ & 0.037 \\
\hline$S-101$ & $4.38 E+06$ & Hardner $+3-\mathrm{Ply}+3 / 4$ Gunite & 38.1 & 0.9525 & 1.905 & 427 & $3.80 \mathrm{E}-03$ & $5.00 \mathrm{E}-07$ & $3.80 \mathrm{E}-03$ & 0.12 & $1.20 \mathrm{E}-03$ & $5.00 \mathrm{E}-07$ & $1.205-03$ & 0.037 \\
\hline$S-102$ & $4.38 \mathrm{E}+06$ & Hardner $+3-\mathrm{Ply}+3 / 4$ Gunite & 38.1 & 0.9525 & 1.905 & 427 & $3.80 \mathrm{E}-03$ & $5.00 \mathrm{E}-07$ & $3.80 \mathrm{E}-03$ & 0.12 & $1.20 \mathrm{E}-03$ & $5.00 \mathrm{E}-07$ & $1.20 \mathrm{E}-03$ & 0.037 \\
\hline$S-103$ & $4.38 \mathrm{E}+06$ & I lardner $+3-P l y+3 / 4$ Gunite & 38.1 & 0.9525 & 1.905 & 427 & $3.80 \mathrm{E}-03$ & $5.00 \mathrm{E}-07$ & $3.80 \mathrm{E}-03$ & 0.12 & $1.20 \mathrm{E}-03$ & $5.00 \mathrm{E}-07$ & $1.20 \mathrm{E}-03$ & 0.037 \\
\hline S-104 & $4.38 E+06$ & Hardner $+3-P l y+3 / 4$ Gunite & 38.1 & 0.9525 & 1.905 & 427 & $3.80 \mathrm{E}-03$ & $5.00 \mathrm{E}-07$ & $3.80 \mathrm{E}-03$ & 0.12 & $1.20 \mathrm{E}-03$ & $5.00 \mathrm{E}-07$ & $1.20 \mathrm{E}-03$ & 0.037 \\
\hline S-105 & $4.38 \mathrm{E}+06$ & Hardner $+3-\mathrm{Ply}+3 / 4$ Gunite & 38.1 & 0.9525 & 1.905 & 427 & $3.80 \mathrm{E}-03$ & $5.00 \mathrm{E}-07$ & $3.80 \mathrm{E}-03$ & 0.12 & $1.20 \mathrm{E}-03$ & $5.00 \mathrm{E}-07$ & $1.20 \mathrm{E}-03$ & 0.037 \\
\hline S-106 & $4.38 \mathrm{E}+06$ & Hardner $+3-\mathrm{Ply}+3 / 4$ Gunite & 38.1 & 0.9525 & 1.905 & 427 & $3.80 \mathrm{E}-03$ & $5.00 \mathrm{E}-07$ & $3.80 \mathrm{E}-03$ & 0.12 & $1.20 \mathrm{E}-03$ & $5.00 \mathrm{E}-07$ & $1.20 \mathrm{E}-03$ & 0.037 \\
\hline S-107 & $4.38 \mathrm{E}+06$ & llardner + 3-Ply + 3/4 Gunite & 38.1 & 0.9525 & 1.905 & 427 & $3.80 \mathrm{E}-03$ & $5.00 \mathrm{E}-07$ & $3.80 \mathrm{E}-03$ & 0.12 & $1.20 \mathrm{E}-03$ & $5.00 \mathrm{E}-07$ & $1.20 \mathrm{E}-03$ & 0.037 \\
\hline S- 108 & $4.38 \mathrm{E}+06$ & Hardner $+3-\mathrm{Ply}+3 / 4$ Gunite & 38.1 & 0.9525 & 1.905 & 427 & $3.80 \mathrm{E}-03$ & $5.00 \mathrm{E}-07$ & $3.80 \mathrm{E}-03$ & 0.12 & $1.20 \mathrm{E}-03$ & $5.00 \mathrm{E}-07$ & $1.20 \mathrm{E}-03$ & 0.037 \\
\hline S-109 & $4.38 \mathrm{E}+06$ & Hardner $+3-P l y+3 / 4$ Gunite & 38.1 & 0.9525 & 1.905 & 427 & $3.80 \mathrm{E}-03$ & $5.00 \mathrm{E}-07$ & $3.80 \mathrm{E}-03$ & 0.12 & $1.20 \mathrm{E}-03$ & $5.00 \mathrm{E}-07$ & $1.20 \mathrm{E}-03$ & 0.037 \\
\hline $\mathrm{S}-110$ & $4.38 \mathrm{E}+06$ & Hardner + 3-Ply $+3 / 4$ Gunitc & 38.1 & 0.9525 & 1.905 & 427 & $3.80 \mathrm{E}-03$ & $5.00 \mathrm{E}-07$ & $3.80 \mathrm{E}-03$ & 0.12 & $1.20 \mathrm{E}-03$ & $5.00 \mathrm{E}-07$ & $1.20 \mathrm{E}-03$ & 0.037 \\
\hline$S-111$ & $4.38 \mathrm{E}+06$ & Hardner $+3-\mathrm{Ply}+3 / 4$ Gunite & 38.1 & 0.9525 & 1.905 & 427 & $3.80 \mathrm{E}-03$ & $5.00 \mathrm{E}-07$ & $3.80 \mathrm{E}-03$ & 0.12 & $1.20 \mathrm{E}-03$ & $5.00 \mathrm{E}-07$ & $1.20 \mathrm{E}-03$ & 0.037 \\
\hline S-112 & $4.38 \mathrm{E}+06$ & Hardner $+3-\mathrm{Ply}+3 / 4$ Gunite & 38.1 & 0.9525 & 1.905 & 427 & $3.80 \mathrm{E}-03$ & $5.00 \mathrm{E}-07$ & $3.80 \mathrm{E}-03$ & 0.12 & $1.20 \mathrm{E}-03$ & $5.00 \mathrm{E}-07$ & $1.20 \mathrm{E}-03$ & 0.037 \\
\hline$S X-101$ & $4.39 \mathrm{E}+06$ & Hardner & 38.1 & 0.0000 & 0.000 & 366 & $3.80 \mathrm{E}-03$ & $5.00 \mathrm{E}-07$ & $3.80 \mathrm{E}-03$ & 0.12 & $1.20 \mathrm{E}-03$ & $5.00 \mathrm{E}-07$ & $1.20 \mathrm{E}-03$ & 0.037 \\
\hline$S X-102$ & $4.39 \mathrm{E}+06$ & Hardner & 38.1 & 0.0000 & 0.000 & 366 & $3.80 \mathrm{E}-03$ & $5.00 \mathrm{E}-07$ & $3.80 \mathrm{E}-03$ & 0.12 & $1.20 \mathrm{E}-03$ & $5.00 \mathrm{E}-07$ & $1.20 \mathrm{E}-03$ & 0.037 \\
\hline$S X-103$ & $4.39 \mathrm{E}+06$ & Hardner & 38.1 & 0.0000 & 0.000 & 366 & $3.80 \mathrm{E}-03$ & $5.00 \mathrm{E}-07$ & $3.80 \mathrm{E}-03$ & 0.12 & $1.20 \mathrm{E}-03$ & $5.00 \mathrm{E}-07$ & $1.20 \mathrm{E}-03$ & 0.037 \\
\hline
\end{tabular}


RPP-5926 REV 6

Table F-1. The Thickness of Dome Coating for 149 Single-Shell Tanks and Diffusion Coefficients of Hydrogen and Methane. (6 sheets)

\begin{tabular}{|c|c|c|c|c|c|c|c|c|c|c|c|c|c|c|}
\hline Tank & $\begin{array}{l}\text { Dome } \\
\text { Area } \\
\left(\mathrm{cm}^{2}\right)\end{array}$ & Coatings of the Tank Dome & $\begin{array}{c}\text { Concrete } \\
\text { Thick } \\
(\mathrm{cm})\end{array}$ & $\begin{array}{c}\text { Asphatic } \\
\text { Thick } \\
(\mathrm{cm})\end{array}$ & $\begin{array}{l}\text { Gunite } \\
\text { Thick } \\
(\mathrm{cm})\end{array}$ & $\begin{array}{c}\text { Soil } \\
\text { Thick } \\
(\mathrm{cm})\end{array}$ & $\begin{array}{l}\text { Concrete } \\
\text { De of } \mathrm{H}_{2} \\
\left(\mathrm{~cm}^{2} / \mathrm{sec}\right)\end{array}$ & $\begin{array}{l}\text { Asphatic } \\
\text { De of } \mathrm{H}_{2} \\
\left(\mathrm{~cm}^{2} / \mathrm{sec}\right)\end{array}$ & $\begin{array}{c}\text { Gunite Dc } \\
\text { of } \mathrm{H}_{2} \\
\left(\mathrm{~cm}^{2} / \mathrm{sec}\right)\end{array}$ & $\begin{array}{c}\text { Soil } \\
\text { De of } \mathrm{H}_{2} \\
\left(\mathrm{~cm}^{2} / \mathrm{sec}\right)\end{array}$ & $\begin{array}{c}\text { Concrete De } \\
\text { of } \mathrm{CH}_{4} \\
\left(\mathrm{~cm}^{2} / \mathrm{sec}\right)\end{array}$ & $\begin{array}{c}\text { Asphatic De } \\
\text { of } \mathrm{CH}_{4} \\
\left(\mathrm{~cm}^{2} / \mathrm{sec}\right)\end{array}$ & $\begin{array}{c}\text { Gunite De } \\
\text { of } \mathrm{CH}_{4} \\
\left(\mathrm{~cm}^{2} / \mathrm{sec}\right)\end{array}$ & $\begin{array}{c}\text { Soil } \\
\text { De of } \mathrm{CH}_{4} \\
\left(\mathrm{~cm}^{2} / \mathrm{sec}\right)\end{array}$ \\
\hline$S X-104$ & $4.39 \mathrm{E}+06$ & Hardner & 38.1 & 0.0000 & 0.000 & 366 & $3.80 \mathrm{E}-03$ & $5.00 \mathrm{E}-07$ & $3.80 \mathrm{E}-03$ & 0.12 & $1.20 \mathrm{E}-03$ & $5.00 \mathrm{E}-07$ & $1.20 \mathrm{E}-03$ & 0.037 \\
\hline SX-105 & $4.39 \mathrm{E}+06$ & Hardner & 38.1 & 0.0000 & 0.000 & 366 & $3.80 \mathrm{E}-03$ & $5.00 \mathrm{E}-07$ & $3.80 \mathrm{E}-03$ & 0.12 & $1.20 \mathrm{E}-03$ & $5.00 \mathrm{E}-07$ & $1.20 \mathrm{E}-03$ & 0.037 \\
\hline SX-106 & $4.39 \mathrm{E}+06$ & Hardner & 38.1 & 0.0000 & 0.000 & 366 & $3.80 \mathrm{E}-03$ & $5.00 \mathrm{E}-07$ & $3.80 \mathrm{E}-03$ & 0.12 & $1.20 \mathrm{E}-03$ & $5.00 \mathrm{E}-07$ & $1.20 \mathrm{E}-03$ & 0.037 \\
\hline SX-107 & $4.39 \mathrm{E}+06$ & Hardner & 38.1 & 0.0000 & 0.000 & 366 & $3.80 \mathrm{E}-03$ & $5.00 \mathrm{E}-07$ & $3.80 \mathrm{E}-03$ & 0.12 & $1.20 \mathrm{E}-03$ & $5.00 \mathrm{E}-07$ & $1.20 \mathrm{E}-03$ & 0.037 \\
\hline $\mathrm{SX}-108$ & $4.39 \mathrm{E}+06$ & Hardner & 38.1 & 0.0000 & 0.000 & 366 & $3.80 \mathrm{E}-03$ & $5.00 \mathrm{E}-07$ & $3.80 \mathrm{E}-03$ & 0.12 & 1.20E-03 & $5.00 \mathrm{E}-07$ & $1.20 \mathrm{E}-03$ & 0.037 \\
\hline SX-109 & $4.39 \mathrm{E}+06$ & Hardner & 38.1 & 0.0000 & 0.000 & 366 & $3.80 \mathrm{E}-03$ & $5.00 \mathrm{E}-07$ & $3.80 \mathrm{E}-03$ & 0.12 & $1.20 \mathrm{E}-03$ & $5.00 \mathrm{E}-07$ & $1.20 \mathrm{E}-03$ & 0.037 \\
\hline$S X-110$ & $4.39 \mathrm{E}+06$ & Hardner & 38.1 & 0.0000 & 0.000 & 366 & $3.80 \mathrm{E}-03$ & $5.00 \mathrm{E}-07$ & $3.80 \mathrm{E}-03$ & 0.12 & $1.20 \mathrm{E}-03$ & $5.00 \mathrm{E}-07$ & $1.20 \mathrm{E}-03$ & 0.037 \\
\hline$S X-111$ & $4.39 \mathrm{E}+06$ & Hardner & 38.1 & 0.0000 & 0.000 & 366 & $3.80 \mathrm{E}-03$ & $5.00 \mathrm{E}-07$ & $3.80 \mathrm{E}-03$ & 0.12 & $1.20[-03$ & $5.00 \mathrm{E}-07$ & $1.20 \mathrm{E}-03$ & 0.037 \\
\hline $\mathrm{SX}-112$ & $4.39 \mathrm{E}+06$ & Hardner & 38.1 & 0.0000 & 0.000 & 366 & $3.80 \mathrm{E}-03$ & $5.00 \mathrm{E}-07$ & $3.80 \mathrm{E}-03$ & 0.12 & $1.20 \mathrm{E}-03$ & $5.00 \mathrm{E}-07$ & $1.20 \mathrm{E}-03$ & 0.037 \\
\hline$S X-113$ & $4.39 \mathrm{E}+06$ & Hardner & 38.1 & 0.0000 & 0.000 & 366 & $3.80 \mathrm{E}-03$ & $5.00 \mathrm{E}-07$ & $3.80 \mathrm{E}-03$ & 0.12 & $1.20 \mathrm{E}-03$ & $5.00 \mathrm{E}-07$ & $1.20 \mathrm{E}-03$ & 0.037 \\
\hline SX-114 & $4.39 E+06$ & Hardner & 38.1 & 0.0000 & 0.000 & 366 & $3.80 \mathrm{E}-03$ & $5.00 \mathrm{E}-07$ & $3.80 \mathrm{E}-03$ & 0.12 & $1.20 \mathrm{E}-03$ & $5.00 \mathrm{E}-07$ & $1.20 \mathrm{E}-03$ & 0.037 \\
\hline $\mathrm{sX}-115$ & $4.39 \mathrm{E}+06$ & Hardner & 38.1 & 0.0000 & 0.000 & 366 & $3.80 \mathrm{E}-03$ & $5.00 \mathrm{E}-07$ & $3.80 \mathrm{E}-03$ & 0.12 & $1.20 \mathrm{E}-03$ & $5.00 \mathrm{E}-07$ & $1.20 \mathrm{E}-03$ & 0.037 \\
\hline$\Gamma-101$ & $4.43 \mathrm{E}+06$ & 3-Ply + 3/4 Mortar & 38.1 & 0.9525 & 1.905 & 404 & $3.80 \mathrm{E}-03$ & $5.00 \mathrm{E}-07$ & $3.80 \mathrm{E}-03$ & 0.12 & $1.20 \mathrm{~F}-03$ & $5.00 \mathrm{E}-07$ & $1.20 \mathrm{E}-03$ & 0.037 \\
\hline $\mathrm{T}-102$ & $4.43 \mathrm{E}+06$ & 3-Ply + 3/4 Mortar & 38.1 & 0.9525 & 1.905 & 404 & $3.80 \mathrm{E}-03$ & $5.00 \mathrm{E}-07$ & $3.80 \mathrm{E}-03$ & 0.12 & 1.20E-03 & $5.00 \mathrm{E}-07$ & $1.20 \mathrm{E}-03$ & 0.037 \\
\hline $\mathrm{T}-103$ & $4.43 E+06$ & 3-Ply $+3 / 4$ Mortar & 38.1 & 0.9525 & 1.905 & 404 & $3.80 \mathrm{E}-03$ & $5.00 \mathrm{E}-07$ & $3.80 \mathrm{~F}-03$ & 0.12 & $1.20 \mathrm{E}-03$ & $5.00 \mathrm{E}-07$ & $1.20 \mathrm{E}-03$ & 0.037 \\
\hline $\mathrm{T}-104$ & $4.43 \mathrm{E}+06$ & 3-Ply $+3 / 4$ Mortar & 38.1 & 0.9525 & 1.905 & 404 & $3.80 \mathrm{E}-03$ & $5.00 \mathrm{E}-07$ & $3.80 \mathrm{E}-03$ & 0.12 & $1.20 \mathrm{E}-03$ & $5.00 \mathrm{E}-07$ & $1.20 \mathrm{E}-03$ & 0.037 \\
\hline$T-105$ & $4.43 \mathrm{E}+06$ & 3-Ply $+3 / 4$ Mortar & 38.1 & 0.9525 & 1.905 & 404 & $3.80 \mathrm{E}-03$ & $5.00 \mathrm{E}-07$ & $3.80 \mathrm{E}-03$ & 0.12 & $1.20 \mathrm{E}-03$ & $5.00 \mathrm{E}-07$ & $1.20 \mathrm{E}-03$ & 0.037 \\
\hline$T-106$ & $4.43 \mathrm{E}+06$ & 3-Ply + 3/4 Mortar & 38.1 & 0.9525 & 1.905 & 404 & $3.80 \mathrm{E}-03$ & $5.00 \mathrm{E}-07$ & $3.80 \mathrm{E}-03$ & 0.12 & $1.20 \mathrm{E}-03$ & $5.00 \mathrm{E}-07$ & $1.20 \mathrm{E}-03$ & 0.037 \\
\hline $\mathrm{T}-107$ & $4.43 \mathrm{E}+06$ & 3-Ply $+3 / 4$ Mortar & 38.1 & 0.9525 & 1.905 & 404 & $3.80 \mathrm{E}-03$ & $5.00 \mathrm{E}-07$ & $3.80 \mathrm{E}-03$ & 0.12 & $1.20 \mathrm{E}-03$ & $5.00 \mathrm{E}-07$ & $1.20 \mathrm{E}-03$ & 0.037 \\
\hline$T-108$ & $4.43 \mathrm{E}+06$ & 3-Ply $+3 / 4$ Mortar & 38.1 & 0.9525 & 1.905 & 404 & $3.80 \mathrm{E}-03$ & $5.00 \mathrm{E}-07$ & $3.80 \mathrm{E}-03$ & 0.12 & $1.20 \mathrm{E}-03$ & $5.00 \mathrm{E}-07$ & $1.20 \mathrm{E}-03$ & 0.037 \\
\hline T-109 & $4.43 \mathrm{E}+06$ & 3-Ply $+3 / 4$ Mortar & 38.1 & 0.9525 & 1.905 & 404 & $3.80 \mathrm{E}-03$ & $5.00 \mathrm{E}-07$ & $3.80 \mathrm{E}-03$ & 0.12 & $1.20 \mathrm{E}-03$ & $5.00 \mathrm{E}-07$ & $1.20 \mathrm{E}-03$ & 0.037 \\
\hline $\mathrm{T}-110$ & $4.43 \mathrm{E}+06$ & 3-Ply $+3 / 4$ Mortar & 38.1 & 0.9525 & 1.905 & 404 & $3.80 \mathrm{E}-03$ & $5.00 \mathrm{E}-07$ & $3.80 \mathrm{E}-03$ & 0.12 & $1.20 \mathrm{E}-03$ & $5.00 \mathrm{E}-07$ & $1.20 \mathrm{E}-03$ & 0.037 \\
\hline$T-111$ & $4.43 \mathrm{E}+06$ & 3-Ply $+3 / 4$ Mortar & 38.1 & 0.9525 & 1.905 & 404 & $3.80 \mathrm{E}-03$ & $5.00 \mathrm{E}-07$ & $3.80 \mathrm{E}-03$ & 0.12 & $1.20 \mathrm{E}-03$ & $5.00 \mathrm{E}-07$ & $1.20 \mathrm{E}-03$ & 0.037 \\
\hline$T-112$ & $4.43 E+06$ & 3-Ply $+3 / 4$ Mortar & 38.1 & 0.9525 & 1.905 & 404 & $3.80 \mathrm{E}-03$ & $5.00 \mathrm{E}-07$ & $3.80 \mathrm{E}-03$ & 0.12 & 1.20E-03 & $5.00 \mathrm{E}-07$ & $1.20 \mathrm{E}-03$ & 0.037 \\
\hline $\mathrm{T}-201$ & $1.46 \mathrm{E}+05$ & Hardner + 3-Ply & 30.48 & 0.9525 & 0.000 & 335 & $3.80 \mathrm{E}-03$ & $5.00 \mathrm{E}-07$ & $3.80 \mathrm{E}-03$ & 0.12 & $1.20 \mathrm{E}-03$ & $5.00 \mathrm{E}-07$ & $1.20 \mathrm{E}-03$ & 0.037 \\
\hline $\mathrm{T}-202$ & $1.46 \mathrm{E}+05$ & Hardner + 3-Ply & 30.48 & 0.9525 & 0.000 & 335 & $3.80 \mathrm{E}-03$ & $5.00 \mathrm{E}-07$ & $3.80 \mathrm{E}-03$ & 0.12 & $1.20 \mathrm{E}-03$ & $5.00 \mathrm{E}-07$ & $1.20 \mathrm{E}-03$ & 0.037 \\
\hline $\mathrm{T}-203$ & $1.46 \mathrm{E}+05$ & Hardner + 3-Ply & 30.48 & 0.9525 & 0.000 & 335 & $3.80 \mathrm{E}-03$ & $5.00 \mathrm{E}-07$ & $3.80 \mathrm{E}-03$ & 0.12 & $1.20 \mathrm{E}-03$ & $5.00 E-07$ & $1.20 \mathrm{E}-03$ & 0.037 \\
\hline
\end{tabular}


RPP-5926 REV 6

Table F-1. The Thickness of Dome Coating for 149 Single-Shell Tanks and Diffusion Coefficients of Hydrogen and Methane. (6 sheets)

\begin{tabular}{|c|c|c|c|c|c|c|c|c|c|c|c|c|c|c|}
\hline Tank & $\begin{array}{l}\text { Dome } \\
\text { Area } \\
\left(\mathrm{cm}^{2}\right)\end{array}$ & Coatings of the Tank Dome & $\begin{array}{c}\text { Concrete } \\
\text { Thick } \\
(\mathrm{cm})\end{array}$ & $\begin{array}{c}\text { Asphatic } \\
\text { Thick } \\
(\mathrm{cm})\end{array}$ & $\begin{array}{l}\text { Gunite } \\
\text { Thick } \\
(\mathrm{cm})\end{array}$ & $\begin{array}{c}\text { Soil } \\
\text { Thick } \\
(\mathrm{cm})\end{array}$ & $\begin{array}{l}\text { Concretc } \\
\text { De of } \mathrm{H}_{2} \\
\left(\mathrm{~cm}^{2} / \mathrm{sec}\right)\end{array}$ & $\begin{array}{l}\text { Asphatic } \\
\text { De of } \mathrm{H}_{2} \\
\left(\mathrm{~cm}^{2} / \mathrm{sec}\right)\end{array}$ & $\begin{array}{c}\text { Gunite De } \\
\text { of } \mathrm{H}_{2} \\
\left(\mathrm{~cm}^{2} / \mathrm{sec}\right)\end{array}$ & $\begin{array}{c}\text { Soil } \\
\text { De of } \mathrm{H}_{2} \\
\left(\mathrm{~cm}^{2} / \mathrm{sec}\right)\end{array}$ & $\begin{array}{c}\text { Concrete De } \\
\text { of } \mathrm{CH}_{4} \\
\left(\mathrm{~cm}^{2} / \mathrm{sec}\right)\end{array}$ & $\begin{array}{c}\text { Asphatic De } \\
\text { of } \mathrm{CH}_{4} \\
\left(\mathrm{~cm}^{2} / \mathrm{sec}\right)\end{array}$ & $\begin{array}{c}\text { Gunite De } \\
\text { of } \mathrm{CH}_{4} \\
\left(\mathrm{~cm}^{2} / \mathrm{sec}\right)\end{array}$ & $\begin{array}{c}\text { Soil } \\
\text { De of } \mathrm{CH}_{4} \\
\left(\mathrm{~cm}^{2} / \mathrm{sec}\right)\end{array}$ \\
\hline $\mathrm{T}-204$ & $1.46 \mathrm{~F}+05$ & Hardner + 3-Ply & 30.48 & 0.9525 & 0.000 & 335 & $3.80 \mathrm{~F}-03$ & $5.00 \mathrm{E}-07$ & $3.80 \mathrm{E}-03$ & 0.12 & $1.20 \mathrm{E}-03$ & $5.00 \mathrm{E}-07$ & $1.20 \mathrm{E}-03$ & 0.037 \\
\hline $\mathrm{TX}-101$ & $4.38 \mathrm{E}+06$ & Hardner + 3-Ply $+3 / 4$ Gunite & 38.1 & 0.9525 & 1.905 & 427 & $3.80 \mathrm{E}-03$ & $5.00 \mathrm{E}-07$ & $3.80 \mathrm{E}-03$ & 0.12 & $1.20 \mathrm{E}-03$ & $5.00 \mathrm{E}-07$ & $1.20 \mathrm{E}-03$ & 0.037 \\
\hline $\mathrm{TX}-102$ & $4.38 \mathrm{E}+06$ & Hardner + 3-Ply + 3/4 Gunite & 38.1 & 0.9525 & 1.905 & 427 & $3.80 \mathrm{E}-03$ & $5.00 \mathrm{E}-07$ & $3.80 \mathrm{E}-03$ & 0.12 & $1.20 \mathrm{E}-03$ & $5.00 \mathrm{E}-07$ & $1.20 \mathrm{E}-03$ & 0.037 \\
\hline $\mathrm{TX}-103$ & $4.38 \mathrm{E}+06$ & Hardner + 3-Ply $+3 / 4$ Gunite & 38.1 & 0.9525 & 1.905 & 427 & $3.80 \mathrm{E}-03$ & $5.00 \mathrm{E}-07$ & $3.80 \mathrm{E}-03$ & 0.12 & $1.20 \mathrm{E}-03$ & $5.00 \mathrm{E}-07$ & $1.20 \mathrm{E}-03$ & 0.037 \\
\hline $\mathrm{TX}-104$ & 4.38E+06 & Hardner $+3-\mathrm{Ply}+3 / 4$ Gunite & 38.1 & 0.9525 & 1.905 & 427 & $3.80 \mathrm{E}-03$ & $5.00 \mathrm{E}-07$ & $3.80 \mathrm{E}-03$ & 0.12 & $1.20 \mathrm{E}-03$ & $5.00 \mathrm{E}-07$ & $1.20 \mathrm{E}-03$ & 0.037 \\
\hline $\mathrm{TX}-105$ & $4.38 E+06$ & Hardner + 3-Ply $+3 / 4$ Gunite & 38.1 & 0.9525 & 1.905 & 427 & $3.80 \mathrm{E}-03$ & $5.00 \mathrm{E}-07$ & $3.80 \mathrm{E}-03$ & 0.12 & $1.20 \mathrm{E}-03$ & $5.00 \mathrm{E}-07$ & $1.20 \mathrm{E}-03$ & 0.037 \\
\hline TX-106 & 4.38E+06 & Hardner $+3-\mathrm{Ply}+3 / 4$ Gunite & 38.1 & 0.9525 & 1.905 & 427 & $3.80 \mathrm{E}-03$ & $5.00 \mathrm{E}-07$ & $3.80 \mathrm{E}-03$ & 0.12 & $1.20 \mathrm{E}-03$ & $5.00 \mathrm{E}-07$ & $1.20 \mathrm{E}-03$ & 0.037 \\
\hline TX-107 & $4.38 \mathrm{E}+06$ & Hardner $+3-\mathrm{Ply}+3 / 4$ Gunite & 38.1 & 0.9525 & 1.905 & 427 & $3.80 \mathrm{E}-03$ & $5.00 \mathrm{E}-07$ & $3.80 \mathrm{E}-03$ & 0.12 & $1.20 \mathrm{E}-03$ & $5.00 \mathrm{E}-07$ & $1.20 \mathrm{E}-03$ & 0.037 \\
\hline $\mathrm{TX}-108$ & $4.38 \mathrm{E}+06$ & Hardner $+3-P l y+3 / 4$ Gunite & 38.1 & 0.9525 & 1.905 & 427 & $3.80 \mathrm{E}-03$ & $5.00 \mathrm{E}-07$ & $3.80 \mathrm{E}-03$ & 0.12 & $1.20 \mathrm{E}-03$ & $5.00 \mathrm{E}-07$ & $1.20 \mathrm{E}-03$ & 0.037 \\
\hline $\mathrm{TX}-109$ & $4.38 \mathrm{E}+06$ & Hardner + 3-Ply $+3 / 4$ Gunite & 38.1 & 0.9525 & 1.905 & 427 & $3.80 \mathrm{E}-03$ & $5.00 \mathrm{E}-07$ & $3.80 \mathrm{E}-03$ & 0.12 & $1.20 \mathrm{E}-03$ & $5.00 \mathrm{E}-07$ & $1.20 \mathrm{E}-03$ & 0.037 \\
\hline TX-110 & $4.38 \mathrm{E}+06$ & Hardner $+3-\mathrm{Ply}+3 / 4$ Gunite & 38.1 & 0.9525 & 1.905 & 427 & $3.80 \mathrm{E}-03$ & $5.00 \mathrm{E}-07$ & $3.80 \mathrm{E}-03$ & 0.12 & $1.20 \mathrm{E}-03$ & $5.00 \mathrm{E}-07$ & $1.20 \mathrm{E}-03$ & 0.037 \\
\hline$T X-111$ & $4.38 \mathrm{E}+06$ & Hardner + 3-Ply + 3/4 Gunite & 38.1 & 0.9525 & 1.905 & 427 & $3.80 \mathrm{E}-03$ & $5.00 \mathrm{E}-07$ & $3.80 \mathrm{E}-03$ & 0.12 & $1.20 \mathrm{E}-03$ & $5.00 \mathrm{E}-07$ & $1.20 \mathrm{E}-03$ & 0.037 \\
\hline$T X-112$ & $4.38 \mathrm{E}+06$ & Hardner $+3-\mathrm{Ply}+3 / 4$ Gunite & 38.1 & 0.9525 & 1.905 & 427 & $3.80 \mathrm{E}-03$ & $5.00 \mathrm{E}-07$ & $3.80 \mathrm{E}-03$ & 0.12 & 1.20E-03 & $5.00 \mathrm{E}-07$ & $1.20 \mathrm{E}-03$ & 0.037 \\
\hline $\mathrm{TX}-113$ & $4.38 \mathrm{E}+06$ & Hardner + 3-Ply $+3 / 4$ Gunite & 38.1 & 0.9525 & 1.905 & 427 & $3.80 \mathrm{E}-03$ & $5.00 \mathrm{E}-07$ & $3.80 \mathrm{E}-03$ & 0.12 & $1.20 \mathrm{E}-03$ & $5.00 \mathrm{E}-07$ & $1.20 \mathrm{E}-03$ & 0.037 \\
\hline $\mathrm{TX}-114$ & $4.38 \mathrm{E}+06$ & Hardner + 3-Ply + 3/4 Gunite & 38.1 & 0.9525 & 1.905 & 427 & $3.80 \mathrm{E}-03$ & $5.00 \mathrm{E}-07$ & $3.80 \mathrm{E}-03$ & 0.12 & $1.20 \mathrm{E}-03$ & $5.00 \mathrm{E}-07$ & $1.20 \mathrm{E}-03$ & 0.037 \\
\hline $\mathrm{TX}-115$ & $4.38 \mathrm{E}+06$ & Hardner $+3-P l y+3 / 4$ Gunite & 38.1 & 0.9525 & 1.905 & 427 & $3.80 \mathrm{E}-03$ & $5.00 \mathrm{E}-07$ & $3.80 \mathrm{E}-03$ & 0.12 & $1.20 \mathrm{E}-03$ & $5.00 \mathrm{E}-07$ & $1.20 \mathrm{E}-03$ & 0.037 \\
\hline$T X-116$ & $4.38 \mathrm{E}+06$ & Hardner + 3-Ply $+3 / 4$ Gunite & 38.1 & 0.9525 & 1.905 & 427 & $3.80 \mathrm{E}-03$ & $5.00 \mathrm{E}-07$ & $3.80 \mathrm{E}-03$ & 0.12 & $1.20 \mathrm{E}-03$ & $5.00 \mathrm{E}-07$ & $1.20 \mathrm{E}-03$ & 0.037 \\
\hline TX-117 & $4.38 \mathrm{E}+06$ & Hardner + 3-Ply $+3 / 4$ Gunite & 38.1 & 0.9525 & 1.905 & 427 & $3.80 \mathrm{E}-03$ & $5.00 \mathrm{E}-07$ & $3.80 \mathrm{E}-03$ & 0.12 & $1.20 \mathrm{E}-03$ & $5.00 \mathrm{E}-07$ & $1.20 \mathrm{E}-03$ & 0.037 \\
\hline $\mathrm{TX}-118$ & $4.38 \mathrm{E}+06$ & Hardner + 3-Ply $+3 / 4$ Gunite & 38.1 & 0.9525 & 1.905 & 427 & $3.80 \mathrm{E}-03$ & $5.00 \mathrm{E}-07$ & $3.80 \mathrm{E}-03$ & 0.12 & $1.20 \mathrm{E}-03$ & $5.00 \mathrm{E}-07$ & $1.20 \mathrm{E}-03$ & 0.037 \\
\hline TY-101 & $4.38 \mathrm{E}+06$ & Hardner + 3-Ply +3/4 Gunite & 38.1 & 0.9525 & 1.905 & 427 & $3.80 \mathrm{E}-03$ & $5.00 \mathrm{E}-07$ & $3.80 \mathrm{E}-03$ & 0.12 & $1.20 \mathrm{E}-03$ & $5.00 \mathrm{E}-07$ & $1.20 \mathrm{E}-03$ & 0.037 \\
\hline TY-102 & $4.38 \mathrm{E}+06$ & Hardner + 3-Ply $+3 / 4$ Gunite & 38.1 & 0.9525 & 1.905 & 427 & $3.80 \mathrm{~F}-03$ & $5.00 \mathrm{E}-07$ & $3.80 \mathrm{E}-03$ & 0.12 & $1.20 \mathrm{E}-03$ & $5.00 \mathrm{E}-07$ & $1.20 \mathrm{E}-03$ & 0.037 \\
\hline TY- 103 & $4.38 \mathrm{~F}+06$ & Hardner + 3-Ply $+3 / 4$ Gunite & 38.1 & 0.9525 & 1.905 & 427 & $3.80 \mathrm{E}-03$ & $5.00 \mathrm{E}-07$ & $3.80 \mathrm{E}-03$ & 0.12 & $1.20 \mathrm{E}-03$ & $5.00 \mathrm{E}-07$ & I.20E-03 & 0.037 \\
\hline TY-104 & $4.38 \mathrm{E}+06$ & Hardner + 3-Ply $+3 / 4$ Gunite & 38.1 & 0.9525 & 1.905 & 427 & $3.80 \mathrm{E}-03$ & $5.00 \mathrm{E}-07$ & $3.80 \mathrm{E}-03$ & 0.12 & $1.20 \mathrm{E}-03$ & $5.00 \mathrm{E}-07$ & $1.20 \mathrm{E}-03$ & 0.037 \\
\hline TY-105 & $4.38 \mathrm{E}+06$ & Hardner $+3-\mathrm{Ply}+3 / 4$ Gunite & 38.1 & 0.9525 & 1.905 & 427 & $3.80 \mathrm{E}-03$ & $5.00 \mathrm{E}-07$ & $3.80 \mathrm{E}-03$ & 0.12 & $1.20 \mathrm{E}-03$ & $5.00 \mathrm{E}-07$ & $1.20 \mathrm{E}-03$ & 0.037 \\
\hline TY-106 & $4.38 \mathrm{E}+06$ & Hardner + 3-Ply + 3/4 Gunite & 38.1 & 0.9525 & 1.905 & 427 & $3.80 \mathrm{E}-03$ & $5.00 \mathrm{E}-07$ & $3.80 \mathrm{E}-03$ & 0.12 & $1.20 \mathrm{E}-03$ & $5.00 \mathrm{E}-07$ & $1.20 \mathrm{E}-03$ & 0.037 \\
\hline$U-101$ & $4.43 \mathrm{E}+06$ & 3-Ply $+3 / 4$ Mortar & 38.1 & 0.9525 & 1.905 & 404 & $3.80 \mathrm{E}-03$ & $5.00 \mathrm{E}-07$ & $3.80 \mathrm{E}-03$ & 0.12 & $1.20 \mathrm{E}-03$ & $5.00 \mathrm{E}-07$ & $1.20 \mathrm{E}-03$ & 0.037 \\
\hline $\mathrm{U}-102$ & $4.43 \mathrm{E}+06$ & 3-Ply +3/4 Mortar & 38.1 & 0.9525 & 1.905 & 404 & $3.80 \mathrm{E}-03$ & $5.00 \mathrm{E}-07$ & $3.80 \mathrm{E}-03$ & 0.12 & $1.20 \mathrm{E}-03$ & $5.00 \mathrm{E}-07$ & $1.20 \mathrm{E}-03$ & 0.037 \\
\hline
\end{tabular}


Table F-1. The Thickness of Dome Coating for 149 Single-Shell Tanks and Diffusion Coefficients of Hydrogen and Methane. (6 sheets)

\begin{tabular}{|c|c|c|c|c|c|c|c|c|c|c|c|c|c|c|}
\hline Tank & $\begin{array}{l}\text { Dome } \\
\text { Area } \\
\left(\mathrm{cm}^{2}\right)\end{array}$ & Coatings of the Tank Dome & $\begin{array}{c}\text { Concretc } \\
\text { Thick } \\
\text { (cm) }\end{array}$ & $\begin{array}{c}\text { Asphatic } \\
\text { Thick } \\
(\mathrm{cm})\end{array}$ & $\begin{array}{l}\text { Gunite } \\
\text { Thick } \\
(\mathrm{cm})\end{array}$ & $\begin{array}{c}\text { Soil } \\
\text { Thick } \\
(\mathrm{cm})\end{array}$ & $\begin{array}{l}\text { Concrete } \\
\text { De of } \mathrm{H}_{2} \\
\left(\mathrm{~cm}^{2} / \mathrm{sec}\right)\end{array}$ & $\begin{array}{l}\text { Asphatic } \\
\text { De of } \mathrm{H}_{2} \\
\left(\mathrm{~cm}^{2} / \mathrm{scc}\right)\end{array}$ & $\begin{array}{c}\text { Gunite De } \\
\text { of } \mathrm{H}_{2} \\
\left(\mathrm{~cm}^{2} / \mathrm{sec}\right)\end{array}$ & $\begin{array}{c}\text { Soil } \\
\text { De of } \mathrm{H}_{2} \\
\left(\mathrm{~cm}^{2} / \mathrm{sec}\right)\end{array}$ & $\begin{array}{c}\text { Concrete De } \\
\text { of } \mathrm{CH}_{4} \\
\left(\mathrm{~cm}^{2} / \mathrm{sec}\right)\end{array}$ & $\begin{array}{c}\text { Asphatic De } \\
\text { of } \mathrm{CH}_{4} \\
\left(\mathrm{~cm}^{2} / \mathrm{scc}\right)\end{array}$ & $\begin{array}{c}\text { Gunite De } \\
\text { of } \mathrm{CH}_{\mathrm{a}} \\
\left(\mathrm{cm}^{2} / \mathrm{sec}\right)\end{array}$ & $\begin{array}{c}\text { Soil } \\
\text { De of } \mathrm{CH}_{4} \\
\left(\mathrm{~cm}^{2} / \mathrm{sec}\right)\end{array}$ \\
\hline $\mathrm{U}-103$ & $4.43 E+06$ & 3-Ply + 3/4 Mortar & 38.1 & 0.9525 & 1.905 & 404 & $3.80 \mathrm{E}-03$ & $5.00 \mathrm{E}-07$ & $3.80 \mathrm{E}-03$ & 0.12 & $1.20 \mathrm{E}-03$ & $5.00 \mathrm{E}-07$ & $1.20 \mathrm{E}-03$ & 0.037 \\
\hline $\mathrm{U}-104$ & $4.43 E+06$ & 3-Ply + 3/4 Mortas & 38.1 & 0.9525 & 1.905 & 404 & $3.80 \mathrm{E}-03$ & $5.00 \mathrm{E}-07$ & $3.80 \mathrm{E}-03$ & 0.12 & $1.20 \mathrm{E}-03$ & $5.00 \mathrm{E}-07$ & $1.20 \mathrm{E}-03$ & 0.037 \\
\hline 0.105 & $4.43 E+06$ & 3-Ply $+3 / 4$ Mortar & 38.1 & 0.9525 & 1.905 & 404 & $3.80 \mathrm{E}-03$ & $5.00 \mathrm{E}-07$ & $3.80 \mathrm{E}-03$ & 0.12 & $1.20 \mathrm{E}-03$ & $5.00 \mathrm{E}-07$ & $1.20 \mathrm{E}-03$ & 0.037 \\
\hline $\mathrm{U}-106$ & $4.43 E+06$ & 3-Ply $+3 / 4$ Mortar & 38.1 & 0.9525 & 1.905 & 404 & $3.80 \mathrm{E}-03$ & $5.00 \mathrm{E}-07$ & $3.80 \mathrm{E}-03$ & 0.12 & $1.20 \mathrm{E}-03$ & $5.00 \mathrm{E}-07$ & $1.20 \mathrm{E}-03$ & 0.037 \\
\hline $\mathrm{U}-107$ & $4.43 \mathrm{E}+06$ & 3-Ply $+3 / 4$ Mortar & 38.1 & 0.9525 & 1.905 & 404 & $3.80 \mathrm{E}-03$ & $5.00 \mathrm{E}-07$ & $3.80 \mathrm{E}-03$ & 0.12 & $1.20 \mathrm{E}-03$ & $5.00 \mathrm{E}-07$ & $1.20 \mathrm{E}-03$ & 0.037 \\
\hline U-108 & $4.43 \mathrm{E}+06$ & 3-Ply $+3 / 4$ Mortar & 38.1 & 0.9525 & 1.905 & 404 & $3.80 \mathrm{E}-03$ & $5.00 \mathrm{E}-07$ & $3.80 \mathrm{E}-03$ & 0.12 & $1.20 \mathrm{E}-03$ & $5.00 \mathrm{E}-07$ & $1.20 \mathrm{E}-03$ & 0.037 \\
\hline $\mathrm{U}-109$ & $4.43 E+06$ & 3-Ply $+3 / 4$ Mortar & 38.1 & 0.9525 & 1.905 & 404 & $3.80 \mathrm{E}-03$ & $5.00 \mathrm{E}-07$ & $3.80 \mathrm{E}-03$ & 0.12 & $1.20 \mathrm{E}-03$ & $5.00 \mathrm{E}-07$ & $1.20 \mathrm{E}-03$ & 0.037 \\
\hline U.110 & $4.43 E+06$ & 3-Ply + 3/4 Mortar & 38.1 & 0.9525 & 1.905 & 404 & $3.80 \mathrm{E}-03$ & $5.00 \mathrm{E}-07$ & $3.80 \mathrm{E}-03$ & 0.12 & $1.20 \mathrm{E}-03$ & $5.00 \mathrm{E}-07$ & $1.20 \mathrm{E}-03$ & 0.037 \\
\hline U-11] & $4.43 \mathrm{E}+06$ & 3-Ply + 3/4 Mortar & 38.1 & 0.9525 & 1.905 & 404 & $3.80 \mathrm{E}-03$ & $5.00 \mathrm{E}-07$ & $3.80 \mathrm{E}-03$ & 0.12 & $1.20 \mathrm{E}-03$ & $5.00 \mathrm{E}-07$ & $1.20 \mathrm{E}-03$ & 0.037 \\
\hline $\mathrm{U}-112$ & $4.43 E+06$ & 3-Ply $+3 / 4$ Mortar & 38.1 & 0.9525 & 1.905 & 404 & $3.80 \mathrm{E}-03$ & $5.00 \mathrm{E}-07$ & $3.80 \mathrm{E}-03$ & 0.12 & $1.20 \mathrm{E}-03$ & $5.00 \mathrm{E}-07$ & $1.20 \mathrm{E}-03$ & 0.037 \\
\hline U.201 & $1.46 \mathrm{E}+05$ & Hardner + 3-Ply & 30.48 & 0.9525 & 0.000 & 335 & $3.80 \mathrm{E}-03$ & $5.00 \mathrm{E}-07$ & $3.80 \mathrm{E}-03$ & 0.12 & $1.20 \mathrm{E}-03$ & $5.00 \mathrm{E}-07$ & $1.20 \mathrm{E}-03$ & 0.037 \\
\hline U-202 & $1.46 E+05$ & Hardner + 3-Ply & 30.48 & 0.9525 & 0.000 & 335 & $3.80 \mathrm{E}-03$ & $5.00 \mathrm{E}-07$ & $3.80 \mathrm{E}-03$ & 0.12 & $1.20 \mathrm{E}-03$ & $5.00 \mathrm{E}-07$ & $1.20 \mathrm{E}-03$ & 0.037 \\
\hline $\mathrm{U}-203$ & $1.46 \mathrm{E}+05$ & Hardner + 3-Ply & 30.48 & 0.9525 & 0.000 & 335 & $3.80 \mathrm{E}-03$ & $5.00 \mathrm{E}-07$ & $3.80 \mathrm{E}-03$ & 0.12 & $1.20 \mathrm{E}-03$ & $5.00 \mathrm{E}-07$ & $1.20 \mathrm{E}-03$ & 0.037 \\
\hline U-204 & $1.46 \mathrm{E}+05$ & Hardner + 3-Ply & 30.48 & 0.9525 & 0.000 & 335 & $3.80 \mathrm{E}+03$ & $5.00 \mathrm{E}-07$ & $3.80 \mathrm{E}-03$ & 0.12 & $1.20 \mathrm{E}-03$ & $5.00 \mathrm{E}-07$ & $1.20 \mathrm{E}-03$ & 0.037 \\
\hline
\end{tabular}


Table F-2. The Diffusion Resistance and Gas Diffusion Rates of Hydrogen and Methane for 149 Single-Shell Tanks. (6 sheets)

\begin{tabular}{|c|c|c|c|c|c|c|c|c|c|c|c|c|}
\hline Tank & $\mid \begin{array}{c}\text { Concrete } \\
\text { Resist of } \mathrm{H}_{2} \\
(\mathrm{sec} / \mathrm{cm})\end{array}$ & $\begin{array}{c}\text { Asphatic } \\
\text { Resist of } \mathrm{H}_{2} \\
(\mathrm{sec} / \mathrm{cm})\end{array}$ & $\begin{array}{c}\text { Gunite Resist } \\
\text { of } \mathrm{H}_{2} \\
\text { (sec/cm) }\end{array}$ & $\begin{array}{c}\text { Soil Resist of } \\
\mathrm{H}_{2}(\mathrm{sec} / \mathrm{cm})\end{array}$ & $\begin{array}{c}\text { Concrete } \\
\text { Resist of } \mathrm{CH}_{4} \\
(\mathrm{sec} / \mathrm{cm})\end{array}$ & $\begin{array}{c}\text { Asphatic } \\
\text { Resist of } \mathrm{CH}_{4} \\
(\mathrm{sec} / \mathrm{cm})\end{array}$ & $\begin{array}{c}\text { Gunite Resist } \\
\text { of } \mathrm{CH}_{4} \\
(\mathrm{sec} / \mathrm{cm})\end{array}$ & $\begin{array}{l}\text { Soil Resist of } \\
\mathrm{CH}_{4}(\mathrm{sec} / \mathrm{cm})\end{array}$ & $\left|\begin{array}{c}\text { Total Resist of } \\
\mathrm{H}_{2}(\mathrm{sec} / \mathrm{cm})\end{array}\right|$ & $\left|\begin{array}{l}\text { Total Resist of } \\
\mathrm{CH}_{4}(\mathrm{sec} / \mathrm{cm})\end{array}\right|$ & $\begin{array}{c}\mathrm{H}_{2} \text { Dilf Rate } \\
\mathrm{Ai} / \mathrm{Ri} \\
(\mathrm{cfm})\end{array}$ & $\begin{array}{c}\mathrm{CH}_{4} \text { Diff Rate } \\
\mathrm{Ai} / \mathrm{Ri} \\
\text { (cfm) }\end{array}$ \\
\hline A-101 & $1.00 \mathrm{E}+04$ & $1.27 \mathrm{E}+06$ & $0.00 \mathrm{E}+00$ & $3.05 \mathrm{E}+03$ & $3.18 \mathrm{E}+04$ & $1.27 \mathrm{E}+06$ & $0.00 \mathrm{E}+00$ & $9.89 \mathrm{E}+03$ & $1.28 \mathrm{E}+06$ & $1.31 \mathrm{E}+06$ & $7.25 \mathrm{E}-03$ & $7.09 \mathrm{E}-03$ \\
\hline $\mathrm{A}-102$ & $1.00 \mathrm{E}+04$ & $1.27 \mathrm{E}+06$ & $0.00 \mathrm{E}+00$ & $3.05 E+03$ & $3.18 \mathrm{E}+04$ & $1.27 \mathrm{E}+06$ & $0.00 \mathrm{E}+00$ & $9.89 \mathrm{E}+03$ & $1.28 \mathrm{E}+06$ & $1.31 \mathrm{E}+06$ & $7.25 \mathrm{E}-03$ & $7.09 \mathrm{E}-03$ \\
\hline A-103 & $1.00 \mathrm{E}+04$ & $1.27 \mathrm{E}+06$ & $0.00 \mathrm{E}+00$ & $3.05 \mathrm{E}+03$ & $3.18 \mathrm{E}+04$ & $1.27 \mathrm{E}+06$ & $0.00 \mathrm{E}+00$ & $9.89 \mathrm{E}+03$ & $1.28 \mathrm{E}+06$ & $1.31 \mathrm{E}+06$ & $7.25 \mathrm{E}-03$ & $7.09 \mathrm{E}-03$ \\
\hline A-104 & $1.00 \mathrm{E}+04$ & $1.27 \mathrm{E}+06$ & $0.00 \mathrm{E}+00$ & $3.05 E+03$ & $3.18 \mathrm{E}+04$ & $1.27 \mathrm{E}+06$ & $0.00 \mathrm{E}+00$ & $9.89 \mathrm{E}+03$ & $1.28 \mathrm{E}+06$ & $1.31 \mathrm{E}+06$ & $7.25 \mathrm{E}-03$ & $7.09 \mathrm{E}-03$ \\
\hline A-105 & $1.00 \mathrm{E}+04$ & $1.27 \mathrm{E}+06$ & $0.00 \mathrm{E}+00$ & $3.05 E+03$ & 3. $18 \mathrm{E}+04$ & $1.27 \mathrm{E}+06$ & $0.00 \mathrm{E}+00$ & $9.89 \mathrm{E}+03$ & $1.28 \mathrm{E}+06$ & $1.31 \mathrm{E}+06$ & $7.25 \mathrm{E}-03$ & $7.09 \mathrm{E}-03$ \\
\hline A-106 & $1.00 \mathrm{E}+04$ & $1.27 \mathrm{E}+06$ & $0.00 \mathrm{E}+00$ & $3.05 E+03$ & $3.18 \mathrm{E}+04$ & $1.27 \mathrm{E}+06$ & $0.00 \mathrm{E}+00$ & $9.89 \mathrm{E}+03$ & $1.28 \mathrm{E}+06$ & $1.31 E+06$ & $7.25 \mathrm{E}-03$ & $7.09 \mathrm{E}-03$ \\
\hline $\mathrm{AX}-101$ & $1.00 \mathrm{E}+04$ & $0.00 \mathrm{E}+00$ & $0.00 \mathrm{E}+00$ & $3.05 \mathrm{E}+03$ & $3.18 \mathrm{E}+04$ & $0.00 \mathrm{E}+00$ & $0.00 E+00$ & $9.89 \mathrm{E}+03$ & $1.31 E+04$ & $4.16 \mathrm{E}+04$ & $7.11 \mathrm{E}-01$ & $2.23 \mathrm{E}-01$ \\
\hline $\mathrm{AX}-102$ & $1.00 \mathrm{E}+04$ & $0.00 \mathrm{E}+00$ & $0.00 \mathrm{E}+00$ & $3.05 E+03$ & $3.18 \mathrm{E}+04$ & $0.00 \mathrm{E}+00$ & $0.00 \mathrm{E}+00$ & $9.89 \mathrm{E}+03$ & $1.31 E+04$ & 4. $16 \mathrm{E}+04$ & $7.11 \mathrm{E}-01$ & $2.23 \mathrm{E}-01$ \\
\hline AX-103 & $1.00 E+04$ & $0.00 \mathrm{E}+00$ & $0.00 \mathrm{E}+00$ & $3.05 \mathrm{E}+03$ & $3.18 \mathrm{E}+04$ & $0.00 \mathrm{E}+00$ & $0.00 \mathrm{E}+00$ & $9.89 \mathrm{E}+03$ & $1.31 E+04$ & $4.16 \mathrm{E}+04$ & $7.11 \mathrm{E}-0 \mathrm{I}$ & $2.23 \mathrm{E}-0 \mathrm{I}$ \\
\hline $\mathrm{AX}-104$ & $1.00 \mathrm{E}+04$ & $0.00 \mathrm{E}+00$ & $0.00 \mathrm{E}+00$ & $3.05 \mathrm{E}+03$ & $3.18 \mathrm{E}+04$ & $0.00 \mathrm{E}+00$ & $0.00 \mathrm{E}+00$ & $9.89 \mathrm{E}+03$ & $1.31 \mathrm{E}+04$ & $4.16 \mathrm{E}+04$ & $7.11 \mathrm{E}-01$ & $2.23 \mathrm{E}-01$ \\
\hline $\mathrm{B}-101$ & $1.00 \mathrm{E}+04$ & $1.91 \mathrm{E}+06$ & $5.01 \mathrm{E}+02$ & $3.37 \mathrm{E}+03$ & $3.18 E+04$ & $1.91 \mathrm{E}+06$ & $1.59 \mathrm{E}+03$ & $1.09 \mathrm{E}+04$ & $1.92 E+06$ & $1.95 \mathrm{E}+06$ & $4.89 \mathrm{E}-03$ & $4.82 \mathrm{E}-0.3$ \\
\hline B-102 & $1.00 \mathrm{E}+04$ & $1.91 \mathrm{E}+06$ & $5.01 \mathrm{E}+02$ & $3.37 E+03$ & $3.18 \mathrm{E}+04$ & $1.91 \mathrm{E}+06$ & $1.59 \mathrm{E}+03$ & $1.09 \mathrm{E}+04$ & $1.92 \mathrm{E}+06$ & $1.95 \mathrm{E}+06$ & $4.89 \mathrm{E}-03$ & $4.82 \mathrm{E}-03$ \\
\hline$B-103$ & $1.00 \mathrm{E}+04$ & $1.91 \mathrm{E}+06$ & $5.01 \mathrm{E}+02$ & $3.37 \mathrm{E}+03$ & $3.18 \mathrm{E}+04$ & $1.91 \mathrm{E}+06$ & $1.59 \mathrm{E}+03$ & $1.09 E+04$ & $1.92 E+06$ & $1.95 \mathrm{E}+06$ & $4.89 \mathrm{E}-03$ & $4.82 \mathrm{E}-03$ \\
\hline B-104 & $1.00 \mathrm{E}+04$ & $1.91 \mathrm{E}+06$ & $5.01 \mathrm{E}+02$ & $3.37 \mathrm{E}+03$ & $3.18 \mathrm{E}+04$ & $1.91 \mathrm{E}+06$ & $1.59 \mathrm{E}+03$ & $1.09 \mathrm{E}+04$ & $1.92 \mathrm{E}+06$ & $1.95 \mathrm{E}+06$ & $4.89 \mathrm{E}-03$ & $4.82 \mathrm{E}-03$ \\
\hline B-105 & $1.00 \mathrm{E}+04$ & $1.91 E+06$ & $5.01 \mathrm{E}+02$ & $3.37 \mathrm{E}+03$ & $3.18 \mathrm{E}+04$ & $1.91 \mathrm{E}+06$ & $1.59 \mathrm{E}+03$ & $1.09 \mathrm{E}+04$ & $1.92 \mathrm{E}+06$ & $1.95 \mathrm{E}+06$ & $4.89 \mathrm{E}-03$ & $4.82 \mathrm{E}-03$ \\
\hline B-106 & $1.00 \mathrm{E}+04$ & $1.91 \mathrm{~F}+06$ & $5.01 \mathrm{E}+02$ & $3.37 \mathrm{E}+03$ & $3.18 \mathrm{E}+04$ & $1.91 \mathrm{E}+06$ & $1.59 \mathrm{E}+03$ & $1.09 \mathrm{E}+04$ & $1.92 \mathrm{E}+06$ & $1.95 \mathrm{E}+06$ & $4.89 \mathrm{E}-03$ & $4.82 \mathrm{E}-03$ \\
\hline B-107 & $1.00 \mathrm{E}+04$ & $1.91 \mathrm{E}+06$ & $5.01 \mathrm{E}+02$ & $3.37 E+03$ & $3.18 \mathrm{E}+04$ & $1.91 E+06$ & $1.59 \mathrm{E}+03$ & $1.09 \mathrm{E}+04$ & $1.92 \mathrm{E}+06$ & $1.95 \mathrm{E}+06$ & 4.89E-03 & $4.82 \mathrm{E}-03$ \\
\hline B-108 & $1.00 \mathrm{E}+04$ & $1.91 \mathrm{E}+06$ & $5.01 \mathrm{E}+02$ & $3.37 \mathrm{E}+03$ & $3.18 \mathrm{E}+04$ & $1.91 \mathrm{E}+06$ & $1.59 \mathrm{E}+03$ & $1.09 \mathrm{E}+04$ & $1.92 \mathrm{~F}+06$ & $1.95 \mathrm{E}+06$ & $4.89 \mathrm{E}-03$ & $4.82 \mathrm{E}-03$ \\
\hline B-109 & $1.00 \mathrm{E}+04$ & $1.91 \mathrm{E}+06$ & $5.01 \mathrm{E}+02$ & $3.37 \mathrm{E}+03$ & $3.18 \mathrm{E}+04$ & $1.91 \mathrm{E}+06$ & $1.59 \mathrm{E}+03$ & $1.09 \mathrm{E}+04$ & $1.92 \mathrm{E}+06$ & $1.95 E+06$ & $4.89 \mathrm{E}-03$ & $4.82 \mathrm{E}-03$ \\
\hline B-110 & $1.00 \mathrm{E}+04$ & $1.91 \mathrm{E}+06$ & $5.01 \mathrm{E}+02$ & $3.37 \mathrm{E}+03$ & $3.18 \mathrm{E}+04$ & $1.91 \mathrm{E}+06$ & $1.59 \mathrm{E}+03$ & $1.09 \mathrm{E}+04$ & $1.92 \mathrm{E}+06$ & $1.95 \mathrm{E}+06$ & $4.89 \mathrm{E}-03$ & $4.82 \mathrm{E}-03$ \\
\hline B-111 & $1.00 \mathrm{E}+04$ & $1.91 \mathrm{E}+06$ & $5.01 \mathrm{E}+02$ & 3.37E+03 & $3.18 \mathrm{E}+04$ & $1.91 \mathrm{E}+06$ & $1.59 \mathrm{E}+03$ & $1.09 \mathrm{E}+04$ & $1.92 \mathrm{E}+06$ & $1.95 \mathrm{E}+06$ & $4.89 \mathrm{E}-03$ & $4.82 \mathrm{E}-03$ \\
\hline B-112 & $1.00 \mathrm{E}+04$ & $1.91 E+06$ & $5.01 E+02$ & $3.37 \mathrm{E}+03$ & $3.18 E+04$ & $1.91 \mathrm{E}+06$ & $1.59 \mathrm{E}+03$ & $1.09 \mathrm{E}+04$ & $1.92 E+06$ & $1.95 E+06$ & $4.89 \mathrm{E}-03$ & $4.82 \mathrm{E}-03$ \\
\hline B-201 & $8.02 \mathrm{E}+03$ & $1.91 \mathrm{E}+06$ & $0.00 \mathrm{E}+00$ & $2.79 E+03$ & $2.54 \mathrm{E}+04$ & $1.91 \mathrm{E}+06$ & $0.00 \mathrm{E}+00$ & $9.05 \mathrm{E}+03$ & $1.92 \mathrm{E}+06$ & $1.94 \mathrm{E}+06$ & $1.61 \mathrm{E}-04$ & $1.60 \mathrm{E}-04$ \\
\hline B-202 & $8.02 \mathrm{E}+03$ & $1.91 \mathrm{E}+06$ & $0.00 \mathrm{E}+00$ & $2.79 \mathrm{E}+03$ & $2.54 \mathrm{E}+04$ & $1.91 \mathrm{E}+06$ & $0.00 \mathrm{E}+00$ & $9.05 \mathrm{E}+03$ & $1.92 \mathrm{E}+06$ & $1.94 \mathrm{E}+06$ & $1.61 \mathrm{E}-04$ & $1.60 \mathrm{E}-04$ \\
\hline B-203 & $8.02 E+03$ & $1.91 E+06$ & $0.00 \mathrm{E}+00$ & $2.79 \mathrm{E}+03$ & $2.54 \mathrm{E}+04$ & $1.91 \mathrm{E}+06$ & $0.00 \mathrm{E}+00$ & $9.05 E+03$ & $1.92 \mathrm{E}+06$ & $1.94 \mathrm{E}+06$ & $1.61 \mathrm{E}-04$ & $1.60 \mathrm{E}-04$ \\
\hline B-204 & $8.02 \mathrm{E}+03$ & $1.91 E+06$ & $0.00 \mathrm{E}+00$ & $2.79 \mathrm{E}+03$ & $2.54 \mathrm{E}+04$ & $1.91 \mathrm{E}+06$ & $0.00 \mathrm{E}+00$ & $9.05 \mathrm{E}+03$ & $1.92 \mathrm{E}+06$ & $1.94 \mathrm{E}+06$ & $1.61 \mathrm{E}-04$ & $1.60 \mathrm{E}-04$ \\
\hline BX-101 & $1.00 \mathrm{E}+04$ & $1.91 \mathrm{E}+06$ & $5.01 \mathrm{E}+02$ & $3.37 E+03$ & $3.18 E+04$ & $1.91 \mathrm{E}+06$ & $1.59 \mathrm{E}+03$ & $1.09 \mathrm{E}+04$ & $1.92 \mathrm{E}+06$ & $1.95 \mathrm{E}+06$ & $4.89 \mathrm{E}-03$ & $4.82 \mathrm{E}-03$ \\
\hline
\end{tabular}


Table F-2. The Diffusion Resistance and Gas Diffusion Rates of Hydrogen and Methane for 149 Single-Shell Tanks. (6 sheets)

\begin{tabular}{|c|c|c|c|c|c|c|c|c|c|c|c|c|}
\hline Tank & $\begin{array}{c}\text { Concrete } \\
\text { Resist of } \mathrm{H}_{2} \\
(\mathrm{sec} / \mathrm{cm})\end{array}$ & $\begin{array}{c}\text { Asphatic } \\
\text { Resist of } \mathrm{H}_{2} \\
(\mathrm{sec} / \mathrm{cm})\end{array}$ & $\begin{array}{c}\text { Gunite Resist } \\
\text { of } \mathrm{H}_{2} \\
(\mathrm{sec} / \mathrm{cm})\end{array}$ & $\begin{array}{c}\text { Soil Resist of } \\
\mathrm{H}_{2}(\mathrm{sec} / \mathrm{cm})\end{array}$ & $\begin{array}{c}\text { Concrete } \\
\text { Resist of } \mathrm{CH}_{4} \\
(\mathrm{sec} / \mathrm{cm})\end{array}$ & $\begin{array}{c}\text { Asphatic } \\
\text { Resist of } \mathrm{CH}_{4} \\
(\mathrm{sec} / \mathrm{cm})\end{array}$ & $\begin{array}{c}\text { Gunite Resist } \\
\text { of } \mathrm{CH}_{4} \\
(\mathrm{sec} / \mathrm{cm})\end{array}$ & $\begin{array}{l}\text { Soil Resist of } \\
\mathrm{CH}_{4}(\mathrm{sec} / \mathrm{cm})\end{array}$ & $\begin{array}{c}\text { Total Resist of } \\
\mathrm{H}_{2}(\mathrm{sec} / \mathrm{cm})\end{array}$ & $\begin{array}{l}\text { Total Resist of } \\
\mathrm{CH}_{4}(\mathrm{sec} / \mathrm{cm})\end{array}$ & $\begin{array}{c}\mathrm{H}_{2} \text { Diff Rate } \\
\quad \mathrm{Ai} / \mathrm{Ri} \\
(\mathrm{cfm})\end{array}$ & $\begin{array}{c}\mathrm{CH}_{4} \text { Diff Rate } \\
\mathrm{Ai} / \mathrm{Ri} \\
(\mathrm{cfm})\end{array}$ \\
\hline BX-102 & $1.00 \mathrm{E}+04$ & $1.91 \mathrm{E}+06$ & $5.01 \mathrm{E}+02$ & $3.37 \mathrm{E}+03$ & $3.18 \mathrm{E}+04$ & $1.91 \mathrm{E}+06$ & $1.59 \mathrm{E}+03$ & $1.09 \mathrm{E}+04$ & $1.92 \mathrm{E}+06$ & $1.95 E+06$ & $4.89 \mathrm{E}-03$ & $4.82 \mathrm{E}-03$ \\
\hline BX-103 & $1.00 \mathrm{E}+04$ & $1.91 \mathrm{E}+06$ & $5.01 \mathrm{E}+02$ & $3.37 \mathrm{E}+03$ & $3.18 \mathrm{E}+04$ & $1.91 \mathrm{E}+06$ & $1.59 \mathrm{E}+03$ & $1.09 \mathrm{E}+04$ & $1.92 \mathrm{E}+06$ & $1.95 E+06$ & $4.89 \mathrm{E}-03$ & $4.82 \mathrm{E}-03$ \\
\hline$B X-104$ & $1.00 \mathrm{E}+04$ & $1.91 \mathrm{E}+06$ & $5.01 \mathrm{E}+02$ & $3.37 \mathrm{E}+03$ & $3.18 \mathrm{E}+04$ & $1.91 \mathrm{E}+06$ & $1.59 \mathrm{E}+03$ & $1.09 \mathrm{E}+04$ & $1.92 \mathrm{E}+06$ & $1.95 \mathrm{E}+06$ & $4.89 \mathrm{E}-03$ & $4.82 \mathrm{E}-03$ \\
\hline $\mathrm{BX}-105$ & $1.00 \mathrm{E}+04$ & $1.91 \mathrm{E}+06$ & $5.01 \mathrm{E}+02$ & $3.37 \mathrm{E}+03$ & $3.18 E+04$ & $1.91 \mathrm{E}+06$ & $1.59 \mathrm{E}+03$ & $1.09 \mathrm{E}+04$ & $1.92 \mathrm{E}+06$ & $1.95 \mathrm{E}+06$ & $4.89 \mathrm{E}-03$ & $4.82 \mathrm{E}-03$ \\
\hline BX-106 & $1.00 \mathrm{E}+04$ & $1.91 \mathrm{E}+06$ & $5.01 \mathrm{E}+02$ & $3.37 \mathrm{E}+03$ & $3.18 \mathrm{E}+04$ & $1.91 \mathrm{E}+06$ & $1.59 \mathrm{E}+03$ & $1.09 \mathrm{E}+04$ & $1.92 \mathrm{E}+06$ & $1.95 \mathrm{E}+06$ & $4.89 \mathrm{E}-03$ & $4.82 \mathrm{E}-03$ \\
\hline$B X-107$ & $1.00 \mathrm{E}+04$ & $1.91 \mathrm{E}+06$ & $5.01 \mathrm{E}+02$ & $3.37 \mathrm{E}+03$ & $3.18 \mathrm{E}+04$ & $1.91 \mathrm{E}+06$ & $1.59 \mathrm{E}+03$ & $1.09 \mathrm{E}+04$ & $1.92 \mathrm{~F}+06$ & $1.95 \mathrm{E}+06$ & 4.89E-03 & $4.82 \mathrm{E}-03$ \\
\hline BX-108 & $1.00 \mathrm{E}+04$ & $1.91 \mathrm{E}+06$ & $5.01 \mathrm{E}+02$ & $3.37 \mathrm{E}+03$ & $3.18 \mathrm{E}+04$ & $1.9 ! E+06$ & $1.59 \mathrm{E}+03$ & $1.09 \mathrm{E}+04$ & $1.92 \mathrm{E}+06$ & $1.95 \mathrm{E}+06$ & $4.89 \mathrm{E}-03$ & $4.82 \mathrm{E}-03$ \\
\hline BX-109 & $1.00 \mathrm{E}+04$ & $1.91 \mathrm{E}+06$ & $5.01 \mathrm{E}+02$ & $3.37 \mathrm{E}+03$ & $3.18 \mathrm{E}+04$ & $1.91 \mathrm{E}+06$ & $1.59 \mathrm{E}+03$ & $1.09 \mathrm{E}+04$ & $1.92 \mathrm{E}+06$ & $1.95 \mathrm{E}+06$ & $4.89 \mathrm{E}-03$ & $4.82 \mathrm{E}-03$ \\
\hline$B X-110$ & $1.00 \mathrm{E}+04$ & $1.91 \mathrm{E}+06$ & $5.01 \mathrm{E}+02$ & $3.37 \mathrm{E}+03$ & $3.18 \mathrm{E}+04$ & $1.91 \mathrm{E}+06$ & $1.59 \mathrm{E}+03$ & $1.09 \mathrm{E}+04$ & $1.92 \mathrm{E}+06$ & $1.95 E+06$ & $4.89 \mathrm{E}-03$ & $4.82 \mathrm{E}-03$ \\
\hline BX-111 & $1.00 \mathrm{E}+04$ & $1.91 \mathrm{E}+06$ & $5.01 \mathrm{E}+02$ & $3.37 \mathrm{E}+03$ & $3.18 \mathrm{E}+04$ & $1.91 \mathrm{E}+06$ & $1.59 \mathrm{E}+03$ & $1.09 \mathrm{E}+04$ & $1.92 \mathrm{E}+06$ & $1.95 \mathrm{E}+06$ & $4.89 \mathrm{E}-03$ & $4.82 \mathrm{E}-03$ \\
\hline$B X-112$ & $1.00 \mathrm{E}+04$ & $1.91 \mathrm{E}+06$ & $5.01 \mathrm{E}+02$ & $3.37 \mathrm{E}+03$ & $3.18 \mathrm{E}+04$ & $1.91 \mathrm{E}+06$ & $1.59 \mathrm{E}+03$ & $1.09 \mathrm{E}+04$ & $1.92 \mathrm{E}+06$ & $1.95 \mathrm{E}+06$ & $4.89 \mathrm{E}-03$ & $4.82 \mathrm{E}-03$ \\
\hline$B Y-101$ & $1.00 \mathrm{E}+04$ & $1.91 \mathrm{E}+06$ & $5.01 E+02$ & $3.56 \mathrm{E}+03$ & $3.18 \mathrm{E}+04$ & $1.91 \mathrm{E}+06$ & $1.59 \mathrm{E}+03$ & $1.15 \mathrm{E}+04$ & $1.92 \mathrm{E}+06$ & $1.95 \mathrm{E}+06$ & $4.84 \mathrm{E}-03$ & $4.76 \mathrm{E}-03$ \\
\hline BY-102 & $1.00 \mathrm{E}+04$ & $1.91 \mathrm{E}+06$ & $5.01 \mathrm{E}+02$ & $3.56 \mathrm{E}+03$ & $3.18 \mathrm{E}+04$ & $1.91 \mathrm{E}+06$ & $1.59 \mathrm{E}+03$ & $1.15 \mathrm{E}+04$ & $1.92 \mathrm{E}+06$ & $1.95 \mathrm{E}+06$ & $4.84 \mathrm{E}-03$ & $4.76 \mathrm{E}-03$ \\
\hline BY-103 & $1.00 \mathrm{E}+04$ & $1.91 \mathrm{E}+06$ & $5.01 \mathrm{E}+02$ & $3.56 \mathrm{E}+03$ & $3.18 \mathrm{E}+04$ & $1.91 \mathrm{E}+06$ & $1.59 \mathrm{E}+03$ & $1.15 \mathrm{E}+04$ & $1.92 \mathrm{E}+06$ & $1.95 \mathrm{E}+06$ & $4.84 \mathrm{E}-03$ & $4.76 \mathrm{E}-03$ \\
\hline BY-104 & $1.00 \mathrm{E}+04$ & $1.9 t E+06$ & $5.01 \mathrm{E}+02$ & $3.56 \mathrm{E}+03$ & $3.18 E+04$ & $1.91 \mathrm{E}+06$ & $1.59 \mathrm{E}+03$ & $1.15 \mathrm{E}+04$ & $1.92 \mathrm{E}+06$ & $1.95 \mathrm{E}+06$ & $4.84 \mathrm{E}-03$ & $4.76 \mathrm{E}-03$ \\
\hline BY-105 & $1.00 \mathrm{E}+04$ & $1.91 \mathrm{E}+06$ & $5.01 \mathrm{E}+02$ & $3.56 \mathrm{E}+03$ & $3.18 \mathrm{E}+04$ & $1.91 E+06$ & $1.59 \mathrm{E}+03$ & 1. $15 \mathrm{E}+04$ & $1.92 \mathrm{E}+06$ & $1.95 \mathrm{E}+06$ & $4.84 \mathrm{E}-03$ & $4.76 \mathrm{E}-03$ \\
\hline BY-106 & $1.00 \mathrm{E}+04$ & $1.91 E+06$ & $5.01 \mathrm{E}+02$ & $3.56 \mathrm{E}+03$ & $3.18 \mathrm{E}+04$ & $1.91 \mathrm{E}+06$ & $1.59 \mathrm{E}+03$ & $1.15 \mathrm{E}+04$ & $1.92 \mathrm{E}+06$ & $1.95 \mathrm{E}+06$ & 4.84E-03 & $4.76 \mathrm{E}-03$ \\
\hline$B Y-107$ & $1.00 E+04$ & $1.91 E+06$ & $5.01 E+02$ & $3.56 \mathrm{E}+03$ & $3.18 \mathrm{E}+04$ & $1.91 \mathrm{E}+06$ & $1.59 \mathrm{E}+03$ & $1.15 \mathrm{E}+04$ & $1.92 \mathrm{E}+06$ & $1.95 E+06$ & 4.84E-03 & $4.76 \mathrm{E}-03$ \\
\hline BY-108 & $1.00 \mathrm{E}+04$ & $1.91 E+06$ & $5.01 \mathrm{E}+02$ & $3.56 \mathrm{E}+03$ & $3.18 \mathrm{E}+04$ & $1.91 \mathrm{E}+06$ & $1.59 \mathrm{E}+03$ & $1.15 \mathrm{E}+04$ & $1.92 \mathrm{E}+00$ & $1.95 \mathrm{E}+06$ & $4.84 \mathrm{E}-03$ & $4.76 \mathrm{E}-03$ \\
\hline BY-109 & $1.00 \mathrm{E}+04$ & $1.91 \mathrm{E}+06$ & $5.01 \mathrm{E}+02$ & $3.56 \mathrm{E}+03$ & $3.18 \mathrm{E}+04$ & $1.91 \mathrm{E}+06$ & $1.59 \mathrm{E}+03$ & $1.15 E+04$ & $1.92 \mathrm{E}+06$ & $1.95 \mathrm{E}+06$ & $4.84 \mathrm{E}-03$ & $4.76 \mathrm{E}-03$ \\
\hline$B Y+110$ & $1.00 \mathrm{E}+04$ & $1.91 \mathrm{E}+06$ & $5.01 \mathrm{E}+02$ & $3.56 \mathrm{E}+03$ & $3.18 \mathrm{E}+04$ & $1.91 \mathrm{E}+06$ & $1.59 \mathrm{E}+03$ & $1.15 \mathrm{E}+04$ & $1.92 \mathrm{E}+06$ & $1.95 \mathrm{E}+06$ & 4.84E- 03 & $4.76 \mathrm{E}-03$ \\
\hline BY-111] & $1.00 \mathrm{E}+04$ & $1.91 \mathrm{E}+06$ & $5.01 \mathrm{E}+02$ & $3.56 \mathrm{E}+03$ & $3.18 \mathrm{E}+04$ & $1.91 \mathrm{E}+06$ & {$[.59 \mathrm{E}+03$} & $1.15 \mathrm{E}+04$ & $1.92 \mathrm{E}+06$ & $1.95 \mathrm{E}+06$ & 4.84E-03 & $4.76 \mathrm{E}-03$ \\
\hline BY-112 & $1.00 \mathrm{E}+04$ & $1.91 \mathrm{E}+06$ & $5.01 \mathrm{E}+02$ & $3.56 \mathrm{E}+03$ & $3.18 \mathrm{E}+04$ & $1.91 \mathrm{E}+06$ & $1.59 \mathrm{E}+03$ & $1.15 \mathrm{E}+04$ & $1.92 \mathrm{E}+06$ & $1.95 \mathrm{E}+06$ & 4.84E- 03 & $4.76 \mathrm{E}-03$ \\
\hline C-101 & $1.00 \mathrm{E}+04$ & $1.91 \mathrm{E}+06$ & $5.01 \mathrm{E}+02$ & $3.37 \mathrm{E}+03$ & $3.18 \mathrm{E}+04$ & $1.91 \mathrm{E}+06$ & $1.59 \mathrm{E}+03$ & $1.09 \mathrm{E}+04$ & $1.92 \mathrm{E}+06$ & $1.95 \mathrm{E}+06$ & $4.89 \mathrm{E}-03$ & $4.82 \mathrm{E}-03$ \\
\hline C. 102 & $1.00 \mathrm{E}+04$ & $1.91 \mathrm{E}+06$ & $5.01 \mathrm{E}+02$ & $3.37 \mathrm{E}+03$ & $3.18 \mathrm{E}+04$ & $1.91 \mathrm{E}+06$ & $1.59 \mathrm{E}+03$ & $1.09 \mathrm{E}+04$ & $1.92 \mathrm{E}+06$ & $1.95 \mathrm{E}+06$ & $4.89 \mathrm{E}-03$ & $4.82 \mathrm{E}-03$ \\
\hline C- -103 & $1.00 \mathrm{E}+04$ & $1.91 \mathrm{E}+06$ & $5.01 \mathrm{E}+02$ & $3.37 \mathrm{E}+03$ & $3.18 \mathrm{E}+04$ & $1.91 \mathrm{E}+06$ & $1.59 \mathrm{E}+03$ & $1.09 \mathrm{E}+04$ & $1.92 \mathrm{E}+06$ & $1.95 \mathrm{E}+06$ & $4.89 \mathrm{E}-03$ & $4.82 \mathrm{E}-03$ \\
\hline $\mathrm{C}-104$ & $1.00 \mathrm{E}+04$ & $1.91 \mathrm{E}+06$ & $5.01 \mathrm{E}+02$ & $3.37 \mathrm{E}+03$ & $3.18 \mathrm{E}+04$ & $1.91 \mathrm{E}+06$ & $1.59 \mathrm{E}+03$ & $1.09 \mathrm{E}+04$ & $1.92 \mathrm{E}+06$ & $1.95 \mathrm{E}+06$ & $4.89 \mathrm{E}-03$ & $4.82 \mathrm{E}-03$ \\
\hline
\end{tabular}


Table F-2. The Diffusion Resistance and Gas Diffusion Rates of Hydrogen and Methane for 149 Single-Shell Tanks. (6 sheets)

\begin{tabular}{|c|c|c|c|c|c|c|c|c|c|c|c|c|}
\hline Tank & $\begin{array}{c}\text { Concrete } \\
\text { Resist of } \mathrm{H}_{2} \\
(\mathrm{sec} / \mathrm{cm} \text { ) }\end{array}$ & $\begin{array}{c}\text { Asphatic } \\
\text { Resist of } \mathrm{H}_{2} \\
(\mathrm{sec} / \mathrm{cm})\end{array}$ & $\begin{array}{c}\text { Gunite Resist } \\
\text { of } \mathrm{H}_{2} \\
\text { (sec/cm) }\end{array}$ & $\begin{array}{c}\text { Soil Resist of } \\
\mathrm{H}_{2}(\mathrm{sec} / \mathrm{cm})\end{array}$ & $\begin{array}{c}\text { Concrete } \\
\text { Resist of } \mathrm{CH}_{4} \\
(\mathrm{sec} / \mathrm{cm})\end{array}$ & $\begin{array}{c}\text { Asphatic } \\
\text { Resist of } \mathrm{CH}_{4} \\
(\mathrm{sec} / \mathrm{cm})\end{array}$ & $\begin{array}{c}\text { Gunite Resist } \\
\text { of } \mathrm{CH}_{4} \\
(\mathrm{sec} / \mathrm{cm})\end{array}$ & $\begin{array}{l}\text { Soil Resist of } \\
\mathrm{CH}_{4}(\mathrm{sec} / \mathrm{cm})\end{array}$ & $\begin{array}{c}\text { Total Resist of } \\
\mathrm{H}_{2}(\mathrm{sec} / \mathrm{cm})\end{array}$ & $\begin{array}{l}\text { Total Resist of } \\
\mathrm{CH}_{4}(\mathrm{sec} / \mathrm{cm})\end{array}$ & $\begin{array}{c}\mathrm{H}_{2} \text { Diff Rale } \\
\mathrm{Ai} / \mathrm{Ri} \\
\text { (cfm) }\end{array}$ & $\begin{array}{c}\mathrm{CH}_{4} \text { Diff Rate } \\
\mathrm{Ai} / \mathrm{Ri} \\
(\mathrm{cfm})\end{array}$ \\
\hline C- -105 & $1.00 \mathrm{E}+04$ & $1.91 E+06$ & $5.01 \mathrm{E}+02$ & $3.37 \mathrm{E}+03$ & $3.18 \mathrm{E}+04$ & $1.91 \mathrm{E}+06$ & $1.59 \mathrm{E}+03$ & $1.09 \mathrm{E}+04$ & $1.92 \mathrm{E}+06$ & $1.95 \mathrm{E}+06$ & $4.89 \mathrm{E}-03$ & $4.82 \mathrm{E}-03$ \\
\hline C-106 & $1.00 \mathrm{E}+04$ & $1.91 \mathrm{E}+06$ & $5.01 \mathrm{E}+02$ & $3.37 E+03$ & $3.18 E+04$ & $1.91 \mathrm{E}+06$ & $1.59 \mathrm{E}+03$ & $1.09 E+04$ & $1.92 \mathrm{E}+06$ & $1.95 E+06$ & $4.89 \mathrm{E}-03$ & $4.82 \mathrm{E}-03$ \\
\hline$C-107$ & $1.00 \mathrm{E}+04$ & $1.91 \mathrm{E}+06$ & $5.01 \mathrm{E}+02$ & $3.37 \mathrm{E}+03$ & 3.18E+04 & $1.91 \mathrm{E}+06$ & $1.59 \mathrm{E}+03$ & $1.09 \mathrm{E}+04$ & $1.92 \mathrm{E}+06$ & $1.95 \mathrm{E}+06$ & $4.89 \mathrm{E}-03$ & $4.82 \mathrm{E}-03$ \\
\hline$C-108$ & $1.00 \mathrm{E}+04$ & $1.91 E+06$ & $5.01 \mathrm{E}+02$ & $3.37 \mathrm{E}+03$ & $3.18 \mathrm{E}+04$ & $1.91 \mathrm{E}+06$ & $1.59 \mathrm{E}+03$ & $1.09 \mathrm{E}+04$ & $1.92 \mathrm{E}+06$ & $1.95 \mathrm{E}+06$ & $4.89 \mathrm{E}-03$ & $4.82 \mathrm{E}-03$ \\
\hline$C-109$ & $1.00 E+04$ & $1.91 E+06$ & $5.01 \mathrm{E}+02$ & $3.37 \mathrm{E}+03$ & $3.18 \mathrm{E}+04$ & $1.91 \mathrm{E}+06$ & $1.59 \mathrm{E}+03$ & $1.09 \mathrm{E}+04$ & $1.92 \mathrm{E}+06$ & $1.95 E+06$ & $4.89 \mathrm{E}-03$ & $4.82 \mathrm{E}-03$ \\
\hline $\mathrm{C}-110$ & $1.00 \mathrm{E}+04$ & $1.91 E+06$ & $5.01 \mathrm{E}+02$ & $3.37 \mathrm{E}+03$ & $3.18 \mathrm{E}+04$ & $1.91 \mathrm{E}+06$ & $1.59 \mathrm{E}+03$ & $1.09 \mathrm{E}+04$ & $1.92 \mathrm{E}+06$ & $1.95 \mathrm{E}+06$ & $4.89 \mathrm{E}-03$ & $4.82 \mathrm{E}-03$ \\
\hline $\mathrm{C}-111$ & $1.00 \mathrm{E}+04$ & $1.91 E+06$ & $5.01 \mathrm{E}+02$ & $3.37 \mathrm{E}+03$ & $3.18 \mathrm{E}+04$ & $1.91 \mathrm{E}+06$ & $1.59 \mathrm{E}+03$ & $1.09 \mathrm{E}+04$ & $1.92 \mathrm{E}+06$ & $1.95 \mathrm{E}+06$ & $4.89 \mathrm{E}-03$ & $4.82 \mathrm{E}-03$ \\
\hline $\mathrm{C}-112$ & $1.00 \mathrm{E}+04$ & $1.91 \mathrm{E}+06$ & $5.01 \mathrm{E}+02$ & $3.37 \mathrm{E}+03$ & $3.18 \mathrm{E}+04$ & $1.91 \mathrm{E}+06$ & $1.59 \mathrm{E}+03$ & $1.09 \mathrm{E}+04$ & $1.92 \mathrm{E}+06$ & $1.95 \mathrm{E}+06$ & $4.89 \mathrm{E}-03$ & $4.82 \mathrm{E}-03$ \\
\hline $\mathrm{C}-201$ & $8.02 \mathrm{E}+03$ & $1.91 \mathrm{E}+06$ & $0.00 \mathrm{E}+00$ & $2.79 \mathrm{E}+03$ & $2.54 \mathrm{E}+04$ & $1.91 \mathrm{E}+06$ & $0.00 \mathrm{E}+00$ & $9.05 \mathrm{E}+03$ & $1.92 \mathrm{E}+06$ & $1.94 \mathrm{E}+06$ & $1.61 \mathrm{E}-04$ & $1.60 \mathrm{E}-04$ \\
\hline C-202 & $8.02 \mathrm{E}+03$ & $1.91 \mathrm{E}+06$ & $0.00 \mathrm{E}+00$ & $2.79 \mathrm{E}+03$ & $2.54 \mathrm{E}+04$ & $1.91 \mathrm{E}+06$ & $0.00 \mathrm{E}+00$ & $9.05 \mathrm{E}+03$ & $1.92 \mathrm{E}+06$ & $1.94 \mathrm{E}+06$ & $1.61 \mathrm{E}-04$ & $1.60 \mathrm{E}-04$ \\
\hline C-203 & $8.02 \mathrm{E}+03$ & $1.91 E+06$ & $0.00 \mathrm{E}+00$ & $2.79 \mathrm{E}+03$ & $2.54 \mathrm{E}+04$ & $1.91 \mathrm{E}+06$ & $0.00 \mathrm{E}+00$ & $9.05 E+03$ & $1.92 \mathrm{E}+06$ & $1.94 \mathrm{E}+06$ & $1.61 \mathrm{E}-04$ & $1.60 \mathrm{E}-04$ \\
\hline C-204 & $8.02 \mathrm{E}+03$ & $1.91 E+06$ & $0.00 \mathrm{E}+00$ & $2.79 \mathrm{E}+03$ & $2.54 \mathrm{E}+04$ & $1.91 \mathrm{E}+06$ & $0.00 \mathrm{E}+00$ & $9.05 \mathrm{E}+03$ & $1.92 \mathrm{E}+06$ & $1.94 \mathrm{E}+06$ & $1.61 \mathrm{E}-04$ & $1.60 \mathrm{E}-04$ \\
\hline S-101 & $1.00 \mathrm{E}+04$ & $1.91 E+06$ & $5.01 E+02$ & $3.56 \mathrm{E}+03$ & $3.18 \mathrm{E}+04$ & $1.91 \mathrm{E}+06$ & $1.59 \mathrm{E}+03$ & $1.15 E+04$ & $1.92 \mathrm{E}+06$ & $1.95 \mathrm{E}+06$ & $4.84 \mathrm{E}-03$ & $4.76 \mathrm{E}-03$ \\
\hline$S-102$ & $1.00 \mathrm{E}+04$ & $1.91 \mathrm{E}+06$ & $5.01 \mathrm{E}+02$ & $3.56 \mathrm{E}+03$ & $3.18 \mathrm{E}+04$ & $1.91 \mathrm{E}+06$ & $1.59 \mathrm{E}+03$ & $1.15 \mathrm{E}+04$ & $1.92 \mathrm{E}+06$ & $1.95 \mathrm{E}+06$ & $4.84 \mathrm{E}-03$ & $4.76 \mathrm{E}-03$ \\
\hline S-103 & $1.00 \mathrm{E}+04$ & $1.91 E+06$ & $5.01 \mathrm{E}+02$ & $3.56 \mathrm{E}+03$ & $3.18 \mathrm{~F}+04$ & $1.91 \mathrm{E}+06$ & $1.59 \mathrm{~F}+03$ & $1.15 \mathrm{E}+04$ & $1.92 \mathrm{E}+06$ & $1.95 \mathrm{E}+06$ & $4.84 \mathrm{E}-03$ & $4.76 \mathrm{E}-03$ \\
\hline S-104 & $1.00 \mathrm{E}+04$ & $1.91 E+06$ & $5.01 \mathrm{E}+02$ & $3.56 \mathrm{E}+03$ & $3.18 \mathrm{E}+04$ & $1.91 \mathrm{E}+06$ & $1.59 \mathrm{E}+03$ & $1.15 E+04$ & $1.92 E+06$ & $1.95 \mathrm{E}+06$ & $4.84 \mathrm{E}-03$ & $4.76 \mathrm{E}-03$ \\
\hline S-105 & $1.00 \mathrm{E}+04$ & $1.91 \mathrm{E}+06$ & $5.01 \mathrm{E}+02$ & $3.56 \mathrm{E}+03$ & $3.18 \mathrm{E}+04$ & $1.91 \mathrm{E}+06$ & $1.59 \mathrm{E}+03$ & 1.15E+04 & $1.92 \mathrm{E}+06$ & $1.95 \mathrm{E}+06$ & 4.84E-03 & $4.76 \mathrm{E}-03$ \\
\hline S-106 & $1.00 \mathrm{E}+04$ & $1.91 \mathrm{E}+06$ & $5.01 \mathrm{E}+02$ & $3.56 \mathrm{E}+03$ & $3.18 \mathrm{E}+04$ & $1.91 \mathrm{E}+06$ & $1.59 \mathrm{E}+03$ & $1.15 \mathrm{E}+04$ & $1.92 \mathrm{E}+06$ & $1.95 \mathrm{E}+06$ & $4.84 \mathrm{E}-03$ & $4.76 \mathrm{E}-03$ \\
\hline S-107 & $1.00 \mathrm{E}+04$ & $1.91 \mathrm{E}+06$ & $5.01 \mathrm{E}+02$ & $3.56 \mathrm{E}+03$ & $3.18 \mathrm{E}+04$ & $1.91 \mathrm{E}+06$ & $1.59 \mathrm{E}+03$ & $1.15 E+04$ & $1.92 \mathrm{E}+06$ & $1.95 \mathrm{E}+06$ & $4.84 \mathrm{E}-03$ & $4.76 \mathrm{E}-03$ \\
\hline S-108 & $1.00 \mathrm{E}+04$ & $1.91 \mathrm{E}+06$ & $5.01 \mathrm{E}+02$ & $3.56 \mathrm{E}+03$ & $3.18 \mathrm{E}+04$ & $1.91 \mathrm{E}+06$ & $1.59 \mathrm{E}+03$ & $1.15 E+04$ & $1.92 \mathrm{E}+06$ & $1.95 \mathrm{E}+06$ & 4.84E- 03 & $4.76 \mathrm{E}-03$ \\
\hline $\mathrm{S}-109$ & $1.00 \mathrm{E}+04$ & $1.91 \mathrm{E}+06$ & $5.01 \mathrm{E}+02$ & $3.56 \mathrm{E}+03$ & $3.18 \mathrm{E}+04$ & $1.91 \mathrm{E}+06$ & $1.59 \mathrm{E}+03$ & $1.15 E+04$ & $1.92 \mathrm{E}+06$ & $1.95 \mathrm{E}+06$ & $4.84 \mathrm{E}-03$ & $4.76 \mathrm{E}-03$ \\
\hline $\mathrm{S}-110$ & $1.00 \mathrm{E}+04$ & $1.91 E+06$ & $5.01 \mathrm{E}+02$ & $3.56 \mathrm{E}+03$ & $3.18 \mathrm{E}+04$ & $1.91 \mathrm{E}+06$ & $1.59 \mathrm{E}+03$ & $1.15 \mathrm{E}+04$ & $1.92 \mathrm{E}+06$ & $1.95 \mathrm{E}+06$ & 4.84E-03 & $4.76 \mathrm{E}-03$ \\
\hline S-111 & $1.00 \mathrm{E}+04$ & $1.91 \mathrm{E}+06$ & $5.01 \mathrm{E}+02$ & $3.56 \mathrm{E}+03$ & $3.18 \mathrm{E}+04$ & $1.9] \mathrm{E}+06$ & $1.59 \mathrm{E}+03$ & $1.15 E+04$ & $1.92 \mathrm{E}+06$ & $1.95 \mathrm{E}+06$ & $4.84 \mathrm{E}-03$ & $4.76 \mathrm{E}-03$ \\
\hline$S-112$ & $1.00 \mathrm{E}+04$ & $1.91 \mathrm{E}+06$ & $5.01 \mathrm{E}+02$ & $3.56 \mathrm{E}+03$ & $3.18 \mathrm{E}+04$ & $1.91 \mathrm{E}+06$ & $1.59 \mathrm{E}+03$ & $1.15 \mathrm{E}+04$ & $1.92 \mathrm{E}+06$ & $1.95 \mathrm{E}+06$ & $4.84 \mathrm{E}-03$ & $4.76 \mathrm{E}-03$ \\
\hline$S X-101$ & $1.00 \mathrm{E}+04$ & $0.00 \mathrm{E}+00$ & $0.00 \mathrm{E}+00$ & $3.05 E+03$ & $3.18 \mathrm{E}+04$ & $0.00 \mathrm{E}+00$ & $0.00 \mathrm{E}+00$ & $9.89 \mathrm{E}+03$ & $1.31 \mathrm{E}+04$ & 4.16E+04 & 7.11E-01 & $2.23 \mathrm{E}-01$ \\
\hline SX-102 & $1.00 \mathrm{E}+04$ & $0.00 \mathrm{E}+00$ & $0.00 \mathrm{E}+00$ & $3.05 \mathrm{E}+03$ & $3.18 \mathrm{E}+04$ & $0.00 \mathrm{E}+00$ & $0.00 \mathrm{E}+00$ & $9.89 \mathrm{E}+03$ & $1.31 \mathrm{E}+04$ & 4.16E+04 & $7.11 \mathrm{E}-01$ & $2.23 \mathrm{E}-01$ \\
\hline SX-103 & $1.00 \mathrm{E}+04$ & $0.00 E+00$ & $0.00 \mathrm{E}+00$ & $3.05 \mathrm{E}+03$ & $3.18 \mathrm{E}+04$ & $0.00 \mathrm{E}+00$ & $0.00 \mathrm{E}+00$ & $9.89 E+03$ & $1.31 \mathrm{E}+04$ & 4.16E+04 & $7.11 \mathrm{E}-01$ & $2.23 \mathrm{E}-01$ \\
\hline
\end{tabular}


RPP-5926 REV 6

Table F-2. The Diffusion Resistance and Gas Diffusion Rates of Hydrogen and Methane for 149 Single-Shell Tanks. (6 sheets)

\begin{tabular}{|c|c|c|c|c|c|c|c|c|c|c|c|c|}
\hline Tank & $\begin{array}{c}\text { Concrete } \\
\text { Resist of } \mathrm{H}_{2} \\
(\mathrm{sec} / \mathrm{cm})\end{array}$ & $\begin{array}{c}\text { Asphatic } \\
\text { Resist of } \mathrm{H}_{2} \\
(\mathrm{sec} / \mathrm{cm})\end{array}$ & $\begin{array}{c}\text { Gunite Resist } \\
\text { of } \mathrm{H}_{2} \\
(\mathrm{sec} / \mathrm{cm})\end{array}$ & $\begin{array}{c}\text { Soil Resist of } \\
\mathrm{H}_{2}(\mathrm{sec} / \mathrm{cm})\end{array}$ & $\begin{array}{c}\text { Concrete } \\
\text { Resist of } \mathrm{CH}_{4} \\
(\mathrm{sec} / \mathrm{cm})\end{array}$ & $\begin{array}{c}\text { Asphatic } \\
\text { Resist of } \mathrm{CH}_{4} \\
(\mathrm{sec} / \mathrm{cm})\end{array}$ & $\begin{array}{c}\text { Gunite Resist } \\
\text { of } \mathrm{CH}_{4} \\
(\mathrm{sec} / \mathrm{cm})\end{array}$ & $\left|\begin{array}{l}\text { Soil Resist of } \\
\mathrm{CH}_{4}(\mathrm{sec} / \mathrm{cm})\end{array}\right|$ & $\begin{array}{c}\text { Total Resist of } \\
H_{2}(\mathrm{sec} / \mathrm{cm})\end{array}$ & $\begin{array}{l}\text { Total Resist of } \\
\mathrm{CH}_{4}(\mathrm{sec} / \mathrm{cm})\end{array}$ & $\begin{array}{c}\mathrm{H}_{2} \text { Diff Ratc } \\
\mathrm{Ai} / \mathrm{Ri} \\
(\mathrm{cfm})\end{array}$ & $\begin{array}{c}\mathrm{CH}_{4} \text { Diff Rate } \\
\mathrm{Ai} / \mathrm{Ri} \\
(\mathrm{cfm})\end{array}$ \\
\hline SX-104 & $1.00 E+04$ & $0.00 \mathrm{E}+00$ & $0.00 \mathrm{E}+00$ & $3.05 E+03$ & $3.18 \mathrm{E}+04$ & $0.00 \mathrm{E}+00$ & $0.00 \mathrm{E}+00$ & $9.89 \mathrm{E}+03$ & $1.31 \mathrm{E}+04$ & $4.16 \mathrm{E}+04$ & $7.11 \mathrm{E}-01$ & $2.23 \mathrm{E}-01$ \\
\hline SX-105 & $1.00 \mathrm{E}+04$ & $0.00 \mathrm{E}+00$ & $0.00 \mathrm{E}+00$ & $3.05 E+03$ & $3.18 \mathrm{E}+04$ & $0.00 \mathrm{E}+00$ & $0.00 \mathrm{E}+00$ & $9.89 \mathrm{E}+03$ & $1.31 \mathrm{E}+04$ & 4. $16 \mathrm{E}+04$ & $7.11 \mathrm{E}-01$ & $2.23 \mathrm{E}-01$ \\
\hline SX-106 & $1.00 \mathrm{E}+04$ & $0.00 \mathrm{E}+00$ & $0.00 \mathrm{E}+00$ & $3.05 \mathrm{E}+03$ & $3.18 \mathrm{E}+04$ & $0.00 \mathrm{E}+00$ & $0.00 \mathrm{E}+00$ & $9.89 \mathrm{E}+03$ & $1.31 \mathrm{E}+04$ & $4.16 \mathrm{E}+04$ & $7.11 \mathrm{E}-01$ & $2.23 \mathrm{E}-01$ \\
\hline SX-107 & $1.00 \mathrm{E}+04$ & $0.00 \mathrm{E}+00$ & $0.00 \mathrm{E}+00$ & $3.05 \mathrm{E}+03$ & $3.18 \mathrm{E}+04$ & $0.00 \mathrm{E}+00$ & $0.00 \mathrm{E}+00$ & $9.89 \mathrm{E}+03$ & $1.31 \mathrm{E}+04$ & 4.16E +04 & $7.11 \mathrm{E}-01$ & $2.23 \mathrm{E}-01$ \\
\hline SX-108 & $1.00 \mathrm{E}+04$ & $0.00 \mathrm{E}+00$ & $0.00 \mathrm{E}+00$ & $3.05 E+03$ & $3.18 \mathrm{E}+04$ & $0.00 \mathrm{E}+00$ & $0.00 \mathrm{E}+00$ & $9.89 \mathrm{E}+03$ & $1.31 \mathrm{E}+04$ & $4.16 \mathrm{E}+04$ & $7.11 \mathrm{E}-01$ & $2.23 \mathrm{E}-01$ \\
\hline$S X \cdot 109$ & $1.00 \mathrm{E}+04$ & $0.00 \mathrm{E}+00$ & $0.00 \mathrm{E}+00$ & $3.05 \mathrm{E}+03$ & $3.18 \mathrm{E}+04$ & $0.00 \mathrm{E}+00$ & $0.00 \mathrm{E}+00$ & $9.89 \mathrm{E}+03$ & $1.31 \mathrm{E}+04$ & $4.16 \mathrm{E}+04$ & $7.11 \mathrm{E}-01$ & $2.23 \mathrm{E}-01$ \\
\hline SX-110 & $1.00 E+04$ & $0.00 \mathrm{E}+00$ & $0.00 \mathrm{E}+00$ & $3.05 E+03$ & $3.18 \mathrm{E}+04$ & $0.00 \mathrm{E}+00$ & $0.00 \mathrm{E}+00$ & $9.89 \mathrm{E}+03$ & $1.31 \mathrm{~F}+04$ & $4.16 \mathrm{E}+04$ & $7.11 \mathrm{E}-01$ & $2.23 \mathrm{E}-01$ \\
\hline$S x-111$ & $1.00 \mathrm{E}+04$ & $0.00 \mathrm{E}+00$ & $0.00 \mathrm{E}+00$ & $3.05 \mathrm{E}+03$ & $3.18 \mathrm{E}+04$ & $0.00 \mathrm{E}+00$ & $0.00 \mathrm{E}+00$ & $9.89 \mathrm{E}+03$ & $1.31 \mathrm{E}+04$ & 4.16E+04 & $7.11 \mathrm{E}-01$ & $2.23 \mathrm{E}-01$ \\
\hline SX-112 & $1.00 \mathrm{E}+04$ & $0.00 \mathrm{E}+00$ & $0.00 \mathrm{E}+00$ & $3.05 \mathrm{E}+03$ & $3.18 \mathrm{E}+04$ & $0.00 \mathrm{E}+00$ & $0.00 \mathrm{E}+00$ & $9.89 \mathrm{E}+03$ & $1.31 \mathrm{E}+04$ & $4.16 \mathrm{E}+04$ & $7.11 \mathrm{E}-01$ & $2.23 \mathrm{E}-01$ \\
\hline $5 X-113$ & $1.00 \mathrm{E}+04$ & $0.00 \mathrm{E}+00$ & $0.00 \mathrm{E}+00$ & $3.05 \mathrm{E}+03$ & $3.18 \mathrm{E}+04$ & $0.00 \mathrm{E}+00$ & $0.00 \mathrm{E}+00$ & $9.89 \mathrm{E}+03$ & $1.31 \mathrm{E}+04$ & $4.16 \mathrm{E}+04$ & $7.11 \mathrm{E}-0 \mathrm{I}$ & $2.23 \mathrm{E}-0 \mathrm{l}$ \\
\hline$S X-114$ & $1.00 \mathrm{E}+04$ & $0.00 \mathrm{E}+00$ & $0.00 \mathrm{E}+00$ & $3.05 \mathrm{E}+03$ & $3.18 \mathrm{E}+04$ & $0.00 \mathrm{E}+00$ & $0.00 \mathrm{E}+00$ & $9.89 \mathrm{E}+03$ & $1.31 \mathrm{E}+04$ & 4. $16 \mathrm{E}+04$ & $7.11 \mathrm{E}-01$ & $2.23 \mathrm{E}-01$ \\
\hline SX-115 & 1.00E+04 & $0.00 \mathrm{E}+00$ & $0.00 \mathrm{E}+00$ & $3.05 \mathrm{E}+03$ & $3.18 \mathrm{E}+04$ & $0.00 \mathrm{E}+00$ & $0.00 \mathrm{E}+00$ & $9.89 E+03$ & $1.31 \mathrm{E}+04$ & $4.16 \mathrm{E}+04$ & $7.11 \mathrm{E}-0 \mathrm{l}$ & $2.23 \mathrm{E}-0 \mathrm{I}$ \\
\hline T-101 & $1.00 \mathrm{E}+04$ & $1.91 \mathrm{E}+06$ & $5.01 \mathrm{E}+02$ & $3.37 \mathrm{E}+03$ & $3.18 \mathrm{E}+04$ & $1.91 E+06$ & $1.59 \mathrm{E}+03$ & $1.09 \mathrm{E}+04$ & $1.92 \mathrm{E}+06$ & $1.95 \mathrm{E}+06$ & $4.89 \mathrm{E}-03$ & $4.82 \mathrm{E}-03$ \\
\hline $\mathrm{T}-102$ & $1.00 \mathrm{E}+04$ & $1.91 \mathrm{E}+06$ & $5.01 \mathrm{E}+02$ & $3.37 E+03$ & $3.18 \mathrm{E}+04$ & $1.91 \mathrm{E}+06$ & $1.59 \mathrm{E}+03$ & $1.09 \mathrm{E}+04$ & $1.92 \mathrm{E}+06$ & $1.95 \mathrm{E}+06$ & $4.89 \mathrm{E}-03$ & $4.82 \mathrm{E}-03$ \\
\hline$T-103$ & $1.00 \mathrm{H}+04$ & $1.91 \mathrm{~F}+06$ & $5.01 \mathrm{E}+02$ & $3.37 \mathrm{E}+03$ & $3.18 \mathrm{E}+04$ & $1.91 \mathrm{E}+06$ & $1.59 \mathrm{E}+03$ & $1.09 \mathrm{E}+04$ & $1.92 \mathrm{E}+06$ & $1.95 \mathrm{E}+06$ & $4.89 \mathrm{~F},-03$ & $4.82 \mathrm{E}-03$ \\
\hline T-104 & $1.00 E+04$ & $1.91 \mathrm{E}+06$ & $5.01 \mathrm{E}+02$ & $3.37 \mathrm{E}+03$ & $3.18 \mathrm{E}+04$ & $1.91 \mathrm{E}+06$ & $1.59 \mathrm{E}+03$ & $1.09 E+04$ & $1.92 \mathrm{E}+06$ & $1.95 \mathrm{E}+06$ & $4.89 \mathrm{E}-03$ & $4.82 \mathrm{E}-03$ \\
\hline T-105 & $1.00 E+04$ & $1.91 \mathrm{E}+06$ & $5.01 \mathrm{E}+02$ & $3.37 \mathrm{E}+03$ & $3.18 \mathrm{E}+04$ & $1.91 \mathrm{E}+06$ & $1.59 \mathrm{E}+03$ & $1.09 \mathrm{E}+04$ & $1.92 \mathrm{E}+06$ & $1.95 \mathrm{E}+06$ & 4. $89 \mathrm{E}-03$ & $4.82 \mathrm{E}-03$ \\
\hline$T-106$ & $1.00 \mathrm{E}+04$ & $1.91 \mathrm{E}+06$ & $5.01 \mathrm{E}+02$ & 3.37E+03 & $3.18 \mathrm{E}+04$ & $1.91 \mathrm{E}+06$ & $1.59 \mathrm{E}+03$ & $1.09 \mathrm{E}+04$ & $1.92 \mathrm{E}+06$ & $1.95 \mathrm{E}+06$ & $4.89 \mathrm{E}-03$ & $4.82 \mathrm{E}-03$ \\
\hline $\mathrm{T}-107$ & $1.00 E+04$ & $1.91 \mathrm{E}+06$ & $5.01 \mathrm{E}+02$ & $3.37 \mathrm{E}+03$ & $3.18 \mathrm{E}+04$ & $1.91 \mathrm{E}+06$ & $1.59 \mathrm{E}+03$ & $1.09 \mathrm{E}+04$ & $1.92 \mathrm{E}+06$ & $1.95 \mathrm{E}+06$ & 4.89E-03 & $4.82 \mathrm{E}-03$ \\
\hline T-108 & $1.00 \mathrm{E}+04$ & $1.91 \mathrm{E}+06$ & $5.01 \mathrm{E}+02$ & $3.37 \mathrm{E}+03$ & $3.18 \mathrm{E}+04$ & $1.91 \mathrm{E}+06$ & $1.59 \mathrm{E}+03$ & $1.09 \mathrm{E}+04$ & $1.92 \mathrm{E}+06$ & $1.95 \mathrm{E}+06$ & $4.89 \mathrm{E}-03$ & $4.82 \mathrm{E}-03$ \\
\hline T-109 & $1.00 \mathrm{E}+04$ & $1.91 \mathrm{E}+06$ & $5.01 \mathrm{E}+02$ & $3.37 \mathrm{E}+03$ & $3.18 \mathrm{E}+04$ & $1.91 \mathrm{E}+06$ & $1.59 \mathrm{E}+03$ & $1.09 \mathrm{E}+04$ & $1.92 \mathrm{E}+06$ & $1.95 \mathrm{E}+06$ & 4.89E-03 & $4.82 \mathrm{E}-03$ \\
\hline $\mathrm{T}-110$ & $1.00 \mathrm{E}+04$ & $1.91 \mathrm{E}+06$ & $5.0 ! \mathrm{E}+02$ & $3.37 \mathrm{E}+03$ & $3.18 \mathrm{E}+04$ & $1.91 \mathrm{E}+06$ & $1.59 \mathrm{E}+03$ & $1.09 \mathrm{E}+04$ & $1.92 \mathrm{E}+06$ & $1.95 \mathrm{E}+06$ & $4.89 \mathrm{E}-03$ & 4.82E-03 \\
\hline T-111 & $1.00 \mathrm{E}+04$ & $1.91 \mathrm{E}+06$ & $5.01 \mathrm{E}+02$ & $3.37 \mathrm{E}+03$ & $3.18 \mathrm{E}+04$ & $1.91 \mathrm{E}+06$ & $1.59 \mathrm{E}+03$ & $1.09 \mathrm{E}+04$ & $1.92 \mathrm{E}+06$ & $1.95 \mathrm{E}+06$ & $4.89 \mathrm{E}-03$ & $4.82 \mathrm{E}-03$ \\
\hline $\mathrm{T}-112$ & $1.00 \mathrm{E}+04$ & $1.91 \mathrm{E}+06$ & $5.01 \mathrm{E}+02$ & $3.37 \mathrm{E}+03$ & $3.18 \mathrm{E}+04$ & $1.91 \mathrm{E}+06$ & $1.59 \mathrm{E}+03$ & $1.09 \mathrm{E}+04$ & $1.92 \mathrm{E}+06$ & $1.95 \mathrm{E}+06$ & $4.89 \mathrm{E}-03$ & $4.82 \mathrm{E}-03$ \\
\hline $\mathrm{T}-201$ & $8.02 \mathrm{E}+03$ & $1.91 \mathrm{E}+06$ & $0.00 \mathrm{E}+00$ & $2.79 \mathrm{E}+03$ & $2.54 \mathrm{E}+04$ & $1.91 \mathrm{E}+06$ & $0.00 \mathrm{E}+00$ & $9.05 \mathrm{E}+03$ & $1.92 \mathrm{E}+06$ & $1.94 \mathrm{E}+06$ & $1.61 \mathrm{E}-04$ & $1.60 \mathrm{E}-04$ \\
\hline $\mathrm{T}-202$ & $8.02 \mathrm{E}+03$ & $1.91 \mathrm{E}+06$ & $0.00 \mathrm{E}+00$ & $2.79 \mathrm{E}+03$ & $2.54 \mathrm{E}+04$ & $1.91 \mathrm{E}+06$ & $0.00 \mathrm{E}+00$ & $9.05 \mathrm{E}+03$ & $1.92 \mathrm{E}+06$ & $1.94 \mathrm{E}+06$ & $1.61 \mathrm{E}-04$ & 1.60F-04 \\
\hline$[-203$ & $8.02 \mathrm{E}+03$ & $1.91 \mathrm{E}+06$ & $0.00 \mathrm{E}+00$ & $2.79 \mathrm{E}+03$ & $2.54 \mathrm{E}+04$ & $1.9 ! \mathrm{E}+06$ & $0.00 \mathrm{E}+00$ & $9.05 \mathrm{E}+03$ & $1.92 E+06$ & $1.94 \mathrm{E}+06$ & $1.61 \mathrm{E}-04$ & 1.60F-04 \\
\hline
\end{tabular}


RPP-5926 REV 6

Table F-2. The Diffusion Resistance and Gas Diffusion Rates of Hydrogen and Methane for 149 Single-Shell Tanks. (6 sheets)

\begin{tabular}{|c|c|c|c|c|c|c|c|c|c|c|c|c|}
\hline Tank & $\begin{array}{c}\text { Concrete } \\
\text { Resist of } \mathrm{H}_{2} \\
(\mathrm{sec} / \mathrm{cm})\end{array}$ & $\begin{array}{c}\text { Asphatic } \\
\text { Resist of } \mathrm{H}_{2} \\
(\mathrm{sec} / \mathrm{cm})\end{array}$ & $\begin{array}{c}\text { Gunite Resist } \\
\text { of } \mathrm{H}_{2} \\
(\mathrm{sec} / \mathrm{cm})\end{array}$ & $\begin{array}{c}\text { Soil Resist of } \\
\mathrm{H}_{2}(\mathrm{sec} / \mathrm{cm})\end{array}$ & $\begin{array}{c}\text { Concrete } \\
\text { Resist of } \mathrm{ClH}_{4} \\
(\mathrm{sec} / \mathrm{cm})\end{array}$ & $\begin{array}{c}\text { Asphatic } \\
\text { Resist of } \mathrm{CH}_{4} \\
(\mathrm{sec} / \mathrm{cm})\end{array}$ & $\begin{array}{c}\text { Gunite Resist } \\
\text { of } \mathrm{CH}_{4} \\
(\mathrm{sec} / \mathrm{cm})\end{array}$ & $\begin{array}{l}\text { Soil Resist of } \\
\mathrm{CH}_{4}(\mathrm{sec} / \mathrm{cm})\end{array} \mid$ & $\begin{array}{c}\text { Total Resist of } \\
\mathrm{H}_{2}(\mathrm{sec} / \mathrm{cm})\end{array}$ & $\begin{array}{l}\text { Total Resist of } \\
\mathrm{CH}_{4}(\mathrm{sec} / \mathrm{cm})\end{array}$ & $\begin{array}{c}\mathrm{H}_{2} \text { Diff Rate } \\
\mathrm{Ai} / \mathrm{Ri} \\
(\mathrm{cfm})\end{array}$ & $\begin{array}{c}\mathrm{CH}_{4} \text { Dill Rate } \\
\mathrm{Ai} / \mathrm{Rj} \\
(\mathrm{cfm})\end{array}$ \\
\hline T.204 & $8.02 \mathrm{E}+03$ & $1.91 \mathrm{E}+06$ & $0.00 E+00$ & $2.79 \mathrm{E}+03$ & $2.54 \mathrm{E}+04$ & $1.91 \mathrm{E}+06$ & $0.00 \mathrm{E}+00$ & $9.05 E+03$ & $1.92 \mathrm{E}+06$ & $1.94 \mathrm{E}+06$ & $1.61 \mathrm{E}-04$ & $1.60 \mathrm{E}-04$ \\
\hline TX-10! & $1.00 \mathrm{E}+04$ & $1.91 E+06$ & $5.01 \mathrm{E}+02$ & $3.56 \mathrm{E}+03$ & 3. $18 \mathrm{E}+04$ & $1.91 \mathrm{E}+06$ & $1.59 \mathrm{E}+03$ & $1.15 \mathrm{E}+04$ & $1.92 \mathrm{E}+06$ & $1.95 \mathrm{E}+06$ & $4.84 \mathrm{E}-03$ & $4.76 \mathrm{E}-03$ \\
\hline TX-102 & $1.00 \mathrm{E}+04$ & $1.91 E+06$ & $5.01 \mathrm{E}+02$ & $3.56 \mathrm{E}+03$ & $3.18 \mathrm{E}+04$ & $1.91 \mathrm{E}+06$ & $1.59 \mathrm{E}+03$ & $1.15 E+04$ & $1.92 \mathrm{E}+06$ & $1.95 \mathrm{E}+06$ & $4.84 \mathrm{E}-03$ & $4.76 \mathrm{E}-03$ \\
\hline TX-103 & $1.00 \mathrm{E}+04$ & $1.91 \mathrm{E}+06$ & $5.01 \mathrm{E}+02$ & $3.56 \mathrm{E}+03$ & $3.18 \mathrm{E}+04$ & $1.91 \mathrm{E}+06$ & $1.59 \mathrm{E}+03$ & $1.15 \mathrm{E}+04$ & $1.92 \mathrm{E}+06$ & $1.95 \mathrm{E}+06$ & $4.84 \mathrm{E}-03$ & $4.76 \mathrm{E}-03$ \\
\hline TX-104 & $1.00 \mathrm{E}+04$ & $1.91 \mathrm{E}+06$ & $5.01 \mathrm{E}+02$ & $3.56 \mathrm{E}+03$ & $3.18 \mathrm{E}+04$ & $1.91 \mathrm{E}+06$ & $1.59 \mathrm{E}+03$ & $1.15 \mathrm{E}+04$ & $1.92 E+06$ & 1.95E+06 & $4.84 \mathrm{E}-03$ & $4.76 \mathrm{E}-03$ \\
\hline TX-105 & $1.00 \mathrm{E}+04$ & $1.91 \mathrm{E}+06$ & $5.01 E+02$ & $3.56 \mathrm{E}+03$ & $3.18 \mathrm{E}+04$ & $1.91 \mathrm{E}+06$ & $1.59 \mathrm{E}+03$ & $1.15 \mathrm{E}+04$ & $1.92 \mathrm{E}+06$ & $1.95 \mathrm{E}+06$ & $4.84 \mathrm{E}-03$ & $4.76 \mathrm{E}-03$ \\
\hline TX-106 & $1.00 \mathrm{E}+04$ & $1.91 \mathrm{E}+06$ & $5.01 \mathrm{E}+02$ & $3.56 \mathrm{E}+03$ & $3.18 \mathrm{E}+04$ & $1.91 \mathrm{E}+06$ & $1.59 \mathrm{E}+03$ & $1.15 \mathrm{E}+04$ & $1.92 \mathrm{E}+06$ & $1.95 \mathrm{E}+06$ & $4.84 \mathrm{E}-03$ & $4.76 \mathrm{E}-03$ \\
\hline TX-107 & $1.00 \mathrm{E}+04$ & $1.91 \mathrm{E}+06$ & $5.01 \mathrm{E}+02$ & $3.56 \mathrm{E}+03$ & $3.18 \mathrm{E}+04$ & $1.91 \mathrm{E}+06$ & $1.59 \mathrm{E}+03$ & $1.15 \mathrm{E}+04$ & $1.92 \mathrm{E}+06$ & $1.95 \mathrm{E}+06$ & $4.84 \mathrm{E}-03$ & $4.76 \mathrm{E}-03$ \\
\hline TX-108 & $1.00 \mathrm{E}+04$ & $1,91 E+06$ & $5.01 \mathrm{E}+02$ & $3.56 \mathrm{E}+03$ & $3.18 \mathrm{E}+04$ & $1.91 \mathrm{E}+06$ & $1.59 \mathrm{E}+03$ & $1.15 \mathrm{E}+04$ & $1.92 \mathrm{E}+06$ & $1.95 \mathrm{E}+06$ & $4.84 \mathrm{E}-03$ & $4.76 \mathrm{E}-03$ \\
\hline$T X-109$ & $1.00 \mathrm{E}+04$ & $1.91 E+06$ & $5.01 \mathrm{E}+02$ & $3.56 \mathrm{E}+03$ & $3.18 \mathrm{E}+04$ & $1.91 \mathrm{E}+06$ & $1.59 \mathrm{E}+03$ & $1.15 E+04$ & $1.92 E+06$ & $1.95 \mathrm{E}+06$ & $4.84 \mathrm{E}-03$ & $4.76 \mathrm{E}-03$ \\
\hline TX-110 & $1.00 \mathrm{E}+04$ & $1.91 E+06$ & $5.01 E+02$ & $3.56 \mathrm{E}+03$ & $3.18 \mathrm{E}+04$ & $1.91 \mathrm{E}+06$ & $1.59 \mathrm{E}+03$ & $1.15 \mathrm{E}+04$ & $1.92 E+06$ & $1.95 \mathrm{E}+06$ & $4.84 \mathrm{E}-03$ & $4.76 \mathrm{E}-03$ \\
\hline$T X-111$ & $1.00 \mathrm{~F}+04$ & $1.91 E+06$ & $5.01 \mathrm{E}+02$ & $3.56 \mathrm{E}+03$ & $3.18 \mathrm{E}+04$ & $1.91 \mathrm{E}+06$ & $1.59 \mathrm{E}+03$ & $1.15 \mathrm{E}+04$ & $1.92 \mathrm{E}+06$ & $1.95 \mathrm{E}+06$ & 4.84E- 03 & $4.76 \mathrm{E}-03$ \\
\hline $\mathrm{TX}-112$ & $1.00 \mathrm{E}+04$ & $1.91 \mathrm{E}+06$ & $5.01 \mathrm{E}+02$ & $3.56 \mathrm{E}+03$ & $3.18 \mathrm{~F}+04$ & $1.91 \mathrm{E}+06$ & $1.59 \mathrm{E}+03$ & $1.15 \mathrm{E}+04$ & $1.92 \mathrm{E}+06$ & $1.95 E+06$ & $4.84 \mathrm{E}+03$ & $4.76 \mathrm{E}-03$ \\
\hline $\mathrm{TX}-113$ & $1.00 \mathrm{E}+04$ & $1.91 \mathrm{E}+06$ & $5.01 \mathrm{E}+02$ & $3.56 \mathrm{E}+03$ & $3.18 \mathrm{E}+04$ & $1.91 \mathrm{E}+06$ & $1.59 \mathrm{E}+03$ & $1.15 \mathrm{E}+04$ & $1.92 E+06$ & $1.95 \mathrm{E}+06$ & $4.84 \mathrm{E}-03$ & $4.76 \mathrm{E}-03$ \\
\hline $\mathrm{TX}-114$ & $1.00 \mathrm{E}+04$ & $1.91 \mathrm{E}+06$ & $5.01 \mathrm{E}+02$ & $3.56 \mathrm{E}+03$ & $3.18 \mathrm{E}+04$ & $1.91 \mathrm{E}+06$ & $1.59 \mathrm{E}+03$ & $1.15 \mathrm{E}+04$ & $1.92 \mathrm{E}+06$ & $1.95 \mathrm{E}+06$ & $4.84 \mathrm{E}-03$ & $4.76 \mathrm{E}-03$ \\
\hline TX-115 & $1.00 \mathrm{E}+04$ & $1.91 \mathrm{E}+06$ & $5.01 \mathrm{E}+02$ & $3.56 \mathrm{E}+03$ & $3.18 \mathrm{E}+04$ & $1.91 \mathrm{E}+06$ & $1.59 \mathrm{E}+03$ & $1.15 \mathrm{E}+04$ & $1.92 \mathrm{E}+06$ & $1.95 \mathrm{E}+06$ & $4.84 \mathrm{E}-03$ & $4.76 \mathrm{E}-03$ \\
\hline TX-116 & $1.00 \mathrm{E}+04$ & $1.91 \mathrm{E}+06$ & $5.01 \mathrm{E}+02$ & $3.56 \mathrm{E}+03$ & $3.18 \mathrm{E}+04$ & $1.91 \mathrm{E}+06$ & $1.59 \mathrm{E}+03$ & $1.15 \mathrm{E}+04$ & $1.92 \mathrm{E}+06$ & $1.95 \mathrm{E}+06$ & $4.84 \mathrm{E}-03$ & $4.76 \mathrm{E}-03$ \\
\hline $\mathrm{TX}-117$ & $1.00 \mathrm{E}+04$ & $1.91 \mathrm{E}+06$ & $5.01 \mathrm{~F}+02$ & $3.56 \mathrm{E}+03$ & $3.18 \mathrm{E}+04$ & $1.91 \mathrm{E}+06$ & $1.59 \mathrm{E}+03$ & $1.15 \mathrm{E}+04$ & $1.92 \mathrm{E}+06$ & $1.95 \mathrm{E}+06$ & $4.84 \mathrm{E}-03$ & $4.76 \mathrm{E}-03$ \\
\hline TX-118 & $1.00 \mathrm{E}+04$ & $1.91 E+06$ & $5.01 \mathrm{E}+02$ & $3.56 \mathrm{E}+03$ & $3.18 E+04$ & $1.91 E+06$ & $1.59 \mathrm{E}+03$ & $1.15 \mathrm{E}+04$ & $1.92 \mathrm{E}+06$ & $1.95 \mathrm{E}+06$ & $4.84 \mathrm{E}-03$ & $4.76 \mathrm{E}-03$ \\
\hline$T Y-101$ & $1.00 \mathrm{~F}+04$ & $1.91 \mathrm{E}+06$ & $5.01 \mathrm{E}+02$ & $3.56 \mathrm{E}+03$ & $3.18 \mathrm{E}+04$ & $1.91 \mathrm{E}+06$ & $1.59 \mathrm{E}+03$ & $1.15 \mathrm{E}+04$ & $1.92 \mathrm{E}+06$ & $1.95 \mathrm{E}+06$ & $4.84 \mathrm{E}-03$ & $4.76 \mathrm{E}-03$ \\
\hline TY -102 & $1.00 \mathrm{E}+04$ & $1.91 \mathrm{E}+06$ & $5.01 \mathrm{E}+02$ & $3.56 \mathrm{E}+03$ & $3.18 \mathrm{E}+04$ & $1.91 \mathrm{E}+06$ & $1.59 \mathrm{E}+03$ & $1.15 \mathrm{E}+04$ & $1.92 \mathrm{E}+06$ & $1.95 \mathrm{E}+06$ & $4.84 \mathrm{E}-03$ & $4.76 \mathrm{E}-03$ \\
\hline TY-103 & $1.00 \mathrm{E}+04$ & $1.91 \mathrm{E}+06$ & $5.01 \mathrm{E}+02$ & $3.56 \mathrm{E}+03$ & $3.18 \mathrm{~F}+04$ & $1.91 \mathrm{E}+06$ & $1.59 \mathrm{E}+03$ & $1.15 E+04$ & $1.92 \mathrm{E}+06$ & $1.95 \mathrm{E}+06$ & $4.84 \mathrm{E}-03$ & $4.76 \mathrm{E}-03$ \\
\hline$T Y \cdot 104$ & $1.00 \mathrm{E}+04$ & $1.91 \mathrm{E}+06$ & $5.01 \mathrm{E}+02$ & $3.56 \mathrm{~F}+03$ & $3.18 \mathrm{E}+04$ & $1.91 \mathrm{E}+06$ & 1.59E+03 & $1.15 \mathrm{E}+04$ & $1.92 \mathrm{E}+06$ & $1.95 \mathrm{E}+06$ & $4.84 \mathrm{E}-03$ & $4.76 \mathrm{E}-03$ \\
\hline$T Y-105$ & $1.00 \mathrm{E}+04$ & $1.91 \mathrm{E}+06$ & $5.01 \mathrm{E}+02$ & $3.56 \mathrm{E}+03$ & $3.18 \mathrm{E}+04$ & $1.91 \mathrm{E}+06$ & $1.59 \mathrm{E}+03$ & $1.15 \mathrm{E}+04$ & $1.92 \mathrm{E}+06$ & $1.95 \mathrm{E}+06$ & $4.84 \mathrm{E}-03$ & $4.76 \mathrm{E}-03$ \\
\hline TY-106 & $1.00 \mathrm{E}+04$ & $1.91 \mathrm{E}+06$ & $5.01 \mathrm{E}+02$ & $3.56 \mathrm{E}+03$ & 3. $18 \mathrm{E}+04$ & $1.91 \mathrm{E}+06$ & $1.59 \mathrm{E}+03$ & $1.15 \mathrm{E}+04$ & $1.92 \mathrm{E}+06$ & $1.95 \mathrm{E}+06$ & $4.84 \mathrm{E}-03$ & $4.76 \mathrm{E}-03$ \\
\hline $\mathrm{U}-101$ & $1.00 \mathrm{E}+04$ & $1.91 E+06$ & $5.01 \mathrm{E}+02$ & $3.37 \mathrm{E}+03$ & $3.18 \mathrm{E}+04$ & $1.91 \mathrm{E}+06$ & $1.59 \mathrm{E}+03$ & $1.09 \mathrm{E}+04$ & $1.92 \mathrm{E}+06$ & $1.95 \mathrm{E}+06$ & $4.89 \mathrm{E}-03$ & $4.82 \mathrm{E}-03$ \\
\hline $\mathrm{U}-102$ & $1.00 \mathrm{E}+04$ & $1.91 E+06$ & $5.01 E+02$ & $3.37 E+03$ & $3.18 E+04$ & $1.91 \mathrm{E}+06$ & $1.59 \mathrm{E}+03$ & $1.09 \mathrm{E}+04$ & $1.92 \mathrm{E}+06$ & $1.95 \mathrm{E}+06$ & $4.89 \mathrm{E}-03$ & $4.82 \mathrm{~F}-03$ \\
\hline
\end{tabular}


Table F-2. The Diffusion Resistance and Gas Diffusion Rates of Hydrogen and Methane for 149 Single-Shell Tanks. (6 sheets)

\begin{tabular}{|c|c|c|c|c|c|c|c|c|c|c|c|c|}
\hline Tank & $\begin{array}{c}\text { Concrete } \\
\text { Resist of } \mathrm{H}_{2} \\
(\mathrm{sec} / \mathrm{cm})\end{array}$ & $\begin{array}{c}\text { Asphatic } \\
\text { Resist of } \mathrm{H}_{2} \\
\text { (sec/cm) }\end{array}$ & $\begin{array}{c}\text { Gunite Resist } \\
\text { of } \mathrm{H}_{2} \\
(\mathrm{sec} / \mathrm{cm})\end{array}$ & $\begin{array}{c}\text { Soil Resist of } \\
\mathrm{H}_{2}(\mathrm{sec} / \mathrm{cm})\end{array}$ & $\begin{array}{c}\text { Concrete } \\
\text { Resist of } \mathrm{CH}_{4} \\
(\mathrm{sec} / \mathrm{cm})\end{array}$ & $\begin{array}{c}\text { Asphatic } \\
\text { Resist of } \mathrm{CH}_{4} \\
\text { (sec/cm) }\end{array}$ & $\begin{array}{c}\text { Gunite Resist } \\
\text { of } \mathrm{CH}_{4} \\
(\mathrm{sec} / \mathrm{cm})\end{array}$ & $\begin{array}{l}\text { Soil Resist of } \\
\mathrm{CH}_{4}(\mathrm{sec} / \mathrm{cm})\end{array} \mid$ & $\begin{array}{c}\text { Total Resist of } \\
\mathrm{H}_{2}(\mathrm{sec} / \mathrm{cm} 1)\end{array}$ & $\begin{array}{l}\text { Total Resist of } \\
\mathrm{CH}_{4}(\mathrm{sec} / \mathrm{cm})\end{array} \mid$ & $\begin{array}{c}\mathrm{H}_{2} \text { Diff Rate } \\
\mathrm{Ai} / \mathrm{Ri} \\
(\mathrm{cfm})\end{array}$ & $\begin{array}{c}\mathrm{CH}_{4} \text { Diff Rate } \\
\mathrm{Ai} / \mathrm{Ri} \\
(\mathrm{cfm})\end{array}$ \\
\hline U.103 & $1.00 \mathrm{E}+04$ & $1.91 \mathrm{E}+06$ & $5.01 \mathrm{E}+02$ & $3.37 \mathrm{E}+03$ & $3.18 E+04$ & $1.91 E+06$ & $1.59 \mathrm{E}+03$ & $1.09 E+04$ & $1.92 \mathrm{E}+06$ & $1.95 \mathrm{E}+06$ & $4.89 \mathrm{E}-03$ & $4.82 \mathrm{E}-03$ \\
\hline U-104 & $1.00 \mathrm{~F}+04$ & $1.91 \mathrm{E}+06$ & $5.01 E+02$ & $3.37 \mathrm{E}+03$ & $3.18 \mathrm{E}+04$ & $1.91 E+06$ & $1.59 E+03$ & $1.09 \mathrm{E}+04$ & $1.92 \mathrm{E}+06$ & $1.95 E+06$ & $4.89 \mathrm{E}-03$ & $4.82 \mathrm{E}-03$ \\
\hline U-105 & $1.00 \mathrm{E}+04$ & $1.91 \mathrm{E}+06$ & $5.01 E+02$ & $3.37 \mathrm{E}+03$ & $3.18 \mathrm{E}+04$ & $1.91 \mathrm{E}+06$ & $1.59 \mathrm{E}+03$ & $1.09 \mathrm{E}+04$ & $1.92 E+06$ & $1.95 E+06$ & $4.89 E-03$ & $4.82 \mathrm{E}-03$ \\
\hline $\mathrm{U}-106$ & $1.00 \mathrm{E}+04$ & $1.91 \mathrm{E}+06$ & $5.01 \mathrm{E}+02$ & $3.37 E+03$ & $3.18 E+04$ & $1.91 E+06$ & $1.59 \mathrm{E}+03$ & $1.09 E+04$ & $1.92 \mathrm{E}+06$ & $1.95 \mathrm{E}+06$ & $4.89 \mathrm{E}+03$ & $4.82 \mathrm{E}-03$ \\
\hline U-107 & $1.00 \mathrm{~F}+04$ & $1.91 \mathrm{E}+06$ & $5.01 \mathrm{E}+02$ & $3.37 E+03$ & $3.18 \mathrm{E}+04$ & $1.91 \mathrm{E}+06$ & $1.59 E+03$ & $1.09 \mathrm{E}+04$ & $1.92 \mathrm{E}+06$ & $1.95 \mathrm{E}+06$ & $4.89 \mathrm{E}-03$ & $4.82 \mathrm{E}-03$ \\
\hline U-108 & $1.00 \mathrm{E}+04$ & $1.91 \mathrm{E}+06$ & $5.01 \mathrm{E}+02$ & $3.37 \mathrm{E}+03$ & $3.18 \mathrm{E}+04$ & $1.91 \mathrm{E}+06$ & $1.59 \mathrm{E}+03$ & $1.09 \mathrm{E}+04$ & $1.92 \mathrm{E}+06$ & $1.95 \mathrm{E}+06$ & $4.89 \mathrm{E}-03$ & $4.82 \mathrm{E}-03$ \\
\hline U-109 & $1.00 \mathrm{E}+04$ & $1.91 \mathrm{E}+06$ & $5.01 \mathrm{E}+02$ & $3.37 \mathrm{E}+03$ & $3.18 \mathrm{E}+04$ & $1.91 \mathrm{E}+06$ & $1.59 \mathrm{E}+03$ & $1.09 \mathrm{E}+04$ & $1.92 E+06$ & $1.95 \mathrm{E}+06$ & $4.89 \mathrm{E}-03$ & $4.82 \mathrm{E} \cdot 03$ \\
\hline U-1 10 & $1.00 \mathrm{E}+04$ & $1.91 \mathrm{E}+06$ & $5.01 \mathrm{E}+02$ & $3.37 \mathrm{E}+03$ & $3.18 \mathrm{E}+04$ & $1.91 \mathrm{E}+06$ & $1.59 \mathrm{E}+03$ & $1.09 \mathrm{E}+04$ & $1.92 \mathrm{E}+06$ & $1.95 E+06$ & $4.89 \mathrm{E}-03$ & $4.82 \mathrm{E}-03$ \\
\hline $\mathrm{U}-111$ & $1.00 \mathrm{E}+04$ & $1.91 \mathrm{E}+06$ & $5.01 \mathrm{E}+02$ & $3.37 \mathrm{E}+03$ & $3.18 \mathrm{E}+04$ & $1.91 \mathrm{E}+06$ & $1.59 \mathrm{E}+03$ & $1.09 \mathrm{E}+04$ & $1.92 \mathrm{E}+06$ & $1.95 \mathrm{E}+06$ & $4.89 \mathrm{E}-03$ & $4.82 \mathrm{E}-03$ \\
\hline $\mathrm{U}-112$ & $1.00 \mathrm{E}+04$ & $1.91 \mathrm{E}+06$ & $5.01 \mathrm{E}+02$ & $3.37 \mathrm{E}+03$ & $3.18 \mathrm{E}+04$ & $1.91 \mathrm{E}+06$ & $1.59 \mathrm{E}+03$ & $1.09 \mathrm{E}+04$ & $1.92 \mathrm{E}+06$ & $1.95 \mathrm{E}+06$ & $4.89 \mathrm{E}-03$ & $4.82 \mathrm{E}-03$ \\
\hline (U-201 & $8.02 \mathrm{E}+03$ & $1.91 E+06$ & $0.00 \mathrm{E}+00$ & $2.79 \mathrm{E}+03$ & $2.54 \mathrm{E}+04$ & $1.91 \mathrm{E}+06$ & $0.00 \mathrm{E}+00$ & $9.05 E+03$ & $1.92 E+06$ & $1.94 E+06$ & $1.61 \mathrm{E}-04$ & $1.60 \mathrm{E}-04$ \\
\hline U-202 & $8.02 \mathrm{E}+03$ & $1.91 \mathrm{E}+06$ & $0.00 \mathrm{E}+00$ & $2.79 \mathrm{E}+03$ & $2.54 \mathrm{E}+04$ & $1.91 \mathrm{E}+06$ & $0.00 \mathrm{E}+00$ & $9.05 \mathrm{E}+03$ & $1.92 \mathrm{E}+06$ & $1.94 \mathrm{E}+06$ & $1.61 \mathrm{E}-04$ & $1.60 \mathrm{E}-04$ \\
\hline $\mathrm{U}-203$ & $8.02 \mathrm{E}+03$ & $1.91 E+06$ & $0.00 \mathrm{E}+00$ & $2.79 E+03$ & $2.54 \mathrm{E}+04$ & $1.91 \mathrm{E}+06$ & $0.00 \mathrm{E}+00$ & $9.05 \mathrm{E}+03$ & $1.92 \mathrm{E}+06$ & $1.94 E+06$ & $1.61 \mathrm{E}-04$ & $1.60 \mathrm{E}-04$ \\
\hline U.204 & $8.02 \mathrm{E}+03$ & $1.91 \mathrm{E}+06$ & $0.00 \mathrm{E}+00$ & $2.79 \mathrm{E}+03$ & $2.54 \mathrm{E}+04$ & $1.91 \mathrm{E}+06$ & $0.00 \mathrm{E}+00$ & $9.05 \mathrm{E}+03$ & $1.92 \mathrm{E}+06$ & $1.94 \mathrm{E}+06$ & $1.61 \mathrm{E}-04$ & $1.60 \mathrm{E}-04$ \\
\hline
\end{tabular}


RPP-5926 REV 6

APPENDIX G

FLAMMABILITY EVALUATION OF TIME TO 25\% OF THE LOWER FLAMMABILITY LIMIT TO SUPPORT THE VACUUM RETRIEVAL PROJECT 
RPP-5926 REV 6

This page intentionally left blank.

G-ii 


\section{CONTENTS}

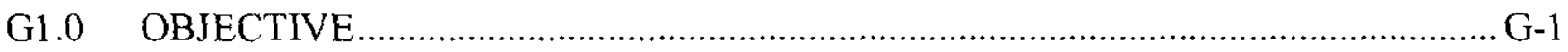

G2.0 SUMMARY OF RESULTS AND CONCLUSIONS …..................................... G-1

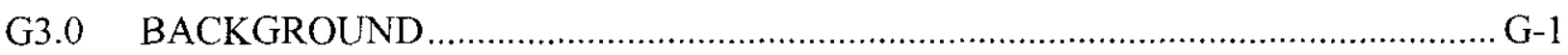

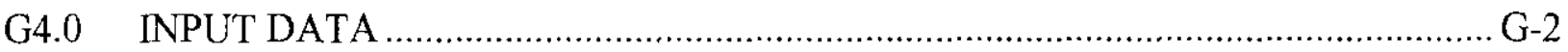

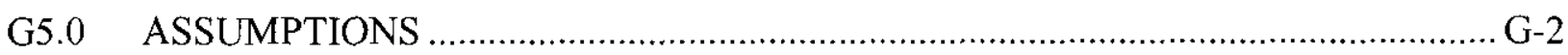

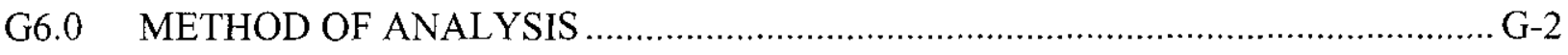

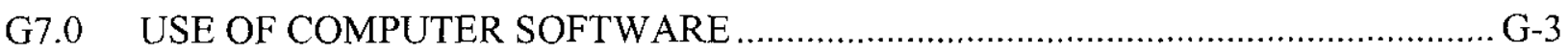

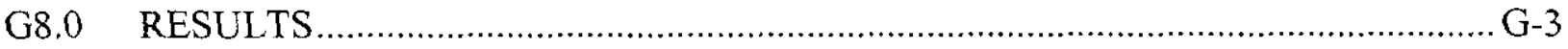

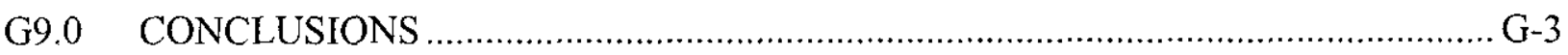

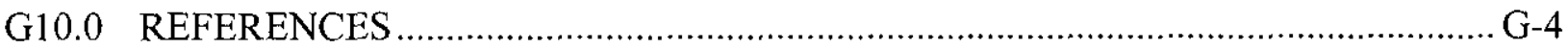

\section{LIST OF TABLES}

Table G1-A. Input Data for Hydrogen Generation Rate Model Calculations. ...................... G-5

Table G1-B. Derived Data for Hydrogen Generation Rate Model Calculations .................... G-5

Table G1-C. Calculated Hydrogen Generation Rate as Mole Per Unit Waste Weight and

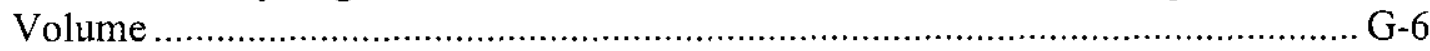

Table G1-D. Calculated Total Hydrogen Generation Rate In Dome Space .......................... G-6

Table G2-A. Steady-State Gas Concentrations, Release Rates and Flammability Levels Under Normal Ventilation Conditions.

Table G2-B Steady-State Flammability Under Barometric Breathing and Time to $25 \%$ of the LFL under Zero Vent. 


\section{G1.0 OBJECTIVE}

This case study provides an evaluation to support the determination that the potential risk associated with using the single-shell tank (SST) waste retrieval system vacuum system for retrieval of the 241-C Tank Farm 200-series SSTs is adequately addressed for steady-state gas accumulation by the current tank farm safety basis. The evaluation provides an upper limit for water additions to the designated SST receiver tank to ensure that the time to $25 \%$ of the lower flammability limit (LFL) is 365 days or greater.

The following calculation is issued in Rev. 3-A and updated in Rev. 4 of the document using the new hydrogen generation rate (HGR) model. The evaluation assumed the total waste of the 241-C Tank Farm 200-series SSTs put on the receiver tank. Since then, retrieval of tank 241-C-201, 241-C-202 and 241-C-203 has been completed. The total waste of 241-C Tank Farm 200 -series is reduced by more than $50 \%$. The total HGR of the source waste has been decreased. Thus, the maximum water addition determined below is bounding and there is no intention to update this calculation in Appendix G, Rev. 6 of this document.

\section{G2.0 SUMMARY OF RESULTS AND CONCLUSIONS}

The evaluation shows that the maximum water addition to the SST receiver is equal to $112 \mathrm{~kL}$ or $29.6 \mathrm{kgal}$. This is the water volume that would cause the time to $25 \%$ of the LFL in the dome space of the designated SST receiver to be 365 days, as specified for this case study.

\section{G3.0 BACKGROUND}

Waste from the 241-C Tank Farm 200-series tanks is planned to be transferred in to the double-shell tank (DST) system after accumulation in one of the four SSTs. A description of the system design and overall process can be found in RPP-17190, Safety Evaluation of the Waste Retrieval System Vacuum System for 241-C Tank Farm 200-Series Tanks. During the retrieval of the 241-C Tank Farm 200-series SSTs, the retrieval solution will be routinely drained to the designated SST receiver tank; in this case, SST 241-C-203. The retrieval solution is essentially water in the water separator with small amounts of entrained tank waste.

For the bounding case study, the time to $25 \%$ of the LFL for the SST receiver tank is 365 days, a limit specified by Retrieval/Closure Nuclear Safety. Currently, the 241-C 200-series SSTs contain very little waste. The time to $25 \%$ of the LFL is over 1,500 days for SSTs 241-C-201, 241-C-202, 241-C-203, and 241-C-204, respectively, under zero ventilation condition (see Table 4-5 of the main text). These times to $25 \%$ of the LFL are much longer than 365 days. This evaluation of steady-state flammable gas accumulation analyzes how much water could be added to the designated SST receiver tank before the time to $25 \%$ of the LFL would decrease to 365 days. 


\section{G4.0 INPUT DATA}

The bounding case evaluation assumes that all the solid waste in the four 241-C 200-series SSTs is accumulated in one of the tanks. Also as the bounding case, the HGR calculations and flammability evaluations assume the waste condition of SST 241-C-204 represents all the waste. SST 241-C-204 is used because it has the waste that contains the highest HGR per unit volume.

The steady-state HGR input data for SST 241-C-204 is documented in Appendix B, Table B-5. The volume of all the waste is the sum of the solid waste in each tank. These data are used in Table F-1 A. For the time to LFL calculations, the required data are taken from Appendix D, Table D-1. Because a verified spreadsheet is used, only input data needs to be checked for accuracy.

\section{G5.0 ASSUMPTIONS}

All of the waste from the four 241-C 200-series tanks is assumed to have the characteristics of SST 241-C-204 waste. This evaluation assumes a hypothetical 1,000-gal water addition, documented in Table B-5, has been made to the SST 241-C-204 prior accumulation in the SST receiver and prior to those tanks receiving any retrieval waste or water. The 1,000-gal water addition to SST 241-C-204 waste is assumed to form a thin liquid layer and has the waste composition of the interstitial liquid.

The additional water used for retrieval is assumed to be well mixed with the hypothetical liquid layer in the SST receiver.

\section{G6.0 METHOD OF ANALYSIS}

The methodology and equations for the flammability evaluation used in this study is discussed in Chapter 3.0 and 4.0 of the main text of this document. This methodology is developed to calculate the gas release rate, gas generation rate, flammability level, and time to $25 \%$ and $100 \%$ of the LFL for a given ventilation rate.

Given the calculated HGRs along with historical gas release rates of ammonia and methane from the identified waste, the time to $25 \%$ of the LFL are calculated. Note that water additions will decrease the volume of the dome space, and therefore, the time to reach $25 \%$ of the LFL for a given ventilation rate will decrease. In order to find the upper limit of water addition, the volume of water is gradually increased until the time to $25 \%$ of the LFL is 365 days. 


\section{G7.0 USE OF COMPUTER SOFTWARE}

This case study uses a validated Excel ${ }^{7}$ spreadsheet, documented in RPP-5926, Rev. 4, to conduct the calculations (RPP-5926-8050-R3-LFL-CAL-T2-1102004.xIs). Refer to the spreadsheet verification form in Appendix I, page I-3. This spreadsheet used the input data to calculate the gas generation rates, then calculates the flammability and time to LFL for a given ventilation rate.

\section{G8.0 RESULTS}

Appendix B, Table B-1 shows that the total waste volume of the four 241-C 200-series SST is $17 \mathrm{~kL}$. The waste composition, physical properties, and waste temperature for SST 241-C-204, along with the total waste volume of $17 \mathrm{~kL}$, is used to calculate the HGR and flammability for the solid waste layer in the SST receiver.

For the liquid layer, the volume of water added is used to calculate the dilution factor, and then the dilution factor is used to recalculate the waste composition and physical properties based on the input data for the liquid layer listed in Table B-5 of Appendix B, which includes a hypothetical 1,000 gal of water addition to the waste. The dilution factor is determined by the ratio of total water addition and supernatant volume of 1,000 gal. Details of the input data and HGR calculation results are given in Table G-1A to Table G-1D. Note that in Table G-1D, note that HGR is primarily coming from the corrosion (about $99 \%$ ) for both liquid and solid layers. So in this case it will make not much difference whether we treat the liquid layer as pure water or assume the added water is well mixed with waste.

It is found that when total water additions are $112 \mathrm{~kL}$ or $29.6 \mathrm{kgal}$ including the hypothetical supernatant of 1,000 gal, the time to $25 \%$ of the LFL is 365 days. Also, note that the steady-state flammability of the system, which is $17 \mathrm{~kL}$ of solid waste and $112 \mathrm{~kL}$ of water, under barometric breathing condition is $15.4 \%$. Details of calculated input data and time to $25 \%$ of the LFL under zero ventilation are given in Table G-2A to Table G-2B.

\section{G9.0 CONCLUSIONS}

For the given maximum solid waste volume of $17 \mathrm{~kL}$, as long as the total water additions to the SST receiver tank are less than $112 \mathrm{~kL}$ or $29.6 \mathrm{kgal}$, the time to $25 \%$ of the LFL will not reach 365 days during the waste retrieval activities.

\footnotetext{
${ }^{7}$ Excel is a registered trademark of Microsoft Corporation, Redmond, Washington.
} 
RPP-5926 REV 6

\section{G10.0 REFERENCES}

RPP-17190, 2003, Safety Evaluation of the Waste Retrieval System Vacuum System for 241-C Tank Farm 200-Series Tanks, Rev 0, CH2M HILL Hanford Group, Inc., Richland, Washington. 
RPP-5926 REV 6

Table G1-A. Input Data for Hydrogen Generation Rate Model Calculations.

\begin{tabular}{|c|c|c|c|c|c|c|c|c|c|c|c|c|c|c|c|}
\hline Tank & $\begin{array}{l}\text { Volume } \\
\text { of Water } \\
\text { Addition } \\
\text { (kL) and } \\
\text { Dilution } \\
\text { factor }\end{array}$ & $\begin{array}{c}\text { TOC } \\
\text { in liquid } \\
\text { [TOC] } \\
(\mu \mathrm{g} / \mathrm{mL})\end{array}$ & $\begin{array}{c}\mathrm{NO}_{2} \\
\text { in liquid } \\
{\left[\mathrm{NO}_{2}\right]} \\
(\mu \mathrm{g} / \mathrm{mL})\end{array}$ & $\begin{array}{c}\mathrm{NO}_{3} \\
\text { in liquid } \\
{\left[\mathrm{NO}_{3}\right]} \\
(\mu \mathrm{g} / \mathrm{mL})\end{array}$ & $\begin{array}{c}\mathrm{A}] \\
\text { in liquid } \\
{[\mathrm{Al}]} \\
(\mu \mathrm{g} / \mathrm{mL})\end{array}$ & $\begin{array}{c}\mathrm{Na} \\
\text { in liquid } \\
{[\mathrm{Na}]} \\
(\mu \mathrm{g} / \mathrm{mL})\end{array}$ & $\begin{array}{c}{ }^{90} \mathrm{Sr} \\
\text { in waste } \\
{[\mathrm{Sr}]} \\
(\mu \mathrm{Ci} / \mathrm{g})\end{array}$ & $\begin{array}{c}{ }^{137} \mathrm{Cs} \\
\text { in waste } \\
{[\mathrm{Cs}]} \\
(\mu \mathrm{Ci} / \mathrm{g})\end{array}$ & $\begin{array}{c}\text { Bulk } \\
\text { density } \\
\text { D } \\
(\mathrm{g} / \mathrm{mL})\end{array}$ & $\begin{array}{c}\text { Liquid } \\
\text { Density } \\
D_{\mathrm{L}} \\
(\mathrm{g} / \mathrm{ml})\end{array}$ & $\begin{array}{c}\text { Non-RGS } \\
\text { waste } \\
\text { volume } \\
\text { V } \\
(\mathrm{kL})\end{array}$ & $\begin{array}{c}\text { Bulk } \\
\text { water } \\
{\left[\mathrm{H}_{2} \mathrm{O}\right]} \\
(\mathrm{wt} \%)\end{array}$ & $\begin{array}{c}\text { Liquid } \\
\text { water } \\
{\left[\mathrm{H}_{2} \mathrm{O} \mid\right.} \\
\left(\mathrm{wt}^{\circ} \%\right)\end{array}$ & $\begin{array}{c}\text { Waste } \\
\text { temp. } \\
T_{w} \\
\left({ }^{\circ} \mathrm{C}\right)\end{array}$ & $\begin{array}{c}\text { Dome } \\
\text { temp. } \\
T_{\mathrm{d}} \\
\left({ }^{\circ} \mathrm{C}\right)\end{array}$ \\
\hline Supernatant & 111.9 & $2.98 \mathrm{E}+03$ & $3.60 \mathrm{E}+04$ & $7.96 \mathrm{E}+04$ & $8.20 \mathrm{E}+04$ & $4.94 \mathrm{E}+03$ & $1.09 \mathrm{E}+01$ & $4.72 \mathrm{E}+00$ & 1.17 & 1.17 & 112 & $75 \%$ & $75 \%$ & 17 & 16 \\
\hline Solid Layer & & $2.98 \mathrm{E}+03$ & $3.60 E+04$ & $7.96 \mathrm{E}+04$ & $8.20 \mathrm{E}+04$ & $4.94 E+03$ & $1.09 E+01$ & $4.72 \mathrm{E}+00$ & 1.62 & 1.17 & 17 & $41 \%$ & $75 \%$ & 17 & 16 \\
\hline
\end{tabular}

Table G1-B. Derived Data for Hydrogen Generation Rate Model Calculations

\begin{tabular}{|c|c|c|c|c|c|c|c|c|c|c|c|c|c|c|c|}
\hline Tank & $\begin{array}{l}\text { Waste } \\
\text { level } \\
\text { D }_{w} \\
\text { (inch) }\end{array}$ & $\begin{array}{c}\text { Total } \\
\text { mass } \\
M \\
(\mathrm{~kg})\end{array}$ & $\begin{array}{c}\text { Liquid } \\
\text { in waste } \\
(w t \%)\end{array}$ & $\begin{array}{c}\text { Wetted } \\
\text { area } \\
A_{\text {wer }} \\
\left(\mathrm{ft}^{2}\right)\end{array}$ & $\begin{array}{c}\text { Heat load } \\
\text { per kg } \\
\mathrm{H}_{\mathrm{L}} \mathrm{B} / \gamma \\
\text { (watt } / \mathrm{kg} \text { ) }\end{array}$ & $\begin{array}{c}\text { Tank } \\
\text { heat load } \\
\mathrm{H}_{\mathrm{L}}^{\text {alpha }} \\
\text { (wattKg) }\end{array}$ & $\begin{array}{c}\mathrm{NO}_{3} \\
\text { in liquid } \\
{\left[\left.\mathrm{NO}_{3}\right|_{\mathrm{M}}\right.} \\
(\mathrm{mole} / \mathrm{L})\end{array}$ & $\begin{array}{c}\mathrm{NO}_{2} \\
\text { in liquid } \\
{\left[\mathrm{NO}_{2}\right]_{\mathrm{M}}} \\
(\text { mole } / \mathrm{L})\end{array}$ & $\begin{array}{c}\text { Excess } \mathrm{Na} \\
\text { in liquid } \\
{[\mathrm{Na}]_{\mathrm{ex}}} \\
(\mathrm{mole} / \mathrm{L})\end{array}$ & $\begin{array}{c}\mathrm{OH} \\
\text { in liquid } \\
{\left[\left.\mathrm{NO}_{2}\right|_{M}\right.} \\
(\mathrm{mole} / \mathrm{L})\end{array}$ & $\begin{array}{c}\text { TOC } \\
\text { in liquid } \\
{[\text { TOC }]_{\%}} \\
(w t \%)\end{array}$ & $\begin{array}{c}\mathrm{Al} \\
\text { in liquid } \\
{\left[\mathrm{A}||_{\%}\right.} \\
(\mathrm{wt} \%)\end{array}$ & $\begin{array}{c}E_{\mathrm{H} 2} \\
\text { Efficiency } \\
\mathrm{H} 2 \text { by } \\
\text { corrosion }\end{array}$ & $\begin{array}{c}\text { Total } \\
\text { G values } \\
\text { GB/ } / \gamma_{\text {ToT }} \\
\left(\mathrm{H}_{2} / 100 \mathrm{eV}\right)\end{array}$ & $\begin{array}{c}\text { Total } \\
\text { G values } \\
\text { G }^{\text {a }} \\
\left(\mathbf{H}_{2} / 100 \mathrm{eV}\right)\end{array}$ \\
\hline Supernatant & 150.8 & $1.31 \mathrm{E}+05$ & $100 \%$ & 789 & $9.52 \mathrm{E}-05$ & $2.63 \mathrm{E}-07$ & 1.28 & 0.78 & 1.50 & 1.00 & 0.26 & 0.42 & 0.20 & 0.063 & 0.230 \\
\hline Solid Layer & 22.2 & $2.67 \mathrm{E}+04$ & $55 \%$ & 431 & $9.52 \mathrm{E}-05$ & $2.63 \mathrm{E}-07$ & 1.28 & 0.78 & 1.50 & 1.00 & 0.26 & 0.42 & 0.20 & 0.063 & 0.230 \\
\hline
\end{tabular}


RPP-5926 REV 6

Table G1-C. Calculated Hydrogen Generation Rate as Mole Per Unit Waste Weight and Volume

\begin{tabular}{|c|c|c|c|c|c|c|c|c|c|c|}
\hline Tank & $\begin{array}{l}\text { HGR from } \\
\text { radiolysis } \\
\text { RC B } / \gamma_{\text {rad }} \\
\text { (mole } / \mathrm{kg}-\mathrm{d})\end{array}$ & $\begin{array}{l}\text { HGR from } \\
\text { radiolysis } \\
\text { RC }^{\text {alpha }} \text { rad } \\
\text { (mole } / \mathrm{kg}-\mathbf{d})\end{array}$ & $\begin{array}{l}\text { HGR from } \\
\text { thermolysis } \\
\text { RC }_{\text {therm }} \\
\text { (mole } / \mathrm{kg}-\mathrm{d} \text { ) }\end{array}$ & $\begin{array}{l}\text { HGR from } \\
\text { corrosion } \\
\text { RC }_{\text {corr }} \\
\text { (mole } / \text { kg-d) }\end{array}$ & $\begin{array}{c}\text { Total HGR } \\
\text { from model } \\
\text { RC }_{\text {tot }} \\
\text { (mole } / \mathrm{kg}-\mathrm{d})\end{array}$ & $\begin{array}{l}\text { HGR from } \\
\text { radiolysis } \\
\text { RC B } / \gamma_{\text {rad }} \\
\left(\text { mole } / \mathbf{m}^{3}-\mathbf{s}\right)\end{array}$ & 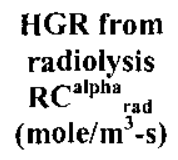 & $\begin{array}{c}\text { HGR from } \\
\text { thermolysis } \\
\mathrm{RC}_{\text {therm }} \\
\left(\mathrm{mole}^{3} \mathrm{~m}^{3}-\mathrm{s}\right)\end{array}$ & $\begin{array}{l}\text { HGR from } \\
\text { corrosion } \\
\text { RC }_{\text {corr }} \\
\left(\mathrm{mole}^{3} \mathrm{~m}^{3}-\mathrm{s}\right)\end{array}$ & $\begin{array}{c}\text { Total HGR } \\
\text { from model } \\
\text { RC }_{\text {tot }} \\
\left.\text { (mole } / \mathbf{m}^{3}-\mathbf{s}\right)\end{array}$ \\
\hline Supernatant & $5.36 \mathrm{E}-08$ & $5.42 \mathrm{E}-10$ & $1.64 \mathrm{E}-08$ & $4.84 \mathrm{E}-07$ & $5.55 \mathrm{E}-07$ & $7.26 \mathrm{E}-10$ & $7.34 \mathrm{E}-12$ & $2.23 \mathrm{E}-10$ & $6.55 \mathrm{E}-09$ & $7.51 \mathrm{E}-09$ \\
\hline Solid Layer & $2.94 \mathrm{E}-08$ & $2.98 \mathrm{E}-10$ & $9.03 \mathrm{E}-09$ & $1.29 \mathrm{E}-06$ & $1.33 \mathrm{E}-06$ & $5.52 \mathrm{E}-10$ & $5.58 \mathrm{E}-12$ & $1.69 \mathrm{E}-10$ & $2.42 \mathrm{E}-08$ & $2.50 \mathrm{E}-08$ \\
\hline
\end{tabular}

Table G1-D. Calculated Total Hydrogen Generation Rate In Dome Space

\begin{tabular}{|c|c|c|c|c|c|c|c|c|c|c|}
\hline Tank & $\begin{array}{l}\text { HGR from } \\
\text { radiolysis } \\
\mathbf{G}_{\text {rad }} B / \gamma \\
\text { (cfm) }\end{array}$ & $\begin{array}{l}\text { HGR from } \\
\text { radiolysis } \\
G_{\text {rad }} \\
\text { (cfm) }\end{array}$ & $\begin{array}{c}\text { HGR from } \\
\text { thermolysis } \\
G_{\text {therm }} \\
\text { (cfm) }\end{array}$ & $\begin{array}{c}\text { HGR from } \\
\text { corrosion } \\
G_{\text {corr }} \\
(\mathrm{cfm})\end{array}$ & $\begin{array}{l}\text { Percent of } \\
\text { HGR from } \\
\text { radiolysis } \\
\left(G_{\text {rad }} B / \gamma\right)_{\%}\end{array}$ & $\begin{array}{l}\text { Percent of } \\
\text { HGR from } \\
\text { radiolysis } \\
\left(G_{\text {rad }}^{\text {alpha }}\right)_{\%}\end{array}$ & $\begin{array}{l}\text { Percent of } \\
\text { HGR from } \\
\text { thermolysis } \\
\left(G_{\text {therm }}\right)_{\%}\end{array}$ & $\begin{array}{c}\text { Percent of } \\
\text { HGR from } \\
\text { corrosion } \\
\left(\mathrm{G}_{\text {corr }}\right)_{\%}\end{array}$ & $\begin{array}{c}\text { Total HGR } \\
\text { from model } \\
\mathrm{G}_{\text {mod }} \\
(\mathrm{cfm})\end{array}$ & $\begin{array}{c}\text { Total HGR } \\
\text { from model } \\
\mathbf{G}_{\text {mod }} \\
(\mathrm{L} / \text { day })\end{array}$ \\
\hline Supernatant & $4.07 \mathrm{E}-06$ & 4. $12 \mathrm{E}-08$ & $1.25 \mathrm{E}-06$ & $3.68 \mathrm{E}-05$ & $9.7 \%$ & $0.10 \%$ & $3.0 \%$ & $87.3 \%$ & $4.21 \mathrm{E}-05$ & 2 \\
\hline Solid Layer & $4.57 \mathrm{E}-07$ & $4.62 \mathrm{E}-09$ & $1.40 \mathrm{E}-07$ & $2.01 \mathrm{E}-05$ & $2.2 \%$ & $0.02 \%$ & $0.7 \%$ & $97.1 \%$ & $2.07 \mathrm{E}-05$ & 1 \\
\hline
\end{tabular}


Table G2-A. Steady-State Gas Concentrations, Release Rates and Flammability Levels Under Normal Ventilation Conditions.

\begin{tabular}{|c|c|c|c|c|c|c|c|c|c|c|c|}
\hline Tank & $\begin{array}{c}\text { Ammonia } \\
\text { headspace } \\
\text { concen. } \\
{\left[\mathrm{NH}_{3}\right]} \\
(\%)\end{array}$ & $\begin{array}{c}\text { Methane } \\
\text { headspace } \\
\text { concen. } \\
{\left[\mathrm{CH}_{4}\right]} \\
(\%)\end{array}$ & $\begin{array}{c}\mathrm{N}_{2} \mathrm{O} \\
\text { headspace } \\
\text { concen. } \\
{\left[\mathrm{N}_{2} \mathrm{O}\right]} \\
(\%)\end{array}$ & $\begin{array}{c}\text { Waste } \\
\text { volume } \\
\mathrm{V}_{\mathrm{w}} \\
\text { (kgal) }\end{array}$ & $\begin{array}{c}\text { Headspace } \\
\text { volume } \\
V_{\mathrm{h}} \\
\left(\mathrm{ft}^{3}\right)\end{array}$ & $\begin{array}{c}\text { Normal } \\
\text { ventilation } \\
\text { rate } \\
\text { (Vr) norm } \\
\text { (cfm) }\end{array}$ & $\begin{array}{c}\text { Steady-state } \\
\text { flammability } \\
\text { at normal } \\
\text { condition } \\
\text { (\% LFL) }\end{array}$ & $\begin{array}{l}\text { Ammonia } \\
\text { release } \\
\text { rate } \\
\operatorname{Rr}\left(\mathrm{NH}_{3}\right) \\
(\mathrm{cfm})\end{array}$ & $\begin{array}{c}\mathrm{N}_{2} \mathrm{O} \\
\text { release } \\
\text { rate } \\
\operatorname{Rr}\left(\mathrm{N}_{2} \mathrm{O}\right) \\
(\mathbf{c f m})\end{array}$ & $\begin{array}{c}\text { Model } \\
\text { hydrogen } \\
\text { release } \\
\text { rate } \\
\operatorname{Rr}\left(\mathrm{H}_{2}\right) \\
(\mathrm{cfm})\end{array}$ & $\begin{array}{c}\text { Methane } \\
\text { release } \\
\text { rate } \\
\mathrm{Rr}^{\left(\mathrm{CH}_{4}\right)} \\
(\mathrm{cfm})\end{array}$ \\
\hline 241-C-204 & 0.0001 & 0.0001 & 0.0014 & 34 & 3,408 & 3 & $0.05 \%$ & $2.18 \mathrm{E}-06$ & $4.31 \mathrm{E}-05$ & $6.28 \mathrm{E}-05$ & $2.76 \mathrm{E}-06$ \\
\hline
\end{tabular}

Table G2-B Steady-State Flammability Under Barometric Breathing and Time to $25 \%$ of the LFL under Zero Vent

\begin{tabular}{|c|c|c|c|c|c|c|c|c|c|c|c|}
\hline Tank & $\begin{array}{c}\text { Off-Normal } \\
\text { Barometric } \\
\text { Breathing } \\
\text { Rate }(\mathrm{Vr})_{\mathrm{bb}} \\
\text { (cfm) }\end{array}$ & $\begin{array}{c}\text { Steady } \\
\text { State } \\
\text { Hydrogen } \\
\text { Concen. } \\
{\left[\mathrm{H}_{2}\right](\%)}\end{array}$ & $\begin{array}{c}\text { Steady } \\
\text { State } \\
\text { Ammonia } \\
\text { Concen. } \\
{\left[\mathrm{NH}_{3}\right]} \\
(\%)\end{array}$ & $\begin{array}{c}\text { Steady } \\
\text { State } \\
\text { Methane } \\
\text { Concen. } \\
{\left[\mathrm{CH}_{4}\right]} \\
(\%)\end{array}$ & $\begin{array}{c}\text { Steady } \\
\text { State } \\
\mathrm{N}_{2} \mathrm{O} \\
\text { Concen. } \\
{\left[\mathrm{N}_{2} \mathrm{O}\right]} \\
(\%)\end{array}$ & $\begin{array}{c}\text { Steady } \\
\text { State } \\
\text { Hydrogen } \\
\text { Flammab. } \\
(\%)\end{array}$ & $\begin{array}{c}\text { Steady } \\
\text { State } \\
\text { Ammonia } \\
\text { Flammab. } \\
\qquad \%)\end{array}$ & $\begin{array}{c}\text { Steady } \\
\text { State } \\
\text { Methane } \\
\text { Flammab. } \\
(\%)\end{array}$ & $\begin{array}{c}\text { Steady } \\
\text { State } \\
\text { Total } \\
\text { Flammab } \\
(\%)\end{array}$ & $\begin{array}{c}\text { Time to } \\
\text { Reach } \\
25 \% \\
\text { of the } \\
\text { LFL } \\
\text { under } \\
\text { Diffusion } \\
\text { (days) }\end{array}$ & $\begin{array}{c}\text { Time to } \\
\text { Reach } \\
100 \% \\
\text { of the } \\
\text { LFL } \\
\text { under } \\
\text { diffusion } \\
\text { (days) }\end{array}$ \\
\hline 241-C-204 & 0.01 & 0.59 & 0.02 & 0.03 & 0.40 & 14.7 & 0.14 & 0.52 & 15.4 & 365 & 1,500 \\
\hline
\end{tabular}


RPP-5926 REV 6

APPENDIX H

FLAMMABILITY EVALUATION OF TIME TO REACH 25\% AND 100\% OF THE LOWER FLAMMABILITY LIMIT FOR DOUBLE-SHELL TANK 241-AY-102 AT VARIOUS WASTE LEVELS WHILE RECEIVING EXHAUSTER CONDENSATE COLLECTED FROM CATCH TANK 241-AZ-151 
RPP-5926 REV 6

This page intentionally left blank. 


\section{CONTENTS}

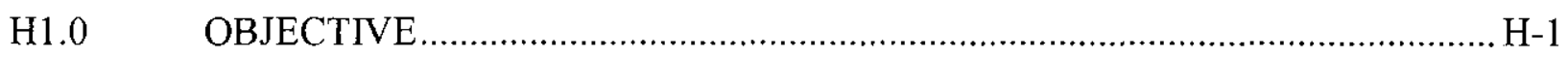

H2.0 SUMMARY OF RESULTS AND CONCLUSIONS ................................. H-1

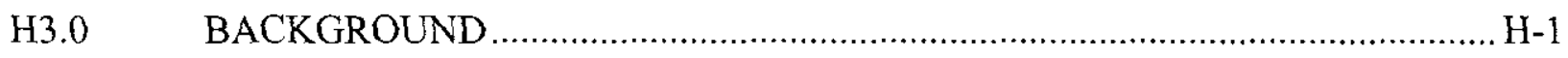

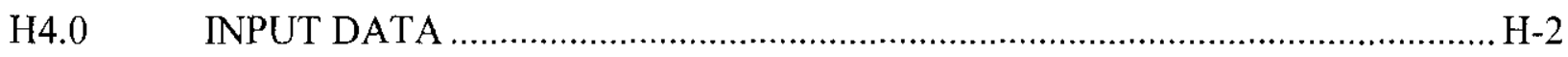

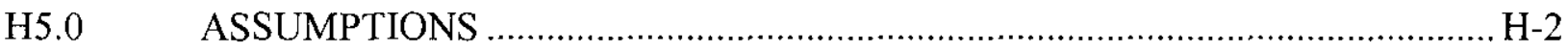

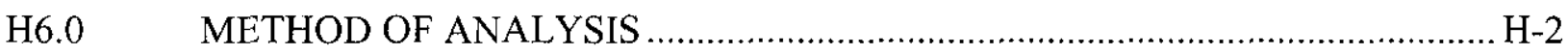

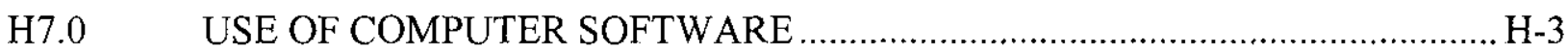

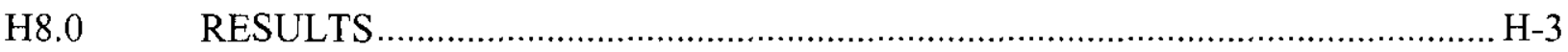

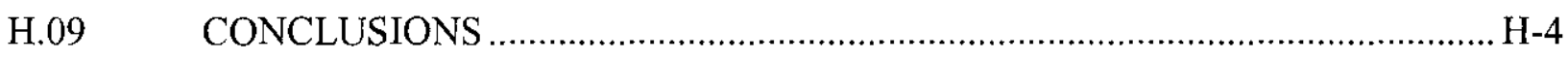

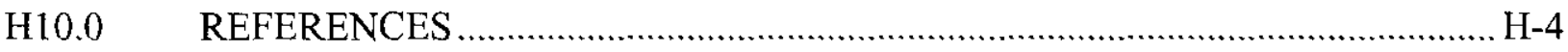

\section{LIST OF TABLES}

Table H1-A. Input Data for Hydrogen Generation Rate Model Calculations. ........................ H-5

Table H1-B. Derived Data for Hydrogen Generation Rate Model Calculations ......................H-6

Table H1-C. Calculated Hydrogen Generation Rate as Mole Per Unit Waste Weight and Volume

Table H1-D. Calculated Total Hydrogen Generation Rate In Dome Space ...........................H-8

Table H2-A. Steady-State Gas Concentrations, Release Rates and Flammability Levels Under Normal Ventilation Conditions.

Table H2-B Steady-State Flammability Under Barometric Breathing and Time to $25 \%$ of the LFL under Zero Vent. 
RPP-5926 REV 6

This page intentionally left blank.

H-iv 
RPP-5926 REV 6

\section{APPENDIX H}

\section{FLAMMABILITY EVALUATION OF TIME TO REACH 25\% AND 100\% OF THE LOWER FLAMMABILITY LIMIT FOR DOUBLE-SHELL TANK 241-AY-102 AT VARIOUS WASTE LEVELS WHILE RECEIVING EXHAUSTER CONDENSATE COLLECTED FROM CATCH TANK 241-AZ-151}

\section{H1.0 OBJECTIVE}

This calculation provides the technical basis for an amendment to the HNF-SD-WM-TSR-006, Technical Safety Requirements, LCO 3.2.1, "DST Primary Ventilation Systems, Surveillance Requirement 3.2.1.4." The amendment is needed because DST 241-AY-102 will continue receiving condensate. The calculation provides the time to reach $25 \%$ and $100 \%$ of the lower flammability limit (LFL) as function of waste level. The evaluation determines the hydrogen generation rate (HGR) and the minimum ventilation rate required to keep below $25 \%$ and $100 \%$ of the LFL for the given waste level.

The following calculation was issued in Rev. 3-D and updated in Rev. 4 of this document. In Table B-4, Appendix B, Rev. 6 of this document, it is found that HGR has dropped by $30 \%$ from Rev. 4 calculation based on latest core sample results (August 2005). So the following evaluation is bounding and there is no intention to update the calculation for Rev. 6 of this document.

\section{H2.0 SUMMARY OF RESULTS AND CONCLUSIONS}

The evaluation shows that the times required to reach $25 \%$ of the LFL are $12,10,8$ and 6 days for the waste levels of 313.8 in. (current waste level), 333.0 in., 366.3 in., and 399.1 in., respectively, under zero ventilation. For all calculated waste level cases, the HGR is $3.93 \mathrm{E}-2 \mathrm{ft}^{3} / \mathrm{min}$, and the minimum ventilation rates to stay within $25 \%$ and $100 \%$ of the LFL level are around 4.0 and $1.0 \mathrm{ft}^{3} / \mathrm{min}$, respectively, for all waste levels calculated.

\section{H3.0 BACKGROUND}

Condensate from tanks in the 241-AY/AZ Tank Farms is collected in catch tank 241-AZ-151 and directed to DST 241-AY-102. Currently, the waste level of DST 241-AY-102 is about 313.8 in., and it will increase approximately $3 \mathrm{in}$. per month because of condensate received from DST 241-AZ-151. LCO 3.2.1, "Surveillance Requirement 3.2.1.4," specifies that the surveillance time for flammable gas in the 241-AY tanks is 5 days. Surveillance times plus the time needed to complete Actions described in LCO 3.2.1 must be equal to or less than the time it takes the flammable gas concentration in the tank to reach $25 \%$ of the LFL. At the current waste level in DST 241-AY-102, the combined Surveillance time and Action time is equal to the time 
to $25 \%$ of the LFL. To continue receiving condensate in DST $241-\mathrm{AY}-102$, the Surveillance time must be changed to reflect the changes in the time to reach $25 \%$ of the LFL.

This appendix calculation determines the corresponding time for DST $241-\mathrm{AY}-102$ to reach $25 \%$ of the LFL as condensate received from DST 241-AZ-151 increases the waste level in DST 241-AY-102. The results will be used for an amendment to RPP-13033, Tank Farms Documented Safety Analysis, that specifies a new surveillance requirement for the 241-AY Tank Farm such that the condensate can be added until the operational level limit of 364 in., documented in OSD-T-151-00007, Operating Specifications for the Double-Shell Storage Tanks, is reached.

\section{H4.0 INPUT DATA}

The input data for HGR calculation, such as waste compositions of total organic carbon (TOC), $\mathrm{NO}_{3}, \mathrm{NO}_{2}, \mathrm{Al}, \mathrm{Sr}-90, \mathrm{Cs}-137$ and waste density, are exactly the same as the previous evaluation listed in Appendix B, Table B-1 of this document, except the condensate addition dilutes the composition and density of supernatant. The composition of condensate is treated as water although the condensate is slightly contaminated. The volume of condensate (Table $\mathrm{H}-1 \mathrm{a}$, column 2) represents the amount of condensate added on top of the supernatant since the last evaluation (Appendix H, Table B-1). The condensate additions of 24, 200, 375, 548, 719, and $889 \mathrm{~kL}$ correspond to the final waste levels of $316.0,333.0,349.7,366.3,382.7$ and $399.1 \mathrm{in}$., respectively, and are listed in Table $\mathrm{H}-2 \mathrm{~b}$. The waste compositions and temperatures after the condensate additions also are given in Table $\mathrm{H}-\mathrm{la}$. The temperature is the same as the previous calculation, following the rule of using the maximum temperature from the past year. The temperature reflects $5^{\circ} \mathrm{C}$ higher temperature than the baseline temperature for double-shell tanks (DST) as discussed in Chapters 3.0 and 4.0 .

\section{H5.0 ASSUMPTIONS}

The condensate is treated as if it is contaminated water. The condensate addition is assumed to be well mixed with the supernatant layer of DST 241-AY-102. The waste composition and density of supernatant are corrected by the dilution factor.

\section{H6.0 METHOD OF ANALYSIS}

The methodology and equations for the flammability evaluation used in this study are discussed in Chapter 3.0. This methodology is developed to calculate the gas release rate, gas generation rate, flammability level, and times to reach $25 \%$ and $100 \%$ of the LFL for a given ventilation rate. 
Given the calculated HGRs along with historical gas release rates of ammonia and methane from the identified waste, the time to reach $25 \%$ of the LFL is calculated. Note that condensate additions will decrease the volume of the dome space; and, therefore, the time to reach $25 \%$ of the LFL for a given ventilation rate will decrease. To find the waste level of water addition, the volume of condensate is gradually increased until the time to reach $25 \%$ of the LFL is $11,10,9$, 8,7 and 6 days. Also, the time to reach $25 \%$ of the LFL is calculated at the current waste level of $313.8 \mathrm{in}$. and the operational waste level of $364 \mathrm{in.}$

\section{H7.0 USE OF COMPUTER SOFTWARE}

This case study uses a validated Excel ${ }^{8}$ spreadsheet, documented in RPP-5926, Rev. 4, to conduct the calculations (RPP-5926-8050-R3-LFL-CAL-T2-102004.xls). Refer to the spreadsheet verification form in Appendix I, page I-3. This spreadsheet used the input data to calculate the gas generation rates and then calculate the flammability and time to reach the LFL for a given ventilation rate.

\section{H8.0 RESULTS}

Tables $\mathrm{H}-1 \mathrm{~b}$ to $\mathrm{H}-\mathrm{l}$ d provide the derived term for the HGR model calculation from input data (Table $\mathrm{H}-1 \mathrm{a}$ ), the unit HGRs, and total HGRs, respectively. The HGRs are roughly $3.9 \mathrm{E}-02 \mathrm{ft}^{3} / \mathrm{min}$ (about $1,598 \mathrm{~L} /$ day) for all calculated waste levels as shown in Table H-2B. The condensate addition increases the volume of supernatant and also slightly reduces concentrations, resulting in almost no change in the total HGR for the supernatant layer. All of the calculations include $5{ }^{\circ} \mathrm{C}$ higher temperature than the waste temperature as discussed in Chapter 3.0 and 4.0 .

The times to reach $25 \%$ of the LFL at the current waste level of 313.8 in. and the operational waste level of $364 \mathrm{in}$. are 12 and 9 days, respectively. The condensate additions increasing the waste level to $316.0,333.0,349.7,366.3,382.7$ and $399.1 \mathrm{i}$. result in $11,10,9,8,7$, and 6 days, respectively, for the time to reach $25 \%$ of the LFL; and are $43,40,37,33,30$, and 26 days, respectively, for the time to reach $100 \%$ of the LFL under zero ventilation. The minimum ventilation rates to keep the headspace below $25 \%$ and $100 \%$ of the LFL are about 4.0 and $1.0 \mathrm{ft}^{3} / \mathrm{min}$, respectively.

The 241-AY Tank Farm tanks cannot be filled to 390 in. due to physical piping limitations. As part of the parametric study, times to LFL down to 7 days was calculated.

\footnotetext{
${ }^{8}$ Excel is a registered trademark of Microsoft Corporation, Redrnond, Washington.
} 


\section{H.09 CONCLUSIONS}

The time to reach $25 \%$ of the LFL for the current waste level of $313.8 \mathrm{in}$. is 12 days, which is still within the current flammable gas surveillance requirement for the 241-AY Tank Farm given in HNF-SD-WM-TSR-006, LCO 3.2.1, "DST Primary Ventilation Systems." The time to reach $25 \%$ of the LFL is 9 days for the operational waste limit of 364 in. under zero ventilation.

\section{H10.0 REFERENCES}

HNF-SD-WM-TSR-006, Tank Farms Technical Safety Requirements, as amended, CH2M HILL Hanford Group, Inc., Richland, Washington.

OSD-T-151-00007, 2003, Operating Specifications for the Double-Shell Storage Tanks, Rev. I-7, CH2M HILL Hanford Group, Inc., Richland, Washington.

RPP-13033, Tank Farms Documented Safety Analysis, as amended, CH2M HILL Hanford Group, Inc., Richland, Washington. 
RPP-5926 REV 6

Table H1-A. Input Data for Hydrogen Generation Rate Model Calculations for 241-AY-102 Condensate Addition.

\begin{tabular}{|c|c|c|c|c|c|c|c|c|c|c|c|c|c|c|c|c|}
\hline Cases & $\begin{array}{l}\text { Waste } \\
\text { layer }\end{array}$ & $\begin{array}{c}\text { Volume of } \\
\text { water } \\
\text { addition } \\
(k L) \text { and } \\
\text { Dilution } \\
\text { factor }\end{array}$ & $\begin{array}{c}\text { TOC } \\
\text { in liquid } \\
{[\mathrm{TOC}]} \\
(\mu \mathrm{g} / \mathrm{mL})\end{array}$ & $\begin{array}{c}\mathrm{NO}_{2} \\
\text { in liquid } \\
{\left[\mathrm{NO}_{2}\right]} \\
(\mu \mathrm{g} / \mathrm{mL})\end{array}$ & $\begin{array}{c}\mathrm{NO}_{3} \\
\text { in liquid } \\
{\left[\mathrm{NO}_{3}\right]} \\
(\mu \mathrm{g} / \mathrm{mL})\end{array}$ & $\begin{array}{c}\text { Al } \\
\text { in liquid } \\
\text { [Al] } \\
(\mu \mathrm{g} / \mathrm{mL})\end{array}$ & $\begin{array}{c}\text { Na } \\
\text { in liquid } \\
\text { [Na] } \\
(\mu \mathrm{g} / \mathbf{m L})\end{array}$ & $\begin{array}{c}{ }^{90} \mathrm{Sr} \\
\text { in waste } \\
{[\mathrm{Sr}]} \\
(\mu \mathrm{Ci} / \mathrm{g})\end{array}$ & $\begin{array}{c}{ }^{137} \mathrm{Cs} \\
\text { in waste } \\
{[\mathrm{Cs}]} \\
(\mu \mathrm{Ci} / \mathrm{g})\end{array}$ & $\begin{array}{c}\text { Bulk } \\
\text { density } \\
D \\
(\mathrm{~g} / \mathrm{mL})\end{array}$ & $\begin{array}{c}\text { Liquid } \\
\text { Density } \\
\mathrm{D}_{\mathrm{L}} \\
(\mathrm{g} / \mathrm{ml})\end{array}$ & $\mid \begin{array}{c}\text { Non-RGS } \\
\text { waste } \\
\text { volume } \\
V \\
(k L)\end{array}$ & $\begin{array}{c}\text { Bulk } \\
\text { water } \\
{\left[\mathrm{H}_{2} \mathrm{O}\right]} \\
(\mathbf{w t} \%)\end{array}$ & $\begin{array}{c}\text { Liquid } \\
\text { water } \\
{\left[\mathrm{H}_{2} \mathrm{O}\right]} \\
\left(\mathrm{wt}^{2} \%\right)\end{array}$ & $\begin{array}{c}\text { Waste } \\
\text { temp. } \\
\mathbf{T}_{w} \\
\left({ }^{\circ} \mathrm{C}\right)\end{array}$ & $\begin{array}{c}\text { Dome } \\
\text { temp. } \\
T_{d} \\
\left({ }^{\circ} \mathrm{C}\right)\end{array}$ \\
\hline 1 & $\begin{array}{l}\text { Supernatant } \\
\text { Solid Layer }\end{array}$ & 0 & $\begin{array}{l}8.67 \mathrm{E}+02 \\
1.66 \mathrm{E}+03\end{array}$ & $\begin{array}{l}3.17 \mathrm{E}+04 \\
8.33 \mathrm{E}+02\end{array}$ & $\begin{array}{l}4.15 \mathrm{E}+02 \\
3.81 \mathrm{E}+01\end{array}$ & $\begin{array}{l}6.04 \mathrm{E}+04 \\
7.33 \mathrm{E}+04\end{array}$ & $\begin{array}{l}\text { I. } 22 \mathrm{E}+03 \\
2.69 \mathrm{E}+02\end{array}$ & $\begin{array}{l}1.02 E+00 \\
9.85 E+03\end{array}$ & $\begin{array}{c}1.80 \mathrm{E}+01 \\
2.70 \mathrm{E}+02\end{array}$ & $\begin{array}{l}1.18 \\
1.55\end{array}$ & $\begin{array}{l}1.18 \\
1.16\end{array}$ & $\begin{array}{l}2700 \\
571\end{array}$ & $\begin{array}{l}82 \% \\
45 \%\end{array}$ & $\begin{array}{l}82 \% \\
79 \%\end{array}$ & $\begin{array}{l}53 \\
75\end{array}$ & $\begin{array}{l}49 \\
49\end{array}$ \\
\hline 2 & $\begin{array}{l}\text { Supernatant } \\
\text { Solid Layer }\end{array}$ & 24 & $\left\{\begin{array}{l}8.67 \mathrm{E}+02 \\
1.66 \mathrm{E}+03\end{array}\right.$ & $\begin{array}{l}3.15 \mathrm{E}+04 \\
8.33 \mathrm{E}+02\end{array}$ & $\begin{array}{l}\text { 4. } 11 \mathrm{E}+02 \\
3.81 \mathrm{E}+01\end{array}$ & $\begin{array}{l}5.98 E+04 \\
7.33 E+04\end{array}$ & $\begin{array}{l}1.21 \mathrm{E}+03 \\
2.69 \mathrm{E}+02\end{array}$ & $\begin{array}{l}1.01 \mathrm{E}+00 \\
9.85 \mathrm{E}+03\end{array}$ & $\begin{array}{l}1.78 \mathrm{E}+01 \\
2.70 \mathrm{E}+02\end{array}$ & $\begin{array}{l}1.18 \\
1.55\end{array}$ & $\begin{array}{l}1.18 \\
1.16\end{array}$ & $\begin{array}{l}2724 \\
571\end{array}$ & $\begin{array}{l}83 \% \\
45 \%\end{array}$ & $\begin{array}{l}83 \% \\
79 \%\end{array}$ & $\begin{array}{l}53 \\
75\end{array}$ & $\begin{array}{l}49 \\
49\end{array}$ \\
\hline 3 & $\begin{array}{l}\text { Supernatant } \\
\text { Solid Layer }\end{array}$ & 200 & $\left\{\begin{array}{l}8.67 \mathrm{E}+02 \\
1.66 \mathrm{E}+03\end{array}\right.$ & $\begin{array}{l}2.95 E+04 \\
8.33 E+02\end{array}$ & $\begin{array}{l}3.86 \mathrm{E}+02 \\
3.81 \mathrm{E}+01\end{array}$ & $\begin{array}{l}5.62 E+04 \\
7.33 E+04\end{array}$ & $\begin{array}{l}1.14 \mathrm{E}+03 \\
2.69 \mathrm{E}+02\end{array}$ & $\begin{array}{l}9.56 \mathrm{E}-01 \\
9.85 \mathrm{E}+03\end{array}$ & $\begin{array}{l}1.69 \mathrm{E}+01 \\
2.70 \mathrm{E}+02\end{array}$ & $\begin{array}{l}1.17 \\
1.55\end{array}$ & $\begin{array}{l}1.17 \\
1.16\end{array}$ & $\begin{array}{l}2900 \\
571\end{array}$ & $\begin{array}{l}83 \% \\
45 \%\end{array}$ & $\begin{array}{l}83 \% \\
79 \%\end{array}$ & $\begin{array}{l}53 \\
75\end{array}$ & $\begin{array}{l}49 \\
49\end{array}$ \\
\hline 4 & $\begin{array}{l}\text { Supernatant } \\
\text { Solid Layer }\end{array}$ & 375 & $\left\{\begin{array}{l}8.67 \mathrm{E}+02 \\
1.66 \mathrm{E}+03\end{array}\right.$ & $\begin{array}{l}2.79 \mathrm{E}+04 \\
8.33 \mathrm{E}+02\end{array}$ & $\begin{array}{l}3.64 \mathrm{E}+02 \\
3.81 \mathrm{E}+01\end{array}$ & $\begin{array}{l}5.30 \mathrm{E}+04 \\
7.33 \mathrm{E}+04\end{array}$ & $\begin{array}{l}1.07 E+03 \\
2.69 E+02\end{array}$ & $\begin{array}{l}9.09 \mathrm{E}-01 \\
9.85 \mathrm{E}+03\end{array}$ & $\begin{array}{l}1.61 E+01 \\
2.70 E+02\end{array}$ & $\begin{array}{l}1.16 \\
1.55\end{array}$ & $\begin{array}{l}1.16 \\
1.16\end{array}$ & $\begin{array}{l}3075 \\
571\end{array}$ & $\begin{array}{l}84 \% \\
45 \%\end{array}$ & $\begin{array}{l}84 \% \\
79 \%\end{array}$ & $\begin{array}{l}53 \\
75\end{array}$ & $\begin{array}{l}49 \\
49\end{array}$ \\
\hline 5 & $\begin{array}{l}\text { Supernatant } \\
\text { Solid Layer }\end{array}$ & 524 & $\left\{\begin{array}{l}7.26 \mathrm{E}+02 \\
1.66 \mathrm{E}+03\end{array}\right.$ & $\begin{array}{l}2.66 \mathrm{E}+04 \\
8.33 \mathrm{E}+02\end{array}$ & $\begin{array}{l}3.47 \mathrm{E}+02 \\
3.81 \mathrm{E}+01\end{array}$ & $\begin{array}{l}5.06 \mathrm{E}+04 \\
7.33 \mathrm{E}+04\end{array}$ & $\begin{array}{l}1.02 \mathrm{E}+03 \\
2.69 \mathrm{E}+02\end{array}$ & $\begin{array}{l}8.72 \mathrm{E}-01 \\
9.85 \mathrm{E}+03\end{array}$ & $\begin{array}{l}1.54 \mathrm{E}+01 \\
2.70 \mathrm{E}+02\end{array}$ & $\begin{array}{l}1.15 \\
1.55\end{array}$ & $\begin{array}{l}1.15 \\
1.16\end{array}$ & $\begin{array}{l}3224 \\
571\end{array}$ & $\begin{array}{l}85 \% \\
45 \%\end{array}$ & $\begin{array}{l}85 \% \\
79 \%\end{array}$ & $\begin{array}{l}53 \\
75\end{array}$ & $\begin{array}{l}49 \\
49\end{array}$ \\
\hline 6 & $\begin{array}{l}\text { Supernatant } \\
\text { Solid Layer }\end{array}$ & 548 & $\left\{\begin{array}{l}8.67 \mathrm{E}+02 \\
1.66 \mathrm{E}+03\end{array}\right.$ & $\begin{array}{l}2.64 \mathrm{E}+04 \\
8.33 \mathrm{E}+02\end{array}$ & $\begin{array}{l}3.45 \mathrm{E}+02 \\
3.81 \mathrm{E}+01\end{array}$ & $\begin{array}{l}5.02 \mathrm{E}+04 \\
7.33 \mathrm{E}+04\end{array}$ & $\begin{array}{l}1.02 \mathrm{E}+03 \\
2.69 \mathrm{E}+02\end{array}$ & $\begin{array}{l}8.67 \mathrm{E}-01 \\
9.85 \mathrm{E}+03\end{array}$ & $\begin{array}{l}1.53 \mathrm{E}+01 \\
2.70 \mathrm{E}+02\end{array}$ & $\begin{array}{l}1.15 \\
1.55\end{array}$ & $\begin{array}{l}1.15 \\
1.16\end{array}$ & $\begin{array}{l}3248 \\
571\end{array}$ & $\begin{array}{l}85 \% \\
45 \%\end{array}$ & $\begin{array}{l}85 \% \\
79 \%\end{array}$ & $\begin{array}{l}53 \\
75\end{array}$ & $\begin{array}{l}49 \\
49\end{array}$ \\
\hline 7 & $\begin{array}{l}\text { Supernatant } \\
\text { Solid Layer }\end{array}$ & 719 & $\left\{\begin{array}{l}8.67 \mathrm{E}+02 \\
1.66 \mathrm{E}+03\end{array}\right.$ & $\begin{array}{l}2.51 E+04 \\
8.33 E+02\end{array}$ & $\begin{array}{l}3.28 \mathrm{E}+02 \\
3.81 \mathrm{E}+0 \mathrm{l}\end{array}$ & $\begin{array}{l}4.77 \mathrm{E}+04 \\
7.33 \mathrm{E}+04\end{array}$ & $\begin{array}{l}9.65 \mathrm{E}+02 \\
2.69 \mathrm{E}+02\end{array}$ & $\begin{array}{l}8.29 \mathrm{E}-01 \\
9.85 \mathrm{E}+03\end{array}$ & $\begin{array}{l}1.46 \mathrm{E}+01 \\
2.70 \mathrm{E}+02\end{array}$ & $\begin{array}{l}1.14 \\
1.55\end{array}$ & $\begin{array}{l}1.14 \\
1.16\end{array}$ & $\begin{array}{l}3419 \\
571\end{array}$ & $\begin{array}{l}86 \% \\
45 \%\end{array}$ & $\begin{array}{l}86 \% \\
79 \%\end{array}$ & $\begin{array}{l}53 \\
75\end{array}$ & $\begin{array}{l}49 \\
49\end{array}$ \\
\hline 8 & $\begin{array}{l}\text { Supernatant } \\
\text { Solid Layer }\end{array}$ & 889 & $\left\{\begin{array}{l}8.67 \mathrm{E}+02 \\
1.66 \mathrm{E}+03\end{array}\right.$ & $\begin{array}{l}2.39 \mathrm{E}+04 \\
8.33 \mathrm{E}+02\end{array}$ & $\begin{array}{l}3.12 \mathrm{E}+02 \\
3.81 \mathrm{E}+01\end{array}$ & $\begin{array}{l}4.54 \mathrm{E}+04 \\
7.33 \mathrm{E}+04\end{array}$ & $\begin{array}{l}9.19 E+02 \\
2.69 E+02\end{array}$ & $\begin{array}{l}7.94 E-01 \\
9.85 E+03\end{array}$ & $\begin{array}{l}1.40 \mathrm{E}+01 \\
2.70 \mathrm{E}+02\end{array}$ & $\begin{array}{l}1.13 \\
1.55\end{array}$ & $\begin{array}{l}1.13 \\
1.16\end{array}$ & $\begin{array}{l}3589 \\
571\end{array}$ & $\begin{array}{l}86 \% \\
45 \%\end{array}$ & $\begin{array}{l}86 \% \\
79 \%\end{array}$ & $\begin{array}{l}53 \\
75\end{array}$ & $\begin{array}{l}49 \\
49\end{array}$ \\
\hline
\end{tabular}


Table H1-B. Derived Data for Hydrogen Generation Rate Model Calculations for 241-AY-102 on Condensate Addition.

\begin{tabular}{|c|c|c|c|c|c|c|c|c|c|c|c|c|c|c|c|c|}
\hline Case & $\begin{array}{l}\text { Waste } \\
\text { layer }\end{array}$ & $\begin{array}{l}\text { Waste } \\
\text { level } \\
D_{w} \\
\text { (inch) }\end{array}$ & $\begin{array}{c}\text { Total } \\
\text { mass } \\
\mathbf{M} \\
(\mathrm{kg})\end{array}$ & $\begin{array}{c}\text { Liquid } \\
\text { in } \\
\text { waste } \\
(\text { wt } \%)\end{array}$ & $\begin{array}{c}\text { Wetted } \\
\text { area } \\
\mathbf{A}_{\text {wet }} \\
\left.\text { (ft }^{2}\right)\end{array}$ & $\begin{array}{c}\text { Heat load } \\
\text { per kg } \\
\text { H }_{\mathrm{L}-\beta / \gamma} \\
\text { (watt } / \mathrm{kg} \text { ) }\end{array}$ & $\begin{array}{c}\text { Tank } \\
\text { heat load } \\
\mathrm{H}_{\mathrm{L}-\alpha} \\
\text { (watt } / \mathrm{kg} \text { ) }\end{array}$ & $\begin{array}{c}\mathrm{NO}_{3} \\
\text { in liquid } \\
{\left[\mathrm{NO}_{3}\right]_{\mathrm{M}}} \\
\left(\mathrm{mole}_{\mathrm{L}} \mathrm{L}\right)\end{array}$ & $\begin{array}{c}\mathrm{NO}_{2} \\
\text { in liquid } \\
{\left[\mathrm{NO}_{2} \mathrm{l}_{\mathrm{M}}\right.} \\
(\mathrm{mole} / \mathrm{L})\end{array}$ & $\begin{array}{c}\text { Excess } \mathrm{Na} \\
\text { in liquid } \\
{\left[\left.\mathrm{Na}\right|_{\mathrm{ex}}\right.} \\
(\mathrm{mole} / \mathrm{L})\end{array}$ & $\begin{array}{c}\mathrm{OH} \\
\text { in liquid } \\
{\left[\mathrm{NO}_{2}\right]_{\mathrm{M}}} \\
(\mathrm{mole} / \mathrm{L})\end{array}$ & $\begin{array}{c}\text { TOC } \\
\text { in liquid } \\
{[\text { TOC }]_{\%}} \\
(w t \%)\end{array}$ & $\begin{array}{c}\text { AI } \\
\text { in } \\
\text { liquid } \\
{[A]_{\%}} \\
(\mathbf{w t} \%)\end{array}$ & $\begin{array}{c}\mathbf{E}_{\mathrm{H} 2} \\
\text { Efficiency } \\
\mathbf{H} 2 \text { by } \\
\text { corrosion }\end{array}$ & $\begin{array}{c}\text { Total } \\
\text { G values } \\
\mathbf{G}_{\text {tot }-\beta / \gamma} \\
\left(\mathrm{H}_{2} / 100 \mathrm{eV}\right)\end{array}$ & $\begin{array}{c}\text { Total } \\
\text { G values } \\
\mathbf{G}_{\text {tot- } \alpha} \\
\left(\mathrm{H}_{2} / 100 \mathrm{eV}\right)\end{array}$ \\
\hline \multirow[t]{2}{*}{1} & Supernatant & 259.0 & $3.18 \mathrm{E}+06$ & $100 \%$ & 5085 & $9.18 \mathrm{E}-05$ & $6.65 \mathrm{E}-08$ & 0.01 & 0.69 & 1.93 & 0.23 & 0.07 & 0.10 & 0.50 & 0.134 & 0.729 \\
\hline & Solid Layer & 54.8 & $8.83 E+05$ & $57 \%$ & 5493 & $6.71 \mathrm{E}-02$ & $2.55 \mathrm{E}-04$ & 0.00 & 0.02 & 3.17 & 0.23 & 0.14 & 0.02 & 0.50 & 0.184 & 1.092 \\
\hline \multirow[t]{2}{*}{2} & Supernatant & 261.3 & $3.20 \mathrm{E}+06$ & $100 \%$ & 5130 & $9.11 \mathrm{E}-05$ & $6.60 \mathrm{E}-08$ & 0.01 & 0.68 & 1.91 & 0.23 & 0.07 & 0.10 & 0.50 & 0.135 & 0.731 \\
\hline & Solid Layer & 54.8 & $8.83 \mathrm{E}+05$ & $57 \%$ & 5493 & $6.71 \mathrm{E}-02$ & $2.55 \mathrm{E}-04$ & 0.00 & 0.02 & 3.17 & 0.23 & 0.14 & 0.02 & 0.50 & 0.184 & 1.092 \\
\hline \multirow[t]{2}{*}{3} & Supernatant & 278.2 & $3.38 \mathrm{E}+06$ & $100 \%$ & 5463 & $8.63 \mathrm{E}-05$ & $6.25 \mathrm{E}-08$ & 0.01 & 0.64 & 1.79 & 0.23 & 0.07 & 0.10 & 0.50 & 0.144 & 0.745 \\
\hline & Solid Layer & 54.8 & $8.83 E+05$ & $57 \%$ & 5493 & $6.71 \mathrm{E}-02$ & $2.55 \mathrm{E}-04$ & 0.00 & 0.02 & 3.17 & 0.23 & 0.14 & 0.02 & 0.50 & 0.184 & 1.092 \\
\hline \multirow[t]{2}{*}{4} & Supernatant & 295.0 & $3.55 \mathrm{E}+06$ & $100 \%$ & 5792 & $8.21 \mathrm{E}-05$ & $5.95 \mathrm{E}-08$ & 0.01 & 0.61 & 1.69 & 0.23 & 0.07 & 0.09 & 0.50 & 0.152 & 0.757 \\
\hline & Solid Layer & 54.8 & $8.83 E+05$ & $57 \%$ & 5493 & $6.71 \mathrm{E}-02$ & $2.55 \mathrm{E}-04$ & 0.00 & 0.02 & 3.17 & 0.23 & 0.14 & 0.02 & 0.50 & 0.184 & 1.092 \\
\hline \multirow[t]{2}{*}{5} & Supernatant & 309.2 & $3.70 \mathrm{E}+06$ & $100 \%$ & 6117 & $7.88 \mathrm{E}-05$ & $5.71 \mathrm{E}-08$ & 0.01 & 0.58 & 1.61 & 0.23 & 0.06 & 0.09 & 0.50 & 0.157 & 0.810 \\
\hline & Solid Layer & 54.8 & $8.83 E+05$ & $57 \%$ & 5493 & $6.71 \mathrm{E}-02$ & $2.55 \mathrm{E}-04$ & 0.00 & 0.02 & 3.17 & 0.23 & 0.14 & 0.02 & 0.50 & 0.184 & 1.092 \\
\hline \multirow[t]{2}{*}{6} & Supernatant & 311.5 & $3.73 E+06$ & $100 \%$ & 6117 & $7.83 \mathrm{E}-05$ & $5.67 \mathrm{E}-08$ & 0.01 & 0.57 & 1.60 & 0.23 & 0.08 & 0.09 & 0.50 & 0.159 & 0.769 \\
\hline & Solid Layer & 54.8 & $8.83 E+05$ & $57 \%$ & 5493 & $6.71 \mathrm{E}-02$ & $2.55 \mathrm{E}-04$ & 0.00 & 0.02 & 3.17 & 0.23 & 0.14 & 0.02 & 0.50 & 0.184 & 1.092 \\
\hline \multirow[t]{2}{*}{7} & Supernatant & 328.0 & $3.90 \mathrm{E}+06$ & $100 \%$ & 6440 & 7.48E-05 & $5.42 \mathrm{E}-08$ & 0.01 & 0.54 & 1.52 & 0.23 & 0.08 & 0.08 & 0.50 & 0.166 & 0.780 \\
\hline & Solid Layer & 54.8 & $8.83 E+05$ & $57 \%$ & 5493 & $6.71 \mathrm{E}-02$ & $2.55 \mathrm{E}-04$ & 0.00 & 0.02 & 3.17 & 0.23 & 0.14 & 0.02 & 0.50 & 0.184 & 1.092 \\
\hline \multirow[t]{2}{*}{8} & Supernatant & 344.3 & $4.07 \mathrm{E}+06$ & $100 \%$ & 6761 & 7.17E-05 & $5.19 \mathrm{E}-08$ & 0.01 & 0.52 & 1.45 & 0.23 & 0.08 & 0.08 & 0.50 & 0.173 & 0.789 \\
\hline & Solid Layer & 54.8 & $8.83 \mathrm{E}+05$ & $57 \%$ & 5493 & $6.71 \mathrm{E}-02$ & $2.55 \mathrm{E}-04$ & 0.00 & 0.02 & 3.17 & 0.23 & 0.14 & 0.02 & 0.50 & 0.184 & 1.092 \\
\hline
\end{tabular}




\section{RPP-5926 REV 6}

Table H1-C. Calculated Hydrogen Generation Unit Rate for 241-AY-102 Condensate Addition.

\begin{tabular}{|c|c|c|c|c|c|c|c|c|c|c|c|c|}
\hline Case & $\begin{array}{l}\text { Waste } \\
\text { layer }\end{array}$ & $\begin{array}{l}\text { Waste } \\
\text { level } \\
D_{w} \\
\text { (inch) }\end{array}$ & $\begin{array}{l}\text { HGR from } \\
\text { radiolysis } \\
\text { RC } \beta / \gamma_{\mathrm{rad}} \\
\text { (mole } / \mathrm{kg-d} \text { ) }\end{array}$ & $\begin{array}{l}\text { HGR from } \\
\text { radiolysis } \\
\text { RC }^{\alpha} \text { rad } \\
\text { (mole } / \mathrm{kg}-\mathrm{d} \text { ) }\end{array}$ & $\begin{array}{c}\text { HGR from } \\
\text { thermolysis } \\
\mathrm{RC}_{\text {therm }} \\
\text { (mole } / \mathrm{kg}-\mathrm{d} \text { ) }\end{array}$ & $\begin{array}{c}\text { HGR from } \\
\text { corrosion } \\
\text { RC }_{\text {corr }} \\
\text { (mole } / \mathrm{kg}-\mathrm{d})\end{array}$ & $\begin{array}{c}\text { Total HGR } \\
\text { from model } \\
\mathbf{R C}_{\text {tot }} \\
(\text { mole } / \mathbf{k g - d})\end{array}$ & $\begin{array}{l}\text { HGR from } \\
\text { radiolysis } \\
\text { RC } \beta / \gamma_{\text {rad }} \\
\left(\mathrm{mole} / \mathrm{m}^{3}-\mathbf{s}\right)\end{array}$ & $\begin{array}{c}\text { HGR from } \\
\text { radiolysis } \\
\mathbf{R C}^{\alpha} \\
\left(\mathbf{m o l e} / \mathbf{m}^{3}-s\right)\end{array}$ & $\begin{array}{l}\text { HGR from } \\
\text { thermolysis } \\
\mathbf{R C}_{\text {therm }} \\
\left(\mathrm{mole}^{3} \mathrm{~m}^{3}-\mathrm{s}\right)\end{array}$ & $\begin{array}{c}\text { HGR from } \\
\text { corrosion } \\
\text { RC }_{\text {carr }} \\
\left(\mathrm{mole}^{2} \mathrm{~m}^{3}-\mathrm{s}\right)\end{array}$ & $\begin{array}{c}\text { Total HGR } \\
\text { from mode } \\
\mathbf{R C}_{\mathrm{rol}} \\
\left(\mathrm{mole} / \mathrm{m}^{3}-\mathrm{s}\right)\end{array}$ \\
\hline \multirow[t]{2}{*}{1} & Supernatant & 259.0 & $1.10 \mathrm{E}-07$ & $4.34 \mathrm{E}-10$ & $3.08 \mathrm{E}-07$ & $8.01 \mathrm{E}-08$ & $4.99 \mathrm{E}-07$ & $1.50 \mathrm{E}-09$ & $5.91 \mathrm{E}-12$ & $4.20 \mathrm{E}-09$ & $1.09 \mathrm{E}-09$ & $6.80 \mathrm{E}-09$ \\
\hline & Solid Layer & 54.8 & $6.32 \mathrm{E}-05$ & $1.42 \mathrm{E}-06$ & $1.46 \mathrm{E}-06$ & 3. $11 \mathrm{E}-07$ & $6.64 \mathrm{E}-05$ & $1.13 \mathrm{E}-06$ & $2.54 \mathrm{E}-08$ & $2.61 \mathrm{E}-08$ & $5.58 \mathrm{E}-09$ & $1.19 \mathrm{E}-06$ \\
\hline \multirow[t]{2}{*}{2} & Supernatant & 261.3 & $1.10 \mathrm{E}-07$ & $4.32 \mathrm{E}-10$ & $3.08 \mathrm{E}-07$ & $8.02 \mathrm{E}-08$ & $4.99 \mathrm{E}-07$ & $1.50 \mathrm{E}-09$ & $5.88 \mathrm{E}-12$ & $4.19 \mathrm{E}-09$ & $1.09 \mathrm{E}-09$ & $6.79 \mathrm{E}-09$ \\
\hline & Solid Layer & 54.8 & $6.32 \mathrm{E}-05$ & $1.42 \mathrm{E}-06$ & $1.46 \mathrm{E}-06$ & $3.11 \mathrm{E}-07$ & $6.64 \mathrm{E}-05$ & $1.13 \mathrm{E}-06$ & $2.54 \mathrm{E}-08$ & $2.61 \mathrm{E}-08$ & $5.58 \mathrm{E}-09$ & $1.19 \mathrm{E}-06$ \\
\hline \multirow[t]{2}{*}{3} & Supernatant & 278.2 & $1.11 \mathrm{E}-07$ & $4.17 \mathrm{E}-10$ & $3.04 \mathrm{E}-07$ & $8.10 \mathrm{E}-08$ & $4.96 \mathrm{E}-07$ & $1.50 \mathrm{E}-09$ & $5.62 \mathrm{E}-12$ & $4.10 \mathrm{E}-09$ & $1.09 \mathrm{E}-09$ & $6.69 \mathrm{E}-09$ \\
\hline & Solid Layer & 54.8 & $6.32 \mathrm{E}-05$ & $1.42 \mathrm{E}-06$ & $1.46 \mathrm{E}-06$ & $3.11 \mathrm{E}-07$ & $6.64 \mathrm{E}-05$ & $1.13 \mathrm{E}-06$ & $2.54 \mathrm{E}-08$ & $2.61 \mathrm{E}-08$ & $5.58 \mathrm{E}-09$ & 1.19E-06 \\
\hline \multirow[t]{2}{*}{4} & Supernatant & 295.0 & I. $12 \mathrm{E}-07$ & $4.03 \mathrm{E}-10$ & $3.00 \mathrm{E}-07$ & $8.16 \mathrm{E}-08$ & $4.94 \mathrm{E}-07$ & $1.49 \mathrm{E}-09$ & $5.40 \mathrm{E}-12$ & 4.02E-09 & $1.09 \mathrm{E}-09$ & $6.61 \mathrm{E}-09$ \\
\hline & Solid Layer & 54.8 & $6.32 \mathrm{E}-05$ & $1.42 \mathrm{E}-06$ & $1.46 \mathrm{E}-06$ & $3.11 \mathrm{E}-07$ & $6.64 \mathrm{E}-05$ & $1.13 \mathrm{E}-06$ & $2.54 \mathrm{E}-08$ & $2.61 \mathrm{E}-08$ & $5.58 \mathrm{E}-09$ & $1.19 \mathrm{E}-06$ \\
\hline \multirow[t]{2}{*}{5} & Supernatant & 309.2 & $1.11 \mathrm{E}-07$ & $3.92 \mathrm{E}-10$ & $2.49 \mathrm{E}-07$ & $8.21 \mathrm{E}-08$ & $4.42 \mathrm{E}-07$ & $1.47 \mathrm{E}-09$ & $5.21 \mathrm{E}-12$ & $3.31 \mathrm{E}-09$ & $1.09 \mathrm{E}-09$ & $5.88 \mathrm{E}-09$ \\
\hline & Solid Layer & 54.8 & $6.32 \mathrm{E}-05$ & $1.42 \mathrm{E}-06$ & $1.46 \mathrm{E}-06$ & $3.11 \mathrm{E}-07$ & $6.64 \mathrm{E}-05$ & $1.13 \mathrm{E}-06$ & $2.54 \mathrm{E}-08$ & $2.61 \mathrm{E}-08$ & $5.58 \mathrm{E}-09$ & 1.19E-06 \\
\hline \multirow[t]{2}{*}{6} & Supernatant & 311.5 & $1.12 \mathrm{E}-07$ & $3.91 \mathrm{E}-10$ & $2.97 \mathrm{E}-07$ & $8.22 \mathrm{E}-08$ & $4.91 \mathrm{E}-07$ & $1.48 \mathrm{E}-09$ & $5.19 \mathrm{E}-12$ & $3.94 \mathrm{E}-09$ & $1.09 \mathrm{E}-09$ & $6.52 \mathrm{E}-09$ \\
\hline & Solid Layer & 54.8 & $6.32 \mathrm{E}-05$ & $1.42 \mathrm{E}-06$ & $1.46 \mathrm{E}-06$ & $3.11 \mathrm{E}-07$ & $6.64 \mathrm{E}-05$ & $1.13 \mathrm{E}-06$ & $2.54 \mathrm{E}-08$ & $2.61 \mathrm{E}-08$ & $5.58 \mathrm{E}-09$ & $1.19 \mathrm{E}-06$ \\
\hline \multirow[t]{2}{*}{7} & Supernatant & 328.0 & $1.12 \mathrm{E}-07$ & $3.79 \mathrm{E}-10$ & $2.93 \mathrm{E}-07$ & $8.27 \mathrm{E}-08$ & $4.88 \mathrm{E}-07$ & $1.47 \mathrm{E}-09$ & $5.00 \mathrm{E}-12$ & $3.87 \mathrm{E}-09$ & $1.09 \mathrm{E}-09$ & $6.44 \mathrm{E}-09$ \\
\hline & Solid Layer & 54.8 & $6.32 \mathrm{E}-05$ & $1.42 \mathrm{E}-06$ & $1.46 \mathrm{E}-06$ & $3.11 \mathrm{E}-07$ & $6.64 \mathrm{E}-05$ & $1.13 \mathrm{E}-06$ & $2.54 \mathrm{E}-08$ & $2.61 \mathrm{E}-08$ & $5.58 \mathrm{E}-09$ & 1.19E-06 \\
\hline \multirow[t]{2}{*}{8} & Supernatant & 344.3 & $1.11 \mathrm{E}-07$ & $3.67 \mathrm{E}-10$ & $2.90 \mathrm{E}-07$ & $8.32 \mathrm{E}-08$ & $4.85 \mathrm{E}-07$ & $1.46 \mathrm{E}-09$ & $4.82 \mathrm{E}-12$ & $3.80 \mathrm{E}-09$ & $1.09 \mathrm{E}-09$ & $6.36 \mathrm{E}-09$ \\
\hline & Solid Layer & 54.8 & $6.32 \mathrm{E}-05$ & $1.42 \mathrm{E}-06$ & $1.46 \mathrm{E}-06$ & $3.11 \mathrm{E}-07$ & $6.64 \mathrm{E}-05$ & $1.13 \mathrm{E}-06$ & $2.54 \mathrm{E}-08$ & $2.61 \mathrm{E}-08$ & $5.58 \mathrm{E}-09$ & 1.19E-06 \\
\hline
\end{tabular}


Table H1-D. Calculated Total Hydrogen Generation Rate In Dome Space for 241-AY-102 Condensate Addition.

\begin{tabular}{|c|c|c|c|c|c|c|c|c|c|c|c|c|}
\hline Case & $\begin{array}{l}\text { Waste } \\
\text { layer }\end{array}$ & $\begin{array}{c}\text { Waste } \\
\text { level } \\
D_{w} \\
\text { (inch) }\end{array}$ & $\begin{array}{c}\text { HGR } \\
\text { from } \\
\text { radiolysis } \\
\text { Grad }_{\text {rar }} \gamma \\
(\mathrm{cfm})\end{array}$ & $\begin{array}{c}\text { HGR from } \\
\text { radiolysis } \\
G_{\text {rad }}{ }^{2} \\
(\mathbf{c f m})\end{array}$ & $\begin{array}{c}\text { HGR from } \\
\text { thermolysis } \\
\mathbf{G}_{\text {therm }} \\
(\mathbf{c f m})\end{array}$ & $\begin{array}{l}\text { HGR from } \\
\text { corrosion } \\
G_{\text {corr }} \\
(\mathrm{cfm})\end{array}$ & $\begin{array}{l}\text { Percent of } \\
\text { HGR from } \\
\text { radiolysis } \\
\left(\mathrm{G}_{\mathrm{rad}} \beta / \gamma\right)_{\%}\end{array}$ & $\begin{array}{l}\text { Percent of } \\
\text { HGR from } \\
\text { radiolysis } \\
\left(G_{\text {rad }}^{\text {alpha }}\right)_{\%}\end{array}$ & $\begin{array}{c}\text { Percent of } \\
\text { HGR from } \\
\text { thermolysis } \\
\left(G_{\text {therm }}\right)_{\%}\end{array}$ & $\begin{array}{l}\text { Percent of } \\
\text { HGR from } \\
\text { corrosion } \\
\left(\mathrm{G}_{\text {corr }}\right)_{\%}\end{array}$ & $\begin{array}{c}\text { Total HGR } \\
\text { from model } \\
\mathbf{G}_{\text {mod }} \\
(\mathrm{cfm})\end{array}$ & $\begin{array}{c}\text { Total HGR } \\
\text { from model } \\
\mathrm{G}_{\text {mod }} \\
(\mathrm{L} / \mathrm{day})\end{array}$ \\
\hline \multirow[t]{2}{*}{1} & Supernatant & 259.0 & $2.27 \mathrm{E}-04$ & $8.93 \mathrm{E}-07$ & $6.34 \mathrm{E}-04$ & $1.65 \mathrm{E}-04$ & $22.1 \%$ & $8.70 \mathrm{E}-04$ & $61.8 \%$ & $16.1 \%$ & $1.03 \mathrm{E}-03$ & 42 \\
\hline & Solid Layer & 54.8 & $3.61 \mathrm{E}-02$ & $8.12 \mathrm{E}-04$ & $8.34 \mathrm{E}-04$ & $1.78 \mathrm{E}-04$ & $95.2 \%$ & $2.14 \mathrm{E}-02$ & $2.2 \%$ & $0.5 \%$ & $3.80 \mathrm{E}-02$ & 1548 \\
\hline \multirow[t]{2}{*}{2} & Supernatant & 261.3 & $2.29 \mathrm{E}-04$ & $8.95 \mathrm{E}-07$ & $6.38 \mathrm{E}-04$ & $1.66 \mathrm{E}-04$ & $22.1 \%$ & $8.66 \mathrm{E}-04$ & $61.7 \%$ & $16.1 \%$ & $1.03 \mathrm{E}-03$ & 42 \\
\hline & Solid Layer & 54.8 & $3.61 \mathrm{E}-02$ & $8.12 \mathrm{E}-04$ & $8.34 \mathrm{E}-04$ & $1.78 \mathrm{E}-04$ & $95.2 \%$ & $2.14 \mathrm{E}-02$ & $2.2 \%$ & $0.5 \%$ & $3.80 \mathrm{E}-02$ & 1548 \\
\hline \multirow[t]{2}{*}{3} & Supernatant & 278.2 & $2.43 \mathrm{E}-04$ & $9.13 \mathrm{E}-07$ & $6.65 \mathrm{E}-04$ & $1.77 \mathrm{E}-04$ & $22.4 \%$ & $8.40 \mathrm{E}-04$ & $61.2 \%$ & $16.3 \%$ & $1.09 \mathrm{E}-03$ & 44 \\
\hline & Solid Layer & 54.8 & $3.61 \mathrm{E}-02$ & $8.12 \mathrm{E}-04$ & $8.34 \mathrm{E}-04$ & $1.78 \mathrm{E}-04$ & $95.2 \%$ & $2.14 \mathrm{E}-02$ & $2.2 \%$ & $0.5 \%$ & $3.80 \mathrm{E}-02$ & 1548 \\
\hline \multirow[t]{2}{*}{4} & Supernatant & 295.0 & $2.57 \mathrm{E}-04$ & $9.28 \mathrm{E}-07$ & $6.91 \mathrm{E}-04$ & $1.88 \mathrm{E}-04$ & $22.6 \%$ & $8.17 \mathrm{E}-04$ & $60.8 \%$ & $16.5 \%$ & $1.14 \mathrm{E}-03$ & 46 \\
\hline & Solid Layer & 54.8 & $3.61 \mathrm{E}-02$ & $8.12 \mathrm{E}-04$ & $8.34 \mathrm{E}-04$ & $1.78 \mathrm{E}-04$ & $95.2 \%$ & $2.14 \mathrm{E}-02$ & $2.2 \%$ & $0.5 \%$ & $3.80 \mathrm{E}-02$ & 1548 \\
\hline \multirow[t]{2}{*}{5} & Supernatant & 309.2 & $2.66 \mathrm{E}-04$ & $9.40 \mathrm{E}-07$ & $5.97 \mathrm{E}-04$ & $1.97 \mathrm{E}-04$ & $25.1 \%$ & $8.86 \mathrm{E}-04$ & $56.3 \%$ & $18.6 \%$ & $1.06 \mathrm{E}-03$ & 43 \\
\hline & Solid Layer & 54.8 & $3.61 \mathrm{E}-02$ & $8.12 \mathrm{E}-04$ & 8.34E-04 & $1.78 \mathrm{E}-04$ & $95.2 \%$ & $2.14 \mathrm{E}-02$ & $2.2 \%$ & $0.5 \%$ & $3.80 \mathrm{E}-02$ & 1548 \\
\hline \multirow[t]{2}{*}{6} & Supernatant & 311.5 & $2.70 \mathrm{E}-04$ & $9.42 \mathrm{E}-07$ & $7.16 \mathrm{E}-04$ & $1.98 \mathrm{E}-04$ & $22.7 \%$ & $7.95 \mathrm{E}-04$ & $60.4 \%$ & $16.7 \%$ & $1.18 \mathrm{E}-03$ & 48 \\
\hline & Solid Layer & 54.8 & $3.61 \mathrm{E}-02$ & $8.12 \mathrm{E}-04$ & $8.34 \mathrm{E}-04$ & $1.78 \mathrm{E}-04$ & $95.2 \%$ & $2.14 \mathrm{E}-02$ & $2.2 \%$ & $0.5 \%$ & $3.80 \mathrm{E}-02$ & 1548 \\
\hline \multirow[t]{2}{*}{7} & Supernatant & 328.0 & $2.82 \mathrm{E}-04$ & $9.55 \mathrm{E}-07$ & $7.40 \mathrm{E}-04$ & $2.09 \mathrm{E}-04$ & $22.9 \%$ & $7.76 \mathrm{E}-04$ & $60.1 \%$ & $17.0 \%$ & $1.23 \mathrm{E}-03$ & 50 \\
\hline & Solid Layer & 54.8 & $3.61 \mathrm{E}-02$ & $8.12 \mathrm{E}-04$ & $8.34 \mathrm{E}-04$ & I. $78 \mathrm{E}-04$ & $95.2 \%$ & 2.14E-02 & $2.2 \%$ & $0.5 \%$ & $3.80 \mathrm{E}-02$ & 1548 \\
\hline \multirow[t]{2}{*}{8} & Supernatant & 344.3 & $2.93 \mathrm{E}-04$ & $9.68 \mathrm{E}-07$ & $7.64 \mathrm{E}-04$ & $2.19 \mathrm{E}-04$ & $22.9 \%$ & $7.58 \mathrm{E}-04$ & $59.8 \%$ & $17.2 \%$ & $1.28 \mathrm{E}-03$ & 52 \\
\hline & Solid Layer & 54.8 & $3.61 \mathrm{E}-02$ & 8.12E-04 & $8.34 \mathrm{E}-04$ & $1.78 \mathrm{E}-04$ & $95.2 \%$ & $2.14 \mathrm{E}-02$ & $2.2 \%$ & $0.5 \%$ & $3.80 \mathrm{E}-02$ & 1548 \\
\hline
\end{tabular}


RPP-5926 REV 6

Table H2-A. Steady-State Gas Concentrations, Release Rates and Flammability Levels Under Normal Ventilation Conditions.

\begin{tabular}{|c|c|c|c|c|c|c|c|c|c|c|c|}
\hline Case & $\begin{array}{c}\text { Ammonia } \\
\text { headspace } \\
\text { concen. } \\
{\left[\mathrm{NH}_{3}\right]} \\
(\%)\end{array}$ & $\begin{array}{c}\text { Methane } \\
\text { headspace } \\
\text { concen. } \\
{\left[\mathrm{CH}_{4}\right]} \\
(\%)\end{array}$ & $\begin{array}{c}\mathrm{N}_{2} \mathrm{O} \\
\text { headspace } \\
\text { concen. } \\
{\left[\mathrm{N}_{2} \mathrm{O}\right]} \\
(\%)\end{array}$ & $\begin{array}{c}\text { Waste } \\
\text { volume } \\
V_{w} \\
\text { (kgal) }\end{array}$ & $\begin{array}{c}\text { Headspace } \\
\text { volume } \\
V_{\mathbf{h}} \\
\left(\mathbf{f t}^{\mathbf{3}}\right)\end{array}$ & $\begin{array}{c}\text { Normal } \\
\text { ventilation } \\
\text { rate } \\
(\mathbf{V r})_{\text {norn }} \\
(\mathbf{c f m})\end{array}$ & $\begin{array}{l}\text { Steady-state } \\
\text { flammability } \\
\text { at normal } \\
\text { condition } \\
\text { (\% LFL) }\end{array}$ & $\begin{array}{l}\text { Ammonia } \\
\text { release } \\
\text { rate } \\
\mathrm{Rr}_{\left(\mathrm{NH}_{3}\right)} \\
(\mathrm{cfm})\end{array}$ & $\begin{array}{c}\mathrm{N}_{2} \mathrm{O} \\
\text { release } \\
\text { rate } \\
\mathrm{Rr}_{\left(\mathrm{N}_{2} \mathrm{O}\right)} \\
(\mathrm{cfm})\end{array}$ & $\begin{array}{c}\text { Model } \\
\text { hydrogen } \\
\text { release } \\
\text { rate } \\
\operatorname{Rr}\left(\mathrm{H}_{2}\right) \\
(\mathrm{cfm})\end{array}$ & $\begin{array}{c}\text { Methane } \\
\text { release } \\
\text { rate } \\
\operatorname{Rr}\left(\mathrm{CH}_{4}\right) \\
(\mathrm{cfm})\end{array}$ \\
\hline 1 & 0.0004 & 0.0001 & 0.0010 & 864 & 72,584 & 250 & $0.40 \%$ & $1.07 \mathrm{E}-03$ & $2.50 \mathrm{E}-03$ & $3.90 \mathrm{E}-02$ & $3.61 \mathrm{E}-04$ \\
\hline 3 & 0.0004 & 0.0001 & 0.0010 & 917 & 65,500 & 250 & $0.40 \%$ & $1.07 \mathrm{E}-03$ & $2.50 \mathrm{E}-03$ & $3.91 \mathrm{E}-02$ & $3.61 \mathrm{E}-04$ \\
\hline 4 & 0.0004 & 0.0001 & 0.0010 & 963 & 59,337 & 250 & $0.40 \%$ & $1.07 \mathrm{E}-03$ & $2.50 \mathrm{E}-03$ & $3.91 \mathrm{E}-02$ & $3.61 \mathrm{E}-04$ \\
\hline 5 & 0.0004 & 0.0001 & 0.0010 & 1,002 & 54,084 & 250 & $0.40 \%$ & $1.07 \mathrm{E}-03$ & $2.50 \mathrm{E}-03$ & $3.90 \mathrm{E}-02$ & $3.61 \mathrm{E}-04$ \\
\hline 6 & 0.0004 & 0.0001 & 0.0010 & 1,009 & 53,242 & 250 & $0.40 \%$ & $1.07 \mathrm{E}-03$ & $2.50 \mathrm{E}-03$ & $3.92 \mathrm{E}-02$ & $3.61 \mathrm{E} \cdot 04$ \\
\hline 8 & 0.0004 & 0.0001 & 0.0010 & 1,099 & 41,171 & 250 & $0.40 \%$ & $1.07 \mathrm{E}-03$ & $2.50 \mathrm{E}-03$ & $3.93 \mathrm{E}-02$ & $3.61 \mathrm{E}-04$ \\
\hline
\end{tabular}


RPP-5926 REV 6

Table H2-B. Steady-State Flammability Under Barometric Breathing and Time to $25 \%$ of the LFL under Zero Vent.

\begin{tabular}{|c|c|c|c|c|c|c|c|c|c|c|c|}
\hline Case & $\begin{array}{c}\text { Off-Normal } \\
\text { Barometric } \\
\text { Breathing } \\
\text { Rate }(\mathrm{Vr})_{\mathrm{bb}} \\
(\mathrm{cfm})\end{array}$ & $\begin{array}{c}\text { Steady } \\
\text { State } \\
\text { Hydrogen } \\
\text { Concen. } \\
{\left[\mathrm{H}_{2} \mid(\%)\right.}\end{array}$ & $\begin{array}{c}\text { Steady } \\
\text { State } \\
\text { Ammonia } \\
\text { Concen. } \\
{\left[\mathrm{NH}_{3}\right]} \\
(\%)\end{array}$ & $\begin{array}{c}\text { Steady } \\
\text { State } \\
\text { Methane } \\
\text { Concen. } \\
{\left[\mathrm{CH}_{\mathbf{4}} \mid\right.} \\
(\%)\end{array}$ & $\begin{array}{c}\text { Steady } \\
\text { State } \\
\mathrm{N}_{2} \mathrm{O} \\
\text { Concen. } \\
{\left[\mathrm{N}_{2} \mathrm{O}\right]} \\
(\%)\end{array}$ & $\begin{array}{c}\text { Steady State } \\
\text { Total } \\
\text { Flamma-bility } \\
(\%)\end{array}$ & $\begin{array}{c}\text { Time to } \\
\text { Reach } \\
25 \% \\
\text { LFL } \\
\text { (days) }\end{array}$ & $\begin{array}{c}\text { Time to } \\
\text { Reach } \\
100 \% \\
\text { LFL } \\
\text { (days) }\end{array}$ & $\begin{array}{l}\text { Minimum } \\
\text { Vent Rate } \\
\text { to Keep } \\
\text { below } \\
25 \% \text { LFL } \\
\text { (cfm) }\end{array}$ & $\begin{array}{c}\text { Minimum } \\
\text { Vent Rate } \\
\text { to Keep } \\
\text { below } \\
100 \% \text { LFL } \\
\text { (cfm) }\end{array}$ & $\begin{array}{l}\text { Waste } \\
\text { level } \\
\text { D }_{w} \\
\text { (inch) }\end{array}$ \\
\hline 1 & 0.23 & 17.19 & 0.02 & 0.16 & 1.10 & 433.2 & 12 & 43 & 3.94 & 0.98 & 313.8 \\
\hline 2 & 0.22 & 17.40 & 0.02 & 0.16 & 1.12 & 438.3 & 11 & 43 & 3.94 & 0.98 & 316.0 \\
\hline 3 & 0.20 & 19.08 & 0.02 & 0.18 & 1.22 & 480.7 & 10 & 40 & 3.95 & 0.98 & 333.0 \\
\hline 4 & 0.19 & 21.09 & 0.02 & 0.19 & 1.35 & 531.3 & 9 & 37 & 3.95 & 0.99 & 349.7 \\
\hline 5 & 0.17 & 23.10 & 0.02 & 0.21 & 1.48 & 577.4 & 9 & 38 & 3.95 & 0.99 & 364.0 \\
\hline 6 & 0.17 & 23.54 & 0.02 & 0.22 & 1.50 & 592.9 & 8 & 33 & 3.96 & 0.99 & 366.3 \\
\hline 7 & 0.15 & 26.59 & 0.02 & 0.24 & 1.70 & 669.7 & 7 & 30 & 3.96 & 0.99 & 382.7 \\
\hline 8 & 0.13 & 30.51 & 0.02 & 0.28 & 1.94 & 768.4 & 6 & 26 & 3.97 & 0.99 & 399.1 \\
\hline
\end{tabular}


RPP-5926 REV 6

APPENDIX I

PEER REVIEW CHECKLIST 
RPP-5926 REV 6

This page intentionally left blank. 


\section{Calculation Review Checklist}

Calculation Reviewed: __ RPP-5926 Rev. 6

Scope of Review: Main document (excluding equation derivation) and input data (Appendices A and B) (e.g., document section or portion of calculation)

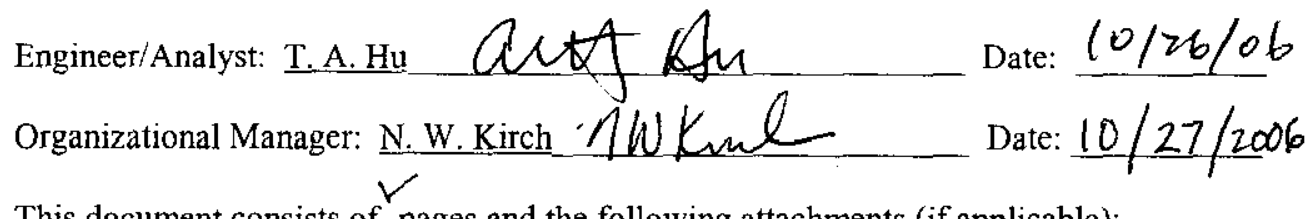

This document consists of pages and the following attachments (if applicable): 321

Yes No NA*

[X] [ ] [ ] 1. Analytical and technical approaches and results are reasonable and appropriate.

[X] [ ] [ ] 2. Necessary assumptions are reasonable, explicitly stated, and supported.

[X] [ ] [ ] 3. Ensure calculations that use software include a paper printout, microfiche, CD ROM, or other electronic file of the input data and identification to the computer codes and versions used, or provide alternate documentation to uniquely and clearly identify the exact coding and execution process.

[X] [ ] [ ] 4. Input data were checked for consistency with original source information.

[] [ ] [X] 5. Key input data (e.g., dimensions, performance characteristics) that may affect equipment design is identified.

[] [] [X] 6. For both qualitative and quantitative data, uncertainties are recognized and discussed and the data is presented in a manner to minimize design interpretations.

[ ] [ ] [X] 7. Mathematical derivations were checked, including dimensional consistency of results.

[X] [ ] [ ] 8. Calculations are sufficiently detailed such that a technically qualified person can understand the analysis without requiring outside information.

[X] [ ] [ ] 9. Software verification and validation are addressed adequately.

[X] [ ] [ ] 10. Limits/criteria/guidelines applied to the analysis results are appropriate and referenced. Limits/criteria/guidelines were checked against references.

[X] [ ] [ ] 11. Conclusions are consistent with analytical results and applicable limits.

[X] [] [] 12. Results and conclusions address all points in the purpose.

[ ] [X] [] 13. Referenced documents are retrievable or otherwise available.

[X] [ ] [ ] 14. The version or revision of each reference is cited as appropriate.

[X] [] [] 15. The document was prepared in accordance with TFC-ENG-DESIGN-C-10, Attachment A, "Calculation Format and Preparation Instructions."

[ ] [ ] [X] 16. Impacts on requirements have been assessed and change documentation initiated to incorporate revisions to affected documents, as appropriate.

[X] [ ] [ ] 17. All checker comments have been dispositioned and the design media matches the calculations.

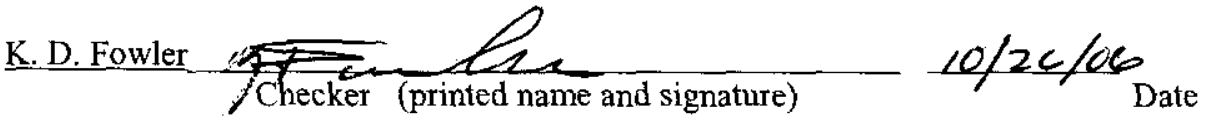

- If No or NA is chosen, an explanation must be provided on or attached to this form. 


\section{Calculation Review Checklist (cont'd)}

\begin{tabular}{|l|l|}
\hline Item & Disposition \\
\hline 5,6 & $\begin{array}{l}\text { This evaluation is based on data for waste stored in the Hanford Tank Farms. It } \\
\text { is not intended for equipment design. Best Basis Inventory data is used where } \\
\text { available as reported as representative of the tank waste. }\end{array}$ \\
\hline 7 & $\begin{array}{l}\text { Mathematical derivations were not included in the scope of this review; however, } \\
\text { references to the documented formula derivations are included in the document. }\end{array}$ \\
\hline 13 & $\begin{array}{l}\text { One reference, TFC-ENG-CHEM-D-32, has been superseded. The Reference } \\
\text { section cites the reference that was actually used and includes a footnote } \\
\text { indicating that the document has been replaced and the document number and } \\
\text { title of the new engineering procedure. }\end{array}$ \\
\hline 16 & $\begin{array}{l}\text { This document will be used as a supporting technical evaluation to aid in the } \\
\text { development of requirements for the DBVS facility. }\end{array}$ \\
\hline
\end{tabular}




\section{CHECKLIST FOR TECHNICAL PEER REVIEW}

Documcnt Revicwed: RPP-5926, Steady State Flammable Gas Release Rate Calculation and Lower Flammability Evaluation for Hanford Tank Waste, Rev. 6

Scopc of Review (c.g., document section or portion of calculation): Technical edit

Yes No NA*

[ ] [] [x] 1. Previous reviews are complete and cover the analysis, up to the scope of this review, with no gaps.

[] [ ] [x] 2. Problem is completely defined.

[ ] [ ] [x] 3. Accident scenarios are developed in a clear and logical manner.

[ ] [ ] [x] 4. Analytical and technical approaches and results are reasonable and appropriate. (ORP QAPP criterion 2.8)

[ ] [ ] [x] 5. Necessary assumptions are reasonable, explicitly stated, and supported. (ORP QAPP criterion 2.2)

[ ] [ ] $[\mathrm{x}]$ 6. Computer codes and data files are documented.

[] [ ] [x] 7. Data used in calculations are explicitly stated.

[ ] [ ] [x] 8. Bases for calculations, including assumptions and data, are consistent with the supported safety basis document (e.g., the Tank Fams Documented Safety Analysis).

[ ] [ ] [x] 9. Data were checked for consistency with original source information as applicable. (ORP QAPP criterion 2.9)

[] [] [x] 10. For both qualitative and quantitative data, uncertainties are recognized and discussed, as appropriate. (ORP QAPP criterion 2.17)

[] [] [x] 11. Mathematical derivations were checked including dimensional consistency of results. (ORP QAPP criterion 2.16)

[ ] [ ] [x] 12. Models are appropriate and were used within their established range of validity or adequate justification was provided for use outside their established range of validity.

[ ] [ ] [x] 13. Spreadsheet results and all hand calculations were verified.

[ ] [ ] [x] 14. Calculations are sufficiently detailed such that a technically qualified person can understand the analysis without requiring outside information. $(O R P$ QAPP criterion 2.5)

[ ] [ ] [x] 15. Software input is correct and consistent with the document reviewed.

[ ] [ ] [x] 16. Software output is consistent with the inpul and with the results reported in the document reviewed.

[ ] [ ] [x] 17. Software verification and validation are addressed adequately. (ORP QAPP criterion 2.6)

[ ] [ ] [x] 18. Limits/criteria/guidelines applied to the analysis results are appropriate and referenced. Limits/criteria/guidelines were checked against references. (ORP QAPP criterion 2.9)

[ ] [ ] $[\mathrm{x}]$ 19. Safety margins are consistent with good engineering practices.

[] [ ] [x] 20. Conclusions are consistent with analytical results and applicable limits.

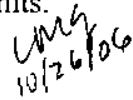




\section{RPP-5926 REV 6}

\section{CHECKLIST FOR TECHNICAL PEER REVIEW}

[ ] [ ] [x] 21. Results and conclusions address all points in the purpose. (ORP QAPP criterion 2.3)

[x] [ ] [ ] 22. All references cited in the text, figures, and tables arc containcd in the reference list.

[x] [ ] [ ] 23. Reference citations (c.g., title and number) are consistent between the text callout and the refcrence list.

[x] [ ] [ ] 24. Only releascd (i.e., not draft) references arc cited. (ORP QAPP criterion 2.1)

[x] [ ] [ ] 25. Referenced documents are retrievable or otherwise available.

[x] [ ] [] 26. The most recent version of each reference is cited, as appropriate. (ORP QAPP criterion 2.1)

[x] [ ] [ ] 27. There are no duplicate citations in the reference list.

[x] [ ] [ ] 28. Referenced documents are spelled out (title and number) the first time they are cited.

[x] [ ] [ ] 29. All acronyms are spclled out the first time they are used.

[x] [] [ ] 30. The Table of Contents is correct.

[x] [ ] [] 31. All figure, table, and section callouts are correct.

[x] [] [ ] 32. Unit conversions are correct and consistent.

[x] [] [] 33. The number of significant digits is appropriatc and consistent.

[ ] [ ] [x] 34. Chemical reactions are correct and balanced.

[x] [ ] [ ] 35. All tables are formatted consistently and are free of blank cells.

[x] [ ] [ ] 36. The document is complete (pages, attachments, and appendices) and in the proper order.

[x] [ ] [ ] 37. The document is free of typographical errors

[x] [ ] [] 38. The tables are intemally consistent.

[x] [ ] [ ] 39. The document was prepared in accordance with HNF-2353, Section 4.3, Attachment B, "Calculation Note Format and Preparation Instructions".

[ ] [ ] [x] 40. Impacted documents are appropriately identified in Blocks 7 and 25 of the Engineering Change Notice (form A-6003-563.1).

[x] [ ] [ ] 41. If more than one Technical Peer Reviewer was designated for this document, an overall review of the entire document was performed after resolution of all Technical Peer Revicw comments and confirmed that the document is selfconsistent and complete.

[x] [] [] Concurrence

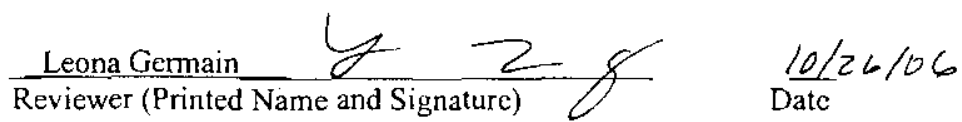

* If No or NA is chosen, provide an explanation on this form.

Technical Edit 